

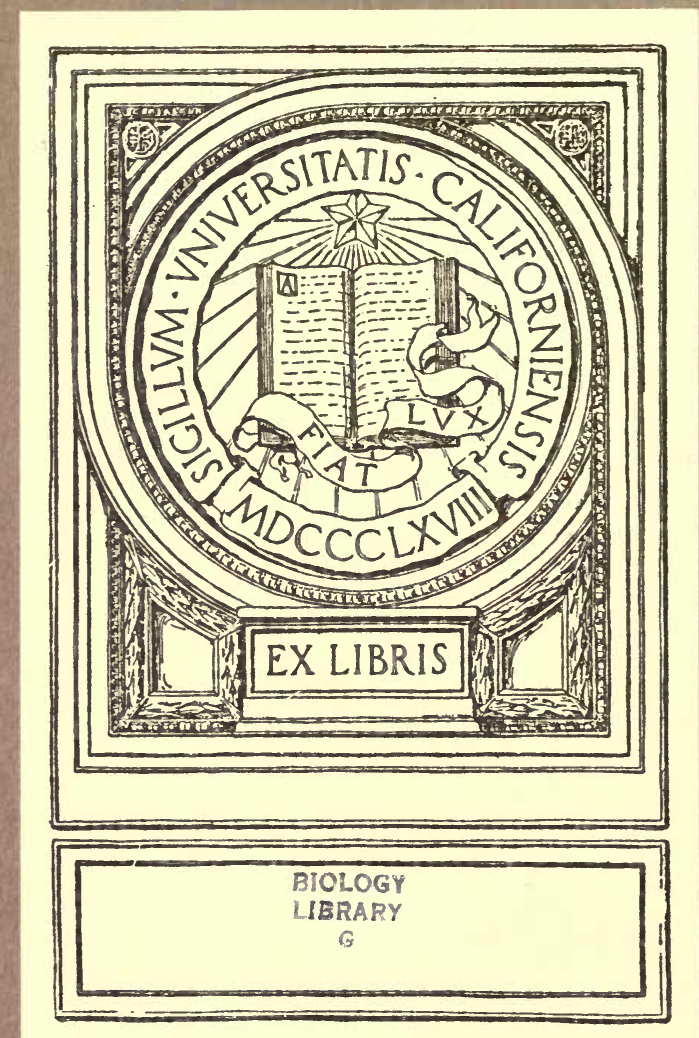




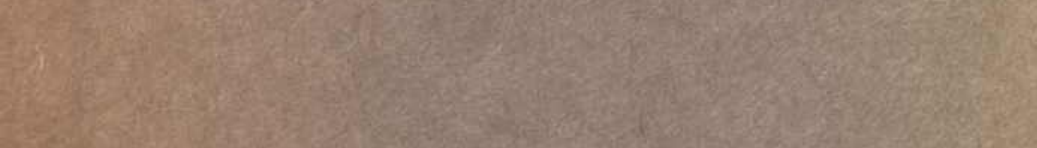

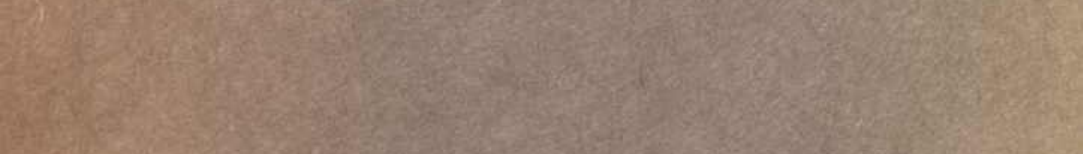

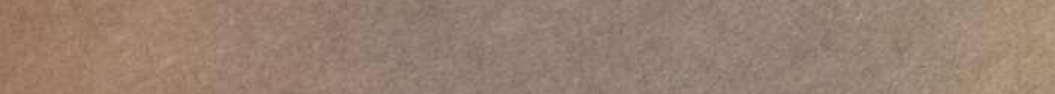

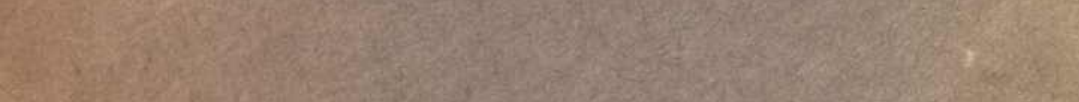
- 



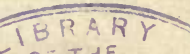

UNIVERSITY

OF

CALIFORNIA 
BLOUD-SPECTRA CDMPARED WITH SPECTRUM DF ARGAND- LAMP.

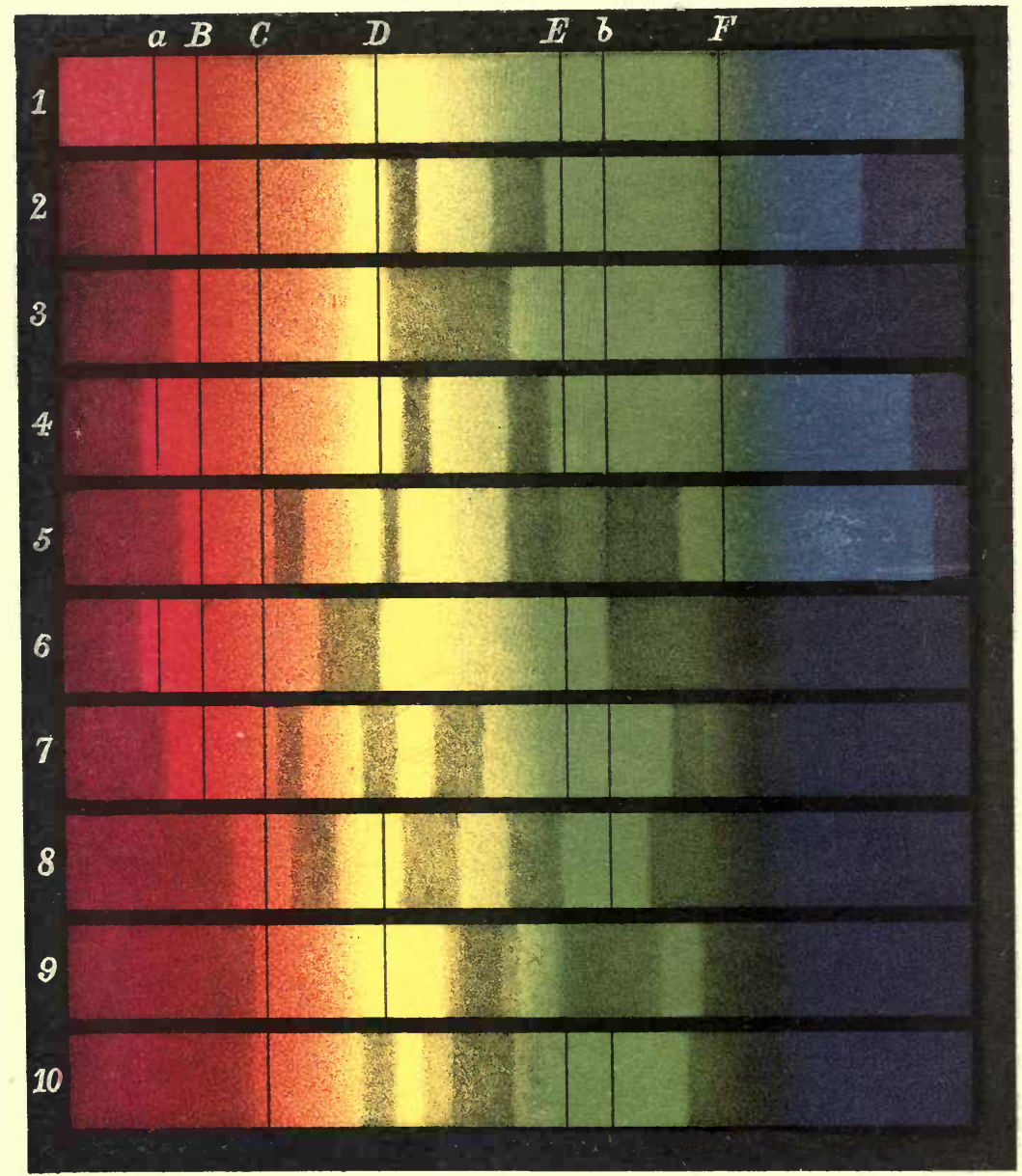

1 Spectrum of Ardand-lamp with Fraunhofers lines in position.

2 5pectrum of Qxyhzmaglabin in diluted blad.

:3 Spectrum of Reduced $\mathrm{H} \boxplus$ maglabin.

4. 5 pectrum of Carbonic axide Hamaglabin.

5 Spectrum of Acid $\mathrm{Hzmatin}$ in etherial solutian.

6 spectrum of Alkaline $\mathrm{H} \nexists$ matin.

7 spectrum of Chlarafarm extract af acidulated Dx-Bile.

8 spectrum of Methemaglabin.

9 spectrum oF Hæmachromagen.

IO 5 pectrum of Himatoparphyrin.

.Yost of the above Spectra have been drawn from observations by MT W.Lepmik F.C.S: 


\section{KIRKES -HANDBOOK OF PHYSIOLOGY}

\section{HANDBOOK}

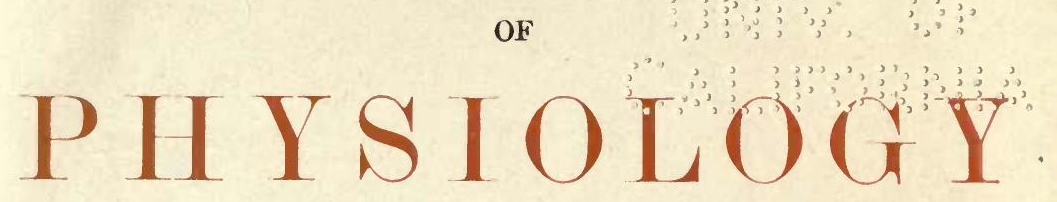

REVISED BY

WILLIAM H. ROCKWELL, JR., M.D.

AND

CHARLES L. DANA, A.M., M.D.

Professor of Diseases of the Nervous System, Cornell University Medical College, New York ; Physician to Bellevue Hospital ; Neurologist to the Montefiore Home.

\section{seventeenth Emerican Edition}

WITH UPWARDS OF FIVE HUNDRED ILLUSTRATIONS INCLUDING MANY IN COLORS

NEW YORK 


\section{GENERAL}

COPYRIGHT, 190\%,

By WILIIAM WOOD AND COMPANY.

$$
103701
$$

THE PUBLISHERS' PRINTING COMPANY 32-34 LAFAYETTE PLACE 


\section{PREFATORY NOTE.}

IN this edition the chief changes will be found in the articles relating to physiological chemistry, the revision having been deemed necessary because of recent advances in this branch of physiology. Chapters IV., IX., and XI. are those most affected, while minor alterations have been made elsewhere as required.

A few changes have also been made in the chapter on the Blood, in conformance with the more prominent of the present views on the subject.

In many instances euts have been replaced by others which illustrate the text more clearly.

W. H. ROCKWELL, JR.

August, 1902. 



\section{CONTENTS.}

CHAPTER I.

The Phenomena of Life,

Properties of Protoplasm,

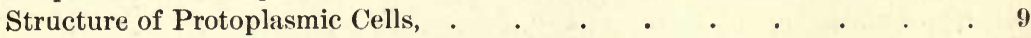

The Cell Nucleus,

Attraction Sphere,

Cell Division,

Plants Compared with Animals,

CHAPTER II.

The Functions of Organized Cells,

CHAPTER III.

The Structure of the Elementaky Tissues,

Epithelial Tissues,

Connective Tissues,

The Teeth,

Development of the Teeth,

Muscular Tissues,

Nervous Tissues,

CHAPTER IV.

The Chemical Composition of the Body, Organic Substances,

Inorganic Substances,

TIIE BLOOD, .

Coagulation, .

The Corpuscles,

Chemical Composition,

Gases of the Blood,

Development of the Corpuscles,

CHAPTER VI.

The Crrculation of the Blood,

The Heart,

The Arteries, .

The Capillaries, 
The Circulation of The Blood (Continued).

The Veins,

The Action of the Heart,

The Sounds of the Heart.

The Impulse of the Heart,

Endocardiac Pressure,

Frequency of Heart's Action, . $\quad . \quad$. $\quad . \quad$. $\quad . \quad$. . . . 203

Blood Pressure,

The Arterial Flow,

The Pulse,

The Capillary Flow,

The Venous Flow,

The Velocity of the Flow,

Local Peculiarities of the Circulation, . . . . . . . . . . . . 228

Regulation of the Flow,

Proofs of the Circulation of the Blood,

CHAPTER VII.

Respiration,

The Respiratory Apparatus,

The Respiratory Mechanism,

Respiratory Changes,

Special Respiratory Acts,

Nervous Mechanism,

Effect on Circulation,

Asphyxia,

CHAPTER VIII.

Secretion,

Organs and Tissues of Secretion,

Secreting Glands,

The Mammary Glands,

Milk,

The Ductless Glands and Internal Secretions,

CHAPTER IX.

Food And Digestion,

Organic Nitrogenous Foods,

Organic Non-Nitrogenous Foods, .

Mineral Foods,

Liquid Foods,

Enzymies,

The Mouth and Mastication,

The Salivary Glands,

Saliva,

The Tongue, .

The Pharynx,

The Esophagus,

Deglutition, 
Food AND Drgestion (Continued).

PAGE

The Stomach,

The Gastric Juice,

Vomiting,

The Intestines,

The Pancreas,

The Liver,

The Intestinal Secretion,

Digestion in the Small Intestine,

Digestion in the Large Intestine,

Micro-organisms in the Intestine,

Movements of the Intestines,

The Frces,

Gases of the Alimentary Canal,

CHAPTER X.

Absorption,

Methods,

The Lymphatic System,

The Lymph Flow,

The Lymph and Chyle,

Channels of Absorption,

Where Absorption May Take Place,

CHAPTER XI.

Metabolism, Nuthition, and Diet,

Requisites of Normal Diet,

Variations in Diet Tables,

Income and Output of Energy,

CHAPTER XII.

Animat, Heat,

Production of Heat,

Regulation of Body Temperature,

CHAPTER XIII.

Excretion,

The Kidneys,

The Urine,

Method of Excretion of the Urine,

The Passage of Urine into the Bladder, . . . . . . . 488

The Skin,

Functions of the Skin,

CHAPTER XIV.

Muscle-Nerve Pirsiology,

Chemical Composition of Muscle, . . . . . . . . . . 500

Muscle at Rest, 
Muscle-Nerve Physiology (Continued).

Muscle in Activity,

Accompaniments of Muscular Contraction,

Muscle in Rigor Mortis,

Action of Voluntary Muscles,

Action of Involuntary Muscles,

Electrical Currents in Nerves,

Battery Currents and Human Nerves, . . . . . . . . . 531

Muscular and Nervous Metabolism,

CHAPTER XV.

The Production of the Voice,

The Larynx,

Movements of the Vocal Cords,

The Voice in Singing and Speaking,

CHAPTER XVI.

The Nervous System,

Functions of Nerve Fibres,

Functions of Nerve-Centres

The Spinal Cord and its Nerves, . . . . . . . . . . . . . . . . . . . . . . .

Functions of the Spinal Nerve-Roots, . . . . . . . . . . . 571

Functions of the Spinal Cord,

Relations of the Different Parts of the Brain,

The Bulb,

Functions of the Bulb,

The Cranial Nerves.

The Pons Varolii,

The Crura Cerebri,

Corpora Striata,

Optic Thalami,

Corpora Quadrigemina,

The Cerebrum,

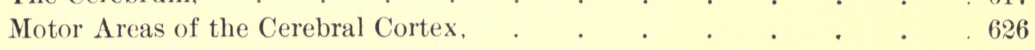

Functions of the Cerebrum,

Sensory Centres,

The Cerebellum,

Functions of the Cerebellum,

The Sympathetic System,

The Senses,

The Sense of Touch,

The Sense of Taste,

The Sense of Smell,

The Sense of Hearing,

The Sense of Sight, 


\section{CHAPTER XVHI.}

The Reproducture Organs, . . . . . . . . . . 744

Of the Female, . . . . . . . . . . . . . . . . . . . 744

Of the Male, . . . . . . . . . . . . 750

Physiology of the Sexual Organs, . . . . . . . . 757

\section{CHAPTER XIX.}

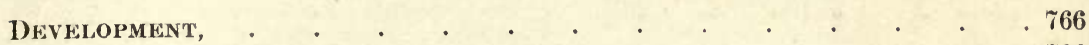

The Changes in the Ovum, . . . . . . . . . . . . 766

The Fotal Membranes, . . . . . . . . . . . . 779

The Development of the Organs, . . . . . . . . . . . 788

APPENDIX, . . . . . . . . . . . . 825

INDEX, . . . . . . . . . . . . . . 839 



\section{FAHRENHEIT \\ and. \\ CENTIGRADE SCATES.}

\begin{tabular}{|c|c|}
\hline $\begin{array}{l}\text { F. } \\
500^{\circ} \\
401 \\
392 \\
383 \\
374 \\
356 \\
347 \\
338 \\
329 \\
320 \\
311 \\
302 \\
284 \\
275 \\
266 \\
248 \\
239 \\
230 \\
212 \\
203 \\
194 \\
176 \\
167 \\
140 \\
122 \\
113 \\
105 \\
104 \\
100\end{array}$ & $\begin{array}{l}\text { C. } \\
260^{\circ} \\
205 \\
200 \\
195 \\
190 \\
180 \\
175 \\
170 \\
165 \\
160 \\
155 \\
150 \\
140 \\
135 \\
130 \\
120 \\
115 \\
110 \\
100 \\
95 \\
90 \\
80 \\
75 \\
60 \\
50 \\
45 \\
40.54 \\
40 \\
37.8\end{array}$ \\
\hline $\begin{array}{r}98.5 \\
95 \\
86 \\
77 \\
68 \\
50 \\
41 \\
32 \\
23 \\
14 \\
+5 \\
-44 \\
-13 \\
-22 \\
-40 \\
-76\end{array}$ & $\begin{array}{r}36.9 \\
35 \\
30 \\
25 \\
20 \\
10 \\
5 \\
0 \\
-5 \\
-10 \\
-15 \\
-20 \\
-25 \\
-30 \\
-40 \\
-60\end{array}$ \\
\hline $\begin{array}{l}1 \\
1.8 \\
3.6 \\
4.5 \\
5.4\end{array}$ & $\begin{array}{l}=.54^{\circ} \mathrm{C} . \\
=1^{\circ} \mathrm{C} . \\
=2^{\circ} \mathrm{C} . \\
=2.5^{\circ} \mathrm{C} \\
=3^{\circ} \mathrm{C} .\end{array}$ \\
\hline
\end{tabular}

To convert degrees F. into degrees C., subtract 32 , and multiply by $\frac{5}{9}$.

To convert degrees C. into degrees F., multiply by 8 , and add $32^{\circ}$.

\section{MEAS UREMENTS.}

\section{FRENCH INTO ENGLISH.}

1 mètre 10 decimètres $\}=39.37$ English

100 centimetres $\}=39.37$ inches.

1,000 millimetres (or 1 yd. and $31 / 3$ in.)

1 décimètre

10 centimetres $\}=3.937$ inches

100 millimetres $\}$ (or nearly 4 inches.)

$\left.\begin{array}{c}1 \text { centimètre } \\ 10 \text { millimetres }\end{array}\right\}=\begin{aligned} & .3937 \text { or about } \\ & \text { (nearly o inch.) }\end{aligned}$

1 millimetre = neariy of inch.

\section{Or,}

(It is the ten-millionth part of a quarter of the meridian of the earth.)

1 Decimètre $=4$ in.

1 Centimetre $=$ 4. in.

1 Millimetre $=$ in.

Décametre $=32.80$ feet.

Hectomètre $=109.36 \mathrm{yds}$.

Kilomètre $=0.62$ miles.

One inch $=2.539$ Centimetres.

One foot $=3.047$ Decimètres.

One yard $=0.91$ of a Mètre.

One mile $=1.60$ Kilometre.

The cubic centimetre (15.432 grains-1 gramme) is a standard at $4^{\circ} \mathrm{C}$., the grain at $16^{\circ} .66 \mathrm{C}$.

\section{WEIGHT.}

(One gramme is the weight of a cubic centimetre of water at $4^{\circ} \mathrm{C}$. at Paris).

1 gramme

to décigrammes $=15.432349$ grs.

100 centigrammes $\}$ (or nearly 151/2).

1,000 milligrammes

1 décigramme

10 centigrammes $=$ rather more

100 milligrammes than $11 / 2$ grain.

1 centigramme $\}=$ rather more

10 décigrammes $\}$ than $\frac{3}{20}$ grain.

1 milligramme = rather more

than $\frac{3}{200}$ grain. OR

1 Décigramme $=2$ dr. $34 \mathrm{gr}$.

1 Hectogrm. = 31/2 oz. (Avoir.)

1 Kilogrm. $=21 \mathrm{~b} .3 \mathrm{oz} .2 \mathrm{dr}$. (Avoir.)

\section{LENGTH.}

ONE METRE $=39.37079$ inches.
A grain equals about 1.16 gram., a Troy oz. about 31 gram.,

a lb. Avoirdupois about 1/ Kilogrm. and 1 cwt. about 50 Kilogrms.

\section{CAPACITY.}

$1,000$ cubic décimètres $\}=1$ cubic $1,000,000$ cubic centimetres $\}$ mètre.

1 cubic décimètre $\left.\begin{array}{l}\text { or } \\ \text { centimètres }\end{array}\right\}=1$ litre.

1,000 cubic centimètres Or

ONe LITRE $=1$ pt. $15 \mathrm{oz} .1 \mathrm{dr} .40$.

(For simplicity, Litre is used to signify 1 cubic décimetre, a little less than 1 English quart.)

Décilitre (100 c.c.) $\quad=31 / 2 \mathrm{oz}$.

Centilitre (10 c.c.) $=24 \mathrm{dr}$.

Millilitre (1 c.c.) $\quad=17 \mathrm{~m}$.

Décalitre $\quad 2 \mathrm{fg}$ gal.

Hectolitre $\quad=22$ gals

Kilolitre (cubic mètre) $=27 \%$ bushels.

A cubic inch $=16.38$ c.c. $; a$ cubic foot $=28.315$ cubic déc., and a gallon $=$ 4.54 litres.

\section{CONVERSION SCALE.}

TO convert GRAMMES to OUNCES avoirdupois, multiply by 20 and divide by $56 \%$.

To convert Kilogrammes to Pounds, multiply by 1,000 and divide by 454 .

To convert Litres to GaLloNs, multiply by 22 and divide by 100 .

To conver't Litres to Pints, muitiply by 88 and divide by 50 .

To convert MILlimetres to INCHES, multiply by 10 and divide by 254 .

To convert MLTREs to YARDS, multiply by 70 and divide by 64 .

\section{SURFACE MEASURE.}

1 square mètre $=$ about $1550 \mathrm{sq}$. inches. Or 10,000 sq. centimetres, or 10.75 sq. ft.

$1 \mathrm{sq}$. inch $=$ about $6.4 \mathrm{sq}$. centimètres.

1 sq. foot $=$ "6 930

\section{ENERGY MEASURE.}

1 kilogrammètre=about $7.24 \mathrm{ft}$. pounds 1 foot pound $=6 . .1381 \mathrm{kgm}$. 1 foot ton $=" 310 \mathrm{kgm}$.

HEAT EQUIVALENT.

1 kilocalorie $=424$ kilogrammètres.
ENGLISH MEASURES.
Apothecaries Weight.

7000 grains $=1 \mathrm{lb}$.

Or

437.5 grains $=1 \mathrm{oz}$.
Avoirdupois Weight.

$16 \mathrm{drams}=1 \mathrm{oz}$.

$16 \mathrm{oz} . \quad=1 \mathrm{lb}$.

28 lbs. $\quad=1$ quarter.

4 quarters $=1$ cwt.

Measure of 1 décimètre, or 10 centimetres, or 100 millimètres.

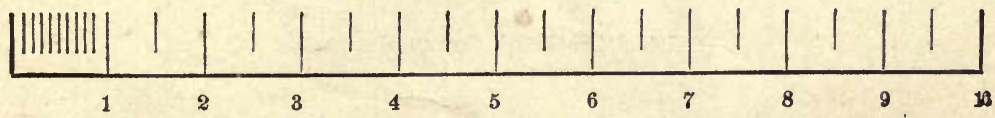




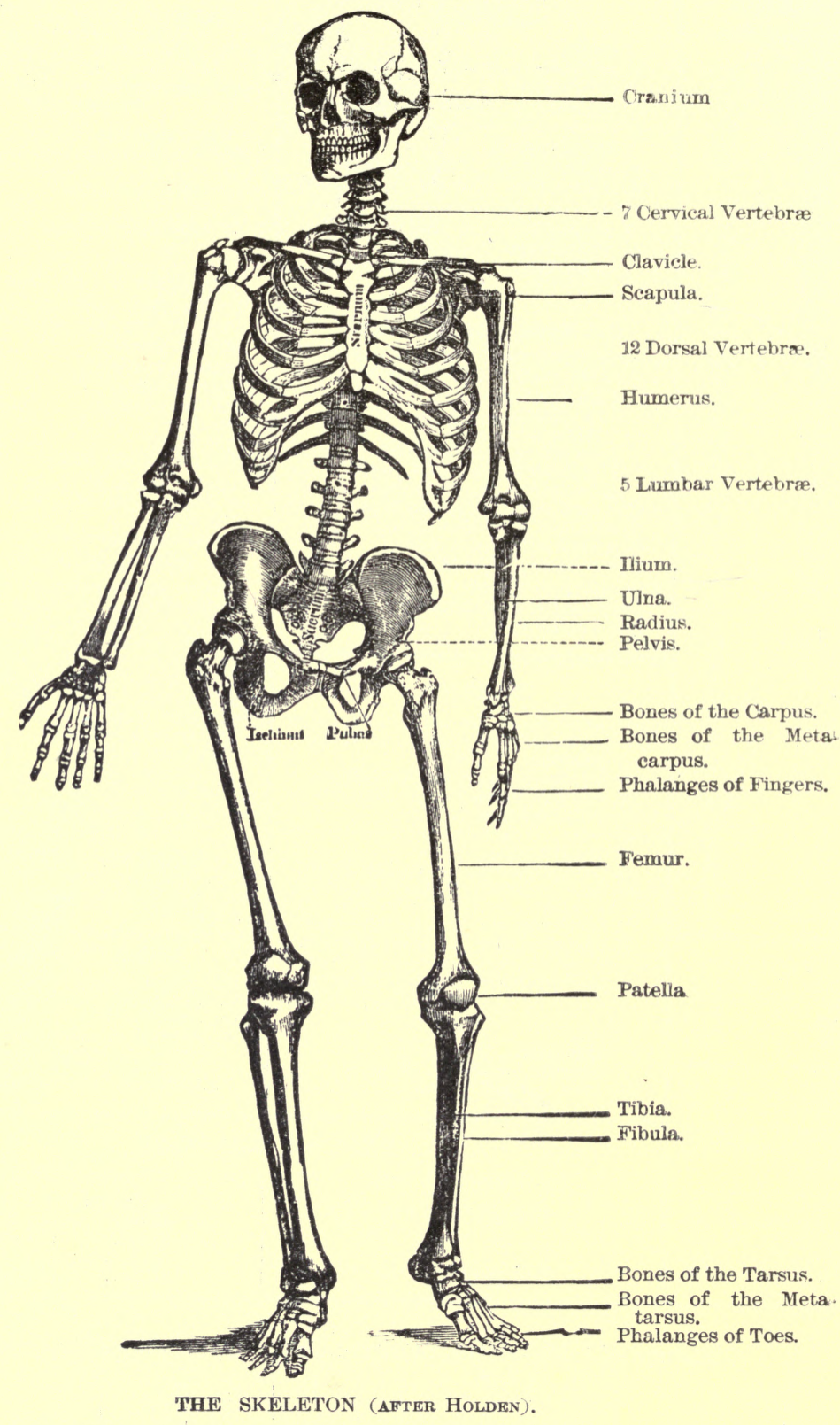




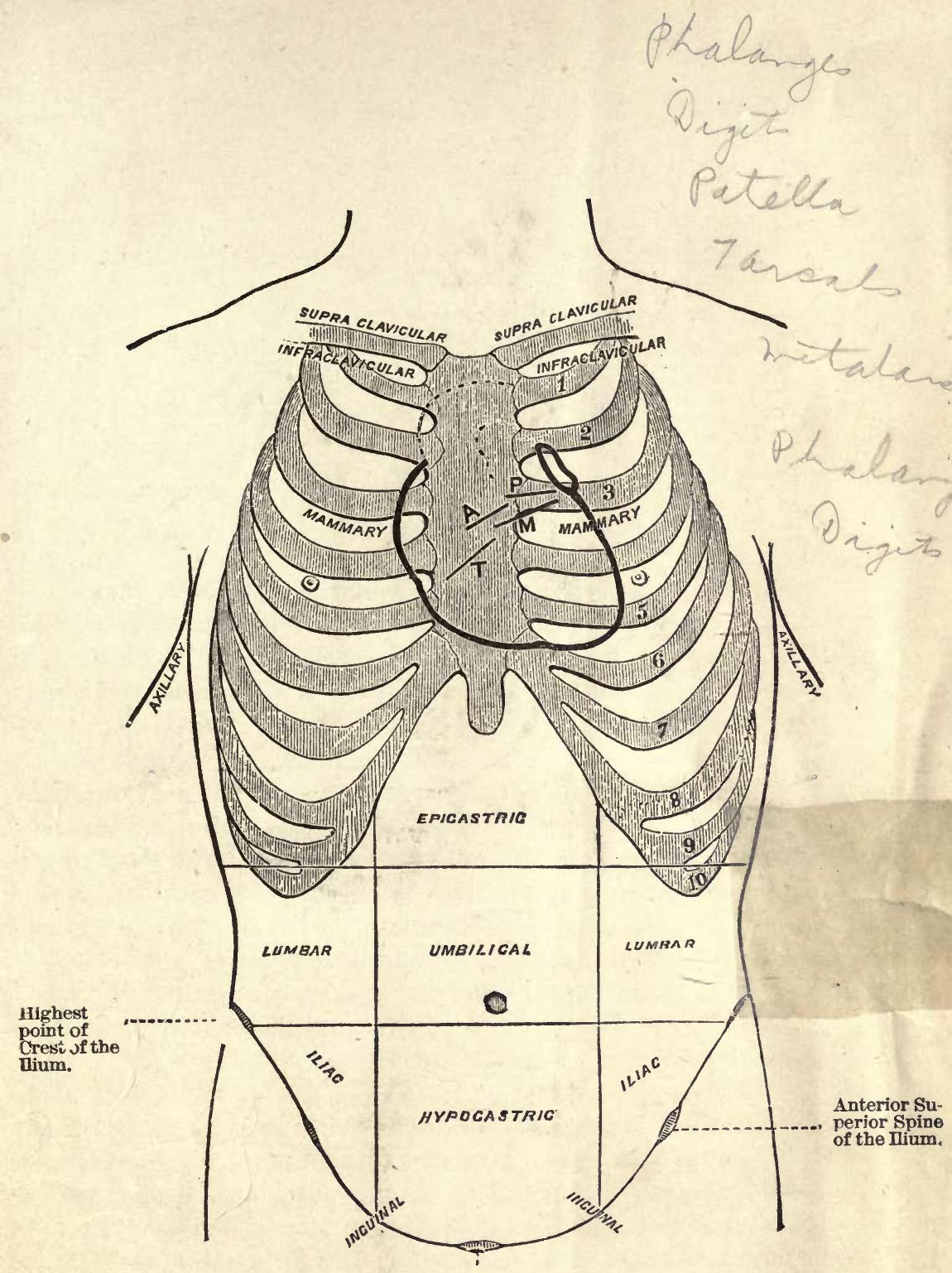

Symphysis Pubis.

DIAGRAM OF THORACIC AND ABDOMTNAL REGIONS.

A. Aortic Valve.

M. Mitral Valve.
$P$. Pulmonary Valve.

T. Tricuspid Valve. 
C 


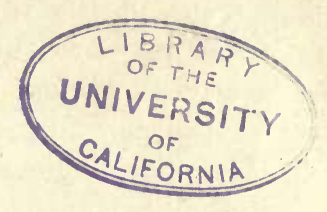

\section{Handbook of Physiology.}

\section{CHAPTER I.}

\section{THE PHENOMENA OF LIFE.}

Humas Physiology is the science which treats of the various processes or changes which take place during life in the organs and tissues of the body of man. These processes, however, must not be considered as by any means peculiar to the human organism since, putting aside the properties which serve to distinguish man from other animals, as well as those which mark out one animal from another, the changes which go on in the tissues of man go on much in the same way in the tissues of all other animals as long as they live. Furthermore it is found that similar changes proceed in all living vegetable tissues; they indeed constitute what are called vital phensmena, and are those properties which mark out living from non-living material.

The lowest types of life, whether animal or vegetable, are found to consist of minute masses of a jelly-like substance, which is now generally known under the name of protoplasm. Each such minute mass is called a cell, so that these minute elementary organisms are designated unicellular. Not only is it true that the lowest types of life are made up of protoplasm, but it has also been shown that the tissues of which the most complex organisms are composed consist of protoplasmic cells.

Thus, for example, the human body can be shown by dissection to be constructed of various dissimilar parts, bones, muscles, brain, heart, lungs, intestines, etc., and these on more minute examination with the aid of the microscope, are found to be composed of different tissues, such as epithelial, connective, nervous, muscular, and the like. Each of these tissues is made up of cells or of their altered equivalents. Again, we are taught by Embryology, the science which treats of the growth and structure of organisms from their first coming into being, that the human body, made up of all these dissimilar structures, commenced its life as a minute cell or ovum (fig. 2) about $\frac{1}{120}$ th of an inch in diameter, consisting of a spherical mass of protoplasm in the midst of which was contained a smaller spherical body, the nucleus or germinal vesicle. 
'The phenomena of life then are exhibited in cells, whether existing alone or developed into the organs and tissues of animals and plants. It must be at once evident that a correct knowledge of the nature and activities of the cell forms the very foundation of physiology; cells being, in fact, physiological no less than morphological units.

The prime importance of the cell as an element of structure was first established by the researches of the botanist Schleiden, and his conclusions, drawn from the study of vegetable histology, were at once ex-

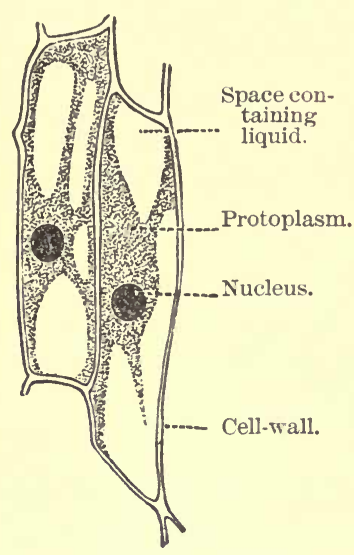

Fig. 1.-Vegetable cells. tended by Theodor Schwann to the animal kingdom. The earlier observers defined a cell as a more or less spherical body limited by a membrane, and containing a smaller body

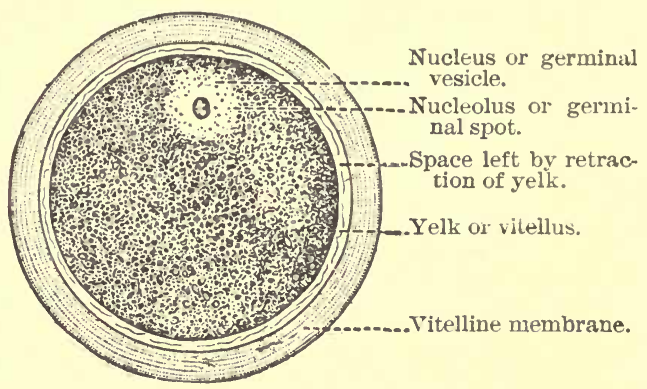

Fig. 2.-Semidiagrammatic representation of a human ovum, showing the parts of an animal cell. (Cadiat.)

termed a nucleus, which in its turn incloses one or more still smaller bodies or nucleoli. Such a definition applied admirably to most vegetable cells, but the more extended investigation of animal tissues soon showed that in many cases no limiting membrane or cell-wall could be demonstrated.

The presence or absence of a cell-wall, therefore, was now regarded as quite a secondary matter, while at the same time the cell-substance came gradually to be recognized as of primary importance. Many of the lower forms of animal life, e.g., the Rhizopoda, were found to consist almost entirely of matter very similar in appearance and chemical composition to the cell-substance of higher forms; and this from its chemical resemblance to flesh was termed Sarcode by Dujardin. When recognized in vegetable cells it was called Protoplasm by Mulder, while Remak applied the same name to the substance of animal cells. As the presumed formative matter in animal tissues it was termed Blastema, and in the belief that, wherever found, it alone of all substances has to do with generation and nutrition, Beale has named it Germinal matter or Bioplasm. Of these terms the one most in vogue at the present day, as we have already said, is Protoplasm, and inasmuch as all life, both in the animal and vegetable kingdoms, is associated with protoplasm, we 
are justified in describing it, with Huxley, as the "physical basis of life," or simply "living matter."

A cell may now be defined as a nucleated mass of protoplasm, of microscopic size, varying in the human body from the red blood-cell which is about $\frac{1}{3000}$ of an inch in diameter to the ganglion cell, $\frac{1}{300}$ of an inch, which possesses sufficient individuality to have a life-history of its own. Each cell originates from a pre-existing cell, grows, produces other cells, and dies, going through the same, though briefer, cycle as the whole organism. Some of the lower forms of life seem to consist of nonnucleated protoplasm, but the above definition holds good for all the higher plants and animals, though some few cells lose their nuclei in the course of development, e.g., the red blood-cells of all mammals.

\section{Properties of Protoplasm.}

Protoplasm is a semi-fluid substance, which swells up but does not mix with water. It is transparent and generally colorless, with refractive index higher than that of water but lower than that of oil. It is neutral or weakly alkaline in reaction, but may under special circumstances be acid, as, for example, after activity. It undergoes stiffening or coagulation at a temperature of about $54.5^{\circ} \mathrm{C} .\left(130^{\circ} \mathrm{F}\right.$.), and hence no organism can live when its own temperature is raised above that point; it is also coagulated and therefore killed by alcohol, by solutions of many of the metallic salts, by strong acids and alkalies, and by many other substances.

Under the microscope it is seen almost universally to be granular, the granules consisting of different substances, either albuminous, or fatty, or glycogenous matters, or more rarely of inorganic salts. The granules are not equally distributed throughout the whole cell-mass, as they are sometimes absent from the outer part or layer, and very numerous in the interior. The granules may exhibit an irregular shaking, dancing movement, which is not vital and is known as the Brownian movement. In addition to granules, protoplasm generally exhibits spaces or vacuoles, generally globular in shape, excepting during movement when they may be irregular, flled with a watery fluid. These vacuoles are more numerous and pronounced in vegetable than in animal cells. Gas bubbles also sometimes exist in cells.

It is impossible to make any definite statement as to the exact chemical composition of living protoplasm, since the methods of chemical analysis necessarily imply the death of the cell; it is, however, stated that protoplasm contains 75 to 85 per cent of water, and of the 15 to 25 per cent of solids, the most important part belongs to the classes of substances called proteids or albumins. Proteids contain the chemical elements carbon, hydrogen, nitrogen, oxygen, sulphur, and phosphorus, the 
last two in small quantities only. A proteicl-like substance, nuclein, found in the nuclei of cells, contains phosphorus in greater abundance. In cell protoplasm a compound of nuclein with proteid, called nucleoproteid, forms the most abundant proteid substance. Other bodies are frequently found associated with the proteids, such as glycogen, starch, cellulose, which contain the elements carbon, hydrogen, and oxygen, the last two in the proportion to form water, and hence are termed carbohydrates; fatty bodres, containing carbon, hydrogen, and oxygen, but not in proportion to form water; lecithin, a complicated fatty body containing phosphorus; cholesterin, a monatomic alcohol; chlorophyll, the coloring matter of plants; inorganc salts, particularly the chlorides and phosphates of calcium, sodium, and potassium; ferments, and other substances.

The vital or physiological characteristics of protoplasm may be well studied in the microscopic animal called the amøba, a unicellular organism found chiefly in fresh water, but also in the sea and in damp
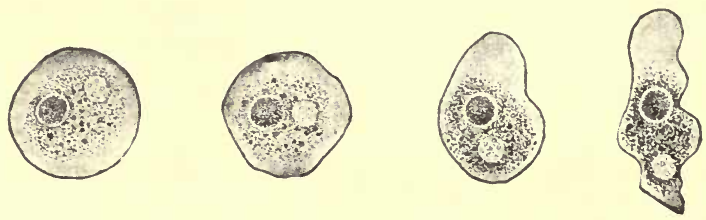

Fig. 3.-Phases of amo's id movement.

earth. These properties may be conveniently studied under the following heads:-

1. The Power of Spontaneous Movement.-When an amœba is observed with a high power of the microscope, it is found to consist of an irregular mass of protoplasm probably containing one or more nuclei, the protoplasm itself being more or less granular and vacuolated. If watched for a minute or two, an irregular projection is seen to be gradually thrust out from the main body and retracted; a second mass is then protruded in another direction, and gradually the whole protoplasmic substance is, as it were, drawn into it. The amœba thus comes to occupy a new position, and when this is repeated severai times we have locomotion in a definite direction, together with a continual change of form. These movements, when observed in other cells, such as the colorless blood-corpuscles of higher animals (fig. 3), in the branched cornea cells of the frog and elsewhere, are hence termed amoboid.

The remarkable movement of pigment granules observed in the branched pigment cells of the frog's skin by Lister are also probably due to amœboid movement. These granules are seen at one time distributed uniformly through the body and branched processes of the cell, while at another time they collect in the central mass leaving the branches quite colorless. 
This movement within the pigment cells might also be considered an example of the so-called streaming movement not infrequently seen in certain of the protozoa, in which the mass of protoplasm extends long and fine processes, themselves very little movable, but upon the surface of which freely moving or streaming granules are seen. A gliding movement has also been noticed in certain animal cells; the motile

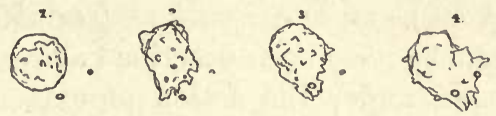

Fig. 4.-Changes of form of a white corpuscle of newt's blood, sketched at brief intervals. :The figures show also the intussusception of two small granules. (Schäfer.)

part of the cell being composed of protoplasm bounding a central and more compact mass. By means of the free movement of this layer, the cell may be observed to move along.

In vegetable cells the protoplasmic movement can be well seen in the hairs of the stinging-nettle and Tradescantia and the cells of Vallisneria and Chara; it is marked by the movement of the granules nearly always imbedded in it. For example, if part of a hair of Tradescantia (fig. 5) be viewed under a high magnifying powor, streams of protoplasm containing crowds of granules hurrying along, like the footpassengers in a busy street, are seen flowing steadily in definite directions, some coursing round the film which lines the interior of the cellwall, and others flowing toward or away from the irregular mass in the centre of the cell-cavity. Many of these streams of protoplasm run
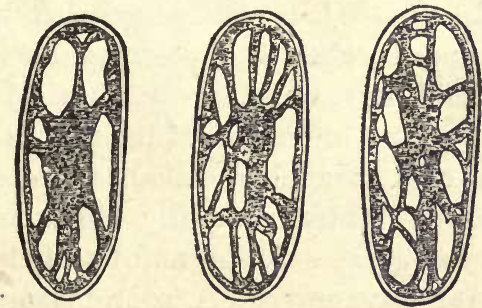

Fig. 5.-Cell of Tradescantia drawn at successive intervals of two minutes.-The cell-conte ts consist of a central mass connected by many irregular processes to a peripheral film, the wl ole forming a vacuolated mass of protoplasm, which is continually changing its shape. (Schofield.)

together into larger ones and are lost in the central mass, and thus ceaseless rariations of form are produced. The movement of the protoplasmic granules to or from the periphery is sometimes called regetable circulation, whereas the movement of the protoplasm round the interior of the cell is called rotation.

The first account of the movement of protoplasm was given by Rösel in 1755, as occurring in a small Proteus, probably a large freshwater amœba. His description was followed twenty years later by 
Corti's demonstration of the rotation of the cell sap in characeæ, and in the earlier part of the century by Meyer in Vallisneria, 1827; Robert Brown, 1831, in "Staminal Hairs of Tradescantia." Then came Dujardin's description of the granular streaming in the pseudopodia of Rhizopods and movement in other cells of animal protoplasm (Planarian eggs, v. Siebold, 1841; colorless blood-corpuscles, Wharton Jones, 1846).

2. The Power of Response to Stimuli, or Irritability.-Although the movements of the amœba have been described above as spontaneous, yet they may be increased under the action of external agencies which excite them and are therefore called stimuli, and if the movement has ceased for the time, as is the case if the temperature is lowered beyond a certain point, movement may be set up by raising the temperature. Again, contact with foreign bodies, gentle pressure, certain salts, and electricity, produce or increase the movement in the amcba. The protoplasm is, therefore, sensitive or irritable to stimuli, and shows its irritability by movement or contraction of its mass.

The effects of some of these stimuli may be thus further detailed:-

a. Changes of Temperature.-Moderate heat acts as a stimulant; the movement stops below $0^{\circ} \mathrm{C} .\left(32^{\circ} \mathrm{F}\right.$.), and above $40^{\circ} \mathrm{C} .\left(104^{\circ} \mathrm{F}\right.$.) ; between these two points the morements increase in actirity; the optimum temperature is about $37^{\circ}$ to $38^{\circ} \mathrm{C}$. Exposure to a temperature even below $0^{\circ} \mathrm{C}$. stops the movement of protoplasm, but does not prevent its reappearance if the temperature is raised; on the other hand, prolonged exposure to a temperature of over $40^{\circ} \mathrm{C}$. altogether kills the protoplasm and causes it to enter into a condition of coagulation or heat rigor.

b. Mechanical Stimuli.-When gently squeezed between a cover and object-glass under proper conditions, a colorless blood-corpuscle is stimulated to active amøobid movement.

c. Nerve Influence.-By stimulation of the nerves of the frog's cornea, contraction of certain of its branched cells has been produced.

d. Chemical Stimuli.-Water generally stops amœboid movement, and by imbibition causes great swelling and finally bursting of the cells. In some cases, however (myxomycetes), protoplasm can be almost entirely dried up, but remains capable of renewing its movements when again moistened. Dilute salt-solution and many dilute acids and alkalies stimulate the movements temporarily. Strong acids or alkalies permanently stop the movements; ether, chloroform, veratria, and quinine also stop it for a time.

Movement is suspended in an atmosphere of hydrogen or carbonic acid and resumed on the admission of air or oxygen, but complete withdrawal of oxygen will after a time kill the protoplasm.

e. Electrical.-Weak currents stimulate the movement, while strong currents cause the cells to assume a spherical form and to become motionless. 
3. The Power of Digestion, Respiration, and Nutrition.-This consists in the power which is possessed by the amceba and similar animal cells of taking in food, modifying it, building up tissue by assimilating it, and rejecting what is not assimilated. These various processes are effected by the protoplasm simply flowing round and inclosing within itself minute organisms such as diatoms and the like, from which it extracts what it requires, and then rejects or excretes the remainder, which has never formed part of the body. This latter proceeding is done by the cell withdrawing itself from the material to be excreted. The assimilation constantly taking place in the body of the amœba, is for the purpose of replacing waste of its tissue consequent upon manifestation of energy. The respiratory process of absorbing oxygen goes on at the same time.

The processes which take place in cells, both animal and regetable, are summed up under the term metabolism (from $\mu \varepsilon \tau \alpha \beta o \lambda \eta$, change). The changes which go on are of two kinds, viz., assimilation, or building up, and disassimilation, or breaking down; they may be also called composition or decomposition, or, using the nomenclature of Gaskell, anabolism or constructive metabolism, and katabolism or destructive metabolism. In the direction of anabolism two processes occur, viz., the building up of materials which it takes in, and secondly, the building up of its own substance by those or other materials. As we shall see in a subsequent paragraph, the process of anabolism differs to some extent in vegetable and animal cells. The katabolism of the cell consists in chem-

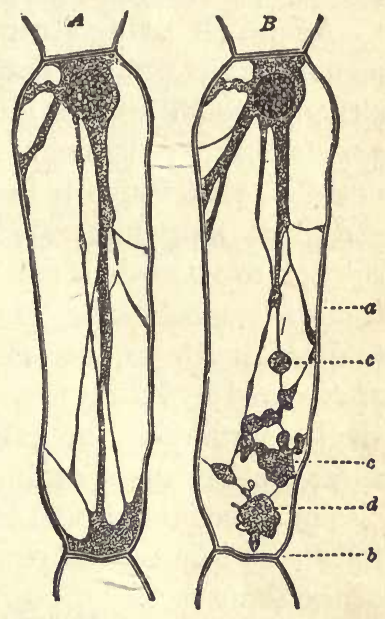

Fig. 6.-Cells from the staminal hairs of Tradescantia. $A$, Fresh in water; $B$, the same cell after slight electrical stimulation; $a, b$, region stimulation; $c, d$, clumps and knobs of contracted protoplasm. (Kühne.) ical changes which occur in the cell-substance itself, or in substances in contact with it.

The destructive metabolism of a cell is increased by its activity, but goes on also during quiescence. It is probably of the nature of oxidation, and results in the evolution of carbonic anhydride and water on the one hand, and in the formation of various substances on the other, some of which may be stored up in the cell for future use, and are called secretions, and others, like the carbonic anhydride and certain bodies containing nitrogen, are eliminated as excretions.

4. The Power of Growth.-In protoplasm then, it is seen that the two processes of waste and repair go on side by side, and as long as they are equal the size of the animal remains stationary. If, however, the building up exceed the waste, then the animal grows; if the waste ex- 
ceed the repair, the animal decays; and if decay go on beyond a certain point, life becomes impossible, and the animal dies.

Growth, or the inherent power of increasing in size, although essential to our idea of life, is not, it must be recollected, confined to living beings. A crystal of common salt, for example, if placed under appropriate conditions for obtaining fresh material, will grow in a fashion as definitely characteristic and as easily to be foretold as that of a living creature; but the growth of a crystal takes place merely by additions to its outside; the new matter is laid on particle by particle, and layer by layer, and, when once laid on, it remains unchanged. In a living structure, where growth occurs, it is by addition of new matter, not to the surface only, but throughout every part of the mass.

Again, all living structures are subject to constant decay. Thus, a man's body is not composed of exactly the same particles day after day, although to all intents he remains the same individual. Almost every part is changed by degrees; but the change is so gradual, and the renewal of that which is lost so exact, that no difference may be noticed, except at long intervals of time. A lifeless structure, as a crystal, is subject to no such laws; neither decay nor repair is a necessary condition of its existence. That which is true of structures which never had to do with life is true also with respect to those which, although they are formed by living parts, are not themselves alive. Thus, an oystershell is formed by the liring animal which it incloses, but it is as lifeless as any other mass of inorganic matter; and in accordance with this circumstance its growth takes place layer by layer, and it is not subject to constant decay and reconstruction. The hair and nails are examples of the same fact.

In connection, too, with the growth of lifeless masses there is no alteration in the chemical composition of the material which is taken up and alded to the previously existing mass. For example, when a crystal of common salt grows on being placed in a fluid which contains the same material, the properties of the salt are not changed by being taken out of the liquid by the crystal and added to its surface in a solid form. But the case is essentially different in living beings, both animal and vegetable, as the materials which serve ultimately to build them up are much altered before they are finally assimilated by the structures they are destined to nourish.

The growth of all living things has a definite limit, and the law which governs this limitation of increase in size is so invariable that we should be as much astonished to find an individual plant or animal without limit as to growth as without limit to life.

5. The Power of Reproduction.-The amœba, to return to our former illustration, when the growth of its protoplasm has reached a certain point, manifests the power of reproduction, by splitting up into (or in 
some other way producing) two or more parts, each of which is capable of independent existence. The new amœbæ manifest the same properties as their parent, perform the same functions, grow and reproduce in their turn. This cycle of life is being continually passed through.

In more complicated structures than the amœba, the life of individual protoplasmic cells is probably very short in comparison with that of the organism they compose; and their constant decay and death necessitate constant reproduction.

The mode in which this takes place has long been the subject of great controversy.

It is now very generally believed that every cell is descended from some pre-existing (mother-) cell. This derivation of cells from cells
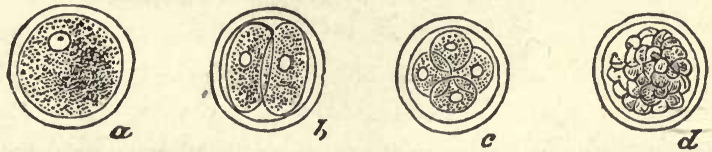

Fig. 7.-Diagram of an ovum (a) undergoing segmentation-In (b) it has divided into two, in $(c)$ into four; and in $(d)$ the process has ended in the production of the so called "mulberry mass." (Frey.)

takes place by (1) gemmation, which essentially consists in the budding off and separation of a portion of the parent cell; or (2) fission or division.

The exact manner of the division of cells is a matter of some difficulty, and will not be described until the subject of the structure of protoplasmic cells has been considered.

\section{Structure of Protoplasmic Cells.}

Protoplasm was formerly thought to be homogeneous: though this may be true in some cases, it is now generally found to consist of two substances, spongioplasm and hyaloplasm. The spongioplasm or reticulum forms a fine network, increases in relative amount as the cell grows older, and has an affinity for staining reagents. The hyaloplasm is less refractile, elastic, or extensile, and has no affinity for stains; it predominates in young cells, is thought to be fluid, and fills the interspaces of the reticulum. The nodal points of the reticulum, with the granules (microsomes) found in the protoplasm, cause the granular appearance. Butschli has recently asserted that protoplasm is an emulsion made up of numerous microscopic vacuoles whose walls are in close apposition and are seen under the microscope in optical section only, thus causing the reticular appearance. This idea is accepted by few.

The arrangement of the reticulum varies considerably in different cells, and even in different parts of the same cell. Sometimes, for example (fig. 8), the meshwork has an elongated radial arrangement from 
the nucleus; at others, the meshwork is more evenly disposed, as in fig. 9. At the junctions of the fibrils there are usually slight enlargements or nodes.

In some cells, particularly in plants, but also in some animal cells, there is a tendency toward a formation of a firmer external envelope,

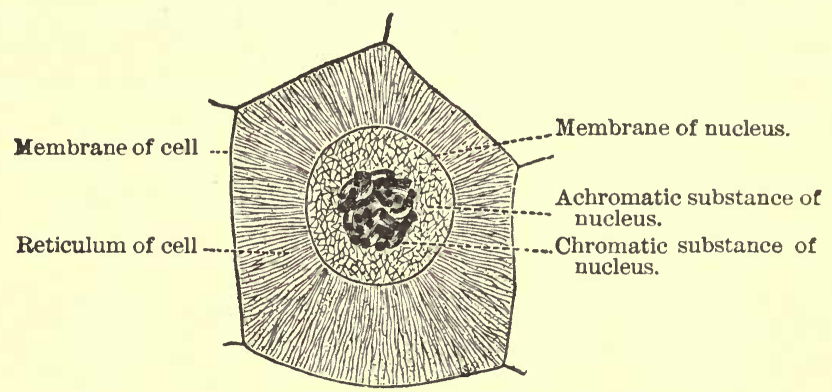

Fig. 8.-Cell with its reticulum disposed radially; from the intestinal epithelium of a worm. (Carnoy.)

constituting in vegetable cells a membrane distinct from the more central and more fluid part of the protoplasm. In such cases the reticulum at the periphery of the cell is made up of very fine meshes. The membrane when formed is usually pierced with pores by which fluid may pass in, or through which protrusion of the protoplasmic filaments forming the cell's connection with other cells surrounding it may take place.

It is an exceedingly interesting question whether in cells the one
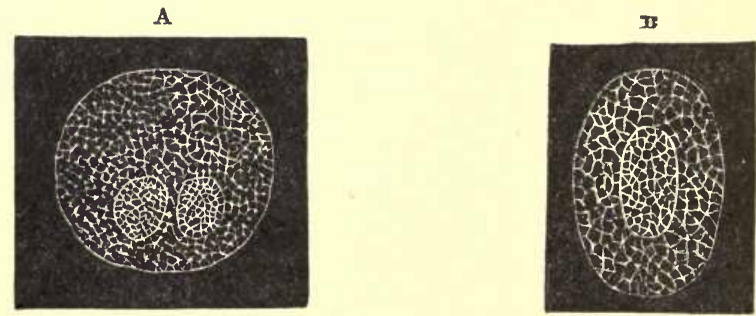

Fig. 9.-(A.) The colorless blood-corpuscle showing the intra-cellular network, and two nuclei with intra-nuclear network. (B.) Colored blood-corpuscle of newt showing the intra-cellular network of fibrils. Also oval nucleus composed of limiting membrane and fine intra-nuclear network of fibrils. $\times 800$. (Klein and Noble Smith.)

part of the protoplasm can exist without the other. Schäfer summarizes the matter thus:- " There are cells, and unicellular organisms both animal and vegetable, in which no reticular structure can be made out, and these may be formed of hyaloplasm alone. In that case, this must be looked upon as the essential part of protoplasm. So far as amœboid phenomena are concerned it is certainly so; but whether the chemical 
changes which occur in many cells are effected by this or by spongioplasm is another matter."

Another question about which there is some difference of opinion is, which part of the protoplasm is chiefly contractile. It is usually concluded that this property rests in the meshwork, but there seems a considerable amount of evidence in favor of the view that part if not all of the contractility resides in the hyaloplasm; for example, in amœboid cells the pseudopodial protoplasm are certainly made of this and not of spongioplasm, and when the corpuscle is stimulated the hyaloplasm flows back into the reticular network. If the view that the hyaloplasm is chiefly contractile be a correct one, the special condition of an amoboid cell must be considered to be condition of contraction, and the flowing out of the process to be relaxation.

\section{The Cell Nucleus.}

All cells at some period of their existence possess nucler. As has been incidentally suggested the origin of a nucleus in a cell is the first trace of the differentiation of protoplasm. The existence of nuclei was first pointed out in the year 1833 by Robert Brown, who observed them in vegetable cells. They are either small transparent vesicular bodies containing one or more smaller particles (nucleoli), or they are semi-solid masses of protoplasm always in the resting condition bounded by a well-defined envelope. In their relation to the life of the cell they are certainly hardly second in importance to the protoplasm itself, and thus Beale is fully justified in comprising both under the term "germinal matter." They control the nutrition of the cell, and probably initiate the process of subdivision. If a cell be mechanically divided, that portion not containing the nucleus dies.

Histologists have long recognized nuclei by two important characters :-

(1.) Their power of resisting the action of various acids and alkalies, particularly acetic acid, by which their outline is more clearly defined, and they are rendered more easily visible. This indicates some chemical difference between the protoplasm of the cells and nuclei, as the former is destroyed by these reagents.

(2.) Their quality of staining in solutions of carmine, hæmatoxylin, etc. Nuclei are most commonly oval or round, and do not generally conform to the diverse shapes of the cells; they are altogether less variable elements than cells, even in regard to size, of which fact one may see a good example in the uniformity of the nuclei in cells so multiform as those of epithelium. But sometimes nuclei occupy almost the whole of the cell, as in the lymph corpuscles of lymphatic glands, and in some small nerve cells, and may even project above the surface. 
Their position in the cell is very variable. In many cells, especially where active growth is progressing, two or more nuclei are present.

\section{Structure of Nuclei.}

The nucleus when in a condition of rest is bounded by a distinct membrane, the nuclear membrane, possibly derived from the spongioplasm of the cell, which encloses the nuclear contents or karyoplasm. The membrane consists of an inner, or chromatic, and an of onter, or

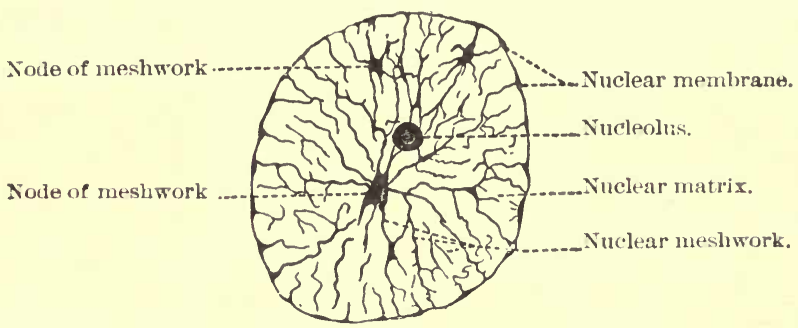

Fig. 10.-The resting nucleus-diagrammatic. (Waldeyer.)

achromatic layer, so called from their reaction to stains. The karyoplasm is made up of a reticular network, or chromoplasm, whose interspaces are filled by the karyolymph, or muclear matrix, a homogeneous substance which is rich in proteids, has but slight affinity for stains, and is supposed to be fluid.

The network is composed of linin, or achromatin, a transparent unstainable framework; and of chromatin, which stains deeply, is sup-
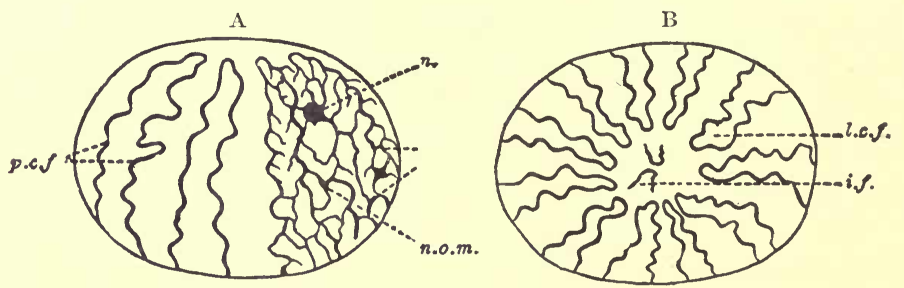

Fig. 11.-Diagram of nucleus showing the arrangement of chief chromatic filaments. A. Tiewed from the side, the polar end being uppermost. p.c.f., Primary chromatic filaments; n., nucleolus; n.o.m., node of meshwork. B. Viewed at the polar end. l.c.f., Looped chromatic filament; i.f., irregular filament. (Rabl.)

ported by the linin, and occurs sometimes in the form of granules, but usually as irregular anastomosing threads, both thicker primary fibres and thinner connecting branches. The threads often form thickened nodes, karyosemes or false nucleoli, at their points of intersection. It 
is now quite generally believed that the chromatin occurs as short, rodlike and highly refractive masses, which are embedded in the linin in a regular series.

The nucleoli, or plasmosomes, are spherical bodies of unknown function. They stain deeply, and may either lie free in the nuclear matrix or be attached to the threads of the network.

\section{Attraction Sphere.}

In addition to the nucleus, a minute spherical body called the centrosome is believed to be constantly present in animal cells, though sometimes too small to be demonstrated. The centrosome is smaller than the nucleus, close to which it lies, and exerts a peculiar attraction for

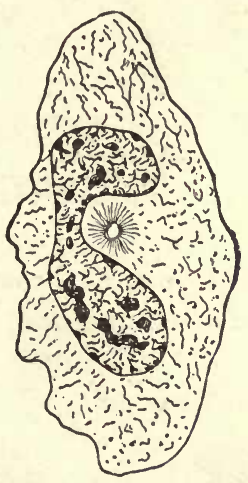

Fig. 11. - Leucocyte of Salamander Larva, showing attraction sphere. (After Flemming.)

the protoplasmic filaments and granules in its vicinity, so that it is surrounded by a zone of fine radiating fibrils, forming the attraction sphere or archoplasm. Some authorities assert that the centrosome lies within the nucleus in the resting state, and only passes into the cell proper in the earlier stages of cell division. The attraction sphere is most distinctly seen in cells about to divide. It plays an important rôle in nuclear division, but it is doubted if it gives the initial impulse to the process.

\section{Cell Division.}

The division of a cell is preceded by division of its nucleus, which may be either direct or indirect. Direct or simple division, amitosis or akinesis (xiv $\sigma \iota s$, movement), occurs without any change in the arrangement of the intranuclear network; it is probably limited to the amœbæ. 
A constriction develops at the centre of the nucleus, possibly preceded by division of the nucleoli, and gradually divides it into two equal daughter nuclei. A similar constriction of the protoplasm of the cell occurs between the daughter nuclei and divides it in two parts.

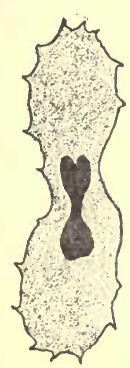

$a$

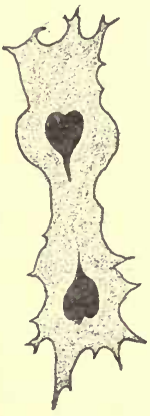

$B$

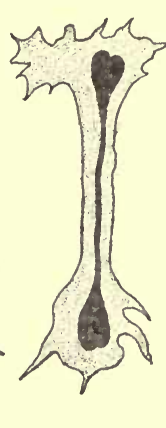

C

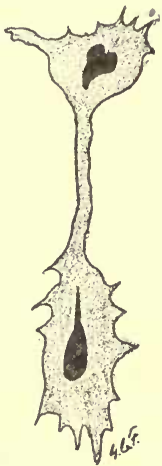

$D$

Fig. 12.-Akinesis, amitosis, or direct cell division. $A$, Constriction of nucleus; $B$, division of nucleus and constriction of cell body; $C$, daughter nuclei still connected by a thread, division being delayed: $D$, division of cell body nearly complete. (After Arnold.)

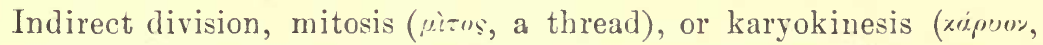
a kernel), is the almost universal method, and consists of a series of
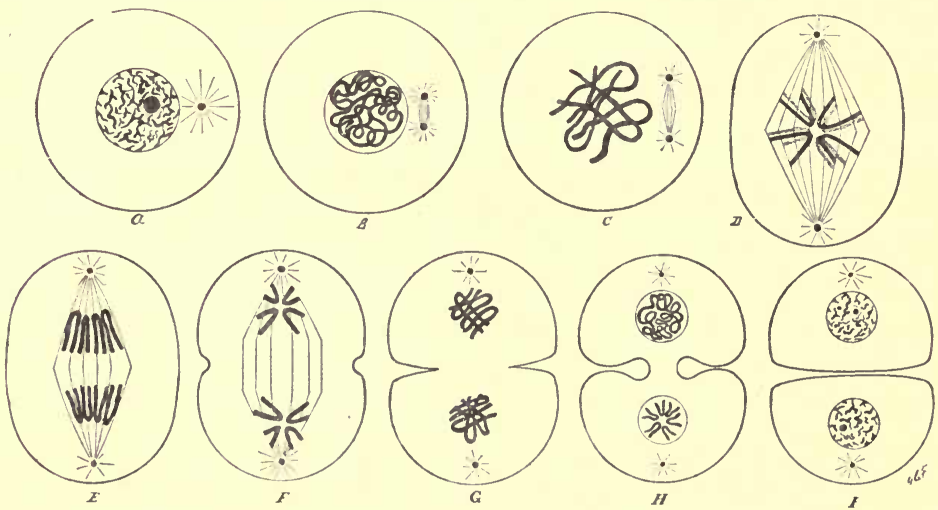

Fig. 12A.-Karyokinesis, mitosis, or indirect cell division (diagrammatic), $A$, Cell with resting nucleus; $B$, wreath, daughter centrosomes and early stage of achromatic spindle; $C$, chromosomes; $D$, monaster stage, achromatic spindle in long axis of nucleus, chromosomes dividing: $E$, chromosomes moving toward centrosomes; $F$, diaster stage, chromosomes at poles of nucleus. commencing constriction of cell body; $G$, daughter nuclei beginning return to resting state: $H$. daughter nuclei showing monaster and wreath; 1 , complete division of cell body into daughter cells whose nuclei have returned to the resting state. (After Böhm and von Davidoff.)

changes in the arrangement of the intranuclear network, resulting in the exact division of the chromatic fibres into two parts, which form the 
chromoplasm of the daughter nuclei. The changes follow a closely similar course in both plant and animal cells. The process has been divided by different authorities into a varying number of stages, with varying names, but for the sake of simplicity it seems best to accept the
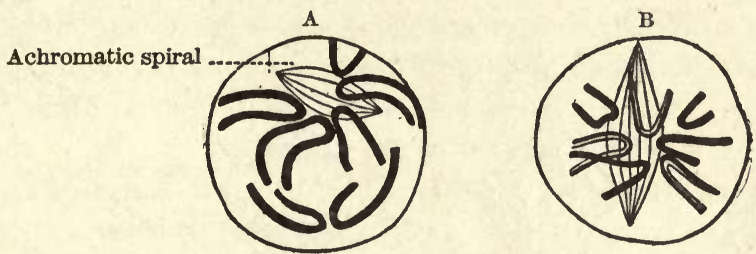

Fig. 13. - Early stages of karyokinesis. $\Delta$. The thicker primary fibres remain and the achromatic spindle appears. B. The thick fibres split into two and the achromatic spindle becomes longitudinal. (Waldeyer.)

anthority of Verworn and recognize two stages only-a progressive one in which the changes in the nucleus advance to a maximum, and a retrogressive one in which the resulting nuclear halves revert to the resting state.

Progressive stage. The resting nucleus becomes somewhat enlarged, and the centrosome (according to those who regard it as lying normally

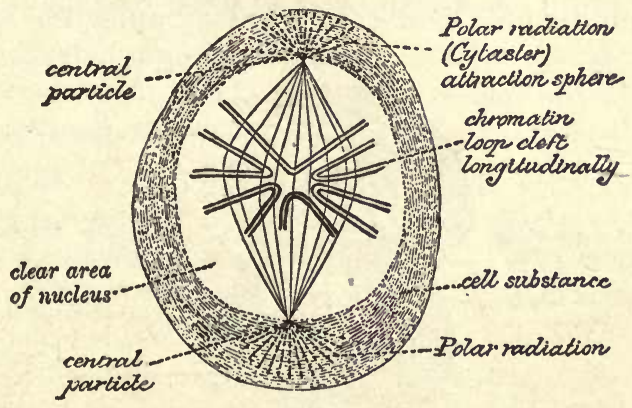

Fig. 14.-Monaster stage of karyokinesis. (Rabl.)

within the nucleus) migrates into the cell protoplasm. The centrosome then divides into two daughter centrosomes which lie near the nucleus but are separated by a considerable interval. Each is surrounded by the radiating fibrils of the attraction sphere, and some of these fibrils pass: continuously from one centrosome to the other, forming the achromatic spindle. At the same time (prophases) the intranuclear network becomes converted into a fine convoluted coil (spirem or skein) which may. be either continuous or else broken up into several threads. The thread: 
or threads then shorten and become thicker, while the convolutions, which have become less numerous, arrange themselves in a series of connecting loops, forming the wreath. The nuclear membrane and the nucleolus disappear, the latter passing at times into the cell protoplasm and disintegrating. The wreath then breaks up into $\mathrm{V}$-shaped segments,
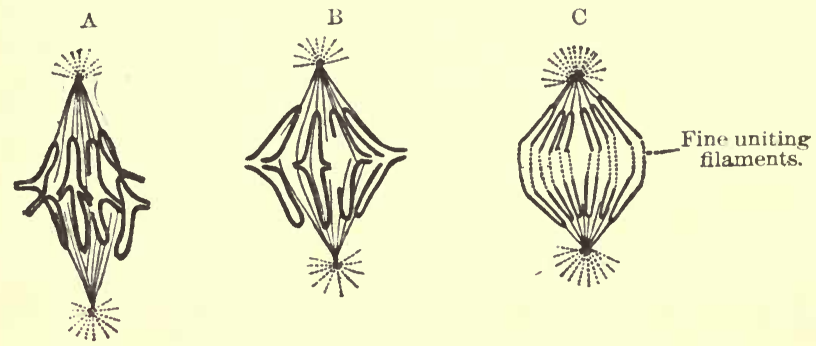

Fig. 15. - Stages of karyokinesis. (Rabl.) A. Commeneing separation of the split chromosomes. B. The separation further advanced. ( . The separated chromosomes passing along the tlbres of the achromatic spindle.

the chromosomes, of which each species of animal has a constant and characteristic number. This varies from two to thirty-six in the different animals, but is sixteen in man.

The two centrosomes migrate to the poles of the nucleus, while the achromatic spindle which connects them occupies the long axis of the

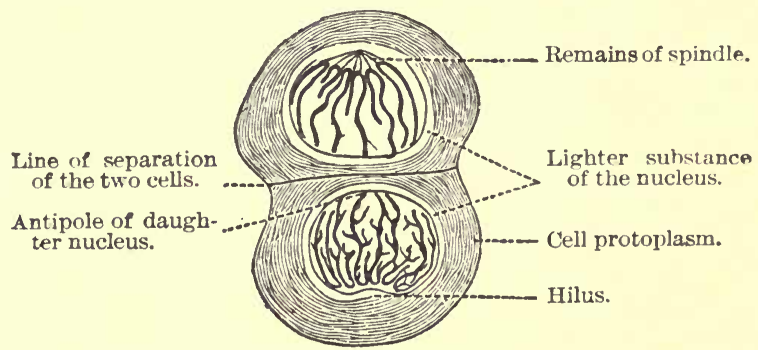

Fig. 16. - Final stages of karyokinesis. In the lower figure the changes are still more advanced than in the upper. (Waldeyer.)

nucleus. 'The chromosomes, becoming much shorter and thicker, gather' around the spindle in its equatorial plane, with their angles directed toward the centre, forming the aster or monaster.

The actual division of the uucleus is begun at this time (metaphases) by the splitting of each chromosome longitudinally into halves which lie at first close together so that each seems doubled. Soon afterward the fibrils of the achromatic spindle begin to contract, and thus separate the 
halves of the chromosomes in such a way that one-half of each is turned toward one pole, and the other half toward the other. As this continues, the two groups, which are equal in size, draw away from each other and from the equator, each group being formed of daughter chromosomes.

Retrogressive stage (anaphases and telophases). 'The two groups (daughter chromosomes) now gradually approach their respective poles, or centrosomes, and the equator becomes free. On reaching the pole, each group gathers in a form which is similar in arrangement to the monaster and is known as the diaster. During this time the cell body becomes slightly constricted by a circular groove at its equatorial plane. Soon afterward the fibrils of the achromatic spindle which connect the two groups begin to grow dim and finally disappear. The daughter chromosomes assume the form of threads twisted in a coil and develop each a nuclear membrane and a nucleolus, forming a daughter nucleus. The nuclei enlarge and the nuclear threads assume the appearance of the resting state of the nucleus. Meanwhile, the constriction about the body of the cell has become deeper and deeper until the protoplasm is divided into two equal parts, or daughter cells, each with its daughter nucleus, and the process of karyokinesis is completed.

\section{Differences between Animals and Plants.}

Having considered at some length the vital properties of protoplasm, as shown in cells of vegetable as well as of animal organisms, we are now in a position to discuss the question of the differences between plants and animals. It might at the outset of our inquiry have seemed an unnecessary thing to recount the distinctions which exist between an animal and a vegetable as they are in many cases so obvious, but, however great the differences may be between the higher animals and plants, in the lowest of them the distinctions are much less plain.

In the first place, it is important to lay stress upon the differences between vegetable and animal cells, first as regards their structure and next as regards their functions.

(1.) It has been already mentioned that in animal cèlls an envelope or cell-wall is by no means always present. In adult vegetable cells, on the other hand, a well-defined cellulose wall is highly characteristic; this, it should be remembered, is non-nitrogenous, and thus differs chemically as well as structurally from the contained protoplasmic mass.

Moreover, in vegetable cells (fig. 1\%, B), the protoplasmic contents of the cell fall into two subdivisions: (1) a continuous film which lines the interior of the cellulose wall; and (2) a reticulate mass contain- 
ing the nucleus and occupying the cell cavity; its interstices are filled with fluid. In young vegetable cells such a distinction does not exist; a finely granular protoplasm occupies the whole cell-cavity (fig. 17, A).

Another striking difference is the frequent presence of a large quantity of intercellular substance in animal tissues, while in regetables it is comparatively rare, the requisite consistency being given to their tissues by the tough cellulose walls, often thickened by deposits of lignin. As an example of the manner in which this end is attained in animal tissues, may be mentioned the deposition of lime salts in a matrix of intercellular substance which occurs in the formation of bone.

(2.) As regards the respective functions of animal and vegetable cells, one of the most important differences consists in the power which vegetable cells possess of being able to build up new complicated nitrogenous
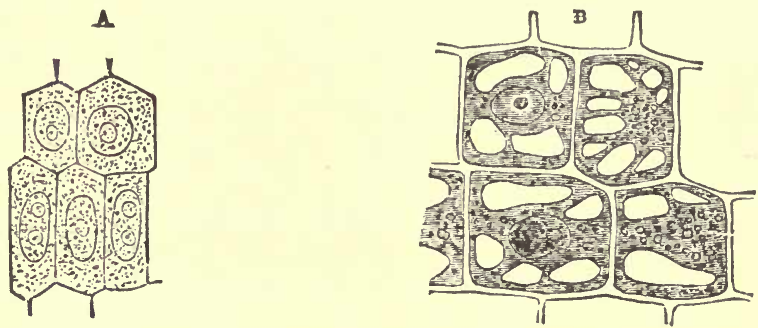

Fig. 17.-(A.) Young vegetable cells, showing cell-cavity entirely filled with granular protoplasm inclosing a large oval nucleus, with one or more nucleoli. (B.) Older cells from same plant, showing distinct cellulose-wall and vacuolation of protoplasm.

and non-nitrogenous bodies out of very simple chemical substances obtained from the air and from the soil. They obtain from the air, oxygen, carbonic anhydride, and water, as well as traces of ammonia gas; and from the soil they obtain water, ammonium salts, nitrates, sulphates, and phosphates, and such bases as potassium, calcium, magnesium, so.. dium, iron, and others. The majority of plants are able to work up these elementary compounds into other and more complicated bodies. This they are able to do in consequence of their containing a certain coloring matter called chlorophyll, the presence of which is the cause of the green hue of plants. In all plants which contain chlorophyll two processes are constantly going on when they are exposed to light: one, which is called true respiration and is a process common to animal and vegetable cells alike, consists in the taking of the oxygen from the atmosphere and the giving out of carbon dioxide; the other, which is peculiar apparently to bodies containing chlorophyll, consists in the taking in of carbon dioxide and the giving out of oxygen. It seems that the chlorophyll is capable of decomposing the carbon dioxide gas and of fixing the carbon in the structures in the form of some new com- 
pound, one of the most rapidly formed of which is starch. The first step in the formation of starch is the union of carbon dioxide and water to form formic aldehyde, $\mathrm{CO}_{2}+\mathrm{H}_{2} \mathrm{O}=\mathrm{CH}_{2} \mathrm{O}+\mathrm{O}_{2}$, oxygen being evolved; then by polymerization the formation of sugar thus, $6 \mathrm{CH}_{2} \mathrm{O}=\mathrm{C}_{6} \mathrm{H}_{12} \mathrm{O}_{6}$; and by dehydration, $\mathrm{C}_{6} \mathrm{H}_{12} \mathrm{O}_{6}-\mathrm{H}_{2} \mathrm{O}=\mathrm{C}_{6} \mathrm{H}_{10} \mathrm{O}_{5}$, the production of starch. In this way is starch synthesized or built up. Vegetable protoplasm by the aid of its chlorophyll is able to build up a large number of bodies besides starch, the most interesting and important being proteid or albumin. It appears to be a fact that the power which bodies possess of being able to synthesize is to a large extent dependent upon the chlorophyll they contain. Thus the power is only present to any marked extent in the plants in which chlorophyll is found and is absent in those which do not possess it; while on the other hand it is present in the extremely few animals which contain it and is absent except in certain rare instances as one of the properties of animal protoplasm.

It must be recollected, however, that chlorophyll without the aid of the light of the sun can do nothing in the way of building up substances, and a plant containing chlorophyll when placed in the dark, as long as it lives, and that is not as a rule long, acts as though it did not contain any of that substance. It is an interesting fact that certain of the bacteria have the chlorophyll replaced by a similar pigment which is able to decompose carbon dioxide gas.

Animal cells, except in the very rare cases above alluded to, do not possess the power of building up from simple materials; their activity is chiefly exercised in the opposite direction, viz., they have brought to them as food the complicated compounds produced by the vegetable kingdom, and with them they are able to perform their functions, setting free energy in the direction of heat, motion, and electricity, and at the same time eliminating such bodies as carbon dioxide and water, and producing other bodies, many of which contain nitrogen, but which are derived from decomposition, and only in very rare cases from building up.

It must be distinctly understood, however, that there are instances of animal cells performing synthetic functions and of combining two simpler compounds to produce one more complex, and it is quite possible that many of the processes performed by the cells of certain organs are instances of synthesis, and not as they have been described of breaking down; and the reverse is undoubtedly the case with vegetable cells, so that it is impossible to generalize to a greater extent than to say that the tendency of the activity of the vegetable cell is chiefly toward synthesis, and of the animal cell toward analysis.

With reference to the substance chlorophyll it is necessary to say a few words. It has been noted that the synthetical operations of rege- 
table cells are peculiarly associated with the possession of chlorophyll and that these operations are dependent upon the light of the sun. It has been further shown that a solution of chlorophyll has a definite absorption spectrum when examined with the spectroscope, and that it is particularly those parts of the solar spectrum corresponding to these absorption bands which are chiefly active in the decomposition of carbonic anhydride, and that, moreover, the position of the maximum absorption corresponds with the maximum of energy of light. In the synthetical processes of the plant then, by aid of its chlorophyll, the radiant energy of the sun's rays becomes stored up or rendered potential in the products formed. The potential energy is set free, or is again made kinetic, when these products simply by combustion produce heat, or when they are taken into the animal organism and used as food and to produce heat and motion.

The influence of light is not an absolute essential to animal life; indeed, it is said not to increase the metabolism of animal tissue to any extent, and the animal cell does not receive its energy directly from the sun's light, nor yet to any extent from the sun's heat, but from the products formed by vegetable metabolism supplied as food, either directiy, as in the case of herbivora, or indirectly in the case of carnivora. The potential energy of these food stuffs is set free in the destructive metabolism of the animal cell already alluded to. But it must be always recollected that anabolism is not peculiar to vegetable, or katabolism to animal cells; both processes go on in each, but the chief function, as far as we know at present of the former, is to transform kinetic into potential energy, and of the latter to render potential energy kinetic, as in heat, motion, and electricity.

With reference to the food of plants, it should not be forgotten that some of the lowest forms of vegetable life, e.g., the bacteria, will live only in a highly albuminous medium, and in fact seem to require for their growth elements of food stuffs which we shall see later on are es. sential to animal life. In their metabolism, too, they very closely approximate to animal cells, not only requiring an atmosphere of oxygen, but giving out carbonic anhydride freely, and secreting and excreting many very complicated nitrogenous bodies, as well as forming protejd. carbohydrates, and fat, requiring heat but not light for the due performance of their functions. It must be added, however, that certain bacteria grow only in the absence of oxygen.

(3.) There is, commonly, a difference in general chemical composition between vegetables and animals, even in their lowest forms; for associated with the protoplasm of the former is a considerable amount of cellulose, a substance closely allied to starch and containing carbon, lydrogen, and oxygen only. 'The presence of cellulose in animals is much more rare 
than in vegetables, but there are many animals in which traces of it may be discovered, and some, the Ascidians, in which it is found in considerable quantity. The presence of starch in vegetable cells is very characteristic, though, as we have seen above, it is not distinctive, and a substance, glycogen, similar in composition to starch, is very common in the organs and tissues of animals.

(4.) Inherent power of movement is a quality which we so commonly consider an essential indication of animal nature, that it is difficult at first to conceive it existing in any other. The capability of simple motion is now known, however, to exist in so many vegetable forms, that it can no longer be held as an essential distinction between them and animals, and ceases to be a mark by which the one can be distinguished from the other. Thus the zoöspores of many of the Cryptogamia exhibit ciliary or amœboid movements of a like kind to those seen in amœbæ; and even among the higher orders of plants, many, e.g., Dioncea Muscipula (Venus's fly-trap), and Mimosa sensitiva (Sensitive plant), exhibit such motion, either at regular times, or on the application of external irritation, as might lead one, were this fact taken by itself, to regard them as sentient beings. Inherent power of movement, then, although especially characteristic of animal nature, is, when taken by itself, no proof of it. 


\section{CHAPTER II.}

\section{THE FUNCTIONS OF ORGANIZED CELIS.}

As we proceed upward in the scale of life from unicellular organisms, we find that another phenomenon is exhibited in the life history of the higher forms, namely, that of Development. An amœba comes into being derived from a previous amoba; it manifests the properties and performs the functions of its life which have been already enumerated; it grows, it reproduces itself, whereby several amœbæ result in place of one, and it dies. It cannot be said to develop, however, unless the formation of a nucleus can be considered as an indication of such a process. In the higher organisms it is different; they, indeed, begin as a single cell, but this cell on division and subdivision does not form so many

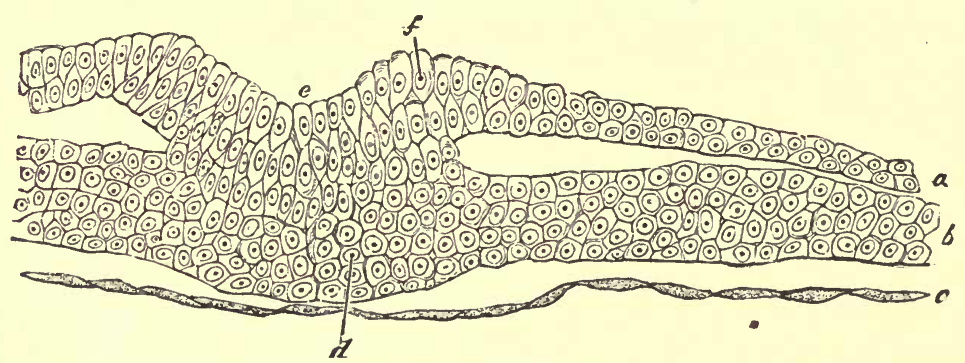

Fig. 18.-Transverse section through embryo chick (26 hours). $a$, Epiblast; $b$, mesoblast; $c$, hypoblast; $d$, central portion of mesoblast, which is here fused with epiblast; $e$, primitive groove; $f$, dorsal ridge. (Klein.)

independent organisms, but produces the material from which, by development, the complete and perfect whole is to be derived. Thus, from the spherical ovum, or germ, which forms the starting-point of animal life and which consists of a protoplasmic cell with a nucleus and nucleolus, in a comparatively short time, by the process of segmentation which has been already mentioned, a complete membrane of cells, polyhedral in shape from mutual pressure, called the Blastoderm, is formed, and this speedily divides into two and then into three layers, chiefly from the rapid proliferation of the cells of the first single layer. These layers are called the Epiblast, the Mesoblast, and the Hypoblast (fig. 18).

It is found in the further development of the animal that from each of these layers is produced a very definite part of its completed body. For example, from the cells of the epiblast are derived, among other 
structures, the skin and the central nervous system; from the mesoblast is derived the flesh or muscles of the body, and from the hypoblast the epithelium of the alimentary canal and some of the chief glands, and so on.

It is obvious that the tissues and organs so derived exhibit in a varying degree the primary properties of protoplasm. The muscles, for example, derived from certain cells of the mesoblast are particularly contractile and respond to stimuli readily, while the cells of the liver, although possibly contractile to a certain extent, have to do chiefly with the processes of nutrition.

Thus, in development, we see that as the cells of the embryo increase in number it speedily becomes necessary for the organism to depute to different groups of cells, or to their equivalents (i.e., to the tissues or organs to which they give rise), special functions, so that the various functions which the original cell may be supposed to discharge, and the various properties it may be supposed to possess, become divided up among various groups of resulting cells. The work of each group is specialized. As a result of this division of labor, as it may be called, these functions and properties are, as might be expected, developed and made more perfect, while the tissues and organs arising from each group of cells are developed also, with a view to the more convenient and effective exercise of their functions and employment of their properties.

In studying the functions of the human body it is necessary first of all to know of what it is composed, of what tissues and organs it is made up; this can of course only be ascertained by the dissection of the dead body, and thus it comes that Anatomy ( $\alpha^{2} \nu \tau \tau \xi \xi \mu \omega \omega$, to cut up) the science which treats of the structure of organized bodies, is closely associated

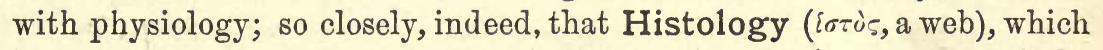
is especially concerned with the minute or microscopic structure of the tissues and organs of the body, and which is strictly speaking a department of anatomy, is usually included in works on physiology. There is much to be said in favor of such an arrangement, since it is impossible to consider the changes which take place in any tissue during life, apart from the knowledge of the structure of the tissues themselves. To understand the structure of the human body in an intelligent way, much help is obtained from the study of the structure of other animals, from the lowest to the highest, which is the province of Comparative Anatomy; while Embryology, which is concerned with the mode of origin of the various tissues in the embryo of each animal, and which is usually studied at the end of physiology, should from some points of view be considered as an introduction to the subject.

A second important essential to the right comprehension of the changes which take place in the living organism is a knowledge of the chemical composition of the body. Here, however, we can only deal 
with the chemical composition of the dead body, and it is as well at once to admit that there may be many chemical differences between living and not living tissues; but as it is impossible to ascertain the exact chemical composition of the living tissues, the next best thing which can be done is to find out as much as possible about the composition of the same tissues after they are dear. This is the assistance which the science of Chemistry can afford to the physiologist, and the same science is concerned with the composition of the ingesta and egesta, as well as with that of the fluids of the body.

Having mastered the structure and composition of the body, we are brought face to face with physiology proper, and have to investigate the vital changes which go on in the tissues, the various actions taking place as long as the organism is at work. The subject includes not only the observation of the manifest processes which are continually taking place in the healthy body, but the conditions under which these are brought about, the laws which govern them and their effeets.

We know from our study of biology that the cells of which the tissues are composed cannot live without food, both solid and liquid. In a complicated organism like the body of man, the tissues cannot supply themselves with food directly like the amoba, and so it comes that the various tissues are furnished with what they require by means of : fluid, the blool, which is carried to them in tubes or canals, the blootressels, which are distributed to every region of the body. In order that the blood shall reach all parts, the system of ressels in which it is contained is supplied with a central pumping organ, the heort. 'Then we find that as the oxygen, which is one of the requisites of the life of the tissues, and which is carried to the tissues by the blood, is used np, a special means is provided by respiration. or breathing, by means of which the blood is exposed to a new supply of oxygen of the air, which is taken irto special organs, the $7 u n g . s$, for the purpose, and which at the same time allows of the elimination of the carbonic anhydride the blood conveys from the tissues. Then again, as the solid food for the tissues cannot be conveyed in the blood in the exact form in which it is introduced into the body, a special and complicated apparatus is provided, that of digestion, by means of which the necessary changes are brought about in the food. The digested food is then absorbed and earried to the blood, either directly with little further change by means of another system of ressels in connection with the blood-ressels, the Tymphatic ressels, or after passing through a special organ or gland, the liver, by means of which some further changes take place. In the digestive apparatus we have the organs, the stomach and intestines, into which the food is received for the purpose of being acted upon by certain chemical agents, of which ferments, bodies which are capable of setting up profound changes in other bodies without themselves under- 
going change, are the most important; there is added the apparatus by means of which the altered food stuffs are absorbed or reach the two systems of blood-vessels already mentioned, and a muscular apparatus contained in the walls of the intestinal tube by means of which that part of the food which is not fit for absorption is removed from the body. In addition to this excretory apparatus we have another, the kidneys, which are concerned with the removal of certain substances from the blood which have served their purpose in the economy.

Then we have the muscular system, which by its special power of contraction is capable of bringing about all the movements of the body - those of the frame, the head, arms, legs, etc., as well as those of the heart, the vessels, the alimentary canal, and the like. The nervous system, by the aid of which the processes of the living body may be regulated and controlled. Lastly, we have a special system-that of the generative system, by means of which the reproduction of the species may take place.

To these subjects, the merest outline of which has been here sketched, our attention has to be given in the succeeding chapters, but it may be well to mention as a preliminary that the information about them which we have at our disposal has been derived from many sources, the chief of which are as follows:-

(1.) From actual observation of the various phenomena occurring in the human body from day to day, and from hour to hour, as, for example, the estimation of the amount and composition of the ingesta and egesta, the respiration, the beat of the heart, and the like;

(2.) From observations upon other animals, the bodies of which we are taught by comparative anatomy approximate to the human body in structure;

(3.) From observations of the changes produced by experiment upon the various processes in such animals;

(4.) From observations of the changes in the working of the human body produced by disease;

(5.) From observations upon the gradual changes which take place in the functions of organs when watched in the embryo from their earliest heginnings to their completed development.

In accordance with the plan sketched out above, the next chapter will be devoted to a consideration of the minute structure of the elementary tissues, and the one after that to a preliminary account of the chemical composition of the body. These two chapters will serve as an introduction to the study of the problems of physiology proper, which will be commenced in Chapter $\mathrm{V}$. 


\section{CHAPTER III.}

THE STRUCTURE OF THE ELEMENTARY TISSUES.

THe careful examination of the minute anatomy of the body has shown that there are certain elementary structures, of which, alone or when combined in varying proportions, the whole of the organs and tissues of the body are made up. These Elementary Tissues are four in number, called: (1.) The Epithelial; (2.) The Connective; (3.) The Muscular, and (4.) The Nervous. To these four, some would add a fifth, looking upon the Blood and Lymph, containing, as they do, formed elements in a fluid menstrum, as a distinct tissue.

All of these elementary tissues consist of cells and of their altered equivalents. It will be as well therefore to indicate some of the differences between the cells of the body. They are named in various ways, according to their shape, situation, contents, origin, and functions.

(a.) From their shape, cells are called spherical or spheroidal, which is the typical shape of the free cell; this may be altered to polyhedral when the pressure on the cells in all directions is nearly the same; of this the primitive segmentation cells afford an example. The discoid form is seen in blood-corpuscles, and the scale-like form in superficial epithelial cells. Some cells have a jagged outline and are then called prickle cells. Cells of cylindrical, conical, or prismatic form occur in various places in the body. Such cells may taper off at one or both ends into fine processes, in the former case being caudate, in the latter fusiform. They may be greatly elongated so as to become fibres. Cells with hair-like processes, or cilia, projecting from their free surfaces, are a special variety. The cilia vary greatly in size, and may even exceed in length the cell itself. Finally, cells may be branched or stellate with long outstanding processes.

(b.) From their situation cells may be called free, as in the blood, or combined, when connected together or with other elements to form organs and tissues.

(c.) From their contents cells are called, when containing fat for example, fat cells; when containing pigment, pigment cells, etc.

(d.) From their function cells are called secreting, protective, sensitive, contractile, and the like.

(e.) From their origin cells are called epiblastic and mesnblastic and hypoblastic. 
Modes of Connection.-Cells are connected together to form tissues in various ways.

(1.) By mean of a cementing intercellular substance. This is probably always present as a transparent, colorless, viscid, albuminous substance, even between the closely apposed cells of epithelium, while in the case of cartilage it forms the main bulk of the tissue, and the cells only appear as imbedded in, not as cemented together by, the intercellular substance. This intercellular substance may be either homogeneous or fibrillated. In many cases (e.g., the cornea) it can be shown to contain a number of irregular branched cavities, which communicate with each other, and in which branched cells lie: through these branching spaces nutritive fluids can find their way into the very remotest parts of a non-vascular tissue.

As a special variety of intercellular substance must be mentioned the basement membrane (membrana propria) which is found at the base of the epithelial cells in most mucous membranes, and especially as an investing tunic of gland follicles which determines their shape, and which may persist as a hyaline saccule after the gland cells have all been discharged.

(2.) By anastomosis of their processes. This is the usual way in which stellate cells, e.g. of the cornea, are united: the individuality of each cell is thus to a great extent lost by its connection with its neighbors to form a reticulum: as an example of a network so produced we may cite the stroma of lymphatic glands.

Sometimes the branched processes breaking up into a maze of minute fibrils, adjoining cells are connected by an intermediate reticulum: this is the case in the nerve cells of the spinal cord.

Derived Tissue-elements. - Besides the Cell, which may be termed the primary tissue-element, there are materials which may be termed secondary or derived tissue-elements. Such are Intercellular substance, Fibres, and Tubules.

a. Intercellular substance is probably in all cases directly derived from the cells themselves. In some cases (e.g. cartilage), by the use of reagents the cementing intercellular substance is, as it were, analyzed into various masses, each arranged in concentric layers around a cell or group of cells, from which it was probably derived.

$\beta$. Fibres. In the case of the crystalline lens, and of muscle both striated and non-striated, each fibre is simply a metamorphosed cell: in the case of a striped fibre, the elongation being accompanied by a multiplication of the nuclei. The various fibres and fibrillæ of connective tissue result from a gradual transformation of an originally homogeneous intercellular substance. Fibres thus formed may undergo great chemical as well as physical transformation: this is notably the case with yellow elastic tissue, in which the sharply defined elastic filres, 
possessing great power of resistance to reagents, contrast strikingly with the homogeneons matter from which they are derived.

r. Tubules, such as the capillary blood-vessels, which were originally supposed to consist of a structureless membrane, have now been proved to be composed of flat, thin cells, cohering along their edges.

Decay and Death of Cells. - There are two chief ways in which the comparatively brief existence of cells is brought to an end. (1) Mechanical abrasion, (2) Chemical transformation.

1. The various epithelia furnish abundant examples of mechanical abrasion. As it approaches the free surface, the cell becomes more and more flattened and scaly in form and more horny in consistency, till at length it is simply rubbed off as in the epidermis. Hence we find epithelial cells in the mucus of the mouth, intestine, and genito-urinary tract.

2. In the case of chemical transformation the cell-contents undergo a degeneration which, though it may be pathological, is very often a normal process.

Thus we have (a) fatty metamorphosis producing oil-globules in the secretion of milk, fatty degeneration of the muscular fibres of the uterus after the birth of the fotus, and of the cells of the Graafian follicle giving rise to the "corpus luteum." (b) Pigmentary degeneration from deposit of pigment, e.g. in the epithelinm of the air vesieles of the lungs. (c) Calcareous degeneration, which is common in the cells of many cartilages.

\section{The Epithelial Tissues.}

The term epithelium is applied to the cells covering the skin, the mucous and serous membranes, and to those forming a lining to other parts of the body as well as entering into the formation of glands. For example:-

Epithelium clothes (1) the whole exterior surface of the body, forming the epidermis with its appendages-nails and hairs; becoming continuous at the chief orifices of the body-nose, mouth, anus, and urethra -with the (2) epithelium which lines the whole length of the (3) respiratory, alimentary, and genito-urinary tracts, together with the ducts of their various glands. Epithelium also lines the cavities of (4) the brain and the central canal of the spinal cord, (5) the serous and synovial membranes, and (6) the interior of all blood-vessels and lymphatics.

Epithelial cells possess an intracellular and an intranuclear network (p. 9 and 10). When combined together to form a tissue, they are held together by a clear, albuminous, cement-substance, scanty in amount. The viscid semi-fluid consistency both of cells and intercellular substance permits such changes of shape and arrangement in the individual 
cells as are necessary if the epithelium is to maintain its integrity in organs the area of whose free surface is so constantly changing, as the stomach, lungs, etc. 'Thus, if there be but a single layer of cells, as in the epithelium lining the air vesicles of the lungs, the stretching of this membrane causes such a thinning out of the cells that they change their shape from spheroidal or short columnar, to squamous, and vice versâ, when the membrane shrinks.

Epithelial tissues are non-vascular, that is to say, do not contain blood-vessels, but in some varieties minute channels exist between the cells of certain layers through which they may be supplied with nourishment from the subjacent blood-vessels. Nerve fibres are supplied to the cells of many epithelia.

Epithelial tissue is classified according as the cells composing it are arranged in a single layer when it is simple, or in several layers when it is called stratified or laminated, or in two or three layers occupying a position between the other two forms, when it is termed transitional. Of each form, when there are several varieties, they are named according to the shape of the cells composing it.

\section{Classification of Epithelium.}

(a) Simple.-(1.) Squamous, scaly, parement, or tessellated; (2.) Spheroidal or glandular: (3.) Columnar, cylindrical, conical or gobletshaped; (4.) Ciliated.

(b) Transitional.

(c) Stratified.

(a) Simple Epithelium.

Squamous Epithelium.-This form of epithelium is found arranged as a single layer of flattened cells, as $(a)$ the pigmentary layer of the retina, and forms the lining of $(b)$ the interior of the serous and synovial sacs, $(c)$ the alveoli of the lungs, and $(d)$ of the heart, blood-and lymph-vessels. It consists of cells, which are flattened and scaly, with a more or less irregular outline.

In the pigment cells of the retina there is a deposit of pigment in the cell-substance. This pigment consists of minute molecules of a colored substance, melanin, imbedded in the cell-substance and almost concealing the nucleus, which is itself transparent.

In white rabbits and other albino animals, in which the pigment of the eye is absent, this layer is found to consist of colorless pavement epithelial cells.

The squamous epithelium which is found as a single layer lining the serous membranes, and the interior of blood-and lymphatic-vessels, is generally called by a distinct name-Endothelium. 
The presence of endothelium in any locality may be demonstrated by staining the part lined by it with silver nitrate, which brings into view

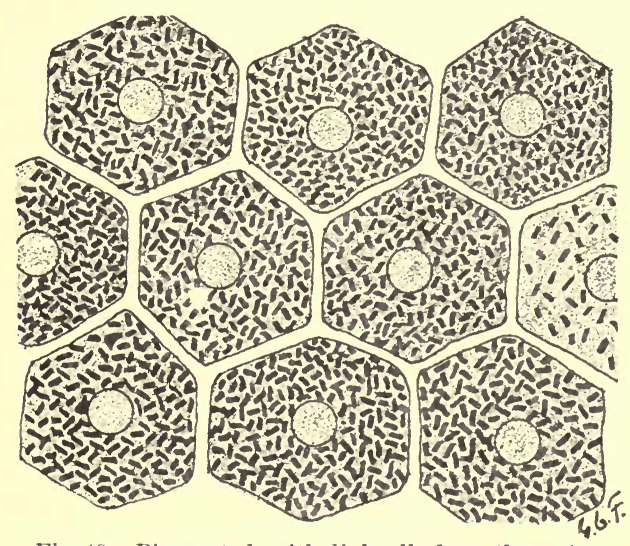

Fig. 19. - Pigmented epithelial cells from the retina.

the intercellular cement substance.

It is found that when a small portion of a perfectly fresh serous membrane for example (fig. 20), is immersed for a few minutes in a solution of silver nitrate, and exposed to the action of light, the silver is precipitated in some form in the intercellular cement substance, and the endothelial cells are thus mapped out by fine, dark, and generally sinuous lines of extreme delicacy. The cells vary in size and shape, and are as a rule irregular in ontline; those lining the interior of blood-

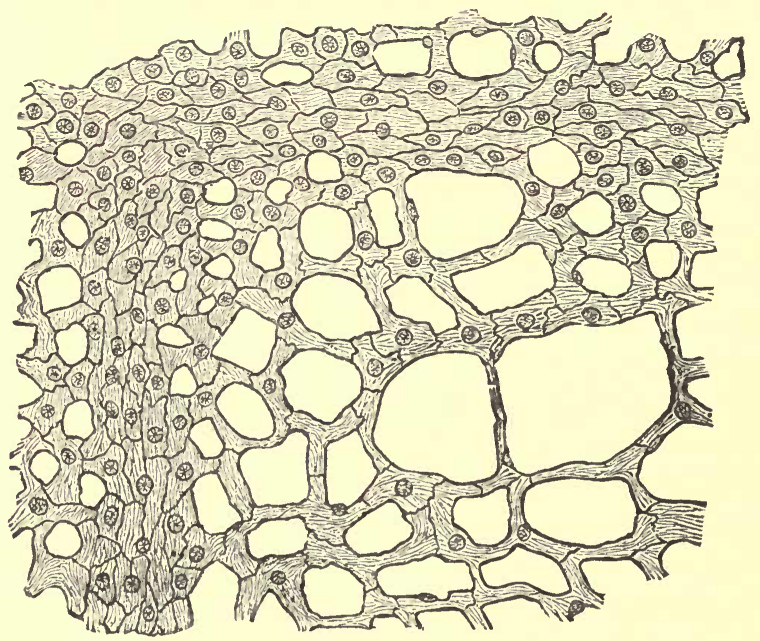

Fig. 20.-A piece of the omentum of a cat, stained in silver nitrate, $\times 100$. The tissue forms a "fenestrated membrane," that is to say, one which is studded with holes or windows. In the figure these are of various shapes and sizes, leaving trabeculæ, the basis of which is fibrous tissue. The trabeculæ are of various sizes and are covered with endothelial cells, the nuclei of which have been made evident by staining with hæmatoxylin after the silver nitrate has outlined the cells by staining the intercellular substance. (V. D. Harris.)

vessels and lymphatics being spindle-shaped with a very wavy outline. They inclose a clear, oval nucleus, which, when the cell is viewed in profile, is seen to project from its surface. The nuclei are not however evident unless the tissue which has been already stained in silver nitrate, 
is placed in another dye, such as hæmatoxylin, which has the property of selecting and staining its nuclei.

Endothelial cells in certain situations may be ciliated, e.g., those of the mesentery of the frog, especially during the breeding season.

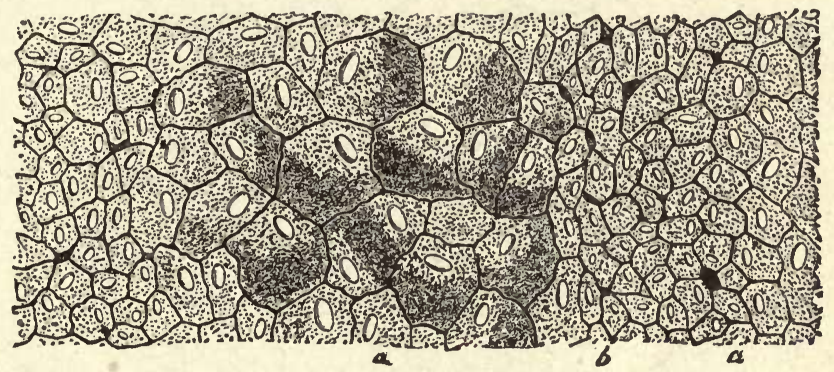

Fig. 21.-Abdominal surface of central tendon of the diaphragm of rabbit, showing the general polygonal shape of the endothelial cells: each cell is nucleated. (Klein.) $\times 300$.

Besides the ordinary endothelial cells above described, there are found on the omentum and parts of the pleura of many animals, little bud-like processes or nodules, consisting of small polyhedral granular cells, rounded on their free surface, which have multiplied very rapidly by division (figs. 22 and 23). These constitute what is known as germinating endothelium. The process of germination doubtless goes on in health, and the small cells which are thrown off in succession are

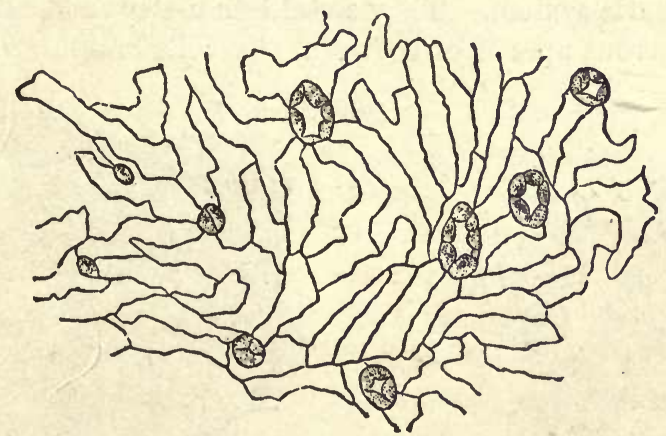

Fig. 22.-Peritoneal surface of a portion of the septum of the great lymph-sacs of frog. The stomata, some of which are open, some collapsed, are surrounded by endothelial cells. (Klein.) $\times 160$.

carried into the lymphatics and contribute to the number of the lymph corpuscles. The buds may be enormously increased both in number and size in certain diseased conditions.

On those portions of the peritoneum and other serous membranes in which lymphatics abound apertures (fig. 2\%) are found surrounded by small, more or less cubical, cells. These apertures are called stomata. They are particularly well seen in the anterior wall of the great lymph 
sac of the frog (fig. 22), and in the omentum of the rabbit. These are really the open mouths of lymphatic vessels or spaces, and through

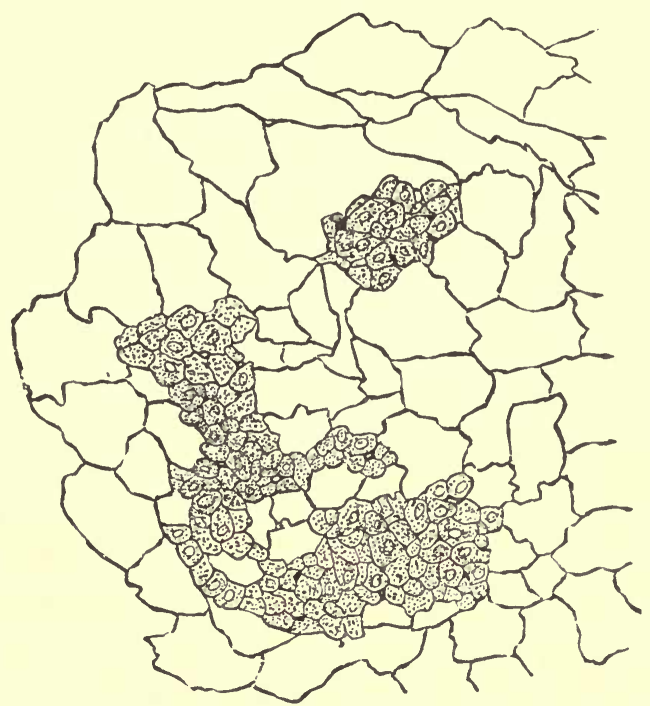

Fig. :33.-A portion of the great omentum of dog, which shows, among the flat endothelium o the surface, small and large groups of germinating endothelium between which are many stomata (Klein.) $\Varangle 300$.

them lymph-corpuseles and the serous fluid from the serous cavity pas: into the lymphatic system. 'They should be distinguished from smalle and more numerous apertures between the cells which are not lined by

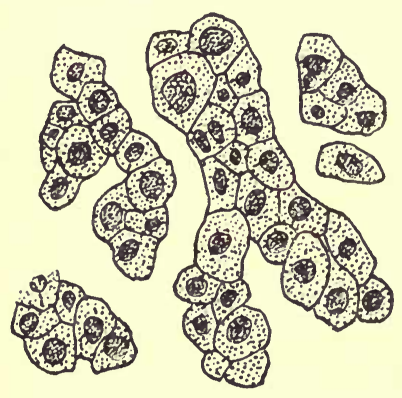

Fig. 24.

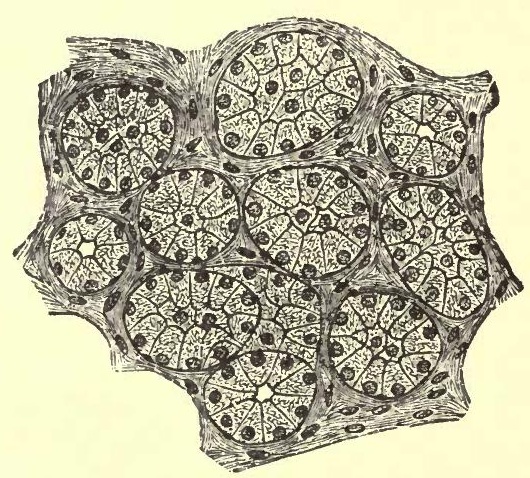

Fig. 25.

Fig. 24.-A small piece of the liver of the horse. (Cadiat.)

Fig. 25.-Glandular epithelium. Small lobule of a mucous gland of the tongue, showing nucleated glandular cells. $\times: 00 . \quad($ V. D. Harris.)

small cells, although the surrounding cells seem to radiate from them, filled up by intercellular substance or by processes of the cells underneath. These are called pseudo-stomata (fig. 23). 
In the neighborhood of the stomata the cells often manifest indications of germinating. They may be either large with two or more nuclei, or about half the size of the generality of cells. Germinating cells of this kind or of the kind above described, are generally very granular.

Splieroidal or glandular epithelium forms the active secreting agent in the glands, the cells are usually spheroidal, but may be polyhedral from mutual pressure, or even columnar; their protoplasm is generally occupied by the materials which the gland secretes.

Examples of glandular epithelium are to be found in the liver (fig. 24 ), in the secreting tubes of the kidney, and in the salivary (fig. 25) and gastric glands.

Columnar epithelium (fig. 28, $a$ and $b$ ) as a single layer lines (a.) the mucous membrane of the stomach and intestines, from the cardiac

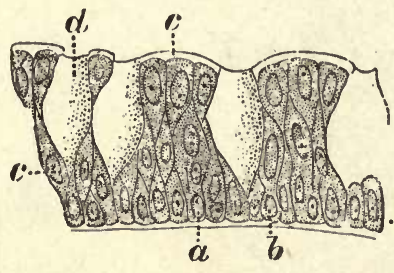

Fig. 26.
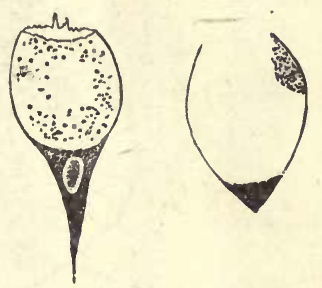

Fig. 2\%.

Fig. 36.-Columnar epithelial cells from the intestinal mucous membrane of a cat. $a$ and $b$, Small cells of the lowest layer; $c$, superficial layer; $d$, goblet cells. (Cadiat.)

Fig. 27.-Goblet cells. (Klein.)

orifice of the stomach to the anus, and (b.) wholly or in part the ducts of the glands opening on its free surface; also (c.) many gland-ducts in other regions of the body, e.g., mammary, salivary, etc.

Columnar epithelium consists of cells which are cylindrical or prismatic in form containing a large oval nucleus. They vary in size and also to a certain extent in shape; the outline is often jagged and irregular from pressure of neighboring cells, but one end of the cell is always narrower than the other, and by this narrower end the cell is as a rule attached to the membrane beneath. The intracellular and intranuclear networks are well developed, and in some cases the spongioplasm is arranged in rods or longitudinal striæ at one part of the cell, generally the attached border, as in some of the cells of the ducts of salivary glands.

This may also be the case with the columnar epithelial cells of the alimentary canal which possess an apparently structureless layer on their free surface: such a layer, appearing striated when viewed in section, is termed the "striated basilar border" (fig. 28, e).

The protoplasm of columnar cells may be vacuolated and may also 
contain fat or ather substances, of which the most likely is mucin or its antecedent mucigen, to be seen in the form of granules. It is to the presence of mucin that a curious transformation which columnar cells may undergo is due, and from which the alteration in their shape whereby "goblet-cells" are produced (fig. 2\%) arises. 'These altered cells are hardly ever evident in a perfoctly fresh specimen; but if such a specimen be watcherl for some time, little knobs are seen gradually to appear on the free surface of the epithelium and are finally detached; these consist of the cell-contents which are discharged by the open mouth of the goblet, leaving the nucleus surrounded by the remains of the protoplasm in its narrow stem.

This transformation is a normal process which is continually going on during life, the discharged cell-contents contributing to form mucus,

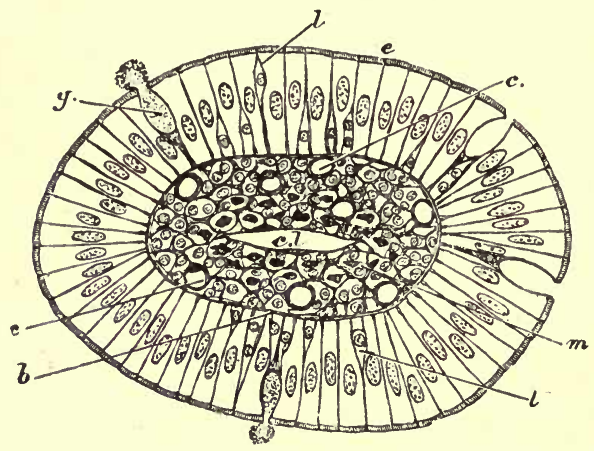

Fig. 28.-Cross section of a villus of the intestine. e. Columnar epithelium with striated border; $g$. goblet cell, with its mucus partly extruded: $i$, lymph-corpuscles between the epithelial cells; $b$, basement membrane; $c$, sections of blood capillaries; $m$, section of plain muscle fibres: c. $l$, central lacteal. (Schäfer.)

the cells themselves being supposed in many cases after discharge to recover their original shape.

Ciliated epithelium consists of cells which are generally cylindrical in form (figs. 29, 30), but may be spheroidal or even almost squamous.

'This form of epithelium lines-(a.) the mucous membrane of the respiratory tract beginning just beyond the nasal aperture and completely covering the nasal passages, except the upper part to which the olfactory nerve is distributed, and also the sinuses and ducts in connection with it and the lachrymal sac; the upper surface of the soft palate and the naso-pharynx, the Eustachian tube and tympanum, the larynx, except over the vocal cords, to the finest subdivisions of the bronchi. In part of this tract, however, the epithelium is in several layers, of which only the most superficial is ciliated, so that it should more accurately be termed transitional (p. 37) or stratified. (b.) Some portions of the generative apparatus in the male, viz., lining the "vasa efferentia" of the testicle, and their prolongations as far as the lower end of the 
epididymis; in the female (c.) commencing about the middle of the neck of the uterus, and extending throughout the uterus and Fallopian tubes to their fimbriated extremities, and even for a short distance on the peritoneal surface of the latter. (d.) The ventricles of the brain and the central canal of the spinal cord are clothed with ciliated epithelium in the child, but in the adult this epithelium is limited to the central canal of the cord. In the embryo the pharynx, osophagus, and part of the stomach may also be lined with ciliated cells. (e.) The excretory ducts of certain small glands in different localities. (f.) In certain animals, especially the lower vertebrates, ciliated cells line the beginning of the tubes of the kidneys.

The Cilia are fine hair-like processes which give the name to this variety of epithelium; they vary a good deal in size in different classes

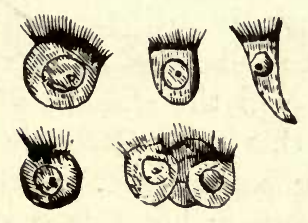

Fig. 29.

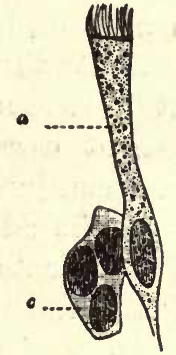

Fig. 30 .

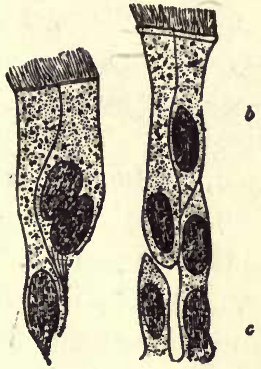

Fig. 29. - Spheroidal ciliated cells from the mouth of the frog. $\times 300$ diameters. (Sharpey.)

Fig. 30.-Ciliated epithelium from the human trachea. $a$, Large, fully formed cell. $b$, Shorter cell; $c$, developing cells with more than one nucleus. (Cadiat.)

of animals, being very much smaller in the higher than among the lower orders, in which they sometimes exceed in length the cell itself.

The number of cilia' on any one cell ranges from ten to thirty, and those attached to the same cell are often of different lengths, in the human trachea measuring $\frac{1}{4000}$ to $\frac{1}{2500}$ of an inch, but nearly ten times the length in the cells of the epididymis.

The cilia themselves are fine rounded or flattened processes, apparently homogeneous, pointed toward their free extremities. According to some observers these processes are connected through intervening knob-like junctions with longitudinal fibres which pass to the other end of the cell, but which are not connected with the nucleus.

When living ciliated epithelium, e.g., from the gill of a mussel, or oyster, or from the mouth of the frog, or from a scraping from a polypus from the human nose, is examined under the microscope in a drop of 0.6 per cent solution of common salt (normal saline solution), the cilia are seen to be in constant rapid motion, each cilium being fixed at one end, and swinging or lashing to and fro. The general impression given 
to the eye of the observer is very similar to that produced by waves in a field of corn, or swiftly ruuning and rippling water, and the result of their movement is to produce a continuous current in a definite direction, and this direction is invariably the same on the same surface, being always, in the case of a cavity, toward its external orifice.

Ciliary Motion.-Ciliary, which is closely allied to amoboid and muscular motion, is alike independent of the will, of the direct influence of the nervous system, and of muscular contraction. It may contiune for several hours after death or removal from the body, provided the portion of tissue under examination be kept moist. Its independence of the nervous system is shown also in its occurrence in the lowest invertebrate animals apparently unprovided with anything analogous to a nervous system, in its persistence in animals killed by prussic acid, by nareotic or other poisons, and after the lirect application of nareoties, such as morphia, opium, and belladomna, to the ciliary surface, or of electricity through it. The vapor of chloroform arrests the motion; but it is renewed on the discontinuance of the application. The movement ceases when the cilia are deprived of oxygen, although it may continue for a time in the absence of free oxygen, but is revived on the admission of this gas. Carbonic acid stops the movement. The contact of various substances, e.y., bile, strong acids, and alkalies, will stop the motion altogether; but this seems to depend chiefly on destruction of the delicate substance of which the cilia are composed. Temperatures above $45^{\circ} \mathrm{C}$. and below $0^{\circ} \mathrm{C}$. stop the movement, whereas moderate heat and dilute alkalies are favorable to the action and revive the movement after temporary cessation. The exact explanation of ciliary movement is not known; whatever may be the exact eause, however, at any rate the movement must depend upon some changes going on in the cell to which the cilia are attached, as when the latter are eut off from the eell the movement ceases, and when severed so that a portion of the ciliu are left attached to the cell, the attached and not the severed portions continue the movement. Some authorities consider it due to actual contraction of the cilia themselves; others assert that it is caused by movements in the cell protoplasm acting upon the rootlets of the cilia. Schäfer suggests a very plausible explanation, viz., that a cilium is either a curved hollow extension of the cell, which is filled by hyaloplasm and invested by a delicate membrane, or else a straight one whose investing membrane is thicker (or otherwise less extensible) along one side than along the other. In either case a rhythmic flowing of the hyaloplasm into and out of the cilium wonld cause its alternate flexion and extension.

As a special subdivision of ciliary action may be mentioned the motion of spermatozoa, which may be regarded as cells with a single cilium. 


\section{(b) Transitional Epithelium.}

This term has been applied to cells, which are neither arranged in a single layer, as is the case with simple epithelium, nor yet in many. superimposed strata as in laminated; in other words, it is employed when epithelial cells are found in two, three, or four superimposed layers.

The upper layer may be either single columnar, columnar ciliated, or squamous. When the upper layer is columnar or ciliated the second layer consists of smaller cells fitted into the inequalities of the cells above them, as in the trachea (fig. 30 ).

The epithelium which is met with lining the urinary bladder and ureters is, however, the transitional par excellence. In this variety.there

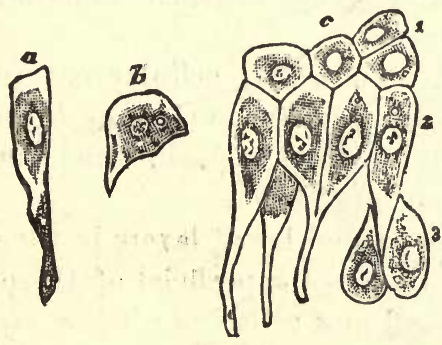

Fig. 31.

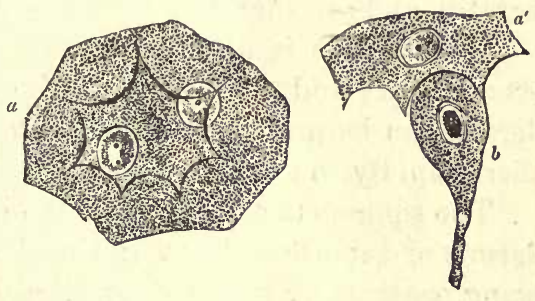

Fig. 32.

Fig. 31.-Epithelium of the bladder. $a$, One of the cells of the first row; $b$, a cell of the second row; $c$, cells in situ, of first, second, and deepest layers. (Obersteiner.)

Fig. 32. - Transitional epithelial cells from the inucous membrane of the bladder of a rabbit. Highly magnified. $a$, Large fiattened cell of superficial layer; $a^{\prime}$, similar cell in profile; $b$, pearshaped cell of second layer. (Klein.)

are two or three layers of cells, the upper being more or less flattened according to the full or collapsed condition of the organ, their under surface being marked with one or more depressions, into which the heads of the next layer of club-shaped cells fit. Between the lower and narrower parts of the second row of cells are fixed the irregular cells which constitute the third row, and in like manner sometimes a fourth row (fig. 31). It can be easily understood, therefore, that if a scraping of the mucous membrane of the bladder be teased, and examined under the microscope, cells of a great variety of forms may be made out (fig. 32). Each cell contains a large nucleus and the larger and superficial cells often possess two.

\section{(c) Stratified Epithelium.}

The term stratified epithelium is employed when the cells forming the epithelium are arranged in a considerable number of superimposed layers. The shape and size of the cells of the different layers, as well as the number of the layers, vary in different situations. Thus the 
superficial cells are as a rule of the squamous, or scaly rariety, and the deepest of the columnar form.

The cells of the intermediate layers are of different shapes, but those of the middle layers are more or less rounded. The superficial cells are broad and overlap by their edges (figs. 33 and 34 ). Their chemical com-

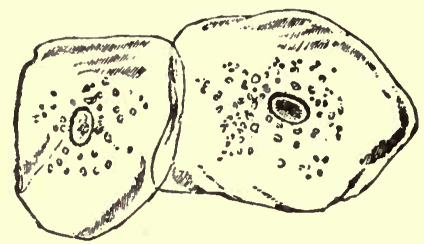

Fig. 33.-Squamous epithelium scales from the inside of the mouth. $\times 260, \quad$ (Henle.)

position is different from that of the underlying cells, as they contain keratin, and are therefore horny in character.

The nucleus is often not apparent. The really cellular nature of even the dry and shrivelled scales cast off from the surface of the epidermis can be proved by the application of caustic potash, which causes them rapidly to swell and assume their original form.

The squamous cells exist in the greatest number of layers in the epidermis or superficial part of the skin; the most superficial of these are being continually removed by friction, and new cells from below supply the place of those cast off.

The intermediate cells approach more to the flat variety the nearer they are to the surface, and to the columnar as they approach the lowest

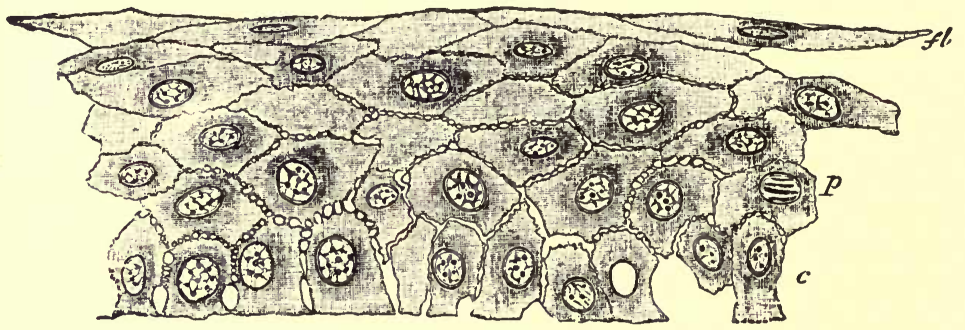

Fig. 34-Vertical section of the stratified epithelium covering the front of the cornea. Highly magnified. (Schäfer.) $c$, Lowermost columnar cells; $p$, polygonal cells above these:

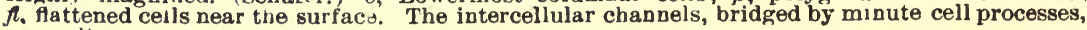
are well seen.

layer. There may be considerable intercellular intervals; and in many of the deeper layers of epithelium in the mouth and skin, the outline of the cells is very irregular, in consequence of processes passing from cell to cell across these intervals.

Such cells (fig. 35) are termed "ridge and furrow," "cogged" or "prickle" cells. These " prickles" are prolongations of the intracellular network which run across from cell to cell, thus joining them together, 
the interstices being filled by the transparent intercellular cement-substance. When this increases in quantity in inflammation the cells are pushed further apart, and the connecting fibrils or "prickles" elongated and therefore more clearly visible.

The columnar cells of the deepest layer are distinctly nucleated; they multiply rapidly by division; and as new cells are formed beneath, they press the older cells forward to be in turn pressed forward themselves toward the surface, gradually altering in shape and chemical composition until they are cast off from the surface.

Stratified epithelium is found in the following situations: (1.) Form. ing the epidermis, covering the whole of the external surface of the body; (2.) Covering the mucous membrane of the nose, tongue, mouth, pharynx, and osophagus; (3.) As the conjunctival epithelium, covering the cornea; (4.) Lining the vagina and the vaginal part of the cervix uteri.

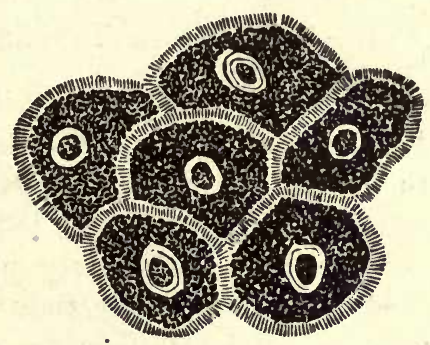

Fig. 35. - Jagged cells from the middle layers of pavement epithelium, from a vertical section of the gum of a new-born infant. (Klein.)

Functions of Epithelium.--According to function, epithelial cells may be classified as: (1.) Protective, e.g., in the skin, mouth, bloodvessels, etc. (2.) Protective and moving-ciliated epithelium. Secreting-glandular epithelium; or, Secreting formed elements-epithelium of testicle secreting spermatozoa. (4.) Protective and secreting, e.g., epithelium of intestine. (5) Sensorial, e.g., olfactory cells, rods and cones of retina, organ of Corti.

Epithelium forms a continuous smooth investment over the whole body, being thickened into a hard, horny tissue at the points most exposed to pressure, and developing various appendages, such as hairs and nails, whose structure and functions will be considered in a future chapter. Epithelium lines also the sensorial surfaces of the eye, ear, nose, and mouth, and thus serves as the medium through which all impressions from the external world-touch, smell, taste, sight, hearing-reach the delicate nerve endings, whence they are conveyed to the brain.

The ciliated epithelium which lines the air-passages serves not only as a protective investment, but also by the movements of its cilia promotes currents of the air in the bronchi and bronchia, and is enabled to propel fluids and minute particles of solid matter so as to aid thoir ex- 
pulsion from the body. In the case of the Fallopian tube, rhis agency assists the progress of the ovum toward the cavity of the uterus. Of the purposes served by eilia in the ventricles of the brain nothing is known.

The epithelium of the various glands, and of the whole intestinal tract, has the power of secretion, i.e., of chemically transforming certain materials of the blood; in the case of mucus and saliva this has been proved to involve the transformation of the epithelial cells themselves; the cell-substance of the epithelial cells of the intestine being discharged by the rupture of their envelopes, as mucus.

Epithelium is likewise concerned in the processes of transudation, diffusion, and absorption.

It is constantly being shed at the free surface and reproduced in the deeper layers. The various stages of its growth and development can be well seen in a section of any laminated epithelium such as the epidermis.

\section{The Connective Tissues.}

This group of tissues forms the Skeleton with its various connections - bones, cartilages, and ligaments - and also affords a supporting framework and inrestment to the various organs composed of nervous, muscular, and glandular tissue. Its chief function is the mechanical one of support, and for this purpose it is so intimately interwoven with nearly all the textures of the body that if all other tissues could be remored, and the connective tissues left, we should have a wonderfully exact model of almost every organ and tissue in the body, correct even to the smallest minutiæ of structure.

\section{Structure of Connective Tissues in General.}

Connective tissue is made up of two chief elements, namely, cells and intercellular substance.

(A.) Cells.-The cells are of two kinds:

(a.) Fixed Cells.- These are of a flattened shape, with branched processes, which are often united together to form a network: they can be most readily observed in the cornea, in which they are arranged, layer above layer, parallel to the free surface. They lie in spaces in the intercellular or ground substance, which are of the same shape as the cells they contain, but rather larger, and which form by anastomosis a system of branching canals freely communicating (fig. 36).

To this class of cells belong the flattened tendon corpuscles which are arranged in long lines or rows parallel to the fibres (fig. 42).

These branched cells, in certain situations, contain a number of pigment granules, giving them a dark appearance; they form one variety of pigment cell. Branched pigment cells of this kind are found in the outer layers of the choroid (fig. 37). In many of the lower animals, 
such as the frog, they are found widely distributed, not only in the skin, but also in internal parts, e.g., the mesentery and sheaths of bloodvessels. In the web of the frog's foot such cells may be seen with pigment granules evenly distributed throughout the body of the cell and its processes; but under the action of light, electricity, and other stimuli, the pigment granules become massed in the body of the cell, leaving the processes quite hyaline; if the stimulus be removed, they will gradually be distributed again throughout the processes. Thus the skin in the frog is sometimes uniformly dusky, and sometimes quite light-colored, with isolated dark spots. In the choroid and retina the pigment cells absorb light.

(b.) Amoboid Cells, of an approximately spherical shape; they have a great general resemblance to colorless blood-corpuscles, with which

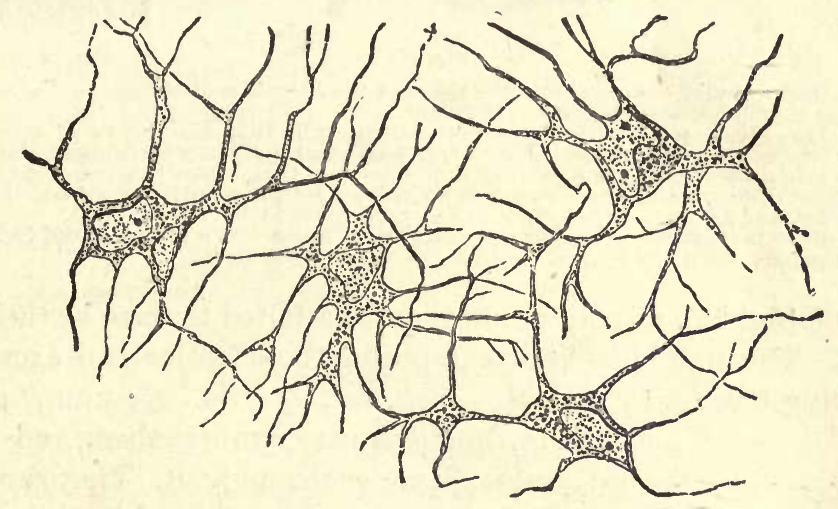

Fig. 36.-Horizontal preparation of the cornea of frog, stained in gold chloride; showing the network of branched cornea corpuscles. The ground substance is completely colorless. $\times 400$. (Klein.)

some of them are probably identical. They consist of finely granular nucleated protoplasin, and have the property, not only of changing their form but also of moving about, hence they are termed migratory. They are readily distinguished from the branched connective-tissue corpuscles by their free condition, and the absence of processes. Some are much larger than others, and are found especially in the sublingual gland of the dog and guinea-pig, and in the mucous membrane of the intestine. A second variety of these cells called plasma cells are larger than the amœboid cells, apparently granular, less active in their movements. They are chiefly to be found in the inter-muscular septa, in the mucous and sub-mucous coats of the intestine, in lymphatic glands, and in the omentum.

(B.) Intercellular Substance.-This may be fibrillar, as in the fibrous tissues, and in certain varieties of cartilage; or homogeneous, as in hyaline cartilage. 
The fibres composing the former are of two kinds-(a.) White fibres (b.) Yellow elastic fibres.

(a.) White Fibres.-These are arranged parallel to each other in wavy bundles of various sizes: such bundles may either have a parallel ar-

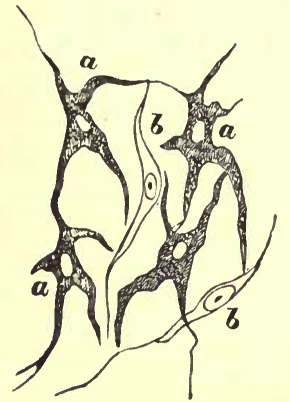

Fig. 37.

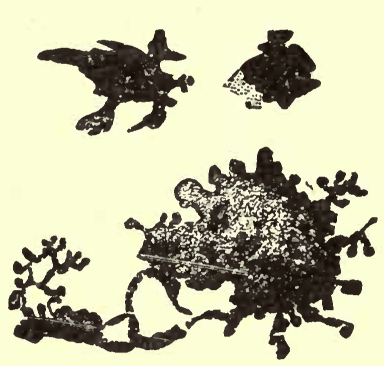

Fig. 38.

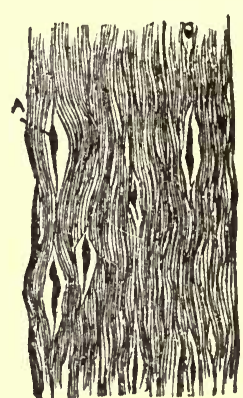

Fig. 39.

Fig. 37.-Ramified pigment cells from the tissue of the choroid coat of the eye. $\times 350 . \quad a$, Cell with pigment; $b$, colorless fusiform cells. (Kölliker.)

Fig. 38.-Flat, pigmented, branched connective-tissue cells from the sheath of a large bloodvessel of the frog's mesentery: the pigment is not distributed uniformly throughout the substance of the larger cell, consequently some parts of it look blacker than others (uncontracted state). In the two smaller cells most of the pigment is withdrawn into the cell-body, so that they appear smaller, blacker, and less branched. $\times 350$. (Klein and Noble Smith.)

Fig. 39.-Fibrous tissue of cornea, showing bundles of fibres with a few scattered fusiform cells (A) lying in the inter-fascicular spaces. $\times 400$. (Klein and Noble Smith.)

rangement (fig. 39), or may produce quite a felted texture by their interlacement. The individual fibres composing these fasciculi are exceedingly fine, varying from $\frac{1}{50000}$ to $\frac{1}{25000}$ inch, i.e., $\frac{1}{2000}$ to $\frac{1}{1000} \mathrm{~mm}$. , $^{*}$ or 0.5 to

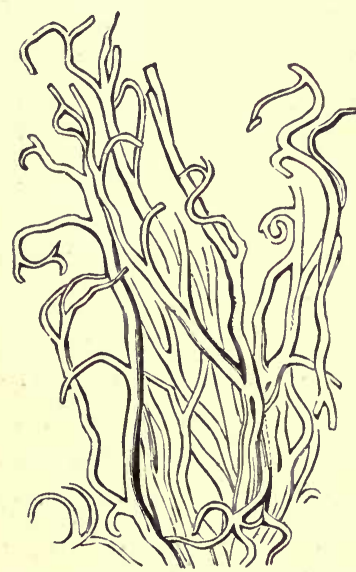

Fig. 40.-Elastic fibres from the ligamenta subflava. $\times 200$ (Shurpey.) $1 \mu$, homogeneous, unbranched, and of the same diameter throughout. They can readily be isolated by macerating a portion of white fibrous tissue (e.g., a small piece of tendon) for a short time in lime, or baryta-water, or in a solution of common salt, or of potassium permanganate: these reagents possess the power of dissolving the cementing interfibrillar substance and of thus separating the fibres from each other. By prolonged boiling the fibres yield gelatin.

(b.) Yellow Elastic Fibres (fig. 40) are of all sizes, from excessively fine fibrils, $2 \frac{1}{2} \frac{1}{000}$ inch, up to fibres of considerable thickness, $\frac{1}{4000}$ inch (i.e., from about $1 \mu$ to $6 \mu$ ): they are distinguished from white fibres by the following characters: (1.) Their great power of resistance even to the prolonged action of chemical reagents, e.g., caustic soda, acetic acid, etc. (2.) Their well-defined outlines. (3.)

* $\frac{1}{1000}$ millimetre $=1$ micron, which is represented by the Greek $\mu$. 
Their great tendency to branch and to form networks by anastomosis. (4.) Their twisted corkscrew-like appearance, and that their free ends usually curl up. (5.) Their yellowish tint and considerable elasticity. (6.) Their resistance to hæmatoxylin and similar reagents, and their affinity for magenta and other aniline staining colors.

These fibres yield on boiling not gelatin, but a gelatinous substance called elastin.

The chief varieties of connective tissues may be thus classified:

I. The Fibrous Connective Tissues.

A.-Chief Forms.

a. White fibrous.

b. Elastic.

c. Areolar.

B.-Special Varieties.

a. Gelatinous.

b. Adenoid or Retiform.

c. Adipose.

II. Cartilage.

III. Bone and dentine.

\section{Fibrous Connective Tissues.}

A.-Chief Forms.-(a.) White Fibrous Tissue.

Distribution.-It is found typically in tendon; also in ligaments, in the periosteum and perichondrium, the dura mater, the pericardium, the sclerotic coat of the eye, the fibrous sheath of the testicle; in the fasciæ and aponeuroses of muscles, and in the sheaths of lymphatic glands.

Structure.-To the naked eye tendons and many of the fibrous membranes, when in a fresh state, present an appearance as of watered silk. This is due to the arrangement of the fibres in wavy parallel bundles. Under the microscope the tissue appears to consist of long, often parallel, bundles of fibres of different sizes. The fibres of the same bundle now and then intersect each other. The cells in tendons (fig. 42) are arranged in long chains in the ground substance separating the bundles of fibres, and are more or less regularly quadrilateral with large round nuclei containing nucleoli, which are generally placed so as to be contiguous in two cells. Fach of these cells consist of a thick body, from which processes pass in various directions into, and partially fil! up the spaces between, the bundles of fibres. The rows of cells are separated from one another by lines of cement substance. The cel! spaces can be brought into view by silver nitrate. The cells are gener- 
ally marked by one or more lines or stripes when viewed longitudinally. This appearance is really produced by the wing-like processes of the cell which project away from the chief part of the cell in different directions. These processes not being in the same plane as the body of the cell are out of focus and give rise to these bright stripes are looked at from above and are in focus.

The branched character of the cells is seen in transverse section in fig. 43.

\section{(b) Yellow Elastic Tissue.}

Distribution.--In the ligamentum nuchæ of the ox, horse, and many other animals; in the ligamenta subflava of man; in the arteries, constituting the fenestrated coat of Henle; in veins; in the lungs and tra-

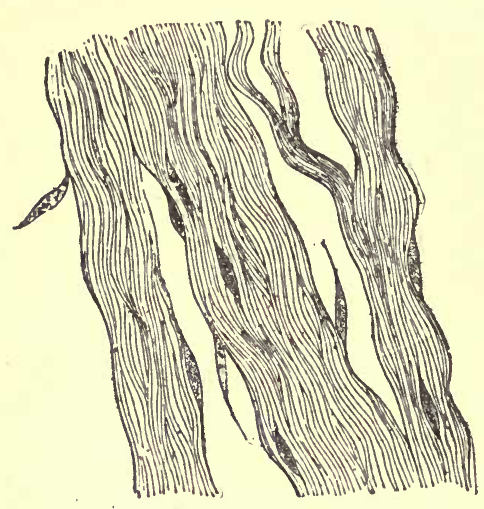

Fig. 41.

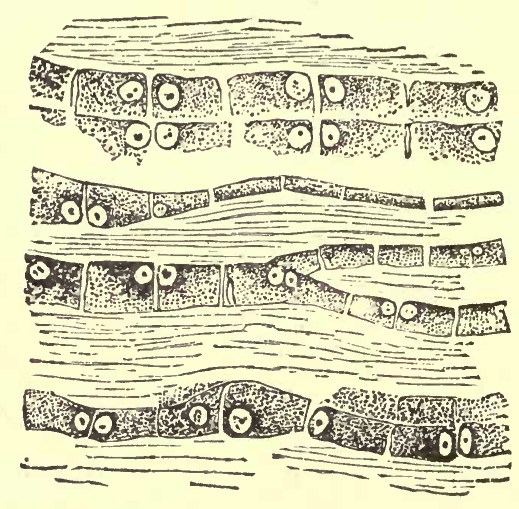

Fig. 42.

Fig. 41.-Mature white fibrous tissue of tendon, consisting mainly of fibres with a few scattered fusiform cells. (Stricker.)

Fig. 42.-Caudal tendon of young rat, showing the arrangement, form, and structure of the tendon cells. $\times 300$. (Klein.)

chea; in the stylo-hyoid, thyro-hyoid, and crico-thyroid ligaments; in the true vocal chords; and in areolar tissue.

Structure.--Elastic tissue occurs in various forms, from a structureless, elastic membrane to a tissue whose chief constituents are bundles of fibres crossing each other at different angles; when seen in bundles elastic fibres are yellowish in color, but individual fibres are not so distinctly colored. The varieties of the tissue may be classified as follows:

(a.) Fine elastic fibrils, which branch and anastomose to form a network: this variety of elastic tissue occurs chiefly in the skin and mucous membranes, in subcutaneous and submucous tissue, in the lungs and true vocal cords.

(b.) Thick fibres, sometimes cylindrical, sometimes flattened like tape, which branch, anastomose and form a network: these are seen most typically in the ligamenta subflava and also in the ligamentum 
nuchæ of such animals as the ox and horse, in which that ligament is largely developed (fig. 40).

(c.) Elastic membranes with perforations, e.g., Henle's fenestrated membrane: this variety is found chiefly in the arteries and veins.

(d.) Continuous, homogenous elastic membranes, e.g., Bowman's anterior elastic lamina and Descemet's posterior elastic lamina, both in the cornea.

A certain number of flattened connective-tissue cells are found in the ground substance between the elastic fibres which make up this variety of connective tissue.

(c.) Areolar Tissue.

Distribution.-This variety of fibrous tissue has a very wide distribu-

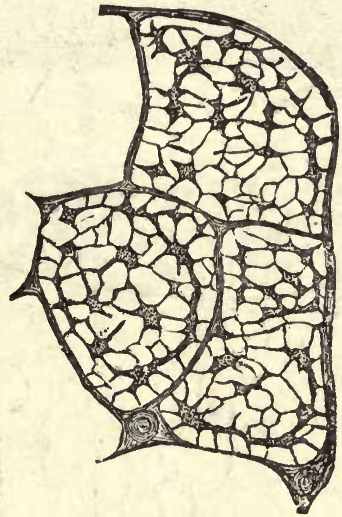

Fig. 43.

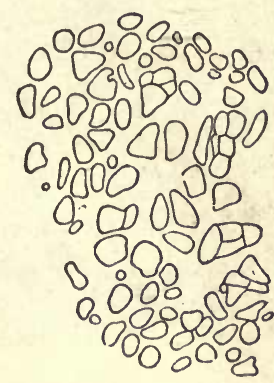

Fig. 44.

Fig. 43.-Transverse section of tendon from a cross section of the tail of a rabbit, showing sheatl, fibrous septa, and branched connective-tissue corpuscles. The spaces left white in the drawing represent the tendinous fibres in transverse section. $\times 250$. (Klein.) Stöhr.)

Fig. 44. - Transverse section of a portion of lig. nuchæ, showing the outline of the fibres. (After

tion and constitutes the subcutaneous, subserous, and submucous tissue. It is found in the mucous membranes, in the true skin, and in the outer sheaths of the blood-vessels. It forms sheaths for muscles, nerves, glands, and the internal organs, and penetrating into their interior, supports and connects the finest parts.

Structure.-To the naked eye it appears, when stretched out, as a fleecy, white, and soft meshwork of fine fibrils, with here and there wider films joining in it, the whole tissue being evidently elastic. The openness of the meshwork varies with the locality from which the specimen is taken. Under the microscope it is found to be made up of fine white fibres, which interlace in a most irregular manner, together with a variable number of elastic fibres. On the addition of acetic acid, the white fibres swell up, and become gelatinous in appearance; but as the elastic fibres resist the action of the acid, they may still be seen arranged in 
Structure.-Adenoid or retiform tissue consists of a very delicate network of minute fibrils, formed originally by the union of processes of branched connective-tissue corpuscles, the nuclei of which, however, are visible only during the early periods of development of the tissue (fig. 46). The network of fibrils is concealed by being covered with flattened connective-tissue corpuscles, which may be readily dissolved in caustic potash, leaving the network bare. The network consists of white fibres, the interstices of which are filled with lymph corpuscles. The cement substance of adenoid tissue is very fluid.

Some authors make a distinction between retiform and adenoid tissues, the former being the meshwork, and the latter the meshwork with its contained lymph cells.

Development of Fibrous Tissues.-In the embryo the place of the fibrous tissues is at first occupied by a mass of roundish cells, derived from the "mesoblast."

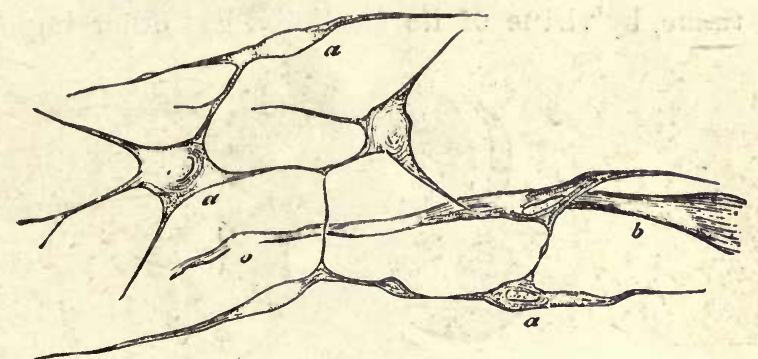

Fin. 4\%.-Portion of submucous tissue of gravid uterus of sow. $a$, Branched cells, more or less spirdle-shaped; $b$, bundles of connective tissue. (Klein.)

These develop either into a network of branched cells or into groups of fusiform cells (fig. 47).

The cells are imbedded in a semi-fluid albuminous substance derived either from the cells themselves or from the neighboring blood-vessels; this afterward forms the cement substance. In it fibres are developed, either by some of the cells becoming fibrils, the others remaining as connective-tissue corpuscles, or by the fibrils being developed from the outside layers of the protoplasm of the cells, which grow up again to their original size and remain imbedded among the fibres. The process gives rise to fibres arranged in the one case in interlacing networks (areolar tissue), in the other in parallel bundles (white fibrous tissue). In the mature forms of purely fibrous tissue not only the remnants of the cellsubstance, but even the nuclei, may disappear. The embryonic tissue, from which elastic fibres are developed, is composed of fusiform cells, and a structureless intercellular substance by the gradual fibrillation of which elastic fibres are formed. The fusiform cells dwindle in size and eventually disappear so completely that in mature elastic tissue hardly a trace of them is to be found: meanwhile the elastic fibres steadily increase in size. 
Another theory of the development of the connective-tissue fibrils supposes that they arise from deposits in the intercellular substance and not from the cells themselves; these deposits, in the ease of elastic fibres, appearing first of all in the form of rows of granules, which, joining together, form long fibrils. It seems probable that even if this view be correct, the cells themselves have a considerable influence in the production of the deposits outside them.

Functions of Areolar and Fibrous Tissue.-The main function of connective tissue is mechanical rather than vital: it fulfils the subsidiary but important use of supporting and connecting the various tissues and organs of the body.

In glands the trabeculæ of connective tissue form an interstitial framework in which the parenehyma or seereting gland-tissue is lodged: in muscles and nerves the septa of connective tissue support the bundles of fibres which form the essential part of the structure.

Elastic tissue, by virtue of its elasticity, has other important uses:

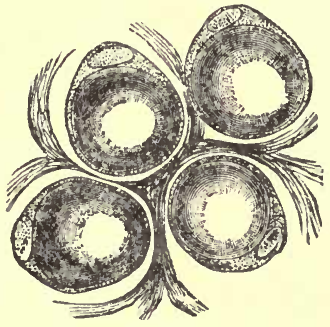

Fig. 48.-Ordinary fat cells of a fat tract in the omentum of a rat. (Klein.)

these, again, are mechanical rather than vital. 'Thus the ligamentum nuche of the horse or ox acts very much as an India-rubber band in the same position wonld; being stretched when the heal is lowered for feeding or other purposes and aiding the muscles materially afterward by its contraction, in raising the head to its normal position and keeping it there.

\section{(c.) Adipose Tissue.}

Distribution.- In almost all regions of the human body a larger or smaller quantity of adipose or fatty tissue is present; the chief exceptions being the subcutaneous tissue of the eyelids, penis, and serotum, the nymphæ, and the eavity of the cranium. Adipose tissue is also absent from the substance of many organs, as the lungs, liver, and others.

Fatty matter, but not in the form of a distinct tissue, is also widely present in the body, e.g., in the liver and brain, and in the blood and chyle.

Adipose tissue is almost always found seated in areolar tissue, and forms in its meshes little masses of unequal size and irregular shape, to which the term lobules is commonly applied. 
Structure.-Under the microscope adipose tissue is found to consist essentially of little vesicles or cells which present dark, sharply-defined edges when viewed with transmitted light: they are about $\frac{1}{400}$ or $\frac{1}{500}$ of an inch in diameter, each consisting of a structureless and colorless membrane or bag formed of the remains of the original protoplasm of the cell, filled with fatty matter, which is liquid during life, but in part solidified after death (fig. 48). A nucleus is always present in some part or other of the cell-protoplasm, but in the ordinary condition of the cell it is not easily or always visible (fig. 49).

This membrane and the nucleus can generally be brought into view by staining the tissue: it can be still more satisfactorily demonstrated by extracting the contents of the fat-cells with ether, when the shrunken, shrivelled membranes remain behind. By mutual pressure, fat-cells

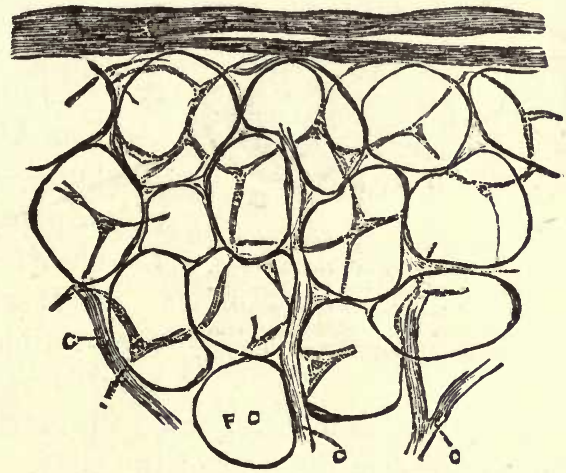

Fig. 49.-Group of fat cells (F c) with capillary vessels (c). (Noble Smith.)

come to assume a polyhedral figure (fig. 49). When stained with osmic acid fat-cells appear black.

The ultimate cells are held together by capillary blood-vessels (fig. 50); while the little clusters thus formed are grouped into small masses, and held so, in most cases, by areolar tissue.

The oily matter contained in the cells is composed chiefly of the compounds of fatty acids with glycerin, which are named olein, stearin, and palmitin.

Development of Adipose Tissue.-Fat cells are developed from connective-tissue corpuscles: in the infra-orbital connective-tissue cells may be found exhibiting every intermediate gradation between an ordinary branched connective-tissue corpuscle and mature fat-cell. The process of development is as follows: a few small drops of oil make their appearance in the protoplasm and by their confluence a larger drop is produced (fig. 51): this gradually increases in size at the expense of the original protoplasm of the cell, which becomes correspondingly diminished in quantity till in the mature cell it only forms a thin crescentic 
film, closely pressed against the cell-wall, and with a nucleus imbedded in its substance (figs. 48 and 49 ).

Under certain circumstances this process may be reversed and fatcells may be changed back into connective-tissue corpuscles.

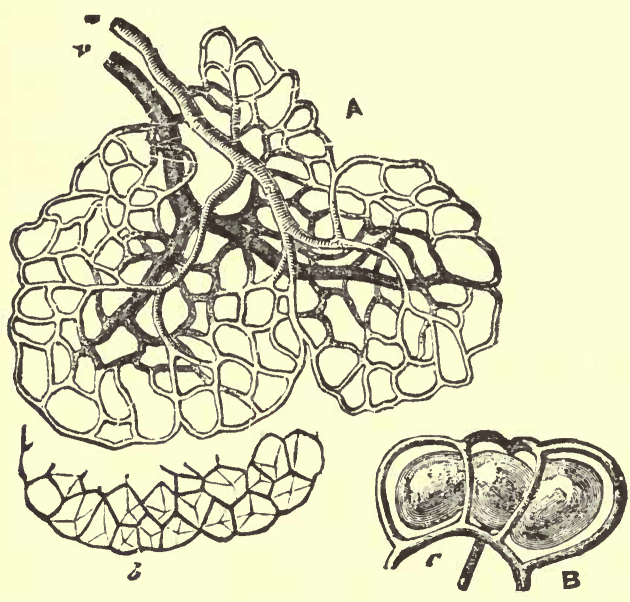

Fig. 50.-Blood-vessels of adipose tissue. A. Minute flattened fat-lobule, in which the vessels only are represented. $a$, The terminal artery; $v$, the primitive vein; $b$, the fatvesicles of one border of the lobule separately represented. $\times 100$. B. Plan of the arrangement of the capillaries $(c)$ on the exterior of the vesleles; more highly magnitied. ('Iodd and Bowman.)

blood when occasion requires, and, being used up in the metabolism of the tissues, may help to preserve the heat of the body.

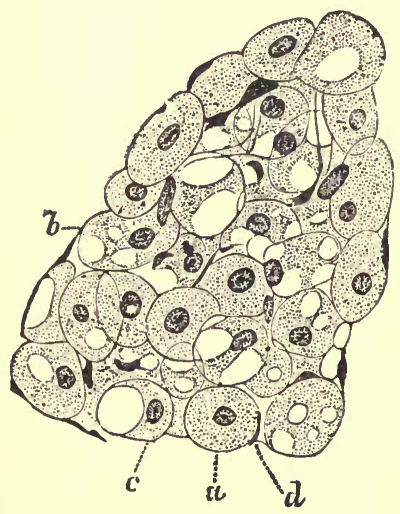

Fig. 51.

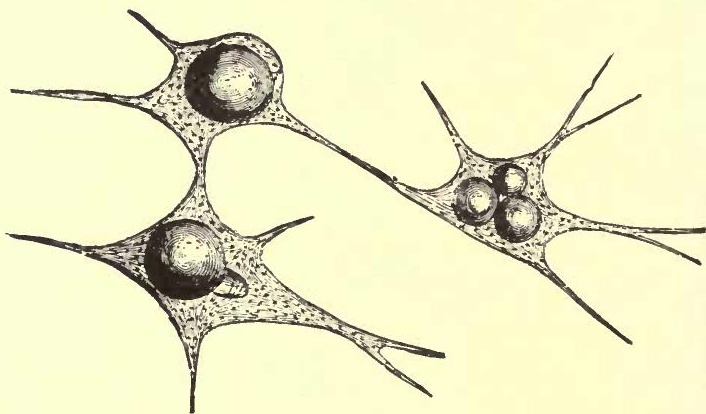

Fig. 52.

Fig. 51.-A lobule of developing adipose tissue from all eight months' fœetus. $a$, Spherical or, from pressure, polyhedral cells with large central nucleus, surrounded by a finely reticulated substance staining uniformly with hæmatoxylin. $b$, Similar cells with spaces from which the fat has been remored by oil of cloves. $c$, Similar cells showing how the nucleus with inclosing protoplasm is being pressed toward periphery. $d$, Nucleus of endothelium of investing capillaries. (IeCarthy.) Drawn by Treves.

Fig. 52.-Branched connective-tissue corpuscles, developing into fat-cells. (Klein.) 
b. That part of the fat which is situate beneath the skin must, by its want of conducting power, assist in preventing undue waste of the heat of the body by escape from the surface.

c. As a packing material, fat serves very admirably to fill up spaces, to form a soft and yielding yet elastic material wherewith to wrap tender and delicate structures, or form a bed with like qualities on which such structures may lie, not endangered by pressure. As examples of situations in which fat serves such purposes may be mentioned the palms of the hands and soles of the feet and the orbits.

d. In the long bones fatty tissue, in the form known as yellow marrow, fills the medullary canal, and supports the small blood-vessels which are distributed from it to the inner part of the substance of the bone.

\section{Basement Membranes.}

Basement membranes are a special structure upon which the epithelium of mucous membranes rests. They are of homogeneous appearance, and are developed from flattened connective-tissue corpuscles, joined at their edges, or from a concentrated cement substance. Some basement membranes possess elasticity, e.g., in the cornea.

\section{Cartilage.}

General Structure of Cartilage.-All kinds of cartilage are composed of cells imbedded in a substance called the matrix : the apparent differences of structure met with in the various kinds of cartilage are more due to differences in the character of the matrix than of the cells. Among the latter, however, there is also considerable diversity of form and size.

With the exception of the articular variety, cartilage is invested by a thin but tough firm fibrous membrane called the perichondrium. On the surface of the articular cartilage of the fotus, the perichondrium is represented by a film of epithelium; but this is gradually worn away up to the margin of the articular surfaces when by use the parts begin to suffer friction.

Nerves are probably not supplied to any variety of cartilage.

Cartilage exists in three different forms in the human body, viz., 1, Hyaline cartilage, 2, Yellow elastic-cartilage, and 3, White fibro-cartilage.

\section{Hyaline Cartilage.}

Distribution.-This variety of cartilage is met with largely in the human body-investing the articular ends of bones, and forming the costal cartilages, the nasal cartilages, and those of the larynx with the exception of the epiglottis and cornicula laryngis, as well as those of the trachea and bronchi.

Structure.-Like other cartilages it is composed of cells imbedded in 
a matrix. The cells, which contain a nucleus with nucleoli, are irregular in shape, and generally grouped together in patches (fig. 53). The patches are of various shapes and sizes and placed at unequal distances apart. They generally appear flattened near the free surface of the mass of cartilage in which they are placed and more or less perpendicular to the surface in the more-deeply seated portions.

The matrix of hyaline cartilage has a dimly granular appearance like that of ground glass, and in man and the higher animals has no apparent structure. In some cartilages of the frog, however, even when examined in the fresh state, it is seen to be mapped out into polygonal blocks or cell-territories, each containing a cell in the centre, and repre-

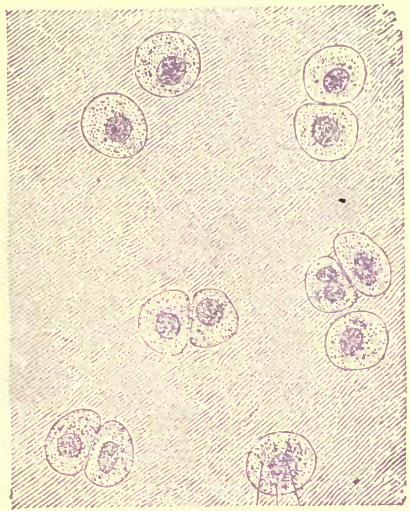

Fig. 53.

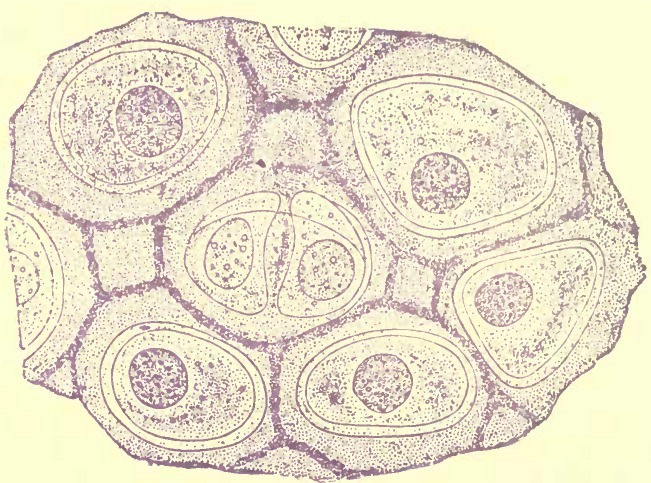

Fig. 54.

Fig. 53. - Hyaline articular cartilage (human). The cell bodies entirely fill the spaces in the matrix. $\times 340$ diams. (Schäfer.)

Fig. 54.-Fresh cartilage from the Triton. (A. Rollett.)

senting what is generally called the capsule of the cartilage cells (fig. 54). Hyaline cartilage in man has really the same structure, which can be demonstrated by the use of certain reagents. If a piece of human hyaline cartilage be macerated for a long time in diluted acid or in hot water $35^{\circ}-45^{\circ} \mathrm{C}$. $\left(95^{\circ}-113^{\circ} \mathrm{F}\right.$.), the matrix, which previously appeared quite homogeneous, is found to be resolved into a number of concentric lamellæ, like the coats of an onion, arranged round each cell or group of cells. It is thus shown to consist of nothing but a number of large systems of capsules which have become fused with one another.

The cavities in the matrix in which the cells lie are connected together by a series of branching canals, very much resembling those in the cornea: through these canals fluids may make their way into the depths of the tissue.

In the hyaline cartilage of the ribs the cells are mostly larger than in the articular variety and there is a tendency to the development of fibres in the matrix (fig. 55). The costal cartilages also frequently be- 
come calcified in old age, as also do some of those of the larynx. Fatglobules may also be seen in many cartilages (fig. 55).

In articular cartilage the cells are smaller and arranged vertically in narrow lines like strings of beads.

In the fotus cartilage is the material of which the bones are first constructed; the "model" of each bone being laid down, so to speak, in this substance. In such cases the cartilage is termed temporary. It closely resembles the ordinary hyaline kind; the cells, however, are not grouped together after the fashion just described, but are more uniformly distributed throughout the matrix.

A variety of temporary hyaline cartilage which has scarcely any ma-

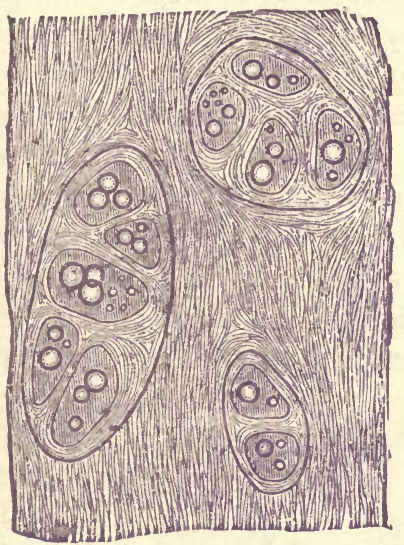

Fig. 55,

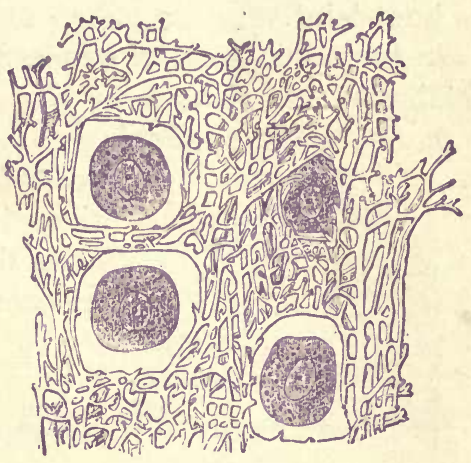

Fig. 56.

Fig. 55.-Costal cartilage from an adult dog, showing the fat globules in the cartilage cells. (Cadiat.)

Fig. 56. - Yellow elastic cartilage of the ear. Highly magnifled. (Hertwig.)

trix is found in the human subject and in the higher animals generally, in early foetal life, when it constitutes the chorda dorsalis.

Nutrition.-Hyaline cartilage is reckoned among the so-called nonvascular structures, no blood-vessels being supplied directly to its own substance; it is nourished by those of the bone beneath. When hyaline cartilage is in thicker masses, as in the case of the cartilages of the ribs, a few blood-vessels traverse its substance. The distinction, however, between all so-called vascular and non-vascular parts is at the best a very artificial one.

\section{Yellow Elastic Cartilage.}

Distribution.-In the external ear, in the epiglottis and cornicula laryngis, and in the Eustachian tube.

Structure.-The cells in this variety of cartilage are rounded or oval, with well-marked nuclei and nucleoli (fig. 56). The matrix in which they are seated is composed almost entirely of fine elastic fibres, which 
form an intricate interlacement about the cells, and in their general characters are allied to the yellow variety of fibrous tissue: a small and variable quantity of hyaline intercellular substance is also usually present.

A variety of elastic cartilage, sometimes called cellular, is found to form the framework of the external ears of rats, mice, or other small mammals. It is composed, as its name implies, almost entirely of cells which are packed very closely with little or no matrix. When present the matrix consists of very fine fibres which twine about the cells in various directions and inclose them in a kind of network. Elastic cartilage seldom or never ossifies.

\section{White Fibro-Cartilage.}

Distribution. - White fibro-cartilage is found to occur:-

1. As inter-articular fibro-cartilage, e.g., the semilunar cartilages of the knee-joint.

2. As circumferential or marginal cartilage, as on the edges of the acetabulum and glenoid cavity.

3. As connecting cartilage, e.g., the inter-vertebral fibro-cartilages.

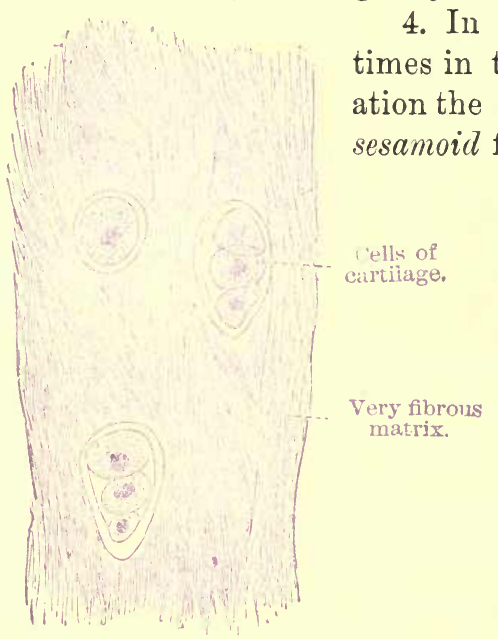

Fig. 57.

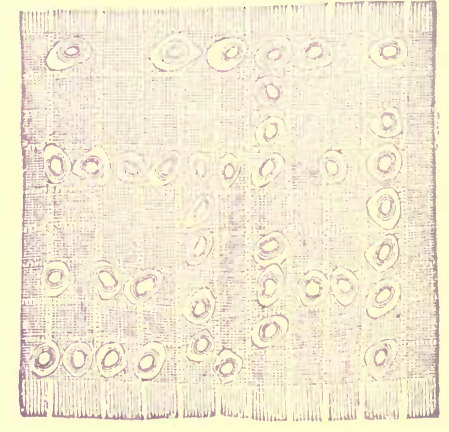

Fig. 58 .

Fig. 57.-White fibro-cartilage. (Cadiat.)

Fig. 58.-White fibro-cartilage from an inter-vertebral ligament. (Klein and Noble Smith.)

may be found in the tendon of the tibialis posticus in the sole of the foot, and usually in the neighboring tendon of the peroneus longus.

Structure.-White fibro-cartilage (fig. 58), which is much more widely distributed throughout the body than the foregoing kind, is composed, like it, of cells and a matrix; the latter, however, being made up almost entirely of fibres closely resembling those of white fibrous tissue.

In this kind of fibro-cartilage it is not unusual to find a great part of its mass composed almost exclusively of fibres, and deriving the name 
of cartilage only from the fact that in another portion, continuous with it, cartilage cells may be pretty freely distributed.

By prolonged boiling, cartilage yields a substance called chondrinwhich gelatinizes on cooling. The cells of white fibro-cartilage are as a rule rounded or somewhat flattened but in some places are distinctly branched.

Functions of Cartilage.-Cartilage not only represents in the fœtus the bones which are to be formed (temporary cartilage) but also offers a firm, yet more or less yielding, framework for certain parts in the developed body, possessing at the same time strength and elasticity. It maintains the shape of tubes as in the larynx and trachea. It affords attachment to muscles and ligaments; it binds bones together, yet allows a certain degree of movement, as between the vertebræ; it forms a firm framework and protection, yet without undue stiffness or weight, as in the pinna, larynx, and chest walls; it deepens joint cavities, as in the acetabulum, without unduly restricting the movements of the bones.

Development of Cartilage.-Cartilage is developed out of an embryonal tissue, consisting of cells with a very small quantity of intercellular substance: the cells multiply by fission within the cell-capsules, while the capsule of the parent cell becomes gradually fused with the surrounding intercellular substance. A repetition of this process in the young cells causes a rapid growth of the cartilage by the multiplication of its cellular elements and corresponding increase in its matrix. Thus we see that the matrix of cartilage is chiefly derived from the cartilage cells.

\section{BONE.}

Chemical Composition.-Bone is composed of earthy and animal matter in the proportion of about 67 per cent of the former to 33 per cent of the latter. The earthy matter is composed chiefly of calcium phosphate, but besides there is a small quantity (about 11 of the 67 per cent) of calcium carbonate and calcium fluoride, and magnesium phosphate.

The animal matter called collagen is resolved into gelatin by boiling.

The earthy and animal constituents of bone are so intimately blended and incorporated the one with the other that it is only by chemical action, as for instance by heat in one case and by the action of acids in another, that they can be separated. Their close union too is further shown by the fact that when by acids the earthy matter is dissolved out, or on the other hand when the animal part is burnt out, the shape of the bone is alike preserved.

The proportion between these two constituents of bone varies in different bones in the same individual and in the same bone at different ages.

Structure.-To the naked eye there appear two kinds of structure 
in different bones, and in different parts of the same bone, namely, the dense or compact, and the spongy or cancellous tissue.

Thus, in making a longitudinal section of a long bone, as the humerus or femur, the articular extremities are found capped on their surface by a thin shell of compact bone, while their interior is made up of the spongy or cancellous tissue. The shaft, on the other hand, is formed almost entirely of a thick layer of the compact bone, and this surrounds a central canal, the medullary cavity - so called from its containing the medulla or marrow.

In the flat bones, as the parietal bone or the scapula, one layer of the cancellous structure lies between two layers of the compact tissue, and in the short and irregular bones, as those of the carpus and tarsus, the cancellous tissue alone fills the interior, while a thin shell of compact bone forms the outside.

Marrow.-There are two distinct varieties of marrow-the red and yellow.

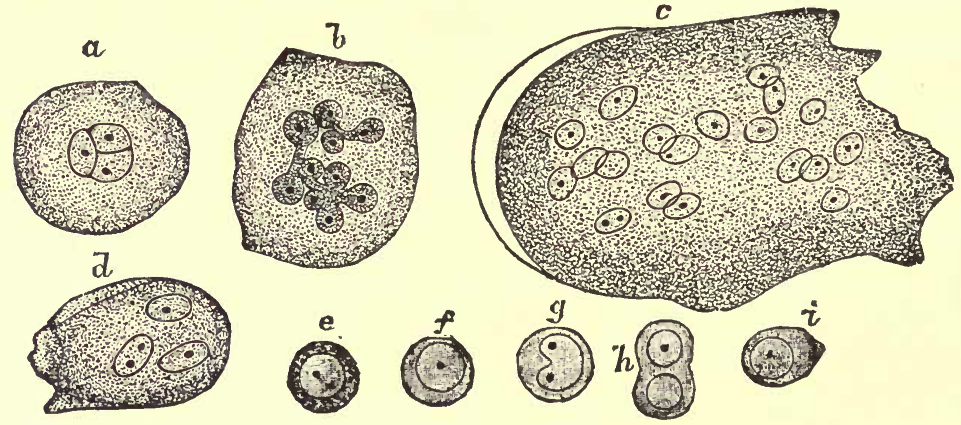

Fig. 59.-Cells of the red marrow of the guinea-pig, highly maguified. $a$, A large cell, the nucleus of which appears to be partly divided into three by constrictions; $b$, a cell, the nucleus of which shows an appearance of being constricted into a number of smaller nuclei; $c$, a so-called giant cell, or myeloplaxe, with many nuclei; $d$, a smaller myelo-plaxe, with three nuclei; $e-i$, proper cells of the marrow. (E. A. Schäfer.)

Red marrow is that variety which occupies the spaces in the cancellous tissue; it is highly vascular, and thus maintains the nutrition of the spongy bone, the interstices of which it fills. It contains a few fat-cells and a large number of marrow-cells, many of which are undistinguishable from lymphoid corpuscles, and has for a basis a small amount of fibrous tissue. Among the cells are some nucleated cells of very much the same tint as colored blood-corpuscles. There are also a few large cells with many nuclei, termed giant-cells or myeloplaxes, which are derived from over-growth of the ordinary marrow-cells (fig. 59).

Yellow marrow fills the medullary cavity of long bones, and consists chiefly of fat-cells with numerous blood-vessels; many of its cells also are in every respect similar to lymphoid corpuscles. 
From these marrow-cells, especially those of the red marrow, are derived, as we shall presently show, large quantities of red blood-corpuscles.

Periosteum and Nutrient Blood-vessels.-The surfaces of bones, except the part covered with articular cartilage, are clothed by a tough, fibrous membrane, the periosteum; and it is from the blood-ressels which are distributed in this membrane, that the bones, especially their more compact tissue, are in great part supplied with nourishment,minute branches from the periosteal vessels entering the little foramina on the surface of the bone, and finding their way to the Haversian cunals to be immediately described. The long bones are supplied also by a proper nutrient artery which, entering at some part of the shaft so

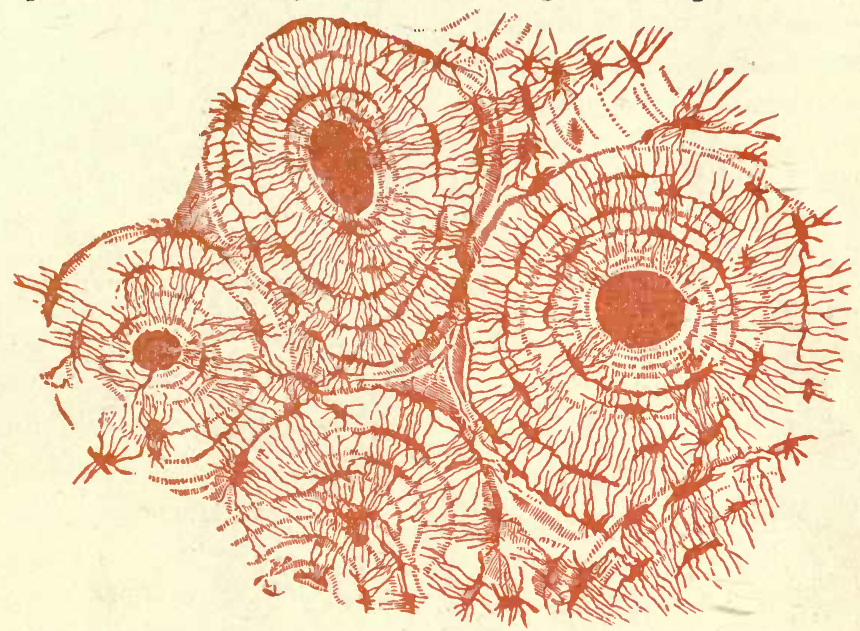

Fig. 60.-Transverse section of compact bony tissue (of humerus). Three of the Haversian canals are seen, with their concentric rings; also the lacunæ, with the canaliculi extending from them across the direction of the lamellæ. The Haversian apertures were filled with débris in grinding down the section, and therefore appear black in the figure, which represents the object as viewed with transmitted light. The Haversian systems are so closely packed in this section, that scarcely any interstitial lamellæ are visible. $\times 150$. (Sharpey.)

as to reach the medullary canal, breaks up into branches for the supply of the marrow, from which again small vessels are distributed to the interior of the bone. Other small blood-ressels pierce the articular extremities for the supply of the cancellous tissue.

Microscopic Structure of Bone.-Notwithstanding the differences of arrangement just mentioned, the structure of all bone is found under the microscope to be essentially the same.

Examined with a rather high power its substance is found to contain a multitude of small irregular spaces, approximately fusiform in shape, called lacuno, with very minute canals or canaliculi, as they are termed, leading from them, and anastomosing with similar little prolongations from other lacunæ (fig. 60). In very thin layers of bone, no other canals than these may be visible; but on making a transverse section of 
the compact tissue as of a long bone, e.g., the humerus or ulna, the arrangement shown in fig. 60 can be seen.

The bone seems mapped out into small circular districts, at or about the centre of each of which is a hole, around which is an appearance as of concentric layers-the lacunce and canaliculi following the same concentric plan of distribution around the small hole in the centre, with which indeed they communicate.

On making a longitudinal section, the central holes are found to be simply the cut extremities of small canals which run lengthwise through the bone, anastomosing with each other by lateral branches (fig. 61),

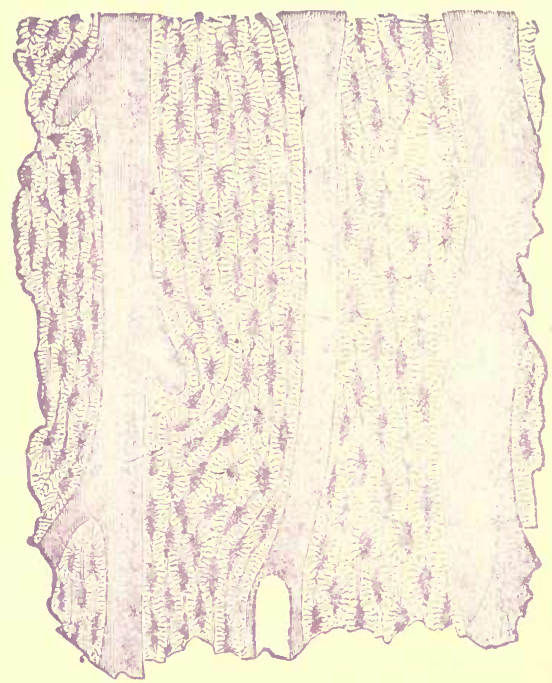

Fig. 61.-Longitudinal section from the human ulna, showing Haversian canal, lacunæ, and canaliculi. (Rollett.)

and are called Haversian canals, after the name of the physician, Clopton Havers, who first accurately described them.

The Haversian canals, the average diameter of which is $\frac{1}{500}$ of an inch, contain blood-vessels, and by means of them blood is conveyed to all, even the densest parts of the bone; the minute canaliculi and lacunæ absorbing nutrient matter from the Haversian blood-vessels and conveying it still more intimately to the very substance of the bone which they traverse.

The blood-vessels enter the Haversian canals both from without, by traversing the small holes which exist on the surface of all bones beneath the periosteum, and from within by means of small channels which extend from the medullary cavity, or from the cancellous tissue. The arteries and veins usually occupy separate canals, and the veins, which are the larger, often present, at irregular intervals, small pouchlike dilatations. 
The lacunæ are occupied by branched cells, which are called bonecells, or bone-corpuscles (fig. 62), which very closely resemble the ordinary branched connective-tissue corpuscles; each of these little masses of protoplasm ministering to the nutrition of the bone immediately surrounding it, and one lacunar corpuscle communicating with another, and with its surrounding district, and with the blood-vessels of the Haversian canals, by means of the minute streams of fluent nutrient matter which occupy the canaliculi.

It will be seen from the above description that bone is essentially connective-tissue impregnated with lime salts: it bears a very close re-

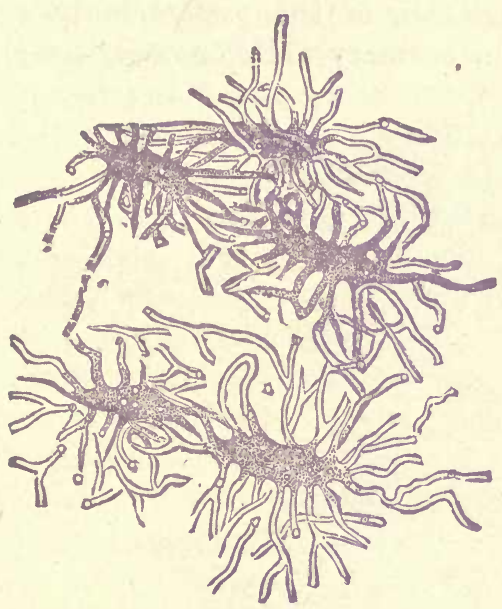

Fig. 62. semblance to what may be termed typical connective-tissue such as the substance of the cornea. The bone-corpuscles with their pro-

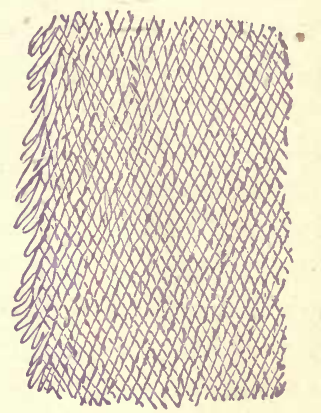

Fig. 63.

Fig. 62.-Bone-corpuscles with their processes as seen in a thin section of human bone. (Rollett.) Fig. 63.-Thin layer peeled off from a softened bone. This figure, which is intended to represent the reticular structure of a lamella. gives a better idea of the object when held rather farther off than usual from the eye. $\times 400$. (Sharpey.)

cesses occupying the lacunæ and canaliculi correspond exactly to the cornea-corpuscles lying in branched spaces.

Lamellæ of Compact Bone.-In the shaft of a long bone three distinct sets of lamellæ can be clearly recognized.

(1.) General or fundamental lamellæ; which are most easily traceable just beneath the periosteum, and around the medullary cavity, forming around the latter a series of concentric rings. At a little distance from the medullary and periosteal surfaces (in the deeper portions of the bone) they are more or less interrupted by

(2.) Special or Haversian lamellæ, which are concentrically arranged around the Haversian canals to the number of six to eighteen around each.

(3.) Interstitial lamellæ, which connect the system of Haversian lamellæ, filling the spaces between them, and consequently attaining 
their greatest development where the Haversian systems are few, and vice versâ.

The ultimate structure of the lamellæ appears to be reticular. If a thin film be peeled off the surface of a bone, from which the earthy matter has been removed by acid, and examined with a high power of the microscope, it will be found composed of a finely reticular structure, formed apparently of very slender fibres decussating obliquely, but coalescing at the points of intersection, as if here the fibres were fused rather than woven together (fig. 63).

In many places these reticular lamellæ are perforated by tapering fibres called the Claviculi of Gagliardi, or the perforating fibres of Sharpey, resembling in character the ordinary white or rarely the elastic

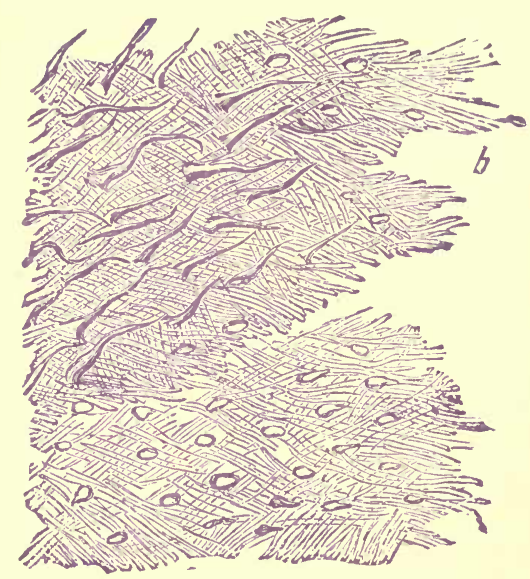

Fig. 64.-Lamellæ torn off from a decalcified human parietal bone at some depth from the sur. face. $a, a$, Lamellæ, showing reticular fibres; $b, b$, darker part, where several lamellæ are superposed; c , perforating fibres. Apertures through which perforating fibres had passed, are seen especially in the lower part, $a, a$, of the figure. (Allen Thomson.)

fibrous tissue, which bolt the neighboring lamellæ together, and may be drawn out when the latter are torn asunder (fig. 64). These perforating fibres originate from ingrowing processes of the periosteum, and in the adult still retain their connection with it.

Development of Bone.-From the point of view of their development, all bones may be subdivided into two classes.

(a.) Those which are ossified directly or from the first in membrane or fibrous tissue, e.g., the bones forming the vault of the skull, parietal, frontal, and a certain portion of the occipital bones.

(b.) Those whose form, previous to ossification, is laid down in hyaline cartilage, e.g., humerus, femur.

The process of development, pure and simple, may be best studied in bones which are not preceded by cartilage, i.e., membrane-formed (e.g., 
parietal); and without a knowledge of this process (ossification in membrane), it is impossible to understand the much more complex series of changes through which such a structure as the cartilaginous femur of the fotus passes in its transformation into the bony femur of the adult (ossification in cartilage).

Ossification in Membrane.-The membrane, afterward forming the periosteum, from which such a bone as the parietal is developed, consists of two layers-an external fibrous, and an internal cellular or osteo-genetic.

The external layer is made up of ordinary connective-tissue, being composed of layers of fibrous tissue with branched connective-tissue corpuscles here and there between the bundles of fibres. The internal layer consists of a network of fine fibrils with a large number of nucleated cells with a certain addition of albuminous ground or cement substance between the fibrous bundles, some of which are oval, others drawn out into long branched processes: it is more richly supplied with capillaries than the outer layer. The relatively large number of its cellular elements, which vary in size and shape, together with the abundance of its blood-vessels, clearly mark it out as the portion of the periosteum which is immediately concerned in the formation of bone.

In such a bone as the parietal, which is represented then when ossification commences by the species of fibrous connective tissue with many cells above indicated, the deposition of bony matter, which is preceded by increased vascularity, takes place in radiating spiculæ, starting from a centre of ossification, and shooting out in all directions toward the periphery. These primary bony spiculæ consist of the fibres of the tissue which are termed osteogenetic fibres, composed of a soft transparent substance called osteogen, in which calcareous granules are deposited. The fibres are said to exhibit in their precalcified state indications of a fibrillar structure, and are likened to bundles of white fibrous tissue, to which they are similar in chemical composition, but from which they differ in being stiffer and less wavy. The deposited granules after a time hecome so numerous as to fill up the substance of the fibres and bony opiculæ result. Calcareous granules are deposited also in the interfibrillar matrix. By the junction of the osteogenetic fibres and their resulting bony spiculæ a meshwork of bone is formed. The osteogenetic fibres, which become indistinct as calcification proceeds, are believed to persist in the lamellæ of adult bone. The osteoblasts, being in part retained within the bone trabeculæ thus produced, form bone corpuscles. On the bony trabeculæ first formed, layers of osteoblastic cells from the osteo-genetic layer of the periosteum are developed side by side, lining the irregular spaces like an epithelium (fig. 65, b). Limesalts are deposited in the circumferential part of each osteoblast, and thus a ring of osteoblasts gives rise to a ring of bone with the remaining 
uncalcified portions of the osteoblasts imbedded in it as bone corpuscles, as in the first formation; then the central portion of the bony plate becomes harder and less cancellous. At the same time, the plate increases at the periphery not only by the extension of the bony spiculæ, but also by deposits taking place from the osteogenetic layer of the periosteum.

The primitive spongy bone is formed, and its irregular branching spaces are occupied by processes from the osteogenetic layer of the periosteum consisting of numerous blood-vessels and osteoblasts. Portions of this primitive spongy bone are re-absorbed. The osteoblasts are arranged in concentric successive layers and give rise to concentric Haversian lamellæ of bone, while the irregular space in the centre is reduced to a well-formed Haversian canal, containing the usual bloodvessels, the portions of the primitive spongy bone between the Haversian

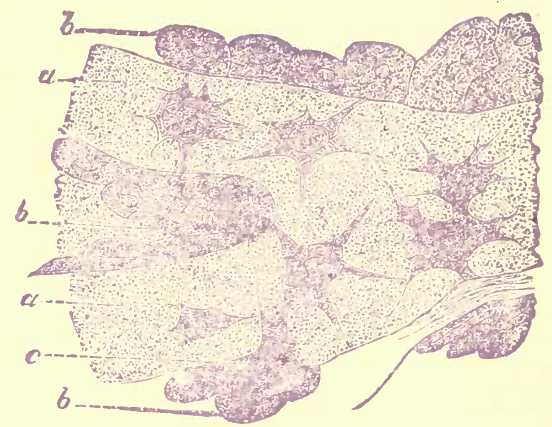

Fig. 65.-Osteoblasts from the parietal bone of a human embryo, thirteen weeks old. $a$, Bony septa with the cells of the lacunæ; $b$, layers of osteoblasts; $c$, the latter in transition to bone corpuscles. Highly maguified. (Gegenbaur.)

systems remaining as interstitial or ground-lamellæ (p. 59). The bulk of the primitive spongy bone is thus gradually converted into compact bony-tissue of Haversian systems. Those portions of the ingrowths from the deeper layer of the periosteum which are not converted into bone remain in the spaces of the cancellous tissue as the red marrow.

Ossification in Cartilage.-Under this heading, taking the femur as a typical example, we may consider the process by which the solid cartilaginous rod which represents the bone in the fœtus is converted into the hollow cylinder of compact bone with expanded ends formed of cancellous tissue of which the adult femur is made up. We must bear in mind the fact that this fotal cartilaginous femur is many times smaller than the medullary cavity even of the shaft of the mature bone, and, therefore, that not a trace of the original cartilage can be present in the femur of the adult. Its purpose is indeed purely temporary; and, after its calcification, it is gradually and entirely absorbed as will be presently explained. 
The cartilaginous rod which forms the fotal femur is sheathed in a membrane termed the perichondrium, which so far resembles the periosteum described above, as to consist of two layers, in the deeper one of which spheroidal cells predominate and blood-vessels abound, while the outer layer consists mainly of fusiform cells which are in the mature tissue gradually transformed into fibres. Thus, the differences between the fotal perichondrium and the periosteum of the adult are such as

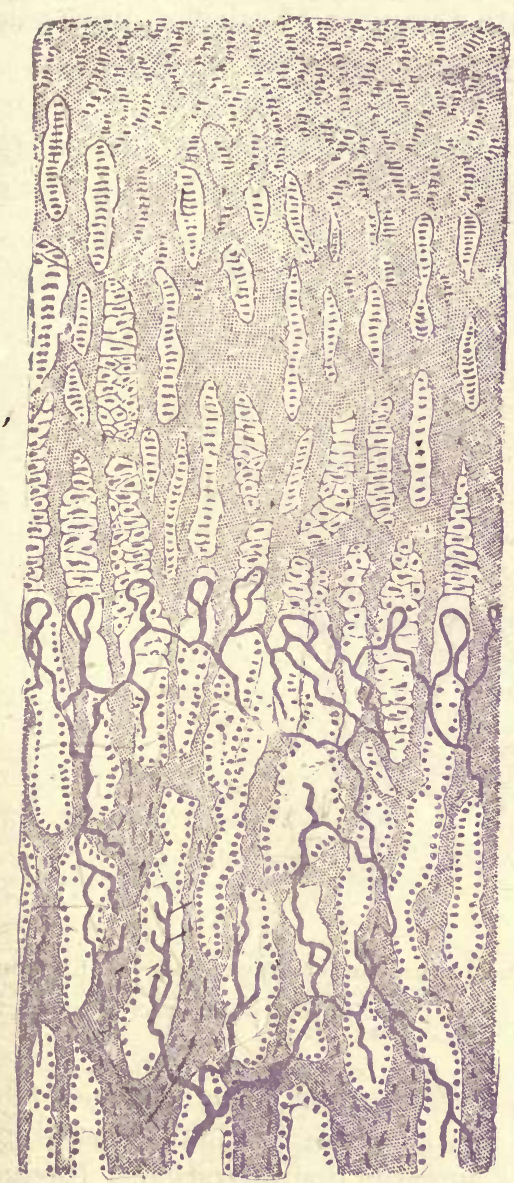

Fig. 66. usually exist between the embryonic and mature forms of connective tissue.

Between the hyaline cartilage of which the foetal femur consists and the bony tissue forming the adult femur, there are two chief intermediate stages - viz. (1) of

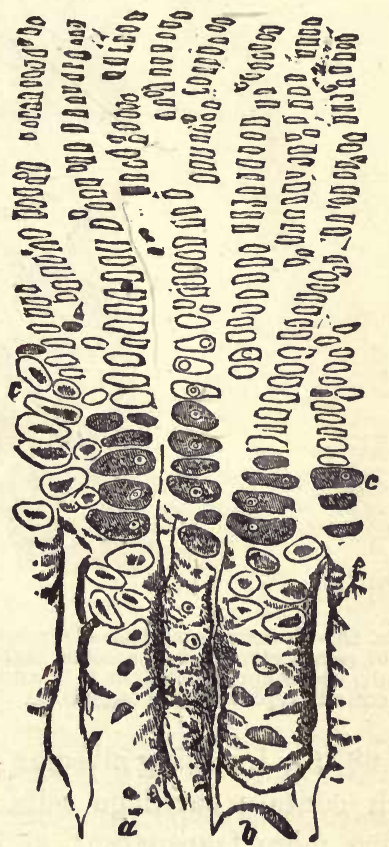

Fig. 67.

Fig. 66.- Ossifying cartilage showing loops of blood-vessels.
Fig. 67.-Longitudinal section of ossifying cartilage from the humerus of a foetal sheep. Calcified trabeculæ are seen extending between the columns of cartilage cells. cartilage cells. $\times 140$. (Sharpey.)

calcified cartilage, and (2) of embryonic spongy bone. These materials, which successively occupy the place of the foetal cartilage, are in succession entirely absorbed, and their place is taken by true bone.

The process by which the cartilaginous is transformed into the 
bony femur may however be divided for the sake of clearness into the following six stages:-

Stage 1.-Proliferation and Calcification.-As ossification is commencing the cartilage cells in and near the centre of ossification become enlarged and proliferate, arranging themselves in rows corresponding to the long axis of the bone (fig. 67 ). Lime salts are next deposited in the form of fine granules in the hyaline matrix of the cartilage, and this gradually becomes transformed into a number of calcified trabecula,

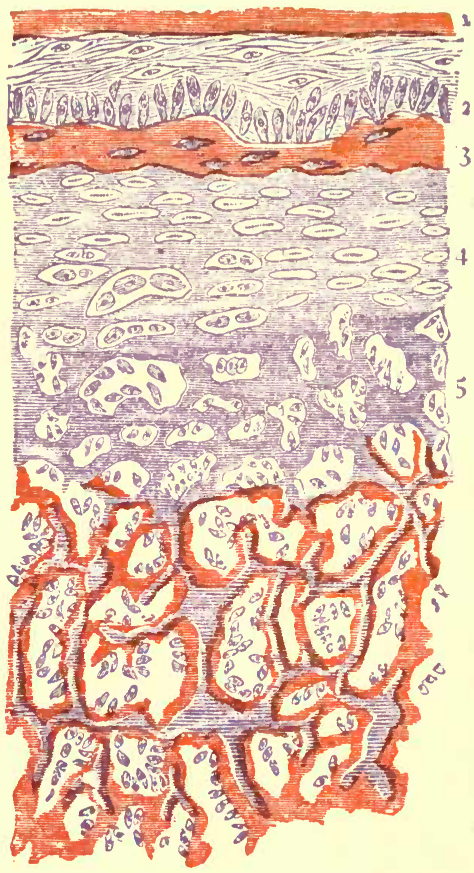

FIG. 68. - Transverse section of a portion of a metacarpal bone of a fotus, showing-1, fibrous layer of periosteum; 2 , osteogenetic layer of ditto; 3 , periosteal bone; 4, cartilage. with matrix gradually becoming calcified, as at 5 , with cells in primary areolæ; beyond 5 the calcified matrix is being entirely replaced by spongy bone. $\times 200$. (V. D. Harris.)

(fig. 68,5 ), inclosing alveolar spaces, which are the primary areolee, and which contain cartilage cells. The cartilage cells, gradually enlarging, become more transparent, and finally undergo disintegration. During this stage the perichondrium has become the periosteum, and is beginning to deposit bone on the outside of the cartilage.

Stage 2.- Vascularization of the Cartilage.-Processes from the osteogenetic or cellular layer of the periosteum containing bloodvessels break into the substance of the cartilage and grow much as ivy insinuates itself into the cracks and crevices of a wall. This begins ai the "centres of ossification," from which the blood-vessels spread chiefly 
up and down the shaft, ete. Thus the substance of the cartilage, which previously contained no vessels, is traversed by a number of branched anastomosing channels formed by the enlargement and coalescence of the spaces in which the cartilage-cells lie, and containing loops of bloodvessels (fig. 66) and spheroidal cells which will become osteoblasts. By further absorption of some of the trabeculæ larger spaces are developed, which contain cartilage-cells for a very short time only, their places being taken by the so-called osteogenetic layer of the periosteum which constitutes the primary marrow.

\section{Stage 3.-Substitution of Embryonic Spongy Bone for Carti-} lage.-The cells of the primary marrow arrange themselves as a continuous layer like epithelium on the calcified trabeculæ and deposit a layer

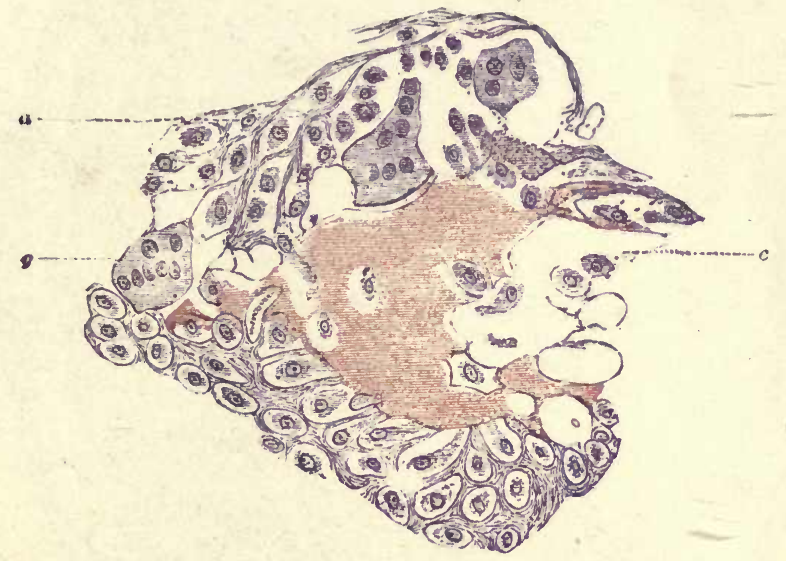

FIG. 69. - A small isolated mass of bone next the periosteum of the lower jaw of human fœetus. $a$. Osteogenetic layer of periosteum. $g$, multinuclear giant cells, the one on the left acting liere probably like an osteoclast. A bove $c$, the osteoblasts are seen to becume surrounded by an osseous matrix. (Klei.s and Noble Smith.)

of bone, and ensheath them: the calcified trabeculæ, encased in the sheaths of joung bone, become gradually absorbed, so that finally we have trabeculæ composed entirely of spongy bone, all trace of the original calcified cartilage having disappeared. It is probable that the large multinucleated giant-cells termed osteoclasts by Kölliker, which are derived from the osteoblasts by the multiplication of their nuclei, are the agents by which the absorption of calcified cartilage, and subsequently of embryonic spongy bone, is carried on (fig. $69, g$ ). At any rate, they are almost always found wherever absorption is in progress.

These stages are precisely similar to what goes on in the growing shaft of a bone which is increasing in length by the adrance of the process of ossification into the intermediary cartilage between the diaphysis and epiphysis. In this case the cartilage-cells become flattened and, multiplying by division, are grouped into regular columns at right 
angles to the plane of calcification, while che process of calcification extends into the hyaline matrix between them (figs. 67 and 68).

Stage 4.-Substitution of Periosteal Bone for the Primary Embryonic Spongy Bone.-The embryonic spongy bone, formed as above described, is simply a temporary tissue occupying the place of the fœtal rod of cartilage, once representing the femur; and the stages 1 ,

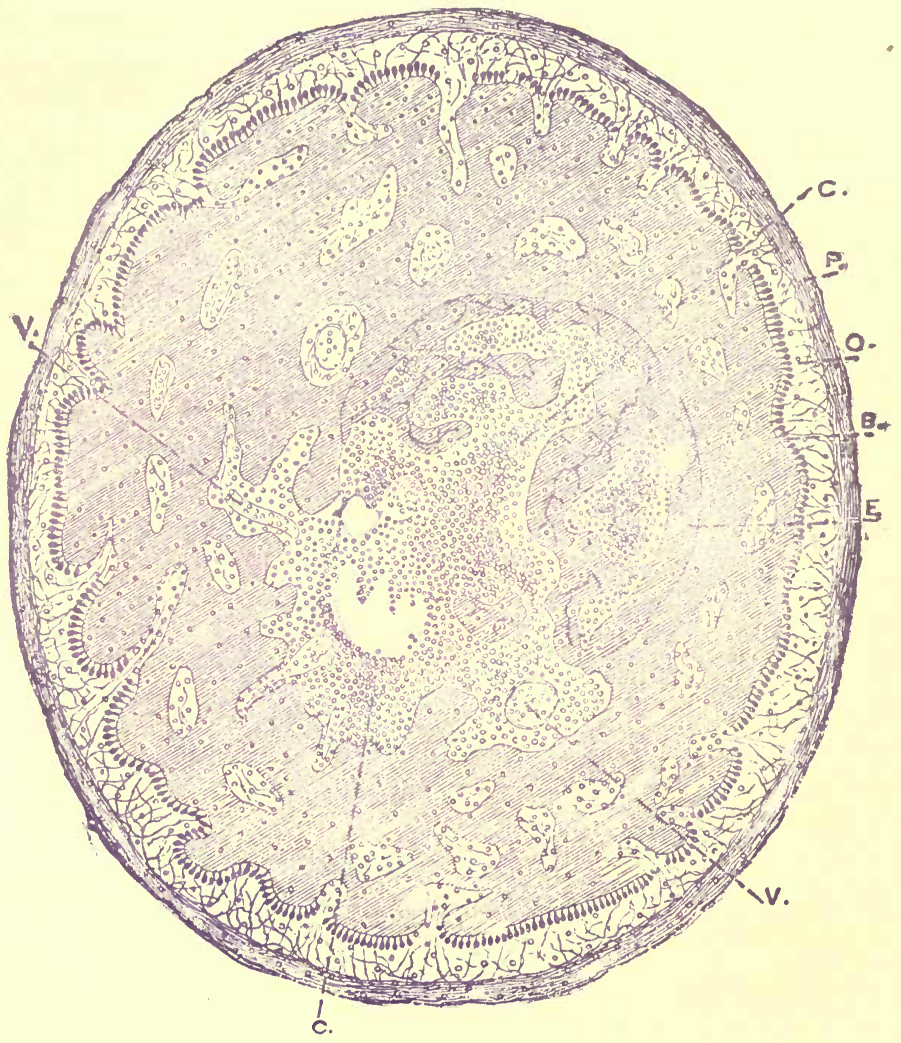

Fig. 70.-Transverse section through the tibia of a fœetal kitten, semi-diagrammatic. $\times 60$. $\mathrm{P}$, Periosteum. O, Osteogenetic layer of the periost um showing the osteoblasts arranged side by side, represented as pear-shaped black dots on the surface of the newly-formed bone. B, The periosteal bone deposited in successive layers beneath the periosteum and ensheathing $\mathrm{E}$, the spongy endochondral bone; represented as more deeply shaded. Within the trabeculæ of endochondral spongy bone are seen the remains of the calcified eartilage trabeculæ represented as dark wavy lines. C, The medulla, with V, V, veins. In the lower half of the figure the endochondral spongy bone has been completely absorbed. (Klein and Noble Smith.)

2, and 3 show the successive changes which occur at the centre of the shaft. Periosteal bone is at the same time deposited in successive layers beneath the periosteum, i.e., at the circumference of the shaft, exactly as described in the section on ossification in membrane, and thus a casing of periosteal bone is formed around the embryonic endochondral spongy bone: this casing is thickest at the centre, where it is first formed, and 
thins out toward each end of the shaft. 'The embryonic spongy bone is absorbed, through the agency of osteoclasts, its trabeculæ becoming gradually thinned and its meshes enlarging, and finally coalescing into one great cavity - the medullary cavity of the shaft.

\section{Stage 5.-Absorption of the Inner Layers of the Periosteal} Bone.-The absorption of the endochondral spongy bone is now complete, and the medullary cavity is bounded by periosteal bone: the inner layers of this periosteal bone are next absorbed, and the medullary cavity is thereby enlarged, while the deposition of bone beneath the periosteum

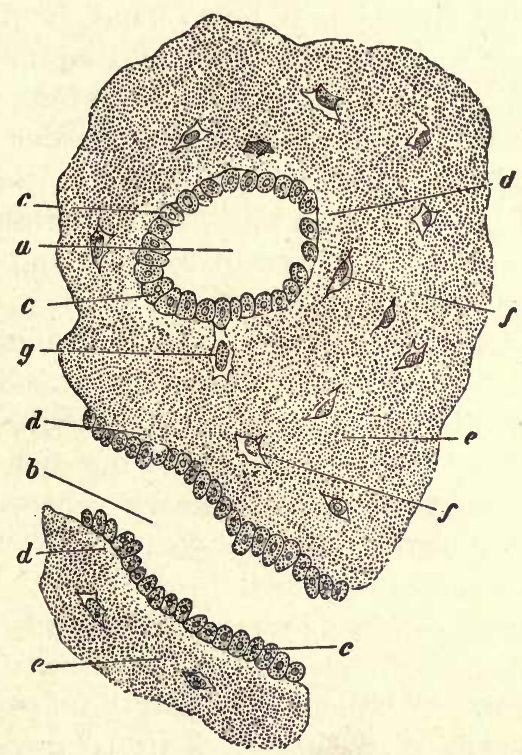

Fig. 71.-Tranverse section of femur of a human embryo about eleven weeks old. $a$, Rudimentary Haversian canal in cross-section; $b$, in longitudinal section; $c$, osteoblasts; $d$, newly formed osseous substance of a lighter color; $e$, that of greater age; $f$, lacunæ with their cells; $g$, a cell still united to an osteoblast. (Frey.)

continues as before. The first-formed periosteal bone is spongy in character.

Stage 6.-Formation of Compact Bone.-The transformation of spongy periosteal bone into compact bone is effected in a manner exactly similar to that which has been described in connection with ossification in membrane (p. 61). The irregularities in the walls of the areolæ in the spongy bone are absorbed, while the osteoblasts which line them are developed in concentric layers, each layer in turn becoming ossified till the comparatively large space in the centre is reduced to a well-formed Haversian canal (fig. 71). When once formed, bony tissue grows to some extent interstitially, as is evidenced by the fact that the lacunæare rather further apart in full-formed than in young bone. 
From the foregoing description of the development of bone, it will be seen that the common terms ossification in cartilage and ossification in membrane are apt to mislead, since they seem to imply two processes radically distinct. The process of ossification, however, is in all cases one and the same, all true bony tissue being formed from membrane (perichondrium or periosteum); but in the development of such a bone as the femur, which may be taken as the type of so-called ossification in cartilage, lime-salts are first of all deposited in the cartilage; this calcified cartilage, however, is gradually and entirely re-absorbed, being ultimately replaced by bone formed from the periosteum, till in the adult structure nothing but true bone is left. Thus, in the process of "ossification in cartilage," calcification of the cartilaginous matrix precedes the real formation of bone. We must, therefore, clearly distinguish between calcification and ossification. The former is simply the infiltration of an animal tissue with lime-salts, and is, therefore, a change of chemical composition rather than of structure; while ossification is the formation of true bone-a tissue more complex and more highly organized than that from which it is derived.

Centres of Ossification.-In all bones ossification commences at one or more points, termed centres of ossification. The long bones, e.g., femur, humerus, etc., have at least three such points-one for the ossification of the shaft or diaplyysis, and one for each articular extremity or epiphysis. Besides these three primary centres which are always present in long bones, various secondary centres may be superadded for the ossification of different processes.

Growth of Bone.-Bones increase in length by the advance of the process of ossification into the cartilage intermediate between the diaphysis and epiphysis. The increase in length indeed is due entirely to growth at the two ends of the shaft. This is proved by inserting two pins into the shaft of a growing bone: after some time their distance apart will be found to be unaltered though the bone has gradually in. creased in length, the growth having taken place beyond and not be. tween them. If now one pin be placed in the shaft, and the other in the epiphysis of a growing bone, their distance apart will increase as the bone grows in length.

Thus it is that if the epiphyses with the intermediate cartilage be removed from a young bone, growth in length is no longer possible; while the natural termination of growth of a bone in length takes place when the epiphyses become united in bony continuity with the shaft.

Increase in thickness in the shaft of a long bone occurs by the deposition of successive layers beneath the periosteum.

If a thin metal plate be inserted beneath the periosteum of a growing bone it will soon be covered by osseous deposit, but if it be put be- 
tween the fibrous and osteogenetic layers it will never become enveloped in bone, for all the bone is formed beneath the latter.

Other varieties of connective tissue may become ossified, e.g., the tendons in some birds.

Functions of Bones.-Bones form the framework of the body; for this they are fitted by their hardness and solidity together with their comparative lightness; they serve both to protect internal organs in the trunk and skull, and as levers worked by muscles in the limbs; notwithstanding their hardness they possess a considerable degree of elasticity, which often saves them from fracture.

The material of which the chief portion of the teeth is made up, called Dentine, is frequently classed with bone and as one of the connective tissues. 'The other constituents of the teeth also resemble bone in structure to a considerable degree; it will be as well therefore to give in this place some account of the teeth.

\section{The Teeth.}

During the course of his life, man, in common with most other mammals, is provided with two sets of teeth; the first set, called the

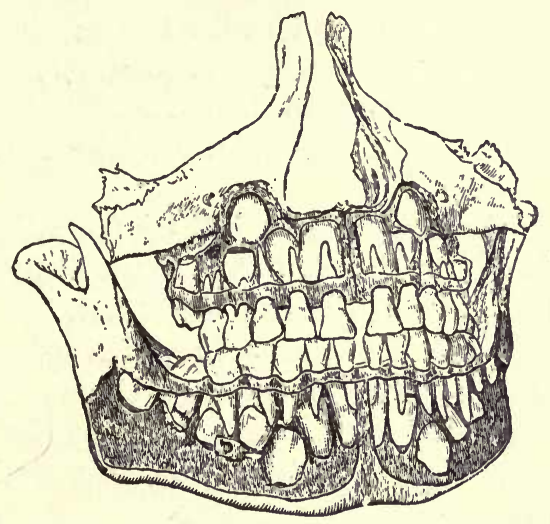

Fig. 72. - Normal well-formed jaws, from which the alveolar plate has been in great part removed, so as to expose the developing permanent teeth in their crypts in the jaws. (Tomes.)

temporary or milk teeth, makes its appearance in infancy, and is in the course of a few years shed and replaced by the second or permanent set.

The temporary or milk teeth have only a very limited term of existence.

They are ten in number in each jaw, namely, on either side from the middle line two incisors, one canine, and two deciduous molars, and are replaced by ten permanent teeth. The number of permanent teeth in 
each jaw is, however, increased to sixteen by the development of three molars on each side of the jaw, which are called the permanent or true molars.

The following formula shows, at a glance, the comparative arrangement and number of the temporary and permanent teeth:-

\section{Temporary Teeth.}

Middle Line of JAw.

\begin{tabular}{ccc|ccc} 
MOLARS. & CANINE. & INCISORS. & INCISORS. & CANINE. & MOLARS. \\
2 & 1 & 2 & 2 & 1 & $2=10$ \\
\hline 2 & 1 & 2 & 2 & 1 & $2=10$
\end{tabular}

\section{Permanent Teeth.}

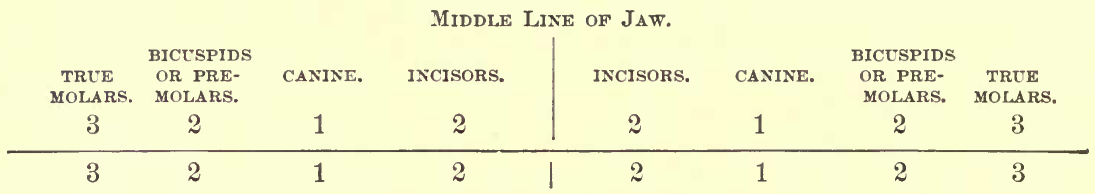

From this formula it will be seen that the two bicuspid or pre-molar teeth in the adult are the successors of the two deciduous molars in the child. They differ from them, however, in some respects, the temporary molars having a stronger likeness to the permanent than to their immediate descendants the so-called bicuspids, besides occupying more space in the jaws.

The temporary incisors and canines differ from their successors but little except in their smaller size and the abrupt manner in which their enamel terminates at the necks of the teeth, forming a ridge or thick edge. Their color is more of a bluish-white than of a yellowish shade.

The following tables show the average times of eruption of the Temporary and Permanent teeth. In both cases the eruption of any given tooth of the lower precedes, as a rule, that of the corresponding tooth of the upper jaw.

\section{Temporary or Milk Teeth.}

The figures indicate in months the age at which each tooth appears.

\begin{tabular}{c|c|c|c|c}
\hline $\begin{array}{c}\text { LOWER CENTRAL } \\
\text { INCISOR. }\end{array}$ & UPPER INCISORS. & $\begin{array}{c}\text { FIRST MOLARS AND LOWER } \\
\text { LATERAL INCISORS. }\end{array}$ & CANINES. & SECOND MOLARS. \\
\hline 6 to 9 & 8 to 12 & 12 to 15 & 18 to 24 & 24 to 30. \\
\hline
\end{tabular}




\section{Permanent Teeth.}

The age at which each tooth is cut is indicated in this table in years.

\begin{tabular}{|c|c|c|c|c|c|c|c|}
\hline \multirow{2}{*}{$\begin{array}{l}\text { FIRST } \\
\text { MOLARS. }\end{array}$} & \multicolumn{2}{|c|}{ INCISORS. } & \multirow{2}{*}{\multicolumn{2}{|c|}{$\begin{array}{l}\text { BICUSPIDS OR PRE- } \\
\text { MOLARS. } \\
\text { FIRST. SECOND. }\end{array}$}} & \multirow{2}{*}{ CANINES. } & \multirow{2}{*}{$\begin{array}{l}\text { SECOND } \\
\text { MOLARS. }\end{array}$} & \multirow{2}{*}{$\begin{array}{l}\text { THIRD } \\
\text { MOLARS OR } \\
\text { WISDONS. }\end{array}$} \\
\hline & CENTRALS. & LATERALS. & & & & & \\
\hline 6 & 7 & 8 & 9 & 10 & 12 to 14 & 12 to 15 & 17 to 25 \\
\hline
\end{tabular}

The times of eruption given in the above tables are only approximate: the limits of variation being tolerably wide. Some children may cut their first teeth before the age of six months, and others not till nearly the twelfth month. In nearly all cases the two central incisors of the lower jaw are cut first, these being succeeded after a short interval by the four incisors of the upper jaw; next follow the lateral incisors of the lower jaw, and so on as indicated in the table till the completion of the milk dentition at about the age of two years. Certain diseases affecting the bony skeleton, e.g., Rickets, retard the eruptive period considerably.

The milk-teeth usually come through in batches, each period of eruption being succeeded by one of quiescence lasting sometimes several months. The milk-teeth should be in use from the age of two up to within a few months of the time for their successors to appear. Their retention serves the purpose of preserving the necessary space sufficient for the succeeding permanent teeth to occupy.

It is important to notice that it is a molar which is the first tooth to be cut in the permanent dentition, not an incisor as in the case of the temporary set, and also that it appears behind the last deciduous molar on each side.

The third molars, often called Wistoms, are sometimes unerupted through life from want of sufficient jaw space and the presence of the other teeth: and in highly civilized races there are evidences to show that they are in process of suppression from the dental series; cases of whole families in which their absence is a characteristic feature being occasionally met with.

When the teeth are fully erupted it will be observed that the upper incisors and canines project obliquely over the lower front teeth and the external cusps of the upper bicuspids and molars lie outside those of the corresponding teeth in the lower jaw. This arrangement allows to some extent of a scissor-like action in.dividing and biting food in the case of incisors; and a grinding motion in that of the bicuspids and molars when the side to side movements of the lower jaw bring the external cusps of the lower teeth into direct articulation with those of the 
upper, and then cause them to glide down the inclined surfaces of the external and up the internal cusps of these same upper teeth during the act of mastication.

'The work of the canine teeth in man is similar to that of his incisors. Besides being a firmly implanted tooth and one of stronger substance than the others, the canine tooth is important in preserving the shape of the angle of the mouth, and by its shape, whether pointed or blunt, long or short, becomes a character tooth of the dentition as a whole in both males and females.

Another feature in the fully developed and properly articulated set of teeth is that no two teeth oppose each other only, but that each tooth antagonizes with two, except the upper Wisdom, usually a small tooth. This is the result of the greater width of the upper incisors, which so arranges the "bite" of the other teeth that the lower canine closes in front of the upper one.

Should a tooth be lost, therefor?, it does not follow that its former opponent remaining in the mouth is rendered useless and thereby liable to be removed from the jaw by a gradual process of extrusion commonly seen in teeth that have no work to perform by reason of absence of antagonists.

It is worthy of note that from the age of four years to the shedding of the first milk-tooth the child has no fewer than forty-eight teetn, twenty milk-teeth and twenty-eight calcified germs of permanent teeth (all in fact except the four wisdom teeth, which show no signs of development until the third year).

\section{Structure of a Tooth.}

A tooth is generally described as possessing a crown, neck, and root or roots.

The crown is the portion which projects beyoud the level of the gum. The neck is that constricted portion just below the crown which is embraced by the free edges of the gum, and the root includes all below this.

On making longitudinal and transverse sections through its centre (fig. $\% 3, \mathrm{~A}, \mathrm{~B}$ ), a tooth is found to be principally composed of a hard material, dentine or ivory, which is hollowed out into a central cavity which resembles in general shape the outline of the tooth, and is called the $p u l p$ cavity from its containing the very vascular and sensitive pulp.

The tooth pulp is composed of fibrous connective tissue, blood-vessels, nerves, and large numbers of cells of varying shapes, e.g., fusiform, stellate, and on the surface in close comnection with the dentine a specialized layer of cells called odontoblasts, which are elongated columnar-looking cells with a large nucleus at the tapering ends or those farthest from 
the dentine (the layer is sometimes mentioned as the membrana eboris, from the tenacity with which it clings to the dentine), all are imbedded in a mucoid gelatinous matrix.

'The blood-vessels and nerves enter the pulp through a small opening at the apical extremity of each root. The exact terminations of the nerves are not definitely known. They have never been observed to enter the dentinal tubes, but they are probably connected with the fibrils in those tubes through the intervention of the odontoblasts and deeper linyer of cells. No lymphatics have been traced to the pulp.

A layer of very hard calcareous matter, the enamel, caps that part of the dentine which projects beyond the level of the gum; while sheath-
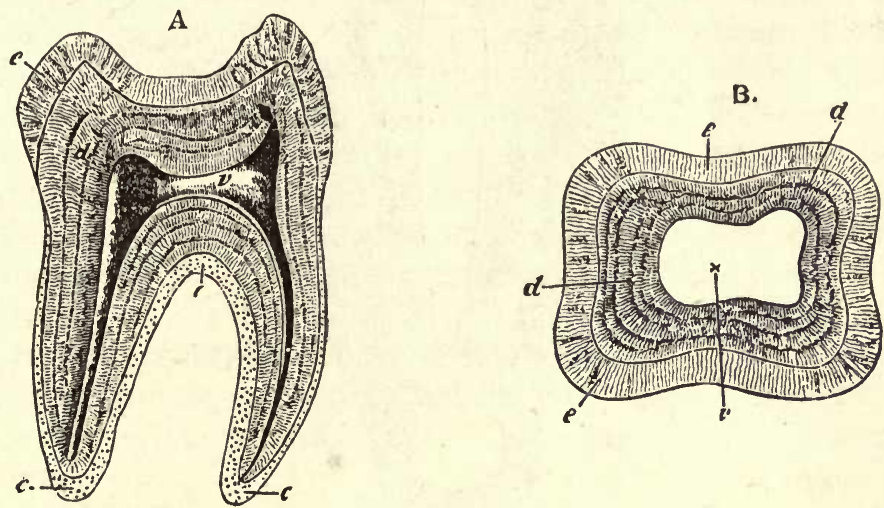

Fig. 73.-A. Longitudinal section of a human molar tooth; $c$, cement; $d$, dentine; $e$, enamel; $v$, pulp cavity (Uwen). B. Trausverse section. The letters indicate the same as in $A$.

ing the portion of dentine which is beneath the level of the gum, is a layer of true bone, called the cement or crusta petrosa.

The enamel and cement are very thin at the neck of the tooth where they come in contact, the cement overlapping the enamel. A thin epithelial and horny membrane (enamel cuticle, or Nasmyth's membrane) covers the outer surface of the enamel on unworn teeth. It is formed of short flattened prisms which are the remains of the uncalcified lastformed portions of the enamel prisms. The enamel becomes thicker toward the crown, and the cement toward the lower end or apex of the root.

\section{I.-Dentine or Ivory.}

Chemical Composition.-Dentine closely resembles bone in chemical composition. It contains, however, rather less animal matter; the proportion in a hundred parts being about twenty-eight animal to seventytwo of earthy. The former, like the animal matter of bone, may be resolved into gelatin by boiling. It also contains a trace of fat. The earthy matter is made up chiefly of calcium phosphate, with a small por- 
tion of the carbonate, and traces of calcium fluoride and magnesium phosphate.

Structure.-Under the microscope dentine is seen to be finely channelled by a multitude of delicate tubes, which, by their inner ends com-

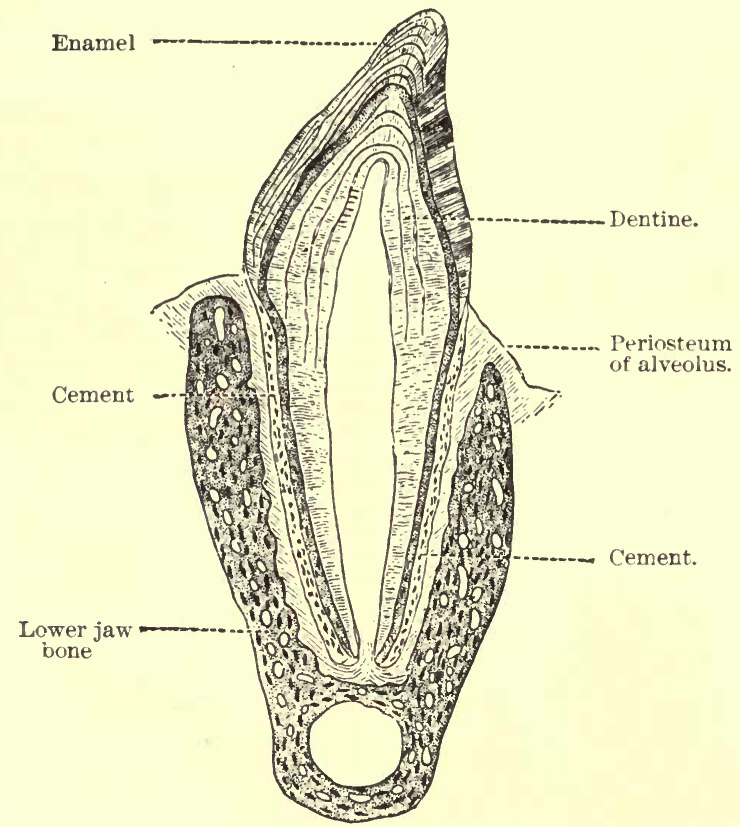

Fig. “4.-Premolar tooth of cat in situ.

municate with the pulp-cavity, and by their outer extremities come into contact with the under part of the enamel and cement, and sometimes

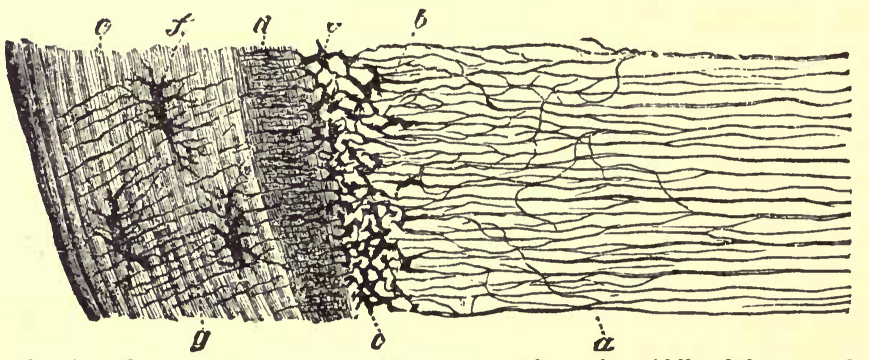

Fig. 75. - Section of a portion of the dentine and cement from the middle of the root of an incisor tootb. $a$, Dental tubuli ramifying and terminating, some of them in the interglobular spaces $b$ and $c$, which somewhat resemble bone lacunæ; $d$, inner layer of the cement with numerous closely set canaliculi; $e$, outer layer of cement; $f$, lacunæ; $g$, canaliculi. $\times 350$. (Kölliker.)

even penetrate them for a greater or less distance (figs. $75,7 \%$ ). The matrix in which these tubes lie is composed of "a reticulum of fine fibres of connective tissue modified by calcification, and where that pro- 
cess is complete, entirely hidden by the densely deposited lime salts" (Mummery).

In their course from the pulp-cavity to the surface the minute tubes form gentle and nearly parallel curves and divide and subdivide dichotomously, but without much lessening of their calibre until they are approaching their peripheral termination.

From their sides proceed other exceedingly minute secondary canals, which extend into the dentine between the tubules and anastomose with each other. The tubules of the dentine, the average diameter of which at their inner and larger extremity is $\frac{1}{4 \frac{1}{500}}$ of an inch, contain fine prolongations from the tooth-pulp, which give the dentine a certain faint sensitiveness under ordinary circumstances and, without doubt, have to do also with its nutrition. These prolongations from the tooth-pulp are probably processes of the dentine-cells or odontoblasts which are branched cells lining the pulp-cavity; the relation of these processes to the tubules in which they lie being precisely similar to that of the processes of the bone-corpuscles to the canaliculi of bone. The outer portion of the dentine, underlying the cement, and the enamel to a much lesser degree, forms a more or less distinct layer termed the granular or interglobular layer. It is characterized by the presence of a number of irregular minute cell-like cavities, much more closely packed than the lacunæ in the cement, and communicating with one another and with the ends of the dentine-tubes (fig. $75, b, c$ ), and cantaining cells like bonecorpuscles.

\section{II.-Enamel.}

Chemical Composition.- The enamel, which is by far the hardest portion of a tooth, is composed, chemically, of the same elements that enter into the composition of dentine and bone. Its animal matter, however, amounts only to about 2 or 3 per cent. It contains a larger proportion of inorganic matter and is harder than any other tissue in the body.

Structure.-Examined under the microscope, enamel is found composed of fine hexagonal fibres (figs. $76,7 \%)^{\frac{1}{500}}$ of an inch in diameter, which are set on end on the surface of the dentine, and fit into corresponding depressions in the same.

They radiate in such a manner from the dentine that at the top of the tooth they are more or less vertical, while toward the sides they tend to the horizontal direction. Like the dentine tubules, they are not straight, but disposed in wavy and parallel curves. The fibres are marked by transverse lines, and are mostly solid, but some of them may contain a very minute canal.

The enamel-prisms are connected together by a very minute quantity of hyaline cement-substance. In the deeper part of badly formed en- 
amels, between the prisms, are small lacunce, or "interglobular spaces" which have the processes or fibrils of the dentine tubes in connection with them (fig. $7 \%, c$ ).

A

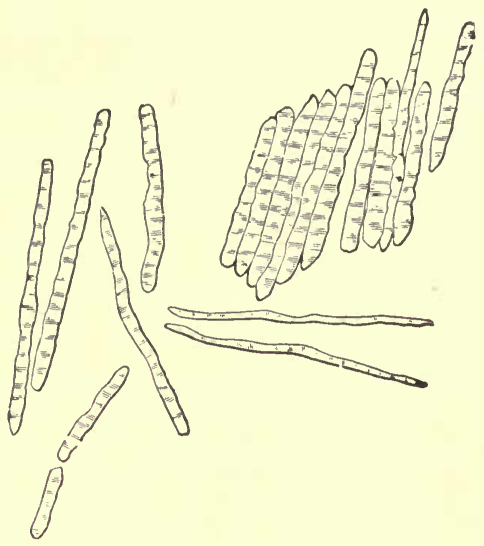

$\mathrm{B}$

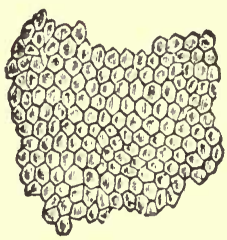

Fig. 76.-Enamel fibres. A, Fragments and single fibres of the transversely-striated enamel, isolated by the action of hydrochloric acid. B, Surface of a small fragment of enamel, showing the hexagonal ends of the fibres with darker centres, or not so highly calcified. $\times 350$. (Kölliker.)

\section{III.-Crusta Petrosa.}

The crusta petrosa, or cement (fig. $75, e, d$ ), is composed of true bone, and in it are lacunæ $(f)$ and canaliculi $(g)$, which sometimes communicate with the outer finely branched ends of the dentine tubules, and generally with the interglobular spaces. Its laminæ are as it were bolted together by perforating fibres like those of ordinary bone (Sharpey's fibres). Cement differs from ordinary bone in possessing no Haversian canals, or, if at all, only in the thickest part. Such canals are more often met with in teeth with the cement hypertrophied than in the normal tooth.

\section{Development of the Teeth.}

Development of the Teeth.-The first step in the development of the teeth consists in a downward growth (fig. $78, \mathrm{~A}, 1$ ) from the Rete Malpighi or the deeper layer of stratified epithelium of the mucous membrane of the mouth, which first becomes thickened in the neighborhood of the maxillæ or jaws now in the course of formation. This process passes downward into a recess of the imperfectly developed tissue of the embryonic jaw. The downward epithelial growth forms the primary enamel organ or enamel germ, and its position is indicated by a slight groove in the mucous membrane of the jaw. The next step in the process consists in the elongation downward of the enamel groove and of 
the enamel germ and the inclination outward of the deeper part (fig. 78, $\left.\mathrm{s}, f^{\prime}\right)$, which is now inelined at an angle with the upper portion or neck $(f)$, and has become bulbous. After this there is an increased development at certain points corresponding to the situations of the future milk-teeth. The enamel germ, or common enamel germ, as it may be called, becomes divided at its deeper portion, or extended by further

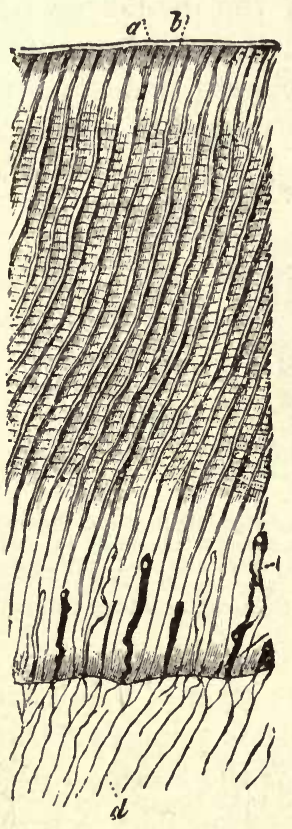

Fig. 77 .

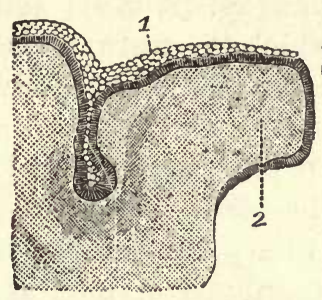

A

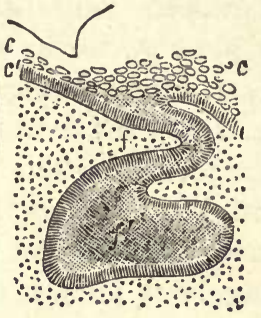

B

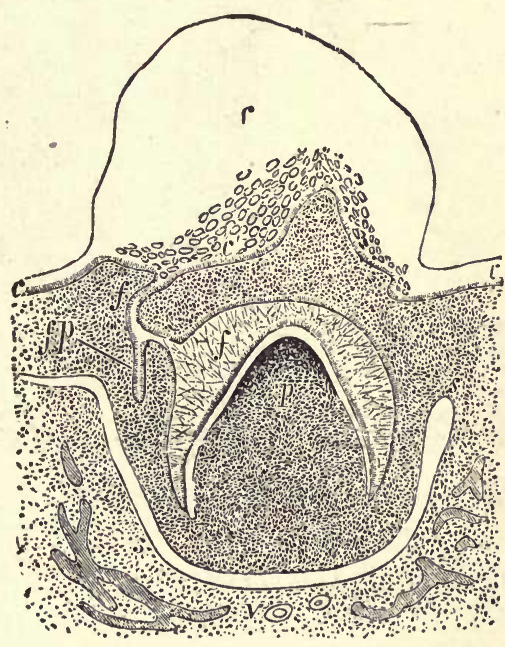

C

Fig. 78

Fig. 77.-Thin section of the enamel and a part of the dentine. $a$, Cuticular pellicle of the enamel (Nasmyth's membrane); $b$. enamel fibres, or columns with fissures between them and cross striæ; $c$, larger cavities in the enamel, communicating with the extremities of some of the dentinal tubuli $(d)$. $\times 350$. (Kölliker.)

Fig. 78. - Section of the upper jaw of a fotal sheep. A.-1, Common enamel germ dipping down into the mucous membrane; 2 , palatine process of jaw; 3 , rete Malpighi. B.-Section similar to A, but passing through one of the special enamel germs here becoming flask-shaped; $c, c^{\prime}$, epithelium of mouth; $f$, neck; $f^{\prime}$, body of special enamel germ. C.-A later stage; $c$, outline of epithelium of gıım: $f$, neck of enamel germ: $f^{\prime}$, enamel organ; $p$, papilla; $s$, dental sac forming: $f p$, the enamel germ of permanent tooth; $m$, bone of jaw; $v$, vessels cut across. (Waldeyer and Kölliker.) Copied from Quain's Anatomy.

growth, into a number of special enamel germs corresponding to each of the above-mentioned milk-teeth, and connected to the common germ by a narrow neck. Each tooth is thus placed in its own special recess in the embryonic jaw (tig. $78, \mathrm{~B}, f f^{\prime}$ ). 
As these changes proceed, there grows up from the underlying tissue into each enamel germ (fig. 78, c, p), a distinct rascular papilla (dental papilla), and upon it the enamel germ becomes moulded, and presents the appearance of a cap of two layers of epithelium separated by an interval (fig. 88, c, $f^{\prime}$ ). While part of the sub-epithelial tissue is elevated to form the dental papillæ, the part which bounds the embryonic teeth forms the dental sacs (fig. 78, c, s); and the rudiment of the jaw, at first a bony gutter in which the teeth germs lie, sends up processes forming partitions between the teeth. In this way small chambers are produced in which the dental sacs are contained, and thus the sockets of the teeth are formed. The papilla, which is really part of the dental sac (if one thinks of this as the whole of the sub-epithelial tissue surrounding the enamel organ and interposed between the enamel germ and the developing bony jaw), is composed of nucleated cells arranged in a meshwork,

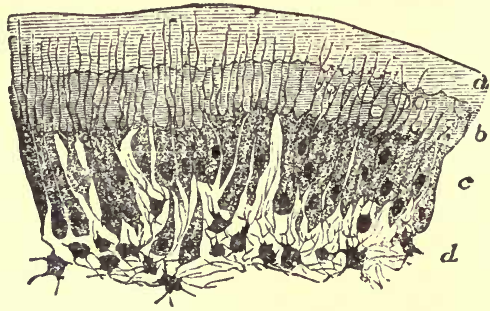

Fig 79.-Part of section of developing tooth of a young rat, showing the mode of deposition of the dentine. Highly magnified. $a$, Outer layer of fully formed dentine; $b$, uncalcified natrix with one or two nodules of calcareous matter near the calcified parts; $c$, odontoblasis sending processes in to the dentine: $d$, pulp; $e$, fusiform or wedge-shape cells found between odontoblasts; $f$. stellate cells of pulp in fibrous connective tissue. The section is stained in carmine, which colors the uncalcified matrix but not the calcified part. (E. A. Schäfer.)

the outer or peripheral part being covered with a layer of columnar nucleated cells called odontoblasts. The odontoblasts possibly form the dentine, while the remainder of the papilla forms the tooth-pulp. The method of the formation of the dentine from the odontoblasts is said to be as follows: The cells elongate at their outer part, and these processes are directly converted into the tubules of dentine (fig. $79, c$ ), and, according to some, into the contained fibrils as well. The continued formation of dentine proceeds by the elongation of the odontoblasts, and their subsequent conversion by a process of calcification into dentine tubules. The most recently formed tubules are not immediately calcified. The dentine fibrils contained in the tubules are said, by others, to be formed from processes of the deeper layer of odontoblasts, which are wedged in between the cells of the superficial layer (fig. 79,e) which form the tubules only. There are several theories upon these points. The matrix, according to more recent views, is formed by a calcification of the fibrous connective tissue developed in the papilla.

Since the papillæ are to form the main portion of each tooth, i.e., the 
dentine, each of them early takes the shape of the crown of the tooth to which it corresponds. As the dentine increases in thickness the p.pillæ diminish, and at last when the tooth is cut only a small amount of the papilla remains as the dental pulp, and is supplied by vessels and nerves which enter at the end of the root. The shape of the crown of the tooth is taken by the corresponding papilla, and that of the single or double root by the subsequent constriction below the crown, or by division of the lower part of the papilla. The number of roots being foreshadowed by the number of arteries going to the papilla. The roots

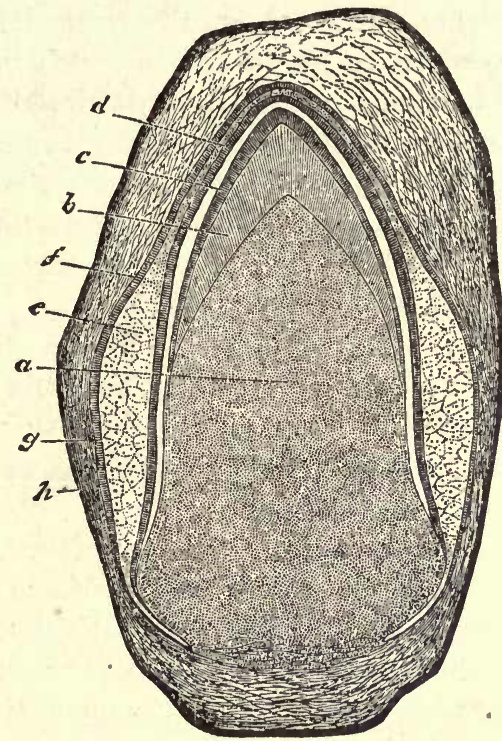

Fig. 80.-Vertical transverse section of the dental sac, pulp, etc, of a kitten. $a$, Dental papilla or pulp; $b$, the cap of dentine formed upon the summit; $c$, its covering of enamel; $d$, inner layer of epithelium of the enamel organ; $e$, gelatinous tissue; $f$, outer epithelial layer of the enamel organ; $g$, inner layer, and $h$, outer layer of dental sac. $\times 14$. (Thiersch.)

are not completely formed at the time of the eruption of the teeth, but subsequently.

The enamel cap is found later on to consist (fig. $80, d, e, f$ ) of four parts: (1) an inner membrane, composed of a layer of columnar epithelium in contact with the dentine, called enamel cells; (2) outside of these one or more layers of small polyhedral nucleated cells (stratum intermedium of Hannover); (3) an outer membrane of several layers of epithelium; (4) a middle membrane formed of a matrix of non-vascular gelatinous tissue, containing stellate cells. The enamel is formed by the enamel cells of the inner membrane, by the deposit of a keratin-like substance, which subsequently undergoes calcification and forms the first layer. Other layers are formed in the same manner, the cells retiring 
meanwhile, until when the tooth breaks throngh the gum it is covered by an uncalcified layer of the keratin-like substance which is called Nasmyth's membrane. At this time the other layers of the enamel cap have disappeared.

The cement or crusta petrosa is formed from the internal tissue of the tooth sac, the structure and function of which are identical with those of the osteogenetic layer of the periosteum, or, in other words, ossification in membrane occurs in it.

The onter layer or portion of the membrane of the tooth sac forms. the fibrous dental periosteum.

This periosteum, when the tooth is fully formed, is not only a means of attachment of the tooth to its socket, but also in conjunction with the pulp a source of nourishment to it. Arditional lamina of cement are added to the root from time to time during the life of the tooth, as especially well seen in the abnormal condition called exostosis, by the process of calcification taking place in the periosteum. On the other hand absorption of the root may equally occur through the same membrane.

In this manner the first set of teeth, or the milk-teeth, are formed; and each tooth, by degrees developing, presses at length on the w:ll of the sac inclosing it, and, causing its absorption, is cut, to use a fumiliar phrase.

The temporary or milk-teeth are speedily replaced by the growth of the permanent teeth, which push their way up from beneath them.

Each temporary tooth is replaced by a tooth of the permanent set which is developed from a small sac set by, so to speak, from the sac of the temporary tooth which precedes it, and called the cavity of reserve (fig. $78, c, f p$ ). Thus the temporary incisors and canines are succeeded by the corresponding permanent ones, the temporary first molar by the first bicuspid, the temporary second molar derelops two offshoots, one for the second bicuspid, the other for the permanent first molar. 'The permanent second molar is budded off from the first permanent molar and the wisdom from the permanent second molar.

The development of the temporary teeth is said to commence about the sixth week of intra-uterine life, after the laying down of the bony structure of the jaws. Their permanent successors begin to form about the sixteenth week of intra-uterine life.

The second permanent molars are believed to originate about the third month after birth, and the wisdom teeth about the third year. 


\section{Muscular Tissue.}

There are two chief kinds of muscular tissue, differing both in minute structure as well as in mode of action, viz., (1.) the plain or nonstriated, and (2.) the striated.

\section{Unstriped or Plain Muscle.}

Distribution.-Unstriped muscle forms the proper muscular coats (1.) of the digestive canal from the middle of the oesophagus to the internal sphincter ani; (2.) of the ureters and urinary bladder; (3.) of the trachea and bronchi; (4.) of the ducts of glands; (5.) of the gall-bladder; (6.) of the vesiculæ seminales; (\%.) of the pregnant uterus; (8.) of blood-vessels and lymphatics; (9.) of the iris, and some other parts of

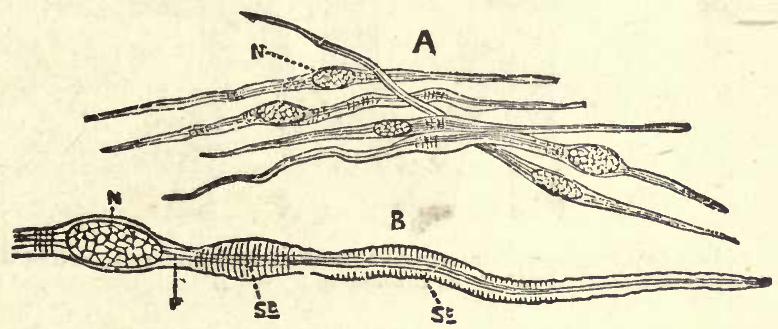

Fig. 81.-A, Unstriped muscle cells from the mesentery of a newt. The sheath exhibits transverse markings. $\times 180$. B, From a similar preparation, showing that each muscle cell consists of a central bundle of fibrils, $F$ (conıractile par't), connected with the intra-nuclear network, $\mathrm{N}$, and a sheath with annular thickenings, St. The cells show varicosities due to local contraction, and on these the annular thickenings are most marked. $\times 450$. (Klein and Noble Smith.)

the eye. This form of tissue also enters largely into the composition (10.) of the tunica dartos, the contraction of which is the principal cauise of the wrinkling and contraction of the scrotum on exposure to cold. Unstriped muscular tissue occurs largely also in the true skin generally, being especially abundant in the interspaces between the bases of the papillæ. Hence when it contracts under the influence of cold, fear, electricity, or any other stimulus, the papillæ are made unusually prominent, and give rise to the peculiar roughness of the skin termed cutis anserina, or goose skin. It occurs also in the superficial portion of the cutis, in all parts where hairs occur, in the form of flattened roundish bundles, which lie alongside the hair-follicles and sebaceous glands. They pass obliquely from without inward, embrace the sebaceous glands, and are attached to the hair-follicles near their base.

Structure.-Unstriated muscles are made up of elongated, spindleshaped, nucleated cells (fig. 81), which in their perfect form are flat, from about $\frac{x}{4500}$ to $\frac{1}{3500}$ of an ineh broad ( 7 to $8 \mu$ ), and $\frac{1}{600}$ to $\frac{1}{300}$ of an inch $\left(\frac{1}{12}\right.$ to $\left.\frac{1}{24} \mathrm{~mm}\right)$ in length-very clear, granular, and brittle, so that 
when they break they often have abruptly rounded or square extremities. Each cell of these consists of a fine sheath, probably elastic; of a central bundle of fibrils representing the contractile substance; and of an oblong nucleus, which includes within a membrane a fine network anastomosing at the poles of the nucleus with the contractile fibrils. 'The ends of fibres are usually single, sometimes divided. Between the fibres is an albuminous cementing material or endomysium in which are found

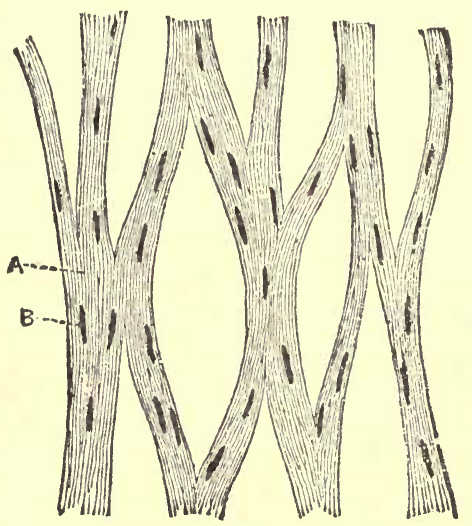

Fig. 8\%.-Plexus of bundles of unstriped muscle cells from the pulnonary pleura of the Guinea-pig. $\times 180$. (Klein and Noble Smith.) A, Branching fibres; B, their long central nuclei.

connective-tissue corpuscles, and a few fibres. The perimysium is continuous with the endomysium in the fibrous connective tissue surrounding and separating the bundles of muscle cells.

\section{Striated Muscle.}

Distribution.--Striated or striped muscle is found in the following situations. It constitutes the whole of the muscular apparatus of the skeleton, of the walls of the abdomen, etc., the whole of those muscles which are under the control of the will and hence termed voluntary, as well as certain other muscles, e.g., of the internal ear and pharynx not directly under the control of the will, and the heart.

Structure.-For the sake of description, striated muscular tissue may be divided into two classes, (a.) skeletal, which comprises the whole of the striated muscles of the body except (b.) the heart:-

(a.) Skeletal Muscle.-In the majority of cases a skelotal muscle is inclosed in a sheath of areolar tissue called the epimysium, which in some cases is a very thick and distinct investment, while in other cases it is much thinner. The sheath sends in partitions which serve to support the fascicali or bundles of fibres, of which the muscle is made up, forming more or less distinct sheaths for them, called perimysium. The 
fibres themselves are supported in their fasciculus by a scanty amount of areolar tissue containing plasma cells and termed endomysium. Within the areolar tissue supporting the fasciculi and between the fibres are contained the blood-vessels and nerves of the tissue.

The muscular fibres of each fasciculus are parallel to one another, and generally speaking so are the fasciculi themselves, except that toward

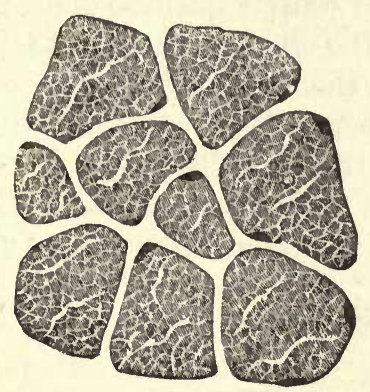

Fig. 83. their terminations they may converge to their insertion into the tendon of the muscle. 'The fascienli extend throughout the whole length of the muscle, but they vary in size and in the number of their con-

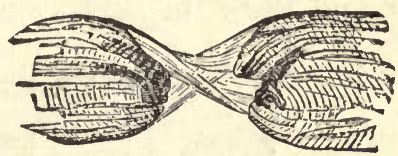

Fig. 84.

Fig. 83.-Transverse section through muscular fibres of human tongue. The muscle-corpuscles are in licated by their deeply-stained nuclei situated at the inside of the sarcolemma. Each musclefibre shows "Cohnheim's fields." that is, the sarcous elements in transverse section separated by clear (apparently linear) interstitial substance. $\times 450$. (Klein and Noble Smith.)

Fig. 84. - Muscular fibre torn across; the sarcolemma still connecting the two parts of the fibre. (Todd and Bownan.)

tained fibres, both in different muscles and also in the same muscle, some muscles having coarse, others fine fasciculi. In some cases it would seem that the perimysium is altogether independent of the external sheath of the muscle. As to the fibres of which the bundles are made up, they have a distinct elastic sheath, the sarcolemma; their size varies considerably, their cross-section being from $100 \mu$ to $10 \mu$, and as regards their

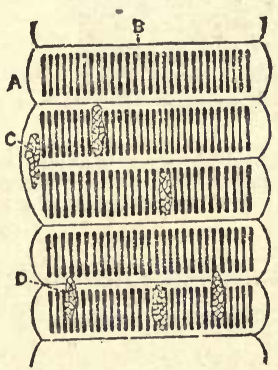

Fig. 85. - Part of a striped muscle-fibre of a water beetle prepared with absolute alcohol. A, Sarcolenina; B, Krause's nembrane. The sarcolemma shows regular bulgings. Above and below Kruuse's membrane are seen the transparent "lateral discs." The chief mass of a muscular comjartment is occupied by the contractile disc composed of sarcous elements. The substance of the i.dividual sarcous elements has collected more at the extremity than in the centre: hence this latter is more transparent. The optical effect of this is that the contractile disc appears to possess " "median disc" (Disc of Hensen). Several nuclei of muscle corpuscles, C and D, are shown, and in them a minute network. $\times 300$. (Klein and Noble Smith.)

shape, it is cylindrical or is triangular, quadrilateral, or pentangular with rounded angles. In length the fibres seldom exceed an inch and a half 
$(3.75 \mathrm{~cm})$. It is thus evident that the same fibre does not extend from one end of a muscle to the other, and indeed it is known that in a fasciculus fibrils are joined together by rounded or angular extremities invested with their proper sheath the sarcolemmil.

Each muscular fibre then is thus constructed:-Externally is a fine, transparent, structureless membrane, the sarcotemma, which in the form of a tubular investing sheath forms the outer wall of the fibre and which contains the contractile material of which the fibre is chiefly made up. Sometimes, from its comparative toughness, the sarcolemma will remain untorn, when by extension the contained part eim be broken (fig. 84), and its presence is in this way best demonstrated. The fibres are of a pale yellow eolor, and apparently marked by fine striæ which pass transversely round them, in slightly eurved or wholly parallel lines. The

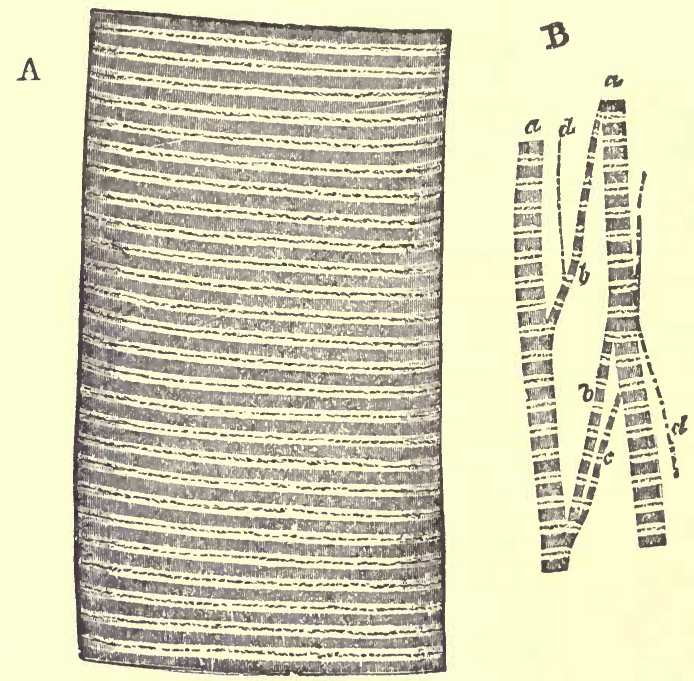

Fig. 86.-A. Portion of a medium-sized human muscular fibre. $\times 800 . \quad$ B. Separated bundles of fibrils equally magnified; $a$, $a$, larger, and $b, b$, smaller collections; $c$, still smaller; $d, d$, the smallest which could be detached, possibly representing a single series of sarcous element. (Sharpey.)

sarcolemma is a transparent structureless elastic sheath of great resistance which surrounds each fibre (fig. 84). There is still some doubt regarding the nature of the fibrils.

A striated muscle fibre, when examined with a suffiejently high power of the mieroseope, presents the following appearances, longitudinally :-

(a.) Alternate dark and light parallel transverse stripes, to which this variety of muscle owes its name, the depth of the stripes not always being the same.

(b.) With still higher powers of the microscope, the bright stripes 
may be seen to be divided in the middle line by other very fine transverse dark lines, sometimes called Dobie's line.

(c.) Each dark stripe may also sometimes be seen to be divided by a clear line, called Henson's disc.

(d.) Each fibre presents an appearance of longitudinal striation and after hardening in alcohol may be divided by teasing with needles into longitudinal fibrils, more or less cylindrical or angular, which are named muscle columns or sarcostyles, and extend throughout each fibre. Each of these appears to consist of short columns connected together by bright intervals, the former are the sarcous elements of Bowman. They may possibly be further longitudinally striated, and so made up of finer fibrillæ still.

After treatment with reagents the fibre may be split up into transverse discs.

(e.) On Transverse Section.-The fibre presents most externally, the outline of the sarcolemma.

(f.) The muscular substance proper appears to be mapped out into

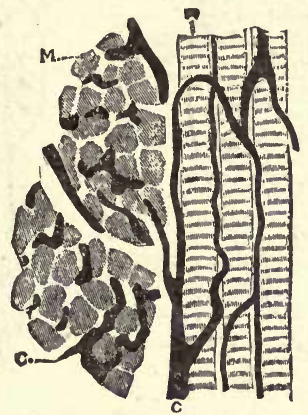

Fig. 87.-Three muscular fibres running longitudinally, and two bundles of fibres in transverse section, M, from the tongue. The capillaries, C, are injected. $\times 150$. (Klein and Noble Smith.)

small polygonal areas by clear lines (fig. 83) called Cohnheim's fields, the lines giving the appearance of a meshwork. The lines represent the transverse section of the cementing material between the sarcostyles, which is called sarcoplasm.

(g.) Immediately within the sarcolemma in ordinary muscle or in the centre of the fibre as in the muscle of some insects, are seen clear oval nuclei called muscle nuclei or muscle corpuscle, surrounding which is a certain amount of granular protoplasm (fig. 85).

The appearances of the muscle fibre when seen under the microscope, cannot be said to be yet thoroughly understood, and have given rise to various theories as to the structure of striped muscle, to several of which it will be as well to allude.

- Muscle Caskets (Krause) Theory.-According to this view a muscle fibre is made up of transverse compartments, bounded laterally by the sarcolemma, and above and below by a fine membrane, called 
Krause's membrane, which passes from side to side from the sarcolemma across the light stripe. This membrane corresponds to Dobie's line. 'The transverse compartments are divided longitudinally into smaller ones by lines which correspond with the boundaries of Cohnheim's areas, and each such compartment is termed a muscle casket. Within the middle part of the casket is a muscle prism made up of darker rods of contractile material called muscle rods, and above and below the muscle prism is a more fluid substance. When the muscle contracts, the fluid substance is pressed more between the muscle rods, causing them to be further away from one another.

Muscle Reticulum Theory.-According to the views of certain observers (Retzius, Melland. Marshall, van Gehuchten, and Carnoy), the

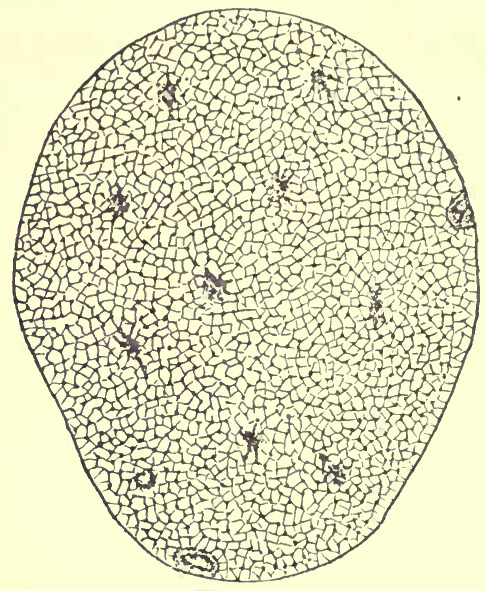

Fig. 88

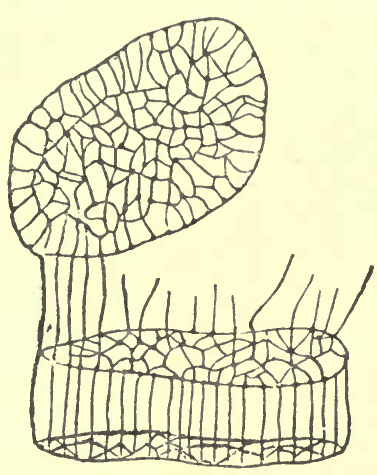

Fig. $88 \mathrm{~A}$.

Fiz. 88.-Transverse section of one of the truuk muscles of the Hippocampus, stained in chloride of gold. (Rollett.)

Fig. 88A.- Portion of muscle-fibre of Dytiscus. showing network very plainly. One of the transverse networks is split off, and some of the longitudiual bars are shown broken off. (After Melland.)

part of fresh muscle which is stained in chloride of gold, is a meshwork of fibrils which corresponds to the intracellular meshwork of ordinary protoplasmic cells, i.e., the spongioplasm, and is the part which is the contractile element in muscle. The meshwork on one level is connected with the meshwork on another level by means of longitudinal fibres, at the junction of which the meshes appear more or less knotted (figs. 88 and $88 \mathrm{~A}$ ). The longitudinal fibres of the network are, according to this theory, the chief agents in the active contraction. The transverse meshwork is more passively elastic, and may be the cause of the speedy relaxation of muscle after contraction has ceased. The material filling up the meshwork is a more fluid and non-contractile material.

Rollett has minutely criticised the idea of the gold-staining substance of the fibre being the contractile portion. His views are the 
following:-That the muscle-fibre consists of longitudinal fibrillæ grouped together into muscle columns, which are seen in the transverse section as Cohnheim's fields, and that the intercolumnar material is semi-fluid sarcoplasm. A muscle column consists of segments alternately thin and thick, while in the centre of the thin portion is a dark enlargement forming a dot, these dots in Cuhnheim's arrangement correspond to Krause's membrane.

In fresh muscle, at low focus, according to this view, the musclecolumns appear dark and the sarcoplasma appears light, the former are in a line with the granules. At high focus, the reverse is the case, but the dark sarcoplasma is now seen in line with two rows of granules (fig. 89).

Also, that in gold-stained preparations, the dark row of granules are thicknesses of the sarcoplasma between the thin segments of the muscle

A.

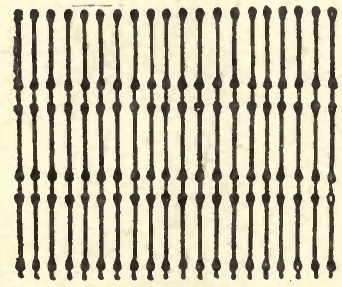

$\mathbf{B}$

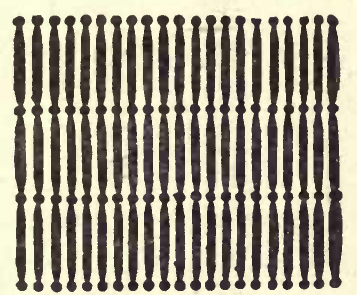

Fig. 89.-Diagram of the appearances in fresh muscle-fibre. A. At low focus (B) the muscle columns appear dark and in a line with the granules, sarcoplasm light. At high focus (A) the sarcoplasm is dark, muscle columns light, and two rows of granules appear in a line with the sarcoplasm and alternating with the muscle columns. (Marshall, after Rollett.)

columns, whereas the two rows of granules do not correspond with these, but alternate with them, belonging as they do to the muscle columns, and not to the sarcoplasm.

Schäfer has thrown considerable light upon the controversy by having actually observed that when a small portion of the living wingmuscle of insects is teased up with needles in a small drop of white of egg, the sarcostyles may easily be separated from their surrounding sarcoplasm, and may be actually seen to contract, whereas the sarcoplasm shows no such property. According to this observer such a sarcostyle may be examined thus isolated, both living and after treatment with various reagents, and it shows alternate bright and light stripes, the latter being bisected by a line which corresponds wtih Krause's membrane. Krause's membrane divides the sarcostyle into sarcomeres, which contain in the middle the strongly refractive disc-like sarcous element, and above and below it hyaline material, which is bounded by Krause's membrane. The sarcous substance is penetrated by canals, which extend upward and downward from the hyaline substance to the middle. 
The sarcous substance stains with hæmatoxylin. A light interval may bisect the sarcous substance if the fibre is stretched, which corresponds with Hensen's disc.

Appearances under Polarized Light.-The appearances which muscle presents when viewed under polarized light vary according as the fibres are looked at, as fresh in their own plasma, or as hardened fibres prepared and mounted in Canada balsam.

'The whole of the living fibre may be doubly refracting, the isotropous part appearing as rows of dots separating transversely the principal material of the fibre. Shortly, according to Schäfer, it may be said that the sarcoplasm is singly refracting, and that the sarcostyle is in great part doubly refracting. In a fibre which is extended, after it has been hardened in alcohol and mounted in Cainda balsam, there are

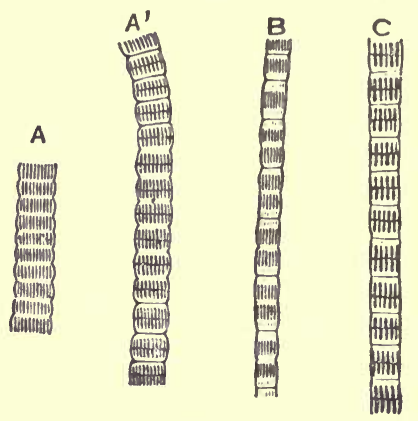

Fig. 90 .
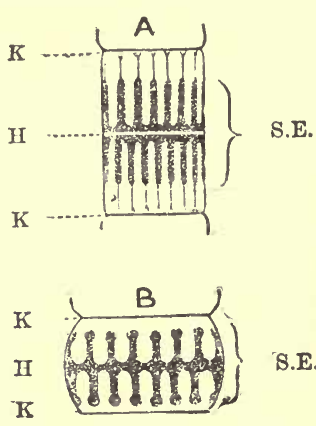

Fig. 91.

Fig. 90.-Sarcostyles from the wing-muscles of a wasp. A, $\mathrm{A}^{\prime}$. Sarcostyles showing degrees of retraction (? contraction). B. A sarcostyle extended with the sarcous elements separated into two parts. c. Sarcostyles moderately extencled (semidiagrammatic). (E. A. Schäfer.)

Fig. 91. - Diagram of a sarcomere in a moderately extencled condition, $\mathbf{A}$, and in a contracted condition, B. K, K, Krause's membranes; H, plane of Henson; s.E., poriferous sarcous element. (E. A. Schäfer.)

alternate dark and light bands, the former corresponding to the light intervals as seen in ordinary light, and the latter to the various elements. When the fibre is more contracted the dark line becomes narrower, and the anisotropous intervals broader, but there is no interval of the bands on contraction. It appears further that the chromatic portion only of the sarcostyles is anisotropous, and the sarcoplasm and the remainder of the fibre is isotropous.

(b.) Heart Muscle.--The muscular fibres of the heart, unlike those of most of the inroluntary muscles, are striated; but although, in this respect, they resemble the skeletal muscles, they hare distinguishing characteristics of their own. The fibres which lie side by side are united at frequent intervals by short branches (fig. 92). The fibres are smaller than those of the ordinary striated muscles, and their striation is less marked. No sareolemma can be discerned. 'The musele-corpuscles are situate in the middle of the substance of the fibre; and in correspond- 
ence with these the fibres appear under certain conditions subdivided into oblong portions or "cells," the offsets from which are the means by which the fibres branch and anastomose one with another.

It should be noted, however, that the heart muscular fibres are not the only ones which branch, since the fibres of the tongue of the frog, especially where they are attached to the mucous membrane, present this peculiarity; branching muscular fibres have also been noted in the tongue, and in the facial muscles of other animals. And again, in the animals in which two kinds of skeletal muscles occur, red and pale, in the red muscles the fibres are much less distinctly striated transversely, whereas their longitudinal striation is more marked than in the pale variety. They are also finer than other skeletal muscles. It should also

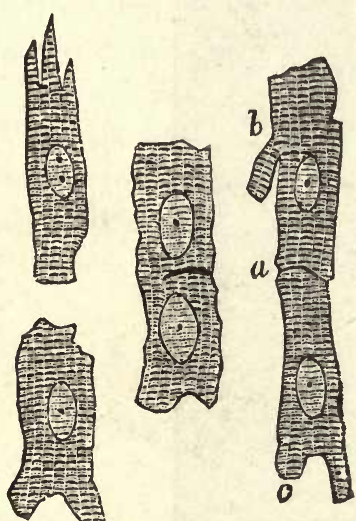

Fig. 92.

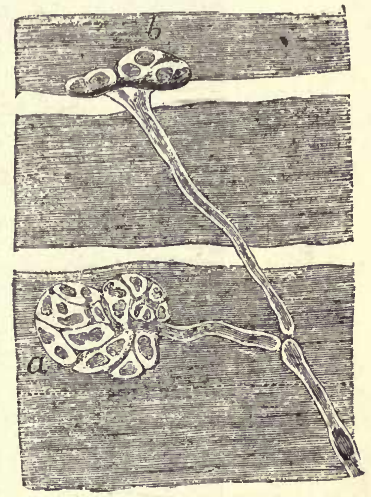

Iig. 93.

Fig. 92.-Muscular fibre cells from the heart. (E. A. Schäfer.)

Fig. 93.- From a preparation of the nerve-termination in the muscular fibres of a snake. $a$, End plate seen only broad surfaced. $b$, End plate seen as narrow surface. (Lingard and Klein.)

be added that in these red muscles the sarcoplasm is much developed, and the muscle nuclei are very numerous, and may be situated in the middle of the fibre, as is the case with heart muscle fibres.

Blood and Nerve Supply.-The voluntary muscles are freely supplied with blood-vessels; the capillaries form a network with oblong meshes around the fibres on the outside of the sarcolemma. No vessels penetrate the sarcolemma to enter the interior of the fibre. Nerves also are supplied freely to muscles; the voluntary muscles receiving them from the cerebro-spinal system, and the unstriped muscles from the sympathetic or ganglionic system.

The nerves terminate in the muscular fibre in the following ways:(1.) In unstriped muscle, the nerves first of all form a plexus, called the ground plexus (Arnold), corresponding to each group of muscle bundles; the plexus is made by the anastomosis of the primitive fibrils of the axis-cylinders. From the ground plexus, branches pass off, and 
again anastomosing, form plexuses which correspond to each muscle bundle-intermediary plexuses. From these plexuses branches consisting of primitive fibrils pass in between the individual fibres and anastomose. These fibrils either send off finer branches, or terminate themselves in the nuclei of the muscle cells.

(2.) In striped muscle the nerves end in motorial end-plates, having first formed, as in the case of unstriped fibres, ground and intermediary

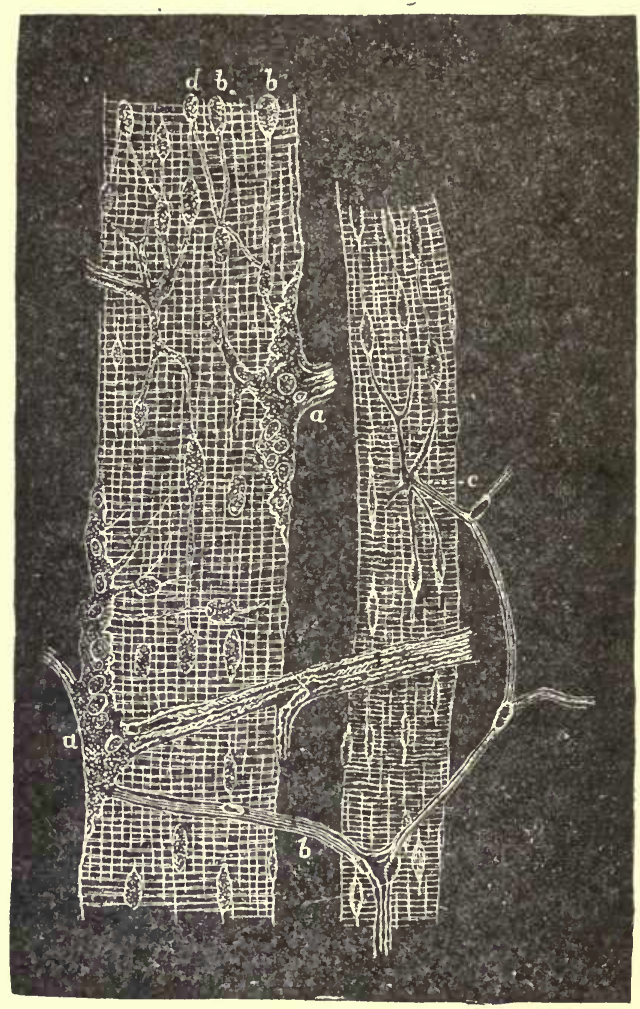

Fig. 94.-Two striped muscle-fibres of the hyoglossus of frog. $a$, Nerve-end plate; $b$, nervefibres leaving the end-plate; $c$, nerve-fibres, terminating after dividing into branches $d$, a nucleus in
which two nerve-fibres anastomose. $\times 600$. (Arndt.)

plexuses. The fibres are, however, medullated, and when a branch of the intermediary plexus passes to enter a muscle-fibre, its primitive sheath becomes continuous with the sarcolemma, and the axis-cylinder forms a network of its fibrils on the surface of the fibre. This network lies embedded in a flattened granular mass containing nuclei of several kinds; this is the motorial end-plate (figs. 93 and 94). In batrachia, besides end-plates, there is another way in which the nerves end in the muscle fibres, viz., by rounded extremities, to which oblung nuclei are attached. 
Development.-(1.) Unstriped. - The cells of unstriped muscle are derived directly from embryonic cells, by an elongation of the cell, and its nucleus; the latter changing from a vesicular to a rod shape.

(2.) Striped.-Formerly it was supposed that striated fibres were formed by the coalescence of several cells, but recently it has been proved, that each fibre is formed from a single cell, the process involving an enormous increase in size, a multiplication of the nucleus by fission, and a differentiation of the cell-contents. This view differs but little from another, that the muscular fibre is produced, not by multiplication of cells, but by arrangement of nuclei in a growing mass of protoplasm (answering to the cell in the theory just referred to), which becomes gradually differentiated so as to assume the characters of a fully developed muscular fibre.

Growth of Muscle.-The growth of muscles, both striated and nonstriated, is the result of an increase both in the number and size of the individual elements. In the pregnant uterus the fibre-cells may become enlarged to ten times their original length. In involution of the uterus after parturition the reverse changes occur, accompanied generally by some fatty infiltration of the tissue and degeneration of the fibres.

\section{Nervous Tissue.}

Nervous tissue has usually been described as being composed of two distinct substances, nerve-fibres and nerve-cells. The modern view of the nature of nerve-tissue is, however, that it is composed of one element alone, called the neuron or nerve unit, embedded in and supported by a substance called neuroglia. This neuron consists of a cell-body, a number of branching processes termed dendrites, and a long process running out from it, the neuraxon, which becomes eventually a nerve-fibre. The nerve-cell and the nerve-fibre, are really parts of the same anatomical unit, and the nervous centres are made up of these units, arranged in different ways throughout the nervous system (fig 94A). The different neurons do not unite anatomically with each other, but form independent units. A further description of these structures will be given later.

\section{Nerve-Fibres.}

While the nerve-fibre is really to be considered as a process of the nerve-cell, it is convenient to describe it separately.

Varieties.-Nerve-fibres are of two kinds, medullated or white fibres, and non-medullated or gray fibres.

Medullated Fibres. - Each medullated nerve-fibre is made up of 
the following parts:-(1.) An external sheath called the primitive nerv sheath, or nucleated sheath of Schwann; (2.) An intermediate or pac ing substance known as the medullary or myelin sheath, or white su stance of Schwann; and (3) internaily the axis-cylinder, primitive ban axis band, or axial fibre.

Although these parts can be made out in nerves examined som time after death, in a recent specimen the contents of the nerve-sheat appear to be homogenenus. But by degrees they undergo changes whic

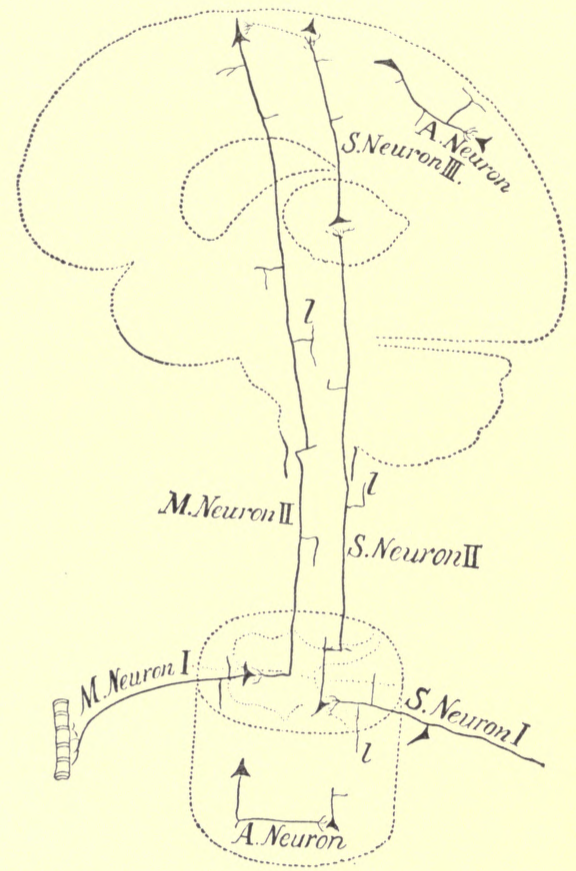

Fig. 94A.-Diagram showing the arrangement of the neurons or nerve-ing sensory ne nervons $I$. and $I$. sensory neurons; A. Neur, associative or commissural neuron. (Dana.) Neurons 1., II., III.,

show them to be composed of two different materials. The internal or central part, occupying the axis of the tube, viz., the axis-cylinder, becomes grayish, while the outer or cortical portion, or white substance of Schwann, becomes opaque and dimly granular or grumous, as if from a kind of coagulation. At the same time the fine outline of the previously transparent cylindrical tube is exchanged for a dark double contour (fig. 95, B), the outer line being formed by the sheath of the fibre, the inner by the margin of curdled or coagulated medullary substance. 
The granular material shortly collects into little masses, which distend portions of the tubular membrane; while the intermediate spaces collapse, giving the fibres a varicose, or beaded appearance (fig. 95, c and D), instead of the previous cylindrical form. The whole contents of the nerve-tubules are extremely soft, for when subjected to pressure they readily pass from one part of the tubular sheath to another, and often cause a bulging at the side of the membrane. They also readily escape, on pressure, from the extremities of the tubule, in the form of a grumous or granular material.

The external nucleated sheath of Schwann, also called the neurilemma, is a pellucid membrane forming the outer investment of the

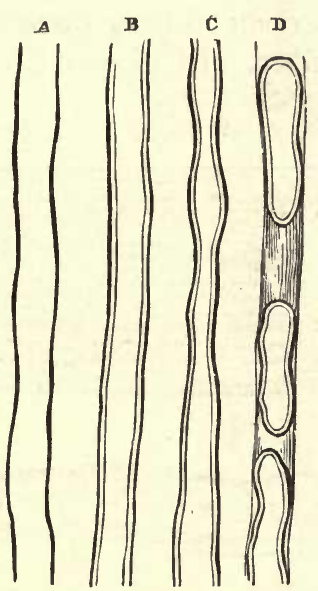

Fig. 95.

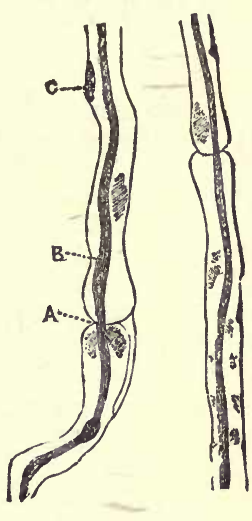

Fig. 96.

Fig. 95.--Primitive nerve-fibres. A. A perfectly fresh tubule with a single dark outline. B. A tubule or fibre with a double contour from commencing post-mortem change. c. The changes further advanced, producing a varicose or beaded appearance. D. A tubule or fibre, the central part of which, in consequence of still further changes, has accumulated in separate portions within the sheath (Wagner).

Fig. 96. - Two nerve-fibres of sciatic nerve. A. Node of Ranvier. B. Axis-cylinder. c. Sheath of Schwann, with nuclei. $\times 300$. (Klein and Noble Smith.)

nerve-fibre. Within this delicate structureless membrane nuclei are seen at intervals, surrounded by a variable amount of protoplasm. The sheath is structureless, like the sarcolemma, and the nuclei appear to be within it: together with the protoplasm which surrounds them they are the relics of embryonic cells, and from their resemblance to the muscle corpuscles of striated muscle may be termed nerve-corpuscles. They are easily stained with logwood and other dyes.

The medullary or myelin sheath or white substance of Schwann is the part to which the peculiar opaque white aspect of medullated nerves is due. The thickness of this layer in nerve-fibres varies consid- 
erably, at one time being very well developed, at another forming but a very thin investment of the axis cylinder. It is a semi-fluid, fatty substance, and in the fibre possesses a double contour. It is said to be made up of a fine reticulum (Stilling, Klein), in the meshes of which is embedded the bright fatty material. It stains well with osmic acid.

According to $\mathrm{M}^{\circ}$ Carthy this sheath is composed of small rods radiating from the axis-cylinder to the external sheath of Schwann. Sometimes the whole space is occupied by them, while at other times the rods appear shortened and compressed laterally into bundles embedded

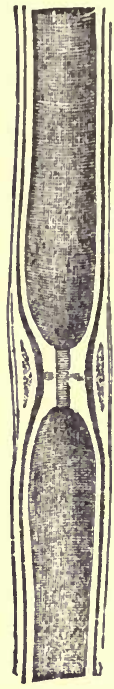

Fig. 97 . in some homogeneous substance. Aceording to other observers the sheath is made up of segments which are either cylindrical or funnel-shaped (sections of Lanter$\operatorname{mann})$. It is not definitely decided that these divisions exist naturally in the nerve-fibre. In nerves hardened in

$\Delta$

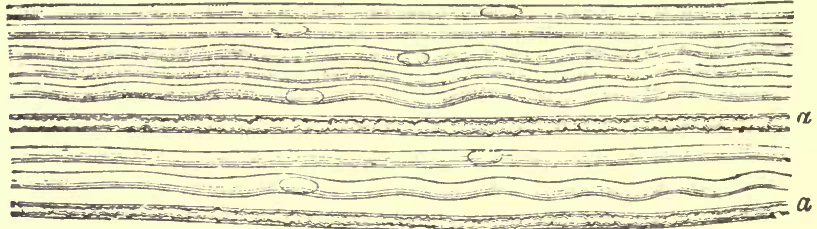

B

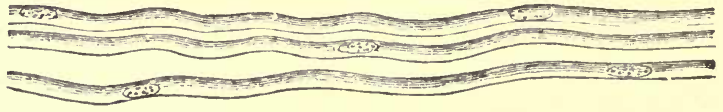

Fig. 98.

Fig. 97.-A node of Ranvier in a medullated nerve-fibre, viewed from above. The medullary sheath is interrupted, and the primitive sheatl thickened. Copied from Axel Key and Retzius. $\times 750$. (Klein and Noble smith.)

Fig. 98. - Gray, pale, or gelatinous nerve-fibres. A. From a branch of the olfactory nerve of the sheep; two dark-bordered or white fibres from the fifth pair are associated with the pale olfactory fibres. B. From the sympathetic nerve. $\times 450$. (Mlax schultze.)

alcohol, it is possible to demonstrate a very chromatic recticulum in the medullary sheath, which is supposed to be of a horny nature, since it offers much resistance both to chemical reagents and to digestive fluids (horny reticulum or neuro-keratin networl).

The axis-cylinder consists of a large number of primitive fibrillce. This is well shown in the cornea, where the axis-cylinders of nerves break up into minute fibrils which form terminal networks, and also in the spinal cord, where these fibrillæ form a large part of the gray matter. From various considerations, such as its invariable presence and unbroken continuity in all nerves, though the primitive sheath or the medullary sheath may be absent, there can be little doubt that the axis- cylinder is the essential part of the fibre, the other parts having the subsidiary function of support and possibly of insulation. 
Nodes of Ranvier.-At regular intervals in most medullated nerves the nucleated sheath of Schwann possesses annular constrictions; these. ure called nodes of Ranvier. At these points (fig. 97), the continuity of the medullary white substance is interrupted, and the primitive sheath comes into immediate contact with the axis-cylinder. The segment of the fibre between two nodes is termed an internode, and the length of the internodes varies in different nerves; their average is said to be $1 \mathrm{~mm}$. T'here is only one nerve nucleus to each internode. At each node the internodes are united within the external sheath by a band, constricting band of Ranvier (fig. 101), and this stains black with silver nitrate; the axis-cylinders at the nodes also are capable of being

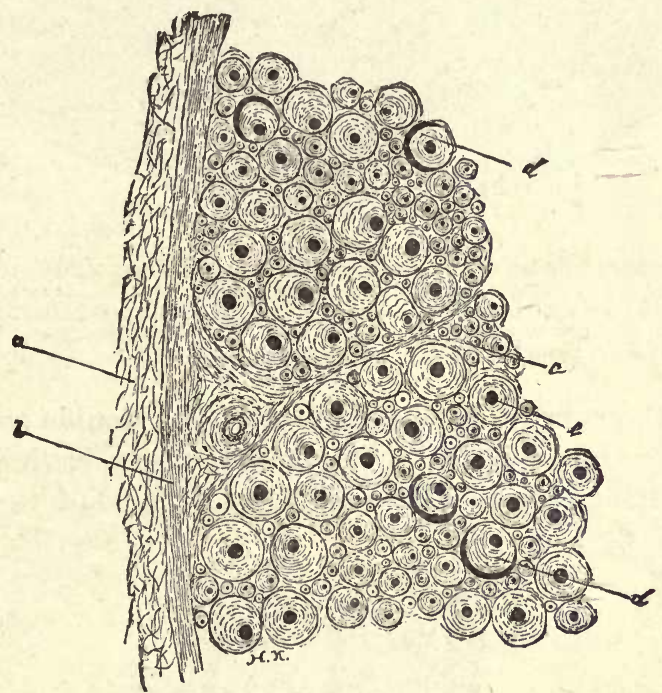

Fig. 99.-Transverse section of sciatic nerve of the rabbit, hardened in chromic acid and stained with picro-carmine, and showing lamellar sheath, reripheric connective tissue, and intra. fascicular connective tissue. $\times 550$ and reduced one-half. $a$, Perifasicular connective tissue; $b$, lamellar beath; $c$, intra-fascicular connective tissue; $d$, nerve-fibre cut across, showing nuclei of the same; $e$, axis-cylinder.

stained with the same reagent, and so a node-of Ranvier when stained with silver nitrate is marked by a black cross.

Size. - The size of the nerve-fibres varies (fig. 99); it is said that the same fibres may not preserve the same diameter through their whole length. The largest fibres are found within the trunks and branches of the spinal nerves, in which the majority measure from $14.4 \mu$ to $19 \mu$ in diameter. In the so-called visceral nerves of the brain and spinal cord medullated nerves are found, the diameter of which varies from $1.8 \mu$ to $3.6 \mu$. In the hypoglossal nerve they are intermediate in size, and generally measure $\% .2 \mu$ to $10.8 \mu$.

Non-medullated Fibres.-The fibres of the second kind (fig. 98) which are also called fibres of Remak, constitute the principal part of the trunk and branches of the sympathetic nerves, the whole of the 
olfactory nerve, and are mingled in various proportions in the cerebiuspinal nerves. 'They differ from the preceding chiefly in their fineness, being only about $\frac{1}{2}$ to $\frac{1}{3}$ as large in their course within the trunks and

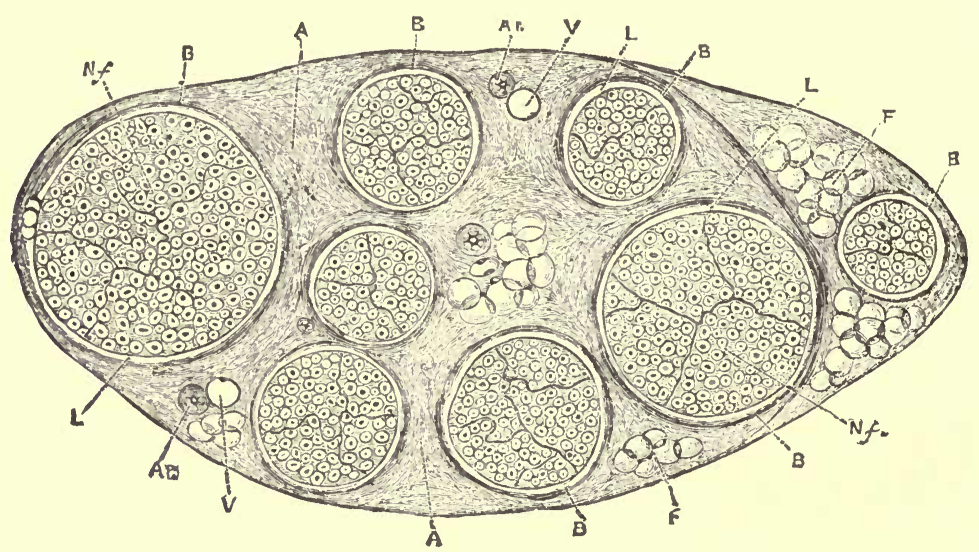

Fig. 100.-Transverse section of the sciatic nerve of a cat about $\times 100$. - It consists of bundles (Funiculi) of nerve-fibres ensheathed in a fibrous supporting capsule, epineurium, A; each bundle has a special sheath (not sufficiently marked out from the epineurium in the figure) or perineurium $\mathrm{B}$; the nerve-fibres $\mathrm{N} f$ are separated from one another by endoneurium; L, lymph spaces; Ar, artery; V, vein; F, fat. Somewhat diagrammatic. (V. I. Harris.)

branches of the nerves; in the absence of the double contour; in their contents being apparently uniform; and in their having, when in bundles, a yellowish-gray hue instead of the whiteness of the cerebro-spinal

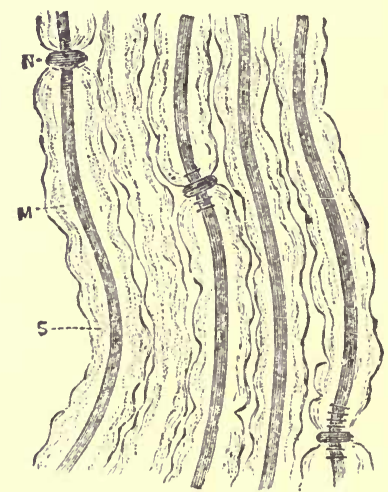

Fig. 101.-Several fibres of a bundle of medullated nerve-fibres acted upon hy silver nitrate to show peculiar behavior of nodes of lianvier. N, toward this reagent, The silver has penetrated at the nodes. and has stained the axis-cylinder, M, for a short distance. $s$, the white substance. (Klein and Noble smith.)

nerves. These peculiarities depend on their not possessing the outer layer of medullary substance; their contents being composed exclusively of the axis-cylinder. Yet, since many nerve-fibres may be found which appear intermediate in character between these two kinds, and since the 
large fibres, as they approach both their central and their peripheral end, lose their medullary sheath and assume many of the other characters of the fine fibres of the sympathetic system, it is not necessary to suppose that there is any material difference in the two kinds of fibres. The non-medullated fibres frequently branch.

- It is worthy of note that in the foetus, at an early period of development, all nerve-fibres are non-medullated.

Nerve-trunks. - Each nerve-trunk is composed of a variable number of different-sized bundles (funiculi) of nerve-fibres which have a special sheath (perineurium). The funiculi are inclosed in a firm fibrous sheath (epineurium); this sheath also sends in processes of connective

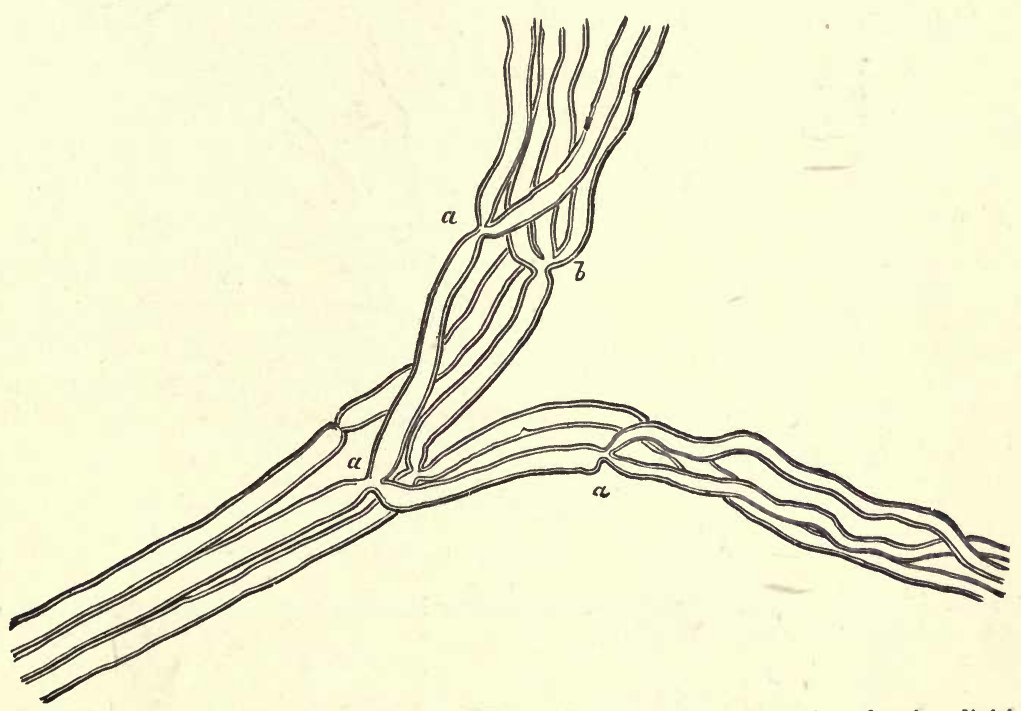

Fig. 102.-Small branch of a muscular nerve of the frog, near its termination, showing divisions of the fibres. $a$, into two; $b$, into three. $\times 350$. (Kölliker.)

tissue which connect the bundles together. In the funiculi between the fibres is a delicate supporting tissue (the endoneurium).

There are numerous lymph-spaces both beneath the connective tissue investing individual nerve-fibres and also beneath that which surrounds the funiculi.

Every nerve-fibre in its course proceeds uninterruptedly from its origin in a nerve-centre to near its destination, whether this be the periphery of the body, another nervous centre, or the same centre whence it issued.

Bundles of fibres run together in the nerve-trunk, but merely lie in apposition to each other; they do not unite: even when they anastomose, there is no union of fibres, but only an interchange of fibres between the anastomosing funiculi. Although each nerve-fibre is thus 
single and undivided through nearly its whole course, yet as it approaches the region in which it terminates, individual fibres break up into several subdivisions before their final ending.

Nerve Collaterals. - It has been discovered through the researches of Golgi, and confirmed by the further studies of Cajal and other anatomists, that each individual nerve-fibre in the central nervous system gives off in its course branches which pass out from it at right angles for a short distance, and then turn and run in various directions. These branches are called collaterals. They end in fine, brush-like terminations, known as end-brustles, or in little bulbous swellings which come in close contact with some nerve cell (fig. 103).

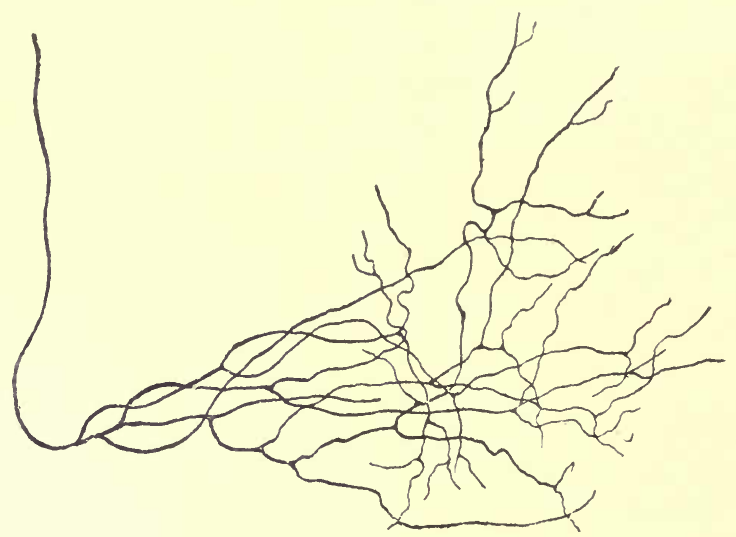

Fig. 103.-Terminal ramifications of a collateral branch belonging to a fibre of the posterior column in lumbar cord of an embryo calf.

These collaterals form a very important part of the nerve-unit. At the point where they are given off, there is usually a little swelling of the neuraxon proper.

The nerve-fibre itself continues on and finally ends in various ways, according to its function and the organ with which it is connected. In the nerve-centres, that is, in the brain and spinal-cord, the different nerve-fibres end just as the collaterals do, by splitting up into fine branches which form the end-brushes. Collaterals of the nerve-fibres and end-brushes are chiefly found in the nervous centres. The nerve-fibres of the peripheral nerves end in the muscles, glands, or special sensory organs, such as the eye and ear. Here, however, some analogy to the end-brush can also be discovered. As the peripheral nerve-fibres approach their terminations, they lose their medullary sheath, and consist. then merely of an axis-cylinder and primitive sheath. They then lose also the latter, and only the axis-cylinder is left. Finally, the axis- 
cylinder breaks up into its elementary fibrillæ, to end in various ways to be described later.

Plexuses.-At certain parts of their course, nerves form plexuses, in which they anastomose with each other, as in the case of the brachial and lumbar plexuses. The objects of such interchange of fibres are:(a), to give to each nerve passing off from the plexus a wider connection with the spinal cord than it would have if it proceeded to its destination without such communication with other nerves. Thus, each nerve by the wideness of its connections is less dependent on the integrity of any single portion, whether of nerve-centre or of nerve-trunk, from which it may spring. (b) Each part supplied from a plexus has wider relations with the nerve-centres, and more extensive sympathies; and, by means of the same arrangement, groups of muscles may be coordinated, every member of the group receiving motor filaments from the same parts of the nervecentre. (c) Any given part, say a limb, is less dependent upon the integrity of any one nerve.

\section{Nerve-Cells.}

The nerve-cell is the nodal and important part of the neuron, and from it are given off the dendrites and axis-cylinder process or neuraxon. It consists of a mass of protoplasm, of varying shape and size, containing within it a nucleus and wucleolus. All nerve-

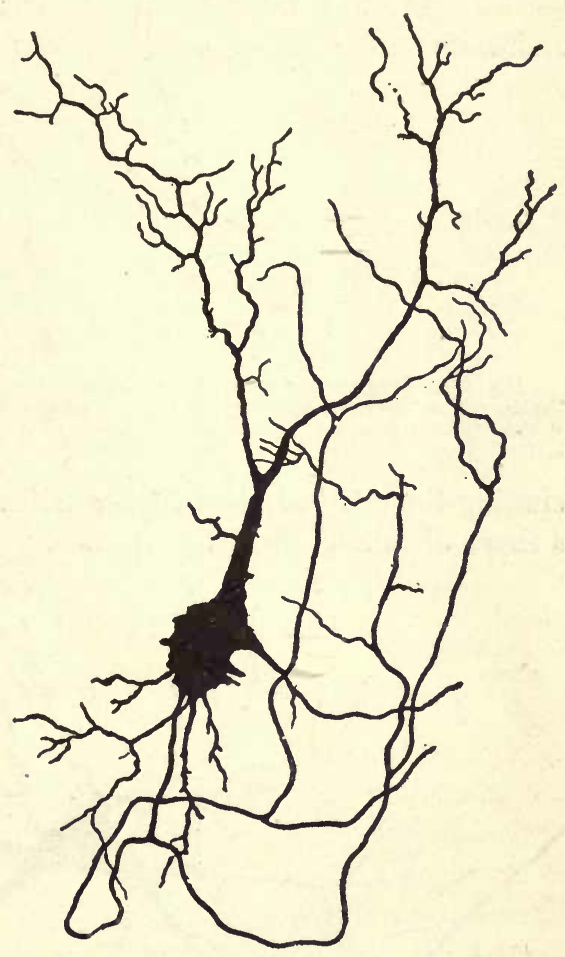

Fig. 103A.-Nerve-cell with short axis-cylinder from the posterior horn of the lumbar cord of an embryo calf measuring $0.55 \mathrm{~cm}$. (After v. Gehuchten.) cells give off a number of processes which branch out in various directions, dividing and subdividing like the branches of a tree, but never anastomosing with each other or with other cells. These branches are what have already been referred to as the dendrites of the cell. They were formerly called the protoplasmic processes (figs. $103 \mathrm{~A}, 104)$. It is thas seen that the neuron or nerve-unit consists of a number of subdivisions, namely, the cell-body? with its nucleus and nucleolus, the dendrites, or protoplasmic processes, 
and the neuraxon or axis-cylinder process, which is continued on to form what is known as a nerve-fibre. The nerve-cell is often spoken of as in.

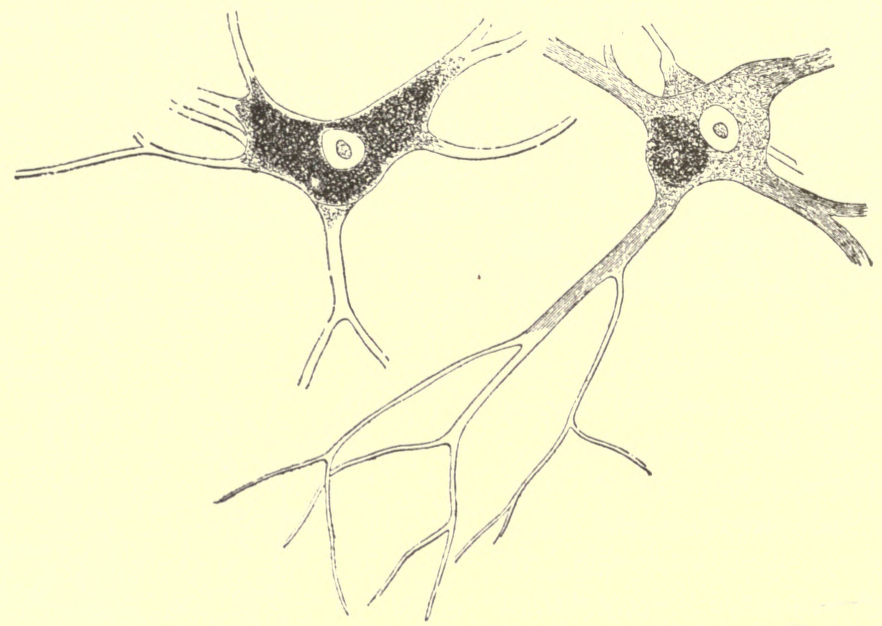

Fig. 104.-Large nerve cells with processes, from the ventral cornua of the cord of man, $\times 350$. On the cell at the right two short processes of the cell-body are present, one or the other of which may. have been an axis-cylinder process (Deiters). A similar process appears also on the cell at theleft.

cluding the cell-body and its dendrites and the axis-cylinder process for a short distance. Strictly speaking, however, the name should be ap-

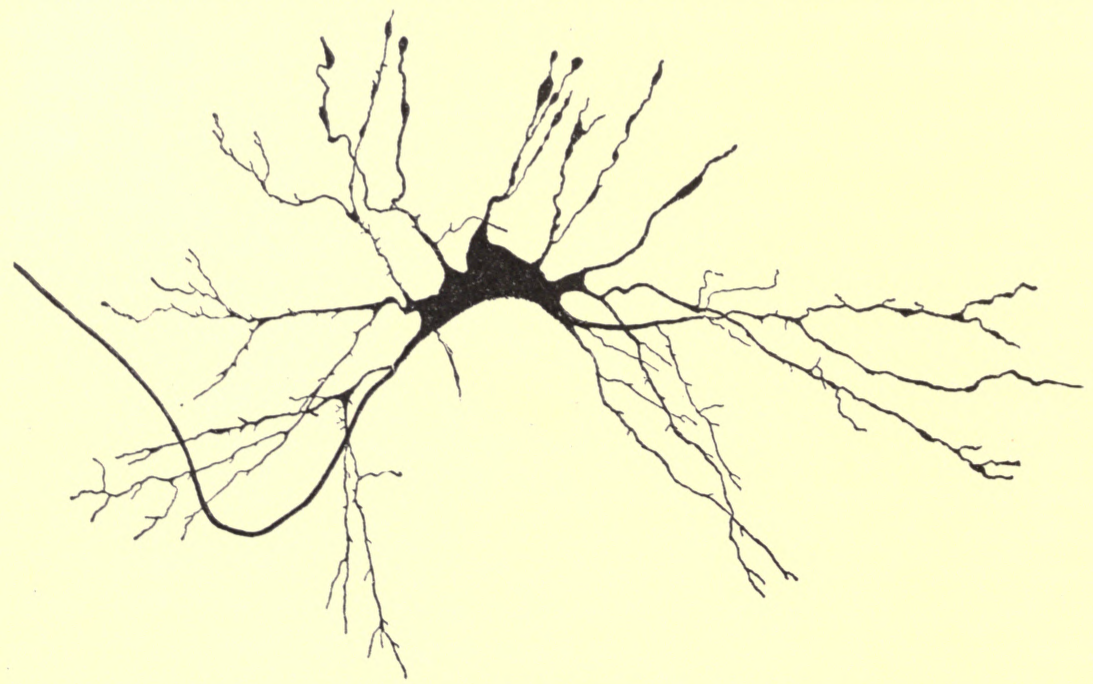

Fig. 104.-Multipolar nerve-cell of the cord of an embryo calf.

plied only to the body of the cell. The nerve-cell is provided with a very large round nucleus in which one or more nucleoli are visible (fig. 104). 


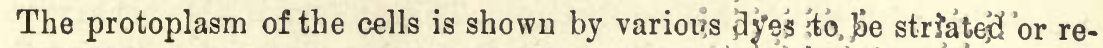
ticulated. The network which makes up, this cell-body stains more

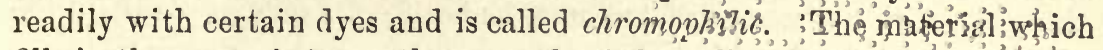
fills in the spaces between the network of the cell-body is called the paraplasm. The cells often contain deposits of yellowish-brown pigment (lig. 105). The nucleus of the cell is sometimes reticulated. Within the nucleus is sometimes seen a nucleolus, and within the nucleolus are bright spots, which are known as nucleolules.

Nerve-cells are not generally present in nerve-trunks, but are found

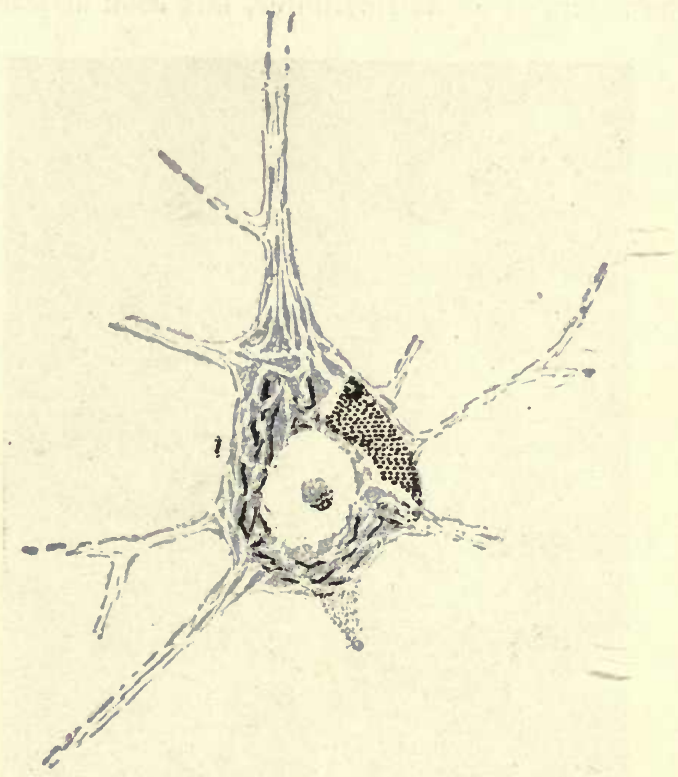

Fig. 105. - Cell of the anterior horn of the human spinal-cord, stained by Nissl's Method. (After Edinger.)

in collections of nerrous tissue called ganglia. They vary considerably in shape, size, and structure in different situations.

a. Some nerve-cells are small, generally spherical or oroid, and have a regular uninterrupted outline. These single nerve-cells are most numerous in the sympathetic ganglia; each is inclosed in a nucleated sheath. b. Others (fig. 105A) are larger, and have one, two, or more long processes issuing from them, the cells being called respectively unipolar, bipolar, or multipolar, which processes often divide and subdivide, and appear tubular and filled with the same kind of granular material that is contained within the cell. These processes are the dendrites. Generally only one process from each cell is continuous with a nerve-fibre, the prolongation from the cell by degrees assuming the characters of the 
nerve-fibre with which it is continuous. This process is the neuraxon. In bipolär cells óne pole inay be continuous with a medullated fibre, and theother: inth of the one or the other kind.

Ganglion-cells are generally inclosed in a transparent membranous capsule similar in appearance to the external nucleated sheath of nervefibres; within this capsule is a layer of small flattened cells.

The process of a nerve-cell or neuraxon which becomes continuous with a nerve-fibre is always unbranched as it leaves the cell. It at first has all the characters of an axis-cylinder, but soon acquires a medullary

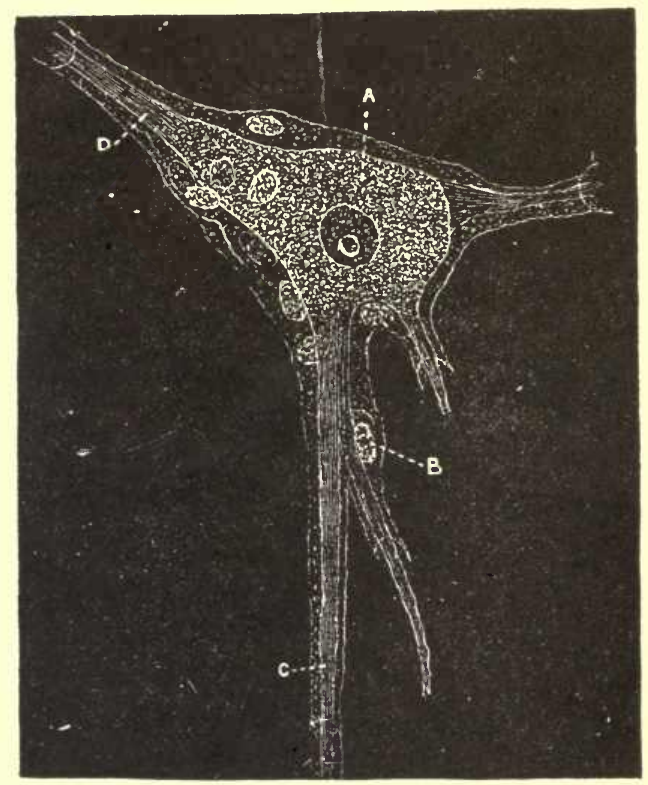

Fig. 105A.-An isolated sympathetic ganglion-cell of man, showing sheath with nucleated-cell lining, B. A. Ganglion-cell. with nucleus and nucleolus. C. Branched process or dendrite. D. Unbranched process or neuraxon. (Key and Retzius.) × 750 .

sheath, and then may be termed a nerve-fibre. This continuity of nervecells and fibres may be readily traced out in the anterior cornua of the gray matter of the spinal cord. In many large branched nerve-cells a distinctly fibrillated appearance is observable; the fibrillæ are probably continuous with those of the axis-cylinder of a nerve.

Other points in the structure of nerve-cells will be mentioned under the account of the central nervous system.

\section{Nerve Terminations.}

Nerve-fibres terminate peripherally in four different ways: 1 , by the terminal subdivisions which pass in between epithelial cells, and are 
known as inter-epithelial arborizations; 2 , by motor-plates which lie in the muscles; 3 , by special end-organs, connected with the senses of sight, hearing, smell, and taste; and, 4 , by various forms of tactile corpuscles.

1. The inter-epithelial arborizations form a most common mode of termination of the sensory nerves of the body. The nerve-fibres pass to the surface of the skin or mucous membrane; they then lose their neu-

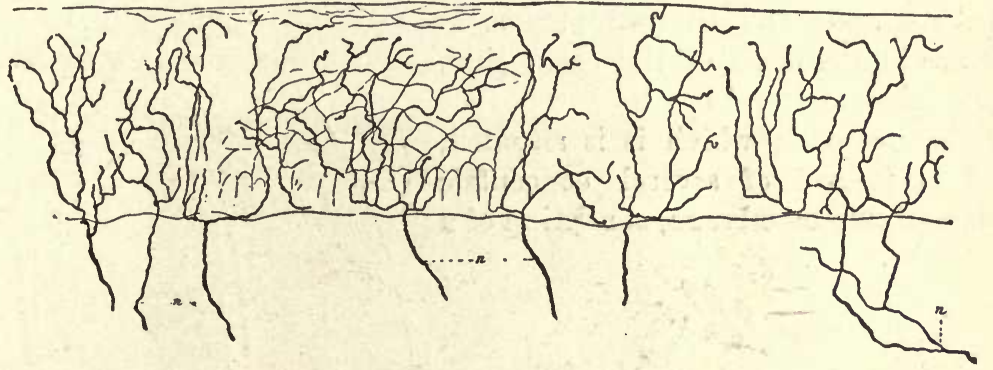

Fig. 106.-Sensory nerve terminations in stratified pavement epithelium. (After G. Retzius.) Golgi's rapid method.

rilemma and myeline sheath, the bare axis-cylinder divides and subdivides into minute ramifications which pass among the epithelial cells of the skin and mucous membrane. In the various glands of the body this form of termination also prevails. The hair-bulbs, the teeth, and the tendons of the body are supplied by this same process of terminal arborization (figs. 106, 10\%).

2. The motor-nerves passing to the muscles end in what are known

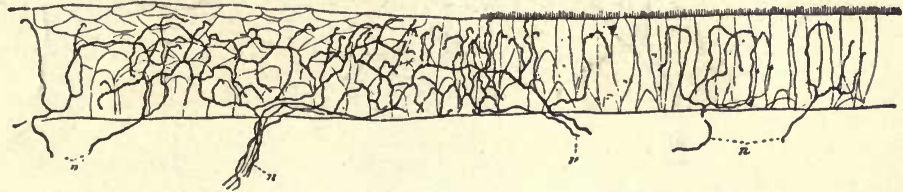

Fig. 107.-Sensory nerve terminations in the epithelium of the mucosa of the inferior vocal cord and in the ciliated epithelium of the subglottic region of the larynx of a cat four weeks old. (After G. Retzius.) Golgi's rapid method. n, Nerve-fibres rising from the connective-tissue layer into the epithelial layer, where they terminate in ramified and free arborizations.

as muscle-plates, the details of whose structure have been already described.

3. The special sensory end-organs will be described later in the chapter on the Special Senses.

4. A fourth form of termination consists of corpuscles that are more or less encapsulated, and these are known as the corpuscles of Pacini, the tactile corpuscles of Meissner, the tactile corpuscles of Krause, the tactice menisques and the corpuscles of Golgi. 
The Pacinian bodies or corpuscles (figs. 108 and 109), named after their discoverer Pacini, also called corpuscles of Vator, are little elongated oval bodies, situated on some of the cerebro-spinal and sympathetic nerves, especially the cutaneous nerves of the hands and feet; and on branches of the large sympathetic plexus about the abdominal aorta. They often occur also on the nerves of the mesentery, and are especially well seen even by the naked eye in the mesentery of the cat. They have been observed also in the pancreas, lymphatic glands, and thyroid glands, as well as in the penis of the cat. Each corpuscle is attached by a narrow pedicle to the nerve on which it is situated, and is formed of several concentric layers of fine membrane, consisting of a

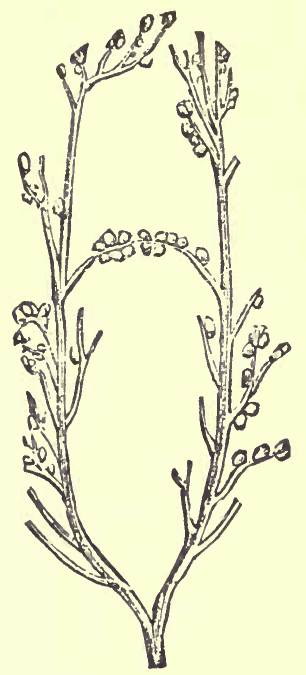

Fig. 108.

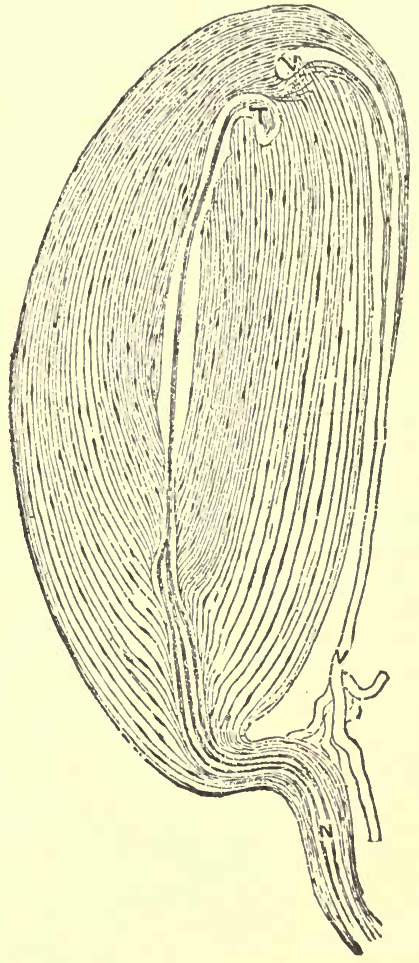

Fig. 109.

Fig. 108.-Extremities of a nerve of the finger with Pacinian corpuscles attached, about the natural size (adapted from Henle and Kölliker).

Fig. 109.-Pacinian corpuscle of the cat's mesentery. The stalk consists of a nerve-fibre (N) with its thick outer sheath. The peripheral capsules of the Pacinian corpuscle are continuous with the outer sheath of the stalk. The intermediary part becomes much narrower near the entrance of the axis-cylinder into the clear central mass. A hook-shaped termination with the end-bulb (T) is seen in the upper part. A blood-vessel (V) enters the Pacinian corpuscle, and approaches the endbulb; it possesses a sheath which is the continuation of the peripheral capsules of the Pacinian corpuscle. $\times 100$. (Klein and Noble Smith.)

hyaline ground membrane with connective-tissne fibres, each layer being lined by endothelium (fig. 109); through its pedicle passes a single nervefibre, which, after traversing the several concentric layers and their immediate spaces, enter's a central cavity and, gradually losing its dark border and becoming smaller, terminates at or near the distal end of the cavity, in a knob-like enlargement or in a bifurcation. The enlarge- 
ment commonly found at the end of the fibre is said by Pacini to resemble a ganglion corpuscle; but this observation has not been confirmed. In some cases two nerves have been seen entering one Pacinian body, and in others a nerve after passing unaltered through one has been ob-

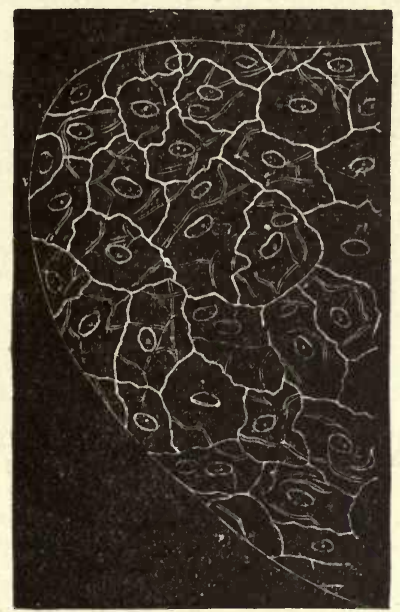

Fig. 110.-Summit of a Pacinian corpuscle of the human finger, showing the endothelial membranes lining the capsules. $\times 220$. (Kleln and Noble Smith.)

served to terminate in a second Pacinian corpuscle. The physiological import of these bodies is still obscure.

2. The tactile corpuscles of Meissner (figs. 111, 112) are found in the

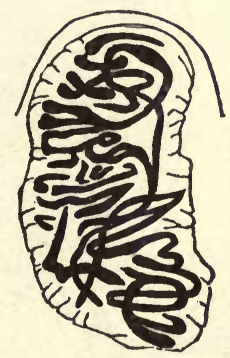

Fig.111. A touch-corpuscle of Meissner, from the skln of the human hand.

papillæ of the skin of the fingers and toes, or among its epithelium. They may be simple or compound. When simple they are small, slightly flattened transparent bodies composed of nucleated cells enclosed in a capsule. When compound, the capsule contains several small cells. The corpuscles are about $\frac{1}{290}$ of an inch long to $\frac{1}{350}$ of an inch wide. The nerve-fibre penetrates the corpuscle, loses its myeline sheath, and divides 
and subdivides to form a series of arborizations, more or less distinct and destined for the different parts of the corpuscle. The terminal arborizations occupy the central part of the corpuscle, and are surrounded by a great number of marginal cells. The touch, or tactile corpuscles

B

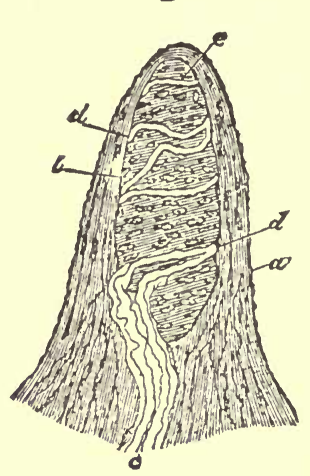

$\Delta$

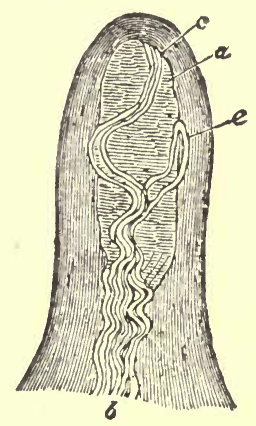

Fig. 112.-Papillæ from the skin of the hand, freed from the cuticle and exhibiting tactile corpuscles. A. Simple papilla with four nerve-fibres; $a$, tactile corpuscles; $b$, nerves with winding fibres $c$ and $e . \quad B$. Papilla treated with acetic acid; $a$. cortical layer with cells and fine elastic filaments; $b$, tactile corpuscle with tranverse nuclei; $c$, entering nerve with neurilemma or perineurium; $d$ and $e$, nerve-fibres winding round the corpuscle. $\times 350$. (Kölliker.)

of Meissuer, have been regarded at one time as epithelial, at another time as nervous, but they are to-day proved to be mesodermic cells, and differentiated for the special purpose of the sense of touch (Dejerine).

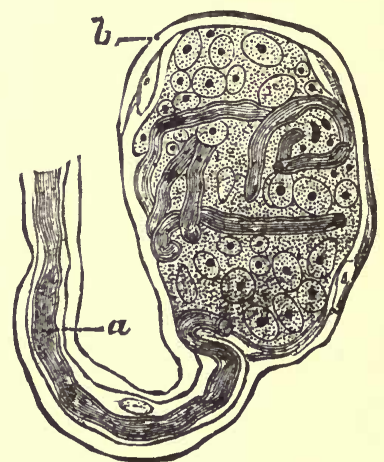

Fig. 113.-End-bulb of Krause. $\quad a$, Medullated nerve-fibre; $b$, capsule of corpuscle.

3. The Corpuscles of Krause or End-Bulbs.-These exist in great numbers in the conjunctiva, the glans penis, clitoris, lips, skin, and tendon of man; they resemble the corpuscles of Pacini, but have much fewer concentric layers to the corpuscle, and contain a relatively voluminous central mass composed of polyhedral cells. In man these 
corpuscles are spherical in shape, and receive many nervous fibres which wind through the corpuscle, and end in the free extremities (fig. 113).

4. Tactile Menisques. - In different regions of the skin of man, one meets, in the superficial layers and in the Malpighian layers, nerves which, after having lost their myeline sheath, divide and subdivide to form extremely beautiful arborizations. The branches of these arborizations are flattened down, forming the tactile menisques. These menisques, which simulate the form of a leaf, represent a mode of terminal nervous arborization (Ranvier).

5. The corpuscles of Golgi are small terminal placques placed at the union of tendons and muscles, but belonging more properly to the tendon.

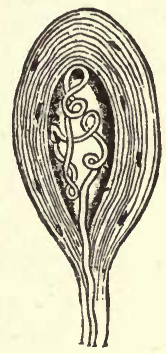

Fig. 114.-A termination of a medullated nerve-fibre in tendon, lower half with convoluted medullated nerve-fibre. (Golgi.)

They are fusiform in shape and are flattened upon the surface of the tendon close to its insertion into the muscular fibres. They are composed of a granular substance, enveloped in several concentric hyaline membranes which contain some nuclei. The nerve-fibre passes into this little corpuscle, splitting itself up into fine terminals. The corpuscles of Golgi are believed to be related to the muscular sense (fig. 114).

In addition to the special end-organs, sensory fibres may terminate in plexuses, as in the sub-epithelial and intra-epithelial plexus of the cornea.

\section{The Neuroglia.}

The neuroglia, while not a nervous tissue, is closely mingled with it and forms an important constituent of the nervous system. It consists of cells giving off a fine network of richly branching fibres. Neuroglia was at one time considered to be a form of connective tissue, and it is in its functions strictly comparable to the connective tissue which supports the special structures of other organs, like the lungs and kidney (fig. 116). It is, however, derived from the epiblastic cells, i.e., the same cells from which the nerve-tissue proper also develops. In the adult animal the neuroglia-tissue is composed of cells from which are given off immense numbers of fine processes. These extend out in every 
direction, and intertwine among the nerve-fibres and nerve-cells (fig. 115). The neuroglia-cell differs in size and shape very much in different parts

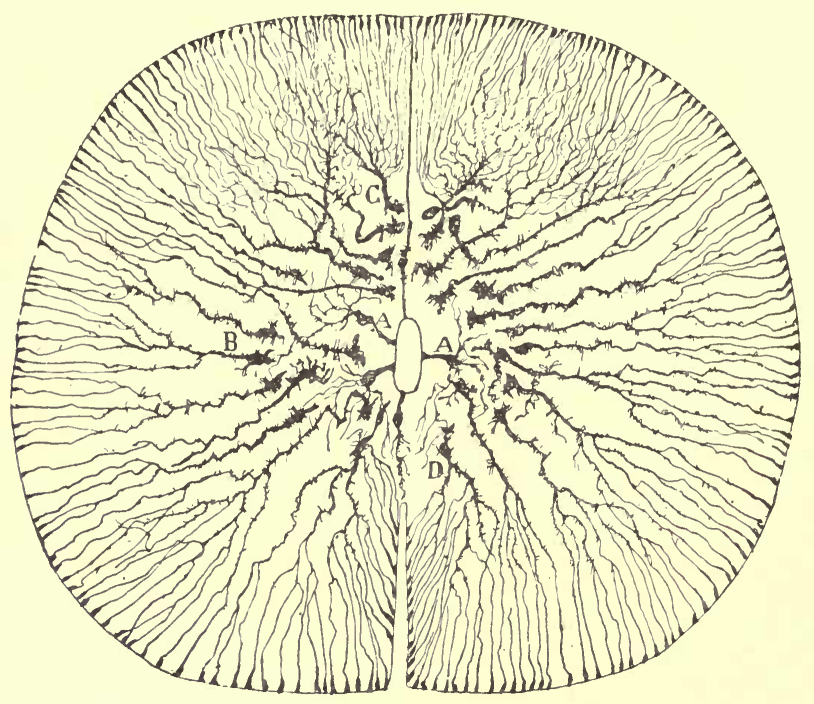

Fig. 115.-Neuroglia cells in the cord of an adult frog. (After Cl. Sala.) A, Ependyma cells with their peripheral extremities atrophied and ramified; $B, C, D$, neuroglia cells in different degrees of emigration and separation from the ependymal canal; their central extremity is atrophied and much contracted; their pe"ipheral extremity, on the other hand, is greatly extended; the ramifications of the latter terminating in conical buttons, $I$, end under the pia mater.
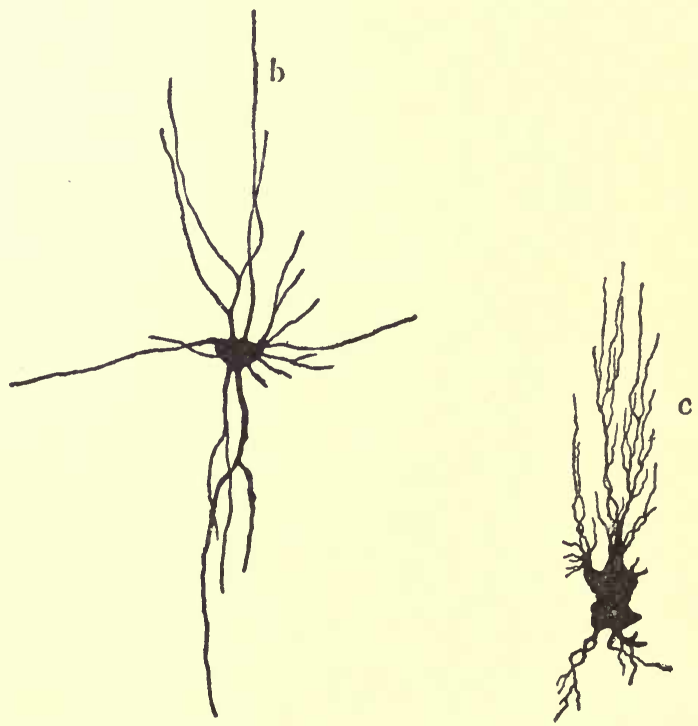

Fig. 116.-Different types of neuroglia cells. (After v. Gehuchten.) $b$, Neuroglia cells of the white substance, and $c$, of the gray substance of the cord of an embryo calf. 
of the nervous system in accordance with the arrangement of the nervous structures about it. The cell is composed of granular protoplasm, and lying in it is a large nucleus, within which is a nucleolus. The body of the cell is small in amount and proportion to the nucleus.

Weigert has shown that the processes of the neuroglia-cells branch and prolong themselves, forming in many places an extremely thick network. These processes become changed in their chemical and physical characters, so that they take a different stain from that of the cell-body itself, and they thus form a really separate structure, distinct almost from the mother-cell, just as the muscle tissue is distinct from its original cell-protoplasm, or just as the substance of cartilage is distinct from its original cell-body. While neuroglia-tissue is distributed throughout the whole of the nervous centres, it is especially deposited in certain places. It is found around the central canal of the spinal cord, and upon the superficial surface of the spinal cord. It was formerly thought to compose part of the gelatinous substance of Rolando in the spinal cord, but this has been shown by Weigert not to be the case.

In the brain a deposit of neuroglia is found beneath the ependymal lining of the ventricles, and upon the superficial surface of the gray matter of the cortex beneath the pia mater. It is distributed to some extent in all parts of the brain and spinal cord, but is not found in the peripheral nerves. 


\section{CHAPTER IV.}

\section{THE CHEMICAL COMPOSITION OF THE BODY.}

OF the known chemical elements of which about seventy have been isolated no less than serenteen combine, in larger or smaller quantities, to form the chemical basis of the animal body.

The substances which contribute the largest share are the non-metallic elements, Oxygen, Carbon, Hydrogen, and Nitrogen-oxygen and carbon making up altogether about $8 \tilde{s}$ per cent of the whole. The most abundant of the metallic elements are Culcium, Solium, and Potassium.*

Few of the elements, howerer, appear free or uncombined in the animal body. They are generally united together in variable proportions to form compounds. The only elements which have been found free in the body are oxygen, nitrogen, and hydrogen, the first two in the blood, and hydrogen as well as oxygen and nitrogen in the intestinal canal.

It was formerly thought that the more complex compounds built up by the animal or regetable oryunism were peculiar and could not be made artificially by chemists, and under this idea they were formed into a distinct class, termed orymic. This idea has long been given up, but the name is still in use with a different signification. The term is now applied simply to the compounds of the element carbon, irrespective of their origin.

A large number of the animal organic compounds, particularly those of the albuminous group, are characterized by their complexity. Many elements enter into their composition, thereby distinguishing them from simple inorganic compounds. Many atoms of the same element occur in each molecule. This latter fact no doubt explains the reason of their instubility. Another great cause of the instability is the frequent presence of nitrogen, which may be called negative or undecided in its affinities and may be easily separated from combination with other elements.

* The following table represents the relative proportion of the various elements.-(Marshall.)

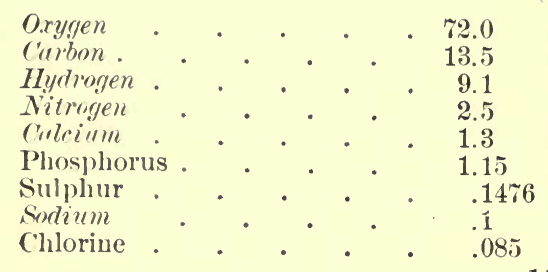

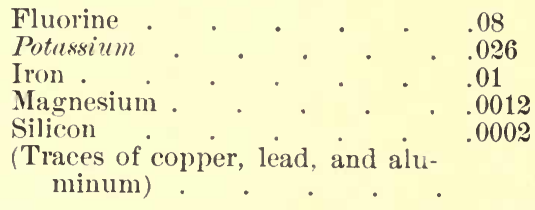


Animal tissues, contaiming as they do these organic nitrogenous compounds, are extremely prone to undergo decomposition. They also contain much water, a circumstance very favorable to the breaking up of such substances. It is due to this tendency to decomposition that we meet with so large a number of decomposition products among the chemical substances forming the basis of the animal body.

The various substances found in the animal organism may be conveniently considered according to the following classifieation: 1. Organica. Nitrogenous and b. Non-Nitrogenous. 2. Inorganic.

\section{Organic Substances.}

Nitrogenous organic bodies take the chief part in forming the solid tissues of the body, and are found also to a considerable extent in the circulating fluids (blood, lymph, chyle), the secretions and excretions. They often contain in addition to carbon, hydrogen, nitrogen, and oxygen, the elements sulphur and phosphorus; but although the composition of most of them is approximately known, no general rational formula can at present be given.

It will be convenient to give an account of the Proteid substances in this Chapter, as these constitute the most important classes of nitrogenous organic substances. According to their chemical composition or superficial differences (e.g., solubility) they are divided into three main classes, viz.: (1) Simple proteids, (2) compound proteids, and (3) albumenoids or proteoids. The other members are Decomposition products, the chief of which is Urea, found for the most part in the urine; Ferments ; Pigments; and other bodies and will be more appropriately treated of later on.

Proteids (simple proteids), are also called Albuminous substances. They are the chief of the nitrogenous organic compounds and exist in both plants and animals, one or more of them entering as an essential part into the formation of all living tissue. In the lymph, chyle, and blood, they exist abundantly. Very little is known with any certainty about their chemical composition. Not a single member of the class has yet been synthesized. Their formula is unknown, the chemists who have attempted to construct it differing very greatly among themselves. In fact the very term proteid is an extremely arbitrary one. It simply means a body which, according to Hoppe-Seyler, contains in its molecule the elements carbon, hydrogen, nitrogen, oxygen, and sulphur, in certain arbitrary but varying amounts, thus-Carbon, from 51.5 to 54.5 ; $\mathrm{Hy}$ drogen, from 6.9 to 7.3 ; Nitrogen, from 15.2 to 17 . Oxygen, from 20.9 to 23.5 ; Sulphur, from .3 to 2 . Some proteids contain from .3 to 1.5 of phosphorus; a small amount of iron is usually associated with 
proteids, but it is not certain whether or not it is an integral part of the molecule. Chittenden defines a proteid as a substance which contains earbon, hydrogen, oxygen, nitrogen, and sulphur, the nitrogen being in a form which serves the physiological needs of the body; and yields, on decomposition, a row of crystalline amido-acids and crystalline nitrogenous bases; nearly all contain 52 per cent of carbon and 16 per cent of nitrogen.

Properties of Proteids. - Proteids are for the most part amorphous and non-crystallizable. Certain of the regetable proteids have, it is said, been crystallized, and according to Hofmeister, egg albumin is also capable of crystallization. They possess as a rule no power (or scarcely any) of passing through animal membranes. They are soluble, but undergo alteration in composition in strong acids and alkalies; some are soluble in water, others in neutral saline solutions, some in dilute acids and alkalies, none in alcohol or ether. Their solutions exereise a left-handed action on polarized light.

The hope that it may be possible in the immediate future to synthesize proteids is rendered all the weaker because of the extraordinary variety of compounds obtained by the decomposition of proteids by various chemical methods, the compounds differing according to the method employed. In the body it seems clear that living proteid is built up by the food supplied to it, which necessarily contains proteid derived either from a vegetable or an animal sonree; how this process takes place we are yet unable to say. In the course of later chapters in this book we shall endeavor to trace the steps of the breaking up of proteid in the body, but we may anticipate by mentioning that it is now generally believed that the ultimate products of this decomposition are urea, a body the formula of which is $\mathrm{CO}\left(\mathrm{NH}_{2}\right)_{2}$, "urbon dioxide and water, while the intermediate substances or by products are probably ammonia compounds (ammonium earbonate). When proteid material is decomposed by putrefaction, by the action of chemical reagents, e.g., acils, alkalies, or by heat, various bodies are produced, of which amido-acids (acids in which one or more of the hydrogen atoms of the radical of the acid are replaced by amidogen, $\mathrm{NH}_{2}$ ) and bodies belonging to the aromatic or benzene series predominate. Hence it comes that various theories of the way in which proteids are built up have arisen. The one which has appeared to have received the greatest support is that of Latham. This observer has suggested that proteid may be considered as made up of a series of cyan-alcohols (bodies obtained by the union of any aldehyde with hydrocyanic acid) with a benzene nucleus. Taking ordinary ethyl alcohol, $\mathrm{CH}_{3} \mathrm{CH}_{2} \mathrm{OH}$, as the type, the aldehyde of which is $\mathrm{CH}_{3} \mathrm{CHO}$, the corresponding cyan-alcohol would be $\mathrm{CH}_{3} \mathrm{CHCNOH}$.

Proteids give certain general chemical reactions. They are a little 
varied in the case of each particular substance. The chief of these are as follows :

i. Xantho-Proteic Reaction.-The addition of strong nitric acid, drop by drop, to a solution of any proteid produces a flocculent precipitate which dissolves in an excess of the acid. The solution becomes canary yellow in color; when heated, this color is more marked; when cooled, the addition of ammonia in excess changes the color to orange. The nitric acid decomposes the proteid to a certain extent and then unites with the decomposition products, forming, among other things, xanthoproteic acid which gives the yellow color. The ammonia unites with this and forms ammonium xanthoproteate which gives the orange color.

ii. Biuret (Piotrowski's) Reaction. - With a trace of cupric sulphate and an excess of potassium or sodium hydrate peptones and proteoses give a rose red; with ammonia instead of the fixed alkalies, a blue coloration. Most proteids, however, give a violet (pinkish purple) color; the color is due to reduced copper, cuprous hydroxide being formed along with other compounds of red, yellow, and blue colors.

iii. Millon's Reaction.-With Millon's reagent (a solution of mercuric nitrate) proteids give a heavy white precipitate of mercuric albuminate which, with an excess of the reagent, becomes brick red when heated. This test is said to be due to the presence of tyrosin, an aromatic compound in the proteid molecule: it is generally used for solids though it may be used for liquids also. With all substances containing the $\mathrm{C}_{6} \mathrm{H}_{5} \mathrm{OH}$ group, e.g., carbolic acid, this reagent gives the same color reaction, though no precipitate is formed, the solution itself becoming red.

iv. Ammonium Sulphate Reaction.-They are, with the exception of peptone, entirely precipitated from their solutions by saturation with ammonium sulphate.

Many of the proteids give, in addition, the following tests:

v. With excess of acetic acid, and potassium ferrocyanide, a white precipitate.

vi. With excess of acetic acid and a saturated solution of sodium sulphate, on boiling, a white precipitate. This test is often used to get rid of all traces of proteids, except peptones, from solutions. 
vii. Boiled with strong hydrochloric acid, they give a violet red coloration.

viii. With cane sugar and strong sulphuric acid, on heating, they give a purplish coloration.

ix. They are precipitated on addition of-citric or acetic acid, and picric acid; or citric or acetic acid, and sodium tungstate; or eitric or acetic acid, and potassio-mercuric iodide; and with many other metallic salts in solution and by alcohol.

Varieties.-Proteids are divided into classes, chiefly on the basis of their solubilities in various reagents. Each class, however, if it contains more than one substance, may often be distinguished by other properties common to its members. Not every one of the proteids enumerated is contained in the animal tissues, some are used as food.

(1.) Native-Albumins. - These substances are soluble in water and in saline solutions, and are coagulated, i.e., turned into coagulated proteid, on heating.

(2.) Albuminates. - These are soluble in acids or alkalies, insoluble in saline solutions and in water, and not coagulated on heating.

(3.) Globulins.-These are soluble in weak saline solutions, in dilute acids and alkalies, and insoluble in water and in strong solutions of neutral salts. They are coagulated on heating.

(4.) Proteoses. - These are soluble in water and dilute saline solutions, precipitated by saturation with ammonium sulphate; precipitated but not coagulated by alcohol; precipitated by picric acid: cannot be coagulated by heat.

(5.) Peptones. - These are soluble in water, saline solutions, acids, or alkalies; not precipitated on saturation with any neutral salt; they are not coagulated on heating.

(6.) Coagulated Proteids. - These are of two classes, either coagulated by (a) action of ferments, or (b) heat. These are soluble only in gastric or pancreatic fluids, forming peptones, or (with difficulty) in strong acids and alkalies.

Native-Albumins. - Of native-albumins there are several varieties: (a) egg-albumin; (b) serum-albumin; (c) lact-albumin, etc.

E.g Albumin is contained in the white of the egg.

When in solution in water it is a transparent, frothy, yellowish fluid, neutral or slightly alkaline in reaction. It gives all of the general proteid reactions. It yields 8 per cent of argenin, 22.6 per cent of leucin, and 2 per cent of tyrosin.

At a temperature not exceeding $40^{\circ} \mathrm{C}$. it is dried up into a yellowish, transparent, glassy mass, soluble in water. At a temperature of $70^{\circ} \mathrm{C}$. it is coagulated, i.e., changed into a new substance, coagulated proteid, 
which is quite insoluble in water. It is coagulated also by the prolonged action of alcohol; by strong mineral acids, especially by nitric acid, also by tannic acid, or carbolic acid; by ethers the coagulum is soluble in eaustic soda.

It is precipitated without coagulation, i.e., forms insoluble compound with the reagent, soluble on removal of the salt by dialysis, with either mercuric chloride, lead acetate, copper sulphate or silver nitrate, the precipitate in each case being soluble in slight excess of the reagent.

With strong nitric acid the albumin is precipitated at the point of contact with the acid in the form of a fine white or yellow ring.

Serum-Albumin is contained in blood-serum, lymph, serous and synovial fluids, and in the tissues generally; it may be prepared from serum, after removal of paraglobulin by saturation with magnesium sulphate, by a further saturation with sodium sulphate. It appears in the urine in the condition known as albuminuria.

It gives similar reactions to egg-albumin, but differs from it in not being coagulated by ether. It also differs from egg-albumin in not bèing easily precipitated by hydrochloric acid, and in the precipitate being easily soluble in excess of that acid. Serum-albumin, either in the coagulated or precipitated form, is more soluble in excess of strong acid than eggalbumin.

Albuminates.-There are two principal substances belonging to this class, a, Acid-Albumin; b, Alkali-Albumin.

Acid-Albumin.-Acid-albumin is made by adding small quantities of dilute acid (of which the best is hydrochloric, .4 per cent to 1 per cent), to either egg- or serum-albumin diluted with five to ten times its bulk of water, and keeping the solution at a temperature not higher than $50^{\circ} \mathrm{C}$. for not less than half an hour. It may also be made by dissolving coagulated native-albumin in strong acid, or by dissolving any of the globulins in acids. Solid acid albuminate may be formed by adding strong acid drop by drop to a strong solution of proteid matter (e.g., undiluted eggalbumin) until solidification occurs.

It is not coagulated on heating, but on exactly neutralizing the solution a flocculent precipitate is produced (if it is then heated to $70^{\circ} \mathrm{C}$. it will coagnlate and cannot then be distinguished from any other form of coagulated proteids). This may be shown by adding to the acid-albumin solution a little aqueous solution of litmus, and then adding, drop by drop, a weak solution of caustic potash from a burette until the red color disappears. The precipitate is the derived-albumin. It is soluble in dilute acid, dilute alkalies, and dilute solutions of alkaline carbonates. The solution of acid-albumin gives the proteid tests. The substance itself is coagulated by strong acids, e.g., nitric acid, and by strong alcohol; it is insoluble in distilled water, and in neutral saline solutions; it is pre- 
cipitated from its solutions by saturation with sodium chloride. On boiling in lime-water it is partially coagulated, and a further precipitation takes place on addition to the boiled solution of calcium chloride, magnesium sulphate, or sodium chloride.

Alkali-Albumin. - If solutions of native-albumin, or coagulated or other proteid, be treated with dilute or strong fixed alkali, alkali-albumin is produced. Solid alkali-albumin (Lieberkiuhn's jelly) may also be prepared by adding eaustic soda or potash, drop by drop, to undiluted eggalbumin, until the whole forms a jelly. This jelly is soluble in an excess of the alkali or in dilute alkalies on boiling. A solution of alkali-albumin gives the tests corresponding to those of acid-albumin. It is not coagulated on heating except after neutralization, as in the case of acid albumin. It is thrown down on neutralizing its solution, except in the presence of alkaline phosphates, in which case the solution must be distinctly acid before a precipitate falls.

To differentiate between Acid- and Alkali-Albumin, the following method may be adopted. Alkali-albumin is not precipitated on exact neutralization, if sodium phosphate has been previously added. Acidalbumin is precipitated on exact neutralization, whether or not sodium phosphate has been previously added.

Globulins. - The globulins give the general proteid tests; are insoluble in water; are soluble in dilute saline solutions; are soluble in acids and alkalies forming the corresponding derived-albumin.

Most of them are precipitated from their solutions by saturation with solid sodium chloride, magnesium sulphate, or other neutral salt. They are coagulated, but at different temperatures, on heating.

Globulin or Crystallin.- It is obtained from the crystalline lens by rubbing it up with powdered glass, extracting with water or with dilute saline solution, and by passing through the extract a stream of carbon iodide. It differs from other globulins in not being precipitated by saturation with sodium chloride.

Myosin. - The relation of myosin to living muscle will be considered under the head of the physiology of muscle. It may, however, be prepared from dead muscle by removing all fat, tendon, etc., and washing repeatedly in water until the washing contains no trace of proteids, mincing it and then treating with 10 per cent solution of sodium chloride, or similar solution of ammonium chloride or magnesium sulphate, which will dissolve a large portion into a viscid fluid, which filters with difficulty. If the viscicl filtrate be dropped little by little into a large quantity of distilled water, a white flocculent precipitate of myosin will occur.

It is soluble in 10 per cent saline solution; it is coagulated at $60^{\circ} \mathrm{C}$. into coagulated proteid; it is soluble without change in very dilute acids; it is precipitated by picric acid, the precipitate being redissolved on 
boiling; it may give a blue color with ozonic ether and tincture of gnaiacum.

Paraglobulin.-Paraglobulin is contained in plasma and in serum, in serous and synovial fluids, and may be precipitated by saturating plasma after removal of fibrinogen or serum with solid sodium chloride or magnesium sulphate, as a bulky flocculent substance which can be removed by filtration.

It may also be prepared by diluting blood serum with ten volumes of water, and passing carbonic acid gas rapidly through it. The fine precipitate may be collected on a filter, and washed with water containing carbonic acid gas.

It is very soluble in dilute saline solutions ( 5 to 8 per cent), from which it is precipitated by carbonic acid gas or by dilute acids; its solution is coagulated at $70^{\circ} \mathrm{C}$.; even dilute acids and alkalies convert it into acid- or alkali-albumin.

Fibrinogen.-Fibrinogen is contained in blood-plasma, from which it may be prepared by addition of sodium chloride to the extent of 13 per cent. It may also be prepared from hydrocele fluid or from other serous transudation by a similar method.

Its general reactions are similar to those of paraglobulin; its solution is coagulated at $52^{\circ}-55^{\circ} \mathrm{C}$. Its characteristic property is that, under certain conditions, it forms fibrin.

Edestrine.-Edestrine is a globulin which is found in many edible vegetables, grain, etc. A solution may be prepared by adding hemp seed to a 10 per cent solution of sodium chloride and heating to $50^{\circ} \mathrm{C}$.

Proteoses are intermediate substances of the digestion of other proteids, the ultimate product of which is peptone. They are produced by the action of the gastric and pancreatic juices and also, slowly, by boiling with dilute acids. The term is a general one, the proteose of albumin being albumose, that of globulin being globulose, etc. They are divided into primary and secondary groups representing the stages of progression from proteids to peptones, so that there may be a primary and a secondary albumose, etc. As digestion is a process of hydration with cleavage, the successive stages present progressively simpler substances. Each group reacts to fewer reagents than the preceding one; e.g., none of the proteoses can be coagulated by boiling, nitric acid will precipitate the primary proteoses but not the secondary ones.

Peptones.-Peptone is formed by the action of the digestive ferments, pepsin, or trypsin, on other proteids, and on gelatin. It is a still simpler form of substance than the proteoses and reacts to still fewer reagents. They will be considered in connection with the physiology of digestion, as will also the intermediate compounds.

Coagulated proteids are formed by the action of heat or of ferments 
upon other proteids; the temperature necessary to produce coagulation varying in the manner previously indicated. They may also be produced by the prolonged action of alcohol upon proteids; the process is one of dehydration. They are soluble in strong acids or alkalies; slightly so in dilute; are soluble in digestive fluids (gastric and pancreatic). Are insoluble in water or saline solutions (except fibrin).

Fibrin.-Fibrin is formed by the action of fibrin ferment on fibrinogen and can be obtained as a soft, white, fibrous, and very elastic substance by whipping blood with a bundle of twigs and washing the adhering mass in a stream of water until all the blood-coloring matter is removed. It is soluble to a certain extent in strong saline solutions.

Compound Proteids. - The compound proteids are compounds of a simple proteid with some other molecule. Acording to their chemical composition and characteristies they are divided into several classes, viz. :

Chromo-proteids.-A combination of a proteid substance with some form of pigment. For example, hæmoglobin is a combination of a globulin with hæmatin, an iron-containing radicle.

Gluco-proteids. - A combination of a proteid substance with a carbohydrate radicle. Examples are mucin, which is found in mucous secretions; and mucoids, which are found in certain tissues, eartilages, etc.

Nucleo-proteids. - A combination of a proteid substance with a nucleic acid: they are divided into two groups according to the character of the acid. The true nucleo-proteids contain true nucleic acid; the para-nucleoproteids, or pseudo-nucleo-proteids contain para-nucleic acid. Both acids, and therefore both groups, contain phosphorus; but the true nuclo-proteids yield nuclein (xanthin) bases while the para-nucleo-proteids do not. They are found in the nucleus and protoplasm of every cell, and also in milk, as caseinogen, and in the yolk of egg, as vitellin.

Gluco-nucleo-proteids. $-\mathrm{A}$ combination of a nucleo-proteid with a carbohydrate radicle.

Mucin.-Mucin is a compound of a globulin with a carbohydrate radicle, and is the characteristic component of mucus; it is contained also in foetal connective tissue, in tendons, and salivary glands. It can be obtained from mucus by diluting it with water, filtering, treating the insoluble portion with weak caustic alkali, and reprecipitating with acetic acil. The mucins derived from different sources probably have different compositions.

Properties.-Mucin has a ropy consistency. It can be coagulated; is insoluble in water, salt solution, and very dilute muriatic acid; is soluble in alkalies and concentrated sulphuric acid. It gives the proteid reaction with Millon's reagent and with nitric acid. Neither mercuric chloride nor tannic acid gives a precipitate with it (?). It does not dialyse. 
When treated with sulphuric acid and then neutralized with solid potassium hydrate, it will give both the Biuret.test, denoting the presence of proteid matter, and also Fehling's test, showing the presence of a sugar: the acid splits it into a globulin and a carbohydrate.

Nucleins.-The substance known as nuclein and found in all cells as well as in milk (caseinogen) and the yolk of egg (vitellin) is really a compound proteid and consists of a whole series of bodies made up of proteid and nucleic acid in varying proportions; there is almost no limit to the possible variations. At one end of the series is nucleic acid $\left(\mathrm{C}_{30}\right.$ $\mathrm{H}_{52} \mathrm{~N}_{9} \mathrm{P}_{3} \mathrm{O}_{17}$, according to Kossel), a body containing the maximum (9 to 11 per cent) of phosphorus, but without any proteid, and found as such only in spermatozoa; in the middle are the nucleins proper; and at the other end are the nucleo-proteids, containing the minimum of phosphorus. As phosphorus is the characteristic component of nucleic acid, its amount will measure the amount of the acid present in any molecule.

The karyoplasm (nucleus) of every cell is richer in the nucleins, while the cytoplasm (cell body) is richer in the nucleo-proteids which contain a smaller proportion of nucleic acid and, therefore, of phosphorus. The difference in staining power of the nucleus and cell body is thus explained as the relative affinity of these substances for a basic dye is proportional to the amount of nucleic acid they contain. The chemical differences in the action of cytoplasm and karyoplasm toward solvents are due also to the proportion of nucleic acid and proteid which they contain. These differences are quantitative and not qualitative. All of the nucleo-proteids in the cell body are true ones in that they yield nuclein bases.

Nuclein Bases.-These are xanthin, hypoxanthin, adenin, and guanin; they are closely related nitrogenous bodies which are always present in every chemical change in the cell, and one may be transformed into another. They are also known as xanthin or purin bases, and all can be derived from the so-called purin nucleus $\mathrm{C}_{5} \mathrm{~N}_{4}$ by substitution of atoms; the purin base, as isolated by Emil Fischer, is $\mathrm{C}_{5} \mathrm{H}_{4} \mathrm{~N}_{4}$.

Hypoxanthin or oxypurin is
Xanthin or dioxypurin is
Adenin or amino-purin is
Guanin or amino-oxypurin is $\cdot \cdot \begin{aligned} & \mathrm{C}_{6} \mathrm{H}_{4} \mathrm{~N}_{4} \mathrm{O} \\ & \mathrm{C}_{5} \mathrm{H}_{4} \mathrm{~N}_{4} \mathrm{O}_{2} \\ & \mathrm{C}_{5} \mathrm{H}_{6} \mathrm{~N}_{5} \\ & \mathrm{C}_{5} \mathrm{H}_{5} \mathrm{~N}_{5} \mathrm{O}\end{aligned}$

Uric acid is closely related, though not one of the nuclein bases, being trioxypurin, $\mathrm{C}_{5} \mathrm{H}_{4} \mathrm{~N}_{4} \mathrm{O}_{3}$. Caffeine, the active principle of coffee, is trimethyl dioxypurin, $\mathrm{C}_{8} \mathrm{H}_{10} \mathrm{~N}_{4} \mathrm{O}_{2}$ - simply xanthin with three atoms replaced by the methyl group $\mathrm{CH}_{3}$.

Caseinogen.-Caseinogen, the chief proteid of milk, is strictly a nucleo-albumin and does not yield the nuclein bases; it bears the same relation to casein that fibrinogen does to fibrin. When acted on by rennin it splits into two parts of which one, the smaller, is peptone-like in 
character. The other, and larger part, is known as soluble casein and does not solidify in the absence of calcium salts; as these are always present in milk, it there unites with them and forms insoluble calcium casein; strictly speaking, therefore, the curcl of milk is the calcium compound of soluble casein. Caseinogen may be prepared by adding dilute hychrochloric acid to milk mitil the mixture is distinctly acid; a flocculent precipitate of caseinogen will be thrown down and may be separated by filtration; the fat which is earried down with this precipitate may be removed by washing with alcohol and then with ether.

Caseinogen may also be prepared by adding to milk an excess of crystallized magnesium sulphate or sodium chloride, either of which salt causes it to separate out.

Caseinogen gives the Biuret and Millon's reactions showing the presence of proteid substances, much the same tests as alkali-albumin. It is soluble in distilled water, dilute or strong alkalies, and sulphuric acid, but insoluble in sodium chloride and .2 per cent of hydrochloric acid.

Vitellin.-Vitellin is prepared from yolk of egg by washing with ether until all the yellow matter has been removed. The residue is dissolved in 10 per cent saline solution, filtered, and poured into a large quantity of distilled water. The precipitate which falls is impure vitellin. It gives the same tests as myosin, but is not precipitated on saturation with sodium chloride; it coagulates between $70^{\circ}$ and $83^{\circ} \mathrm{C}$.

Albumenoids or Proteoids. - The albumenoids belong to the simple tissues of the body which are derived from the epiblast and are characterized by a lack of any degree of activity, either physiological or chemical. They are proteid derivatives, nitrogenous bodies derived from proteid matter in the cells, and give crystalline amido-acids and nitrogenous bases on decomposition, but differ from true proteids in not having their nitrogen in a form fit for the physiological needs of the body. In other words, they are not true foods, though gelatin has a certain indirect food value as it protects the body proteids from work in many ways, at times. The albumenoids are soluble in dilute acids or alkalies; they may be distinguished from albumin or globulin by being insoluble in water or salt solution respectively.

Gelatin.-Gelatin is contained in the form of collegen, its anhydride, in bone (ossein), teeth, fibrous connective tissues, tendons, ligaments, etc. It may be obtained by prolonged action of boiling water in a Papin's digester or of dilute acetic acid at a low temperature $\left(1 \tilde{5}^{\circ} \mathrm{C}\right.$.).

Properties. - The percentage composition is O, 25.24 per cent, H, 6.56 percent, N, 17.81 per cent, C, 50 per cent, SO, 25 per cent. It contains more nitrogen and less carbon and sulphur than proteids. It is amorphous, and transparent when dried. It does not dialyse; it is insoluble 
in cold water, but swells up to about six times its volume: it dissolves readily on the addition of very dilute acids or alkalies. It is soluble in hot water, and forms a jelly on cooling, even when only 1 per cent of gelatin is present; it is also soluble in hot salt solution. Prolonged boiling in dilute acids, or in water alone, destroys this power of forming a jelly on cooling. Its physical properties seem to indicate a closer relationship to albumin than to keratin, but decomposition proves the reverse. On decomposition it gives 2 per cent of leucin and 2.6 per cent of argenin, but no tyrosin; instead there is a large amount of glycocoll (amido-acetic acid or glycin), a crystalline substance.

A fairly strong solution of gelatin-2 per cent to 4 per cent-gives the following reactions :

(A) With proteid tests: (i.) Xanthoproteic test.-A yellow color but no previous precipitate with nitric acid, becoming darker on the addition of ammonia. (ii.) Biuret test. $\mathrm{\Lambda}$ blue color. (iii.) Millon's test-A pink color but no precipitate. (iv.) Potassium ferrocyanide and acetic acid.-No reaction. (v.) Boiling with sodium sulphate and acetic acid. No reaction.

(B) Special reactions: (i.) No precipitate with acetic acid. (ii.) No precipitate with dilute hydrochloric acid. (iii.) A white precipitate with tannic acid, not soluble in excess or in dilute acetic acid. (iv.) No precipitate with mercuric chloride, unlike the reaction with albumose and peptone. (v.) A white precipitate with alcohol. (vi.) A yellowish-white precipitate with picric acid, dissolved on heating and reappearing on cooling.

Collagen is insoluble in almost everything.

Elastin is found in elastic tissue, in the ligamenta subflava, ligamentum nuchæ, etc. It is insoluble in all ordinary reagents, but swells up both in cold and hot water. Is soluble in strong caustic soda slowly, when heated. It is precipitated by tannic acid; does not gelatinize. Gives the proteid reactions with strong nitric acid and ammonia, and imperfectly with Millon's reagent. On decomposition it gives 4.5 per cent of leucin, a small amount of argenin, and a mere trace of tyrosin. It is prepared by boiling with water, then treating with artificial gastric and pancreatic juices, then boiling again in water, and then extracting with acids, alcohol, and ethers; the remainder is elastin.

Chondrin is found in the condition of chondrigen in cartilage.

It is a mixture of gelatin with a mucin-like substance, and is obtained from chondrigen by boiling.

Properties.-It is soluble in hot water, and in solutions of neutral salt, e.g., sulphate of sodium, in dilute mineral acids, caustic potash, and soda. Insoluble in cold water, alcohol, and ether. It is precipitated from its solutions by dilute mineral acids (excess redissolves it), by alum, 
by lead acetate, by silver nitrate, and by chlorine water. On boiling with strong hydrochloric acid, it yields grape-sugar and certain nitrogenous substances. Prolonged boiling in dilute acids, or in water, destroys its power of forming a jelly on cooling.

Keratin is obtained from hair, horns, finger nails, etc. Its composition is very similar to that of ordinary albumin and is approximately C, 49.5, H, 6.5, N, 16.8, S, 4., O, 23.2; the keratins obtained from the various substances are distinct and differ slightly though closely related. Sulphur is the characteristic body found in keratin and occurs as a sulphur-containing radicle; a large amount of mercaptan sulphur can usually be obtained. On decomposition, keratin yields argenin 2.26 per cent, leucin 10 per cent, and tyrosin 4 per cent.

Properties. - Keratin is insoluble in water, salt, sodium carbonate, and dilute hydrochloric acid; is soluble slowly, when warmed, in caustic potash and sulphuric acid; gives Millon's and the xanthoproteic reactions.

Neurokeratin is a form of keratin which is found in the white substance of Schwann around the axis-cylinders of nerves. It yields argenin 5 per cent, leucin 10 per cent, and tyrosin 3.5 per cent.

Non-nitrogenous organic bodies consist of

(a) Oils and Fats, which are for the most part mixtures of tri-palmitin, $\mathrm{C}_{51} \mathrm{H}_{98} \mathrm{O}_{6}$, tri-steurin $\mathrm{C}_{67} \mathrm{H}_{110} \mathrm{O}_{6}$, and tri-olein $\mathrm{C}_{57} \mathrm{H}_{104} \mathrm{O}$, in different proportions. They are formed by the union of three molecules of fatty acid with one molecule of the triatomic alcohol, glycerin $\mathrm{C}_{3} \mathrm{H}_{5}(\mathrm{OH})_{3}$, and are ethereal salts or esters of that alcohol. Palmitic acid is $\mathrm{C}_{16} \mathrm{H}_{32} \mathrm{O}_{2}$; stearic acid is $\mathrm{C}_{18} \mathrm{H}_{30} \mathrm{O}_{2}$; oleic acid is $\mathrm{C}_{18} \mathrm{H}_{34} \mathrm{O}_{2}$. Human fat consists of a mixture of tri-palmitin, tri-stearin, and tri-olein, of which the two former contribute three-quarters of the whole. Olein is the only liquid constituent. The fat of milk (and butter) is tri-butyrine; butyric acid is $\mathrm{C}_{4}, \mathrm{H}_{\varepsilon}, \mathrm{O}_{2}$.

Fats are insoluble in water and in cold alcohol; soluble in hot alcohol, ether, and chloroform. Colorless and tasteless; easily decomposed or saponified by alkalies or super-heated steam into glycerin and the fatty acids.

And (b) Carbohydrates, which are bodies composed of six or twelve atoms of carbon with hydrogen and oxygen, the two latter elements being in the proportion to form water. There are three main classes of carbohydrates.

Monosaccharides or Glucoses, $\mathrm{C}_{6} \mathrm{H}_{12} \mathrm{O}_{6}$, containing one molecule of sugar, and comprising Dextrose or Grape Sugar, Lævulose or Fruit Sugar, Inosite, etc. Disaccharides or Saccharoses, $\mathrm{C}_{12} \mathrm{H}_{22} \mathrm{O}_{11}$, containing two molecules of sugar from which one molecule of water has been withdrawn, and comprising Saccharose or Cane Sugar, Lactose, Maltose, etc. Polysaccharides or Amyloses, $\mathrm{C}_{6} \mathrm{H}_{10} \mathrm{O}_{5}$, containing a large but unknown number of molecules of sugar from which water has been withdrawn, and comprising Starch, Dextrin, Glycogen, ete. 
Properties.-Monosaccharides are especially soluble and polysaccharides are especially insoluble; monosaccharides and disaccharides do not give colored solutions with iodine while polysaccharides do ; monosaccharides and (except saccharose) disaccharides reduce Fehling's solution while polysaccharides do not.

Of these the most important are :

Starch $\left(\mathrm{C}_{6} \mathrm{H}_{10} \mathrm{O}_{5}\right)$, which is contained in nearly all plants, and in many seeds, roots, stems, and some fruits. It is a soft white powder composed of granules having an organized structure, consisting of granulose (soluble in water) contained in a coat of cellulose (insoluble in water); the shape and size of the granules varying according to the source whence the starch has been obtained. It is not crystalline and will not dialyze. It is insoluble in cold water, in alcohol, and in ether; it is soluble after boiling for some time, and may be filtered, in consequence of the swelling up of the granulose, which bursts the cellulose coat, and becoming free, is entirely dissolved in water. This solution is a solution of soluble starch or amydin. It gives a blue coloration with iodine, which disappears on heating and returns on cooling. It is converted into maltose by diastase, and by boiling with dilute acids into dextrose.

Glycogen, which is contained in the liver, is also present in all muscles but especially in those of very young animals, in the placenta, in colorless corpuscles, and in embryonic tissues. It is sometimes called animal starch and gives many reactions proper to starch itself. It is freely soluble in water, and its solution looks opalescent; it gives a portwine coloration with iodine, which disappears on heating and returns on cooling. It is precipitated by basic lead acetate and is insoluble in absolute alcohol and in ether. It exists in the liver during life, but very soon after death is changed into sugar. It may be prepared by grinding muscle with sand till a pasty mass is formed, boiling the mass in water for twenty minutes, filtering, and then precipitating the glycogen from the filtrate by adding a little more than an equal quantity of 95 per cent alcohol. It is converted into sugar by diastase ferments, or into dextrose by boiling with dilute acids.

Dextrin.-This substance is made in commerce by heating dry potato-starch to a temperature of $400^{\circ}$. It is also produced in the process of the conversion of starch into sugar by diastase, and by the salivary and pancreatic ferments. A yellowish amorphous powder, soluble in water, but insoluble in absolute alcohol and in ether. It corresponds almost exactly in tests with glycogen; but one variety (achroo-dextrin) does not give the port-wine coloration with iodine.

Cane Sugar, or Saccharose, is contained in the juices of many plants and fruits, and is as a rule extracted from the sugar cane, from beetroot, or from the maple. It is crystalline and is precipitated from 
eoncentrated solutions by absolute alcohol. It has no power of reducing

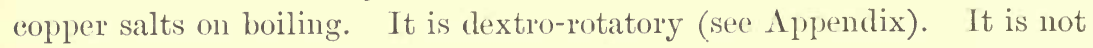
subject to alcoholic fermentation, until by inversion it is converted into glucose, it chars on addition of sulphuric acid, and on heating with potassium or sodium hyclrate.

Lactose is the chief carbohydrate of milk. It is less soluble in water than glucose; not sweet, and is gritty to the taste; but it is insoluble in absolute alcohol. In cligestion it yields a molecule of dextrose and a molecule of galactose. Undergoes alcoholic fermentation with extreme difficulty; gives the tests similar to glucose, but less readily. It is dextro-rotatory $+59^{\circ}$.

Maltose is produced by the action of the saliva and pancreatic juice on starch. It is also formed by the action of malt upon starch by the ferment diastase, and in the formation of glucose from starch. It is converted into dextrose by dilute sulphurie acid. It is dextro-rotatory; ferments with yeast; reduces copper salts, and crystallizes in fine needles.

Glucose oceurs widely diffused in the vegetable kingdom, in diabetic urine, in the blood, ete. ; it is usually obtained from grape-juice, honey, beet-root or carrots. It really is a mixture of two isomeric bodies, Dextrose or grape-sugar, which turns a ray of polarized light to the right $\left(+56^{\circ}\right)$, and Lemelose or fruit-sugar, which turns the ray to the left.

It is easily soluble in water and in alcohol; not so sweet as cane-sugar; the relation of its sweetness to that of cane-sugar is as 3 to 5 . It is not so easily charred by strong sulphuric acid as cane-sugar. It is not entirely soluble in alcohol. It undergoes alcoholic fermentation with yeast.

Dextrose is the characteristic carbohydrate of the blood. It has the power of reducing the salts of silver, bismuth, mercury, and copper, either to the form of the metal in the first three cases, or to the form of the suboxide in the case with cuprous salts. Upon this property the chief tests for the sugar, e.y., Trommer's and Böttcher's, depend (see $\Lambda$ ppendix). When boiled with potash, glucic and melanic acids are formed, aul a yellowish fluid results (Moore's test). It is oxidized by the action of nitric acid to saccharic acid. It forms compounds with acids and with potash and lime. It undergoes alcoholic fermentation with yeast, and lactic-acid fermentation with bacteria lactis. It forms caramel when strongly heated, and is also charred with strong acids. For the method of quantitative estimation, etc., see Appendix.

Lævulose is one of the products of the decomposition of cane-sugar by means of dilute mineral acids, or by means of the ferment invertin in the alimentary canal.

It reacts to the same test as glucose, but is non-crystallizable, and is lævo-rotatory-1060. It is soluble in water and in alcohol. Its compound with lime is solid, whereas that with dextrose is not. 
Galactose is formed from lactose by the action of dilute mineral acids, or inverting ferments; it may also be obtained from cerebrin. It undergoes alcoholic fermentation, and reduces copper salts to the suboxide.

Inosite.-Inosite occurs in the heart and voluntary muscles, as well as in beans and other plants. It crystallizes in the form of large colorless monoclinic tables, which are soluble in water, but insoluble in alcohol or ether. It has the formula of glucose, but is not a sugar. Inosite may be detected by evaporating the solution containing it nearly to dryness, and by then adding a small drop of solution of mercuric nitrate, and afterward evaporating carefully to dryness, a yellowish-white residue is obtained; on further cautiously heating, the yellow changes to a deep rose-color, which disappears on cooling, but reappears on heating. If the inosite be almost pure, its solution may be evaporated nearly to dryness. After the addition of nitric acid, the residue mixed with a little ammonia and calcium chloride, and again evaporated, yields a rose-red coloration.

Certain of the monatomic Fatty Acids are found in the body, viz., Formic $\mathrm{CH}_{2} \mathrm{O}_{2}$, acetic $\mathrm{C}_{2} \mathrm{H}_{4} \mathrm{O}_{2}$, and propionic $\mathrm{C}_{3} \mathrm{H}_{6} \mathrm{O}_{3}$, present in sweat, but normally in no other human secretion. They have been found elsewhere in diseased conditions. Butyric acid, $\mathrm{C}_{4} \mathrm{H}_{8} \mathrm{O}_{2}$, is found in sweat. Various others of these acids have been obtained from blood, muscular juice, fæces and urine.

Of the diatomic fatty acids, one acid, Lactic acid, $\mathrm{C}_{3} \mathrm{H}_{6} \mathrm{O}_{3}$, exists in a free state in muscle plasma, and is increased in quantity by muscular contraction, is never contained in healthy blood, and when present in abnormal amount seems to produce rheumatism.

Of the aromatic series, Benzoic acid, $\mathrm{C}_{3} \mathrm{H}_{6} \mathrm{O}_{2}$, is always found in the urine of herbivora, and can be obtained from stale human urine. It does not exist free elsewhere.

Phenol.-Phenyl alcohol or carbolic acid exists in minute quantity in human urine. It is an alcohol of the aromatic series.

\section{Inorganic Principles.}

The inorganic proximate principles of the human body are numerous. They are derived, for the most part, directly from food and drink, and pass through the system unaltered. Some are, however, decomposed on their way, as chloride of sodium, of which only four-fifths of the quantity ingested are excreted in the same form; and some are newly formed within the body,-as, for example, a part of the sulphates and carbonates, and some of the water.

Much of the inorganic saline matter found in the body is a necessary constituent of its structure,-as necessary in its way as albumin or any 
other organic principle; another part is important in regulating or modifying various physical processes, as absorption, solution, and the like; while a part must be reckoned only as matter, which is, so to speak, accidentally present, whether derived from the food or the tissues, and which will, at the first opportunity, be excreted from the body.

Gases. - The gaseous matters found in the body are Oxygen, Hydrogen, Nitrogen, Carburetted and Sulphuretted hydrogen, and Carbonic acid. The first three have been referred to. Carburetted and sulphuretted hydrogen are found in the intestinal canal. Carbonic acid is present in the blood and other fluids, and is excreted in large quantities by the lungs, and in very minute amount by the skin. It will be specially considered in the chapter on Respiration.

Water, the most abundant of the proximate principles, forms a large proportion,-more than two-thirds of the weight of the whole body. Its relative amount in some of the principal solids and fluids of the body is shown in the following table (from Robin and Verdeil's) :-

\section{Quantity of Water in 1000 Parts.}

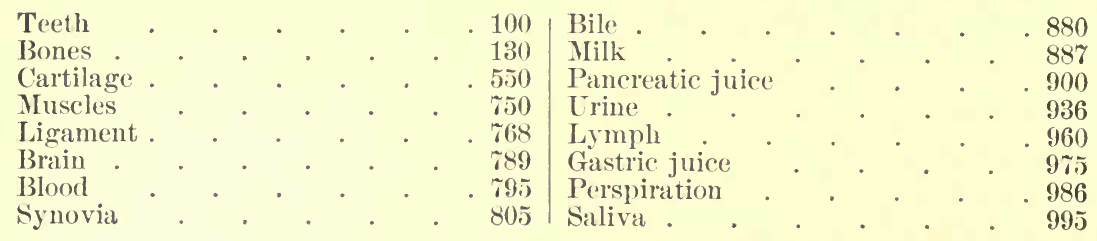

The importance of water as a constituent of the animal body may be assumed from the preceding table, and is shown in a still more striking manner by its withdrawal. If any tissue, as muscle, cartilage, or tendon, be subjected to heat sufficient to drive off the greater part of its water, all its characteristic physical properties are destroyed; and what was previously soft, elastic, and flexible becomes hard and brittle, and horny, so as to be scarcely recognizable.

In all the fluids of the body-blood, lymph, etc.,-water acts the part of a general solvent, and by its means alone circulation of nutrient matter is possible. It is the medium also in which all fluid and solid aliments are dissolved before absorption, as well as the means by which all, except gaseous, excretory products are removed. All the various processes of secretion, transudation, and nutrition depend of necessity on its presence for their performance.

The greater part, by far, of the water present in the body is taken into it as such from without, in the food and drink. A small amount, however, is the result of the chemical union of hydrogen with oxygen in the blood and tissue. The total amount taken into the body every day 
is about $4 \frac{1}{2} \mathrm{lbs}$. ; while an uncertain quantity (perhaps $\frac{1}{2}$ to $\frac{3}{4} \mathrm{lb}$.) is formed by chemical action within it.-(Dalton.)

The loss of water from the body is intimately connected with excretion from the lungs, skin, and kidneys, and, to a less extent, from the alimentary canal. The loss from these various organs may be thus apportioned (quoted by Dalton from various observers).

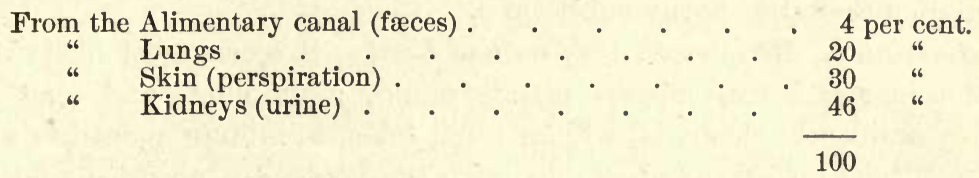

Sodium and Potassium Chlorides are present in nearly all parts of the body. The former seems to be especially necessary, judging from the instinctive craving for it on the part of animals in whose food it is deficient, and from the diseased condition which is consequent on its withdrawal. In the blood, the quantity of sodium chloride is greater than that of all its other saline ingredients taken together. In the muscles, on the other hand, the quantity of sodium chloride is less than that of the chloride of potassium.

Calcium Fluoride, in minute amount, is present in the bones and teeth, and traces have been found in the blood and some other fluids.

Calcium, Potassium, Sodium, and Magnesium Phosphates are found in nearly every tissue and fluid. In some tissues-the bones and teeth-the phosphate of calcium exists in very large amount and is the principal source of that hardness of texture on which the proper performance of their functions so much depends. The phosphate of calcium is intimately incorporated with the organic basis or matrix, but it can be removed by acids without destroying the general shape of the bone; and, after the removal of its inorganic salts, a bone is left soft, tough, and flexible.

Potassium and sodium phosphates with the carbonates, maintain the alkalinity of the blood.

Calcium Carbonate occurs in bones and teeth, but in much smaller quantity than the phosphate. It is found also in some other parts. The small concretions of the internal ear (otoliths) are composed of crystalline calcium carbonate, and form the only example of inorganic crystalline matter existing as such in the body.

Potassium and Sodium Carbonates are found in the blood, and some other fluids and tissues.

Potassium, Sodium, and Calcium Sulphates are met with in small amount in most of the solids and fluids.

Silicon.-A very minute quantity of silica exists in the urine, and in the blood. Traces of it have been found also in bones, hair, and some other parts. 
Iron.-The especial place of iron is in hæmoglobin, the coloring-matter of the blood, of which a full account will be given with the chemistry of the blood. Peroxide of iron is found, in very small quantities, in the ashes of bones, muscles, and many tissues, and in lymph and chyle, albumin of serum, fibrin, bile, milk and other fluids; and a salt of iron, probably a phosphate, exists in the hair, black pigment, and other deeply colored epithelial or horny substances.

Aluminium, Manganese, Copper, and Lead.-It seems most likely that in the human body, copper, manganesium, aluminium, and lead are merely accidental elements, which, being taken in minute quantities with the food, and not excreted at once with the fæces, are absorbed and deposited in some tissue or organ, of which, however, they form no necessary part. In the same manner, arsenic, being absorbed, may be deposited in the liver and other parts. 


\section{CHAPTER V.}

\section{THE BLOOD.}

THE blood is the fluid medium by means of which all the tissues of the body are directly or indirectly nourished; by means of it also such of the materials which result from the metabolism of the tissues as are of no further use in the economy, are carried to the excretory organs to be removed from the body. It is a somewhat viscid fluid, and in man and in all other vertebrate animals with the exception of two,* is red in color. The exact shade of red is variable; that taken from the arteries, from the left side of the heart and from the pulmonary veins is of a bright scarlet hue, that obtained from the systemic veins, from the right side of the heart. and from the pulmonary artery, is of a much darker color, and varies from bluish-red to reddish-black. At first sight, the red color appears to belong to the whole mass of blood, but on further examination this is found not to be the case. In reality blood consists of an almost colorless fluid, called plasma or liquor sanguinis, in which are suspended numerous minute rounded masses of protoplasm, called blood corpuscles, which are, for the most part, colored, and it is to their presence in the fluid that the red color of the blood is due.

Even when examined in very thin layers, blood is opaque, on account of the different refractive powers possessed by its two constituents, viz:, the plasma and the corpuscles. On treatment with chloroform and other reagents, however, it becomes transparent and assumes a lake color, in consequence of the coloring matter of the corpuscles having been discharged into the plasma. The specific gravity of the blood varies slightly at different times in accordance with the changing proportions of its component parts. Of these, water is the most variable constituent, and salts the next, while albumens are the most constant and the last affected by disease. Under normal conditions the average specific gravity in adults is about 1.059 , the normal limits of variation being given by Becquerel and Rodier as 1.054-1.060, by Hammerschlag as $1.056-1.063$, and by Jones as $1.045-1.066$. The physiological variations may be considerable, depending on the age, sex, time of day, amount of exercise or sleep, etc. The specific gravity is increased by residence in 
high altitudes. According to Jones it is very high (1.066) in new-born infants, but, after the second week, sinks throughout the first year of life (to 1.048-1.050) and then rises again, becoming almost as high in old age as in infancy. In pathological conditions the variations may be most marked, e.g., the specific gravity is constantly lowered in anæmia, while in cachexia resulting from malignant new growths it may even be reduced to 1.030 (Lyonnet). Various drugs (e.g., diuretics and diaphoretics) also affect the specific gravity. A rapid and useful method of estimating the specific gravity of blood is the one devised by Hammerschlag as a modification of Roy's method. Chloroform and benzol, which are respectively heavier and lighter than blood, are mixed in such proportions that the resultant specific gravity is about 1.059. A drop of blood is then added to this mixture, with which it does not mix at all, but floats as a red bead. Accordingly as it sinks to the bottom or rises to the top, either chloroform or benzol is added. When the drop remains stationary in the body of the liquid the specific gravity of the mixture will be the same as that of the blood, and can be ascertained by a hydrometer. The reaction of blood is faintly alkaline and the taste saltish. Its temperature varies slightly, the average being $37.8^{\circ} \mathrm{C} .\left(100^{\circ}\right.$ F.). The blood stream is warmed by passing through the muscles, nerve centres, and glands, but is somewhat cooled on traversing the capillaries of the skin. Recently drawn blood has a distinct odor, which in many cases is characteristic of the animal from which it has been taken. It may be further developed also by adding to blood a mixture of equal parts of sulphuric acid and water.

Quantity of the Blood.-The quantity of blood in any animal under normal conditions bears a fairly constant relation to the bodyweight. The methods employed for estimating it are not so simple as might at first sight have been thought, For example, it would not be possible to get any accurate information on the point from the amount obtained by rapidly bleeding an animal to death, for then an indefinite quantity would remain in the vessels, as well as in the tissues; nor, on the other hand, would it be possible to obtain a correct estimate by less rapid bleeding, as, since life would be more prolonged, time would be allowed for the passage into the blood of lymph from the lymphatic vessels and from the tissues. In the former case, therefore, we should under-estimate, and in the latter over-estimate the total amount of the blood.

Of the several methods which have been employed, the most accurate appears to be the following. A small quantity of blood is taken from an animal by renesection; it is defibrinated and measured, and used to make standard solutions of blood. The animal is then rapidly bled to death, and the blood which escapes is collected. The blood-vessels are next washed out with saline solution until the washings are no longer 
colored, and these are added to the previously withdrawn blood; lastly the whole animal is finely minced with saline solution. The fluid obtained from the mincings is carefully filtered, and added to the diluted blood previously obtained, and the whole is measured. The next step in the process is the comparison of the color of the diluted blood with that of standard solutions of blood and water of a known strength, until it is discovered to what standard solution the diluted blood corresponds. As the amount of blood in the corresponding standard solution is known, as well as the total quantity of diluted blood obtained from the animal, it is easy to calculate the absolute amount of blood which the latter contained, and to this is added the small amount which was withdrawn to make the standard solutions. This gives the total amount of blood which the animal contained. It is contrasted with the weight of the animal, previously known.

The result of many experiments shows that the quantity of blood in various animals arerages $\frac{1}{12}$ to $\frac{1}{14}$ of the total body-weight.

An estimate of the quantity in man which corresponded nearly with this proportion, has been more than once made from the following data. A criminal was weighed before and after decapitation; the difference in the weight representing the quantity of blood which escaped. The blood-vessels of the head and trunk were then washed out by the injection of water, until the fluid which escaped had only a pale red or straw color. This fluid was then also weighed; and the amount of blood which it represented was calculated by comparing the proportion of solid matter contained in it with that of the first blood which escaped on decapitation. Two experiments of this kind gave precisely similar results. (Weber and Lehmann.)

It should be remembered, in connection with these estimations, that the quantity of the blood must vary very considerably, even in the same animal, with the amount of both the ingesta and egesta of the period immediately preceding the experiment; it has been found, for example, that the quantity of blood obtainable from the body of a fasting animal rarely exceeds a half of that which is present soon after a full meal.

\section{Coagulation of the Blood.}

One of the most characteristic properties which the blood possesses is that of clotting or coagulating. This phenomenon may be observed under the most favorable conditions in blood which has been drawn into an open vessel. In about two or three minutes, at the ordinary temperature of the air, the surface of the fluid is seen to become semisolid or jelly-like, and this change takes place, in a minute or two afterward at the sides of the vessel in which it is contained, and then extends throughout the entire mass. The time which is occupied in these changes is about eight or nine minutes. The solid mass is of exactly 
the same volume as the previously liquid blood, and adheres so closely to the sides of the containing vessel that if the latter be inverted none of its contents escape. The solid mass is the crassamentum or clot. If the clot be watched for a few minutes, drops of a light, straw-colored fluid, the serum, nay be seen to make their appearance on the surface, and, as they become more and more numerous, to run together, forming a complete superficial stratum above the solid clot. At the same time the fluid begins to transude at the sides and at the under-surface of the clot, which in the course of an hour or two floats in the liquid. The first drops of serum appear on the surface about eleven or twelve minutes after the blood has been drawn; and the fluid continues to transude for from thirty-six to forty-eight hours.

The clotting of blood is due to the development in it of a substance called fibrin, which appears as a meshwork (fig. 11i) of fine fibrils. This meshwork entangles and incloses within itself the blood corpuscles. The first clot formed, therefore, includes the whole of the constituents of the blood in an apparently solid mass, but soon the fibrinous meshwork begins to contract and the serum which does not belong to the clot is squeezed out. When the whole of the serum has transuded the clot is found to be smaller, but firmer and harder, as it is now made up of fibrin and blood corpuscles only. Thus coagulation rearranges the constituents of the blood; liquid blood being made up of plasma and blood corpuscles, and clotted blood of serum and clot.

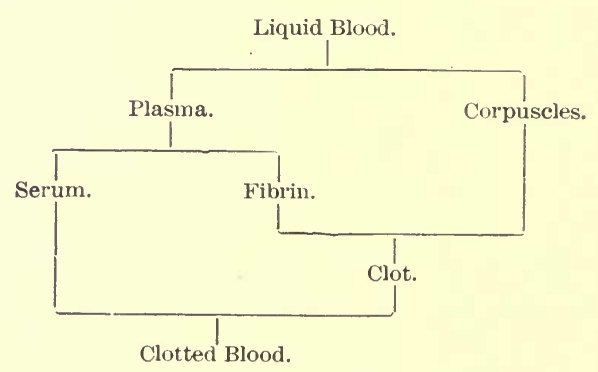

Under ordinary circumstances coagulation occurs before the red corpuscles have had time to subside; and thus from their being entangled in the meshes of the fibrin, the clot is of a deep red color throughout, probably slightly darker at the most dependent part, from greater accumulation of red corpuscles there than elsewhere. When, however, coagulation is from any cause delayed, as when blood is kept at a temperature slightly above $0^{\circ} \mathrm{C}$. $\left(32^{\circ} \mathrm{F}\right.$.), or when clotting is naturally slow, as is the case with horse's blood, or, lastly, in certain diseased conditions, particularly in inflammatory states, time is allowed for the colored corpuscles to sink to the bottom of the fluid. When clotting after a time occurs, the upper layers of the blood are free of colored corpuscles and 
consist chiefly of fibrin. This forms a superficial stratum differing in appearance from the rest of the clot, and is of a grayish yellow color. This is known as the buffy coat or crusta phlogistica. The buffy coat produced in the manner just described, commonly contracts more than the rest of the clot, on account of the absence of colored corpuscles

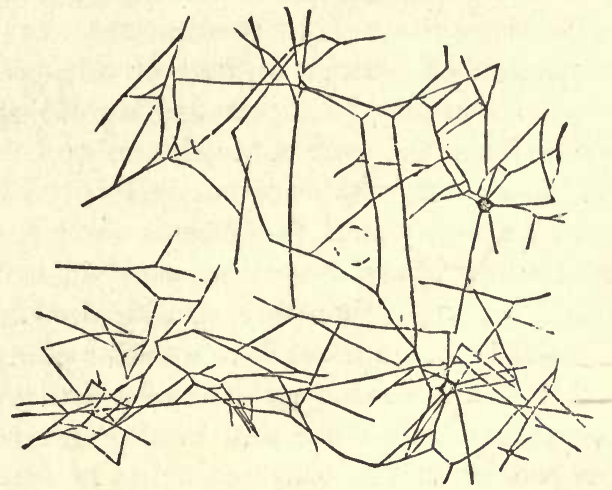

Fig. 117.-Reticulum of fibrin, from a drop of human blood, after treatment with rosanilin. (Ranvier.)

from its meshes, and because contraction is less interfered with by adhesion to the interior of the containing vessel in the vertical than the horizontal direction. A cup-like appearance of the buffy coat results, and the clot is not only buffed but cupped on the surface.

Formation of Fibrin. - That the clotting of blood is due to the gradual appearance in it of fibrin may be easily demonstrated. For example, if recently drawn blood be whipped with a bundle of twigs, the fibrin may be withdrawn from the blood before it can entangle the blood corpuscles within its meshes, as it adheres to the twigs in stringy threads almost free from corpuscles; the blood from which the fibrin has been withdrawn no longer exhibits the power of spontaneous coagulability. Although these facts have long been known, the closely associated problem as to the exact manner in which fibrin is formed is by no means so simple. It will be most convenient to treat of the question step by step.

Fibrin is deriver from the plasma.

Pure plasma may be procured by delaying coagulation in blood by keeping it at a temperature slightly above freezing point, until the colored corpuscles have subsided to the bottom of the containing vessel; the blood of the horse being specially suited for the purposes of this experiment. A portion of the colorless supernatant plasma, if decanted into another vessel and exposed to the ordinary temperature of the air, will coagulate just as though it were the entire blood, producing a clot similar in all respects to blood clot, except that it is almost colorless 
from the absence of red corpuscles. If some of the plasma be diluted with twice or three times its bulk of normal saline solution,* coagulation is delayed, and the stages of the gradual formation of fibrin in it may be conveniently watched. The viscidity which precedes the complete coagulation may be actually seen to be due to the formation of fibrin fibrils-first of all at the edge of the fluid containing vessel, and then gradually extending throughout the mass.

If a further portion of plasma, diluted or not, be whipped with a bundle of twigs, the fibrin may be obtained as a solid, stringy mass, just in the same vay as from the entire blood, and the resulting fluid no longer retains its power of spontaneous coagulability.

It is not indeed necessary that the plasma shall have been obtained by the process of cooling above aescribed, as if it had been separated from the corpuscles in any other way, e.g., by allowing blood to flow direct from the ressels of an animal into a vessel containing a third or a fourth of its bulk of a saturated solution of a neutral salt (preferably of magnesium or sodium sulphate) and mixing carefully, will answer the purpose and, just as in the other case the colored corpuscles will subside leaving the clear superstratum of (salted) plasma. In order that salted plasma may coagulate, however, it is necessary to get rid of the salts by dialysis, or to dilute it with several times its bulk of water.

'The second question which must be considered is, from what materials of the plasma is fibrin formed? If plasma be saturated with solid magnesium sulphate or sodium chloride, a white, sticky precipitate called by Denis, by whom it was first obtained, plasmine, is thrown down, after the removal of which, by filtration, the plasma will not spontaneously coagulate. Plasmine is soluble in dilute neutral saline solutions, and the solution of it speedily coagulates, producing a clot composed of fibrin. Blood plasma therefore contains a substance without which it cannot coagulate, and a solution of which is spontaneously coagulable. This substance is very soluble in dilute saline solutions, and is not, therefore, fibrin, which is insoluble in these fluids.

But there is distinct evidence that plasmine is a compound body made up of two or more substances, not all of which are requisite to form a clot, and that it is not mere soluble fibrin. 'There exists in all the serous cavities of the body in health, e.g., the pericardium, the peritoneum, and the pleura, a certain small amount of transparent fluid, generally of a pale straw color, which in diseased conditions may be greatly increased. It somewhat resembles serum in appearance, but in reality differs from it, being in a measure allied to plasma. This serous fluid is not, as a rule, spontaneously coagulable, but may be made to clot on the addition of serum, which is also a fluid which has no

* Normal saline solution commonly consists of a .6 per cent solution of common salt (sodium chloride) in water. 
tendency of itself to coagulate. The clot produced consists of fibrin, and the clotting is identical with the clotting of plasma. From the serous fluid (that from the inflamed tunica vaginalis testis or hydrocele fluid is mostly used) we may obtain, by half-saturating it with solid sodium chloride, a white viscid substance as a precipitate which is called fibrinogen. If fibrinogen be separated by filtration, it can be dissolved in water, as a certain amount of the neutral salt used in precipitating it is entangled with the precipitate, and is sufficient to produce a dilute ( 6 to 8 per cent) saline solution in which fibrinogen, being a body of the globulin class, is soluble. The solution of fibrinogen has no tendency to clot of itself, but if blood-serum be added to a solution of fibrinogen, the mixture clots.

On the other hand from blood-serum may be obtained, by saturation with one of the neutral salts above-mentioned, a globulin very similar in properties to fibrinogen, which is called serumglobulin or paraglobulin, and it may be separated by filtration and dissolved in a dilute saline solution in a manner similar to fibrinogen.

If the solutions of fibrinogen and paraglobulin be mixed, the mixture cannot be distinguished from a solution of plasmine, and in a great majority of cases firmly clots like that solution, whereas a mixture of the hydrocele fluid and serum, from which these bodies have been respectively taken, no longer manifests the like property.

In addition to this evidence of the compound nature of plasmine, it may be further shown that, if sufficient care be taken, both fibrinogen and paraglobulin may be separately obtained from plasma: the one, fibrinogen, as a flaky precipitate by adding carefully 13 per cent of crystalline sodium chloride to it; and the other, paraglobulin, may be precipitated, after the removal of fibrinogen by filtration, on the further addition (above 20 per cent and to saturation) of the same salt or of magnesium sulphate to the filtrate. It is evident, therefore, that both these substances must be thrown down together when plasma is at once saturated with sodium chloride or magnesium sulphate, and that the mixture of the two corresponds with plasmine.

So far it has been shown that plasmine, the antecedent of fibrin, to the possession of which blood owes its power of coagulating, is not a simple body, but is composed of at least two factors-viz., fibrinogen and paraglobulin; there is reason for believing that yet another body is precipitated with them in plasmine.

It was at one time thought that the reason why hydrocele fluid coagulated, when serum was added to it, was that the latter fluid supplied the paraglobulin which the former lacked; this, however, is not the case, as hydrocele fluid does not lack this body, and moreover, if paraglobulin, obtained from diluted serum by passing a stream of carbonic acid gas through it, be added, no clotting will take place. But if 
paraglobulin, obtained by the saturation method, be added to hydrocele fluid, clotting soon foilows, as it will also in a mixed solution of fibrinogen and paraglobulin, both obtained by the saturation method. From this it is evident that in plasmine there is sornething more than the two bodies above mentioned, and that this something is precipitated with the paraglobulin by the saturation method, and is not precipitated by the carbonic acid method.

The following experiments show that this substance is of the nature of a ferment. If defibrinated blood or serum be kept in a stoppered bottle with its own bulk of alcohol for some weeks, all the proteids are precipitated in a coagulated form; if the precipitate be then removed by filtration, dried over sulphuric acid, finely powdered, and then suspended in water, a watery extract may be obtained by further filtration, containing but little proteid. Yet a little of this watery extract will produce coagulation in fluids, e.g., hydrocele fluid or diluted plasma, which are not spontaneously coagulable, or which coagulate slowly and with difficulty. It will also cause a mixture of fibrinogen and paraglobulin both obtained by the carbonic acid method to clot. The watery extract appears to contain the body which is precipitated with the paraglobulin by the saturation method. Its active properties are entirely destroyed by boiling. The amount of the extract added does not influence the amount of the clot formed, but only the rapidity of clotting, and moreover the active substance contained in the extract evidently does not form part of the clot, as it may be obtained from the serum after blood has clotted. So that the substance contained in the aqueous extract of blood appears to belong to that class of bodies which promote the union of, or cause changes in, other bodies, without themselves entering into union or undergoing change; these are known as ferments. It has, therefore, received the name fibrin ferment or thrombin. This ferment is developed in the blood soon after it has been shed, and its amount contiunes to increase for some little time (p) 133).

So far we have seen that plasmine is a body composed of three substances, viz., fibrinogen, paraglobulin, and fibrin ferment. But we shall see that only two of them are necessary to coagulation.

Relation of Calcium Salts to Coagulation.-Blood will not clot except in the presence of soluble calcium salts. If potassium or sodium oxalate be added to blood as it is drawn from the vessels in quantities sufficient to precipitate the calcinm salts, coagulation will no longer occur. But blood which has thus lost its coagulability may be made to clot upon the addition of soluble calcium salts in proper proportion. This fact has been demonstrated not only for blood, but for solutions of pure fibrinogen.

Theories of Coagulation.-All present theories of coagulation agree 
in that fibrin is formed by a reaction between fibrinogen and thrombin. Beyond this, however, there is some difference of opinion. The chief points at issue are: (1) the origin of fibriuogen; (2) the nature of thrombin; (3) the nature of the reaction between fibrinogen and thrombin.

1. Hammarsten has shown that the presence of paraglobulin is not necessary for coagulation. Schmidt believes, however, that fibrinogen is derived from paraglobulin after the blood is shed from the body.

2. The nature of thrombin is not as yet satisfactorily determined. Pekelharing has concluded from experiment that thrombin is a compound of a nucleo-albumin with the calcium salts of the blood. He has succeeded in separating from blood-plasma a nucleo-albumin, which when brought into solution with fibrinogen and calcium salts will form fibrin. If, however, this nucleo-albumin be brought into contact with either fibrinogen alone or calcium salts alone, no clotting will occur.

Pekelharing further supposes that thrombin is not present in blood circulating in the body - at any rate in greater than minimal quantities; but that when blood is drawn or for other reasons coagulates in the body, the white blood-cells break down, nucleo-albumin is liberated, and then unites with the calcium salts to form thrombin.

3. The nature of the reaction between fibrinogen and thrombin has not been definitely determined. Hammarsten has proved, however, that the entire fibrinogen molecule does not enter into the reaction to become fibrin. A part of it splits off and passes into solution as fibrin-globulin.

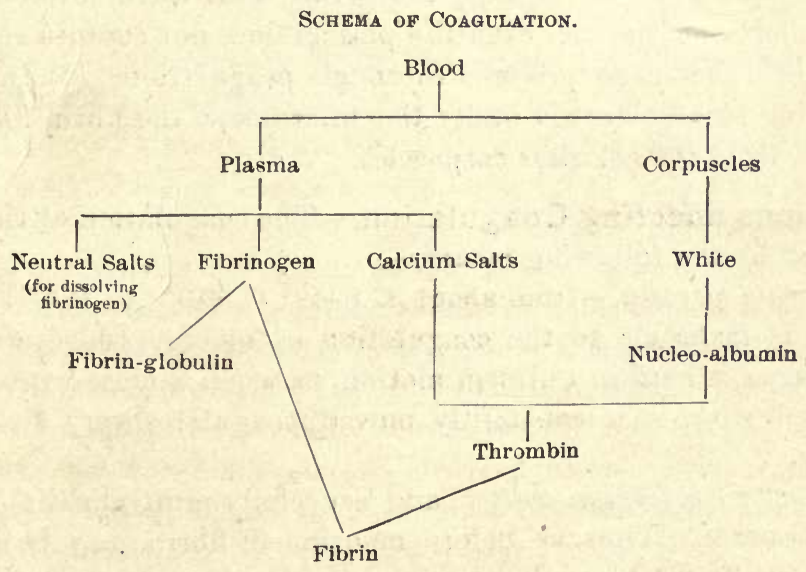

There is strong evidence that fibrin is a compound of calcium with a portion of the fibrinogen molecule. There are three factors, then, in the reaction-fibrinogen; nucleo-albumin, calcium salts. According to Pekelharing, the nucleo-albumin first combines with the calcium salts, 
forming thrombin, which in turn causes a splitting of the fibrinogen molecule-the calcium remaining with one portion of the molecule to form insoluble fibriu, the other portion passing into solution as fibringlobulin. According to Lilienfeld, the reaction first occurs between the fibrinogen molecule and the nucleo-albumin, resulting in a splitting of the former. A portion of the fibrinogen molecule then unites with the calcium salts to form fibrin.

Sources of the Fibrin Ferment.-Fibrin ferment cannot be obtained in any appreciable amount from blood which is allowed to flow direct from the living vessel into absolute alcohol. It is almost certainly a result of the more or less complete disintegration of the colorless corpuscles after blood is shed, or of the third corpuscles which will be described later on under the rame of blood platelets. The proofs of this may be briefly summarized as follows:-(1) That all strongly coagulable fluids contain these corpuscles almost in direct proportion to their coagulability; (2) That clots formed on foreign bodies, such as needles projecting into the interior or lumen of living blood-ressels, are preceded by an aggregation of colorless corpuscles; (3) That plasma in which these corpuscles happen to be scanty clots feebly; (4) That if horse's blood be kept in the cold, so that the corpuscles subside, it will be found that the lowest stratum, containing chiefly colored corpuscles, will, if removed, clat feebly, as it contains little of the fibrin factors; whereas the colorless plasma, especially the lower layers of it in which the colorless corpus les are most numerous, will clot well, but if filtered in the cold will not clot so well, indicating that when filtered nearly free from colorless corpuscles even the plasma does not contain sufficient of all the fibrin factors to produce thorough coagulation; (j) In a drop of coagulating blood observed under the microscope the fibrin fibrils are seen to start from the colorless corpuscles.

Conditions affecting Coagulation. - The coagulation of the blood is hastened by the following means:-

1. Moderate warmth,-from about $3 \% .8-49^{\circ} \mathrm{C}$. $\left(100^{\circ}\right.$ to $120^{\circ} \mathrm{F}$.).

2. Rest is favorable to the coagulation of blood. Blood, of which the whole mass is kept in uniform motion, as when a closed vessel completely filled with it is constantly moved, coagulates very slowly and imperfectly.

3. Contact with foreign matter, and especially multiplication of the points of contact. Thus, as before mentioned, fibrin may be quickly obtained from liquid blood by stirring it with a bundle of small twigs; and even in the living body the blood will coagulate upon rough bodies projecting into the vessels.

4. Injury to the walls of the blood-vessels.

5. The addition of less than twice the buth of uater. 
The blood last drawn is said, from being more watery, to coagulate more quickly than the first.

The coagulation of the blood is retarded, suspended, or prevented by the following means:-

1. Coid retards coagulation; and so long as blood is kept at a tem. perature $0^{\circ} \mathrm{C} .\left(32^{\circ} \mathrm{F}.\right)$, it will not coagulate at all. Freezing the blood, of course, prevents its coagulation; yet it will coagulate, though not firmly, if thawed after being frozen; and it will do so even after it has been frozen for several months. A higher temperature than $49^{\circ} \mathrm{C} .\left(120^{\circ}\right.$ $F$.) retards coagulation by coagulating the albumen of the serum, and a still higher one above $56^{\circ} \mathrm{C}$. $\left(133^{\circ} \mathrm{F}\right.$.) prevents it altogether.

2. The addition of water in greater proportions than twice the bulk of the blood, also the addition of syrup, glycerine, and other viscid substances.

3. Contact with living tissues, and especially with the interior of a living blood-vessel. Blood may be kept fluid in a tortoise's heart after removal from the body for several days, and if the jugular vein of a horse be ligatured in two places so as to include within it blood, and then be removed from the body and placed in a cool place, the contained blood will remain unclotted for hours or even days.

4. The addition of neutral salts in the proportion of 2 or 3 per cent and upward. When added in large proportion most of these saline substances prevent coagulation altogether. Coagulation, however, ensues on dilution with water. The time during which blood can be thus preserved in a liquid state and coagulated by the addition of water, is quite indefinite.

5. In inflammatory states of the system the blood coagulates more slowly although more firmly.

6. The coagulation of the blood is prevented altogether by the addition of strong acids and caustic alkalies, and also by the addition of a 0.1 per cent solution of potassium oxalate, which precipitates the soluble calcium salt present in the blood, in the form of insoluble calcium oxalate. Without the presence of soluble calcium salt, blood does not coagulate.

\%. The injection of commercial peptone containing albumoses, or of various digestive ferments, e.g., trypsin or pepsin, into the vessels of an animal appears to prevent or stay coagulation of its blood if it be killed soon after. The secretion of the mouth of the leech, and possibly the blood squeezed out of its body when full, also prevents the clotting if added to blood.

It is stated that the reason why blood does not coagulate in the living vessels is, that the factors which are necessary for the formation of fibrin are not in the exact state required for its production, and that at any rate the fibrin ferment is not formed or is not free in the living blood, but that it is produced (or set free) at the moment of coagulation by the 
disintegration of the colorless corpuscles. 'This supposition is certainly plausible, and, if it be a true one, it must be assumed either that the living blood-vessels exert a restraining influence upon the disintegration of the corpuscles in sufficient numbers to form a clot, or that they render inert any small amount of fibrin ferment, which may have been set free by the disintegration of a few corpuscles; as it is certain, firstly, that white corpuscles must from time to time disintegrate in the blood without causing it to clot; and, secondly, that shed and defibrinated blood which contains blood corpuscles, broken down and disintegrated, will not, when injected into the vessels of an animal, under ordinary conditions, produce clotting. There must be a distinct difference, therefore, if only in amount, between the normal disintegration of a few colorless corpuscles in the living uninjured blood-vessels and the abnormal disintegration of a large number which occurs whenever the blood is shed without suitable precaution, or when coagulation is unrestrained by the neighborhood of the living uninjured blood-vessels.

\section{The Blood Corpuscles.}

There are two principal forms of corpuseles, the red and the white, or, as they are now frequently named, the colored and the colorless. In the moist state, the red corpuscles form about 45 per cent by weight, of the whule mass of the blood. The proportion of colorless corpuscles is only as 1 to 500 or 600 of the colored.

Red or Colored Corpuscles.-Human red blood-corpuscles are circular, biconcave discs with rounded edges, from $\frac{1}{3000}$ to $\frac{1}{4000}$ inch in diameter $\eta_{\mu}$ to $8 \mu$, and $\frac{1}{12000}$ inch or about $2 \%$ in thickness, becoming flat or convex on addition of water. When viewed singly they appear of a pale yellowish tinge; the deep red color which they give to the blood being observable in them only when they are seen en masse. They are composed of a colorless, structureless, and transparent filmy framework or stroma, infiltrated in all. parts by a red coloring matter termed hamoglobin. The stroma is tough and elastic, so that, as the corpuscles circulate, they admit of elongation and other changes of form, in adaptation to the vessels, yet recover their natural shape as soon as they escape from compression. The term cell, in the sense of a bag or sac, although sometimes applied, is scarcely applicable to the red blood corpuscle; it must be considered, if not solid throughout, yet as having no such marked difference of consistence in different parts as to justify the notion of its being a membranous sac with fluid contents. The stroma exists in all parts of its substance, and the coloring-matter uniformly pervades this; but at the same time it is probable that the consistence of the peripheral part of the protoplasm is more solid than that of the more central mass. 
The red corpuscles have no nuclei, although, in their usual state, the unequal refraction of transmitted light gives the appearance of a central spot, brighter or darker than the border, according as it is viewed in or out of focus. Their specific gravity is about 1088 .

The corpuscles of all mammals with the exception of the camelidx are circular and biconcave. In the camelidæ they are oval and biconvex. In all mammals the corpuscles are non-nucleated, and in all other vertebrates (birds, reptiles, amphibia, and fish), the corpuscles are oval biconvex and nucleated (fig. 121).

Numbers. - The normal number of red blood cells in a cubic millimetre of human blood was estimated by Welcker, in 1854, to be 5,000,000 in men and 4,500,000 in women. Recent observations, however, have shown that these estimates are a little low, especially in men, and the average number has been placed by different authorities at various points between 5,000,000 and 5,500,000, or even $6,000,000$. Still the original numbers as given by Welcker are accepted at the present day as being sufficiently accurate for ordinary purposes. It has also been shown that there are many distinct physiological variations in the number, depending on the time of day, digestion, sex, and pregnancy. The number of red cells usually diminishes in the course of each day, while the leucocytes increase in number. It has been suggested that this is due to the influence of digestion and exercise.

It has generally been found that within half an hour or an hour after a full meal the number of red cells begins to diminish, and that this keeps up for from two to four hours, when it is followed by a gradual rise to the normal. The usual fall is 250,000 to 750,000 per cubic millimetre. These results are most marked after a largely fluid meal, and are probably due to dilution of the blood as a result of the absorption of fluids. In animals the number of red cells is increased by fasting, but in man the results are variable, some authorities claiming an increase and others a decrease. In childhood there is no difference between the sexes in the number of red cells per cubic millimetre, but after menstruation is established, a relative anæmia develops in women. Welcker's original estimate placed the difference at 500,000 per cubic millimetre, and these figures have been generally accepted, though one observer (Leichtenstein) asserted that the difference was 1,000,000. Recent invastigations, however, seem to show that Welcker's estimate is a little too great.

Menstruation in healthy subjects has practically no effect, as not more than 100-200 cubic centimetres are lost normally in the course of several days. Under such circumstances the normal diminution of red cells per cubic millimetre is probably less than 150,000 , though one observer (Sfameni) has placed the loss at about 225,000. Some observers, on the 
other hand, claim an increase. The leucocytes are slightly increased during menstruation. It is now the general opinion that pregnancy has little or no effect on the number of red cells, and that any anæmia must be due to abnormal conditions. Post-partum anæmia should not last longer than two weeks.

Varieties. - The red corpuscles are not all alike. In almost every specimen of blood a certain number of corpuscles smaller than the rest may be observed. They are termed microcytes, or hamatoblasts, and are probably immature corpuscles.

A peculiar property of the red corpuscles, which is exaggerated in inflammatory blood, may be here again noticed, i.e., their great tendency to adhere together in rolls or columns (rouleaux), like piles of coins, These rolls quickly fasten together by their ends, and cluster; so that, when the blood is spread out thinly on a glass, they form a kind of

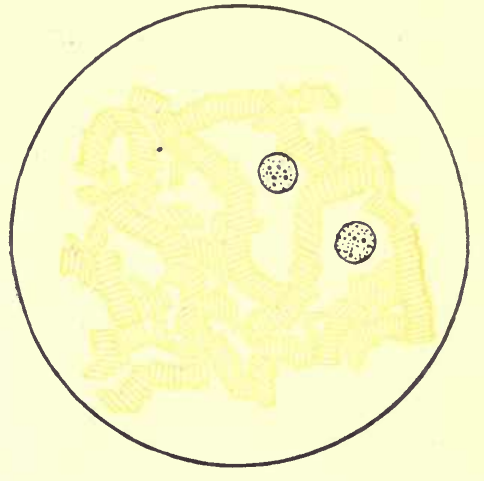

Fig. 118.

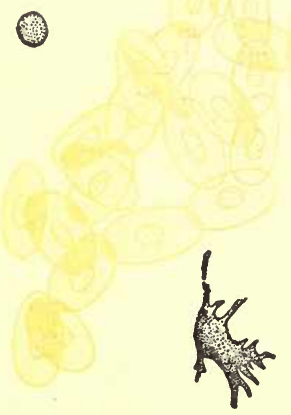

Fig. 119.

Fig. 118.-Red corpuscles in rouleaux. The rounded corpuscles are white or uncolored.

Fig. 119.-Corpuscles of the frog. The central mass consists of nucleated colored corpuscles. The other corpuscles are two varieties of the colorless form.

irregular network, with crowds of corpuscles at the several points corresponding with the knots of the net (fig. 118). Hence the clot formed in such a thin layer of blood looks mottled with blotches of pink upon a white ground, and in a larger quantity of blood such masses help, by the consequent rapid subsidence of the corpuscles, in the formation of the buffy coat already referred to.

Action of Re-agents.-Considerable light has been thrown on the physical and chemical constitution of red blood-cells by studying the effects produced by mechanical means and by various reagents: the following is a brief summary of these re-actions :-

Pressure.-If the red blood-cells of a frog or man are gently squeezed, they exhibit a wrinkling of the surface, which clearly indicates that there is a superficial pellicle partly differentiated from the softer mass within; again, if a needle be rapidly drawn across a drop of blood, several corpuscles will be found cut in two, but this is not accompanied by any escape of cell contents; the two halves, on the contrary, assume a rounded form, proving clearly that the corpuscles are not mere membranous sacs with fluid contents like fat-cells. 
Fluids. i. Water.-When water is added gradually to frog's blood, the oval disc-shaped corpuscles become spherical, and gradually discharge their hæmoglobin, a pale, transpareat stroma being left behind; human red blood-cells change from a discoidal to a spheroidal form, and discharge their cell-contents, becoming quite transparent and all but invisible.

ii. Saline' solution produces no appreciable effect on the red blood-cells of the frog. In the red blood-cells of man the discoid shape is exchanged for a spherical one, with spinous pro-

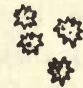

Fig. 120 Effect cf saline solution. jections, like a horse-chestnut (fig. 120). Their original forms can be at once restored by the use of carbonic acid.

iii. Acetic acid (dilute) causes the nucleus of the red blood-cells in the frog to become more clearly defined; if the action is prolonged, the nucleus becomes strongly granulated, and all the coloring matter seems to be concentrated in it,

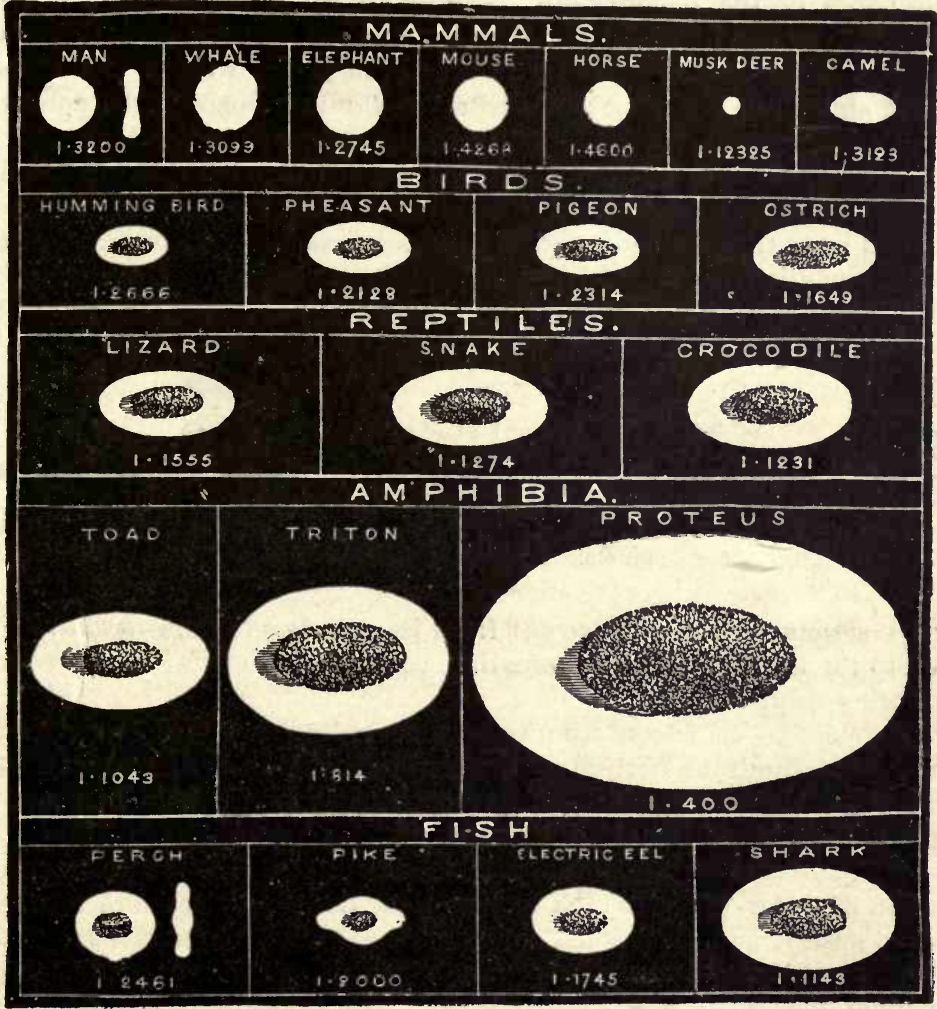

Fig. 121. - The above illustration is somewhat altered from a drawing by Gulliver, in the Proceed. Zool Society, and exhibits the typical characters of the red blood-cells in the main divisions of the Vertebrata. The fractions are those of an inch, and represent the average diameter. In the case of the oval cells, only the long diameter is here given. It is remarkable, that although the size of the red blood-cells varies so much in the different classes of the vertebrate kingdom, that of the white corpuscles remains comparatively uniform, and thus they are, in some animals, much greater, in others much less than the red corpuscle existing side by side with them.

the surrounding cell-substance and outline of the cell becoming almost invisible; after a time the cells lose their color altogether. The cells in the figure 
(fig. 122) represent the successive stages of the change. A similar loss of color occurs in the red cells of human blood, which. however. from the absence of nuclei, seem to disappear entirely.

iv. Alkalies cause the red blood-cells to swell and finally to disappear.

v. Chloroform added to the red blood-cells of the frog causes them to part with their hæmoglobin; the stroma of the cells becomes gradually broken up. A similar effect is produced on the human red blood-cell.

vi. Tannin.-When a 2 per cent fresh solution of tannic acid is applied to frog's blood it causes the appearance of a sharply-defined little knob, projecting from the free surface (Roberts' macula) : the coloring matter becomes at the same time concentrated in the nucleus, which grows more distinct (fig. 123). A somewhat similar effect is produced on the human red blood corpuscle.

vii. Magenta, when applied to the red blood-cells of the frog, produces a similar little knob or knobs, at the same time staining the nucleus and causing the discharge of the hæmoglobin. The first effect of the magenta is to cause the discharge of the hæmoglobin, then the nucleus becomes suddenly stained, and lastly a finely granular matter issues through the wall of the corpuscle, becoming stained by the magenta, and a macula is formed at the point of escape. A similar macula is produced in the human red blood-cells.

viii. Boric acid.-A 2 per cent solution applied to nucleated red blood-cells (frog) will cause the concentration of all the coloring matter in the nucleus; the colored body thus formed gradually quits its central position, and comes to be partly, sometimes entirely, protruded from the surface of the now colorless cell (fig. 124). The result of this experiment led Brücke to distinguish the

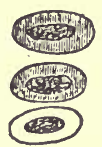

Fig. 122.

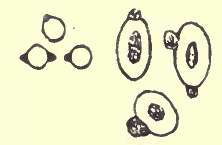

F'ig. 123.

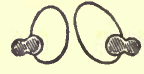

Fig. 124

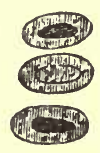

Fig. 125.

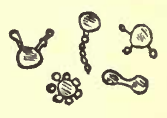

Fig. 126.

Effect of acetic acid. Effect of tannin. Effect of boric acid. Effect of gases. Effect of heat.

colored contents of the cell (zooid) from its colorless stroma (acoid). When applied to the non-nucleated mammalian corpuscle its effect merely resembles that of other dilute acids.

ix. Ammonia.-Its effects seem to vary according to the degree of concentration. Sometimes the outline of the corpuscles becomes distinctly crenated; at other times the effect resembles that of boracic acid, while in other cases the edges of the corpuscles begin to break up.

Gases. Carbonic acid.-If the red blood-cells of a frog be first exposed to the action of water-vapor (which renders their outer pellicle more readily permeable to gases), and then acted on by carbonic acid, the nuclei immediately become clearly defined and strongly granulated; when air or oxygen is admitted the original appearance is at once restored. The upper and lower cell in fig. 125 show the effect of carbonic acid ; the middle one the effect of the re-admission of air. These effects can be reproduced five or six times in succession. If, however, the action of the carbonic acid be much prolonged, the granulation of the nucleus becomes permanent; it appears to depend on a coagulation of the paraglobulin.

Heat. -The effect of heat up to $50^{\circ}-60^{\circ}$ C. $\left(120-140^{\circ}\right.$ F.) is to cause the formation of a number of bud-like processes (fig. 126). 
Electricity causes the red blood-corpuscles to become crenated, and at length mulberry-like. Finally they recover their round form and become quite pale.

The Colorless Corpuscles.-In human blood the white or colorless corpuscles or leucocytes are nearly spherical masses of granular protoplasm without cell wall. In all cases one or more nuclei exist in each corpuscle. The corpuscles vary considerably in size, but average $\frac{1}{2500}$ of an inch $(10 \mu)$ in diameter.

There was for some time a general acceptance of the original estimate of Welcker that there were about 13,500 leucocytes in each cubic millimetre of normal human blood. But improved methods and appliances, especially the introduction of the Thoma hæmatocytometer, soon proved that these figures were too high, and that the number was about $\%, 500$. The latter estimate is upheld by the results of the most recent investigations, Rieder placing the number in adults at $\%, 680$, Limbeck at 8,000 to 9,000 , and Reinert at 5,125 at $6 \mathrm{A.M}$. and 8,262 at 4 P.м. Therefore the proportion of white to red cells (counting 5,000,000 of the latter to each cubic millimetre) is about 1 to 666 . This proportion is in no way to be regarded as a constant one in health, as considerable variations occur frequently, even in the course of the same day. The chief physiological variations are those due to the influence of digestion, of pregnancy, and of infancy.

After a full meal the white cells in a healthy adult are increased in number about one-third (Rieder), the increase beginning within an hour, attaining a maximum in three or four hours, and then gradually falling to normal. This process is frequently modified by the character of the food, the greatest increase occurring with an, exclusively meat diet, while a purely vegetarian diet has usually no effect. The increase is also more marked in children, and especially in infants. The essential factor is probably the absorption of albuminous matter in considerable quantities; this causes proliferation of leucocytes in the adenoid tissue of the gastro-intestinal tract. In pregnancy there is often a moderate increase in the number of white cells during the latter months. This does not begin until after the third month, and is most marked and constant in primiparæ. After parturition the leucocytes gradually diminish under normal conditions, and usually reach the normal within a fortnight. The essential factor is probably the general stimulation in the maternal organism. It is well established that the white cells are very numerous in the new-born, though different observers have made very conflicting estimates. Still all agree that there is a very rapid decrease in their numbers during the first few days, and that this is followed by a less marked increase, which continues for many months. According to Rieder, who is perhaps the most reliable, there are at birth from 14,200 to $2 \%, 400$ 
per cubic millimetre, on the second to fourth day from 8,700 to 12,400 , and after the fourth day from 12,400 to 14,800 .

Varieties.-The colorless corpuscles present greater diversities of form than the red ones. They are usually classified according to their reaction to staining agents, or to the presence or absence of granules in their cytoplasm. Kanthack and Hardy offer the following classification based upon both phenomena:

A Oxyphil (staining with acid dyes). $\left\{\begin{array}{l}\text { 1. Finely granular. } \\ 2 \text {. Coarsely granular. }\end{array}\right.$

B. Basophil (staining with basic dyes).-1. Finely granular.

C. Hyaline ............... 1 . Small.

The finely gramular axyphul constitutes 75 per cent of all leucocytes. It has an average diameter of $10 \%$, and possesses phayocytıc action to a marked degree-that is, it possesses the power of ingesting foreign par-

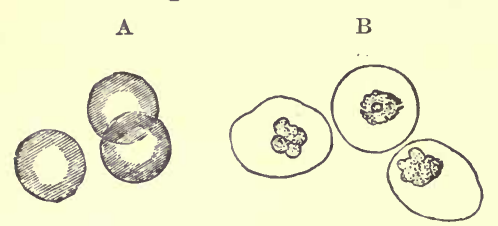

Fig. 12\%.--A. Three colored blood-corpuscles. B. Three colorless hlood-corpuscles acted on by acetic acid: the nuclei are very clearly visible. $\times 900$ ticles. Its mucleus consists of several lobes united by threads of chromatin. This cell was formerly known under the term neutrophul, because of its supposed reaction to neutral dyes.

The coarsely granular form or eosinophal constitutes only 2 per cent of the lencocytes. It has a diameter of $12 \mu$ and a reniform nucleus.

'The basophal cell is rarely found in normal blood. It may occur occasionally during periods of digestion. It is a small, spherical cell, with an irregular nucleus and a diameter of $7 / \mu$.

The small hyalme lencocyte is also called a lymphocyte, because of the large numbers found in adenoid tissue, and is supposed to be an immature form. The nucleus is proportionately large, and is surrounded by but little protoplasm in which no granules can be detected. The cell is about the size of a red blood cell, and constitutes from 10 to 20 per cent of all lencocytes.

The large hyaline or myelocyte varies in diameter from 8.5 to $10 \%$. Its nucleus is spherical or reniform, and is surrounded by more protoplasm than in the case of the lymphocyte. It forms about 10 per cent of the leucocytes.

Amœboid Movement.-The remarkable property of the colorless corpuscles of spontaneously changing their shape was first demonstrated by Wharton Jones in the blood of the skate. If a drop of blood be examined with a high power of the microscope, under conditions by which loss of moisture is prevented, and at the same time the temperature is maintained at about that of the body, $37^{\circ} \mathrm{C}$. $\left(98.5^{\circ} \mathrm{F}\right.$.), the colorless 
corpuscles will be observed slowly to alter their shapes, and to send out processes at various parts of their circumference. The amcboid movement which can be demonstrated in human colorless blood-corpuscles, can be most conveniently studied in the newt's blood. The processes

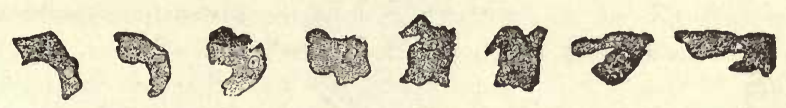

Fig. 128.-Human colorless b!ood-corpuscles, showing its successive changes of outline withir ten minutes when kept moist on a warm stage. (Schofield.)

which are sent out from the corpuscle are either lengthened or withdrawn. If lengthened, the protoplasm of the whole corpuscle flows as it were into its process, and the corpuscle changes its position; if withdrawn, protrusion of another process at a different point of the circumference speedily follows. The change of position of the corpuscle can also take place by a flowing movement of the whole mass, and in this case the locomotion is comparatively rapid. The activity both in the processes of change of shape and also of change in position is much more marked in some corpuscles than in others. Klein states that in the newt's blood the changes are especially noticeable in a variety of the colorless corpuscle, which consists of a mass of finely granular protoplasm with jagged outline, and contains three or four nuclei, or in large irregular masses of protoplasm containing from five to twenty nuclei.

Action of reagents upon the colorless corpuscles.-Feeding the corpuscles.-If some fine pigment granules, e.g., powdered vermilion, be added to a fluid containing colorless blood-corpuscles, on a glass slide, these will be observed, under the microscope, to take up the pigment. In some cases colorless corpuscles have been seen with fragments of colored ones thus embedded in their substance. They have also been seen, in diseased states, to contain micro-organisms, e.g., bacilli, and according to some pathologists are capable of destroying them (phagocytosis). They may too take up other foreign matter or even other colorless corpuscles. This property of the colorless corpuscles is especially interesting as helping still further to connect them with the lowest forms of animal life, and to connect both with the organized cells of which the higher animals are composed.

The property which the colorless corpuscles possess of passing through the walls of the blood-vessels will be described later on.

The Blood-Plates. - A third variety of corpuscle is found in the blood, and is known as the blood-plate. It is circular or elliptical in shape, of nearly homogeneous structure, and varies in size from .5-5.5 $\mu$. Hence it is smaller than the red cell. Though found in the circulating blood, they are not independent cells. It is altogether probable that they are derived chiefly from the red cells, being extruded therefrom in the 
form of masses or chains of globular material. Chemically they contain a nucleo-proteid, and it is supposed that they take part in the phenomenon of coagulation.
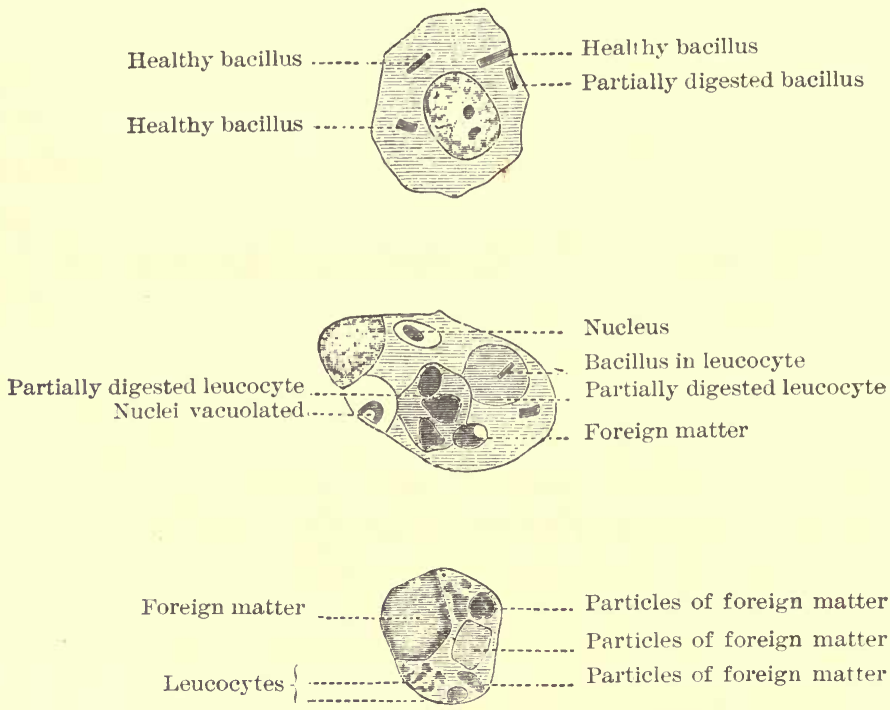

Fig. 129.-Macrophages containing bacilli and other structures supposed to be undergoing digestion. (Ruffer.)

Enumeration of the blood-corpuscles.- Sereral methods are employed for counting the blood-corpuscles, most of them depending upon the same principle, i.e., the dilution of a minute volume of blood with a given volume of a colorless solution similar in specific gravity to blood plasma, so that the size and shape of the corpuscles is altered as little as possible. A minute quantity of the well-mixed solution is then taken, examined under the microscope, either in a flattened capillary tube (Malassez) or in a cell (Hayem \& Nachet, Gowers) of known capacity, and the number of corpuscles in a measured length. of the tube, or in a giren area of the cell is counted. The length of the tube and the area of the cell are ascertained by means of a micrometer scale in the microscope ocular; or in the case of Gowers' modification, by the division of the cell area into squares of known size. Having ascertained the number of corpuscles in the diluted blood, it is easy to find out the number in a given volume of normal blood. Gowers' modification of Hayem \& Nachet's instrument, called by him Hoemacytometer, consists of a small pipette (A), which, when filled up to a mark on its stem, holds 995 cubic millimetres. It is furnished with an india-rubber tube and glass mouth-piece to facilitate filling and emptying; a capillary tube (B) marked to hold 5 cubic millimetres, and also furnished with an india-rubber tube and mouth-piece; a small glass jar (D) in which the dilution of the blood is performed; a glass stirrer (E) for mixing the blood thoroughly, (F) a needle, the length of which can be regulated by a screw; a brass stage plate $(\mathrm{G})$ carrying a glass slide, on which is a cell one- 
fifth of a millimetre deep, and the bottom of which is divided into one-tenth millimetre squares. On the top of the cell rests the cover-glass, which is kept in its place by the pressure of two springs proceeding from the stage plate $A$ standard saline solution of sodium sulphate, or similar salt, of specific gravity 1025 , is made, and 995 cubic millimetres are measured by means of the pipette into the glass jar, and with this five cubic millimetres of blood, obtained by pricking the finger with a needle, and measured in the capillary pipette (B) are thoroughly mixed by the glass stirring-rod. A drop of this diluted blood is then placed in the cell and covered with a cover-glass, which is fixed in position by means of the two lateral springs. The layer of diluted blood between the slide and cover-glass is $\frac{1}{5}$ inch thick. The preparation is then examined under a microscope with a power of about 400 diameters, and focussed until the lines dividing the cell into squares are visible.

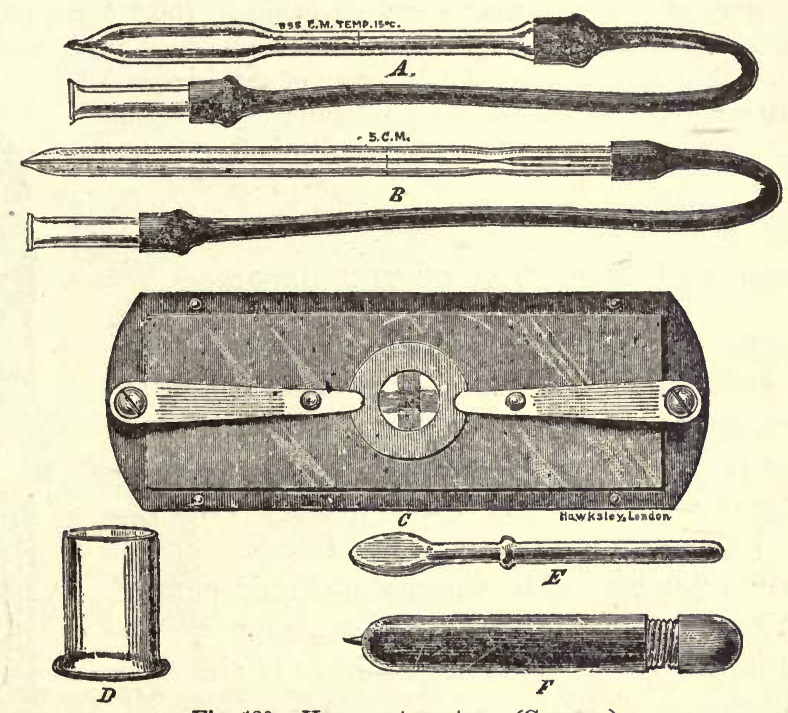

Fig. 130.-Hæmacytometer. (Gowers.)

After a short delay, the red corpuscles which have sunk to the bottom of the cell, and are resting on the squares, are counted in ten squares, and the number of white corpuscles noted. By adding together the numbers counted in ten (one-tenth millimetre) squares, and, as the blood has been diluted, multiplying by ten thousand, the number of corpuscles in one cubic millimetre of blood is obtained. The average number of corpuscles per each cubic millimetre of healthy blood, according to Vierordt and Welcker, is 5,000,000 in adult men, and 4,500,000 in women.

A hæmacytometer of another form, and one that is much used at the present time, is known as the Thoma-Zeiss hæmacytometer. It consists of a carefully graduated pipette, in which the dilution of the blood is done; this is so formed that the capillary stem has a capacity equalling one-hundredth of the ball above it. If the blood is drawn up in the capillary tube to the line marked 1 (fig. 132) the saline solution may afterward be drawn up the stem to the line 101; in this way we have 101 parts of which the blood forms 1 . As the con- 
tents of the stem can be displaced unmixed we shall have in the mixture the proper dilution. The blood and the saline solution are well mixed by shaking the pipette, in the ball of which is contained a small glass bead for the purpose of aiding the mixing. The other part of the instrument consists of a glass slide (fig. 131) upon which is mounted a corered $\cdot d$ isc, $m$, accurately ruled so as to present one square millimetre divided into 400 squares of one-twentieth

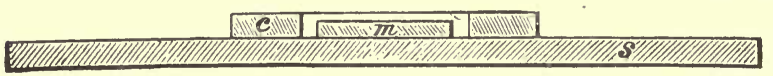

Fig. 131.-Thoma-Zeiss Hæmacytometer.

of a millimetre each. The micrometer thus made is surrounded by another annular cell, $c$, which has such a height as to make the cell project exactly one-tenth millimetre beyond $m$. If a drop of the diluted blood be placed upon $m$, and $c$ be covered with a perfectly flat cover-glass, the volume of the diluted blood above each of the squares of the micrometer, i.e., abore each $\frac{1}{40} \overline{0}$, will be $\frac{1}{4000}$ of a cubic millimetre. An arerage of ten or more squares are then taken, and this number multiplied by $4000 \times 100$ gives the number of corpuscles in a cubic millimetre of undiluted blood.

\section{Chemical Composition of the Blood.}

Before considering the chemical composition of the blood as a whole, it wili be convenient to take in order the composition of the various chief factors which have been set out in the table on p. 132, into which the blood may be separated, viz.:-(1.) The Plasma; (2.) The Serum; (3.) The Corpuscles; (4.) The Fibrin.

(1.) The Plasma.-The Plasma, or liquid part of the blood, in which the corpuscles float, may be obtained free from colored corpuscles in either of the ways mentioned below.

In it are the fibrin factors, inasmuch as when exposed to the ordinary temperature of the air it undergoes coagulation and splits up into fibrin and serum. It differs from the serum in containing fibrinogen, but in appearance and in reaction it closely resembles that fluid; itsalkalinity, however, is greater than that of the

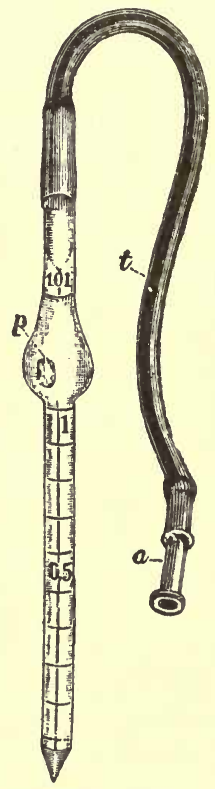

Fig. 132.-ThomaZeiss Hæmacytometer. serum obtained from it. It may be freed from white corpuscles by filtration at a temperature below $5^{\circ} \mathrm{C} .\left(41^{\circ} \mathrm{F}\right.$.) or by the centrifugal machine.

The chief methods of obtaining plasma free from corpuscles may be here epitomized: (1) by cold, the temperature should be about $0^{\circ} \mathrm{C}$. and may be two or three degrees higher, but not lower. (2) The addition of neutral salts, in certain proportions, either solid or in solution, e.g. of sodium sulphate, if solid 1 part to 12 parts of blood; if a saturated solution 1 part to 6 parts of blood; of magnesium sulphate, of a $23 \%$, or if saturated solution 1 part to 4 of 
blood. (3) A third way is to mix frog's blood with an equal part of a $5 \%$ of cane sugar, and to get rid of the corpuscles by filtration; or (4) by the injection of commercial peptone into the veins of certain mammals, previous to bleeding them to death, allowing the corpuscles to subside, and afterward subjecting the supernatant plasma to the action of a centrifugal machine; by
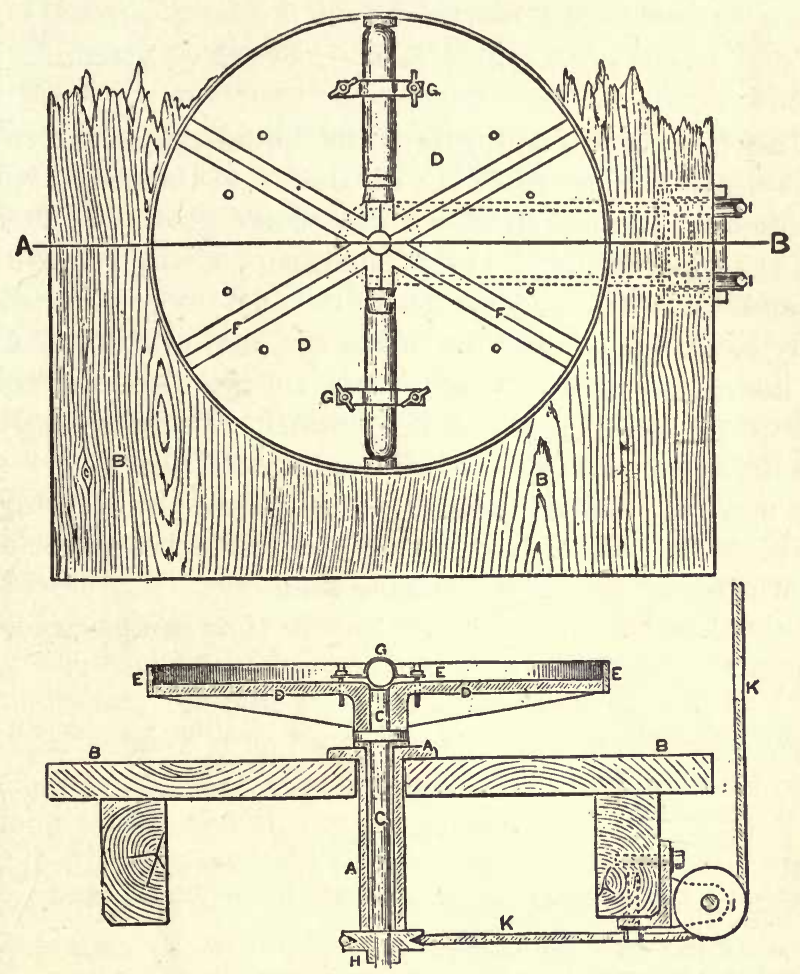

Fig. 133.-Plan and section of centrifugal machine. A, An iron socket secured to top of table B;

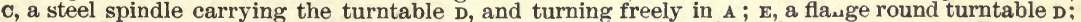
F F, shallow grooves on face of $D$, in which the test tubes are fixed by clamp $G G$; $B$, a pulley fixed to end of spindle $\mathrm{c}$, and turned by the cord $\mathrm{K}$; I I are two guide-pulleys for cord $\mathrm{k}$. (Gamgee.)

the rapid rotation of which (fig. 133) the whole of the remaining solid particles, if any, is driven to the outer end of the test-tubes in which the plasma is placed.

\section{Composition of Plasma.}

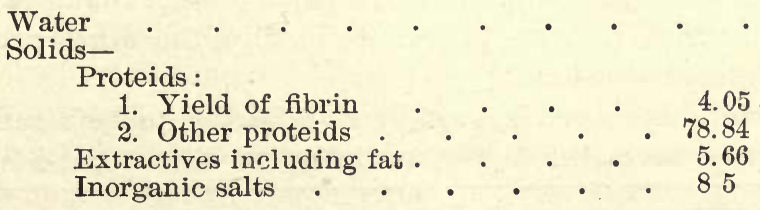

Proteids :

1. Yield of fibrin . . . . 4.05

Extractives including fat . . . . $\quad{ }^{\circ} \quad 5.66$

Inorganic salts . $. \quad . \quad .85$ 
Inorganic Substances - In 1,000 parts of plasma there are -

Clilorine

3.536

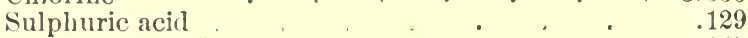

Plosphoric acid . . . . . . . . . . . . . . .

Potassium , . . . . . . . . . . . 314

Sodium, . . . . 3.410

Phosphate of lime , , . . . . . . . . . 298

Phosphate of magnesia . . . . . . . . . 218

Oxygen . . . . . . . . . . . 455

8.505

(2.) The Serum.-The serum is the liquid part of the blood or of the plasma which remains after the separation of the clot. It is a transparent, yellowish, alkaline fluid, with a specific gravity of from 1025 to 1032. In the usual mode of coagulation, part of the serum remains in the clot, and the rest, squeezed from the clot by its contraction, lies aruund it. Since the contraction of the clot may continue for thirty-six or more hours, the quantity of serum in the blood cannot be even roughly estimated till this period has elapsed. There is nearly as much, by weight, of serum as there is clot in coagulated blood.

Serum may be obtained from blood corpuscles by allowing blood to clot in large test tubes, and subjecting the test tubes to the action of a centrifugal machine (fig. 133) for some time.

In tabular form the composition may be thus summarized. In 1000 parts of serum * there are:-

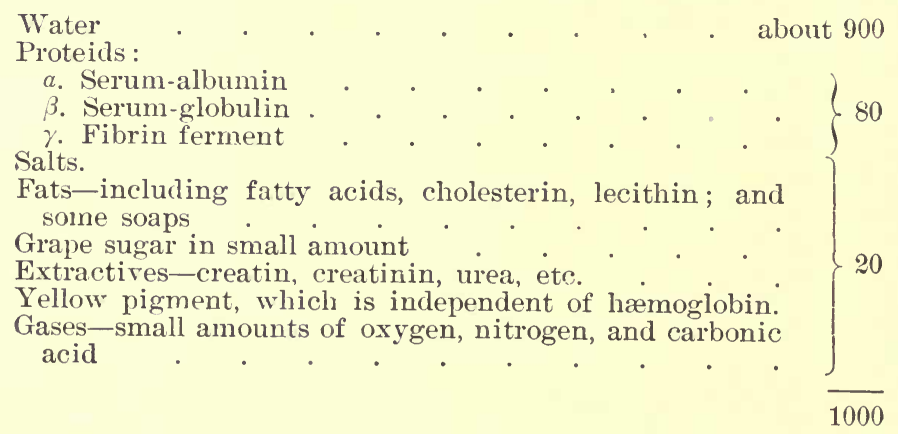

a. Water.-The water of the serum varies in amount according to the amount of food, drink, and exercise, and with many other circumstances.

b. Proteids.- $\alpha$. Serum albumin is the chief proteid found in serum. The proportion which it bears to serum-globulin, the other proteid, is as 3 to 4.5 in human blood.

Serum-albumin has been shown by Halliburton to be a compound body which may be called serine, made up of three proteids, which

* This table is more detailed than that of the plasma giren above. The salts, extractires, etc., are the same in both serum and plasma, but the proteids are somewhat different in nature and amount. 
coagulate at different temperatures, $\alpha$ at $73^{\circ}$ C., $\beta$ at $77^{\circ}$ C., and $\gamma$ at $84^{\circ}$ C. The serine is coagulated by the addition of strong acids, such as nitric and hydrochloric; by long contact with alcohol it is precipitated. It is not precipitated on addition of ether, and so differs from the other native albumin, viz., egg-albumin. When dried at $40^{\circ} \mathrm{C} .\left(104^{\circ} \mathrm{F}\right.$. $)$ serum-albumin is a brittle, yellowish substance, soluble in water, possessing a lævorotary power of $-56^{\circ}$. It is with great difficulty freed from its salts. It is precipitated by solutions of metallic salts, e.g., of mercuric chloride, copper sulphate, lead acetate, sodium tungstate, etc. If dried at a temperature over $75^{\circ} \mathrm{C}$. $\left(167^{\circ} \mathrm{F}\right.$.), the chief part of the residue is insoluble in water, having been changed into coagulated proteid. Serum-albumin may be precipitated from serum, from which the serum-globulin has been previously separated by saturation with magnesium sulphate, by further saturation with sodium sulphate, sodium nitrate, or iodide of potassium.

. Serum-globulin can be obtained as a white precipitate from cold serum by adding a considerable excess of water over ten times its bulk, and passing through the mixture a current of carbonic acid gas or by the cautious addition of dilute acetic acid. It can also be obtained by saturating serum with either crystallized magnesium sulphate, or sodium chloride, nitrate, acetate, or carbonate. When obtained in the latter way, precipitation seems to be much more complete than by means of the former method. Serum-globulin coagulates at $75^{\circ}$ C. $\left(167^{\circ}\right.$ F.). There seems to be more globulin in the serum than in the corresponding plasma, and supposing Halliburton is correct in believing the fibrin ferment to belong to the globulin class, its presence arising from the disintegration of the colorless corpuscles (cell-globulin) would account for part of the increase, while possibly another part might be due, as suggested by Hammersten, to the fact that fibrinogen splits up into fibrin, leaving a globulin residue which appears in the serum.

c. The salts of sodium predominate in serum as in plasma, and of these the chloride generally forms by far the largest proportion.

d. Fats are quite constantly present in a free state (i.e., as palmitin, stearin, and olein), though usually in very small quantities. Gumprecht, however, asserts that they may be present in such quantities as to give the blood a milky hue. Fatty acids have been found by some observers in pathological conditions, but on the other hand it is claimed that this is merely the result of faulty technique. The amount of fatty matter varies according to the time after, and the ingredients of, a meal.

e. Grape sugar is found principally in the blood of the hepatic vein, to the extent of about two parts in a thousand.

f. The extractives vary from time to time; sometimes hippuric acid is found in addition to urea, uric acid, creatin and creatinin, xanthin, hypoxanthin, and cholesterin. Urea exists in proportion from .02 to .04 per cent. 
g. The yellow pigment of the serum and the odorous matter which gives the blood of each particular animal a peculiar smell, have not yet been exactly differentiated. The former is probably of the nature of a lipochrome, and might be called serum lutein. It is soluble in alcohol and ether, and has two hazy absorption bands toward the violet end of the spectrum.

(3.) The Corpuscles.-a. Colored.-Analysis of a thousand parts of moist blood corpuscles shows the following result:-

Water

Solids-

Organic

Mineral
688

303.88

$$
8.12-312=1000
$$

Of the solids the most important is Hemoglobin, the substance to which the blood owes its color. It constitutes, as will be seen from the appended Table, more than 90 per cent of the organic matter of the corpuscles. Besides hæmoglobin there are proteid and fatty matters, the former chiefly consisting of globuiins, and the latter of cholesterin and lecithin.

In 1000 parts organic matter are found:-

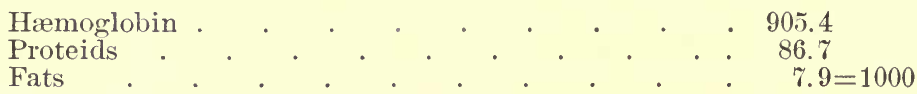

Of the inorganic salts of the corpuscles, with the iron omittedIn 1000 parts corpuscles (Schmidt) are found:-

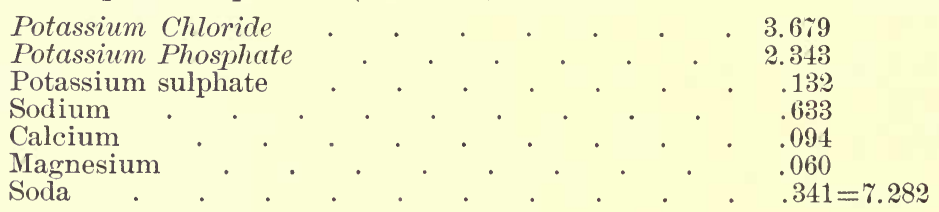

The properties of hæmoglobin will be considered in relation to the Gases of the blood.

b. Colorless.-In consequence of the difficulty of obtaining colorless corpuscles in sufficient number to make an analysis, little is accurately known of their chemical composition; in all probability, however, the protoplasm of the corpuscles is made up of proteid matter, and the nucleus of nuclein, a nitrogenous phosphorus-containing body akin to mucin, capable of resisting the action of the gastric juice. The proteid matter is made up probably of one or more nucleo-albumins, and of one or more globulins with a small amount of serum albumin. There are also present lecithin, a fatty body containing phosphorus, fatty granules staining black with osmic acid, cholesterin, a monatomic alcohol, glycogen, and salts of sodium, potassium, calcium, and magnesium, of which the phosphate of potassium is in greatest amount.

(4.) Fibrin.-The part played by fibrin in the formation of a clot and its tests have been already described, and it is only necessary to 
consider here its general properties. It is a stringy elastic substance belonging to the proteid class of bodies. Blood contains only .2 per cent of fibrin. It can be converted by the gastric or pancreatic juice into peptone. It possesses the power of liberating the oxygen from solutions of hydric peroxide $\mathrm{H}_{2} \mathrm{O}_{2}$ or ozonic ether. This may be shown by dipping a few shreds of fibrin in tincture of guaiacum, and then immersing them in a solution of hydric peroxide. The fibrin becomes of a bluish color, from its having liberated from the solution oxygen, which oxidizes the resin of guaiacum contained in the tincture, and thus produces the coloration.

\section{The Gases of the Blood.}

The gases contained in the blood are carbonic acid, oxygen, and nitrogen, 100 volumes of blood containing from 50 to 60 volumes of these gases collectively.

Arterial blood contains relatively more oxygen and less carbonic acid than venous. But the absolute quantity of carbonic acid is in both kinds of blood greater than that of the oxygen.

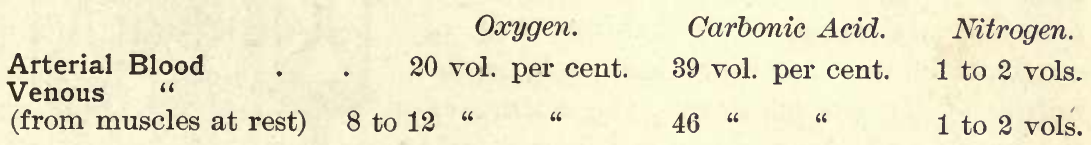

The Extraction of the Gases from the Blood.-As the ordinary air pumps are not sufficiently powerful for the purpose, the extraction of the gases from the blood is accomplished by means of a mercurial air-pump, of which there are many varieties, those of Ludwig, Alvergnidt, Geissler, and Sprengel being the chief. The principle of action in all is much the same. Ludwig's pump, which may be taken as a type, is represented in fig. 134 . It consists of two fixed glass globes, $C$ and $F$, the upper one communicating by means of the stop-cock $D$, and a stout india-rubber tube with another glass globe, $L$, which can be raised or lowered by means of a pulley; it also communicates by means of a stop-cock, $B$, and a.bent glass tube, $A$, with a gas receiver (not represented in the figure), $A$, dipping into a bowl of mercury, so that the gas may be received over mercury. The lower globe, $F$, communicates witl $C$ by means of the stop-cock, $E$, with $I$ in which the blood is contained by the stopcock, $G$, and with a movable glass globe, $M$, similar to $L$, by means of the stopcock, $H$, and the stout india-rubber tube, $K$.

In order to work the pump, $L$ and $M$ are filled with mercury, the blood from which the gases are to be extracted is placed in the bulb $I$, the stopcocks, $H$, $E, D$, and $B$, being open, and $G$ closed. $M$ is raised by means of the pulley until $F$ is full of mercury, and the air is driven out. $E$ is then closed, and $L$ is raised so that $C$ becomes full of mercury, and the air driven off. $B$ is then closed. On lowering $L$ the mercury runs into it from $C$, and a vacuum is established in $C$. On opening $E$ and lowering $M$, a vacuum is similarly established in $F$; if $G$ be now opened, the blood in $I$ will enter ebullition, and the gases will pass off into $F$ and $C$, and on raising $M$ and then $L$, the stopcock $B$ being opened, the gas is driven through $A$, and is received into the receiver 
over mercury. By repeating the expcriment sereral times the whole of the gases of the specimen of blood is obtained, and may be estimated.

A. The Oxygen of the Blood.-It has been found that a very small proportion of the oxygen which can be obtained, by the aid of the mercurial pump from the blood, exists in a state of simple solution in the plasma. If the gas were in simple solution, the amount of oxygen in any given quantity of blood, exposed to any given atmosphere, ought to vary with the amount of oxygen contained in the atmosphere. Since, speaking generally, the amount of any gas absorbed by a liquid such as plasma would depend upon the pro. portion of the gas in the atmosphere to which the liquid is exposed-if the proportion is great, the absorption will be great; if small, the absorption will be similarly small. The absorption continues until the proportions of the gas in the liquid and in the atmosphere are equal. Other things will, of course, influence the absorption, such as the nature of the gas employed, the nature of the liquid and the temperature, but coteris paribus, the amount of a gas which a liquid absorbs depends upon the proportion - the so-called partial pressure-of the gas in the atmosphere to which the liquid is subjected. And conversely, if a liquid containing a gas in solution be exposed to an atmosphere containing none of the gas, the gas will be given up to the atmosphere until the

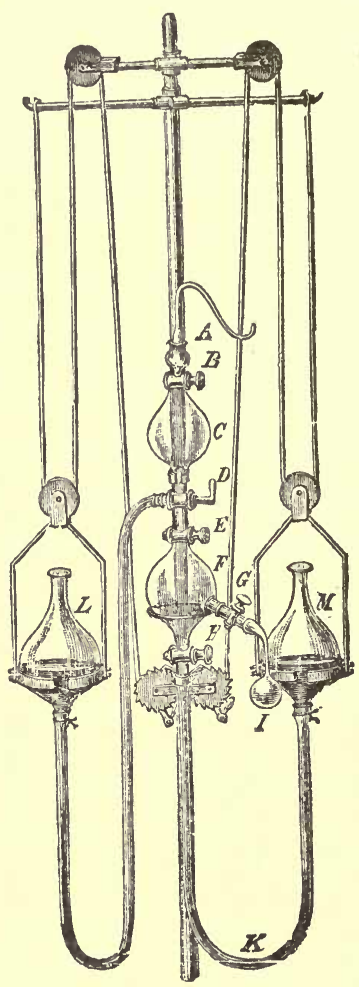

Fig. 134.-Ludwig's Mercurial Pump. amount in the liquid and in the atmosphere becomes equal. This condition is called a condition of equal tensions.

The condition may be understood by a simple illustration. A large amount of carbonic acid gas is dissolved in a bottle of water by exposing the liquid to extreme pressure of the gas, and a cork is placed in the bottle and wired down: The gas exists in the water in a condition of tension, and therefore exhibits a tendency to escape into the atmosphere, in order to relieve the tension; this produces the violent expulsion of the cork when the wire is removed, and if the aërated water is placed in a glass the gas will continue to be evolved until it has almost entirely passed into the atmosphere, and the tension of the gas in the water approximates to that of the atmosphere, in which, it should be remembered, the carbon dioxide is, naturally, in very small amount, viz., .04 per cent. 
The oxygen of the blood does not obey this law of pressure. For if blood which contains little or no oxygen be exposed to a succession of atmospheres containing more and more of that gas, we find that the absorption is at first very great, but soon becomes relatively very small, not being therefore regularly in proportion to the increased amount (or tension) of the oxygen of the atmospheres, and that conversely, if arterial blood be submitted to regularly diminishing pressures of oxygen, at first very little of the contained oxygen is given off to the atmosphere, then suddenly the gas escapes with great rapidity, and again disobeys the law of pressures.

Very little oxygen can be obtained from plasma freed from blood corpuscles, even by the strongest mercurial air-pump, neither can it be made to absorb a large quantity of that gas; but the small quantity which is so given up or so absorbed follows the laws of absorption according to pressure.

It must be, therefore, evident that the chief part of the oxygen is contained in the corpuscles, and not in a state of simple solution. The chief solid constituent of the colored corpuscles is hoomoglobin, which constitutes more than 90 per cent of their bulk. This body has a very remarkable affinity for oxygen, absorbing it to a very definite extent under favorable circumstances, and giving it up when subjected to the action of reducing agents, or to a sufficiently low oxygen pressure. From these facts it is inferred that the oxygen of the blood is combined with hcomoglobin, and not simply dissolved; but inasmuch as it is comparatively easy to cause the hæmoglobin to give up its oxygen, it is believed that the oxygen is but loosely combined with the substance.

Hæmoglobin.-Hæmoglobin is a crystallizable body which constitutes by far the largest portion of the colored corpuscles. It is intimately distributed throughout their stroma, and must be dissolved out before it will undergo crystallization. Its percentage composition is C. 53.85; H. $7.32 ;$ N. $16.17 ; 0.21 .84 ;$ S. .63; Fe. .42. Jacquet gives the empirical formula for the hæmoglobin of the dog, $\mathrm{C}_{758} \mathrm{H}_{1203} \mathrm{~N}_{195} \mathrm{~S}_{3} \mathrm{Fe}$ $\mathrm{O}_{218}$. The most interesting of the properties of hæmoglobin are its power's of crystallizing and its attraction for oxygen and other gases.

Crystais. - The hæmoglobin of the blood of various animals possesses the power of crystallizing to very different extents (hæmoglobin). In some animals the formation of crystals is almost spontaneous, whereas in others it takes place either with great difficulty or not at all. Among the animals whose blood coloring-matter crystallizes most readily are the guinea-pig, rat, squirrel, and dog; and in these cases to obtain crystals it is generally sufficient to dilute a drop of recently-drawn blood with water and to expose it for a few minutes to the air. Light seems to favor the formation of the crystals. In many instances other means 
must be adopted, e.g., the addition of alcohol, ether, or chloroform, rapid freezing, and then thawing, an electric current, a temperature of $60^{\circ} \mathrm{C}$. $\left(140^{\circ} \mathrm{F}.\right)$, the addition of sodium sulphate, or the addition of decomposing serum of another animal.

The hæmoglobin of human blood crystallizes with difficulty, as does also that of the ox, the pig, the sheep, and the rabbit.

The forms of hæmoglobin crystals, as will be seen from the appended figures, differ greatly.

Hæmoglobin crystals are soluble in water. Both the crystals themselves and also their solutions have the characteristic color of arterial blood.

A dilute solution of oxy-hæmoglobin gives a characteristic appearance with the spectroscope. Two absorption bands are seen between

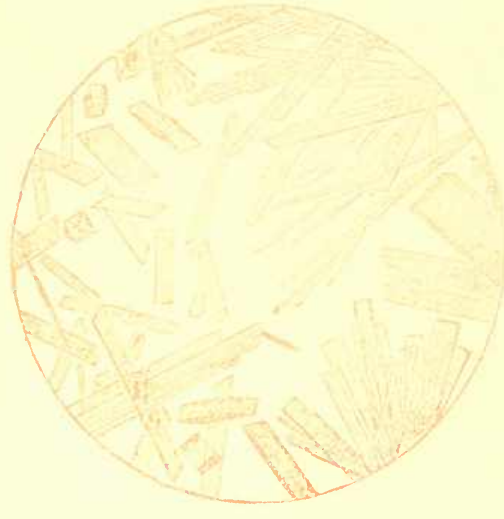

Fig. 135.-Crystals of oxy-hæmoglobinprismatic, from human blood.
Fig. 136.-Oxy-hæmoglobin crystals-tetrahedral, from blood of the guinea-pig.

the solar lines $\mathrm{D}^{*}$ (which is the sodium band in the yellow) and $\mathrm{E} *$ (see plate), one in the yellow, with its middle line some little way to the right of $\mathrm{D}$, is very intense, but narrower than the other, which lies in the green near to the left of $\mathrm{E}$. Each band is darkest in the middle and fades away at the sides. As the strength of the solution increases the bands become broader and deeper, and both the red and the blue ends of the spectrum become encroached upon until the bands coalesce to form one very broad band, and only a slight amount of the green remains unabsorbed, and part of the red; on still further increase of strength the former disappears.

If the crystals of oxy-hæmoglobin be subjected to a mercurial airpump they give off a definite amount of oxygen ( 1 gramme giving off $1.59 \mathrm{ccm}$. of oyxgen), and they become of a purple color; and a solution of oxy-hæmoglobin may be made to give up oxygen, and to become purple in a similar manner. 
This change may be also effected by passing through the solution of blood or of oxy-hæmoglobin, hydrogen or nitrogen gas, or by the action of reducing agents, of which Stokes's fluid * or ammonium sulphide are the most convenient.

With the spectroscope, a solution of deoxidized or reduced homoglobin is found to give an entirely different appearance from that of oxidized hæmoglobin. Instead of the two bands at $\mathrm{D}$ and $\mathrm{E}$ we find a single broader but fainter band occupying a position midway between the two, and at the same time less of the blue end of the spectrum is absorbed. Even in strong solutions this latter appearance is found, thereby differing from the strong solution of oxidized hæmoglobin which lets through only the red and orange rays; accordingly to the naked eye the one (reduced hæmoglobin solution) appears purple, the other (oxy-hæmoglo-

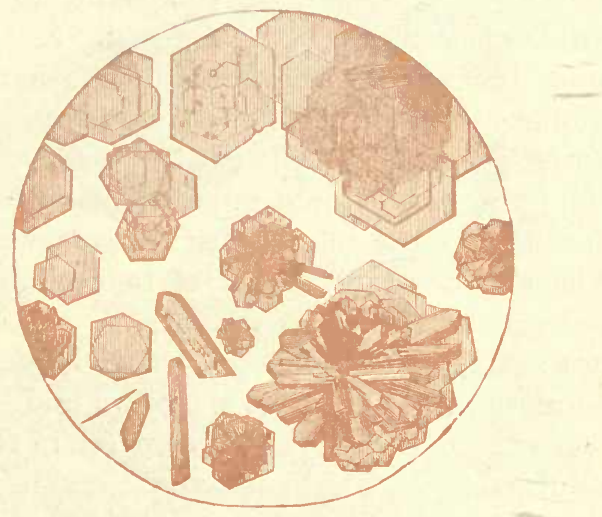

Fig. 137.-Hexagonal oxy-hæmoglobin crystals, from blood of squirrel. On these hexagonal plates prismatic crystals grouped in a stellate manner not unfrequently occur (after Funke).

bin solution) red. The deoxidized crystals or their solutions quickly absorb oxygen on exposure to the air, becoming scarlet. If solutions of blood be taken instead of solutions of hæmoglobin, results similar to the whole of the foregoing can be obtained.

Venous blood never, except in the last stages of asphyxia, fails to show the oxy-hæmoglobin bands, inasmuch as the greater part of the hæmoglobin even in renous blood exists in the more highly oxidized condition.

Action of Gases on Hæmoglobin.-Carbonic oxide gas, passed through a solution of hæmoglobin, causes it to assume a cherry-red color,

* Stokes's Fluid consists of a solution of ferrous sulphate, to which ammonia has been added and sufficient tartaric acid to prevent precipitation. Another reducing agent is a solution of stannous chloride, treated in a way similar to the ferrous sulphate, and a third reagent of like nature is an aqueous solution of yellow ammonium sulphide, $\mathrm{NH}_{4} \mathrm{HS}$. 
and to present a slightly altered spectrum; two bands are still visible, but are slightly nearer the blue end than those of oxy-hæmoglobin (see plate). The amount of carbonic oxide taken up is equal to the amount of the oxygen displaced. Although the carbonic oxide gas readily displaces oxygen, the reverse is not the case, and upon this property depends the dangerous effect of coal-gas poisoning. Coal gas contains much carbonic oxide, and when breathed, the gas combines with the hæmoglobin of the blood, and produces a compound which cannot easily be reduced. This compound (carb-oxy-hæmoglobin) is by no means an oxygen carrier, and death may result from suffocation due to the want of oxygen notwithstanding the free entry of pure air into the lungs. Crystals of carbonic-oxide hæmoglobin closely resemble those of oxyhæmoglobin.

Nitric oxide produces a similar compound to the carbonic-oxide hæmoglobin, which is even less easily reduced.

Nitrous oxide reduces oxy-hæmoglobin, and therefore leaves the reduced hæmoglobin in a condition to actively take up oxygen.

Sulphuretted Hydrogen.-If this gas be passed through a solution of oxy-hæmoglobin, the hæmoglobin is reduced and an additional band appears in the red. If the solution be then shaken with air, the two bands of oxy-hæmoglobin replace that of reduced hæmoglobin, but the band in the red persists.

Methæmoglobin.-If an aqueous solution of oxy-hæmoglobin is exposed to the air for some time, its spectrum undergoes a change; the two $\mathrm{D}$ and $\mathrm{E}$ bands become faint, and a new line in the red at $\mathrm{C}$ is developed. The solution, too, becomes brown and acid in reaction, and is precipitable by basic lead acetate. This change is due to the decomposition of oxy-hæmoglobin, and to the production of methcemoglobin. On adding ammonium sulphide, reduced hæmoglobin is produced, and on shaking this up with air, oxy-hæmoglobin is reproduced. Methæmoglobin is probably a stage in the deoxidation of oxy-hæmoglobin. It appears to contain less oxygen than oxy-hæmoglobin, but more than reduced hæmoglobin. Its oxygen is in more stable combination, however, than is the case with the former compound.

Estimation of Hæmoglobin.- The most exact method is by the estimation of the amount of iron (dry hæmoglobin containing .42 per cent of iron) in a given specimen of blood, but as this is a somewhat complicated process, various methods have been proposed which, though not so exact, have the advantage of simplicity. In Gower's hæmoglobinometer, this consists in comparing the color of a given small amount of diluted blood with glycerine jelly tinted with carmine and picro-carmine to represent a standard solution of blood diluted one hundred times. The amount of dilution which the given blood requires will thus approximately represent the quantity of hæmoglobin it contains. 
But of the several varieties of hæmoglobinometer that which appears to be the best adapted to its purpose is that invented by Professor Fleischl, of Vienna. In this instrument, the anount of hæmoglobin in a solution of blood is estimated by comparing a stratum of diluted blood with a standard solid substance of uniform tint similar spectroscopically to diluted blood. In order that the strength of color in the standard substance may be varied, the red tinted glass is made wedge-shaped. This, which is called the comparison wedge, is cemented on to a colorless plain strip of glass, and is mounted in a frame (fig. 138, P) made to slide in a V-shaped groove, on the under surface of the stage of the instrument. The comparison wedge, $K$, is so placed that one of its longi-

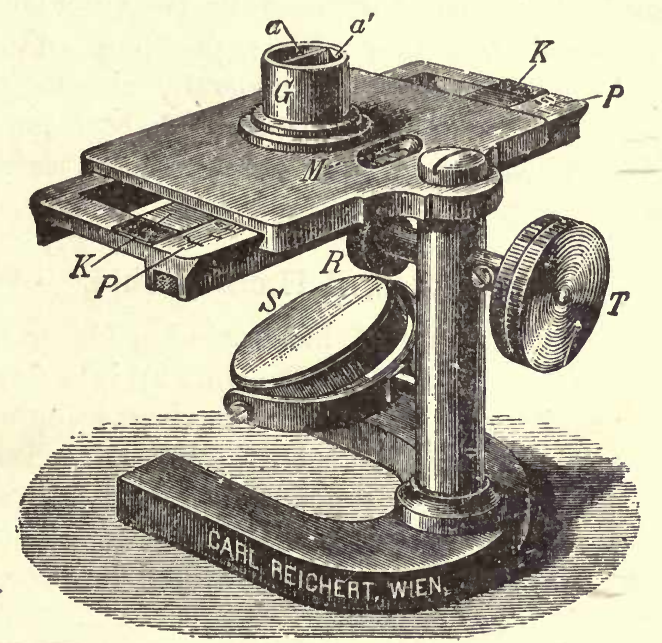

Fig 138.-Fleischl's Hæmoglobinometer.

tudinal edges bisects the circular stage-opening, so that one-half of the latter is cut off by the red-tinted wedge. Into the stage-opening fits a small circular trough, $G$, having a glass bottom, and divided into equal compartments by a thin lamina. One compartment, $a$, is filled in the manner to be presently indicated with diluted blood, and the other, $a^{\prime}$, with water; the trough is so placed that the lamina is in one plane with the edge of the wedge, the water compartment being above the wedge and the blood compartment above the free half of the stage opening. By turning the screw head, $T$, the frame, $P$, with the wedge, $K$, may be moved backward and forward until a position is found where the intensity of the tints due to the stratum of blood on the one hand and the thickness of the wedge on the other appears to be equal. The required degree of dilution is obtained by the use of small capillary tubes of a capacity varying from 6 to $8 \mathrm{cmm}$. The capillary pipette is filled with blood and is held over the blood compartment and its contents thor- 
oughly washed out into that compartment, and the blood and water are mixed with a wire. Water is then added until the blood compartment is quite full. The other compartment is filled with water. Light is then reflected by the mirror, $S$, so as to illuminate both compartments. By moving $K$ by means of the milled head, $T$, a position of $K$ may be found corresponding to the exact intensity of the light passing through the two compartments; this is read off at $M$ on the scale $P$, the division of which corresponds to standard strengths of solutions of hæmoglobin.

Distribution of Hæmoglobin.-Hæmoglobin occurs not only in the red blood-cells of all vertebrata (except amphioxus and leptocephalus whose blood-cells are all colorless,) but also in similar cells in many Worms; moreover, it is found diffused in the vascular fluid of some other worms and certain Crustacea; it also occurs in all the striated muscles of Mammals and Birds. It is generally absert from unstriated muscle except that of the rectum. It has also been found in Mollusca in eertain muscles which are specially active, viz., those which work the rasp-like tongue.

\section{Derivatives of Hæmoglobin.}

Hæmatin.-By the action of heat, or of acids or alkalies in the presence of oxygen, hæmogìbin can be split up into a substance called Homatin, which contains all the iron of the hæmoglobin from which it was derived, and a proteid residue. Of the latter it is impossible to say more than that it probably consists of one or more bodies of the globulin class. If there be no oxygen present, instead of hæmatin a body called hæmochromogen is produced, which, however, will speedily undergo oxidation into hæmatin.

Hæmatin is a dark brownish or black non-crystallizable substance of metallic lustre. Its percentage composition is C. $64.30 ; \mathrm{H} .5 .50 ; \mathrm{N}$. 9.06 ; Fe. 8.82; 0. 12.32; which gives the formula $\mathrm{C}_{68}, \mathrm{H}_{70}, \mathrm{~N}_{8}, \mathrm{Fe}_{2}$, $\mathrm{O}_{10}$ (Hoppe-Seyler). It is insoluble in water, alcohol, and ether; soluble in the caustic alkalies; soluble with difficulty in hot alcohol to which is added sulphuric acid. The iron may be removed from hæmatin by heating it with fuming hydrochloric acid to $160^{\circ} \mathrm{C}$. $\left(320^{\circ} \mathrm{F}\right.$.), and a new body, hæmatoporphyrin, the so-called iron-free hæmatin, is produced. Hæmatoporphyrin $\left(\mathrm{C}_{68}, \mathrm{H}_{74}, \mathrm{~N}_{8}, \mathrm{O}_{12}\right.$, Hoppe-Seyler $)$ may also be obtained by adding blood to strong sulphuric acid, and if necessary filtering the fluid through asbestos. It forms a fine crimson solution, which has a distinet spectrum, viz., a dark band just beyond $\mathrm{D}$, and a second all but midway $\mathrm{D}$ and $\mathrm{E}$. It may be precipitated from its acid solution by adding water or by neutralization, and when redissolved in alkalies presents four bands, a pale band between $\mathrm{C}$ and $\mathrm{D}$, a second between $\mathrm{D}$ and $\mathrm{E}$, nearer $\mathrm{D}$, another nearer $\mathrm{E}$, and a fourth occupying the chief part of the space between $b$ and $\mathrm{F}$. 
Homatin in acid solution.-If an excess of acetic acid is added to blood, and the solution is boiled, the color alters to brown from decomposition of hæmoglobin and the setting free of hæmatin; by shaking this solution with ether, a solution of hæmatin in acid solution is obtained. The spectrum of the ethereal solution (colored plate) shows no less than four absorption bands, viz., one in the red between $\mathrm{C}$ and $\mathrm{D}$, one faint and narrow close to $\mathrm{D}$ and then two broader bands, one between $\mathrm{D}$ and $\mathrm{E}$, and another nearly midway between $b$ and $\mathrm{F}$. The first band is by far the most distinct, and the acid aqueous solution of hæmatin shows it plainly.

Homatin in alkaline solution.-If a caustic alkali is added to blood and the solution is boiled, alkaline hæmatin is produced, and the solution becomes olive green in color. The absorption band of the new compound is in the red, near to $\mathrm{D}$, and the blue end of the spectrum is

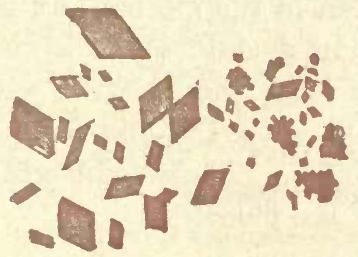

Fig. 139.-Hæmatoidin crystals. (Frey.)

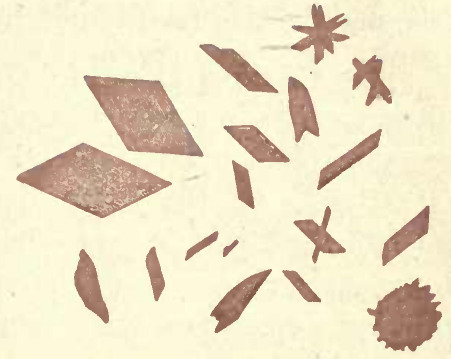

Fig. 140.-Hæmin crystals. (Frey.)

absorbed to a considerable extent. If a reducing agent be added, two bands resembling those of oxy-hæmoglobin, but nearer to the blue, appear; this is the spectrum of reduced hcematin, or hæmochromogen. On violently shaking the reduced hæmatin with air or oxygen the two bands are replaced by the single band of alkaline hæmatin.

Hæmatoidin.-This substance is found in the form of yellowish crystals (fig. 139) in old blood extravasations and is derived from the hæmoglobin. Their crystalline form and the reaction they give with fuming nitric acid seem to show them to be closely allied to Bilirubin, the chief coloring matter of the bile, and in composition they are probably either identical or isomeric with it.

Hæmin.-One of the most important derivatives of hæmatin is hæmin. It is usually called Hydrochlorate of Homatin (or hydrochloride), but its exact chemical composition is uncertain. Its formula is said to be $\mathrm{C}_{32} \mathrm{H}_{30} \mathrm{~N}_{4} \mathrm{FeO}_{3} \mathrm{HCl}$, and it contains 5.18 per cent of chlorine, but by some it is looked upon as simply crystallized hæmatin. Although difficult to obtain in bulk, a specimen may be easily made for the microscope in the following way:-A small drop of dried blood is finely powdered with a few crystals of common salt on a glass slide and 
spread out; a cover glass is then placed upon it, and glacial acetic acid added by means of a capillary pipette. The blood at once turns of a brownish color. The slide is then heated, and the acid mixture evaporated to dryness at a high temperature. The excess of salt is washed away with water from the dried residue, and the specimen may then be dried and mounted. A large number of small, dark, reddish black erystals of a rhombic shape, sometimes arranged in bundles, will be seen if the slide be subjected to microscopic examination (fig. 140).

The formation of these hæmin crystals is of great interest and importance from a medico-legal point of view, as it constitutes the most certain and delicate test we have for the presence of blood (not of necessity the blood of man) in a stain on clothes, etc. It exceeds in delicacy even the spectroscopic test. Compounds similar in composition to hæmin, but containing hydrobromic or hydriodic acid, instead of hydrochloric, may be also readily obtained.

B. The Carbon Dioxide Gas in the Blood.-Of this gas in the blood part exists in a state of simple solution in the plasma, and is given up in vacuo (35.2 per cent), and the rest in a state of weak chemical combination. Of the latter, part is in loose combination with the hæmoglobin and part is more firmly united with the alkalies, possibly with the carbonates in the form of bicarbonate. The amount which can be absorbed depends on the alkalescence of the blood.

C. The Nitrogen in the Blood.-The whole of the small quantity of the nitrogen contained in the blood is simply dissolved in the fluid plasma.

Chemical Composition of the Blood in Bulk.-Analyses of the blood as a whole differ slightly, but the following table may be taken to represent the average composition:

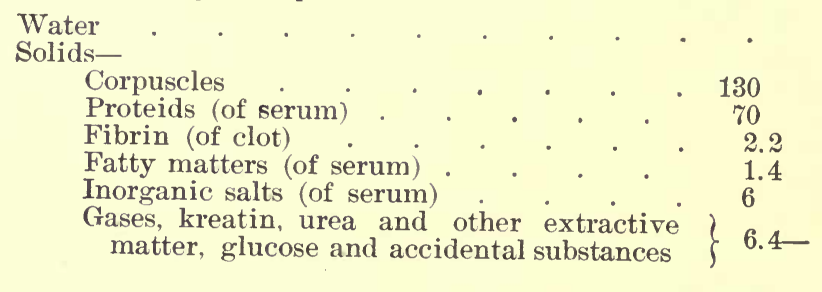

784

\section{Variations in the Composition of healthy Blood.}

The conditions which appear most to influence the composition of the blood in health are these: Sex, Pregnancy, Age, and Temperament. The composition of the blood is also, of course, much influenced by diet.

1. Sex.-The blood of men differs from that of women, chiefly in. 
being of somewhat higher specific gravity, from its containing a relatively larger quantity of red corpuscles.

2. Pregnancy. - The blood of pregnant women is rather lower than the average specific gravity. The quantity of the colorless corpuscles is increased in the latter months, especially in primiparæ; it is also claimed that the fibrin is increased in amount.

3. Age.-The blood of the foetus is very rich in solid matter, and especially in colored corpuscles; and this condition, gradually diminishing, continues for some weeks after birth. The quantity of solid matter then falls during childhood below the average, rises during adult life, and in old age falls again.

4. Temperament. - There appears to be a relatively large quantity of solid matter in those of a plethoric or sanguineous temperament.

5. Diet.-Such differences in the composition of the blood as are due to the temporary presence of various matters absorbed with the food and drink, as well as the more lasting changes which must result from generous or poor diet respectively, need be here only referred to.

6. Effects of Bleeding. - The result of bleeding is to diminish the specific gravity of the blood; and so quickly, that in a single venesection, the portion of blood last drawn has often a less specific gravity than that of the blood that flowed first. This is, of course, due to absorption of fluid from the tissues of the body. The physiological import of this fact, namely, the instant absorption of liquid from the tissues, is the same as that of the intense thirst which is so common after either loss of blood, or the abstraction from it of watery fluid, as in cholera, diabetes, and the like.

For some little time after bleeding the want of colored corpuscles is well marked, but with this exception, no considerable alteration seems to be produced in the composition of the blood for more than a very short time; the loss of the other constituents, including the colorless corpuscles, being very quickly repaired.

Variations in different parts of the Body.-The composition of the blood, as might be expected, is found to vary in different parts of the body. Thus arterial blood differs from venous; and although its composition and general characters are uniform throughout the whole course of the systemic arteries, they are not so throughout the venous system - the blood contained in some veins differing remarkably from that in others.

Differences between Arterial and Venous Blood.-The differences between arterial and venous blood are these:-

(a.) Arterial blood is bright red, from the fact that almost all its hæmoglobin is combined with oyxgen (Oxy-hæmoglobin, or scarlet hæmoglobin), while the purple tint of venous blood is due to the decxida- 
tion of a certain quantity of its oxy-hæmoglobin, and its consequent reduction to the purple variety (Deoxidized, or purple hæmoglobin).

(b.) Arterial blood coagulates somewhat more quickly.

(c.) Arterial blood contains more oxygen than venous, and less carbonic acid.

Some of the veins contain blood which differs from the ordinary standard considerably. These are the Portal, the Hepatic, and the Splenic veins.

Portal vein. - The blood which the portal rein conveys to the liver is supplied from two chief sources; namely, from the gastric and mesenteric veins, which contain the soluble elements of food absorbed from the stomach and intestines during digestion, and from the splenic vein; it must, therefore, combine the qualities of the blood from each of these sources.

The blood in the gastric and mesenteric veins will vary much according to the stage of digestion and the nature of the food taken, and can therefore be seldom exactly the same. Speaking generally, and without considering the sugar, and other soluble matters which may have been absorbed from the alimentary canal, this blood appears to be deficient in solid matters, especially in colored corpuscles, owing to dilution by the quantity of water absorbed, to contain an excess of proteid matter, and to yield a less tenacious kind of fibrin than that of blood generally.

The blood from the splenic vein is generally deficient in colored corpuscles, and contains an unusually large proportion of proteids. The fibrin obtainable from the blood seems to vary in relative amount, but to be almost always above the average. The proportion of colorless corpuscles is also unusually large. The whole quantity of solid matter is decreased, the diminution appearing to be of colored corpuscles. The plasma is said to be colored in consequence of its containing dissolved hæmatin.

The blood of the portal vein, combining the peculiarities of its two factors, the splenic and mesenteric venous blood, is usually of lower specific gravity than blood generally, is more watery, contains fewer colored corpuscles, more proteids, and yields a less firm clot than that yielded by other blood, owing to the deficient tenacity of its fibrin.

Guarding (by ligature of the portal vein) against the possibility of an error in the analysis from regurgitation of hepatic blood into the portal vein, recent observers have determined that hepatic venous blood contains less water, proteids, and salts than the blood of the portal veins; but that it yields a much larger amount of extractive matter, in which is one constant element, namely, grape-sugar, which is found, whether saccharine or farinaceous matter has been present in the food or not. 


\section{Development of the Blood-Corpuscles.}

The first formed blood-corpuscles of the human embryo differ much in their general characters from those which belong to the later periods of intra-uterine, and to all periods of extra-nterine life. Their manner of origin is at first very simple.

Surrounding the early embryo is a sircular area, called the vascular area, in which the first rudiments of the blood-vessels and blood-corpuscles are developed. Here the nucleated embryonal cells of the mesoblast, from which the blood-vessels and corpuscles are to be formed, send out processes in various directions, and these joining together, form an irregular meshwork. The nuclei increase in number, and collect chiefly in the larger masses of protoplasm, but partly also in the

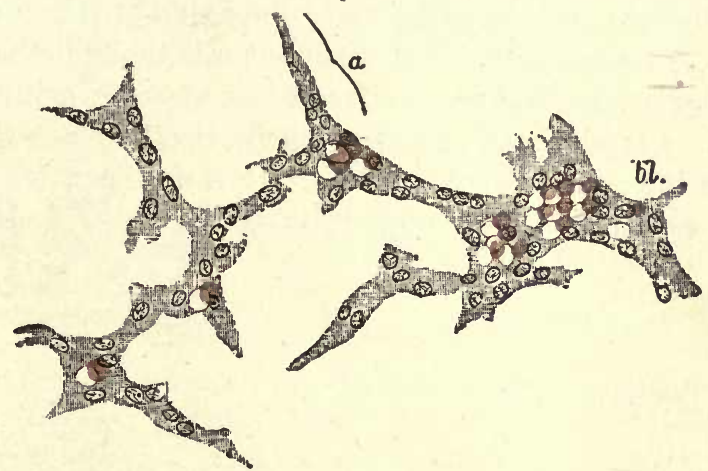

Fig. 141.-Part of the network of developing blood-vessels in the vascular area of a guinea-pig. $b l$, blood-corpuscles becoming free in an enlarged and hollowed-out part of the network; $a$, process
of protoplasm. (E. A. Schäfer.)

processes. It appears that hæmoglobin then makes its appearance in certain of these nucleated embryonal cells, which thus become the earliest red blood-corpuscles. The protoplasm of the cells and their branched net-work in which these corpuscles lie then become hollowed out into a system of canals inclosing fluid, in which the red nucleated corpuscles float. The corpuscles at first are from about $\frac{1}{2500}$ to $\frac{1}{1500}$ of an inch $(10 \mu$ to $16 \mu)$ in diameter, mostly spherical, and with granular contents, and a well-marked nucleus. Their nuclei, which are about $\frac{1}{500} \overline{0}$ of an inch $(5 \mu)$ in diameter, are central, circular, very little prominent on the surfaces of the corpuscles, and apparently slightly granular or tuberculated.

The corpuscles then strongly resemble the colorless corpuscles of the fully developed blood, but are colored. They are capable of amœboid movement and multiply by division.

When, in the progress of embryonic development, the liver begins to be formed, the multiplication of blood-cells in the whole mass of blood ceases, and new blood-cells are produced by this organ, and also by the 
spleen. These are at first colorless and nucleated, but afterward acquire the ordinary blood-tinge, and resemble very much those of the first set. 'They also multiply by division. About this time the bone marrow also begins to form red corpuscles, though at first in small amounts only. This function develops rapidly, however, so that at birth the marrow represents the chief seat of production of the red cells. Nevertheless, nucleated red cells are usually found at birth, sometimes in considerable quantities, in the liver, and, less often, the spleen. Nonnucleated red cells begin to appear soon after the first month of foetal life, and gradually increase, so that at the fourth month they form onefourth of the whole amount of colored corpuscles; at the end of foetal life they almost completely replace the nucleated cells. In late fotal life the red cells are formed in almost the same way as in extra-uterine life.

Various theories have prevailed as to the mode of origin of the nonnucleated colored corpuscles. For a time it was thought that they were of endoglobular origin, and merely fragments of some original cell, being produced by subdivision of the cell body itself. This theory easily accounted for the absence of the nuclei, but it has not been supported by recent investigations. At present it is the general belief that the

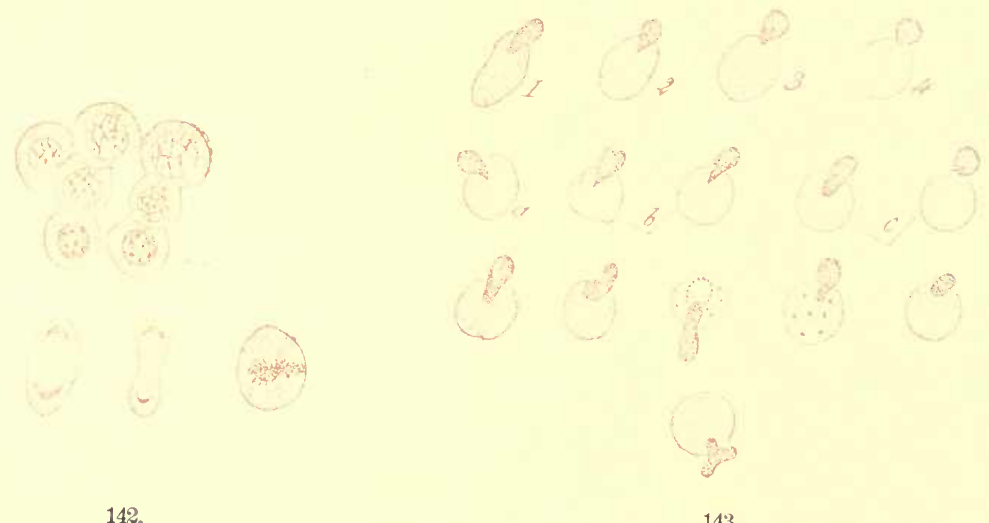

142.

143.

Fig. 14:-Multiplication of the nucleated red corpuscles. Marrow of young kitten after bleeding, showing above karyokinetic division of erythroblast, and below the formation of mature from immature erythrocysts. (Adapted from Howell.)

Fig. 143. Shows the way in which the nucleus escapes from the nucleated red corpuscles. Specimen from the circulating blood of the extrusion noticed upon the living corpuscles. $a$, culating blood of a kitten forty days old, bled twice: $c$, specimens from the blood of a fœetal cat, $9 \mathrm{~cm}$. long. Others from the marrow of an adult cat, $t$ wo of the figures showing the granules present in the corpuscles which have been interpreted erroneousiy as a sign of the disintegration
of the nucleus. (After Howell.)

non-nucleated cells are derived from nucleated cells by a process of mitotic division, and further that their nuclei gradually shrink or fade and are then extruded. Extrusion undoubtedly occurs with great frequency, but the use of some of the more recent stains seems to prove that there 
are traces of nuclear material in the non-nucleated corpuscles. Owing to these facts and other recent investigations, Maximow asserts that while the greater part of the nucleus is extruded, still a small portion usually remains in a finely granular form and gives a basic staining quality to the centres, especially of the young red cells.

Origin of the Mature Colored Corpuscles.-It has already been shown that during uterine life the marrow gradually assumes more and more completely the function of forming red cells. This function prevails after birth, and most authorities now regard the red marrow as the exclusive seat, under normal conditions, of the production of red corpuscles. The original cell, or erythroblast, is generally considered to be a large colorless cell which is devoid of hæmoglobin, is larger than the ordinary red cell, and has a single nucleus but no nucleolus; it differs but very slightly from the original mesoblastic cell.

By mitotic division of these original cells there are derived several series of cells which approach, more and more completely, the type of. nucleated red corpuscles, becoming rich in hæmoglobin. The nucleus

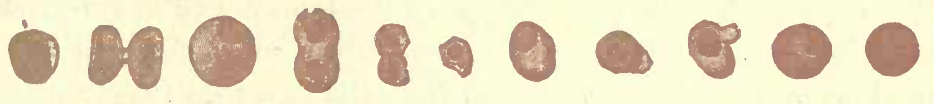

Fig. 144.-Colored nucleated corpuscles, from the red marrow of the guinea-pig. (E. A. Schäfer.)

is then extruded (or partly extruded and partly broken up) and the normal non-nucleated red corpuscle results. A few authorities, however, in tracing the red cells back to colorless cells, think that all the lymphoid tissues are also probable sources of the erythroblasts. In infancy and early childhood the red marrow, which produces the colored corpuscles, is found in large amount in the cavities of almost all the bones. In adult life it is normally confined to the ribs, flat bones, vertebræ, and upper and lower thirds of the long bones. In pathological conditions it has been found that the spleen in the adult, or both the spleen and the liver in infancy and early childhood, can resume the function of producing red corpuscles.

Without doubt, the red corpuscles have, like all other parts of the organism, a tolerably definite term of existence, and in a like manner die and waste away when the portion of work allotted to them has been performed. Neither the length of their life, however, nor the fashion of their decay has been yet clearly made out. It is generally believed that a certain number of the colored corpuscles undergo disintegration in the spleen; and indeed corpuscles in various degrees of degeneration have been observed in that organ.

Origin of the Colorless Corpuscles.-In fœtal life the white corpuscles are not found in the blood until the vascular system has been 
very extensively developed, long after the appearance of red cells; the exact time of their appearance, howerer, has not yet been fully determined. It is now quite generally believed that in the fortus both red and white cells are derived from a common origin, and that they become differentiated in the course of development. 'The earliest known progenitors of the leucocytes are the primary wandering cells of mesodermal origin, which are found chiefly in the connective tissues, thuslying ontside of the vessels. Gathering in groups, partly at the sites of the future lymph nodes, but chiefly in the embryonal liver, these wandering cells pass through several generations of mitotic division, and thus gradually assume the type of lencocytes. According to a few observers the leucocytes are also formed in the circulating blood and lymph by amitotic, less frequently by mitotic, division. Later on in foetal life the function of forming lencocytes is gradually transferred from the liver to the lymphoid and adenoid tissues, i.e., the lymph norles, spleen, marrow, and thymus.

In adult life, under normal conditions, the leucocytes are only formed in the lymphoid tissues, including the lymph nodes, spleen and marrow. The process is also one of mitotic division (a few authorities claim that it is amitotic), and the resulting cells pass into the circulation by way of the thoracic duct.

\section{Uses of the Blood.}

1. To be a medium for the reception and storing of matter, e.g., oxygen and digested food material, from the outer world, and for its conveyance to all parts of the body.

2. To be a source whence the various tissues of the body may take the materials necessary for their nutrition and maintenance; and whence the secreting organs may take the constituents of their various secretions.

3. To be a medium for the absorption of refuse matters from all the tissues, and for their conveyance to those organs whose function it is te separate them and cast them out of the body.

4. To warm and moisten all parts of the body. 


\section{CHAPTER VI.}

\section{THE CIRCULATION OF THE BLOOD.}

The blood is made to circulate within the system of closed tubes in which it is contained by means of the alternate contraction and relaxation of the heart. The heart is a hollow muscular organ consisting of four chambers, two auricles and two ventricles, arranged in pairs. On

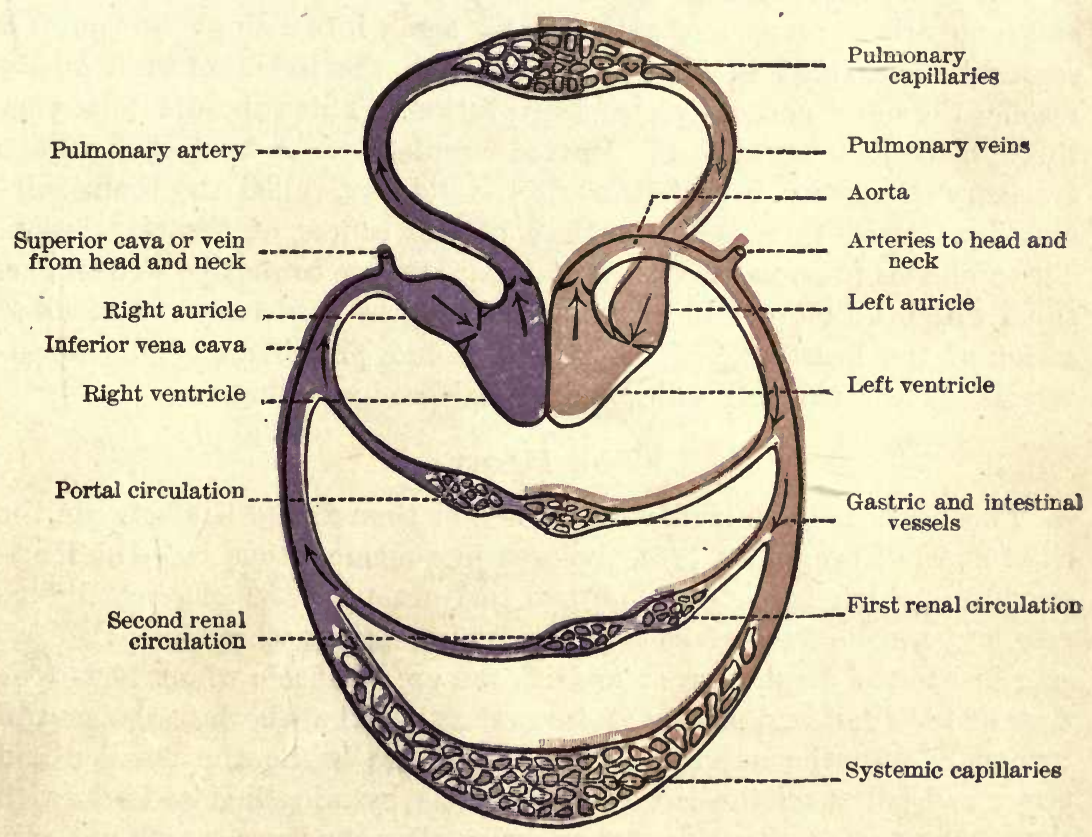

Fig. 145.-Diagram of the circulation.

the right and left sides is an auricle joined to and communicating with a ventricle, but the chambers on the right side do not directly communicate with those on the left side. The blood is conveyed away from the left side of the heart (as in the diagram, fig. 145) by the arteries, and returned to the right side of the heart by the veins, the arteries and veins being continuous with each other at one end by means of the heart, and at the other by a fine network of vessels called the capillaries.

From the right side of the heart the blood passes to the lungs 
through the pulmonary artery, then through the pulmonary capillaries, and through the pulmonary veins to the left side of the heart (Fig. 145). Thus there are two circulations through which the blood must pass; the one, a shorter circuit from the right side of the heart to the lungs and back again to the left side of the heart; the other and larger circuit, from the left side of the heart to all parts of the body and back again to the right side; strictly speaking, however, there is but one complete circulation, which may be diagrammatically represented by a double loop, as in fig. 145, in which there is one continuous stream, the whole of which must, at one part of its course, pass through the lungs. Subordinate to the circulations through the lungs and through the system generally, respectively named the Pulmonary and Systemic, it will be noticed also in the same figure that a portion of the stream of blood having been diverted once into the capillaries of the intestinal canal, and some other organs, and gathered up again into a single stream, is a second time divided in its passage through the liver, before it finally reaches the heart and completes a revolution. This subordinate stream through the liver is called the Portal circulation. A somewhat similar accessory circulation is that through the kidneys, called the Renal circulation. Such then is the outline of the course of the circulation. The problems in connection with its maintenance cannot be well understood withont a more detailed knowledge of the structure and mode of action of the heart, and of the structure and properties of the bloodressels. These subjects will now be considered seriatim.

\section{The Heart.}

The heart is contained in the chest or thorax, and lies between the right and left lungs (fig. 146), inclosed in a membranous sac-the Pericardium, which is made up of two distinct parts, an external fibrous membrane, composed of closely interlacing fibres, which has its base attached to the diaphragm or midriff, the great muscle which forms the floor of the chest and divides it from the abdomen-both to the central tendon and to the adjoining muscular fibres, while the smaller and upper end is lost on the large blood-vessels by mingling its fibres with that of their external coats; and an internal serous layer, which not only lines the fibrous sac, but also is reflected on to the heart, which it completely invests. The part which lines the fibrous membrane is called the parietal layer, and that inclosing the heart, the visceral layer or eptcardium, and these being continuous for a short distance along the great vessels of the base of the heart, form a closed sac, the cavity of which in health contains just enough fluid to lubricate the two surfaces, and thus to enable them to glide smoothly over each other during the movements of the heart. The vessels passing in and out of the heart receive investments from this sac to a greater or less degree. 
The heart is situated in the chest behind the sternum and costal cartilages, being placed obliquely from right to left, quite two-thirds of it being to the left of the mid-sternal line. It is of pyramidal shape, with the apex pointing downward, outward, and toward the left, and the base backward, inward, and toward the right. It rests upon the diaphragm, and its pointed apex, formed exclusively of the left side of the heart, is in contact with the chest wall, and during life beats against it at a point called the apex beat, sitnated in the fifth left intercostal space, and about three inches from the mid-sternal line. The heart is, as it were, suspended in the chest by the large vessels which proceed from its base, but, excepting at this part, the organ itself lies free within the sac of the pericardium. The part which rests upon the diaphragm

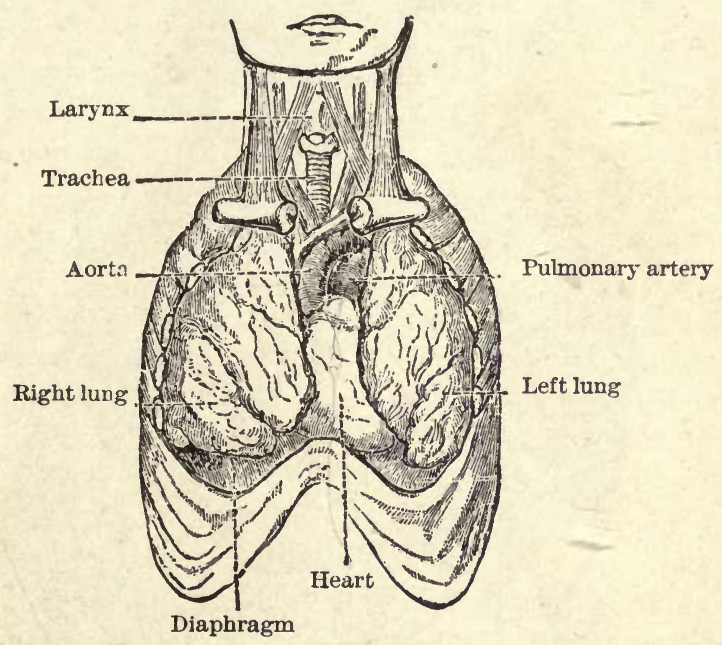

Fig. 146.-View of heart and lungs in situ. The front portion of the chest-wall, and the outer or parietal layers of the pleuræ and pericardium have been removed. The lungs are partly collapsed.

is flattened, and is known as the posterior surface, while the free upper part is called the anterior surface. The margin toward the left is thick and obtuse, while the lower margin toward the right is thin and acute.

On examination of the external surface the division of the heart into parts which correspond to the chambers inside of it may be traced, for a deep transverse groove called the auriculo-ventricular groove divides the auricles which form the base of the heart from the ventricles which form the remainder, including the apex, the ventricular portion being by far the greater; and, again, the inter-ventricular groove runs between the ventricles both front and back, and separates the one from the other. The anterior groove is nearer the left margin and the posterior nearer the right, as the front surface of the heart is made up chiefly of the right ventricle and the posterior surface of the left ventricle. In the 
furrows or grooves run the coronary vessels, which supply the tissue of the heart with blood, as well as nerves and lymphatics imbedded in more or less fatty material.

The Chambers of the Heart.-The interior of the heart is divided by a longitudinal partition in such a manner as to form two chief cham. bers or cavities-right and left. Each of these chambers is again sub-

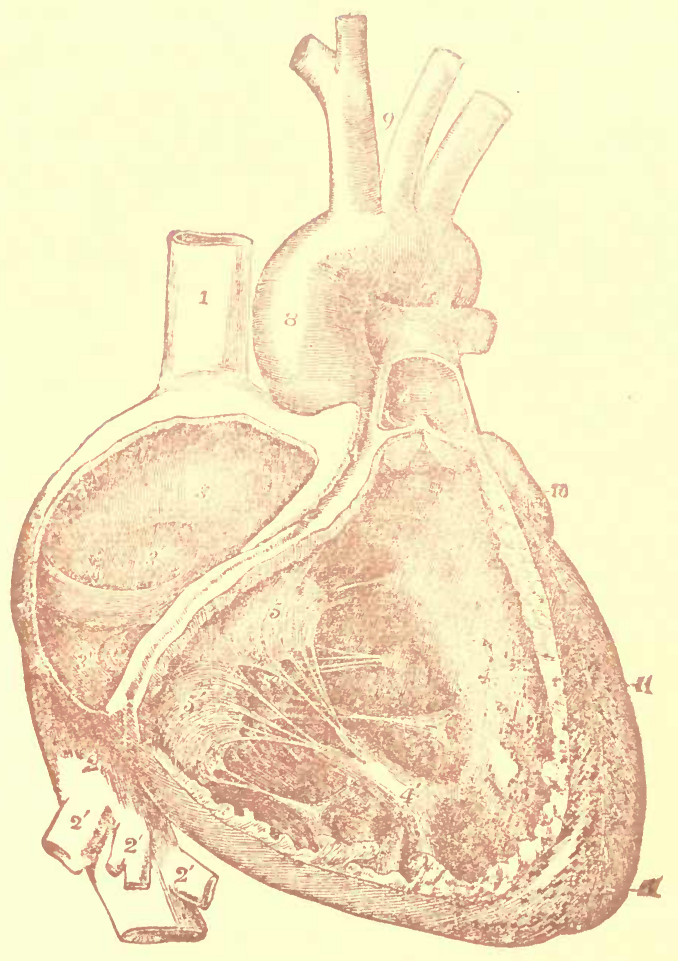

Fig. 147. - The right auricle and ventricle opened, and a part of their right and anterior walls removed, so as to show their interior. $1 / 2 .-1$, Superior vena cava ; 2 , inferior vena cava ; $2^{\prime}$, hepatic veins cut short; 3 , right auricle ; $3^{\prime}$, placed in the fossa ovalis, below which is the Eustachian valve ; $3^{\circ}$, is placed close to the aperture of the coronary vein;,,++ placed in the auriculo-ventricular groove, where a narrow portion of the adjacent walls of the auricle and ventricle has been preserved; 4,4 , cavity of the right ventricle, the upper figure is immediately below the semilunar valves; $4^{\prime}$, large columna carnea or musculus papillaris $; 5,5^{\prime}, 5^{\circ}$, tricuspid valve ; 6 , placed in the interior of the pulmonary artery, a part of the anterior wall of that vessel having been removed, and a narrow portion of it preserved at its commencement, where the semilunar valves are attached; 7 , concavity of the aortic arch close to the cord of the ductus arteriosus ; 8 , ascending part or sinus of the arch covered at its commencement by the auricular appendix and pulmonary artery ; 9 . placed between the iunominate and left carotid arteries ; 10 , appendix of the left auricle; 11 , 11, outside of the left ventricle, the lower figure near the apex. (Allen Thomson.)

divided transrersely into an upper and a lower portion, called respectively, as already incidentally mentioned, auricle and ventricle, which freely communicate one with the other; the aperture of communication, however, is guarded by valves, so disposed as to allow blood to pass freely from the auricle into the ventricle, but not in the opposite direc- 
tion. There are thus four cavities in the heart-the auricle and ventricle of one side being quite separate from those of the other (fig. 14\%).

Right Auricle.-The right auricle is situated at the right part of the base of the heart as viewed from the front. It is a thin-walled cavity of more or less quadrilateral shape, prolonged at one corner into a

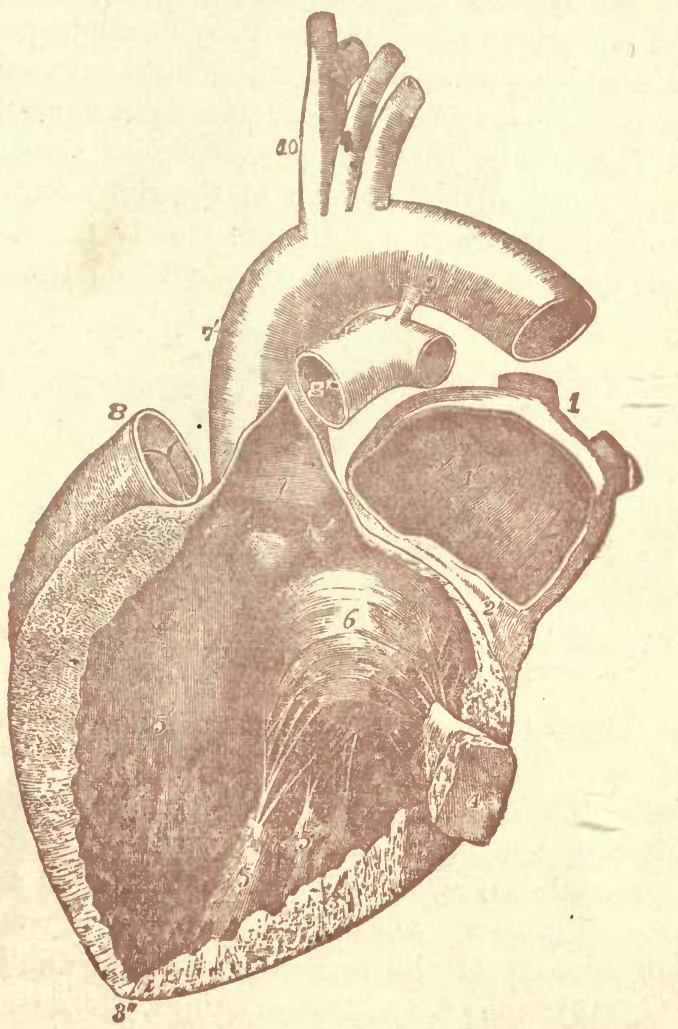

Fig. 148.-The left auricle and ventricle opened and a part of their anterior and left walls removed. 1/2-The pulmonary artery has been divided at its commencement; the opening into the left ventricle is carried a short distance into the aorta between two of the segments of the semilunar valves; and the left part of the auricle with its appendix has been removed. The right auricle is out of view. 1, The two right pulmonary veins cut short; their openings are seen within the auricle; $1^{\prime}$, placed within the cavity of the auricle on the left side of the septum and on the part which forms the remains of the valve of the foramen ovale, of which the crescentic fold is seen toward the left hand of $1^{\prime} ; 2$, a narrow portion of the wall of the auricle and ventricle preserved round the auriculoventricular orifice; $3,3^{\prime}$, the cut surface of the walls of the ventricle, seen to become very much thinner towards $3^{n}$, at the apex ; 4, a small part of the anterior wall of the left ventricle which has been preserved with the principal anterior columna carnea or musculus papillaris attached to it ; 5,5 , musculi papillares ; 5 ', the left side of the septum, between the two ventricles, within the cavity of the left ventricle; $6,6^{\prime}$, the mitral valve; 7 , placed in the interior of the aorta near its commencement and above the three segments of its semilunar valve which are hanging loosely together ; $7^{\prime}$, the exterior of the great dortic sinus ; 8 , the root of the pulmonary artery and its semilunar valves: $8^{\prime}$, the separated portion of the pulmonary artery remaining attached to the aorta by 9 , the cord of the ductus arteriosus ; 10, the arteries rising from the summit of the aortic arch. (Allen Thomsen.)

tongue-shaped portion, the right auricular appendix, which slightly overlaps the exit of the great artery, the aorta, from the heart.

'The interior is smooth, being lined with the general lining 'of the 
heart, the endocardium, and into it open the superior and inferior venæ cavæ, or great veins, which convey the blood from all parts of the body to the heart. The former is directed downward and forward, the latter upward and inward; between the entrances of these vessels is a slight tubercle called tubercle of Lower. The opening of the inferior cava is protected and partly covered by a membrane called the Eustachian valve. In the posterior wall of the auricle is a slight depression called the fossa oralis, which corresponds to an opening between the right and left auricles which exists in fotal life. The right auricular appendix is of oval form, and admits three fingers. Various veins, including the coronary sinus, or the dilated portion of the right coronary vein, open into this chamber. In the appendix are closely set elevations of the muscular tissue corered with endocardium, and on the anterior wall of

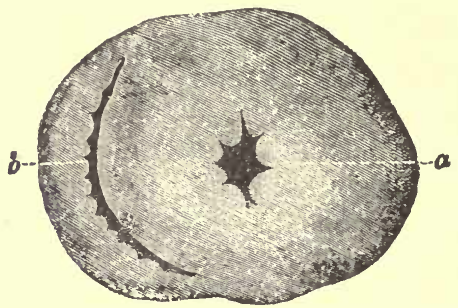

Fig. 149.-Transverse section of bullock's heart in a state of cadaveric rigidity. (Dalton.) $b$, Cavity of right ventricle. $a$, Cavity of left ventricle.

the auricle are similar elevations arranged parallel to one another, called musculi pectinati.

Right Ventricle.-The right ventricle occupies the chief part of the anterior surface of the heart, as well as a small part of the posterior surface: it forms the right margin of the heart. It takes no part in the formation of the apex. On section its cavity, in consequence of the encroachinent upon it of the septum ventriculorum, is semilunar or crescentic (fig. 149); into it are two openings, the auriculo-ventricular at the base and the opening of the pulmonary artery also at the base, but more to the left; the part of the ventricle leading to it is called the conus arteriosus or infundibulum; both orifices are guarded by valves, the former called tricuspid and the latter semilunar or sigmoid. In this ventricle are also the projections of the muscular tissue called colunnce carnece (described at length p. 179).

Left Auricle.-The left auricle is situated at the left and posterior part of the base of the heart, and is best seen from behind. It is quadrilateral, and receives on either side two pulmonary veins. The auricular appendix is the only part of the auricle seen from the front, and corresponds with that on the right side, but is thicker, and the interior is more smooth. The left auricle is only slightly thicker than the right. The left auriculo-ventricular orifice is oval, and a little smaller than 
that on the right side of the heart. There is a slight vestige of the foramen between the auricles, which exists in fœtal life, on the septum between them.

Left Ventricle.-Though taking part to a comparatively slight extent in the anterior surface, the left ventricle occupies the chief part of the posterior surface. In it are two openings very close together, viz. the auriculo-ventricular and the aortic, guarded by the valves corresponding to those of the right side of the heart, viz. the bicuspid or mitral and the semilunar or sigmoid. The first opening is at the left and baek part of the base of the ventricle, and the aortic in front and toward the right. In this ventricle, as in the right, are the columnæ carneæ, which are smaller but more closely reticulated. They are chiefly found near the apex and along the posterior wall. They will be again referred to in the description of the valves. The walls of the left ven-

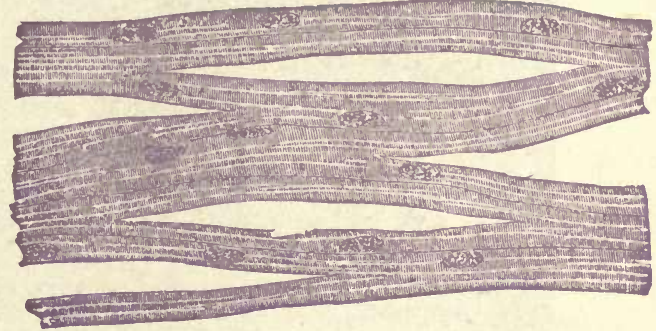

Fig. 150,-Network of muscular fibres from the heart of a pig. The nuclei of the muscle-corpus. cles are well shown. $\times 450$. (Klein and Noble Smith.)

tricle, which are nearly half an inch in thickness, are, with the exception of the apex, twice or three times as thick as those of the right.

Capacity of the Chambers.-During life each ventricle is capable of containing about four to six ounces (about $180 \mathrm{grms}$.) of blood. The capacity of the auricles after death is rather less than that of the ventricles: the thickness of their walls is considerably less. The latter condition is adapted to the small amount of force which the auricles require in order to empty themselves into their adjoining ventricles; the former to the circumstance of the ventricles being partly filled with blood before the auricles contract.

Size and Weight of the Heart.-The heart is about 5 inches long (about $12.6 \mathrm{~cm}$.), $3 \frac{1}{2}$ inches $\left(8 \mathrm{~cm}\right.$.) greatest width, and $2 \frac{1}{2}$ inches $(6.3 \mathrm{~cm}$. ) in its extreme thickness. The average weight of the heart in the adult is from 9 to 10 ounces (about 300 grms.); its weight gradually increasing thrọughout life till middle age; it diminishes in old age.

Structure.-The walls of the heart are constructed almost entirely of layers of muscular fibres; but a ring of connective tissue, to which some of the muscular fibres are attached, is inserted between each auricle and ventricle, and forms the boundary of the auriculo-ventricular 
opening. Fibrous tissue also exists at the origins of the pulmonary artery and aorta.

The muscular fibres of each auricle are in part continuous with those of the other, and partly separate; and the same remark holds true for the ventricles. The fibres of the auricles are, however, quite separate from those of the ventricles, the bond of connection between them being only the fibrous tissue of the auriculo-ventricular openings.

The minute structure of the striated muscular fibres of the heart has been already described (p. 88).

Endocardium.-As the heart is clothed on the outside by a thin transparent layer of pericardium, so its cavities are lined by a smooth

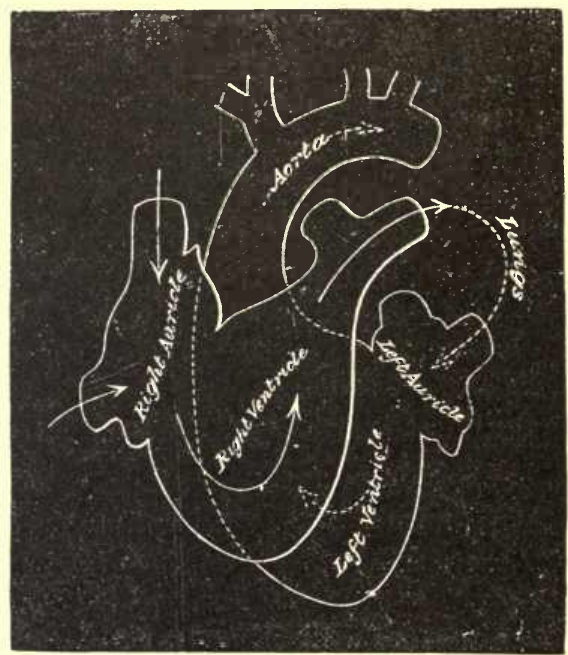

Fig. 151.-Diagram of the circulation through the heart (Dalton)

and shining membrane, or endocardium, which is directly continuous with the internal lining of the arteries and veins. The endocardium is composed of connective tissue with a large admixture of elastic fibres; and on its inner surface is laid down a single tesselated layer of flattened endothelial cells. Here and there unstriped muscular fibres are sometimes found in the tissue of the endocardium.

Valves.-The arrangement of the heart's valves is such that the blood can pass only in one direction (fig. 151).

The tricuspid valve (5, fig. 14\%) presents three principal cusps or subdivisions, and the mitral or bicuspid valve has two such portions (6, fig. 148). But in both valves there is between each two principal portions a smaller one; so that more properly, the tricuspid may be described as consisting of six, and the mitral of four, portions. Each portion is of triangular form. Its base is continuous with the bases of the neighbor- 
ing portions, so as to form an annular membrane around the auriculoventricular opening, and is fixed to a tendinous ring which encircles the orifice between the auricle and ventricle and receives the insertions of the muscular fibres of both. In each principal cusp may be distinguished a central part, extending from base to apex, and including about half its width. It is thicker and much tougher than the border pieces or edges.

While the bases of the cusps of the valves are fixed to the tendinous rings, their ventricular surface and borders are fastened by slender tendinous fibres, the chordoe tendinere, to the internal surface of the walls of the ventricles, the muscular fibres of which project into the ventricular cavity in the form of bundles or columns-the columnce carnece. These columns are not all alike, for while some are attached along their whole length on one side, and by their extremities, others are attached only by their extremities; and a third set, to which the name musculi papillares has been given, are attached to the wall of the ventricle by one extremity only, the other projecting, papilla-like, into the cavity of the ventricle (4, fig. 148), and having attached to it chordæ tendineæ. Of the tendinous cords, besides those which pass from the walls of the ventricle and the musculi papillares to the margins of the valves, there are some of especial strength, which pass from the same parts to the edges of the middle and thicker portions of the cusps before referred to. The ends of these cords are spread out in the substance of the valve, giving its middle piece its peculiar strength and toughness; and from the sides numerous other more slender and branching cords are given off, which are attached all over the ventricular surface of the adjacent border-pieces of the principal portions of the valves, as well as to those smaller portions which have been mentioned as lying between each two principal ones. Moreover, the musculi papillares are so placed that, from the summit of each, tendinous cords proceed to the adjacent halves of two of the principal divisions, and to one intermediate or smaller division, of the valve.

The preceding description applies equally to the mitral and tricuspid valve; but it should be added that the mitral is considerably thicker and stronger than the tricuspid, in accordance with the greater force which it is called upon to resist.

The semilunar valves guard the orifices of the puimonary artery and of the aorta. They are nearly alike on both sides of the heart; but the aortic valves are altogether thicker and more strongly constructed than the pulmonary valves, in accordance with the greater pressure which they have to withstand. Each valve consists of three parts which are of semilunar shape, the convex margin of each being attached to a fibrous ring at the place of junction of the artery to the ventricle, and the concave or nearly straight border being free, so as to form a little pouch 
like a watch-pocket ( $\%$, fig. 148). In the centre of the free edge of the pouch, which contains a fine cord of fibrous tissue, is a small fibrous nodule, the corpus Arantii, and from this and from the attached border fine fibres extend into every part of the mid substance of the valve, except a small lunated space just within the free edge, on each side of the corpus Arantii. Here the valve is thinnest, and composed of little more than the endocardium. Thus constructed and attached, the three semilunar pouches are placed side by side around the arterial orifice of each ventricle, which can be separated by the blood passing out of the ventricle, but which immediately afterward are pressed together, so as to prevent any return $(6$, fig. $14 \tau$, and $\%$, fig. 148$)$. This will be again referred to. Opposite each of the semilunar cusps, both in the aorta and pulmonary artery, there is a bulging outward of the wall of the vessel: these bulgings are called the sinuses of Valsalva.

Structure.-The valves of the heart are formed essentially of thick layers of closely woven connective and elastic tissue, over which, on every part, is reflected the endocardium.

\section{The Arteries.}

Distribution.-The arterial system begins at the left ventricle in a single large trunk, the aorta, which almost immediately after its origin gives off in the thorax three large branches for the supply of the head, neck, and upper extremities; it then traverses the thorax and abdomen, giving off branches, some large and some small, for the supply of the various organs and tissues it passes on its way. In the abdomen it divides into two chief branches, for the supply of the lower extremities. The arterial branches wherever given off divide and subdivide, until the calibre of each subdivision becomes very minute, and these minute vessels pass into capillaries. Arteries are, as a rule, placed in situations protected from pressure and other dangers, and are, with few exceptions, straight in their course, and frequently communicate (anastomose or inosculate) with other arteries. The branches are usually given off at an acute angle, and the areas of the branches of an artery generally exceed that of the parent trunk; and as the distance from the origin is increased, the area of the combined branches is increased also. After death, arteries are usually found dilated (not collapsed as the veins are) and empty, and it was to this fact that their name (à $\rho$ r $\rho i^{\alpha} \alpha$, the windpipe) was given them, as the ancients believed that they conveyed air to the various parts of the body. As regards the arterial system of the lungs, the pulmonary artery is distributed much as the arteries belonging to the general systemic circulation.

Structure.-The walls of the arteries are composed of three principal coats, termed $(a)$ the external or tunica adrentitia, $(b)$ the middle or tunica media, and $(c)$ the internal or tunica intima. 
(a) The external coat or tunica adventitia (figs. 152 and 153, a), the strongest and toughest part of the wall of the artery, is formed of areolar tissue, with which is mingled throughout a network of elastic fibres. At the inner part of this outer coat the elastic network forms in most arteries so distinct a layer as to be sometimes called the external elastic coat (fig. 153, e).

(b) The middle coat (fig. 153, $m$ ) is composed of both muscular and elastic fibres, with a certain proportion of areolar tissue. In the larger arteries (fig. 153) its thickness is comparatively as well as absolutely much greater than in the small, constituting, as it does, the greater part

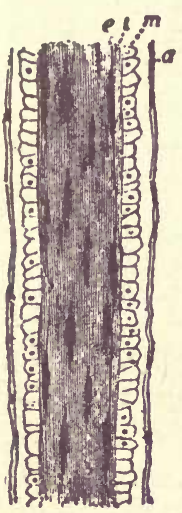

Fig. 152

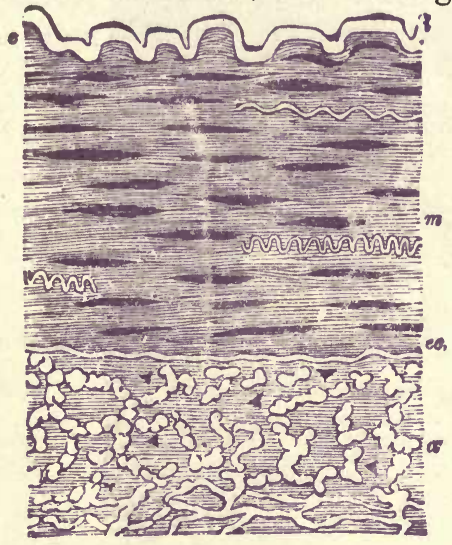

Fig. 153.
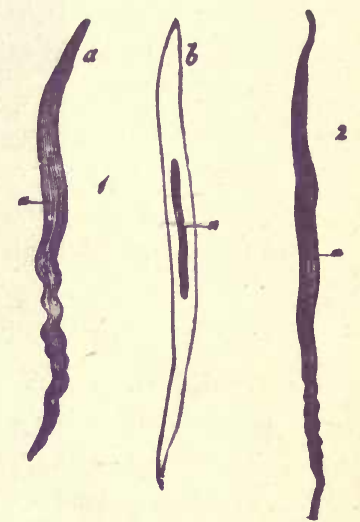

Fig. 154.

Fig. 152.-Minute artery viewed in longitudinal section. $e$, Nucleated endothelial membrane, with faint nuclei in lumen, looked at from above; $i$, thin elastic tunica intima; $m$, muscular coat or tunica media ; $a$, tunica adventitia. (Klein and Noble Smith.) $\times 250$.

Fig. 153. - Transverse section through a large branch of the inferior mesenteric artery of a pig. $e$. Endothelial membrane ; $i$, tunica elastica interna, no subendothelial layer is seen; $m$, muscular tunica media, containing only a few wavy elastic flbres; $e, c$, tunica elastica externa, dividing the media from the connective tissue adventitia $a$. (Klein and Noble Smith.) $\times 350$.

Fig. 154.-Muscular fibre-cells from human arteries, magnified 350 diameters. (Kölliker.) $a$, Nucleus. $b$, a fibre-cell treated with acetic acid.

of the arterial wall. The muscular fibres are unstriped (fig. 154), and are arranged for the most part transversely to the long axis of the artery (fig. 155, $m$ ); while the $\epsilon$ lastic element, taking also a transverse direction, is disposed in the form of closely interwoven and branching fibres, which intersect in all parts the layers of muscular fibre. In arteries of. various size there is a difference in the proportion of the muscular and elastic element, elastic tissue preponderating in the largest arteries, and unstriped muscle in those of medium and small size.

(c) The internal coat is formed by a layer of elastic tissue, called the fenestrated coat of Henle. It is peculiar in its tendency to curl up, when peeled off from the artery, and in the perforated and streaked appearance which it presents under the microscope. Its inner surface is lined with a delicate layer of elongated endothelial cells (fig. 153,e), 
which make it smooth and polished, and furnish a nearly impermeable surface, along which the blood may flow with the smallest possible amount of resistance from friction.

Immediately external to the endothelial lining of the artery is fine connective tissue, the sub-endotheluat layer, with branched corpuscles. Thus the internal coat consists of three parts, $(a)$ an endothelial lining, (b) the sub-endothelial layer, and (c) elastic layers.

Vasa Vasorum.-The walls of the arteries, with the exception of

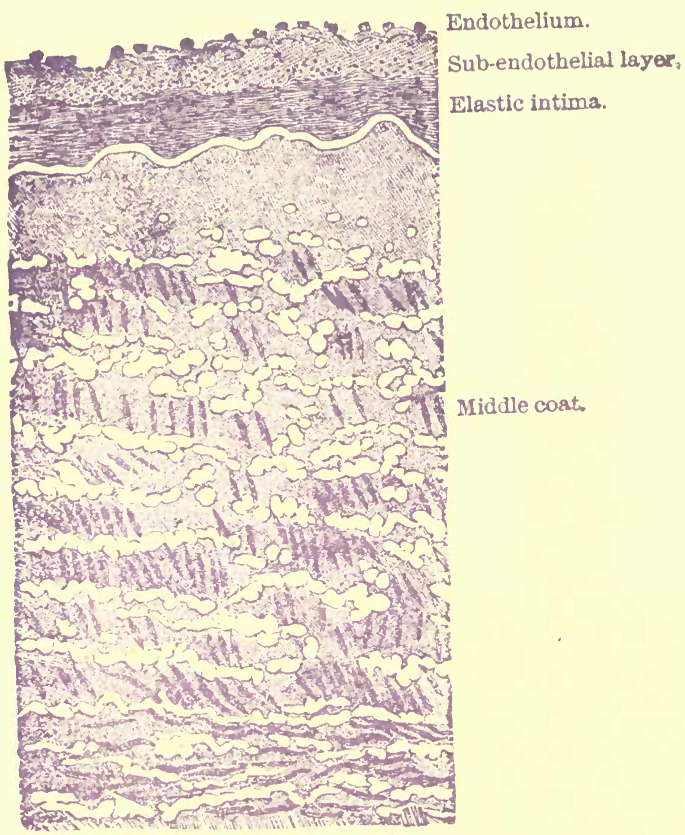

Fig. 155. - Transverse section of aorta through internal and about half the middle coat.

the endothelial lining and the layer's of the internal coat immediately outside it, are not nourished by the blood which they convey, but are, like other parts of the body, supplied with little arteries, ending in capillaries and veins, which, branching throughout the external coat, extend for some distance into the middle, but do not reach the internal coat. These nutrient vessels are called vasa vasorum.

Nerves.-Most of the arteries are surrounded by a plexus of sympathetic nerves, which twine around the vessel very much like ivy round a tree: and ganglia are found at frequent intervals. The smaller arteries are also surrounded by a very delicate network of similar nerve-fibres, many of which appear to end near the nuclei of the transverse muscular fibres (fig. 156). 


\section{The Capillaries.}

Distribution.-In all vascular textures except some parts of the corpora cavernosa of the penis, and of the uterine placenta, and of the spleen, the transmission of the blood from the minute branches of the arteries to the minute veins is effected through a network of capillaries. They may be seen in all minutely injected preparations.

The point at which the arteries terminate and the minute veins commence, cannot be exactly defined, for the transition is gradual; but the

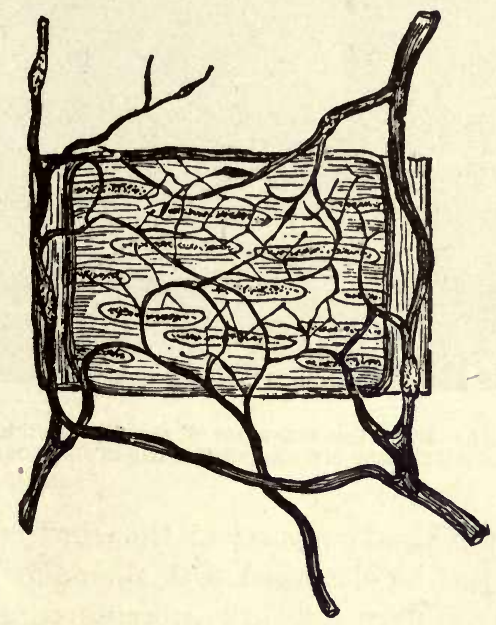

Fig. 156.-Ramification of nerves and termination in the muscular coat of a small artery of the frog. (Arnold.)

capillary network has, nevertheless, this peculiarity, that the small vessels which compose it maintain the same diameter throughout: they do not diminish in diameter in one direction, like arteries and veins; and the meshes of the network that they compose are more uniform in shape and size than those formed by the anastomoses of the minute arteries and veins.

Structure.-This is much more simple than that of the arteries or veins. Their walls are composed of a single layer of elongated or radiate, flattened and nucleated cells, so joined and dovetailed together as to form a continuous transparent membrane (fig. 15\%). Outside these cells. in the larger capillaries, there is a structureless or very finely fibrillated membrane, on the inner surface of which they are laid down. In some cases this external membrane is nucleated, and may then be regarded as a miniature representative of the tunica adventitia of arteries. Here and there at the junction of two or more of the delicate endothelial cells which compose the capillary wall, pseudo-stomata may be seen. 
The diameter of the capillary vessels varies somewhat in the different textures of the body, the most common size being about $\frac{1}{3000}$ th of an inch, 12 $\%$. Among the smallest may be mentioned those of the brain, and of the follicles of the mucous membrane of the intestines; among the largest, those of the skin, and especially those of the medulla of bones.

The size of capillaries varies necessarily in different animals in rela-

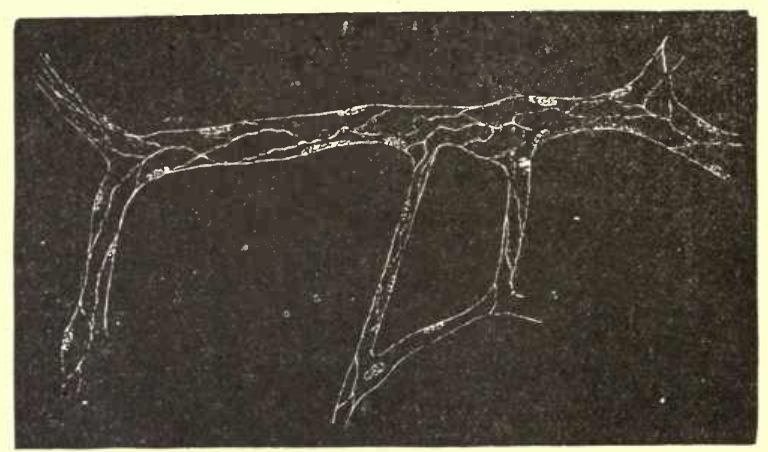

Fig. 15\%.-Capiilary blood-vessels from the omentum of rabbit, showing the nucleated endothelial membrane of which they are composed. (Klein and Noble Smith.)

tion to the size of their blood corpuscles: thus, in the Proteus, the capillary circulation can just be discerned with the naked eye.

The form of the capillary network presents considerable variety in the different textures of the body: the varieties consisting principally of modifications of two chief kinds of mesh, the rounded and the elongated. That kind in which the meshes or interspaces have a roundish form is the most common, and prevails in those parts in which the capillary network is most dense, such as the lungs (fig. 158), most glands, and mucous membranes, and the cutis. The meshes of this kind of network are not quite circular but more or less angular, sometimes presenting a nearly regular quadrangular or polygonal form, but being more frequently irregular. The capillary network with elongated meshes is observed in parts in which the vesseis are arranged among bundles of fine tubes or fibres, as in muscles and nerves. In such parts, the meshes form parallelograms, the short sides of which may be from three to eight or ten times less than the long ones; the long sides being more or less parallel to the long axis of the fibre. The rounded and elongated meshes vary according as the vessels composing them are straight or tortuous.

The number of the capillaries and the size of the meshes in different parts determine in general the degree of vascularity of those parts. 
The capillary network is closest in the lungs and in the cheroid coat of the eye. In the iris and ciliary body, the interspaces are somewhat wider, yet very small. In the human liver the interspaces are of the same size, or even smaller than the capillary vessels themselves. In the human lung they are smaller than the vessels; in the human kidney, and in the kidney of the dog, the diameter of the injected capillaries, compared with that of the interspaces, is in the proportion of one to four, or of one to three. The brain receives a very large quantity of blood; but its capillaries are very minute, and are less numerous than in some other parts. In the mucous membranes-for example in the conjunctiva and in the cutis vera, the capillary vessels are much larger than in the brain, and the interspaces narrower,-namely, not more

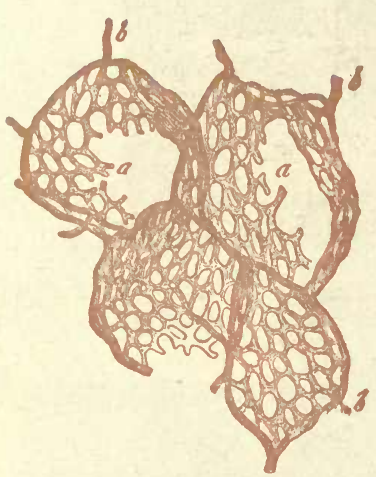

Fig. 158.

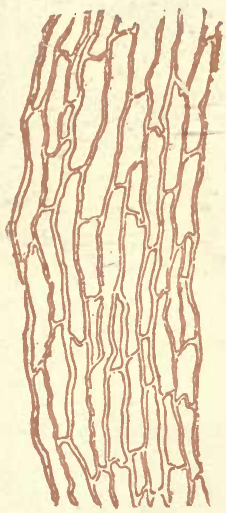

Fig. 159 .

Fig. 158.-Network of capillary vessels of the air-cells of the horse's lung magnified. $a, a$, Capillaries proceeding from $b, b$, terminal branches of the pulmonary artery. (Frey.)

Fig. 159.-Injected capillary vessels of muscle seen with a low magnifying power. (Sharpey.)

than three or four times wider than the vessels. In the periosteum the meshes are much larger. In the external coat of arteries, the width of the meshes is ten times that of the vessels.

It may be held as a general rule, that the more active the functions of an organ are, the more vascular it is. Hence the narrowness of the interspaces in all glandular organs, in mucous membranes, and in growing parts; their much greater width in bones, ligaments, and other very tough and comparatively inactive tissues; and the usually complete absence of vessels in cartilage, and such parts as those in which, probably, very little vital change occurs after they are once formed. 


\section{The Veins.}

Distribution.-The venous system begins in small vessels which are slightly larger than the capillaries from which they spring. These vessels are gathered up into larger and larger trunks until they terminate (as regards the systemic circulation) in the two venæ cavæ and the coronary veins, which enter the right auricle, and (as regards the pulmonary circulation) in four pulmonary veins, which enter the left auricle. The total capacity of the veins diminishes as they approach

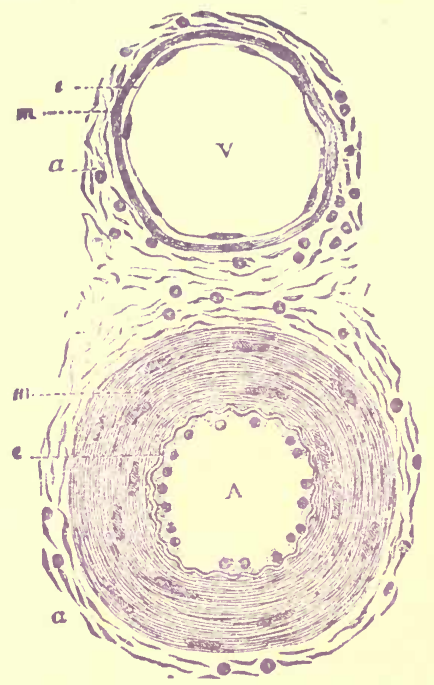

Fig. 160.-Transverse section through a small artery and vein of the mucous membrane of a child's epiglottis : the artery is thick-walled and the vein thin-walled. A. Artery, the 'etter is placed in the lumen of the vessel. $e$. Endothelial cells with nuclei clearly visible; these cells appear very thick from the contracted state of the vessel. Outside it a double wavy line narks the elastic tunica intina. $m$. Tunica media consisting of unstriped muscular fibres circularly arranged; their nuclei are well seen. $a$. Part of the tunica adventitia showing bundles of connective-tissue fibre in section, with the circular nuclei of the connective-tissue corpuscles. This coat gradually merges into the surrounding connective-tissue. v. In the lumen of the vein. The other lecters indicate the same as in the artery. The muscular coat of the vein $(m)$ is seen to be much thinner than that of the artery. $\times 350$. (Klein and Noble Smith.)

the heart; but, as a rule, their capacity exceeds by twice or three times that of their corresponding arteries. The pulmonary veins, however, are an exception to this rule, as they do not exceed in capacity the pulmonary arteries. The veins are found after death more or less collapsed, and often contain blood. They are usually distributed in a superficial and a deep set which communicate frequently in their course.

Structure.-In structure the coats of veins bear a general resemblance to those of arteries (fig. 160). Thus, they possess outer, middle, and internal coats.

The outer coat is constructed of areolar tissue like that of the 
arteries, but is thicker. In some veins it contains muscular fibre-cells, which are arranged longitudinally.

The middle coat is considerably thinner than that of the arteries; it contains circular unstriped muscular fibres, mingled with a large pro-
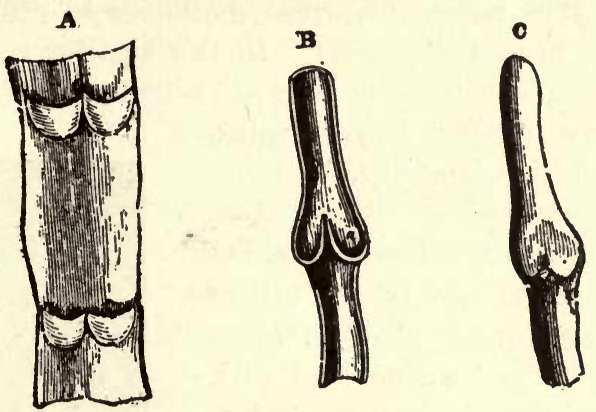

Fig. 161.-Diagram showing valves of veins. A, part of a vein laid open and spread out, with two pairs of valves. B, longitudiual section of a vein, showing the apposition of the edges of the valves in their closed state. $c$, portion of a distended vein, exhibiting a swelling in the situation of a pair of valves.

portion of yellow elastic and white fibrous tissue. In the large veins, near the heart, namely the vence cavce and pulmonary veins, the middle coat is replaced, for some distance from the heart, by circularly arranged striped muscular fibres, continuous with those of the auricles.
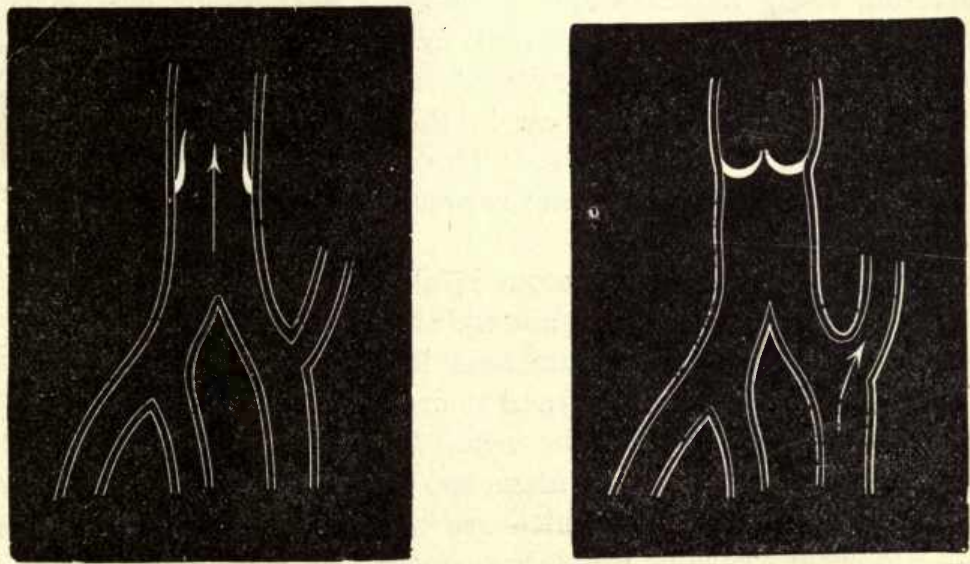

Fig. 162.-A, vein with valves open. B, vein with valves closed: stream of blood passing of by lateral channel. (Dalton.)

The internal coat of veins consists of a fenestrated membrane, which may be absent in the smaller ones, lined by endothelium.

Valves. - The chief influence which the veins have in the circulation, is effected with the help of the valves, contained in all veins subject to local pressure from the muscles between or near which they run. 
The general construction of these valves is similar to that of the semilunar valves of the arta and pulmonary artery, already described; but their free margins are turned in the opposite direction, i. e., toward the heart, so as to prevent any movement of blood backward. They are commonly placed in pairs, at various distances in different veins, but almost uniformly in each (fig. 161). In the smaller veins single valves are often met with; and three or four are sometimes placed together, or near one another, in the largest reins, such as the subclavian, and at their junction with the jugular reins. The valves are semilunar; the unattached edge being in some examples concare, in others straight. They are composed of inextensile fibrous tissue, and are covered with endothelium like that lining the reins. During the period of their inaction, when the renous blood is flowing in its proper direction, they lie by the sides of the reins; but when in action, they come together like the valves of the arteries (figs. 161 and 162). Their situation in the superficial reins of the forearm is reauily discorered by pressing along its surface, in a direction opposite to the venous current, i.e., from the elbow toward the wrist; when little swellings (fig. 161, c) appear in the position of each pair of valves. These swellings at once disappear when the pres. sure is removed.

Valves are not equally numerous in all veins, and in many they are absent altogether. They are most numerous in the veins of the extremities, and more

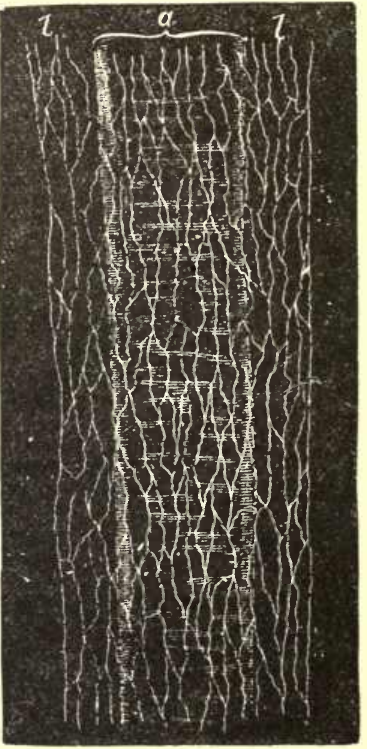

Fig. 163.-Surface view of an artery from the mesentery of a frog, ensheathed in a peri-vascular lymphatic vessel. $a$. The artery, with its circular muscular coat (media) indicated by broad, transverse markings, with an indication of the adventitia outside. $l$. Lymphatic vessel, its wall is a simple endothelial membrane. (Klein and Yoble Smith.

so in those of the leg than the arm. They are commonly absent in veins of less than a line in diameter, and, as a general rule there are few or none in those which are not subject to muscular pressure. Among those veins which have no valves may be mentioned the superior and inferior vena cava, the trunk and branches of the portal vein, the hepatic and renal veins, and the pulmonary veins; those in the interior of the cranium and vertebral column, those of the bones, and the trunk and branches of the umbilical vein are also destitute of valves.

Lymphatics of Arteries and Veins.-Lymphatic spaces are present in the coats of both arteries and veins; but in the tunica adventitia or external coat of large vessels they form a distinct plexus of more or less 
tubular vessels. In smaller vessels they appear as sinous spaces lined by endothelium. Sometimes, as in the arteries of the omentum, mesentery, and membranes of the brain, in the pulmonary, hepatic, and splenic arteries, the spaces are continuous with vessels which distinctly ensheath them-perivascular lymphatic sheaths (fig. 163). Lymph channels are said to be present also in the tunica media.

\section{The Action of the Heart.}

The heart's action in propelling the blood consists in the successive alternate contraction (systole) and relaxation (diastole) of the muscular walls of its two auricles and two ventricles.

Action of the Auricles. - The description of the action of the heart may be commenced at that period in each cycle which immediately precedes the beat of the heart against the chest wall. The whole heart is then in a passive state; the auricles are gradually filling with blood flowing into them from the veins; and a portion of this blood is passing at once through them into the ventricles, the opening between the cavity of each auricle and that of its corresponding ventricle being, during all the pause, free and patent. The auricles, however, receiving more blood than at once passes through them to the ventricles, become, near the end of the pause, fully distended; and at the end of the pause, they contract and expel their contents into the ventricles.

The contraction of the auricles is sudden and very quick; it commences at the entrance of the great veins into them, and is thence propagated toward the auriculo-ventricular opening, forcing the contained blood into the ventricle. The reflux of blood into the great veins during the auricular systole is resisted not only by the mass of blood within them, but also by the simultaneous contraction of the muscular coats with which the large veins are provided near their entrance into the auricles. Any slight regurgitation from the right auricle is limited by the valves at the junction of the subclavian and internal jugular veins, beyond which the blood cannot move backward; and the coronary vein is preserved from it by a valve at its mouth.

The force of the blood propelled into the ventricle at each auricular systole is transmitted in all directions, but being insufficient to open the semilunar valves, it is expended in distending the ventricle.

Action of the Ventricles.-The dilatation of the ventricles which proceeds during the chief part of the dilatation of the auricles is completed by the forcible injection of the contents of the latter. Thus distended, the ventricles immediately contract: so immediately, indeed, that their systole looks as if it were continuous with that of the auricles. The rentricles contract much more slowly than the auricles, and in their contraction probably always thoroughly empty themselves, 
differing in this respect from the auricles, in which, even after their complete contraction, a small quantity of blood remains. The shape of both ventricles during systole uudergoes an alteration when the chest is opened, the diameter in the plane of the base being diminished, but the length of the heart as a whole is not altered (Ludwig). Haycraft states that the heart undergoes no change of shape in the unopened chest. During the systole of the ventricles, too, the aorta and pulmonary artery, being filled with blood by the force of the ventricular action against considerable resistance, elongate as well as expand, and the whole heart moves slightly toward the right and forward, twisting on its long axis, and exposing more of the left ventricle anteriorly than is usually in front. When the systole ends the heart resumes its former position, rotating to the left again as the aorta and pulmonary artery contract. After the whole of the blood has been expelled from the ventricles, the walls are believed to remain contracted for a short period before the rapid re-dilatation of the chamber's begin.

Action of the Valves.-(1) The Auriculo-Ventricular.-The distention of the ventricles with blood continues throughout the whole period of their diastole. The auriculo-ventricular valves are gradually brought into place by some of the blood getting behind the cusps and forcing them up; and by the time that the diastole is complete, the valves are no doubt in apposition, the completion of this being brought about by the reflux current caused by the systole of the auricles. This elevation of the auriculo-ventricular valves is materially aided by the action of the elastic tissue which has been shown to exist so largely in their structure, especially on the ventricular surface. At any rate at the commencement of the ventricular systole they are completely closed. It should be recollected that the diminution in the breadth of the base of the heart in its transverse diameters during ventricular systole is especially marked in the neighborhood of the auriculo-ventricular rings, and this aids in rendering the auriculo-ventricular valves competent to close the openings, by greatly diminishing their diameter. The margins of the cusps of the valves are still more secured in apposition with another, by the simultaneous contraction of the musculi papillares, whose chordæ tendineæ have a special mode of attachment for this object. The cusps of the auriculo-ventricular valves meet not by their edges only, but by the opposed surfaces of their thin outer borders.

The form and position of the fleshy columns on the internal walls of the ventricle no doubt help to produce the obliteration of the ventricu. lar cavity during contraction; and the completeness of the closure may often be observed on making a transverse section of a heart shortly after death, in any case in which rigor mortis is very marked (fig. 149) In such a case only a central fissure may be discernible to the eye in tha place of the cavity of each ventricle. 
If there were only circular fibres forming the ventricular wall, it is evident that on systole the ventricle would elongate; if there were only longitudinal fibres, the ventricle would shorten on systole; but there are both. The tendency to alter in length is thus counterbalanced, and the whole force of the contraction is expended in diminishing the cavity of the ventricle; or, in other words, in expelling its contents.

On the conclusion of the systole the ventricular walls tend to expand by virtue of their elasticity, and a negative pressure is set up, which tends to suck in the blood. This negative or suctional pressure on the left side of the heart is of the highest importance in helping the pulmonary circulation. It has been found to be equal to $23 \mathrm{~mm}$. of mercury, and is quite independent of the aspiration or suction power of the thorax itself, which will be described in a later chapter.

The musculi papillares prevent the auriculo-ventricular valves from being everted into the auricle. For the chordæ tendineæ might allow the valves to be pressed back in to the auricle, were it not that when the wall of the ventricle is brought by its contraction nearer the auriculoventricular orifice, the musculi papillares more than compensate for this by their own contraction-holding the chords tight, and, by pulling down the valves, adding slightly to the force with which the blood is expelled.

These statements apply equally to the auriculo-ventricular valves on both sides of the heart; the closure of both is generally complete every time the ventricles contract. But in some circumstances the tricuspid valve does not completely close, and a certain quantity of blood is forced back in to the auricle. This has been called the safety-valve action. The circumstances in which it usually happens are those in which the véssels of the lung are already completely full when the right ventricle contracts, as, e.g., in certain pulmonary diseases, in very active exertions, and in great efforts. In these cases, the tricuspid valve does not completely close, and the regurgitation of the blood may be indicated by a pulsation in the jugular veins synchronous with that in the carotid arteries.

(2.) The Semitunar. - It has been shown that the commencement of the ventricular systole precedes the opening of the semilunar valves by a fraction of a second. This would seem to show that the intraventricular pressure does not exceed the arterial pressure until the systole has actually begun, for the opening of the valves takes place at once when there is a distinct difference in favor of the intraventricular over the arterial pressure, and continues open only as long as this difference continues. When the arterial begins to exceed the intraventricular pressure, there is, as it were, a reflux of blood toward the heart, and the valves close. The dilatation of the arteries is, in a peculiar manner, adapted to bring this about. 
The lower borders of the semilunar valves are attached to the inner surface of the tendinous ring, which is, as it were, inlaid at the orifice of the artery, between the muscular fibres of the ventricle and the elastic fibres of the walls of the artery. The tissue of this ring is tough, and does not admit of extension under such pressure as it is commonly exposed to; the valves are equally inextensile, being, as already mentioned, formed mainly of tough, close-textured, fibrous tissue, with strong interwoven cords. Hence, when the ventricle propels blood through the orifice and into the canal of the artery, the lateral pressure which it exercises is sufficient to dilate the walls of the artery, but not enough to stretch in an equal degree, if at all, the unyielding valves and the ring to which their lower borders are attached. The effect, therefore, of each such propulsion of blood from the ventricle is, that the wall of the first portion of the artery is dilated into three pouches behind
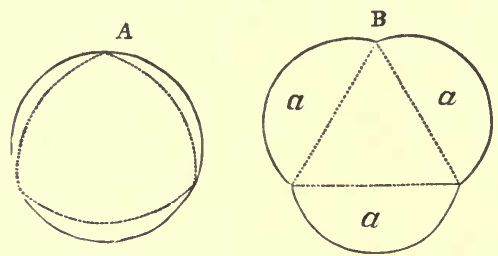

Fig. 164.-Sections of aorta, to show the action of the semilunar valves. $\Delta$ is intended to show the valves, represented by the dotted lines, lying near the arterial walls, represented by the continuous outer line. B (after Hunter) shows the arterial wall distended into three pouches $(a)$, and drawn away from the valves, which are straightened into the form of an equilateral triangle as represented by the dotted lines.

the valves, while the free margins of the valves are drawn inward toward its centre (fig. 164, B). Their positions may be explained by the diagrams, in which the continuous lines represent a transverse section of the arterial walls, the dotted ones the edges of the valves, firstly, when the valves are nearest to the walls (A), as in the dead heart, and, secondly, when, the walls being dilated, the valves are drawn away from them (B).

This position of the valves and arterial walls is retained so long as the ventricle continues in contraction: but as soon as it relaxes, and the dilated arterial walls can recoil by their elasticity, the blood is forced backward toward the ventricles and onward in the course of the circulation. Part of the blood thus forced back lies in the pouches (sinuses of Valsalva) ( $a$, fig. 164, B) between the valves and the arterial walls; and the valves are by it pressed together till their thin lunated margins meet in three lines radiating from the centre to the circumference of the artery ( 7 and 8 , fig. 148).

The contact of the valves in this position and the complete closure 
of the arterial orifice are secured by the peculiar construction of their borders before mertioned. Among the cords which are interwoven in the substance of the valve are two of greater strength and prominence than the rest; of which one extends along the free border of each valve, and the other forms a double curve or festoon just below the free border. Each of these cords is attached by its outer extremities to the outer end of the free margin of its valve, and in the middle to the corpus Arantii; they thus enclose a lunated space from a line to a line and a half in width, in which space the substance of the valve is much thinner and more pliant than elsewhere. When the valves are pressed down, all these parts or spaces of their surfaces come into contact, and the closure of the arterial orifice is thus secured by the apposition not of the mere edges of the valves, but of all those thin lunated parts of each which lie between the free edges and the cords next below them. These parts are firmly pressed together, and the greater the pressure that falls on them the closer and more secure is their apposition. The corpora Arantii meet at the centre of the arterial orifice when the valves are down, and they probably assist in the closure; but they are not essential to it, for, not unfrequently, they are wanting in the valves of the pulmonary artery, which are then extended in larger, thin, flapping margins. In valves of this form, also, the inlaid cords are less distinct than in those with corpora Arantii; yet the closure by contact of their surfaces is not less secure.

Cardiac Cycle.-Taking 72 as the average number of cardiac evolutions per minute, each revolution may be considered to occupy $\frac{5}{6}$ of a second, or about .8, which may be approximately distributed in the following way:-

$\begin{array}{ccc}\begin{array}{c}\text { Auricular systole, about } \\ \text { Ventricular systole " }\end{array} & .1+\text { Auricular diastole } & .3+\text { Ventricular diastole } \\ \begin{array}{c}\text { Period of joint auricular } \\ \text { and ventricular diastole }\end{array} .4+\begin{array}{c}\text { Period of systole of } \\ \text { auricles or ventricles }\end{array} & .7=.8 \\ & .5=.8 \\ & .4=.8\end{array}$

If the speed of the heart be quickened, the time occupied by each cardiac revolution is of course diminished, but the diminution affects only the diastole and pause. The systole of the ventricles occupies very much the same time, whatever the pulse-rate.

The exact period in which the several valves of the heart are in action is a matter of some uncertainty; the auriculo-ventricular valves are probably closed during the whole time of the ventricular contraction, while, during the dilatation and distention of the ventricles, they are open. The semilunar valves are only certainly open during the middle period of the ventricular contraction. 


\section{The Sounds of the Heart.}

When the ear is placed over the region of the heart, two sounds may be heard at every beat of the heart, which follow in quick succession, and are succeeded by a pause or period of silence. The first sound is dull and prolonged; its commencement coincides with the impulse of the heart against the chest wall, and just precedes the pulse at the wrist. The second is shorter and sharper, with a somewhat flapping character, and follows close after the arterial pulse. The periods of time occupied respectively by the two sounds taken together and by the pause between the second and the first, are unequal. According to Foster, the interval of time between the beginning of the first sound and the second sound is .3 second, while between the second and the succeeding first it is nearly .5 (see fig. 165). The relative length of time occupied by each sound, as compared with the other, may be best appreciated by considering the different forces concerned in the production of the two sounds. In one case there is a strong, comparatively slow, contraction of a large mass of muscular fibres, urging forward a certain quantity of fluid against considerable resistance; while in the other it is a strong but shorter and sharper recoil of the elastic coat of the large arteries-shorter because there is no resistance to the flapping back of the semilunar valves, as there was to their opening. The sounds may be expressed by the words $l \bar{u} b b-d \grave{u} p$.

The events which correspond, in point of time, with the first sound, are (1) the contraction of the ventricles, (2) the first part of the dilatation of the auricles, (3) the tension of the auriculo-ventricular valves, (4) the opening of the semilunar valves, and (5) the propulsion of blood into the arteries. The sound is succeeded, in about one-thirtieth of a second, by the pulsation of the facial arteries, and in about one-sixth of a second, by the pulsation of the arteries at the wrist. The second sound, in point of time, immediately follows the cessation of the ventricular contraction, and corresponds with $(a)$ the tension of the semilunar valves, $(b)$ the continued dilatation of the auricles, $(c)$ the commencing dilatation of the ventricles, and $(d)$ the opening of the auriculo-ventricular valves. The pause immediately follows the second sound, and corresponds in its first part with the completed distention of the auri. cles, and in its second with their contraction, and the completed distention of the ventricles; the auriculo-ventricular valves being all the time of the pause open, and the arterial valves closed.

Causes.-The exact cause of the first sound of the heart is not known. Two factors probably enter into it, viz., firstly the vibration of the auriculo-ventricular valves and of the chordæ tendinøæ. This vibration is produced by the increased intraventricular pressure set up 
when the ventricular systole commences, which puts the valves on the stretch. The question whether this stretched condition of the valve continues throughout the whole of the ventricular systole cannot be definitely settled, but if it does not, the valvular element may possibly take part in the production of the first part of the first sound only. It is not unlikely too that the vibration of the ventricular walls themselves, and of the aorta and pulmonary artery, all of which parts are suddenly

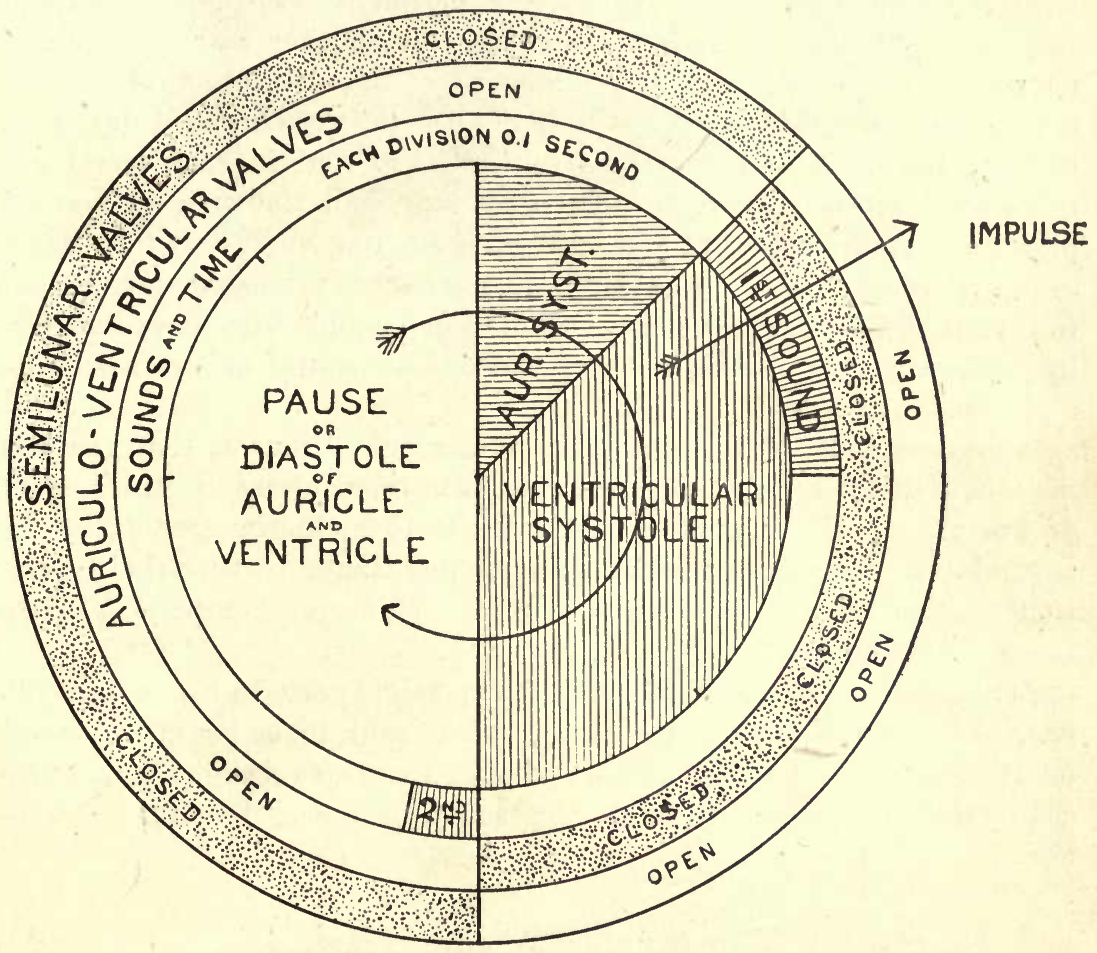

Fig. 165.-Diagrammatic representation of the events of the cardiac cycle. For events which occur in sequence, read in the direction of the curved arrow; for synchronous events, read from the centre to the periphery in any direction. (Coleman.)

put into a state of tension at the moment of ventricular contraction, may have some part in producing the first sound. Secondly, the muscular sound produced by contraction of the mass of muscular fibres which form the ventricle. Looking upon the contraction of the heart as a single contraction and not as a series of contractions or tetanus, it is at first sight difficult to see why there should be any muscular sound at all when the heart contracts, as contraction of a single muscle does not produce sound. It has been suggested, however, that it arises from the repeated unequal tension produced when the wave of muscular contractions passes along the very intricately arranged fibres of the ventric- 
ular walls. 'The valvular element is probably the more important of the two factors.

'The cause of the second sound is more simple than that of the first. It is entirely due to the vibration consequent on the sudden closure of the semilunar valves when they are pressed down across the orifices of the aorta and pulmonary artery. The influence of these valves in producing the sound was first demonstrated by Hope who experimented with the hearts of calves. In these experiments two delicate curved needles were inserted, one into the aorta, and another into the pulmonary artery, below the line of attachment of the semilunar valves, and, after being carried upward about half an inch, were brought out again through the coats of the respective vessels, so that in each vessel one valve was included between the arterial walls and the wire. Upon applying the stethoscope to the vessels, after such an operation, the second sound had ceased to be audible. Disease of these valves, when sufficient to interfere with their efficient action, also demonstrates the same fact by modifying the valvular cause of the second sound or destroying its distinctness.

One reason that the second sound is clearer and sharper than the first may be, that the semiluuar valves are not covered in by the thick layer of fibres composing the walls of the heart to such an extent as are the auriculo-rentricular. It might be expected therefore that their vibration would be more easily heard by means of a stethoscope applied to the walls of the chest.

The contraction of the auricles which takes place in the end of the pause is inaudible outside the chest, but is said to be heard, when the heart is exposed and the stethoscope placed on it, as a slight sound preceding and continued into the louder sound of the ventricular contraction.

\section{The Impulse of the Heart.}

With each contraction the heart may be felt to beat with a slight shock or impulse against the walls of the chest. The force of the impulse and the extent to which it may be perceived beyond this point vary considerably in different individuals, and in the same individual under different circumstances. It is felt more distinctly, and over a larger extent of surface, in emaciated than in fat and robust persons, and more during a forced expiration than in a deep inspiration; for, in the one case, the intervention of a thick layer of fat or muscle between the heart and the surface of the chest, and in the other the inflation of the portion of lung which overlaps the heart, prevents the impulse from being fully transmitted to the surface. An excited action of the heart, 
and especially a hypertrophied condition of the ventricles, will increase the impulse; while a depressed condition, or an atrophied state of the ventricular walls, will diminish it.

Cause of the Impulse.-During the period which precedes the ven-

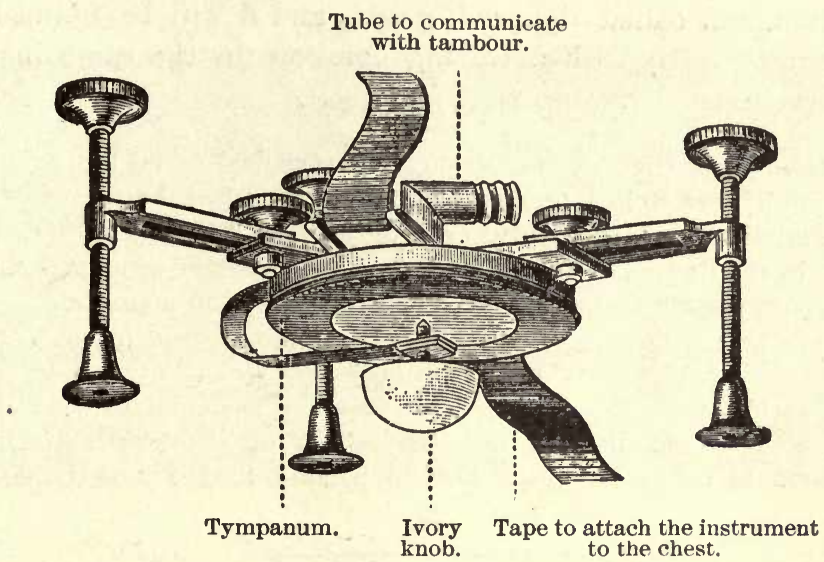

Fig. 166.-Cardiograph. (Sanderson's.)

tricular systole the apex of the heart is situated upon the diaphragm and against the chest-wall in the fifth intercostal space. When the ventricles contract, their walls become hard and tense, since to expel their contents into the arteries is a distinctly laborious action, as it is resisted

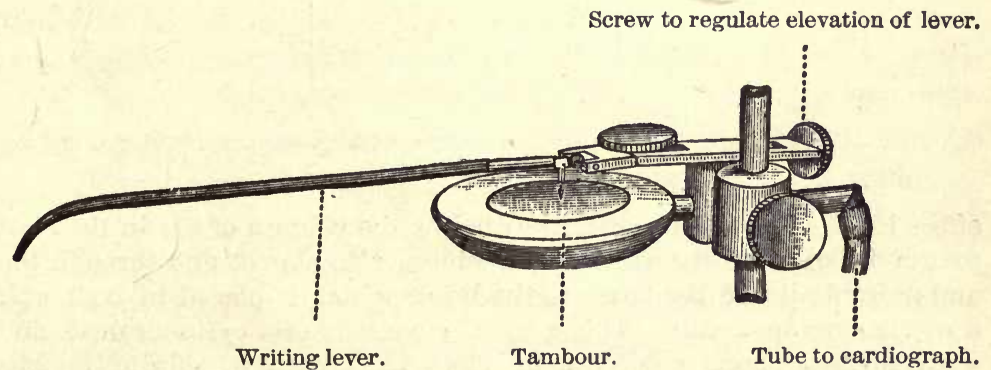

Fig. 167.-Marey's Tambour, to which the movement of the column of air in the first tympanum is conducted by a tube, and from which it is communicated by the lever to a revolving cylinder, so that the tracing of the movement of the impulse beat is obtained.

by the elasticity of the vessels. It is to this sudden hardening that the impulse of the heart against the chest-wall is due, and the shock of the sudden tension may be felt not-only externally, but also internally, if the abdomen of an animal be opened and the finger be placed upon the 
under surface of the diaphragm, at a point corresponding to the under surface of the ventricle. The shock is felt, and possibly seen more distinctly because of the partial rotation of the heart, already spoken of, along its long axis toward the right. The movement produced by the ventricular contraction against the chest-wall may be registered by means of an instrument called the cardiograph, and it will be found to correspond almost exactly with a tracing obtained by the same instrument applied over the contracting ventricle itself.

The Cardiograph (fig. 166) consists of a cup-shaped metal box over the open front of which is stretched an elastic India-rubber membrane, upon which is fixed a small knob of hard wood or ivory. This knob, however, may be attached, as in the figure, to the side of the box by means of a spring, and may be made to act upon a metal disc attached to the elastic membrane.

The knob is for application to the chest-wall over the place of the greatest impulse of the heart. The box or tympanum communicates by means of an air-tight tube with the interior of a second tympanum, in connection with which is a long and light lever. The shock of the heart's impulse being communicated to the ivory knob, and through it to the first tympanum, the

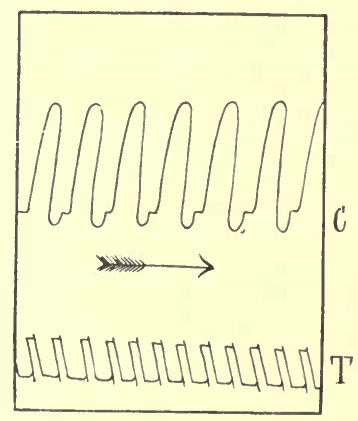

Fig. $16 \tau$ A.-Cardiogram of Frog's Heart. c, Tracing of auricular and ventricular systole; T, time in half seconds.

effect is, of course, at once transmitted by the column of air in the elastic tube to the interior of the second tympanum, also closed, and through the elastic and movable lid of the latter to the lever, which is placed in connection with a registering apparatus. This generally consists of a cylinder or drum covered with smoked paper, revolving by clock-work with a definite velocity. The point of the lever writes upon the paper, and a tracing of the heart's impulse or cardiogram is thus obtained.

\section{Endocardiac Pressure.}

It cannot be considered, however, that the cardiogram represents what is actually occurring within the heart itself. For determining this, communication must be established with the cavities of the heart. 
By placing three small India-rubber air-bags or cardiac sounds in the interior respectively of the right auricle and the right ventricle, and in an intercostal space in front of the heart of living animals (horse), and placing these bags, by means of long, narrow tubes, in communication with three levers, arranged one over the other in connection with a reg-

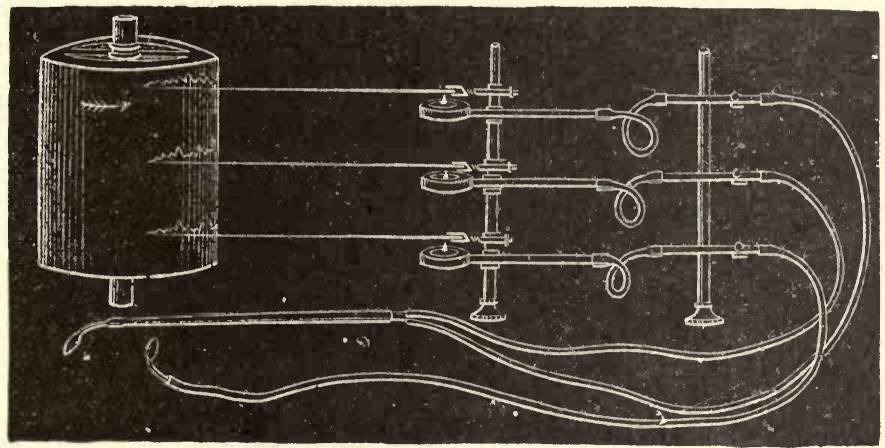

Fig. 168.-Apparatus of MM. Chauveau and Marey for estimating the varlations of endocardial pressure, and production of impulse of the heart.

istering apparatus (fig. 168), Chauveau and Marey have been able to record and measure with much accuracy the variations of the endocardial pressure and the comparative duration of the contractions of the auricles and ventricles. By means of the same apparatus, the synchronism of the impulse with the contraction of the ventricles, is also well shown; and the causes of the several vibrations of which it is really composed, have been demonstrated.

In the tracing (fig. 169), the intervals between the vertical lines represent periods of a tenth of a second. The parts on which any given vertical line falls represent simultaneous events. It will be seen that the contraction of the auricle, indicated by the marked curve at $\mathbf{A}$ in first tracing, causes a slight increase of pressure in the ventricle which is shown at $\mathbf{A}^{\prime}$ in the second tracing, and produces also a slight impulse, which is indicated by $A^{\prime \prime}$ in the third tracing. The closure of the semilunar valves causes a momentarily increased pressure in the ventricle at $\mathrm{D}^{\prime}$, affects the pressure in the auricle $\mathrm{D}$, and is also shown in the tracing of the impulse $\mathrm{D}^{\prime \prime}$.

The large curve of the ventricular and the impulse tracings, between $\mathrm{A}^{\prime}$ and $\mathrm{D}^{\prime}$, and $\mathrm{A}^{\prime \prime}$ and $\mathrm{D}^{\prime \prime}$, are cansed by the ventricular contraction, while the smaller undulations, between $\mathrm{B}$ and $\mathbf{c}, \mathrm{B}^{\prime}$ and $\mathrm{C}^{\prime}, \mathrm{B}^{\prime \prime}$ and $\mathrm{C}^{\prime \prime}$, are caused by the vibrations consequent on the tightening and closure of the auriculo-ventricular valves.

It seems by no means certain that Marey's curves properly represent the variations in intraventricular pressure. Much objection has been 
taken to his method of investigation. Firstly, because his tambour arlamgement does not admit of both positive and negative pressure being simultaneously recorded. Secondly, becanse the method is only applicable to large animals, such as the horse. And thirdly, becanse the intraventricular changes of pressure are communicated to the recording tambour ly a long elastic column of air; and fourthly, because the tambour arrangement has a tendency to record inertia vibrations. H. D. Rolleston, who has pointed ont the above imperfections of Marey's method, has reinvestigated the subject with a more suitable apparatus. The method

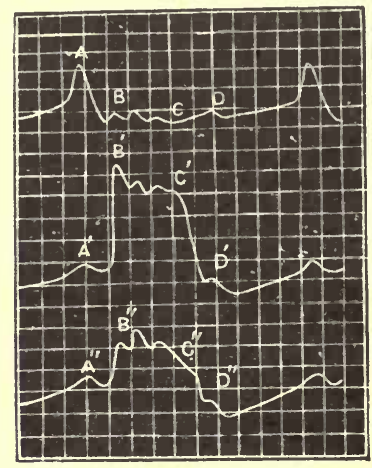

Fig. 169.-Tracings of (1), Intra-auricular, and (2), Intra-ventricular pressures, and (3), of the im. pulse of the heart. to be real from left to right, obtained by (Chaureau and Marey's apparatus.

adopted by Rolleston is as follows: a window is made in the chest of an anæsthetized and curarized animal, and an appropriately curved glass canula introduced throngh an opening in the auricular appendix. 'The canula is then passed through the auriculo-ventricular orifice without causing any appreciable regurgitation, into the auricle, or it may be introduced into the carity of the right or left rentricle by an opening made in the apex of the heart. In some experiments the trocar is pushed through the chest wall into the ventricular cavity. The apparatus is filled with a solution of leech extract in .75 per cent saline solution, or with a solution of sodium bicarbonate of specific gravity 1083 . The animals employed were chiefly dogs. The movement of the column of blood is communicated to the writing lever by means of a vulcanite piston which moves with little friction in a brass tube comnected with the glass canula by means of a short comnecting tube.

When the lower part of the tube $(A)$ is placed in communication with one of the cavities of the heart, the movements of the piston are recorded by means of the lever (c). Attached to the lever is a section of i pulley $(\mathrm{H})$, the axis of which coincides with that of the steel ribbon (E); while, firmly fixed to the piston, is the curved steel piston rod (1), 
from the top of which a strong silk thread (J) passes downward into the groove on the pulley.

This thread (J), after being twisted several times round a small pin at the side of the lever, enters the groove in the pulley from above downward, and then passes to be fixed to the lower part of the curve on the piston-rod as shown in the smaller figure.

The rise and fall of the lever (c) is controlled by the resistance to

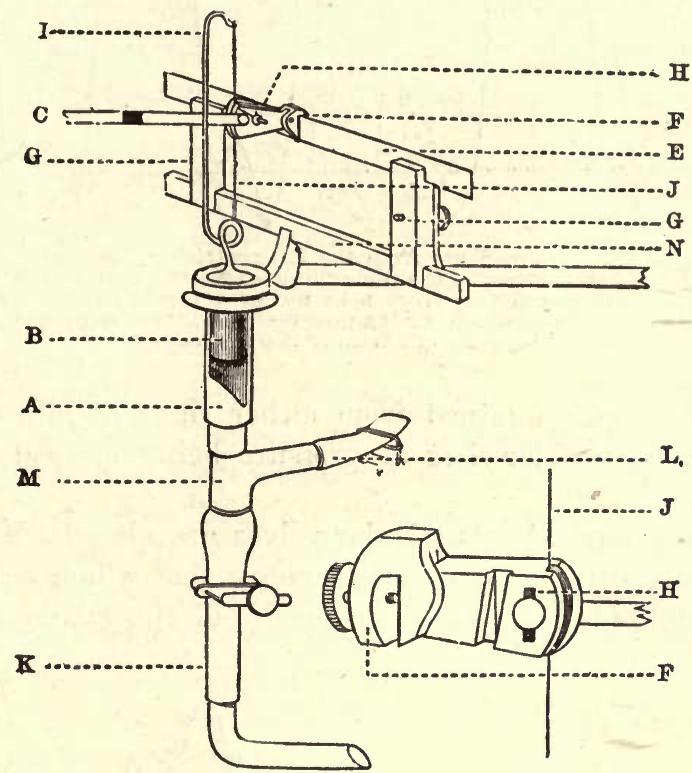

Fig. 170.-Apparatus for recording the endocai dial pressure. (Rolleston.)

torsion of the steel ribbon (E), to the middle of whish one end of the lever is securely fixed by a light screw clamp (F). At some distance from this clamp-the distance varying with the degree of resistance which it is desired to give to the movements of the lever-are two holders (G.G') which securely clamp the steel ribbon.

As the torsion of a steel wire or strip follows Hooke's law, the torsion being proportional to the twisting force-the movements of the lever point are proportional to the force employed to twist the steel strip or ribbon-in other words to the pressures which act on the piston (B).

To make it possible to record satisfactorily the very varying ventricular and auricular pressures, the resistance to torsion of a steel ribbon adapts itself very conveniently.

This resistance can be varied in two ways, 1st, by using one or more pieces of steel ribbon or by using strips of different thicknesses; or $2 d$, 
by varying the distance between the holders (G.G.) and the central part of the steel ribbon to which the lever is attached.

Rolleston's conclusions are as follows:-

1. That there is no distinct and separate auricular contraction

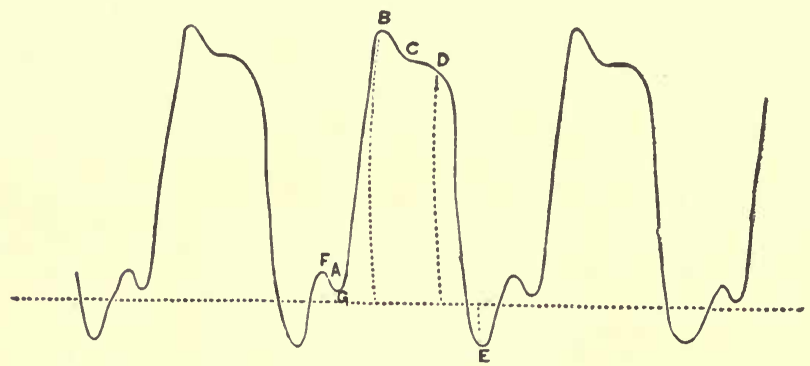

Fig. 171.-Endocardial pressure-curve from the left ventricle. The thorax was opened and a canula introduced through the apex of the ventricle: abscissa is line of atmospheric pressure. G to $\mathrm{D}$ represents veutricular contraction; from $\mathrm{D}$ to the next rise at $\mathrm{G}$ represents the ventricular diastole. The notch at the top of which is $\mathrm{F}$ is a post-ventricular rise in pressure from below that of the atmosphere and not a pre-systolic or auricular rise in pressure.

marked in the curves obtained from either right or left ventricles, the auricular and ventricular rises of pressure being merged into one continuous rise.

2 . That the auriculo-ventricular valves are closed before any great rise of pressure within the ventricle above that which results from the auricular systole ( $a$, fig. 172). The closure of the valve occurs probably

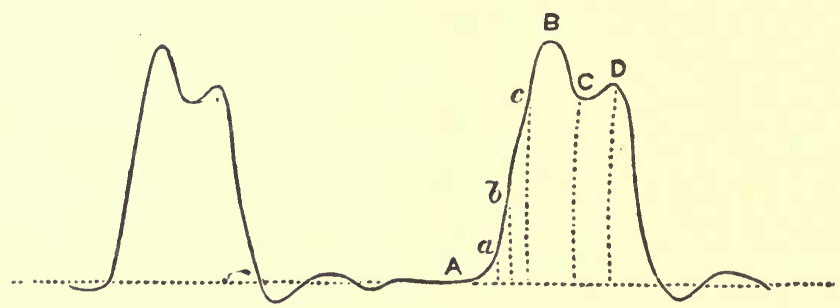

Fig. 172.-Curve with dicrotic summit from left ventricle; abscissa shows atmospheric pressure.

in the lower third of the rise A B (fig. 172), and does not produce any notch or wave.

3. That the semilunar valves open at the point in the ventricular systole, situated (at $\mathrm{G}$ ) about or a little above the junction of the middle or upper third of the ascending line (A B), and the closure about or a little before the shoulder (D).

4. That the minimum pressure in the ventricle may fall below that of the atmosphere, but that the amount varies considerably. 


\section{Frequency of the Heart's Action.}

The heart of a healthy adult man contracts about 72 times in a minute; but many circumstances cause this rate, which of course corresponds with that of the arterial pulse, to vary even in health. The chief are age, temperament, sex, food and drink, exercise, time of day, posture, atmospheric pressure, temperature; as follows:-

(1.) Age.-The frequency of the heart's action gradually diminishes from the commencement to near the end of life, but is said to rise again somewhat in extreme old age, thus:-

Before birth the average number of pulsations per minute is 150

Just after birth . from 140 to 130

During the first year

During the second year . .

During the third year
130 to 115

115 to 100

100 to 90
About the seventh year . . from 90 to 85 About the fourteenth year . $\quad$. In adult age . $\quad 80$ to 70 In old age $\quad . \quad 70$ to 60 In decrepitude . . . 75 to 65

(2.) Temperament and Sex.-In persons of sanguine temperament, the heart acts somewhat more frequently than in those of the phlegmatic; and in the female sex more frequently than in the male.

(3 and 4.) Food and Drink. Exercise.-After a meal the heart's action is accelerated, and still more so during bodily exertion or mental excitement; it is slower during sleep.

(5.) Diurnal Variation.-In health the pulse is most frequent in the morning, and becomes gradually slower as the day advances: and this diminution of frequency is both more regular and more rapid in the evening than in the morning.

(6.) Posture.-The pulse, as a general rule, especially in the adult male, is more frequent in the standing than in the sitting posture, and in the latter than in the recumbent position; the difference being greatest between the standing and the sitting postures. The effect of change of posture is greater as the frequency of the pulse is greater, and, accordingly, is more marked in the morning than in the evening. By supporting the body in different positions, without the aid of muscular effort of the individual, it has been proved that the increased frequency of the pulse in the sitting and standing positions is dependent upon the muscular exertion engaged in maintaining them; the usual effect of these postures on the pulse being almost ent..ely prevented when the usually attendant muscular exertion was rendered unnecessary.

(7.) Atmospheric Pressure.-The frequency of the pulse increases in a corresponding ratio with the elevation above the sea.

(8.) Temperature.-The rapidity and force of the heart's contractions are largely influenced by variations of temperature. The frog's heart, when excised, ceases to beat if the temperature be reduced to 
$0^{\circ} \mathrm{C}$. $\left(32^{\circ} \mathrm{F}\right.$.). When heat is gradually applied to it, both the speed and force of the contractions increase till they reach a maximum. If the temperature is still further raised, the beats become irregular and feeble, and the heart at length stands still in a condition of "heatrigor." Similar effects are produced in warm-blooded animals. In the rabbit, the number of heart-beats is more than doubled when the temperature of the air was maintained at $40^{\circ} .5 \mathrm{C} .\left(105^{\circ} \mathrm{F}\right.$. $)$. At $45^{\circ} \mathrm{C} .\left(113^{\circ}\right.$ $-114^{\circ} \mathrm{F}$.), the rabbit's heart ceases to beat.

In health there is observed a nearly uniform relation between the frequency of the beats of the heart and of the respirations; the proportion being, on an average, 1 respiration to 3 or 4 beats. The same relation is generally maintained in the cases in which the action of the heart is natura!ly accelerated, as after food or exercise; but in disease this relation may cease. In many affections accompanied with increased frequency of the heart's contraction, the respiration is, indeed, also accelerated, yet the degree of its acceleration may bear no definite proportion to the increased number of the heart's actions: and in many other cases, the heart's contraction becomes more frequent without any accompanying increase in the number of respirations; or, the respiration alone may be accelerated, the number of pulsations remaining stationary, or even falling below the ordinary-standard.

\section{The Force of the Cardiac Action.}

(a.) Ventricular.-The force of the left ventricular systole is more than double that exerted by the contraction of the right ventricle: this difference results from the walls of the left ventricle being about twice or three times as thick as those of the right. And the difference is adapted to the greater degree of resistance which the left rentricle has to orercome, compared with that to be overcome by the right: the former having to propel blood through every part of the body, the latter only through the lungs. The actual amount of the intraventricular pressures during systole in the dog has been found to be 2.4 inches ( 60 $\mathrm{mm}$.) of mercury in the right ventricle, and 6 inches $(150 \mathrm{~mm}$.) in the left.

During diastole there is in the right ventricle a negative or suction pressure of about $\frac{2}{3}$ of an inch $(-17$ to $-16 \mathrm{~mm}$.), and in the left ventricle from 2 inches to $\frac{4}{5}$ of an inch $(-52$ to $-20 \mathrm{~mm}$.). Part of this fall in pressure, and possibly the greater part, is to be referred to the influence of respiration; but without this the negative pressure of the left ventricle caused by its active dilatation is about equal to $\frac{4}{5}$ of an inch (20 mm. )of mercury.

The right ventricle is undoubtedly aided by this suction power of the left, so that the whole of the work of conducting the pulmonary 
circulation does not fall upon the right side of the heart, but is assisted by the left side.

(b.) Auricular.-The maximum pressure within the right auricle is equal to about $\frac{4}{5}$ of an inch $(20 \mathrm{~mm}$.) of mercury, and is probably somewhat less in the left. It has been found that during diastole the pressure within both auricles sinks considerably below that of the atmosphere; and as some fall in pressure takes place, even when the thorax of the animal operated upon has been opened, a certain proportion of the fall must be due to active auricular dilatation independent of respiration in the right auricle, this negative pressure is equal to about $-10 \mathrm{~mm}$.

In estimating the work done by any machine it is usual to express it in terms of the unit of work. In England, the unit of work is the foot-pound, and is defined to be the energy expended in raising a unit of weight (1 lb.) through a unit of height (1 ft.): in France, the kilogram-metre. The work done by the heart at each contraction can be readily found by multiplying the weight of blood expelled by the ventricles by the height to which the blood rises in a tube tied into an artery. This height is probably about $9 \mathrm{ft} .3 .21$ metres in man. Taking the weight of blood expelled from the left ventricle at each systole at 6 oz., i.e., $\frac{3}{8} \mathrm{lb}$., we have $9 \times \frac{3}{8}=3.375$ foot-pounds, or $3.21 \times 180 \mathrm{grms}$. or 578 gram-metres, as the work done by the left ventricle at each systole; and adding to this the work done by the right ventricle (about one-fourth that of the left) we have $3.3 \% 5+.822=4.19$ foot-pounds, or 722 gram-metres as the work done by the heart at each contraction.

\section{Blood Pressure.}

The subject of blood-pressure has been already incidentally mentioned more than once in the preceding pages, the time has now arrived for it to receive more detailed consideration.

That the blood exercises pressure upon the walls of the vessels containing it, is due to the following facts:-

Firstly, that the heart at each contraction forcibly injects a considerable amount of blood, viz., 4 to $6 \mathrm{oz}$. (120 to $180 \mathrm{grms}$.) suddenly and quickly into the arteries.

Secondly, that the arteries are already full of blood at the commencement of the ventricular systole, since there is not sufficient time between the heart beats for the blood to pass into the veins.

Thirdly, that the arteries are highly distensible and stretch to accommodate the extra amount of blood forced into them; and

Fourthly, that there is a distinct resistance interposed to the passage of the blood from the arteries into the veins, from the enormous number of minute vessels, small arteries (arterioles) and capillaries into 
which the main artery has been ultimately broken up. The sectional area of the capillaries is several hundred times that of the aorta, and the friction generated by the passage of the blood through these minute channels opposes a considerable hindrance or resistance in its course. The resistance thus set up is called peripheral resistance. 'The friction is greater in the arterioles where the current is comparatively rapid than in the capillaries where it is slow.

That the blood exerts considerable pressure upon the arterial walls in keeping them in a stretched or distended condition, may be readily shown by puncturing any artery; the blood is instantly projected with great force through the opening, and the jet rises to a considerable height, the exact level of which varies with the size of the artery experimented with. If a large artery be punctured, the blood may be projected upward for many feet, whereas if a small artery be similarly dealt with the jet does not rise to such a height. Another marked feature of the jet of blood from a cut artery, particularly well marked if the vessel be a large one, and near the heart, is the jerky character of the outflow. If the artery be cut across, the jet issues with force, chiefly from the central end, unless there is considerable anastomosis of vessels in the neighborhood, when the jet from the peripheral end may be as forcible and as intermittent as that from the other end. The intermittent flow in the arteries which is due to the intermittent action of the heart, and which represents the systolic and diastolic alterations of blood pressure, may be felt if the finger be placed upon a sufficiently superficial artery. The finger is apparently raised and lowered by the intermittent systolic distention of the vessel, occurring at each heart beat. This intermittent distention of the artery is what is known as the Pulse, to the further consideration of which we shall persently return, but we may say here, that in a normal condition the pulse is a characteristic of the arterial, and is absent from the venous flow. At the same time it must be recollected that in the veins the blood exercises a pressure on its containing vessel, but as we shall see presently this is small when compared with the arterial blood-pressure. As might be expected, therefore, the blood is not expelled with so much force if a vein be punctured or cut, and further, the flow from the cut vein is continuous and not intermittent, and the greater amount of blood comes from the peripheral and not from the central end as is the case when an artery is severed.

The result produced by the experiment of cutting or puncturing a blood vessel may be modified by introducing into the vessel a glass tube of a calibre corresponding to that of the vessel, and allowing the blood to rise in it. If the vessel be an artery, the blood will rise several feet, according to the distance of the vessel from the heart, and when it has reached its highest point will be seen to oscillate with the heart's beats. 'Ihis experiment shows that the pressure which the 
blood exerts upon the walls of the contained artery, equals the pressure of a column of blood of a certain height; in the case of the rabbit's carotid it is equal to 3 feet of blood, or rather more than 3 feet of water. In the case of the vein, if a similar experiment be performed, blood will rise in the tube for an inch or two only.

The usual method of estimating the amount of blood pressure differs somewhat from the foregoing simple experiment. Instead of a simple straight tube of glass inserted into the vessel, a U-shaped tube contain-

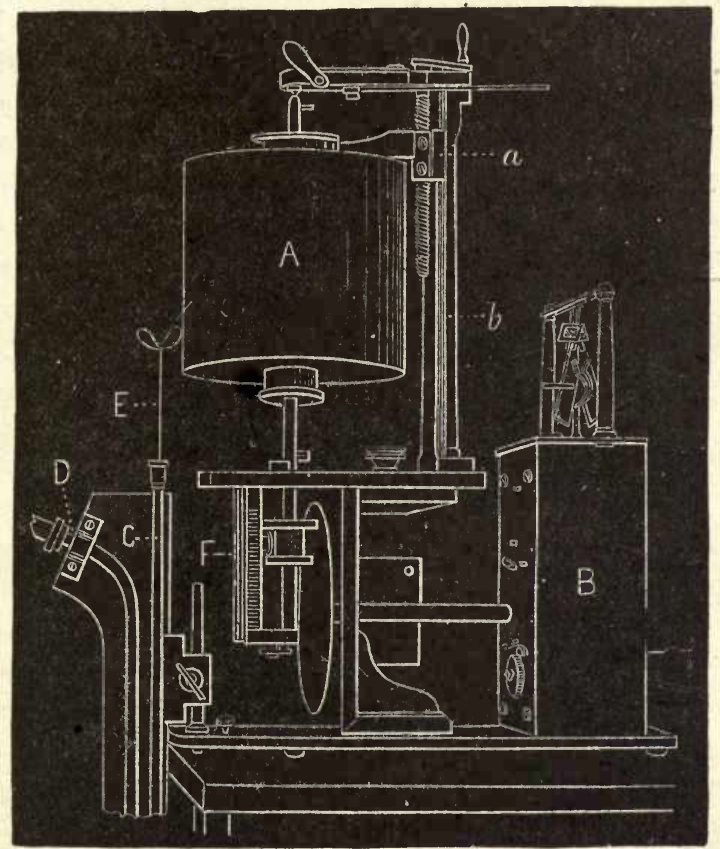

Fig. 173.-Diagram of mercurial kymograph. A, revolving cylinder, worked by a clock-work arrangement contained in the box (B), the speed being regulated by a fan above the box; cylinder supported by an upright $(b)$, and capable of being raised or lowered by a screw $(a)$, by a handle attached to it; $\mathrm{D}, \mathrm{C}, \mathrm{E}$, represent mercurial manometer, a somewhat different form of which is shown in next figure.

ing mercury, or a mercurial manometer is employed, and the artery is made to communicate with it by means of a small canula which is inserted into the vessel, and a connecting tube, an arrangement being made whereby the canula, tubes, etc., are filled with a saturated saline solution to prevent the clotting of blood when it is allowed to pass from the artery into the apparatus. The passage of blood is prevented during the arrangement of the details of the experiment by a pair of clamp or bull-dog forceps. The free end of the U-tube of mercury contains a very fine glass piston, the bulbous end of which floats upon the surface of the mercury, rising with its rise and oscillating with its oscillations. 
As soon as there is free communication between the artery and the tube of mercury, the blood rushes out and pushes before it the column of mercury. 'The mercury will therefore rise in the free limb of the tube, and will continue to do so until a point is reached which corresponds to the mean pressure of the blood-vessel used. The blood-pressure is thus communicated to the upper part of the mercurial column; and the depth to which the latter sinks, added to the height to which it rises in the other, will give the height of the mereurial column which the bloodpressure balances; the weight of the saline solution being subtracted. For the estimation of the amount of blood pressure at any given moment, no further apparatus than this, which is called Poiseuilles's ho-

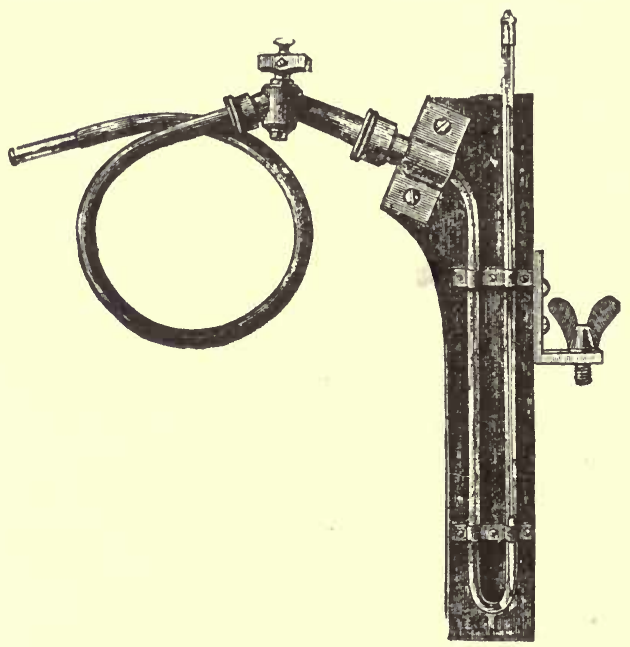

Fig. 174.-Ludwig's Kymograph. The manometer is shown in fig. 173, D. C. E. The mercury which partially fills the tube supports a float in form of a piston, nearly filling the tube; a wire is fixed to the float, and the writing style or pen is guided by passing through the brass cap of the tube fixed to the wire; the pressure is communicated to the mercury by means of a flexible metal tube filled with fluid.

madynamometer, is necessary; but for noting the variations of pressure in the arterial system, as well as its absolute amount, the instrument is usually combined with a recording apparatus, in this form called a kymograph (fig. 1ri3).

The recording apparatus consists of a revolving cylinder (fig. 173, A), which is moved by clockwork, and the speed of which is capable of regulation. The cylinder is covered with glazed paper blackened in the flame of a lamp, and the mercurial manometer is so fixed (fig. 173, D) that its float provided with a style writes on the cylinder as it revolves. There are many ways in which the mercurial manometer may be varied; in fig. 114 is seen a form, which is known as Ludwig's Kymograph. In order to obviate the necessity of a large quantity of blood entering the tube of the apparatus, it is usual to have some arrangement by means 
of which the mercury may be made to rise in the tube of the manometer to the level corresponding to the mean pressure of the artery experimented with, so that the writing style simply records the variations of the blood pressure above and below the mean pressure. This is done by causing the saline solution, generally a saturated solution of sodium carbonate or sulphate, to fill the apparatus from a bottle suspended at a height, and capable of being raised or lowered as required for the purpose, or by injecting the saline solution into the tube by means of a syringe. The canula inserted and tied into the artery may be of two kinds. In one case a fine glass tube is used with the end drawn out and eut so that its end is oblique, and provided with a shoulder to prevent its coming out easily, the peripheral end of the cut artery being tied to obviate the escape of blood. By this means, the pressure communicated to the column of mercury is the forward and not the lateral pressure of blood, or a T-canula may be employed and may be tied into the two ends of a divided artery, and the free arm of the $T$ piece being made

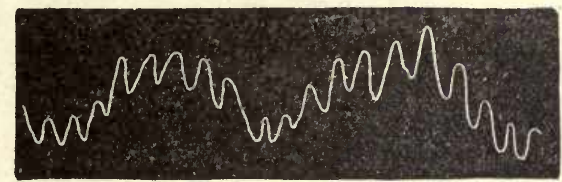

Fig. 175.-Normal tracing of arterial pressure in the rabbit obtained with the mercurial kymograph. The smaller undulations correspond with the heart beats; the larger curves with the respiratory movements. (Burdon-Sanderson.)

to communicate with the manometer. This communicates the lateral blood pressure.

As soon as the experiment is completed, the writing float is seen to oscillate in a regular manner, and a curve of blood pressure is traced upon the smoked paper by the style (or, if a continuous roll of unsmoked paper be used instead, by an inked pen), when a figure similar to fig. 175 will be obtained.

This indicates two main variations of the blood pressure; the smaller excursions of the lever corresponds with the systole and diastole of the heart, and the large curves correspond with the respirations, being called .the respiratory undulations of blood pressure, to which attention will be directed in the next chapter. Of course, the undulations spoken of are only seen in records of arterial blood pressure; they are more clearly marked in the arteries nearer the heart than in those more remote, in the smaller arteries the amount of the pressure as well as the indication of the systolic rise of pressure, being, comparatively speaking, small.

In order to record the undulations of arterial pressure, for some purposes it is better to use Fick's Spring Kymograph than the mercurial manometer. Two forms of this instrument are shown in figs. 176 and 
17\%. It consists of a hollow C-spring, filled with fluid, the interior of which is made to communicate with the artery by means of a flexible metal tube and canula. In response to the pressure, transmitted to its interior, the spring tends to straighten itself. and the movement thus produced is communicated by means of a lever to a writing style and so to a recording apparatus. This instrument obviates the errors which might be caused by the inertia of the mercury in the mercurial manometer; it also shows in more detail the rariations of the blood pressure in the vessel during and after each incividual beat of the heart.

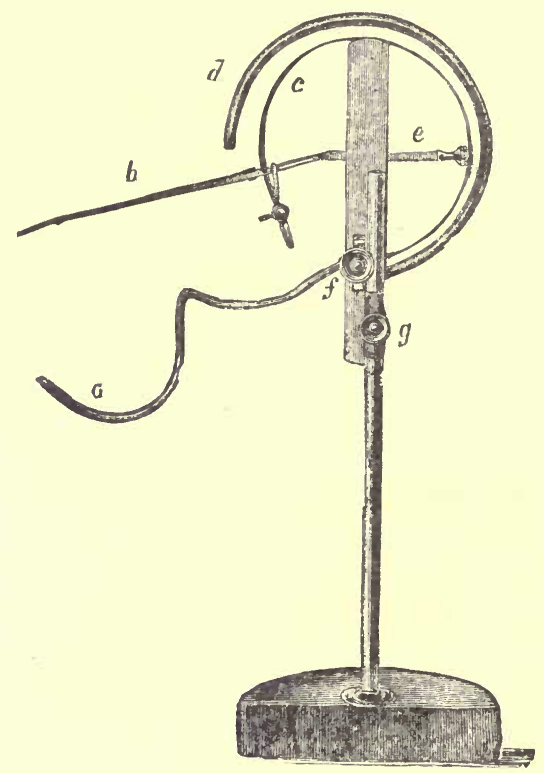

Fig. 176.-A form of Fick's Spring Kymograph. a, Tube to be connected with artery; $c$, hollow spring, the movement of which moves $b$, the writing lever'; $e$, screw to regulate height of $b ; d$, outside protective spring: $g$, serew to fix on the u right of the support.

In fig. 178 is seen a tracing taken with Fick's Kymograph from an artery of a dog.

As regards the actual amount of blood pressure, from observations which have been made by means of the mercurial manometer, it has. been found that the pressure of blood in the carotid of a rabbit is capable of supporting a column of 2 to 3.5 inches ( 50 to $90 \mathrm{~mm}$.) of mercury, in the dog 4 to 7 inches (100 to $175 \mathrm{~mm}$.), in the horse 5 to 8 inches (152 to $200 \mathrm{~mm}$.), and in man the pressure is estimated to be about the same.

To measure the absolute amount of this pressure in any artery, it is necessary merely to multiply the area of its transverse section by the height of the column of mercury which is already known to be sup- 
ported by the blood-pressure in any part of the arterial system. The weight of a column of mercury this found will represent the pressure of the blood. Calculated in this way, the blood-pressure in the human aorta is equal to $4 \mathrm{lbs} .4 \mathrm{oz}$. avoirdupois; that in the aorta of the horse being $11 \mathrm{lb} .9 \mathrm{oz}$; and that in the radial artery at the human wrist only 4 drs. Supposing the muscular power of the right ventricle to be only one-half that of the left, the blood-pressure in the pulmonary artery will be only $2 \mathrm{lb} .2 \mathrm{oz}$. avoirdupois. The amounts above stated represent the arterial tension to the time of the ventricular contraction.

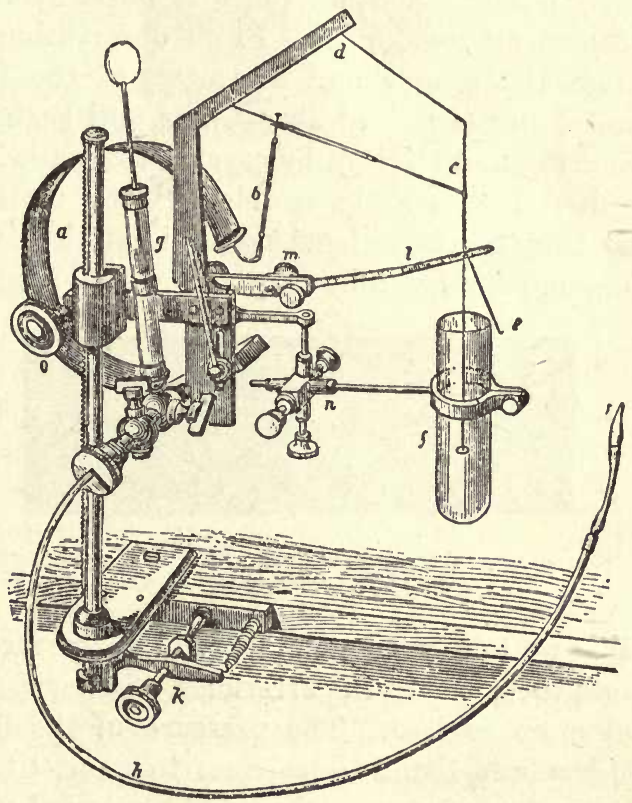

Fig. 177.-Fick's Kymograph, improved by Hering (after McKendrick). $a$, Hollow spring filled with alcohol, bearing lever arrangement $b, d, c$, to which is attached the marker $e$; the rod $c$ passes downward into the tube $f$, containing castor oil, which offers resistance to the oscillations of $c ; g$, syringe for filling the leaden tube $b$ with saturated sulphate of sodium solution, and to apply sufficient pressure as to prevent the blood from passing into the tube $h$ at $i$, the canula inserted into the vessel; $l$, abscissa-marker, which can be applied to the moving surface by turning the screw $m$; $k$, screw for adjusting the whole apparatus to the moving surface; $o$, screw for elevating or depressing by a rack and pinion movement the Kymograph; $n$, screw for adjusting the position of the tube $f$.

The blood-pressure is greatest in the left ventricle and at the beginning of the aorta, and decreases toward the capillaries. It is greatest in the arteries at the period of the ventricular systole. The blood-pressure gradually lessens then as we proceed from the arteries near the heart to those more remote, and again from these to the capillaries, and thence along the veins to the right auricle. The blood-pressure in the veins is nowhere very great, but is greatest in the small veins, while in the large veins toward the heart the pressure becomes negative, or, in other words, when a vein is put in connection with a mercurial man- 
ometer the mereury will iall in the arm furthest away from the vein and will rise in the arm nearest the vein, the action being that of suction rather than pressure forward. In the large veins of the neek the tendency to suek in air is especially marked, and is the eause of death in some surgieal operations in that region. The amount of pressure in the brachial rein is said to support $9 \mathrm{~mm}$. of merenry, whereas the pressure in the reins of the neck may fall to a negative pressure of rather more than $\frac{1}{3}$ inch or - about $\frac{1}{5}$ to $\frac{1}{3}$ inch or -3 to $-8 \mathrm{~mm}$.

The variations of venous pressure during systole and diastole of the heart are very slight, and a distinct pulse is never seen in veins except under extraordinary eircumstances. From observations upon the web of the frog's foot, the tongue and mesentery of the frog, the tails of newts, and small fishes (Roy and Brown), as well as upon the skin of the finger behind the nail (Kries), by careful estimation of the amount of pressure required to empty the ressels of blood under varions conditions, it appears that the blood-pressure is subject to variations in the capillaries, apparently following the variations of that of the

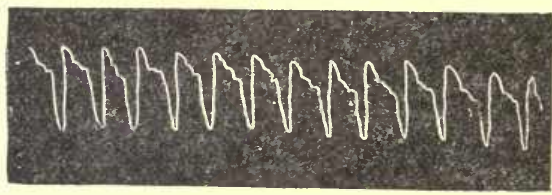

Fig. 178.-Normal arterial tracing obtained with Fick's kymograph in the dog. (Burdon-Sanderson.)

arteries; and that up to a certain point, as the extravascular pressure is increased, so does the pulse in the arterioles, capillaries, and venules become more and more evident. The pressure in the first case (web of the frog's foot) has been found to be equal to about $\frac{1}{2}$ to $\frac{4}{5}$ inch or 14 to $20 \mathrm{~mm}$. of mercury; in other experiments to be equal to about $\frac{1}{5}$ to $\frac{1}{2}$ of the ordinary arterial pressure.

The arterial blood-pressure may be made to vary by variations of either of the two ehief factors upon which the pressure in the ressels depends, viz., the cardiac eontractions and the peripheral resistance. Thus, increase of blood-pressure may be brought about by either (a) a more frequent or more foreible action of the heart, or (b) by inerease of the peripheral resistance; and on the other hand, diminution of the blood-pressure may be produced, either by (a) a diminished force or frequency of the contractions of the heart, or by (b) a diminished peripheral resistance. These different factors, however, although varying constantly, are so combined that the general arterial pressure remains fairly constant; for example, the heart may, by increased force or frequeney of its contractions, distinetly inerease the blood-pressure, but this increased action is almost certainly followed by diminished peripheral 
resistance, and thus the two altered conditions may balance, with the result of bringing back the blood-pressure to what it was before the heart began to beat more rapidly or more forcibly.

It will be clearly seen that the circulation of the blood within the blood-vessels must depend upon the diminution of the pressure from the heart to the capillaries, and from the capillaries to the veins, the blood flowing in the direction of least resistance; we shall presently see further that the general or local flow also depends upon the relations between the heart's action and the peripheral resistance, general or local.

\section{The Arterial Flow.}

The character of the flow of blood through the arterial system depends to a very considerable extent upon the structure of the arterial walls, and particularly upon the elastic tissue which is so highly developed in them.

The elastic tissue first of all guards the arteries from the suddenly exerted pressure to which they are subjected at each contraction of the ventricles. In every such contraction as is above seen the contents of the ventricles are forced into the arteries more quickly than they can be discharged through the capillaries. The blood, therefore, being, for an instant, resisted in its onward course, a part of the force with which it was impelled is directed against the sides of the arteries; under this force their elastic walls dilate, stretching enough to receive the blood, and, as they stretch, becoming more tense and more resisting. Thus, by yielding they break the shock of the force impelling the blood. On the subsidence of the pressure, when the ventricles cease contracting, the arteries are able, by the same elasticity, to resume their former calibre; the elastic tissue also equalizes the cnrrent of blood by maintaining pressure on it in the arteries during the period at which the ventricles are at rest or are dilating. If the arteries were rigid tubes, the blood, instead of flowing, as it does, in a constant stream, would be propelled through the arterial system in a series of jerks corresponding to the ventricular contractions, with intervals of almost complete rest during the inaction of the ventricles. But in the actual condition of the vessels, the force of the successive contractions of the ventricles is expended partly in the direct propulsion of the blood, and partly in the dilatation of the elastic arteries; and in the intervals between the contractions of the ventricles, the force of the recoil is employed in continuing the onward flow. Of course the pressure exercised is eqnally diffused in every direction, and the blood tends to move backward as well as onward; all movement backward, however, is prevented by the closure of the semilunar valves, which takes place at the very commencement of the recoi] of the arterial walls. 
Thus by the exercise of the elasticity of the arteries, all the force of the ventricles is expended upon the circulation; for that part of the force which is used up or rendered potential in dilating the arteries is restored or made active or kinetic, in full when they recoil. There is no loss of force; neither is there any gain, for the elastic walls of the artery cannot originate any force for the propulsion of the blood-they only restore that which they received from the ventricles. It is by this equalizing influence of the successive branches of every artery that at length the intermittent accelerations produced in the arterial current by the action of the heart, cease to be observable, and the jetting stream is converted into the continuous and equable movement of the blood which we see in the capillaries and veins. In the production of a continuous stream of blood in the smaller arteries and capillaries, the resistance which is offered to the blood-stream in these vessels is a necessary agent. Were there no greater obstacle to the escape of blood from the larger arteries than exists to its entrance into them from the heart, the stream would be intermittent, notwithstanding the elasticity of walls of the arteries.

By means of the elastic and muscular tissue in their walls again the arteries are enabled to dilate and contract readily in correspondence with any temporary increase or diminution of the total quantity of blood in the body; and within a certain range of diminution of the quantity, still to exercise due pressure on their contents. The elastic tissue further assists in restoring the normal channel after diminution of its calibre, whether this has been caused by a contraction of the muscular coat, or by the temporary application of a compressing force from without. This action is well shown in arteries which, having contracted by means of their muscular element, after death regain their average potency on the cessation of post-mortem rigidity.

The office of the muscular coat also is employed to adjust the flow of the blood locally, to regulate the quantity of blood to be received by each part or organ, and to adjust it to the requirements of each, according to various circumstances, but, chiefly, according to the activity with which the functions of each are at different times performed. The amount of work done by each organ of the body varies at different times, and the variations often quickly succeed each other, so that, as in the brain, for example, during sleep and waking, within the same hour a part may be now very active and then inactive. In all its active exercise of function, such a part requires a larger supply of blood than is sufficient for it during the times when it is comparatively inactive. It is evident that the heart cannot regulate the supply to each part at different periods; neither could this be regulated by any general and uniform contraction of the arteries; but it may be regulated by the power which the arteries of each part have, in their muscular tissue, of cor- 
tracting so as to diminish, and of passively dilating or yielding so as to permit an increase of, the supply of blood, according to the requirements of the part to which they are distributed. And thus, while the ventricles of the heart determine the total quantity of blood, to be sent onward at each contraction, and the force of its propulsion, and while the large and merely elastic arteries distribute it and equalize its stream, the smaller arteries, in addition, regulate and determine, by means of their muscular tissue, the proportion of the whole quantity of blood which shall be distributed to each part.

This regulating function of the arteries is governed and directed by the nervous system in the way to be presently described.

The muscular element of the middle coat also co-operates with the elastic in adapting the calibre of the vessels to the quantity of blood which they contain. For the amount of fluid in the blood-vessels varies very considerably even from hour to hour, and can never be quite constant; and were the elastic tissue only present the pressure exercised by the walls of the containing vessels on the contained blood would be sometimes very small, and sometimes inordinately great. The presence of a muscular element, however, provides for a certain uniformity in the amount of pressure exercised; and it is by this adaptive, uniform, gentle, muscular contraction, that the normal tone of the blood-vessels is maintained. Deficiency of this tone is the cause of the soft and yielding pulse, and its unnatural excess of the hard and tense one.

The elastic and muscular contraction of an artery may also be regarded as fulfilling a natural purpose when, the artery being cut, it first limits and then, in conjunction with the coagulated fibrin, arrests the escape of blood. It is only in consequence of such contraction and coagulation that we are free from danger through even very slight wounds; for it is only when the artery is closed that the processes for the more permanent and secure prevention of bleeding are established. But there appears no reason for supposing that the muscular coat assists, to more than a very small degree, in propelling the onward current of blood.

\section{The Pulse.}

The most characteristic feature, then, of the arterial flow, is its intermittency, and this intermittent flow is seen or felt as the Pulse.

The pulse is generally described as an expansion of the artery produced by the wave of blood set in motion by the injection of blood at each ventricular systole into the already full aorta. As the force of the left ventricle, however, is not expended in dilating the aorta only, the wave of blood passes on, expanding the arteries as it goes, running as it were on the surface of the more slowly travelling blood already contained in them, and producing the pulse as it proceeds. 
The distention of eich artery increases both its length and its diameter. In their elongation, the arteries change their form, the straight ones becoming slightly curved, and those already eurved becoming more so; but they recover their previous form as well as their diameter when the ventricular contraction ceases, and their elastic walls recoil. The increase of their curves which accompanies the distention of arteries, and the succeeding recoil, may be well seen in the prominent temporal artery of an old person. In feeling the pulse, the finger cannot distinguish the sensation produced by the dilatation from that produced by the elongation and curving; that which it perceives most plainly, however, is the dilatation, or return, more or less, to the cylindrical form, of the artery which has been partially flattened by the finger.

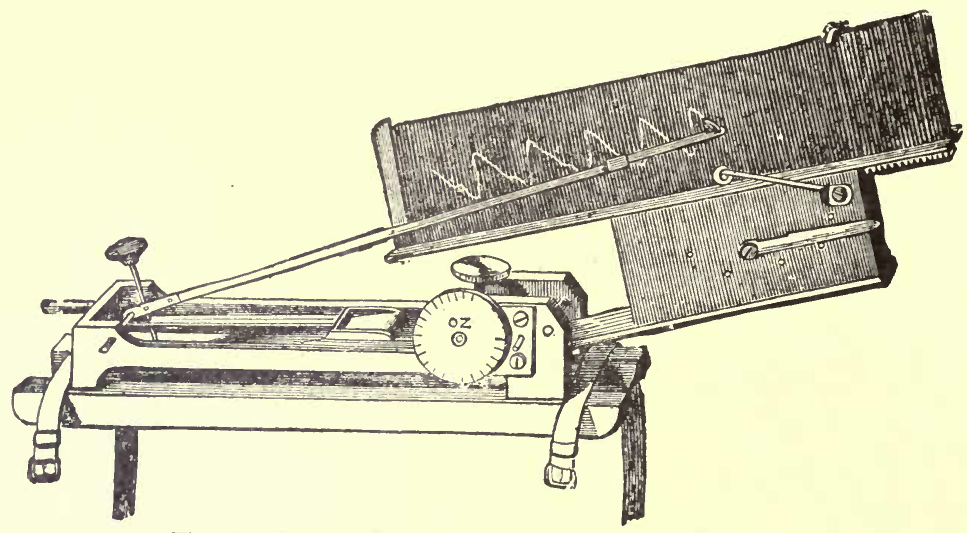

Fig. 1\%9.-Marey's Sphygmograph, modified by Mahomed.

The pulse--due to any given beat of the heart-is not perceptible at the same moment in all the arteries of the body. Thus, it can be felt in the carotid a very short time before it is perceptible in the radial artery, and in this ressel again before it occurs in the dorsal artery of the foot. The delay in the beat is in proportion to the distance of the artery from the heart, but the difference in time between the beat of any two arteries probably never exceeds $\frac{1}{6}$ to $\frac{1}{8}$ of a second.

A distinction must be carefully made between the passage of the wave along the arteries and the arterial flow itself. Both wave and current are present; but the rates at which they travel are very different, that of the wave 16.5 to 33 feet per second (5 to 10 metres), being twenty or thirty times as great as that of the current.

The Sphygmograph.-Much light has been thrown on what may be called the form of the pulse wave by the sphygmograph (figs. 179 and 182). The principle on which it acts will be seen on reference to figs.

The small button replaces the finger in the act of taking the pulse, 
and is made to rest lightly on the artery, the pulsations of which it is desired to investigate. The up-and-down movement of the button is communicated to the lever, to the hinder end of which is attached a slight spring, which allows the lever to move up, at the same time that

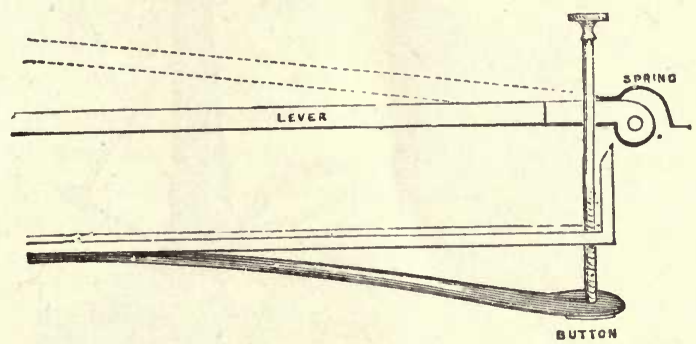

Fig. 180.-Diagram of the lever of the Sphygmograph.

it is just strong enough to resist its making any sudden jerk, and in the interval of the beats also to assist in bringing it back to its original position. For ordinary purposes the instrument is bound on the wrist (fig. 181).

It is evident that the beating of the pulse with the reaction of the spring will cause an up-and-down movement of the lever, the pen of which will write the effect on a smoked eard, which is made to move by clockwork in the direction of the arrow. Thus a tracing of the pulse is obtained, and in this way much more delicate effects can be seen than can be felt on the application of the finger.

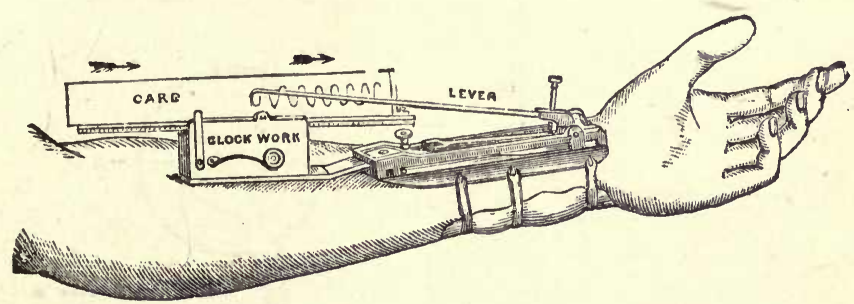

Fig. 181.-The Sphygmograph applied to the arm.

Two forms of sphygmograph are shown in figs. 179, 182, viz., a modification of the original instrument of Marey and Dudgeon's. Marey's instrument, and indeed all modifications of it, suffer from the defect that there is no adequate method of measuring the pressure exercised by the button of the instrument upon the artery, and that it is difficult to be certain of the exact position it occupies over the artery. Dudgeon's sphygmograph, although very convenient to use, is, according to Roy and Adami, even less satisfactory, and the tracings obtained by it are so disfigured by inertia vibrations as to render them more or less worthless. "The mechanical construction of the instrument is such as to render great inertia vibrations unavoidable." These authors have 
invented an instrument called a sphygmometor, in which these defects of the sphygmograph are corrected.

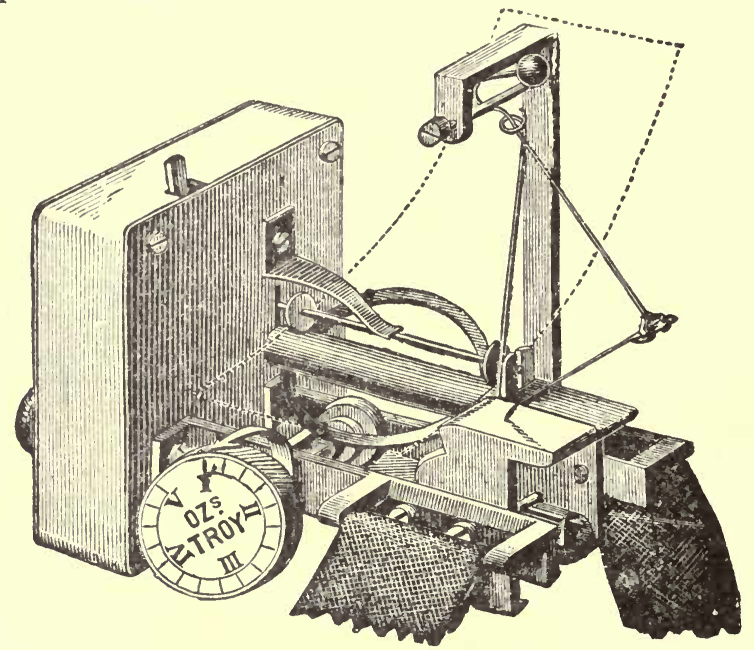

Fig. 182.-Dudgeon's Sphygmograph.

The principle of the sphygmometer of Roy and Adami is shown in the diagram (fig. 183).

The apparatus consists of a box $(a)$ which is moulded to fit over the end of the radius so as to bridge over the radial artery. Within this is a flexible bag (b) filled with water, and connected by a $T$ tube with a rubber bag $(h)$ and

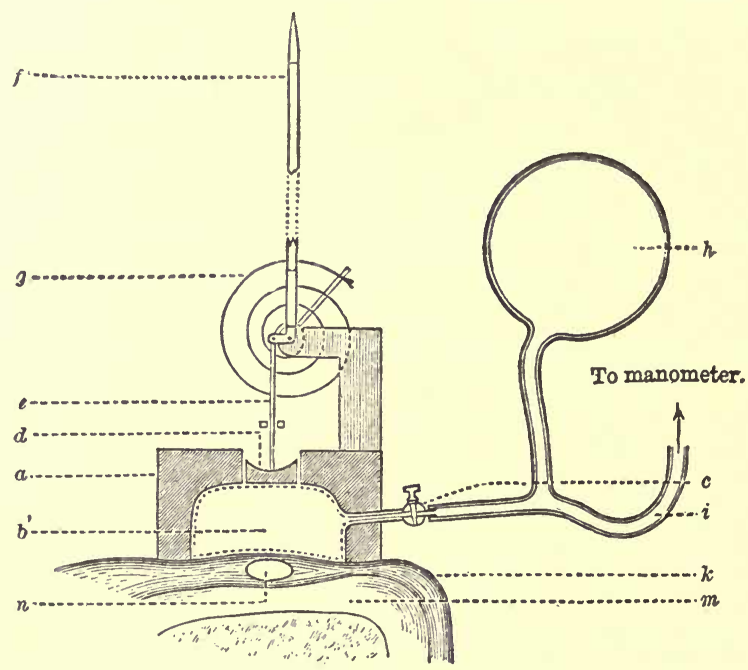

Fig. 183.-Diagrammatic sectional representation of the sphygmometer (Roy and Adami). $a$, Box in which the portion of the artery is jnclosed; $b$, thin-walled india-rubber bag filled with water, and communicating through tap, $c$, with mannmeter and thick-walled rubber bag, $h ; d$, piston con. nected by rod, $e$, with recording lever, $f: g$. spiral spring attached to axis of lever, and by which the pressure in $b$, against the piston, $d$, is counterbalanced; $k$, skin and subcutaneous tissue; $m$, end of radius seen in section; $n$, radial artery seen in section. 
mercurial manometer. The fluid in the box may be raised to any desired pressure, and may then be shut off by tap (c). At the upper part of the box is a circular opening, and resting upon $(b)$ is a flat button $(d)$, which by means of a short light rod $(e)$ communicates the movement of $(b)$ to the lever $(f)$. To the axis of rotation of this lever is a spiral watch-spring $(g)$ which can be tightened at will, so that the lever can be made to take a vertical position at any desired hydrostatic pressure within the box. The movements of the lever are recorded upon a piece of blackened glazed paper made to move in a vertical direction past it. When in use, the box is fixed upon the end of the radius by an appropriate holder, and the pressure is raised to any desired height to which the lever is adapted by tightening or slackening the spring. The tap $(c)$ is then closed. The pressure within the box acts in all directions, and is correctly indicated by the manometer.

The tracing of the pulse (sphygmogram), obtained by the use of the sphygmograph, differs somewhat according to the artery upon which it is applied, but its general characters are much the same in all cases. It consists of:-A sudden upstroke (fig. 184, A), which is somewhat

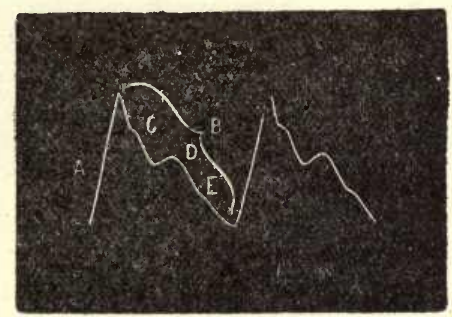

Fig. 184.-Diagram of pulse tracing. A, Up-stroke; B, down-stroke; c, pre-dicrotic wave; D, dicrotic; E, post-dicrotic wave.

higher and more abrupt in the pulse of the carotid and of other arteries near the heart than in the radial and other arteries more remote; and a gradual decline (B), less abrupt, and therefore taking a longer time than (A). It is seldom, however, that the decline is an uninterrupted fall; it is usually marked about half-way by a distinct notch (c), called the dicrotic notch, which is caused by a second more or less marked ascent of the lever at that point and by a second wave called the dicrotic wave (D); not unfrequently there is also soon after the commencement of the descent a slight ascent previous to the dicrotic notch: this is called the pre-dicrotic wave (c), and in addition there may be one or more slight ascents after the dicrotic, called post-dicrotic (E).

The interruptions in the downstroke are called the katacrotic waves, to distinguish them from an interruption in the upstroke, the anacrotic wave, which is sometimes met with.

The explanation of these tracings presents some difficulties, not, however, as regards the two primary factors, viz., the upstroke and downstroke, because they are universally taken to mean the sudden in- 
jection of blood into the already full arteries, and the gradual fall of the lever signifying the recovery of the arteries by their recoil. These points may be demonstrated on a system of clastic tubes, with a syringe to pump in water at regular intervals, just as well as on the radial artery, or on the more complicated system of tubes in which the heart, the arteries, the capillaries and veins are represented, which is known as an arterial scheme. If we place two or more sphygmographs upon such a system of tubes at increasing distances from the pump, we may demoustrate first, that the rise of the lever commences earliest in that nearest the pump, and secondly, that it is higher and more sudden, while at a longer distance from the pump the ware is less marked, and a little later. So in the arteries of the body the wave of blood gradually gets less and less as we approach the periphery of the arterial system, and is lost in the capillaries.

The origin of the secondary waves is still a matter of uncertainty.

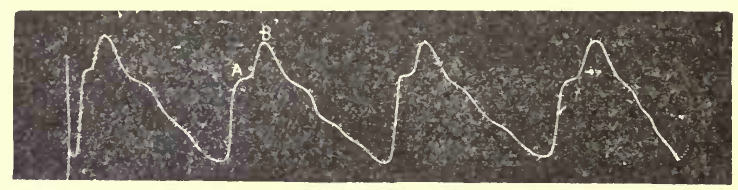

Fig. 185.-Anacrotic pulse from a case of aortic aneurism.

The anacrotic wave occurs when the peripheral resistance is high; that is, when, for some tine during the systole, the flow from the aorta toward the periphery is slower than the flow from the ventricle into the aorta. Thus, it is seen in some cases of nephritis where the arteries are rigid and the peripheral resistance high.

'The dicrotic wave is the most important of the secondary waves, and has been the subject of much discussion. It is constantly present in pulse-tracings, but varies in height. In point of time the dicrotic wave occurs immediately after the closure of the aortic semilunar valves. In certain conditions, generally of disease, it becomes so marked as to be quite plain to the unaided finger. Such a pulse is called dicrotic. The most generally accepted view of the cause of the dicrotic wave is that it represents a rebound from the closed aortic valves. During systole, as the blood is forcibly injected into the aorta, there is as it were an overdistention of the artery. The systole suddenly ends, the aorta by reason of its elasticity tends to recover itself, the blood is driven back against the semilunar valves, closing them and at the same time giving rise to a wave-the dicrotic wave-which begins at the heart and travels onward toward the periphery like the primary wave. According to Fos- 
ter, the conditions favoring the development of dicrotism are: (1) a highly extensible and elastic arterial wall; (2) a comparatively low mean blood pressure, leaving the extensible reaction free scope to act; (3) a vigorous and rapid stroke of the ventricle, discharging into the aorta a considerable quantity of blood.

The other secondary waves are probably due to the elastic recoil of the arteries, though some of them at least may be due to the inertia of the instruments used.

A

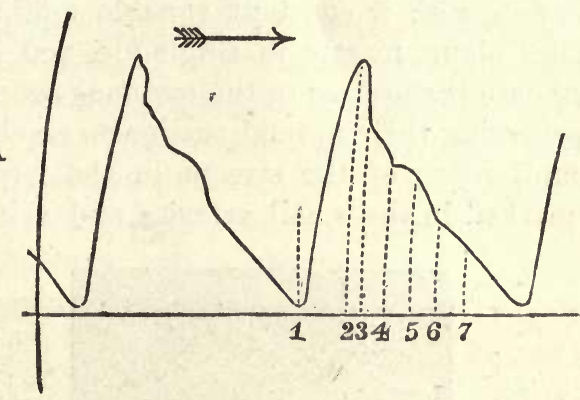

B

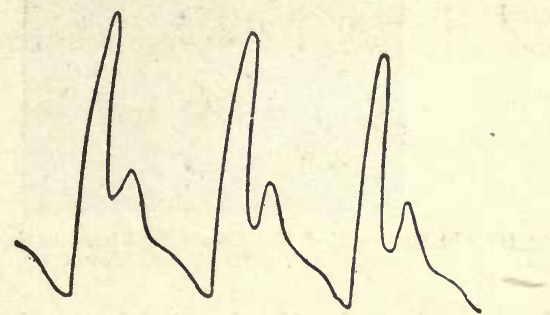

Fig. 186.-A, Normal pulse-tracing from radial of healthy adult, obtained by the sphygmometer. B, From same artery, with the same extra-arterial pressure, taken during acute nasal catarrh.

In the use of the sphygmograph care must be taken as to the careful regulation of the pressure. If the pressure be too great, the characters of the pulse may be almost entirely obscured, or the artery may be entirely obstructed, and no tracing is obtained; and on the other hand, if the pressure is too slight, a very small part of the characters may be represented on the tracing. 


\section{The Capillary Flow.}

It is in the capillaries that the chief resistance is offered to the prog. ress of the blood; for in them the friction of the blood is greatly in. creased by the enormous multiplication of the surface with which it is brought in contact.

When the capillary circulation is examined in any transparent part of a full-grown living animal by means of the microscope (fig. 18\%), the blood is seen to flow with a constant equable motion; the red bloodcorpuscles moving along, mostly in single file, and bending in various ways to accommodate themselves to the tortuous course of the capillary, but instantly recovering their normal outline on reaching a wider ressel.

At the circumference of the stream in the larger capillaries, but sepecially well marked in the small arteries and reins, in contact with

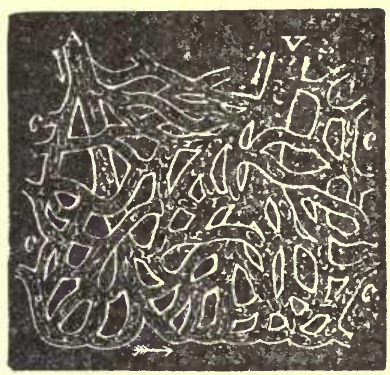

Fig. 18\%.-Capillaries (C.) in the web of the frog`s foot connecting a small artery (A) with a small vein V (after Allen Thomson).

the walls of the vessel, and adhering to them, there is a layer of liquor sanguinis which appears to be motionless. The existence of this still layer, as it is termed, is inferred both from the general fact that such an one exists in all fine tubes traversed by fluid, and from what can be seen in watching the morements of the blood-corpuscles. The red corpuscles occupy the middle of the stream and move with comparative rapidity; the colorless corpuscles run much more slowly by the walls of the vessel; while next to the wall there is often a transparent space in which the fluid appears to be at rest; for if any of the corpuscles happen to be forced within it, they move more slowly than before, rolling lazily along the side of the vessel, and often adhering to its wall. Part of this slow movement of the colorless corpuscles and their occasional stoppage may be due to their haring a natural tendency to adhere to the walls of the vessels. Sometimes, indeed, when the motion of the blood is not strong, many of the white corpuscles collect in a capillary vessel, and for a time entirely prevent the passage of the red corpuscles. 
The white corpuscles pass through the capillary wall chiefly by the amoboid movement with which they are endowed. This migration occurs to a limited extent in health, but in inflammatory conditions is much increased.

The process of diapedesis of the red corpuscles, which occurs under circumstances of impeded renous circulation, and consequently increased blood-pressure, resembles elosely the migration of the lencocytes, with the exception that they are squeezed through the wall of the vessel, and do not, like them, work their way through by amoboid morement.

Varions explanations of tr:se remarkable phenomena have been suggested. Some beliere that psendo-stomata between contignous endothelial cells provide the means of escape for the blood-corpuscles. But the chief share in the process is probably to be found in the vital endowments with respect to mobility and contraction of the parts concerned-both of the corpuscles and of the capillary wall itself.

The eireulation throngh the eapillaries must. of neeessity, be largely influenced by that which oceurs in the ressels on either side of themin the arteries or the reins; their intermediate position cansing them to feel at once, so to speak, any alteration in the size or rate of the arterial or venous blood-stream. Thus, the apparent contraction of the eapillaries, on the application of certain irritating snbstances, and during fear, and their dilatation in blushing may be referred primarily to the action of the small arteriss.

\section{The Venous Flow.}

The blood-current in the veins is maintained (a) primarily by the vis a tergo of the contraction of the left ventricle; but very effectual assistance to the flow is afforded (b) by the action of the muscles capable of pressing on the veins with valves, as well as (c) by the suction action of the heart, and (e) aspiration of the thorax.

'The effect of muscular pressure upon the circulation may be thus explained. When pressure is applied to any part of a vein, and the current of blood in it is obstrneted, the portion behind the seat of pressure becomes swollen and distended as far back as the next pair of valves, which are in consequence closed. Thus, whatever force is exercised by the pressure of the muscles on the veins, is distributed partly in pressing the blood onward in the proper course of the circulation, and partly in pressing it backward and closing the valves behind.

The circulation might lose as much as it gains by such an action, if it were not for the numerous communieations, one with another; for through these, the closing up of the renous ehamel by the backward 
pressure is prevented from being any serious hindrance to the circulation, since the blood, of which the onward course is arrested by the closed valves, ean at once pass through some anastomosing channel, and proceed on its way by another vein. Thus, therefore, the effect of muscular pressure upon veins which have valves, is turned almost entirely to the advantage of the circulation; the pressure of the blood onward is all advantageous, and the pressure of the blood backward is prevented from being a hindrance by the closure of the valves and the anastomoses of the veins.

The venous flow is also assisted by the suction action of the heart, since at some time during every cardiac cycle the pressure falls below that of the atmosphere.

The aspiration of the thorax will be considered more fully in the chapter on Respiration. In this connection it may be said, however, that the pressure in the great veins falls during inspiration and rises during expiration.

\section{The Velocity of the Flow.}

The velocity of the blood-current at any given point in the various divisions of the circulatory system is inversely proportional to their sectional area at that point. If the sectional area of all the branches of a vessel united were always the same as that of the vessel from which they arise, and if the aggregate sectional area of the capillary vessels were equal to that of the aorta, the mean rapidity of the blood's motion in the capillaries would be the same as in the aorta; and if a similar correspondence of capacity existed in the veins and arteries, there would be an equal correspondence in the rapidity of the circulation in them. But the arterial and venous systems may be represented by two truncated cones with their apices directed toward the heart; the area of their united base (the sectional area of the capillaries) being 400-800 times as great as that of the truncated apex representing the aorta. Thus the velocity of blood in the capillaries is not more than $\frac{1}{400}$ of that in the aorta.

In the Arteries. - The velocity of the stream of blood is greater in the arteries than in any other part of the circulatory system, and in them it is greatest in the neighborhood of the heart, and during the ventricular systole. The rate of movement diminishes during the diastole of the ventricles, and in the parts of the arterial system most distant from the heart. Chauveau has estimated the rapidity of the blood-stream in the carotid of the horse at over 20 inches per second during the heart's systole, and nearly 6 inches during the diastole (520-150 mm.). 
Estimation of the Velocity.-Various instruments have been devised for measuring the velocity of the blood-stream in the arteries. Ludwig's Stromuhr (fig. 189) consists of a $U$-shaped glass tube dilated at $a$ and $a^{\prime}$, the ends of which, $h$ and $i$, are of known calibre. The bulbs can be filled by a common opening at $k$. The instrument is so contrived that at $b$ and $b^{\prime}$ the glass part is

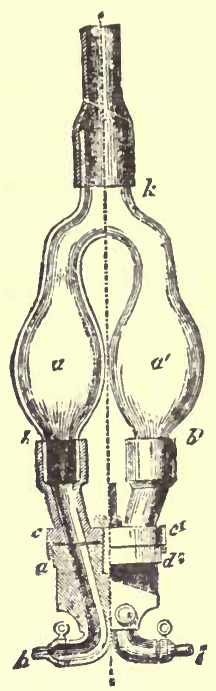

Fig. 189. firmly fixed into metal cylinders, attached to a circular horizontal table, $c c^{\prime}$, capable of horizontal movement on a similar table $d d^{\prime}$ about the vertical axis marked in figure by a dotted line. The opening in $c c$, when the instrument is in position, as in fig., corresponds exactly with those in $d d^{\prime}$; but if $c c^{\prime}$ be

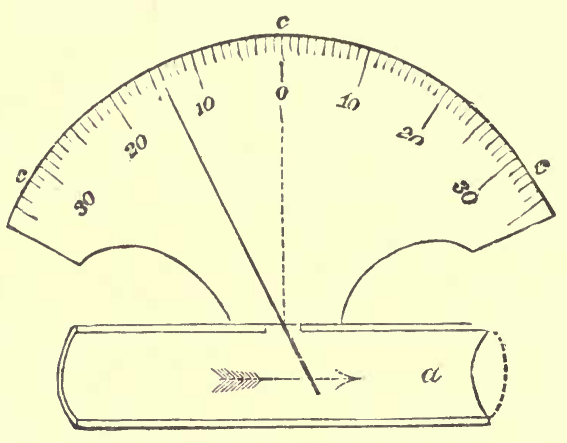

Fig. 190.

Fig. 189.-Ludwig's Stromuhr.

Fig. 190.-Diagram of Chauveau's Instrument. $a$. Brass tube for introduction into the lumen of the artery, and containing an index needle, which passes through the elascic membrane in its side, and moves by the impulse of the blood-current. $c$. Graduated scale, for measuring the extent of the oscillations of the needle.

turned at right angles to its present position, there is no communication between $h$ and $a$, and $i$ and $a^{\prime}$, but $h$ communicates directly with $i$; and if turned through two right angles $c^{\prime}$ communicates with $d$, and $c$ with $d$, and there is no direct communication between $h$ and $i$. The experiment is performed in the following way :-The artery to be experimented upon is disided and connected with two canulæ and tubes which fit it accurately with $h$ and $i-h$ the central end, and $i$ the peripheral ; the bulb $a$ is filled with olive oil up to a point rather lower than $k$, and $\alpha^{\prime}$ and the remainder of $a$ is filled with defibrinated blood; the tube on $k$ is then carefully clamped: the tubes $d$ and $d^{\prime}$ are also filled with defibrinated blood. When everything is ready, the blood is allowed to flow into $a$ through $h$, and it pushes before it the oil, and that the defibrinated blood into the artery through $i$. and replaces it in $\alpha^{\prime}$; when the blood reaches the former level of the oil in $a^{\prime}$, the disc $c c^{\prime}$ is turned rapidly through two right angles, and the blood flowing through $d$ into $a^{\prime}$ again displaces the oil which is driven into $a$. This is repeated several times, and the duration of the experiment noted. The capacity of $a$ and $a^{\prime}$ is known; the diameter of the artery is also known by its corresponding with the canulæ of known diameter, and as the number of times $a$ has been filled in a giren time is known, the velocity of the current can be calculated. 
Chauveau's instrument, fig. 190, consists of a thin brass tube, $a$ in one side of which is a small perforation closed by thin vulcanized india-rubber. Passing through the rubber is a fine lever, one end of which, slightly flattened, extends into the lumen of the tube, while the other moves over the face of a dial. The tube is inserted into the interior of an artery, and ligatures applied to fix it, so that the movement of the blood may, in flowing through the tube, be indicated by the movement of the outer extremity of the lever on the face of the dial.

The Hoematochometer of Vierordt, and the instrument of Lortet, resemble in principle that of Chauveau.

In the Capillaries. - The observation of Hales, E. H. Weber, and Valentin agree very closely as to the rate of the blood-current in the capillaries of the frog; and the mean of their estimates gives the velocity of the systemic capillary circulation at about one inch $(25 \mathrm{~mm}$.) per minute. The velocity in the capillaries of warm-blooded animals is greater. In the $\operatorname{dog} \frac{1}{50}$ to $\frac{3}{100}$ inch $(.5$ to $.75 \mathrm{~mm}$.) a second. This may seem inconsistent with the facts, which show that the whole circulation is accomplished in about half a minute. But the whole length of capillary vessels, through which any given portion of blood has to pass, probably does not exceed from $\frac{1}{30}$ th to $\frac{1}{50}$ th of an inch $(.5 \mathrm{~mm}$.); and therefore the time required for each quantity of blood to traverse its own appointed portion of the general capillary system will scarcely amount to a second.

In the Veins. - The velocity of the blood is greater in the veins than in the capillaries, but less than in the arteries: this fact depending upon the relative capacities of the arterial and venous systems. If an accurate estimate of the proportionate areas of arteries and the veins corresponding to them could be made, we might, from the velocity of the arterial current, calculate that of the venous. A nsual estimate is, that the capacity of the veins is about twice or three times as great as that of the arteries, and that the velocity of the blood's motion is, therefore, about twice or three times as great in the arteries as in the veins, 8 inches (200 mm.) a second. The rate at which the blood moves in the veins gradually increases the nearer it approaches the heart, for the sectional area of the venous trunks, compared with that of the branches opening into them, becomes gradually less as the trunks advance toward the heart.

Of the Circulation as a Whole.-It would appear that a portion of blood can traverse the entire course of the circulation, in the horse, in half a minute. Of course it would require longer to traverse the vessels of the most distant part of the extremities than to go through those of the neck; but taking an average length of vessels to be traversed, it may be concluded that half a minute represents the average rate. Stewart states that the circulation time in man is probably not less than twelve nor more than fifteen seconds. 
Satisfactory data for these estimates are afforded by the results of experiments to ascertain the ripidity with which poisons introduced into the blood are transmitted from one jart of the vascular system to another. The time required for the passage of a solution of potassium ferrocyanide, mixed with the blood, from one jugular vein (through the right side of the heart, the pulmonary vessels, the left carities of the heart, and the general circulation) to the jugular vein of the opposite side, varies from twenty to thirty seeonds. The same substance was transmitted from the jugular vein to the great saphena in twenty seconds; from the jugular vein to the masseteric artery in between fifteen and thirty seconds; to the facial artery, in one experiment, in letween ten and fifteen seconds; in another experiment in between twenty and twenty-five seconds; in its transit from the jugular vein to the metatarsal artery, it ocenpied between twenty and thirty seconds, and in one instance more than forty seconds. The result was nearly the same whatever was the rate of the heart's action.

In all these experiments, it is assumed that the substance injected moves with the blood, and at the same rate, and does not move from one part of the organs of eirculation ts another by diffusing itself through the blood or tissues more quickly than the blood mores. The assumption is sufficiently probable to be considered nearly certain, that the times above mentioned, as occupied in the passage of the injeeted substances, are those in which the portion of blood, into which each was injected, was earried from one part to another of the vascular system.

Another mode of estimating the general velocity of the circulating blood, is by calculating it from the quantity of blood supposed to he contained in the body, and from the quantity which can pass through the heart in each of its actions. But the conclusions arrived at by this method are less satisfactory. For the total quantity of blood, and the capacity of the cavities of the heart, have as yet been only approximately ascertained. Still tise most careful of the estimates thus made accord very nearly with those already mentioned; and it may be assumed that the blood may all pass through the heart in abont twenty-five seconds.

\section{Local Peculiarities of the Circulation.}

The most remarkable peculiarities attending the cireulation of llood throngh different organs are observed in the cases of the brain, the arectile organs, the lungs, the liver, and the kidneys.

In the Brain.-For the due performance of its functions the hrain requires a large supply of blood. 'Ihis object is effected through the number and size of its arteries, the two internal carotids, and the two vertebrals. It is further necessary that the force with which this blood 
is sent to the brain should be less, or at least should be subject to less variation from external circumstances than it is in other parts, and so the large arteries are very tortuous and anastomose freely in the circle of Willis, which thus insures that the supply of blood to the brain is uniform, though it may by an accident be diminished, or in some way changed, through one or more of the principal arteries. The transit of the large arteries through bone, especially the carotid canal of the temporal bone, may prevent any undue distention; and uniformity of supply is further insured by the arrangement of the vessels in the pia mater, in which, previous to their distribution to the substance of the brain, the large arteries break up and divide into innumerable minute branches ending in capillaries, which, after frequent communication with one another, enter the brain, and carry into nearly every part of it uniform and equable streams of blood. The arteries are also enveloped in a special lymphatic sheath. The arrangement of the veins within the cranium is also peculiar. The large venous trunks or sinuses are formed so as to be scarcely capable of change of size; and composed, as they are, of the tough tissue of the dura mater, and, in some instances bounded on one side by the bony cranium, they are not compressible by any force which the fulness of the arteries might exercise through the substance of the brain; nor do they admit of distention when the flow of venous blood from the brain is obstructed.

The general uniformity in the supply of blood to the brain, which is thus secured, is well adapted, not only to its functions, but also to its condition as a mass of nearly incompressible substance placed in a cavity with unyielding walls. These conditions of the brain and skull formerly appeared, indeed, enough to justify the opinion that the quantity of blood in the brain must be at all times the same. But it was found that in animals bled to death, without any aperture being made in the cranium, the brain became pale and anæmic like other parts. And in death from strangling or drowning, there was congestion of the cerebral vessels; while in death by prussic acid, the quantity of blood in the cavity of the cranium was determined by the position in which the animal was placed after death, the cerebral vessels being congested when the animal was suspended with its head downward, and comparatively empty when the animal was kept suspended by the ears. Thus, it was concluded, although the total volume of the contents of the cranium is probably nearly always the same, yet the quantity of blood in it is liable to variation, its increase or diminution being accompanied by a simultaneous diminution or increase in the quantity of the cerebro-spinal fluid, which, by readily admitting of being removed from one part of the brain and spinal cord to another, and of being rapidly absorbed, and 
as readily effused, would serve as a kind of supplemental fluid to the other contents of the cranium, to keep it uniformly filled in case of variations in their quantity. And there can be no doubt that, although the arrangements of the blood-vessels, to which reference has been made, insure to the brain an amount of blood which is tolerably uniform, yet, inasmuch as with every beat of the heart and erery act of respiration and under many other circumstances, the quantity of blood in the cavity of the cranium is constantly varying, it is plain that, were there not provision made for the possible displacement of some of the contents of the unyielding bony case in which the brain is contained, there would be often alternations of excessive pressure with insufficient supply of blood.

Chemical Composition of Cerebro-spinal Fluid.-The cerebro-spinal fluid is transparent, colorless, not viscid, with a saline taste and alkaline reaction, and is not affected by heat or acids. It contains 981-984 parts water, sodium chloride, traces of potassium chloride, of sulphates, carbonates, alkaline and earthy phosphates, minute traces of urea, sugar, sodium lactate, fatty matter, cholesterin, and albumen (Flint).

In Erectile structures.-The instances of greatest variation in the quantity of blood contained, at different times, in the same organs, are found in eertain struetures which, under ordinary circumstances, are soft and flaceid, but, at certain times, receive an unusually large quantity of blood, become distended and swollen by it, and pass into the state which has been termed erection. Such structures are the corpora cavernosa and corpus spongiosum of the penis in the male, and the clitoris in the female; and, to a less degree, the nipple of the mammary gland in both sexes. The corpus eavernosum penis, which is the best example of an erectile structure, has an external fibrous membrane or sheath; and from the inner surface of the latter are prolonged numerous fine lamellæ which divide its cavity into small compartments looking like cells when they are inflated. Within these is situated the plexus of veins upon which the peculiar erectile property of the organ mainly depends. It consists of short veins which very closely interlace and anastomose with each other in all directions, and admit of great variations of size, collapsing in the passive state of the organ, but, for erection, capable of an amount of dilatation which exceeds beyond comparison that of the arteries and reins which convey the blood to and from them. The strong fibrous tissue lying in the intervals of the venous plexuses, and the external fibrous membrane or sheath with which it is connected, limit the distention of the vessels, and, during the state of erection, give to the penis its condition of tension and firmness. 'T'he same general condition of vessels exists in the corpus spongiosum urethræ, but around the urethra the fibrous tissue is much weaker than around the body of the penis, and around the glans there is none. The venous blood is 
returned from the plexuses by comparatively small veins; those from the glans and the fore part of the urethra empty themselves into the dorsal veins of the penis; those from the cavernosum pass into deeper veins which issue from the corpora cavernosa at the crura penis; and those from the rest of the urethra and bulb pass more directly into the plexus of the veins about the prostate. For all these veins one condition is the same; namely, that they are liable to the pressure of muscles when they leave the penis. The muscles chiefly concerned in this action are the erector penis and accelerator urinæ. Erection results from the distention of the venous plexuses with blood. The principal exciting cause in the erection of the penis is nervous irritation, originating in the part itself, or derived from the brain and spinal cord. The nervous influence is communicated to the penis by the pudic nerves, which ramify in its vascular tissue; and after their division in the horse the penis is no longer capable of erection.

This influx of the blood is the first condition necessary for erection, and through it alone much enlargement and turgescence of the penis may ensue. But the erection is probably not complete, nor maintained for any time except when, together with this influx, the muscles already mentioned contract, and, by compressing the veins, stop the efflux of blood, or prevent it from being as great as the influx.

It appears to be only the most perfect kind of erection that needs the help of muscles to compress the veins; and none such can materially assist the erection of the nipples, or that amount of turgescence, just falling short of erection, of which the spleen and many other parts are capable. For such turgescence nothing more seems necessary than a large plexiform arrangement of the veins, and such arteries as may admit, upon occasion, augmented quantities of blood.

The circulation in the Lungs, Liver, and Kidneys will be described under their respective heads.

\section{The Regulation of the Blood-Flow.}

The flow of blood is not always the same, but varies:-

(a.) With alterations in the force and frequency of the contractions of the heart; and.

(b.) With variations of the peripheral resistance.

It is obvious that the flow of blood may be increased under the following circumstances:-

(a.) If the force and frequency of the heart's beats be increased, and the peripheral resistance be (1) unchanged, or be (2) diminished.

(b.) If the force and frequency of the heart be unchanged, and the peripheral resistance be diminished, 
And may on the other hand be diminished:-

(c.) If the force and frequency of the heart's beats be diminished, and if the peripheral resistance be (1) unchanged, or be (2) increissed.

(d.) If the force and frequency of the heart's beats be unchanged, and the peripheral resistance be increased.

When the force and frequency of the heart's contractions are increased and at the same time the peripheral resistance is increased, the flow may be increased, diminished, or unchanged, according as either of the two factors, one of which tends to increase the flow, and the other to diminish it, is more markedly increased, or if they are balanced. The complemental proposition is also true, that the flow may be increased, diminished, or unchanged, when the force and frequency of the heart's contractions are diminished, and the peripheral resistance is diminished.

The conditions of increased flow and of inerease of blood pressure are not the same. Indeed, the greatest blood-flow may oceur when the blood pressure is low, i.e., when the peripheral resistance is diminished and the heart's beat is increased or is unchanged. In fact there is only one condition in which increased blood-flow is accompanied by increased blood-pressure, viz., when the heart's beat is increased and the peripheral resistance is unchanged.

It will be necessary now to consider (a) the unys in which the force and frequeney of the heeret's beats are regulated; and also (b) the way.s in which the peripheral resistance is increased or diminished. We shall afterward be in a better position to discuss the variations of bloodpressure produced by different combinations of cardiac and arterial alterations.

(a.) The force and frequency of the contractions of the heart may be considered to depend upon:

1. The properties and condition of the heart-muscle itself;

2. 'The influence of the central nervous system;

3. 'The amount of the blood passing into the heart's cavities;

4. The amount of pressure to be overcome.

5. The coronary circulation.

Each of these factors must be considered seriatim.

1. The properties of the heart-muscle. - It has already been pointed out that in strueture the muscular fibres of the heart differ from skeletal muscle on the one hand, and from unstriped muscle on the other, occupying, as it were, an intermediate position between the two varieties. The heart-muscle, however, possesses a property which is not possessed by skeletal muscle, or by unstriped muscle to such a degree, namely, the property of rhythmical contractility. The property of rhythmic contraction 
is shown by the action of the heart within the body; its systole is followed by its diastole in regular sequence throughout the life of the individual. The force and frequency of the systole may vary from time to time as occasion requires, but there is no interruption to the action of the normal heart, or any interference with its rhythmical contractions. Further, we find that in an animal rapidly bled to death, the heart continues to beat for a time, varying in duration with the kind of animal experimentally dealt with, and in the entire absence of blood within the heart-chambers; and still further, if the heart of an animal be removed from the body, it still, for a varying time, continues its alternate systolic and diastolic movements. Thus we see that the power of rhythmic contraction depends neither upon connection with the central

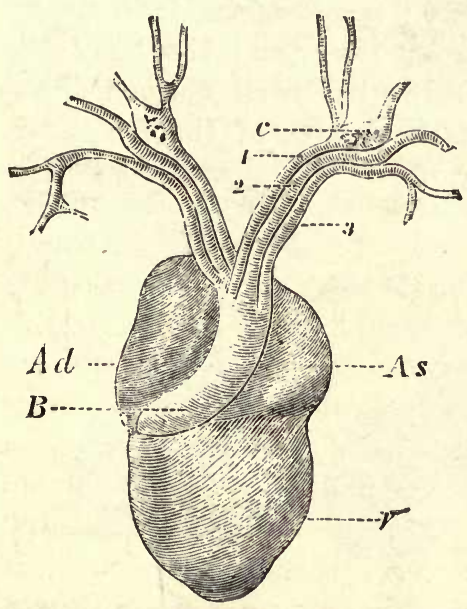

Fig. 191.

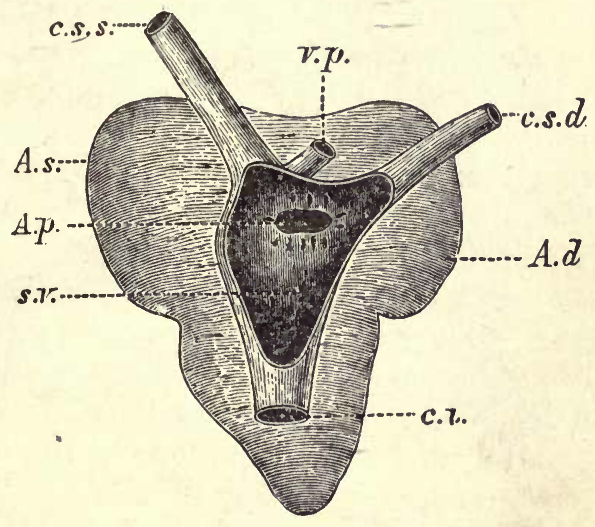

Fig $191 \mathrm{~A}$.

Fig. 191. - The heart of a frog (Rana esculenta) from the front. $\not s$, left auricle; $B$, bulbus arterio-us, dividing into right and left aortæ. (Ecker.)

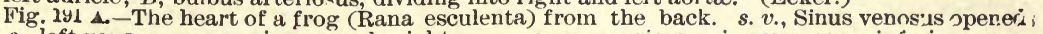
$c . s$. $s$., left vella cava superior; $c, s$. $d$., right vena cava superior; $c$. $i$, vena cava in fr.rivr; $v, p$, vena pulmonales; A.d., right auricle; A.s., left auricle; A.p., opening of communicatior. let ween the. right auricle and the sinus venosus, $\times 21 / 2-3$. (Ecker.)

nervous system, nor yet upon the stimulation produced by the presence of blood within its chambers-it is automatic. The cause of this rhythmic power has been the subject of much discussion and experimental observation. Up to a comparatively short time ago, the remarkable property of the heart to continue its rhythmical contractions after removal from the body was believed to be connected in some way or other with the presence of collections of nerve cells, or ganglia in several parts of its tissue. Although this idea, as we shall presently see, has now been very generally given up, it may be as well to describe shortly these ganglia in this place; they have been studied more particularly 
in the heart of the frog, of the tortoise, and of other cold-blooded animals.

In the frog's heart (fig. 191) these ganglia consist of three chief groups. The first group is situated in the wall of the sinus venosus at the junction of the sinus with the right auricle (Remak's); the second group is placed near the junction between the auricles and ventricle (Bidder's); and the third in the septum between the auricles (v. Bezold's).

The nerve cells of which these ganglia are composed are generally unipolar, and seldom bipolar; sometimes two cells are said to exist in the same envelope, constituting the twin cells of Dogiel. The cells are large, and have very large round nuclei and nucleoli (fig. 193). Ganglion cells have not been found in the lower part of the ventricle.

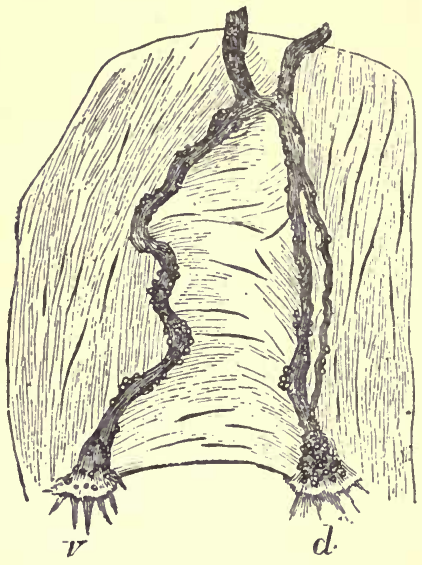

Fig. 19:.

As regards the automatic morements of the heart removed from the body our chief knowledge has been derived from the study of the hearts of the frog and tortoise.

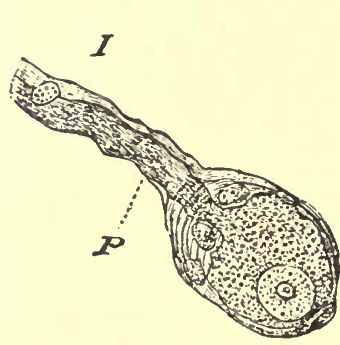

Fig. 192.-Course of the nerves in the auricular partition wall of the heart of a frog. $d$. Dorsal branch: $v$, ventral branch. (Ecker.)

Fig. 193. - Isolated nerve-cells from the frog's heart. I. Usual form. II. Twin cell. C, Capsule; $\mathrm{N}$, nucleus; $N^{\prime}$, nucleolus; $P$, process. (From Ecker.)

If removed from the body entire, the frog's heart will continue to beat for many hours and even days, and the beat has no apparent difference from the beat, of the heart before removal; it will take place, as we have mentioned, without the presence of blood or other fluid within its. chambers. Not only is this the case, but the auricles and ventricle may be cut off from the sinus, and both parts continue to pulsate, and further the auricles may be divided from the ventricle with the same result. If the heart be divided lengthwise, its parts will continue to pulsate rhythmically, and the auricles may be cut up into pieces, and the pieces will continue their movements of rhythmical contraction.

It will be thus seen that the rhythmical movements appear to be more marked in the parts supplied by the ganglia, as the apical portion 
of the ventricle, in which ganglia have not been found, does not, under ordinary circumstances, possess the power of automatic movement.

It has, however, been shown by Gaskell that the extreme apex of the ventricle of the heart of the tortoise, which contains no ganglia, may under appropriate stimuli be made to contract rhythmically. This proves that the muscular tissue of the heart itself is capable of rhythmical contraction independent of the ganglia. Thus it seems probable that the rhythmic contractility of the heart is a power inherent in the muscular tissue, which is quite independent as far as its commencement, at any rate, is concerned of nerve influence.

The heart-muscle exhibits another property which distinguishes it from ordinary skeletal muscle, viz., the way in which it reacts to stimuli. The latter as will be described at greater length in its appropriate place, reacts slightly to a slight stimulus above the minimal, and with an increase of the strength of the stimuli will give increasingly ample contractions until the maximum contraction is reached; in the case of the heart-beats this is not so, since the minimum stimulus which has any effect is followed by the maximum contraction; in other words the weakest effectual stimulus brings out as great a beat as the strongest. "There is another great difference between the contraction of the heart and of skeletal muscle, viz., the inability of the former to enter into a state of tetanus under the influence of stimuli repeated very rapidly. If the heart be stimulated by a series of rapid interrupted induction shocks, there is no summation of the contractions, as there would besupposing an ordinary skeletal muscle were stimulated in the same way. This phenomenon is said to be due to the following fact, viz., that in order to produce an extra contraction of the excised frog's heart, the stimulus must be applied during diastole or period of rest or relaxation, and in that case the next contraction happens at an earlier period than if the stimulus were not applied. If applied during the systole, the stimulus has scarcely any effect; the period during which the muscle is refractory to stimuli is much longer in the case of the heart than in the case of other muscles. In order to produce a tetanus in skeletal muscle, the second stimulus must be sent into the muscle before it has had time to recover from the effect of the first stimulus and relax, and so on with the third, fourth, and other stimuli. If, as we may suppose, the same conditions for the production of tetanus are necessary in heart-muscle, the reasons of the impossibility of producing tetanus, i.e. that a stimulus applied during contraction is ineffectual, are sufficiently obvious. It appears, however, that if the stimuli are sufficiently strong and rapidly repeated, the refractory period during which the muscle is practically insensible to stimuli diminishes, and a very rapid repetition of the beats occurs. As a rule the beats are fewer with rapid stimulation. 
In connection with the rhythmic contraction of muscle, it is neessary to allude briefly to what is known as Stannius' experiment. 'This experiment consisted originally of applying a tight ligature to the heart between the sinus and the right auricle, the effect of which is to stop the beat of the heart below the ligature, while the sinus and the veins leading into it continue to beat. If a second ligature be applied at the junction of the auricles and rentricle, the ventricle maly begin to beat slowly, while the auricles continue quiescent. In both cases the quiescent parts of the heart may be made to give single contractions in response to mechanical stimulation. A considerable amount of discussion has arisen as to the explanation of these phenomena. It was suggested that the action of the ligature is to stimulate some inhibitory nervous mechanism in the sinus, whereby the auricles and rentricle can no longer continue to contract, but this suggestion must certainly be given up if the present theory as to the functions of the nerve ganglia be correct. It may be that the effect of St:mnius' ligature is simply an example of what has been called by Gaskell blocking. The explanation of this term is as follows:-it appears that under normal conditions the wave of contraction in the heart starts at the sinus and travels downward over the anricles to the ventricle, the irritability of the muscle and the power of rhythmic contractility being greatest in the sinus, less in the auricles and still less in the ventricle, whle under ordinary conditions the apical portion of the ventricle exhibits very slight irritability and still less power of spontaneous contraction. Thus it may be supposed that the wave of contraction beginning at the sinus is more or less blocked by a ring of muscle of lower irritability at its junction with the auricles, and again the wave in the auricles is similarly delayed in its passage over to the ventricle by a ring of lesser irritability, and thus the ware of contraction starting at the sinus is broken as it were both at the auricles and at the ventricle. By an arrangement of ligatures, or better, of a system of clamps, one part of the heart may be isolated from the other portion, and the contraction when stimulated by an induction shock maly be made to stop in the portion of the heart-muscle in which it begins. It is not unlikely that the contraction of one portion of the heart acts as a stimulus to the next portion, and that the sinus contraction generally begins first, since the sinus is the most irritable to stimuli, and possesses the power of rhythmic contractility to the most highly developed degree. It must not be thought, however, that the wave of contraction is incapable of passing orer the heart in any other direction than from the sinus downward; it has been shown that by application of appropriate stimuli at appropriate instants, the natural sequence of beats may be reversed, and the contraction starting at the arterial part of the ventricle may pass upward to the auricles and then to the sinus in order. 
An exceedingly interesting fact with regard to the passage of the wave in any direction has been made out by partial division of the muscular fibres at any point, whereby one part of the wall of the heart is left connected with the other parts by a small portion of undivided muscular tissue, and the wave of contraction then being only able to pass to the next portion of the wall every second or third beat. Thus division of the muscle has much the same effect as partial clamping it in the same position, or of a ligature similarly applied, but not tied tightly. It may, therefore, be suggested that Stannius' ligature acts as a partial or complete block, and prevents the stimulus of the sinus-beat from passing further down the heart, but that the parts below the ligature may be made to contract by stimuli applied to them directly. Nearly all the information to be obtained as to the phenomena of the contraction of heart-muscle apart from the rhythmic action of the organ itself, may be obtained from a heart to which a Stannius' ligature has been applied; indeed, the effect of minimal stimuli, the effect of rapidly repeated shocks, and the refractory period of heart-muscle may all be studied from a heart in this condition.

The velocity of the wave of contraction in frog's heart-muscle has been shown to be $\frac{2}{5}$ to $\frac{3}{5}$ inch, or $10-15 \mathrm{~mm}$. a second.

In pointing out the differences between the phenomena of contraction in skeletal and heart-muscle, the similarities between the two are not to be overlooked; thus it has been shown that the effect of cold, heat, fatigue, and other influences have very much the same effect in both cases.

2. The influence of the central nervous system.

The heart is capable of automatic rhythmic movement, as has been clearly shown by its behavior when removed from the body, and it has been shown further that there is reason for believing that the power resides in the inherent property of its muscle fibres themselves. While in the body, however, the heart's beats are under control of the central nervous system. To this nervous control, we must next direct our attention. The influence which is exerted by the central nervous system appears to be of two kinds, firstly, in the direction of slowing or inhibiting the beats, and secondly, in the direction of accelerating or augmenting the beats. The influence of the first kind is brought to bear upon the heart through the fibres of the vagi nerves, and that of the second kind through the sympathetic fibres.

Influence of the Vagi.-It has long been known, indeed ever since the experiments of the Bros. Weber in 1845, that stimulation of one or both vagi produces slowing of the beats of the heart. It has since been shown in all of the vertebrate animals experimented with, that this is the normal action of vagus stimulation. Moreover, section of one nerve; 
or at any rate of both vagi, produces acceleration of the pulse, and stimulation of the distal or peripheral end of the divided nerve produces normally slowing or stopping of the heart's beats.

It appears that any kind of stimulus produces the same effect, either chemical, mechanical, electrical, or thermal, but that of these the most potent is a rapidly interrupted induction current. A certain amount of confusion has arisen as to the effect of vagus stimulation in consequence of the fact that within the trunk of the nerve is contained, in some animals, fibres of the sympathetic, and it depends to some extent upon the exact position of the application of the stimulus, as to the exact effect produced. Speaking generally, however, excitation of any part of the trunk of the vagus produces inhibition, the stimulus being particularly potent if applied to the termination of the vagi in the heart itself, where they enter the substance of the organ at the situation of the sinus ganglia. The stimulus may be applied to either vagus with effect, although it is frequently more potent if applied to the nerve on the right side. The effect of the stimulus is not immediately seen; one or more beats may occur before stoppage of the heart takes place, and slight stimulation may produce only slowing and not complete stoppage

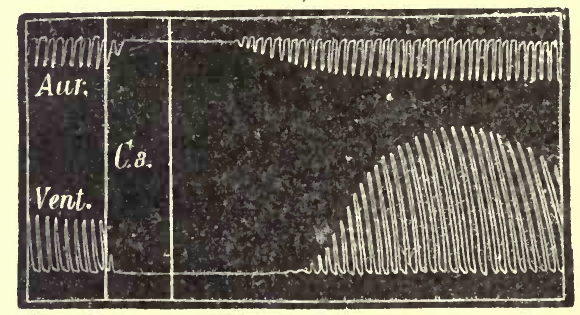

Fig. 194.-Tracing showing the actions of the vagus on the heart. Aur., Auricular; vent., ventricular tracing. The part between perpendicular lines indicates period of vagus stimulation. $C .8$ indicates that the secondary coil was $8 \mathrm{~cm}$. from the primary. The part of tracing to the left shows the regular contractious of moderate height before stimulation. During stimulation. and for some time after, the beats of auricle and ventricle are arrested. After they commence again they are single at first, but soon acquire a much greater amplitude than before the application of the
stimulus. (From Brunton, after Gaskell.)

of the heart. 'The stoppage may be due either to prolongation of the diastole, as is usually the case, or to diminution of the systole. Vagus stimulation inhibits the spontaneous beats of the heart only, it does not do away with the irritability of the heart-muscle, since mechanical stimulation may bring out a beat during the still-stand caused by vagus stimulation. The inhibition of the beats varies in duration, but if the stimulation be a prolonged one, the beats may reappear before the current is shut off. When the beats reappear, the first few are usually feeble, and may be auricular only; after a time the contractions become more and more strong, and very soon exceed both in amplitude and 
frequency those which occurred before the application of the stimulus (fig. 194).

Influence of the Sympathetic.-The influence of the sympathetic may be considered, to a certain extent, as the reverse of that of the vagus. Stimulation of the sympathetic, even of one side, produces acceleration of the heart-beats, and according to certain observers, section. of the same nerve produces slowing. The acceleration produced by stimulation of the sympathetic fibres is accompanied by increased force, and so the action of the nerve is more properly termed augmentor. The action of the sympathetic differs from that of the vagus in several particulars besides the augmentation which is produced: firstly, the stimulus required to produce any effect must be more powerful than is the case with the vagus stimulation; secondly, a longer time lapses before the effect is manifest; and thirdly, the augmentation is followed by exhaustion, the beats being after a time feeble and less frequent.

Origin of the cardiac nerve-fibres. - The fibres of the sympathetic system, which influence the heart-beat in the frog, leave the spinal cord by the anterior root of the third spinal nerve, and pass thence by the ramus communicans to the third spinal ganglion, thence to the second spinal ganglion, and thence by the annulus of Vieussens

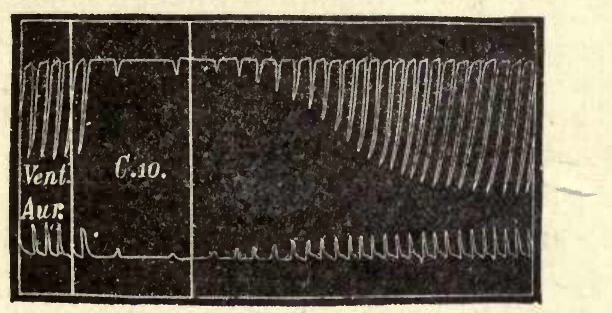

Fig. 195.-Tracing showing diminished amplitude and slowing of the pulsations of the auricle and ventricle without complete stoppage during irritation of the vagus. (From Brunton, after Gaskell.)

(round the subclavian artery) to the first spinal ganglion, and thence in the main trunk of the sympathetic, to near the exit of the vagus from the cranium, where it joins that nerve and runs down to the heart within its sheath, forming the joint vago-sympathetic trunk.

In the dog, the augmentor fibres leave the cord by the second and third dorsal nerves, and possibly by anterior roots of two or more lower nerves, passing by the rami communicantes to the ganglion stellatum, or first thoracic ganglion, thence by the annulus of Vieussens to the inferior cervical ganglion of the sympathetic fibres from the annulus, or from the inferior cervical ganglion proceed to the heart.

From the fact that the augmentor fibres are joined to the vagus 
trunk, it may be understood that the effect of the stimulation of the vagus in the frog is not in all cases purely inhibitory, but may be augmentor, according to the position where the stimulus is applied, the intensity of the stimulus, and the condition of the heart; if it is beating strongly a slight vagus stimulation will produce immediate inhibition.

The fibres of the vagus which pass to the heart arise in the medulla oblongata, in the floor of the fourth ventricle, and in a nuelens of gray matter, the exact position of which will be indicated in a future chapter. It was formerly thought that the inhibitory fibres in the vagus trunk were derived from the spinal accessory nerve, but this view has been largely abandoned. The spinal accessory fibres probably supply certain muscles of the larynx. It has been found that stimulation of this nucleus, which is ealled the cardio-inhibitory centre, produces inhibition of the heart-beat.

Thus there is no doubt that the vagi nerves are simply the media of an inhibitory or restraining influence over the action of the heart, which is conveyed through them from the centre in the medulla oblongata which is always in operation. The restraining influence of the centre in the medulla may be reflexly increased by stimulation of almost any afferent nerve, particularly of the abdominal sympathetic, so as to produce slowing or stoppage of the heart, through impulses from it passing down the vagi. As an example of this reflex stimulation, the wellknown effect on the heart of a violent blow on the epigastrium may be referrod to. 'The stoppage of the heart's action in this case is due to the conveyance of the stimulus by fibres of the sympathetic (afferent) to the medulla oblongata, and its subsequent reflection through the ragi (efferent) to the muscular substance of the heart. It is possible that the power of the medullary inhibitory centre may in a similar manner be reflexly lessened so is to produce accelerated action of the heart.

The course of the augmentor fibres in the spinal cord is not known, but it is thought that in all probability they are connected with an angmentor centre in the medulla. The circulation of venous blood appears to stimulate the inhibitory centre, and of highly oxygenated the augmentor centre.

In addition to direct and reflex stimulation it is almost certain that impulses passing down from the cerebrum may have a similar effect.

Other Influences Affecting the Heart-Beat.-Alteration of temperature.-The effect of cold is to slow the heart-beats, and if the heart be cooled down to $3^{\circ} \mathrm{C}$. $\left(38^{\circ} \mathrm{F}\right.$.) it will stop beating. 'The heart may be frozen, and when thawed will continue its spontaneous beats. The effect of heat is to quicken and shorten the heirt-beats, but át a moderate temperature, $20^{\circ} \mathrm{C} .\left(68^{\circ} \mathrm{F}\right.$.), the contractions are increased in force. 
At or below $40^{\circ} \mathrm{C}$. $\left(104^{\circ} \mathrm{F}\right.$.) the contrations are so rapid as to pass into heart-rigor; this may be stopped by cooling.

Poisons and other chemical substances.-A large number of chemical substances have a distinct effect upon the cardiac contractions. Of these the most important are atropin and muscarin.

Atropin produces considerable augmentation of the heart-beats, and when acting upon the heart prevents the results of vagus stimulation.

Muscarin (obtained from various species of poisonous fungi) produces marked slowing of the heart-beats, and, in larger doses, stoppage of the heart. It produces a similar effect to that of prolonged vagus stimulation, and as in that case the effect can be removed by the action of atropin.

Digitalin (the active principle of digitalis purpurea), slows the heart and appears to act by stimulating the vagi. Later on the muscle is also more excitable. Veratrin and aconitin have a similar effect. Nicotine prevents the effect of vagus stimulation.

\section{Methods of investigating the Heart-beat.}

(1) The simplest form of an apparatus to be used for recording the contractions of the frog's heart consists of a small closed cylindrical box fixed to a stand. At the bottom of the box are two tubes by means of which water at various temperatures may be made to circulate through it, one tube being the inlet and the other the outlet, and they are connected with india-rubber, suitable for the purpose of conducting water to and from the apparatus. The lever is made of a piece of glass rod which is softened and drawn out in the flame of the blow-pipe a very fine thread, leaving a small piece unaltered to act as a counterpoise. The lever is then passed through a piece of cork and through this cork a fine needle is inserted at right angles to the lever. The needle is made to rest on a support attached above one side of the box in such a way that the lever has free novement up and down. Another small piece of cork is passed along the lever arm and is adjusted and cut so that its point directed downward can rest upon the frog's heart, which is removed from the body and placed upon the top of the box in serum or defibrinated blood. In this way the contractions of the auricles and ventricle are communicated to the lever, and this may be made to write upon a recording cylinder.

(2) The variations of endocardial pressure, which correspond, of course, with the various phases of the cardiac cycle, may be recorded by a modification of the ordinary mercurial manometer. The apparatus is best used with a large frog (Rana esculenta), and the heart is exposed in the usual manner, the pericardium opened. A cut is made into the bulb, and by this means a double or perfusion canula (fig. 196) is passed into the ventricle, a ligature is passed round the heart, and the canula is tied in tightly. The vessels are then divided beyond the ligature, and the canula, with the heart attached, is removed. To one stem of the canula a tube is attached, communicating with a reservoir of a solution of dried blood in .6 saline solution, and filtered, which is capable of being raised or lowered in temperature by being surrounded by a metal box which contains hot, cold, or iced water. Attached to the other end is a I6 
similar tube, which communicates by a $T$ piece with a small mercurial manometer, provided with a writing style, and also with a ressel into which the serum is received. The apparatus being arranged so that the movements of the mercury can be recorded by the float and the writing style on a slowly revolving drum, and after some serum has been allowed to pass freely through the ventricle, both tubes are clipped, the second one beyond the $T$ piece, and the alterations in the pressure are recorded. The effects of fluids at various temperatures and of poisons may be recorded in the manner indicated above.

(3) By Roy's Tonometer (fig. 197) the alterations in volume which a frog's heart undergoes during contraction are recorded by the following means: A small bell-jar, open above, but provided with a firmly fitting cork, in which is fixed a double canula, is adjustable by a smoothly ground base upon a circular brass plate,

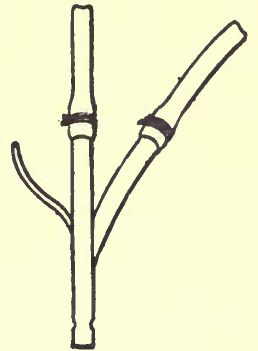

Fig. 196.

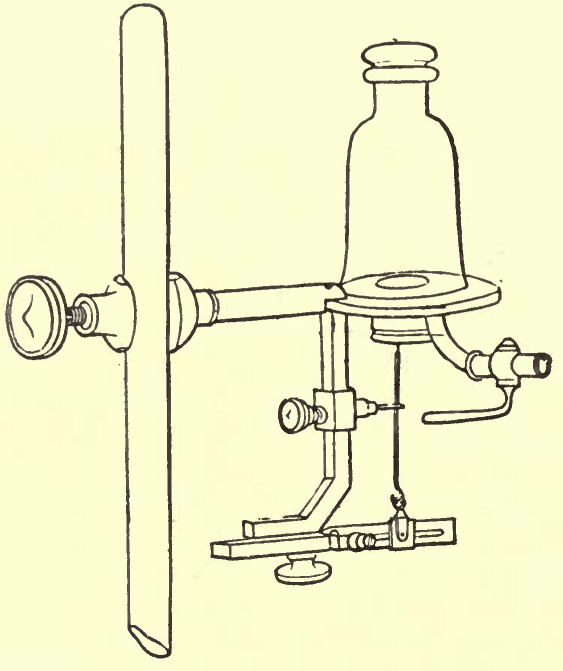

Fig. 197.

Fig. 196.--Kronecker's Perfusion Canula. for supplying Fluids to the interior of the Frog's Heart. It consists of a double tube, one outside the other; the end view is shown in the engraving. The inner tube branches out to the left: thus, when the ventricle is tied to the outer tube of the canula, a current of liquid can be made to pass into the heart by one tube and out through the other.

Fig. 197.-Roy's Tonometer.

about 2 to 3 inches in diameter. The junction is made complete by greasing the base with lard. In the plate, which is fixed to a stand adjustable on an upright, are two holes, one in the centre, a large one about one-third of an inch in diameter, to which is fixed below a brass grooved collar, about half an inch deep; the other hole is the opening into a pipe provided with a tap (stopcock). The opening provided with the collar is closed at the lower part with a membrane of animal tissue, which is loosely tied by means of a ligature around the groove at the lower edge of the collar. To this membrane a piece of cork is fastened by sealing-wax, from which passes a wire, which can be attached to a lever, fixed on a stage below the apparatus.

When using the apparatus, the bell-jar is fixed by means of lard, and the jar is filled with olive oil. In the way above described, the heart of a large frog is prepared and the canula fixed in the cork is firmly tied into the heart; the tubes of the canula communicating with the reservoir of serum on the ose hand, and with a vessel to contain the serum after it has run through on the other. The canula with heart attached is passed into the oil, and the cork firmly secured. By these means the lever will be found to be adjusted to 
a convenient elevation. The lever is allowed to write on a moving drum, and serum is passed through at various temperatures. After a short time the heart may stop beating; but two wires are arranged, the one in the canula, the other projecting from the plate in such a way that the heart can be moved against them by shifting the position of the bell-jar a little. The wires act as electrodes, and can be made to communicate with an induction apparatus, so that single induction shocks can be sent into the heart to produce contractions, and if need be, by means of the trigger key, at one definite point in the revolution of the recording cylinder.

Electrical Phenomena of the Heart-beat.-The phenomena of the natural beat of the heart are generally considered to indicate that the systolic contraction is a single and not a tetanic one. The electrical changes support this view. During the contraction a distinct electrical change occurs which is similar to that which happens in skeletal muscle with each contraction. It has been demonstrated that a stanniused frog heart undergoes two changes or phases as regards its electrical condition, the first immediately before the contraction, in which the excited part becomes negative to the other parts, contraction following the wave of excitation, and the second during relaxation, in which the current flows in an opposite way.

The Metabolism of the Heart.-Whatever view may be taken of the nature of the rhythmic cardiac contractions, it will be generally acknowledged that the contractions cannot long be maintained without a due supply of blood or of a similar nutritive fluid. Some very remarkable facts have been made out about this, in the case of the frog's heart. For instance, it has been shown that normal saline solution is insufficient to maintain the contractions, and that in experiments in which it is necessary to maintain the beats for any length of time failing serum or saline solution of dried blood, the solution should contain some serum-albumin, and that there should also be present some potassium chloride, and Dr. Ringer has composed a nutritive fluid which contains chlorides of sodium, potassium, and calcium in small amounts, which is able to maintain the normal beats of the heart. It is therefore very reasonable to suppose that the amount and quality of the blood supplied to the human heart has the greatest influence in maintaining the force and frequency of the rhythmic activity. The view that is taken at present of the action of the heart is one propounded by Gaskell, viz., that in heart muscle as in protoplasm generally, the metabolic processes are those of anabolism or building up, which takes place during the diastole of the heart, that vagus stimulation helps on this process, and of catabolism or discharge, which is manifested in the contraction of the heart, and which is accelerated by stimulation of the sympathetic fibres. That vagus stimulation is therefore ultimately beneficial to the contractions. The electrical currents set up on the stimulation of the 
vagus and of the sympathetic are in opposite directions, and so if Gaskell's contention is correct that the negative variation of the muscle current occurring on sympathetic stimulation is a sign of catabolism, the result of vagus stimulation, viz., a positive variation of the muscle current, may be supposed to indicate the complementary condition of anabolism.

3. The Amount of Blood Passing into the Heart's Cavities.-It is found that in the body at any rate the amount of blood which passes into the cavities of the heart distinctly affects the strength of its beat. Thus if from any cause the blood is diminished the contractions become much

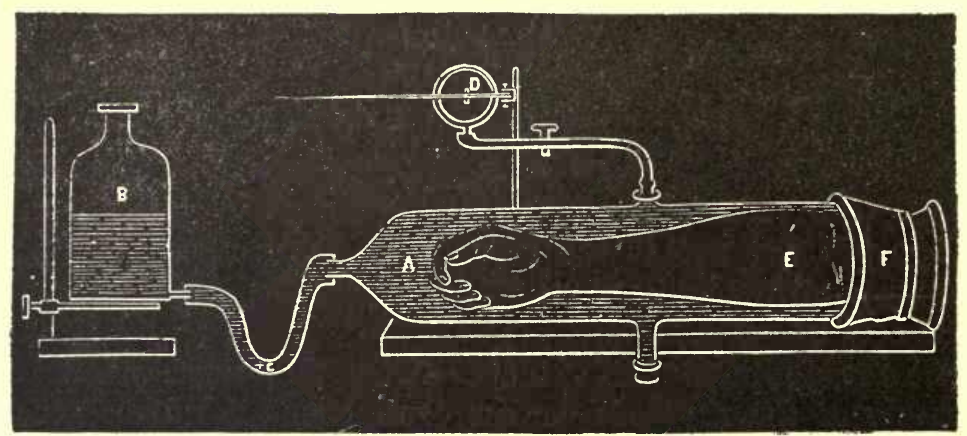

Fig. 198.-Plethysmograph. By means of this apparatus, the alteration in volume of the arm, E, which is inclosed in a glass tube, $\Delta$, filled with fluid, the opening through which it passes being firmly closed by a thick gutta-percha band, $F$, is communicated to the lever, $D$, and registered by a recording apparatus. The fluid in $\mathbf{A}$ communicates with that in $\mathbf{B}$, the upper limit of which is above that in $\mathrm{A}$. The chief alterations in volume are due to alteration in the blood contained in the arm. When the volume is increased, fluid passes out of the glass cylinder, and the lever, $D$, also is raised, and when a decrease takes place the fluid returns again from $\mathbf{B}$ to $\mathrm{A}$. It will therefore be evident that the apparatus is capable of recording alterations of blood-pressure in the arm. Apparatus founded upon the same principle have been used for recording alterations in the volume of the spleen and kidney.

more feeble, although they may possibly be increased in rapidity. Similarly with

4. The Amount of Pressure to be Overcome.-If the aortic pressure is too low the muscle contractions of the heart is not so powerful or effective as if the pressure is normal, whereas too great arterial pressure may be sufficient to delay if not to stop altogether the heart's beats, dilatation of its cavities taking place and a condition of asystolism (Beau) resulting.

Another condition sometimes forgotten should be added as influencing the potency of the cardiac contraction, viz., the heart must have sufficient room to contract, it must not be unduly pressed upon.

5. The Coronary Circulation. - The nutrition of the heart wall has been fully investigated by Porter and others. The coronary arteries are terminal arteries; that is, they do not permit the establishment of a collateral circulation when one of their branches is blocked. If the 
block be complete, that portion of the heart wall supplied by the branch dies. The immediate effect of the closure of a large branch in the dog may be occasional and transient irregularity, or arrest of the ventricular contractions, preceded by irregularities in the force of the contractions and a diminution in the amount of work performed. The force, rather than the rate, of the ventricular contractions is closely dependent upon the blood-supply to the coronary arteries.

(b.) The Peripheral Resistance. - The regulation of the amount of resistance to the passage of blood at the periphery is principally done by the alteration of the calibre of the arterioles. This regulating power is chiefly invested in the nervous system. Its influence is exerted upon the muscular coat of the arteries and not upon the elastic element, which possesses, as must be obvious, rather physical than vital properties. The muscular tissue in the walls of the vessels increases in amount relatively to the other coats as the arteries grow smaller, so that in the arterioles it is developed out of all proportion to the other elements; in fact, in passing from capillary vessels, made up as we have seen of endothelial cells with a ground substance, the first change which occurs as the vessels become larger (on the side of the arteries) is the appearance of muscular fibres. Thus the nervous system is more powerful in regulating the calibre of the smaller than of the larger arteries.

It was long ago shown by Claude Bernard that if the cervical sympathetic nerve is divided in a rabbit, the blood-vessels of the corresponding side of the head and neck become dilated. This effect is best seen in the ear, which if held up to the light is seen to become redder, and the arteries are seen to become larger. The whole ear is distinctly warmer than the opposite one. This effect is produced by removing the arteries from the influence of the central nervous system, which influence normally passes down the divided nerve; for if the peripheral end of the divided nerve (i.e., that farthest from the brain) be stimulated, the arteries which were before dilated return to their natural size, and the parts regain their primitive condition. And, besides this, if the stimulus is very strong or very long continued, the point of normal constriction is passed, and the vessels become much more contracted than normal. The natural condition, which is about midway between extreme contraction and extreme dilatation, is called the natural tone of an artery; if this is not maintained, the vessel is said to have lost tone, or if it is exaggerated, the tone is said to be too great. The effects described as having been produced by section of the cervical sympathetic and by subsequent stimulation are not peculiar to that nerve, as it has been found that for every part of the body there exists a nerve the division of which produces the same effects, viz., dilatation of the vessels; such may be cited as the case with the sciatic, the splanchnic nerves, and the 
nerves of the brachial plexus: when these are divided, dilatation of the blood-vessels in the parts supplied by them takes place. It appears. therefore, that nerves exist which have a distinct control over the rascular supply of every part of the body. These nerves are called vasomotor.

Recently Mall has shown that veins possess a vaso-motor nerre-supply as well as arteries.

Vaso-motor nerres may be dirided into two classes, according to their function of causing contraction or dilatation of the blood-ressels, into vaso-constrictor and vaso-dilator nerres.

Vaso-motor Centres. Bulbar Centre.-The bulbar vaso-constrictor centre in the rabbit lies in the floor of the fonth ventricle, a millimetre or two caudal to the corpora quadrigemina, and extends longitudinally orer an area of about 3 millimetres. Owsjannikow has: shown that the centre is composed of two halres, each half lying in the lateral column to the side of the median line. This centre is in constant action, as is shown by dilatation of the blood-vessels when removed from its action by section of the spinal cord.

The existence of a raso-dilator centre in the spinal bulb has not been proved.

Spinal Centres.-Secondary raso-motor centres are present in the spinal cord (Goltz). Under normal couditions they do not act independently of the bulbar centre, but when the action of the latter has been interrupted by section of the cord, certain spinal cells below the section take on central functions and bring about a re-establishment of the lost vascular tone. Moreover, the central functions disappear if the cord below the section be destroyed.

Sympathetic Taso-motor Centres.-The existence of sympathetic vaso-motor centres has been proved by the experiments of Goltz and Ewald. It was found by these observers that even after destruction of the lower part of the spinal cord, the tone of the vessels of the hind. limbs, lost as a result of the operation, was re-established later.

Vaso-motor Reflexes. - The secondary vaso-motor centres, when removed from the influence of the bulbar centre, respond to afferent impulses by vaso-motor action. But under normal conditions the bulbar centre controls vaso-motor reflexes. The afferent impulses which excite reflex raso-motor action may proceed from the terminations of. sensory nerves in general or from the blood-ressels themselves, and the constriction or dilatation which follows generally occurs in the area whence the impulses arise. Yet the reflex may appear elsewhere, e.g., the vessels of the submaxillary gland dilate when the tongue is stimulated-an association in function. Impulses proceeding to the raso-motor centre from the 
cerebrum may cause vaso-dilatation as in blushing, or vaso-constriction as in the pallor of fear. An important reflex association exists between the vessels of the skin and those of subjacent parts. It is generally an inverse relation; that is, when the superficial vessels are dilated the deep are contracted. This reflex is made use of in medical practice when poultices are applied to the chest in pneumonia, the lung being in a state of inflammation.

Afferent influence upon the vaso-motor centre is well shown by the action of a nerve called the depressor, the existence of which was demonstrated by Cyon and Ludwig.

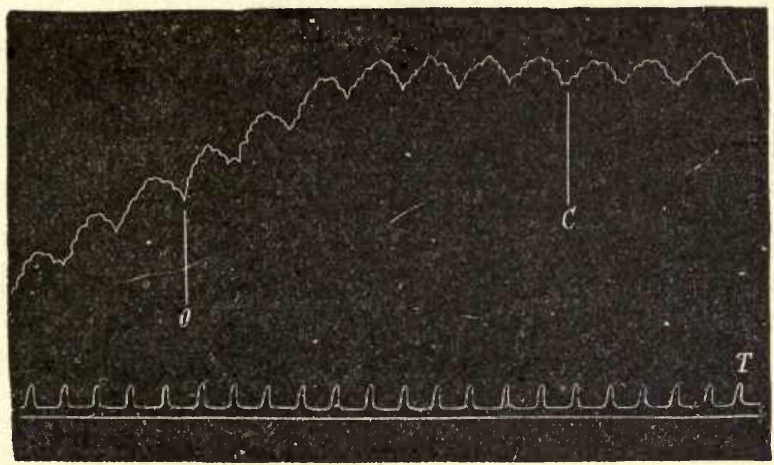

Fig. 199. - Tracing showing the effect on blood-pressure of stimulating the central end of the Depressor nerve in therabbit. To be read from right to left. $T$, indicates the rate at which the recording surface was travelling, the intervals correspond to seconds; $C$. the moment of entrance of current; $O$, moment at which it was shut off. The effect is some time in developing and lastc after the current has been taken off. The larger undulations are the respiratory nerves; the pulse osci:Ltions are very small. (Foster.)

It is a small afferent nerve and passes up from the heart in which it takes its origin, and in the rabbit goes upward in the sheath of the superior laryngeal branch of the vagus or with that branch and the vagus itself, communicating by filaments with the inferior cervical ganglion as it proceeds from the heart.

If during a blood-pressure observation in a rabbit this nerve be divided, and the central end (i.e., that nearest the brain) be stimulated, a remarkable fall of blood-pressure takes place (fig. 199).

The cause of the fall of blood-pressure is found to proceed from the dilatation of the vascular district within the abdomen supplied by the splanchnic nerves, in consequence of which the vessels hold a much larger quantity of blood than usu:al. The engorgement of the splanchnic area very greatly diminishes the amount of blood in the ressels elsewhere, and so materially diminishes the blood-pressure. The function of the depressor nerve is that of conveying to the vaso-motor centre indications of such conditions of the heart as require a diminution of the 
tension in the blood-vessels; as, for example, that the heart cannot, with sufficient ease, propel blood into the already too full or too tense arteries.

The action of the depressor nerve in causing an inhibition of the vaso-motor centre illustrates the more unusual effect of afferent impulses. As a rule, the stimulation of the central end of an afferent nerve produces a reverse or pressor effect, and increases the tonic influence of the centre, and by causing constriction of the arterioles, either locally or generally, raises the blood-pressure. 'Thus the effect of stimulating an afferent nerre may be either to dilate or to constrict the arteries. Stimnlation of an afferent nerve too may produce a kind of paradoxical effect, causing general rascular constriction and so general increase of bloodpressure, but at the same time local dilatation which must evilently have an immense influence in increasing the flow of blood through the part.

Course of the Vaso-motor Nerves. - The cell bodlies forming the bulbar vaso-motor centre give off neuraxons (axis-cylinder processes), some of which go to the nuclei of certain cranial nerves, while others pass down the cord to end at different levels in contact with cells-probably small cells in the anterine horn and lateral part of the gray matter. These cells constitute the spinal centres. The neuraxous of the spinal cells leare the cord in certain cranial nerves and in the anterior roots, and end in sympathetic ganglia in contact with their cell bodies. From these latter, neuraxons pass uninterruptedly to their termination in the vessel wall.

Besides the regulation of the heart beat and of the peripheral resistance, it must be recollected that other circumstances may affect the blood pressure, of which changes in the blood are the chief. First of alla. As regards quantity. At first sight it would appear probable that one of the easiest ways to diminish the blood-pressure would be to remove blood from the ressels by bleeding. It has been found by experiment, however, although the blood-pressure sinks while large abstractions of blood are taking place, that as soon as the bleeding ceases it rises rapidly, and speedily becomes normal; that is to say, unless so large an amount of blood has been taken as to be positively dangerous to life, abstraction of blood has little effect upon the blood-pressure. The rapid return to the normal pressure is due not so much to the withdrawal of lymph and other fluids from the body into the blood, as was formerly supposed, as to the regulation of the peripheral resistance by the vasomotor nerves; in other words, the small arteries contract, and in so doing maintain pressure on the blood and favor its accumulation in the arterial system. This is due to the stimulation of the vaso-motor centre from diminution of the supply of blood, and therefore of oxygen. The failure of the blood-pressure to return to normal in the too great 
abstraction must be taken to indicate a condition of exhaustion of the centre, and consequently of want of regulation of the peripheral resistance. In the same way it might be thought that injection of blood into the already full vessels would be at once followed by rise in the bloodpressure, and this is indeed the case up to a certain point-the pressure does rise, but there is a limit to the rise. Until the amount of blood injected equals about 2 to 3 per cent of the body-weight, the pressure continues to rise gradually; but if the amount exceed this proportion, the rise does not continue. In this case, therefore, as in the opposite when blood is abstracted, the vaso-motor apparatus must counteract the great increase of pressure, but now by dilating the small vessels, and so diminishing the peripheral resistance, for after each rise there is a partial fall of pressure; and after the limit is reached the whole of the injected blood displaces, as it were, an equal quantity which passes into the small veins, and remains within them. It should be remembered that the veins are capable of holding the whole of the blood of the body.

Further, as we have seen, the amount of blood supplied to the heart, both to its substance and to its chambers, has a marked effect upon the blood-pressure.

b. As regards quality. The quality of the blood supplied to the heart has a distinct effect upon its contraction, as too watery or too little oxygenated blood must interfere with its action. Thus it appears that blood containing certain substances affects the peripheral resistance by acting upon the muscular fibres of the arterioles, and so directly altering the calibre of the vessels.

\section{Proofs of the Circulation of the Blood.}

It seems hardly necessary at the present time to bring forward the proofs that during life the blood circulates within the body; they are so well known. It is interesting, however, to recount the main arguments by which Harvey in the first instance established the fact of the circulation; they were as follows:-

1. That the heart in half an hour propels more blood than the whole mass of blood in the body;

2. That the blood spurts with great force and in a jerky manner from an opened artery, such as the carotid, with every beat of the heart;

3. That if true, the normal course of the circulation would explain why after death the arteries are commonly found empty and the veins full;

4. That if the large veins near the heart be tied in a fish or snake, the heart becomes pale, flaccid, and bloodless; and that on moving the ligature, the blood again flows into the heart. If the artery is tied, the 
heart becomes distended, the distention lasting until the ligature is removed;

5. That if a ligature round a limb be drawn very tight, no blood can enter the limb, and it becomes pale and cold. If the ligature be somewhat relaxed, blood can enter but cannot leave the limb; hence it becomes swollen and congested. If the ligature be removed, the limb soon regains its natural appearance;

6. That the valves in the veins only permit the blood to flow toward the heart;

\%. That there is general constitutional disturbance resulting from the introduction of a poison at a single point, e.g., snake poison;

To these may now be added many further proofs which have accumulated since the time of Harvey, e.g.:-

8. That in wounds of arteries and veins; in the former case hemorrhage may be almost stopped by pressure between the heart and the wound, in the latter by pressure beyond the seat of injury;

9. 'That the passage of blood-corpuscles from small arteries through capillaries into veins in all transparent rascular parts, as the mesentery, tongue, or web of the frog, the tail or gills of a tadpole, etc., may actually be observed under the microscope.

Further, it is obvious that the mere fact of the existence of a hollow muscular organ (the heart) with valves so arranged as to permit the blood to pass only in one direction, of itself suggests the course of the circulation. The only part of the circulation which Harvey could not follow was that through the capillaries, for the simple reason that he had no lenses sufficiently powerful to enable him to see it. Malpighi (1661) and Leeuwenhoek (1668) demonstrated this in the tail of the tadpole and lung of the frog. 


\section{CHAPTER VII.}

\section{RESPIRATION.}

THe maintenance of animal life necessitates the continual absorption of oxygen and excretion of carbonic acid; the blood being, in all animals which possess a well-developed blood-vascular system, the medium by which these gases are carried. By the blood, oxygen is absorbed from without and conveyed to all parts of the organism; and, by the blood, carbonic acid, which comes from within, is carried to those parts by which it may escape from the body. The two processes,-absorption of oxygen and excretion of carbonic acid, are complementary, and their sum is termed the process of Respiration.

In all Vertebrata, and in a large number of Invertebrata, certain parts, either lungs or gills, are specially constructed for bringing the blood - into proximity with the aërating medium (atmospheric air, or water containing air in solution). In some of the lower Vertebrata (frogs and other naked Amphibia) the skin is important as a respiratory organ, and is capable of supplementing, to some extent, the functions of the proper breathing apparatus; but in all the higher animals, including man, the respiratory capacity of the skin is so infinitesimal that it may be practically disregarded.

Essentially a lung or gill is constructed of a fine transparent membrane, one surface of which is exposed to the air or water, as the case may be, while, on the other, is a network of blood-vessels, - the only separation between the blood and aërating medium being the thin wall of the blood-vessels, and the fine membrane on one side of which vessels are distributed. The difference between the simplest and the most complicated respiratory membrane is one of degree only.

The various complexity of the respiratory membrane, and the kind of aërating medium, are not, however, the only conditions which cause a difference in the respiratory capacity of different animals. The number and size of the red blood-corpuscles, the mechanism of the breathing apparatus, the presence or absence of a pulmonary heart, physiologically distinct from the systemic, are, all of them, conditions scarcely second in importance.

It may be as well to state here that the lungs are only the medium for the exchange, on the part of the blood, of carbonic acid for oxygen. They are not the seat, in any special manner, of those combustion-pro- 
cesses of which the production of carbonic acid is the final result. These processes occur in all parts of the body in the substance of the tissues.

\section{Of the Respiratory Apparatus.}

The object of respiration being the interchange of gases in the lungs, it is necessary that the atmospheric air shall pass into them and that the changed air should be expelled from them. The lungs are contained in the chest or thorax, which is a closed cavity having no communica-

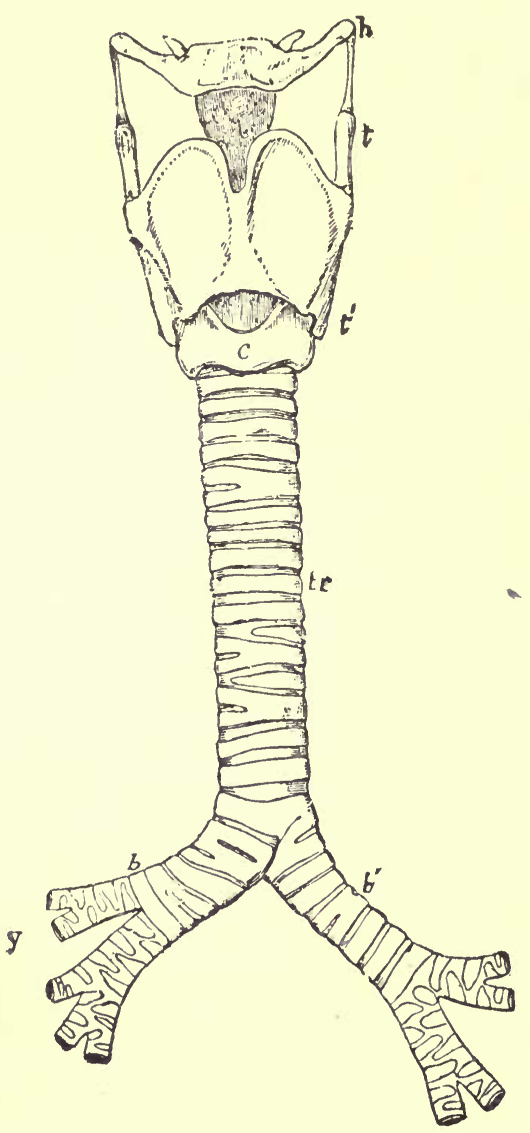

Fig. 200.

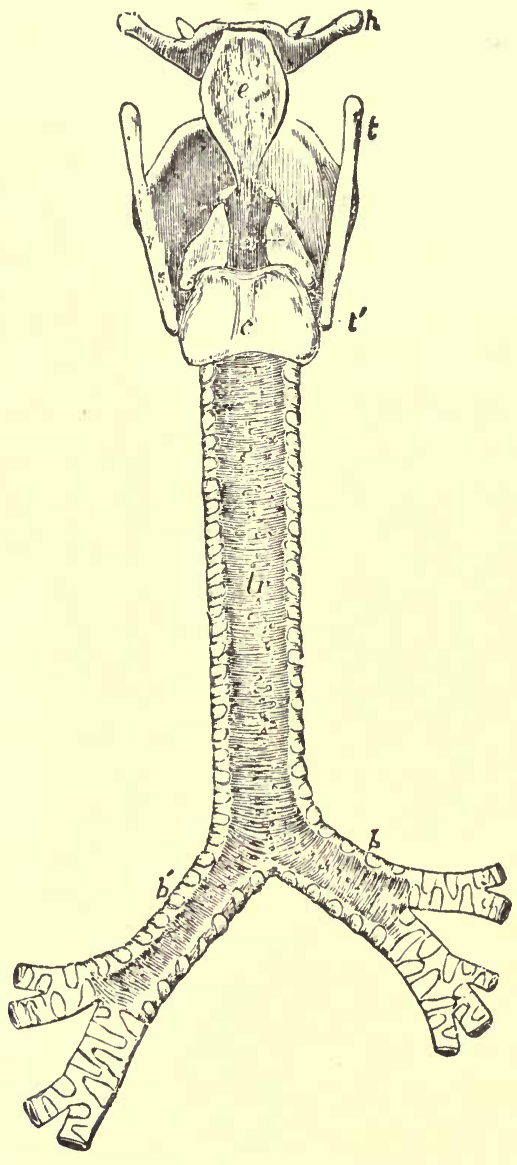

Fig. 201.

Fig. 200.-Outline showing the general form of the larynx, trachea, and bronchi, as seen from before. $h$, The great cornu of the hyoid bone; $e$, epiglottis; $t$, superior, and $t^{\prime}$, inferior cornu of the thyroid cartilage; $c$. middle of the cricoid cartilage; $t r$, the trachea, showing sixteen cartilaginous rings; $b$, the right, and $b^{\prime}$, the left bronchus. (Allen Thomson.) $\times 1 / 2$.

Fig. 201. - Outline showing the general form of the larynx. trachea, and bronchi, as seen from behind. $h$, Great cornu of the hyoid bone; $t$, superior and $t^{\prime}$, the inferior cornu of the thyroid cartilage ; $e$, epiglottis; $a$, points to the back of both the arytenoid cartilages, which are surmounted by the cornicula; $c$, the middle ridge on the back of the cricoid cartilage; $t r$, the posterior membranous part of the trachea; $b, b^{\prime}$, right and left bronchi. (Allen Thomson.) $\times 1 / 2$. 
tion with the outside, except by means of the respiratory passages. The air enters these passages through the nostrils or through the mouth, thence it passes through the larynx into the trachea or windpipe, which about the middle of the chest divides into two tubes, bronchi, one to each (right and left) lung.

The Larynx is the upper part of the passage which leads exclusively to the lung; it is formed by the thyroid, cricoid, and arytenoid cartilages (fig. 200), and contains the vocal cords, by the vibration of which the voice is chiefly produced. These vocal cords are ligamentous bands attached to certain cartilages capable of movement by muscles. By their approximation the cords can entirely close the entrance into the larynx; but under ordinary conditions, the entrance of the larynx is formed by a more or less triangular chink between them, called the rima glottidis. Projecting at an acute angle between the base of the tongue and the larynx, to which it is attached, is a leaf-shaped cartilage, with its larger extremity free, called the epiglottis (fig. 201,e). The whole of the larynx is lined by mucous membrane, which, however, is extremely thin over the vocal cords. At its lower extremity the larynx joins the trachea. With the exception of the epiglottis and the socalled cornicula laryngis, the cartilages of the larynx are of the hyalin variety.

The Epiglottis.-The supporting cartilage of the epiglottis is composed of yellow elastic cartilage, inclosed in a fibrous sheath (perichon drium), and covered on both sides with mucous membrane. The anterior surface, which looks toward the back of the tongue, is covered with mucous membrane, the basis of which is fibrous tissue, elevated toward both surfaces in the form of rudimentary papillæ, and covered with several layers of squamous epithelium. In it ramify capillary bloodvessels, and in its meshes are a large number of lymphatic channels. Under the mucous membrane, in the less dense fibrous tissue of which it is composed, is a number of tubular glands. The posterior or laryngeal surface of the epiglottis is covered by a mucous membrane, similar in structure to that on the other surface, but its epithelial coat is thin. ner, the number of strata of cells is less, and the papillæ few and less distinct. The fibrous tissue which constitutes the mucous membrane is in great part of the adenoid variety, and is here and there collected into. distinct masses or follicles. The glands of the posterior surface are smaller but more numerous than those of the other surface. In many places the glands which are situated nearest to the perichondrium are directly continuous through apertures in the cartilage with those on the other side, and often the ducts of the glands from one side of the carti-

* A detailed account of the structure and function of the Larynx wili ine found in a later chapter. 
lage pass tnrough and open upon the mucous surface of the other side. Taste goblets have been found in the epithelium of the posterior surface of the epiglottis, and in several other situations in the laryngeal mucous membrane.

The Trachea and Bronchi.-The trachea extends from the cricoid cartilage, which is on a level with the fifth cerrical vertebra, to a point opposite the third dorsal vertebra, where it divides into the two bronchi

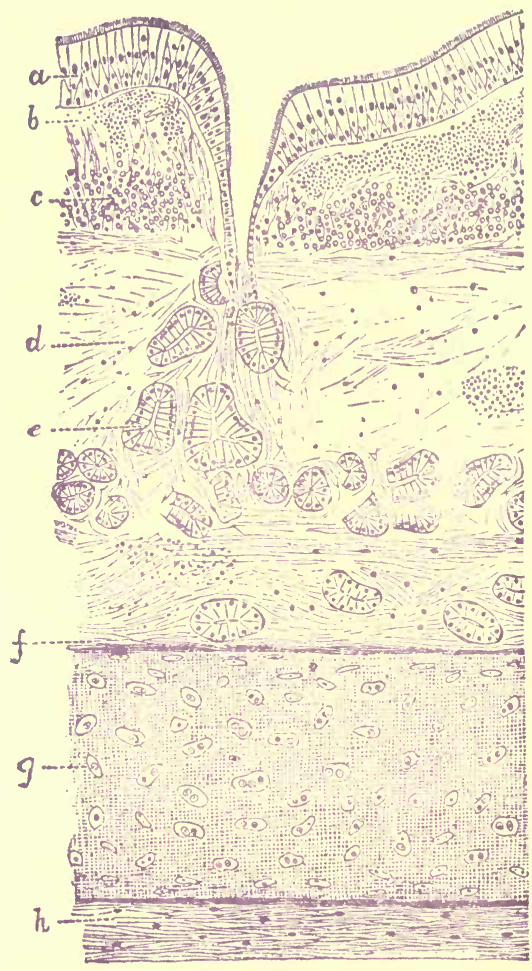

Fig. 202.-Section of the trachea. $a$, Columnar ciliated epithelium; $b$ and $c$, proper structure of the mucous membrane, containing elastic fibres cut across transversely; $d$, submucuous tissue containing mucous glands, $e$, separated from the hyaline cartilage, $g$, by a fine fibrous tissue, $f ; h$, external investment of fine fibrous tissue. (S. K. Alcock.)

one for each lung (fig. 201). It measures, on an average, four or fourand-a-half inches in length, and from three-quarters of an inch to an inch in diameter, and is essentially a tube of fibro-elastic membrane, within the layers of which are enclosed a series of cartilaginous rings, from sixteen to twenty in number. These rings extend only around the front and sides of the trachea (about two-thirds of its circumference), and are deficient behind; the interval between their posterior extremities being bridged over by a continuation of the fibrous mem- 
brane in which they are closed (fig. 202). The cartilages of the trachea and bronchial tubes are of the hyaline variety.

Immediately within this tube, at the back, is a layer of unstriped muscular fibres, which extends, transversely, between the ends of the cartilaginous rings to which they are attached, and opposite the intervals between them, also; their evident function being to diminish, when required, the calibre of the trachea by approximating the ends of the cartilages. Outside there are a few longitudinal bundles of muscular tissue, which, like the preceding, are attached both to the fibrous and cartilaginous framework.

The mucous membrane consists to a great extent of adenoid tissue, separated from the stratified columnar epithelium which lines it by a homogeneous basement membrane. This is penetrated here and there by channels which connect the adenoid tissue of the mucosa with the intercellular substance of the epithelium. The stratified columnar epithelium is formed of several layers, of which the most superficial layer is ciliated, and is often branched downward to join connective tissue corpuscles; while between these branched cells are smaller elongated cells prolonged up toward the surface and down to the basement membrane. Beneath these are one or more layers of more irregularly-shaped cells. Many of the superficial cells are of the goblet variety. In the deeper part of the mucosa are many elastic fibres between which lie connective-tissue corpuscles and capillary blood-vessels.

Numerous mucous glands are situated on the exterior and in the substance of the fibrous framework of the trachea; their ducts perforating the various structures which form the wall of the trachea, and opening through the mucous membrane in to the interior.

The two bronchi into which the trachea divides, of which the right is shorter, broader, and more horizontal than the left (fig. 200), resemble the trachea exactly in structure, with the difference that in them there is a distinct layer of unstriped muscle arranged circularly beneath the mucous membrane, forming the muscularis mucosce. On entering the substance of the lungs the cartilaginous rings, although they still form only larger or smaller segments of a circle, are no longer confined to the front and sides of the tubes, but are distributed impartially to all parts of their circumference.

The bronchi divide and subdivide, in the substance of the lungs, into a number of smaller and smaller branches, which penetrate into every part of the organ, until at length they end in the smaller subdivisions of the lungs, called lobules.

All the larger branches have walls, formed of tough membrane, containing portions of cartilaginous rings, by which they are held open, and unstriped muscular fibres, as well as longitudinal bundles of elastic tissue. They are lined by mucous membrane, the surface of which, like 
that of the larynx and trachea, is covered with ciliated epithelium, but the several layers become less and less distinct until the lining consists of a single layer of more or less cubical cells covered with cilia (fig. 203). The mucous membrane is abundantly provided with mucous glands.

As the bronchi become smaller and smaller, and their walls thinner, the cartilaginous rings become scarcer and more irregular, until, in the smaller bronchial tubes, they are represented only by minute and scattered cartilaginous flakes. And when the bronchi, by successive branches are reduced to about $\frac{1}{40}$ of an inch $(.6 \mathrm{~mm}$.) in diameter, they lose their cartilaginous element altogether, and their walls are formed only of a tough fibrous elastic membrane, with circular muscular fibres; they are still lined, however, by a thin mucous membrane, with ciliated epithelium, the length of the cells bearing the cilia haring become so far diminished that the cells are now almost cubical. In the smaller bron-

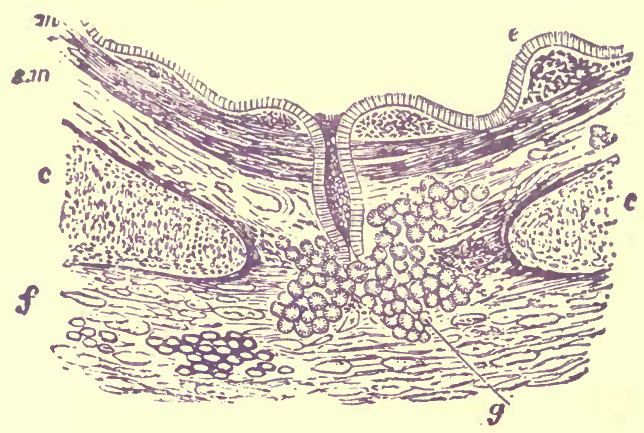

Fig. 203. - Transverse section of a bronchus, about $1 / 2$ inch in diameter. $e$. Epithelium (ciliated); immediately beneath it is the mucous membrane or internal fibrous layer, of varying thickness; $m$, muscular layer; $s . m$, submucous tissue; $f$, fibrous tissue ; $c$, cartilage enclosed within the layers of fibrous tissue; $g$, mucous gland. (F. E. Schulze.)

chi the circular muscular fibres are relatively more abundant than in the larger bronchi, and form a distinct circular coat.

The Lungs and Pleurce.-The Lungs occupy the greater portion of the thorax. They are of a spongy elastic texture, and on section appear to the naked eye as if they were in great part solid organs, except here and there, at certain points, where branches of the bronchi or air-tubes may have been cut across, and show, on the surface of the section, their tubular structure. In fact, however, the lungs are hollow organs, each of which communicates by a separate orifice with a common air-tube, the trachea.

Each lung is enveloped by a serous membrane-the pleura, one layer of which adheres closely to its surface, and provides it with its smooth and slippery covering, while the other adheres to the inner surface of the chest-wall. The continuity of the two layers, which form a closed sac, as in the case of other serous membranes, will be best understood by reference to fig. 204. The appearance of a space, howerer, between 
the pleura which covers the lung (visceral layer), and that which lines the inner surface of the chest (parietal layer), is inserted in the drawing only for the sake of distinctness. These layers are, in health, everywhere in contact, one with the other; and between them is only just so much fluid as will insure gliding easily, in their expansion and contraction, on the inner surface of the parietal layer, which lines the chestwall. While considering the subject of normal respiration, we may discard altogether the notion of the existence of any space or cavity between the lungs and the wall of the chest.

If, however, an opening be made so as to permit air or fluid to enter the pleural sac, the lung, in virtue of its elasticity, recoils, and a considerable space is left between it and the chest-wall. In other words, the natural elasticity of the lungs would cause them at all times to contract

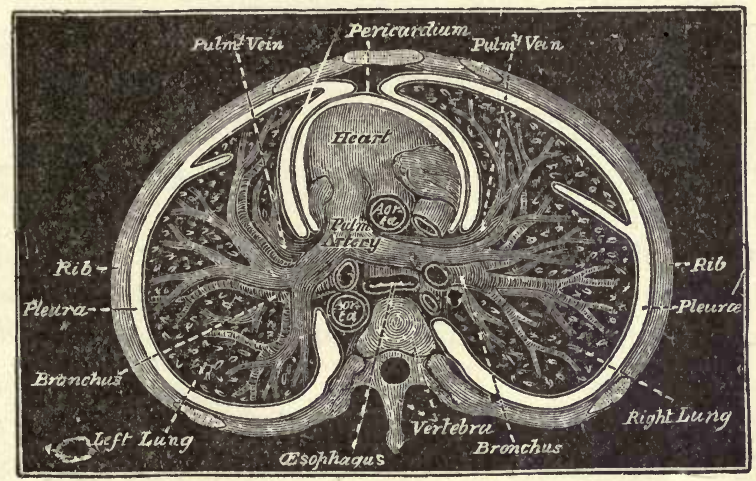

Fig. 204.-Transverse section of the chest.

away from the ribs were it not that the contraction is resisted by atmospheric pressure which bears only on the inner surface of the air-tubes and air-cells. On the admission of air into the pleural sac, atmospheric pressure bears alike on the inner and outer surfaces of the lung, and their elastic recoil is thus no longer prevented.

The pulmonary pleura consists of an outer or denser layer and an inner looser tissue. The former or pleura proper consists of dense fibrous tissue with elastic fibres, covered by endothelium, the cells of which are large, flat, hyaline, and transparent when the lung is expanded, but become smaller, thicker, and granular when the lung collapses. In the pleura is a lymph-canalicular system; and connective tissue corpuscles are found in the fibrous tissue which forms its groundwork. The inner, looser, or sub-pleural tissue contains lamellæ of fibrous connective tissue and connective-tissue corpuscles between them. $\mathrm{Nu}$ merous lymphatics are to be met with, which form a dense plexus of vessels, many of which contain valves. They are simple endothelial 
tubes, and take origin in the lymph-canalicular system of the pleura. proper. Scattered bundles of unstriped muscular fibre occur in the pulmonary pleura. They are especially strongly developed on the anterior and internal surfaces of the lungs, the parts which move most

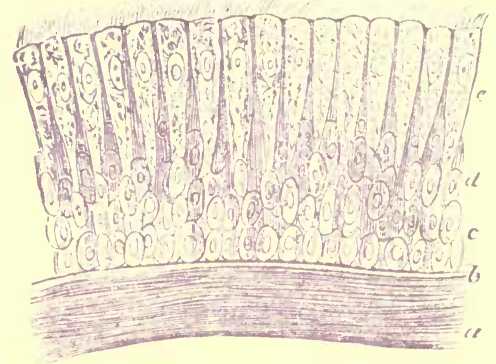

Fig. 205.-Ciliary epithelium of the human trachea. $\quad a$, Layer of longitudinally arranged elastic tibres ; $b$, basement membrane ; $c$, deepest cells, circular in form ; $d$, intermediate elongated cells ; $e$, outermost layer of cells fully developed and bearing cilia. $\times 350$. (Kölliker.)

freely in respiration: their function is doubtless to aid in expiration. The structure of the parietal portion of the pleura is very similar to that of the visceral layer.

Each lung is partially subdivided into separate portions called lobes; the right lung into three lobes, and the left into two. Each of these lobes, again, is composed of a large number of minute parts, called lob-

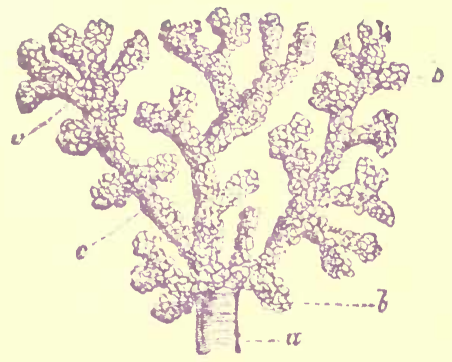

Fig. 206.

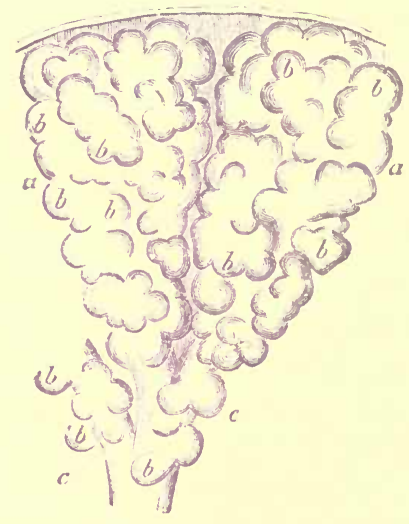

Fig. 207.

Fig. 206.-Terminal oranch of a bronchial tube, with its infundibula and air-cells, from the margin of the lung of a monkey, injected with quicksilver. $a$, Terminal bronchial twig; $b b$, infundibula

Fig. 207. - Two small infundibula or groups of air-cells, $a a$, with air-cells, $b b$, and the ultimate bronchial tubes, $c$, with which the air-cells communicate. From a new-born child. (Kölliker.)

ules. Each pulmonary lobule may be considered to be a lung in miniature, consisting, as it does, of a branch of the bronchial tube, of air-cells, blood-vessels, nerves, and lymphatics, with a sparing amount of areolar tissue. 
On entering a lobule, the small bronchial tube, the structure of which has been just described ( $a$, fig. 206), divides and subdivides; its walls at the same time becoming thinner and thinner, until at length they are formed only of a thin membrane of areolar and elastic tissue, lined by a layer of squamous epithelium, not provided with cilia. At the same time, they are altered in shape; each of the minute terminal branches widening out funnel-wise, and its walls being pouched out irregularly into small saccular dilatations, called air-cells (fig. 206, b). Such a funnel-shaped terminal branch of the bronchial tube, with its

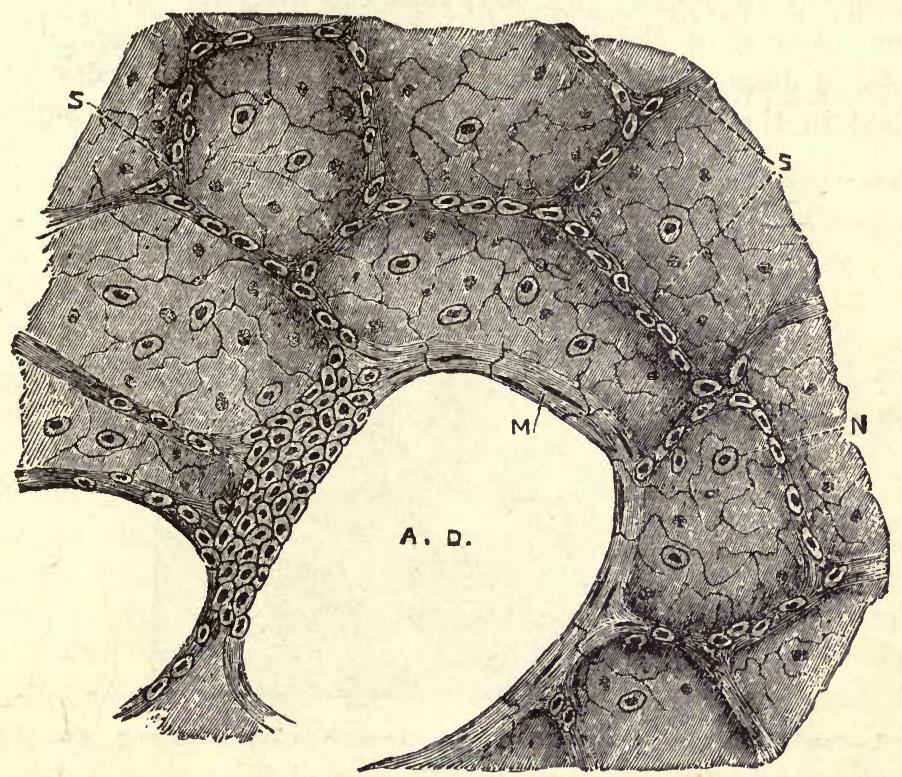

Fig. 208.-From a section of the lung of a cat, stained with silver nitrate. A. D. Alveolar duct or intercellular passage. S. Alveolar septa. N. Alveoli or air-cells, lined with large flat, nucleated cells, with some smaller polyhedral nucleated cells. M. Unstriped muscular fibres. Circular muscular fibres are seen surrounding the intelior of the alveolar duct, and at one part is seen a group of small polyhedral cells continued from the bronchus. (Klein and Noble Smith.)

group of pouches or air-cells, has been called an infundibulum (figs. 206, $207)$, and the irregular oblong space in its centre, with which the aircells communicate, an intercellular passage.

The air-cells, or air-vesicles, may be placed singly, like recesses from the intercellular passage, but more often they are arranged in groups or even in rows, like minute sacculated tubes; so that a short series of vesicles, all communicating with one another, open by a common orifice into the tube. The vesicles are of various forms, according to the mutual pressure to which they are subject; their walls are nearly in contact, and they vary from $\frac{x}{50}$ to $\frac{1}{70}$ of an inch $(.5$ to $.3 \mathrm{~mm}$.) in diameter. Their walls are formed of fine membrane, similar to that of the 
intercellular passages, and continuous with it, which membrane is folded on itself so as to form a sharp-edged border at each circular orifice of communication between contiguous air-vesicles, or between the vesicles and the bronchial passages. Numerous fibres of elastic tissue are spread out between contiguous air-cells, and many of these are attached to the outer surface of the fine membrane of which each cell is composed, imparting to it additional strength, and the power of recoil after distention. The cells are lined by a layer of epithelium (fig. 208), not provided with cilia. Outside the cells, a network of pulmonary capillaries is spread out so densely (fig. 209) that the interspaces or meshes are even narrower than the vessels, which are, on an average, $\frac{1}{3000}$ of an inch $(8 \mu)$ in diameter. Between the atmospheric air in the cells and the blood in these ressels, nothing intervenes but the thin walls of the

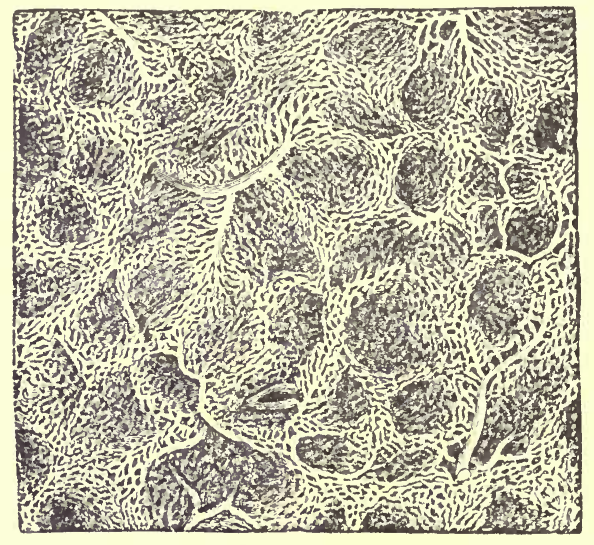

Fig. 209. - Capillary network of the pulmonary blood-vessels in the human lung. $\times 60$. (Kölliker.)

cells and capillaries; and the exposure of the blood to the air is the more complete, because the folds of membrane between contiguous cells, and often the spaces between the walls of the same, contain only a single layer of capillaries, both sides of which are thus at once exposed to the air.

The air-vesicles situated nearest to the centre of the lung are smaller and their networks of capillaries are closer than those nearer to the circumference. The resicles of adjacent lobules do not communicate; and those of the same lobule or proceeding from the same intercellular passage, do so as a general rule only near angles of bifurcation; so that, when any bronchial tube is closed or obstructed, the supply of air is lost for all the cells opening into it or its branches.

Blood-supply. - The lungs receive blood from two sources, $(a)$ the pulmonary artery, $(b)$ the bronchial arteries. The former conveys venous blood to the lungs for its arterialization, and this blood takes no share 
in the nutrition of the pulmonary tissues through which it passes. The branches of the bronchial arteries ramify for nutrition's sake in the walls of the bronchi, of the larger pulmonary vessels, in the interlobular connective tissue, etc.; the blood of the bronchial vessels being returned chiefly through the bronchial and partly through the pulmonary veins.

Lymphatics. - The lymphatics are arranged in three sets:-1. Irregular lacunæ in the walls of the alveoli or air-cells. The lymphatic vessels which lead from these accompany the pulmonary vessels toward the root of the lung. 2. Irregular anastomosing. spaces in the walls of the bronchi. 3. Lymph-spaces in the pulmonary pleura. The lymphatic vessels from all these irregular sinuses pass in toward the root of the lung to reach the bronchial glands.

Nerves. - The nerves of the lung are to be traced from the anterior and posterior pulmonary plexuses, which are formed by branches of the vagus and sympathetic. The nerves follow the course of the vessels and bronchi, and in the walls of the latter many small ganglia are situated.

\section{The Respiratory Mechanism.}

Respiration consists of the alternate expansion and contraction of the thorax, by means of which air is drawn into or expelled from the lungs. These acts are called Inspiration and Expiration respectively.

For the inspiration of air into the lungs it is evident that all that is necessary is such a movement of the side-walls or floor of the chest, or of both, that the capacity of the interior shall be enlarged. By such increase of capacity there will be of course a diminution of the pressure of the air in the lungs, and a fresh quantity will enter through the larynx and trachea to equalize the pressure on the inside and outside of the chest.

For the expiration of air, on the other hand, it is also evident that, by an opposite movement which shall diminish the capacity of the chest, the pressure in the interior will be increased, and air will be expelled, until the pressure within and without the chest are again equal. In both cases the air passes through the trachea and larynx, whether in entering or leaving the lungs, there being no other communication with the exterior of the body; and the lung, for the same reason, remains inder all the circumstances described closely in contact with the walls and floor of the chest. To speak of expansion of the chest, is to speak also of expansion of the lung.

We have now to consider the means by which the respiratory movements are effected.

Inspiration.-The enlargement of the chest in inspiration is a muscular act; the effect of the action of the inspiratory muscles being an increase in the size of the chest-cavity $(a)$ in the vertical, and $(b)$ 
in the lateral and antero-posterior diameters. The muscles engaged in ordinary inspiration are the diaphragm; the scaleni ; the external intercostals; parts of the internal intercostals; the levatores costarum; and serratus posticus superior.

(a.) The vertical diameter of the chest is increased by the contraction and consequent descent of the diaphragm-the sides of the muscle descending most, but the central tendon also-descends to some extent, while the intercostal and other muscles, by acting at the same time, prevent the diaphragm, during its contraction, from drawing in the sides of the chest.

(b.) The increase in the lateral and antero-posterior cliameters of the

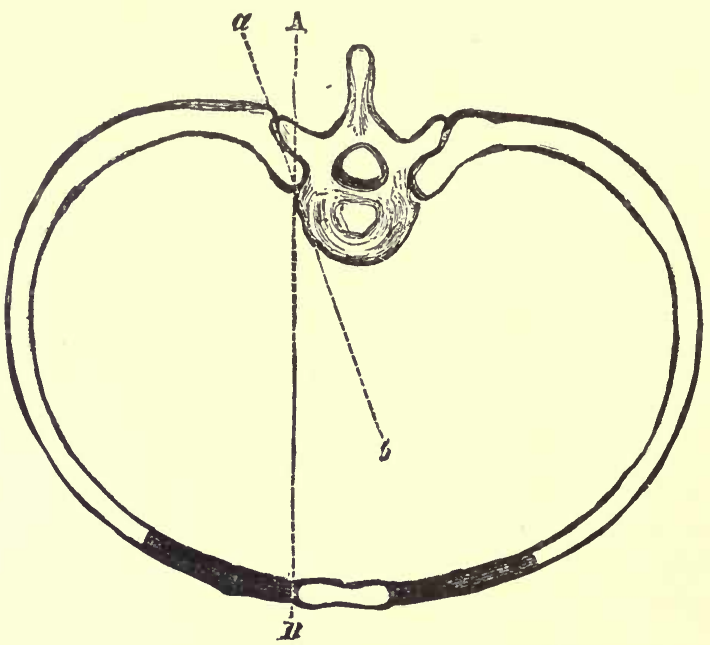

Fig. 210. -Diagram of axes of movement of ribs.

chest is effected by the raising of the ribs, the greater number of which are attached very obliquely to the spine and sternum.

The elevation of the ribs takes place both in front and at the sides - the hinder ends being prevented from performing any upward movement by their attachment to the spine. The movement of the front extremities of the ribs is of necessity accompanied by an upward and forward movement of the sternum to which they are attached, the movement being greater at the lower end than at the upper end of the latter bone.

The axes of rotation in these movements are two; one corresponding with a line drawn through the two articulations which the rib forms with the spine ( $a, b$, fig. 210); and the other with a line drawn from one of these (head of rib) to the sternum (A B, fig. 210); the motion of the rib around the latter axis being somewhat after the fashion of raising the handle of a bucket. 
The elevation of the ribs is accompanied by a slight opening out of the angle which the bony part forms with its cartilage (fig. 211, A); and thus an additional means is provided for increasing the anteroposterior diameter of the chest.

The muscles by which the ribs are raised, in ordinary quiet inspiration, are external intercostals, and that portion of the internal intercostals which is situate between-the costal cartilages; and these are assisted by the scaleni, which fix the first and second ribs, the levatores costarum, and the serratus posticus superior. The action of the levatores and the serratus is very simple. Their fibres, arising from the spine as a fixed point, pass obliquely downward and forward to the ribs, and necessarily raise the latter when they contract. The action of the intercostal muscles is not quite so simple, inasmuch as, passing merely from rib to rib, they seem at irst sight to have no fixed point toward which they can pull the bones to which they are attached.

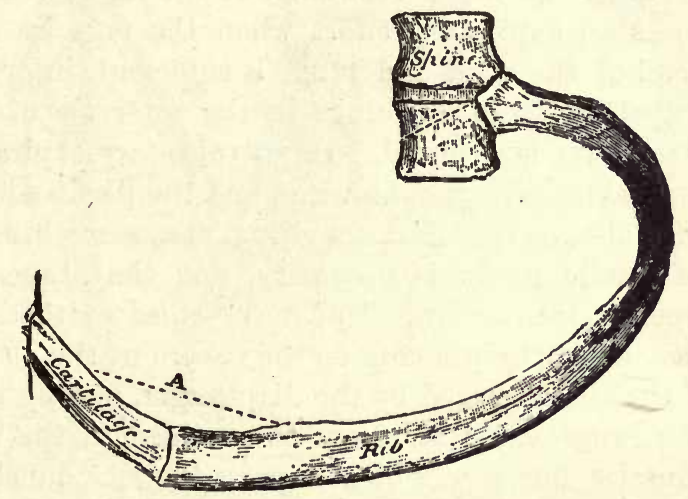

Fig. 211.-Diagram of movement of a rib in inspiration.

In tranquil breathing, the expansive movements of the lower part of the chest are greater than those of the upper. In forced inspiration, on the other hand, the greatest extent of movement appears to be in the upper antero-posterior diameter.

In extraordinary or forced inspiration, as in violent exercise, or in cases in which there is some interference with the due entrance of air into the chest, and in which, therefore, strong efforts are necessary, other muscles than those just enumerated, are pressed into the service. It is very difficult or impossible to separate by a hard and fast line the socalled muscles of ordinary from those of extraordinary inspiration; but there is no doubt that the following are but little used as respiratory agents, except in cases in which unusual efforts are required-the sternomastoid, the serratus magnus, the pectorales, and the trapezius. 
The expansion of the chest in inspiration presents some peculiarities in different persons. In young children, it is effected chiefly by the diaphragm, which being highly arched in expiration, becomes flatter as it contracts, and, descending, presses on the abdominal viscera, and pushes forward the front walls of the abdomen. The movement of the abdominal walls being here more manifest than that of any other part, it is usual to call this the abdominal type of respiration. In men, together with the descent of the diaphragm, and the pushing forward of the front wall of the abdomen, the chest and the sternum are subject to a wide movement in inspiration (inferior costal type). In women, the morement appears less extensive in the lower, and more so in the upper, part of the chest (superior costal type).

Expiration.-From the enlargement produced in inspiration, the chest and lungs return in ordinary tranquil expiration, by their elasticity; the force employed by the inspiratory muscles in distending the chest and overcoming the elastic resistance of the lungs and chest-walls, being returned as an expiratory effort when the muscles are relaxed. This elastic recoil of the chest and lungs is sufficient, in ordinary quiet breathing, to expel air from the lungs in the intervals of inspiration, and no muscular power is required. In all voluntary expiratory efforts, however, as in speaking, singing, blowing, and the like, and in many involuntary actions also, as sneezing, coughing, etc., something morc than merely passive elastic power is necessary, and the proper expiratory muscles are brought into action. By far the chief of these are the abdominal muscles, which, by pressing on the viscera of the abdomen, push up the floor of the chest formed by the diaphragm, and by thus making pressure on the lungs, expel air from them through the trachea and larynx. All muscles, however, which depress the ribs, must act also as muscles of expiration, and therefore we must conclude that the abdominal muscles are assisted in their action by the greater part of the internal intercostals, the triangularis sterni, the serratus posticus inferior and quadratus lumborum. When by the efforts of the expiratory muscies, the chest has been squeezed to less than its arerage diameter, it again, on relaxation of the muscles, returns to the normal dimensions by virtue of its elasticity. The construction of the chest-walls, therefore, admirably adapts them for recoiling against and resisting as well undue contraction as undue dilatation.

In the natural condition of the parts the lungs can never contract to the utmost, but are always more or less " on the stretch," being kept closely in contact with the inner surface of the walls of the chest by cohesion as well as by atmospheric pressure, and can contract away from these only when, by some means or other, as by making an opening into the pleural cavity, or by the effusion of fluid there, the pressure on the exterior and interior of the lungs becomes equal. 'Thus, under ordinary 
circumstances, the degree of contraction or dilatation of the lungs is dependent on that of the boundary walls of the chest, the outer surface of the one being in close contact with the inner surface of the other, and obliged to follow it in all its movements.

\section{Methods of recording Respiratory Movements.}

The movements of respiration may be recorded graphically in several ways. The ordinary method is to introduce a tube into the trachea of an animal, and to connect this tube by some gutta-percha tubing with a $T$ piece introduced into the cork of a large-sizer bottle, the other end of the $T$ having attached to it a second piece of tubing, which can remain open or can be partially or completely closed by means of a screw clamp. Into the cork is inserted a second piece of glass tubing connected with a Marey's tambour by suitable tubing. This second tube communicates any alteration of the pressure in the bottle of

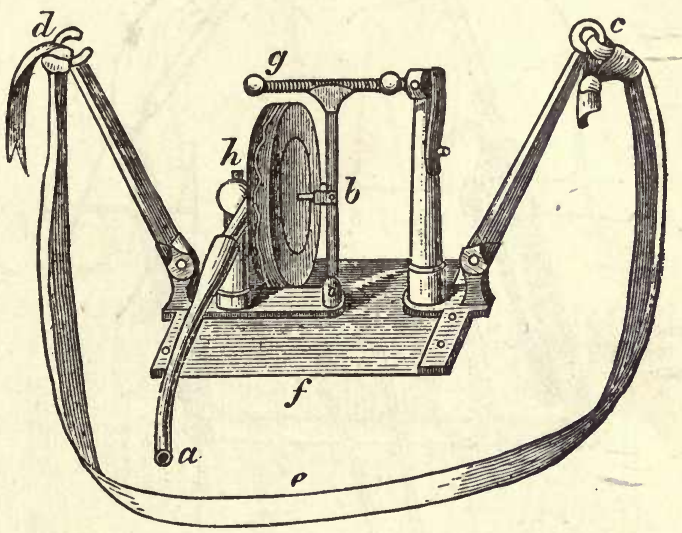

Fig. 212.-Stethograph or Pneumograph. $h$, tambour fixed at right angles to plate of steel $f$; $c$ and $d$. sums by which instrument is attached to chest by belt $e$. When the chest expands, the arms are pulled asunder, which bends the steel plate, and the tambour is affected by the pressure of $b$ which is attached to it on the one hand, and to the upright in connection with horizontal screw g. (Modified from Marey's instrument.)

the tambour, and this may be made to write on a recording surface (fig. 173). If the tube attached to the $T$ piece be closed the movements of inspiration and expiration are larger than if it were closed. The alteration of the pressure within the lungs on inspiration and expiration is shown by the movement of the column of air in the trachea. By these means a record of the respiratory morements may be obtained.

Various instruments for recording the movements of the chest by application of apparatus to the exterior. Such is the stethometer of Burton Sanderson. This consists of a frame formed of two parallel steel bars joined by a third at one end. At the free end of the bars is attached a leather strap, by means of which the apparatus may be suspended from the neck. Attached to the inner end of one bar is a tambour and ivory button, to the end of the other an ivory button. When in use, the apparatus is suspended with the transverse bar posteriorly, the button of the tambour is placed on the part of the chest the movement of which it is desired to record, and the other button 
is made to press upon the corresponding side of the chest, so that the chest is, as it were, held between a pair of calipers. The tambour is connected by tubing and a $T$ piece with a recording tambour of Marey's, and with a ball, by means of which air can be squeezed into the cavity of the tympanum. When in work the tube connected with the air ball is shut off by means of a screw clamp. The movement of the chest is thus communicated to the recording tambour.

A simpler form of this apparatus, called a pneumograph or stethograph, consisting of a thick India-rubber bag of elliptical shape about three inches long, to one end of which a rigid gutta-percha tube is attached. This bag may be fixed at any required place on the chest by means of a strap and buckle. By means of the gutta-percha tube the variations of the presssure of air in the

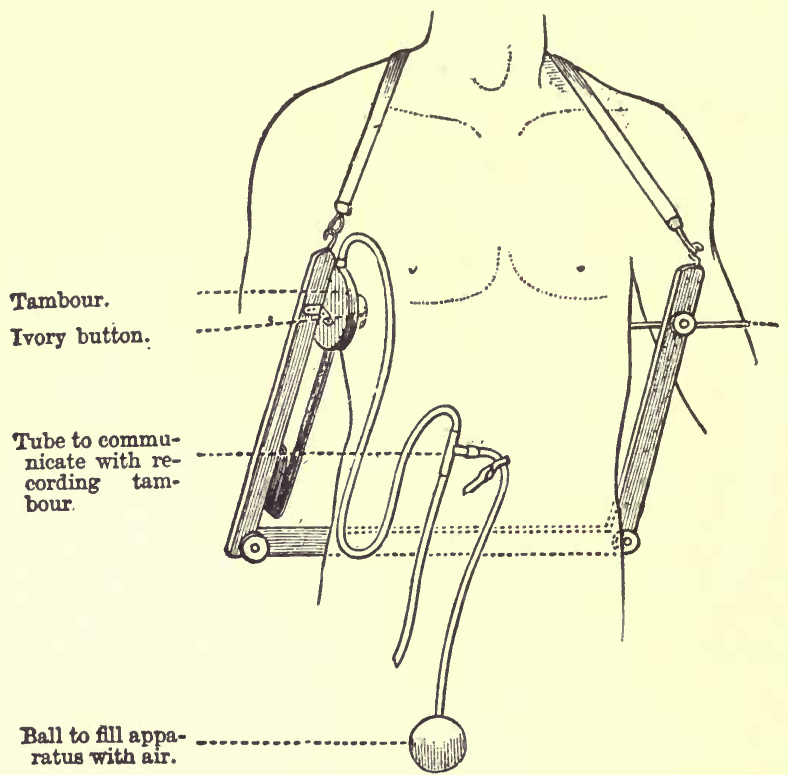

Fig. 213. -Stethometer. (Burdon Sanderson.)

bag produced by the movements of the chest are communicated to a recording tambour. This apparatus is a simplified form of Marey's pneumograph (fig. 212).

The variations of intrapleural pressure may be recorded by the introducton of a canula into the pleural or pericardial cavity, which is connected with a mercurial manometer.

Finally, it has been found possible in various ways to record the diaphragmatic movements by the insertion of an elastic bar connected with a tambour into the abdomen below it (phrenograph), by the insertion of needles into different parts of its structure, or by recording the contraction of isolated strips of the diaphragm.

The acts of expansion and contraction of the chest take up under ordinary circumstances a nearly equal time. The act of inspiring air, 
however, especially in women and children, is a little shorter than that of expelling it, and there is commonly a very slight pause between the end of expiration and the beginning of the next inspiration. The respiratory rhythm may be thus expressed:-

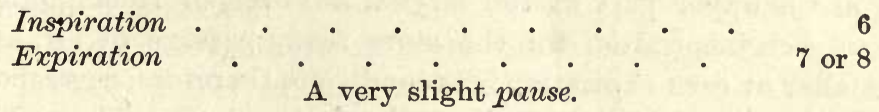

If the ear be placed in contact with the wall of the chest, or be separated from it only by a good conductor of sound or stethoscope, a faint respiratory murmur is heard during inspiration. This sound varies

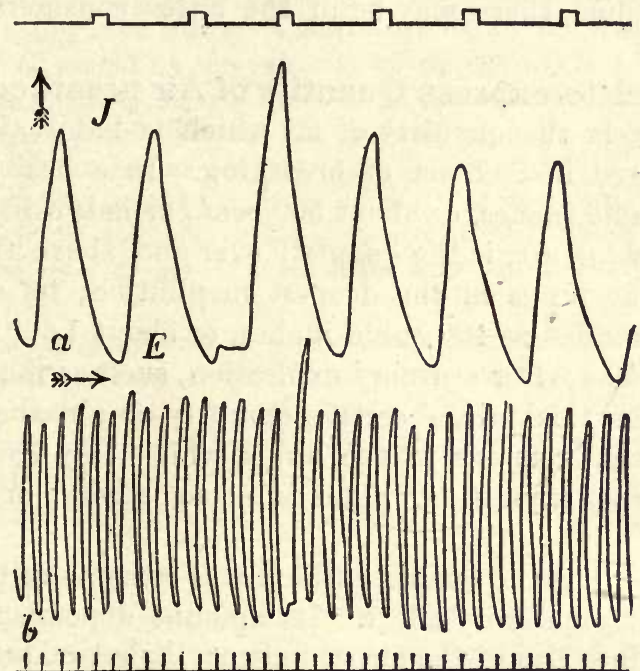

Fig. 214. Tracing of the normal diaphragm respirations of rabbit. $a$, with quick movement of drum. $b$, with slow movement. $J$, inspiration, $E$, expiration. To be read from left to right. (Marckwald.)

somewhat in different parts-being loudest or coarsest in the neighborhood of the trachea and large bronchi (tracheal and bronchial breathing), and fading off into a faint sighing as the ear is placed at a distance from these (vesicular breathing). It is best heard in children, and in them a faint murmur is heard in expiration also. The cause of the vesicular murmur has received various explanations. Most observers hold that the sound is produced in the glottis and larger bronchial tubes, but that it is modified in its passage to the pulmonary alveoli. In disease of the lungs the vesicular murmur undergoes various modifications, for a description of which one must consult text-books on physical diagnosis.

Respiratory Movements of the Nostrils and of the Glottis. -During 
the action of the muscles which directly draw air into the chest, those which guard the opening through which it enters are not passive. In hurried breathing the instinctive dilatation of the nostrils is well seen, although under ordinary conditions it may not be noticeable. The opening at the upper part of the larynx, however, or rima glottidis, is dilated at each inspiration for the more ready passage of air, and becomes smaller at each expiration; its condition, therefore, corresponding during respiration with that of the walls of the chest. There is a further likeness between the two acts in that, under ordinary circumstances, the dilatation of the rima glottidis is a muscular act and its contraction chiefly an elastic recoil; although, under various conditions to be hereafter mentioned, there may be in the latter considerable muscular power exercised.

Terms used to express Quantity of Air breathed.-a. Breathing or tidal air, is the quantity of air which is habitually and almost uniformly changed in each act of breathing. In a healthy adult man it is about 30 eubic inches, or about $500 \mathrm{ccm}$., or half a litre.

b. Complemental air, is the quantity over and above this which can be drawn into the lungs in the deepest inspiration; its amount varies, but may be reckonded as 100 cubic inches, or about 1,600 ecm.

c. Reserve air.-After ordinary expiration, such as that which expels the breathing or tidal air, a certain quantity of air, about 100 cubic inches $(1,600 \mathrm{ccm}$.) remains in the lungs, which may be expelled by a forcible and deeper expiration. This is termed reserve or supplemental air.

d. Residual air is the quantity which still remains in the lungs after the most violent expiratory effort. Its amount depends in great measure on the absolute size of the chest, but may be estimated at about 100 cubic inches, or about $1,600 \mathrm{ccm}$. to $2,000 \mathrm{ccm}$.

The total quantity of arr which passes into and out of the lungs of an adult, at rest, in 24 hours, is about 686,000 cubic inches. This quantity, however, is largely increased by exertion; the average amount for a hard-working laborer in the same time being 1,568,390 cubic inches.

e. Respiratory Capacity. - The greatest respiratory capacity of the chest is indicated by the quantity of air which a person can expel from his lungs by a forcible expiration after the deepest inspiration possible; it expresses the power which a person has of breathing in the emergencies of active exercise, violence, and disease. The average capacity of an adult, at $15.4^{\circ} \mathrm{C}$. $\left(60^{\circ} \mathrm{F}\right.$.), is about 225 to 250 cubic inches, or 3,500 to $4,000 \mathrm{ccm}$.

The respiratory capacity, or as John Hutchinson called it, vital capacity, is usually measured by a modified gasometer or spirometer, into which the experimenter breathes, -making the most prolonged expiration possible after the deepest possible inspiration. The quantity of air which is thus expelled 
from the lungs is indicated by the height to which the air chamber of the spirometer rises; and by means of a scale placed in connection with this, the number of cubic inches is read off.

In healthy men, the respiratory capacity varies chiefly with the stature, weight, and age.

It was found by Hutchinson, from whom most of our information on this subject is derived, that at a temperature of $15.4^{\circ} \mathrm{C}$. $\left(60^{\circ} \mathrm{F}\right.$.), 225 cubic inches is the average vital or respiratory capacity of a healthy person, five feet seven inches in height.

Circumstances affecting the amount of respiratory capacity.-For every inch of height above this standard the capacity is increased, on an average, by eight inches; and for every inch below, it is diminished by the same amount.

The influence of weight on the capacity of respiration is less manifest and considerable than that of height: and it is difficult to arrive at any definite conclusions on this point, because the natural average weight of a healthy man in relation to stature has not yet been determined. As a general statement, however, it may be said that the capacity of respiration is not affected by weights under 161 pounds, or $11 \frac{1}{2}$ stones; but that, above this point, it is diminished at the rate of one cubic inch for every additional pound up to 196 pounds or 14 stones.

By age, the capacity appears to be increased from about the fifteenth to the thirty-fifth year, at the rate of five cubic inches per year; from thirty-five to sixty-five it diminishes at the rate of about one and a half cubic incls per year; so that the capacity of respiration of a man of sixty years old would be about 30 cubic inches less than that of a man of forty years old, of the same height and weight. (John Hutchinson.)

'The number of respirations in a healthy adult person usually ranges from 14 to 18 per minute. It is greater in infancy and childhood. It varies also much according to different circumstances, such as exercise or rest, health, or disease, etc. Variations in the number of respirations correspond ordinarily with similar variations in the pulsations of the heart. In health the proportion is about 1 to 4 , or 1 to 5 , and when the rapidity of the heart's action is increased, that of the chest movement is commonly increased also; but not in every case in equal proportion. It happens occasionally in disease, especially of the lungs or air-passages, that the number of respiratory acts increases in quicker proportion than the beats of the pulse; and, in other affections, much more commonly, that the number of the pulses is greater in proportion than that of the respirations.

The Force of Inspiratory and Expiratory Muscles.-The force with which the inspiratory muscles are capable of acting is greatest in individuals of the height of from five feet seven inches to five feet eight inches, and will elevate a column of three inches of mercury. Above this height the force decreases as the stature increases; so that the average of men of six feet can eleviate only about two and a half inches of 
mercury. The force manifested in the strongest expiratory acts is, on the average, one-third greater than that exercised in inspiration. But this difference is in great measure due to the power exerted by the elastic reaction of the walls of the chest; and it is also much influenced by the disproportionate strength which the expiratory muscles attain, from their being called into use for other purposes than that of simple expiration. The force of the inspiratory act is, therefore, better adapted than that of the expiratory for testing the muscular strength of the body. (John Hutchinson.)

The instrument used by Hutchinson to gauge the inspiratory and expiratory power was a mercurial manometer, to which was attached a tube fitting the nostrils, and through which the inspiratory or expiratory effort was made. The following table represents the results of numerous experiments:

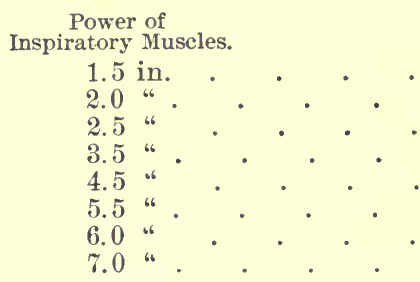

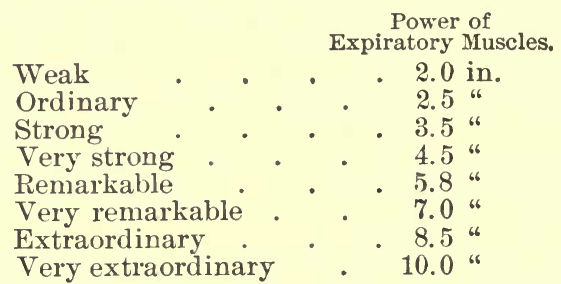

The greater part of the force exerted in deep inspiration is employed in overcoming the resistance offered by the elasticity of the lungs.

The amount of this elastic resistance was estimated by observing the eleration of a column of mercury raised by the return of air forced, after dearh, into the lungs, in quantity equal to the known capacity of respiration during life; and Hutchinson calculated, according to the well-known hydrostatic law of equality of pressures (as shown in the Bramah press), that the total force to be overcome by the muscles in the act of inspiring 200 cubic inches of air is more than $450 \mathrm{lbs}$.

The elastic force overcome in ordinary inspiration is, according to the same authority, equal to about $170 \mathrm{lbs}$.

Douglas Powell has shown that within the limits of ordinary tranquil respiration the elastic resilience of the walls of the chest favors inspiration; and that it is only in deep inspiration that the ribs and ribcartilages offer an opposing force to their dilatation. In other words, the elastic resilience of the lungs, at the end of an act of ordinary breathing, has drawn the chest-walls within the limits of their normal degree of expansion. Under all circumstances, of course, the elastic tissue of the lungs opposes inspiration and favors expiration.

It is possible that the contractile power which the bronchial tukes and air-vesicles possess, by means of their muscular fibres may (1) assist in expiration; but it is more likely that its chief purpose is (2) to regulate and adapt, in some measure, the quantity of air admitted to the 
lungs, and to each part of them, according to the supply of blood; (3) the muscular tissue contracts upon and gradually expels collections of mucus, which may have accumulated within the tubes, and which cannot be ejected by forced expiratory efforts, owing to collapse or other morbid conditions of the portion of lung connected with the obstructed tubes (Gairdner). (4) Apart from any of the before-mentioned functions, the presence of muscular fibre in the walls of a hollow viscus, such as a lung, is only what might be expected from analogy with other organs. Subject as the lungs are to such great variation in size, it might be anticipated that the elastic tissue, which enters so largely into their composition, would be supplemented by the presence of much muscular fibre also.

\section{Respiratory Changes in the Air Breathed.}

Composition of the Atmosphere.-The atmosphere we breathe has, in every situation in which it has been examined in its natural state, a nearly uniform composition. It is a mixture of oxygen, nitrogen, carbon dioxide, argon, and watery vapor, with, commonly, traces of other gases, as ammonia, sulphuretted hydrogen, etc. Of every 100 volumes of pure atmospheric air, 79 volumes (on an average) consist of nitrogen, the remaining 21 of oxygen. By weight the proportion is N. 7\%, 0. 23. The proportion of carbon dioxide is extremely small; 10,000 volumes of atmospheric air contain only about 4 or 5 of that gas.

The quantity of watery vapor varies greatly according to the temperature and other circumstances, but the atmosphere is never without some. In this country, the average quantity of watery vapor in the atmosphere is 1.40 per cent.

Composition of Air which has been breathed.-The changes effected by respiration in the atmospheric air are: 1 , an increase of temperature; 2 , an increase in the quantity of carbonic acid; 3 , a diminution in the quantity of oxygen; 4 , a diminution of volume; 5 , an increase in the amount of watery vapor; 6 , the addition of a minute amount of organic matter and of free ammonia.

1. The expired air, heated by its contact with the interior of the lungs, is (at least in most climates) hotter than the inspired air. Its temperature varies between $36^{\circ}-3 \% .5^{\circ} \mathrm{C}$. (97 $9^{\circ}$ and $99.5^{\circ} \mathrm{F}$.), the lower temperature being observed when the air has remained but a short time in the lungs. Whatever may be the temperature of the air when inhaled, it acquires nearly that of the blood before it is expelled from the chest.

2. The Carbonic dioxide is increased; but the quantity exhaled in a given time is subject to change from various circumstances. From every volume of air inspired, from 4 to 5 per cent of oxygen is abstracted; 
while a rather smaller quantity, 4.0 of carbon dioxide is added in its place: the expired air will contain, therefore, 400 vols. of carbon dioxide in 10,000 . The quantity of carbon dioxide exhaled into the air breathed by a healthy adult man. calculating that $20 \mathrm{~cm}$. of the $500 \mathrm{ccm}$. of the air breathed out at each expiration consists of carbon dioxide, and that the rate of respiration is on an average 16, the total amount would be about 460 litres in the 24 hours. From actual experiment this amount seems to be too high, since from the average of many investigations the total amount of carbon dioxide excreted per diem has been found to be about 400 litres, weighing 800 grms., consisting of 218 grms. of C., and 582 grms. of $\mathrm{O}$. From this has to be deducted about $10 \mathrm{grms}$. excreted in any other way than by the lungs, it leares about $215 \mathrm{grms}$. as the amount of $\mathrm{C}$. excreted by the average healthy man by respiration each day and night, that is about 8 oz., about half a pound. 'These quantities must be considered approximate only, inasmuch as various cireumstances, even in health, influence the amount of carbon clioxide excreted, and, correlatively, the amount of oxygen absorbed.

Circumstances influencing the amount of carbon dioxide excreted.-a. Age and Sex.-The quantity of carbon dioxide exhaled into the air breathed by males, regularly increases from 8 to 30 years of age; from 30 to 50 the quantity, after remaining stationary for a while, gradually diminishes, and from 50 to extreme age it goes on diminishing, till it scarcely exceeds the quantity exhaled at ten years old. In females (in whom the quantity exhaled is always less than in males of the same age) the same regular increase in quantity goes on from the 8th year to the age of puberty, when the quantity abruptly ceases to increase, and remains stationary so long as they continue to menstruate. When menstruation has ceased, it soon decreases at the same rate as it does in old men.

b. Respiratory Movements. - The quicker the respirations, the smaller is the proportionate quantity of carbon dioxide contained in each volume of the expired air. Although the proportionate quantity of carbon dioxide is thus diminished, the absolute amount exhaled within a given time is increased thereby, owing to the iarger quantity of air which is breathed in the time. The last half of a rolume of expired air contains more carbonic acid than the half first expired; a circumstance which is explained by the one portion of air coming from the remote part of the lungs, where it has been in more immediate and prolonged contact with the blood than the other has, which comes chiefly from the larger bronchial tubes.

c. External temperature.-The observation made by Vierordt at various temperatures between $3.4^{\circ}-23.8^{\circ} \mathrm{C} .\left(38^{\circ} \mathrm{F}\right.$. and $75^{\circ} \mathrm{F}$. $)$ show, for warm-blooded animals, that within this range, every rise equal to $5.5^{\circ} \mathrm{C}$. $\left(10^{\circ} \mathrm{F}\right.$.) causes a diminution of about $33 \mathrm{ccm}$. ( 2 cubic inches) in the quantity of carbonic acid exhaled per minute.

d. Season of the Year.-The season of the year, independently of temperature, materially influences the respiratory phenomena; spring being the season of the greatest, and autumn of the least activity of the respiratory and other functions.

e. Purity of the Respired Air.-The average quantity of carbon dioxide 
given out by the lungs constitutes about 4.3 per cent. of the expired air: but if the air which is breathed be previously impregnated with carbon dioxide (as is the case when the same air is frequently respired), then the quantity of carbon dioxide exhaled becomes relatively much less.

f. Hygrometric State of Atmosphere.-The amount of carbon dioxide exhaled is considerably influenced by the degree of moisture of the atmosphere, much more being given off when the air is moist than when it is dry.

g. Period of the Day.-During the day-time more carbon dioxide is exhaled than corresponds to the oxygen absorbed; while, on the other hand, at night very much more oxygen is absorbed than is exhaled in carbon dioxide. There is, thus, a reserve fund of oxygen absorbed by night to meet the requirements of the day. If the total quantity of carbon dioxide exhaled in 24 hours be represented by 100,52 parts are exhaled during the day, and 48 at night. While similarly, 33 parts of the oxygen are absorbed during the day, and the remaining 67 by night.

$h$. Food and Drink.-By the use of food the quantity is increased, while by fasting it is diminished; it is greater when animals are fed on farinaceous food than when fed on meat. The effects produced by spirituous drinks depend much on the kind of drink taken. Pure alcohol tends rather to increase than to lessen respiratory changes, and the amount therefore of carbon dioxide expired; rum, ale, and porter, also sherry, have very similar effects. On the other hand, brandy, whiskey, and gin, particularly the latter, almost always lessened the respiratory changes, and consequently the amount of the gas exhaled.

$i$. Exercise.-Bodily exercise, in moderation, increases the quantity to about $\frac{1}{8}$ more than it is during rest: and for about an hour after exercise the volume of the air expired in the minute is increased nearly $2,000 \mathrm{ccm}$., or 118 cubic inches : and the quantity of carbon dioxide about $125 \mathrm{ccm}$., or 7.8 cubic inches per minute. Violent exercise, such as full labor on the tread-wheel, still further increases the amount of the acid exhaled.

A larger quantity is exhaled when the barometer is low than when it is high.

3. The oxygen is diminished. Pettenkofer and Voit have found that the mean consumption of oxygen during 24 hours, by a man weighing 70 kilos, is about 700 grms., or 490 litres. The quantity of oxygen absorbed increases with muscular exercise, and falls during rest. In general terms the quantity absorbed varies with the activity of the metabolic processes.

4. The volume of air is diminished (allowance being made for the expansion in heating), the loss being due to the fact that a portion of the oxygen absorbed is not returued in the form of carbon dioxide. Since the oxygen of a given volume of carbon dioxide would have the same volume as the carbon dioxide itself at a given temperature and pressure, a portion of the oxygen absorbed must be used for other purposes than the formation of carbon dioxide. In fact, some of it is used in the formation of urea, some in the formation of water, etc. The oxygen in 
the carbon dioxide exhaled, divided by the oxygen absorbeci, gives what is known as the respiratory quotient; thus

$$
\frac{\mathrm{CO}_{2} \text { exhaled }}{\mathrm{O}_{2} \text { absorbed }} \text {. }
$$

Normally in man on a mixed diet the respiratory quotient is

$$
\frac{4.0-4.5}{5}=0.8-0.9 \text {. }
$$

But it is subject to variation through sereral causes; for example, through variation in diet. On a carbohydrate diet the respiratory quotient may rise above 0.9 , since carbohydrates contain enough oxygen to oxidize the carbon in their molecnle. On a diet containing much fat it is lowest, since oxygen is needed to completely oxidize it. And the same is true, but to a less degree, in the case of proteids. Muscular exertion raises the respiratory quotient, because in its performance carbohydrates are used np.

5. The uatery vapor is increased.-The quantity emitted is, as a general rule, sufficient to saturate the expired air, or very nearly so. Its absolute amount is, therefore, influenced by the following circumstances, (1), by the quantity of air respired; for the greater this is, the greater also will be the quantity of moisture exhaled; (2), by the quantity of watery vapor contained in the air previous to its being inspired; because the greater this is, the less will be the amount to complete the saturation of the air; (3), by the temperature of the expired air; for the higher this is, the greater will be the quantity of watery vapor required to saturate the air; (4), by the length of time which each volume of inspired air is allowed to remain in the lungs; for although, during ordinary respiration, the expired air is always saturated with watery vapor, yet when respiration is performed very rapidly the air has scarcely time to be raised to the highest temperature, or be fully charged with moisture ere it is expelled.

The quantity of water exhaled from the lungs in twenty-four hours ranges (according to the various modifying circumstances already mentioned) from about 5 to 27 ounces, the ordinary quantity being about 9 or 10 ounces. Some of this is probably formed by the chemical combination of oxygen with hydrogen in the system; but the far larger proportion of it is water which has been absorbed, as such, into the blood from the alimentary canal, and which is exhaled from the surface of the air-passages and cells, as it is from the free surfaces of all moist animal membranes, particularly at the high temperature of warm-blooded animals.

6. A small quantity of ammonia is added to the ordinary constituents of expired air. It seems probable, however, both from the fact that 
this substance cannot be always detected, and from its minute amount when present, that the whole of it may be derived from decomposing particles of food left in the mouth, or from carious teeth or the like; and that it is, therefore, only an accidental constituent of expired air.

7. The quantity of organic matter in the breath is increased. It was formerly supposed that this organic matter was injurious and gave rise to the unpleasunt symptoms which come on in badly ventilated rooms. But this has been proved erroneous.

Method of Experiment. - The experiments are conducted in such a manner that comparative analyses may be made between the air inspired and that expired. Generally au animal is placed in a chamber, called the respiratory chamber, having but two openings-one for the entrance of the inspired air, the other for the escape of expired air. Some form of pump is used for renewing the air in the chamber. Both the inspired and expired air is made to pass through agents which will absorb the contained carbon dioxide, such as baryta water or soda lime, and in turn through agents which will absorb the watery vapor. When the experiment is completed the differences between the two are determined. The difference in oxygen has to be calculated, and is open to error. The famous respiratory chamber of Pettenkofer is large enough to perform such experiments on man, and is of very elaborate construction.

How the Changes in the Air are effected.-The method by which fresh air is inhaled and expelled from the lungs has been explained. It remains to consider how it is that the blood absorbs oxygen from, and gives up carbonic acid to, the air of the alveoli. In the first place, it must be remembered that the tidal air only amounts to about $25-30$ cubic inches (about $500 \mathrm{ccm}$.) at each inspiration, and that this is of course insufficient to fill the lungs, but it mixes with the stationary air by diffusion, and so supplies to it new oxygen. The amount of oxygen in expired air, which may be taken as the average composition of the mixed air in the lungs, is about 16 to 17 per cent; in the pulmonary alveoli it may be rather less than this. From this air the venous blood has to take up oxygen in the proportion of 8 to 12 vols. per cent of blood, as the difference between the amount of oxygen in arterial and venous blood is no less. It seems therefore somewhat difficult to understand how this can be accomplished at the low partial pressure of oxygen in the pulmonary air. But as was pointed out in a previous Chapter (V.), the oxygen is not simply dissolved in the blood, but is to a great extent chemically combined with the hæmoglobin of the red corpuscles; and when a fluid contains a body which enters into loose chemical combination in this way with a gas, the tension of the gas in the fluid is not directly proportional to the total quantity of the gas taken up by the fluid, but to the excess above the total quantity which the substance dissolved in the fluid is capable of taking up (a known quantity in the case of hæmoglobin, viz., $1.59 \mathrm{~cm}$. for 1 grm. hæmoglobin). On the 
other hand, if the substance be not saturated, i.c., if it be not combined with as much of the gas as it is capable of taking up, further combination leads to no increase of its tension. However, there is a point at which the hæmoglobin gives up its oxygen when it is exposed to a low partial pressure of oxygen, and there is also a point at which it neither takes up nor gives out oxygen; in the case of arterial blood of the dog, this is found to be when the oxygen tension of the atmosphere is equal to 3.9 per cent (or $29.6 \mathrm{~mm}$. of mercury), which is equivalent to saying that the oxygen tension of arterial blood is 3.9 per cent; venous blood, in a similar manner, has been found to have an oxygen tension of 2.8 per cent. At a higher temperature, the tension is raised, as there is a greater tendency at a high temperature for the chemical compound to undergo dissociation. It is therefore easy to see that the oxygen tension of the air of the pulmonary alveoli is quite sufficient, even supposing it much less than that of the expired air, to enable the venous blood to take up oxygen, and what is more, it will take it up until the hæmoglobin is very nearly saturated with the gas.

As regards the elimination of carbon dioxide from the blood, there is evidence to show that it is given up by a process of simple diffusion, the only condition necessary for the process being that the tension of the carbonic acid of the air in the pulmonary alveoli should be less than the tension of the carbonic acid in venous blood. The carbonic acid tension of the alveolar air probably does not exceed (in the dog) 3 or 4 per cent, while that of the venous blood is 5.4 per cent, or equal to 41 mm. of mercury.

\section{Respiratory Changes in the Blood.}

Circulation of Blood in the Respiratory Organs.-To be exposed to the air thus alternately moved into and out of the air-cells and minute bronchial tubes, the blood is propelled from the right ventricle through the pulmonary capillaries in steady streams, and slowly enough to permit every minute portion of it to be for a few seconds exposed to the air, with only the thin walls of the capillary vessels and the air-cells intervening. 'The pulmonary circulation is of the simplest kind: for the pulmonary artery branches regularly; its successive branches run in straight lines, and do not anastomose: the capillary plexus is uniformly spread over the air-cells and intercellular passages; and the veins derived from it proceed in a course as simple and uniform as that of the arteries, their branches converging but not anastomosing. The veins have no valves, or only small imperfect ones prolonged from their angles of junction, and incapable of closing the orifice of either of the veins between which they are placed. The pulmonary circulation also is unaffected by changes of atmospheric pressure, and is not exposed to the 
influence of the pressure of muscles: the force by which it is accomplished, and the course of the blood are alike simple.

Changes in the Blood. - The most obvious change which the blood of the pulmonary artery undergoes in its passage through the lungs is 1st, that of color, the dark crimson of venous blood being exchanged for the bright scarlet of arterial blood. The cause of this has been already shown to be that the arterial blood contains a greater quantity of scarlet or oxyhæmoglobin; $2 d$, and in connection with the preceding change it gains oxygen ; $3 d$, it loses carbon dioxide. It was incidentally mentioned in the Chapter on the Blood that the carbon dioxide which is carried by the blood to be eliminated by the lungs is not simply dissolved in the plasma. It is combined with some substance in the blood, and when it is carried to the lungs this substance must undergo decomposition. What is the nature of the compound it forms is not known, but it appears most likely that the gas is combined in the plasma with the sodium carbonate which it contains. It has also been suggested that as the carbon dioxide of the entire blood is more easily given up to the vacuum of a mercurial air-pump than is the gas of the serum corresponding to the blood taken, that the corpuscles of the blood exercise some power in promoting the decomposition of the substance with which the gas is combined in the plasma. The plasma or serum will not give up the whole of its carbon dioxide until the addition of an acid, when the last portion, 2 to 5 per cent, comes off, the entire blood gives up the whole of its carbon dioxide to the action of the mercurial pump, and does not require the action of an acid. It may be mentioned that, according to some, the carbon dioxide is combined with proteid, either in the plasma.or in the red blood-corpuscles; 4th, it becomes slightly cooler; 5th, it coagulates sooner and more firmly, apparently containing more fibrin. The oxygen absorbed into the blood from the atmospheric air in the lungs is combined chemically with the hæmoglobin of the red blood-corpuscles. In this condition it is carried in the arterial blood to the various parts of the body, and brought into near relation or contact with the tissues. In these tissues, a certain portion of the oxygen which the arterial blood contains, disappears, and a proportionate quantity of carbon dioxide and water is formed. The venous blood, containing the new-formed carbon dioxide, returns to the lungs, where a portion of the carbon dioxide is exhaled, and a fresh supply of oxygen is taken in.

In what way these changes are brought about will be next discussed.

\section{Respiratory Changes in the Tissues.}

The changes which occur in the composition of the blood during its circulation are believed to take place in the tissues, and particularly in the muscles. The changes are, as we have just mentioned, chiefly the 
removal of oxygen from and the addition of carbon dioxide to the blood. These changes are sometimes spoken of as internal respiration. The oxygen carried by the corpuscles of the blood in the form of oxyhwmoglobin is given up to the tissues, as the tension of the gas within them is very small. 'The gas thus set free is apparently seized upon by the protoplasm of the tissues and built up into its molecule, and thus assists in the process of anabolism, possibly uniting with some compound somewhat in the same manner but more firmly than it does with hæmoglobin. The low oxygen pressure of the tissues thus allows a constant abstraction of the gas from the blood. 'The process of katabolism, or breaking down, is always associated with the evolution of earbon dioxide, so that as the blood passes through the tissues containing little of this gas, the high tension of the gas in the tissues permits of its passage into the blood. It has been proved that the process of the evolution of carbon dioxide from living muscle will go on for a time in the absence of a supply of free oxygen, and so it is clear that the former gas is not derived directly from the combustion of the carbon in the presence of the latter gas. It was at one time beliered that the earbon dioxide of venous blood resulted from the oxidation of substances in the blood itself. It has, however, been shown that the blood itself has very slight oxidizing powers, and that in the frog the whole of the blood may be replaced by saline solution without producing any marked effect upon the metabolism of the body. It is obviously unlikely that any but very slight oxidation could go on in such a medium. It has moreover been demonstrated that the tension of carbon dioxide in the tissues is considerably greater in the tissues than it is in the venous blood.

\section{Special Respiratory Acts.}

It will be well here, perhaps, to explain eertain special respiratory acts, which appear at first sight somewhat complicated, but cease to be so when the mechanism by which they are performed is clearly understood. The diagram (fig. 215) shows that the eavity of the chest is separated from that of the abdomen by the diaphragm, which, when acting, will lessen its curve, and thus descending, will push downward and forward the abdominal viscera; while the abdominal muscles have the opposite effect, and in acting will push the viscera upward and backward, and with them the diaphragm, supposing its ascent to be not from any cause interfered with. It will also be seen that the lungs communicate with the exterior of the body through the trachea and larynx, and further on through the mouth and nostrils--through either of them separately, or through both at the same time, according to the position of the soft palate. The stomach communicates with the exterior of the body through the cesophagus, pharynx, and mouth; while 
below the rectum opens at the anus, and the bladder through the urethra. All these openings, through which the hollow viscera communicate with the exterior of the body, are guarded by muscles, called sphincters, which can act independently of each other.

Sighing.-In sighing there is a somewhat prolonged inspiration; the air almost noiselessly passing in through the glottis, and by the elastic recoil of the lungs and chest-walls, and probably also of the abdominal walls, being suddenly expelled.

In the first, or inspiratory part of this act, the descent of the diaphragm presses the abdominal viscera downward, and of course this pressure tends to evacuate the contents of such of them as communicate with the exterior of the body. Inasmuch, however, as their various openings are guarded by sphincters, in a state of constant tonic contraction, there is no escape of their contents, and the air simply enters the lungs. In the second, or expiratory part of the act, pressure is also made on the abdominal viscera in the opposite direction, by the recoil of the abdominal.walls; but the pressure is relieved by the escape of air through the open glottis, and the relaxed diaphragm is pushed up again into its original position. The sphincters of the stomach, rectum, and bladder, act in the same manner as before.

Hiccough resembles sighing in that it is an inspiratory act: but the inspiration is sudden instead of gradual, the diaphragm acting suddenly and spasmodically; and the air, rushing through the unprepared rima glottidis, is suddenly arrested and produces the peculiar sound.

Coughing.--In the act of coughing there is most often first of all a deep inspiration, followed by an expiration; but the latter, instead of being easy and uninterrupted, as in normal breathing, is obstructed, the glottis being momentarily closed by the approximation of the vocal cords. The abdominal muscles, then strongly acting, push up the viscera against the diaphragm, and thus make pressure on the air in the lungs until its tension is sufficient to noisily open the vocal cords which oppose its outward passage. In this way considerable force is exercised, and mucus or any other matter that may need expulsion from the airpassages is quickly and sharply expelled by the outstreaming current of air. It will be evident on reference to fig. 215 , that pressure exercised by the abdominal muscles in the act of coughing, acts as foreibly on the abdominal viscera as on the lungs, inasmuch as the viscera form the medium by which the upward pressure on the diaphragm is made, and there is of necessity quite as great a tendency to the expulsion of their contents as of the air in the lungs. The instinctive and if necessary voluntarily increased contraction of the sphincters, however, prevents any escape at the openings guarded by them, and the pressure is effective at one part only, at the rima glottidis. 
Sneezing.-The same remarks that apply to conghing, are almost exactly applicable to the act of sneezing; but in this instance the blast of air, on escaping from the lungs, is directed, by an instinctive contraction of the pillars of the fauces, and descent of the soft palate, chiefly through the nose, and any offending matter is thence expelled.

speaking.-In speaking, there is a voluntary expulsion of air through the glottis by means of the expiratory muscles. The rocal coris, hy the

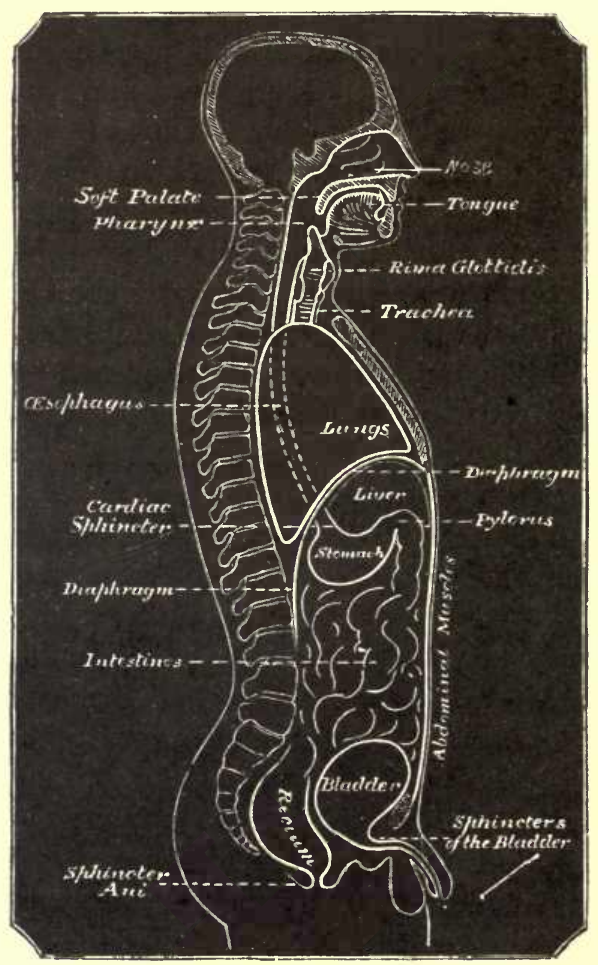

Fig. 215.

muscles of the larynx, are put in a proper position and state of tension for vibrating as the air passes over them, and sound is produced. 'The sound is moulded into articnlate speech by the tongue, teeth, lips, etc. -the vocal cords producing the sound only, and having nothing to do with articulation.

Singing.-Singing resembles speaking in the manner of its production; the laryngeal muscles, by variously altering the position and degree of tension of the vocal cords, producing the different notes. Words used in the act of singing are of course framed, as in speaking, by the tongue, teeth, lips, etc.

Sniffing.-Sniffing is produced by a rapidly repeated but incomplete 
action of the diaphragm and other inspiratory muscles. The mouth is closed, and the whole stream of air is made to enter the air-passages through the nostrils. 'The alæ nasi are commonly at the same time instinctively dilated.

Sobbing.-Sobbing consists of a series of convulsive inspirations, at the moment of which the glottis is usually more or less closed.

Laughing.-Laughing is made up of a series of short and rapid expirations.

Yawning.-Yawning is an act of inspiration but is unlike most of the preceding actions as it is always more or less involuntary. It is attended by a stretching of varions muscles about the palate and lower jaw, which is probably analogous to the stretching of the muscles of the limbs in which a weary man finds relief, as a voluntary act, when they have been some time out of action. The involuntary and reflex character of yawning probably depends on the fact that the muscles concerned are themselves at all times more or less used involuntarily, and require, therefore, something beyond the exercise of the will to set them in action. For the same reason, yawning, like sneezing, cannot be well performed voluntarily.

Sucking.-Sucking is not properly a respiratory act, but it may be most conveniently considered in this place. It is caused chiefly by the depressor muscles of the os hyoides. These, by drawing downward and backward the tongue and floor of the mouth, produce a partial vacuum in the latter: and the weight of the atmosphere then acting on all sides tends to produce equilibrium on the inside and outside of the mouth as best it may. The communication between the mouth and pharynx is completely shut off by the contraction of the pillars of the soft palate and descent of the latter so as to touch the back of the tongue; and the equilibrium, therefore, can be restored only by the entrance of something through the mouth. The action, indeed, of the tongue and floor of the mouth in sucking may be compared to that of the piston in a syringe, and the muscles which pull down the os hyoides and tongue, to the power which draws the handle.

\section{The Nervous Apparatus of Respiration.}

Like all other functions of the body, the discharge of which is necessary to life, respiration is essentially an involuntary act. Unless these were the case, life would be in constant danger, and would cease on the loss of consciousness for a few moments, as in sleep. It is, however, also necessary that respiration should be to some extent under the control of the will. For were it not so, it would be impossible to perform those respiratory acts which have been just discussed, such as speaking, singing, and the like. 
It has been known for centuries that there exists a district of the central nervous system on the destruction of which both respiration and life cease. All attempts to localize this district, however, before those of Flourens were unsuccessful. Flourens, after many series of experiments as to the exact position of what he called the "knot of life" (nœud vital), placed it in the fourth ventricle, at the point of the $\mathrm{V}$ in the gray matter at the lower end of the calamus scriptorius; a district of considerable size, viz., $5 \mathrm{~mm}$., on both sides of the middle line. Observers subsequent to Flourens have attempted to show that the chief respiratory centre on the one hand is situated higher up in the nervous system, e.g., in the floor of the third ventricle (Christiani), or in the corpora quadrigemina (Martin and Booker, Christiani, and Stanier), or on the other hand, lower down in the spinal cord, and that the medullary centres, if they exist, are either accessory or subservient to such centres. The balance of experimental evidence, however, is to prove that the sole centres for respiration is a limited district in the medulla oblongata in close connection with the vagus nucleus on each side, with which they are probably identical. The destruction of this district stops respiration forever; whereas, if it be left in connection with the muscles of respiration by their nerves, although the remainder of the central nervous system be separated from it, respiration continues. It may be considered almost certain that the medullary centre is the only true respiratory centre, and that the observations of Langendorff, that in newly-born animals in which the medulla has been cut immediately or a few millimetres below the point of the calamus scriptorius respiration continues for some time as in normal animals cannot be received. We are indebted to Marckwald for much information on this subject, and he has come to the conclusion that normal respiration does not occur after division of the bulb from the cord, and that the so-called respiratory movements noticed by Langendorff are merely tetanic contractions of the respira. tory muscles with which often enough other muscles take part.

The action of the medullary centre is to send out impulses during inspiration, which cause respiratory movements of the muscles- $(a)$ of the nostrils, and jaws through the facial and inferior division of the fifth nerves; $(b)$ of the glottis, chiefly through the inferior laryngeal branches of the vagi; $(c)$ of the intercostal and other muscles which produce raising of the ribs, chiefly through the intercostal nerves, ard $(d)$ of the diaphragm through the phrenic nerves.

If any one of these sets of nerves be divided, respiratory morements of the corresponding part cease.

Similarly it may be supposed that the centre sends out impulses during expiration to certain other muscles. It has been suggested, however, that the centre consists of two parts, or is double, and that it is made up of an inspiratory centre, which is constantly in action, and of an ex- 
piratory centre, which acts less generally, inasmuch as ordinary tranquil expiration is seldom more than an elastic recoil, and not a muscular act to any marked degree.

Assuming this view of the double centres to be correct, of their exact mode of action there is some difference of opinion; it is now thought that they may act automatically, but normally are influenced by afferent impulses from the periphery, as well as by impulses passing down from the cerebrum. The centre is, in other words, both automatic and reflex. It will be simplest to discuss its reflex function first of all.

Action of Afferent Stimuli.-(a) Action of the vagi.-If both vagi be divided in the neck, the respirations become much slower and deeper; this may be the case, but to a less marked degree, if one of the nerves is divided instead of both. If the central end of the divided nerve be stimulated with a weak interrupted current, the most constant effect is that the respirations are quickened, and if the stimuli are properly regulated, the normal rhythm of respiration may be resumed. If the stimuli be repeated with sufficient quickness, after a while the breathing is brought to a stand-still at the height of inspiration by tetanus of the diaphragm. Sometimes, however, stimulation of the central end of the divided vagi produces still greater slowing than that which follows the division, so that if it be continued, the respirations cease, with the diaphragm in a condition of complete relaxation. Marckwald considers that the differences in the effects of vagus stimulation are due to the stimulus being applied to the nerve at different periods in the respiratory cycle, and that the action of the vagus may be to call forth either inspiration or expiration - the impulses passing up the vagi being necessary to the production of the normal respiratory rhythm. The fibres of the vagus are used under the following circumstances, those fibres which tend to inhibit expiration and to stimulate inspiration are stimulated at their distribution in the lung when the lung is empty and in a condition of expiration, and the fibres which tend to inhibit inspiration and to promote expiration are stimulated when the lung is fully expanded. The afferent impulses are the results of mere mechanical stimulation, and do not depend upon the chemical nature of the gases within the pulmonary alveoli. The vagus always acts upon the centres as a stimulator of discharge, or exciter of catabolism.

(b) Action of the superior laryngeal nerves.-If the superior laryngeal branch of the vagus be divided, which usually produces no apparent effect, and the central end be stimulated, the effect is very constant, respirations are slowed, but there is a tendency toward expiration, as is shown by the contraction of the abdominal muscles. Thus if the vagus fibres contain fibres which stimulate inspiration and inhibit expiration, as well as other fibres which have the reverse effect, the superior laryngeal fibres inhibit inspiration and stimulate expiration. 
'I'he superior laryngeal nerves are true expiratory nerves, and may ne set in action when the mucous membrane of the larynx is irritated. 'They are not constantly in action like the vagi.

(c) Action of the glosso-pharyngeal nerves. - It has been ascertained, chiefly by the researches of Marckwald, that while division of the glosso-pharyngeal nerves produces no effect upon respiration, stimulation of them causes inhibition of inspiration for a short period. This action accounts for the very necessary cessation of breathing during swallowing. The effect of the stimulation is only temporary, and is followed by normal breathing movements.

(d) Action of other sensory nerves.-The respiratory centres are as a rule stimulated to produce respiration by impressions conveyed by sensory nerves, e.g., the nerves of the skin; cold water applied to the surface is almost invariably followed by a deep inspiration. Stimulation of the splanchinies and of the abdominal branches of the vagi produce expiration. 'The fifth nerves, as well as the glosso-pharyngeal and the superior laryngeal, inhibit inspiration, but they tend to produce a gradual slowing and not an absolute inhibition, as do the glossopharyngeal.

It must be remembered that although many sensory nerves may on stimulation be made to produce an effect upon the respiratory centres, there is no evidence to show that any one of them, except the vagi, is constantly in action. 'The vagi indeed are, as far as we know, the only normal regulators of respiration.

Automatic Action of the Respiratory Centres.-Although it has been very definitely proved that the respiratory centres may be affected by afferent stimuli, and particularly by those reaching them through the vagi, there is reason for believing that the centres are capable of sending out efferent impulses to the respiratory muscles without the action of any afferent stimuli. Thus, if the brain be removed above the bulb, respiration continues. If the spinal cord be divided below the bulb, the facial and laryngeal respiratory movements continue, although no afferent impulses can reach the centres except through the cranial sensory nerves, and these, as we have seen, are not always in action, and indeed may be divided without producing any effect, when the bulb and cord are intact. As has been shown, too, respiration continues when the vagi are divided. All of these experiments render it highly probable that afferent impulses are not required in order that the respiratory centres should send out efferent impulses of some kind to the respiratory muscles; these centres, then, are automatic. How they act in the absence of afferent stimuli has been demonstrated by Marckwald. He has shown $-(a)$ firstly, that if the bulb be separated from the brain, and the vagi be then cut, there is first of all inspiratory spasm followed by irregular spasm of muscles both of inspiration and expiration, and death; $(b)$ 
secondly, that if the vagi are divided, the respirations, although altered in character, are regular, but that if then the brain is separated from the medulla, the same respiratory spasms occur. From these experiments it is concluded that the automatic action of the centres consists in the liberation of respiratory spasms only, and not of regular rhythmic movements; but that impressions reaching the centres either from the cerebrum or through the vagi, prevent the gathering tension in the centres from becoming too great, and convert the spasms which would otherwise arise into regular movements. The chief difference between the action of the vagi and of the cerebral tracts, is that the former are always in action, whilst the latter are not. When the vagi are in action and the higher centres are not, periodic respiration takes place, that is to say, respirations occurring in groups, each such group being followed by a pause; a type of respiration known as Cheyne-Stokes breathing, to which we shall return presently. It will be thus seen that even the ordinary action of the respiratory centres is to a large extent reflex, and dependent upon vagus or cerebral stimulation.

Method of Stimulation of the Respiratory Centres.-Apart then from afferent impulses, the respiratory centres are capable of working automatically, and this fact has been explained by the supposition that they are stimulated to action by the condition of the blood circulating through them, since when the blood becomes more and more venous the action of the centres becomes more and more energetic, and if the air is prevented from entering the chest, the respiration in a short time becomes very labored. Any obstruction to the entrance of air indeed, whether partial or complete, is followed by an abnormal rapidity of the inspiratory acts. The condition caused by any interference with the free exchange of gases in the lungs, or by any circumstance in consequence of which the oxygen of the blood is used up in an abnormally quick manner, is known as dyspnoea. If the aëration of the blood is much interfered with, not only are the ordinary respiratory muscles employed, but also those muscles of extraordinary inspiration and expiration which have been previously enumerated. Thus as the blood becomes more and more venous, the action of the medullary centres becomes more and more active. The question has been much debated as to what quality of the venous blood it is which causes this increased activity; whether it is its deficiency of oxygen or its excess of carbonic acid. It has been answered to some extent by the experiments, which show on the one hand that dyspnoa occurs when there is no obstruction to the exit of carbonic acid as when an animal is placed in an atmosphere of nitrogen, and that it cannot therefore be due to the accumulation of carbonic acid; and on the other, that if plenty of oxygen is supplied, true dyspnœa does not occur, although the carbonic acid of the blood is in excess. It is highly probable, therefore, that the respiratory centres may be stimu- 
lated to action by the absence of sufficient oxygen in the blood circulating in it, and not by the presence of an excess of carbonic acid.

But this is not all, since it has been proved by Marckwald that the medullary centres are capable of acting for some time in the absence of any circulation, and after excessive bleeding. 'The view taken by this author with regard to the action of the centres is as follows: the respiratory centres are set to act by the condition of their metabolism, much in the same way as the heart is set to beat rhythmically. When anabolism is completed, catabolism or discharge occurs, and this alternate but crude and spasmodic action will occur without a definite bloodsupply, as long as the centres are properly nourished and stimulated by their own intercellular fluid. The afferent impulses brought by the vagi, in consequence of the stimulation of their terminal fibres in the lungs, have a tendency to bring about catabolism, and to convert crude respiratory spasms into regular and rhythmic discharges. In the absence of the vagus stimulation, the impulses from the cerebrum may be effectual for the same purpose.

It is unreasonable to think, however, that the respiratory centres are independent of the character of the blood-supply either as regards quantity or quality. This must have a great influence upon their irritability ; it is certain, for example, that venous blood greatly increases the respiratory movements, first of all both of inspiration and of expiration, and then of the latter to a greater degree. It may be that the diminution of oxygen in the blood acts as a stimulator of catabolism, in both inspiratory and expiratory centres, but particularly in the latter, in a manner similar to but not identical with, that of the vagus. It has also been shown that the presence of the products of great muscular metabolism in the blood will greatly increase the irritability of the respiratory centres, even if the blood itself be not particularly venous in character.

It appears that the inspiratory and expiratory respiratory centres are bilateral, and that each pair may act independently, since the bulb may be divided longitudinally, and then if one vagus be divided, the respiratory rhythm on the two sides of the body becomes unequal, the movements of the side upon which the vagus is divided being slower than on the other side, while stimulation of the divided nerve acts only upon the movements of its own side.

Apnœa.-When we take several deep inspirations in rapid succession by voluntary effort, we find that we can do without breathing for a much longer time than usual; in other words, several rapid respirations seem to inhibit for a time normal respiratory movements. It was thought that the reason for this partial cessation of respiration, which was called apnœa, is that by taking several deep breaths we overcharge our blood with oxygen, and that as the respiratory centre can only be stimulated by blood in which the standard of oxygen is below a certain 
level, no respiratory impulses can occur until the oxygen tension of the blood reach that level. This idea must now be modified, if not given up, in face of the experiments, e.g., those of Hering, on cats' blood during apnœa, which have shown that animals in a condition of apnœa may have less and not more oxygen in their blood than in a normal state, although the carbonic anhydride is less. One view now taken of the cause of apnœa is that by rapid inflations of the lungs impulses pass up by the vagi, by means of which inspiration is after a while inhibited; another view is that by the repeated stimulation of the centre by vagus impulses which result in rapid respiratory morements, anabolism is at last arrested. Apnoa is with difficulty produced, if at all, when the vagi are divided.

Effects of Vitiated Air.-Ventilation.-As the air expired from the lungs contains a large proportion of carbon dioxide and a minute amount of organic putrescible matter, it is obvious that if the same air be breathed again and again, the proportion of carbonic dioxide and organic matter in it will constantly increase till it becomes unfit to breathe; long before this point is reached however, uneasy sensations occur, such as headache, languor, and a sense of oppression. It is a remarkable fact, however, that the organism after a time adapts itself to a very vitiated atmosphere, and that a person soon comes to breathe, without sensible inconvenience, an atmosphere which, when he first enters it, feels intolerable. Such an adaptation, however can only take place at the expense of a depression of all the vital functions, which must be injurious if long continued or often repeated.

This power of adaptation is well illustrated by the experiments of Claude Bernard. A sparrow is placed under a bell-glass of such a size that it will live for three hours. If now at the end of the second hour (when it could have survived another hour) it be taken out and a fresh healthy sparrow introduced, the latter will perish instantly.

It must be evident that provision for a constant and plentiful supply of fresh air, and the removal of that which is vitiated, is of far greater importance than the actual cubic space per head of occupants. Not less than 2,000 cubic feet per head should be allowed in sleeping apartments (barracks, hospitals, etc.), and with this allowance the air can only be maintained at the proper standard of purity by such a system of ventilation as provides for the supply of 1,500 to 2,000 cubic feet of fresh air per head per hour. (Parkes.)

\section{The Effect of Respiration on the Circulation.}

As the heart, the aorta, and pulmonary vessels are situated in the air-tight thorax, they are exposed to a certain alteration of pressure when the capacity of the latter is increased in inspiration; for although the expansion of the lungs tends to counter-balance this increase of area, 
it never does so entirely, since part of the pressure of the air which is drawn into the lungs through the trachea is expended in overeoming their elasticity. The amount thus used up increases as the lungs become more and more expanded, so that the pressure inside the thorax during inspiration, as far as the heart and great vessels are concerned, never quite equals that outside, and at the conclusion of inspiration is considerably less than the atmospheric pressure. It has been ascertained that the amount of the pressure used up in the way above described, varies from 5 or $5 \mathrm{~mm}$. of mercury during the pause, to $30 \mathrm{~mm}$. of mercury when the lungs are expanded at the end of a deep inspiration, so that it will be understood that the pressure to which the heart and great vessels are subjected diminishes as inspiration progresses, and at

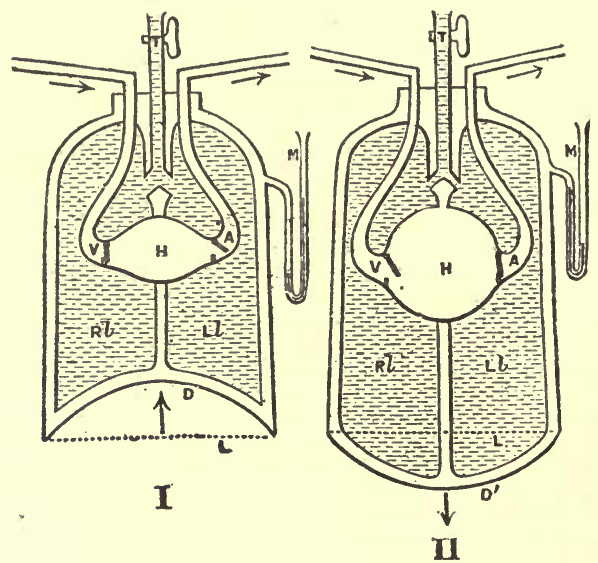

Fig. 216.-Diagram of an apparatus illustratıng the effect of inspiration upon the heart and great vessels within the thorax. I, the thorax at res : II, during inspiration; $D$, represents the diaphragm when relaxed; $\mathrm{D}^{\prime}$, when contracted (it must be remembered that this position is a mere diagram), i.e., when the capacity of the thora" is enla ged; н, the heart; v, the veins entering it. and $\mathrm{A}$, the aorta : $\mathrm{k} l$, $\mathrm{L} l$, the right and left lung: $\mathrm{T}$, th trachea: $\mathrm{M}$, mercurial manometer in eonnection with pleura. The increase in the capacity of the box representing the thorax is seen to dilate the heart as well as the lungs, and so to pump in blood throngh $\mathrm{v}$, whereas the valve prevents reflex through $\mathbf{A}$. The position of the mercury in $\mathbf{M}$ shows also the suction which is taking place. (Landois.)

its minimum is less by $30 \mathrm{~mm}$., than the normal pressure, $760 \mathrm{~mm}$. of mercury. It will be understood from the accompanying diagram how, that if there were no lungs in the chest, if its capacity were increased, the effect of the increase would be expended in pumping blood into the heart from the veins. With the lungs placed as they are, during inspiration the pressure outside the heart and great ressels is diminished, and they have therefore a tendency to expand and to diminish the intravascular pressure. The diminution of pressure within the veins passing to the right auricle and within the right auricle itself, will draw the blood into the thorax, and so assist the circulation. This suction action is independent of the suction power of the diastole of the auricle about which we have previously spoken. The effect of sucking more blood 
into the right auricle will, cceteris paribus, increase the amount passing through the right ventricle, which also exerts a similar suction action, and through the lungs into the left auricle and ventricle, and thus into the aorta. This all tends to increase the blood-pressure. The effect of the diminished pressure upon the pulmonary vessels will also help toward the same end, i.e., an increased flow through the lungs, so that, as far as the heart and its veins are concerned, inspiration increases the blood-pressure in the arteries. The effect of inspiration upon the aorta and its branches within the thorax would be, however, contrary; for as the pressure outside is diminished the vessels would tend to expand, and thus to diminish the tension of the blood within them, but inasmuch as the large arteries are capable of little expansion beyond their natural calibre, the diminution of the arterial tension caused by this means

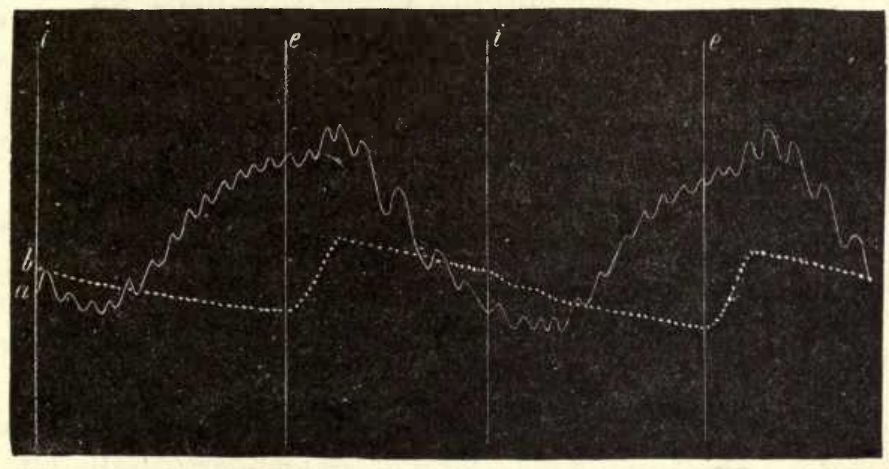

Fig. 217. - Comparison of blood-pressure curve with curve of intra-thoracic pressure. (To be read from left to right.) $a$ is the curve of blood-pressure with its respiratory undulations, the slower beats on the descent being very marked; $b$ is the curve of intra-thoracic pressure obtained by connecting one limb of a manometer with the plural cavity. Inspiration begius at $i$ and expiration at $e$. The intra-thoracic pressure rises very rapidly after the cessation of the inspiratory effort, and then slowly falls as the air issues from the chest; at the beginning of the inspiratory effort the fall becomes more rapid. (M. Foster.)

would be insufficient to counteract the increase of blood-pressure produced by the effect of inspiration upon the veins of the chest, and the balance of the whole action would be in favor of an increase of bloodpressure during the inspiratory period. But if a blood-pressure tracing be taken at the same time that the respiratory movements are being recorded, it will be found that, although speaking generally, the arterial tension is increased during inspiration, the maximum of arterial tension does not correspond with the acme of inspiration (fig. 21\%). In fact, at the beginning of inspiration the pressure continues to fall, then gradually rises until the end of inspiration, and continues to do so for some time after expiration has commenced.

As regards the effect of expiration, the capacity of the chest is diminished, and the intra-thoracic pressure returns to the normal, which is not exactly equal to the atmospheric pressure. The effect of this on 
the veins is to increase their extra-rascular and so their intra-vascular pressure, and to diminish the flow of blood into the left side of the heart, and with it the general blood-pressure, but this is almost exactly balanced by the necessary increase of arterial tension caused by the increase of the extra-vascular pressure of the aorta and large arteries, so that the arterial tension is not much affected during expiration either way. Thus, ordinary expiration does not produce a distinct obstruction to the circulation, as even when the expiration is at an end the intrathoracic pressure is less than the extra-thoracic.

The effect of violent expiratory efforts, however, has a distinct action in obstructing the current of blood through the lungs, as seen in the blueness of the face from congestion in straining, this condition being produced by pressure on the small pulmonary vessels.

We may summarize this mechanical effect of respiration on the bloodpressure therefore, and say that inspiration aids the circulation and so increases the arterial tension, and that although expiration does not materially aid the circulation, yet under ordinary conditions neither docs it obstruct it. Under extraordinary conditions, however, as in violent expiration, the circulation is decidedly obstructed.

We have seen, however, that there is no exact correspondence between the point of highest blood-pressure and the end of inspiration, and we must suppose that there are other mechanical factors, such, for example, as the effect of the abdominal movements, both in inspiration and in expiration, upon the arteries and veins within the abdomen and of the lower extremities, and the influence of the varying intrathoracic pressure upon the pulmonary vessels, both of which ought to be taken into consideration. As regards the first of these, the effect during inspiration-as the cavity of the abdomen is diminished by the descent of the diaphragm - should be two-fold: on the one hand, blood would be sent upward into the chest by compression of the vena cava inferior; on the other hand, the passage of blood downward from the chest in the abdominal aorta, and upward in the veins of the lower extremity, would be to a certain extent obstructed. In ordinary expiration all this would be reversed, but if the abdominal muscles are violently contracted, as in extraordinary expiration, the same effect would be produced as by inspiration. The effect of the varying intrathoracic pressure, which occurs during inspiration upon the pulmonary vessels is to produce an initial dilatation of both artery and veins, and this delays for a short time the passage of blood toward the left side of the heart, and the arterial pressure falls, but the fall of blood-pressure is soon followed by a steady rise, since the flow is increased by the initial dilatation of the ressels: the converse is the case with expiration. As, however, the pulmonary veins are more easily dilatable than the pulmonary artery, their greater distensibility increases the flow of blood as inspiration proceeds, while 
during expiration, except at its beginning, this property of theirs acts in the opposite direction, and diminishes the flow. Thus, at the beginning of inspiration the diminution of blood-pressure, which commenced during expiration, is continued, but after a time the diminution is succeeded by a steady rise; the reverse is the case with expiration-at first a rise and then a fall.

The effect of the nervous system in producing rhythmical alterations quite independent of the mechanically caused undulations of the

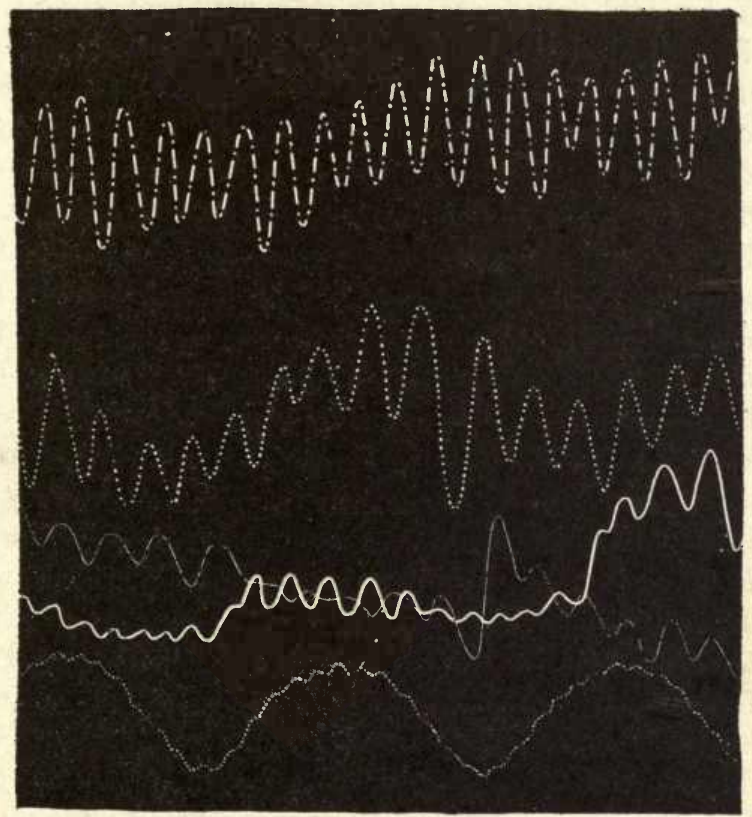

Fig. 218.-Traube-Hering's curves. (To be read from left to right.) The curves 1, 2, 3, 4, and 5 are portions selected from one continuous tracing forming the record of a prolonged observation, so that the several curves represent successive stages of the same experiment. Each curve is placed in its proper position relative to the base line, which is omitted; the blood-pressure rises in stages from 1 to 2,3 , and 4 , but falls again in stage 5 . Curve 1 is taken from a period when artificial respiration was being kept up, but the vagi having been divided, the pulsations on the ascent and descent of the undulations do not differ; when artificial respiration ceased these undulations for a while disappeared, and the blood-pressure rose steadily while the heart-beats became slower. Soon, as at 2 , new undulations appeared ; a little later, the blood-pressure was still rising, the heart beats still slower, but the undulations still more obvious (3); still later (4), the pressure was still higher, but the heart-beats were quicker, and the undulations flatter, the pressure then began to fall rapidly (5), and continued to fall un il some time after artificial respiration was resumed. (M. Foster.)

blood-pressure is two-fold. In the first place the cardio-inhibitory centre is stimulated during the fall of blood-pressure, and produces $a$ slower rate of heart-beat, which will be noticed in the tracing (fig. 218). The undulations during the decline of blood-pressure are therefore longer but less frequent. This effect disappears when, by section of the vagi, the effect of the centre is cut off from the heart. In the second place, the vaso-motor centre sends out rhythmical impulses, by which undulations of blood-pressure are produced, quite independent of the so-called 
respiratory undulations. The action of this centre in producing such undulations is thus demonstrated. In an animal under the influence of urari, a record of whose blood-pressure is being taken, and where artificial respiration has been stopped, and both vagi cut, the bloodpressure curve rises at first almost in a straight line, but after a time rhythmical undulations occur (called Traube's or Traube-Hering's curves); there may be upward of ten of the respiratory undulations in one Traube-Hering curve. They continue as long as the blood-pressure continues to rise, and only cease when the vaso-motor centre and the heart are exhausted, when the pressure falls. The undulations cannot depend upon anything but the vaso-motor centre, as the mechanical effects of respiration have been eliminated by the urari and by the cessation of artificial respiration, .nd the effect of the cardio-inhibitory centre has been removed, by the division of the vagi. The rhythmic rise of blood-pressure is most likely due to a rhythmic constriction of the arterioles followed by a rhythmic fall of pressure and relaxation, both being due to the action of the raso-motor centre. The vaso-motor centre, therefore, as well as the cardio-inhibitory, is capable of producing rhythmical undulations of blood-pressure.

Cheyne-stokes' breathing is a rhythmical irregularity in respirations which has been observed in various discases, and is especially connected with fatty degeneration of the heart. Respirations oecur in groups, at the beginning of each group the inspirations are very shallow, but each successive breath is deeper than the preceding, until a climax is reached, after which the inspirations become less and less deep, until they cease after a slight pause altogether. 'This phenomenon appears to be due to the want of action of some of the usual cerebral influences which pass down to and regulate the discharges of the respiratory centres.

Whatever is the exact quality of the venous blood which exeites the respiratory centre to produce normal respirations, there can be no doubt that as the blood becomes more and more venous from obstruction to the entrance of air into the lung, or from the blood not taking up from the air its usual supply of oxygen, the respiratory centre becomes more active and excitable, and a condition ensues, which passes rapidly from Hyperpnea (excessive breathing) to the state of Dyspmona (difficult breathing), and afterward to dsphyyrice; and the latter, unless relieved, quickly ends in death.

The ways by which this condition of asphyxia may be produced are very numerous:-As, for example, by the prevention of the due entry of oxygen into the blood, either by direct obstruction of the trachea or other part of the respiratory passages, or by introducing instead of ordinary air a gas devoid of oxygen, or, by interference with the due interchange of gases between the air and the blood.

The symptoms of asphyxia may be divided into three groups, which 
correspond with the stages of the condition which are usually recognized, these are (1), the stage of exaggerated breathing; (2), the stage of convulsions; (3), the stage of exhaustion.

In the first stage the breathing becomes more rapid and at the same time more deep than usual, the inspirations at first being especially exaggerated and prolonged. 'The muscles of extraordinary inspiration are called into action, and the effort to respire is labored and painful. 'This is soon followed by a similar increase in the expiratory efforts, which become excessively prolonged, being aided by all the muscles of extraordinary expiration. During this stage, which lasts a varying time, from a minute upward, according as the deprivation of oxygen is sudden or gradual, the lips become blue, the eyes are prominent, and the expression intensely anxious. The prolonged respirations are accompanied by a distinctly audible sound; the muscles attached to the chest stand out as distinct cords. 'This stage includes the two conditions hyperpnœa and dyspnoa already spoken of. It is due to the increasingly powerful stimulation of the respiratory centres by the increasingly venous blood.

In the second stage, which is not marked out by any distinct line of demarcation from the first, the violent expiratory efforts become convulsive, and then give way, in men and other warm-blooded animals at any rate, to general convulsions, which arise from the further stimulation of the centres. The spasms of the muscles of the body in general occur, and not of the respiratory muscles only. The convulsive stage is a short one, and lasts far less than a minute.

The third stage or stage of exhaustion. In it, the respirations all but cease, the spasms give way to flaccidity of the muscles, there is insensibility, the conjunctivæ are insensitive and the pupils are widely dilated. Every now and then a prolonged sighing inspiration takes place, at longer and longer intervals until they cease altogether, and death ensues. During this stage the pulse is scarcely to be felt, but the heart may beat for some seconds after respirations have quite ceased. The condition is due to the gradual paralysis of the respiratory centre by the prolonged action of the increasingly venous blood.

As with the first stage, the duration of the second and third stages depends whether the manner of the deprivation of oxygen is sudden or gradual. The convulsive stage is short, lasting, it may be, only one minute. The third stage may last three minutes and upward.

The conditions of the vascular system in asphyxia are:-(1) More or less interference with the passage of the blood through the systemic and the pulmonary blood-vessels; (2) Accumulation of blood in the right side of the heart and in the systemic yeins; (3) Circulation of impure (nonaërated) blood in all parts of the body.

After death from asphyxia it is found in the great majority of cases that the right side of the heart, the pulmonary arteries, and the systemic 
veins are gorged with dark, almost black blood, and the left side of the heart, the pulmonary veins, and the arteries are empty. 'The explaniltion of these appearances may be thus summarized: when respiration is stopped, venous blood at first passes freely through the lungs to the left heart, and so to the great arteries. When it reaches the arterioles either by its direct action upon their muscular tissue, or more probably through the medium of the vaso-motor centres, the arterioles contract, particularly those of the splanchnic area, the blood-pressure rises and the left side of the heart becomes distended. This latter effect may be from the extra action of the right heart, but is more probably due to the increased peripheral resistance, and its slower beat. Although the arterioles are contracted, a little blood is allowed to pass through them, and this highly venous blood, favored by the labored respiratory movements, arrives at the right side of the heart. When it reaches the pulmonary arterioles it gives rise to the same contraction in them as it did in the systemic vessels. This obstruction to the circulation through the lungs causes a distended condition of the right heart and the pulmonary artery, and on the other hand, produces a greatly diminished blood-flow through the pulmonary veins and to the left side of the heart, resulting after a time in practical emptiness. So that in the third stage of asphyxia it is stated by some observers that the left heart gets into the condition in which it is found after death. Others think that the empty condition of the left heart is a post-mortem phenomenon. In the first and second stages of the condition the blood-pressure continuously rises until it reaches a point far above the normal. 'The veins are greatly engorged, so that when pricked they act as arteries, inasmuch as they eject the blood for some distance. Both sides of the heart and the pulmonary vessels are engorged with blood, at any rate during the greater portion of these stages, and at the third stage blood-pressure falls rapidly.

Cause of death.-The causes of these conditions and the manner in which they act, so as to be incompatible with life, may be here briefly considered.

(1) The obstruction to the passage of blood through the lungs occurs chiefly in the later stages of asphyxia, the obstruction being chiefly in the arterioles, which contract under the influence of the vaso-motor centre, or possibly of a special part of it, which governs the action of the pulmonary blood-vessels.

(2) Accumulation of blood, with consequent distention of the right side of the heart and of the systemic veins, is the direct result, at least in part, of the obstruction to the pulmonary circulation just referred to. Other causes, however, are in operation. (a) The vaso-motor centres stimulated by blood deficient in oxygen, cause contraction of all the small arteries with increase of arterial tension, and as an immediate 
consequence the filling of the systemic veins. (b) The increased arterial tension is followed by inhibition of the action of the heart, and the heart, contracting less frequently, and also gradually enfeebled by deficient supply of oxygen, becomes over-distended with blood which it cannot expel. At this stage the left as well as the right cavities are over-distended.

The ill effects of these conditions are to be looked for partly in the heart, the muscular fibres of which, like those of the urinary bladder or any other hollow muscular organ, may be paralyzed by over-stretching; and partly in the venous congestion, and consequent interference with the function of the higher nerve-centres, especially the medulla oblongata.

(3) The passage of non-aërated blood through the lungs and its distribution over the body are events incompatible with life in one of the higher animals for more than a few minutes; the rapidity with which death ensues in asphyxia being due, more particularly, to the effect of non-oxygenized blood on the medulla oblongata, and, through the coronary arteries, on the muscular substance of the heart. The excitability of both nervous and muscular tissue is dependent on a constant and large supply of oxygen, and, when this is interfered with, excitability is rapidly lost.

Effects of breathing gases other than the atmosphere.-The diminution of oxygen has a more direct influence in the production of the usual symptoms of asphyxia than the increased amount of carbon dioxide. Indeed, the fatal effect of a gradual accumulation of carbon dioxide in the blood, when a due supply of oxygen is maintained, resembles rather the action of a narcotic poison than it does asphyxia.

Then again we must carefully distinguish the asphyxiating effect of an insufficient supply of oxygen from the directly poisonous action of such gases as carbonic oxide, which is contained to a considerable amount in common coal-gas. The fatal effects often produced by this gas (as in accidents from burning charcoal stoves in small, close rooms) are due to its entering into combination with the hæmoglobin of the blood-corpuscles and thus expelling the oxygen. The partial pressure of oxygen in the atmosphere may be considerably increased without much effect. Hydrogen may take the place of nitrogen if the oxygen is in the usual proportion with no marked ill effect. Sulphuretted hydrogen destroys the hæmoglobin of blood. Nitrous oxide acts directly on the nervous system as a narcotic. Certain gases, such as carbon dioxide in more than a certain proportion; sulphurous and other acid gases, ammonia, and chlorine produce spasmodic closure of the glottis, and are irrespirable.

As conditions causing asphyxia in addition to the obstruction to the trachea or elsewhere, and the prevention of the meeting of the blood 
and the air in the lung tissue by the blocking of one or more branches of the pulmonary artery, may be mentioned the following:

Alteration in the atmospheric pressure.-The normal condition of breathing is that the oxygen of the air breathed should be at the pressure of $\frac{1}{5}$ of the atmosphere, viz., $\frac{1}{5}$ of $760 \mathrm{~mm}$. of mercury, or $152 \mathrm{~mm}$., but it is found that life may be carried on by gradual diminution of the oyxgen pressure to considerably less than one half of this, viz., to $76 \mathrm{~mm}$., or $\frac{1}{10}$ partial pressure, which is reached at an altitude above 15,000 feet.* Any pressure less than this may begin to produce altera. tions in the relations of the gases in the blood, and if an animal is subjected suddenly to a marked decrease of barometric pressure, and so of oxygen pressure (below $r$ per cent), it is thrown into convulsions, and it is found that the gases are set free in the blood-vessels, no doubt carbon dioxide and oyxgen as well as nitrogen, althongh the latter is the only one of the three gases the presence of which in the vessels in death from this condition of affairs has been proved; the others are said to be reabsorbed. Other derangements may precede this, e.g., bleeding from the nose, dyspnca, and vascular derangement. On the other hand, the oygxen may be gradually increased to a considerable extent without marked effect, even to the extent of 8 or 10 atmospheres, but when the oxygen pressure is increased up to 20 atmospheres the animals experimented upon by Paul Bert died with severe tetanic convulsions. The alteration of pressure above or below a certain average affects primarily the gaseous interchange in the lungs, and then that in the tissues generally, but signs of dyspnoa may be produced as well either by cutting off the supply of blood to the medullary centres, or by warming the blood of the carotid arteries which supply them. The cause in the former case being the deprivation of oxygen and the accumulation of the carbon dioxide, and of the latter, the increased metabolism of the centre set up by the warmed blood.

* For an interesting account of the symptoms produced by diminished atmospheric pressure in those mounting to very high altitudes, Whymper's "Travels amongst the Andes of the Equator" may be consulted. 


\section{CHAPTER VIII.}

\section{SECRETION.}

IT is the function of gland cells to produce by the metabolism of their protoplasm certain substances called secretions. 'I'hese materials are of two kinds; viz., those which are employed for the purpose of serving some ulterior office in the economy, and those which are discharged from the body as useless or injurious. In the former case, the separated materials are termed true secretions; in the latter they are termed excretions.

The secretions as a rule consist of substanees which do not pre-exist in the same form in the blood, but require special cells and a process of elaboration for their formation, e.g., the liver cells for the formation of bile, the mammary gland-cells for the formation of milk. The excretions, on the other hand, commonly consist of substances which exist ready-formed in the blood, and are merely abstracted therefrom. If from any eause, such as extensive disease or extirpation of an excretory organ, the separation of an exeretion is prevented, and an accumulation of it in the blood ensues, it frequently escapes through other organs, and may be detected in various fluids of the body. But this is never the case with secretions; at least with those that are most elaborated; for after the removal of the special organ by which each of them is manufactured, the secretion is no longer formed. Cases sometimes occur in which the secretion continues to be formed by the natural organ, but not being able to escape toward the exterior, on account of some obstruction, is re-absorbed into the blood, and afterward discharged from it by exudation in other ways; but these are not instances of true vicarious secretions, and must not be so regarded.

The circumstances of their formation, and their final destination, are, however, the only particulars in which secretions and excretions can be distinguished; for, in general, the structure of the parts engaged in eliminating excretions is as complex as that of the parts concerned in the formation of secretions. And since the differences of the two processes of separation, corresponding with those in the several purposes and destinations of the fluids, are not yet ascertained, it will be sufficient to speak in general terms of the process. 
Every secreting apparatus possesses, as essential parts of its structure, a simple and almost textureless membrane, named the primary or basement-membrane; certain cells; and blood-vessels. These three structural elements are arranged together in various ways; but all the varieties may be classed under one or other of two principal divisions, namely, membranes and glands.

\section{Organs and Tissues of Secretion.}

The principal secreting organs are the following:-(1) the serous and synovial membranes; (2) the mucous membranes with their special glands, e.g., the buccal, gastric, and intestinal glands; (3) the salivary glands and pancreas; (4) the mammary glands; (5) the liver; (6) the lachrymal gland; (\%) the kidney and skin; and (8) the testes.

'The structure and functions of the glands secreting materials used in digestion will be considered when we study the alimentary tract. The functions of the kidney and skin will be described in a future chapter.

'The lachrymal gland will be considered with the rest of the optic apparatus and the testes in the Chapter on Generation. There remain, then, the serous and mucous membranes and the mammary gland to be here described.

(1.) Serous and Synovial Membranes. - Serous membranes are of two principal kinds: 1st. Those which line visceral cavities,- the arachnoid, pericardium, pleura, peritoneum, and tunica vaginales. 2d. The synorial membranes lining the joints, and the sheaths of tendons and ligaments, with which, also, are usually included the synovial bursa, or bursce mucosce, whether these be subcutaneous, or situated beneath tendons and glide over bones.

The serous membranes form closed sacs, and exist wherever the free surfaces of viscera come into contact with each other or lie in cavities unattached to surrounding parts. The viscera invested by a serous membrane are, as it were, pressed into the shut sac which it forms, carrying before them a portion of the membrane, which serves as their investment. To the law that serous membranes form shut sacs, there is, in the human subject, one exception, viz.: the opening of the Fallopian tubes into the abdominal cavity, - an arrangement which exists in man and all Vertebrata, with the exception of a few fishes.

The serous membranes are especially distinguished by the characters of the endothelium covering their free surface: it always consists of a single layer of polygonal cells. The ground substance of most serous membranes consists of connective-tissue corpuscles of various forms lying in the branching spaces which constitute the lymph canalicular system, and interwoven with bundles of white fibrous tissue, and nu- 
merous delicate elastic fibrillæ, together with blood-vessels, nerves, and lymphatics. In relation to the process of secretion, the layer of connective tissue serves as a groundwork for the ramification of blood-vessels, nerves, and lymphatics. But in its usual form it is absent in some instances, as in the arachnoid covering the dura mater, and in the interior of the ventricles of the brain. The primary membrane and epithelium

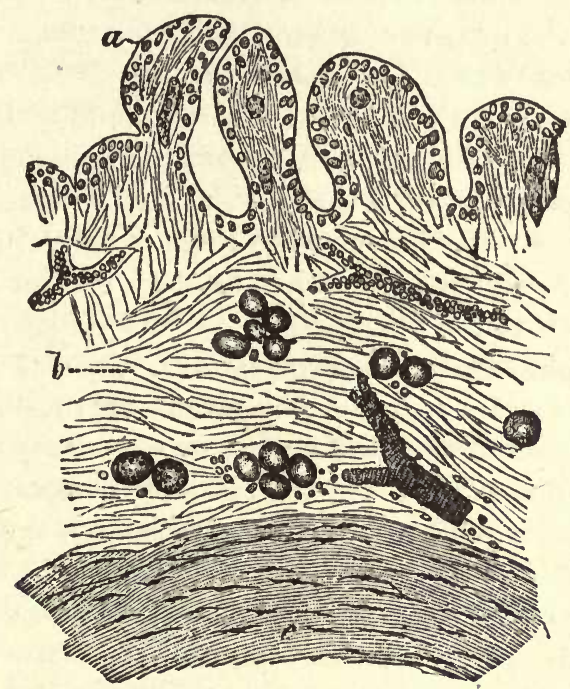

Fig. 219.- Section of synovial membrane. $a$, Endothelial covering of the elevations of the membrane; $b$, subserous tissue containing fat and blood-vessels; $c$, ligament covered by the synovial membrane. (Cadiat.)

are always present, and are concerned in the formation of the fluid by which the free surface of the membrane is moistened.

Functions.-The principal purpose of the serous and synovial membranes is to furnish a smooth, moist surface, to facilitate the movements of the invested organ, and to prevent the injurious effects of friction. This purpose is especially manifested in joints, in which free and extensive movements take place; and in the stomach and intestines, which, from the varying quantity and movements of their contents, are in almost constant motion upon one another and the walls of the abdomen.

Fluid. - The fluid secreted from the free surface of the serous membranes is, in health, rarely more than sufficient to ensure the maintenance of their moisture. The opposed surfaces of each serous sac are at every point in contact with each other. After death, a larger quantity of fluid is usually found in each serous sac; but this, if not the product of manifest disease, is probably such as has transuded after death, or in the last hours of life. An excess of such fluid in any serous sac constitutes dropsy of the sac. 
The flaid naturally secreted by the serous membranes appears to be identical, in general and chemical characters, with very dilute liquor sanguinis. It is of a pale-yellow or straw-color, slightly viscid, alkaline, and on account of the presence of albumen, coagulable by heat. 'Thi similarity of the serous fluid to the liquid part of blood, and to the fluid with which most animal tissnes are moistened, formerly led to the belief that it was a simple transudation; but Ireidenhain has concluded from experiments that the process of separation is one of secretion, dependent upon the vital activity of the entothelial cells. There is reason for supposing that the fluids of the cerebral ventricles and of the arachnoid sac are likewise secretions; for they liffer from the fluids of the other serous sacs not only in being pellucid, colorless, and of much less specific gravity, but in that they seldom receive the tinge of bile when present in the blood, and are not colored by madter, or other simila: substances introduced abundantly into the bloor.

It is also probable that the formation of s!lnowinl fluid is a process of genuine and elaborate secretion, by means of the epithelial cells on the surface of the membrane, and especially of those which are accumulated on the edge and processes of the synovial fringes; for, in its peeuliar density, viscidity, and abundance of albumen, synovia differs alike from the serum of blood and from the fluid of any of the serous cavities.

(2.) Mucous Membranes.-The mucous membranes line all those passages by which internal parts communieate with the exterior, and by which either matters are eliminated from the body or foreign substances taken into it. They are soft and velvety, and extremely vascular. The external surfaces of mucous membranes are attached to various other tissues; in the tongue, for example, to muscle; on cartilaginous parts, to perichondrinm; in the cells of the ethmoid bone, in the frontal and sphenoidal sinuses, as well as in the tympanum, to periosteum; in the intestinal canal, it is connected with a firm submucous membrane, which on its exterior gives attachment to the fibres of the muscular coat. The mucous membranes line certain principal tractsGastro-putmonary and Genito-urinary; the former being subdivided into the Digestive and Respiratory tracts.

1. 'The Digestive tract commences in the cavity of the mouth, from which prolongations pass into the ducts of the salivary glands. From the mouth it passes through the fances, pharynx, and osophagus, to the stomach, and is thence continued along the whole tract of the intestinal canal to the termination of the rectum, being in its course arranged in the various folds and depressions already described, and prolonged into the ducts of the intestinal glands, the pancreas and liver, and into the gall-bladder. 
2. The Respiratory tract includes the mucous membrane lining the cavity of the nose, and the various sinuses communicating with it, the lachrymal canal and sac, the conjunctiva of the eye and eyelids, and the prolongation which passes along the Eustachian tubes and lines the tympanum and the inner surface of the membrana tympani. Crossing the pharynx, and lining that part of it which is above the soft palate, the respiratory tract leads into the glottis, whence it is continued, through the larynx and trachea, to the bronchi and their divisions, which it lines as far as the branches of about $\frac{1}{50}$ of an inch $\left(\frac{1}{2} \mathrm{~mm}\right.$.) in diameter, and continuous with it is a layer of delicate epithelial membrane which extends into the pulmonary cells.

3. The Genito-urinary tract, which lines the whole of the urinary passages, from their external orifice to the termination of the tubuli uriniferi of the kidneys, extends also into the organs of generation in both sexes, and into the ducts of the glands connected with them: and in the female becomes continuous with the serous membrane of the abdomen at the fimbriæ of the Fallopian tubes.

Structure.-These mucous tracts, and different portions of each of them, present certain structural peculiarities, adapted to the functions which each part has to discharge; yet in some essential characters the mucous membrane is the same, from whatever part it is obtained. In all the principal and larger parts of the several tracts, it presents, as just remarked, an external layer of epithelium, situated upon a basement membrane, and beneath this, a stratum of vascular tissue of variable thickness, containing lymphatic vessels and nerves. The vascular stratum, together with the basement membrane and epithelium, in different cases, is elevated into minute papillæ and villi, or depressed into involutions in the form of glands. But in the prolongations of the tracts, where they pass into gland-ducts, these constituents are reduced in the finest branches of the ducts to the epithelium, the primary or basement-membrane, and the capillary blood-vessels spread over the outer surface of the latter in a single layer.

The primary or basement membrane is a thin transparent layer, simple, homogeneous, or composed of endothelial cells. In the minuter divisions of the mucous membranes, and in the ducts of glands, it is the layer continuous and correspondent with this basement-membrane that forms the proper walls of the tubes. The cells also, which, lining the larger and coarser mucous membranes, constitute their epithelium, are continuous with and often similar to those which, lining the gland-ducts, are called gland-cells. No certain distinction can be drawn between the epithelium-cells of mucous membranes and gland-cells.

Mucous Fluid: Mucus. - From all mucous membranes there is secreted either from the surface or from certain special glands, or from both, a 
more or less viscid, grayish, or semi-transparent fluid, of alkaline reaction and high specific gravity, named mucus. It mixes imperfectly with water, but, rapidly absorbing liquid, it swells considerably when water is added. Under the microscope it is found to contain epithelium and leucocytes. It is found to be made up, chemically, of mucin, which forms its chief bulk, of a little albumen, of salts chiefly chlorides and phosphates, and water with traces of fats and extractives.

\section{Secreting Glands.}

The secreting glands present, amid manifold diversities of form and composition, a general plan of structure; all contain, and appear constructed with particular regard to the arrangement of the cells, which, as already expressed, both line their tubes or cavities as an epithelium, and elahorate, as secreting cells, the substances to be discharged from them.

Typpes of Secretiny (ilands. - Secreting glands may be classified according to certain types, which are the following:-1. The simple tubular gland ( $\Lambda$, fig. 220), examples of which are furnished by the follicles of Lieberkühn, and the tubular glands of the stomach. They are simple tubular depressions of the mucous membrane, the wall of which is formed of primary membrane and is lined with secreting cells arranged as an epithelium. To the same class may be referred the elongated and tortuous sudoriferous glends.

2. The componud tubular glands (D, fig. 220) form another division. These consist of main gland-tubes, which divide and subdivide. Each gland may be made up of the subdivisions of one or more main tubes. 'The ultimate subdivisions of the tubes are generally highly convoluted. 'They are formed of a basement-membrane, lined by epithelium of various forms. The larger tubes may have an outside coating of fibrous, areolar, or muscular tissue. 'The kidney, testes, salivary glands, pancreas, Brunner's glands, with the lachrymal and mammary glands, and some mucous glands are examples of this type but present more or less marked variations among themselves.

3. 'The aggregate or racemose glands, in which a number of vesicles or acini are arranged in groups or globules (c, fig. 220). The meibomian follicles are examples of this kind of gland. There seem to be glands of mixed character, combining some of the characters of the tubular with others of the racemose type; these are called tubuto-racemose or tubuloacinous glands. These glands differ from each other only in secondary points of structure: such as, chiefly, the arrangement of their excretory ducts, the grouping of the acini and lobules, their connection by areolar tissue, and supply of blood-vessels. The acini commonly appear to be formed by a kind of fusion of the walls of several vesicles, which thus 
combine to form one cavity lined or filled with secreting cells which also occupy recesses from the main cavity. The smallest branches of the gland-ducts sometimes open into the centres of these cavities; sometimes the acini are clustered round the extremities, or by the sides of the ducts: but, whatever secondary arrangement there may be, all have the same essential character of rounded groups of vesicles containing
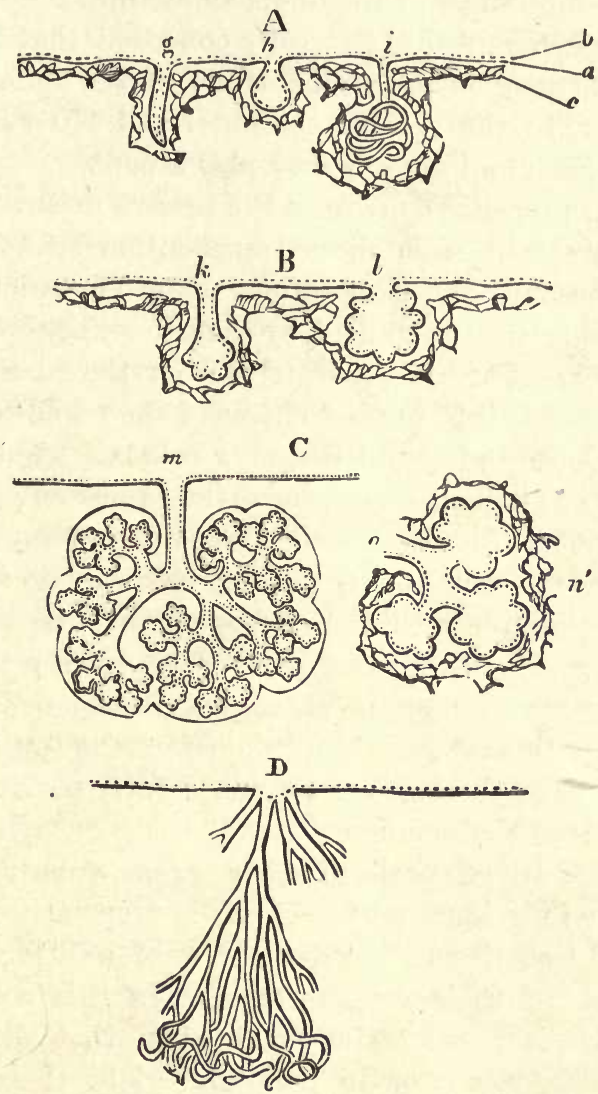

Fig. 220.-Plans of extension of secreting membrane by inversion or recession in form of cav. ities. A, Simple glands, viz., $g$, straight tube; $h$, sac; $i$, coiled tube. B, Multilocular crypts; $k$ of tubular form; $l$, saccular. c, Racemose, or saccular compound gland; $m$, entire gland, showing branched duct and lobular structure; $n$, a lobule, detached with $o$, branch of duct proceeding from it. D, Compound tubular gland (Sharpey).

gland-cells, and opening by a common central cavity into minute ducts, which ducts in the large glands converge and unite to form larger and larger branches, and at length by one common trunk open on a free surface of membrane.

Among these varieties of structure, all the secreting glands are alike in some essential points, besides those which they have in common with 
all truly secreting structures. They agree in presenting a large extent of secreting surface within a comparatively small space; in the circumstance that while one end of the gland-duct opens on a free surface, the opposite end is always closed, having no direct communication with blood-vessels, or any other canal; and in a uniform arrangement of capillary blood-vessels, ramifying and forming a network around the walls and in the interstices of the ducts and acini.

Process of Secretion.- It is generally conceded that the process of secretion is dependent upon the vital activity of the secreting cells. It is possible, however, in the case of the water and salts, that the physical processes of filtration and dialysis may play a part.

The chemical processes constitute the process of secretion, properly so called, as distinguished from mere transudation spoken of above. In the chemical process of secretion various materials which do not exist as such in the blood are manufactured by the agency of the gland-cells from the blood, or to speak more accurately, from the plasma which exudes from the blood-vessels into the interstices of the gland-textures.

The best evidence in favor of this view is: 1st. That cells and nuclei are constituents of all glands, however diverse their outer forms and other characters, and that they are in all glands placed on the surface or in the cavity whence the secretion is poured. 2d. 'That certain materials of secretions are visible with the microscope in the gland cells before they are discharged. Thus, granules probably representing the ferments of the pancreas may be discerned in the cells of that gland; spermatozoids in the eells of the tubules of the testicles; granules of urie acid in those of the kidneys (of fish); fatty particles, like those of milk, in the cells of the mammary gland.

Seereting cells, like the eells of other organs, appear to develop, grow, and attain their individual perfection by appropriating nutriment from the fluid exuded by adjacent blood-vessels and building it up, so that it shall form part of their own substance. In this perfeeted state the cèlls subsist for some brief time, and when that period is over they appear to dissolve, wholly or in part, and yield their contents to the peculiar material of the secretion. And this appears to be the case in every part of the gland that contains the appropriate gland-cells; therefore not in the extremities of the ducts or in the acini alone, but in great part of their length.

We will describe elsewhere the changes which have been noticed from actual experiment in the cells of the salivary glands, pancreas, and peptic glands.

Discharge of secretions from glands may either take place as soon as they are formed; or the secretion may be long retained within the 
gland or its ducts. The former is the case with the sweat glands. But the secretions of those glands whose activity of function is only occasional are usually retained in the cells in an undeveloped form during the periods of the gland's inaction. And there are glands which are like both these classes, such as the lachrymal, which constantly secrete small portions of fluid, and on occasions of greater excitement discharge it more abundantly.

When discharged into the ducts, the further course of secretions is affected (1) partly by the pressure from behind; the fresh quantities of secretion propelling those that were formed before. In the larger ducts, its propulsion is (2) assisted by the contraction of their walls. All the larger ducts, such as the ureter and common bile-duct, possess in their coats plain muscular fibres; they contract when irritated, and sometimes manifest peristaltic movements. Rhythmic contractions in the pancreatic and bile-ducts have been observed, and also in the ureters and vasa deferentia. It is probable that the contractile power extends along the ducts to a considerable distance within the substance of the glands whose secretions can be rapidly expelled. Saliva and milk, for instance, are sometimes ejected with much force.

Circumstances Influencing Secretion.-The principal conditions which influence secretion are (1) variations in the quantity of blood, (2) variations in the quantity of the peculiar materials for any secretion that the blood may contain, and (3) variations in the condition of the nerves of the glands.

(1.) An increase in the quantity of blood traversing a gland, as in nearly all the instances before quoted, coincides generally with an augmentation of its secretion. Thus the mucous membrane of the stomach becomes florid when, on the introduction of food, its glands begin to secrete; the mammary gland becomes much more vascular during lactation; and all circumstances which give rise to an increase in the quantity of material secreted by an organ produce, coincidently, an increased supply of blood; but we have seen that a discharge of saliva may occur under extraordinary circumstances, without increase of blood-supply, and so it may be inferred that this condition of increased blood-supply is not absolutely essential.

(2.) An increase in the amount of the materials which the glands are designed to separate or elaborate, contained in the blood supplied to them, increases the amount of any secretion. Thus, when an excess of nitrogenous waste is in the blood, from destruction of one kidney or whatever cause, a healthy kidney will excrete more urea than it did before.

(3.) Influence of the Nervous System on Secretion.-The process of secretion is largely influenced by the condition of the nervous system. 
The exact mode in which the influence is exhibited must still be regarded as somewhat obscure. In part, it exerts its influence by increasing or diminishing the quantity of blood supplied to the secreting gland, in virtue of the power which it exercises over the contractility of the smaller blood-ressels; while it also has a more direct influence, as is described at length in the case of the submaxillary gland, upon the secreting cells themselves; this may be called trophic influence. Its influence over secretion, as well as over other functions of the body, may be excited by causes acting directly upon the nervous centres, upon the nerves going to the secreting organ, or upon the nerves of other parts. In the latter case, a reflex action is produced: thus the impression produced upon the nervous centres by the contact of food in the mouth is reflected upon the nerves supplying the salivary glands, and produces, through these, a more abundant secretion of the saliva.

Through the nerves, various conditions of the brain also influence the secretions. Thus, the thought of food may be sufficient to excite an abundant flow of saliva. And, probably, it is the mental state which excites the abundant secretion of urine in hysterical paroxysms, as well as the perspirations, and occasionally diarrhœa, which ensue under the influence of terror, and the tears excited by sorrow or excess of joy. The quality of a secretion may also be affected by mental conditions, as in the cases in which, through grief or passion, the secretion of milk is altered, and is sometimes so changed as to produce irritation in the alimentary canal of the child, or even death.

Relations between the Secretions. - The secretions of some of the glands seem to bear a certain relation or antagonism to each other, by which an increased activity of one is usually followed by diminished activity of one or more of the others; and a deranged condition of one is apt to entail a disordered state in the others. Such relations appear to exist among the various mucous membranes; and the close relation between the secretion of the kidney and that of the skin is a subject of constant observation.

\section{The Mammary Glands.}

Structure.-The mammary glands are composed of large divisions or lobes, and these are again divisible into lobules-the lobules being composed of the convoluted and dilated subdivisions of the main ducts (alveoli) held together by connective tissue. The lobes and lobules too are bound together by areolar tissue; penetrating between the lobes and covering the general surface of the gland, with the exception of the nipple, is a considerable quantity of yellow fat, itself lobulated by sheatlıs and processes of tough areolar tissue (fig. 221) connected both with the skin in front and the gland behind; the same bond of connec- 
tion extending also from the under surface of the gland to the sheathing connective tissue of the great pectoral muscle on which it lies. The main ducts of the gland, fifteen to twenty in number, called the lactiferous or galactophorous ducts, are formed by the union of the smaller (lobular) ducts, and open by small separate orifices through the nipple. At the points of junction of lobular ducts to form lactiferous ducts, and just before these enter the base of the nipple, the ducts are dilated (fig.

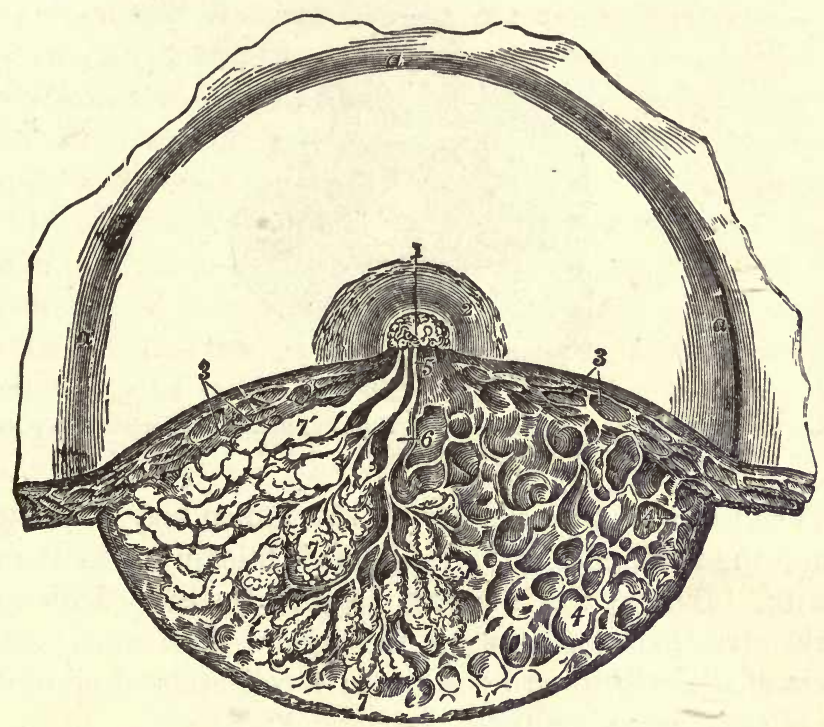

Fig. 221.-Dissection of the lower half of the female mamma, during the period of lactation. 2/. - In the left-hand side of the dissected part the glandular lobes are exposed and partially unravelled; and on the right-hand side, the glandular substance has been removed to show the reticular loculi of the connective tissue in which the glandular lobules are placed: 1, Upper part of the mamilla or nipple; 2 , areola; 3 , subcutaneous masses of fat; 4 , reticular loculi of the connective tissue which support the glandular substance and contain the fatty masses; 5 , one of three lactiferous ducts shown passing toward the mamilla where they open; 6 ; one of the sinus lactei or reservoirs; 7 , some of the glandular lobules which have been unravelled; $7^{\prime}$, others massed together (Luschka).

$221)$; and, during lactation, the period of active secretion by the gland, - the dilatations form reservoirs for the milk, which collects in and distends them. The walls of the gland-ducts are formed of areolar with some unstriped muscular tissue, and are lined internally by short columnar and near the nipple by squamous epithelium. The alveoli consist of a membrana propria of flattened endothelial cells lined by low columnar epithelium, and are filled with fat globules.

The nipple, which contains the terminations of the lactiferous ducts, is composed also of areolar tissue, and contains unstriped muscular fibres. Blood-vessels are also freely supplied to it, so as to give it a species of erectile structure. On its surface are very sensitive papillæ; and around 
it is a small area or areola of pink or dark-tinted skin, on which are to be seen small projections formed by minute secreting glands.

Blood-vessels, nerves, and lymphatics are plentifully supplied to the mammary glands; the calibre of the blood-vessels, as well as the size of the glands, varying very greatly under certain conditions, especially those of pregnancy and lactation.

The alveoli of the glands during the secreting periods are found to be lined with very short columnar cells, with nuclei situated toward the

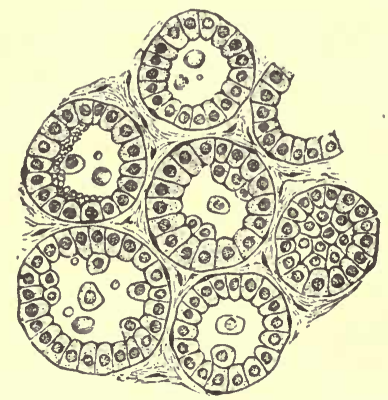

Fig. 2:2.-Section of mammary gland of biteh, showing acini, lined with epithelial cells of a polyhedral or short columnar form. $\times 200$. (V. D. Harris.)

centre. The edges of the cells toward the lumen may be irregular and jagged, and the remainder of the alveolus is filled up with the materials of the milk. During the intervals between the acts of discharge, the cells of the alveoli elongate toward the lumen, their nuclei divide, and in the part of the cells toward the lumen a collection of oil globules and probably of other materials takes place.

The next stage is that the cells divide and the part of each toward the lumen containing a nucleus and the materials of the secretion is, as it were, broken off from the outer part and goes to form the solid part of the milk. The cells also secrete, from the blood supplied to them, the water, salts, and probably sugar. In addition to the actual casting off parts of the cells containing fat and the other materials, oil globules appear to pass out from the cells with the other materials into the lumen of the alveoli. The cast-off parts of the cells disintegrate or break down, andergoing a kind of solution in the more fluid part of the secretion.

In the earlier days of lactation, epithelial cells partially transformed are discharged in the secretion: these are termed colostrum corpuscles, but later on the cells are completely transformed into fat before the secretion is discharged.

After the end of lactation, the mamma gradually returns to its original size (involution). The acini, in the early stages of involution, are lined with cells in all degrees of vacuolation. As involution proceeds the acini diminish considerably in size, and at length, instead of a mosaic 
of lining epithelial cells (twenty to thirty in each acinus), we have five or six nuclei (some with no surrounding protoplasm) lying in an irregular heap within the acinus. During the later stages of involution, large yellow granular cells are to be seen. As the acini diminish in size, the connective tissue and fatty matter between them increase, and in some animals, when the gland is completely inactive, it is found to consist of a thin film of glandular tissue overlying a thick cushion of fat. Many of the products of waste are carried off by the lymphatics.

During pregnancy the mammary glands undergo changes (evolution) which are readily observable. They enlarge, become harder and more distinctly lobulated: the veins on the surface become more prominent. The areola becomes enlarged and dusky, with projecting papillæ; the nipple too becomes more prominent, and milk can be squeezed from the orifices of the ducts. This is a very gradual process, which commences about the time of conception, and progresses steadily during the whole period of gestation. In the gland itself solid columns of cells bud off from the old alveoli to form new alveoli. But these solid columns after a while are converted into tubes by the central cells becoming fatty and being discharged as the colostrum corpuscles above mentioned.

\section{Milk.}

The mammary secretion, or milk, is a bluish-white, opaque fluid with a pleasant, sweet taste, of specific gravity of 1028-1034. It is a true

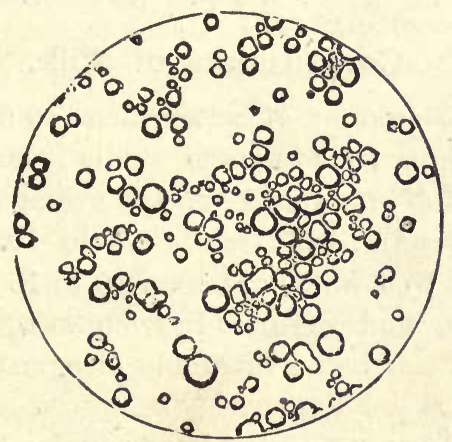

Fig. 223.-Globules and molecules of cow's milk. $\times 400$.

emulsion. Under the microscope, it is found to contain a number of globules of various sizes (fig. 223), the majority about $\frac{1}{10000}$ of an inch $(.25 \mu)$ in diameter. They are composed of oily matter, and are called milk-globules, but the old view that they had an investing membrane of albuminous material is now generally discarded. Accompanying these are numerous minute particles, both oily and albuminous, which exhibit ordinary molecular movements. The milk which is secreted in the first 
few days after parturition is called the colostrum. This contains the granular colostrum corpuscles, which are four or five times the size of milk globules, and differs from ordinary milk in containing a larger quantity of solid matter, and in being deep yellow, less sweet, but far more alkaline, and in having a specific gravity of 1040-1046.

Composition of Colostrum (Pfeiffer).

Proteids

Fat

Sugar

Salts .

Water

$\begin{array}{r}5.71 \\ 2.04 \\ 3.74 \\ 0.28 \\ 88.23 \\ \hline\end{array}$

100.00

Chemical Composition of Milk.-In addition to the oil existing in numberless little globules floating in a large quantity of water, milk contains certain proteids, milk-sugar (lactose), and several varieties of salts. Its percentage composition has been already mentioned, but may be here repeated. Its reaction is slightly alkaline.

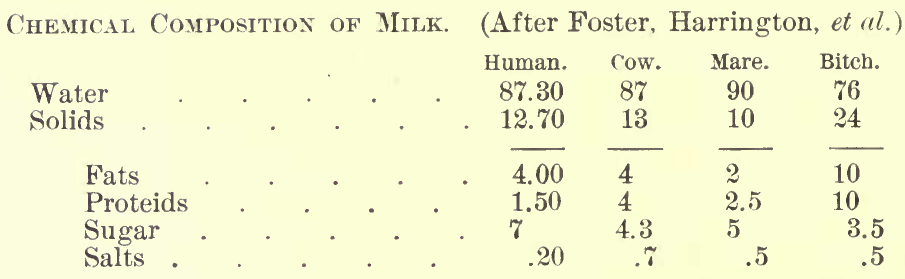

\section{Constituents of Milk.}

(1.) Water.-The amount of water varies in different animals, and in the same animal from time to time. This is seen from the varying specific gravity; that of cow's milk, on the average, varies from 1028 to 1034 in unskimmed milk, and from 1033 to $103 \%$ in skimmed milk. 'The amount secreted by a woman is from 10 to $16 \mathrm{oz}$. at the end of the first week of lactation, and increases to from 30 to $40 \mathrm{oz}$. by the eighth or ninth month. A cow under favorable circumstances secretes at least ten pints a day.

(2.) Proteids. - These are of two kinds at least, viz., caseinogen and lact-albumin. Caseinogen may be obtained from milk either by the addition of an acid, e.g., acetic, or by saturation with crystallized magnesium sulphate or sodium chloride in the way already indicated ( $p$. 119). Caseinogen, as already pointed out, belongs to the class of nucleo-albumins (see p. 119).

Coagulation of Milk. - The clotting of caseinogen is seen when the gastric ferment rennin, or when similar ferments from the pancreas or intestinal juice are added to milk; it will take place when the milk is 
neutral or alkaline. By the clotting, caseinogen is converted into a coagulated proteid, casein, and a proteid residue called whey-proteid. Casein carries down with it the fat, and the two materials form cheese. As in the case of blood, coagulation cannot occur except in the presence of calcium salts. When caseinogen is acted on by rennin, it is split by hydrolytic cleavage into two parts, paracasein and whey-proteid. Paracasein combines with the calcium salts to form the insoluble compound casein; the whey-proteid remains behind in solution in the whey. By reference to the coagulation of the blood, the similarity of the two processes will be seen. Caseinogen is also precipitated from milk in the presence of an excess of acid. When milk curdles after "souring," it is due to the formation of lactic acid from the milk-sugar by microorganisms.

Lact-albumin differs in some of its reactions from serum-albumin ( $p$. 115 ); it coagulates when milk is boiled, but this scum is also partly due to the drying up of the caseinogen on the surface of the milk.

Lactoglobulin, another proteid of milk, is similar to the paraglobulin of the blood.

(3.) Fats. - The fats of milk are those usually found in animal tissues, viz., olein, stearin, and palmatin (p. 122). There are also others, especially that of butyric acid in combination with glycerin. Lecithin and cholesterin and a lipochrome may also be present. The fat, split up into minute particles, which are lighter than the remainder of the constituents, rises to the surface when the milk stands, forming cream; and cream, when its fatty molecules have run together, forms butter.

(4.) Lactose. - This sugar, the reactions of which are mentioned at p. 124 , is apt to undergo lactic-acid fermentation if the milk be exposed to the air, from the action of the organized ferment, the bacterium lactis. When this occurs milk becomes sour and the caseinogen is thrown down.

(5.) Salts. - The chief salt of milk is calcium phosphate. Without its presence caseinogen cannot form casein. The gases are carbon dioxide and nitrogen.

Salts in Woman's Milk (Rotch).

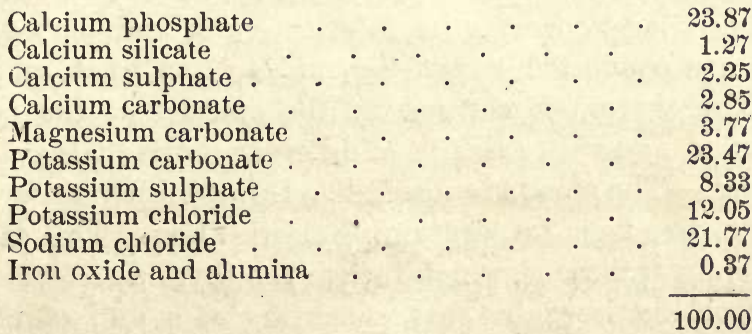




\section{The Ductless Glands}

\section{and Internal Secretioxs.}

The discovery of the remarkable and sometimes fatal effects of the removal of certain of the ductless glands has giren a marked impetus to the study of these organs, so that at the present time they occupy a place of importance in physiology formerly unthought of. The converæe effects of removing certain of these glands and of injections of extracts (aqueous and others) of them into healthy unimals or those operated upon, have led to the belief that they elaborate in the course of their metabolic activity some substance or substances which are of use to the body. Since the parenchyma cells of these glands belong morphologically to the secretory type, and since active constituents may be extracted from the glands, it is assumed that they produce a secretion. But this secretion, whatever its quantity may be, passes either into the blood stream directly (supra-renal) or indirectly by way of the lymphatics (thyroid), instead of discharging through a duct upon a free surface, as in the case of the salivary glands and others. Hence the term internal secretion has come into popular use by way of distinction.

It must be borne in mind, however, that both anabolic and katabolic products are formed by all tissues and are absorbed to a greater or less extent into the circulation. But the term internal secretion does not spply to these. It is confined to such products as are formed by organs of a distinctly glandular type.

The glands which are known certainly to form internal secretions are the thyroid, the supra-renal capsules, the pancreas, and possibly the pituitary body. And Howell has called attention to the fact that to be consistent the glycogen formed by the liver from dextrose (and proteid) should be regarded as an internal secretion. Thus the liver forms both an internal and external secretion, as in the case of the pancreas.

The spleen has been included in this chapter for convenience. It has not been proved to form an internal secretion.

The Thyroid.-The thyroid gland is situated in the neck. It consists of two lobes, one on each side of the trachea, extending upward to the thyroid cartilage, covering its inferior cornu and part of its body; these lobes are connected across the middle line by a middle lobe or isthmus. The thyroid is corered by the muscles of the neck. It is highly vascular, and varies in size in different individuals.

Structures. - The gland is encased in a thin transparent laser of dense areolar tissue, free from fat, containing elastic fibres. This capsule sends in strong fibrous trabeculæ, which inclose the thyroid vesicles-which are rounded or oblong irregular sacs, consisting of a wall of thin hyaline 
membrane lined by a single layer of short cylindrical or cubical cells. These vesicles are filled with transparent nucleo-albuminous colloid material. The colloid substance increases with age, and the cavities appear to coalesce. In the interstitial connective tissue is a round meshed capillary plexus, and a large number of lymphatics. The nerves adhere closely to the vessels.

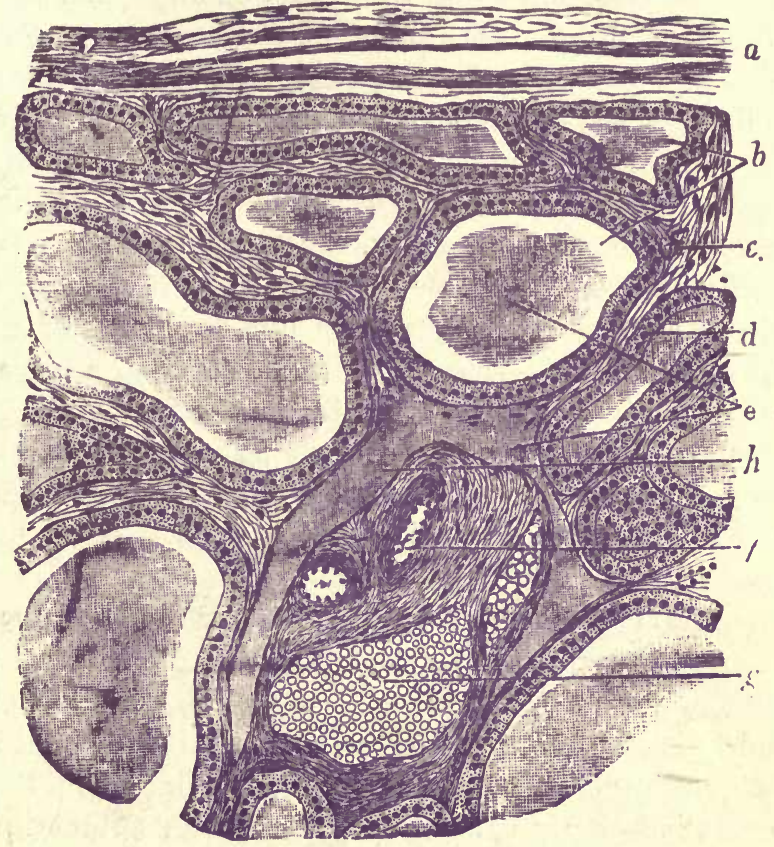

Fig. 224.-Part of a section of the human thyroid. $a$, Fibrous capsule; $b$, thyroid vesicles filled with, $e$, collold substance; $c$, supporting fibrous tissue; $d$ short columnar cells lining vesicles; $f$, arteries; $g$, veins filled with blood; $h$, lymphatic vessel filled with colloid substance. $\times$ (S. $\mathbf{K}$. Alcock.)

In the vesicles there are in addition to the yellowish glassy colloid material, epithelium cells, colorless blood-corpuscles, and also colored corpuscles undergoing disintegration.

Accessory Thyroids. - These are small bodies possessing the structure of the thyroid and apparently performing the same function. They are found in the neck and in the mediastinum as far as the heart. The accessory thyroids undergo hypertrophy when the thyroid has been removed.

Parathyroids. - In addition to the accessory thyroids, parathyroids are found in the neck, lying behind or to the side of the thyroid, or even within its substance (in the rat). They are small bodies, differing from the thyroid in structure in that they consist of solid columns of cells, not of acini; yet they seem capable of performing the function of 
the thyroid when tinat body is removed. They frequently exist in pairs, but there may be more than two, lying along the carotid in the region of the thyroid. The parathyroids are thought to be immature thyroids.

Functions of the Thyroid.-The colloid material which is formed within the thyroid vesicles, and is believed to be their secretion, finally ruptures through their walls into the lymph channels and thus gains entrance to the circulation. The secretion of the thyroid falls into the class known as internal secretions, and exerts a profound influence upon the metabolic processes of the body, probably through the agency of the central nervous system. Complete extirpation of the thyroid, at least in some animals, produces death, preceded by a group of characteristic symptoms. In man and the monkey, the symptoms after removal come on slowly and resemble the disease known in man as myxoedema.

This disease is known definitely to be due to disease of the thyroid, whereby its function is interfered with. Moreover, if a piece of thyroid of sufficient size be grafted into an animal from which the glands have been removed, and the graft takes, the symptoms of thyroid removal are lessened in intensity or disappear altogether. And, likewise, thyroid feeding or the administration of thyroid extracts relieves the symptoms of the disease myxwedema.

The above facts show that the thyroid gland must perform some important function in the animal economy, and it is believed that this is accomplished by virtue of its internal secretion. The colloid material of the gland has been submitted to much chemical study, and a substance called iodothyrin has been isolated as its active principle. Baunann and Roos state that iodothyrin exists in the gland in combination with proteid bodies. Iodothyrin relieves the symptoms of thyroid removal much to the same extent as thyroid feeding. It is a very resistant substance, and is not injured by the action of the gastric juice or by boiling with 10 per cent sulphuric acid for a long time.

The Supra-renal Capsules or Adrenals. - These are two flattened, more or less triangular or cocked-hat shaped bodies, resting by their lower border upon the upper border of the kidneys.

Structure. - The gland is surrounded by an outer sheath of connective tissue, which sometimes consists of two layers, sending in exceedingly fine prolongations forming the framework of the gland. The gland tissue proper consists of an outside firmer cortical portion, and an inside soft dark medullary portion.

The finer structure of the supra-renal capsules is incompletely known.

(1.) The cortical portion is divided into (fig. 225) an external narrow layer of small rounded or oval spaces, the zona glomerulosa, made by the fibrous trabeculæ, containing polyhedral cells $(b)$. The second layer of cells is arranged in columns radiating from the medulla, the zona fascic- 
ulata $(c)$, and separated from each other by fibrous septa. The third layer, that next the medulla, is called from its arrangement the zona reticularis (not shown in fig. 225). The individual cells are polyhedral in shape, each possessing a well-defined nucleus. In man the protoplasm of the cells is especially rich in fat globules, and oftentimes contains in addition larger or smaller granules of a yellowish pigment. The blood-vessels are confined to the septa, and do not penetrate into the cell groups.

(2.) The medullary substance consists of a coarse rounded or irregular meshwork of fibrous tissue, in the alveoli of which are masses of multinucleated protoplasm (fig. 226); numerous blood-vessels; and an abundance of nervous elements. The cells are very irregular in shape and size, poor in fat, and occasionally branched; the nerves run through the cortical substance, and anastomose over the medullary portion.

Nerves. - The adrenals are very abundantly supplied with nerves, chiefly composed of medullated fibres. These fibres are derived from the solar and renal plexuses, vagi and phrenics. Nerve-cells are also numerous in connection with these fibres. The fibres enter the hilum of the gland, but the method of their termination is unknown.

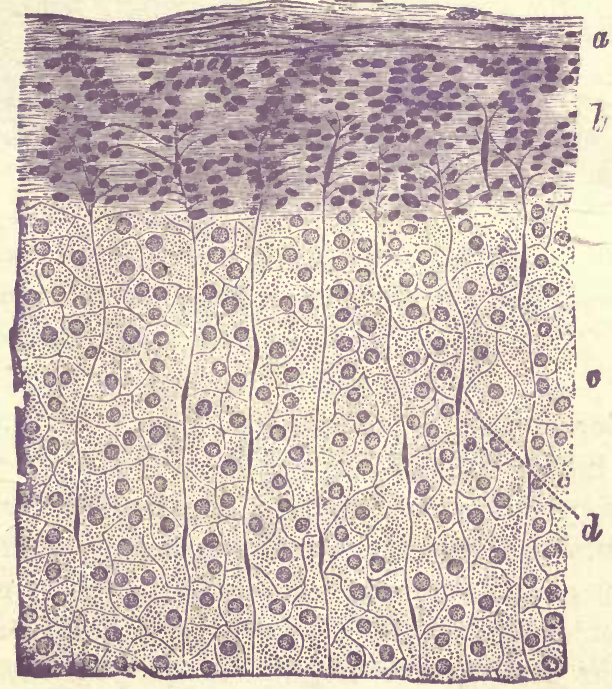

Fig. 225.-Vertical section through part of the cortical portion of supra-renal of guinea-pig. $a$, Capsule; $b$, zona glomerulosa ; $c$, zona fasciculata; $d$, connective tissue supporting the columns of the cells of the latter, and also indicating the positions of the blood-vessels. $X$ ( $\mathrm{S}$. K. Alcock.)

Composition. - In addition to the ordinary extractives, benzoic acid, hippuric acid, and taurin have been found, and also inosite, as well as a peculiar pigmentary substance, soluble in water, becoming red on ex- 
posure to light, and giving with ferric chloride a green or blue color. Hæmochromogen has been found by McMunn. Neurin, apparently from the nervous elements, has also been shown.

Function. - Though formerly unknown, a vast amount of light has been thrown upon the function of the supra-renal capsules within the last few years by the researches of Schäfer and Oliver, Zyboulski, Abel, and others. Brown-Séquard, it is true, showed by experiment as early as 1856 that removal of the supra-renal capsules is followed by the death of the animal, but his experiments were repeated by others who did not obtain the same results; and it was concluded that the supra-renal capsules had no function, or at least that their function was not known. Death was preceded in the case of Brown-Séquard's animals by symptoms

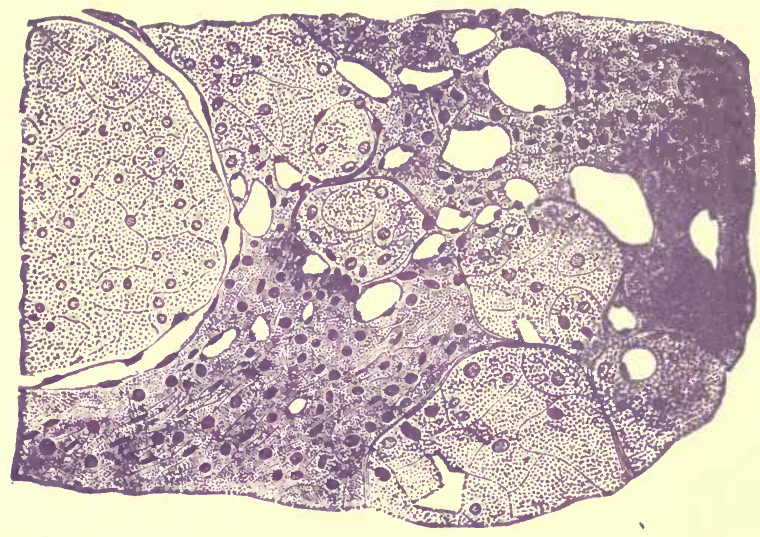

Fig. 226. - Section through a portion of the medullary part of the supra-renal of guinea-pig. The vessels are very numerous, and the fibrous stroma more distinct than in the cortex, and is moreover reticulated. The cells are irregular and larger, clean, and free from oil globules. $\times$
(S. K. Alcock.)

somewhat analogous to those of the disease of man known as Addison's disease. The failures to produce symptoms after attempted removal of the glands have probably resulted from incomplete removal or the presence of accessory bodies. Accessory supra-renal capsules are commonly present in some animals and are sometimes found in man. Further, if one gland is removed, the other hypertrophies. The experiments of all recent observers confirm the original experiments of Brown-Séquard. The presence of the supra-renal capsules is essential to life. Thus the supra-renal capsules are proved to have a very important function, and they perform this function through the agency of an internal secretion.

Schafer and Oliver found that injections of supra-renal extract produced marked cffects upon the muscular layer of the arteries, the muscular tissue of the heart, and the skeletal muscles. The muscular layer of the arteries is markedly contracted, causing a rise of blood-pressure. 
When the heart is freed from nervous control its contractions are increased both in force and frequency, still further raising blood-pressure. The contraction of the skeletal muscles in response to a single stimulus is much prolonged.

Very small doses of supra-renal extract are sufficient to produce murked effects. Thus Schäfer states that less than $\frac{1}{13000}$ gramme $\left(\frac{1}{800}\right.$ grain) of the desiccated gland is sufficient to produce an effect upon the heart and arteries of an adult man.

It is a curious fact that only extracts of the medullary portion of the gland are active.

Abel has succeeded in separating the blood-pressure-raising constituent of the extract, and calls it epinephrin. By nature it is related to the alkaloid group.

Destruction of the supra-renal capsules through disease in man results in the production of a group of symptoms known as Addison's disease. The administration of supra-renal extract to these cases sometimes results beneficially, but not so uniformly as thyroid feeding does in myxœdema. Yet Langlois states that if one-sixth of the supra-renal capsule by weight be left in the dog, the animal survives the operation of removal.

On the whole, the assumption that the supra-renal capsules produce an internal secretion which is essential to life is warranted.

The Pituitary Body.-This body is a small reddish-gray mass, occupying the sella turcica of the sphenoid bone.

Structure.-It consists of two lobes-a small posterior one, consisting of nervous tissue; an anterior larger one, resembling the thyroid in structure. A canal lined with flattened or with ciliated epithelium passes through the anterior lobe; it is connected with the infundibulum. The gland spaces are oval, nearly round at the periphery, spherical toward the centre of the organ; they are filled with nucleated cells of various sizes and shapes not unlike ganglion cells, collected together into rounded masses, filling the vesicles, and contained in a semifluid granular substance. The vesicles are inclosed by connective tissue rich in capillaries.

Function.-The function of the pituitary body has not yet been established. Some observers have found that its removal causes death, preceded by symptoms resembling those of thyroid removal. Hence it has been supposed that the pituitary body has a function identical with or analogous to that of the thyroid. On the other hand, tumors or other disease of the pituitary body have been found after death in association with a disease known as acromegaly, in which the bones and soft parts undergo great hypertrophy. In this connection it must be remembered that the two lobes of the pituitary body are morphologically and embryologically distinct. 


\section{Internal Secretion of the Pancreas.}

Minkowski and von Mering have shown that total extirpation of the pancreas is followed in all cases in the course of a few hours by the appearance of sugar in the urine. The amount of sugar which appears is considerable-from 5-10 per cent. This experimental disease (diabetes mellitus) is accompanied by an increase in the quantity of urine and by abnormal thirst and appetite, and proves fatal in 15 days or less. These results are obtained only when the entire gland or more than nine-tenths of it have been removed. If one-tentl of the gland be left behind, sugar appears in the urine when carbohydrates are eaten, but not otherwise. Nor is it necessary that the remaining portion of the gland be in its normal situation. Successful grafts under the skin of the abdomen or elsewhere will prevent the appearance of sugar in the urine and the other symptoms. If, however, the graft be subsequently removed, the sugar in the urine and the other symptoms reappear, and the experimental disease proceeds to a rapidly fatal issue.

The symptoms produced by total extirpation of the pancreas do not depend upon the loss of the pancreatic juice proper to the organism. This secretion may be diverted from the intestine through a pancreatic fistula without the prodnction of diabetes. Moreover, Hédon and Thiroloix have rendered the acini of the gland functionally inactive, and ultimately destroyed them, by the injection of paraffin or other substances into the duct of Wirsung, without the supervention of diabetes. These experiments have led to the conviction that the little groups of epitheliallike cells situated in the connective-tissue stroma of the pancreas secrete something which is absorbed into the circulation and coustitutes its internal secretion. Lépine and Boulud have recently extracted from the urine of patients suffering from diabetes or pneumonia a crystalline substance which produces glycosuria when injected under the skin or into the jugular vein of animals. This substance loses its power if passed in the blood through the vessels of a living pancreas. They conclude, therefore, that the pancreas, possibly through its internal secretion, has an antitoxic function and favors glycolysis in the tissues by destroying the substance which inhibits the conversion of glucose into glycogen or fat.

\section{Internal Secretion of the Liver.}

This subject will be considered at length when we come to study the formation of glycogen (see p. 435).

The Spleen is the largest of these so-called rascular glands; it is situated to the left of the stomach, between it and the diaphragm. 
is of a deep red color, of a variable shape, gcnerally oval, somewhat concavo-convex. Vessels enter and leave the gland at the inner side or hilus.

Structure. - The spleen is covered externally almost completely by a serous coat derived from the peritoneum, while within this is the proper fibrous coat or capsule of the organ. The latter, composed of connective tissue, with a large preponderance of elastic fibres, and a certain proportion of unstriated muscular tissue, forms the immediate investment of the spleen. Prolonged from its inner surface are fibrous processes or trabeculo, containing much unstriated muscle, which enter the interior of the organ, and, dividing and anastomosing in all parts, form a kind of supporting framework or stroma, in the interstices of which the proper substance of the spleen (spleen-pulp) is contained (fig. 228). At the hilus of the spleen, the blood-vessels, nerves, and lymphatics enter, and the fibrous coat is prolonged into the spleen-substance in the form

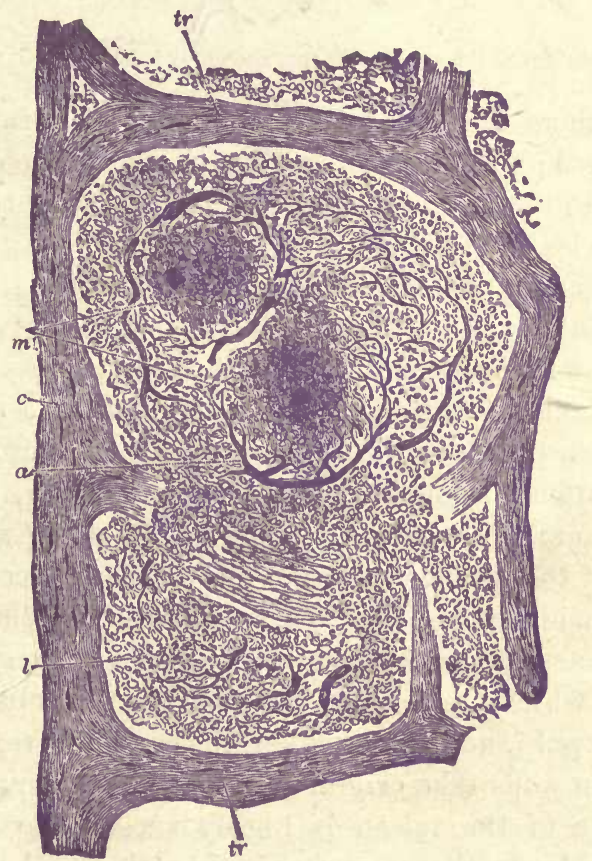

Fig. 227.-Section of injected dog's spleen: $c$, capsule: $t r$, trabeculæ; $m$, two Malpighiax bodies with numerous small arteries and crpillaries; $a$, artery; $l$, lymphoid tissue, consistirg of closely-packed ly mphoid cells supported by very delicate retiform tissue ; a light space unoccupied by culs is seen all round the trabeculø, which corresponds to the "lymph path" in lymp. iatic glands. (Schofield.)

of investing sheaths for the arteries and veins, which sheaths again are continuous with the trabeculæ before referred to. 
Mall has recently described the spleen as consisting of lobules, formed by the trabeculæ and contained masses of spleen-pulp.

The spleen-pulp, which is of a dark red or reddish-brown color, is composed chiefly of cells, imbedded in a matrix of fibres formed of the branching of large flattened nucleated endothelioid cells. The spaces of the network only partially occupied by cells form a freely communicating system. Of the cells some are granular corpuscles resembling the lymph-corpuscles, more or less connected with the cells of the meshwork, both in general appearance and in being able to perform amœboid

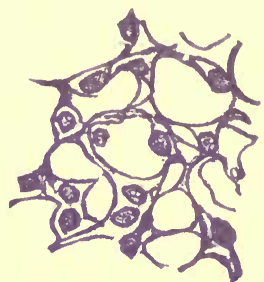

Fig. 228. - Reticulum of the spleen of a cat, shown by injection with geiatine. (Cadiat.)

movements; others are red blood-corpuscles of normal appearance or variously changed; while there are also large cells containing either a pigment allied to the coloring matter of the blood, or rounded corpuscles like red corpuscles.

The splenic artery, after entering the spleen by its concave surface, divides and subdivides, with but little anastomosis between its branches; at the same time its branches are sheathed by the prolongations of the fibrous coat, which they, so to speak, carry into the spieen with them. The arteries then pass into the spleen-pulp, their fibrous coat being replaced by lymphoid tissue, and end in capillaries, which communicate with the lacunar spaces in the spleen-pulp, from which veins arise.

The walls of the smaller veins are more or less incomplete, and readily allow lymphoid corpuscles to be swept into the blood-current. The blood from the arterial capillaries is emptied into a system of intermediate passages, which are directly bounded by the cells and fibres of the network of the pulp, and from which the smallest venous radicles with iheir cribriform walls take origin. The veins are large and distensible: whe whole tissue of the spleen is highly vascular and becomes readily engorged with blood: the amount of distention is, however, limited by the fibrous and muscular tissue of its capsule and trabeculæ, which forms an investment and support for the pulpy mass within.

On the face of a section of the spleen can be usually seen readily with the naked eye, minute, scattered rounded or oval whitish spots, mostly from $\frac{1}{30}$ to $\frac{1}{60}$ inch $\left(\frac{5}{6}\right.$ to $\frac{2}{5} \mathrm{~mm}$.) in diameter. These are the Malpi- 
ghian corpuscles of the spleen, and are situated on the sheaths of the minute splenic arteries, of which, indeed, they may be said to be outgrowths (fig. 229). For while the sheaths of the larger arteries are constructed of ordinary connective tissue, this has become modified where it forms an investment for the smaller vessels, so as to be composed of adenoid tissue, with abundance of corpuscles, like lymph-corpuscles, contained in its meshes, and the Malpighian corpuscles are but small outgrowths of this cytogenous or cell-bearing comwective tissue. They are composed of cylindrical masses of corpuscles, intersected in all parts by a delicate fibrillar tissue, which, though it invests the Malpighian bodies, does not form a complete capsule. Blood-capillaries traverse the Malpighian corpuscles and form a plexus in their interior. The struc-

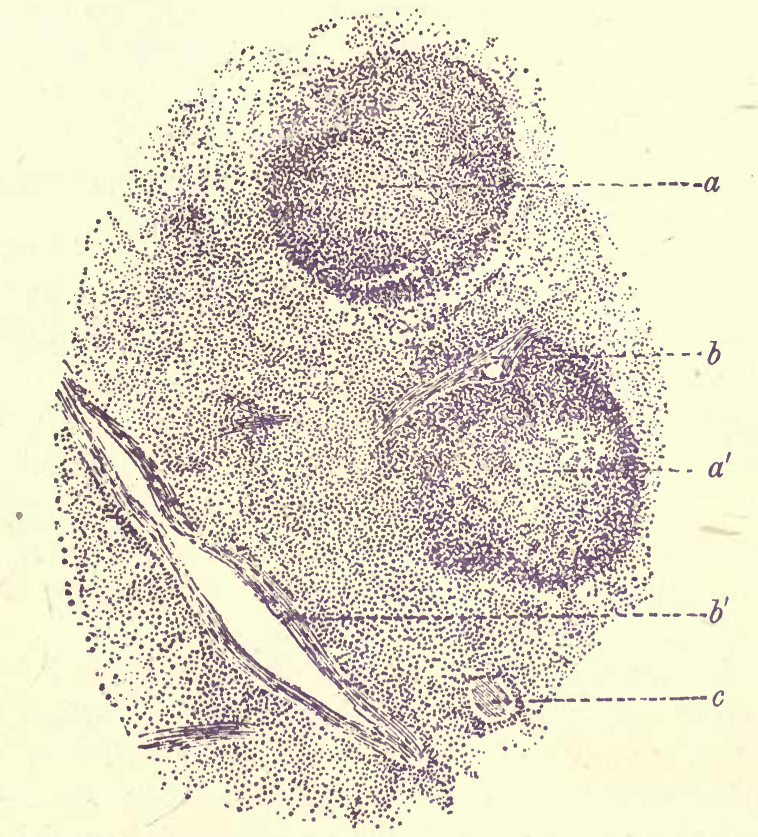

Fig. 229.-Section of spleen of cat. $\alpha, \alpha^{\prime}$, Malpighian corpuscles, in case of $a^{\prime}$, in connection with small artery, $b ; b, b^{\prime}$, small arteries; $c$, section of trabeculæ.

ture of a Malpighian corpuscle of the spleen is, therefore, very similar to that of lymphatic-gland substance.

Functions. -With respect to the office of the spleen, we have the following data: (1.) The large size which it gradually acquires toward the termination of the digestive process, and the great increase observed about this period in the amount of the finely-granular albuminous plasma within its parenchyma, and the subsequent gradual decrease of 
this material, seem to indicate that this organ is concerned in storing up some of the changed and absorbed proteid food, to be gradually introduced into the blood according to the demands of the general system.

(2.) It seems probable that the spleen, like the lymphatic glands, is engaged in the formation of blood-corpuscles. For it is quite certain that the blood of the splenic vein contains an unusually large amount of white corpuscles; and in the disease termed leucocythæmia, in which the pale corpuscles of the blood are remarkably increased in number, there is almost always found an hypertrophied state of the spleen or of the lymphatic glands. In Kölliker's opinion, the development of colorless and also colored corpuscles of the blood is one of the essential functions of the spleen, into the veins of which the new-formed corpuscles pass, and are thus conveyed into the general current of the circulation.

(3.) The formation of red corpuscles. The spleen is concerned in the formation of red corpuscles during fœtal life and shortly after birth, and in some animals during their whole existence. For, if the spleen be removed from such animals, the red marrow undergoes hypertrophy. Moreover, in these animals the cells previously described as hæmatoblasts may be found in the spleen.

It was formerly believed that the spleen exercised the function of destroying red corpuscles that had lived out their allotted time. The evidence of this, however, is not convincing, and the theory has been practically abandoned. It rested chiefly upon the fact that large nucleated cells were found in the spleen, with whole or partially disintegrated red cells in their interior. But the phenomenon is probably of post-mortem occurrence. When the circulation ceases, the red cells come to rest, and, lying alongside these large cells, are probably then ingested.

(4.) From the almost constant presence of uric acid, in larger quantities than in other organs, as well as of the nitrogenous bodies, xanthin, hypoxanthin, and leucin, in the spleen, some special nitrogenous metabolism may be fairly inferred to occur in it. One of the features of the chemical composition of the spleen is the presence of a special proteid, of the nature of alkali-albumin, containing iron. The salts of the spleen consist chiefly of sodium phosphates.

(5.) Besides these, its supposed direct offices, the spleen is believed to fulfil some purpose in regard to the portal circulation, with which it is in close connection. From the readiness with which it admits of being distendea, and from the fact that it is generally small while gastric digestion is going on, and enlarges when that act is concluded, it is supposed to act as a kind of vascular reservoir, or diverticulum to the portal system, or more particularly to the vessels of the stomach. That it may serve such a purpose is also made probable by the enlargement which it 
undergoes in certain affections of the heart and liver, attended with obstruction to the passage of blood through the latter organ, and by its diminution when the congestion of the portal system is relieved by discharges from the bowels, or by the effusion of blood into the stomach. This mechanical influence on the circulation, however, can hardly be supposed to be more than a very subordinate function.

The spleen may be removed without any obvious ill effect.

Influence of the Nervous System upon the Spleen. - When the spleen is enlarged after digestion, its enlargement is probably due to two causes, (1) a relaxation of the muscular tissue which forms so large a part of

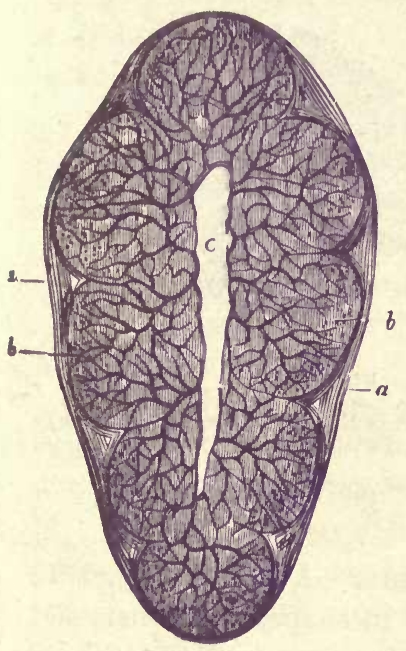

Fig. 230.

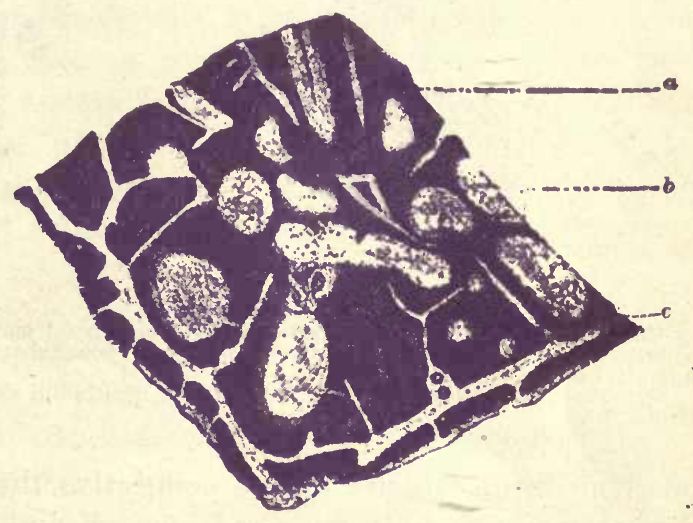

Fig. 231.

Fig. 230.-Transverse section of a lobule of an injected infantile thymus gland. $a$, Capsule of connective-tissue surrounding the lobule; $b$, membrane of the glandular vesicles; $c$, cavity of the lobule, from which the larger blood-vessels are seen to extend toward and ramify in the spheroidal masses of the lobule. $\times 30$. (Kölliker.)

Fig. 231. - Thymus of a calf. $a$, Cortex of follicle; $b$, medulla; $c$, interfollicular tissue, magnified about twelve times. (Watney.)

its framework; (2) a dilatation of the vessels. Both these phenomena are doubtless under control of the nervous system. It has been found by experiment that when the splenic nerves are cut the spleen enlarges, and that contraction can be brought about (1) by stimulation of the spinal cord (or of the divided nerves); (2) reflexly by stimulation of the central stumps of certain divided nerves, e.g., vagus and sciatic; (3) by local stimulation by an electric current; (4) the exhibition of quinine and some other drugs. It has been shown by the oncometer of Roy (fig. 30\%), that the spleen undergoes rhythmical contractions and dilatations, due no doubt to the contraction and relaxation of the muscular tissue in its capsule and trabeculæ. It also shows the rhythmical alteration of the general blood pressure, but to a less extent than the kidney. 
The Thymus. - This gland must be looked upon as a temporary organ, as it attains its greatest size early after birth, and after the second year gradually diminishes, until in adult life hardly a vestige remains. At its greatest development it is a long, narrow body, situated in the front of the chest behind the sternum and partly in the lower part of the neck. It is of a reddish or grayish color, distinctly lobulated.

Structure.-The gland is surrounded by a fibrous capsule, which sends in processes, forming trabeculæ, which divide the glands into lobes, and carry the blood and lymph-ressels. The large trabeculæ branch into small ones, which diride the lobes into lobules. The lobules are further

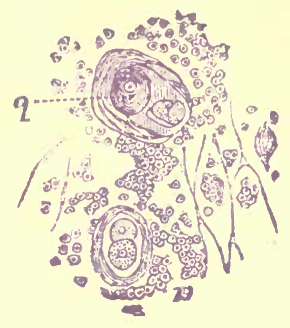

Fig. 232.

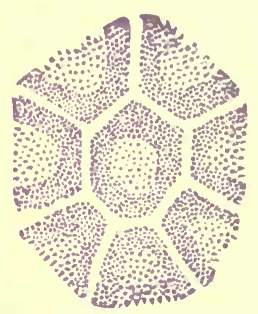

Fig. 233.

Fig. 232.- From a horizontal section through superficial part of the thymus of a calf, slightly magnified. Showing in the centre a follicle of polygonal shape with similarly shaped follicles round it. (Klein and Noble Smith.)

Fig. 233. - The reticulum of the Thymus. $a$, Epithelial elements; $b$, corpuscles of Hassall. (Cadiat.)

subdivided into follicles by fine connective tissue. A follicle (fig. 232) is seen on section to be more or less polyhedral in shape, and consists of cortical and medullary portions, both of which are composed of adenoid tissue, but in the medullary portion the matrix is coarser, and is not so filled up with lymphoid corpuscles as in the cortex. The adenoid tissue of the cortex, and to a less marked extent that of the medulla, consists of the two elements, one with small meshes formed of fine fibres with thickened nodal points, and the other enclosed within the first, composed of branched connective-tissue corpuscles (Watney). Scattered in the adenoid tissue of the medulla are the concentric corpuscles of Hassall, which are protoplasmic masses of various sizes, consisting of a nucleated granular centre, surrounded by flattened nucleated epithelial cells. In the reticulum, especially of the medulla, are large transparent giant cells. In the thymus of the dog and of other animals are to be found cysts, probably derived from the concentric corpuscles, some of which are lined with ciliated epithelium, and others with short columnar cells. The arteries radiate from the centre of the gland. Lymph sinuses may be seen occasionally surrounding a greater or smaller portion of the periphery of the follicles (Klein). The nerves are very minute. 
From the thymus various substances may be extracted, many of them similar to those obtained from the spleen, e.g., xanthin, hypoxanthin, and leucin, as well as certain proteids, especially nucleo-proteid (found in all protoplasm), which on injection into the veins of an animal produces intra-vascular clotting.

Function.-Beard has recently concluded from some experiments on the smooth skate that the important function of the thymus is the formation of the colorless corpuscles-that the thymus, in fact, is the parent source from which all the colorless corpuscles are derived. The first are developed from the thymus cells, and from them all the others arise.

Respecting the thymus gland in the hybernating animals, in which it exists throughout life, as each successive period of hybernation approaches, the thymus greatly enlarges and becomes laden with fat, which accumulates in it and in fat glands connected with it, in even larger proportions than it does in the ordinary seats of adipose tissue. Hence it appears to serve for the storing up of materials which, being re-absorbed in inactivity of the hybernating period, may maintain the respiration and the temperature of the body in the reduced state to which they fall during that time. It is also believed to be a source of the red blood-corpuscles, at any rate in early life.

The Pineal Gland.-This gland, which is a small reddish body, is placed beneath the back part of the corpus callosum, and rests upon the corpora quadrigemina.

Structure. - It contains a central cavity lined with ciliated epithelium. The gland substance proper is divisible into-(1.) An outer cortical layer, analogous in structure to the anterior lobe of the pituitary body; and (2.) An inner central layer, wholly nervous. The cortical layer consists of a number of close follicles, containing (a) cells of variable shape, rounded, elongated, or stellate; $(b)$ fusiform cells. There is also present a gritty matter (acervulus cerebri), consisting of round particles aggregated into small masses. The central substance consists of white and gray matter. The blood-vessels are small, and form a very delicate capillary plexus.

The pineal gland is a restigial structure, being the atrophied third eye which was situated in the median line. It is found in a better developed condition in certain lizards, though it is functionless.

The Coccygeal and Carotid Glands. - These so-called glands are situated, the one in front of the tip of the coccyx, and the other at the point of bifurcation of the common carotid artery on each side. They are made up of a plexus of small arteries, are inclosed and supported by a capsule of fibrous tissue, which contains connective-tissue corpuscles. The blood-vessels are surrounded by one or more layers of cells like secreting cells, which are said to be modified plasma cells of the connective tissue. The function of these bodies is unknown. 


\section{CHAPTER IX.}

\section{FOOD AND DIGESTION.}

THE object of digestion is to bring the materials of the food into such a condition that they may be taken up by the blood and lymphatic vessels, and so rendered available for the wants of the system. It makes the foods soluble and diffusible, and also converts bodies already soluble and diffusible into forms which can be utilized, e.g., cane sugar, although soluble and diffusible, cannot be used by the body until it has been split into two molecules of monosaccharide. Very few of these materials are fit for this purpose when taken into the body, and the majority would therefore be to all intents and purposes quite useless unless digested.

It is unnecessary to mention all the various substances which may have been used as food at some time or another, and we shali confine our attention, therefore, to the chief and most familiar articles of diet.

We find, then, that foods may be divided into classes corresponding closely to those employed to describe the chief substances of which the animal body consists. This classification may be recapitulated as follows:-

\section{ORGANIC.}

I. Foods primarily containing Nitrogenous substances, consisting of Proteids, e.g., albumen, casein, myosin, gluten, legumin and their allies; and Albuminoids, e.g., gelatin, elastin, and chondrin.

II. Food primarily containing Non-Nitrogenous substances, comprising :

(1.) Amyloid or saccharine bodies, chemically kuown as carbo-hydrates; e.g., starches and sugars.

(2.) Oils and fats. - These substances contain carbon, hydrogen, and oxy gen, but the oxygen is less in amount than in the amyloids and saccharine bodies.

\section{INORGANIC.}

I. Foods which supply Mineral and saline matter.

II. Liquid food containing chiefly Water.

Man requires that the chief part of his food should be cooked. Very few organic substances can be properly digested without previous exposure to heat and to other manipulations which constitute the process of cooking.

\section{Organic nitrogenous foods.}

a.-The Flesh of Animals, e.g., of the ox (beef, veal), sheep (mutton, lamb), pig (pork, bacon, ham).

Of these, beef is richest in nitrogenous matters, containing about 20 per cent, whereas mutton contains about 18 per cent, veal 16.5, and 
pork, 10; beef is also firmer, more satisfying, and is supposed to be more strengthening than mutton, whereas the latter is more digestible. The flesh of young animals, such as lamb and veal, is less digestible and less nutritious. Pork is comparatively indigestible, and contains a large amount of fat.

Flesh contains:-(1) Nitrogenous bodies; chiefly myosin, and one or more globulins; serum-albumin, gelatin (from the interstitial fibrous connective tissue); elastin (from the elastic tissue), as well as hamoglobin. (2) Fatty matters, including lecithin and cholesterin. (3) Extractive matters, some of which are agreeable to the palate, e.g. osmazome, and others, which are weakly stimulating, e.g., creatin. Besides, there are sarcolactic and inositic acids, taurin, xanthin, and others. (4) Salts, chiefly of potassium, calcium, and magnesium. (5) Water, the amount of which varies from 15 per cent in dried bacon to 39 in pork, 51 to 53 in fat beef and mutton, to 72 per cent in lean beef and mutton. certain amount of carbo-hydrate material is found in the flesh of some animals, in the form of inosite, dextrin, grape sugar, and (in young animals) glycogen.

'Table of Percentage Composition of Beef, Mutton, Pork, and Veal.(LETHEBY.)

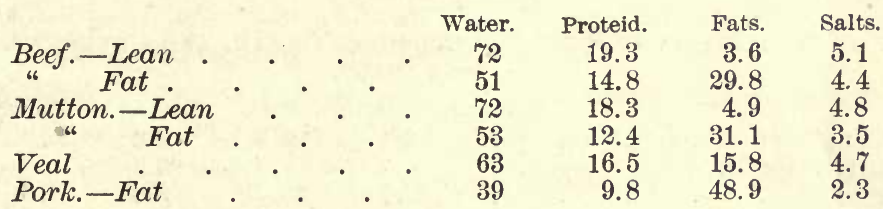

Together with the flesh of the above-mentioned animals, that of the deer, hare, rabbit, and birds, constituting venison, game, and poultry, should be added as taking part in the supply of nitrogenous substances, and also fish-salmon, eels, etc., and shell-fish, e.g., lobster, crab, mussels, oysters, shrimps, scollops, cockles, etc.

Table of Percentage Composition of Poultry and Fish.-(Letheby.)

\begin{tabular}{|c|}
\hline $\begin{array}{lllllllll}\text { Poultry } & . & . & . & . & 74 & 21 & 3.8 & 1.2\end{array}$ \\
\hline
\end{tabular}

(Singularly devoid of fat, and is therefore generally enten with bacon or pork.)

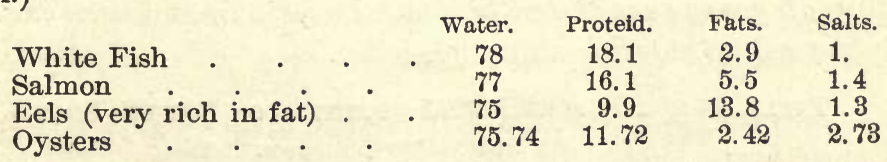

(7.39 consist of non-nitrogenous matter and loss.) (Payen.)

Even now the list of fleshy foods is not complete, as the flesh of nearly all animals has been occasionally eaten, and we may presume 
that except for difference of flavor, etc., the average composition is nearly the same in every case.

b. Milk.*-Is intended as the entire food of young animals, and as such contains, when pure, all the elements of a typical diet. (1) Albuminous substances in the form of caseinogen, and serum or lact-albumin. (2) Fats in the cream. (3) Carbo-hydrates in the form of lactose or milk sugar. (4) Salts, chiefly calcium phosphate; and (5) Water. From it we obtain $(\alpha)$ cheese, which is the clotted caseinogen or casein precipitated with more or less of fat according as the cheese is made of skim milk (skim cheese), of fresh milk with its cream (Cheddar and Cheshire), or of fresh milk plus cream (Stilton and double Gloucester). The precipitated casein is allowed to ripen, by which process some of the albumin is further split up, with formation of fat. ( $\beta$ ) Cream, consists of the fatty globules encased in caseinogen and serum-albumin, and which being of low specific gravity float to the surface. (r) Butter, or the fatty matter deprived of its proteid envelope by the process of churning. ( $\delta)$ Buttermilk, or the fluid obtained from cream after butter has been formed; rery rich therefore in nitrogen. (s) Whey, or the fluid which remains after the precipitation of casein; it contains sugar, salt, and a small quantity of albumin.

Table of Composition of Mrlk, Butter-Milk, Cream, and Cheese.-(Letheby AND PAYEN.)

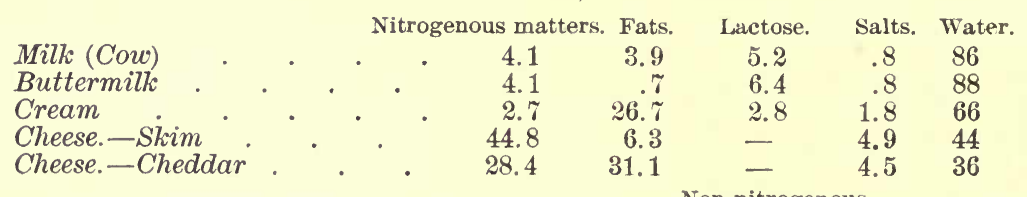

\section{matter and loss.}

$\begin{array}{lllllll}\text { Cheese. -Neufchatel }(\text { Fresh }) . & 8 . & 40.71 & 36.58 & .51 & 36.58\end{array}$

c. Eggs. - The yolk and albumen of eggs are in the same relation as food for the embryos of oviparous animals that milk is to the young of mammalia, and afford another example of the natural admixture of the various alimentary principles. 'I'he proteids of eggs are egg-a lbumin and globulins, of which the vitellin of the yolk is most important; nuclein in combination with iron is also found. In addition to the three common fats there is a yellow fat, lutein (lipochome), a small quantity of grape sugar; lecithin, and cholesterin and inorganic salts, chiefly potassium chloride and phosphates.

Table of the Percentage Composition of Fowls' Eggs.

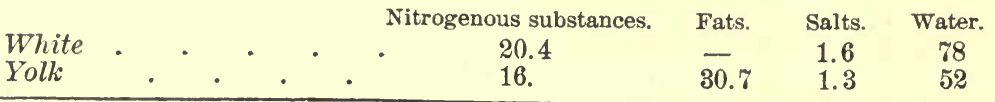

* The details of the composition of milk have been discussed in the Chapter on Secretion. 
d. Leguminous fruits are used by vegetarians, as the chief source of the nitrogen of the food. Those chiefly used are peas, beans, lentils, etc., they contain a nitrogenous substance called legumin, allied to albumen. They contain about 25.30 per cent of this nitrogenous body, and twice as much nitrogen as wheat.

\section{Organic non-nitrogenous foods.}

I. Carbo-hydrates.-a. Bread, made from the ground grain obtained from various so-called cereals, viz., wheat, rye, maize, barley, rice, oats, etc., is the direct form in which the carbo-hydrate is supplied in an ordinary diet. It contains starch, dextrin, and a little sugar. It also, besides these, contains gluten, composed of several vegetable proteids, and a small amount of fat.

\section{Table of Percentage Composition of Bread and Flour.}

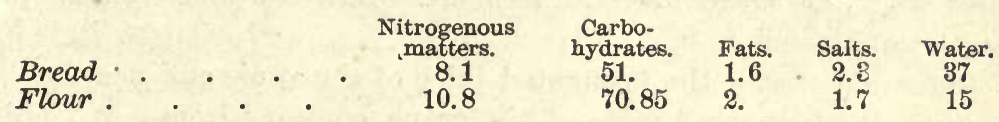

Various articles of course besides bread are made from flour, e.g., sago, macaroni, biscuits, etc. There is dextrine and a small amount of dextrose in bread, particularly in the crust.

b. Vegetables, especially potatoes. They contain starch and sugar. In cabbage, turnips, etc., the salts of potassium are abundant.

c. Fruits contain sugar, and organic acids, tartaric, malic, citric, and others.

d. Sugar, chiefly saccharose, used pure or in various sweetmeats.

II. Oils and fats.-The substances supplying the oils and fats of the food are chiefly butter, bacon and lard (pig's fat), suet (beef and mutton fat), and vegetable oils. These contain olein, stearin, and palmitin. Butter contains others in addition, while vegetable oils, as a rule, contain no stearin.

\section{Mineral or Inorganic Foods.}

The salts of the food.-Nearly all the foregoing substances in the preceding classes, contain a greater or less amount of the salts required in food, but green vegetables and fruit supply certain salts, chiefly potassium, without which the normal health of the body cannot be maintained.

Sodium chloride is an essential food; it is contained in nearly all solids, but so much is required that it has also to be taken as a condiment. Potassium salts are supplied in muscle, nerve, in meats generally, and in potatoes. Calcium salts are supplied in eggs, blood of meat, wheat and vegetables. Iron is contained in hæmoglobin, in milk, eggs, and 
vegetables. It is derived in all cases, so it is supposed, by organic compounds, into which it is built up during plant life, or during the life of other animals (hæmatogens).

\section{Liquid Foods.}

Water is consumed alone, or together with certain other substances used to flavor it, e.g., tea, coffee, etc. Tea in moderation is a stimulant, and contains an aromatic oil to which it owes its peculiar aroma, an astringent of the nature of tannin, and an alkaloid, theine. The composition of coffee is very nearly similar to that of tea. Cocoa, in addition to similar substances contained in tea and coffee, contains fat, albuminous matter and starch, and must be looked upon more as a food.

Beer, in various forms, is an infusion of malt (barley which has sprouted, and in which its starch is converted in great part into sugar), boiled with hops and allowed to ferment. Beer contains from 1.2 to 8.8 per cent of alcohol.

Cider and Perry, the fermented juice of the apple and pear.

Wine, the fermented juice of the grape, contains from 6 or 7 (Rhine wines, and white and red Bordeaux) to 24-25 (ports and sherries) per cent of alcohol.

Spirits, obtained from the distillation of fermented liquors. They contain upward of 40- 60 per cent of absolute alcohol.

The effect of cooking.-In general terms this may be said to make the food more easily digestible; this usually implies two alterations,food is made more agreeable to the palate and also more pleasing to the eye. Cooking consists in exposing the food to various degrees of heat, either to the direct heat of the fire, as in roasting, or to the indirect heat of the fire, as in broiling, baking, or frying, or to hot water, as in boiling or stewing. The effect of heat upon $(a)$ flesh is to coagulate the albumin and coloring matter, to solidify fibrin, and to gelatinize tendons and fibrous connective tissue. Previous beating or bruising (as with steaks and chops) or keeping (as in the case of game), renders the meat more tender. Prolonged exposure to heat also develops on the surface certain empyreumatic bodies, which are agreeable both to the taste and smell. By placing meat in hot water, the external coating of albumin is coagulated, and very little, if any, of the constituents of the meat are lost afterward if boiling be prolonged; but if the constituents of the meat are to be extracted, it should be exposed to prolonged simmering at a much lower temperature, and the "broth" will then contain the gelatin and extractive matters of the meat, as well as a certain amount of albumin. The addition of salt will help to extract myosin.

The effect of boiling $(b)$ an egg is to coagulate the albumen, which helps to render it more easily digestible. Upon $(c)$ milk, the effect of 
heat is to produce a scum composed of albumen and a little caseinogen (the greater part of the caseinogen being uncoagulated) with some fat. Upon $(d)$ vegetables, the cooking produces the necessary effect of rendering them softer, so that they can be more readily broken up in the mouth; it also causes the starch grains to swell up and burst, and so aids the digestive fluids in penetrating into their substance. The albuminous matters are coagulated, and the gummy, saccharine and saline matters are removed. The conversion of flour into dough is effected by mixing it with water, and adding a little salt and a certain amount of yeast. Yeast consists of the cells of an organized ferment (Torula cerevisia), and it is by the growth of this plant, changing by ferment action the sugar produced from the starch of the flour, that a quantity of carbonic acid gas and alcohol is formed. By means of the former the dough rises. Another method of making dough consists in mixing the flour with water containing a large quantity of carbonic acid gas in solution.

By the action of heat during baking $(d)$ the dough continues to expand, and the gluten being coagulated, the bread sets as a permanently vesiculated mass.

\section{Digestion.}

The Enzymes, or unorganized ferments, are the essential factors in digestion, and their predominant action is one of hydrolytic cleavage; that is, the substance acted upon takes up water and then splits into two different substances, usually of the same class. Their chemical nature is as yet undetermined because of the inability of getting absolutely pure specimens, but it is generally admitted that they contain nitrogen, and they are usually classed as proteids. Practically all are secreted in the glands as zymogens, which bear the same relation to enzymes as fibrinogen does to fibrin; they are transformed to enzymes by the proper stimulus but never exist as such in the glands. Some of them pass into the urine, but most are excreted with the fæces.

Each enzyme has a special point of temperature at which it acts best, and any change in the temperature retards its action; the action is suspended at a definite point of low temperature, but the enzyme is not destroyed by cold; the action is also suspended at higher temperatures, and at a still higher point the enzyme is destroyed. Some enzymes act only in an alkaline medium, being destroyed in an acid medium, and vice versa; others act in either alkaline, neutral or acid media. Enzymes are hindered in their action by the accumulation of the products of their activity. Most of them cease acting altogether when these products reach a certain concentration, but will begin acting again on the removal of these products or if the mixture be simply diluted. 
The quantity of the enzyme determines the rapidity of the action but not the amount; a small quantity will digest as much as a large quantity but will take longer. The enzymes are not used up in the course of their activity, as far as can be seen, and do not seem to undergo any change in their composition. They are classified either according to the chemical nature of their action, or according to the class of substances on which they act; the former classification is more logical, but the latter is more convenient and more generally used.

The food is first of all received into the mouth, and is subjected to the action of the teeth and tongue, being at the same time mixed with the first of the digestive juices - the saliva. It is then swallowed, and, passing through the pharynx and resophagus into the stomach, is subjected to the action of the gastric juice-the second digestive juice. Thence it passes into the intestines, where it meets with the bile, the pancreatic juice, and the intestinal juices, all of which exercise an influence upon the portion of the food not already absorbed from the stomach. By this time most of the food is digested, and the residue of undigested matter leaves the body in the form of freces by the external opening of the bowel.

The Mouth is the cavity contained between the jaws and inclosed by the cheeks laterally, the lips anteriorly; behind, it opens into the pharynx by the fauces, and is separated from the nasal cavity above, by the hard palate in front, and the soft palate behind, which forms its roof. The tongue forms the lower part or floor. In the jaws are contained the teeth, and when the mouth is closed these form its anterior boundaries. The whole of the cavity of the mouth is lined with stratified epithelium, of which the superficial layers are squamous. This epithelium is continuous at the lips with that of the skin anteriorly, and posteriorly with that of the pharynx. The mucous membrane itself, varying in thickness in various parts, and consisting of a fine areolar connective, in which is found adenoid tissue in considerable amount, is provided with numerous small tubular glands lined with columnar epithelium, and resembling in structure the mucous salivary glands, to be presently described. Into the buccal cavity open the ducts of the salivary glands, which are three in number on either side.

In the mouth, thell, the food is subjected to the action of the teeth, or is masticated, and is mixed with saliva. These processes of mastication and insalivation must be considered more in detail.

Mastication.-The act of chewing, or mastication, is performed by the biting and grinding movement of the lower range of teeth against the upper. The simultaneous movements of the tongue and cheeks assist partly by crushing the softer portions of the food against the hard palate and gums, and thus supplementing the action of the teeth, and partly by returning the morsels of food to the action of the teeth, again and again, 
as they are squeezed out from between them, until they have been sufficiently chewed.

Muscles.-The simple up and down, or biting movements of the lower jaw, are performed by the temporal, masseter, and internal pterygoid muscles, the action of which in closing the jaws alternates with that of the digastric and other muscles passing from the os hyoides to the lower jaw, which open them. The grinding or side to side movements of the lower jaw are performed mainly by the external pterygoid muscles, the muscle of one side acting alternately with the other. When both external pterygoids act together, the lower jaw is pulled directly forward, so that the lower incisor teeth are brought in front of the level of the upper.

Temporo-maxillary Fibro-cartilage.-The function of the inter-articulo-fibro-cartilage of the temporo-maxillary joint in mastication is to serve : -(1) As an elastic pad to distribute the pressure caused by the exceedingly powerful action of the masticatory muscles. (2) As a joint-surface or socket for the condyle of the lower jaw when the latter has been partially drawn forward out of the glenoid cavity of the temporal bone by the external pterygoid muscle, some of the fibres of the latter being attached to its front surface, and consequently drawing it forward with the condyle which moves on it.

Nervous Mechanism. - The act of mastication is partly voluntary and partly reflex and involuntary. The consideration of such nervous actions will come hereafter. It will suffice here to state that the afferent nerves chiefly concerned are the sensory branches of the fifth and the tenth or glosso-pharyngeal, and the efferent are the motor branches of the fifth and the twelfth (hypoglossal) cerebral nerves. The nerve-centre through which the reflex action occurs, and by which the movements of the various muscles are harmonized, is situated in the medulla oblongata. In so far as mastication is voluntary or mentally perceived, it is under the influence of the cerebral hemispheres.

Insalivation.- The act of mastication is much assisted by the saliva which is secreted by the salivary glands in largely increased amount during the process, and the intimate incorporation of which with the food, as it is being chewed, is termed insalivation.

\section{The Salivary Glands.}

The glands which secrete the saliva in the human subject are the salivary glands proper, viz., the parotid, the sub-maxillary, and the sub-lingual, and numerous smaller bodies of similar structure, and with separate ducts; which are scattered thickly beneath the mucous membrane of the lips, cheeks, soft palate, and root of the tongue.

Structure.-The salivary glands are compound tubular or tubulo-race- 
mose glands. They are made up of lobules. Each lobule consists of the branchings of a subdivision of the main duct of the gland, which is generally more or less convoluted toward its extremities, and sometimes, according to some observers, sacculated or pouched. The convoluted or pouched portions form the alveoli, or proper secreting parts of the gland. The alveoli are composed of a basement membrane of flattened cells joined together by processes to produce a fenestrated membrane, the spaces of which are occupied by a homogeneous ground-substance. Within, upon this membrane, which forms the tube, the nucleated salivary secreting cells, of cubical or columnar form, are arranged parallel to one another enclosing a central canal. The granular appearance frequently seen in the salivary cells is dne to the numerous zymogen granules which they contain. When isolated, the cells not infrequently are found to be branched. Connecting the alveoli into lobules is a eonsiderable amount

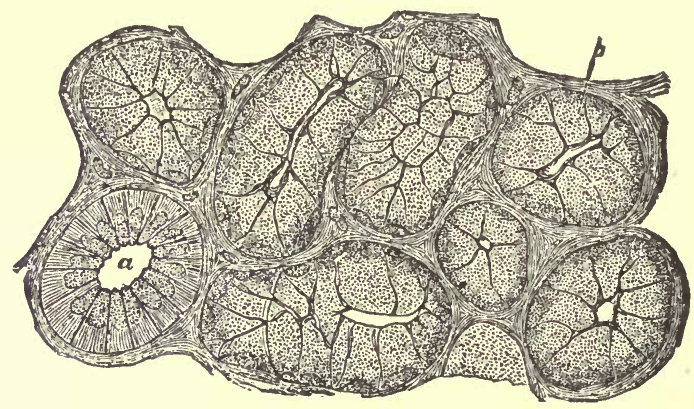

Fig. 234. -Section of sub-maxillary gland of dog. Showing gland cells, $b$, and a duct, $a$, in section. (Kölliker.)

of tibrous connective tissue, which contains both flattened and granular protoplasmic cells, lymph corpuscles, and in some cases fat cells. The lobules are connected to form larger lobules (lobes), in a similar manner. The alveoli pass into the intralobular ducts by a narrowed portion (intercalary), lined with flattened epithelium with elongated nuclei. The intercalary ducts pass into the intralobular ducts by a narrowed neck, lined with enbical cells with small nuclei. The intralobular duct is larger in size, and is lined with large columnar nucleated cells, the parts of which, toward the lumen of the tube, present a fine longtitudinal striation, due to the arrangement of the cell network. It is most marked in the submaxillary gland. The intralobular ducts pass into the larger ducts, and these into the main duct of the gland. As these ducts become larger they acquire an outside coating of connective tissue, and later on some unstriped muscular fibres. The lining of the larger ducts consist of one or more layers of columnar epithelium, the cells of which contain an intracellular network of fibres arranged longitudinally. 
Varieties.-Certain differences in the structure of salivary glands may be observed according as the glands secrete pure saliva, or saliva mixed with mucus, or pure mucus, and therefore the glands have been classified as :-

(1) True salivary glands (called most unfortunately by some, serous glands), e.g., the parotid of man and other animals, and the submaxillary of the rabbit and guinea-pig (fig. 235). In this kind the alveolar lumen is small, and the cells lining the tubule are short granular columnar cells, with nuclei presenting the intranuclear network. During rest the cells become larger, highly granular, with obscured nuclei, and the lumen becomes smaller. During activity, and after stimulation of the sympathetic, the cells become smaller and their contents more opaque; the granules first of all disappearing from the outer part of the cells, and

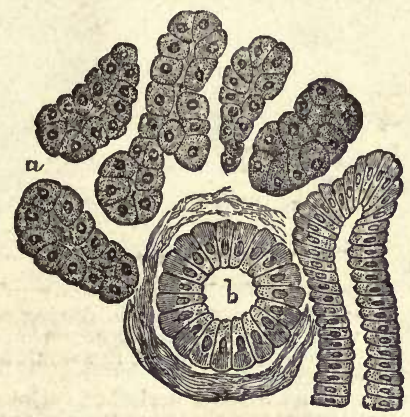

Fig. 235.-From a section through a truesalivary gland, $a$, The gland alveoli, lined with albuminous "salivary cells;" $b$, intralobular duct cut transversely. (Klein and Noble Smith.)

then being found only at the extreme inner part and contiguous border of the cell. The nuclei reappear, as does also the lumen.

(2) In the true mucus-secreting glands, as the sublingual of man and other animals, and in the submaxillary of the dog, the tubes are larger, contain a larger lumen, and also have larger cells lining them. The cells are of two kinds, (a) mucous or central cells, which are transparent columnar cells with irregular or flattened nuclei near the basement membrane. The cell substance is made up of a fine network, which in the resting state contains a transparent substance called mucigen, during which the cell does not stain well with logwood (fig. 236). When the gland is secreting, as well as on stimulation of the nerve, mucigen is converted into mucin, and the cells swell up, appear more transparent, and stain deeply in logwood (fig. 237). After stimulation, the cells become smaller, more granular, and more easily stained, from having discharged their contents. The nuclei appear more distinct. (b) Crescents of Gianuzzi, sometimes called the Semilunes of Heidenhain (fig. 236), which are crescentic masses of granular parietal cells found here and there be- 
tween the basement membrane and the central cells. The cells composing the mass are small, and have a very dense reticulum, the nuclei are spherical, and increase in size during secretion. In the mucous gland there are some large tubes, lined with large transparent central cells, and having besides a few granular parietal cells; other small tubes are lined with small granular parietal cells alone; and a third variety are lined equally with each kind of cell.

(3) In the muco-sativary or mixed glands, as the human submaxillary

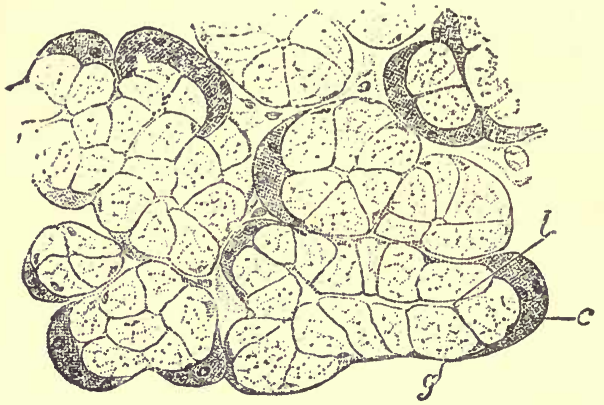

Fig. 236 .

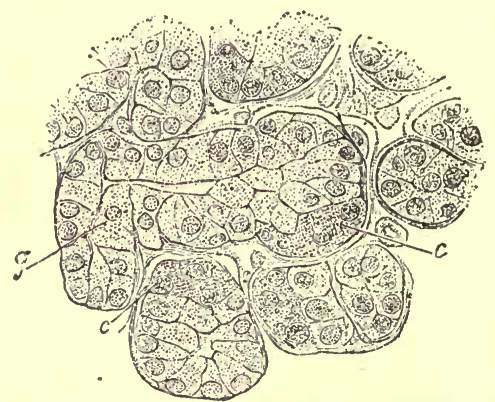

Fig. 23\%.

Fig. 236. - Section of the submaxillary gland of a dog, during rest. Most of the alveolar cells are large and clear, being filled with the material for secretion (in this case, mucigen) which obscures their protoplasm : some of the crlls, however, are small and protoplasmic, forming the crescents seen in most of the alveoli. (Ranvier.)

Fig. 237. - Section of a similar gland after a period of activity. The mucigen has been discharged from the mucin-secreting cells, which consequently appear shrunken and less clear. Both the cells and the alveoli are much smaller, and the protoplasm of the cells is more apparent. The crescents of Gianuzzi are enlarged. (Ranvier.)

$c$, Crescent cells; $g$, mucus-secreting cells; $l$, lumen of alveolus.

gland, part of the gland presents the structure of the mucous gland, while the remainder has that of the salivary glands proper.

Nerves and Blood-ressels. - Nerves of large size are found in the salivary glands; they are principally contained in the connective tissue of the alveoli, and in certain glands, especially in the dog, are provided with ganglia. Some nerves have special endings in Pacinian corpuscles, some supply the blood-vessels, and others penetrate the basement membrane of the alveoli and end upon, but not in, the salivary cells.

The blood-vessels form a dense capillary network around the ducts of the alveoli, being carried in by the fibrous trabeculæ between the alveoli, in which also begin the lymphatics by lacunar spaces.

The so-called mucous glands of the mouth and tongue present in some cases the structures of mucous, in others of serous glands. 


\section{Saliva.}

Saliva, as it commonly flows from the mouth, is the mixed secretion of the salivary glands proper and of the glands of the buccal mucous membrane and tongue; it is often mixed with air, which, being retained by its viscidity, makes it frothy. When obtained from the parotid ducts, and free from mucus, saliva is a transparent watery fluid, the specific gravity of which varies from 1004 to 1008 , and in which, when examined with the microscope, are found floating a number of minute particles, derived from the secreting ducts and vesicles of the glands. In the impure or mixed saliva are found, besides these particles, numerous epithelial scales separated from the surface of the mucous membrane of the mouth and tongue, and the so-called salivary corpuscles, discharged probably from the mucous glands of the mouth and the tonsils, which, when the saliva is collected in a deep vessel, and left at rest, subside in the form of a white opaque matter, leaving the supernatant salivary fluid transparent and colorless, or with a pale bluish-ray tint. It also contains various kinds of micro-organisms (bacteria). In reaction, the saliva, when first secreted, appears to be always alkaline: the alkalinity is about equal to .08 or .10 per cent of sodium carbonate and is due to the presence of disodium hydrogen phosphate $\mathrm{Na}_{2} \mathrm{HPO}_{4}$. During fasting, the saliva, although secreted alkaline, shortly becomes neutral; especially when it is secreted slowly and is allowed to mix with the acid mucus of the mouth, by which its alkaline reaction is neutralized.

\section{Chemical Composition of Human Saliva (Hammerbacher).}

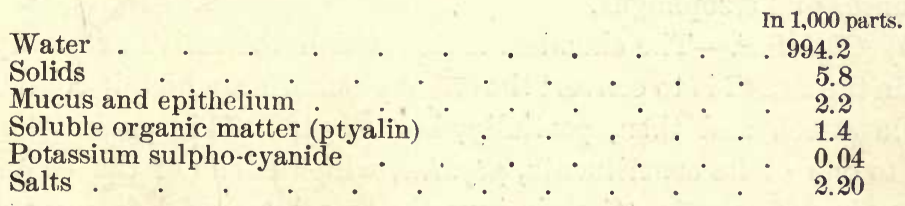

The mucin is the largest representative of the organic nitrogenous class of bodies in the saliva ; it may be thrown down by addition of acetic acid, if sodium chloride be absent. It gives the three chief proteid reactions, and may easily be split up by the action of a dilute mineral acid into globulin and a carbohydrate whose exact character has not yet been established, though it resembles a sugar in reducing copper sulphate solutions.

The presence of potassium sulphocyanide (CNKS) in saliva, may be shown by the blood-red coloration which the fluid gives with a solution of ferric chloride $\left(\mathrm{Fe}_{2} \mathrm{Cl}_{6}\right)$, and which is bleached on the addition of a solution of mercuric chloride $\left(\mathrm{HgCl}_{2}\right)$, but not by hydrochloric acid. 
Rate of Secretion and Quantity. - The rate at which saliva is secreted is subject to considerable variation. When the tongue and muscles concerned in mastication are at rest, and the nerves of the mouth are subject to no unusual stimulus, the quantity secreted is not more than sufficient, with the mucus, to keep the mouth moist. During actual secretion the flow is much accelerated.

The quantity secreted in twenty-four hours varies, but is at least 2 pints (1 litre).

Uses of Saliva. - The purposes served by saliva are (a) mechanical and (b) chemical.

(a). Mechanical.-(1) It keeps the mouth in a due condition of moisture, facilitating the movements of the tongue in speaking, and the mastication of food. (2) It serves also in dissolving sapid substances, and rendering them capable of exciting the nerves of taste. But the principal mechanical purpose of the saliva is, (3) that by mixing with the food during mastication, it makes it a soft pulpy mass, such as may be easily swallowed. To this purpose the saliva is adapted both by quantity and quality. For, speaking generally, the quantity secreted during feeding is in direct proportion to the dryness and hardness of the food. The quality of saliva is equally adapted to this end. It is easy to see how much more readily it mixes with most kinds of food than water alone does; and the saliva from the parotid, labial, and other small glands, being more aqueous than the rest, is that which is chiefly braided and mixed with the food in mastication; while the more viscid mucous secretion of the submaxillary, palatine, and tonsillitic glands is spread over the surface of the softened mass, to enable it to slide more easily through the fauces and œsophagus.

(b) Chemical.-The chemical action which the saliva exerts upon the food in the mouth is to convert the starchy materials which it contains into soluble starch and then, partially, into sugar. This power the saliva owes to one of its constituents, ptyalin, which is one of the enzymes, or unorganized ferments. Certain investigators have of late asserted that saliva contains another enzyme, known as glucase, which has the power of splitting the disaccharides into monosaccharides, or maltose into dextrose. The action of this ferment is certainly very limited. The conversion of the starch under the influence of the ferment into sugar takes place in several stages, and in order to understand it, a knowledge of the structure and composition of starch granules is necessary. A starch granule consists of two parts: an envelope of cellulose, which does not give a blue color with iodine except on addition of sulphuric acid, and of granulose, which is contained within, and which gives a blue with iodine alone. Brücke states that a third body is contained in the granule, which gives a red with iodine, viz., erythro-granulose. On boiling, the granulese swells up, bursts the envelope, and the whole granule is more or less 
completely converted into a paste or gruel, which is called gelatinous starch.

When ptyalin acts upon boiled starch, it first changes the latter (by hydrolysis) into soluble starch, or amidulin; this is more limpid and more like a true solution, though it still gives the blue coloration on the addition of iodine. This stage is very brief, only thirty seconds being sometimes required in laboratory experiments, to render a stiff starch paste completely fluid when a few drops of saliva are added at body temperature. This rapidity of action is of great importance, as under proper conditions of mastication practically all the boiled starch of the food ought to enter the stomach as soluble starch. When the starch has not been previously boiled, the envelope of cellulose retards the action of the ptyalin to a very marked degree.

The further stages of hydrolytic cleavage result in the formation of a variable mixture of maltose and iso-maltose with dextrins, but never result (in laboratory experiments) in the complete conversion of the dextrins into sugars. Gradually, as the starch is converted, the blue coloration with iodine is replaced by a purplish-red and finally by a distinctly red color: the latter color is produced by erythro-dextrin (so-called from the color), a hypothetical substance which has never been isolated. In the later stages no coloration is obtained with iodine, and for this reason the dextrins formed are known as achroö-dextrins; there are probably several of these, but they have not yet been sufficiently isolated. As sugar appears very early in the process, even at the stage of erythro-dextrin, and gradually increases in amount, it is generally concluded that maltose is formed early in the decomposition of the starch molecule: the process is usually represented schematically as follows:

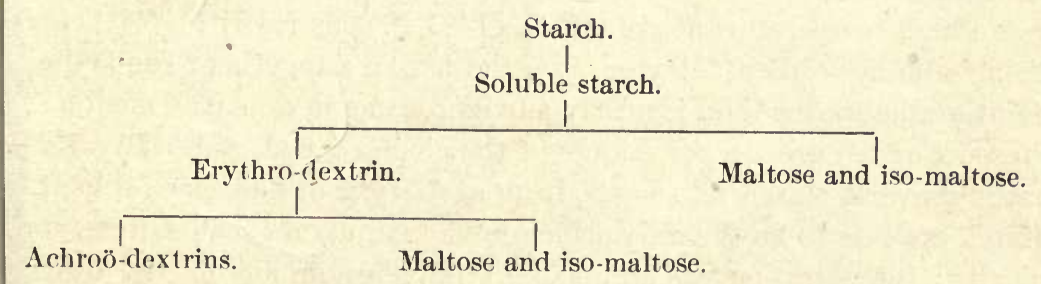

The sugars formed are maltose $\left(\mathrm{C}_{12} \mathrm{H}_{22} \mathrm{O}_{11}\right)$ and a closely allied sugar known as iso-maltose. A small percentage of dextrose has been found by some observers, and this may be due to the action of glucase. Maltose is allied to saccharose or cane-sugar more nearly than to glucose; it is crystalline; its solution has the property of polarizing light to the right to a greater degree than solutions of glucose (3 to 1 ); it is not so sweet, and reduces copper sulphate less easily. It can be converted into glucose by boiling with dilute acids. 
According to Brown and Heron the reactions may be represented thus:(J) molecule of gelatinous starch is converted by the action of an amylolytic ferment into $n$ molecules of soluble starch.

One molecule of soluble starch $=10\left(\mathrm{C}_{12} \mathrm{H}_{20} \mathrm{O}_{10}\right)+8\left(\mathrm{H}_{2} \mathrm{O}\right)$, which is further con verted by the ferment into

1. Erythro-dextrin (giving red with iodine) + Maltose.

$$
\begin{aligned}
& 9\left(\mathrm{C}_{12} \mathrm{H}_{20} \mathrm{O}_{10}\right) \quad\left(\mathrm{C}_{12} \mathrm{H}_{22} \mathrm{O}_{11}\right)
\end{aligned}
$$

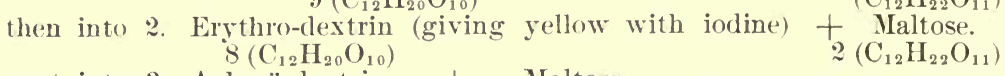

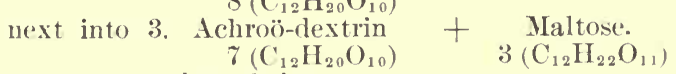

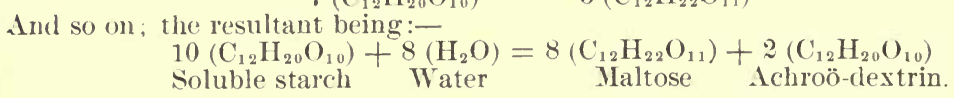

Many observers, however, deny that the maltose simultaneously present with erythro-dextrin is actually split off from the starch molecule in the formation of erythro-dextrin; they claim that it is rather the product of more advanced hydrolysis in other starch molecules, and point out that in such a chemical reaction of considerable time duration, it is improbable that all the starch molecules are attacked at the same rate or are, at any given moment, equally advanced in cleavage. Their theory is that a series of more and more simple dextrins are formed which give rise finally to the disaccharides.

Test for Sugar.-In such an experiment the presence of sugar is at once discovered by the application of Trommer's test, which consists in the addition of a drop or two of a solution of copper sulphate, followed by a larger quantity of caustic potash. When the liquid is boiled, an orange-red precipitate of copper suboxide indicates the presence of sugar.

The action of saliva on starch is facilitated by: (a) Moderate heat, about $37.8^{\circ} \mathrm{C}$. $\left(100^{\circ} \mathrm{F}\right.$.). (b) A neutral medium. (c) Removal of the changed material from time to time. Its action is returded $b_{y}$ : (a) Cold; a temperature of $0^{\circ} \mathrm{C}$. $\left(32^{\circ} \mathrm{F}\right.$. $)$ stops it for a time, but does not destroy it, whereas a high temperature above $60^{\circ} \mathrm{C} .\left(140^{\circ} \mathrm{F}\right.$.) destroys it. (b) Acids or strong alkalies either delay or stop the action altogether; the action in a faintly alkaline medium is nearly as vigorous as in a neutral mediun. (c) Presence of too great a percentage of the changed material. Ptyalin. in that it converts starch into sugar, is an amylolytic or diastasic ferment.

Starch appears to be the only principle of food upon which saliva acts chemically: the secretion has no apparent influence on any of the other ternary principles, such as sugar, gum, cellulose, or on fat, and seems to be equally destitute of power over albuminous and gelatinous substances.

Saliva from the parotid is less viscid; less alkaline, the first few drops discharged in secretion being even acid in reaction; clearer, although it may become cloudy on standing from the precipitation of calcium carbonate from escape of carbon dioxide; and more watery than that from the submaxillary. It has moreover a less powerful action on starch. Sublingual saliva is the most viscid, and contains more solids than either of the other two, but has little diastasic action. 
The salivary glands of children do not become functionally active till the age of 4 to 6 months, and hence the bad effect of feeding them before this age on starchy food, corn-flour, etc., which they are unable to render soluble and capable of absorption. The salivas of the dog, cat, bear, and pig are almost inactive, whereas that of monkeys, rabbits, mice, squirrels, and guinea-pigs, are strongly diastasic.

Salivary Digestion in the Stomach.-Under proper conditions salivary digestion may continue for some time after the foód has entered the stomach. In laboratory experiments it is found that while the addition of even .05 per cent. of hydrochloric acid will inhibit the action of ptyalin on a solution of starch, if any proteids be present in the solution, much more acid must be added before the action of the ptyalin is stopped. The explanation of the latter fact is that the acid unites with the proteids in some loose chemical combination, forming "combined acid" which has little effect, comparatively, on ptyalin. This "combined acid" gives a red color with litmus, but is distinguished from free acid by giving a brownish instead of a bluish color with Congo red.

When food enters an empty stomach, as happens at the beginning of a meal, the acid first secreted combines with the proteid food-stuffs and so does not affect the ptyalin. It usually requires at least 15 to $20 \mathrm{~min}$ utes before the acid is secreted in sufficient quantity to be in excess, as free acid, of the amount which ean combine with the proteids, and during this time salivary digestion may continue. Of course the action of ptyalin on food taken later in a meal is promptly stopped when it reaches the stomach because of the presence of free acid.

\section{The Nervous Mechanism of the Secretion of Saliva.}

The secretion of saliva is under the control of the nervous system. It is a reflex action. Under ordinary conditions it is excited by the stimulation of the peripheral branches of two nerves, viz., the gustatory or lingual branch of the inferior maxillary division of the fifth nerve, and the glosso-pharyngeal part of the eighth pair of nerves, which are distributed to the mucous membrane of the tongue and pharynx conjointly. The stimulation occurs on the introduction of sapid substances into the mouth, and the secretion is brought about in the following way: From the terminations of the above-mentioned sensory nerves distributed in the mucous membrane an impression is conveyed upward (afferent) to the special nerve centre situated in the medulla-oblongata which controls the process, and by it is reflected to certain nerves supplied to the salivary glands, which will be presently indicated. In other words, the centre, stimulated to action by the sensory impressions carried to it, sends out impulses along efferent or secretory nerves supplied to the salivary glands, which cause the saliva to be secreted by and dis- 
charged from the gland cells. Other stimuli, however, besides that of the food, and other sensory nerves besides those mentioned, may produce reflexly the same effects. For example, saliva may be caused to flow by irritation of the mucous membrane of the mouth with mechanical, chemical, electrical, or thermal stimuli, also by the irritation of the mucous membrane of the stomach in some way, as in nausea, which precedes vomiting, when some of the peripheral fibres of the vagi are irritated. Stimulation of the olfuctory nerves by smell of food, of the optic nerves by the sight of it, and of the anditory nerves by the sounds which are known by experience to accompany the preparation of a meal, may also, in the hungry, stimulate the nerve centre to action. In addition to these, as a secretion of saliva follows the movement of the muscles of mastication, it may be assumed that this movement stimulates the secreting nerve fibres of the gland, direct or reflexly. From the fact that the flow of saliva may be increased or diminished by mental emotions, it is evident that impressions from the cerebrum also are capable of stimulating the centre to action or of inhibiting its action.

Salivary secretion may also be exeited by direct stimulation of the centre in the medulla.

On the Submaxillary Giland.- The submaxillary gland has been the gland chiefly employed for the purpose of experimentally demonstrating the influence of the nerrous system upon the secretion of saliva, because of the comparative ficility with which, with its blood-vessels and nerves, it may be exposed to view in the dog, rabbit, and other animals. The chief nerves supplied to the gland are (1) the chorda tympani, a branch given off from the facial (or portio dura of the seventh pair of nerves), in the canal through which it passes in the temporal bone, in its passage from the interior of the skull to the face; and (2) branches of the sympathetic nerve from the plexus around the facial artery and its branches to the glind. The chorda (fig. 238,ch.t.), after quitting the temporal bone, passes downward and forward, under corer of the external pterygoid muscle, and joins at an acute angle the lingual or gustatory nerve, proceeds with it for a short distance, and then passes along the submaxillary gland duct (fig. 238, sm. d.), to which it is distributed, giving branches to the submaxillary ganglion (fig. $238, \mathrm{sm} . \mathrm{gl}$.), and sending others to terminate in the superficial muscles of the tongue. It consists of fine medullated fibres which lose their medulla in the gland. If this nerve be exposed and divided anywhere in its course from its exit from the skull to the gland, no immediate result will follow, nor will stimulation either of the lingual or of the glosso-pharyngeal produce a flow of saliva. But if the peripheral end of the divided nerve be stimulated, an abundant secretion of saliva ensues, and the blood supply is enormously increased, the arteries being dilated. The veins even pulsate, and the blood contained within them is more arterial than venous in character. 
When, on the other hand, the stimulus is applied to the sympathetic filaments (mere division producing no apparent effect), the arteries contract, and the blood stream is in consequence much diminished; and from the veins, when opened, there escapes only a sluggish stream of dark blood. The saliva, instead of being abundant and watery, becomes scanty and tenacious. If both chorda tympani and sympathetic branches be divided, the gland, released from nervous control, may secreté continuously and abundantly ( paralytic secretion).

The abundant secretion of saliva, which follows stimulation of the chorda tympani, is not merely the result of a filtration of fluid from

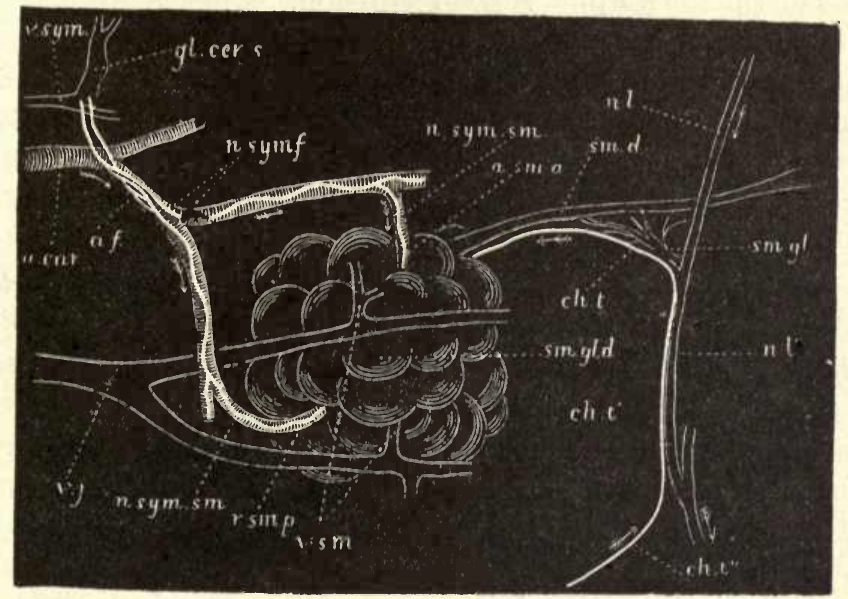

Fig. 238.-Diagrammatic representation of the sub-maxillary gland of the dog with its nerves and blood-vessels. (This is not intended to iliustrate the exact anatomical relations of the several structures.) sm. gld., the sub-maxillary gland into the duct $(\mathrm{sm}$. $d$.) of which a caunla has been tied. The sublingual gland and duct are not shown. n. $l_{\text {., } n . ~} l^{\prime}$., the lingual or gustatory nerve ; ch. $t$., ch. $t^{\prime}$., the chorda tympani proceeding from the facial nerve, becoming conjolned with the lingual at $n$. $l^{\prime}$. , and afterward diverging and passing to the gland along the duct; sm. gl., sub-maxillary ganglion with its roots; $n, l$., the lingual nerve proceeding to the tongue; $a$. car., the carotid artery, two branches of which, $a .8 m$. $a$. and $r .8 \mathrm{~m}$. p.,pass to the anterior and posterior parts of the gland; $v$. $s m$., the anterlor and posterior veins from the gland ending in $v . j .$, the jugular vein ; $v .8 y m$., the conjoined vagus and sympathetic trunks; gl. cer. s., the superior-cervical ganglion, two branches of which forming a plexus, $a$. $f$., over the facial artery are distributed $(n .8 y m .8 m$.) along the two glandular arteries to the anterior and posterior portion of the gland. The arrows indicate the direction taken by the nervous impulses; during reflex stimulations of the gland they ascend to the brain by the lingual and descend by the chorda tympani. (M. Foster.)

the blood-vessels, in consequence of the largely increased circulation through them. This is proved by the fact that, when the main duct is obstructed, the pressure within may considerably exceed the blood-pressure in the arteries, and also that when into the veins of the animal experimented upon some atropin has been previously injected, stimulation of the peripheral end of the divided chorda produces all the vascular effects as before, without any secretion of saliva accompanying them. Again, if an animal's head be cut off, and the chorda be rapidly exposed and stimulated with an interrupted current, a secretion of saliva ensues for a short time, although the blood supply is necessarily absent. These 
experiments serve to prove that the chorda contains two sets of nerve fibres, one set (vaso-dilator) which, when stimulated, act upon a local vaso-motor centre for regulating the blood supply, inhibiting its action, and causing the vessels to dilate, and so producing an increased supply of blood to the gland; while another set, which are paralyzed by injection of atropin, directly stimulate the cells themselves to activity, whereby they secrete and discharge the constituents of the saliva which they produce. These latter fibres very possibly terminate on the salivary cells themselves. If, on the other hand, the sympathetic fibres be divided, stimulation of the tongue by sapid substances, or of the trunk of the lingual, or of the glosso-pharyngeal, continues to produce a flow of saliva. From these experiments it is evident that the chorda tympani nerve is the principal nerve through which efferent impulses proceed from the centre to excite the secretion of this gland.

The sympathetic nerve also contains two sets of fibres, vaso-constrictor and secretory. But the flow of saliva, upon stimulating the sympathetic, is scanty, and the saliva itself viscid. At the same time the vessels of the gland are constricted. The secretory fibres may be paralyzed by the administration of atropine.

On the Parotid Gland.- The nerves which influence secretion in the parotid gland are branches of the facial (lesser superficial petrosal) and of the sympathetic. The former nerve, after passing through the otic ganglion, joins the auriculo-temporal branch of the fifth cerebral nerve, and, with it, is distributed to the gland. The nerves by which the stimulus ordinarily exciting secretion is conveyed to the medulla oblongata, are, as in the case of the submaxillary gland, the fifth, and the glosso-pharyngeal. The pneumogastric nerves convey a further stimulus to the secretion of saliva, when food has entered the stomach; the nerve centre is the same as in the case of the submaxillary gland.

Changes in the Gland Cells. - The method by which the salivary cells produce the secretion of saliva appears to be divided into two stages, which differ somewhat according to the class to which the gland belongs, viz., whether to (1) the true salivary, or (2) to the mucous type. In the former case, it has been noticed, as has been already described, that during the rest which follows an active secretion the lumen of the alveolus becomes smaller, the gland cells larger and very granular. During secretion the alveoli and their cells become smaller, and the granular appearance in the latter to a considerable extent disappears, and at the end of secretion the granules are confined to the inner part of the cell nearest to the lumen, which is now quite distinct (fig. 239).

It is supposed from these appearances that the first stage in the act of secretion consists in the protoplasm of the salivary cell taking up from the lymph certain materials from which it manufactures the ele- 
ments of its own secretion, and which are stored up in the form of granules in the cell during rest, the second stage consisting of the actual discharge of these granules, with or without previous change. The granules are zymogen granules, and represent the chief substance of the salivary secretion, i.e., ptyalin. In the case of the submaxillary gland of the dog, at any rate, the sympathetic nerve-fibres appear to have to do with the first stage of the process, and when stimulated the protoplasm is extremely active in manufacturing the granules, whereas the chorda tympani is concerned in the production of the second act, the actual discharge of the materials of secretion, together with a considerable amount of fluid, the latter being an actual secretion by the protoplasm, as it ceases to occur when atropin has been subcutaneously injected.

In the mucus-secreting gland, the changes in the cells during secretion have been already spoken of. They consist in the gradual secre-

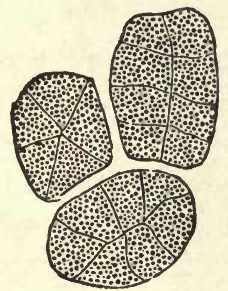

A

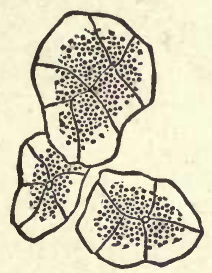

B

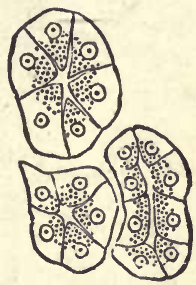

C

Fig. 239. -Alveoli of true salivary gland. A, at rest; B, in the first stage of secretion ; C, after prolonged secretion. (Langley.)

tion by the protoplasm of the cell of a substance called mucigen, which is converted into mucin, and discharged on secretion into the canal of the alveoli. The mucigen is, for the most part, collected into the inner part of the cells during rest, pressing the nucleus and the small portion of the protoplasm which remains, against the limiting membrane of the alveoli.

The process of secretion in the salivary glands is identical with that of glands in general; the cells which line the ultimate branches of the ducts being the agents by which the special constituents of the saliva are formed. The materials which they have incorporated with themselves are almost at once given up again, in the form of a fluid (secretion), which escapes from the ducts of the gland; and the cells, themselves, undergo disintegration - again to be renewed, in the intervals of the active exercise of the functions. The source whence the cells obtain the materials of their secretion is the blood, or, to speak more accurately, the plasma, which is filtered off from the circulating blood into the interstices of the glands as of all living textures. 
The TONgEE.

Structure.-The tongue is a muscular organ covered by mucous membrane. The muscles, which form the greater part of the substance of the tongue (intrinsic muscles) are termed linguales; and by these,

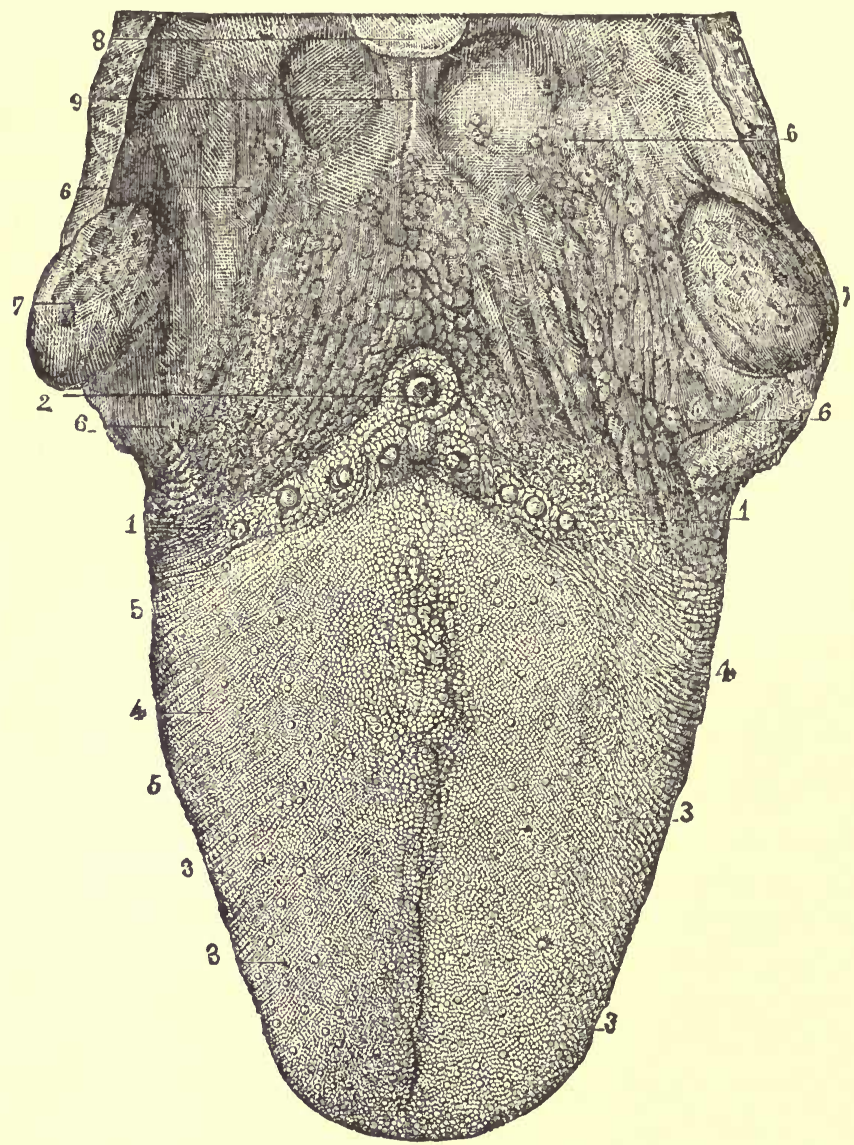

Fig. 240.-Papillar surface of the tongue, with the fauces and tonsils. 1, 1, circumvallate pa pillæ, in front of 2 , the foramen cæcum; 3 , fungiform papillæ ; 4 , filiform and conical papillæ ; 5 , transverse and oblique rugæ ; 6 , mucous glands at the base of the tongue and in the fauces; 7 . tonsils: 8, part of the epiglottis ; 9, median glosso-epiglottidean fold (frænum epiglottidis). (From Sappey.)

which are attached to the mucous membrane chiefly, its smaller and more delicate movements are chiefly performed.

By other muscles (extrinsic muscles), as the genio-hyoglossus, the styloglossus, etc., the tongue is fixed to surrounding parts, and by this group of muscles its larger movements are performed.

The mucous membrane of the tongue resembles other mucous mem- 
branes in essential points of structure, but contains papillae, more or less peculiar to itself; peculiar, howerer, in details of structure and arrangement, not in their nature. The tongue is beset with numerous mucous follicles and glands.

The larger papilla of the tongue are thickly set over the anterior two-thirds of its upper surface, or dorsum (fig. 240), and give to it its characteristic roughness. In carnivorous animals, especially those of the cat tribe, the papillæ attain a large size, and are developed into

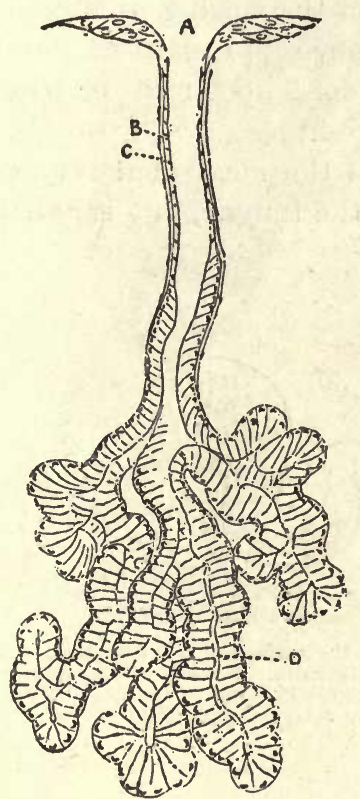

Fig. 241. sharp recurved horny spines. Such papillæ cannot be regarded as sensitive, but they enable the tongue to play the part of a most efficient rasp, as in scraping bones, or of a comb in cleaning fur. Their greater prominence than those of the skin is due to their interspaces not being filled up with epithelium, as the interspaces of the papillæ of the skin are. The papillæ of the tongue present several diversities of form; but

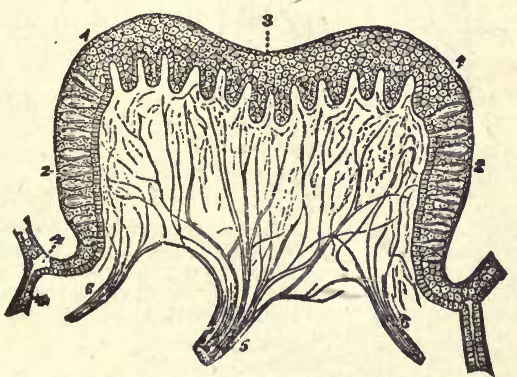

Fig. 242.

Fig. 241.-Section of a mucous gland from the tongue. A, opening of the duct on the free surface; $\mathrm{C}$, basement membrane with nuclei; $\mathrm{B}$, flattened epithelial cells lining duct. The duct divides into several branches, which are convoluted and end blindly, being lined throughout by columnar epithelium. D, lumen of one of the tubuli of the gland. $\times 90$. (Klein and Noble Smith.)

Fig. 242.-Vertical section of a circumvallate papilla of the calf. 1 and 3, epithelial layers covering it ; 2 , taste goblets ; 4 and $4^{\prime}$, duct of serous gland opening out into the pit in which papilla is situated; 5 and 6 , nerves ramifying within the papilla. (Engelmann.)

three principal varieties, differing both in seat and general characters, may usually be distinguished, namely, the (1) circumvallate, the (2) fungiform, and the (3) filiform papillæ. Essentially these have all of them the same structure, that is to say, they are all formed by a projection of the mucous membrane, and contain special branches of bloodvessels and nerves. In details of structure, however, they differ considerably one from another.

The surface of each kind is studded by minute conical processes of mucous membrane, which thus form secondary papillæ.

(1.) Circumvallate.-These papillæ (fig. 242), eight or ten in num- 
ber, are situate in two $\mathrm{V}$-shaped lines at the base of the tongue $(1,1$, fig. 240). They are circular elevations from $\frac{1}{2} \frac{1}{2}$ th to $\frac{1}{12}$ th of an inch wide, ( 1 to $2 \mathrm{~mm}$.), each with a central depression, and surrounded by a circular fissure, at the outside of which again is a slightly elevated ring, both the central elevation and the ring being formed of close-set simple papillæ.

(2.) Fungiform. - The fungiform papillæ (3, fig. 240) are scattered chiefly over the sides and tip, and sparingly over the middle of the dorsum, of the tongue; their name is derived from their being usually narrower at their base than at their summit. They also consist of groups of simple papillæ (A. fig. 243), each of which contains in its interior a loop of capillary blood-vessels (B.), and a nerve-fibre.

(3.) Conical or Filiform.-These, which are the most abundant papillæ, are scattered over the whole surface of the tongue, but especially
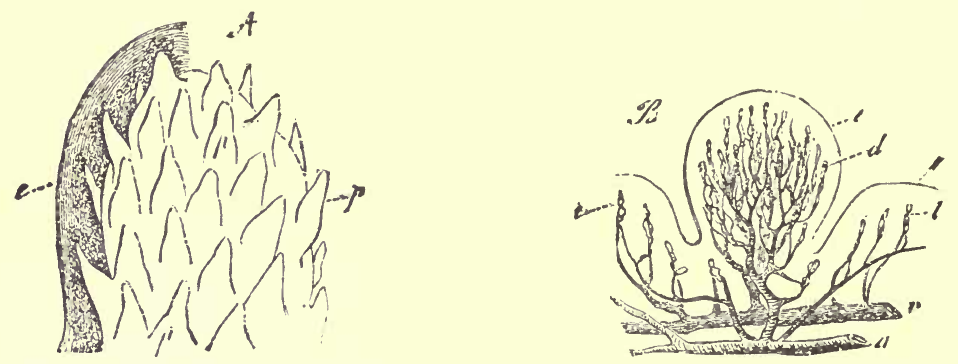

Fig. 243.- Surface and section of the fungiform papillæ. A, the surface of a fungiform papilla, partially denuded of its epithelium: $p$, secondary papillæ; $e$, epithelium. $\mathrm{B}$, section of a fungiform papila with the blood-vessels injected ; $a$, artery $: v$, vein; $c$, capillary loops of similar papillæ in the neighboring structure of the tongue; $d$, capillary loops of the secondary papillæ; $e$, epithelium. (From Kölliker, after Todd and Bowman.)

over the middle of the dorsum. They vary in shape somewhat, but for the most part are conical or filiform, and corered by a thick layer of epidermis, which is arranged over them, either in an imbricated manner, $01^{\circ}$ is prolonged from their surface in the form of fine stiff projections, hair-like in appearance, and in some instances in structure also (fig. 244). From their peculiar structure, it seems likely that these papillæ have a mechanical function, or one allied to that of touch rather than of taste; the latter sense being probably seated especially in the other two varieties of papillæ, the circumvallate and the fungiform.

The epithelium of the tongue is stratified with the upper layers of the squamous kind. It covers every part of the surface; but over the fungiform papillæ forms a thinner layer than elsewhere. 'The epithelium covering the filiform papillæ is extremely dense and thick, and, as before mentioned, projects from their sides and summits in the form of long, stiff, hair-like processes (fig. 244). Many of these processes bear a close resemblance to hairs. Blood-vessels and nerves are supplied freely to 
the papillæ. The nerves in the fungiform and circumvallate papillæ form a kind of plexus, spreading out brushwise (fig. 244), but the exact mode of termination of the nerve-filaments is not certainly known.

In the circumvallate papillæ of the tongue of man peculiar structures known as gustatory buds or taste goblets, have been discovered. They are of an oval shape, and consist of a number of closely packed,

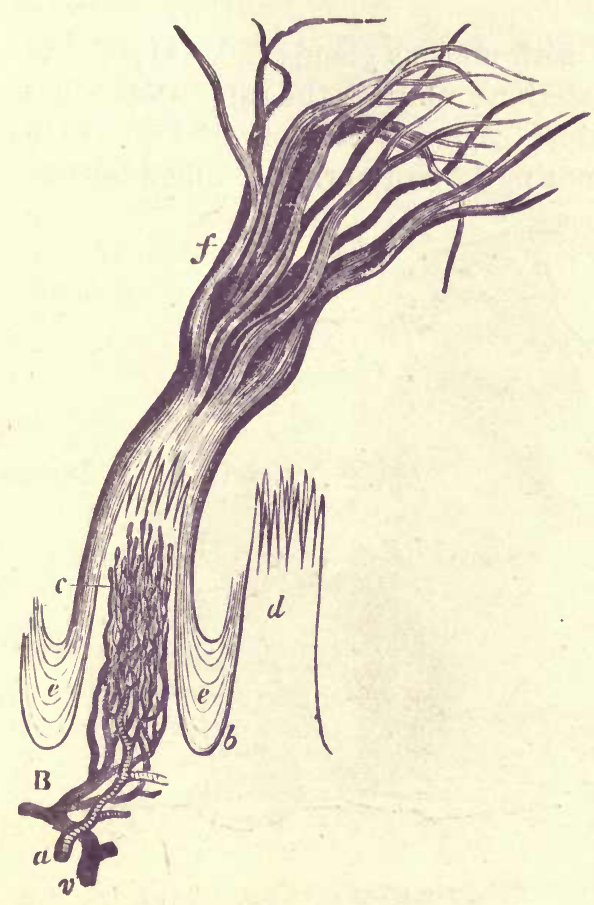

Fig. 244. very narrow and fusiform, cells (gustatory cells). This central core of gustatory cells is inclosed in a single layer of broader fusiform cells (incasing cells). The gustatory cells terminate in fine spikes not unlike cilia, which project on the free surface (fig. $245 a$ ).

These bodies also occur side by side in considerable num-

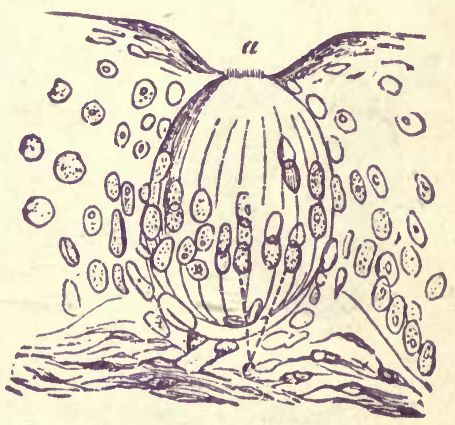

Fig. 245.

Fig. 244. - Two filiform papillæ, one with epithelium, the other without. $\frac{3.5}{1}$. $-d$, the substance of the papillæ dividing at their upper extremities into secondary papillæ; $a$, artery, and $v$, vein dividing into capillary loops; $e$, epithelial covering, laminated between the papillæ, but extended into hair-like processes, $f$, from the extremities of the secondary papillæ. (From Kölliker, after Todd and Bowman.)

Fig. 245. - Taste-goblet from dog's epiglottis (laryngeal surface near the base), precisely similar in structure to those found in the tongue. $a$, depression in epithelium over goblet; below the letter are seen the fine hair-like processes in which the cells terminate; $c$, two nuclei of the axial (gustatory) cells. The more superficial nuclei belong to the superficial (incasing) cells ; the converging lines indicate the fusiform shape of the incasing cells. $\times 400$. (Schofield.)

bers in the epithelium of the papilla foliata, which is situated near the root of the tongue in the rabbit, and also in man. Similar taste-goblets have been observed on the posterior (laryngeal) surface of the epiglottis.

\section{The Pharynx.}

The portion of the alimentary canal which intervenes between the mouth and the esophagus is termed the Pharynx. It will suffice here to mention that it is constructed of a series of three muscles with stri- 
ated fibres (constrictors), which are covered by a thin fascia externally, and are lined internally by a strong fascia (pharyngeal aponeurosis), on the inner aspect of which is areolar (submucous) tissue and mucous membrane, continuous with that of the mouth, and, as regards the part concerned in swallowing, is identical with it in general structure. The epithelium of this part of the pharynx, like that of the mouth, is stratified and squamous.

The pharynx is well supplied with mucous glands (fig. 241).

Between the anterior and posterior arches of the soft palate are situated the Tonsils, one on each side. A tonsil consists of an elevation of the mucous membrane representing 12 to 15 orifices, which lead into
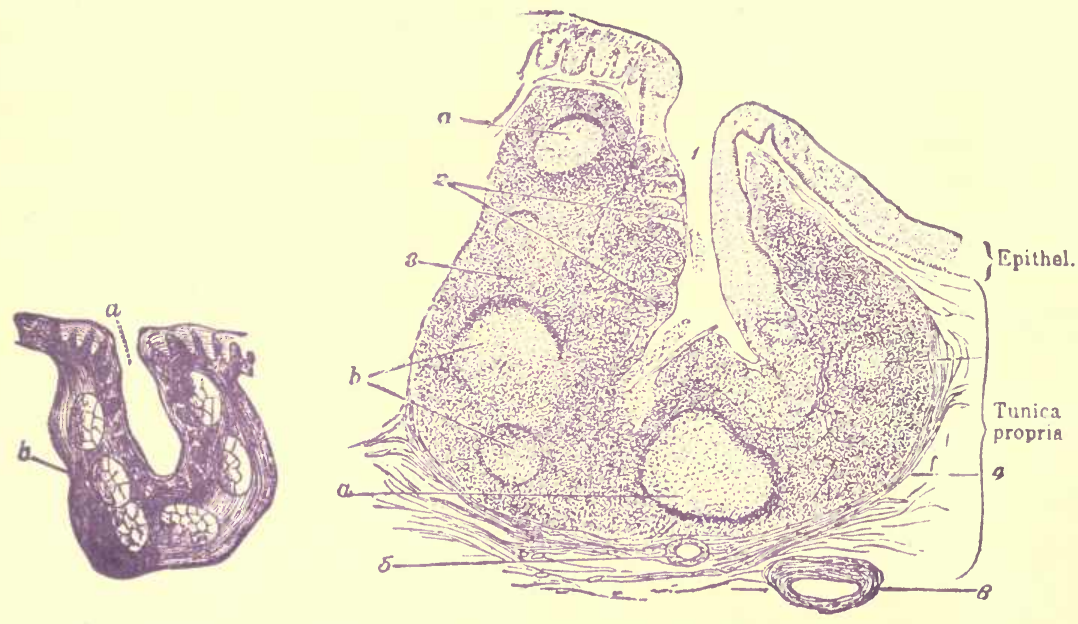

Fig. 246.

Fig. $24 \%$.

Fig. 246,-Lingual follicle or crypt. $a$, involution of mucous membrane with its papillæ; $b$, lymphoid tissues, with several lymphoid sacs. (Frey.)

Fig. 247. - Vertical section through a crypt of the human tonsil. 1, entrance to the crypt; 2 and 3 , the framework or adenoid tissue; 4 , the inclosing fibrous tissue; $a$ and $b$, lymphatic follicles; 5 and 6, blood-vessels. (Stöhr.)

crypts or recesses, in the walls of which are placed nodules of adenoid or lymphoid tissue (fig. 247). These noủules are enveloped in a less dense adenoid tissue which reaches the mucous surface. The surface is covered with stratified squamous epithelium, and the subepithelial or mucous membrane proper may present rudimentary papillæ formed of adenoid tissue. The tonsil is bounded by a fibrous capsule (fig. $24 \%, 4$ ). Into the crypts open the ducts of numerous mucous glands.

The viscid secretion which exudes from the tonsils serves to lubricate the bolus of food as it passes them in the second part of the act of deglutition. 


\section{The Esophagus or Gullet.}

The Esophagus or Gullet, the narrowest portion of the alimentary canal, is a muscular and mucous tube, nine or ten inches in length, which extends from the lower end of the pharynx to the cardiac orifice of the stomach.

Structure.-The esophagus is made up of three coats-viz., the outer, muscular; the middle, submucous; and the inner, mucous. The muscular coat is covered externally by a varying amount of loose fibrous

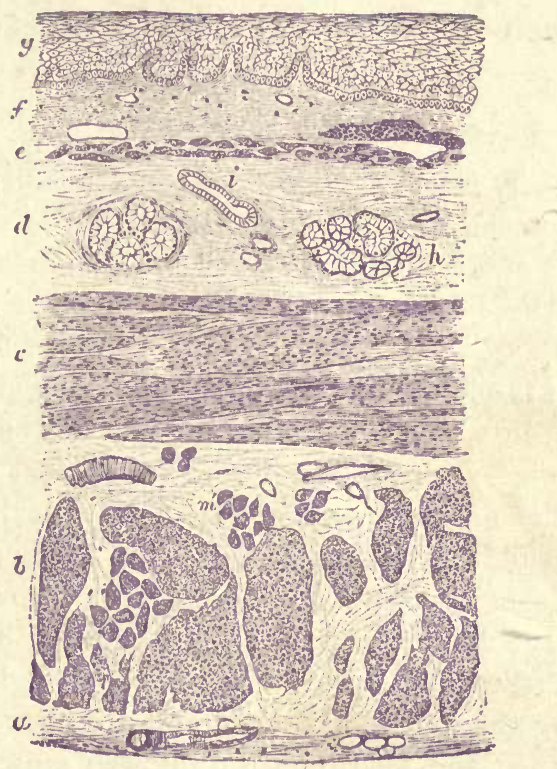

Fig. 248. - Transverse section of the human csophagus. $a$, Fibrous covering; $b$, longitudinal mutcular fibres; $c$, transverse muscular fibres; $d$, areolor or submucous coat; $e$, muscularis mucosæ; $f$, nucous membrane, with part of a lymphoid nodule; $g$, stratifled epithelial lining; $h$, mucous gland; $i$, gland duct; $m^{\prime}$, striated muscle fibres. (V. Horsley.)

tissue. It is composed of two layers of fibres, the outer being arranged longitudinally, and the inmer circularly. At the upper part of the cesophagus this coat is made up principally of striated muscle fibres, as they are continuous with the constrictor muscles of the pharynx; but lower down the unstriated fibres become more and more numerous, and toward the end of the tube form the entire coat. The muscular coat is connected with the mucous coat by a more or less developed layer of areolar tissue, which forms the submucous coat (fig. $248, f$ ), in which is contained in the lower half or third of the tube many mucous glands, the ducts of which, passing through the mucous membrane, open on its surface. Separating this coat from the mucous membrane proper is a well- 
developed layer of longitudinal, unstriated muscle, called the muscularis mucosa. The mucous membrane is composed of a closely felted meshwork of fine connective tissue, which, toward the surface, is elevated into rudimentary papillæ. It is covered with a stratified epithelium, of which the most superficial layers are squamous. The epithelium is arranged upon a basement membrane.

In newly-born children the mucous membrane exhibits, in many parts, the structure of lymphoid tissue (Klein).

Blood-and lymph-vessels, and nerves, are distributed in the walls of the oesophagus. Between the outer and inner layers of the muscular coat, nerve-ganglia of Auerbach are also found (fig. 254).

\section{Deglutition.}

When properly masticated, the food is transmitted in successive portions to the stomach by the act of deglutition or swallowing. The following account of deglutition is based upon the researches of Kronecker and Meltzer, whose experiments seem to disprove the earlier theory of Magendie:

The mouth is closed, and the food is rolled after thorough mixing with the saliva into a bolus on the dorsum of the tongue. The tip of the tongue is pressed upward and forward against the hard palate, thus shutting off the anterior part of the mouth cavity. The mylo-hyoid muscles then suddenly contract, the bolus of food is put under great pressure, and shot backward and downward through the pharynx and œsophagus to the cardiac orifice of the stomach. Coincidently with the contraction of the mylo-liyoid muscles, the hyoglossi are thrown into action, drawing the tongue backward and downward, not only increasing the pressure upon the food, but forcing the epiglottis over the glottis and thus closing the larynx. The interval of time between the commencement of the act of deglutition and the arrival of the food at the cardiac orifice of the stomach is not more than 0.1 second. Usually the food remains at the cardiac orifice without entering the stomach until the first parc of the act of swallowing is reinforced by the subsequent contraction of the constrictors of the pharynx and the passage of a peristaltic wave down the osophagus. This wave, reaching the cardiac orifice about 6 seconds after the commencement of the act of deglutition, forces the food into the stomach, the sphincter having previously relaxed. In some cases, however, the food is not stopped at the cardiac orifice, but is sent through the relaxed sphincter by the original force of the mylo-hyoid contraction.

In man the cesophagus coutracts in three separate segments-the first segment lying in the neck and being abont 6 centimetres long, the 
second being the next 10 centimetres of the tube, and the third the remaining portion to the stomach.

The act of swallowing consists, then, of the contraction in sequence of five muscle-segments: the mylo-hyoids, the constrictors of the pharynx, and the three segments of the osophagus. The computed time of contraction is as follows:

Contraction of mylo-hyoids and constrictors of the pharynx Contraction of the first part of the œsophagus .

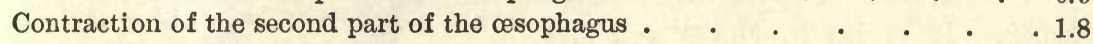

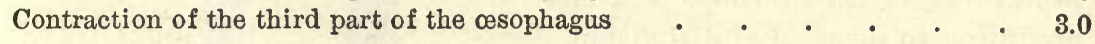

If a second attempt at swallowing be made before the first has been completed (that is, before 6 seconds have elapsed), the remaining portion of the first act is inhibited, and the contraction wave reaches the stomach 6 seconds after the commencement of the second act.

In addition to the above, the following facts must be noted:

During the act of deglutition the posterior nares are closed through the action of the levator palati and tensor palati muscles, which raise the velum; the palato-pharyngei, drawing the posterior pillars of the fauces together; and the azygos uvulæ, which raises the uvula-thus forming a complete curtain. Otherwise the food would pass into the nose, as happens in the case of cleft palate. At the same time the larynx is closed by the adductor muscles of the vocal cords and the descent of the epiglottis, the larynx being drawn upward as a whole through the action of the mylo-hyoid, gonio-hyoid, thyro-hyoid, and digastric muscles. The presence of the epiglottis is not necessary for the completion of the act of deglutition.

Nervous Mechanism. - The nerves engaged in the reflex act of deglutition are:-sensory, branches of the fifth cerebral supplying the soft palate; glosso-pharyngeal, supplying the tongue and pharynx; the superior laryngeal branch of the vagus, supplying the epiglottis and the glottis; while the motor fibres concerned are:-branches of the fifth, supplying part of the digastric and mylo-hyoid muscles, and the muscles of mastication; the facial, supplying the levator palati; the glosso-pharyngeal, supplying the muscles of the pharynx; the vagus, supplying the muscles of the larynx through the inferior laryngeal branch, and the hypoglossal, the muscles of the tongue. The nerve-centre by which the muscles are harmonized in their action, is situate in the medulla oblongata. In the movements of the œsophagus, the ganglia contained in its walls, with the pneumo-gastrics, are the nerve-structures chiefly concerned.

It is important to note that the swallowing both of food and drink is a muscular act, and can, therefore, take place in opposition to the force of gravity. Thus, horses and many other animals habitually drink uphill, and the same feat can be performed by jugglers. 


\section{The Stomach.}

In man and those Mammalia which are provided with a single stomach, it consists of a dilatation of the alimentary canal placed between and continuous with the osophagus, which enters its larger or cardiac end on the one hand, and the small intestine, which commences at its narrowed end or pylorus, on the other. It varies in shape and size according to its state of distention.

Structure.-The stomach is composed of four coats, called respectively-(1) an external or peritoneal, (2) muscular, (3) submucous, and (4) mucous coat; with blood-vessels, lymphatics, and nerves distributed in and between them.

(1) The peritoneal coat has the structure of serous membranes in general, as has been described.

The muscular coat consists of three separate layers or sets of fibre, which, according to their several directions, are named the longitudinal, circular, and oblique. The longitudinal set are the most superficial: they are continuous with the longitudinal fibres of the cesophagus and spread out in a diverging manner over the cardiac end and sides of the stomach. They extend as far as the pylorus, being especially distinct at the lesser or upper curvature of the stomach, along which they pass in several strong bands. The next set are the circular or transverse fibres, which more or less completely encircle all parts of the stomach; they

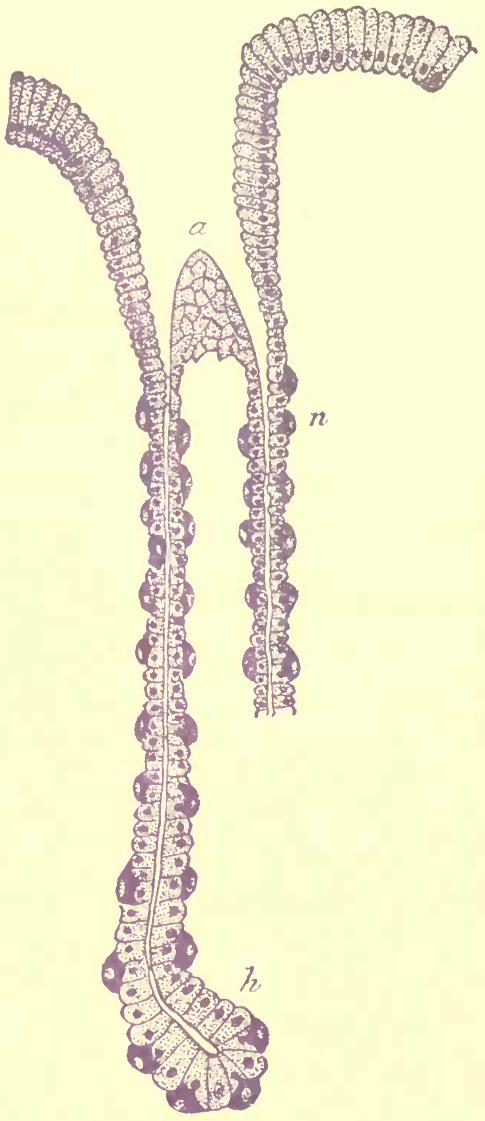

Fig. 249.-From a vertical section through the mucous membrane of the cardiac end of stomach. Two peptic glands are shown with a duct common to both, one gland only in part. $a$, duct with columnar epithelium becoming shorter as the cells are traced downward; $n$, neck of gland tubes, with central and parietal or so-called peptic cells; $b$, fundus with curved cæcal extremity - the parietal cells are not so numerous here. $\times 400$. (Klein and Noble Smith.) are most abundant at the middle and in the pyloric portion of the organ, and form the chief part of the thick projecting ring of the pylorus. These fibres are not simple circles, but form double or figure-of-8 loops, the fibres intersecting very obliquely. The next, and consequently 
deepest set of fibres, are the oblique, continuous with the circular muscular fibres of the cesophagus, and having the same double-looped arrangement that prevails in the preceding layer: they are comparatively few in number, and are placed only at the cardiac orifice and portion of the stomach, over both surfaces of which they are spread, some passing obliquely from left to right, others from right to left, around the cardiac orifice, to which, by their interlacing, they form a kind of sphincter, continuous with that around the lower end of the osophagus. The muscular fibres of the stomach and of the intestinal canal are unstriated, being composed of elongated, spindle-shaped fibre-cells.

(3) and (4) The mucous membrane of the stomach, which rests upon a layer of loose cellular membrane, or submucous tissue, is smooth, level, soft, and velvety; of a pale pink color during life, and in the contracted state thrown into numerous, chiefly longitudinal, folds or rugæ, which disappear when the organ is distended.

The basis of the mucous membrane is a fine connective tissue, which

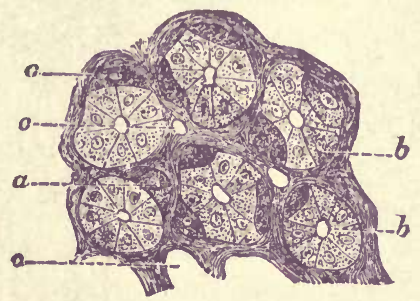

Fig. 250.-Transverse section through lower part of peptic glands of a cat. $a$, pe ptic cells; $b$, small spheroidal or cubical cells; $c$, transverse section of capillaries. (Frey.)

approaches closely in structure to adenoid tissue; this tissue supports the tubular glands of which the superficial and chief part of the mucous membrane is composed, and passing up between them assists in binding them together. Here and there are to be found in this coat, immediately underneath the glands, masses of adenoid tissue sufficiently marked to be termed by some lymphoid follicles. The glands are separated from the rest of the mucous membrane by a very fine homogeneous basement membrane.

At the deepest part of the mucous membrane are two layers (circular and longitudinal) of unstriped muscular fibres, called the muscularis mucose, which separate the mucous membrane from the scanty submucous tissue.

When examined with a lens, the internal or free surface of the stomach presents a peculiar honeycomb appearance, produced by shallow polygonal depressions, the diameter of which varies generally from $\frac{1}{200}$ th to $\frac{1}{3 \frac{1}{0}}$ th of an inch (about $125 \mu$ ); but near the pylorus is as much as $\frac{1}{100}$ th of an inch $(250 \mu)$. They are separated by slightly elevated ridges, which sometimes, especially in certain morbid states of the stomach, bear minute, narrow vascular processes, which look like villi, and 
have given rise to the erroneous supposition that the stomach has absorbing villi, like those of the small intestines. In the bottom of these little pits, and to some extent between them, minute openings are visible, which are the orifices of the ducts of perpendicularly arranged tubular glands (fig. 249), imbedded side by side in sets or bundles, on the surface of the mucous membrane, and composing nearly the whole structure.

The glands of the mucous membrane are of two varieties, $(a)$ Cardiac, (b) Pyloric.

(a) Cardiac glands are found throughout the whole of the cardiac

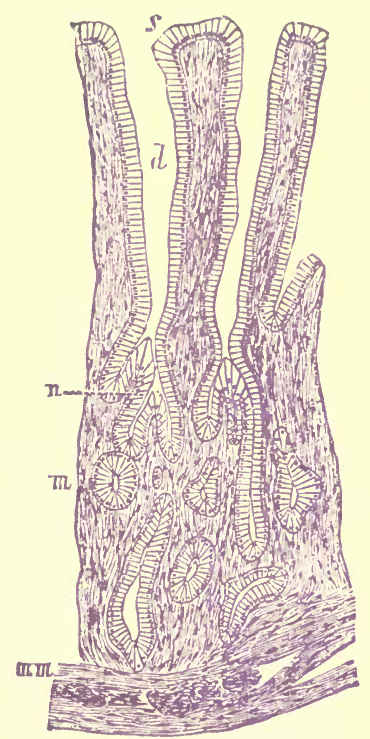

Fig.251. end of the stomach. They are arranged in groups of four or five, which are separated by a fine connective tissue. Two or three tubes often open into one duct,

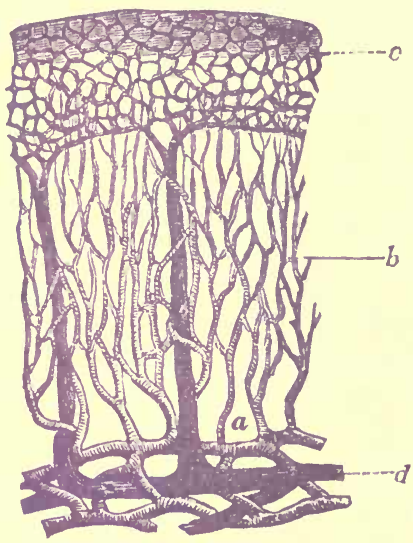

Fig. 252.

Fig. 251.-Section showing the pyloric glands. $s$, free surface: $d$, ducts of pyloric glands; $n$, neck of same; $m$, the gland alveoli; $m m$, muscularis mucosæ. (Klein and Noble Smith.)

Fig. 252. -Plan of the blood-vessels of the stomach, as they would be seen in a vertical section. $a$, arteries, passing up from the vessels of submucous coat; $b$, capillaries branching between and around the tubes; $c$, superficial plexus of capillaries occupying the ridges of the mucoms membrane; $d$, vein formed by the union of veins which, having collected the blood of the superficial capillary plexus, are seen passing down between the tubes. (Brinton.)

which forms about a third of the whole length of the tube and opens on the surface. The ducts are lined with columnar epithelium. Of the gland tube proper, i.e., the part of the gland below the duct, the apper third is the neck and the rest the body. The neck is narrower than the body, and is lined with granular cubical cells which are continuous with the columnar cells of the duct. Between these cells and the membrana propria of the tubes, are large oval or spherical cells, opaque or granular in appearance, with clear oval nuclei, bulging out the membrana propria; these cells are called oxyntic or parietal cells. They do not form a continuous layer. The body, which is broader than the neck and ter- 
minates in a blind extremity or fundus near the muscularis mucosæ, is lined by cells continuous with the cubical or central cells of the neck, but longer, more columnar and more transparent. In this part are a few parietal cells of the same kind as in the neck (fig. 249).

As the pylorus is approached the gland ducts become longer and the tube proper becomes shorter, and occasionally branched at the fundus.

(b) Pyloric Glands. - These glands (fig. 251) liave much longer ducts than the peptic glands. Into each duct two o: three tubes open by very short and narrow necks, and the body of each tube is branched, wavy, and convoluted. The lumen is very large. The ducts are lined with columnar epithelium, and the neck and body with shorter and more granular cubical cells, which correspond with the central cells of the cardiac glands. During secretion the cells become, as in the case of the cardiac glands, larger and the granules restricted to the inner zone of the cell. As they approach the duodenum the pyloric glands become larger, more convoluted and more deeply situated. They are directly continuous with Brunner's glands in the duodenum. (Watney.)

Changes in the gland cells during secretion.-The chief or cubical cells of the cardiac glands, and the corresponding cells of the pyloric glands during the early stage of digestion, if hardened in alcohol, appear swollen and granular, and stain readily. At a later stage the cells become smaller and less granular, and stain even more readily. The parietal cells swell up, but are otherwise not altered during digestion. The granules, however, in the alcohol-hardened specimen, are believed not to exist in the living cells, but to have been precipitated by the hardening reagent; for if examined during life they appear to be confined to the inner zone of the cells, and the outer zone is free from granules, whereas during rest the cell is granular throughout. These granules are thought to be pepsin, or the substance from which pepsin is formed, pepsinogen, which is during rest stored chiefly in the inner zone of the cells and discharged into the lumen of the tube during secretion. (Langley.)

Lymphatics.-Lymphatic vessels surround the gland tubes to a greater or less extent. Toward the fundus of the peptic glands are found masses of lymphoid tissue which may appear as distinct follicles, somewhat like the solitary glands of the small intestine.

Blood-vessels. -The blood-vessels of the stomach, which first break up in the sub-mucous tissue, send branches upward between the closely packed glandular tubes, anastomosing around them by means of a fine capillary network, with oblong meshes. Continuous with this deeper plexus, or prolonged upward from it, so to speak, is a more superficial network of larger capillaries, which branch densely around the orifices of the tubes, and form the framework on which are moulded the small 
elevated ridges of mucous membrane bounding the minute, polygonal pits before referred to. From this superficial network the veins chiefly take their origin. Thence passing down between the tubes, with no very free eonnection with the deeper inter-tubular capillary plexus, they open finally into the venous network in the submucous tissue.

Nerves.-The nerves of the stomach are derived from the pneumogastric and sympathetic, and form a plexus in the sub-mucous and muscular coats containing many ganglia (Remak, Meissner).

\section{Gastric Juice.}

The functions of the stomach are, (i) to afford storage for the food until it can be taken up for digestion and absorption by the intestines; (b) to secrete a digestive fluid, the gastric juice, to the action of which the food is subjected after it has entered the eavity of the stomach from the nsophagus; (c) to thoroughly incorporate the fluid with the food by means of its muscular movements; and (d) to absorb such substances as are ready for absorption. It is not essential to life as has been shown by successful removal of the stomach; but in such cases food has to be given in small quantities frequently until a secondary dilatation of the intestine has formed and can act as a place of storage. While the stomach contains no food, and is inactive, no gastric fluid is secreted; and mucus, which is either neutral or slightly alkaline, covers its surface. But immediately on the introduction of food or other substance, the mucous membrane, previously quite pale, beeomes slightly turgid and reddened with the influx of a larger quantity of blood; the gastric glands commence secreting actively, and an acid fluid is poured out in minute drops, which gradually run together and flow down the walls of the stomach, or soak into the substances within it.

Chemical Composition.-The first accurate analysis of gastric juice was made by Prout: but it does not appear to have been collected in any lirge quantity, or pure and separate from food, until the time when Beaumont was enabled, by a fortunate circumstance, to obtain it from the stomach of a man named St. Martin, in whom there existed, as the result of a gunshot wound, an opening leading directly into the stomach, near the upper extremity of the great curvature, and three inches from the cardiac orifice. The introduction of any mechanical irritant, such as the bulb of a thermometer, into the stomach, through this artificial opening, excited at once the secretion of gastric fluid. This was drawn off, and was often obtained to the extent of nearly an ounce. The introduction of alimentary substances caused a much more rapid and abundant secretion than dirl other mechanical irritants. No increase of temperature could be de- 
tected during the most active secretion; the thermometer introduced into the stomach always stood at $37,8^{\circ} \mathrm{C}$. $\left(100^{\circ} \mathrm{F}\right.$.) except during muscular exertion, when the temperature of the stomach, like that of other parts of the body, rose one or two degrees higher.

The chemical composition of human gastric juice has been also investigated by Schmidt. The fluid in this case was obtained by means of an accidental gastric fistula, which existed for several years below the left mainmary region of a patient between the cartilages of the ninth and tenth ribs. The mucous membrane was excited to action by the introduction of some hard matter, such as dry peas, and the secretion was removed by means of an elastic tube. The fluid thus obtained was found to be acid, limpid, odorless, with a mawkish taste-with a specific gravity of 1002 to 1010. It contained a few cells, seen with the microscope, and some fine granular matter. The analysis of the fluid obtained in this way is given below. Essentially it is a weakly acid fluid containing hydrochloric acid and two enzymes, pepsin and rennin, with possibly a third (glucase). The gastric juice of dogs and other animals obtained by the introduction into the stomach of a clean sponge through an artificially made gastric fistula, shows a decided difference in composition, but possibly this is due, at least in part, to admixture with food.

CHEMICAL COMPOSITION OF GASTRIC JUICE.

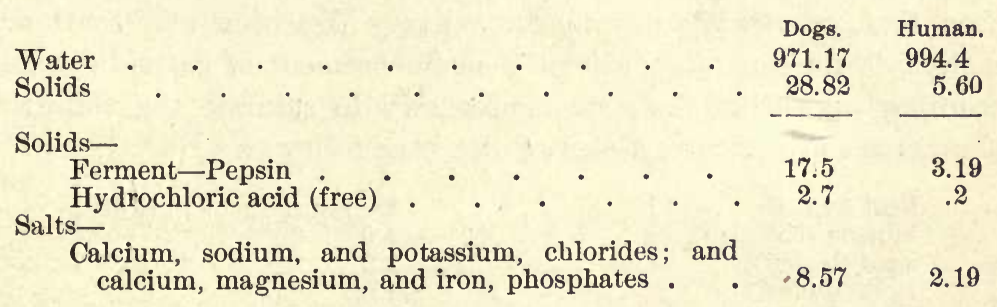

The quantity of gastric juice secreted daily has been variously estimated; but the average for a healthy adult may be assumed to range from ten to twenty pints in the twenty-four hours. The acidity of the fluid is due to free hydrochloric acid, although other acids, e.g., lactic, acetic, butyric, are not infrequently to be found therein as products of gastric digestion or abnormal fermentation. In healthy gastric juice the amount of free hydrochloric acid is usually about 0.2 per cent, but may be as much as 0.3 per cent. In pathological conditions it may be entirely absent, or may amount to 0.5 per cent, or even more.

There is but little doubt that hydrochloric acid is the proper acid of healthy gastric juice, and various tests have been used to prove this; most of these depend upon changes produced in aniline colors by the 
action of hydrochloric acid, even in minute traces, whereas lactic and other organic acids have no such action. Pepsin will act with phosphoric, lactic, and oxalic acids, as proven by laboratory experiments, but the best results are obtained with hydrochloric acid. Of these tests the following may be mentioned.

An aqueous alkaline solution of 00 tropecolin, a bright yellow dye, is turned red on the addition of a minute trace of hydrochloric acid; and aqueous solutions of methyl violet and gentian violet are turned blue under the same eircumstances. The lactic acid sometimes present in the contents of the stomach is derived partly from the sarcolactic acid of muscle, and partly from lactic acid fermentation of carbohydrates. Lactic: acid $\left(\mathrm{C}_{s} \mathrm{H}_{e} \mathrm{O}_{3}\right)$, if present, gives the following test. A solution of $10 \mathrm{ce}$. of a 4 per cent aqueous solution of carbolic acid, $20 \mathrm{cc}$. of water, and one drop of liquor ferri perchloridi is made, forming a blue-colored mixture; a mere trace of free lactic acid added to such a solution causes it to become yellow, whereas hydrochloric acid even in large amount only bleaches it.

The proteid matter in the food combines with part of the hydrochloric acid, which is then known as combined acid and does not redden litmus paper. As this combination is immediate, it follows that no free acid is found in the gastric contents until the amount secreted is more than enough to saturate the various albuminous affinities. It is for this reason that, as already mentioned, salivary digestion may continue in the stomach for some time after the commencement of gastric digestion. According to Ehrlich the amount necessary to saturate the affinities of 100 grammes of various articles of diet is as follows:

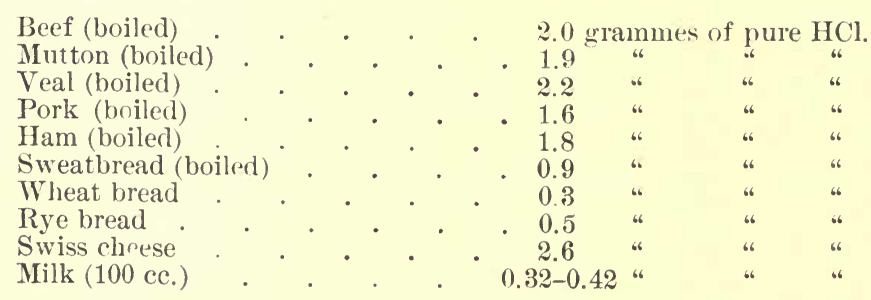

As regards the formation of pepsin and acid, the former is produced by the central or chief cells of the cardiac glands, and also most likely by the similar cells in the pyloric glands; the acid is chiefly found at the surface of the mucous membrane, but is in all probability formed by the parietal cells of the cardiac glands, hence called oxyntic, as no acid is formed by the pyloric glands in which this variety of cell is absent.

The acid is probably formed from materials in the blood and results from a combination of common salt with monosodic orthophosphate 
$\left(\mathrm{NaH}_{2} \mathrm{PO}_{4}+\mathrm{NaCl}=\mathrm{Na}_{2} \mathrm{HPO}_{4}+\mathrm{HCl}\right)$; the disodic orthophosphate is then reconverted by the action of carbonic acid and water $\left(\mathrm{Na}_{2} \mathrm{HPO}_{4}+\right.$ $\left.\mathrm{CO}_{2}+\mathrm{H}_{2} \mathrm{O}=\mathrm{NaH}_{2} \mathrm{PO}_{4}+\mathrm{NaHCO}_{3}\right)$ : all these salts are found in the blood.

The ferment Pepsin can be procured by digesting portions of the mucous membrane of the stomach in cold water, after they have been macerated for some time in water at a temperature $27^{\circ}-37.8^{\circ} \mathrm{C} .\left(80^{\circ}-100^{\circ} \mathrm{F}\right.$. $)$. The warm water dissolves various substances as well as some of the pepsin, but the cold water takes up little else than pepsin, which is contained in a grayish-brown viscid fluid, on evaporating the cold solution. The addition of alcohol throws down the pepsin in grayish-white flocculi. Glycerine also has the property of dissolving out the ferment; and if the mucous membrane be finely minced, and dehydrated by absolute alcohol, a powerful extract may be obtained by macerating it in glycerine.

Functions.-The chief function of gastric juice is such alteration of proteid food-stuffs as will lead to their ready absorption and such modifieation as will favor their further digestion (as far as necessary) in the intestines; gastric digestion is thus both a complete and a preliminary process. Less important functions are the antiseptic action, coagulation of milk, and inversion of disaccharides into monosaccharides. The chief digestive power of the gastric juice depends on the pepsin and acid contained in it, both of which are, under ordinary circumstances, necessary for the process.

The general effect of digestion in the stomach is the conversion of the food into chyme, a substance of varying composition according to the nature of the food, yet always presenting a characteristic thick, pultaceous, grumous consistence, with the undigested portions of the food mixed in a more fluid substance, and a strong, disagreeable acid odor and taste.

This action on proteids may be shown by adding a little gastric juice (natural or artificial) to some diluted egg-albumin, and keeping the mixture at a temperature of about $37.8^{\circ} \mathrm{C} .\left(100^{\circ} \mathrm{F}\right.$. $)$; it is soon found that the albumin cannot be precipitated on boiling, but that if the solution be neutralized with an alkali, a precipitate of acid-albumin is thrown down. After a while the acid-albumin disappears, so that no precipitate results on neutralization, and proper analysis will show that all the albumin has been converted into other proteid substances, viz., proteoses and peptones. The process, as is the case in salivary digestion, is never complete and the final result is always a mixture of peptones with proteoses which cannot be further peptonized: the relative proportions, of course, depend on the duration of the process. A side product is found (as an insoluble residue) in artificial gastric digestion which gives practically all the proteid reactions and is soluble in dilute alkali, though insoluble in water, sodium chloride, or dilute acid. This is known as anti-albumid and may 
be changed into peptone by prolonged digestion; it does not occur in physiological gastric digestion. The commonest proteose is the one formed from albumin and is known as albumose: the class name, however, is proteose, and this name is userl in the subsequent descriptions of the digestive processes.

Characteristics of Peptones.-Peptones have a certain characteristic which distinguishes them from other proteids. They are diffusible, i.e., they possess the property of passing through animal membranes.

In their diffusibility peptones differ remarkably from egg-albumin, and on this diffusibility depends one of their chief uses. Egg-albumin as such, even in a state of solution, would be of little service as food, inasmuch as its indiffusibility would effectually prevent its passing by absorption into the blood-vessels of the stomach and intestinal canal. When completely changed by the action of the gastric juice into peptones, albuminous matters diffuse readily, and are thus quickly absorbed.

After entering the blood the peptones are very soon again modified, so as to reassume the chemical characters of albumin, a change as necessary for preventing their diffusing out of the blood-vessels, as the previous change was for enabling them to pass in. This is effected, probably, in great part by their passage through the vascular walls.

Products of Gastric Digestion.- - The proteid is first changed into syntonin, or acid proteid, by the combined action of the pepsin and acid. Though the acid alone is capable of accomplishing this, the fact that it does not do so physiologically is proven by the great length of time required, in laboratory experiments, for the change. The next change is the conversion of the syntonin into proteoses which, according to Neumeister, occurs in two successive stages. The first of these stages is the conversion of syntonin into the primary proteoses, i.e., proto-proteose and hetero-proteose; the second is the conversion of both proto-proteose and hetero-proteose into the secondary proteoses, i.e., deutero-proteose. The last change is the conversion of the deutero-proteose into peptone; this change does not occur to any great extent physiologically and the proteoses always predominate. Schematically the changes in the proteids may be represented as follows:

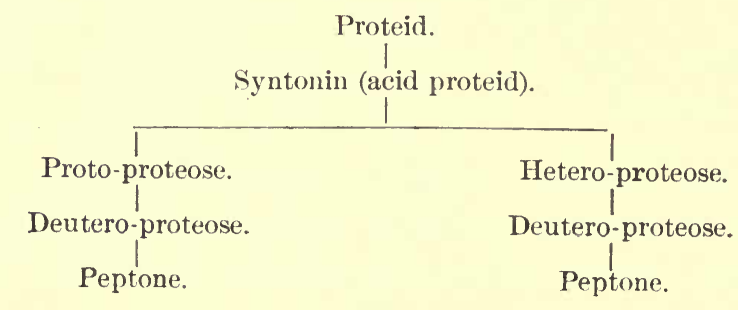


The action of pepsin is one of hydrolysis and the products are hydrated forms of proteid. The acid is not only essential to the action of pepsin, but it also aids digestion by causing the proteids to swell. That this action is important is proven, in laboratory experiments, by the increased length of time required for digestion when fibrin has been wrapped with thread and thus prevented from swelling.

Reactions of Proteoses. - The proteoses cannot be coagulated by heat. All are soiubie in salt solution. All are precipitated by picric acid or by saturation (after neutralizing) with ammonium sulphate. All give the Biuret test, copper sulphate producing a precipitate which redissolves on the addition of caustic potash and forms a rose red solution. The primary proteoses are precipitated by strong nitric acid, also by acetic acid and potassium ferrocyanide, and by saturation with sodium chloride and maguesium sulphate. The secondary proteoses are not precipitated by these reactions just mentioned but are characterized by the fact that their precipitates, when formed, disappear on warming and reappear on cooling. Proto-proteose is distinguished by being soluble in water while hetero-proteose is not.

Peptone reacts to the same test as deutero-proteose, but is not precipitated on saturation with ammonium sulphate.

Circumstances favoring Gastric Digestion.-1. A temperature of about $37.8^{\circ} \mathrm{C} .\left(100^{\circ} \mathrm{F}\right.$. $)$; at $0^{\circ} \mathrm{C}$. $\left(32^{\circ} \mathrm{F}\right.$. $)$ it is delayed, and by boiling is altogether stopped. 2. An acid medium is necessary. Hydrochloric is the best acid for the purpose. Excess of acid or neutralization stops the process. 3. The removal of the products of digestion. Excess of peptone delays the action.

a. Fibrin is first dissolved, forming a solution of globulins. The intermediate products of the digestion of globulins are called globuloses; of vitellin, vitelloses; of casein, caseinoses; of myosin, myosinoses. These are practically the same as albumoses, and are included under the term proteoses.

b. Proteids.-All proteids are converted by the gastric juice into proteoses and peptones, and, therefore, whether they be taken into the body in meat, eggs, milk, bread, or other foods, proteoses and peptone are still the resultant.

c. Milk is curdled, the casein being precipitated, and then dissolved. The curdling is due to a special ferment of the gastric juice, and is not due to the action of the free acid only. The effect of rennet, which is a decoction of the fourth stomach of a calf in brine (rennet), has long been known, as it is used extensively to cause precipitation of casein in cheese manufacture. The ferment which produces this curdling action is distinct from pepsin, and is called rennin. 
d. Upon pure oleaginous principles the gastric juice has no action. In the case of adipose tissue, its effect is to dissolve the areolar tissue, albuminous cell-walls, etc., which enter into its composition, by which means the fat is able to mingle more uniformly with the other constituents of the chyme.

The gastric fluid acts as a general solvent for some of the saline constituents of the food, as, for example, particles of common salt, which may happen to have escaped solution in the saliva; while its acid may enable it to dissolve some other salts which are insoluble in the latter or in water.

e. Upon starches the gastric juice has no action, but by the aid of its hydrochloric acid it inverts the disaccharides into monosaccharides to a certain extent, changing cane sugar into dextrose; the ferment glucose (if existent) may have a similar, though unimportant and slight, action.

$g$. The action of the gastric juice in preventing and checking putrefaction has been often directly demonstrated. Indeed, that the secretion which the food meets with in the stomach is antiseptic in its action, is what might be anticipated from the proneness to decomposition of organic matters, such as those used as food, especially under the influence of warmth and moisture. It is due to the antiseptic action of the gastric juice that disease-germs are often destroyed in the stomach, and the person is saved from an attack of illness.

Time occupied in Gastric Digestion.-Under ordinary conditions, from three to four hours may be taken as the average time occupied by the digestion of a meal in the stomach. But many circumstances will modify the rate of gastric digestion. The chief are: the nature of the food taken and its quantity (the stomach should be fairly filled-not distended); the time that has elapsed since the last meal, which should be at least enough for the stomach to be quite clear of food; the amount of exercise previous and subsequent to a meal (gentle exercise being favorable, over-exertion injurious to digestion); the state of mind (tranquillity of temper being essential, in most cases, to a quick and due digestion), and the bodily health.

Movements of the Stomach. - The gastric fluid is assisted in accomplishing its share in digestion by the movements of the stomach. In granivorous birds, for example, the contraction of the strong muscular gizzard affords a necessary aid to digestion, by grinding and triturating the hard seeds which constitute part of the food. But in the stomachs of man and other Mammalia, the movements of the muscular coat are too feeble to exercise any such mechanical force on the food; neither are they needed, for mastication has already done the mechanical work of a gizzard; and experiments have demonstrated that substances are 
digested even inclosed in perforated tabes, and consequently protected from mechanical influence.

The normal actions of the muscular fibres of the human stomach appear to have a three-fold purpose: (1) to adapt the stomach to the quantity of food in it, so that its walls may be in contact with the food on all sides, and, at the same time, may exercise a certain amount of compression upon it; (2) to keep the orifices of the stomach closed until the food is digested; and (3) to perform certain peristaltic movements, whereby the food, as it becomes chymified, is gradually propelled toward, and ultimately through, the pylorus. In accomplishing this latter end, the movements without doubt materially contribute toward effecting a thorough intermingling of the food and the gastric fluid.

When digestion is not going on, the stomach is uniformly contracted, its orifices not more firmly than the rest of its walls; but, if examined shortly after the introduction of food, it is found closely encircling its contents, and its orifices are firmly closed like sphincters. The cardiac orifice, every time food is swallowed, opens to admit its passage to the stomach, and immediately again closes. The pyloric orifice, during the first part of gastric digestion, is usually so completely closed, that even when the stomach is separated from the intestines, none of its contents escape. But toward the termination of the digestive process, the pylorus seems to offer less resistance to the passage of substances from the stomach; first it yields to allow the successively digested portions go through it; and then it allows the transit of even undigested substances. It appears that food, so soon as it enters the stomach, is subjected to a kind of peristaltic action of the muscular coat, whereby the digested portions are gradually moved toward the pylorus. The movements were observed to increase in rapidity as the process of chymification advanced, and were continued until it was completed.

The contraction of the fibres situated toward the pyloric end of the stomach seems to be more energetic and more decidedly peristaltic than those of the cardiac portion. Thus, it was found in the case of St. Martin, that when the bulb of the thermometer was placed about three inches from the pylorus, through the gastric fistula, it was tightly embraced from time to time, and drawn toward the pyloric orifice for a distance of three or four inches. The object of this movement appears to be, as just said, to carry the food toward the pylorus as fast as it is formed into chyme, and to propel the chyme into the duodenum; the undigested portions of food being kept back until they are also reduced into chyme, or until all that is digestible has passed out. The action of these fibres is often seen in the contracted state of the pyloric portion of the stomach after death, when it alone is contracted and firm, while the cardiac portion forms a dilated sac. Sometimes, by a predominant action of strong circular fibres placed between the cardia and pylorus, the two por- 
tions, or ends as they are called, of the stomach, are partially separated from each other by a kind of hour-glass contraction. By means of the peristaltic action of the muscular coats of the stomach, not merely is chymified food gradually propelled through the pylorus, but a kind of double current is continually kept up among the contents of the stomach, the circumferential parts of the mass being gradually moved onward toward the pylorus by the contraction of the muscular fibres, while the central portions are propelled in the opposite direction, namely toward the cardiac orifice; in this way is kept up a constant circulation of the contents of the viscus, highly conducive to their free mixture with the gastric fluid and to their ready digestion.

Influence of the Nervous System.-The normal movements of the stomach during gastric digestion do not appear to be so closely con-

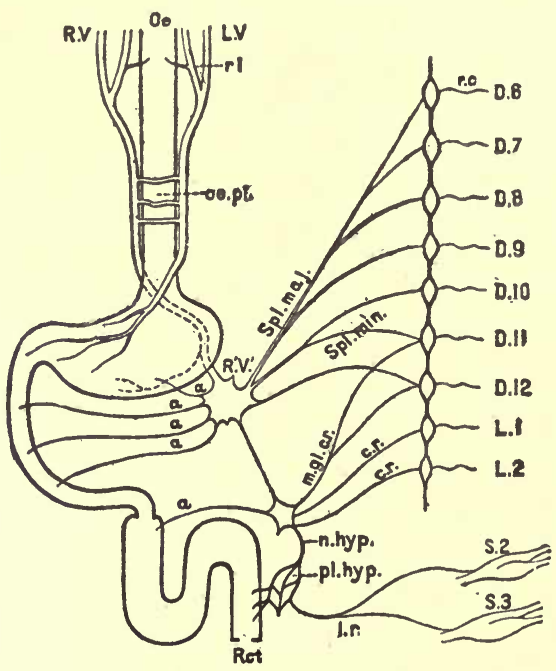

Fig. 253.-Very diagrammatic representation of the nerves of the alimentary canal. Oe to Ret, the various parts of the alimentary canal from oesophagus to rectum; L. V, left vagus, ending on front of stomach; rl, recurrent laryngeal nerve, supplying upper part of cesophagus; R.V, right vagus, joining left vagus in œesophageal plexus; œ.pl, supplying the posterior part of stomach, and continues as $\mathrm{R}^{\prime} \mathrm{V}^{\prime}$ to join the solar plexus, here represented by a single ganglion, and connected with the inferior mesenteric ganglion $\mathrm{m}$.gl; a branches from the solar plexus to stomach and small intestine, and from the mesenteric ganglia to the large intestine; Spl.maj., large splanchnic nerve, arising from the thoracic ganglia and rami communicantes; r.c, belonging to dorsal nerves from the 6 th to the 9 th (or 10th); Spl.min., small splauchnic nerve similarly from the 10th and 11th dorsal nerves. These both join tlie solar plexus, and thence make their way to the alimentary canal; c.r., nerves from the ganglia, etc., belonging to 11 th and 12 th dorsal and 1st and $2 \mathrm{~d}$ lumbar nerves, proceeding to the inferior mesenteric ganglia (or plexus), m.gl., and thence by the hypogastric nerve, n.hyp., and the hypogastric nerve, n.hyp., and the hypogastric plexus, pl.hyp., to the circular muscles of the rectum; l.r., nerves from the $2 \mathrm{~d}$ and $3 \mathrm{~d}$ sacral nerves, S.2, S.3 (nervi erigentes) proceeding by the hypogastric plexus to the longitudinal muscles of the rectum. (M. Foster.)

nected with the plexuses of nerves and ganglia contained in its walls as was formerly supposed. The action, however, appears to be set up by the presence of food within it. The stomach is, moreover, directly connected with the higher nerve-centres by means of branches of tr vagi and of the splanchnic nerves through the solar plexus. 
First as to the function of the ragi in connection with the gastric movements. Irritation of these nerves produces contraction of the stomach, including the sphincter pylori. The vagi, then, are the motor nerves to the stomach.

Secondly as to the other nerve-fibres, which reach the stomach and intestines through the solar plexus. These fibres pass from the spinal cord in the anterior roots of the nerves from the sixth to the twelfth dorsal, passing in the splanchnic nerves to the solar plexus, and thence to the stomach. Stimulation of the splanchnics causes stoppage of the muscular movements as well as relaxation of the sphincter pylori.

It seems probable that automatic peristaltic contraction is inherent in the muscular coat of the stomach, and that the central nervous system is only employed to regulate it by impulses passing down by the vagi or splanchnic nerves.

Next as to the influence of the nerves on the secretion of the gastric juice. It has been known for a long time that the secretion of gastric juice could be reflexly stimulated. For example, Bidder and Schmidt observed in a dog with a gastric fistula that the mere sight of food was sufficient to cause a flow of gastric juice. Quite recently, Pawlow has proved that secretory fibres are carried to the gastric glands in the vagus trunk. His experiment consisted in establishing a gastric fistula, and some days later in dividing the osophagus in the neck in such a manner that any food swallowed would be diverted to the exterior through the cut end. "Fictitious meals" could then be given to the animal, and the effect upon the stomach noted. As long as the vagi were intact, certain foods (meats) caused a flow of gastric juice, though none of the food reached the stomach. When the vagi had been cut, no secretion occurred. Moreover, he found that direct stimulation of the vagus produced a flow of gastric juice.

The subject has been still further elucidated by some experiments of Heidenhain, relative to the normal mechanism of secretion. He cut out a portion of the fundic end of the stomach, converting it into a blind pouch opening to the exterior, while the continuity of the stomach itself was established by sutures. Food given to the animal caused a secretion in the cul-de-sac as well as in the stomach. From the experiments be concludes that normally there occur a primary secretion due to the mechanical stimulation of the mucous membrane and confined to isolated spots, and a secondary secretion due to the absorption of the products of digestion, which comes from the whole mucous membrane.

Khigine has carried these experiments still further and obtained very complete results. $\mathrm{He}$ has investigated the effects of various chemical substances upon the flow of secretion, and has found that peptone is the 
best of all stimuli. How it acts is unknown. Khigine believes that it acts upon the afferent nerve-filaments in the stomach, and that the effect is reflex.

The influence of the higher nerve-centres on gastric digestion, as in the case of mental emotion, is too well known to need more than a reference.

Digestion of the Stomach after Death.-If an animal die during the process of gastric digestion, and when, therefore, a quantity of gastric juice is present in the interior of the stomach, the walls of this organ itself are frequently themselves acted on by their own secretion, and to such an extent that a perforation of considerable size may be produced, and the

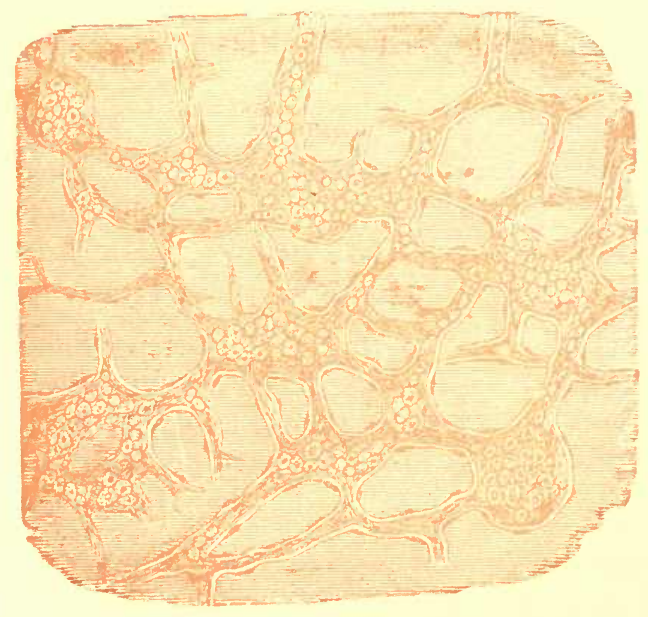

Fig. 254. -Auerbach's nerve-plexus in small intestine. The plexus consists of fibrillated substance, and is made up of trabeculæ of various thicknesses. Nucleus-like elenients and ganglioncells are imbedded in the plexus, the whole of which is inclosed in a nucleated sheath. (Klein.)

contents of the stomach may in part escape into the cavity of the abdomen. This phenomenon is not infrequently observed in post-mortem examinations of the human body. If a rabbit be killed during a period of digestion, and afterward exposed to artificial warmth to prevent its temperature from falling, not only the stomach, but many of the surrounding parts will be found to have been dissolved (Pavy).

From these facts, it becomes an interesting question why, during life, the stomach is free from liability to injury from a secretion, which, after death, is capable of such destructive effects.

It is unly necessary to refer to the idea of Bernard, that the living stomach finds protection from its secretion in the presence of $e_{1}$.ithelium and mucus, which are constantly renewed in the same degree that they are constantly dissolved, in order to remark that although the gastric mucus is probably protective, this theory, so far as the epithelium is 
concerned, has been disproved by experiments of Pary's, in which the mucous membrane of the stomachs of dogs was dissected off for a small space, and, on killing the animals some days afterward, no sign of digestion of the stomach was visible. "Upon one uccasion, after removing the mucous membrane, and exposing the muscular fibres over a space of about an inch and a half in diameter, the animal was allowed to live for ten days. It ate food every day, and seemed scarcely affected by the operation. Life was destroyed while digestion was being carried on, and the lesion in the stomach was found very nearly repaired; new matter had been deposited in the place of what had been removed, and the denuded spot had contracted to much less than its original dimensions."

Pavy believes that the natural alkalinity of the blood, which circulates so freely during life in the walls of the stomach, is sufficient to neutralize the acidity of the gastric juice; and as may be gathered from what has been previously said, the neutralization of the acidity of the gastric secretion is quite sufficient to destroy its digestive powers; but the experiments adduced in favor of this theory are open to many objections, and afford only a negative support to the conclusions they are intended to prove. Again, the pancreatic secretion acts best on proteids. in an alkaline medium; but it has no digestive action on the living intestine. No satisfactory theory of the reason why the stomach does not digest itself has yet been suggested.

\section{Vomiting.}

The expulsion of the contents of the stomach in vomiting, like that of mucus or other matter from the lungs in coughing, is preceded by an inspiration; the glottis is then closed, and immediately afterward the abdominal museles strongly act; but here occurs the difference in the two actions. Instead of the vocal cords yielding to the action of the abdominal muscles, they remain tightly closed. Thus the diaphragm being unable to go up, forms an unyielding surface against which the stomach can be pressed. In this way, as well as by its own contraction, the diaphragm is fixed, to use a technical phrase. At the same time the cardiac sphincter-muscle being relaxed, and the orifice which it naturally guards being actively dilated, while the pylorus is closed, and the stomach itself also contracting, the action of the abdominal muscles, by these means assisted, expels the contents of the organ through the osophagus, pharynx, and mouth. The reversed peristaltic action of the œsophagus probably increases the effect.

It has been frequently stated that the stomach itself is quite passive during vomiting, and that the expulsion of its coutents is effected solely by the pressure exerted upon it when the capacity of the abdomen is diminished by the contraction of the diaphragm, and subsequently of the abdominal muscles. The experiments and observations, however, which 
are aupposed to confirm this statement, only show that the contraction of the abdominal muscles alone is sufficient to expel matters from an unresisting bag through the œsophagus; and that, under very abnormal circumstances, the stomach, by itself, cannot expel its contents. They by no means show that in ordinary vomiting the stomach is passive; and, on the other hand, there are good reasons for believing the contrary.

It is true that facts are wanting to demonstrate with certainty this action of the stomach in vomiting; but some of the cases of fistulous opening into the organ appear to support the belief that it does take place; and the analogy of the case of the stomach with that of the other hollow viscera, as the rectum and bladder, may be also cited in confirmation.

The muscles concerned in the act of vomiting, are chiefly and primarily those of the abdomen; the diaphragm also acts, but usually not as the muscles of the abdominal walls do. They contract and compress the stomach more and more toward the diaphragm; and the diaphragm (which is usually drawn down in the deep inspiration that precedes each act of vomiting) is fixed, and presents an unyielding surface against which the stomach may be pressed. The diaphragm is, therefore, as a rule passive, during the actual expulsion of the contents of the stomach. But there are grounds for believing that sometimes this muscle actively contracts, so that the stomach is, so to speak, squeezed between the descending diaphragm and the retracting abdominal walls.

Some persons possess the power of vomiting at will, without applying any undue irritation to the stomach, but simply by a voluntary effort. It seems also that this power may be acquired by those who do not naturally possess it, and by continual practice may become a habit. There are cases also of rare occurrence in which persons habitually swallow their food hastily, and nearly unmasticated, and then at their leisure regurgitate it, piece by piece, into their mouth, remasticate, and aguin swallow it, like members of the ruminant order of Mammalia.

The various nerve-actions concerned in vomiting are governed by a nerve-centre situated in the medulla oblongata.

The sensory nerves are the fifth, glosso-pharyngeal and vagus principally; but, as well, vomiting may occur from stimulation of sensory nerves from many organs, e.g., kilney, testicle, etc. The centre may also be stimulated by impressions from the cerebrum and cerebellum, so-called central vomiting occurring in disease of those parts. The efferent impulses are carried by the phrenics and other spinal nerves.

\section{The Intestines.}

The Intestinal canal is divided into two chief portions, named from their differences in diameter, the small and large intestine (fig. 215). 
These are continuous with each other, and communicate by means of an opening guarded by a valve, the ileocrecal valve, which allows the passage of the products of digestion from the small into the large bowel, but not, under ordinary circumstances, in the opposite direction.

The Small Intestine.-The Small Intestine, the average length of which in an adult is about twenty feet, has been divided, for convenience of description, into three portions, viz., the duodenum, which extends for eight or ten inches beyond the pylorus; the jejunum, which forms two-fifths, and the ileum, which forms three-fifths of the rest of the canal.

Structure.-The small intestine, like the stomach, is constructed of four principal coats, viz., the serous, muscular, sub-mucous, and mucous.

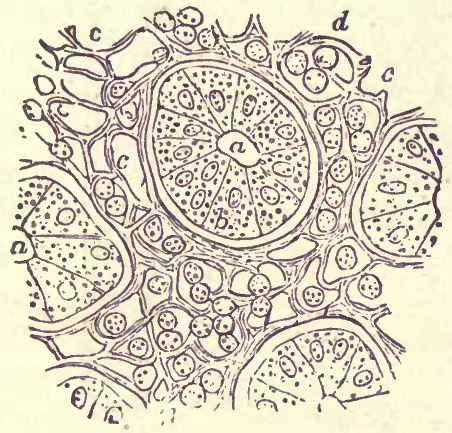

Fig. 255.

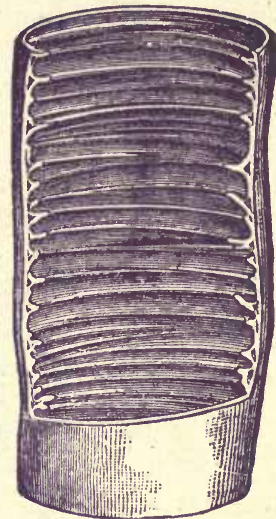

Fig. 256.

Fig. 255-Horizontal section of a small fragment of the mucous membrane, including one entire cryot of Lieberkühn and parts of several others.

Fig. 256.-Piece of small intestine (previously distended and hardened by alcohol), laid open to show the normal position of the valvulæ conniventes.

(1.) The serous coat is formed by the visceral layer of the peritoneum, and has the structure of serous membranes in general.

(2.) The muscular coats consist of an internal circular and an external longitudinal layer: the former is usually considerably the thicker. Both alike consist of bundles of unstriped muscle supported by connective tissue. They are well provided with lymphatic vessels, which form a set distinct from those of the mucous membrane.

Between the two muscular coats is a nerve plexus (Auerbach's plexus) (fig. 254), similar in structure to Meissner's (in the submucous tissue), but with more numerous ganglia.

(3.) Between the mucous and muscular coats is the submucous coat, which consists of connective tissue, in which numerous blood-vessels and lymphatics ramify. A fine plexus, consisting mainly of non-medul- 
lated nerve-fibres, Meissner's plexus, with ganglion cells at its nodes, occurs in the submucous tissue from the stomach to the anus.

(4.) The mucous membrane is the most important coat in relation to the funation of digestion. The following structures, which enter into its composition, may now be successively described:- the valvulre conniventes; the villi; and the glands. The general structure of the mucous membrane of the intestines resembles that of the stomach (p. 34\%), and, like it, is lined on its inner surface by columnar epithelium. Adenoid tissue (fig. 255) enters largely into its construction; and on its deep surface is the muscularis mucosce ( $\mathrm{mm}$, fig. 260), the fibres of which are arranged in two layers: the onter longitudinal and the inner circular.

Valvulce Conniventes.-The valvulæ conniventes (fig. 256) commence in the duodenum, about one or two inches beyond the pylorus, and

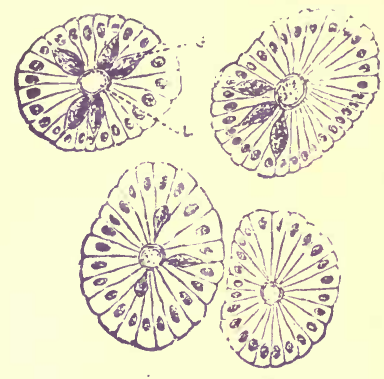

Fig. 25\%.

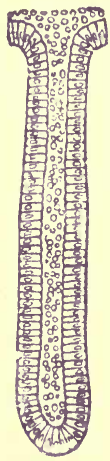

Fig. 258.

Fig. 257. - Transverse section through four crypts of Lieberkühn from the large intestine of the pig. They are lived by columnar epithelial cells, the nuclei being placed in the outer part of the cells. The divisions between the cells are seen as lines radiating fiom L, the lumen of the crypt; G, epithelial erlls, which have become transformed iuto goblet cells. $\times 350$. (Klein and Noble Smith.)

Fig. 258.-A gland of Lieberkühn in longitudinal section. (Brinton.)

becoming larger and more numerous immediately beyond the entrance of the bile duct, continue thickly arranged and well developed throughout the jejunum; then, gradually diminishing in size and number, they cease near the middle of the ileum. They are formed by a doubling inward of the mucous membrane; the crescentic, nearly circular, folds thus formed being arranged transversely to the axis of the intestine, and each individual fold seldom extending around more than $\frac{1}{2}$ or $\frac{2}{3}$ of the bowel's circumference. Unlike the rugæ in the œsopnagus and stomach, they do not disappear on distention of the canal. Only an imperfect notion of their natural position and function can be obtained by looking at them after the intestine has been laid open in the usual manner. To understand them aright, a piece of gut should be distended either with air or alcohol, and not opened until the tissues have become hardened. On then making a section it will be seen that, instead of 
disappearing, they stand out at right angles to the general surface of the mucous membrane (fig. 256). Their functions are (1) to afford a largely increased surface for secretion and absorption, and (2) to prevent the too rapid passage of the very liquid products of gastric digestion, immediately after their escape from the stomach, and (:) to assist in the more perfect mingling of the latter with the secretions poured out to act on them, by their projection, and consequent interference with an uniform and untroubled current of the intestinal contents.

Glands.-The glands are of three principal kinds:-viz., those of (1) Lieberkühn, (2) Brunner, and (3) Peyer.

(1.) The glands or crypts of Lieberkühn are simple tubular depres-

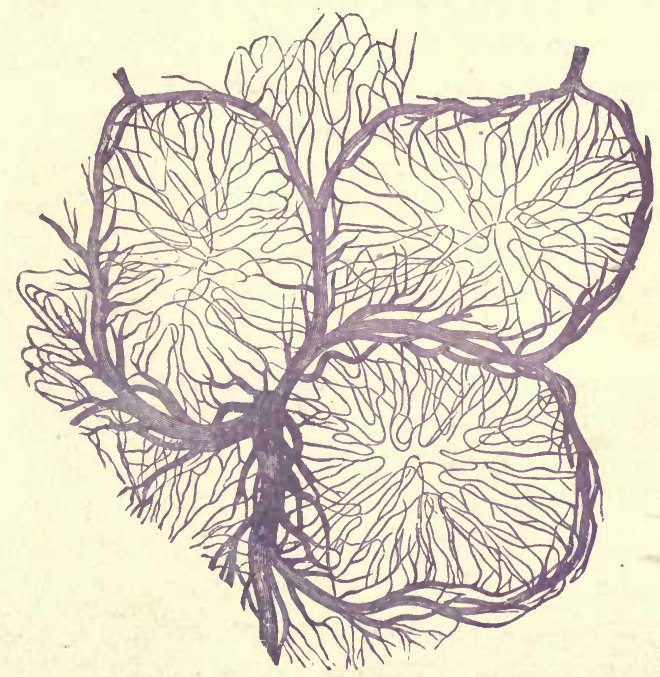

Fig. 259.-Transverse section of injected Peyer's glands (from Kölliker). The drawing was taken from a preparation made by Frey: it represents the fine capillary-looped network spreading from the surrounding blood-vessels into the interior of three of Peyer's capsules from the intestine of the rabbit.

sions of the intestinal mucous membrane, thickly distributed over the whole surface both of the lirge and small intestines. In the small intestine they are visible only with the aid of a lens; and their orifices appear as minute dots scattered between the villi. They are larger in the large intestine, and increase in size the nearer they approach the anal end of the intestinal tube; and in the rectum their orifices may be visible to the naked eye. In length they vary from $\frac{1}{180}$ to $\frac{1}{60}$ of an inch. Each tubule (fig. 258) is constructed of the same essential part as the intestinal mucous membrane, viz., of a fine membrana propria, or basement membrane, a layer of columnar epithelium lining it, many of which are goblet cells, and capillary blood-vessels covering its exterior, the free surface of the columnar cells presenting a striated appearance. 
(2.)-Brunner's glands (fig. 260) are confined to the duodenum; they are most abundant and thickly set at its commencement, diminish gradnally as the duodenum advances. They are situated beneath the muscularis mucosæ, imbedded in the submucous tissue; each gland is a branched and convoluted tube, lined with columnar epithelium. As before said, in structure they are very similar to the pyloric glands of the stomach, and their epithelium undergoes a similar change during secretion; but they are more branched and convoluted snd their ducts are longer. (Watney.) The duct of each gland passes through the muscularis mucosæ, and opens on the surface of the mucous membrane.

(3.) The glands of Peyer occur chiefly but not exclusively in the small intestine. They are found in greatest abundance in the lower part of the ileum near to the ileo-cæcal valve. They are met with in two conditions, viz., either scattered singly, in which case they are termed glandulce solitarice, or aggregated in groups varying from one to three inches in length, and about half-an-inch in width, chiefly of an oval form, their long axis parallel with that of the intestine. In this state, they are named glandulce agminata, the groups being commonly called Peyer's patches (fig. 261), and almost always placed opposite the attachment of the mesentery. In structure, and in function, there is no essential difference between the solitary glands and the individual bodies of which each group or patch is made up. They are really single or aggregated masses of adenoid tissue forming lymph-follicles. In

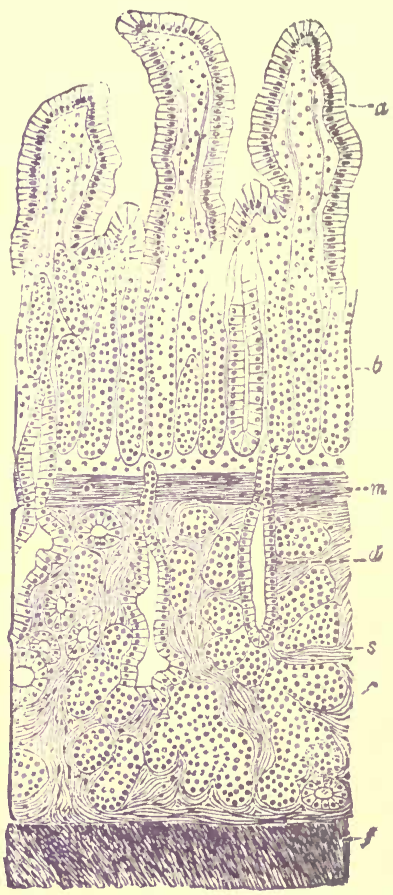

Fig. 260.-Vertical section of duodenum, showing $a$, villi ; $b$, crypts of Lieberkühn, and $c$, Brunner's glands in the submucosa $s$, with ducts, $d$; muscularis mucosæ, $m$; and circular muscular coat, $f$. (Schofield.) the condition in which they have been most commonly examined, each gland appears as a circular opaque-white rounded body, from $\frac{1}{24}$ to $\frac{1}{12}$ inch (1 to $2 \mathrm{~mm}$.) in diameter, according to the degree in which it is developed. They are principally contained in the submucous coat, but sometimes project through the muscularis mucose into the mucous membrane. In the agminate glands, each follicle reaches the free surface of the intestine, and is covered with columnar epithelium. Each gland is surrounded by the openings of Lieberkühn's follicles.

The adjacent glands of a Peyer's patch are connected together by areolar tissue. Sometimes the lymphoid tissue reaches the free surface, 
replacing the epithelium, as is also the case with some of the lymphoid follicles of the tonsil.

Peyer's glands are surrounded by lymphatic sinuses which do not penetrate into their interior; tho interior is, however, traversed by $a$ very rich blood capillary plexus. If the vermiform appendix of a rabbit, which consists largely of Peyer's glands, be injected with blue by pressing the point of a fine syringe into one of the lymphatic sinuses, the Peyer's glands will appear as grayish white spaces surrounded by blue; if now the arteries of the same be injected with red, the grayish patches will change to red, thus proving that they are surrounded by lymphatic spaces but penetrated by blood-vessels. The lacteals passing out of the villi communicate with the lymph sinuses round Peyer's glands. It is to be noted that Peyer's patches are largest and most prominent in children and young persons.

Vill. - The Villi (figs. 260, 262, and 263) are confined exclusively to the mucous membrane of the small intestine. They are minute vascular processes, from a line $\frac{1}{48}$ to $\frac{1}{8}$ of an inch $(.5$ to $3 \mathrm{~mm}$.) in length, covering the surface of the mucous membrane, and giving it a peculiar velvety, fleecy appearance. Krause estimates them at fifty to ninety in number in a square line at the upper part of the small intestine, and

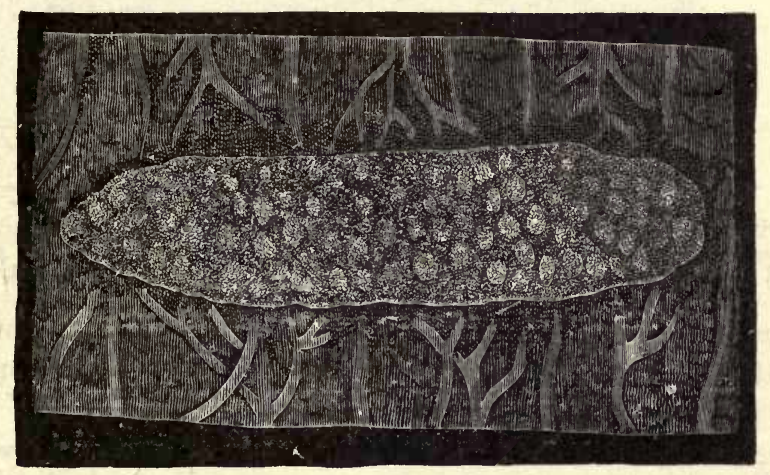

Fig. 261. -Agminate follicles, or Peyer's patch, in the state of distention. $\times 5$. (Boehm.)

at forty to seventy in the same area at the lower part. They vary in form even in the same animal, and differ according as the lymphatic vessels or lacteals which they contain are empty or full; being usually, in the former case, flat and pointed at their summits, in the latter cylindrical or clavate.

Each villus consists of a small projection of mucous membrane; its interior is supported thronghout by fine adenoid tissue, which forms the framework or stroma in which the other constituents are contained.

The surface of the villus is clothed by columnar epithelium, which rests on a fine basement membrane; while within this are found, reckoning from without inward, blood-vessels, fibres of the muscularis mu- 
$\cos \mathfrak{x}$, and a single lymphatic or lacteal vessel rarely looped or branched (fig. 263).

The epithelium is continuous with that lining the other parts of the mucous membrane. The cells are arranged with their long axis radiating from the surface of the villus (fig.260), and their smaller ends resting on the basement membrane. The free surface of the epithelial cells of the villi, like that of the cells which cover the general surface of the mucous membrane, is covered by a fine border which exhibits very delicate striations, whence it derives its name, striated basilar border.

Beneath the basement or limiting membrane there is a rich supply of blood-vessels. Two or more minute arteries are distributed within each villus; and from their capillaries, which form a dense network, proceed one or two small veins, which pass out at the base of the villus.

The layer of the muscularis mucose in the villus forms a kind of

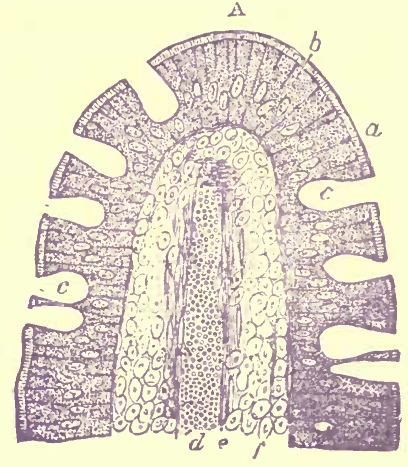

Fig. 262. - Vertical section of a villus of the small intestine of a cat. $a$, striated basilar borcler of the epichelium; $b$, columnar epithelium; $c$, goblet cells; $d$, central lymph-vessel; $e$, smooth muscular fibres; $f$, adenoid stroma of the villus in which lymph corpuscles lie. (Klein.)

thin hollow cone immediately around the central lacteal, and is, therefore, situated beneath the blood-vessels. It is without doubt instrumental in the propulsion of chyle along the lacteal.

The lacteal vessel in each villus is the form of commencement of the lymphatic system of vessels ${ }^{*}$ in the intestines. It begins almost at the tip of the villus commonly by a dilated extremity. In the larger villi there may be two small lacteal vessels which join on (fig. 263), or the lacteals may form a kind of network in the villus. The last method is rarely or never seen in the human subject, although common in some of the lower animals (A, fig. 263).

The Large Intestine.-The Large Intestine, which in an adult is from about 4 to 6 feet long, is subdivided for descriptive purposes into three portions, viz.:- the cacum, a short wide pouch, communicating with the lower end of the small intestine through an opening, guarded by the ileo-crecal valve; the colon, continuous with the cæcum,

* For an account of the Lymphatic System, see Chapter IX. 
which forms the principal part of the large intestine, and is divided into ascending, transverse, and descending portions; and the rectum, which, after dilating at its lower part, again contracts, and immediately afterward opens externally through the anus. Attached to the cæcum is the small appendix vermiformis.

Structure.-Like the small intestine, the large intestine is constructed of four principal coats, viz., the serous, muscular, sub-mucous and mucous. The sercus coat need not be here particularly described. Connected with it are the small processes of peritoneum containing fat, called appendices epiploicce. The fibres of the muscular coat, like those of the small intestine, are arranged in two layers-the outer longitudinal, the inner circular. In the cæcum and colon, the longitudinal fibres, besides being, as in the small intestine, thinly disposed in all parts of the wall of the bowel, are collected, for the most part, into three strong bands, which, being shorter, from end to end, than the other coats of the intestine, hold the canal in folds, bounding intermediate sacculi. On the division of these bands, the intestine can be drawn out to its full length,

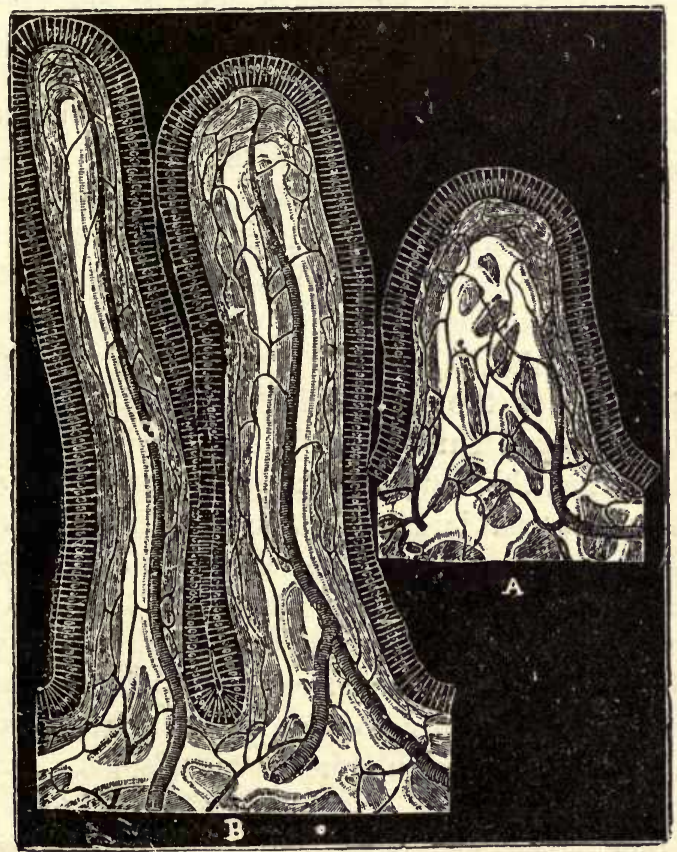

Fig. 203.-A. Villus of sheep. B. Villi of man. (Slightly altered from Teichmann.)

and it then assumes, of course, an uniformly cylindrical form. In the rectum, the fasciculi of these longitudinal bands spread out and mingle with the other longitudinal fibres, forming with them a thicker layer of fibres than exists on any other part of the intestinal canal. The circular muscular fibres are spread over the whole surface of the bowel, but 
are somewhat more marked in the intervals between the sacculi. Toward the lower end of the rectum they become more numerous, and at the anus they form a strong band called the internal sphincter muscle.

The mucous membrane of the large, like that of the small intestine, is lined throughout by columnar epithelium, but, unlike it, is quite destitute of villi, and is not projected in the form of valvula conniventes. Its general microscopic structure resembles that of the small intestine: and it is bounded below by the muscularis mucose.

The general arrangement of ganglia and nerve-fibres in the large intestine resembles that in the small.

Glands.-The glands with which the large intestine is provided are of two kinds, (1) the tubular and (2) the lymphoid.

(1.) The tubular glands, or glands of Lieberkühn, resemble those of the small intestine, but are somewhat larger and more numerous. They also contain many goblet cells.

(2.) Follicles of adenoid or lymphoid tissue are most numerous in the cæcum and vermiform appendix. They resemble in shape and structure, almost exactly, the solitary glands of the small intestine. Peyer's patches are not found in the large intestine.

Ileo-crecal Valve.-The ileo-cæcal valve is situate at the place of junction of the small with the large intestine, and guards against any reflux of the contents of the latter into the ileum. It is composed of two semilunar folds of mucous membrane. Each fold is formed by a doubling inward of the mucous membrane, and is strengthened on the outside by some of the circular muscular fibres of the intestine, which are contained between the outer surfaces of the two layers of which each fold is composed. While the circular muscular fibres, however, of the bowel at the junction of the ileum with the cæcum are contained between the outer opposed surfaces of the folds of mucous membrane which form the ralve, the longitudinal muscular fibres and the peritoueum of the small and large intestine respectively are continuous with each other, without dipping in to follow the circular fibres and the mucous membrane. In this manner, therefore, the folding inward of these two last-named structures is preserved, while on the other hand, by dividing the longitudinal muscular fibres and the peritoneum, the valve can be made to disappear, just as the constrictions between the sacculi of the large intestine can be made to disappec.r by performing a similar operation. The inner surface of the folds is smooth; the mucous membrane of the ileum being continuous with that of the cæcum. That surface of each fold which looks toward the small intestine is covered with villi, while that which looks to the cæcum has none. When the cæcum is distended, the margin of the folds are stretched, and thus are brought into firm apposition one with the other. 


\section{Digestion in the Intestines.}

After the food has been duly acted upon by the gastric juice, such of it as has not been absorbed passes into the duodenum, and is there subjected to the action of the secretions of the pancreas and liver which enter that portion of the small intestine, as well as to the secretion (succus entericus) which is poured out into the intestines from the glands lining them. Mixed with products of gastric digestion is found a certain amount of proteid matter which has not been acted upon at all: the fats are also included and such carbohydrates as have not been acted upon by salivary digestion together with products of this digestion.

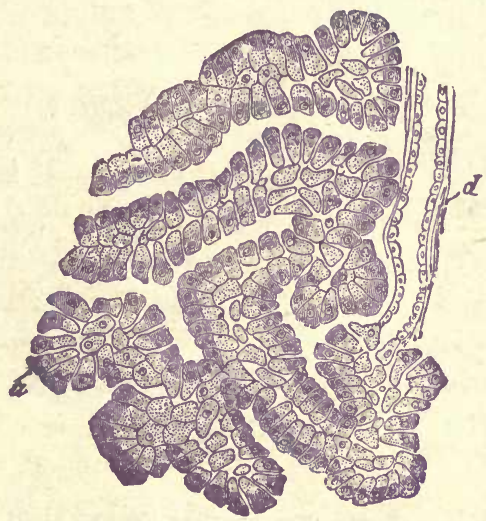

Fig. 264. - Section of the pancreas of a dog during digestion. $a$, alveoli lined with cells, the outer zone of which is well stained with hæmatoxylin; $d$, intermediary duct lined with squamous epithelinm. $\times 350$. (Klein and Noble Smith.)

\section{The Pancreas, and its Secretion.}

The Pancreas is situated within the curve formed by the duodenum; and its main duct opens into that part of the small intestine, through a small opening, or through a duct common to it and to the liver, about two and a halt inches from the pylorus.

Structure. - In structure the pancreas bears some resemblance to the salivary glands. Its capsule and septa, as well as the blood-vessels and lymphatics, are similarly distributed. It is, howerer, looser and softer, the lobes and lobules being less compactly arranged. The main duct divides into branches (lobar ducts), one for each lobe, and these branches subdivide into intra-lobular ducts, and these again by their division and branching form the gland tissue proper. The intralobar ducts correspond to a lobule, while between them and the sesreting tubes or 
alveoli are longer or shorter intermediary ducts. The larger ducts possess a very distinct lumen and a membrana propria lined with columnar epithelinm, the cells of which are longitudinally striated, but are shorter than those found in the ducts of the salivary glands. In the intralobular ducts the epithelium is short and the lumen is smaller. The intermediary ducts opening into the alveoli possess a distinct lumen, with a membrana propria lined with a single layer of fattened elongated cells. The alveoli are branched and convoluted tubes, with a membrana propria lined with a single layer of columvar cells. They have a distinct lumen, though spindle-shaped cells are often seen in the centre of the acini. Heidenhain has observed that the alveolar cells in the pancreas of a fasting dog consist of two zones, an inner or central zone which is finely granular, and which stains feebly, and a

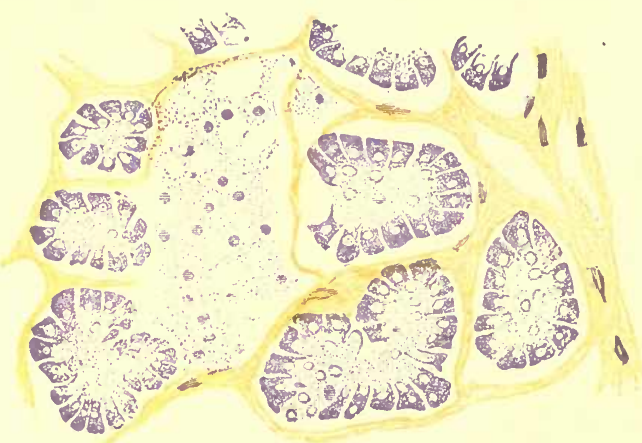

Flg. 265.--Section of the pancreas of armadillo, shuwing the two kinds of gland-structure. (V. D. Harris.)

smaller parietal zone of finely striated protoplasm which stains easily. The nucleus is partly in one, partly in the other zone. During digestion, it is found that the outer zone increases in size, and the central zone diminishes; the cell itself becoming smaller from the discharge of the secretion. At the end of digestion the first condition again appears, the inner zone enlarging at the expense of the outer. It appears that the granules are formed by and stored up in the protoplasm of the cells, from material supplied to it by the blood. The granules are thought to consist of material from which, under certain conditions, the ferments of the gland are developed, and which is therefore called Zymogen. In addition to the ordinary alveoli of the pancreas there are found distributed irregularly in the gland other collections of cells of a different character. They are considerably smaller, their protoplasm is more granular, and is less easily stained with hæmatoxylin, and their nuclei are small and deeply staining, being situated also more toward the centre of the cells. The collections of cells vary in size and shape, and 
sometimes seem to be mere masses of protoplasm with nuclei undifferentiated into cells. These nests of cells are sometimes seen to consist of distinct columns of cells. No distinct basement membrane, however, can be made out as bounding these columns. The special form of nerve terminations, called Pacinian corpuscles, are often found in the pancreas.

The Pancreatic Juice.-The secretion of the pancreas has been obtained for purposes of experiment from the lower animals, especially the dog, by opening the abdomen and exposing the duct of the gland, which is then made to communicate with the exterior. A pancreatic fistula is thus established.

An extract of pancreas made from the gland which has been removed from an animal killed during digestion possesses the active properties of pancreatic secretion. It is made by first dehydrating the gland, cut up into small pieces, by keeping it for some days in absolute alcohol, and then, after the entire removal of the alcohol, by pounding up these pieces into a pulpy mass and placing it in strong glycerin. A glycerin extract is thus obtained. It is a remarkable fact, however, that the amount of the ferment trypsin greatly increases if the gland be exposed to the air for twenty-four hours before placing in alcohol; indeed, a glycerin extract made from the gland immediately upon the removal from the body often appears to contain none of the ferments. This seems to indicate that the conversion of zymogen in the gland into the ferment only takes place during the act of secretion, and that the gland, although it always contains. in its cells the materials (trypsinogen) out of which trypsin is formed, yet the conversion of the one into the other only takes place by degrees. Dilute acid appears to assist and accelerate the conversion, and if a recent pancreas be rubbed up.with dilute acid before dehydration, a glycerin extract made afterward, even though the gland may have been only recently removed from the body, is very active.

Many other vehicles may be employed instead of glycerin, e.g., brine, chloroform, water, dilute methylated spirit acidulated with acetic acid.

Properties.-Pancreatic juice is colorless, transparent, and slightly viscid, alkaline in reaction. It varies in specific gravity from 1010 to 1030 , according as it is obtained from a permanent fistula-then more watery-or from a newly-opened duct. The solids vary in a temporary fistula from 80 to 100 parts per thousand, and in a permanent one from - 16 to 50 per thousand. It is characterized by having three distinct and important enzymes known as trypsin, amylopsin, and steapsin, whose action is, respectively, proteolytic, amylolytic, and lipolytic (fat-splitting); there is also a fourth distinct, though less important, one known as glucase, which inverts the disaccharides. 
Chemical Composition of Pancreatic Juice (C. Schmidt).

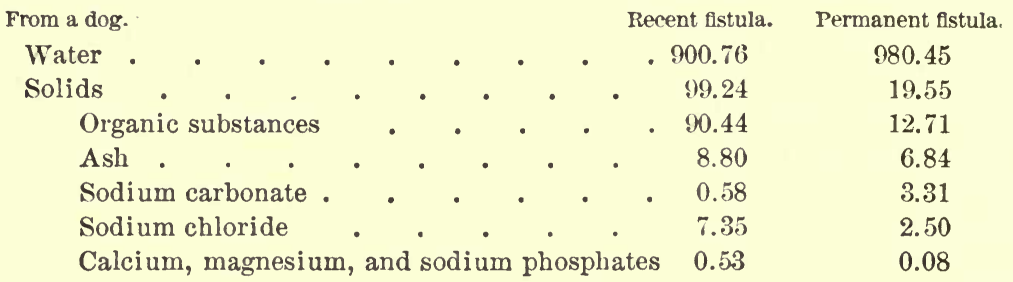

Functions.-(1.) By the aid of its proteolytic or proteid-splitting enzyme, trypsin, it converts proteids into proteoses and peptones, but the process is both more rapid and more complete than in gastric digestion, so that, in the final result, the peptones are greatly in excess of the proteoses. The proteids pass through the same preliminary stages as in gastric digestion, being split at first into alkali-albumin, then into primary proteoses, both proto-proteose and hetero-proteose, and then into deutero-proteose; but the first stages are so transient that it is difficult to detect either the alkali-albumin or primary proteose. For this reason some investigators deny the existence of either alkali-albumin or primary proteose in pancreatic digestion. The deutero-albumoses are easily demonstrated in the earlier stages, but become very scanty later. Anti-albumid is found as a side product in artificial digestion, but is not present in normal digestion. Trypsin also has the power of splitting a certain proportion of peptones into simpler bodies, such as leucin, or amido-caproic acid, tyrosin or paroxyphenyl-amido-propionic acid, lysin, lysatinin, tryptophan, and some other bodies. Leucin and tyrosin have been found in the intestinal contents, so that this destruction of hemipeptone must take place to a certain extent within the body as well as in artificial tryptic digestion.

In laboratory experiments only about oue-half of the peptones can be changed in this way. The more stable portion which canuot be changed is usually known as antipeptone, though it is as yet undecided whether this term represents a single chemical substance or a complex of various bodies; recent experiments, however, tend to show that it represents a mixture of much simpler substances than peptone. There are several theories as to the reason or use of this change into leucin, tyrosin, etc. One of the most plausible is that it saves the body from needless work when too much proteid food has been taken; the breaking down in the intestine of bodies only slightly removed from urea relieres the liver and other glandular organs from the strain of converting an excess of absorbed proteid material into a form in which it can be excreted. Another theory is that leucin, tyrosin, etc., are essential for the physiological 
working of the body, in some unknown way, just as the products of the thyroid gland are.

The formation of the decomposition products indol and skatol is caused by the action of bacteria on proteids, and will be spoken of under another heading.

The albuminous or proteid substances which have not been converted into peptone and absorbed in the stomach, and the partially changed substances, i.e., the proteoses, are converted into peptone by the pancreatic juice, and then in part into leucin and tyrosin.

The ferment trypsin acts best in an alkaline medium, but will act also in a neutral medium, or in the presence of a small amount of combined acid; it will not work in the presence of free acid. It therefore differs from pepsin in being able to act without the aid of any other substance than water. In the process of tryptic digestion, proteid matter does not swell up at first but seems to be corroded.

(2.) Starch is converted into maltose in an exactly similar manner to that which happens with saliva, erythro-dextrine and one or more achroödextrines being the intermediate products. The amylolytic enzyme of the pancreatic juice, which cannot be distinguished from ptyalin, is called amylopsin. The maltose thus formed is converted to dextrose either just before or during its absorption, in which form it passes into the blood. This conversion is in part due to the action of the enzyme glucase.

(3.) Pancreatic juice possesses the property of curdling milk, containing a special (rennet) ferment for that purpose. The ferment is distinct from trypsin, and will act in the presence of an acid (W. Roberts). It is best extracted by brine. The milk-curdling ferment of the pancreas is, in some pancreatic extracts, extremely powerful, insomuch that $1 \mathrm{cc}$. of a brine extract will coagnlate $50 \mathrm{cc}$. of milk in a minute or two.

(t.) Oils and fats are emulsified and saponified by pancreatic secretion. The terms emulsification and saponification may need a little explanation. The former is used to signify an important mechanical change in oils or fats, whereby they are made into an emulsion, or in other words are minutely subdivided into small particles. If a small drop of an cmulsion be looked at under the microscope it, will be seen to be made up of an immense number of minute rounded particles of oil or fat, of varying sizes. The more complete the emulsion the smaller are these particles. An emulsion is formed at once if oil or fat, which when old is slightly acid from the presence of free fatty acid, is mixed with an alkaline solution. Saponification signifies a distinct chemical change in the composition of oils and fats. An oil or a fat being made up chemically of glycerin, a triatomic alcohol, and one or more fatty acid radicles, when an alkali is added to it, and heat is applied, two 
changes take place: firstly, the oil or fat is split up into glycerin, and its corresponding fatty acid; secondly, the fatty acid combines with the alkali, to form a soap which is chemically known as stearate, oleate, or palmitate of potassium or sodium. Thus saponification means a chemical splitting up of oils or fats into new compounds, and emulsification means merely a mechanical splitting of them up into minute particles. The pancreatic juice has been for many years credited with the possession of a special ferment, which was called by Claude Bernard steapsin, and which is a lipolytic or fat-splitting ferment. This ferment has not been isolated, but its presence may be demonstrated by adding portions of the fresh pancreas to butter or other fat and maintaining the proper temperature. Its action is made manifest by the liberation of butyric acid, which smells like rancid butter.

The generally accepted theory is that only a small portion of the fat which is eaten is thus changed into soap, and that the function of the saponified fat is to assist in the emulsitication of the major part, a process which is favorably influenced by the bilc. The proper emulsification of fat is a necessary preliminary to its absorption, for wher in disease the entrance of the pancreatic juice or the bile to the intestine is interfered with, the fæces contains a great excess of fat.

Some recent experiments, however, tend to invalidate the emulsion theory and to prove that the entire fat of the food is changed in the intestine into fatty acids and glycerine; that the fatty acids are entirely, or in part, changed to soaps; and that these soaps, or the mixture of soaps and free fatty acids, are absorbed in solu. tion. The chicf facts favoring this view are that: (1) The action of steapsin is suffieiently rapid to allow the saponitication of a full fatty meal within the ordinary period of digestion; (2) histological examination has never shown that fat particles can pass into a columnar cell, and none have ever becu for nd in the broad striated border of the cell; (3) the fat globules found in columnar colls after a fatty meal grow steadily larger as the period of absorption progresses, indicating that they are deposited from solution; (4) the fatty acids are easily soluble in bile solutions, and the solubility of the soaps is greatly increased by the presence of bile. The fat constituents, according to this theory, are recombined in the columnar cells to form neutral fats.

Conditions facorable to the Action.-These are almost precisely similar to those which have been mentioned as favorable to the action of the saliva, and the reverse. The secretion of the pancreatic juice appears to be, at any rate in some animals, e.g., the rabbit and dog, almost continuous; the flow, however, is not uniform, the amount increases immediately after taking food, and the maximum is reached in from one to one and a half hours, then the amount falls to about one-half, after which a conspicuous rise occurs, and this is followed by a gradual fall to the base line. The nervous mechanism of the pancreatic secretion has only recently been disccvered by Pawlow. Increased flow of secretion will occur on stimulation of the spinal bulb or cord, or of the gland it- 
self, even after division of the vagus. By special methods of investigation Pawlow has found that the innervation of the pancreas is somewhat similar to that of the salivary glands. Stimulation of both the vagus and sympathetic nerves, under proper conditions, will cause a flow of secretion, but the secretion is more abundant in the case of the vagus. Both nerves appear to contain secretory fibres; they are more numerous, however, in the vagus, while trophic fibres or those which cause a building up of the secretion materials in the gland cells are more abundant in the sympathetic. In function the nerves are analogous to the chorda tympani and sympathetic of the submaxillary gland. The gland will continue to secrete after the section of all of its nerves, and in this respect is said to differ from the salivary glands. The secretion appears to be called forth on the introduction of food into the stomach, when the blood-vessels of the gland become much dilated, and the secretion continues, as we have seen, for many hours after a meal; indeed, may be continuous. The pressure of the secretion is not so great as in the case of the salivary glands; the maximum pressure in the duct is said not to exceed $17 \mathrm{~mm}$. of mercury.

The amount of secretion per diem is not definitely known but is approximately estimated to be about half a litre.

\section{The Liver.}

The Liver, the largest gland in the body, situated in the abdomen on the right side chiefly, is an extremely vascular organ, and receives its supply of blood from two distinct sources, viz., from the portal vein and from the hepatic artery, while the blood is returned from it into the vena cava inferior by the hepatic veins. Its secretion, the bile, is conveyed from it by the hepatic duct, either directly into the intestine, or, when digestion is not going on, into the cystic duct, and thence into the gall-bladder, where it accumulates until required. The portal vein, hepatic artery, and hepatic duct branch together throughout the liver, while the hepatic veins and their tributaries run by themselves.

On the outside, the liver has an incomplete covering of peritoneum, and beneath this is a very fine coat of areolar tissue, continuous over the whole surface of the organ. It is thickest where the peritoneum is absent, and is continuous on the general surface of the liver with the fine and, in the human subject, almost imperceptible areolar tissue investing the lobules. At the transverse fissure it is merged in the areolar investment called Glisson's capsule, which, surrounding the portal vein, hepatic artery, and hepatic duct, as they enter at this part, accompanies them in their branches through the substance of the liver.

Structure. The liver is made up of small roundish or oval portions 
called lobules, each of which is about $\frac{1}{20}$ of an inch (about $1 \mathrm{~mm}$.) in diameter, and composed of the minute branches of the portal vein, hepatic artery, hepatic duct, and hepatic vein; while the interstices of these

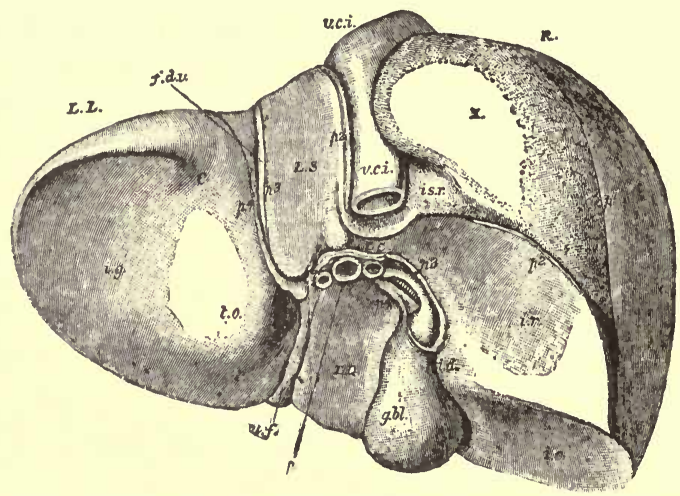

Fig. 266.-The liver from below and behind. L.S., Spigelian lobe; L.C., caudate lobe; L.Q., quadrate lobe: R.L., right lobe; L.L., left lobe; g.bi., gall-bladder; v.c.i., inferior vena cava u.f., umbilical fissure; f.d.v., fissure of the ductus venosus; $p$, portal fissure with portal veut, hepatic artery and bile-duct. (Wesley, from a His model.)

vessels are filled by the liver cells. The hepatic cells (fig. 254), which form the glandular or secreting part of the liver, are of a spheroidal form, somewhat polygonal from mutual pressure about $\frac{1}{800}$ to $\frac{1}{1000}$ inch (about $\frac{1}{32}$ to $\frac{1}{40} \mathrm{~mm}$.) in diameter, possessing one, sometimes two nuclei. The cell-substance contains numerous fatty molecules. and possibly some granules of bile-pigment, as well as a variable amount of glycogcn. The cells sometimes exhibit slow amoboid movements. They are held together by a very delicate sustentacular tissue, continuous with the interlobular connective tissue.

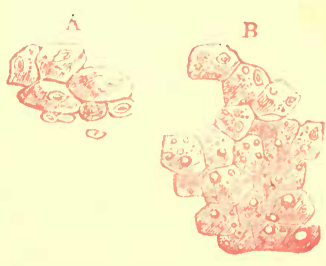

Fig. 267.

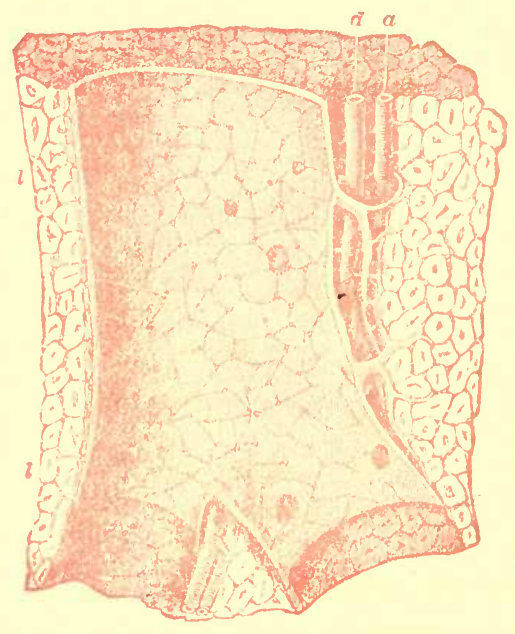

Fig. 268.

Fig. 267.-A. Liver-cells. B. Ditto, containing various-sized particles of fat.

Fig. 268.-Longitudinal section of a portal canal, containing a portal vein, hepatic artery and hepatic duct, from the pig. P, branch of vena portæ, situate in a portal canal formed among the lobules of the liver, $l, l$, and giving off vaginal branches; there are also seen within the large portal vein numerous orifices of the smallest interlobular veins arising directly from it; $a_{1}$ hepatic artery; $d$, hepatic duct. $\times 5$. (Kiernan). 
To understand the distribution of the blood-vessels in the liver, it will be well to trace, first, the two blood-vessels and the duct which enter the organ on the under surface at the transverse fissure, viz., the portal vein, hepatic artery, and hepatic duct. As before remarked, all three run in company, and their appearance on longitudinal section is shown in fig. 268. Running together through the substance of the liver, they are contained in small channels called portal canals, their immediate investment being a sheath of areolar tissue continuous with Glisson's capsule.

To take the distribution of the portal vein first:-In its course through the liver this ressel gives off small branches which divide and subdivide between the lobules surrounding them and limiting them, and from this circumstance called inter-lobular veins. From these small vessels a dense capillary network is prolonged into the substance of the lobule,

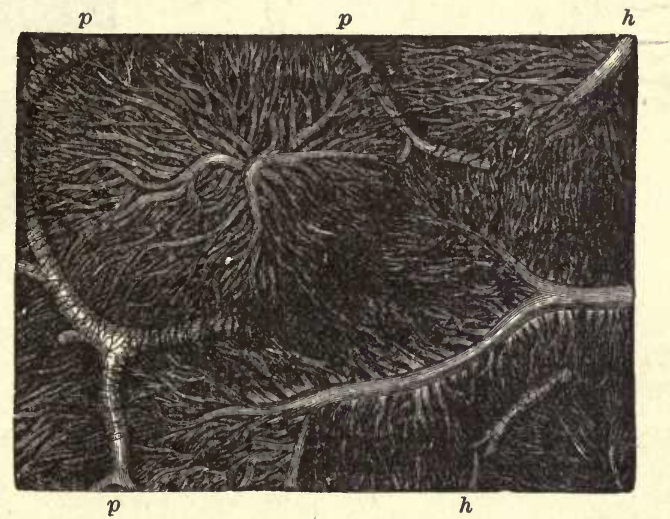

Fig. 269 -Capillary network of the lobules of the rabbit's liver. The figure is taken from a very successful injection of the hepatic veins, made by Harting: it shows nearly the whole of two lobules, and parts of three others; $p$, portal branches running in the interlobular spaces; $h$, hepatic veins penetrating and radiating from the centre of the lobules. $\times 45$. (Kölliker.)

and this network gradually gathering itself up, so to speak, into larger vessels, converges finally to a single small vein, occupying the centre of the lobule, and hence called intra-lobular. This arrangement is well seen in fig. 269, which represents a transverse section of a lobule.

The small intra-lobular veins discharge their contents into veins called sub-lobular ( $h h h$, fig. 270 ), while these again, by their union, form the main branches of the hepatic veins, which leave the posterior border of the liver to end by two or three principal trunks in the inferior vena cava, just before its passage through the diaphragm. The sub-lobular and hepatic veins, unlike the portal vein and its companions, have little or no areolar tissue around them, and their coats being very thin, they form little more than mere channels in the liver substance which closely surrounds them.

The manner in which the lobules are connected with the sublobular 
veins by means of the small intralobular veins has been likened to a twig having leaves without footstalks - the lobules representing the leaves, and the sublobular vein the small branch from which it springs.

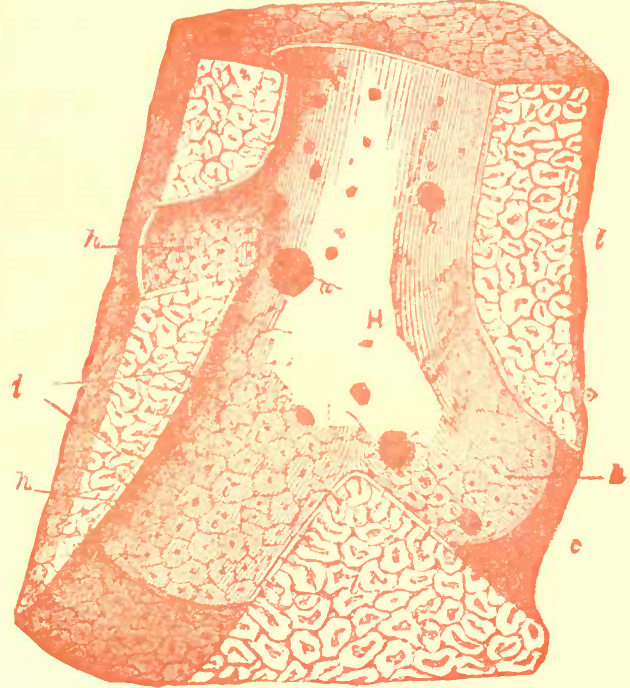

Fig. 270

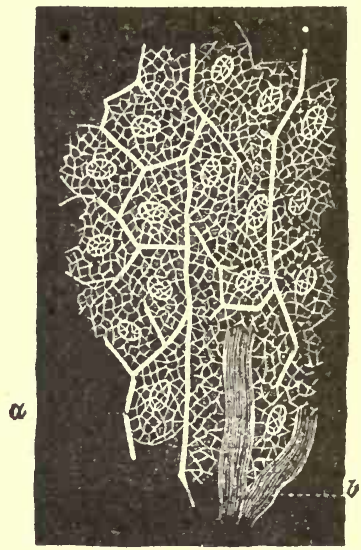

Fig. $2 \% 1$.

Fig. $2 \% 0$-Section of a portion of liver passing longitudinally through a considerable hepatic vein, from the pig. H. hepatic renous trunk, against which the sides of the lobules $(l)$ are applied; $h, h, h$, sublobular hepatic veins, on which the bases of the lobules rest, and through the coats of which they are seen as polygonal figures; $i$, mouth of the intralobular veins, opening into the sublobular veins; $i^{\prime}$, intralobular veins shown passing up the centre of some divided lobules; $l, l$, cut surface of the liver; $c, c$, walls of the hepatic venous canal, formed by the polygonal bases of the lobules. $\times 5$. (Kiernan.)

Fig. 2/1-Portion of a lobule of liver. $a$, bile capillaries between liver-cells, the network in which is well seen; $b$, blood capillaries. $\times 350$. (Klein and Noble Smith.)

The hepatic artery, the chief function of which is to distribute blood for nutrition to Glisson's capsule, the walls of the ducts and blood-vessels, and other parts of the liver, is distributed in a very similar manner
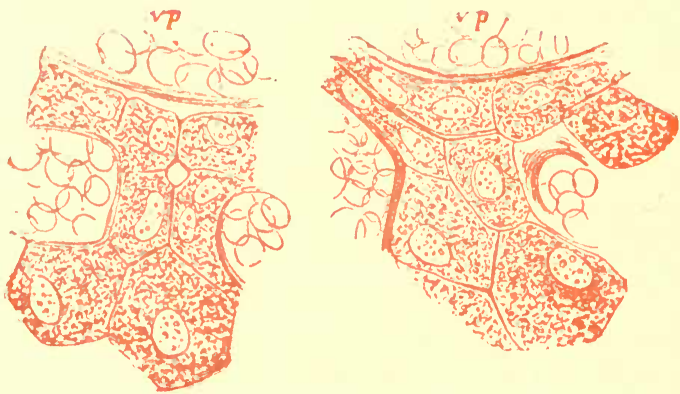

Fig. 272 - Hepatic cells and bile capillaries, from the liver of a child three months' old. Both figures represent fragments of a section carried through the peripliery of a lobule. The red corpuscles of the blood are recognized by their circular contour; vp, corresponds to an interlobular vein in immediate proximity with which are the epithelial cells of the biliary ducts, to which, at the lower part of the figures, the much larger bepatic cells suddenly succed. (E. Hering.)

to the portal vein, its blood being returned by small branches either 
into the ramifications of the portal vein, or into the capillary plexus of the lobules which connect the inter-and intra-lobular veins.

The hepatic duct divides and subdivides in a manner very like that of the portal vein and hepatic artery, the larger branches being lined by cylindrical, and the smaller by small polygonal epithelium.

The bile-capillaries commence between the hepatic cells, and are bounded by a delicate membranous wall of their own. They appear to be always bounded by hepatic cells on all sides, and are thus separated from the nearest blood-capillary by at least the breadth of one cell (figs. 271 and 272$)$.

\section{The Gall-bladder.}

The Gall-bladder ( $g . b l$. fig. 266) is a pyriform bag, attached to the under surface of the liver, and supported also by the peritoneum, which passes below it. The larger end, or fundus, projects beyond the front margin of the liver; while the smaller end contracts into the cystic duct.

Structure.-The walls of the gall-bladder are constructed of three principal coats. (1) Externally (excepting that part which is in contact with the liver) is the serous coat, which has the same structure as the peritoneum, with which it is continuous. Within this is (2) the fibrous or areolar coat, constructed of tough fibrous and elastic tissue, with which is mingled a considerable number of plain muscular fibres, both longitudinal and circular. (3) Internally the gall-bladder is lined by mucous membrane, and a layer of columnar epithelium. The surface of the mucous membrane presents to the naked eye a minutely honeycombed appearance from a number of tiny polygonal depressions with intervening ridges, by which its surface is mapped out. In the cystic duct the mucous membrane is raised up in the form of crescentic folds, which together appear like a spiral valve, and which minister to the function of the gall-bladder in retaining the bile during the interval of digestion.

The gall-bladder and all the main biliary ducts are provided with mucous glands, which open on the internal surface.

\section{Functions of the Liver.}

The function of the liver in connection with digestion is to secrete the bile, and may be now considered. The other functions in connection with the general metabolism of the body, and particularly its glycogenic function, will be discussed later on. First of all it will be as well to take the composition and functions of the bile, and afterward to discuss its mode of secretion. 


\section{The Bile.}

Properties.-The bile is a somewhat viscid fluid, of a yellow, reddishyellow or green color, a strongly bitter taste, and, when fresh, with a scarcely perceptible odor: it has a neutral or slightly alkaline reaction, and its specific gravity is about 1020. Its color and degree of consistence vary much, quite independent of disease; but, as a rule, bile becomes gradually more deeply colored and thicker as it advances along its ducts, or when it remains long in the gall-bladder, wherein, at the same time, it becomes more viscid and ropy, darker, and more bitter, mainly from its greater degree of concentration, on account of partial absorption of its water, but also from being mixed with mucus.

\section{Chemical Composition of Human Bile. (Frerichs.)}

\begin{tabular}{|c|c|c|c|c|}
\hline $\begin{array}{l}\text {-Bile salts } \\
\text { Fat } \\
\text { Cholesterin } \\
\text { Mucus and }\end{array}$ & coloring & matters & . & \\
\hline
\end{tabular}

859.2

140.8

1000.0

(a) Bile salts, sometimes termed Bilin, can be obtained as colorless, exceedingly deliquescent crystals, soluble in water, alcohol, and alkaline solutions, giving to the watery solution the taste and general characters of bile. 'They consist of sodium salts of glycocholic and taurocholic acids. The formula of the former salt being $\mathrm{C}_{26} \mathrm{H}_{43} \mathrm{NaNO}_{6}$, and of the latter $\mathrm{C}_{26} \mathrm{H}_{44} \mathrm{NaNO}_{7} \mathrm{~S}$.

The bile acids are easily decomposed by the action of dilute acids or alkalies thus :

$$
\begin{gathered}
\underset{\text { Glycocholic Acid. }}{\mathrm{C}_{26} \mathrm{H}_{43} \mathrm{NO}_{6}+\mathrm{H}_{2} \mathrm{O}}=\underset{\text { Glycin. }}{\mathrm{C}_{2} \mathrm{H}_{5} \mathrm{NO}_{2}}+\underset{\text { Cholic Acid. }}{\mathrm{C}_{24} \mathrm{H}_{40} \mathrm{O}_{5}} \\
\underset{\text { Taurocholic Acid. }}{\text { and } \mathrm{C}_{26} \mathrm{H}_{45} \mathrm{NO}_{7} \mathrm{~S}}+\mathrm{H}_{2} \mathrm{O}=\underset{\text { Taurin. }}{\mathrm{C}_{2} \mathrm{H}_{7} \mathrm{NO}_{3} \mathrm{~S}}+\underset{\text { Cholic Acid. }}{\mathrm{C}_{24} \mathrm{H}_{40} \mathrm{O}_{5}}
\end{gathered}
$$

Glycin, or glycocin, is amido-acetic acid, i.e., acetic acid $\mathrm{C}_{2} \mathrm{H}_{4} \mathrm{O}_{2}$, with one of the atoms of $\mathrm{H}$ replaced by the radical amidogen $\mathrm{NH}_{2}, \mathrm{C}_{2} \mathrm{H}_{3}\left(\mathrm{NH}_{2}\right) \mathrm{O}_{2}$, $\mathrm{C}_{2} \mathrm{H}_{5} \mathrm{NO}_{3}$. Taurin likewise is amido-isethionic acid. Isethionic acid is sulphurous acid $\mathrm{H}_{2} \mathrm{SO}_{3}$, in which an atom of $\mathrm{H}$ is replaced by the monotomic radicle oxy-ethylene, $\mathrm{C}_{2} \mathrm{H}_{4} \mathrm{OH}$, viz., $\mathrm{H}\left(\mathrm{C}_{2} \mathrm{H}_{4} \mathrm{OH}\right) \mathrm{SO}_{3}$, and in amido-isethionic acid, the $\mathrm{OH}$ hydroxyl in this radicle is replaced by amidogen $\mathrm{NH}_{2}$, thus $\mathrm{H}\left(\mathrm{C}_{2} \mathrm{H}_{4} \mathrm{NH}_{2}\right) \mathrm{SO}_{3}=\mathrm{C}_{2} \mathrm{H}_{7} \mathrm{NSO}_{3}$. The proportion of these two salts in the bile of different animals varies, e.g., in ox bile the glycocholate is in great excess, whereas the bile of the dog, cat, bear, and other carnivora contains taurocholate alone; in human bile the glycocholate is in excess (4.8 to 1.5).

Preparation of Bile Salts. -Bile salts may be prepared in the following manner: mix bile which has been evaporated to a quarter of its bulk with 
animal charcoal, and evaporate to perfect dryness in a water bath. Next ex. tract the mass while still warm with absolute alcohol. Separate the alcoholic extract by filtration, and to it add perfectly anhydrous ether as long as a precipitate is thrown down. The solution and precipitate should be set aside in a closely stoppered bottle for some days, when crystals of the bile salts or bilin will have separated out. The glycocholate may be separated from the taurocholate by dissolving bilin in water, and adding to it a solution of neutral lead acetate, and then a little basic lead acetate, when lead glycocholate separates out. Filter and add to the filtrate lead acetate and ammonia, a precipitate of lead taurocholate will be formed, which may be filtered off. In both cases, the lead may be got rid of by suspending or dissolving in hot alcohol, adding hydrogen sulphide, filtering and allowing the acids to separate out by the addition of water.

The Test for bile salts is known as Pettenkofer's. If to an aqueous solution of the salts strong sulphuric acid be added, the bile acids are first of all precipitated, but on the further addition of the acid are redissolved. If to the solution a drop of solution of cane sugar be added, a fine deep cherry red to purple color is developed.

The reaction will also occur on the addition of grape or fruit sugar instead of cane sugar, slowly with the first, quickly with the last; and a color similar to the above is produced by the action of sulphuric acid and sugar on albumen, the crystalline lens, nerve tissue, oleic acid, pure ether, cholesterin, morphia, codeia and amylic alcohol. The substance which gives the reaction is furfuraldehyde, formed by the action of sulphuric on sugar. Furfur-aldehyde with cholalic acid gives the red color.

The spectrum of Pettenkofer's reaction, when the fluid is moderately diluted, shows four bands-the most marked and broadest at E, and a little to the left; another at $\mathrm{F}$; a third between D and $\mathrm{E}$, nearer to $\mathrm{D}$; and the fourth near $\mathrm{D}$.

(b) The yellow coloring matter of the bile of man and the Carnivora is termed Bilirubin or Bilifulvin $\left(\mathrm{C}_{16} \mathrm{H}_{18} \mathrm{~N}_{2} \mathrm{O}_{3}\right)$ crystallizable and insoluble in water, soluble in chloroform or carbon disulphide; a green coloring matter, Biliverdin $\left(\mathrm{C}_{16} \mathrm{H}_{18} \mathrm{~N}_{2} \mathrm{O}_{4}\right)$ which always exists in large amount in the bile of Herbivora, being formed from bilirubin on exposure to the air, or by subjecting the bile to any other oxidizing agency, as by adding nitrous acid. Biliverdin is soluble in alcohol, glacial acetic acid, and strong sulphuric acid, but insoluble in water, in chloroform and ether. It is usually amorphous but may sometimes crystallize in green rhombic plates. When the bile has been long in the gall-bladder, a third pigment, Biliprasin, may be also found in small amount.

In cases of biliary obstruction, the coloring matter of the bile is reabsorbed and circulates with the blood, giving to the tissues the yellow tint characteristic of jaundice.

The coloring matters of human bile do not appear to give characteristic absorption spectra; but the bile of the Guinea-pig, rabbit, mouse, 
sheep, ox, and crow do so, the most constant of which appears to be a band at F. The bile of the sheep and ox gives three bands in a thick layer, and four or five bands with a thinner layer, one on each side of $\mathrm{D}$, one near $\mathrm{E}$, and a faint line at $\mathrm{F}$. (McMunn.)

'There seems to be a close relationship between the coloring matters of the blood and of the bile, and it may be added, between these and that of the urine (urobitin), and of the fæces (stercobilin) also; it is probable they are, all of them, varieties of the same pigment, or derived from the same source. Indeed it is maintained that Urobilin is identical with Hydrobilirubin, a substance which in alkaline solution gives a green fluorescence with zinc chloride, which is obtained from bilirubin by the action of sodium amalgam, or by the action of sodium amalgam on alkaline hæmatin; both urobilin and hydrobilirubin giving a characteristic absorption band between $\mathrm{b}$ and $\mathrm{F}$. They are also identical with stercobilin, which is formed in the alimentary canal from bile pigments.

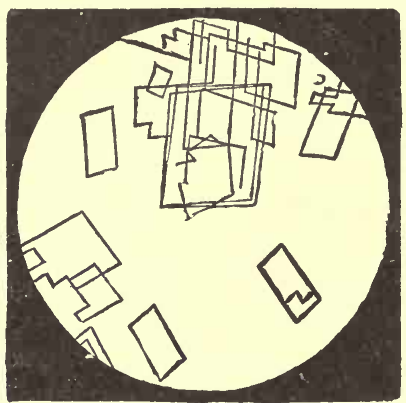

Fig. 273.-Crystalline scales of cholesterin.

The Test (Gmelin's) for the presence of bile-pigment consists of the addition of a small quantity of nitric acid, yellow with nitrous acid; if bile be present, a play of colors is produced, beginning with green and passing throngh blue and violet to red, and lastly to yellow. 'The final yellow substance has been called choletelin. The spectrum of Gmelin's test gives a black band extending from near $\mathrm{b}$ to beyond $\mathrm{F}$.

(c) Fatty substances are found in variable proportions in the bile. Besides these saponifiable fats, there is a small quantity of Cholesterin, which is an alcohol, and, with the free fats, is probably held in solution by the bile salts. It is a body belonging to the class of monatomic alco. hols $\left(\mathrm{C}_{27} \mathrm{H}_{45} \mathrm{OH}\right.$, Obermüller), and crystallizes in rhombic plates (fig. 273). It is insoluble in water and cold alcohol, but dissolves easily in boiling alcohol or in ether. It gives a red color with strong sulphuric acid, and with nitric acid and ammonia; also a play of colors beginning with blood red and ending with green on the addition of sulphuric acid and chloroform. Lecithin $\left(\mathrm{C}_{42} \mathrm{H}_{84} \mathrm{NPO}_{9}\right)$ is also found: it is a combination of cholin with glycerophosphoric acid in which two of the hydrogen 
atoms of the glycerine are replaced by radicals of the fatty acids, usually oleic and palmitic acids.

(d) The Mucus in bile is derived from the mucous membrane and glands of the gall-bladder, and of the hepatic ducts. It constitutes the residue after bile is treated with alcohol. The epithelium with which it is mixed may be detected in the bile with the microscope in the form of cylindrical cells, either scattered or still held together in layers. 'To the presence of the mucus is probably to be ascribed the rapid decomposition of the bile; for, according to Berzelius, if the mucus be separated, it will remain unchanged for many days.

(e) The Saline or inorganic constituents of the bile are similar to those found in most other secreted fluids. It is possible that the carbonate and neutral phosphate of sodium and potassium, found in the ashes of bile, are formed in the incineration, and do not exist as such in the fluid. Oxide of iron is said to be a common constituent of the ashes of bile, and copper is generally found in healthy bile, and constantly in biliary calculi.

(f) Gas.-Small amounts of carbonic acid, oxygen, and nitrogen gases, may be extracted from bile.

Functions of the Bile.-Though it is not a true digestive fluid, in that it has no ferment and digests nothing itself, yet it must be regarded as an important aid to digestion for the following reasons: There is little doubt that it (a) assists in emulsifying the fats of the food, and thus rendering them capable of passing into the acteals by absorption. For it has appeared in some experiments in which the common bile-duct was tied, that, although the process of digestion in the stomach was unaffected, chyle was no longer well formed; the contents of the lacteals consisting of clear, colorless fluid, instead of being opaque and white, as they ordinarily are, after feeding. It is, however, the combined action of the bile with the pancreatic juice to which the emulsification is due ratber than to that of the bile alone. The bile itself has a very feeble emulsifying power. If the theory be accepted that fats are absorbed as fatty acids and soaps, in solution, the action of the bile becomes very important because solutions of bile salts have the power of dissolving the fatty acids.

(b) It is probable, also, that the moistening of the mucous membrane of the intestines by bile facilitates absorption of fatty matters through it.

(c) The bile, like the gastric fluid, has a certain but not very considerable antiseptic power, and may serve to prevent the decomposition of food during the time of its sojourn in the intestines. Experiments show that the contents of the intestines are much more fotid after the common bile-duct has been tied than at other times: moreover, it is found that the mixture of bile with a fermenting fluid stops or spoils the process of fermentation. This function may, very probably, be ex- 
plained by its so aiding fat digestion that the fats are absorbed before they can decompose.

(d) 'The bile has also been considered to act as a natural purgative by promoting an increased secretion of the intestinal glands, and by stimulating the intestines to the propulsion of their contents. This view receives support from the constipation which ordinarily exists in jaundice, from the diarrhœa which accompanies excessive secretion of bile and from the purgative properties of ox-gall.

(e) The bile appears to have the power of precipitating the gastric proteoses and peptones, together with the pepsin, which is mixed up with them, as soon as the contents of the stomach meet it in the duodenum. It thus stops the action of the pepsin. The purpose of this operation is probably both to delay any change in the proteoses until the pancreatic juice can act upon them, and also to prevent the pepsin from exercising its solvent action on the ferments of the pancreatic juice. In some way its presence seems also to aid the action of trypsin.

(f) As an excrementitious substance, the bile may serve especially as a medium for the separation of certain highly carbonaceous substances from the blood; and its adaptation to this purpose is well illustrated by the peculiarities attending its secretion and disposal in the fotus. During intra-uterine life, the lungs and the intestinal canal are almost inactive; there is no respiration of open air or digestion of food; these are unnecessary, on account of the supply of well-elaborated nutriment received by the vessels of the fœtus at the placenta. The liver, during the same time, is proportionately larger than it is after birth, and the secretion of bile is active, although there is no food in the intestinal canal upon which it can exercise any digestive property. At birth, the intestinal canal is full of concentrated bile, mixed with intestinal secretion, and this constitutes the meconium, or fæces of the fœtus. In the fœtus, therefore, the main purpose of the secretion of bile must be directly excretive. Probably all the bile secreted in fœtal life is incorporated in the meconium, and with it discharged, and thus the liver may be said to discharge a function in some sense vicarious of that of the lungs. For, in the fœtus, nearly all the blood coming from the placenta passes through the liver, previous to its distribution to the several organs of the body; and the abstraction of certain substances will purify it, as in extra-uterine life it is purified by the separation of carbon dioxide and water at the lungs.

Mode of Secretion and Discharge.-The secretion of bile is continually going on, but is retarded during fasting, and accelerated on taking food. This has been shown by tying the common bile-duct of a dog, and establishing a fistulous opening between the skin and gall-bladder, whereby all the bile secreted was discharged at the surface. It was noticed that when the animal was fasting, sometimes not a drop of bile 
was discharged for several hours; but that, in about ten minutes after the introduction of food into the stomach, the bile began to flow abundantly, and continued to do so during the whole period of digestion.

The bile is formed in the hepatic cells; thence, being discharged into the minute hepatic ducts, it passes into the larger trunks, and from the main hepatic duct may be carried at once into the duodenum. This probably happens only while digestion is going on, i.e., for 5 to 7 hours after the introduction of food into the stomach; during fasting, it regurgitates from the common bile-duct through the cystic duct, into the gall-bladder, where it accumulates till, in the next period of digestion, it is discharged into the intestine. The gall-bladder thus fulfils its office, that of a reservoir; for its presence enables bile to be constantly secreted, yet insures its employment in the service of digestion, aithough digestion is periodic, and the secretion of bile constant.

The mechanism by which the bile passes into the gall-bladder is simple. The orifice through which the common bile-duct communicates with the duodenum is narrower than the duct, and appears to be closed, except when there is sufficient pressure behind to force the bile through it. The pressure exercised upon the bile secreted during the intervals of digestion appears insufficient to overcome the force with which the orifice of the duct is closed; and the bile in the common duct, finding no exit in the intestine, traverses the cystic duct, and so passes into the gall-bladder, being probably aided in this retrograde course by the peristaltic action of the ducts. The bile is discharged from the gall-bladder and enters the duodenum on the introduction of food into the small intestine: being pressed on by the contraction of the coats of the gall-bladder, and of the common bile-duct also; for both these organs contain unstriped muscular fibre-cells. Their contraction is excited by the stimulus of the food in the duodenum acting so as to produce a reflex movement, the force of which is sufficient to open the orifice of the common bile-duct, which is closed by a sphincter.

Bile is not pre-formed in the blood. As just observed, it is secreted by the hepatic cells, although some of its constituents may be brought to them almost in the condition for immediate secretion. The blood from which the liver cells secrete the bile is that supplied to them by the portal vein. This is shown by the alterations which occur in the process on the alteration of the pressure in the portal system. If the portal vein be obstructed, the amount of bile secreted diminishes, and is ultimately suppressed, death resulting. It has, however, been shown that under extraordinary circumstances bile may be secreted by the aid of the blood from the hepatic artery, since if a branch of the portal vein be tied, the part of the liver supplied by it continues to secrete bile, though in diminished quantity. When the discharge of the bile into the intestine is prevented by an obstruction of some kind, as by a gall- 
stone blocking the hepatic duct, it is reabsorbed in great excess into the blood, and, circulating with it, gives rise to the well-known phenomena of jaundice. This is explained by the fact that the pressure of secretion in the ducts although normally very low, not exceeding 15 $\mathrm{mm}$. in the dog, is still higher than that of the portal veins, and if it exceeds $16 \mathrm{~mm}$. the secretion although formed ceases to be poured out, and if the opposing force be increased, the bile passes into the bloodvessels through the lymphatics, and the yellow color appears in the skin and in the secretions, and constitutes the condition of jaundice. In jaundice the fæces are light colored and highly offensive, there is constipation, the heart beats slowly, and from the presence of bile salts as well as bile pigment in the blood, the red blood corpuscles may be in part dissolved. The latter action results in the presence of hæmoglobin and of an additional amount of bile pigment in the urine.

Disposal of the Bile.-The simple excretion of the fotal bile makes it probable that the bile in extra-uterine life is also, at least in part, destined to be discharged as excrementitious. The analysis of the frees shows, however, that (except when rapidly discharged in purgation) they contain very little of the bile secreted, probably not more than one-sixteenth part of its weight, and that this portion includes chiefly its coloring matter in the form of stercobilin, and some of its fatty matters and mucin, but its salts to only a very slight degree, almost all of which have been reabsorbed from the intestines into the blood. The bilirubin is in part converted into urobilin and is reabsorbed and excreted by the kidneys in the urine.

The elementary composition of bile-salts shows such a preponderance of carbon and hydrogen that probably, after absorption, they combine with oxygen, and are excreted in the form of carbonic acid and water. The change after birth, from the direct to the indirect mode of excretion of the bile may, with much probability, be connected with a purpose in relation to the development of heat. The temperature of the fœtus is largely maintained by that of the parent, but, in extra-uterine life, there is (as one may say) a waste of material for heat when any excretion is discharged unoxidized; the carbon and hydrogen of bilin, therefore, instead of being ejected in the fæces, to a very large extent (viz., $\frac{7}{9}$ ), are reabsorbed, in order that they may be combined with oxygen, and that in the combination heat may be generated. It appears that taurocholic acid may easily be split up in the intestine into taurin and cholalic acid, and the same is probable of glycocholic acid. Taurin, glycin, and cholalic acid have all been detected in small amounts in the fæces. So that the bile is in part excreted, but in part is reabsorbed from the intestine (chiefly the large), and returned to the liver. What may be the ultimate destination of these altered or unaltered constituents is unknown. Glycin is supposed to go partly to form urea, and taurin is ex- 
creted to a slight extent in the urine as tauro-carbamic acid, but it is probable that although part of this may unite to re-form glycocholic or taurocholic acid, the remainder is united with oxygen, and is burnt off in the form of carbonic acid and water.

A substance, contained in the fæces, and named stercorin, is closely allied to cholesterin. Ten grains and a half of stercorin are excreted daily (A. Flint).

From the peculiar manner in which the liver is supplied with much of the blood that flows through it, it is probable that this organ is excretory, not only for such hydro-carbonaceous matters as may need expulsion from the blood, but that it serves for the direct purification of the stream which, arriving by the portal vein, has just gathered up various substances in its course through the digestive organs-substances which may need to be expelled almost immediately after their absorption. For it is easily conceivable that many things may be taken up during digestion, which not only are unfit for purposes of nutrition, but which would be positively injurious if allowed to mingle with the general mass of the blood. The liver, therefore, may be supposed placed in the only road by which such matters can pass unchanged into the general current, jealously to guard against their further progress, and turn them back again into an excretory channel. The frequency with which metallic poisons are either excreted by the liver, or intercepted and retained, often for a considerable time, in its own substance, may be adduced as evidence for the probable truth of this supposition.

The secretion of the bile by the hepatic cells is undoubtedly influenced by the amount of blood supplied to them. This is well seen after a meal, when the amount of blood passing through the portal circulation in consequence of the congestion of the secreting organs of the abdomen is greatly increased, and with it the bile secretion. It is, however, probable that the secretion of the cells is in some more direct way under the control of the nervous system, but how this influence is exercised is unknown. The antecedents of the various substances of the bile from which the cells manufacture its chief constituents are not exactly known. It is surmised that the bilirubin is formed from hæmoglobin brought from the spleen either actually dissolved in the plasma of the blood or in such a condition in the corpuscles as to be easily acted upon by the liver cells, by which the iron is separated. The bile salts are, at any rate in part, formed simply by the conjunction of glycin and taurin with cholalic acid, all of which may be brought to the liver in the portal blood, but failing this it is probable that the hepatic cells can produce these substances anew. 


\section{The Intestinal Secretion, or Succus Entericus.}

On account of the difficulty in isolating the secretion of the glands in the wall of the intestine (Brunner's and Lieberkühn's) from other secretions poured into the canal (gastric juice, bile, and pancreatic secretion), but little is known regarding the composition of the intestinal juice, or succus entericus.

It is said to be a yellowish alkaline fluid with a specific gravity of 1011, and to contain about 2.5 per cent of solid matters (Thiry).

Functions. - The secretion is said to be able to convert proteids into peptones, and to convert starch into sugar, but the evidence in favor of these actions is insufficient. The chief function of the juice is to act upon sugars. It possesses the power of converting cane into grape sugar, and maltose into glucose. It also contains a milk-curdling ferment.

The reaction which represents the conversion of cane sugar into grape sugar may be represented thus:

$$
\underset{\text { Saccharose. }}{2 \mathrm{C}_{12} \mathrm{H}_{22} \mathrm{O}_{11}}+\underset{\text { Water. }}{2 \mathrm{H}_{2} \mathrm{O}}=\underset{\text { Dextrose. }}{\mathrm{C}_{12} \mathrm{H}_{24} \mathrm{O}_{12}}+\underset{\text { Lævulose. }}{\mathrm{C}_{12} \mathrm{H}_{24} \mathrm{O}_{12}}
$$

The conversion is probably effected by means of a hydrolytic ferment, invertin (Bernard).

\section{Summary of the Digestive Changes in the Small Intestine.}

In order to understand the changes in the food which occur during its passage through the small intestine, it will be well to refer briefly to the state in which it leaves the stomach through the pylorus. It has been said before, that the chief office of the stomach is not only to mix into an uniform mass all the varieties of food that reach it through the œsophagus, but especially to dissolve the nitrogenous portion by means of its secretion. The fatty matters, during their sojourn in the stomach, become more thoroughly mingled with the other constituents of the food taken, but are not yet in a state fit for absorption. The conversion of starch into sugar, which began in the mouth, has been interfered with, if not altogether stopped. The soluble matters-both those which were so from the first, as sugar and saline matter, and the gastric peptones-have begun to disappear by absorption into the blood-vessels, and the same thing has befallen such fluids as may have been swallowed.

'The thin pultaceous chyme, therefore, which, during the whole period of gastric digestion, is being constantly squeezed or strained through the pyloric orifice into the duodenum, consists of albuminous matter, broken down, dissolving and half dissolved; fatty matter broken down and 
melted, but not dissolved at all; starch very slowly in process of conversion into sugar, and as it becomes sugar, also dissolving in the fluids with which it is mixed; while with these are mingled gastric fluid, and fluid that has been swallowed, together with such portions of the food as are not digestible, and will be finally expelled as part of the fæces.

On the entrance of the chyme into the duodenum, it is subjected to the influence of the bile and pancreatic juice, which are then poured out, and also to that of the succus entericus. All these secretions have a more or less alkaline reaction, and by their admixture with the gastric chyme, its acidity becomes less and less until at length, at about the middle of the small intestine, the reaction becomes alkaline and continues so as far as the ileo-cæcal valve.

The special digestive functions of the small intestine may be taken in the following order:-

(1.) One important duty of the small intestine is the alteration of the fat in such a manner as to make it fit for absorption; and there is no doubt that this change is chiefly effected in the upper part of the small intestine. What is the exact share of the process, however, allotted respectively to the bile and to the pancreatic secretion, is still uncertain. The fat is changed in two ways. (a.) To a slight extent it is chemically decomposed by the alkaline secretions with which it is mingled, and a soap is the result. (b.) It is emulsionized, i.e., its particles are minutely subdivided and diffused, so that the mixture assumes the condition of a milky fluid, or emulsion. As will be seen in the next Chapter, most of the fat is absorbed by the lacteals of the intestine, but a small part, which is saponified, is also absorbed by the blood-vessels.

(2.) The albuminous substances which have been partly dissolved in the stomach, and have not been absorbed, are subjected chiefly to the action of the pancreatic juice. The pepsin is rendered inert by being precipitated together with the gastric peptones and proteoses, as soon as the chyme meets with bile. By these means the pancreatic ferment trypsin is enabled to proceed with the further conversion of the proteoses into peptones, and part of the peptones (hemipeptone) into leucin and tyrosin. Albuminous substances, which are chemically altered in the process of digestion (peptones) and gelatinous matters similarly changed, are absorbed by the blood-vessels and lymphatics of the intestinal mucous membrane. Albuminous matters, in state of solution, which have not undergone the peptonic change, are probably, from the difficulty with which they diffuse, absorbed, if at all, almost solely by the lymphatics.

(3.) The starchy, or amyloid portions of the food, the conversion of which into maltose was more or less interrupted during their stay in the stomach, are now acted on briskly by the pancreatic juice and the succus entericus; and the sugar in the form of maltose is dissolved in the intes- 
tinal fluids, and is absorbed chiefly by the blood-vessels. During or just prior to its absorption, maltose is converted into dextrose.

(4.) Saline and saccharine matters, such as common salt, and cane sugar, if not in a state of solution beforehand in the saliva or other fluids which may have been swallowed with them, are at once dissolved in the stomach, and if not here absorbed, are soon taken up in the small intestine; the blood-vessels, as in the last case, being chiefly concerned in the absorption. Cane sugar is in part or wholly converted into grape sugar before its absorption. This is accomplished partially in the stomach, but also by a ferment in the succus entericus.

(5.) The liquids, including in this term the ordinary drinks, as water, wine, ale, tea, etc., which may have escaped. absorption in the stomach, are absorbed probably very soon after their entrance into the intestine; the fluidity of the contents of the latter being preserved more by the constant secretion of fluid by the intestinal glands, pancreas, and liver, than by any given portion of fluid whether swallowed or secreted, remaining long unabsorbed. From this fact, therefore, it may be gathered that there is a kind of circulation constantly proceeding from the intestines into the blood, and from the blood into the intestines again; for as all the fluid-a very large amount-secreted by the intestinal glands, must come from the blood, the latter would be too much drained, were it not that the same fluid after secretion is again reabsorbed into the current of blood-going into the blood charged with nutrient products of digestion-coming out again by secretion through the glands in a comparatively unchanged condition.

At the lower end of the small intestine, the chyme, still thin and pultaceous, is of a light yellow color, and has a distinctly fæcal odor. This odor depends upon the formation of indol and other substances to be again alluded to. In this state it passes through the ileo-cæcal opening into the large intestine.

\section{Summary of the Digestive Changes in the Large Intestine.}

The changes which take place in the chyme in the large intestine are probably only the continuation of the same changes that occur in the course of the food's passage throngh the upper part of the intestinal canal. From the abscnce of villi, however, we may conclude that absorption, especially of fatty matter, is in great part completed in the small intestine; while, from the still half-liquid, pultaceous consistence of the chyme when it first enters the cæcum, there can be no doubt that the absorption of liquid is not by any means concluded. The peculiar odor, moreover, which is acquired after a short time by the contents of the large bowel, would seem to indicate a further chemical change in 
the alimentary matters or in the digestive fluids, or both. The acid reaction, which had disappeared in the small bowel, again becomes very manifest in the cæcum-probably from acid fermentation processes in some of the materials of the food.

There seems no reason to conclude that any special secondary digestive process occurs in the cæcum or in any other part of the large intestine. Probably any constituent of the food which has escaped digestion and absorption in the small bowel may be digested in the large intestine; and the power of this part of the intestinal canal to absorb fatty, albuminous, or other matters, may be gathered from the good effects of nutrient enemata, so frequently given when from any cause there is difficulty in introducing food into the stomach. In ordinary healthy digestion, however, the changes which ensue in the chyme after its passage into the large intestine are mainly the absorption of the more liquid parts; the chief function of the large intestine being to act as a reservoir for the residues of digestion before their expulsion from the body.

\section{Action of Micro-organisms in the Intestines.}

Certain changes take place in the intestinal contents independent of, or at any rate supplemental to, the action of the digestive ferments. These changes are brought about by the action of micro-organisms or bacteria. We have indicated elsewhere that the digestive ferments are examples of unorganized ferments, so bacteria are examples of organized ferments. Organized ferments, of which the jeast plant, torula

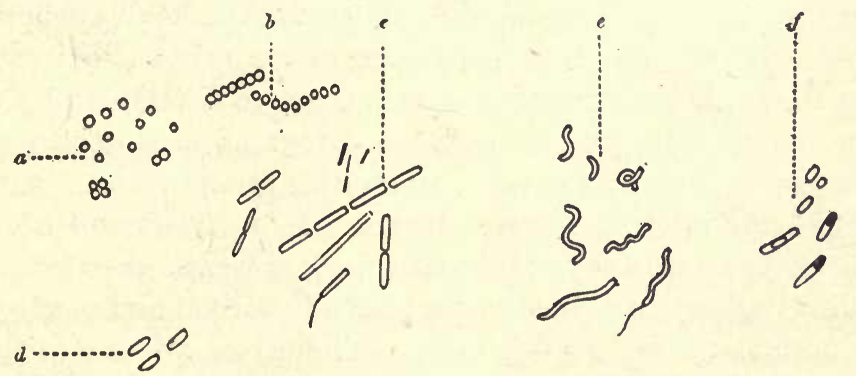

Fig. 274.-Types of micro-organisms. $a$, micrococci arranged singly; in twos, diplococci-if all the micrococci at $a$ were grouped together, they would be called staphylococci-and in fours, sarcinæ; $b$, micrococci, in chains streptococci ; $c$ and $d$, bacilli of various kinds, one is represented with flagellum; $e$, various forms of spirilla; $f$, spores, either free or in bacilli.

(saccharomyces) cerevisice, may be taken as a typical example, consist of unicellular vegetable organisms, which when introduced into a suitable culture medium grow with remarkable rapidity, and by their growth produce new substances from those supplied to them as food. Thus for example, when the yeast cell is introduced into a solution of grape sugar, 
it grows, and on the one hand alcohol, and on the other hand carbon dioxide are produced. These substances are not the direct result of the life of the cell, but probably arise from the formation of some chemical substances allied to the unorganized ferments which greatly increase in amount with the multiplication of the original cell. In all such fermentative processes, organisms analogous to the yeast cell are present, and it is not strange that if the ferment cell is introduced into a suitable medium, it may by its rapid reproduction have power to convert an unlimited amount of one substance into another. Speaking generally a special variety of cell is concerned with each ferment action, thus one variety has to do with alcoholic, another with lactic and another with acetous fermentation. A considerable number of species of bacteria exist in the body during life, chiefly in connection with the nucous membranes, particularly of the digestive tract. These bacteria are unicellular organisms, devoid of chlorophyll, sometimes called fission fungi or schizomycetes. They multiply chiefly by division, but many of them also form spores-whereas the yeast cell multiplies by gemmation. The bacteria are very much smaller than the yeast cells, being only from 1 to $2 \mu$ in width. Morphologically they are classified into i. micrococci or globular bacteria, ii. bacilli or rod-shaped bacteria, and iii. spirilla or sinuous bacteria.

Many forms of bacteria have been isolated from the mouth, a few varieties from the stomach, and a very large number from the intestines. It is only in the last named locality that their multiplication has much effect from a physiological point of view. The normal (hydrochloric acid) acidity of the stomach usually destroys all the micro-organisms taken in with the food, but when the amount of this acid is deficient (and sometimes even when it is normal) some of the spores may escape. On reaching the small intestine these spores begin to develop in its alkaline medium, aud may increase to such an extent as to stop all pancreatic and intestinal digestion; the point where this occurs varies from day to day. The large intestine always swarms with micro-organisms, though the ileo-cæcal valve, in some unknown way, prevents their passage into the small intestine; as a consequence, both intestinal and pancreatic digestion normally cease at this valve. The bacteria found in the intestine are anaerobic, i.e., they do not exist in the presence of free oxygen.

The changes induced in the intestine by the activity of microorganisms are of two kinds, fermentation and putrefaction; the former of these results in the breaking down of carbohydrate matter and the latter in that of proteid matter. The process of fermentation is the least complex and probably occurs normally in the small intestine, to a certain extent. The lactic-acid fermentation is the most important, though the butyric-acid fermentation is next; under their influence the carbohydrates are broken down into lactic and butyric acids, and perhaps 
into acetic acid also. Carbonic acid gas may be formed at the same tine and cause flatulence. Cellulose and other insoluble carbohydrates are decomposed with the formation of marsh gas and hydrogen, which escape by the rectum.

In putrefaction the process is nearly the same as in tryptic digestion, the proteids being broken down into peptones, leucin, tyrosin, and a long row of other substances which have strong odors and belong to the aromatic group. It also results in the production of various gases, such as carbon dioxide, sulphuretted hydrogen, ammonia, hydrogen and methane (marsh gas), and of a high percentage of the volatile fatty acids, valerianic and butyric. Of the aromatic substances the most important are indol and skatol, though their toxicity has been greatly overestimated. Some undergo oxidation, indol and skatol forming indoxyl and skatoxyl; they are usually carried off in the fæces, kut when the bowel is obstructed they are absorbed and eventually appear in the urine, indoxyl and skatoxyl forming respectively indoxyl-and skatoxyl-sulphuric acids and their salts. Tyrosin is further broken down into para-oxyphenol-proprionic acid, paracresol and phenol; para-oxy-phenol-acetic acid is also formed.

\section{Movements of the Intestines.}

It remains only to consider the manner in which the food and the several secretions mingled with it are moved through the intestinal canal, so as to be slowly subjected to the influence of fresh portions of intestinal secretion, and as slowly exposed to the absorbent power of all the villi and blood-vessels of the mucous membrane. The movement of the intestines is peristaltic or vermicular, and is effected by the alternate contractions and dilatations of successive portions of the muscular coats. The contractions, which may commence at any point of the intestine, extend in a wave-like manner along the tube. In any given portion, the longitudinal muscular fibres contract first, or more than the circular; they draw a portion of the intestine upward, or, as it were, backward, over the substance to be propelled, and then the circular fibres of the same portion contracting in succession from above downward, or, as it were, from behind forward, press on the substance into the portion next below, in which at once the same succession of action next ensues. These movements take place slowly, and, in health, commonly give rise to no sensation; but they are perceptible when they are accelerated under the influence of any irritant. The movements of the intestines are sometimes retrograde; and there is no hindrance to the backward movement of the contents of the small intestine. But almost complete security is afforded against the passage of the contents of the large into the small intestine by the ileo-cæcal valve. Besides,-the orifice of 
communication between the ileum and cæcum (at the borders of which orifice are the folds of mucous membrane which form the valve) is encircled with muscular fibres, the contraction of which prevents the undue dilatation of the orifice.

Proceeding from above downward, the muscular fibres of the large intestine become, on the whole, stronger in direct proportion to the greater strength required for the onward moving of the fæces, which are gradually becoming firmer. 'The greatest strength is in the rectum, at the termination of which the circular unstriped muscular fibres form a strong band called the internal sphincter; while an external sphincter muscle with striped fibres is placed rather lower down, and more externally, and as we have seen above, holds the orifice close by a constant slight tonic contraction.

Experimental irritation of the brain or cord produces no evident or constant effect on the movements of the intestines during life; yet in consequence of certain mental conditions the movements are accelerated or retarded; and in paraplegia the intestines appear after a time much weakened in their power, and costiveness, with a tympanitic condition, ensues. Stimulation of pneumo-gastric nerves, if not too strong, induces genuine peristaltic movements of the intestines. Violent irritation stops the movements.

\section{Influence of the Nervous System on Intestinal Digestion.}

As in the case of the œsophagus and stomach, the peristaltic movements of the intestines may be directly set up in the muscular fibres by the presence of chyme acting as the stimulus. Few or no movements occur when the intestines are empty. The intestines are connected with the central nervous system both by the vagi and by the splanchnic nerves, as well as by other branches of the sympathetic which come to them from the cœliac and other abdominal plexuses.

The relations of these nerves respectively to the movements of the intestine and the secretions are probably the same as in the case of the stomach already treated of.

Duration of Intestinal Digestion.-The time occupied by the journey of a given portion of food from the stomach to the anus varies considerably even in health, and on this account probably it is that such different opinions have been expressed in regard to the subject. About twelve hours are occupied by the journey of an ordinary meal through the small intestine, and twenty-four to thirty-six hours by the passage through the large bowel.

The contents of the large intestine, as they proceed toward the rectum, become more and more solid, and losing their more liquid and nutrient parts, gradually acquire the odor and consistence characteristic 
of focces. After a sojourn of uncertain duration in the sigmoid flexure of the colon, or in the rectum, they are finally expelled by the act of defæcation.

The average quantity of solid fæcal matter evacuated by the human adult in twenty-four hours is about six or eight ounces.

\section{Composition of F feces.}

The amount of water varies considerably, from 68 to 82 per cent and upward. The following table is about an average composition:-

Water

Solids, comprising :

733.00

a. Insoluble residues of the food, uncooked starch, cellulose, woody fibres, cartilage, seldom muscular fibres and other proteids, fat, cholesterin, horny matter, and mucin

b. Certain substances resulting from decomposition of foods, indol, skatol, fatty and other acids, calcium and magnesium soaps

c. Special excrementitious constituents :-Excretin, excretoleic acid (Marcet), and stercorin (Austin Flint)

d. Salts :-Chiefly phosphate of magnesium and phosphate of calcium, with small quantities of iron, soda, lime, and silica

e. Insoluble substances accidentally introduced with the food

f. Mucus, epithelium, altered coloring matter of bile, fatty acids, etc.

g. Varying quantities of other constituents of bile, and derivatives from them.

267.00

1000.00

The Gases contained in the Stomach and Intestines.-Under ordinary circumstances, the alimentary canal contains a considerable quantity of gaseous matter. Any one who has had occasion, in a post-mortem examination, either to lay open the intestines, or to let out the gas which they contain, must have been struck by the small space afterward occupied by the bowels, and by the large degree, therefore, in which the gas, which naturally distends them, contributes to fill the cavity of the abdomen. Indeed, the presence of air in the intestines is so constant, and, within certain limits, the amount in health so uniform, that there can be no doubt that its existence here is not a mere accident, but intended to serve a definite and important purpose, although, probably, a mechanical one.

Sources.-The sources of the gas contained in the stomach and bowels may be thus enumerated:-

1. Air introduced in the act of swallowing either food or saliva; $\approx$. Gases developed by the decomposition of alimentary matter, or of the secretions and excretions mingled with it in the stomach and intestines; 3. It is probable that a certain mutual interchange occurs between the 
gases contained in the alimentary canal, and those present in the blood of these gastric and intestinal blood-vessels; but the conditions of the exchange are not known, and it is very doubtful whether anything like a true and definite secretion of gas from the blood into the intestines or stomach ever takes place. There can be no doubt, however, that the intestines may be the proper excretory organs for many odorous and other substances, either absorbed from the air taken into the lungs in inspiration, or absorbed in the upper part of the alimentary canal, again to be excreted at a portion of the same tract lower down-in either ease assuming rapidly a gaseous form after their excretion, and in this way, perhaps, obtaining a more ready egress from the body. It is probable that, under ordinary circumstances, the gases of the stomach and intestines are derived chiefly from the second of the sources which have been enumerited.

\section{Composition of Gases of the Alimentary Canal.}

\section{(Tabulated from various authorities by Brinton.)}

\begin{tabular}{|c|c|c|c|c|c|c|}
\hline \multirow{2}{*}{ Whence obtained. } & \multicolumn{6}{|c|}{ Composition by Volume. } \\
\hline & Oxygen. & Nitrog. & $\begin{array}{l}\text { Carbon. } \\
\text { Acid. }\end{array}$ & Hydrog. & $\begin{array}{l}\text { Carburet. } \\
\text { Hydrogen. }\end{array}$ & $\begin{array}{l}\text { Sulphuret. } \\
\text { Hydrogen. }\end{array}$ \\
\hline Stomach . . & 11 & 71 & 14 & 4 & 一 & - \\
\hline Small Intestines & - & 32 & 30 & 38 & - & \\
\hline Cæcum . . & - & 67 & 12 & 8 & 13 & \\
\hline Colon . & - & 35 & 51 & 6 & 8 & trace. \\
\hline Rectum . . & 一 & 46 & 43 & - & 11 & ) \\
\hline Expelled per anum & - & 22 & 40 & 19 & 19 & $\frac{1}{2}$ \\
\hline
\end{tabular}

The above table differs little from the average obtained by more modern observers, but it cmits an important point to which attention should be drawn, viz., that the amounts of the gases vary with the diet. For all practical purposes oxygen and sulphuretted hydrogen may be omitted. An analysis of the intestinal gases (Ruge, copied by Halliburton) in man is as follows:-

\begin{tabular}{|c|c|c|c|c|}
\hline Gases. & & Milk Diet. & Meat Diet. & Vegetable Diet. \\
\hline $\begin{array}{l}\text { Carbon dioxide . } \\
\text { Hydrogen } \\
\text { Carburetted hydrogen } \\
\text { Nitrogen . }\end{array}$ & $\begin{array}{ll}\cdot & \\
\cdot & \cdot \\
& \cdot \\
\cdot & \cdot\end{array}$ & $\begin{aligned} 9 & \text { to } 16 \\
43 & \text { to } 54 \\
& 0.9 \\
36 & \text { to } 38\end{aligned}$ & $\begin{array}{l}8 \text { to } 13 \\
0.7 \text { to } 3 \\
26 \text { to } 37 \\
45 \text { to } 64\end{array}$ & $\begin{array}{l}21 \text { to } 34 \\
1.5 \text { to } 4 \\
44 \text { to } 55 \\
10 \text { to } 19\end{array}$ \\
\hline
\end{tabular}

Sources of the Carbon Dioxide.-From the carbonates and lactates in food; from alcoholic fermentation of sugar; from putrefaction of carbohydrates and proteids; and from butyric acid fermentation.

Sources of the Hydrogen.-From butyric acid fermentations of lactic acid- 


$$
\underset{\text { Lactic Acid. }}{2 \mathrm{C}_{3} \mathrm{H}_{8} \mathrm{O}_{3}}=\underset{\text { Butyric Acid. }}{\mathrm{C}_{11} \mathrm{H}_{8} \mathrm{O}_{2}}+2 \mathrm{CO}_{3}+2 \mathrm{H}_{2}
$$

Source of the Carburetted Hydrogen.-From the decomposition of acetates and lactates and from cellulose $\left(\mathrm{C}_{6} \mathrm{H}_{10} \mathrm{O}_{5}+\mathrm{H}_{2} \mathrm{O}=3 \mathrm{CO}_{2}+\right.$ $\left.3 \mathrm{CH}_{4}\right)$.

Source of the Nitrogen.-The nitrogen is derived from the swallowed air.

\section{Defæcation.}

The act of the expulsion of fæces is in part due to an increased reflex peristaltic action of the lower part of the large intestine, namely of the sigmoid flexure and rectum, and in part to the more or less voluntary action of the abdominal muscles. In the case of active voluntary efforts, there is usually, first an inspiration, as in the case of coughing, sneezing, and vomiting; the glottis is then closed, and the diaphragm fixed. The abdominal muscles are contracted as in expiration; but as the glottis is closed, the whole of their pressure is exercised on the abdominal contents. The sphincter of the rectum being relaxed, the evacuation of its contents takes place accordingly; the effect being, of course, increased by the peristaltic action of the intestine. As in the other actions just referred to, there is as much tendency to the escape of the contents of the lungs or stomach as of the rectum; but the pressure is relieved only at the orifice, the sphincter of which instinctively or involuntarily yields.

Nervous Mechanism.-The anal sphincter muscle is normally in a state of tonic contraction. The nervous centre which governs this contraction is probably situated in the lumbar region of the spinal cord, inasmuch as in cases of division of the cord above this region the sphincter regains, after a time, to some extent the tonicity which is lost immediately after the operation. By an effort of the will, acting through the centre, the contraction may be relaxed or increased. In ordinary cases the apparatus is set in action by the gradual accumulation of fæces in the sigmoid flexure and rectum, pressing by the peristaltic action of these parts of the large intestine against the sphincter, and causing by reflex action its relaxation; this sensory impulse acting through the brain and reflexly through the spinal centre. At the same time that the sphincter is inhibited or relaxed, impulses pass to the muscles of the lower intestine increasing their peristalsis, and, if necessary, to the abdominal muscles as well. The action of the centre is therefore double. 


\section{CHAPTER X.}

\section{ABSORPTION.}

ABsonptor is generally considered to consist of two processes; the first. having for its object the introduction into the blood of fresh material, and which is called absorption from without, takes place chiefly from the alimentary canal, and to a less extent from the skin and lungs; the second, having for its object the gradual removal of parts of the body itself when they need removal, is called absorption from within, and takes place everywhere within the tissues of the body.

The conditions of absorption from the alimentary canal which may be taken as an example of the first of these processes are the following: on one side is a fluid containing matters which have been so acted upon by the digestive juices as to be in a fit condition to be absorbed. On the other side are blood-capillaries and capillaries of the lymphatic system, and separating the two are epithelium and commective tissue, as well as the endothelium of the vessels themselves. The problem which has to be considered is, how does the fluid on the one side of the organic membrane reach the blood or lymphatic vessel? Until within recent date it was assumed that the passage of the fluid from one side of this membrane to the other came about solely by definite physical laws, and these were practically independent of the vital condition of the tissues. In the first place, it was taught, came in osmosis, the passage of fluids throngh an animai membrane, which occurs independent of vital conditions, and in the next place came in filtration, the passage of fluids through the pores of a membrane under pressure. It is now believed, however, that there is another factor concerned in absorption, viz., the vital and selective action of the epithelium, and possibly of the tissue which separates the fluid to be absorbed, from the blood and lymph stream. About this vital action of the epithelium very little definite is known, but the mere fact that fats are principally absorbed in one part of the intestine, and as we shall see pass through the cells of the intestinal villi, is some evidence in its favor. It will be as well to consider briefly the two physical processes of osmosis and filtration.

\section{Methods of Absorption.}

Osmosis. - The phenomenon of the passage of fluids through animal membrane, which occurs quite independently of vital conditions, was first demonstrated by Dutrochet. The instrument which he employed 
in his experiments was named an endosmometer. One form of this, represented in the figure, consists of a graduated tube expanded into an open-mouthed bell at one end, over which a portion of membrane is tied. If the bell be filled with a solution of a salt-say sodium chloride, and be immersed in water, the water will pass into the solution, and part of the salt will pass out into the water; the water, however, will pass into the solution much more rapidly than the salt will pass out into the water, and the diluted solution will rise in the tube. It is to this passage of fluids through membrane that the term osmosis is applied.

The nature of the membrane used as a septum, and its affinity for the fluids subjected to experiment have an important influence, as might be anticipated, on the rapidity and duration of the osmotic current. Thus, if a piece of ordinary bladder be used as the septum between water and alcohol, the current is almost solely from the water to the alcohol, on account of the much greater affinity of water for this kind of membrane; while, on the other hand, in the case of a membrane of caoutchouc, the alcohol, from its greater affinity for this substance, would pass freely into the water.

Absorption by blood-vessols is the consequence of their walls being, like the membranous septum of the endosmometer, porous and capable of imbibing fluids, and of the blood being so composed that most fluids will mingle with it. Thus the relation of the chyme in the stomach and intestines to the blood circulating in the ressels of the gastric and intestinal mucous membrane is evidently just that which is required for osmosis. The chyme contains

Fig. 275 substances which have been so acted upon by the diEndosmometer. gestive juices as to have become quite able to pass through an animal membrane, or to dialyze as it is called. The thin animal membrane is the coat of the blood-vessels with the intervening mucous membrane. The nature of the fluid within the vessels, the very feeble power of dialyzation which the albuminous blood possesses, determines the direction of the osmotic current, viz., into and not out of the bloodvessels. The current is of course aided by the fact of the constant change in the blood presented to the osmotic surface, as it rapidly circulates within the vessels. As a rule the current is from the stomach or intestine into the blood, but the reversed action may occur, when, for example, sulphate of magnesia is taken into the stomach, in which case there is a rapid discharge of water from the blood-vessels into the alimentary canal resulting in purgation. The presence of various substances in the food has the power of diminishing the rate of absorption; their influence is probably exerted upon the membrane, diminishing its power of permitting osmosis. Whereas the presence of a little hydro- 
chloric acid in the contents of the stomach appears to determine the direction of the osmosis, or at any rate to diminish or prevent exosmosis.

The conditions for osmosis exist not only in the alimentary mucous membrane, but also in the serous cavities and the tissues elsewhere.

Various substances have been classified according to the degree in which they possess the property of passing, when in a state of solution in water, through membrane; those which pass freely, inasmuch as they are usually capable of crystallization, being termed crystalloids, and those which pass with difficulty, on account of their physically glue-like character, colloids.

This distinction, however, between colloids and crystalloids which is made the basis of their classification, is by no means the only difference between them. The colloids, besides the absence of power to assume a crystalline form, are characterized by their inertness as acids or bases, and feebleness in all ordinary chemical relations. Examples of them are found in albumin, gelatin, starch, hydrated alumina, hydrated silicic acid, etc.; while the crystalloids are characterized by qualities the reverse of those just mentioned as belonging to colloids. Alcohol, sugar, and ordinary saline substances are examples of crystalloids.

Filtration, or transudation, means the passage of fluids through the pores of a membrame under pressure. The greater the pressure the greater the amount which passes through the membrane. Colloids will filter, although less easily than crystalloids. The nature of the substance to be filtered and the nature of the membrane which acts as the filter materially affect the activity of the process. No doubt both osmosis and filtration go on together in the process of absorption. An excellent example of filtration or transudation occurs in the pathological condition known as dropsy, in which the connective tissues become infiltrated with serous fluid. The fluid passes out of the vein when the intra-venous pressure passes a certain point, the fluid being, as it were, squeezed through the walls of the vessels by this excess of pressure.

Rapidity of Absorption.- The rapidity with which matters may be absorbed from the stomach, probably by the blood-ressels chiefly, and diffused through the textures of the body, has been found by experiment. It appears that lithium chloride may be diffused into all the vascular textures of the body, and into some of the non-vascular, as the cartilage of the hip-joint, as well as into the aqueous humor of the eye, in a quarter of an hour after being given on an empty stomach. Into the outer part of the crystalline lens it may pass after a time, varying from half an hour to an hour and a half. Lithium carbonate, when taken in five or ten-grain doses on an empty stomach, may be detected in the urine in 5 or 10 minutes; or, if the stomach be full at the time of taking the dose, in 20 minutes. It may sometimes be detected in the urine, moreover, for six, seven, or eight days. 
Some experiments on the absorption of various mineral and vegetable poisons have brought to light the singular fact that, in some cases, absorption takes place more rapidly from the rectum than from the stomach. Strychnia, for example, when in solution, produces its poisonous effects much more speedily when introduced into the rectum than into the stomach. When introduced in the solid form, however, it is absorbed more rapidly from the stomach than from the rectum, doubtless because of the greater solvent property of the secretion of the former than of the latter.

Conditions for Absorption.-1. The diffusibility of the substance to be absorbed is one of the chief conditions for its absorption-a colloid, as we have seen, dialyzes very little. It must be also in the liquid or gaseous state. Mercury may, however, be absorbed even in the metallic state; and in that state may pass into and remain in the blood-vessels, or be deposited from them; and such substances as exceedingly finelydivided charcoal, when taken into the alimentary canal, have been found in the mesenteric veins. Oil, minutely divided, as in an emulsion, will pass slowly into blood-vessels, as it will through a filter moistened with water; but it is doubtful if fatty matfers find their way into the bloodvessels as they do into the lymph-ressels of the intestinal canal.

2. The less dense the fluid to be absorbed, the more speedy, as a general rule, is its absorption by the living blood-vessels. Hence the rapid absorption of water from the stomach; also of weak saline solutions; but with strong solutions, there appears less absorption into, than effusion from, the blood-vessels.

3. The absorption is the less rapid the fuller and tenser the blood-vessels are; and the tension may be so great as to hinder altogether the entrance of more fluid. Thus, if water is injected into a dog's veins to repletion, poison is absorbed very slowly; but when the tension of the vessels is diminished by bleeding, the poison acts quickly. So, when cupping-glasses are placed over a poisoned wound, they retard the absorption of the poison not only by diminishing the velocity of the circulation in the part, but by filling all its vessels too full to admit more.

4. On the same ground, absorption is the quicker the more rapid the circulation of the blood; not because the fluid to be absorbed is more quickly imbibed into the tissues, or mingled with the blood, but because as fast as it enters the blood, it is carried away from the part, and the blood being constantly renewed, is constantly as fit as at the first for the reception of the substance to be absorbed.

These four conditions are physical, but (5) the vital condition of the absorptive epithelium must not be forgotten. It has been shown, for example, that the absorption by the frog's skin is bastened by alcohol and retarded by chloroform. It appears also that absorption is retarded rather than hastened by removal of the intestinal epithelium. 


\section{The Lymphatic System.}

Having now discussed the methods and conditions of absorption in general, we must next turn to the system of vessels in which, on the one hand, materials of the food not taken directly into the blood-vessels of the alimentary canal are received and carried into the blood-stream; and, on the other, fluid which has exuded from the blood-vessels into the

Lymphatics of head and neck, right.

Right internal jugular vein. Right subclavian vein.

Lymphatics of right arm.

Lymphaties of lower extremReceptaculum chyli. ities.

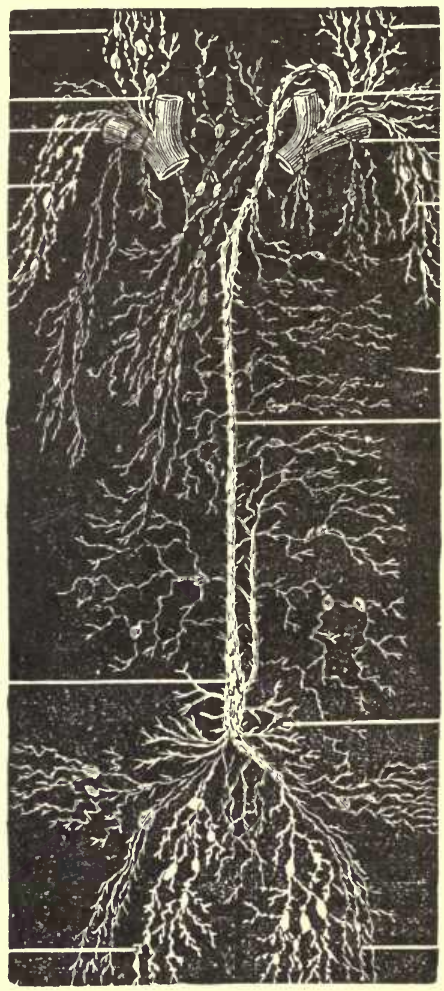

Lymphaties of head and neck, left.

Thoracic duct.

Left subclavian vein.

Thoracic duct.

Lacteals.

Lymphatics of lower ex. tremities.

Fig. 276.-Diagram of the principal groups of Lymphatic vessels (from Quain).

tissues is gathered up and carried back again into the blood. This system of vessels is called the Lymphatic System, and the vessels themselves are named Lymphatics or Absorbents. They have often been incidentally mentioned in former ahapters.

The principal vessels of the lymphatic system are, in structure and general appearance, like very small and thin-walled veins. They are provided with valves. They commence in fine microscopic lymph-capillaries, in the organs and tissues of the body, and they end directly or indirectly in two trunks which open into the large veins near the heart 
(fig. $2 \% 6$ ). The fluid which they contain, unlike the blood, passes only in one direction, namely, from the fine branches to the trunk and so to the large veins, on entering which they are mingled with the stream of blood and form part of its constituents. 'The course of the fluid in the lymphatic vessels is always toward the large veins in the neighborhood of the heart, and in fig. 276 the greater part of the contents of the lym-

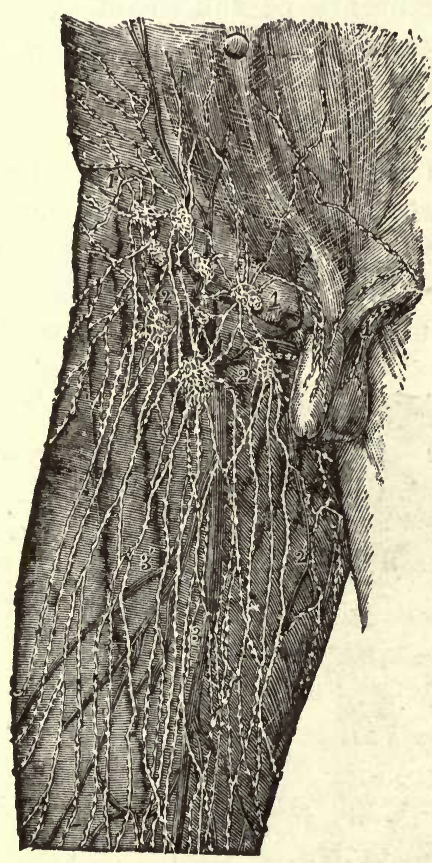

Fig. 277.

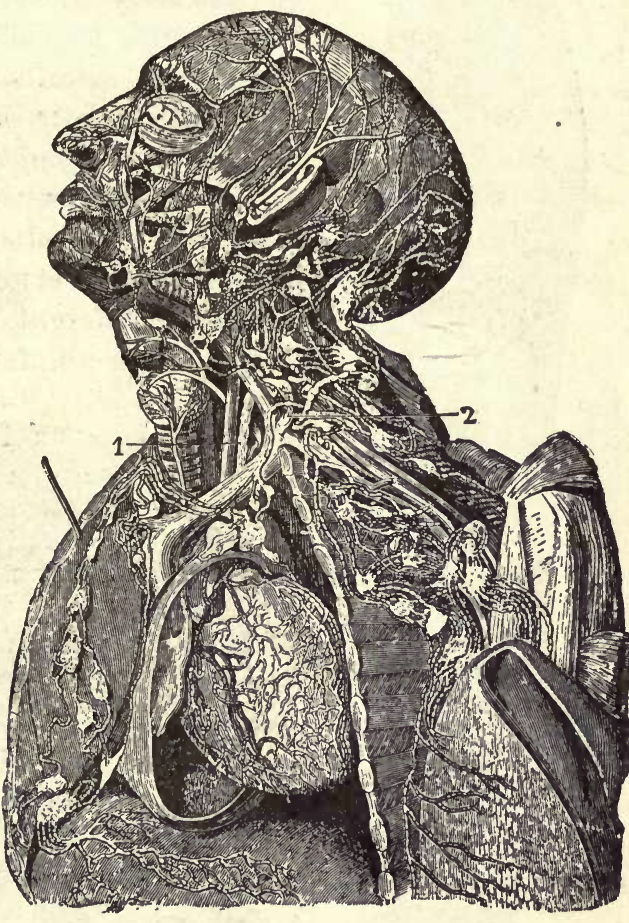

Fig. 278

Fig. 277.-Superficial lymphatics of right groin and upper part of thigh, $\frac{1}{6} .-1$. Upper inguinal glands. 2, $2^{\prime}$. Lower or inguinal or femoral glands. $3,3^{\prime}$. Plexus of lymphatics in the course of the long saphenous vein. (Mascagni.)

Fig. 278.-Lymphatic vessels of the head and neck and the upper part of the trunk (Mascagni). $\frac{1}{6}$. - The chest and pericardium have been opened on the left side, and the left mamma detached and thrown outward over the left arm, so as to expose a great part of its deep surface. The principal ly mphatic vessels and glands are shown on the side of the head and face, and in the neck, axilla, and mediastinum. Between the left internal jugular vein and the common carotid artery, the upper ascending part of the thoracic duct marked $\mathrm{I}$, and above this, and descending to 2 , the arch and last part of the duct. The termination of the upper lymphatics of the diaphragm in the mediastinal glands, as well as the cardiac and the deep mammary lymphatics, is also shown.

phatic system of vessels will be seen to pass through a comparatively large trunk called the thoracic duct, which finally empties its contents into the blood-stream, at the junction of the internal jugular and subclavian veins of the left side. There is a smaller duct on the right side. The lymphatic vessels of the intestinal canal are called lacteals, because during digestion the fluid contained in them resembles milk in appear- 
ance; and the lymph in the lacteals during the period of digestion is called chyle. There is no essential distinction, however, between lacteals and lymphatics. In some parts of its course the lymph-stream must pass through lymphatic glands.

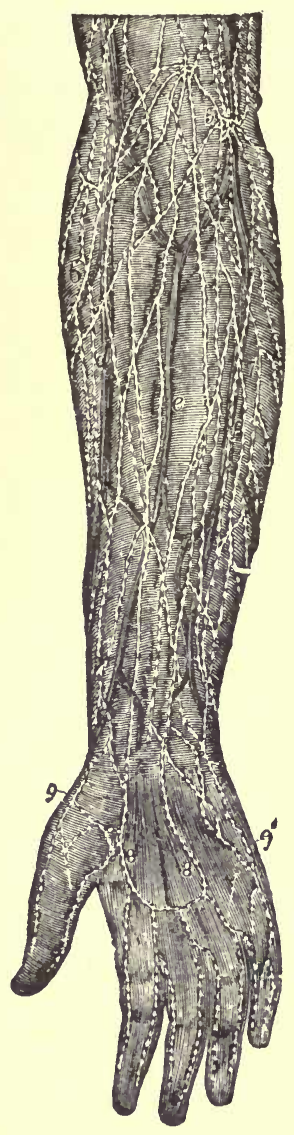

Fig. 279

Lymphatic vessels are distributed in nearly all parts of the body. Their existence, however, has not yet been determined in the placenta, the umbilical cord, the membranes of the ovum, or in any of the so-called non-vascular parts, as the nails, cuticle, hair, and the like.

Origin of Lymph Capillaries.-The lymphatic capillaries commence most commonly either $(a)$ in closely meshed networks, or $(b)$ in irregular lacunar spaces between the various structures of which the different organs are composed. Such irregular spaces, forming what is now termed

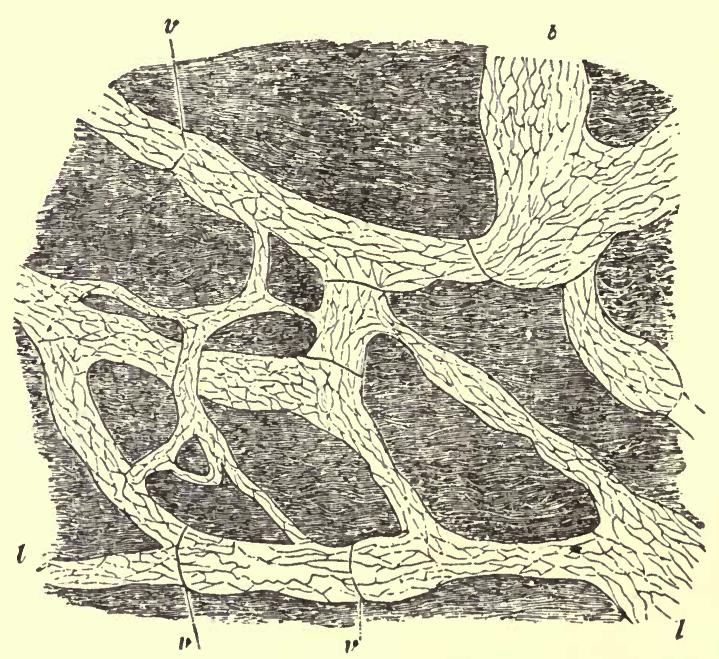

Fig. 280.

Fig. $2 \% 9$-Superficial lymphatics of the forearm and palm of the hand, $\frac{1}{5} .-5$. Two small glands at the bend of the arm. 6. Radial lymphatic vessels. 7. Ulnar lynıhatic vessels. 8, 8. Palmar arch of lymphatics. 9, $9^{\prime}$. Outer and inner sets of vessels. $b$. Cephalic vein. $d$. Radial vein. $e$ Median rein. $f$. Ulnar vein. The lymphatics are represented as lying on the deep fascia. (Mascagni.)

Fig. 280.-Lymphatics of central tendon of rabbit's diaphragm, stained with silver nitrate. The ground sulsstance has been shaded diagrammatically to bring out the lymphatics clearly. $l$. Lymphatics lined by long narrow endothelial cells, and showing $v$, valves at frequent intervals. (Schofield.)

the lymph-canalicular system, have been shown to exist in many tissues. In serous membranes such as the omentum and mesentery they occur as a connected system of very irregular branched spaces partly occupied by connective tissue-corpuscles, and both in these and in many other tissues are found to communicate freely with regular lymphatic 
vessels. In many cases, though they are formed mostly by the chinks and crannies between the blood-vessels, secreting ducts, and other parts which may happen to form the framework of the organ in which they exist, they are lined by a distinct layer of endothelium.

The lacteals offer an illustration of another mode of origin, namely, $(c)$ in blind dilated extremities; but there is no essential difference in structure between these and the lymphatic capillaries of other parts.

Structure of Lymph Capillaries. - The structure of lymphatic capillaries is very similar to that of blood-capillaries: their walls consist of a single layer of elongated endothelial cells with sinuous outline, which cohere along their edges to form a delicate membrane. They differ from blood capillaries mainly in their larger and very variable calibre, and in their numerous communications with the spaces of the lymphcanalicular system.

Communications of the Lymphatics. - The fluid part of the blood constantly exudes from or is strained through the walls of the bloodcapillaries, so as to moisten all the surrounding tissues, and occupies the interspaces which exist among their different elements, which form the beginnings of the lymph-capillaries; and the latter, therefore, are the means of collecting the exuded blood plasma, and returning that part which is not directly absorbed by the tissues into the blood-stream. It is not necessary to assume the presence of any special channels between the blood and lymphatic vessels, inasmuch as even blood-corpuscles can pass bodily, without much difficulty, through the walls of the bloodcapillaries and small veins, and could pass with still less trouble, probably, through the comparatively ill-defined walls of the capillaries which contain lymph.

It has been already mentioned (p. 31) that in certain parts of the body, stomata exist, by which lymphatic capillaries directly communicate with parts hitherto supposed to be closed cavities.

Stomata have been found in the pleura; and as they may be presumed to exist in other serous membranes, it would seem as if the serous cavities, hitherto supposed closed, form but a large lymph-sinus or widening out, so to speak, of the lymph-capillary system with which they directly communicate.

When absorption into the lymphatic system takes place in membranes covered by epithelium or endothelium through the interstitial or intercellular cement-substance, it is said to take place throngh pseudo-stomata, already alluded to (p. 32).

Demonstration of Lymphatics of Diaphragm. - The stomata on the peritoneal surface of the diaphragm are the openings of short vertical canals which lead up into the lymphatics, and are lined by cells like those of germinating endothelium. By introducing a solution of Berlin blue into the peritoneal cavity of an animal shortly after death, and suspending it, head downward, an in. 
jection of the lymphatic vessels of the diaphragm, through the stomata on its peritoneal surface, may readily be obtained if artificial respiration be carried on for about half an hour. In this way it has been found that in the rabbit the lymphatics are arranged between the tendon bundles of the centrum tendineum; and they are hence termed interfascicular. The centrum tendineum is coated by endothelium on its pleural and peritoneal surfaces, and its substance consists of tendon bundles arranged in concentric rings toward the pleural side and in radiating bundles toward the peritoneal side.

The lymphatics of the anterior half of the diaphragm open into those of the anterior mediastinum, while those of the posterior half pass into a lymphatic ressel in the posterior mediastinum, which soon enters the thoracic duct. Both these sets of vessels, and the glands into which they pass, are readily injected by the method abore described; and there can be little doubt that during life the flow of lymph along these channels is chiefly caused by the action of the diaphragm during respiration. As it descends in inspiration, the spaces between the radiating tendon bundles dilate, and lymph is sucked from the peritoneal cavity, through the widely open stomata, into the interfascicular lymphatics. During expiration, the spaces between the concentric tendon bundles dilate, and the lymph is squeezed into the lymphatics toward the pleural surface (Klein). It thus appears probable that during health there is a continued sucking in of lymph from the peritoneum into the lymphatics by the "pumping" action of the diaphragm; and there is doubtless an equally continuous exudation of fluid from the general serous surface of the peritoneum. When this balance of transudation and absorption is disturbed either by increased transudation or some impediment to absorption, an accumulation of fluid necessarily takes place (ascites).

Structure of Lymphatic Vessels.-The larger vessels as before mentioned are very like veins, having an external coat of areolar tissue, with elastic filaments; within this, a thin layer of areolar tissue, with unstriped muscular fibres, which have, principally, a circular direction, and are much more abundant in the small than in the larger vessels; and again, within this, an inner elastic layer of longitudinal tibres, and a lining of epithelium; and numerous valves. The valves, constructed like those of veins, and with the free edges turned toward the heart, are usually arranged in pairs, and, in the small vessels, are so closely placed, that when the vessels are full, the valves constricting them where their edges are attached, give them a peculiar beaded or knotted appearance.

\section{The Lymph Flow.}

'The flow of the lymph toward the point of its discharge into the veins is brought about by several agencies. With the help of the valvular mechanism (1) all occasional pressure on the exterior of the lymphatic and lacteal vessels propels the lymph onward: thus muscular and other external pressure accelerates the flow of the lymph as it does that of the blood in the veins. The actions of (2) the muscular fibres of the. 
small intestine, and probably the layer of unstriped muscle present in each intestinal villus, seem to assist in propelling the chyle: for, in the small intestine of a mouse, the chyle has been seen moving with intermittent propulsions that appeared to correspond with the peristaltic movements of the intestine. But for the general propulsion of the lymph and chyle, it is probable that, together with (3) the vis a tergo resulting from absorption (as in the ascent of sap in a tree), and from external pressure, some of the force may be derived (4) from the contractility of the vessel's own walls. The respiratory movements, also, (5) favor the current of lymph through the thoracic duct as they do the current of blood in the thoracic veins.

Lymph-Hearts. - In reptiles and some birds, an important auxiliary to the movement of the lymph and chyle is supplied in certain muscular sacs, named lymph-hearts, and it has been shown that the caudal heart of the eel is a lymph-heart also. The number and position of these organs vary. In frogs and toads there are usually four, two anterior and two posterior; in the frog, the posterior lymph-heart on each side is situated in the ischiatic region, just beneath the skin; the anterior lies deeper, just over the transverse process of the third vertebra. Into each of these cavities several lymphatics open, the orifices of the vessels being guarded by valves, which prevent the retrograde passage of the lymph. From each heart a single vein proceeds, and conveys the lymph directly into the venous system. In the frog, the inferior lymphatic heart, on each side, pours its lymph into a branch of the ischiatic vein; by the superior, the lymph is forced into a branch of the jugular vein, which issues from its anterior surface, and which becomes turgid each time that the sac contracts. Blood is prevented from passing from the vein into the lymphatic heart by a valve at its orifice.

The muscular coat of these hearts is of variable thickness; in some cases it can only be discovered by means of the microscope; but in every case it is composed of striped fibres. The contractions of the hearts are rhythmical, occurring about sixty times in a minute, slowly, and, in comparison with those of the blood-hearts, feebly. The pulsations of the cervical pair are not always synchronous with those of the pair in the ischiatic region, and even the corresponding sacs of opposite sides are not always synchronous in their action.

Unlike the contractions of the blood-heart, those of the lymph-heart appear to be directly dependent upon a certain limited portion of the spinal cord. For Volkmann found that so long as the portion of spinal cord corresponding to the third vertebra of the frog was uninjured, the cervical pair of lymphatic hearts continued pulsating after all the rest of the spinal cord and the brain were destroyed; while destruction of this portion, even though all other parts of the nervous centres were uninjured, instantly arrested the heart's movements. The posterior, or ischiatic, pair of lymph-hearts were found to be governed, in like manner, by the portion of spinal cord corresponding to the eighth vertebra. Division of the posterior spinal roots did not arrest the movements; but division of the anterior roots caused them to cease at once.

Lymphatic Glands.-Lymphatic glands are small round or oval compact bodies varying in size from a hemp-seed to a bean, interposed 
in the course of the lymphatic vessels, and through which the chief part of the lymph passes in its course to be discharged into the blood-vessels. They are found in great numbers in the mesentery, and along the great vessels of the abdomen, thorax, and neck; in the axilla and groin; a

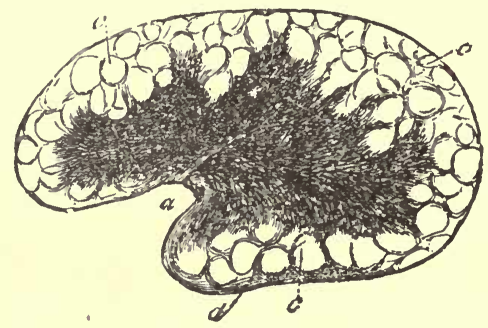

Fig. 281 - Section of a mesenteric gland from the ox, slightly magnified. $a$, Hilus; $b$ (in the central part of the figure), medullary substance ; $c$, cortical substance with indistinct alveoli; $d$, capsule. (Kölliker.)

few in the popliteal space, but not further down the leg, and in the arm as far as the elbow. Some lymphatics do not, however, pass through glands before entering the thoracic duct.

Structure.-A lymphatic gland is covered externally by a capsule of connective tissue, generally containing some unstriped muscle. At the inner side of the gland, which is somewhat concave (hilus), (fig. 281, a),

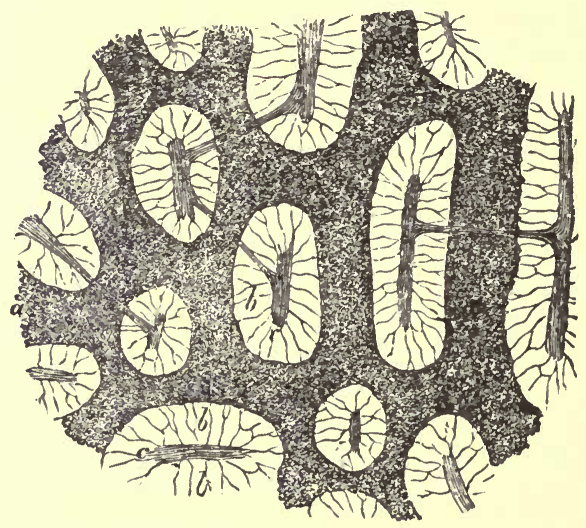

Fig. 282-Section of medullary substance of an inguinal gland of an ox. $a, a$, glandular sub stance or pulp forıning rounded cords joining in a continuous net (dark in the figure); $c, c$, tra veculæ; the space, $b, b$, between these and the glandular substance is the lymph sinus, washed clear of corpuscles and traversed by filaments of retiform connective-tissue. $\times 90$. (Kölliker.)

the capsule sends inward processes called trabeculoe in which the bloodvessels are contained, and these join with other processes prolonged from the inner surface of the part of the capsule covering the convex or outer part of the gland; they have a structure similar to that of the capsule, and entering the gland from all sides, and freely communicating, form 
a fibrous supporting stroma. The interior of the gland is seen on section, even when examined with the naked eye, to be made up of two parts, an outer or cortical (fig. 283, c, c), which is light colored, and an inner of redder appearance, the medullary portion (fig. 281). In the outer or cortical part of the gland (fig. 283) the intervals between the trabeculæ are comparatively large, and form more or less triangular intercommunicating spaces termed alveoli; while in the more central or medullary part is a finer meshwork formed by the more free anastomosis of the trabecular process. Within the alveoli of the cortex and in the meshwork formed by the trabeculæ in the medulla, is contained the

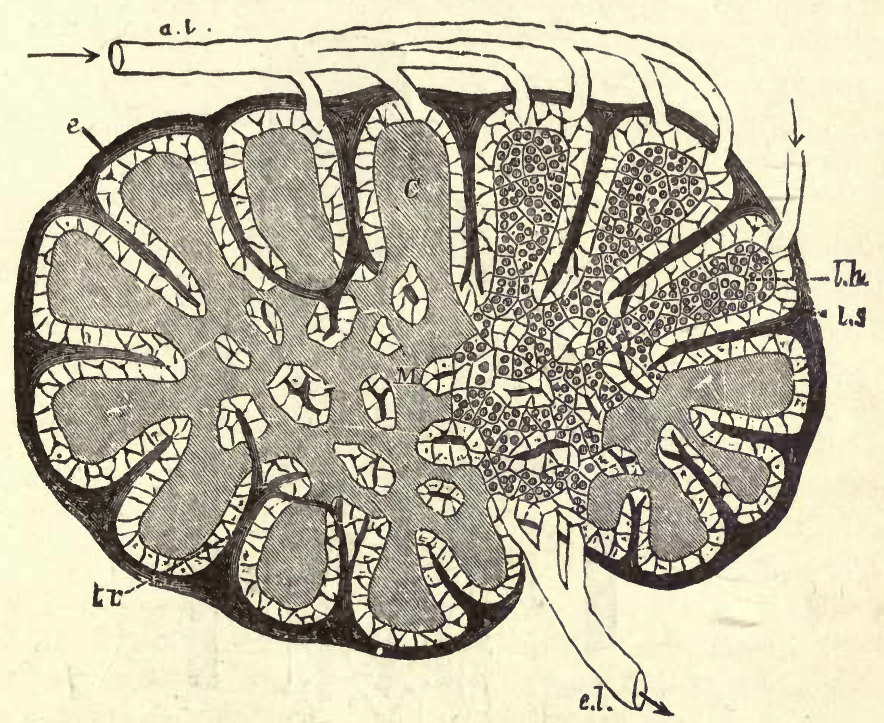

Fig. 283.-Diagrammatic section of lymphatic gland. a.l., afferent ; e.l., efferent lymphatics; $C$, cortical substance; $l . h .$, reticulating cords of medullary substance; $l . s .$, lymph-sinus; $c$., fibrous coat sending in trabeculæ; t.r., into the substance of the gland. (Sharpey.)

proper gland structure. In the former it is arranged as follows: occupying the central and chief part of each alreolus is a more or less wedgeshaped mass of adenoid tissue, densely packed with lymph corpuscles; but at the periphery surrounding the central portion and immediately next the capsule and trabeculæ, is a more open meshwork of adenoid tissue constituting the lymph sinus or channel, and containing fewer lymph-corpuscles. The central mass is inclosed in endothelium, the cells of which join by their processes, the processes of the adenoid framework of the lymph sinus. The trabeculæ are also covered with endothelium. The lining of the central mass does not prevent the passage of fluids and even of corpuscles into the lymph sinus. The framework of adenoid tissue of the lymph sinus is nucleated, that of the central mass is non-nucleated. At the inner part of the alveolus, the wedge-shaped 
central mass divides into two or more smaller rounded or cord-like masses which joining with those from the other alveoli, form a much closer arrangement of the gland tissue than in the cortex; spaces (fig. $284, b$ ), are left within those anastomosing cords, in which are found portions of the trabecular meshwork and the continuation of the lymph sinus.

The essential structure of lymphatic-gland substance resembles that which was described as existing, in a simple form, in the interior of the solitary and agminated intestinal follicles.

The lymph enters the gland by several afferent vessels, which open

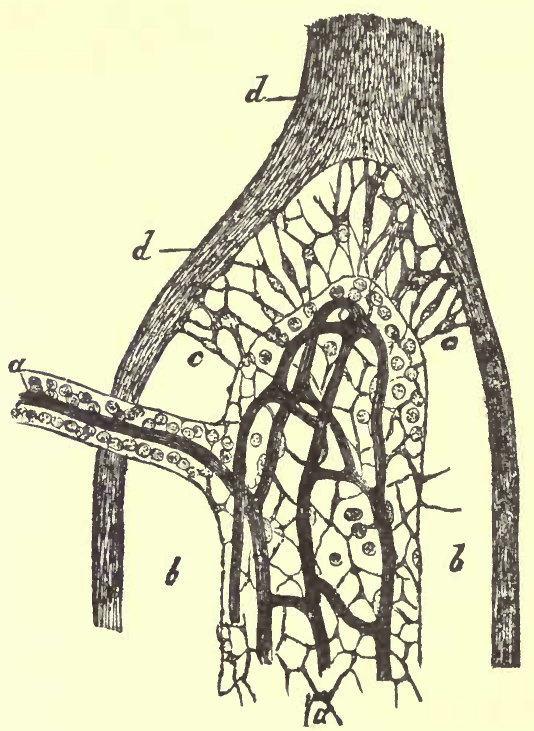

Fig. 284-A small portion of medullary substance from a mesenteric gland of the ox. $d, d$, trabeculæ; $a$, part of a cord of glandular substances from which all but a few of the lymph-corpuscles have been washed out to show its supporting meshwork of retiform tissue and its capillary bloodvessels (which have been injected, and are dark in the figure); $b, b$, lymph-sinus, of which the retiform tissue is represented only at $c, c . \times 300$. (Kölliker.)

beneath the capsule into the lymph-channel or lymph-path; at the same time they lay aside all their coats except the endothelial lining, which is continuous with the lining of the lymph-path. The efferent vessels begin in the medullary part of the gland, and are continuous with the lymph-path here as the afferent vessels were with the cortical portion; the endothelium of one is continuous with that of the other.

The efferent vessels leave the gland at the hilus, the more or less concave inner side of the gland, and generally either at once or very soon after join together to form a single vessel.

Blood-vessels which enter and leave the gland at the hilus are freely distributed to the trabecular tissue and to the gland-pulp. 


\section{The Lymph and Chyle.}

Lymph is, under ordinary circumstances, a clear, transparent, and yellowish fluid, of a specific gravity varying from 1012-1022. It is devoid of smell, is slightly alkaline, and has a saline taste. As seen with the microscope in the small transparent vessels of the tail of the tadpole, it usually contains no corpuscles or particles of any kind; and it is only in the larger trunks that any corpuscles are to be found. These corpuscles are similar to colorless blood-corpuscles. The fluid in which the corpuscles float is albuminous, and contains no fatty particles; but is liable to variations according to the general state of the blood, and to that of the organ from which the lymph is derived. It may clot on exposure to the air. As it advances toward the thoracic duct, after passing through the lymphatic glands, it becomes spontaneously coagulable and the number of corpuscles is much increased.

Chyle, found in the lacteals after a meal, is an opaque, whitish, milky fluid, neutral or slightly alkaline in reaction. Its whiteness and opacity are due to the presence of innumerable particles of oily or fatty matter, of exceedingly minute though nearly uniform size, measuring on the average about $\frac{1}{30000}$ of an inch $(0.8 \mu)$. These constitute what is termed the molecular base of chyle. Their number, and consequently the opacity of the chyle, are dependent upon the quantity of fatty matter contained in the food. The fatty nature of the molecules is made manifest by their solubility in ether. Each molecule probably consists of a droplet of oil coated over with albumen, in the manner in which minute drops of oil always become covered in an albuminous solution. This is proved when water or dilute acetic acid is added to chyle, many of the molecules are lost sight of, and oil-drops appear in their place, as the investments of the molecules have been dissolved, and their oily contents have run together.

Except these molecules, the chyle taken from the villi or from lacteals near them, contains no other solid or organized bodies. The fluid in which the molecules float is albuminous, and does not spontaneously coagulate. But as the chyle passes on toward the thoracic duct, and especially while traversing one or more of the mesenteric glands, it is elaborated. The quantity of molecules and oily particles gradually diminishes; cells, to which the name of chyle-corpuscles is given, appear in it; and it acquires the property of coagulating spontaneously. The higher in the thoracic duct the chyle advances, the greater is the number of chyle-corpuscles, and the larger and firmer is the clot which forms in it when withdrawn and left at rest. Such a clot is like one of blond without the red corpuscles, having the chyle-corpuscles entangled in it. and the fatty matter forming a white creamy film on the surface of the 
serum. But the clot of chyle is softer and moister than that of blood. Like blood, also, the chyle often remains for a long time in its vessels without coagulating, but coagulates rapidly on being removed from them. The existence of the materials which, by their union, form fibrin, is, therefore, certain; and their increase appears to be commensurate with that of the corpuscles.

The structure of the chyle-corpuscles was described when speaking of the white corpuscles of the blood, with which they are identical. The lymph, in chemical composition, resembles diluted plasma, and from what has been said, it will appear that perfect chyle and lymph are, in essential characters, nearly similar, and scarcely differ, except in the preponderance of fatty and proteid matter in the chyle.

Chemical Composition of Lymph and Chyle.

\begin{tabular}{|c|c|c|c|}
\hline $\begin{array}{l}\text { Water } \\
\text { Solids . }\end{array}$ & $\begin{array}{c}\text { I. } \\
\text { Lymph. } \\
\text { (Donkey). } \\
96.536 \\
3.454\end{array}$ & $\begin{array}{c}\text { II. } \\
\text { Chyle. } \\
\text { (Donkey). } \\
90.237 \\
9.763\end{array}$ & $\begin{array}{c}\text { III. } \\
\text { Mixed Lymph \& } \\
\text { Chyle (Human). } \\
90.48 \\
9.52\end{array}$ \\
\hline \multicolumn{4}{|l|}{ Solids- } \\
\hline $\begin{array}{l}\text { Proteids, including Serum-Albu- } \\
\text { min, Fibrinogen, and Globulin. } \\
\text { Extractives, including in (I and }\end{array}$ & 1.320 & 3.886 & 7.08 \\
\hline $\begin{array}{l}\text { II) Sugar, Urea, Leucin and } \\
\text { Cholesterin }\end{array}$ & 1.559 & 1.565 & 1.08 \\
\hline Fatty matter and Soaps . & a trace & 3.601 & .92 \\
\hline Salts $\quad . \quad . \quad$. & .585 & .711 & .44 \\
\hline
\end{tabular}

Quantity.-The quantity which would pass into a cat's blood in twenty-four hours has been estimated to be equal to about one-sixth of the weight of the whole body. And, since the estimated weight of the blood in cats is to the weight of their bodies as 1 to $\%$, the quantity of lymph daily traversing the thoracic duct would appear to be about equal to the quantity of blood at any time contained in the animals. By another series of experiments, the quantity of lymph traversing the thoracic duct of a dog in twenty-four hours was found to be about equal to two-thirds of the blood in the body.

\section{Channels of Absorption.}

The Lacteals.-During the passage of the chyme along the intestinal canal, its completely digested parts are absorbed into the blood and distributed in the mucous membrane. The absorption into both sets of vessels is carried on most actively but not exclusively, in the villi of the small intestine; for in them both the capillary blood-vessels and the lacteals are brought almost into contact with the intestinal contents. There seems to be no doubt that absorption of fatty matters during digestion, from the contents of the intestines, is effected chiefly through 
the epithelial cells which line the intestinal tract, and especially those which clothe the surface of the villi. Thence, the fatty particles are passed on into the interior of the lacteal ressels, but how they pass, and what laws govern their passage, are not at present exactly known. The lymph-corpuscles of the villi are however, in some animals, e.g., the rat and frog, important agents in effecting the passage of fat-particles into the lacteals. These cells take up the fat which has passed through the columnar cells and then, by reason of their amæboid movement, carry

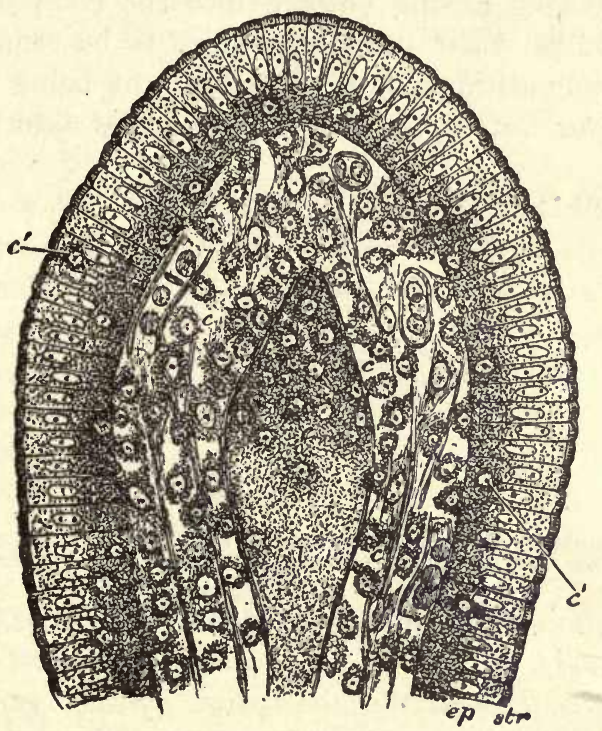

Fig, 285.-Section of the villus of a rat killed during fat absorption. ep, epithelium; str, striated border; $c$, lymph-cells ; $c^{\prime}$, lymph-cells in the epithelium; 1 , central lacteal containing disintegrating lymph-corpuscles. (E. A. Schäfer.)

it in to the lacteal. When arrived there they break up and set free both fat and proteid matter thereby.

The process of absorption is assisted by the pressure exercised on the contents of the intestines by their contractile walls; and the absorption of fatty particles is also facilitated by the presence of the bile, and the pancreatic and intestinal secretions, which moisten the absorbing surface.

The Lymphatics.-The lymph is diluted liquor sanguinis, which is always exuding from the blood-capillaries into the interstices of the tissues in which they lie; and as these interstices form in most parts of the body the beginnings of the lymphatics, the source of the lymph is sufficiently obvious. In connection with this may be mentioned the fact that changes in the character of the lymph correspond very closely with changes in the character of either the whole mass of blood, or of that 
in the vessels of the part from which the lymph is exuded. Thus it appears that the coagulability of the lymph, although always less than, is directly proportionate to that of the blood; and that when fluids are injected into the blood-vessels in sufficient quantity to distend them, the injected substance may be almost directly afterward found in the lymphatics.

Some other matters than those originally contained in the exuded liquor sanguinis may, however, find their way with it into the lymphatic vessels. Parts which having entered into the composition of a tissue, and, having fulfilled their purpose, require to be removed, may not be altogether excrementitious, but may admit of being reorganized and adapted again for nutrition; and these may be absorbed by the lym-

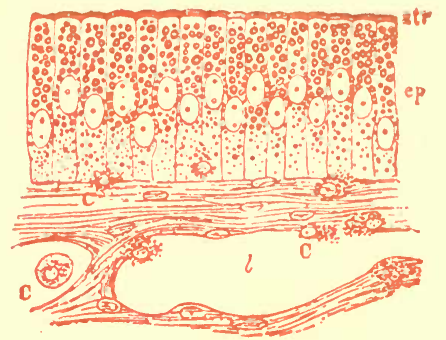

Fig. 286.-Mucous membrane of frog's intestıne during fat absorption. ep, epithelium; str, striated border; C, lymph corpuscles ; $l$, Iacteal. (E. A. Schäfer.)

phatics, and elaborated with the other contents of the lymph in passing through the glands.

The Blood-Vessels. - In the absorption by the lymphatic or lacteal vessels just described there appears something like the exercise of choice in the materials admitted into them. This is not the case with the blood-vessels; it appears that every substance, whether gaseous, liquid, or a soluble, may be absorbed by the blood-vessels, provided it is capable of permeating their walls, and of mixing with the blood.

\section{Where Absorption May Take Place.}

In the Alimentary Canal. - The greatest activity of absorption occurs in the alimentary canal. In it the materials of the duly digested food find their way by means of this process on the one hand into the bloodvessels of the portal circulation, and on the other into the lacteal vessels which are, as we have seen, the commencements of the lymphatic vessels of the intestines.

In the Stomach.-Recent experiments have shown that though absorption does take place in the stomach, it is not as active as was formerly supposed, even in the case of water. Von Mering has found that water begins to pass from the stomach into the intestine almost 
as soon as it is swallowed, and that very little of it is absorbed from the stomach. Of 500 cc. given by mouth to a large dog, only 5 cc. were absorbed in 25 minutes, the rest having passed into the intestine. Peptones and sugars are absorbed in the stomach, but only to a limited extent, and the same is true of salts. Fats are not absorbed at all in the stomach. In all cases absorption from the stomach is much increased by alcohol and condiments, such as pepper and mustard.

In the Small Intestine. - All the products of digestion are absorbed in the small intestine, as is abundantly shown by experiments. The absorption of fats has been already described. Recently absorption from the small intestine has been studied in the human subject in the case of a patient who had a fistulous opening in the lower part of the ileum. Eighty-five per cent of the proteid of a test-meal was absorbed before the food reached the fistula. Though water and salts are freely absorbed, the intestinal contents does not lose much in bulk or fluidity because of the quantity of water added in the alimentary secretions. In absorption, sugar is changed either just before or during its passage through the wall of the intestine from maltose into dextrose.

In the Large Intestine.-A great deal of absorption takes place in the large intestine. This is evident from the fact that the intestinal contents is very fluid when it enters the large intestine, and almost solid when it leaves it. Its contents passes through the large intestine very slowly, usually occupying about 12 hours. In addition to water and salts, the sugar, proteid, and fats not absorbed in the small intestine are almost entirely absorbed here.

The power of absorption in the large intestine sometimes forms an important feature in medical practice. When patients cannot swallow solid or liquid food, or retain what has been swallowed, they may be nourished by rectal feeding. The large intestine shows a remarkable power in its ability to absorb unchanged albumins, such as white of egg, as well as peptones and proteoses.

In the stomach as well as in both the large and small intestine, the absorption of water, salts, proteids, and sugars takes place chiefly into the blood-vessels.

Through the Skin.-It has been shown that metallic preparations rubbed into the skin have the same action as when given internally, only in a less degree. Mercury applied in this manner exerts its specific influence upon syphilis, and excites salivation; potassio-tartrate of antimony may excite vomiting, or an eruption extending over the whole body; and arsenic may produce poisonous effects. Vegetable matters, also, if soluble, or already in solution, give rise to their peculiar effects, as cathartics, narcotics, and the like, when rubbed into the skin. The effect of rubbing is probably to convey the particles of the matter into 
the orifices of the glands, whence they are more readily absorbed than they would be through the epidermis. When simply left in contact with the skin, substances, unless in a fluid state, are seldom absorbed.

It has long been a contested question whether the skin covered with the epidermis has the power of absorbing water; and it is a point the more difficult to determine because the skin loses water by evaporation. But, from the result of many experiments, it may now be regarded as a well-ascertained fact that such absorption really occurs. The absorption of water by the surface of the body may take place in the lower animals very rapidly. Not only frogs, which have a thin skin, but lizards, in which the cuticle is thicker than in man, after having lost weight by being kept for some time in a dry atmosphere, are found to recover both their weight and plumpness very rapidly when immersed in water. When merely the tail, posterior extremities, and posterior part of the body of the lizard are immersed, the water absorbed is distributed throughout the system. And a like absorption through the skin, though to a less extent, may take place also in man.

In severe cases of dysphagia, when not even flujds cam be taken into the stomach, immersion in a bath of warm water or of milk and water may assuage the thirst; and it has been found in such cases that the weight of the body is increased by the immersion. Sailors also, when destitute of fresh water, find their urgent thirst allayed by soaking their clothes in salt water, and wearing them in that state; but these effects are in part due to the hindrance to the evaporation of water from the skin.

Through the Lungs.-It is a remarkable fact that not only is the epithelium of the pulmonary air vesicles able to allow the passage through it of gases and volatile substances, but that also under certain conditions fluids such as water may also be absorbed, and besides this, the presence of carbon particles in the bronchial glands and elsewhere in connection with the lungs must point to the pulmonary epithelium as the only possible channel of their absorption. 


\section{CHAPTER XI.}

\section{METABOLISM, NUTRITION, AND DIET.}

IT is not only necessary that the animal body should be supplied with food in order that its natural functions may go on without interruption, but it is also equally requisite that the food should consist of proper materials. It may be supposed that each kind of animal by instinct keeps itself supplied with the substances which supply the needs of its own metabolism the best, and it is a matter of every-day experience that in the case of man, each endeavors to supply himself with food according to the circumstances of his surroundings. We may therefore accept such data as we can obtain from the observation of numerous examples of such selection in the way of diet when we are in the act of drawing up a diet-scale, relying upon such empiric knowledge alone, or, on the other hand, we may proceed more scientifically, and endeavor to plan a diet-scale from our experimental observation of the loss which takes place in the body in the course of the twenty-four hours by the excreta. If we do this we assume that the food is taken in to supply what is generally called the waste of the tissues. The term is scarcely an accurate one, but if we take it to mean in a restricted sense,-what the tissues and organs of the body give out to be eliminated by the excretory organs in the course of the day,-we may continue to use it.

The food then may be supposed as intended to supply the place of that which is given out by the body. But in the choice of a diet this is not enough; the food should be sufficient to supply such need without waste and without unduly increasing the output of excreta, while at the same time the body should be maintained in health, withont increase or loss of weight.

These requisites of a diet scale then allow for wide alterations in the amount of different kinds of foods under different circumstances.

Careful analyses of the excreta, many of which we have already had occasion to call attention to, show that they are made up, besides water, chiefly of the chemical elements carbon, hydrogen, oxygen, and nitrogen, but that they also contain, to a less extent, sulphur, phosphorus, chlorine, potassium, sodium, and certain other of the elements. Since this is the case it must be evident that to balance this waste, foods must be supplied containing all these elements to a certain degree, but some of them, viz., those which take a principal part in forming the excreta, in large amount. 
Of the excreta the carbon dioxide and ammonia, which are made up of the elements carbon, oxygen, nitrogen, hydrogen, are given off from the lungs. By the urine many elements are eliminated from the blood, especially nitrogen, hydrogen, and oxygen. In the sweat, the elements chiefly represented are carbon, hydrogen, and oxygen, and these are also those of which the frces are made. By all the excretions large quantities of water are got rid of daily, but chiefly by the urine.

The relations between the amounts of the chief elements contained in these various excreta in twenty-four hours may be thus summarized:-

\begin{tabular}{|c|c|c|c|c|c|}
\hline$\longrightarrow$ & water. & C. & H. & $\mathrm{N}$. & o. \\
\hline 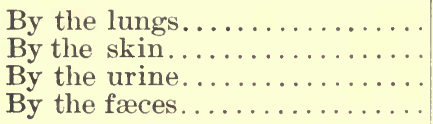 & $\begin{array}{r}330 \\
660 \\
1700 \\
128\end{array}$ & $\begin{array}{l}248.8 \\
2.6 \\
9.8 \\
20 .\end{array}$ & $\begin{array}{l}- \\
\overline{3.3} \\
3 .\end{array}$ & $\begin{array}{c}? \\
\overline{15.8} \\
3 .\end{array}$ & $\begin{array}{l}651.15 \\
7.2 \\
11.1 \\
12 .\end{array}$ \\
\hline Grammes ....... & 2818 & 281.2 & 6.3 & 18.8 & 681.41 \\
\hline
\end{tabular}

From this should be subtracted the 296 grms. water, which are produced by the union of hydrogen and oxygen in the body during the process of oxidation (i.e., 33 hydrogen and 262 oxygen). There are 26 grms. of salts got rid of by the urine, and 6 by the fæces; total, 32 grms.

The quantity of carbon daily lost from the body amounts to about 281.2 grms. (nearly 4,500 grains), and of nitrogen 18.8 grms. (nearly 300 grains), and if a man could be fed by these elements, as such, the problem would be a very simple one; a corresponding weight of charcoal and, allowing for the oxygen in it, of atmospheric air, would be all that is necessary. But an animal can live only upon these elements when they are arranged in a particular manner with others, in the form of such food-stuffs as we have already enumerated, p. 326 et seq.; moreover, the relative proportion of carbon to nitrogen in either of these compounds alone is, by no means, the proportion required in the diet of man. Thus, in proteid, the proportion of carbon to nitrogen is only as 3.5 to 1 . If, therefore, a man took into his body, as food, sufficient proteid to supply him with the needful amount of carbon, he would receive more than four times as much nitrogen as he wanted; and if he took only sufficient to supply him with nitrogen, he would be starved for want of carbon. It is plain, therefore, that he should take with the albuminous part of his food, which contains so large a relative amount of nitrogen in proportion to the carbon he needs, substances in which the nitrogen exists in much smaller quantities relatively to the carbon. 
It is therefore evident that the diet must consist of several substances, not of one alone.

Many valuable observations have been made with a view of ascertaining the effect upon the metabolism of a variation in the amount and nature of food. These are of great assistance in the consideration of dietetics.

Effect of a Proteid Diet.-Experiments have been made, to a considerable extent upon dogs, which demonstrate the effect of proteid food. After a period without food, during which the output of nitrogen, as shown by the urea, had diminished to a certain amount, the animal is fed with a diet of lean meat which would suffice to produce the amount of urea, and so of flesh, which it had been losing during its starvation period. The effect of this, however, is at once to send up the amount of urea excreted to a point above that which it has been previous to the commencement of its flesh diet, so that again the output of nitrogen would exceed its income, and the weight of the animal would continue slowly to diminish. It is only after a considerable increase of the flesh given that a point is reached where the income and expenditure are equal, and at which the animal is not using up quickly or slowly the nitrogen of his own tissue, and is no longer losing flesh. 'This condition in which the nitrogen of the egesta equals the nitrogen of the ingesta is known as nitrogenous equilibrium. In the dog, according to Waller, it does not occur until the amount of flesh of the food is over three times as great as would be necessary to supply the nitrogen of the urea during a period of starvation. Thus a dog excretes during a starvation period 0.5 grms. of urea per kilo of body weight; in order to satisfy this it would be necessary to administer 1.5 grms. per kilo of meat; this at once increases urea excreted to about 0.75 grms. per kilo of body weight, and nitrogenous equilibrium is not attained until over three times-viz., 5 grms. per kilo of body weight of meat is given. Foster gives even a larger figure. The effect, therefore, of proteid food is largely to increase the excretion of urea, which indicates increase of the metabolism of the tissues.

It must not be thought however that during nitrogenous equilibrium there is, of necessity, equilibrium of carbon. On the contrary, it is very possible that the carbon, as supplied by the large amount of meat, is not entirely eliminated, but may be partially retained in the body. If retained in the body it is probably retained in the form of fat, although possibly it might be retained partially as some carbohydrate, e.g., glycogen; but the amount of glycogen obtained from the body is too small for the latter to be appreciable. The animal in nitrogenous equilibrium, therefore, may gain weight, although not in the form of flesh. The converse may also be the case, the animal getting rid of more carbon 
than the meat supplies, in which case he would lose weight but would not lose flesh.

The proteids of food are described by Voit as having two relations to the proteid metabolism and to outgoing urea; the first part going to maintain the ordinary and quiet metabolism of the tissues, for which purpose it is actually built up into their molecule, and the second part causing a more rapid formation of urea and rapid proteid mefabolism, but never forming a part of the actual protoplasmic molecule. The former proteids are called morphotic or tissue proteids, the latter circulating or floating proteids. Normally more proteid is eaten than is needed to supply proteid waste. Pflüger has pointed ont, however, that the tissues must have an excess of proteid to destroy in order to perform their metabolic processes normally. This use of the proteids to form by their oxidation heat and not to produce tissue was looked upon by the older physiologists as a wasteful use of good material, and was called a luxus consumption.

The condition of nitrogenous equilibrium (i.e., the income and output being equal) is one which may be maintained even if the amount of proteid taken as diet far exceeds the necessities of the economy, the urea being excreted in excessive amount, and the wasteful use of proteid food which is so common may not be attended with harmful consequences, so long as the liver is able to do its work in the formation of urea. The body may or may not increase in weight, but if the liver strikes work from any cause, a condition of lithiasis, or of gout, follows.

It has not actually been proved, but it is not unlikely, that even in the condition of lithiasis, the nitrogen of the ingesta may not greatly exceed that of the egesta, but that the mode of elimination is different. It is only in cases of growth or putting on of flesh, as in growing children, that nitrogen is retained in the body, except to a very small amount, in health.

According to calculations which have been made, it appears that the body puts on thirty grammes of flesh for every gramme of nitrogen so retained.

As regards the retention of carbon in the body, it is calculated that one gramme and a half of weight is put on for each gramme by which the ingesta of carbon is greater than the egesta.

The Effect of an Albuminoid Diet.-The albuminoid which is eaten in greatest quantity is gelatin. Though gelatin closely resembles the proteid molecule chemically, it cannot replace the proteid of the food. In other words, nitrogenous equilibrium cannot be maintained on a diet consisting of gelatin, carbohydrates, and fats. Proteid food is absolutely essential to the reconstruction of the proteid molecule. Gelatin is one of the proteid substances which does not have any food value, 
strictly speaking, as the following experiments will prove: In one case, when $500 \mathrm{grms}$. of food, without any gelatin, formed the diet, the subject lost 22 grms., but when 200 grms. of gelatin were added, the subject gained 54 grms. In another experiment, when the diet consisted of 2,000 grms. of meat without gelatin, the gain was 30 grms., but when 200 grms. of gelatin were added, the gain became $376 \mathrm{grms}$. The lack of real food value is proven by a third experiment in which the diet consisted at first of 200 grms. each of meat and of gelatin; here the gain was 25 grms., but when the meat was omitted and the gelatin alone given, there was a loss of $118 \mathrm{grms}$. In these cases gelatin did not take the place of proteid in any sense, but rather saved it from work. The proteid was so protected that, instead of being used up, it helped to form tissues and increased the body weight. Gelatin, therefore, saves other material for constructive processes.

Formation of Urea. - Having studied the uses of proteids in the body, we may next turn our attention to their conversion to urea, the form in which the used-up proteids chiefly leave the body. The method of formation of urea, as well as the place where this occurs, has given rise to great controversy, while most of the intermediate products between proteids and urea have not as yet been determined. We can state with certainty that urea is not formed in the kidneys, since it is not only found in the blood of the renal artery, but it accumulates in the blood if the kidneys are diseased or removed and the separation of the urine is interfered with. Thus it seems reasonable to assume that the function of the kidneys, so far as the more important solid-urea-is concerned, is only one of separation. This will be discussed under the heading of the method of the secretion of the urine. It remains to consider here the question of the origin of the urea which is found in the blood, and its method of formation.

At the present time it is believed that urea is formed in the liver. This conclusion is borne out by a number of experiments. The power of the liver cells to form urea is shown by the increase of urea in the blood leaving an isolated (and living) liver, through which an artificial circulation is kept up, when ammonium carbonate, or other ammonium salts, are added to the blood. The same change occurs even when liver is chopped up and simply mixed with the ammonium compounds in a beaker; this shows that the change is due to the metabolic activity of liver cells. The reaction is probably as follows:

$$
\left(\mathrm{NH}_{4}\right)_{2} \mathrm{CO}_{3}-2 \mathrm{H}_{2} \mathrm{O}=\mathrm{CON}_{2} \mathrm{H}_{4} \text {. }
$$

If blood from a well-fed animal be circulated through the isolated liver, there is a distinct increase in the amount of urea it contains. On 
the other hand, if the blood be from a fasting animal, there is no increase of urea. Evidently, then, the blood from a well-fed animal contains something which the liver cells are capable of transforming to urea. And, finally, if the liver be removed and the animal kept alive, as has been done (Pawlow), there is a marked diminution in the quantity of urea in the urine. The power of the liver to form urea is thus demonstrated, and, moreover, the fact that the liver forms from some antecedent substance the greater part of the urea eliminated.

The question which now presents itself is, What is this antecedent substance or substances?

Urea is the end-product of the oxidation of proteids. It was formerly thought that urea was formed directly from some antecedent among the closely related products of proteid metabolism, such as creatin, creatinin, leucin, tyrosin, xanthin, hypoxanthin, etc. Creatinin at one time seemed the most probable source, because in laboratory experiments it decomposes into urea and sarcosin. Attention was also directed to leucin and tyrosin, which are found in practically all the glandular organs of the body. It was found that when leucin was fed to a dog, the amount of urea in the urine was considerably increased, but that leucin itself did not appear; the same phenomena were noticed with glycin, sarcosin, and the amido acids. It was also known that in acute yellow atrophy of the liver, a disease characterized by degeneration of the liver cells with consequent loss of functional power, the urea of the urine was replaced by leucin and tyrosin. Experimental investigation, however, did not justify any of these theories.

Finally it was found that when ammonia was fed to animals, the nitrogen appeared in the urine in the form of urea. Due investigation of this fact led to the belief that proteids were first broken down to an ammonia stage and then again built up into urea by the liver. For a long time it was thought that this stage was represented by ammonium carbonate, but in view of recent experiments this idea has been given up, and it is now believed that ammonium carbamate is the true antecedent.

In these experiments the liver was first shut out of the general circulation by (Eck's fistula) connecting the portal vein with the hepatic artery; the results of this operation are, for all practical purposes, equivalent to actual removal of the liver. When animals survived this operation it was found that they could live if fed very carefully on a mixed diet from which proteids were almost entirely eliminated, but that if the food contained an excess of proteids, convulsions ensued and proved fatal. Further investigation of the composition of the urine and blood showed that proteid metabolism was represented in them by ammonium carbamate and not by urea. Ammonium carbamate was then 
injected into the blood of other animals; when a larger quantity was used than the liver could dispose of, death ensued, following couvulsions of the same nature as those produced by an excess of proteid food in the animals which had been operated on.

Ammonium carbamate is thus shown to be, in part at least, the direct antecedent of urea; it is also shown to be a toxic substance which may cause death by accumulating in excess. The reaction by which the liver changes it to the inert form of urea is as follows:

$$
\mathrm{CO}<\underset{\mathrm{ONH}_{4}}{\mathrm{NH}_{2}}-\mathrm{H}_{2} \mathrm{O}=\mathrm{CO}<\underset{\mathrm{NH}_{2}}{\mathrm{NH}_{2}} \text {. }
$$

(Ammonium carbamate.) (Urea.)

The manner in which absorbed proteids are changed to ammonium carbamate, etc., is as yet undecided. According to one theory, while still in the circulating medium, they are metabolized by direct contact with the living bioplasm of the tissues; according to another, they must first be incorporated in the body tissues and then changed. The intermediate steps occur chiefly in muscle tissue, and there is great reason to suppose that some of the steps are represented by various muscle extractives such as creatinin, hypoxanthin, etc. These substances probably break down into carbon dioxide, ammonia, and amido-acids, and are then built up by synthetic processes into ammonium carbonate, and then by dehydration changed to ammoniun carbamate. Another possible antecedent is ammonium lactate; this is derived from the lactic acid which is produced in large quantities in the muscles. Muscular activity increases the elimination of urea, but the increase is very slight, and there is no direct relationship between the amount of work done and the amount of nitrogen excreted.

There is experimental evidence to show that while the liver produces the major part of the urea eliminated, other organs or tissues are capable of forming it to a limited degree.

Formation of Uric Acid.-Uric acid probably arises much in the same way as urea. The relation which uric acid and urea bear to each other, as we have seen, is still obscure. The fact that they often exist together in the same urine, makes it seem probable that they have different origins; but the entire replacement of one by the other, as of urea by uric acid in the urine of birds, serpents, and many insects, and of uric acid by urea, in the urine of the feline tribe of Mammalia, shows their close relationship. But although it is true that one molecule of uric acid is capable of splitting up into two molecules of urea and one of mes-oxalic acid, this is no evidence that uric acid is an antecedent of urea in the nitrogenous metabolism of the body.

The intimate relations which exist between several other of the ni28 
trogenons extractives and uric acid will be seen by a reference to their IUl'uulæ:-

Hypoxanthin or Carnin
Xanthin

Uric Acid
$\mathrm{C}_{3} \mathrm{H}_{4} \mathrm{~N}_{4} \mathrm{O}$.

$\mathrm{C}_{5} \mathrm{H}_{4} \mathrm{~N}_{4} \mathrm{O}_{2}$.

$\mathrm{C}_{5} \mathrm{H}_{4} \mathrm{~N}_{4} \mathrm{O}_{3}$.

Formation of Hippuric Acid.-The source of hippuric acid is not satisfactorily determined; in part it is probably derived from some constituents of vegetable diet, though man has no hippuric acid in his food, nor, commonly, any benzoic acid that might be converted into it; in part from the natural disintegration of tissues, independent of vegetable food, for Weismann constantly found an appreciable quantity, even when living on an exclusively animal diet. Hippuric acid arises from the union of benzoic acid with glycin $\left(\mathrm{C}_{2} \mathrm{H}_{5} \mathrm{NO}_{2}+\mathrm{C}_{7} \mathrm{H}_{6} \mathrm{O}_{2}=\mathrm{C}_{9} \mathrm{H}_{9} \mathrm{NO}_{3}+\right.$ $\mathrm{H}_{2} \mathrm{O}$ ), which union probably takes place in the kidneys themselves. It is possible that the aromatic radicle in this reaction is obtained from the splitting up of tyrosin, which appears so frequently as a result of the decomposition of proteid, the ammonia radicle with which it is associated going to form urea.

The source of the extractives of the urine is probably in chief part metabolism of the nitrogenous tissues, but we are unable to say whether these nitrogenous bodies are merely accidental, having resisted further decomposition into urea, or whether they are the representatives of the decomposition of special tissues, or of special forms of metabolism of the tissues. There is, however, one exception, and that is in the case of kreatinin; this represents not only the kreatinin which enters the body in ordinary flesh food, but nitrogenous waste as well.

Effects of Fats and Carbohydrates as Food.-Experiments illustrating the ill-effects produced by feeding animals upon one or two alimentary substances only have been often performed.

Dogs were fed exclusively on sugar and distilled water. During the first seven or eight days they were brisk and active, and took their food and drink as usual; but in the course of the second week they began to get thin, although their appetite continued good, and they took daily between six and eight ounces of sugar. The emaciation increased during the third week, and they became feeble, and lost their activity and appetite. At the same time an ulcer formed on each cornea, followed by an escape of the humors of the eye: this took place in repeated experiments. The animals still continued to eat three or four ounces of sugar daily; but became at length so feeble as to be incapable of motion, and died on a day varying from the thirty-first to the thirty-fourth. On dissection their bodies presented all the appearances produced by death from starvation; indeed, dogs will live almost the same length of time without any food at all.

When dogs were fed exclusively on gum, results almost similar to the 
above ensued. When they were kept on olvve-oil and water, all the phenomena produced were the same, except that no ulceration of the cornea took place; the effects were also the same with butter. The experiments of Chossat and Letellier prove the same; and in men, the same is shown by the various diseases to which those who consume but little nitrogenous food are liable, and especially by the affection of the cornea which is observed in Hindus feeding almost exclusively on rice.

The nutritive function of fats and carbohydrates in the body is to serve as a source of energy. They are oxidized, with the ultimate production of carbon dioxide and water, and must liberate the same amount of energy as when burned outside the body. A given amount of fat, however, furnishes more energy than a corresponding amount of either proteid or carbohydrate. The stock of fat in the animal body will delay the fatal consequences of the deprivation of food. The percentage loss of fat in a starving animal is given on page 440 .

The Formation of Glycogen (Glycogenesis). - The important fact that the liver normally forms sugar, or a substance readily convertible into it, was discovered by Claude Bernard in the following way: he fed a dog for seven days with food containing a large quantity of sugar and starch; and, as might be expected, found sugar in both the portal and hepatic blood. But when this dog was fed with meat only, to his surprise, sugar was still found in the blood of the hepatic veins. Repeated experiments gave invariably the same result; no sugar being found, under a meat diet, in the portal vein, if care were taken, by applying a ligature on it at the transverse fissure, to prevent reflux of blood from the hepatic venous system. Bernard found sugar also in the substance of the liver. It thus seemed certain that the liver formed sugar, even when, from the absence of saccharine and amyloid matters in the food, none could be brought directly to it from the stomach or intestines.

Bernard found, subsequently to the before-mentioned experiments, that a liver, removed from the body, and from which all sugar had been completely washed away by injecting a stream of water through its blood-vessels, after the lapse of a few hours contained sugar in abundance. This post-mortem production of sugar was a fact which could only be explained on the supposition that the liver contained a substance readily convertible into sugar; and this theory was proved correct by the discovery of a substance in the liver allied to starch, and now generally termed glycogen.

We may believe that glycogen is first formed and stored in the liver cells, and that the sugar, when present, is the result of its transformation.

Source of Glycogen.--Although, as before mentioned, the greatest amount of glycogen is produced by the liver upon a diet of starch or sugar, a certain quantity is produced upon a proteid diet. The glycogen when stored in the liver cells may readily be demonstrated in sec- 
tions of liver containing it by its reaction (red or port-wine color) with iodine, and moreover, when the hardened sections are so treated that the glycogen is dissolved out, the protoplasm of the cell is so racuolated as to appear little more than a framework. There is no doubt that in the liver of a hibernating frog the amount of glycogen stored up in the outer parts of the liver cells is very considerable.

Average ainount of Glycogen in the Liver of Dogs under Varous Diets (Pavy).

Diet.

Amount of Glycogen in Liver.

Animal food

7.19 per cent.

Animal food with sugar (about $\frac{1}{4} \mathrm{lb}$. of sugar daily)

Vegetable diet (potatoes, with bread or barley-meal)

17.23

The dependence of the formation of glycogen on the kind of food taken is also well shown by the following results, obtained by the same experimenter:

Average quantity of Glycogen found in the Liver of Rabbits after Fasting, and after a diet of Starch and Sugar respectively.

After fasting for three days

" diet of starch and grape-sugar

" " cane-sugar
Arerage Amount of Glycogen in Liver.

Practically absent.

15.4 per cent.

16.9

Glycogen is also formed on a gelatin diet, but fats taken in as food do not increase its amount in the cells. The diet most favorable to the production of a large amount of glycogen is a mixed diet containing a large amount of carbo-hydrate, but with some proteid. Glycerin injected into the alimentary canal may also increase the glycogen of the liver.

Destination of Glycogen.-There are two chief theories as to the destination of hepatic glycogen. (1.) That the glycogen is converted into sugar during life by the agency of a ferment (liver diastase) also formed in the liver; and that the sugar is conveyed away by the blood of the hepatic veins, to undergo combustion in the tissues. (2.) That the conversion into sugar only occurs after death, and that during life no sugar exists in healthy livers; glycogen not undergoing this transformation. The chief arguments advanced in support of this view are, $(a)$ that scarcely a trace of sugar is found in blood drawn during life from the right ventricle, or in blood collected from the right side of the heart immediately after an animal has been killed; while if the examination be delayed for a very short time after death, sugar in abundance may be found in such blood; $(b)$, that the liver, like the venous blood in the heart, is, at the moment of death, completely free from sugar, although afterward its tissue speedily becomes saccharine, unless the formation of 
sugar be prevented by boiling, or other means calculated to interfere with the action of a ferment.

Instead of adopting the view that normally, during life, glycogen acts as a store of carbo-hydrate material to be converted, little by little, into sugar as occasion requires, and that it passes as sugar into the hepatic venous blood, to be conveyed to the tissues to be further disposed of, Pavy inclines to the belief that it may represent an intermediate stage in the formation of fat from materials absorbed from the alimentary canal. There is little evidence in favor of this view, and although it is possible that the liver cells may, in some way or other (not at present understood), be able to convert part of its store of glycogen into fat, the consensus of opinion inclines to the belief that most of the glycogen leaves the liver as sugar.

Indeed, wherever glycogen is found, in the muscles, in the placenta, or elsewhere, it must be looked upon as a store of carbo-hydrate material which may be oxidized to furnish energy to the body. Whether the glycogen which probably reaches the muscles as sugar is reconverted into glycogen before it is built up as it were into the protoplasmic molecule is not known.

The relation of glycogen to the cell metabolism. - It is not exactly known whether the glycogen is formed simply by a process of dehydration of the sugar which reaches the cells in the portal blood, or whether the cells by their metabolism are usually in the habit of forming glycogen or sugar which, during fasting and other similar conditions, is at once discharged into the hepatic blood to be used up by the tissues, but which is stored up in the cells as glycogen as long as there is sufficient sugar in the blood without it, or as long as the tissues are so quiescent as not to require more than a small quantity of the total amount of carbo-hydrate secreted by the hepatic cells.

Glycosuria.-Sugar may be present not only in the hepatic reius, but in the systemic blood to excess, and when such is the case, the sugar is excreted by the kidneys, and appears in variable quantities in the urine. This condition is known as glycosuria.

Influence of the Nervous System.-Glycosuria may be experimentally produced by puncture of the medulla oblongata in the region of the vaso-motor centre. The better fed the animal the larger is the amount of sugar found in the urine; in the case of a starving animal no sugar appears. It is, therefore, highly probable that the sugar comes from the hepatic glycogen, since in the one case glycogen is in excess, and in the other it is almost absent. The nature of the influence is uncertain. It may be exercised in dilating the hepatic vessels, or possibly may be exerted on the liver cells themselves. The whole course of the nervous 
stimulus cannot be traced to the liver, but, at any rate, it is not conducted by the vagi or by the splanchnics, but at first it passes from the lower part of the floor of the fourth ventricle and medulla down the spinal cord as far as-in rabbits - the fourth dorsal vertebra, and hence to the first thoracic ganglion. The formation of sugar by the liver is also not a vaso-dilator effect, since it will occur when the vessels are constricted.

Many other circumstances will cause glycosuria. It has been observed after the administration of various drugs-e.g., strychnine (in frogs), phloridzin, a glucoside, and phloretin, a derivative of phloridzin, not a glucoside, morphine, nitrite of amyl, etc.-after the injection of urari, poisoning with carbonic oxide gas, the inhalation of ether, chloroform, etc., the injection of oxygenated blood into the portal venous system. It has been observed in man after injuries to the head, and in the course of various diseases.

In all such cases, at any rate, the glycosuria appears to be due to some abnormal activity of the liver cells themselves set up by the direct action of the secretory nerves upon them.

The well-known disease, diabetus mellitus, in which a large quantity of sugar is persistently secreted daily with the urine, has, doubtless, some close relation to the normal functions of the pancreas. The nature of the relationship has not yet been determined, thongh some recent experiments seem to be pertinent (see p. 318).

Effect of too much Food.-All the three classes of food-stuffs mentioned-fats, carbohydrates, and gelatin-have their distinct uses when combined with proteids. A small amount of fat or a larger amount of carbohydrate (starch or sugar) added to some proteid diminishes the amount of proteid required before nitrogenous equilibrium is attained (in a $\log$ to the extent of 50 per cent or more), but if the carbohydrate exceed a certain minimum it is retained in the body as fat.* If the proteid be increased, the metabolism is increased likewise, and so fat may not be deposited, even if the carbohydrate of the diet be excessive. It is even possible that some of the already stored-up fat may be used up, and so loss of weight (fat) might result.

Persistent excess of carbohydrate food produces an accumulation of fat, which may not only be an inconvenience causing obesity, but may interfere with the proper nutrition of muscles, and a feebleness of the action of the heart, with other troubles. Starches when taken in great

* The result of various feeding experiments, e.g., of the milch cow fed upon grass, have proved beyond all doubt that fat is formed by the tissues chiefly from carbohydrate food, but to a less extent from proteids. Fatty foods, even if they indirectly lead to the deposition of fats, are not as such deposited in the tissues. Fat is every where in the body an effect of actual protoplasmic metabolism. 
excess are almost certain to give rise to dyspepsia, with acidity and flatulence. Excess of starch or of sugar in the food may, however, be got rid of by the urine in the form of sugar. There is evidently a limit to the absorption of fat as well as of starch, since if in excessive amount they may appear in the fæces.

That salts are necessary as food is proved by the presence of scurvy when they are not present, and we know that there is a consant excretion of chlorides, phosphates and sulphates in the urine, so that in order to balance the income and output, these salts in combination with sodium, potassium, calcium, etc., must be taken in.

The necessity for the taking in of water, in order to balance the ex cretion, is sufficiently obvious.

'To summarize what has been said:-

Proteid.-i. If the nitrogen of the income is less than that of the output, the animal loses flesh and starves, gradually or quickly, according to the extent of the deficiency.

ii. If the nitrogen of the income be evenly balanced, the proteid being only just sufficient, the animal does not lose flesh, but may increase or diminish in weight (fat).

iii. If the nitrogen of the ingesta exceed that of the egesta, the excess is mainly retained in the form of flesh.

iv. If the proteid be in great excess, although there be a condition of nitrogenous equilibrium, there may be increase in weight, but also a likelihood of gout and similar affections.

Fatty and Carbohydrate Foods are of no use either together or separately without the addition of the other food-stuffs. In moderation, either may diminish the amount of proteid necessary to produce nitrogenous equilibrium. If the quantity of either be increased beyond a certain amount, it is retained in the body in form of fat (and, in the case of the carbohydrate, as glycogen). If in great excess, disorders of digestion occur. Fats have more potential energy than carbohydrates, but are less digestible. Fatty foods need more oxygen than carbohydrates when they are used up in the body.

Gelatin will not entirely, but will partly replace the proteid in a diet.

Salts of sodium, potassium, calcium, etc., are necessary in food, the chlorides, phosphates and sulphates, and possibly the citrates, being the most important of those required.

Water is absolutely essential to life-an animal will not survive deprivation for longer than a few days.

Effects of Deprivation of Food. - The animal body deprived of all food in the course of a variable time dies from starvation. The length of time that any given animal will live in such a condition depends upon many circumstances; the chief may be supposed to be the nature and activity of the metabolism of its tissues. 
The effect of starvation on the lower animals, as recorded by varion: experimenters is:-(1.) One of the most notable effects of starvation, at might be expected, is loss of weight; the loss being greatest at first, as at rule, but afterward not varying very much, day by day, until death ensues. Chossat found that the ultimate proportional loss was, in different animals experimented on, almost exactly the same; death occurring when the body had lost two-fifths (forty per cent) of its original weight. Different parts of the body lose weight in very different proportions. The following most noteworthy losses are taken, in round numbers, from the table given by Chossat:-

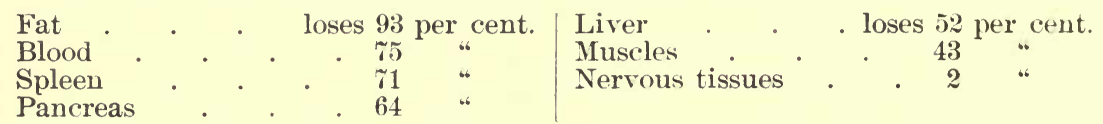

These figures are in practical agreement with those of later experimenters. They show that the chief losses are sustained by the adipose tissue, the muscles and glands.

(2.) The effect of starvation on the temperature of the various animals experimented on by Chossat was very distinct. For some time the variation in the daily temperature was more marked than its absolute and continuous diminution, the daily fluctuation amounting to $3^{\circ} \mathrm{C} .\left(5^{\circ}\right.$ or $6^{\circ} \mathrm{F}$.), instead of $5^{\circ}$ to $1^{\circ} \mathrm{C}$. $\left(1^{\circ}\right.$ or $2^{\circ} \mathrm{F}$. $)$, as in health. But a short time before death, the temperature fell very rapidly, and death ensued when the loss had amounted to about $16.2^{\circ} \mathrm{C}$. $\left(30^{\circ} \mathrm{F}\right.$.). It has been often said, and with truth, although the statement requires some qualification, that death by starvation is really death from want of heat; for not only has it been found that differences of time with regard to the period of the fatal result are attended by the same ultimate loss of heat, but the effect of the application of external warmth to animals cold and dying from starvation, is more effectual in reviving them than the administration of food.

The symptoms produced by starvation in the human subject are hunger, accompanied, or it may be replaced, by pain, referred to the region of the stomach; insatiable thirst; sleeplessness; general weakness and emaciation. The exhalations both from the lungs and skin are foetid, indicating the tendency to decomposition which belongs to badly nctirished tissues; and death occurs, sometimes after the additional exhaustion caused by diarrhoa, often with symptoms of nerrous disorder, delirium or convulsions.

In the human subject death commonly occurs within six to ten days after total ceprivation of food. But this period may be considerably prolonged by taking a very small quantity of food, or even water only. The cases so frequently related of survival after many days, or even some weeks, of abstinence, have been due either to the last-mentioned circum- 
stances, or to others no less effectual, which prevented the loss of heat and moisture. Cases in which life has continued after total abstinence from food and drink for many weeks, or months, exist only in the imagination of the vulgar.

(3.) During the starvation period the excreta diminish. The urea, as representing the nitrogen, falls quickly in amount, reaches a minimum and remains constant at this point for several days, and then rises again and finally falls rapidly immediately before death; the sulphates and phosphates undergo much the same form of reduction. The carbon dioxide given out and the oxygen taken in diminish. The fæces diminish, as well as the bile. It has been concluded as highly probable that the greater part of the urea represents the loss of weight of the muscles.

The appearances presented after death from starvation are those of general wasting and bloodlessness, the latter condition being least noticeable in the brain. The stomach and intestines are empty and contracted, and the walls of the latter appear remarkably thinned and almost transparent. The various secretions are scanty or absent, with the exception of the bile, which, not being discharged, usually fills the gall-bladder. All parts of the body readily decompose.

In starvation, then, we see that the only income consists of the inspired oxygen. The whole of the energy of the body given out in the direction of heat and mechanical labor is obtained at the expense of the using up of its own tissues, there being as a result a constant drain of the nitrogen and carbon, not to mention the other elements of which they are made up. It is obvious that such a condition cannot be endured for any length of time.

\section{Requisites of a Normal Diet.}

It will have been understood that it is necessary that a normal diet should be be made up of various articles, that they should be well cooked, and that they should contain about the same amount of carbon and nitrogen as are got rid of by the excreta. No doubt these desiderata may be satisfied in many ways, and it would be unreasonable to expect the diet of every adult to be unvarying. The age, sex, strength, and circumstances of each individual must ultimately determine what he takes as food. A dinner of bread and cheese with an onion contains all the requisites for a meal, but such diet would be suitable only for those possessing strong digestive powers. It is a well-known fact that the diet of the continental nations differs from that of our own country, and that of cold from that of hot climates, but the same principle underlies them all, viz., the replacement of the loss of the excreta in the most convenient and economical way possible. Without going into detail in 
the matter here, it may be said that any one in active work requires more food than one at rest, and that children and women require less food than do adult men.

Of the various diet-scales which have been drawn out with the object of supplying the proximate principles in the required proportions, the foregoing is slightly modified from Moleschott:-

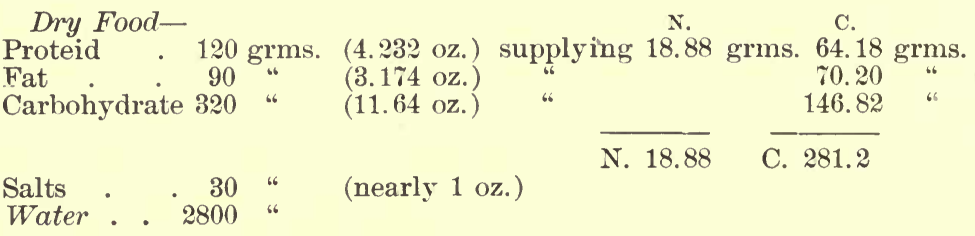

Two other diet-scales may be mentioned, which are often quoted, viz:-

Ranke's Diet-Scale.

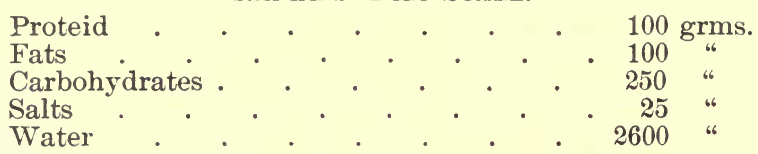

PetTenkofer \& Voit's Diet-Scale is as follows :-

Proteids
Fats
Carbohydrates
Salts . . . . . . . . . . . . . . . 352 to 500 "
Water .

The amount of the excreted carbon and nitrogen is not, of course, always the same, it having been unfortunately proved possible, for example, to subsist on 9 or 10 grms. of nitrogen and 200 grms. of carbon per diem (the ordinary diet for needle-women in London, and the average of the cotton operatives in Lancashire during the famine, 1862), the amount of these elements excreted falling to figures corresponding to such an income. Of course, upon such a diet the metabolism is low, and persistent weakness must be the result.

The 9 or 10 grms. of $\mathrm{N}$ in such a semi-starvation diet would be equivalent to 58.5 to 65 grms. of proteids, whereas the amount of proteids in some diets may be as high as 150-159 grms. per diem (English navvies), or 165 gms. (Munich brewer's' men). 'The English and Bavarian soldier in time of peace consumes 126 grms. of proteid per diem (4.4 oz.).

Not only the proteids but also the fats may vary; the amount may be as low as 56 grms. and as high as 117 grms. The carbohydrates may vary from 200 grms. to 500 grms. and upward. Sometimes, with a small proportion of fat, the carbohydrate may be correspondingly increased to make up the necessary carbon. A useful table after Payen 
will help to show in what ways it is possible to obtain the requisite amount of nitrogen and carbon from the most common food-stuffs.

In 100 parts of the following substances the proportion of $\mathrm{N}$ and $\mathrm{C}$ is indicated:

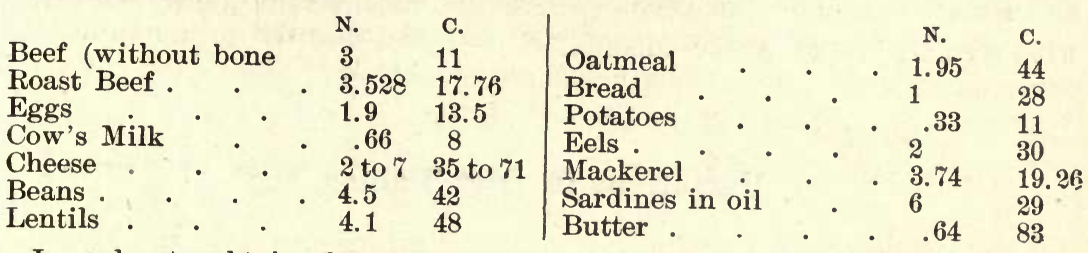

In order to obtain the amount of proteid present from the proportion of nitrogen, multiply by 6.5 .

From these data it is possible to form various diet-scales which shall supply the needs of different conditions. Assuming that the average amount of carbon and nitrogen required is about $300 \mathrm{grms}$. and $20 \mathrm{grms}$. respectively, this may be obtained as follows:-

340 grms. $\left\{\begin{array}{l}12 \text { oz. avoirdupois } \\ \text { g. } l \text { lb. }\end{array}\right.$ lean uncooked meat $* 10$ grms. 37 grms. 906 “ (32 oz. or 2 lbs. avoirdupois) bread . . 9 " 252 "

19 grms. 289 grms.

But this diet is not a usual one; a certain proportion of the carbon is usually supplied as butter, or bacon, and so if 90 grms. (3.1 oz.) of butter or bacon be used they would supply about 72 grms. of carbon, and the carbohydrate would be diminished nearly one-third; but the nitrogen would also be diminished from 9 grms. to 6 grms. It would be necessary to supply some extra nitrogenous principle, and this might be done by the addition of eggs, milk, cheese, beans, or of any of the food-stuffs already enumerated at p. 326 et seq., as supplying nitrogenous food chiefly. For example, 56 grms. (2 oz.) cheese, would supply, on an average, 3 grms. nitrogen and 20 grms. carbon; or 28 grms. cheese, supplying 1.5 grms. nitrogen and about 10 grms. carbon, and 225 grms. ( $\frac{1}{2} \mathrm{lb}$.) potatoes, and 225 grms. ( $\frac{1}{2} \mathrm{lb}$.) carrots, supplying together about $1 \mathrm{grm}$. of nitrogen and $35 \mathrm{grms}$. of carbon. The diet would then read as follows:-

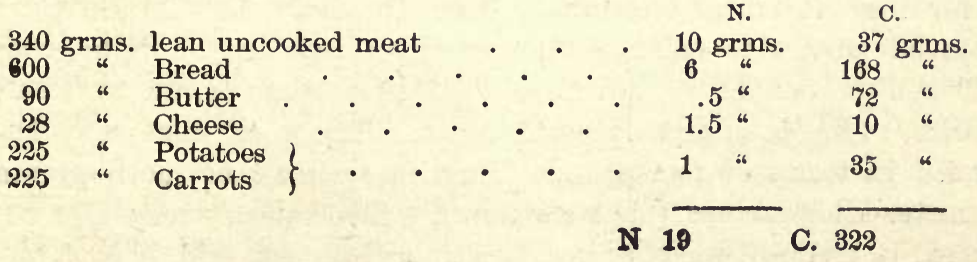

* As meat loses 23 to 34 per cent on cooking, the weight of cooked meat would be proportionately be less. 
The salts, over 20 grms., would be supplied by the meat 16 grms., the bread 12 grms., and regetables about 4 grms. The fluids should consist of about 2,500-2,800 grms., and might be given as water, with or without tea, coffee, or cocoa (which are chiefly stimulants), together with a small proportion of alcohol.

\section{Variations in Diet Tables.}

For infancy. - Milk affords a natural and perfect diet for infants. 'The amount which an infant during the first month should take is not less than 1 kilogramme ( $2 \frac{1}{4} \mathrm{lbs}$.) per diem. In 1,000 grms. there would be about 6.6 grms. nitrogen and 80 to 90 of carbon. This allows for a gain of weight of 2 to $5 \mathrm{oz}$. in the time.

For climate.-Very slight alteration is necessary. For warm climates, slightly increase the carbohydrates.

For hard labor. - All the articles of diet should be increased to make up for the increased metabolism.

Fattening diet. - In such a diet an excess of carbohydrates should be present.

To reduce obesity.- The fats and carbohydrates should be diminished, but the proteids should be relatively increased.

To increase muscle. - It has been found that a diet consisting largely of proteids in considerable amount combined with such passive exercise as that obtained by massage, will cause the body to put on flesh.

For training. - The whole diet should be increased, possibly preceded by a diet in which the proteid is in excess.

For brain work. - The chief essential is that the diet should consist of easily digestible materials.

\section{Income and Output of Energy.}

The food must be considered from another point of view in addition to that from which we have been considering it. It not only makes up for the substances eliminated from the body, but it also supplies potential energy to balance the energy set free in the living body as heat and movement. The amount of heat is measured in terms of calories, as has been already pointed out. 'The work done may be expressed in terms of foot-pounds (Euglish system), or metre-grammes, or metre-kilogrammes (metric system). The calories may also be expressed in terms of work, as heat is also, as has been said, a mode of motion. The heat-unit $\mathrm{Ca}$, may be transformed into metric work-unit by multiplying by 42 and diriding ly 1000 , and the converse. 
Manifestations of Force in the form either of Heat or Motion. - In the former case (Heat), the combustion must be sufficient to maintain a temperature of about $37.8^{\circ} \mathrm{C}$. $\left(100^{\circ} \mathrm{F}\right.$.) throughout the whole substance of the body, in all varieties of external temperature, notwithstanding the large amount continually lost in the ways previously enumerated. In the case of Motion, there is the expenditure involved in the $(a)$ Ordinary muscular movements, as in Prehension, Mastication, Locomotion, and numberless other ways: as well as in (b) Various involuntary movements, as in Respiration, Circulation, Digestion, etc.

Manifestation of Nerve-force; as in the general regulation of all physiological processes, e.g., Respiration, Circulation, Digestion; and in Volition and all other manifestations of cerebral activity.

The energy expended in all physiological processes, e.g., Nutrition, Secretion, Growth, and the like.

The total expenditure or total manifestation of energy by an animal body can be measured, with fair accuracy. All statements, however, must be considered for the present approximate only, and especially is this the case with respect to the comparative share of expenditure to be assigned to the various objects just enumerated.

The amount of energy daily manifested by the adult human body in (a) the maintenance of its temperature; (b) in internal mechanical work, as in the movements of the respiratory muscles, the heart, etc.; and $(c)$ in external mechanical work, as in locomotion, and all other voluntary movements, is made up, according to McKendrick, as follows:-

\begin{tabular}{|c|c|c|}
\hline $\begin{array}{l}\text { Work of heart per diem } \\
\text { Work of respiratory muscle } \\
\text { Eight hours' active work }\end{array}$ & $\begin{array}{l}\text { Metre- } \\
\text { kilogrammes. } \\
88,000 \\
14,000 \\
213,344\end{array}$ & $\begin{array}{l}\text { Gramme- } \\
\text { calories. }\end{array}$ \\
\hline Amount of heat produced in 24 hours & $\begin{array}{r}315,334 \text { or } \\
1,582,700 \text { or }\end{array}$ & $\begin{array}{r}743,000 \\
3,724,000\end{array}$ \\
\hline & $1,898,034$ or & $4,467,000$ \\
\hline
\end{tabular}

So that 4, 467 kilogramme calories represent the total energy manifested in 24 hours, 8 of which were employed in mechanical work, one-sixth of the total energy being work. This estimation considerably exceeds those of others, and the most general view is that the total energy exhibited in 24 hours by the average adult is rather under than over $1,000,000 \mathrm{kilog}$. metres.

Taking the diet-scale as given above (modified from Moleschott), we may see how this supplies the energy which is given out, remembering that $1 \mathrm{grm}$. proteid $=5,000$ to 5,500 calories ; minus the value of $\frac{1}{8} \mathrm{grm}$. urea $=700$ or 800 calories, $=$ say 4,$500 ; 1$ grm. fat $=9,000$ calories : and 1 grm. carbohydrate $=$ 4,000 calories. 


\begin{tabular}{|c|c|c|c|}
\hline & & & $\begin{array}{l}\text { Gramme- } \\
\text { calories. }\end{array}$ \\
\hline & Proteid at 4,500 per grm. & $=$ & 544,500 \\
\hline & Fat at 9,000 per grm. & $=$ & 810,000 \\
\hline “ & Carbohydrate at 4000 per grm. & $=$ & $1,320,000$ \\
\hline & & & 2.694 \\
\hline
\end{tabular}

Or roughly, 2,694 kilog. calories, equivalent to 1, 144,950 metre-kilogrammes of energy. This shows, although the calculation is only rough, that the diet which from other reasons was considered to be correct contains the potential energy to set free one million metre-kilogrammes of kinetic energy, and to leare a fair margin for errors of calculation.

'T'o the foregoing amounts of expenditure must be added the quite unknown quantity expended in the various manifestations of nerve-force, and in the work of nutrition and growth (using these terms in their widest sense). By comparing the amount of energy which should be produced in the body from so much food of a given kind, with that which is actually manifested (as shown by the various products of combustion, in the excretions), attempts have been made, indeed, to estimate, by a process of exclusion, these unknown quantities; but all such calculations must be at present considered only very doubtfully approximate.

Sources of Error.-Among the sources of error in any such calculations as the one above given must be reckoned, as a chief one, the, at present, entirely unknown extent to which forces external to the body (mainly heat) can be utilized by the tissues. We are too apt to think that the heat and light of the sun are directly correlated, as far as living beings are concerned, with the chemico-vital transformations involved in the nutrition and growth of the members of the regetable world only. But animals, although comparatively independent of external heat and other forces, probably utilize them, to the degree occasion offers. And although the correlative manifestation of energy in the body, due to external heat and light, may still be measured in so far as it may take the form of mechanicai work; yet, in so far as it takes the form of expenditure in nutrition or nerve-force, it is evidently impossible to include it by any method of estimation yet discovered; and all accounts of it must be matters of the purest theory. These considerations may help to explain the apparent discrepancy between the amount of energy which is capable of being produced by the usual daily amount of food, with that which is actually manifested daily by the body; the former leaving but a small margin for anything beyond the maintenance of heat, and mechanical work.

It is of much interest to consider the way in which protoplasm acts in converting food into energy plus decomposition products. It is certain that the substance itself does not undergo much change in the process except a slight amount of wear and tear. We may assume that it is the 
property of protoplasm to separate from the blood the materials which it may require to produce secretions, in the case of the protoplasm of secreting glands, or to enable it to evolve heat and energy as in the case of the protoplasm of muscle. The properties of the protoplasm are very possibly differently developed in each case, and the decomposition products, too, may be different in quality or quantity. Proteid materials appear to be specially needed, as is shown by the invariable presence of urea in the urine even during starvation; and as in the latter case there has been no food from which these materials could have been derived, the urea is considered to be derived from the disintegration of the nitrogenous tissues themselves. Which, if not all, of the three varieties of proteid of the blood, viz., serum-albumin, serum-globulin, and fibrinogen, is necessary for muscular metabolism is not certainly known, opinion appears to incline toward the first as the most important. The removal of all fat from the body in a starvation period, as the first apparent change, would lead to the supposition that fat is also a specially necessary pabulum for the production of protoplasmic energy; and the fact that, as mentioned above, with a diet of lean meat an enormous amount appears to be required, suggests that in that case protoplasm obtains the fat it needs from the proteid food, which process must be evidently a source of much waste of nitrogen. The fat which is deposited in the tissues has for its origin, as we have before remarked, in great part carbohydrate food, and is looked upon as a store of carbonaceous material; it has been suggested that as it leaves the tissue to be used up, it is reconverted into a carbohydrate, viz., dextrose. Salts appear to be absolutely essential for protoplasmic life. The idea that proteid food has two destinations in the economy, viz., to form organ or tissue proteid which builds up organs and tissues, and circulating proteid, from which the organs and tissues derive the materials of their secretions or for producing their energy, is a convenient one, but cannot be said to rest upon any very certain facts. Except in the possible case of the appearance of leucin and tyrosin in pancreatic digestion, already fully discussed, it must not be looked upon as more than a convenient hypothesis.

One question which has been little considered by physiologists, is what relationship, if any, there is between each tissue and the waste products of other tissues, or perhaps it should be said, the products of the metabolism of other tissues. It is not known whether, as the result of the katabolism of one tissue, products, proteid or otherwise, are not taken up by the blood and carried to other tissues, supplying exactly what is necessary for their complete anabolism; whether, for example, proteid residue does not arise from the metabolism of muscle which 
may be used further by glands. One step, at all events, in this direction has been taken; it has been suggested that the sarco-lactic acid continually produced by muscle is carried to the liver, either to be converted itself into glycogen, or by its influence on the hepatic cells to cause them to store up that substance. 


\section{CHAPTER XII.}

ANIMAL HEAT.

ONE of the most important results of the metabolism of the tissues is the production of the heat of the body. It is by this means that the bodily temperature is raised to such a point as to make life possible. In man and in such animals as are called warm-blooded, including only mammals and birds, it is found on the one hand, that there is an average temperature which is maintained with only slight variations in spite of changes in their environment, and on the other hand, that the possible variations above and below this average are comparatively slight. It must not be thought, however, that the average temperature in all mammals and birds is the same; for example, as we shall see. the average temperature of man is just $37^{\circ} \mathrm{C}$. $\left(98.6^{\circ} \mathrm{F}\right.$.), in some birds it is as high as $44^{\circ} \mathrm{C}$. $\left(111^{\circ} \mathrm{F}\right.$.), whereas in the wolf it is said to be under $36^{\circ} \mathrm{O}$ $\left(96^{\circ} \mathrm{F}.\right)$.

The average temperature of the human body in those internal parts which are most easily accessible, as the mouth and rectum, is from $36.9^{\circ}$ $-3 \% .4^{\circ} \dot{\mathrm{C}}$. $\left(98.5^{\circ}\right.$ to $99.5^{\circ} \mathrm{F}$.). In different parts of the external surface of the human body the temperature varies only to the extent of one or two degrees (C.), when all are alike protected from cooling influences; and the difference which under these circumstances exists, depends chiefly upon the different degrees of blood-supply. In the axilla-the most convenient situation, under ordinary circumstances, for examination by the thermometer-the average temperature is $36.9^{\circ} \mathrm{C}$. $\left(98.6^{\circ} \mathrm{F}\right.$.). In different internal parts, the variation is one or two degrees; those parts and organs being warmest which contain most blood, and in which there occurs the greatest amount of chemical change, e.g., the muscles and the glands; and the temperature is highest, when they are in a condition of activity: while those tissues which, subserving only a mechanical function, are the seat of least active circulation and chemical change, are the coolest. These differences of temperature, however, are actually but slight, on account of the provisions which exist for maintaining uniformity of temperature in different parts.

Circumstances causing Variations in Temperature. - The chief circumstances by which the temperature of a healthy body is influenced are the following:-

Age. - The average temperature of the new-born child is only about half a degree C. $\left(1^{\circ} \mathrm{F}.\right)$ above that of the adult; and the uifference becomees still 
more trifling during infancy and early childhood. The temperature falls to the extent of about $.2^{\circ} \mathrm{C}$. $\left(.5^{\circ} \mathrm{F}\right.$.) from early infancy to puberty, and by about the same amount from puberty to fifty or sixty years of age. In old age the temperature again rises, and approaches that of infancy.

Sex.-The average temperature of the female is slightly higher than that of the male.

Period of the Day. - The temperature undergoes a gradual alteration, to the extent of about $.54^{\circ}-.8^{\circ} \mathrm{C}$. $\left(1^{\circ}\right.$ to $1.5^{\circ} \mathrm{F}$. $)$ in the course of the day and night; the minimum being at night or in the early morning, the maximum late in the afternoon.

Exercise.-Active exercise raises the temperature of the body from $.54^{\circ}-1.08^{\circ}$ C. $\left(1^{\circ}\right.$ to $2^{\circ}$ F. $)$.

Climate and Season.-The temperature of the human body is practically the same in temperate as in tropical climates. In summer the temperature of the body is a little higher than in winter; the difference amounting to about a fifth of a degree C.

Food and Drink. - The effect of a meal upon the temperature of a body is but small. A very slight rise usually occurs. Cold alcoholic drinks slightly depress the temperature about, half a degree C. Warm alcoholic drinks, as well as warm tea and coffee, raise the temperature about a third of a degree $\mathrm{C}$.

Disease.-In disease the temperature of the body deviates from the normal standard to a greater extent than would be anticipated from the slight effect of external conditions during health. Thus, in some disease, as pneumonia and typhus, it occasionally rises as high as $41^{\circ}-41.6^{\circ} \mathrm{C}$. $\left(106^{\circ}\right.$ or $107^{\circ} \mathrm{F}$.), and considerably higher temperatures have been noted. In Asiatic cholera, on the other hand, a thermometer placed in the mouth may sometimes rise only to $25^{\circ}-26.2^{\circ}$ C. $\left(77^{\circ}\right.$ or $79^{\circ}$ F. $)$.

The temperature maintained by Mammalia in an active state of life, accord. ing to the tables of Tiedemann and Rudolphi, averages $38.3^{\circ} \mathrm{C}$. (101 ${ }^{\circ} \mathrm{F}$.). The extremes recorded by them were $34.6^{\circ}$ C. $\left(96^{\circ} \mathrm{F}\right.$. $)$ and $41^{\circ} \mathrm{C}$. $\left(106^{\circ} \mathrm{F}\right.$. $)$, the former in the narwhal, the latter in a bat (Vespertilio pipistrella). In Birds, the average is as high as $41.2^{\circ} \mathrm{C} .\left(107^{\circ} \mathrm{F}\right.$.) ; the highest temperature, $46.2^{\circ} \mathrm{C}$. $\left(111.25^{\circ}\right.$ F.) being in the small species, the linnets, etc. Among Reptiles, while the medium they were in was $23.9^{\circ} \mathrm{C}$. $\left(75^{\circ} \mathrm{F}\right.$.) their average temperature was $31.2^{\circ} \mathrm{C}$. $\left(82.5^{\circ} \mathrm{F}.\right)$. As a general rule, their temperature, though it falls with that of the surrounding medium, is, in temperate media, two or more degrees higher; and though it rises also with that of the merlium, yet at very high degrees it ceases to do so, and remains even lower than that of the medium. Fish and invertebrata present, as a general rule, the same temperature as the medium in which they live, whether that be high or low ; only among fish, the tunny tribe, with strong hearts and red meat-like muscles, and more blood than the average of fish have, are generally $3.8^{\circ} \mathrm{C} .\left(7^{\circ} \mathrm{F}\right.$.) warmer than the water around them.

The difference, therefore, between what are commonly called the warm and the cold-blooded animals, or homoiothermal (" $\mu \circ \iota s$, like, $\theta \dot{\varepsilon} \rho \mu \eta$, heat) and poikilo-

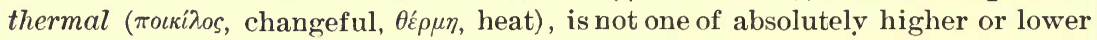
temperature; for the animals which to us in a temperate climate feel cold (being like the air or water, colder than the surface of our bodies), would in an external temperature of $37.8^{\circ} \mathrm{C}$. $\left(100^{\circ} \mathrm{F}\right.$. $)$ have nearly the same temperature and feel hot to us. The real difference is that warm-blooded animals have a certain permanent heat in all atmospheres, while the temperature of coldblooded animals is variable with every atmosphere. 


\section{The Production on the Body Heat.}

The heat which is produced in the body arises from the metabolic changes of the tissues, the chief part of which are of the nature of oxidation, since it may be supposed that the oxygen of the atmosphere taken into the system is ultimately combined with carbon and hydrogen, and discharged from the body as carbonic acid and water. Any changes, indeed, which occur in the protoplasm of the tissues, resulting in an exhibition of their function, are attended by the evolution of heat and the formation of carbonic acid and water. The more active the changes the greater is the heat produced and the greater is the amount of the carbonic acid and water formed. But in order that the protoplasm may perform its function, the waste of its own tissue (destructive metabolism), must be repaired by the due supply of food material to be built up in some way into the protoplasmic molecule. For the production of heat, therefore, food is necessary. In the tissues, as we have several times remarked, two processes are continually going on: the building up of the protoplasm from the food (constructive metabolism) which is not accompanied by the evolution of heat, possibly even by its storing, and the oxidation of the protoplastic materials resulting in the production of energy, by which heat is set free and carbonic acid and water are evolved.

It is not necessary to assume that the combustion processes, indeed, are as simple as the bare statement of the fact might seem to indicate; and, we have indicated, in treating of muscular metabolism, the process appears to consist first of all of building up of the oxygen into the molecule. But complicated as the various stages may be, the ultimate result is as simple as in ordinary combustion outside the body, and the products are the same.

This theory that the maintenance of the temperature of the living body depends on continual chemical change, chiefly by oxidation of combustible materials in the tissues, has long been established by the demonstration that the quantity of carbon and hydrogen as supplied as food, which, in a given time, unites in the body with oxygen, is sufficient to account for the amount of heat generated in the animal within the same period: an amount capable of maintaining the temperature of the body at from $36.8^{\circ}-3.87^{\circ} \mathrm{C}$. $\left(98^{\circ}-100^{\circ} \mathrm{F}\right.$.), notwithstanding a large loss by radiation and evaporation. This estimation depends upon the chemical axiom that when a body undergoes a chemical change the amount of energy set free is the same, supposing the resulting products are the same, whether the change takes place suddenly or gradually. If a certain number of grammes of different substances are introduced as food, and if they undergo complete oxidation, the amount of kinetic 
energy as shown in the amount of heat, and mechanical work, is the same if the same bodies are completely oxidized outside the body; so that if 1 gramme of fat be taken into the body and the oxidation completely oxidized, resulting in the production of a definite amount of carbon dioxide and water, it may be supposed to have produced the same amount of heat as it would have produced outside the body. In the case of proteid food it is a little different, since it is never completely oxidized within the body, but may be supposed to give rise to a definite amount of urea, not a completely oxidized body. In this case the gramme of proteid may be considered to perform the same amount of heat as the proteid would outside the body minus the amount which would be obtained from the complete oxidation of the resulting urea.

The actual amount of heat produced per diem has been experimentally ascertained in the case of small animals by the aid of an apparatus called a Calorimeter. The animal is inclosed in a metal box completely contained in a second box containing water, and air is led into and out of the inner box by means of metal tubes; the one through which the air is led out of the chamber has sereral coils in it. The heat given out by the animal warms the water in the outside box, and may be estimated by the rise of its temperature, the amount of which is known.

'The amount of heat produced and of energy in the form of mechanical work set free in a given time arise from the oxidation of the substances taken in as food in so far as they are oxidized. In order that there may be correct data to assist in the consideration of the subject, the amount of heat evolved by the oxidation of various food-stuffs has been carefully measured. The results may be set down in terms of gramme-calories (Ca), a calorie being the heat unit, and meaning the amount of heat required to raise 1 gramme of water 1 degree C., or, more strictly, from $15^{\circ} \mathrm{C}$. to $16^{\circ} \mathrm{C}{ }^{*}$ 'The number of gramme-calories which 1 gramme of the following substances equals will be seen in the annexed table.

\begin{tabular}{|c|c|c|c|}
\hline $\begin{array}{l}\text { Hydrogen } \\
\text { Carbon. }\end{array}$ & $\begin{array}{r}3450 \\
. \quad 8100\end{array}$ & $\begin{array}{l}\text { Fat } \\
\text { Carbohydrate } \\
\text { Proteid }\end{array}$ & $\begin{array}{l}9000 \quad \text { Urea } \\
4000 \\
5000-5500\end{array}$ \\
\hline
\end{tabular}

1 gramme of proteid giving rise to $\frac{1}{3}$ gramme of urea.

The relation between the income and expenditure of the body has been already considered in detail in the preceding chapter. We may now turn to the question of the chief heat-producing tissues.

Heat-producing Tissues.-(1.) The Muscles.-As the muscles form so large a part of the body, and as in them metabolism is particularly active, it is only reasonable to consider the muscular as the chief heat-

* Sometimes the term kilogramme-calorie is used; one kilogramme-calorie being equal to 1000 gramme-calories. 
producing tissue. It will shortly be pointed out that the manifestation of muscular energy is always accompanied by the evolution of heat and the production of carbon dioxide. This production of carbon dioxide goes on while the muscles are at rest, only in a less degree to that which is noticed during muscular activity, and so it is certain that an active metabolism is going on in resting as well as in contracting muscles. This metabolism is a source of much heat, and so the total amount of heat produced in the muscular tissues per diem must be very great. It has been calculated that, even neglecting the heat produced by the quiet metabolism of muscular tissue, the amount of heat generated by muscular activity would supply the principal part of the total heat produced within the body. (2.) The Secreting glands, and principally the liver, as being the largest and most active, come next to the muscles as heat-producing tissue. It has been found by experiment that the blood leaving the glands is considerably warmer than that entering them. The metabolism in the glands is very active, and, as we have seen, the more active the metabolism the greater the heat produced. (3.) The Brain; the venous blood has a higher temperature than the arterial. It must be remembered, however, that although the organs above mentioned are the chief heat-producing parts of the body, all living tissues contribute their quota, and this in direct proportion to their activity. The blood itself is also the seat of metabolism, and, therefore, of the production of heat; but the share which it takes in this respect, apart from the tissues in which it circulates, is very inconsiderable. There are two other means by which the heat produced by metabolism of the tissues is alded to in slight degree, viz., by friction, i.e., in the movements of muscles, in the circulation of blood, and elsewhere. This contributes a slight but undetermined amount of heat, and by the taking in of warm foods, solid or liquid, a further small amount of heat is at the same time acquired.

\section{Regulation of the Temperature of the Human Body.}

The average temperature of the body is maintained under different conditions of external circumstances by mechanisms which permit of (1) variation in the loss of heat, and (2) variations in the production of heat. In healthy warm-blooded animals the loss and gain of heat are so nearly balanced one by the other that, under all ordinary circumstances, an uniform temperature, within a degree or two, is preserved.

Variation in the Loss of Heat.-The loss of heat from the human body is principally regulated by the amount given off (1) by radiation and conduction from its surface, and by means of the (2) constant evaporation of water from the same part, heat being thus rendered latent, and 
to a much less degree (3) from the air-passages; in each act of respiration, heat is lost to a greater or less extent according to the temperature of the atmosphere; unless indeed the temperature of the surrounding air exceed that of the blood. We must remember too that (4) all food and drink which enter the body at a lower temperature than itself abstract a small measure of heat; (5) while the urine and fæces which leave the body at about its own temperature are also means by which a small amount is lost.

(a.) From the Surface of the Body.-By far the most important loss of heat from the body,-probably 90 per cent and upward of the whole amount, is that which takes place by radiation, conduction, and evaporation from the skin. The actual figures are as follows:-of 100 calories of heat produced, 2.6 are lost in heating food and drink; 2.6 in heating air inspired; 14.7 in evaporation; and 80.1 by radiation and conduction. The means by which the skin is able to act as one of the most important organs for regulating the temperature of the blood, are-(1), that it offers a large surface for radiation, conduction, and evaporation; ( 2$)$, that it contains a large amount of blood; (3), that the quantity of blood contained in it is the greater under those circumstances which demand a loss of heat from the body, and vice versa. For the circumstance which directly determines the quantity of blood in the skin, is that which governs the supply of blood to all the tissues and organs of the body, namely, the power of the vaso-motor nerves to cause a greater or less tension of the muscular element in the walls of the arteries, and, in correspondence with this, a lessening or increase of the calibre of the vessel, accompanied by a less or greater current of blood. A warm or hot atmosphere so acts on the nerve fibres of the skin, as to lead them to cause in turn a relaxation of the muscular fibre of the bloodvessels; and, as a result, the skin becomes full-blooded, hot, and sweating; and much heat is lost. With a low temperature, on the other hand, the blood-vessels shrink, and in accordance with the consequently diminished blood-supply, the skin becomes pale, and cold, and dry; and no doubt a similar effect may be produced through the vaso-motor centre in the medulla and spinal cord. Thus, by means of a self-regulating apparatus, the skin becomes the most important of the means by which the temperature of the body is regulated.

In connection with loss of heat by the skin, reference has been made to that which occurs both by radiation and conduction, and by evaporation; and the subject of animal heat has been considered almost solely with regard to the ordinary case of man living in a medium colder than his body, and therefore losing heat in all the ways mentioned. The importance of the means however, adopted, so to speak, by the skin for regulating the temperature of the body, will depend on the conditions 
by which it is surrounded; an inverse proportion existing in most cases between a loss by radiation and conduction on the one hand, and by evaporation on the other. Indeed, the small luss of heat by evaporation in cold climates may go far to compensate for the greater loss by radiction; as, on the other hand, the great amount of fluid evaporated in hot air may remove nearly as much heat as is commonly lost by both radiation and evaporation together in ordinary temperatures; and thus, it is possible that the quantities of heat required for the maintenance of a uniform proper temperature in various climates and seasons are not so different as they, at first sight, seem.

Many examples may be given of the power which the body possesses of resisting the effects of a high temperature, in virtue of evaporation from the skin. Blagden and others supported a temperature varying between $92^{\circ}-100^{\circ} \mathrm{C}$. $\left(198^{\circ}-212^{\circ}\right.$ F. $)$ in dry air for several minutes; and in a subsequent experiment he remained eight minutes in a temperature of $126.5^{\circ} \mathrm{C}$. $\left(260^{\circ} \mathrm{F}\right.$.). "The workmen of Sir F. Chantrey were accustomed to enter a furnace, in which his moulds were dried, while the floor was red-hot, and a thermometer in the air stood at $177.8^{\circ} \mathrm{C} .\left(350^{\circ} \mathrm{F}\right.$. $)$, and Chabert, the fire-king, was in the habit of entering an oven, the temperature of which was from $205^{\circ}-315^{\circ} \mathrm{C} .\left(400^{\circ}-600^{\circ}\right.$ F.)." (Carpenter.)

But such heats are not tolerable when the air is moist as well as hot, so as to prevent evaporation from the body. C. James states, that in the vapor baths of Nero he was almost suffocated in a temperature of $44.5^{\circ} \mathrm{C} .\left(112^{\circ} \mathrm{F}\right.$.), while in the caves of Testaccio, in which the air is dry, he. was but little incommoded by a temperature of $80^{\circ} \mathrm{C}$. $\left(176^{\circ} \mathrm{F}\right.$.). In the former, evaporation from the skin was impossible; in the latter it was abundant, and the layer of vapor which would rise from all the surface of the body would, by its very slowly conducting power, defend it for a time from the full action of the external heat.

We are able by suitable clothing to increase or to diminish the amount of heat lost by the skin.

The ways by which the skin may be rendered more efficient as a cooling-apparatus too, by exposure, by baths, and by other means which man instinctively adopts for lowering his temperature when necessary, are too well known to need more than passing mention.

Although under any ordinary circumstances the external application of cold only temporarily depresses the temperature to a slight extent, it is otherwise in cases of high temperature in fever. In these cases a tepid bath may reduce the temperature several degrees, and the effect so produced last in some cases for many hours.

(b) From the Lungs. - As a means for lowering the temperature, the lungs and air-passages are very inferior to the skin; although, by giving heat to the air we breathe, they stand next to the skin in importance. As a regulating power, the inferiority is still more marked. The air which is expelled from the lungs leaves the body at about the tempera- 
ture of the blood, and is always saturated with moisture. No inverse proportion, therefore, exists, as in the case of the skin, between the loss of heat by radiation and conduction on the one hand, and by evaporation on the otber. The colder the air, for example, the greater will be the loss in all ways. Neither is the quantity of blood which is exposed to the cooling influence of the air diminished or increased, so far as is known, in accordance with any need in relation to temperature. It is true that by varying the number and depth of the respirations, the quantity of heat given off by the lungs may be made, to some extent, to vary also. But the respiratory passages, while they must be considered important means by which heat is lost, are altogether subordinate, in the power of regulating the temperature, to the skin.

(c) By Warming Cold Foods. - This is an obrious method of expenditure of heat which may be resorted to, but the loss of heat by the excreta discharged from the body at a high temperature, must be of little use as a means of regulating the temperature, since the amount so lost must be capable of little variation.

Variation in the Production of Heat.-It may seem to have been assumed, in the foregoing pages, that the only regulating apparatus for temperature required by the human body is one that shall, more or less, produce a cooling effect; and as if the amount of heat produced were always, therefore, in excess of that which is required. Such an assumption would be incorrect. We have the power of regulating the production of heat, as well as its loss.

The regulation of the production of heat in the body is apparently different for each animal, as the absolute amount of heat set free by different animals in a given period varies; in one the production of heat exceeds that in another. It is even said that each individual has his own coefficient of heat production. From all that has been said on the subject it will be seen that the amount of heat for all practical purposes depends upon the metabolism of the tissues of the body, everything therefore which increases that metabolism will increase the heat production, so therefore the absolute amount of heat produced by a large animal, having a larger amount of tissues in which metabolism may go on, will be, cceteris paribus, greater than that of a small animal. But of course the activity of the tissue change in a small animal may be greater than in a large one, and naturally no strict line can be drawn between the two.

The ingestion of food has been proved to increase the metabolism of the tissues, and so, as one would expect, the rate of heat production is found by experiment upon the dog to be increased after a meal, and in this animal the heat production reaches its height about 6 to 9 hours after a meal. 
It has also been experimentally ascertained that the rate of heat production varies somewhat with the kind of food taken, for example, if sugar be added to the meal of meat given to the dog, the height of maximum production is reached. It was always said that various nations had found by experience what food was most suitable for the climate in which they lived, and that such experience could be trusted to regulate the quantity consumed. Although there have been no very conclusive experiments to prove this view, yet it is a matter of general observation that in northern climates and in colder seasons the quantity of food taken is greater than in warmer climates or in warmer seasons. Moreover, the kind of food is different. For example, persons living in the colder climates require much fat in order to produce the requisite amount of heat.

In exercise, we have an important means of raising the temperature of our bodies, by it the muscular metabolism is increased, as is shown by the increased output of carbon dioxide.

Influence of the Nervous System.-The influence of the nervous system in modifying the production of heat must be very important, as upon nervous influence depends the amount of the metabolism of the tissues. The experiments and observations which best illustrate it are those showing, first, that when the supply of nervous influence to a part is cut off, the temperature of that part after a time falls below its ordinary degree; and, secondly, that when death is caused by severe injury to, or removal of, the nervous centres, the temperature of the body rapidly falls, even though artificial respiration be performed, the circulation maintained, and to all appearance the ordinary chemical changes of the body be completely effected. It has been repeatedly noticed, that after division of the nerves of a limb its temperature ultimately falls; and this diminution of heat has been remarked still more plainly in limbs deprived of nervous influence by paralysis.

With equal certainty, though less definitely, the influence of the nervous system on the production of heat is shown in the rapid and momentary increase of temperature, sometimes general, at other times quite local, which is observed in states of nervous excitement; in the general increase of warmth of the body, excited by passions of the mind; in the sudden rush of heat to the face, which is not a mere sensation; and in the equally rapid diminution of temperature in the depressing passions. All of these examples, however, are explicable, on the supposition that the nervous system alters, by its power of controlling the calibre of the blood-vessels, the quantity of blood supplied to a part.

A part, however, from this vaso-motor power of increasing the bloodsupply to internal organs, and to the tissues in general, by means of which it is possible to increase their metabolism and so their production of heat, there is evidence to suppose that there is another nervous appa- 
ratus closely comparable to that which regulates the secretion of saliva or of sweat, by means of which the production of heat in the warmblooded animals is increased or diminished as occasion requires. This apparatus probably consists of a centre or centres which may be reflexly stimulated, as for example by impulses from the skin, and which act through special nerves supplied to the various tissues. The evidence upon which the existence of this regulating apparatus depends is the marked effect in the increase of the oxygen taken in by a warm-blooded animal when exposed to cold and the corresponding increase in the output of carbon dioxide, indicating that there is an increase of the metabolism and so an increased production of heat, under such circumstances and not a mere diminution of the amount of heat lost by the skin, etc. A coldblooded animal reacts very differently to exposure to cold; instead of as in the case of the warm-blooded animal, increasing the metabolism, cold diminishes the metabolism of its tissues. It appears clear, therefore, that in warm-blooded animals there is some extra apparatus which counteracts the effects of cold which in cold-blooded animals eauses diminished metabolism. In warm-blooded animals poisoned by urari, or in which section of the bulb has been done, it has been found that this regulating apparatus is no longer in action, and under such circumstances no difference appears to exist between such animals and those which are naturally cold-blooded. Warmth increases their temperature and cold lowers it, and with this there is of course evidence of diminished metabolism. The explanation of these experiments as given by modern physiologists is that in such animals the connection which naturally exists between the skin and the muscles through the nervous chain, such as a thermotaxic nervous apparatus might be supposed to afford, is broken either at the termination of the nerves in the muscles or at the section point of the bulb. The position of this hypothetical centre is a matter of some difference of opinion. It has been demonstrated that stimulation of different parts of the brain may, among other symptoms, produce increased metabolism of the tissues with increased output of carbon dioxide and a raised temperature: the parts of which this may be asserted are parts of the corpus striatum and of the optic thalamus. 'The exact situation of the heat centres, however, is at present not known with certainty.

Experimental observations such as have been made upon animals receive confirmation from the observations of patients who suffer from fever or pyrexia; in them the temperature of the body may be raised several degrees, as we have already pointed out (p. 450.) This increase of temperature might of course be due to diminished loss of heat from the skin, but this although in all probability entering into its causation, is not the only cause. The amount of oxygen taken in and the amount 
of carbon dioxide given out are both increased, and with this there must be increased metabolism of the tissues, and particularly of the muscular tissues, since at the same time the amount of urea in the urine is increased. Every one is familiar with the rapid wasting which is such a characteristic of high fever; it must indicate not only too rapid metabolism of the body, but also insufficient time for the tissues to build themselves up. In fever then there may be supposed to be some interference in the ordinary channel by which the skin is able to communicate to the nervous system the necessity of an increased or diminished production of heat in the muscles and other tissues. In consequence of this, and in spite of the condition of heat of the surface of the body, the production of heat goes on at an abnormal rate. It is not certain in what way the centre acts, whether it is one which keeps the metabolism in check, and when out of gear it is no longer able to do this, or whether, on the other hand, it is a centre by means of which the metabolism of the tissues may be increased by stimuli proceeding from it. Impulses from the skin would, according to these two possible modes of action, act either in the direction of increasing its inhibitory action, or in the direction of increasing or of diminishing the different stimuli causing increased production.

Influence of Extreme Heat and Cold.-In connection with the regulation of animal temperature, and its maintenance in health at the normal height, may be noted the result of circumstances too powerful, either in raising or lowering the heat of the body, to be controlled by the proper regulating apparatus. Walther found that rabbits and dogs kept exposed to a hot sun, reached a temperature of $46^{\circ} \mathrm{C} .\left(114.8^{\circ} \mathrm{F}\right.$.), and then died. Cases of sunstroke furnish us with several examples in the case of man; for it would seem that here death ensues chiefly or solely from elevation of the temperature.

The effect of mere loss of bodily temperature in man is less well known than the effect of heat. "From experiments by Walther, it appears that rabbits can be cooled down to $8.9^{\circ} \mathrm{C}$. $\left(48^{\circ} \mathrm{F}\right.$.), before they die, if artificial respiration be kept up. Cooled down to $1 \% .8^{\circ} \mathrm{C}$. $\left(64^{\circ} \mathrm{F}\right.$.), they cannot recover unless external warmth be applied together with the employment of artifical respiration. Rabbits not cooled below $25^{\circ} \mathrm{C}$. $\left(77^{\circ} \mathrm{F}.\right)$ recover by external warmth alone. 


\section{CHAPTER XIII.}

\section{EXCRETION.}

We have now considered the methods by which the food is digested and prepared for absorption, as well as the methods by which the changed materials reach the general blood-stream, either by means of the lymphatics of the intestinal wall or by the capillaries of the portal circulation. We have also discussed the most difficult problems of physiology, viz., those concerned with the exact changes which take place in the tissues and organs of the body, when they are supplied with the food necessary for life. We have mentioned the chief forms in which the waste materials resulting from the metabolism of the tissues leave the body. We have seen how carbon dioxide and other matters are eliminated by the lungs, and, further, we have devoted some time to the consideration of the amount and composition of the fæces. The highly important function of the kidneys, in excreting the urine, and thus removing certain waste materials, and the functions of the skin remain, and it is to these that we must now direct our attention.

\section{The Structure and Functions of the Kidneys.}

The kidneys are two in number, and are situated deeply in the lumbar region of the abdomen on either side of the spinal column behind the peritoneum. They correspond in position to the last two dorsal and two upper lumbar vertebræ; the right being slightly below the left in consequence of the position of the liver on the right side of the abdomen. They are about 4 inches long, $2 \frac{1}{2}$ inches broad, and $1 \frac{1}{2}$ inches thick. The weight of each kidney is about $4 \frac{1}{2} \mathrm{oz}$.

Structure.-The kidney is covered by a tough fibrous capsule, which is slightly attached by its inner surface to the proper substance of the organ by means of very fine fibres of areolar tissue and minute bloodvessels. From the healthy kidney, therefore, it may be easily torn off without injury to the subjacent cortical portion of the organ. At the hilus or notch of the kidney, it becomes continuous with the external coat of the upper and dilated part of the ureter (fig. 287).

On dividing the kidney into two equal parts by a section carried 
through its long convex border (fig. 287), the main part of its substance is seen to be composed of two chief portions called respectively cortical and medullary, the latter being also sometimes called pyramidal, from the fact of its being composed of about a dozen conical bunales of urine tubes, each bundle forming what is called a pyramid. The upper part of the ureter or duct of the organ, is dilated into the pelvis; and this, again, after separating into two or three principal divisions, is finally subdivided into still smaller portions, varying in number from about 8 to 12 , or even more, and called calyces. Each of these little calyces or cups, which are often arranged in a double row, receives the pointed

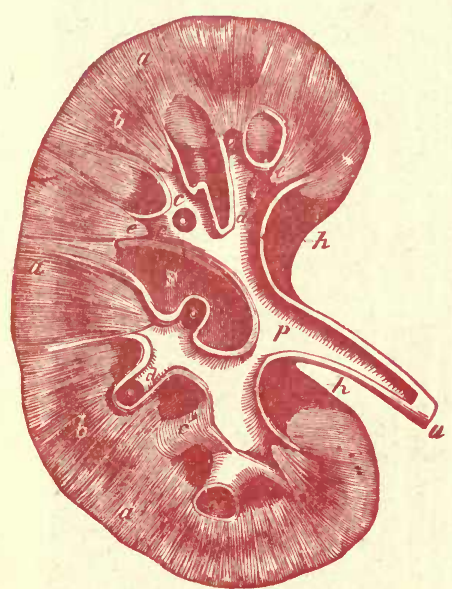

Fig. 287.

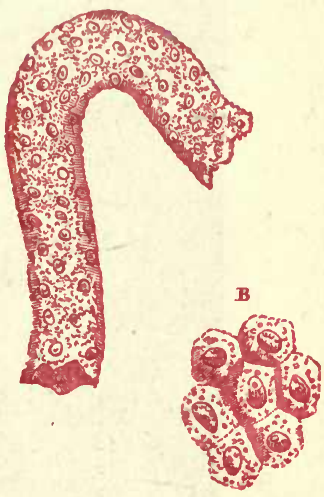

Fig. 288

Fig. 287.-Plan of a longitudinal section through the pelvis and substance of the right kidney, 1/2; $a$, the cortical substance : $b, b$, broad part of the pyramids of Malpighi; $c, c$, the divisions of the pelvis named calyces, laid open ; $c^{\prime}$, one of those unopened; $d$, summit of the pyramids of papillæ projecting into calyces; $e, e$, section of the narrow part of two pyramids near the calyces; $p$, pelvis or enlarged divisions of the ureter within the kidney; $u$, the ureter; $s$, the sinus; $h$, the hilus.

Fig. 288. - A. Portion of a secreting tubule from the cortical substance of the kidney. B. The epithelial or gland-cells. $\times 700$ times.

extremity or papilla of a pyramid. Sometimes, however, more than one papilla is received by a calyx.

The kidney is a compound tubular gland, and both its cortical and medullary portions are composed essentially of tubes, the tubuli uriniferi, which, by one extremity, in the cortical portion, end commonly in little saccules containing blood-vessels, called Malpighian bodies, and, by the other, opened through the papillæ into the pelvis of the kidney, and thus discharge the urine which flows through them.

In the pyramids the tubes are chiefly straight-dividing and diverging as they ascend through these into the cortical portion; while in the latter region they spread out more irregularly, and become much branched and convoluted.

Tubuli Uriniferi.-The tubuli uriniferi (fig. 288) are composed of 
a nearly homogeneous membrane, and are lined internally by epithelium. They vary considerably in size in different parts of their course, but are, on an ayerage, about $\frac{1}{600}$ of an inch $\left(\frac{1}{2}-\mathrm{mm}\right.$.) in diameter, and are found

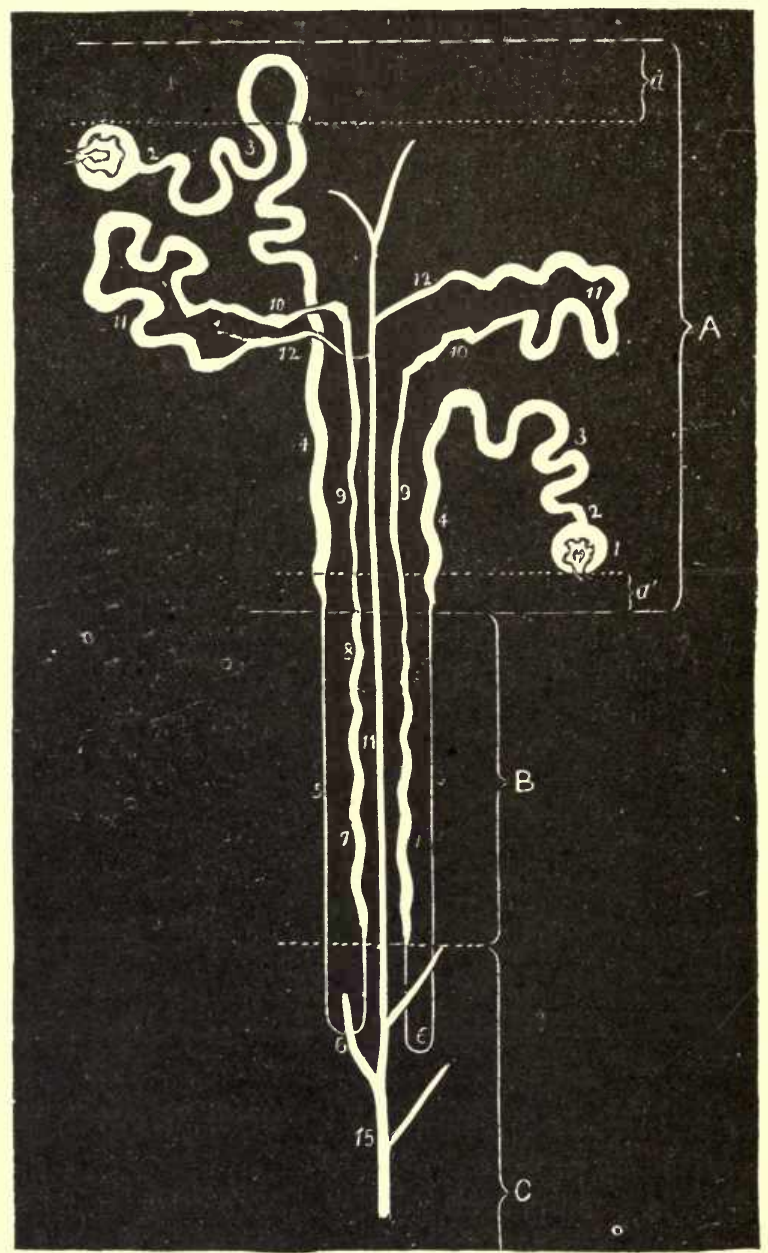

Fig. 289.-A diagram of the sections of uriniferous tubes. A, Cortex limited externally by the capsuie; $a$, subcapsular layer not containing Malpighian corpuscles; $a^{\prime}$, inner stratum of cortex, also without Malpighian capsules; B, boundary layer; C, papillary part next the boundary layer; 1, Bowman's capsule of Malpighian corpuscle; 2 , neck of capsule; 3 , proximal convoluted tubule; 4, spiral tubule; 5 . descending limb of Henle's locp; 6, the loop proper; $\%$, thick part of the ascending limb $; 8$, spiral part of ascending limb; 9. narrow ascending limb in the medullary ray; 10, the irregular tubule; 11 , the intercalated section, or the distal convoluted tubule; 12 , the curved collecting tubule; 13 , the straight collecting tubule of the medullary ray; 14 , the collecting tube of the boundary layer; 15, the large collecting tube of the papillary part which, joining with similar tubes, forms the duct. (Klein.)

to be made up of several distinct sections which differ from one another very markedly, both in situation and strueture. According to Klein, the following segments may be made out: (1) The Malpighian corp s. $^{-}$ 
rle (figs. 289,294 ), composed of a hyaline membrana propria, thickened by a varying amount of fibrous tissue, and lined by flattened nucleated epithelial plates. This capsule is the dilated extremity of the uriniferous tubule, and contains within it a glomerulus of convoluted capillary blood-vessels supported by connective tissues, and covered by flattened epithelial plates. The glomerulus is connected with an efferent and an afferent vessel. (2) The constricted neck of the capsule (fig. 289, 2), lined in a similar manner, connects it with (3) The Proximal convoluted tubule, which forms sereral distinct curves and is lined with

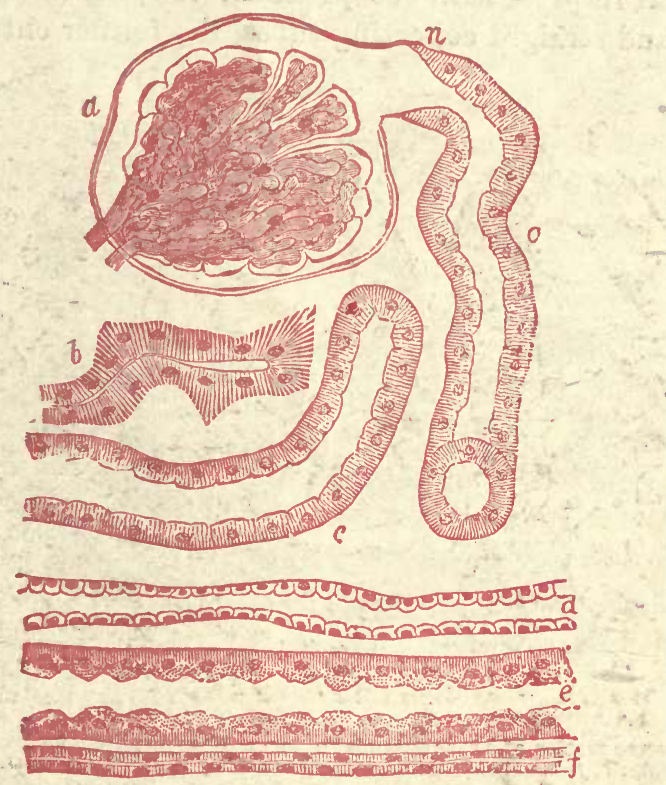

Fig. 290.-From a vertical section through the kidney of a dog-the capsule of which is supposed to be on the right. $a$, the capillaries of the Malpighian corpuscle-viz., the glomerulus, are arranged in lobules; $n$, neck of capsule ; $c$, convoluted tubes cut in various directions ; $b$, irregular $d$ colle;,$e$, and $f$, are straight tubes running toward capsules forming a so-called medullary ray; $d$, collecting tube ; $e$, spiral tube; $f$, narrow section of ascending limb. $\times 380 . \quad$ (Klein and Noble
Smith.)

short columnar cells, which vary somewhat in size. The tube next passes almost vertically downward, forming (4) The Spiral Tubule, which is of much the same diameter, and is lined in the same way as the convoluted portion. 'So far the tube has been contained in the cortex of the kidney; it now passes vertically downward through the most external part (boundary layer) of the Malpighian pyramid into the more internal part (papillary layer), where it curves up sharply, forming altogether the (5 and 6) Loop of Henle, which is a very narrow tube lined with flattened nucleated cells. Passing vertically upward just as the tube reaches the boundary layer ( 7$)$, it suddenly enlarges and becomes lined with polyhedral cells. (8) About midway in the boundary 
layer the tube again narrows, forming the ascending spiral of Henle's loop, but is still lined with polyhedral cells. At the point where the tube enters the cortex (9) the ascending limb narrows, but the diameter varies considerably; here and there the cells are more flattened, but both in this as in (8), the cells are in many places very angular, branched, and imbricated. It then joins (10) the "irregular tubule," which has a very irregular and angular outline, and is lined with angular and imbricated cells. The tube next becomes convoluted (11), forming the distal convoluted tube or intercalated section of Schwergger-Seidel, which is identical in all respects with the proximal convoluted tube (12 and 13). The curved and straight collecting tubes, the former entering the latter

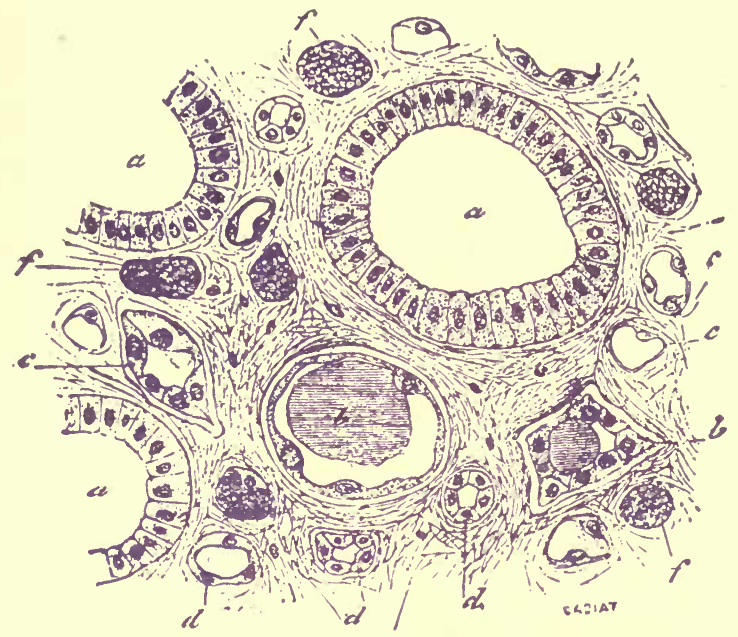

Fig. 291.-Transverse section of a renal papilla; $a$, large tubes or papillary ducts; $b, c$, and $d$, smaller tubes of Henle; $e, f$, blood capillaries. distinguished by their flatter epithelium. (Cadiat.)

at right angles, and the latter passing vertically downward, are lined with polyhedral, or spindle-shaped, or flattened, or angular cells. The straight collecting tube now enters the boundary layer (14) and passes on to the papillary layer, and, joining with other collecting tubes, forms larger tubes, which finally open at the apex of the papilla. These collecting tubes are lined with transparent nucleated columnar or cubical cells $(14,15)$.

The cells of the tubules with the exception of Henle's loop and all parts of the collecting tubules, are, as a rule, possessed of the intranuclear as well as of the intra-cellular network of fibres, of which the vertical rods are most conspicuous.

In some places, it is stated that a distinct membrane of flattened cells can be made out lining the lumen of the tubes (centrotubular membrane). 


\section{Blood-Vessels.}

Blood-supply. - In connection with the general distribution of bloodvessels to the kidney, the Malpighian Corpuscles must be further considered. They (fig. 293) are found only in the cortical part of the kid.

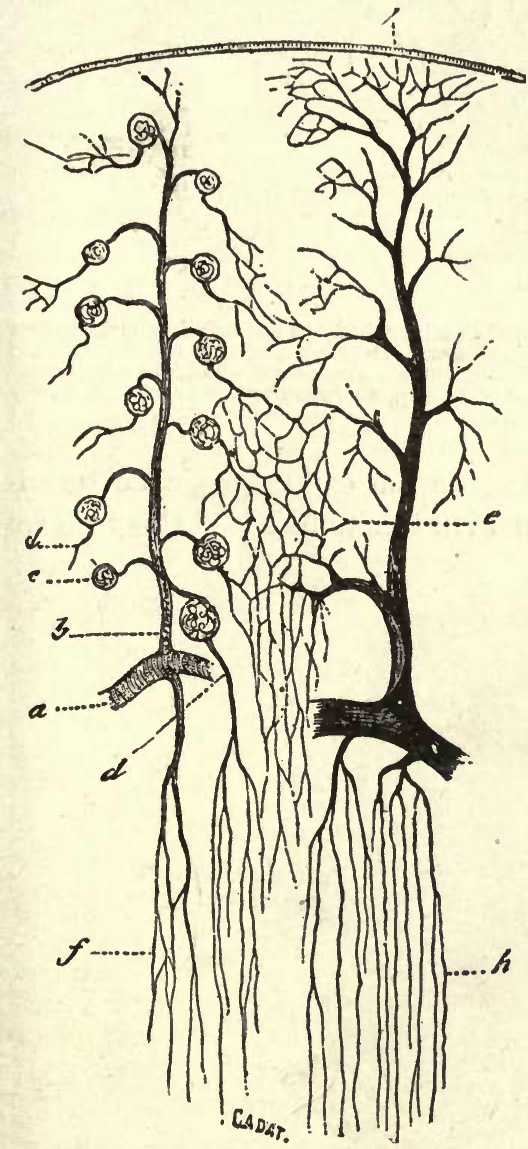

Fig. 292-Vascular supply of kidney. $a$, part of arterial arch; $b$, interlobular artery; $c$, glomerulus; $d$, efferent vessels passing to the medulla as false arteria recta: $e$, capillaries of cortex ; $f$, capillaries of medulla; $g$, venous arch ; $h$, straight veins of medulla; $j$, vena stellula: $i$, interlobular vein. (Cadiat.)

The small afferent artery (figs. 293 and 294) which enters the Malpighian corpuscle, breaks up in the interior as before mentioned into a dense convoluted and looped capillary plexus, which is ultimately gathered up again into several small efferent vessels, comparable to minute veins, which leave the capsule at one or more places near the point at

ney, and are confined to the central part, which, however, makes up about seven-eighths of the whole cortex. On a section of the organ, some of them are just visible to the naked eye as minute red points; others are too small to be thus seen. Their average diameter is about $\frac{1}{120}$ of an inch $\left(\frac{1}{5} \mathrm{~mm}\right.$.). Each of them is composed, as we have seen above, of the dilated extremity of an uriniferous tube, or Malpighian capsule, which encloses a tuft of blood-vessels.

The renal artery divides into several branches, which, passing in at the hilus of the kidney, and covered by a fine sheath of areolar tissue derived from the capsule, enter the substance of the organ chiefly in the intervals between the papillæ, and at the junction between the cortex and the boundary layer. The main branches then pass almost horizontally, forming more or less complete arches and giving off branches upward to the cortex and downward to the medulla. The former are for the most part straight; they pass almost vertically to the surface of the kidney, giving off laterally in all directions longer and shorter branches, which ulti. mately supply the Malpighian bodies. 
which the afferent artery enters it. On leaving, they do not immediately join other small veins as might have been expected, but again breaking up into a network of capillary vessels, are distributed on the exterior of

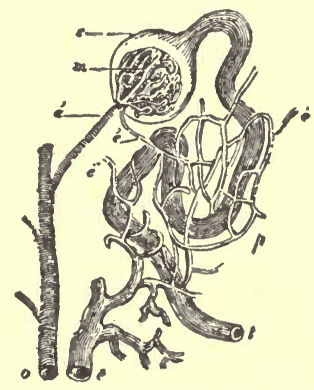

Fig. 293.-Diagram showing the relation of the Malpighian body to the uriniferous ducts and blood-vessels. $a$, one of the interlobular arteries; $a^{\prime}$, afferent artery passing into the glomerulus ; $c$, capsule of the Malpighian body, forming the termination of and continuous with $t$, the uriniferous tube $; e^{\prime}, e^{\prime}$, efferent vessels which subdivide in the plexus, $p$, surrounding the tube, and finally terminate in the branch of the renal vein $e$ (after Bowman).

the tubule. After this second breaking up the capillary plexus terminates in a small vein, which, by union with others like it, helps to form

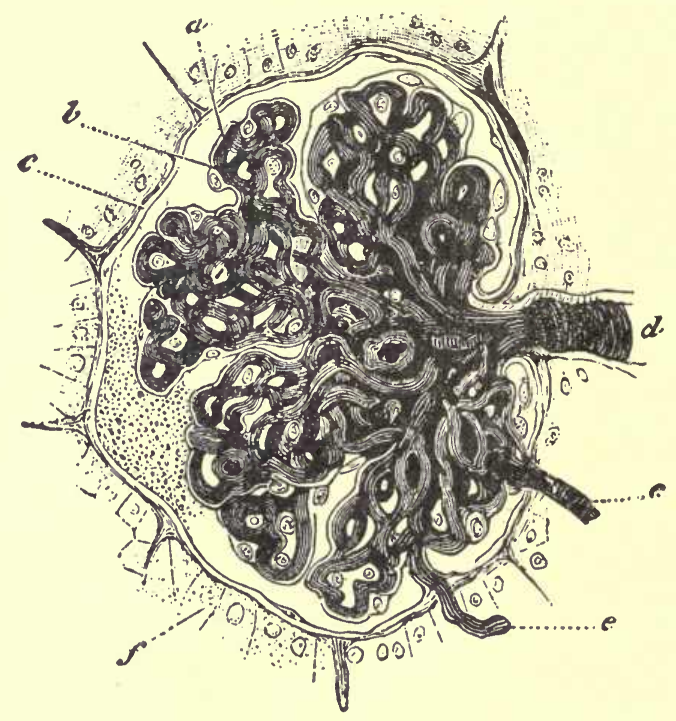

Fig. 294.-Malpighian capsule and tuft of capillaries, injected through the renal artery with colored gelatin. $a$, glomerular vessels ; $b$, capsule ; $c$, anterior capsule; $d$, glomerular artery ; $e$, efferent veins; $f$, epithelium of tubes. (Cadiat.)

the radicles of the renal vein. These small veins pass into others which form venous arches corresponding to the arterial arches, but which are more distinct, situated between the medulla and cortex. 
Thus, in the kidney, the blood entering by the renal artery, traverses two sets of capillaries before emerging by the renal vein, an arrangement which may be compared to the portal system in miniature.

The tuft of vessels within the Malpighian capsule in the course of development has been thrust into the dilated extremity of the urinary tubule, which finally completely invests it. Thus within the Malpighian capsule there are two layers of squamous epithelium, a parietal layer lining the capsule proper, and a visceral or reflected layer immediately covering the vascular tuft (fig. 295), and sometimes dipping down into its interstices. This reflected layer of epithelium is readily seen in young subjects, but cannot always be demonstrated in the adult. (See figs. 295 and 296.)

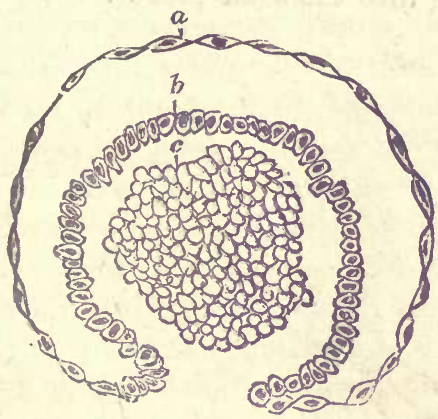

Fig. 295.

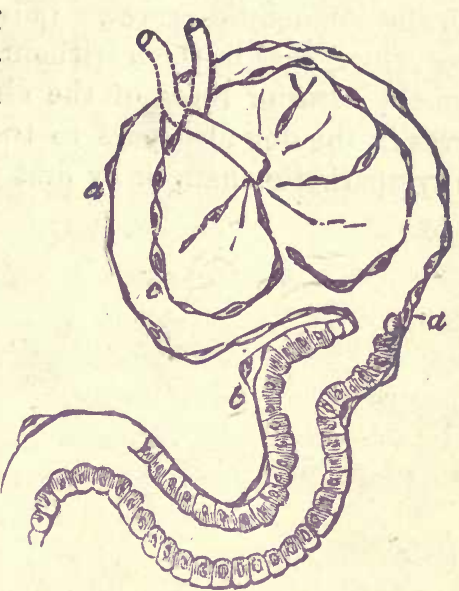

Fig. 296.

Fig. 295.-Transverse section of a developing Malpighian capsule and tuft (human). $\times 300$. From a foetus at about the fourth month; $a$, flattened cells growing to form the capsule; $b$, more rounded cells, continuous with the above, reflected round $c$, and finally enveloping it; $c$, mass of embryonic cells which will later become developed into blood-vessels. (W. Pye.)

Fig. 296.-Epithelial elements of a Malpighian capsule and tuft, with the commencement of a urinary tubule showing the afferent and efferent vessel; $a$, layer of flat epithelium forming the capsule; $b$, similar, but rather larger epithelial cells, placed in the walls of the tube; $c$, cells, covering the vessels of the capilary tuft; $d$, commencement of the tubule, somewhat narrower that the rest of it. (W. Pye.)

The vessels which enter the medullary layer break up into smaller arterioles, which pass through the boundary layer, and proceed in a straight course between the tubules of the papillary layer, giving off on their way branches, which form a fine arterial meshwork around the tubes, and ending in a similar plexus from which the venous radicles arise.

Besides the small afferent arteries of the Malpighian bodies, there are, of course, others which are distributed in the ordinary manner, for the nutrition of the different parts of the organ; and in the pyramids, between the tubes, there are numerous straight vessels, the vasa recta, some of which are branches of vasa efferentia from Malpighian bodies, and therefore comparable to the venous plexus around the tubules in 
the cortical portion, while others arise directly as small branches of the renal arteries.

Between the tubes, vessels, etc., which make up the substance of the kidney, there exists, in small quantity, a fine matrix of areolar tissue.

Nerves.-The nerves of the kidney are derived from the renal plexus of each side. This consists of both medullated and non-medullated nerve-fibres, the former of varying size, and of nerve-cells. The renal plexus is derived from the solar plexus, particularly from the semilunar ganglion. The renal plexus is thus indirectly connected with the vagi and with the splanchnic nerves. It is also directly connected with them by fibres which pass to them without first joining the solar plexus. Fibres from the anterior roots of the eleventh, twelfth, and thirteenth dorsa! nerves in the dog also pass to the same plexus, either directly through the sympathetic chain or by first passing into the solar plexus.

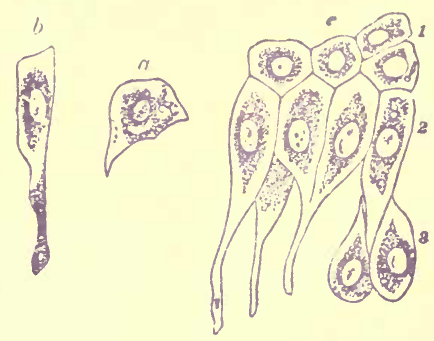

Fig. 297.-Epithelium of the bladder; $a$, one of the cells of the first row; $b$, a cell of the second row; $c$, cells in situ, of first, second, and deepest layers. (Obersteiner.)

The Ureters. - The duct of each kidney, or ureter, is a tube about the size of a goose-quill, and from twelve to sixteen inches in length, which, continuous above with the pelvis of the kidney, ends below by perforating obliquely the walls of the bladder, and opening on its internal surface.

Structure.-It is constructed of three principal coats $(a)$ an onter, tough, fibrous and elastic coat; (b) a middle muscular coat, of which the fibres are unstriped, and arranged in three layers-the fibres of the central layer being circular, and those of the other two longitudinal in direction; and $(c)$ an internal mucous lining continuous with that of the pelvis of the kidney above, and of the urinary bladder below. The epithelium of all these parts (fig. 297) is alike stratified and of a somewhat peculiar form; the cells on the free surface of the mucous membrane being usually spheroidal or polyhedral with one or more spherical or oval nuclei; while beneath these are pear-shaped cells, of which the broad ends are directed toward the free surface, fitting in beneath the cells of the first row, and the apices are prolonged into processes of $\mathrm{va}-$ 
rious lengths, among which, again, the deepest cells of the epithelium are found spheroidal, irregularly oval, spindle-shaped or conical.

The Urinary Bladder.-The urinary bladder, which forms a receptacle for the temporary lodgment of the urine in the intervals of its expulsion from the body, is more or less pyriform, its widest part, which is situate above and behind, being termed the fundus; and the narrow constricted portion in front and below, by which it becomes continuous with the urethra, being called its cervix or neck.

Structure.-It is constructed of four principal coats- serous, muscular, areolar or submucous, and mucous. (a.) The serous coat, which covers only the posterior and upper part of the bladder, has the same structure as that of the peritoneum, with which it is continuous. (b) The fibres of the muscular coat, which are unstriped, are arranged in three principal layers, of which the external and internal have a general longitudinal, and the middle layer a circular direction. The latter are especially developed around the cervix of the organ, and are described as forming a sphincter vesica. The muscular fibres of the bladder, like those of the stomach, are arranged not in simple circles, but in figureof-8 loops. (c) The areolar or submucous coat is constructed of connective tissue with a large proportion of elastic fibres. (d) The mucous membrane, which is rugose in the contracted state of the organ, does not differ in essential structure from mucous membranes in general. Its epithelium is stratified and closely resembles that of the pelvis of the kidney and the ureter (fig. 297).

The mucous membrane is provided with mucous glands, which are more numerous near the neck of the bladder.

The bladder is well provided with blood-and lymph-vessels, and with nerves. The latter are both medullated and non-medullated fibres, both branches from the sacral plexus (spinal) and hypogastric plexus (sympathetic). Ganglion-cells are found, here and there, in the course of the nerve-fibres.

\section{The Urine.}

Physical Properties.-Healthy urine is a perfectly transparent, amber-colored liquid, with a peculiar, but not disagreeable odor, a bitterish taste, and slight acid reaction. Its specific gravity varies from 1015 to 1025. On standing for a short time, a little mucus appears in it as a flocculent cloud, consisting chemically, it is said, of nucleo-albumin and not mucin.

Chemical Composition. - The urine consists of water, holding in solution certain organic and saline matters as its ordinary constituents, and occasionally various other matters; some of the latter are indications of diseased states of the system, and others are derived from unusual articles of food or drugs taken into the stomach. 
Chemical Composition of the URINe.

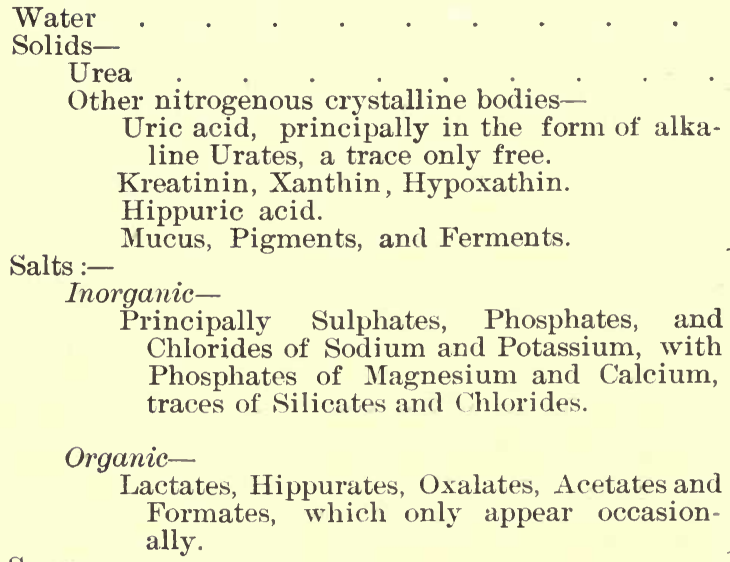
Chlorides of Sodium and Potassium, with Phosphates of Magnesium and Calcium, traces of Silicates and Chlorides.

Organic-

Lactates, Hippurates, Oxalates, Acetates and Formates, which only appear occasionally. Sugar .

Gases (nitrogen and carbonic acid principally).

Reaction.-The normal reaction of the urine is slightly acid. This acidity is due to acid phosphate of sodium, and is less marked soon after meals. The urine contains no appreciable amount of free acid, as it gives no precipitate of sulphur with sodium hyposulphite. After standing for some time the acidity increases from a kind of acid fermentation, due in all probability to the presence of mucus and fungi, and acid urates or free uric acid is deposited. After a time, varying in length according to the temperature, the reaction becomes strongly alkaline from the change of urea into ammonium carbonate, due to the presence of one or more specific micro-organisms (micrococcus urece). 'The urea takes up two molecules of water-a strong ammoniacal and fœtid odor appears, and deposits of triple phosphates and alkaline urates take place. This does not occur unless the urine is freely exposed to the air, or, at least, until air has had access to it.

Reaction of Urine in Different Classes of Animals. - In most herbivorous animals the urine is alkaline and turbid. The difference depends not on any peculiarity in the mode of secretion, but on the difference in the food on which the two classes subsist; for when carnivorous animals, such as dogs, are restricted to a vegetable diet, their urine becomes pale, turbid, and alkaline like that of an herbivorous animal, but resumes its former acidity on the return to an animal diet: while the urine voided by herbivorous animals, e.g., rabbits, fed for some time exclusively upon animal substances, presents the acid reaction and other qualities of the urine of Carnivora, its ordinary alkalinity being restored only on the substitution of a regetable for the animal diet. Human urine is not usually rendered alkaline by vegetable diet, but it becomes so after the free use of alkaline medicines, or of the alkaline salts with car- 
bonic or vegetable acids; for these latter are changed into alkaline carbonates previous to elimination by the kidneys.

Average daily quantity of the chief urinary constituents (modified from Parkes).

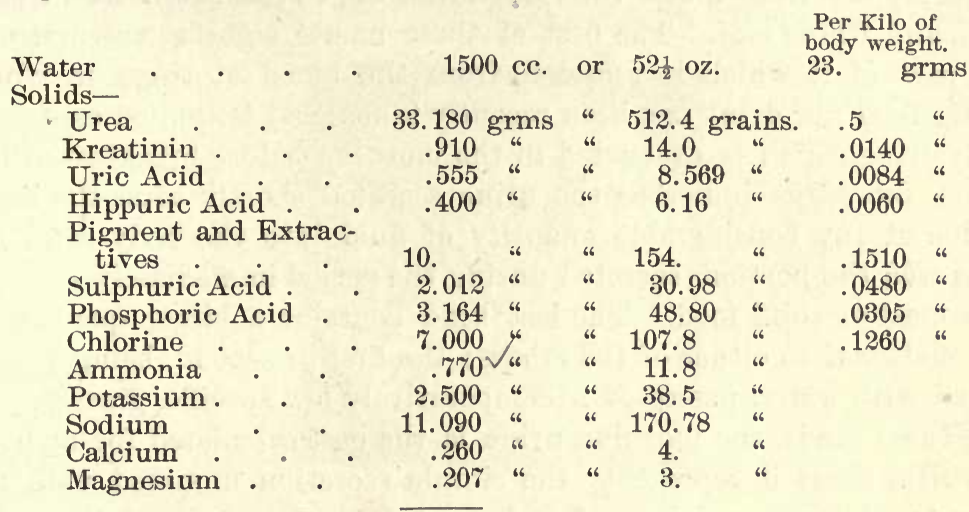

72.

Variations in the Quantity of the Constituents.-From the proportions given in the above table, most of the constituents are, even in health, liable to variations. The variations of the water in different seasons, and according to the quantity of drink and exercise, have already been mentioned. The water of the urine is also liable to be influenced by the condition of the nervous system, being sometimes greatly increased, e.g., in hysteria and in some other nervous affections; and at other times diminished. In some diseases it is enormously increased; and its increase may be either attended with an augmented quantity of solid matter, as in ordinary diabetes, or may be nearly the sole change, as in the affection termed diabetes insipidus. In other diseases, e.g., the various forms of albuminuria, the quantity may be considerably diminished. A febrile condition almost always diminishes the quantity of water; and a like diminution is caused by any affection which draws off a large quantity of fluid from the body through any other channel than that of the kidneys, e.g., the bowels or the skin.

Method of Estimating the Solids. - A useful rule for approximately estimating the total solids in any given specimen of healthy urine is to multiply the last two figures representing the specific gravity by 2.33. Thus, in urine of sp. gr. $1025,2.33 \times 25=58.25$ grains of solids, are contained in 1000 grains of the urine. In using this method it must be remembered that the limits of errors are much wider in diseased than in healthy urine.

Variations in the Specific Gravity.-The average specific gravity of the human urine is about 1020 . The relative quantity of water and of solid constituents of which it is composed is materially influenced by the condition and occupation of the body during the time at which it is 
secreted; by the length of time which has elapsed since the last meal; and by several other accidental circumstances. The existence of these causes of difference in the composition of the urine has led to the secretion being describer under the three heads of Urina sanguinis, Urina potus, and Urina cibi. The first of these names signifies the urine, or that part of it which is secreted from the blood at times in which neither food nor drink has been recently taken, and is applied especially to the urine which is evacuated in the morning before breakfast. The term urina potuis indicates the urine secreted shortly after the introduction of any considerable quantity of fluid into the body: and the urina cibi, the portions secreted during the period immediately succeeding a meal of solid food. The last kind contains a larger quantity of solid matter than either of the others; the first or second, being largely diluted with water, possesses a comparatively low specific gravity. Of these three kinds, the morning urine is the best calculated for analysis in health, since it represents the simple secretion unmixed with the elements of food or drink; if it be not used, the whole of the urine passed during a period of twenty-four hours should be taken. The specific gravity of the urine may thus, consistently with health, range widely on both sides of the usual average. It may vary from 1015 in the winter to 1025 in the summer; but variations of diet and exercise, and many other circumstances, may make even greater differences than these. In disease, the variation may be greater; sometimes descending, in albuminuria, to 1004, and frequently ascending in diabetes, when the urine is loaded with sugar, to 1050 , or even to 1060 .

Quantity.-The total quantity of urine passed in twenty-four hours is affected by numerous circumstances. On taking the mean of many observations by several experiments, the average quantity voided in twenty-four hours by healthy male adults from 20 to 40 years of age has been found to amount to about $52 \frac{1}{2}$ fluid ounces ( $1 \frac{1}{2}$ to 2 litres).

Abnormal Constituents. - In disease, or after the ingestion of special foods, various abnormal substances occur in urine, of which the following may be mentioned-Serum-albumin, Globulin, Ferments (apparently present in health also), Proteoses, Blood, Sugar, Bile acids and pigments, Casts, Fats, various Salts taken as a medicine, Micro-organisms of various kinds, and other matters.

\section{The Solids of the Urine.}

Urea $\left(\mathrm{CH}_{4} \mathrm{~N}_{2} \mathrm{O}\right)$. - Urea is the principal solid constituent of the urine, forming nearly one-half of the total quantity. It is also the most important ingredient, since it is the chief substance by which the nitrogen which is derived from the metabolic changes in the tissues as well as that which is derived from any superfluous food is excreted 
from the body. For its removal, the secretion of urine seems especially provided, though urea itself is not toxic.

Properties.-Urea, like the other solid constituents of the urine, exists in a state of solution. When in the solid state, it appears in the

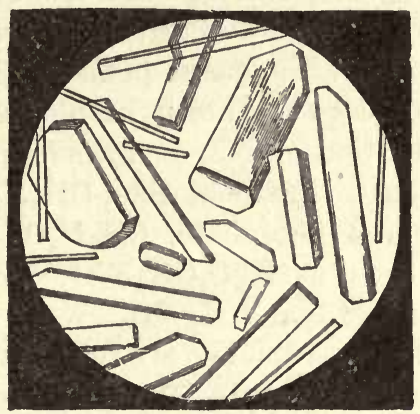

Fig. 298.-Crystals of Urea.

form of delicate silvery acicular crystals, which, under the microscope, appear as four-sided prisms (fig. 298). It may be obtained in this state by evaporating urine carefully to the consistence of honey, acting on the inspissated mass with four parts of alcohol, then evaporating the alcoholic solution to dryness, and purifying the residue by repeated solution in water or in alcohol, and finally allowing it to crystallize. It readily combines with some acids, like a weak base: and may thus be conveniently procured in the form of crystals of nitrate or oxalate of urea (figs. 299 and 300).

Urea is colorless when pure; when impure it may be yellow or

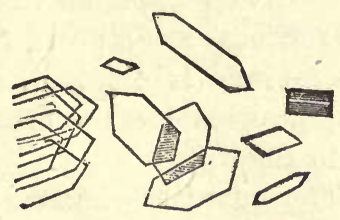

Fig. 299.-Crystals of Urea nitrate.

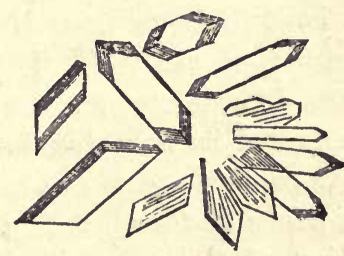

Fig. 300.-Crystals of Urea oxalate.

brown: it is without smell, and of a cooling nitre-like taste; it has neither an acid nor an alkaline reaction, and deliquesces in a moist and warm atmosphere. At $15^{\circ} \mathrm{C} .\left(59^{\circ} \mathrm{F}\right.$.) it requires for its solution less than its own weight of water; it is dissolved in all proportions by boiling water; but it requires five times its weight of cold alcohol for its solution. It is insoluble in ether. At $120^{\circ} \mathrm{C}$. ( $248^{\circ} \mathrm{F}$.) it melts with- 
out undergoing decomposition; at a still higher temperature ebullition takes place, and carbonate of ammonium sublimes. When heated with water in a sealed tube to $100^{\circ} \mathrm{C}$., urea splits up into carbonic acid and ammonia; when heated to a high temperature urea loses ammonia and first yields biuret, $\mathrm{C}_{2} \mathrm{H}_{5} \mathrm{~N}_{3} \mathrm{O}_{2}$, which gives a rose color with caustic potash and a trace of copper sulphate, and afterward cyanuric acid, $\mathrm{C}_{3} \mathrm{H}_{3} \mathrm{O}_{3} \mathrm{~N}_{3}$, which gives a violet color with caustic potash and a trace of copper sulphate. It is decomposed by sodium hypochlorite or hypobromite or by nitrous acid, with evolution of $\mathrm{N}$. It forms compounds with acids, of which the chief are urea hydrochloride, $\mathrm{CH}_{4} \mathrm{~N}_{2} \mathrm{O}$.HCL; urea nitrate, $\mathrm{CH}_{4} \mathrm{~N}_{2} \mathrm{OHNO}_{3}$; and urea phosphate, $\mathrm{CH}_{4} \mathrm{~N}_{2} \mathrm{O} \cdot \mathrm{H}_{3} \mathrm{PO}_{4}$. It forms compounds with metals such as $\mathrm{HgO} . \mathrm{CH}_{4} \mathrm{~N}_{2} \mathrm{O}$; with silver $\mathrm{CH}_{2} \mathrm{~N}_{2} \mathrm{OAg}_{2}$; and with salts such as $\mathrm{HgCl}_{2}$ and $\mathrm{HgNO}_{3}$.

Chemical Nature.-Urea is isomeric with ammonium cyanate $\mathrm{NH}_{4}, \mathrm{CNO}$. It was first of all artificially prepared from that substance.

It may also be produced artificially by treating carbonyl chloride $\left(\mathrm{CO} \mathrm{Cl}_{2}\right)$ with ammonia; or by heating ethyl carbonate with ammonia $\mathrm{CO} \underset{\mathrm{OC}_{2} \mathrm{O}_{5}}{\mathrm{OC}_{2} \mathrm{H}_{5}}+2 \mathrm{NH}_{3}=$ $\mathrm{CON}_{2} \mathrm{H}_{4} \quad 2 \mathrm{C}_{2} \mathrm{H}_{6} \mathrm{O}$; by heating ammonium carbonate $\mathrm{CO} \underset{\mathrm{ONH}_{4}}{\mathrm{NH}_{2}}=\mathrm{CON}_{2} \mathrm{H}_{4}+$ $\mathrm{H}_{2} \mathrm{O}$; by adding water to cyanamide $\mathrm{CN} . \mathrm{NH}_{2}$, or by evaporating ammonium cyanate in aqueous solution.

It is usually considered to be a diamide of carbonic acid, in other words, carbonic acid, $\mathrm{CO}(\mathrm{OH})_{2}{ }_{2}$, with two of hydroxyl, $(\mathrm{OH})_{2}^{\prime}$, replaced by two of amidogen $\left(\mathrm{NH}_{2}\right)^{\prime}{ }_{2}$. It may also be written as if it were a monamide of carbamic acid $\left(\mathrm{COOHNH}_{2}\right)$, thus $\mathrm{CONH}_{2} \cdot \mathrm{NH}_{2}$; one of amidogen, $\mathrm{NH}_{2}$, in the latter replacing one of hydroxyl in the former. Decomposition of the urea with development of ammonium carbonate takes place from the action of the bacteria (micrococcus ureæ), when urine is kept for some days after being voided, and explains the ammoniacal odor then evolved. The urea is sometimes decomposed before it leaves the bladder, when the mucous membrane is diseased, and the mucus secreted by it is abundant; but decomposition does not often oceur unless atmospheric germs have had access to the urine.

Variations in the Quantity excreted.-The quantity of urea excreted is, like that of the urine itself, subject to considerable variation. For a healthy adult about 512.4 grains (about 33.18 grms.) per diem may be taken as rather a high average. Its percentage in healthy urine is from 1.5 to 2.5. Its amount is materially influenced by diet, being greater when animal food is exclusively used, less when the diet is mixed, and least of all with a vegetable diet. As a rule, men excrete a larger quantity than women, and persons in the middle periods of life a larger quantity than infants or old people. The quantity of urea excreted by 
children, relatively to their body-weight, is much greater than by adults; Thus the quantity of urea excreted per kilogram of weight was found to be, in a child, $0.8 \mathrm{grm}$; in an adult only $0.4 \mathrm{grm}$. Regarded in this way, too, the excretion of carbonic acid gives similar results, the proportions in the child and adult being as $82: 34$.

The quantity of urea does not necessarily increase and decrease with that of the urine, though on the whole it would seem that whenever the amount of urine is much augmented, the quantity of urea also is usually increased; and it appears that the quantity of urea, as of urine, may be especially increased by drinking large quantities of water. In various diseases the quantity is reduced considerably below the healthy standard, while in other affections it is above it.

Quantitative Estimation.-There are two chief methods of estimating the amount of urea in the urine. (1.) By decomposing it by means of an alkaline solution of sodium hypobromite, or hypochlorite, and calculating the amount in a measured quantity, by collecting and measuring the amount of nitrogen evolved under such circumstances. Urea contains nearly half its weight of nitrogen, hence the amount of the gas collected may be taken as a measure of the urea decomposed, remembering that 1 litre of nitrogen at the standard temperature and pressure weighs $14 \times .08936$, or $1.251 \mathrm{grms}$. The percentage of urea can thus be readily calculated from the volume of nitrogen evolved from a measured quantity of the urine, but this calculation is avoided by graduating the tube in which the nitrogen is collected with numbers which indicate the corresponding percentage of urea. The reaction is $\mathrm{CON}_{2} \mathrm{H}_{4}+$ $3 \mathrm{NaBrO}+2 \mathrm{NaHO}=3 \mathrm{NaBr}+3 \mathrm{H}_{2} \mathrm{O}+\mathrm{Na}_{2} \mathrm{CO}_{3}+\mathrm{N}_{2}$. (2.) By precipitating the urea by adding to a given amount of urine, freed from sulphates and phosphates, a standard solution of mercuric nitrate from a burette, until the whole of it has been thrown down in an insoluble form; then reading off the exact amount of the mercuric nitrate solution, which it was necessary to use. As the amount of urea which each cubic centimetre of the standard solution will precipitate is previously known, it is easy to calculate the amount in the sample of urine taken. The precipitate which is formed was generally said to be composed of mercuric oxide and urea. Some, however, now consider that it is a mixture of mercuric nitrate itself and urea.

Uric Acid $\left(\mathrm{C}_{5} \mathrm{H}_{4} \mathrm{~N}_{4} \mathrm{O}_{3}\right)$. - Uric or lithic acid is rarely absent from the urine of man or animals, though in the feline tribe it seems to be sometimes entirely replaced by urea.

Properties.-Uric acid when pure is colorless, but when deposited from the urine is yellowish-brown. It crystallizes in various forms, of which the most common are smooth transparent, rhomboid plates, diamond-shaped plates, hexagonal tables, etc. (fig. 301). It is odorless and tasteless. It is very slightly soluble in cold water, and a little more so in hot water, quite insoluble in alcohol and ether. It dissolves freely in solution of the alkaline carbonates and other salts. 
The proportionate quantity of uric acid varies considerably in different animals. In man, and Mammalia generally, especially the Herbivora, it is comparatively small. In the whole tribe of birds, and of serpents, on the other hand, the quantity is very large, greatly exceeding that of the urea. In the urine of granivorous birds, indeed, urea is rarely if ever found, its place being entirely supplied by uric acid.

Variations in Quantity.-The quantity of uric acid, like that of urea, in human urine, is increased by the use of animal food, and decreased by the use of food free from nitrogen, or by an exclusively vegetable diet. In most febrile diseases, and in plethora, it is formed in unnaturally large quantities; and in gout it is deposited in and around joints, in the form of urate of soda, of which the so-called chalk-stones of this disease are principally composed. The average amount secreted in twenty-four hours is about one-third of a gramme.

Condition in the Urine.-The condition in which uric acid exists in solution in the urine has formed the subject of some discussion. The uric acid exists as urate of soda, produced by the uric acid as soon as it is formed combining with part of the base of the alkaline sodium phosphate of the blood. Hippuric acid, which exists in human urine also, acts upon the alkaline phosphate in the same way, and increases still more the quantity of acid phosphate, on the presence of which it is probable that a part of the natural acidity of the urine depends. It is scarcely possible to say whether the union of uric acid with the bases sodium and ammonium takes place in the blood, or in the act of secretion in the kidney: the latter is more likely; but the quantity of either uric acid or urates in the blood is probably too small to allow of this question being solved.

Owing to its existence in combination in healthy urine, uric acid for examination must generally be precipitated from its bases by a stronger acid, e.g., hydrochloric or nitric. When excreted in excess, however, it is deposited in a crystalline form (fig. 301), mixed with large quantities of ammonium or sodium urate. In such cases it may be procured for microscopic examination by gently warming the portion of urine containing the sediment; this dissolves urate of ammonium and sodium, while the comparatively insoluble crystals of uric acid subside to the bottom.

The most common form in which uric acid is deposited in urine, is that of a brownish or yellowish powdery substance, consisting of granules of ammonium or sodium urate. When deposited in crystals, it is most frequently in rhombic or diamond-shaped laminæ, but other forms are not uncommon (fig. 301). When deposited from urine, the crystals are generally more or less deeply colored, from being combined with the coloring principles of the urine.

Tests.-There are two chief tests for uric acid besides the micro- 
scopic evidence of its crystalline structure: (1) The Murexide test, which consists of evaporating to dryness a mixture of strong nitric acid and uric acid in a water bath. This leaves a yellowish-red residue of Alloxan $\left(\mathrm{C}_{4} \mathrm{H}_{2} \mathrm{~N}_{2} \mathrm{O}_{4}\right)$ and urea, and on addition of ammonium hydrate, a 'seautiful purple color (ammonium purpurate, $\left.\mathrm{C}_{8} \mathrm{H}_{4}\left(\mathrm{NH}_{4}\right) \mathrm{N}_{5} \mathrm{O}_{6}\right)$, deepened on addition of caustic potash, takes place. (2) Schiff's test consists of dissolving the uric acid in sodium carbonate solution, and of dropping some of it on a filter paper moistened with silver nitrate. A black spot appears, which corresponds to the reduction of silver by the uric acid.

Hippuric Acid $\left(\mathrm{C}_{9} \mathrm{H}_{9} \mathrm{NO}_{3}\right)$ has long been known to exist in the urine of herbivorous animals in combination with soda. It also exists

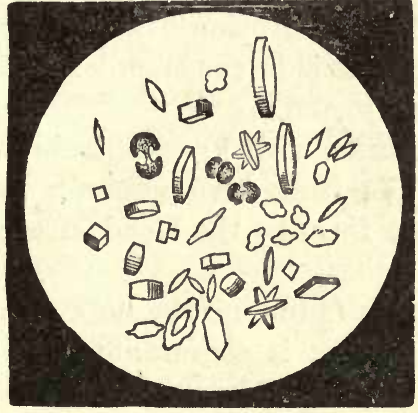

Fig. 301.-Various forms of uric acid crystals.

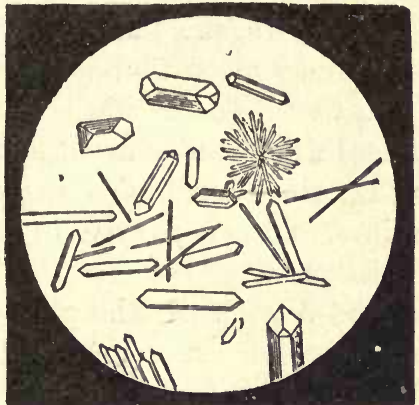

Fig. 302.-Crystals of hippuric acid.

naturally in the urine of man, in a quantity equal or rather exceeding that of the uric acid.

The quantity of hippuric acid excreted is increased by a vegetable diet. It appears to be formed in the body from benzoic acid or from some allied substance. The benzoic acid unites with glycin, probably in the kidneys, and hippuric acid and water are formed thus, $\mathrm{C}_{7} \mathrm{H}_{6} \mathrm{O}_{2}$ (Benzoic acid) $+\mathrm{C}_{2} \mathrm{H}_{5} \mathrm{NO}_{2}$ (Glycin) $=\mathrm{C}_{9} \mathrm{H}_{9} \mathrm{NO}_{3}$ (Hippuric acid) $+\mathrm{H}_{2} \mathrm{O}$ (water). It may be decomposed by acids into benzoic acid and glycin.

Properties.-It is a colorless and odorless substance of bitter taste, crystallizes in semi-transparent rhombic prisms (fig. 302). It is more soluble in cold water than uric acid, and much more soluble in hot water. It is soluble in alcohol.

Pigments. - The pigments of the urine are the following:-1. Urochrome, a yellow coloring matter, giving no absorption band; of which but little is known. Urine owes its yellow color mainly to the presence of this body. 2. Urobilin, an orange pigment, of which traces may be found in nearly all urines, and which is especially abundant in the urines passed by febrile patients. It is characterized by a well-marked spectroscopic absorption band at the junction of green and blue, best 
seen in acid solutions; and by giving a green fluorescence when excess of ammonia with a little chloride of zinc is added to it. The very vexed question of the relation of the pigments of urine to bile pigments turns largely upon the spectroscopic appearances of urobilin; for orangecolored solutions having the same absorption band as urobilin may be prepared from bile pigments in two different ways-i, by reduction with sodium amalgam-Hydrobilirubin (Maly); ii, by oxidation with nitric acid-Choletelin (Jaffé), and both these bile derivatives give a fluorescence with ammonia and a drop of chloride of zinc. It is not satisfactorily settled which of these, if either, is the same as urobilin of urine. It is worth noting that choletelin may be oxidized a stage further; it then loses its absorption band, remaining however of a yellow color. It is very possible that the urochrome of normal urine may be this oxidized choletelin, and that the presence of the absorption band of urobilin in urines may mean that some of the pigment is in the stage of choletelin; i.e., that its oxidation is not quite completed.

Those who believe urobilin to be identical with hydrobilirubin suppose that the bilirubin is reduced by the putrefactive processes in the intestines, and is conveyed in its reduced form by the blood stream to the kidneys.

3. Cro-erythrin is the pigment which is found in the pink deposits of urates which are sometimes seen in urines; it communicates a rich red-orange color to urine when in solution, and its solutions have two broad faint absorption bands in the green.

4. Uromelanin. When urine is boiled with strong acids it darkens to a reddish-brown color. This change, once ascribed to the formation of a new pigment uromelanin, is now believed to be due to the presence in urine of pyrocatechin and allied bodies which are capable of taking up oxygen when boiled with acids, yielding $\mathrm{CO}_{2}$ and brown or black residual products.

5. Indigo is rarely found in urines, to which it may communicate a blue or green color. Urine frequently contains a compound which is either a glucoside, Indican; or more probably a salt of indoxyl-sulphuric acid. It yields indigo blue when treated with strong hydrochloric acid and left to stand for some hours exposed to the air; the indigo may be separated by treatment with boiling chloroform, which takes it up, forming a blue solution.

There is a similar compound of skatol and sulphuric acid which is sometimes recognized in the urine, by the production of a red color when nitric acid is added to it.

Many medicinal substances color the urine, for instance Rhubarb, Santonin, Senna, Fuchsine, Carbolic Acid.

Bromides and Iodides yield Bromine or Iodine, when nitric acid is added to the urine of patients taking these drugs. In the case of iodides 
the liberated iodine communicates a strong mahogany color to the urine thus treated.

Mucus.-Mucus in the urine consists principally of the epithelial débris from the mucous surface of the urinary passages. Particles of epithelium, in greater or less abundance, may be detected in most samples of urine, especially if it has remained at rest for some time, and the lower strata are then examined (fig. 303). As urine cools, the mucus is sometimes seen suspended in it as a delicate opaque cloud, but generally it falls. In inflammatory affections of the urinary passages, especially of the bladder, mucus in large quantities is poured forth, and speedily undergoes decomposition. The presence of the decomposing mucus excites chemical changes in the urea, whereby carbonate of ammonium is formed, which, combining with the excess of acid in the superphosphates in the urine, produces insoluble neutral or alkaline phosphates of calcium and magnesium, and phosphate of ammonium and magnesium. These, mixing with the mucus, constitute the peculiar white, viscid, mortar-like substance which collects upon the mucous surface of the bladder, and is often passed with the urine, forming a thick tenacious sediment.

Extractives.-In addition to those already considered, urine contains a considerable number of nitrogenous compounds. These are usually described under the generic name of Extractives. Of these, the chief are: (1) Kreatinin $\left(\mathrm{C}_{4} \mathrm{H}_{7} \mathrm{~N}_{3} \mathrm{O}\right)$, a substance derived almost entirely from muscle taken as food, crystallizing in colorless oblique rhombic prisms; a fairly definite amount of this substance, about 15 grains ( 1 grm.), appears in the urine daily, so that it must be looked upon as a normal constituent; it is increased by increasing the nitrogenous constituents of the food; (2) Xanthin $\left(\mathrm{C}_{6} \mathrm{~N}_{4} \mathrm{H}_{4} \mathrm{O}_{2}\right)$, when isolated, is an amorphous powder soluble in hot water; (3) Sarkin, or hypo-xanthin $\left(\mathrm{C}_{6} \mathrm{~N}_{4} \mathrm{H}_{4} \mathrm{O}\right)$; (4) Oxaluric acid $\left(\mathrm{C}_{3} \mathrm{H}_{4} \mathrm{~N}_{2} \mathrm{O}_{4}\right)$, in combination with ammonium in the urine of the new-born child; (5) Allantoin $\left(\mathrm{C}_{4} \mathrm{H}_{6} \mathrm{~N}_{4} \mathrm{O}_{3}\right)$. All these extractives are chiefly interesting as being closely connected with urea, and mostly yielding that substance on oxidation. Leucin and tyrosin can scarcely be looked upon as normal constituents of urine.

Saline Matter.-(a) The Sulphuric acid in the urine is combined chiefly or entirely with sodium or potassium; forming salts which are taken in very small quantity with the food, and are scarcely found in other fluids or tissues of the body; for the sulphates commonly enumerated among the constituents of the ashes of the tissues and fluids are for the most part, or entirely, produced by the changes that take place in the burning. Only about one-third of the sulphuric acid found in the urine is derived directly from the food (Parkes). Hence the greater part of the sulphuric acid which the sulphates in the urine contain, 
must be formed during the metabolism of nitrogenous foods; the sulphur of which the acid is formed being probably derived from the decomposing nitrogenous tissues, the other elements of which are resolved into urea and uric acid. It may be in part derived also from the sulphur-holding taurin and cystin, which can be found in the liver, lungs, and other parts of the body, but not generally in the excretions; and which, therefore, must be broken up. The oxygen is supplied through the lungs, and the heat generated during combination with the sulphur is one of the subordinate means by which the animal temperature is maintained.

Besides the sulphur in these salts, some also appears to be in the urine uncombined with oxygen; for after all the sulphates have been removed from urine, sulphuric acid may be formed by drying and burn-

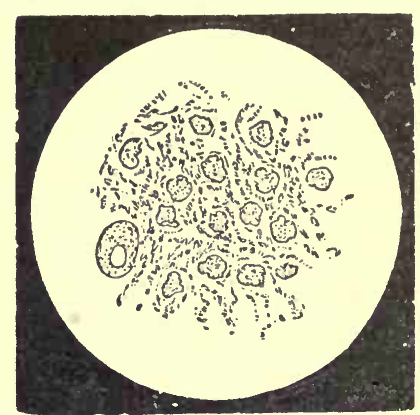

Fig. 303.

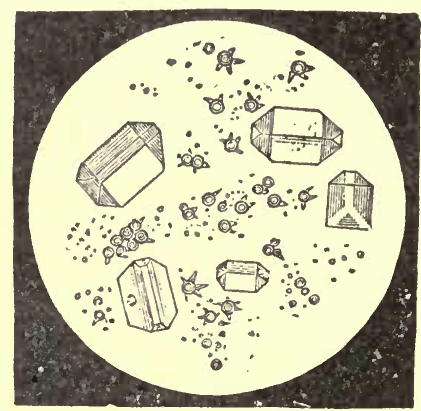

Fig. 304.

Fig. 303.-Mucus deposited from urine. Fig. 304.-Urinary sediment of triple phosphates (large 'prismatic erystais) and urate of ammo-
nium, from urine which had undergone alkaline fermentation.

ing it with nitre. From three to five grains of sulphur are thus daily excreted. The combination in which it exists is uncertain: possibly it is in some compound analogous to cystin or cystic oxide. Sulphuric acid also exists normally in the urine in combination with phenol $\left(\mathrm{C}_{6} \mathrm{H}_{6} \mathrm{O}\right)$ as phenol-sulphuric acid or its corresponding salts, with sodium, etc.

(b) The phosphoric acid in the urine is combined partly with the alkalies, partly with the alkaline earths-about four or five times as much with the former as with the latter. In blood, saliva, and other alkaline fluids of the body, phosphates exist in the form of alkaline, neutral, or acid salts. In the urine they are acid salts, viz., the sodium, ammonium, calcium, and magnesium phosphates, the excess of acid being (Liebig) due to the appropriation of the alkali with which the phosphoric acid in the blood is combined, by the several new acids which are formed or discharged at the kidneys, namely, the uric, hippuric, and sulphuric acids, all of which are nentralized with soda. 
The phosphates are taken largely in both vegetable and animal food; some thus taken are excreted at once; others, after being transformed and incorporated with the tissues. Calcium phosphate forms the principal earthy constituent of bone, and from the decomposition of the osseous tissue the urine derives a large quantity of this salt. The decomposition of other tissues also, but especially of the brain and nervesubstance, furnishes large supplies of phosphorus to the urine, which phosphorus is supposed, like the sulphur, to be united with oxygen, and then combined with bases. The quantity is, however, liable to considerable variation. Any undue exercise of the brain and all circumstances producing nervous exhaustion increase it. The earthy phosphates are more abundant after meals, whether of animal or vegetable food, and are diminished after long fasting. The alkaline phosphates are in-

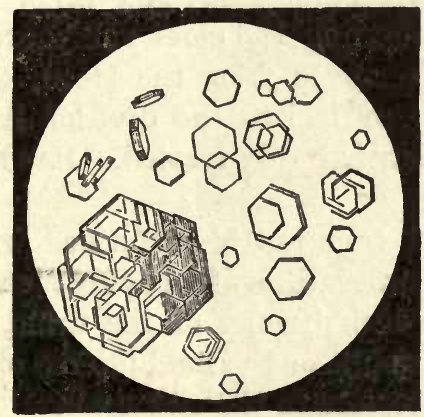

Fig. 305.-Crystals of Cystin.

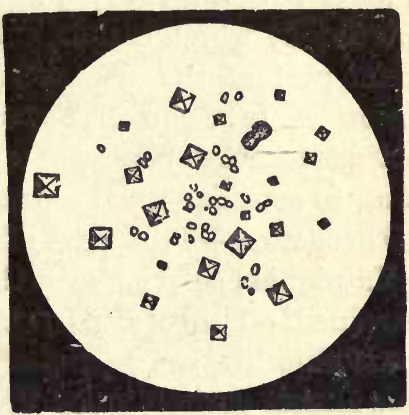

Fig. 306.-Crystals of Calcium Oxalate.

creased after animal food, diminished after vegetable food. Exercise increases the alkaline, but not the earthy phosphates. Phosphorus uncombined with oxygen appears, like sulphur, to be excreted in the urine. When the urine undergoes alkaline fermentation phosphates are deposited in the form of a urinary sediment, consisting chiefly of ammonio-magnesium phosphates (triple phosphate) (fig. 304). The compound does not, as such, exist in healthy urine. The ammonia is chiefly or wholly derived from the decomposition of urea.

(c.) The Chlorine of the urine occurs chiefly in combination with sodium (next to urea, sodium chloride is the most abundant solid constituent of the urine), but slightly also with ammonium, and, perhaps, potassium. As the chlorides exist largely in food, and in most of the animal fluids, their occurrence in the urine is easily understood.

Occasional Constituents.-Cystin $\left(\mathrm{C}_{3} \mathrm{H}_{7} \mathrm{~N} \mathrm{SO}_{2}\right)$ (fig. 305) is an occasional constituent of urine. It resembles taurin in containing a large quantity of sulphur-more than 25 per cent. It does not exist in healthy urine.

Another common morbid constituent of the urine is Oxalic acid, 
which is frequently deposited in combination with calcium (fig. 306) as a urinary sediment. Like cystin, but much more commonly, it is the chief constituent of certain calculi.

Of the other abnormal constituents of the urine which were mentioned on $\mathrm{p} .472$, it will be unnecessary to speak at length in this work.

Gases.-A small quantity of gas is naturally present in the urine in a state of solution. It consists of carbonic acid (chiefly) and nitroger and a snall quantity of oxygen.

\section{The Method of the Excretion of Urine.}

The excretion of the urine by the kidney is believed to consist of two more or less distinct processes-viz., (1) of Filtration, by which the water and the ready-formed salts are eliminated; and (2) of True Secretion, by which certain substances forming the chief and more important part of the urinary solids are removed from the blood. This division of function corresponds more or less to the division in the functions of other glands of which we have already treated. It will be as' well to consider them separately.

Filtration.-This part of the renal function is performed within the Malpighian corpuscles by the renal glomeruli. By it not only the water is strained off, but also certain other constituents of the urine, e.g., sodium chloride, are separated. The amount of the fluid filtered off depends almost entirely upon the blood-pressure in the glomeruli.

The greater the blood-pressure in the arterial system generally, and consequently in the renal arteries, the greater, cateris paribus, will be the blood-pressure in the glomeruli, and the greater the quantity of urine separated; but even without increase of the general blood-pressure, if the renal arteries be locally dilated, the pressure in the glomeruli will be increased and with it the secretion of urine. All the causes, therefore, which increase the general blood-pressure will secondarily increase the secretion of urine. Of these-

(1) The heart's action is among the most important. When the cardiac contractions are increased in force or frequency, increased diuresis is the result.

(2) Since the connection between the general blood-pressure and the nervous system is so close it will be evident that the amount of urine secreted depends greatly upon the influence of the latter. This may be demonstrated experimentally. Thus, division of the spinal cord, by producing general vascular dilatation, causes a great diminution of bloodpressure, and so diminishes the amount of water passed; since the local dilatation in the renal arteries is not sufficient to counteract the general diminution of pressure. Stimulation of the cut cord produces, strangely enough, the same results-i.e., a diminntion in the amount of the urine 
passed, but in a different way, viz., by constricting the arteries generally, and, among others, the renal arteries; the diminution of blood-pressure resulting from the local resistance in the renal arteries being more potent to diminish blood-pressure in the glomeruli than the general increase of blood-pressure is to increase it. Section of the renal nerves which produces local dilatation without greatly diminishing the general blood-pressure will cause an increase in the quantity of fluid passed.

(3) The fact that in summer or in hot weather the urine is diminished may be attributed partly to the copious elimination of water by the skin in the form of sweat which occurs in summer, as contrasted with the greatly diminished functional activity of the skin in winter,

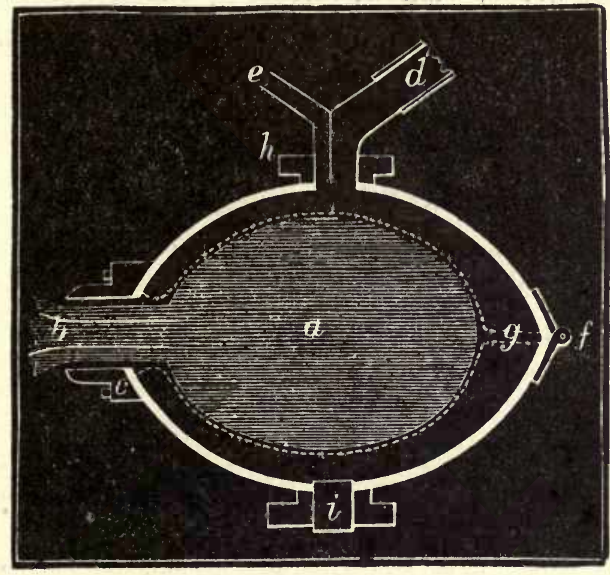

Fig. 307.-Diagram of Roy's Oncometer. $a$, represents the kidney inclosed in a metal box, which opens by hinge $f ; b$, the renal vessels and duct. Surrounding the kidney are two chambers formed by membranes, the edges of which are firmly fixed by being clamped between the outside metal capsule, and one (not represented in the figure) inside, the two being firmly screwed together by screws at $h$, and below. The membranous chamber below is filled with a varying amount of warm oil, according to the size of the kidney experimented with, through the opening then closed with the plug $i$. After the kidney has been inclosed in the capsule, the membrano'is chamber above is filled with warm oil through the tube $e$, which is then closed by a tap (not represented in the diagram); the tube $d$ communicates with a recording apparatus, and any alteration in the volume of the kidney is communicated by the oil in the tube to the chamber $d$ of the Oncograph, fig. 295 .

but also to the dilated condition of the vessels of the skin causing a decrease in the general blood-pressure. Thus we see that in regard to the elimination of water from the system, the skin and kidneys perform similar functions, and are capable to some extent of acting vicariously, one for the other. Their relative activities are inversely proportional to each other.

The intimate connection which exists between the volume of the kidney and the variations of blood-pressure is exceedingly well shown with the Oncometer, introduced by Roy, which is a modification of the plethysmograph, fig. 307. By means of this apparatus any alteration in the volume of the kidney is communicated to an apparatus [oncograph], capable of recording graphically, with a writing lever, such variations. 
It has been found that the kidney is extremely sensitive to any alteration in the general blood-pressure, every fall in the general bloodpressure being accompanied by a decrease in the volume of the kidney, and every rise, unless produced by considerable constriction of the peripheral vessels, including those of the kidney, being accompanied by a corresponding increase of volume. Increase of volume is followed by an increase in the amount of urine secreted, and decrease of volume by a decrease in the secretion. In addition, however, to the response of the kidney to alterations in the general blood-pressure, it has been further observed that certain substances, when injected into the blood, will also produce an increase in volume of the kidney, and consequent increased flow of urine, without affecting the general blood-pressure-

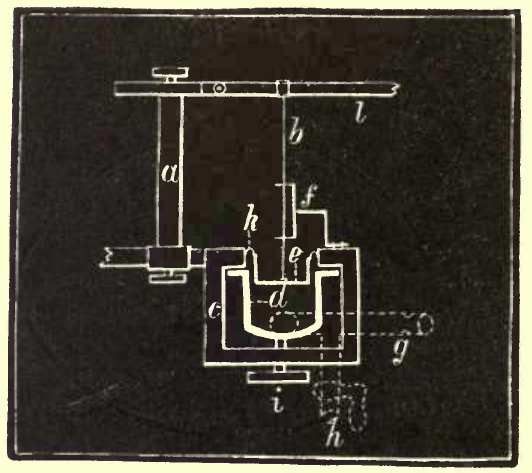

Fig. 308.-Roy's Oncograph, or apparatus for recording alterations in the volume of the kiduey, etc., as shown by the oncometer $-a$, upright, supporting recording lever $l$, which is raised or lowered by needle $b$, which works through $f$, and which is attached to the piston $e$, working in the chamber $d$, with which the tube from the oncometer communicates. The oil is prevented from being squeezed out as the piston descends by a membrane, which is clamped between the ring-shaped surfaces of cylinder by the screw $i$ working upward; the tube $h$ is for filling the instrument.

such bodies as sodium acetate and other diuretics. These observations appear to prove that local dilatation of the renal vessels may be produced by alterations in the blood acting upon a local nervous mechanism, as this happens when all of the renal nerves have been divided. The alterations are not only produced by the addition of drugs, but also by the introduction of comparatively small quantities of water or saline solution. To this alteration of the blood acting upon the renal vessels (either directly or) through a local vaso-motor mechanism, and not to any great alteration in the general blood-pressure, must we attribute the effects of meals, etc., observed by Roberts. The renal excretion is increased after meals and diminished during fasting and sleep. The increase begins within the first hour after breakfast, and continues during the succeeding two or three hours; then a diminution sets in, and continues until an hour or two after dinner. The effect of dinner does not appear until 
two or three hours after the meal; and it reaches its maximum about the fourth hour. From this period the excretion steadily decreuses until bed-time. During sleep it sinks still lower, and reaches its minimum-being not more than one-third of the quantity excreted during the hours of digestion. The increased amount of urine passed after drinking large quantities of fluid depends upon the temporary increase of blood-pressure thus caused.

'T'he following table* will help to explain the dependence of the inltration function upon the blood-pressure and the nervous systern:-

Table of the relation of the secretion of Urine to Arterial Pressure.

A. Secretion of urine may be increased-

a. By increasing the general blood-pressure; by

1. Increase of the force or frequency of heart-beat.

2. Constriction of the small arteries of areas other than that of the kidney.

b. By increasing the local blood-pressure, by relaxation of the renal artery, without compensating relaxation elsewhere; by

1. Division of the renal nerves (causing polyuria).

2. Division of the renal nerves and stimulation of the cord, below the medulla (causing greater polyuria).

3. Division of the splanchnic nerves; but the polyuria produced is less than in 1 or 2 , as these nerves are distributed to a wider area, and the dilatation of the renal artery is accompanied by dilatation of other vessels, and therefore with a somewhat diminished general blood supply.

4. Puncture of the floor of fourth ventricle or mechanical irritation of the superior cervical ganglion of the sympathetic, possibly from the production of dilatation of the renal arteries.

B. Secretion of urine may be diminished-

a. By diminishing the general blood-pressure; by

1. Diminution of the force or frequency of the heart-beats.

2. Dilatation of capillary areas other than that of the kidney.

3. Division of spinal cord below the medulla, which causes dilatation of general abdominal area, and urine generally ceases being secreted.

b. By increasing the blood-pressure, by stimulation of the spinal cord below the medulla, the constriction of the renal artery, which follows, not being compensated for by the increase of general blood-pressure.

c. By constriction of the renal artery, by stimulating the renal or splanchnic nerves, or the spinal cord.

'Lhough the quantity of urine secreted corresponds closely with the local blood-pressure, it must be stated that it is more directly dependent on the quantity of blood flowing through the kidney in a given unit of time. Under normal conditions increased blood-pressure and increased blood-flow go hand in hand. But the local pressure may be enormously 
increased by clamping the renal vein, in which circumstance the secretion of urine is suspended.

Although it is convenient to call the processes which go on in the renal glomeruli, filtration, there is reason to believe that they are not absolutely mechanical, as the term might seem to imply, since, when the epithelium of the Malpighian capsule has been, as it were, put out of order by ligature of the renal artery, on removal of the ligature, the urine has been found temporarily to contain albumen, indicating that a selective power resides in the healthy epithelinm, which allows certain constituent parts of the blood to be filtered off, and not others.

Secretion.-That there is a second part in the process of the exeretion of urine, which is true secretion, is suggested by the structure of the tubuli uriniferi, and the idea is supported by various experiments. It will be remembered that the convoluted portions of the tubules are lined with an epithelium, which bears a close resemblance to the secretory epithelium of other glands, whereas the Malpighian capsules and portions of the loops of Henle are lined simply by flattened epithelium. The two functions of the different parts of an uriniferous tube are, then, suggested by the differences of epithelium, and also by the fact that the blood supply to the different parts is different, since, as we have seen,

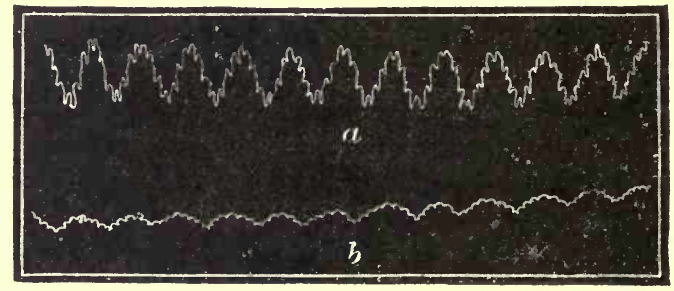

Fig. 309.-Curve taken by renal oncometer compressed-with that of ordinary blood-pressure. $a$, Kidney curve; $b$, blood-pressure curve. (Roy.)

the convoluted tubes are surrounded by capillary vessels derived from the breaking up of the efferent vessels of the Malpighian tufts. As to the functions of the different parts of the uriniferous tubes in the secretion of urine, two chief theories have been brought forward. The first, suggested by Bowman (1842), and still generally accepted, is that the cells of the convoluted tubes, by a process of true secretion, separate from the blood substances such as urea, whereas from the glomeruli are separated the water and the inorganic salts. The second, suggested by Ludwig (1844), is that in the glomeruli are filtered off from the blood all the constituents of the urine in a very diluted condition. When this passes along the tortuous uriniferous tube, part of the water is re-absorbed into the vessels surrounding them, learing the urine in a more concentrated condition-retaining all its proper constituents. This osmosis is promoted by the high specific gravity of the blood in 
the capillaries surrounding the convoluted tubes, but the return of the area and similar substances is prevented by the secretory epithelium of the tubules. The first theory is, however, more strongly supported by direct experiment.

By using the kidney of the newt, which has two distinct vascular supplies, one from the renal artery to the glomeruli, and the other from the renal-portal vein to the convoluted tubes, Nussbaum has shown that certain substances, e.g., peptones and sugar, when injected into the blood, are eliminated by the glomeruli, and so are not got rid of when the renal arteries are tied; whereas certain other substances, e.g., urea, when injected into the blood, are eliminated by the convoluted tubes, even when the renal arteries have been tied. This evidence is very direct that urea is excreted by the convoluted tubes, that is to say, if it is certain that ligature of the renal artery assists the circulation through the glomeruli, which, however, is denied by Adami.

Heidenhain also has shown by experiment that if a substance (sodium sulph-indigotate), which ordinarily produces blue urine, be injected into the blood after section of the medulla which causes lowering of the blood-pressure in the renal glomeruli, that when the kidney is examined, the cells of the convoluted tubules (and of these alone) are stained with the substance, which is also found in the lumen of the tubules. This appears to show that under ordinary circumstances the pigment at any rate is eliminated by the cells of the convoluted tubules, and that when by diminishing the blood-pressure, the filtration of urine ceases, the pigment remains in the convoluted tubes, and is not, as it is under ordinary circumstances, swept away from them by the flushing of them which ordinarily takes place with the watery part of urine derived from the glomeruli. It therefore is probable that the cells, if they excrete the pigment, excrete urea and other substances also. But urea acts sornewhat differently to the pigment, as when it is injected into the blood of an animal in which the medulla has been divided, and the secretion of urine stopped, a copious secretion of urine results, which is not the case when the pigment is used instead under similar conditions. The flow of urine, independent of the general blood-pressure, might be supposed to be due to the action of the altered blood upon some local vaso-motor mechanism; and, indeed, the local blood-pressure is directly affected in this way, but there is reason for believing that part of the increase of the secretion is due to the direct stimulation of the cells by the urea contained in the blood.

To sum up, then, the relation of the two functions: (1.) The process of filtration, by which the chief part, if not the whole, of the fluid is eliminated, together with certain inorganic salts and possibly other solids, is indirectly dependent upon blood-pressure, is accomplished by the renal glomeruli, and is accompanied by a free discharge of solids from the tubules. (2.) The process of secretion proper, by which urea 
and the principal urinary solids are eliminated, is accomplished by the cells of the convoluted tubes, and is sometimes (as in the case of the elimination of urea and similar substances) accompanied by the elimination of copious fluid, produced by the chemical stimulation of the epithelium of tise same tubules.

\section{The Passage of Urine into the Bladder.}

As each portion of urine is secreted it propels that which is already in the uriniferous tubes onward into the pelvis of the kidney. Thence through the ureter the urine passes into the bladder, into which its rate and mode of entrance has been watched in cases of ectopia vesicce, i.e., of such fissures in the anterior or lower part of the walls of the abdomen, and of the front wall of the bladder, as expose to view its hinder wall together with the orifices of the ureters. The urine does not enter the bladder at any regular rate, nor is there a synchronism in its movement through the two ureters. During fasting, two or three drops enter the bladder every minute, each drop as it enters first raising up the little papilla on which, in these cases, the ureter opens, and then passing slowly through its orifice, which at once again closes like a sphincter. In the recumbent posture, the urine collects for a little time in the ureters, then flows gently, and, if the body be raised, runs from them in a stream till they are empty. Its flow is aided by the peristaltic contractions of the ureters, and is increased in deep inspiration, or by straining, and in active exercise, and in fifteen or twenty minutes after a meal. The urine collecting is prevented from regurgitation into the ureters by the mode in which these pass through the walls of the bladder, namely, by their lying for between half and three-quarters of an inch between the muscular and mucous coats before they turn rather abruptly forward, and open through the latter into the interior of the bladder.

Micturition.-The contraction of the muscular walls of the bladder may by itself expel the urine with little or no help from other muscles. In so far, however, as it is a voluntary act, it is performed by means of the abdominal and other expiratory muscles, which in their contraction, as before explained, press on the abdominal viscera, the diaphragm being fixed, and cause the expulsion of the contents of those whose sphincter muscles are at the same time relaxed. The muscular coat of the bladder co-operates, in micturition, by reflex involuntary action, with the abdominal muscles; and the act is completed by the accelerator urince, which, as its name implies, quickens the stream, and expels the last drop of urine from the urethra. The act, so far as it is not directed by volition, is under the control of a nervous centre in the lumbar spinal cord, through which, as in the case of the similar centre for defæcation, the various muscles concerned are harmonized in their action. It is well known that the act may be reflexly induced, e.g., in children who 
suffer from intestinal worms, or other such irritation. Generally the afferent impulse which calls into action the desire to micturate is excited by over-distention of the bladder, or even by a few drops of urine passing into the urethra. This passes up to the lumbar centre (or centres) and produces on the one hand inhibition of the sphincter and on the other hand contraction of the necessary muscles for the expulsion of the contents of the bladder.

\section{The Structure and Functions of the Skin.}

'The skin serves-(1), as an external integument for the protection of the deeper tissues, and (2), as a sensitive organ in the exercise of touch, a subject to be considered in the Chapter on the Special Senses; it is also (3), an important secretory and excretory, and (4), an absorbing organ, already noticed, p. 425 ; while it plays an important part in (5) the regulation of the temperature of the body. (See the Chapter on Animal Heat.)

Structure.-The skin consists principally of a vascular tissue named the corium, derma, or cutis vera, and of an external covering of epithelium termed the epidermis or cuticle. Within and beneath the corium are imbedded several organs with special functions, namely, sudoriferous glands, sebaceous glands, and hair follicles; and on its surface are sensitive papillce. The so-called appendages of the skin-the hair and nails -are modifications of the epidermis.

Epidermis.-The epidermis is composed of several strata of cells of various shapes and sizes; it closely resembles in its structure the epithelium of the mucous membrane that lines the mouth. The following four layers may be distinguished in a more or less developed form: 1 . Stratum corneum (fig. 310, a), consisting of superposed layers of horny scales. The different thickness of the epidermis in different regions of the body is chiefly due to variations in the thickness of this layer; e.g., on the horny parts of the palms of the hands and soles of the feet it is of great thickness. The stratum corneum of the buccal epithelium chiefly differs from that of the epidermis in the fact that nuclei are to be distinguished in some of the cells even of its most superficial layers.

2. Stratum lucidum, a bright homogeneous membrane consisting of squamous cells closely arranged, in some of which a nucleus can be seen.

3. Stratum granulosum, consisting of one layer of flattened cells which appear fusiform in vertical section: they are distinctly nucleated, and a number of granules extend from the nucleus to the margins of the cell.

4. Stratum Malpighii or Rete mucosum consists of many strata. The deepest cells, placed immediately above the cutis vera, are columnar with oval nuclei: this layer of columnar cells is succeeded by a number of tayers of more or less polyhedral cells with spherical nuclei; the cells 
of the more superficial layers are considerably flattened. The deeper surface of the rete mucosum is accurately adapted to the papillæ of the true skin, being, as it were, moulded on them. It is very constant in thickness in all parts of the skin. The cells of the middle layers of the stratum Malpighii are almost all connected by processes, and thus form prickle cells (fig. 35). The pigment of the skin, the varying quantity of which causes the various tints observed in different individuals and different races, is contained in the deeper cells of rete mucosum; the pigmented cells as they approach the free surface gradually losing their color. Epidermis maintains its thickness in spite of the constant

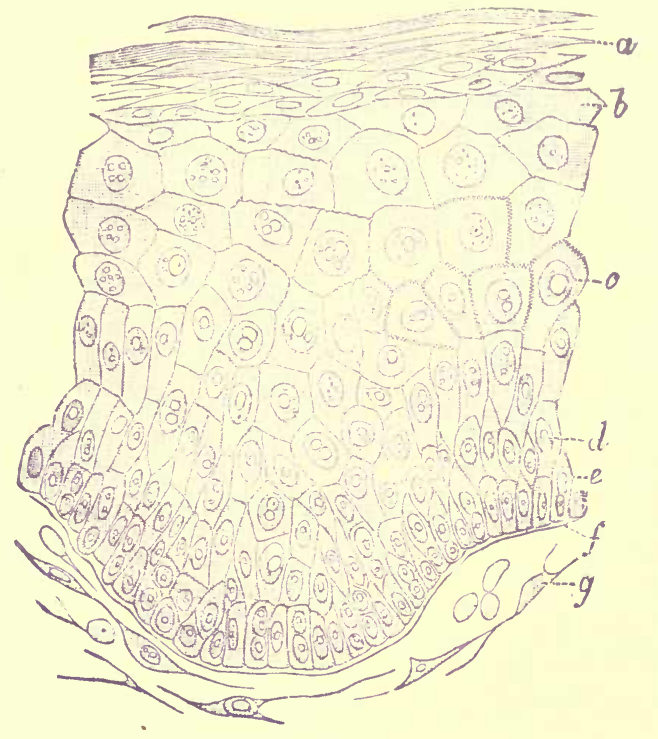

Fig. 310.-Vertical section of the epidermis of the prepuce. $a$, stratum corneum, of very few layers, the stratum lucidum and stratum granulosum not being distinctly represented; $b, c, d$, and $e$, the layers of the stratum Malpighii. a certain number of the cells in layers $d$ and $e$ showing signs of segmentation; layer $c$ consists chiefly of prickle or ridge and furrow cells; $f$, basement membrane: $g$, cells in cutis vera. (Cadiat.)

wear and tear to which it is subjected. The columnar cells of the deepest layer of the rete mucosum elongate, and their nuclei divide into two (fig. 310,e). Lastly the upper part of the cell divides from the lower; thus from a long columnar cell are produced a polyhedral cell and a short columnar cell: the latter elongates and the process is repeated. The polyhedral cells thus formed are pushed up toward the free surface by the production of fresh ones beneath them, and become flattened from pressure: they also become gradually horny by evaporation and transformation of their protoplasm into keratin, till at last by rubbing in ordinary wear and tear they are detached as dry horny scales at the free surface. There is thus a constant production of fresh cells in the 
deeper layers, and a constant throwing off of old ones from the free surface. When these two processes are accurately balanced, the epidermis maintains its thickness. When, by intermittent pressure a more active cell-growth is stimulated, the production of cells exceeds their waste and the epidermis increases in thickness, as we see in the horny hands of the laborer.

The thickness of the epiderınis on the different portions of the skin is directly proportioned to the friction, pressure, and other sources of injury to which it is exposed; for it serves as well to protect the sensitive and vascular cutis from injury from without, as to limit the evaporation of fluid from the blood-vessels. The adaptation of the epidormis to the latter purposes may be well shown by exposing to the air two dead hands or feet, of which one has its epidermis perfect, and the other is deprived of it; in a day, the skin of the latter will become brown, dry and horn-like, while that of the former will almost retain its natural moisture.

Cutis vera.-The corium or cutis vera, which rests upon a layer of adipose and cellular tissue of varying thickness, is a dense and tough, but yielding and highly elastic structure, composed of fasciculi of areolar tissue, interwoven in all directions, and forming, by their interlacements, numerous spaces or areolæ. These areolæ are large in the deeper layers of the cutis, and are there usually filled with little masses of fat (fig. 298) : but, in the superficial parts, they are small or entirely obliterated. Unstriped muscular fibres are also abundantly present.

Papillæ.-The cutis vera presents numerous conical papillæ, with a single or divided free extremity, which are more prominent and more densely set at some parts than at others. This is especially the case on the palmar surface on the hands and fingers, and on the soles of the feet -parts, therefore, in which the sense of touch is most acute. On these parts they are disposed in double rows, in parallel curved lines, separated from each other by depressions. Thus they may be easily seen on the palm, whereon each raised line is composed of a double row of papillæ, and is intersected by short transverse lines or furrows corresponding with the interspaces between the successive pairs of papillæ. Over other parts of the skin they are more or less thinly scattered, and are scarcely elevated above the surface. Their average length is about $\frac{1}{100}$ of an inch ( $\frac{1}{4} \mathrm{~mm}$.), and at their base they measure about $\frac{1}{250}$ of an inch in diameter. Each papilla is abundantly supplied with blood, receiving from the vascular plexus in the cutis one or more minute arterial twigs, which divide into capillary loops in its substance, and then reunite into a minute vein, which passes out at its base. This abundant supply of blood explains the turgescence or kind of erection which they undergo when the circulation through the skin is active. The majority, but not all, of the papillæ contain also one or more terminal 
nerve-fibres, from the ultimate ramifications of the cutaneous plexus, on which their exquisite sensibility depends.

The nerve-terminations in the skin have been described under the Sensory Nerve Terminations (p. 102 et sèq.).

Glands of the Skin.- The skin possesses glands of two kinds: (a) Sudoriferous, or Sweat Glands; (b) Sebaceous glands.

(a) Sudoriferous, or Sweat Glands.-Each of these glands consists of a small lobular mass, formed of a coil of tubular gland-duct, sur-

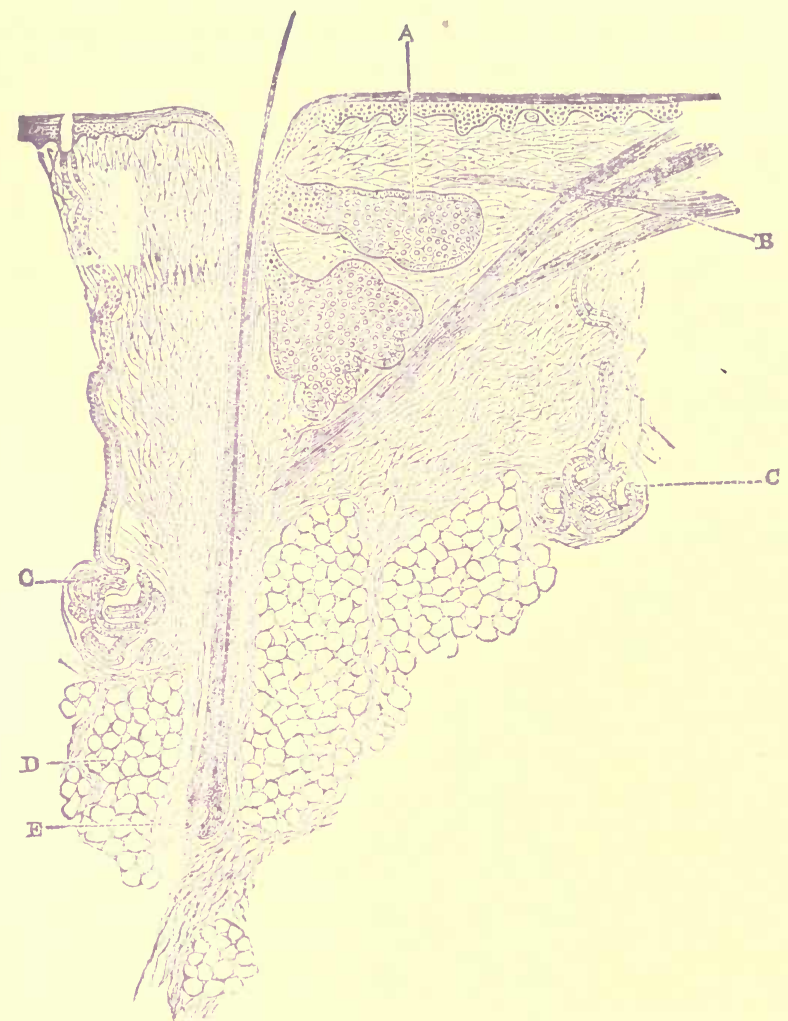

Fig. 311.-Vertical section of skin. A. Sebaceous gland opening into hair follicle. B. Muscular fibres. C. Sudoriferous or sweat-gland. D. Subcutaneous fat. E. Fundus of hair-follicle, with hair-papillæ. (Klein.)

rounded by blood-vessels and embedded in the subcutaneous adipose tissue (fig. 311, C). From this mass, the duct ascends, for a short distance in a spiral manner through the deeper part of the cutis, then passing straight, and then sometimes again becoming spiral, it passes through the epidermis and opens by an oblique valve-like aperture. In the parts where the epidermis is thin, the ducts themselves are thinner and more nearly straight in their course (fig. 311). The duct, which maintains nearly the same diameter throughout, is lined with a 
layer of columnar epithelium (fig. 311) continuous with the epidermis; while the part which passes through the epidermis is a mere passage through the epidermal cells not being bounded by any special lining; but the cells which immediately form the boundary of the canal in this part are somewhat differently arranged from those of the adjacent cuticle. The coils or terminal portions of the gland are lined with at least two layers of short columnar cells with very distinct nuclei (fig. 312), and possess a large lumen distinctly bounded by a special lining of cuticle.

The sudoriferous glands are abundantly distributed over the whole surface of the body; but are especially numerous, as well as very large, in the skin of the palm of the hand and of the sole of the foot. The glands by which the peculiar odorous matter of the axillæ is secreted

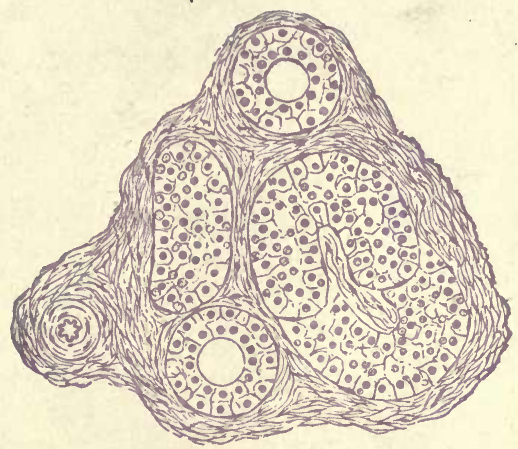

Fig. 812.-Terminal tubules of sudoriferous glands, cut in various directions from the skin of the pig's ear. (V. D. Harris.)

form a nearly complete layer under the cutis, and are like the ordinary sudoriferous glands, except in being larger and having rery short ducts.

The peculiar bitter yellow substance secreted by the skin of the external auditory passage is named cerumen, and the glands themselves ceruminous glands; but they do not much differ in structure from the ordinary sudoriferous glands.

(b) Sebaceous Glands.-The sebaceous glands (figs. 311, 316), like sudoriferous glands, are abundant in most parts of the surface of the body, particularly in parts largely supplied with hair, as the scalp and face. They are thickly distributed about the entrances of the various passages into the body, as the anus, nose, lips, and external ear. They are entirely absent from the palmar surface of the hand and the plantar surfaces of the feet. They are racemose glands composed of an aggregate of small tubes or sacculi lined with columnar epithelium and filled with an opaque white substance, like soft ointment, which consists of broken-up epithelial cells which have undergone fatty degeneration. Minute capillary vessels overspread them; and their ducts open on 
either the surface of the skin, close to a hair, or, which is more usual, directly into the follicle of the hair. In the latter ease, there are generally two or more glands to each hair (fig. 312).

Hair.-A hair is produced by a peculiar growth and modification of the epidermis. Externally it is covered by a layer of fine scales closely imbricated, or overlapping like the tiles of a house, but with the free edges turned upward (fig. 314, A). It is called the cutccle of the hair. Beneath this is a much thicker layer of elongated horny cells, closely packed together so as to resemble a fibrous structure. This, very commonly, in the human subject, occupies the whole inside of the hair; but

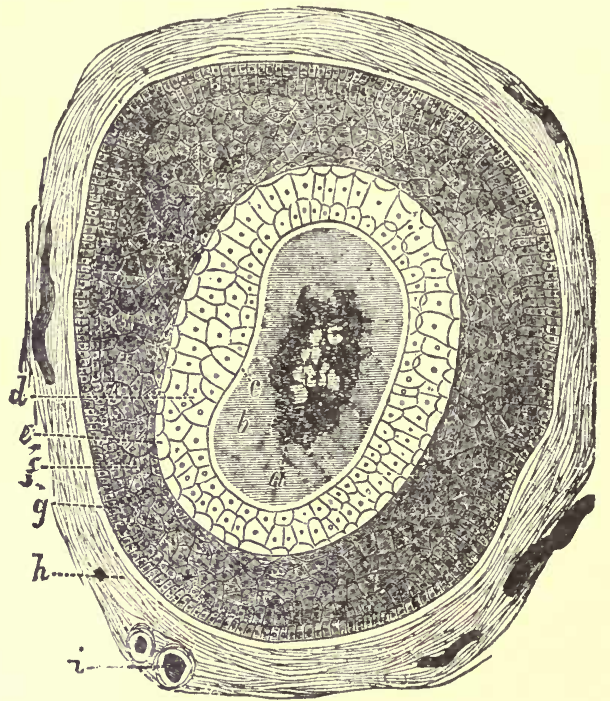

Fig. 313-Transverse section of a hair and.hair-follicle made below tle opening of the sebaceous gland. $a$, medulla or pith of the hair; $b$, fibrous layer or cortex; $c$, cuticle; $d$, Huxley's layer: $c$, Henle's layer of internal ront-sheatl: $f$ and $g$. layers of external root-sheath, outside of $g$ is $a$ light layer, or "glassy membrane," which is equivalent to the basement membrane; $h$, fibrous coat. of hair sac; $i$, vessels. (Cadiat.)

in some cases there is left a small central space filled by a substance called the medulla or pith, composed of small collections of irregularly shaped cells, containing sometimes pigment granules or fat, but mostly air.

The follicle, in which the root of each hair is contained (fig. 315), forms a tubular depression from the surface of the skin,-descending into the subcutaneous fat, generally to a greater depth than the sudoriferous glands, and at its deepest part enlarging in a bulbous form, and often curving from its previous rectilinear course. It is lined throughout by cells of epithelium, continuous with those of the epidermis, and its walls are formed of pellucid membrane, which commonly in the follicles of the largest hairs has the structure of vascular fibrous tissue. 
At the bottom of the follicle is a small papilla, or projection of true skin, and it is by the production and outgrowth of epidermal cells from the surface of this papilla that the hair is formed. The inner wall of the follicle is lined by epidermal cells continuous with those covering

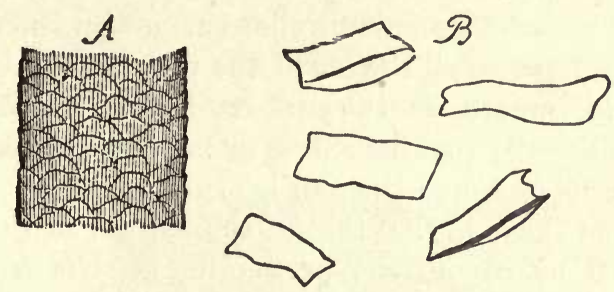

Fig. 314. - Surface of a white hair, magnifled 160 diameters. The wave lines mark the upper or free edges of the cortical scales. $B$, separated scales, magnified 350 diameters. (Kölliker.)

the general surface of the skin; as if indeed the follicle had been formed by a simple thrusting in of the surface of the integument (fig. 315). 'This epidermal lining of the hair-follicle, or root-sheath of the hair, is composed of two layers, the inner one of which is so moulded on the imbricated scaly cuticle of the hair, that its inner surface becomes imbricated also, but of course in the opposite direction. When a hair is

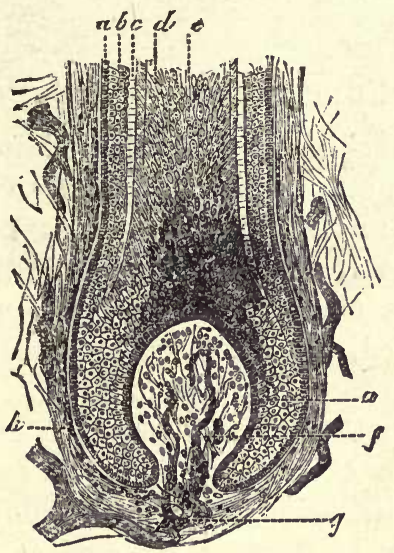

Fig. 815. - Longitudinal section of a hair follicle. a, Stratum of Malpighi, deep layer forming the external root-sheath, and continued to the surface of the papilla to form the medullary sheath of the hair; $b$, second external sheath; $c$, internal root-sheath; $d$, fibroid sheath of the hair; $e$, medullary sheath or medulla; $f$, hair papilla; $g$, blood-vessels of the hair-papilla; $h$, fibro-vascular sheath. (Cadiat.)

pulled out, the inner layer of the root-sheath and part of the outer layer also are commonly pulled out with it.

Nails. - A nail, like a hair, is a peculiar arrangement of epidermal cells, the undermost of which, like those of the general surface of the integument, are rounded or elongated, while the superficial are flattened, and of more horny consistence. That specially modified portion 
of the corium, or true skin, by which the nail is secreted is called the matrix.

The back edge of the nail, or the root as it is termed, is received into a shallow crescentic groove in the matrix, while the front part is free and projects beyond the extremity of the digit. The intermediate portion of the nail rests by its broad under surface on the front part of the matrix, which is here called the bed of the nail. This part of the matrix is not uniformly smooth on the surface, but is raised in the form of longitudinal and nearly parallel ridges or laminæ, on which are moulded the epidermal cells of which the nail is made up.

The growth of the nail, like that of the hair, or of the epidermis generally, is effected by a constant production of cells from beneath and behind, to take the place of those which are worn or cut away. Inasmuch, however, as the posterior edge of the nail, from its being lodged in a groove of the skin, cannot grow backward, on additions being made to it, so easily as it can pass in the opposite direction, any growth at its hinder part pushes the whole forward. At the same time fresh cells are added to its under surface, and thus each portion of the nail becomes gradually thicker as it moves to the front, until, projecting beyond the surface of the matrix, it can receive no fresh addition from beneath, and is simply moved forward by the growth at its root, to be at last worn away or cut off.

\section{Functions of THE SkIN.}

The function of the skin to be considered in this chapter is that of the excretion of the sweat. The fluid secreted by the sweat-glands is usually formed so gradually that the watery portion of it escapes by evaporation as fast as it reaches the surface. But during strong exercise, exposure to great external warmth, in some diseases, and when evaporation is prevented, the secretion becomes more sensible, and collects on the skin in the form of drops of fluid.

The perspiration, as the term is sometimes employed in physiology, includes all that portion of the secretions and exudations from the skin which passes off by evaporation; the sweat includes that which may be collected only in drops of fluid on the surface of the skin. The two terms are, however, most often used synonymously; and for distinction, the former is called insensible perspiration; the latter, sensible perspiration. The fluids are the same, except that the sweat is commonly mingled with various substances lying on the surface of the skin. The contents of the sweat are, in part, matters capable of assuming the form of vapor, such as carbonic acid and water, and in part, other matters which are deposited on the skin, and mixed with the sebaceous secretions.

The secretion of the sebaceous glands and hair-follicles consists of cast-off epithelium cells, with nuclei and granules, together with an oily 
matter, extractive matter, and stearin; in certain parts, also, it is mixed with a peculiar odorous principle, which contains caproic, butyric, and rutic acids. It is, perhaps, nearly similar in composition to the unctuous coating, or vernix caseosa, which is formed on the body of the fœtus while in the uterus, and which contains large quantities of ordinary fat. Its purpose seems to be that of keeping the skin moist and supple, and, by its oily nature, of both hindering the evaporation from the surface, and guarding the skin from the effects of the long-

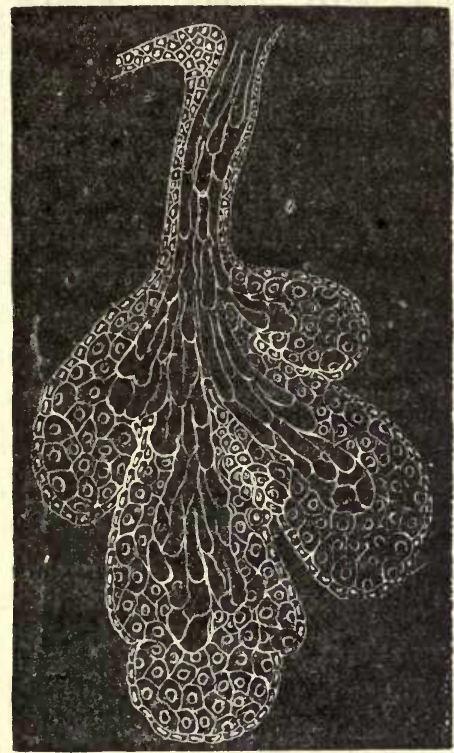

Fig. 316. -Sebaceous gland from human skin. (Klein and Noble Smith.)

continued action of moisture. But while it thus serves local purposes, its removal from the body entitles it to be reckoned among the excretions of the skin.

Chemical Composition of Sweat.

Water

Solids :-

Organic Acids (formic, acetic, butyric, pro- $\quad .9$ pionic, caproic, caprylic)

Salts, chiefly sodium chloride . . . 1.8

Neutral fats and cholesterin

.7

Extractives (including urea), with epithelium 1.6

The sweat is a colorless, slightly turbid fluid, alkaline, neutral or acid in reaction, of a saltish taste, and peculiar characteristic odor.

Of the several substances it contains, however, only the carbonic acid and water need particular consideration.

Watery Vapor. - The quantity of watery vapor excreted from the 
skin is on an average between $1 \frac{1}{2}$ and $2 \mathrm{lb}$. daily (about 1 kilo). This subject has been very carefully investigated by Lavoisier and Sequin. The latter chemist enclosed his body in an air-tight bag, with a mouthpiece. The bag being closed by a strong band above, and the mouthpiece adjusted and gummed to the skin around the mouth, he was weighed, and then remained quiet for several hours, after which time he was again weighed. The difference in the two weights indicated the amount of loss by pulmonary exhalation. Having taken off the airtight dress, he was immediately weighed again, and a fourth time after a certain interval. The difference between the two weights last ascertained gave the amount of the cutaneous and pulmonary exhalation together; by subtracting from this the loss by pulmonary exhalation alone, while he was in the air-tight dress, he ascertained the amount of cutaneous transpiration. During a state of rest, the average loss by cutaneous and pulmonary exhalation in a minute, is eighteen grains,the minimum eleven grains, the maximum thirty-two grains; and of the eighteen grains, eleven pass off by the skin, and seven by the lungs.

'The quantity of watery vapor lost by transpiration is of course influenced by all external circumstances which affect the exhalation from other evaporating surfaces, such as the temperature, the hygrometric state, and the stillness of the atmosphere. But, of the variations to which it is subject under the influence of these conditions, no calculation has been exactly made.

Carbonic Acid.-The quantity of carbonic acid exhaled by the skin on an average is about $\frac{1}{150}$ to $\frac{1}{200}$ of that furnished by the pulmonary respiration.

The cutaneous exhalation is most abundant in the lower classes of animals, more particularly the naked Amphibia, as frogs and toads, whose skin is thin and moist, and readily permits an interchange of gases between the blood circulating in it, and the surrounding atmosphere. Bischoff found that, after the lungs of frogs had been tied and cut out, about a quarter of a cubic inch of carbonic acid gas was exhaled by the skin in eight hours. And this quantity is very large, when it is remembered that a full-sized frog will generate only about half a cubic inch of carbonic acid by his lungs and skin together in six hours.

The importance of the respiratory function of the skin, which was once thought to be proved by the speedy death of animals whose skins, after removal of the hair, were covered with an impermeable varnish, has been shown by further observations to have no foundation in fact; the immediate cause of death in such cases being the loss of temperature. A rarnished animal is said to have suffered no harm when surrounded by cotton wadding, and to have died when the wadding was removed.

\section{Influence of the Nervous System.}

The secretion of sweat is closely connected with the quantity of blood flowing through the cutaneous ressels. The quantity of sweat increases with vaso-dilatation and diminishes with vaso-constriction. It 
is practically certain that the sweat-glands are also under the control of efferent impulses passing to them from the special sweat centres in the brain and spinal cord throngh special sweat nerves. Thus, if the sciatic nerve be divided in a cat and the peripheral end be stimulated, beads of sweat are seen to appear upon the pad of the corresponding foot, although at the same time the blood-vessels are constricted or while the aorta is pressed upon, whereas if atropin have been injected previously to the stimulation, no sweat appears, although dilatation of the vessels be present. Secretion of sweat, too, may be reflexly brought about.

The circulation of venous blood in the spinal bulb causes the sweating of phthisis and of dyspnca generally, by stimulating the sweat centre. If the cat whose sciatic nerve is divided be rendered dyspnœic, abundant sweat occurs upon the foot of the uninjured, and none on the injured side. The effect of heat in producing sweating may be both local and general, and again, the various drugs which produce an increased secretion of sweat do not all act in the same way; thus, there is reason for thinking that pilocarpin acts upon the local apparatus, that strychnia and picrotoxin act upon the sweat centres, and that nicotin acts both upon the central and npon the local apparatus.

The special sweat-nerves appear to issue from the spinal cord, in the case of the hind limb of the cat by the last two or three dorsal and first two or four lumbar nerves, pass to the abdominal sympathetic and from thence to the sciatic nerve. In the case of the fore limb, the nerves leave the cord by the 5th and 6th cervical nerves into the thoracic sympathetic, and then join the brachial plexus, reaching the arm through the median and ulnar nerves.

It will be as well to repeat here the other functions which the skin subserves. In addition to its excretory office, we have seen that it acts as a channel for absorption. It is also concerned with a special sense, viz., that of touch, to the consideration of which as well as to its function of regulating the temperature of the body we shall presently return. It should be recollected, however, that apart from these special functions, by means of its toughness, flexibility and elasticity, the skin is eminently qualified to serve as the general integument of the body, for defending the internal parts from external violence, while readily yielding and adapting itself to their various movements and changes of position. 


\section{CHAPTER XIV.}

\section{MUSCLE-NERVE PHYSIOLOGY.}

\section{Chemical Composition of Muscle.}

THE muscles make up about one-half of the total body weight. The principal substance which can be extracted from muscle, when examined after death, is the proteid body, Myosin, some of the reactions of which have been already discussed, p. 116. This body appear's to bear somewhat the same relation to the living muscle as fibrin does to the living blood, since the coagulation of muscle after death is due to the formation of myosin. Thus, if coagulation be delayed in muscles removed immediately from recently killed animals, by subjecting them to a temperature below $0^{\circ} \mathrm{C}$, it is possible to obtain from them by expression a viscid fluid of slightly alkaline reaction, called muscle-plasma (Kühne, Halliburton). And musele plasma, if exposed to the ordinary temperature of the air (and more quickly at $37^{\circ}-40^{\circ} \mathrm{C}$.), undergoes coagulation much in the same way as, under similar circumstances, does blood plasma, separated from the blood corpuscles by the action of a low temperature. 'The appearances presented by the fluid during the process are also very similar to the phenomena of blood-clotting, viz., that first of all an increased viscidity appears on the surface of the fluid, and at the sides of the containing vessel, which gradually extends throughout the entire mass, until a fine transparent clot is obtained. In the course of some hours the clot begins to contract, and to squeeze out of its meshes a fluid corresponding to blood-serum. In the course of coagulation, therefore, muscle plasma separates into muscle-clot and muscle-serum. The muscle clot is the substance myosin. It differs from fibrin in being easily soluble in a 2 per cent solution of hydrochloric acid, and in a 10 per cent solution of sodium chloride. It is insoluble in distilled water, and its solutions coagulate on application of heat. It is in short a globulin. During the process the reaction of the fluid becomes distinctly acid.

The coagulation of muscle plasma cannot only be prevented by cold, but also, as Halliburton has shown, by the presence of neutral salts in certain proportions; for example, of sodium chloride, of magnesium sulphate, or of sodium sulphate. It will be remembered that this is also the case with blood plasma. Dilution of the salted muscle plasma will produce its slow coagulation, which is prevented by the presence of the neutral salts in strong solution.

It is highly probable that the formation of muscle-clot is due to the 500 
presence of a ferment (myosin-ferment). The antecedent myosin in liv. ing muscle has received the name of myosinogen, in the same way as the fibrin-forming element in the blood is called fibrinogen. Myosinogen is, however, made up of two globulins, which coagulate at the temperatures $47^{\circ} \mathrm{C}$. and $56^{\circ} \mathrm{C}$. respectively. Myosin may also, as we have before mertioned, p. 482, be obtained from dead muscle by subjecting it, after all the blood, fat, and fibrous tissue, and substances soluble in water have been removed, to a 10 per cent solution of sodium chloride, or 5 per cent solution of magnesium sulphate, or 10 to 15 por cent solution of ammonium chloride, filtering and allowing the filtrate to drop into a large quantity of water; the myosin separates out as a white flocculent precipitate.

A very remarkable fact with regard to the properties of myosin has been demonstrated by Halliburton, namely, that a solution of dead muscle in strong neutral saline solution, possesses very much the same properties as muscle plasma, and that if diluted with twice or three times its bulk of water, myosin will separate out as a clot, which clot can be again dissolved in a strong neutral saline solution, and the solution can be again made to clot on dilution. This process can often be repeated; but in the fluid which exudes from the clot there is no proteid present. Myosin when dissolved in neutral saline fluids is converted into myosinogen, but reappears on dilution of the fluid. Muscle clot is almost pure myosin; but it appears to be combined with a certain amount of salts, for if it be freed of salts, especially of those of calcium, by prolonged dialysis, it losies its solubility. If a small amount of calcium salts be added, however, it regains that property.

Muscle serum is acid in reaction, and almost colorless. It contains three proteid bodies, viz.-(a) A globulin (myoglobulin), which can be precipitated by saturation with sodium chloride, or magnesium sulphate, and which can be coagulated at $63^{\circ} \mathrm{C} .\left(145^{\circ} \mathrm{F}\right.$ ). (b) Serum-albumin (myoalbumin), which coagulates at $73^{\circ} \mathrm{C} .\left(163^{\circ} \mathrm{F}\right.$.), but is not precipitated by saturation with either of those salts. And (c) Myo-albumose, which is neither precipitated by heat, nor by saturation with sodium chloride or magnesium sulphate, but may be precipitated by saturation with ammonium sulphate. It is closely connected with, even if it is not itself, myosin ferment. Neither casein nor peptone has been found by Halliburton in muscle extracts. In extracts of muscles, especially of red muscles, there is a certain amount of Homoglobin, and also of a pigment special to muscle, called by McMunn Myo-hcematin, which has a spectrum quite distinct from hæmoglobin, viz., a narrow band just before $\mathrm{D}$, two very narrow between $\mathrm{D}$ and $\mathrm{E}$, and two other faint bands, nearly violet, $\mathrm{E} \mathrm{b}$, and between $\mathrm{E}$ and $\mathrm{F}$ close to $\mathrm{F}$.

In addition to muscle ferments, already mentioned, muscle extracts 
contain certain small amounts of pepsin and fibrin ferment, and also an amylolytic ferment.

Certain acids are also present, particularly sarco-lactic, as well as acetic and formic.

Of carbohydrates, glycogen and glucose (or maltose), also inosite.

Nitrogenous crystalline bodies, such as kreatin, kreatinin, xanthin, hypo-xanthin, or carnin, taurin, urea, in very small amount, uric acid and inosinic acid.

Salts, the chief of which is potassium phosphate.

\section{Muscle at Rest.}

Physical Condition.-During rest or inactivity a muscle has a slight but very perfect Elasticity; it admits of being considerably stretched, but returns readily and completely to its normal condition. In the living body the muscles are always stretched somewhat beyond their natural length, they are always in a condition of slight tension; an arrangement which enables the whole force of the contraction to be utilized in approximating the points of attachment. It is obvious that if the muscles were lax, the first part of the contraction until the muscle became tight would be wasted.

There is no doubt that even in a condition of rest Oxygen is abstracted from the blood, and carbonic acid is given out by a muscle; for the blood becomes venous in the transit, and since the muscles form by far the largest element in the composition of the body, chemical changes must be constantly going on in them as in other tissues and organs, although not necessarily accompanied by contraction. When cut out of the body such muscles retain their contractility longer in an atmosphere of oxygen than in an atmosphere of hydrogen or carbonic acid, and during life, an amount of oxygen is no doubt necessary to the manifestation of energy as well as for the metabolism going on in the resting condition.

The reaction of living muscle in a resting or inactive condition is neutral or faintly alkaline.

In muscles which have been removed from the body, it has been found that for some little time electrical currents can be demonstrated passing from point to point on their surface; but as soon as the muscles die or enter into rigor mortis, these currents disappear.

The demonstration of muscle currents is usually done as follows:-The frog's muscles are the most convenient for experiment; and a muscle of regular shape, in which the fibres are parallel, is selected. The ends tre cut off by clean vertical cuts, and the resulting piece of muscle is called a regular muscle prism. The muscle prism is insulated, and a pair of non-polarizable electrodes connected with a very delicate galvanometer (fig. 317 ) is applied to various points of the prism, and by a deflection of the needle to a greater or less extent 
in one direction or another, the strength and direction of the currents in the piece of muscle can be estimated. It is necessary to use non-polarizable and not metallic electrodes in this experiment, as otherwise there is no certainty that the whole of the current observed is communicated from the muscle itself, and is not derived from the metallic electrodes arising in consequence of the action of the saline juices of the tissues upon them. The form of the nonpolarizable electrodes is a modification of Du Bois Reymond's apparatus (fig. 318), which consists of a somewhat flattened glass cylinder, $a$, drawn abruptly
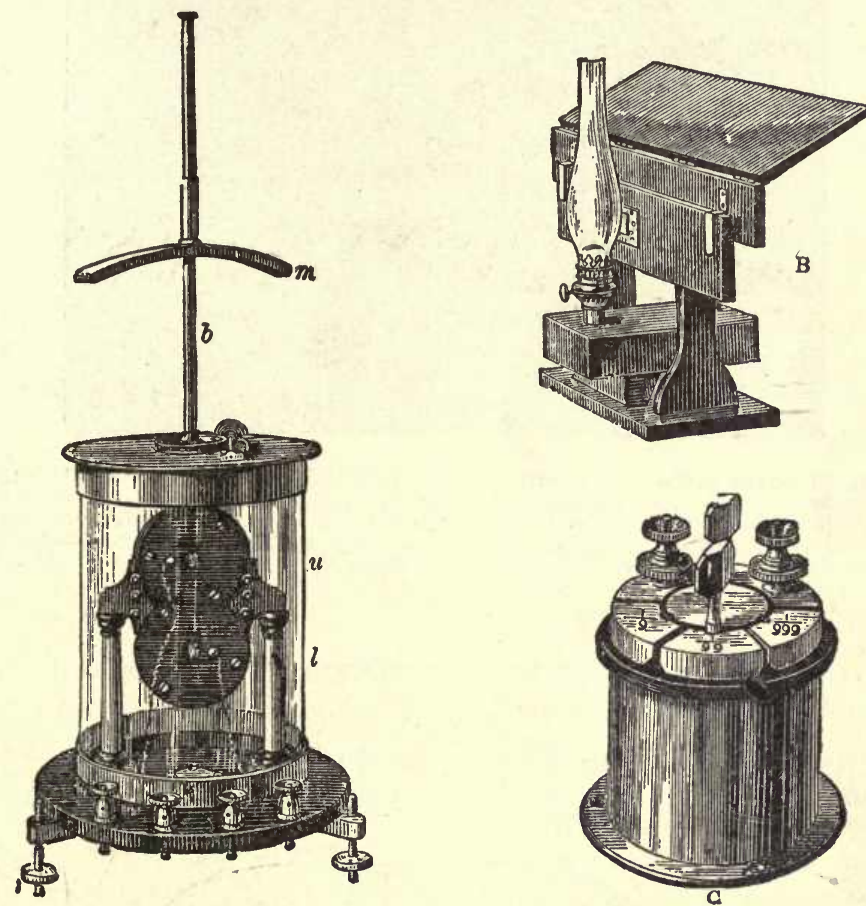

Fig. 317.-Reflecting galvanometer. (Thomson.) A. The galvanometer, which consists of two systems of small astatic needles suspended by a fine hair from a support, so that each set of needles is within a coil of fine insulated copper wire, that forming the lower coil is wound in an opposite direction to the upper. Attached to the upper set of needles is a small mirror about 1 inch in diameter; the light from the lamp at B is thrown upon this little mirror, and is reflected upon the scale on the other side of B, not shown in figure. The coils $l l$ are arranged upon brass uprights, and their ends are carried to the binding screws. The whole apparatus is placed upon a vulcanite plate capable of being levelled by the screw supports, and is covered by a brass-bound glass sliade, $\mathrm{L}$, the cover of which is also of brass, and supports a brass rod, $b$, on which moves a weak curved magnet, $m$. C is the shunt by means of which the amount of the current sent into the galvanometer may be regulated. When in use the scale is placed about three feet from the galvanometer, which is arranged east and west, the lamp is lighted, the mirror is made to swing, and the light from the lamp is adjusted to fall upon it, and it is then regulated until the reflected spot of light from it falls upon the zero of the scale. The wires from the non-polarizable electrodes touching the muscle are attached to the outer binding screws of the galvanometer, a key intervening for short-circuiting, or if a portion only of the current is to pass into the galvanometer, the shunt should intervene as well with the appropriate plug in. When a current passes into the galvanometer the needles and, with them, the mirror, are turned to the right or left according to the direction of the current. The amount of the deflection of the needle is marked on the scale by the spot of light travelling along it.

to a point, and fitted to a socket capable of movement, and attached to a stand, $A$, so that it can be raised or lowered as required. The lower portion of the cylinder is filled with china clay moistened with saline solution, part of which projects through its drawn-out point; the rest of the cylinder is fitted with a 
saturated solution of zinc sulphate into which dips a well amalgamated piece of zinc connected by means of a wire with the galvanometer. In this way the zinc sulphate forms a homogeneous and non-polarizable conductor between the zinc and the china clay. A second electrode of the same kind is, of course, necessary.

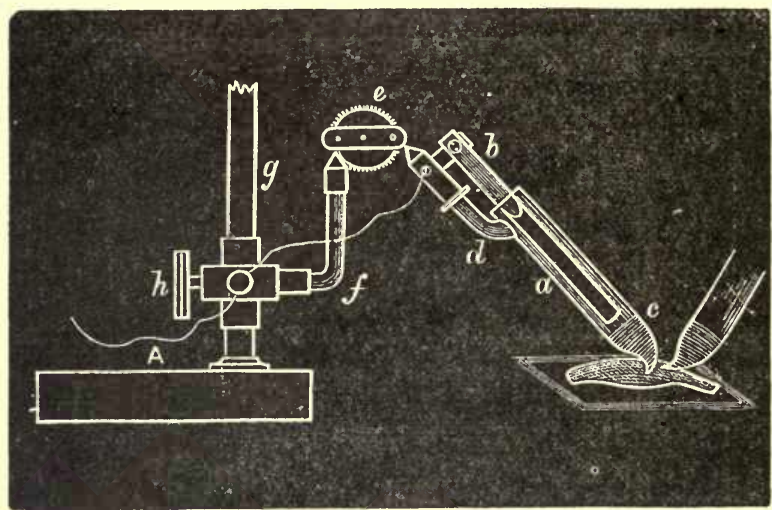

Fig. 318, -Diagram of Du Bois Reymond's non-polarizable electrodes. $a$, Glass tube filled with a saturated solution of zinc sulphate, in the end, $c$, of which is china clay drawn out to a point; in the solution a well amalgamated zinc rod is immersed and connected, by means of the wire which passes through $\mathrm{A}$, with the galvanometer. The remainder of the apparatus is simply for convenience of application. The muscle and the end of the second electrode are to the right of the figure.

In a regular muscle prism the currents are found to be as follows:If from a point in the surface a line-the equator-be drawn across the muscle prism equally dividing it, currents pass from this point to points away from it, which are weak if the points are near, and increased in

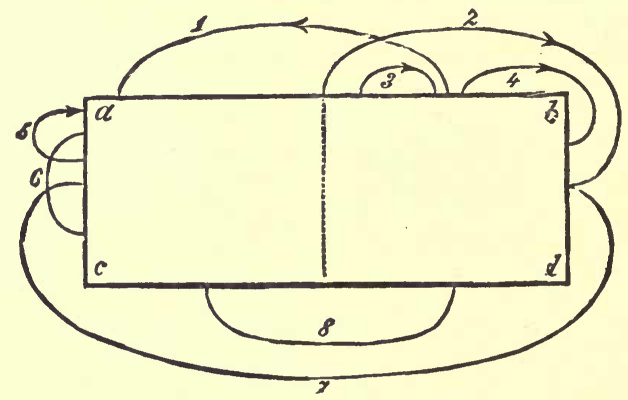

Fig. 319.-Diagram of the currents in a musele prism. (Du Bois Reymond.)

strength as the points are further and further away from the equator; the strongest passing from the equator to a point representing the middle of the cut ends (fig. 319,2 ); currents also pass from points nearer the equator to those more remote (fig. $319,1,3,4$ ), but not from points 
equally distant or iso-electrio points (fig. $319,6,7,8$ ). The cut ends are always negative to the equator. These currents are constant for some time after removal of the muscle from the body, and in fact remain as long as the muscle retains its life. They are in all probability due to chemical changes going on in the muscles.

The currents are diminished by fatigue and are increased by an increase of temperature within natural limits. If the uninjured tendon be used as the end of the muscle, and the muscle be examined without removal from the body, the currents are very feeble, but they are at once much increased by injuring the muscle, as by cutting off its tendon. The last observation appears to show that they are right who believe that the currents do not exist in uninjured muscles in situ, but that injury, either mechanical, chemical or thermal, will render the injured part electrically negative to other points on the muscle. In a frog's heart it has been shown, too, that no currents exist during its inactivity, but that as soon as it is injured in any way they are developed; the injured part being negative to the rest of the muscle. The currents which have been above described are called either natural muscle currents or currents of rest, according as they are looked upon as always existing in muscle or as developed when a part of the muscle is subjected to injury; in either case, up to a certain point, it is agreed that the strength of the currents is in direct proportion to the injury.

\section{Muscle in Activity.}

The property of muscular tissue, by which its peculiar functions are exercised, is its Contractility, which is excited by all kinds of stimuli applied either directly to the muscles, or indirectly to them through the medium of their motor nerves. This property, although commonly brought into action through the nervous system, is inherent in the muscular tissue. For-(1.) it may be manifested in a muscle which is isolated from the influence of the nervous system by division of the nerves supplying it, so long as the natural tissue of the muscle is duly nourished; and (2.) it is manifest in a portion of muscular fibre, in which, under the microscope, no nerve-fibre can be traced. (3.) Substances such as urari, which paralyze the nerve-endings in muscles, do not at all diminish the irritability of the muscle itself.

(4.) When a muscle is fatigued, a lccal stimulation is followed by a contraction of a small part of the fibre in the immediate vicinity without any regard to the distribution of nerve-fibres.

The Conditions which Affect the Irritability of Muscle-that is, its readiness of response to stimuli-are numerous. The chief causes of variation in irritability are the following:

Blood-Supply. - The irritability of muscles is also soon lost, unless a 
supply of arterial blood to them is kept up. Thus, after ligature of the main arterial trunk of a limb, the power of moving the muscles is partially or wholly lost, until the collateral circulation is established; and when, in animals, the abdominal aorta is tied, the hind legs are rendered almost powerless.

The same fact may be readily shown by compressing the abdominal aorta in a rabbit for about 10 minutes; if the pressure be released and the animal be placed on the ground, it will work itself along with its front legs, while the hind legs sprawl helplessly behind. Gradually the muscles recover their power and become quite as cfficient as before.

So, also, it is to the imperfect supply of arterial blood to the muscular tissue of the heart that the cessation of the action of this organ in asphyxia is in some measure due.

Fatigue. - The irritability of muscle is decreased by undue functional activity. The cause of the diminished irritability is twofold-when a muscle contracts, part of its substance is expended, part of its store of nutriment is exhausted, and it cannot readily contract again until the loss is made up. To this extent fatigue is much the same in its effect as cutting off or diminishing the blood-supply. The other cause for the diminution of irritability is the accumulation of poisonous products in the lymphatics of the muscle-substances generated during contraction.

Separation from Central Nervous System.-Generally a muscle begins to lose its irritability to all forms of stimuli about two weeks after its nerve is severed. Within a short time, however, its readiness of response to mechanical stimuli and to direct battery currents is heightened, while to induction shocks it is lessened. The increase of irritability reaches its maximum in about seven weeks, after which the irritability to all forms of stimuli diminishes, until it is completely lost toward the end of the serenth or eighth month.

The loss of irritability in muscle is due to degenerative changes in its protoplasm. But the cause of the degeneration is a matter of controversy, being considered due to loss of trophic influences from the central nervous system on the one hand, and to circulatory disturbances on the other.

Use.-Not only irritability but strength and power of endurance in muscle are increased by use. The effect of properly regulated exercises on muscles is too well known to need more than bare mention. And, on the contrary,

Disuse leads to diminution or loss of irritability. This fact is familiarly shown when a limb is disabled for a time, as through breaking a bone, in the stiffness of the muscles and the slowness with which they respond to the will. 
Temperature.-The irritability of muscle is increased by raising its temperature slightly above that of the animal from which it has been taken, while it is decreased by cooling. If, however, the temperature be raised too high $\left(45^{\circ} \mathrm{C}\right.$. for frog, $50^{\circ} \mathrm{C}$. for mammal), the muscle enters into a condition of heat rigor and its irritability is forever lost. After cooling, unless the cold be too severe and prolonged, the irritability returns as the temperature is raised. The effect of cold on irritability is shown in the superficial muscles of the face in winter.

Chenicals and Drugs.-Most chemical substances cause a marked alteration of irritability in muscle. In general terms, it may be said that those which produce any eflect at all at first increase and then diminish irritability.

Mechanical stimuli at first increase and then diminish the irritability of muscle. If they are powerful enough, the muscle is destroyed.

\section{The Phenomena of Muscular Contraction.}

The power which muscles possess of contraction may then be called forth by stimuli of various kinds, and these stimuli may also be applied directly to the muscle or indirectly to the nerve supplying it. There are distinct advantages, however, in applying the stimulus to the nerve, as it is more convenient, as well as more potent. The stimuli are of four kinds, viz.:-

(1.) Mechanical stimuli, as by a blow, pinch, prick of the muscle or its nerve, will produce a contraction, repeated on the repetition of the stimulus; but if applied to the same point for a limited number of times only, as such stimuli will soon destroy the irritability of the preparation.

(2.) Thermal Stimuli. - If a needle be heated and applied to a muscle or its nerve, the muscle will contract. A temperature of over $45^{\circ} \mathrm{C}$. $\left(113^{\circ} \mathrm{F}\right.$.) will cause the muscles of a frog to pass into a condition known as heat rigor.

(3.) Chemical Stimuli.-A great variety of chemical substances will excite the contraction of muscles, some substances being more potent in irritating the muscle itself, and other substances having more effect upon the nerve. Of the former may be mentioned, dilute acids, salts of certain metals, e.g., zinc, copper and iron; to the latter belong strong glycerin, strong acids, ammonia and bile salts in strong solution.

(4.) Electrical Stimuli.-For the purpose of experiment electrical stimuli are most frequently used, as the strength of the stimulus may be more conveniently regulated. Any form of electrical current may be employed for this purpose, but galvanism or the induced current is usually chosen. 
Galvanic currents are usually obtained by the employment of a continuous current battery such as that of Daniell, by which an electrical current which varies but little in intensity is obtained. The battery (fig. 320) consists of a positive plate of well-amalgamated zinc immersed in a porous cell, containing dilute sulphuric acid; and this cell is again contained within a large copper vessel (forming the negative plate), containing besides a saturated solution of copper sulphate. The electrical current is made continuous by the use of the two fluids in the following manner. The actinn of the dilute sulphuric acid upon the zinc plate partly dissolves it, and liberates hydrogen, and this gas passes through the porous vessel, and decomposes the copper sulphate into copper

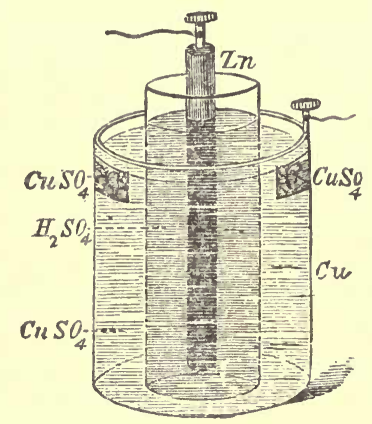

Fig. 320.-Diagram of a Daniell's battery.

and sulphuric acid. The former is deposited upon the copper plate, and the latter passes through the porous ressel to renew the sulphuric acid which is being used up. The copper sulphate solution is renewed by spare crystals of the salt, which are kept on a little shelf attached to the copper plate, and slightly below the level of the solution in the ressel. The current of electricity supplied by this battery will continue without variation for a considerable time. Other continuous current batteries, such as Grore's, may be used in place of Daniell's. The way in which the apparatus is arranged is to attach wires to the copper and zinc plates, and to bring them to a key, which is a little apparatus for connecting the wires of a battery. One often employed is Du Bois Reymond's (fig. 321) ; it consists of two pieces of brass about an inch long, in tach of which are two holes for wires and binding screw, to hold them tightly ; these pieces of brass are fixed upon a vulcanite plate, to the under surface of which is a screw clamp by which it can be secured to the table. The interval between the pieces of brass can be bridged over by means of a third thinner piece of similar metal fixed by a screw to one of the brass pieces, and capable of morement by a handle at right angles, so as to touch the other piece of brass. If the wires from the battery are brought to the inner binding screws, and the bridge connects them, the current passes across it and back to the battery. Wires are connected with the outer binding screws, and the other ends are joined together for about two inches, but, being corered except at their points, are insulated; the uncovered points are about an eighth of an inch apart. These wires are the electrodes, and the electrical stimulus is applied to the muscle through them, if they are placed behind its nerve. When the connection between the two brass plates of the key is broken by depressing the frandle of the bridge, the key is then said to be opened.

An induced current is developed by means of an apparatus, called an induc- 
the primary circuit, an instantaneous current of electricity is induced in the secondary coil, $g$, if it be sufficiently near and in line with the primary coil; and the nearer it is to $c$, the stronger is the current induced. The current is only momentary in duration and does not continue during the whole of the period while the primary circuit is complete. When, however, the primary current is broken by opening the key, a second, also momentary, current is induced in $g$. The former induced current is called the making and the latter the breaking shock; the former is in the opposite direction to, and the latter in the same as, the primary current.

The induction coil may be used to produce a rapid series of shocks by means of another and accessory part of the apparatus at the right of the fig., called the magnetic interrupter. If the wires from a battery are connected with the two pillars by the binding screws, one below $c$, and the other, $a$, the course of

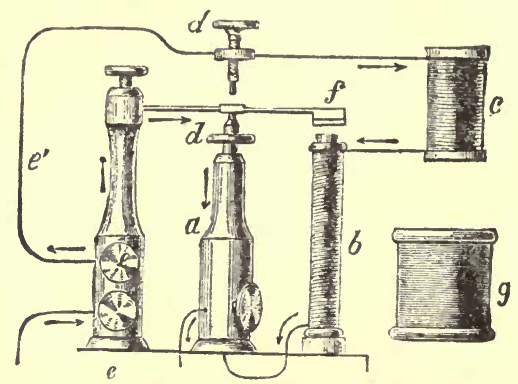

Fig. 324.-Diagram of the course of the current in the magnetic interrupter of Du Bois Reymond's induction coil. (Helmholz's modification.)

the current is indicated in fig. 324, the direction being indicated by the arrows. The current passes up the pillar from $e$, and along the springs if the end of $d$ is close to the spring, the current passes to the primary coil $c$, and to wires covering two upright pillars of soft iron, from them to the pillar $a$, and out by the wires to the battery ; in passing along the wire, $b$, the soft iron is converted into a magnet, and so attracts the hammer, $f$, of the spring, breaks the connection of the spring with $d^{\prime}$, and so cuts off the current from the primary coil, and also from the electro-magnet. As the pillars, $b$, are no longer magnetized the spring is released, and the current passes in the first direction, and is in like manner interrupted. At each make and break of the primary current, currents corresponding are induced in the secondary coil. These currents are opposite in direction, but are not equal in intensity, the break shock being greater. In order that the shocks should be nearly equal at the make and break, a wire (fig. 324, $e^{\prime}$ ) connects $e$ and $d^{\prime}$, and the screw $d^{\prime}$ is raised out of reach of the spring, and $d$ is raised (as in fig. 324), so that part of the current always passes through the primary coil and electro-magnet. When the spring touches $d$, the current in $b$ is diminished, but never entirely withdrawn, and the primary current is altered in intensity at each contact of the spring with $d$, but never entirely broken.

Record of Muscular Contraction under Stimuli.-The muscles of the frog are most convenient for the purpose of recording contractions. The frog is pithed, that is to say, its central nervous system is entirely destroyed by the insertion of a stout needle into the spinal cord, and the parts above it. One of its lower extremities is used in the following manner. The large trunk of the sciatic nerve is dissected out at the back of the thigh, and a pair of electrodes is 


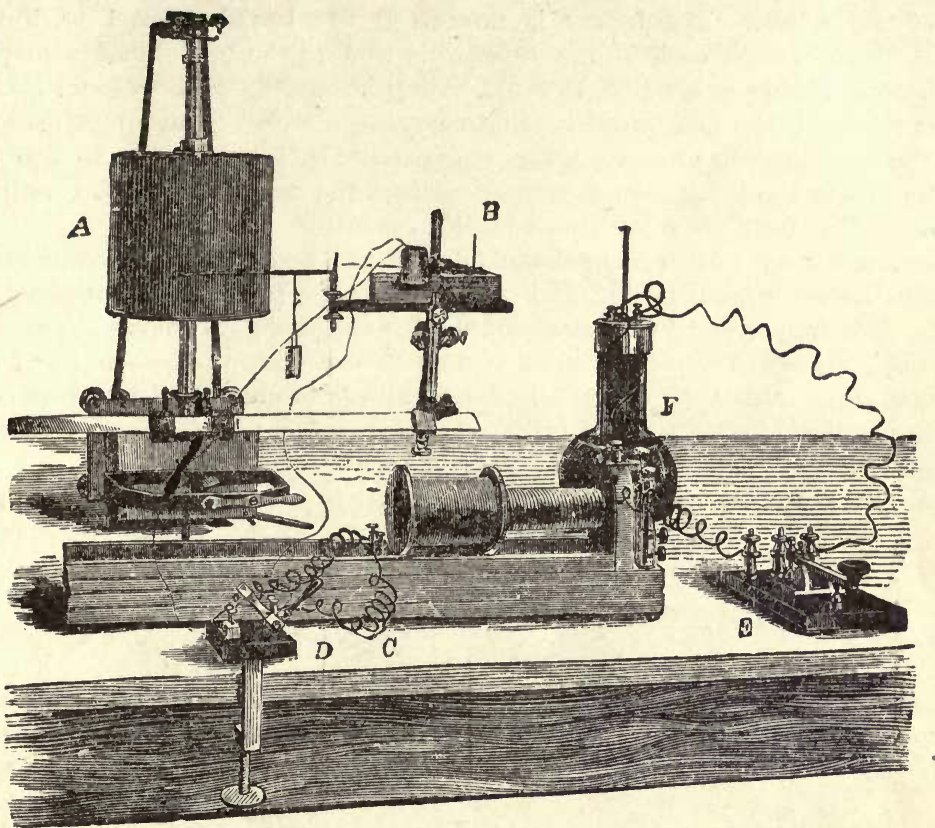

Fig. 325.-Arrangement of the apparatus necessary for recording muscle contractions with a revolving cylinder carrying smoked paper. A, Revolving cylinder; $\mathbf{B}$, the frog arranged upon a cork-covered board which is capable of being raised or lowered on the upright, which also can be moved along a solid triangular bar of metal attached to the base of the recording apparatus - the tendon of the gastrocnemius is attached to the writing lever, properly weighted, by a ligature. The electrodes from the secondary coil pass to the apparatus-being, for the sake of convenience, first of all brought to a key, D (Du Bois Reymond's); C, the induction coil; F, the battery (in this fig. a bichromate one); E, the key (Morse's) in the primary circuit.

inserted behind it. The tendo-achillis is divided from its attachment to the os calcis, and a ligature is tightly tied round it. This tendon is part of the broad muscle of the thigh (gastrocnemius), which arises from above the con.

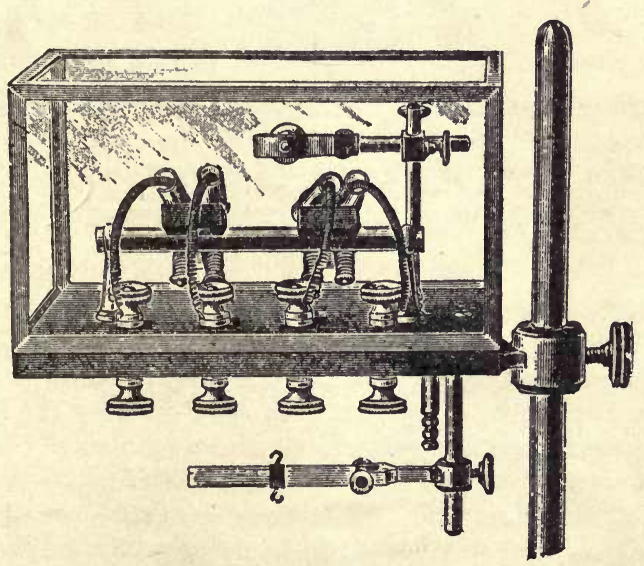

Fig. 326.-Moist Chamber. 
dyles of the femur. The femur is now fixed to a board covered with cork, and the ligature attached to the tendon is tied to the upright of a piece of metal bent at right angles (fig. $325, \mathrm{~B}$ ), which is capable of movement about a pivot at its knee, the horizontal portion carrying a writing lever (myograph). When the muscle contracts, the lever is raised. It is necessary to attach a small weight to the lever. In this arrangement the muscle is in situ, and the nerre disturbed from its relations as little as possible.

The muscle may, however, be detached from the body with the lower end of the femur trom which it arises, and the nerre going to it may be taken away with it. The femur is divided at about the lower third. The bone is held in a firm clamp, the nerve is placed upon two electrodes connected with an induction apparatus, and the lower end of the muscle is connected by means of a ligature attached to its tendon with a lever which can write on a recording apparatus.

To prevent evaporation this so-called nerve-muscle preparation is placed under

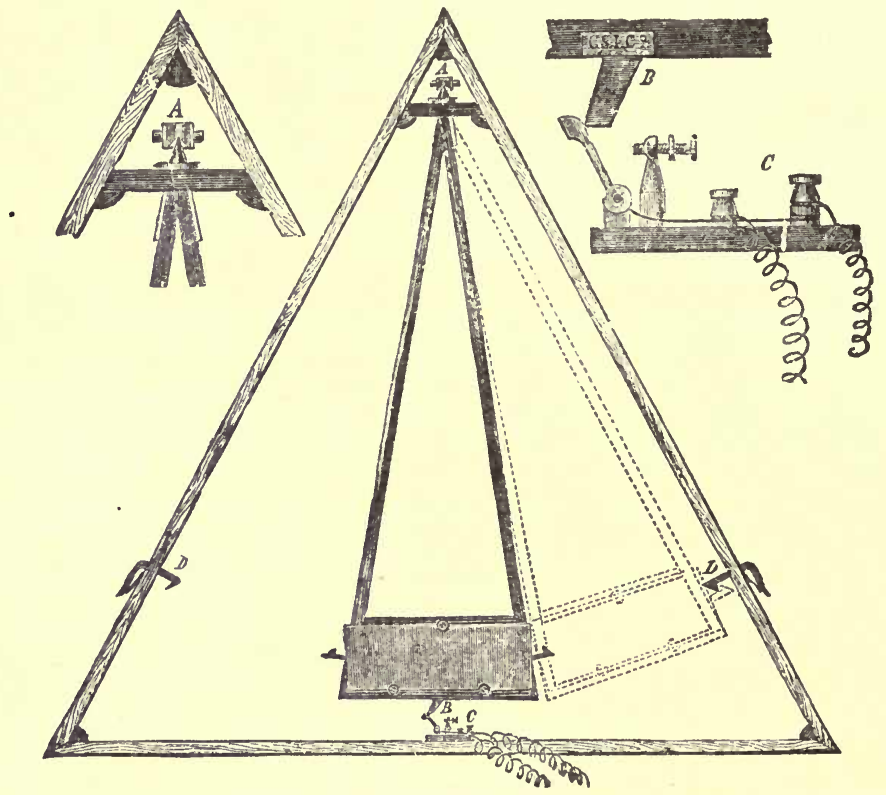

Fir. 32\% --Simple form of pendulum myograph and accessory parts. $A$, Pivot upon which penfufum swings: $B$, eatch on lower end of myograph opening the key, $C$, in its swing: $D$, a spring-catch which retains myograph, as indicated by dotted lines. and on pressing down the handle of which the pendulum swings along the arc to $D$ on the left of figure, and is caught by its spring.

a glass shade (moist chamber, fig. 326), the air in which is kept moist by means of blotting paper saturated with saline solution.

Effects of a Single Induction Shock.-With a nerve-muscle preparation arranged in either of the above ways, on closing or opening the key in the primary circuit, we obtain and can record a contraction, and if we use the clock. work apparatus revolving rapidly, a curve is traced such as is shown in fig.

Another way of recording the contraction is by the use of the pendulum myograph (fig. 327). Here the movement of the pendulum along a certain are is substituted for the clockwork morement of the other apparatus. The jen- 
dulum carries a smoked glass plate upon which the writing lever of a myograph is made to mark. The opening or breaking shock is sent into the nerve-muscle preparation by the pendulum in its swing opening a key (fig. 327, C.) in the primary circuit.

Single Muscle Contractions.-The tracing (muscle curve) obtained of a single muscle contraction or twitch is seen in fig. 328, and may be thus explained.

The upper line $(m)$ represents the curve traced by the end of the lever in connection with a muscle after stimulation of the muscle by a single

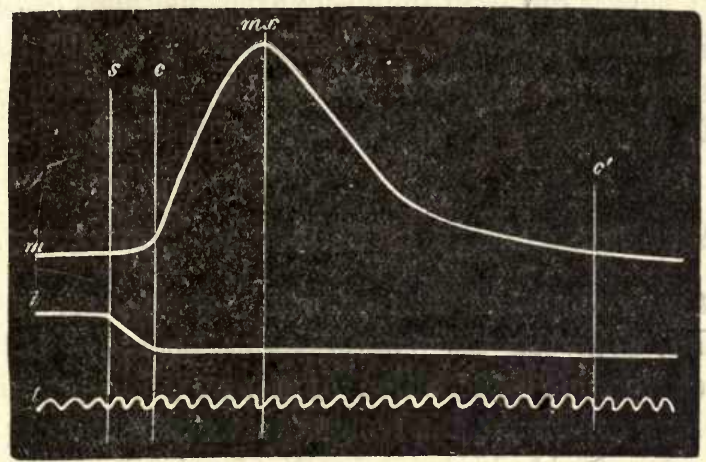

Fig. 328.-Muscle-curve obtained by means of the pendulum myograph. $s$, indicates the exact instant of the induction shock; $c$, commencement; and $m x$, the maximum elevation of lever; $t$, the line of a vibrating tuning-fork. (M. Foster.)

induction-shock: the middle-line $(l)$ is that described by the markinglever, and indicates by a sudden drop the exact instant at which the induction-shock was given. The lower wavy line $(t)$ is traced by a vibrating tuning-fork, and serves to measure precisely the time occupied in each part of the contraction.

It will be observed that after the stimulus has been applied, as indicated by the vertical line $s$, there is an interval before the contraction commences, as indicated by the line $c$. This interval, termed $(a)$ the latent period, when measured by the number of vibrations of the tuning-fork between the lines $s$ and $c$, is found to be about $\frac{1}{100} \mathrm{sec}$. The latent period is longer in some muscles than in others, and differs also according to the condition of the muscle, being longer in fatigued muscles, and the kind of stimulus employed. During the latent period there is no apparent change in the muscle.

The second part is the $(b)$ stage of contraction proper. The lever is raised by the sudden contraction of the muscle. The contraction is at first very rapid, but then progresses more slowly to its maximum, indicated by the line $m x$, drawn through its highest point. It occupies in the figure $\frac{4}{100} \mathrm{sec}$. (c) The next stage, stage of elongation. After 
reaching its highest point, the lever begins to descend, in consequence of the elongation of the muscle. At first the fall is rapid, but then becomes more gradual until the lever reaches the abscissa or base line, and the muscle attains its pre-contraction length, indicated in the figure by the line $c^{\prime}$. The stage occupies $\mathbf{x}_{\frac{5}{0}} \frac{5}{0}$ second. Very often after the main contraction the lever rises once or twice to a slight degree, producing curves, one of which is seen in fig. 330. These contractions, due to the elasticity of the muscle, are called most properly $(d)$ stage of elastic after-vibration, or contraction remainder.

The latent period has been found by exact methods of determination to be only $\frac{1}{40} \sigma$ second in length. The remainder of the time indicated above is occupied in the propagation of the impulse along the nerve and in overcoming the resistance of the apparatus used for recording the curve.

\section{Accompaniments of Muscular Contraction.}

(1.) Heat is developed in the contraction of muscles. Becquerel and Breschet found, with the thermo-multiplier, about $.5^{\circ} \mathrm{C}$. of heat produced by each forcible contraction of a man's biceps; and when the actions were long continued, the temperature of the muscle increased $1^{\circ}$. This estimate is probably high, as in the frog's muscle a considerable contraction has been found to produce an elevation of temperature equal on an average to less than $\frac{1}{5}^{\circ} \mathrm{C}$. The cause of the rise of temperature is the increased chemical activity at the time of contraction. As we have already seen (Animal Heat), muscles produce heat even when uncontracted.

(2.) Sound is produced, as mentioned above, when voluntary muscles contract. Wollaston showed that this sound might be easily heard by placing the tip of the little finger in the ear, and then making some muscles contract, as those of the ball of the thumb, whose sound may be conducted to the ear through the substance of the hand and finger. A low shaking or rumbling sound is heard. The sound is due to the vibration of the individual muscle fibres. Experimentally it has been found that the number of vibrations corresponds to the number of excitations, and that muscle exhibits no normal rate of vibration, except in so far as a rate is expressed in the discharge of nerve impulses from the cells controlling the muscle. Nerve cells do not send out a single, but a series of impulses. Moreover, the muscle sound corresponds to the rate at which the muscle is stimulated.

Helmholtz found that, in the voluntary contraction of muscle, only reeds having a vibration of 18-20 per second were thrown into motion; and since this rate is too slow to produce a tone, he concluded that the 
sound heard was the first overtone. But this rate has been called into question by later experiments, in which a tambour, connected with a recording apparatus, is placed on a contracting muscle. The rate of vibration thus obtained is stated to be from 8-12 per second, according to the muscle investigated aud its condition. Tremors are shown by a muscle in fatigue and in mauy conditions of disease. Since the resonance tone of the membrana tympani corresponds to $36-40$ vibrations a second, the muscle sound does not indicate the number of vibrations in a contracting muscle.

(3.) Changes in Shape.-There is a considerable difference of opinion as to the mode. in which the transversely striated muscular fibres contract. The most probable account is, that the contraction is effected by an approximation of the constituent parts of the fibrils, which, at the instant of contraction, without any alteration in their general direction, become closer, flatter, and wider; a condition which is rendered evident by the approximation of the transverse striæ seen on the surface of the fasciculus, and by its increased breadth and thickness. The appearance of the zigzag lines into which it was supposed the fibres are thrown in contraction, is due to the relaxation of a fibre which has been recently contracted, and is not at once stretched again by some antagonist fibre, or whose extremities are kept close together by the contractions of other fibres. The contraction is therefore a simple and, according to Ed. Weber, a uniform, simultaneous, and steady shortening of each fibre and its contents. What each fibril or fibre, loses in length, it gains in thickness: the contraction is a change of form not of size; it is, therefore, not attended with any diminution in bulk, from condensation of the tissue. This has been proved for entire muscles, by making a mass of muscles, or many fibres together, contract in a vessel full of water, with which a fine, perpendicular, graduated tube communicates. Any diminution of the bulk of the contracting muscle would be attended by a fall of fluid in the tube; but when the experiment is carefully performed, the level of the water in the tube remains the same, whether the muscle be contracted or not.

In thus shortening, muscles appear to swell up, becoming rounder, more prominent, harder, and apparently tougher. But this hardness of muscle in the state of contraction is not due to increased firmness or condensation of the muscular tissue, but to the increased tension to which the fibres, as well as their tendons and other tissues, are subjected from the resistance ordinarily opposed to their contraction. When no resistance is offered, as when a muscle is cut off from its tendon, not only is no hardness perceived during centraction, but the muscular tissue is even softer, more extensile, and less elastic than in its ordinary uncontracted state. During contraction in each fibre it is said that the anisotropous 
or doubly refractive elements become less refractive and the singly refractive more so (tig. 329).

(4.) Chemical Changes.- $(a)$ The reaction of the muscle which is normally alkaline or neutral becomes decidedly acid, from the development of sarcolactic acid. (b) The muscle gives out carbonic acid gas and takes up oxygen, the amount of the $\mathrm{CO}_{2}$ given out not appearing to be entirely dependent upon the 0 taken in, and so doubtless in part arising from some other source. $(c)$ Certain imperfectly understood chemical changes occur, in all probability connected with $(a)$ and $(b)$. Glycogen is diminisher, and glucose, or muscle sugar (inosite) appears; the extractives are increased.

(5.) Electrical Changes. - When a muscle contracts the natural muscle current or currents of rest undergo a distinct diminution, which is due to the appearance in the actively contracting muscle of currents in an opposite direction to those existing in the muscle at rest. This causes a temporary deflection of the needle of a galvanometer in a direction opposite to the original current, and is called by some the negative variation of the muscle current, and by others a current of action.
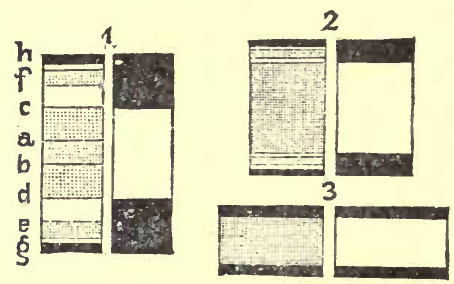

Fig. 329.-The microscopic appearances during a muscular contraction in the individual fibrillæ, after Engelmann. 1. A passive muscle-fibre; $\mathrm{c}$ to $\mathrm{d}=$ doubly refractive discs, with median dise $\mathrm{a} b$ in it; $\mathrm{h}$ and $\mathrm{g}$ are lateral dises; $\mathrm{f}$ and e are secondary dises, only slightly doubly refractive; fig. on right same fibre in polarized light; bright part is doubly refracted, black ends not so. 2. Transition stage; and 3 . Stage of entire contraction: in each case the right-hand figure represents the effect of polarized light. (Landois after Engelmann.)

\section{Conditions which Affect the Characters of the Contraction.} -In addition to the factors already considered which influence the irritability of muscle as such, these and others may affect the characters of its contraction and hence the curve produced.

Effect of Load. - Within certain limits a muscle contracts more powerfully when acting against resistance-that is, when it is loaded. Beyond this point of maximum contraction, however, increase of load diminishes the height and duration of contraction and increases the length of the latent period.

Effect of Fatrgue.-As already stated, exercise increases the strength of muscles, so that the first effect of contraction is to increase the height of the curve; but if the stimulation be kept up and the muscle be made to contract frequently, both the height and form of the curve are altered. The latent period is lengthened, the height of the curve is lessened, and 
the duration of the contraction is much prolonged. Later a condition is reached in which the muscle remains more or less contracted for a considerable time. This condition is called contracture.

Effect of Temperature.-Heat up to a certain point increases the irritability of muscle and favors rapidity in chemical activity, with the result that when it contracts the latent period is shortened, the height of the wave is increased, and the duration of the contraction is lessened. Cold produces contrary effects.

Effect of Drugs. - Veratrine does not alter the rapidity with which contraction occurs, but enormously prolongs the stage of relaxation. The salts of barium act similarly, and to a less extent those of calcium and strontium. In this connection it is interesting to recall that suprarenal extract acts likewise on voluntary muscles.

Effect of Strength of Stimulus. - A strength of current that is just sufficient to give a contraction is called a minimal stimulus. As the strength of the current is increased, the height of the contraction curve increases until the maximal stimulus is reached, beyond which no increase occurs. The latent period shortens with increased strength of stimulus.

Effect of Rate of Stimulation.-If we stimulate the nerve-muscle preparation with two induction shocks, one immediately after the other, when the point of stimulation of the second one corresponds to the maximum of the first, a second curve (fig. 330) will occur, which will

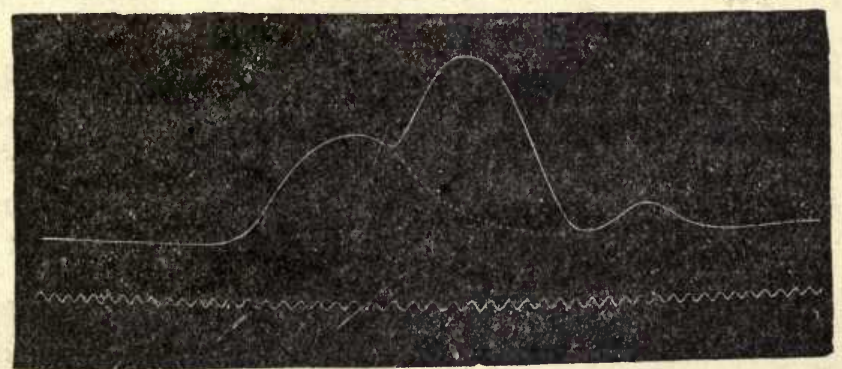

Fin the muscle was engaged in the first contraction (whose complete course, had nothing intervened, is muscle was engaged in the first contraction (whose complete course, ha at such a time that the indicated by the dotted line), a second ind was beginning to decline. The second curve is seen to start from the first, as does the first from the base line. (M. Foster.)

commence at the highest point of the first and will rise nearly as high, so that the sum of the height of the two curves almost exactly equals twice the height of the first. If a third and fourth shock be passed, a similar effect will ensue, and curves one above the other will be traced, the third being slightly lower than the second, and the fourth than the third. If a more numerous series of shocks occur, however, the lever 
after a time ceases to rise any further, and the contraction, which has reached its maximum, is maintained. The condition which ensures is called Tetanus. A tetanus is really a summation of contractions, but unless the stimuli become very rapid indeed, the muscle will still be in a condition of vibratory contraction and not of unvarying contraction.

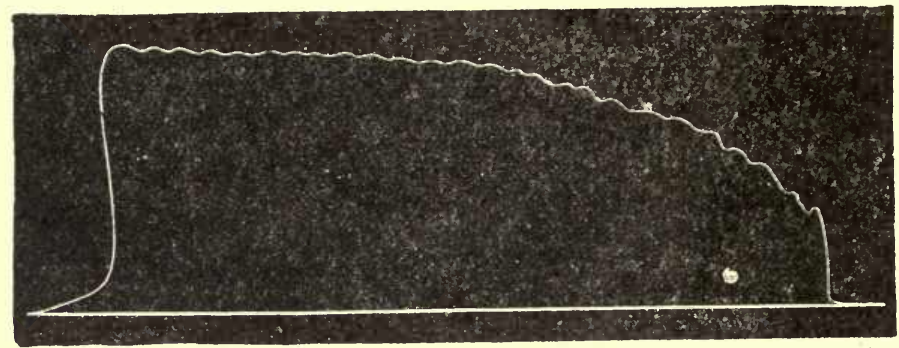

Fig. 331.-Curve of tetanus, obtained from the gastrocnemius of a frog, where the shocks were sent in from an induction coil, about sixteen times a second, by the interruption of the primary current by means of a vibrating spring, which dipped into a cup of mercury, and broke the primary current at each vibration.

If the shocks, howerer, be repeated at rery short intervals, being 15 per second for the frog's muscle, but rarying in each animal, the muscle contracts to its utmost suddenly and continues at its maximum contraction for some time and the lever rises almost perpendicularly, and then describes a straight line (fig. 332). If the stimuli are not quite so rapid the line of maximum contraction becomes somewhat wary, indicating a slight tendency of the muscle to relax during the intervals between the stimuli (fig. 331).

Muscular Work.-We have seen that work is estimated by multiplying the weight raised, by the height through which it has been lifted. It has been found that in order to obtain the maximum of work a muscle must be moderately loaded: if the weight is increased beyond a certain point, however, the muscle becomes strained and raises it through

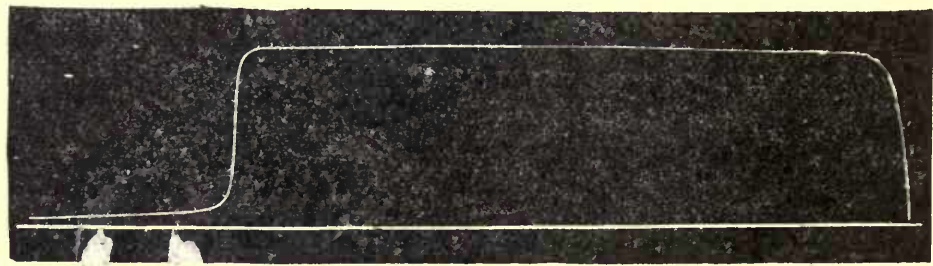

Fig. 332.-Curve of : canus, from a series of very rapid shocks from a magnetic interrupter.

so small a distance that less work is accomplished. If the load is still further increased, the muscle is completely overtaxed and cannot raise the weight. No work is then done at all. Practical illustrations of these facts must be familiar to every one. 
The power of a muscle is usually measured by the maximum weight which it will support without stretching. In man this is readily determined by weighting the body to such an extent that it can no longer be raised on tiptoe: thus the power of the calf-muscles is determined. The power of a muscle thus estimated depends of course upon its cross-section. The power of a human muscle is from two to three times as great as a frog's muscle of the same sectional area.

Fatigue of Muscle.-A muscle becomes rapidly exhausted from repeated stimulation, and the more rapidly, the more quickly the induction-shocks succeed each other. This is indicated by the diminished height of the muscular contractions.

A fatigued muscle has a much longer latent period than a fresh one. The slowness with which muscles respond to the will when fatigued must be familiar to every one.

In a muscle which is exhausted, stimulation only causes a contraction producing a local bulging near the point irritated. A similar effect may be produced in a fresh muscle by a sharp blow, as in striking the biceps smartly with the edge of the hand, when a hard muscular swelling is instantly formed.

As we have seen in discussing the irritability of muscle, the cause of fatigue is twofold, being in part due to its nutritive condition, and in part to the accumulation of poisonous products formed during contraction-probably sarcolactic acid, chiefly. In a living animal these poisowous products exert their influence not only upon the muscle or muscles immediately concerned in contraction, but upon the musculature of the body generally, and the effect remains until they are eliminated from the body. Massage of the muscles increases the passage of them in to the general blood-stream and the rapidity of their elimination.

Under normal circumstances muscles do not become completely fatigued, for the reason that the nerve cells which send out the impulses for contraction become fatigued sooner than the muscles thernselves do. Nerve cells, however, recover from fatigue more quickly than muscles. These facts are sometimes shown when one feels utterly exhausted and scarcely able to drag one foot after another, yet under a strong effort of will, as from fright, is able to make unwonted effort.

Response to Stimuli in Voluntary and Involuntary Muscles. - The two kinds of fibres, the striped and the unstriped, hav? characteristic differences in the mode in which they act on the apnlication of the same stimulus; differences which may be ascribed $a g_{1}$ it part to their respective differences of structure, but in some di, iee, possibly, to their respective modes of connection with the nervous system. When irritation is applied directly to a muscle with striated fibres, or to the motor nerve supplying it, contraction of the part irritated, and of that only, ensues; and this contraction is instantaneons, and ceases on the instant of withdrawing the irritation. But when any part with unstriped muscular fibres, e.g., the intestines or bladder, is irritated, the subse- 
quent contraction ensues more slowly, extends beyond the part irritated, and, with alternating relaxation, continues for some time after the withdrawal of the irritation. The difference in the modes of contraction of the two kinds of muscular fibres may be particularly illustrated by the effects of the repeated stimuli with the magnetic interrupter.

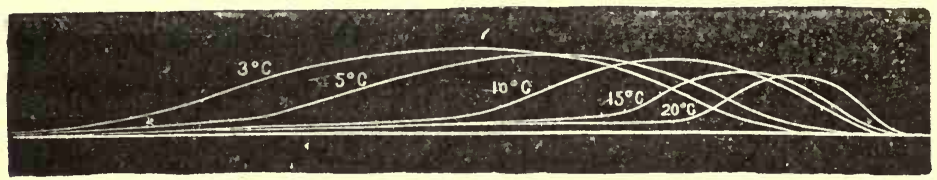

Fig. 333.-Muscle-curves from the gastrocnemius of a frog, illustrating effects of alterations in temperature.

The rapidly succeeding shocks given by this means to the nerres of muscles excite in all the transversely striated muscles, except in the case of the heart, a fixed state of tetanic contraction as previously described, which lasts as long as the stimulus is continued, and on its withdrawal instantly ceases; but in the muscles with unstriped fibres they excite a slow vermicular movement, which is comparatively slight and alternates with rest. It continues for a time after the stimulus is withdrawn.

In their mode of responding to these stimuli, all the skeletal muscles, or those with transverse striæ, are alike; but among those with unstriped fibres there are many differences-a fact which tends to confirm the opinion that their peculiarity depends as well on their connection with nerres and ganglia as on their own properties. The ureters and gall-bladder are the parts least excited by stimuli ; they do not act at all till the stimulus has been long applied, and then contract feebly, and to a small extent. The contractions of the cæcum and stomach are quicker and wider spread: still quicker those of the iris, and of the urinary bladder if it be not too full. The actions of the small and large intestines, of the ras deferens, and pregnant uterus, are yet more vivid, more regular, and more sustained; and they require no more stimulus than that of the air to excite them. The heart, on account, doubtless, of its striated muscle, is the quickest and most vigorous of all the muscles of organic life in contracting upon irritation, and appears in this, as in nearly all other respects, to be the connecting member of the two classes of muscles.

All the muscles retain their property of contracting under the influence of stimuli applied to them or to their nerves for some time after death. the period being longer in cold-blooded than in warm-blooded Vertebrata, and shorter in Birds than in Mammalia. It would seem as if the more active the respiratory process in the living animal, the shorter is the time of duration of the irritability in the muscles after death; and this is confirmed by the comparison of different species in the same order of Vertebrata. But the period during which this irritability lasts is not the same in all persons, nor in all the muscles of the same person. In a man it ceases, according to Nysten, in the following order:-first in the left ventricle, then in the intestines and stomach, the urinary bladder, right ventricle, œesophagus, iris ; then in the voluntary muscles of the trunk, lower and upper extremities; lastly, in tha right and left auricle of the heart. 


\section{Muscle in Rigor Mortis.}

After the muscles of the dead body have lost their irritability or capa bility of being excited to contraction by the application of a stimulus, they spontaneously pass into a state of contraction, apparently identical with that which ensues during life. It affects all the muscles of the body; and, when external circumstances do not prevent it, commonly fixes the limbs in that which is their natural posture of equilibrium or rest. Hence, and from the simultaneous contraction of all the muscies of the trunk, is produced a general stiffening of the body, constituting the rigor mortis or post-mortem rigidity.

When this condition has set in, the muscle $(a)$ becomes acid in reaction (due to development of sarcolactic acid), (b) gives off carbonic acid in great excess, $(c)$ diminishes in volume slightly, $(d)$ becomes shortened and opaque, its substance setting firm. Rigor comes on much more rapidly after muscular activity, and is hastened by warmth. It may be brought on, in muscles exposed for experiment, by the action of distilled water and many acids, also by freezing and thawing.

Cause. -The immediate cause of rigor seems to be a chemical one, namely, the coagulation of the muscle plasma. We may distinguish three main stages-(1.) Gradual coagulation. (2.) Corstraction of coagulated muscle-clot (myosin), and squeezing out of muscle-serum. Putrefaction. After the first stage, restoration is possible through the circulation of arterial blood through the muscles, and even when the second stage has set in, vitality may ke restored by dissolving the coagulum of the muscle in salt solution, and passing arterial blood through the vessels. In the third stage recovery is impossible.

It has been noticed that the relaxation in muscles after rigor sometimes occurs too quickly to be caused by putrefaction, anc the suggestion that in such cases at any rate such relaxation is due to a ferment-actior. is very plausible. It is known that pepsin is present in muscles, and that this ferment will act in an acid medium. The conditions for the solution of the coagulated myosin are therefore present as the reaction of rigored muscle is acid.

Order of Occurrence. - The muscles are not affected simultaneously by rigor mortis. It affects the neck and lower jaw first; next, the upper extremities, extending from above downward; and lastly, reaches the lower limbs; in some rare instances only, it affects the lower extremities before, or simultaneously with, the upper extremities. It usually ceases in the order in which it begins: first at the head, then in the upper extremities, and lastly in the lower extremities. It never commences earlier than ten minutes, and never later than seven hours after death; and its duration is greater in proportion to the lateness of its accession. 
Heat is developed during the passage of a muscular fibre into the condition of rigor mortis.

Since rigidity does not ensue until muscles have lost the capacity of being excited by external stimuli, it follows that all circumstances which cause a speedy exhaustion of muscular irritability, induce an early occurrence of the rigidity, while conditions by which the disappearance of the irritability is delayed, are succeeded by a tardy onset of this rigidity. Hence its speedy occurrence, and equally speedy departure in the bodies of persons exhausted by chronic diseases; and its tardy onset and long continuance after sudden death from acute diseases. In some cases of sudden death from lightning, violent injuries, or paroxysms of passion, rigor mortis has been said not to occur at all; but this is not always the case. It may, indeed, be doubted whether there is really a complete absence of the post-mortem rigidity in any such cases; for the experiments of Brown-Séquard make it probable that the rigidity may supervene immediately after death, and then pass away with such rapidity as to be scarcely observable.

The occurrence of rigor mortis is not prevented by the previous existence of paralysis in a part, provided the paralysis has not been attended with very imperfect nutrition of the muscular tissue.

The rigidity affects the involuntary as well as the voluntary muscles, whether they be constructed of striped or unstriped fibres. The rigidity of involuntary muscles with striped fibres is shown in the contraction of the heart after death. The contraction of the muscles with unstriped fibres is shown by an experiment of Valentin, who found that if a graduated tube connected with a portion of intestine taken from a recentlykilled animal, be filled with water, and tied at the opposite end, the water will in a few hours rise to a considerable height in the tube, owing to the contraction of the intestinal walls. It is still better shown in the arteries, of which all that have muscular coats contract after death, and thus present the roundness and cord-like feel of the arteries of a limb lately removed, or those of a body recently dead. Subsequently they relax, as do all the other muscles, and feel lax and flabby, and lie as if flattened, and with their walls nearly in contact.

\section{Action of the Voluntary Muscles.}

The greater part of the voluntary muscles of the body act as sources of power for moving levers, - the latter consisting of the various bones to which the muscles are attached.

Examples of the three orders of levers in the Human Body.-All levers have been divided into three kinds, according to the relative position of the power. the weight to be removed, and the axis of motion or fulcrum. In a lever of the first kind the power is at one extremity of the lever, the weight at the other, 
and the fulcrum between the two. If the initial letters only of the power, weight, and fulcrum be used, the arrangement will stand thus:-P. F. W. A poker as ordinarily used, or the bar in fig. 334, may be cited as an example of this variety of lever; while, as an instance in which the bones of the human

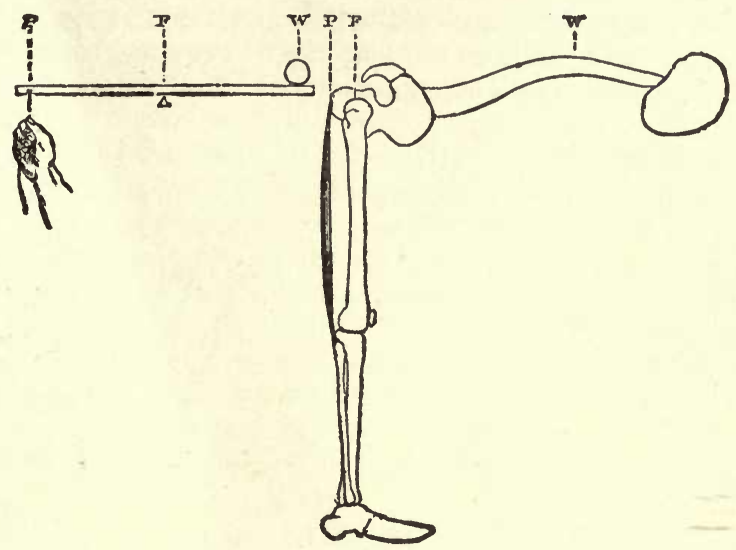

Fig. 334 .

skeleton are used as a lever of the same kind, may be mentioned the act of raising the body from the stooping posture by means of the hamstring muscles attached to the tuberosity of the ischium (fig. 334).

In a lever of the second kind, the arrangement is thus:-P. W. F. ; and this leverage is employed in the act of raising the handles of a wheelbarrow, or in stretching an elastic band, as in fig. 335. In the human hody the act of opening the mouth by depressing the lower jaw is an example of the same kind-
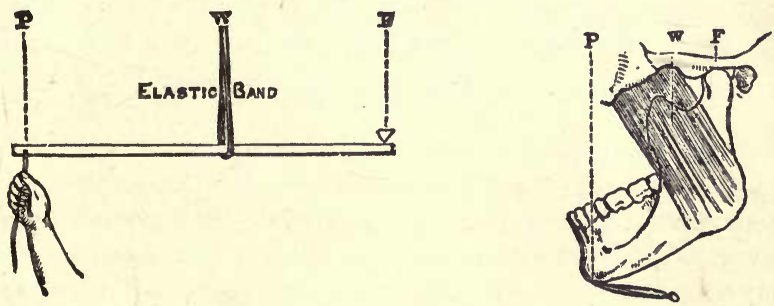

Fig. 335.

the tension of the muscles which close the jaw representing the weight (fig. 335).

In a lever of the third kind the arrangement is-F. P. W., and the act of raising a pole, as in fig. 336, is an example. In the human body there are numerous examples of the employment of this kind of leverage. The act of bending the fore arm may be mentioned as an instance (fig. 336). The act of biting is another example.

At the ankle we have examples of all three kinds of lever. 1st kind-Extending the foot. $3 \mathrm{~d}$ kind-Flexing the foot. In both these cases the foot represents the weight: the ankle joint the fulcrum, the power being the calf muscles in the first case and the tibialis anticus in the second case. $2 d$ kind- 
When the body is raised on tiptoe. Here the ground is the fulcrum, the weight of the body acting at the ankle joint the weight, and the calf muscles the power.

In the human body, levers are most frequently used at a disadvantage as regards power, the latter being sacrificed for the sake of a greater range of motion. Thus in the diagrams of the first and third kinds it is evident that the power is so close to the fulcrum, that great force must be exercised in order to produce motion. It is also evident. however, from the same diagrams, that
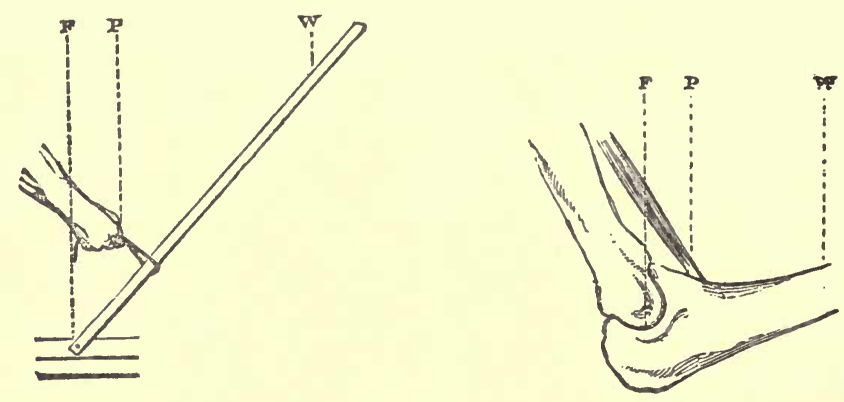

Fig. 338 .

by the closeness of the power to the fulcrum a great range of morement can be obtained by means of a comparatively slight shortening of the muscular fibres.

The greater number of the more important muscular actions of the human body-those, namely, which are arranged harmoniously so as to subserve some definite purpose or other in the animal economy-are describer in rarious parts of this work, in the sections which treat of the physiology of the processes by which these muscular actions are resisted or carried out. 'There are, however, one or two very important and somewhat complicated muscular acts which may be best described in this place.

Walking.-In the act of walking, almost erery roluntary muscle in the body is brought into play, either directly for purposes of progression, or indirectly for the proper balancing of the head and trunk. The muscles of the arms are least concerned; but eren these are for the most part instinctively in action to some extent.

Among the chief muscles engaged directly in the act of walking are those of the calf, which, by pulling up the heel, pull up also the astragalus, and with it, of course, the whole body, the weight of which is transmitted through the tibia to this bone (fig. 337). When starting to walk, say with the left leg, this raising of the body is not left entirely to the muscles of the left calf, but the trunk is thrown forward in such a way, that it would fall prostrate were it not that the right foot is brought forward and planted on the ground to support it. Thus the muscles of the left calf are assisted in their action by those muscles on the front of the trunk and legs which, by their contraction, pull the body forward; and, of course, if the trunk form a slanting line, with the inclination forward, it is plain that when the heel is raised by the calf-muscles, 
the whole body will be raised, and pushed obliquely forward and upward. The successive acts in taking the first step in walking are represented in fig. $337,1,2,3$.

Now it is evident that by the time the body has assumed the position No. 3 , it is time that the right leg should be brought forward to support it and prevent it from falling prostrate. This advance of the other leg (in this case the right) is effected partly by its mechanically swinging forward, pendulumwise, and partly by muscular action; the muscles used being-1st, those on the front of the thigh, which bend the thigh forward on the pelvis, especially the rectus femoris, with the psoas and the iliacus ; 2dly, the hamstring muscles, which slightly bend the leg on the thigh; and, $3 d l y$, the muscles on the front of the leg, which raise the front of the foot and toes, and so prevent the latter in swinging forward from hitching in the ground.

The second part of the act of walking, which has been just described, is shown in the diagram (4, fig. 337).

When the right foot has reached the ground the action of the left leg has not ceased. The calf-muscles of the latter continue to act, and by pulling $u_{p}$ the heel, throw the body still more forward over the right leg, now bearing nearly the whole weight, until it is time that in its turn the left leg should swing forward, and the left foot be planted on the ground to prevent the body from falling prostrate. As at first, while the calf-muscles of one leg and foot are preparing, so to speak, to push the body forward and upward from behind by raising the heel, the muscles on the front of the trunk and the same leg (and of the other leg, except when it is swinging forward) are helping the

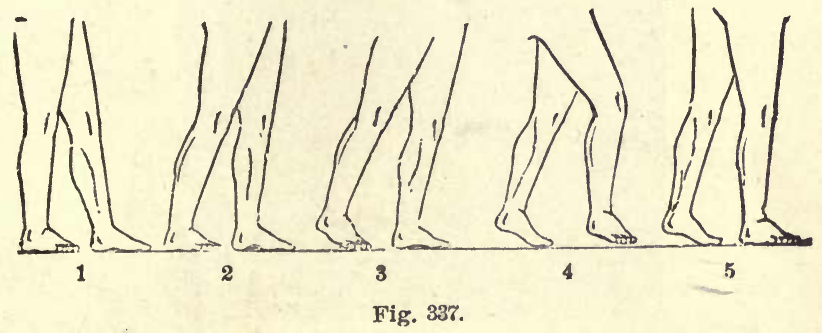

act by pulling the legs and trunk, so as to make them incline forward, the rotation in the inclining forward being effected mainly at the ankle joint. Two main kinds of leverage are, therefore, employed in the act of walking, and if this idea be firmly grasped, the details will be understood with comparative ease. One kind of leverage employed in walking is essentially the same with that employed in pulling forward the pole, as in fig. 336. And the other, less exactly, is that employed in raising the handles of a wheelbarrow. Now, supposing the lower end of the pole to be placed in the barrow, we should have a very rough and inelegant, but not altogether bad representation of the two main levers employed in the act of walking. The body is pulled forward by the muscles in front. minh in the same way that the pole might be by the force applied at P., while the raising of the heel and pushing forward of the trunk by the calf-muscles is roughly represented on raising the handles of the barrow. The manner in which these actions are performed alternately by each leg, so that one after the other is swung forward to support the trunk, which is at the same time pushed and pulled forward by the muscles of the other, may be gathered from the the previous description. 
There is one more thing to be especially noticed in the act of walking. Inasmuch as the body is being constantly supported and balanced on each leg alternately, and therefore on only ove at the same moment, it is erident that there must be some provision made for throwing the centre of gravity over the line of support formed by the bones of each leg, as, in its turn, it supports the weight of the body. This may be done in various ways, and the manner in which it is effected is one element in the differences which exist in the walking of different people. Thus it may be done by an instinctive slight rotation of the pelvis on the head of each femur in turn, in such a manner that the centre of gravity of the body shall fall over the foot of this side. Thus when the body ' pushed onward and upward by the raising, say, of the right heel, as in fig. 337,3 , the pelvis is instinctively by various muscles made to rotate on the itead of the left femur at the acetabulum, to the left side, so that the weight may fall over the line of support formed by the left leg at the time that the

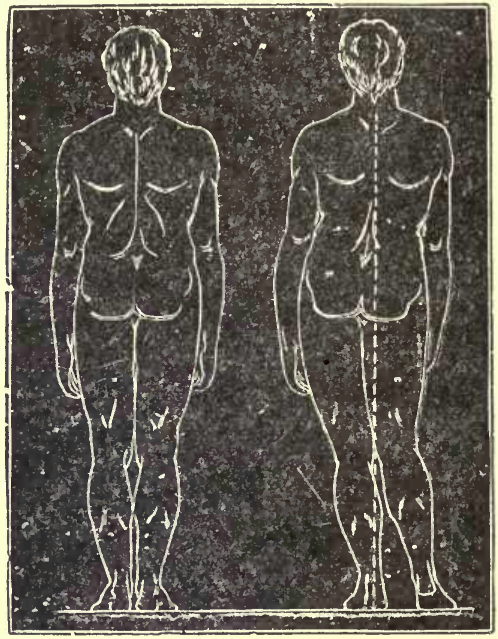

Fig. 338.

right leg is swinging forward, and leaving all the work of support to fall on its fellow. Such a "rocking" movement of the trunk and pelvis, however, is accompanied by a movement of the whole trunk and leg over the foot which is being planted on the ground (fig. 338) : the action being accompanied with a compensatory outward morement at the hip, more easily appreciated by looking at the figure (in which this movement is shown exaggerated) than described.

Thus the body in walking is continually rising and swaying alternately from one side to the other, as its centre of gravity has to be brought alternately over one or other leg; and the curvatures of the spine are altered in correspondence with the varying position of the weight which it has to support. The extent to which the body is raised or swayed differs much in different people.

In walking, one foot or the other is always on the ground. The act of leaping or jumping, consists in so sudden a raising of the heels by the sharp and strong contraction of the calf-muscles, that the body is jerked off the ground. At the same time the effect is much increased by first bending the thighs on the pel- 
vis, and the legs on the thighs, and then suddenly straightening out the angles thus formed. The share which this action has in producing the effect may be easily known by attempting to leap in the upright posture, with the legs quite straight.

Running is performed by a series of rapid low jumps with each leg alternately : so that, during each complete muscular act concerned, there is a moment when both feet are off the ground.

In all these cases, however, the description of the manner in which any given effect is produced, can give but a very imperfect idea of the infinite number of combined and harmoniously arranged muscular contractions which are necessary for even the simplest acts of locomotion.

Action of the Involuntary Muscles.-The involuntary muscles are for the most part not attached to bones arranged to act as levers, but enter into the formation of such hollow parts as require a diminution of their calibre by muscular action, under particular circumstances. Examples of this action are to be found in the intestines, urinary bladder, heart and blood-vessels, gall-bladder, gland-ducts, etc.

The difference in the manner of contraction of the striated and nonstriated fibres has been already referred to (p. 529); and the peculiar vermicular or peristaltic action of the latter fibres has also been described.

\section{Electrical Currents in Nerves.}

The electrical condition of nerves is so closely connected with the phenomena of muscular contraction, that it will be convenient to consider it in the present chapter.

If a piece of nerve be removed from the body and subjected to examination in a way similar to that adopted in the case of muscle, which has been described, electrical currents are found to exist which correspond exactly to the natural muscle currents, and which are called natural nerve currents or currents of rest, according as one or other theory of their existence be adopted, as in the case with muscle. One point (equator) on the surface being positive to all other points nearer to the cut ends, and the greatest deflection of the needle of the galvanometer taking place when one electrode is applied to the equator and the other to the centre of either cut end. As in the case of muscle, these nerve currents undergo a negative variation when the nerve is stimulated, the variation being momentary and in the opposite direction to the natural currents; and are similarly known as the currents of action. The currents of action are propagated in both directions from the point of the application of the stimulus, and are of momentary duration.

Rheoscopic Frog.-This negative variation may be demonstrated by means of the following experiment. The new current produced by stimulating the nerve of one nerve-muscle preparation may be used to stimulate the nerve of a second nerve-muscle preparation. The foreleg of a frog with the nerve going to the 
gastrocnemius cut long is placed upon a glass plate, and arranged in such way that its nerve touches in two places the sciatic nerve, exposed but preserver in situ in the opposite thigh of the frog. The electrodes from an induction coil are placed behind the sciatic nerve of the second preparation, high up. On stimulating it with a single induction shock, the muscles not only of the same leg are found to undergo a twitch, but also those of the first preparation, although this is not near the electrodes, and so the stimulation cannot be due to an escape of the current into the first nerve. This experiment is known under the name of the rheoscopic frog.

Nerve-stimuli.-Nerve-fibres require to be stimulated before they can manifest any of their properties, since they have no power of themselves of generating force or of originating impulses. The stimuli which are capable of exciting nerves to action are, as in the case of muscle, very diverse. They are very similar in each case. The mechanical, chemical, thermal, and electric stimuli which may be used in the one case are also, with certain differences in the methods employed, efficacious in the other. The chemical stimuli are chiefly these: withdrawal of water, as by drying, strong solutions of neutral salts of potassium, sodium, etc., free inorganic acids, except phosphoric; some organic acids; ether, chloroform, and bile salts. The electrical stimuli employed are the induction and continuous currents concerning which the observations in reference to muscular contraction should be consulted. Weaker electrical stimuli will excite nerve than will excite muscle; the nerve stimuli appears to gain strength as it descends, and a weaker stimulus applied far from the muscle will have the same effect as a stronger one applied to the nerve near the muscle.

It will be only necessary here to add some account of the effect of a constant current, such as that obtained from a Daniell's battery, upon a nerve. This effect may be studied with the apparatus described before. A pair of electrodes is placed behind the nerve of the nerve-muscle preparation, with a Du Bois Reymond's key arranged for short circuiting the battery current, in such a way that when the key is opened the current is sent into the nerve, and when closed the current is cut off. It will be found that with a current of moderate strength there will be a contraction of the muscle both at the opening and at the closing of the key (called respectively making and breaking contractions), but that during the interval between these two events the muscle remains flaccid, provided the battery current continues of constant intensity. If the current be a very weak or a very strong one the effect is not quite the same; one or other of the contractions may be absent. Which of these contractions is absent depends upon another circumstance, viz., the direction of the current. The direction of the current may be ascending or descending: if ascending, the anode or positive pole is nearer the muscle than the cathode or negative pole, and the current to return to 
the battery has to pass up the nerve; if descending, the position of the electrodes is reversed. It will be necessary before considering this question further to return to the apparent want of effect of the constant current during the interval between the make and break contraction: to all appearances no change is produced, but in reality a very important alteration of the irritability is brought about in the nerve by the passage of this constant (polarizing) current. This may be shown in two ways, first of all by the galvanometer. If a piece of nerve be taken, and if at either end an arrangement be made to test the electrical condition of the nerve by means of a pair of non-polarizable electrodes connected with a galvanometer, while to the central portion a pair of electrodes connected with a Daniell's battery be applied, it will be found that the natural nerve-currents are profoundly altered on the passage of the constant current in the neighborhood. If the polarizing current be in the same direction as the latter the natural current is increased, but if in the direction opposite to it, the natural current is diminished. This change, produced by the continual passage of the battery-current through a portion of the nerve, is to be distinguished from the negative variation of the natural current to which allusion has been already made, and which is a momentary change occurring on the sudden application of the stimulus. The condition produced by the passage of a constant current is known by the name of Electrotonus.

A second way of showing the effect of the polarizing current is by taking a nerve-muscle preparation and applying to the nerve a pair of electrodes from an induction coil, while at a point further removed from the muscle, electrodes from a Daniell's battery are arranged with a key for short circuiting and an apparatus (reverser) by which the battery current may be reversed in direction. If the exact point be ascertained to which the secondary coil should be moved from the primary coil in order that a minimum contraction be obtained by the induction shock, and the secondary coil be removed slightly further from the primary, the induction current cannot now produce a contraction; but if the polarizing current be sent in a descending direction, that is to say, with the cathode nearest the other electrodes, the induction current, which was before insufficient, will prove sufficient to cause a contraction; whereby indicating that with a descending current the irritability of the nerve is increased. By means of a somewhat similar experiment it may be shown that an ascending current will diminish the irritability of a nerve. Similarly, if instead of applying the induction electrodes below the other electrodes they are applied between them, like effects are demonstrated, indicating that in the neighborhood of the cathode the irritability of the nerve is increased by the passage of a constant current, and in the neighborhood of the anode diminished. This in- 
crease in irritability is called katelectrotonus, and similarly the decrease is called anelectrotonus. As there is between the electrodes both an increase and a decrease of irritability on the passage of a polarizing current, it must be evident that the increase must shade off into the decrease, and that there must be a neutral point where there is neither increase nor decrease of irritability. The position of this neutral point is found to vary with the intensity of the polarizing current-when the current is weak the point is nearer the anode, when

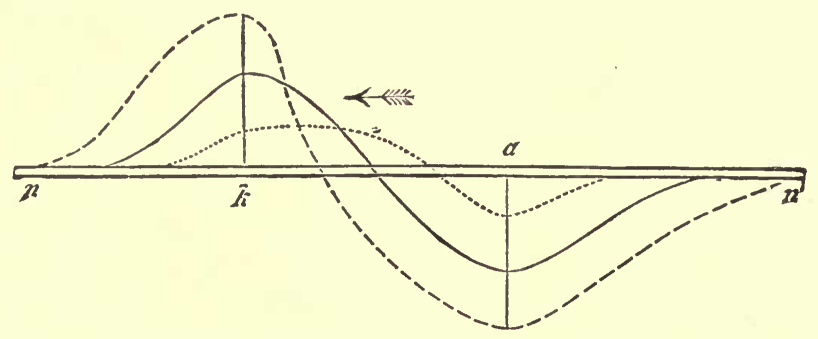

Fig. 339. -Diagram illustrating the effects of various intensities of the polarizing currents. $n$, $n^{\prime}$, nerve; $a$, anode: $k$. kathode; the curves above indicate increase, and those below decrease of irritability, and when the current is small the increase and decrease are both small, with the neutral point near $a$, and so on as the current is increased in strength.

strong nearer the kathode (fig. 339); when a constant current passes into a nerve, therefore, if a contraction result, it may be assumed that it is due to the increased irritability produced in the neighborhood of the kathode, but the breaking contraction must be produced by a rise in irritability from a lowered state to the normal in the neighborhood of the anode. The contractions produced in the muscle of a nerremuscle preparation by a constant current have been arranged in a table which is known as Pflüger's Law of Contractions. It is really only a statement as to when a contraction may be expected:-

\begin{tabular}{|c|c|c|c|c|}
\hline \multirow[t]{2}{*}{ - Strexgth of Current usfid. } & \multicolumn{2}{|c|}{ Descending Current. } & \multicolumn{2}{|c|}{ Ascending Current. } \\
\hline & Make. & Break. & Iake. & Break. \\
\hline 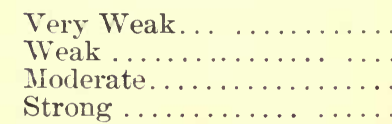 & $\begin{array}{l}\text { Yes. } \\
\text { Yes. } \\
\text { Yes. } \\
\text { Yes. }\end{array}$ & $\begin{array}{l}\text { No. } \\
\text { No. } \\
\text { Yes. } \\
\text { No. }\end{array}$ & $\begin{array}{l}\text { No. } \\
\text { Yes. } \\
\text { Yes. } \\
\text { No. }\end{array}$ & $\begin{array}{l}\text { No. } \\
\text { No. } \\
\text { Yes. } \\
\text { Yes. }\end{array}$ \\
\hline
\end{tabular}

The difficulty in this table is chiefly in the effect of a weak ascending current, but the following statement may remove it. The increase of irritability at the kathode when the current is made is more potent to produce a contraction than the rise of irritability at the anode when the current is broken; and so with weak currents the only effect is a contraction at the make of both currents. The descending current is more 
notent than the ascending (and with still weaker currents is the only one which produces any effect), since the kathode is near the muscle. In the case of the ascending current the stimulus has to pass through a district of diminished irritability, which with a very strong current acts as a block, being of considerable amount and extent, but with a weak current being less considerable both in intensity and extent, only slightly affects the contraction. As the current is stronger however, recovery from anelectrotonus is able to produce a contraction as well as katelectrotonus; a contraction occurs both at the make and the break of the current. The absence of contraction with a very strong current at the break of the ascending current may be explained by supposing that the region of fall in irritability at the kathode blocks the stimulus of the rise in irritability at the anode.

Thus we have seen that two circumstances influence the effect of the constant current upon a nerve, viz., the strength and direction of the current. It is also necessary that the stimulus should be applied suddenly and not gradually, and that the irritability of the nerve should be normal; not increased or diminished. Sometimes (when the preparation is specially irritable?) instead of a simple contraction a tetanus occurs at the make or break of the constant current. - This is especially liable to occur at the break of a strong ascending current which has been passing for some time into the preparation; this is called Ritter's tetanus, and may be increased by passing a current in an opposite direction or stopped by passing a current in the same direction.

\section{The Effect of Battery Currents on Normal Human Nerves.}

The following account is condensed from Lombard in "An American Text-book of Physiology."

As an electric current cannot be applied to living human nerves directly, it is applied to the skin along the course of the nerve. The current passes from the anode or positive pole through the skin, and spreads out in the tissues much as the bristles of a brush; it then gradually concentrates and leaves the skin at the kathode or negative pole.

In addition to the physical anode and kathode of the battery, there are what are called physiological anodes and kathodes. There is a physiological anode at every point where the current enters a nerve, and a physiological kathode at every point where it leaves it.

Generally when the current is applied to nerves through the skin, only part of it flows longitudinally along the nerves; most of it passes diagonally through them to the tissues below. Thus it happens that in that part of the nerve beneath either the physical anode or kathode, groups of physiological anodes and kathodes are found. 
The contraction which occurs when the current is closed (closing contraction) represents irritation at the physiological kathode, while the opening contraction represents irritation at the physiological anode.
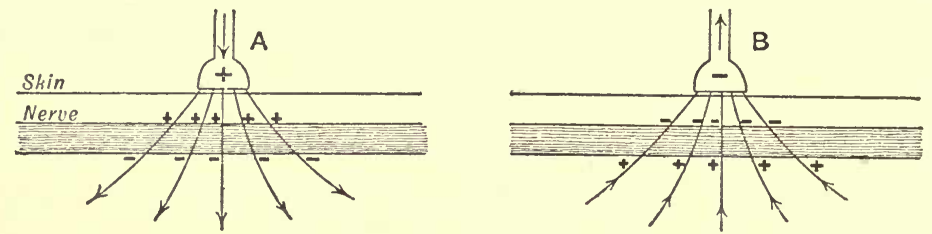

Fig. 340.-Diagram of skin and subjacent nerve. A, the positive electrode or physical anode; $B$, the negative electrode or physical kathode. Signs + , physiological anodes; signs - , phy siological kathodes. (After Waller.)

Since there are physiological anodes and kathodes beneath each electrode, one or more of four conditions may arise:

1. Anorlic closing contraction, i.e., the effect of the change developed at the physiological kathode, beneath the physical anode (positive pole).

2. Anodic opening contraction, i.e., the effect of the change dereloped at the physiological anode, beneath the physical anode (positive pole).

3. Kathodic closing contraction, i.e., the effect of the change developed at the physiological kathode, beneath the physical kathode (negative pole).

4. Kathodic opening contraction, i.e., the effect of the change developed at the physiological anode, beneath the physical kathode (negative pole).

The following abbreviations of these contractions are used: ACC, $\mathrm{AOC}, \mathrm{KCO}, \mathrm{KOC}$.

The closing contractions, $\mathrm{KCC}$ and $\mathrm{ACC}$, are stronger than the opening contractions, $\mathrm{KOC}$ and $\mathrm{AOC}$. Of the closing contractions, $\mathrm{KCC}$ is stronger than ACC. Of the opening contractions, AOC is stronger than KOC. These facts are also shown in a table of the effects of gradually increasing the strength of the current.

$\begin{array}{ccc}\text { Weak currents. } & \text { Medium currents. } & \text { strong currents. } \\ \mathrm{KCC} & \mathrm{KCC} & \mathrm{KCC} \\ - & \mathrm{ACC} & \mathrm{ACC} \\ - & \mathrm{AOC} & \mathrm{AOC} \\ - & - & \mathrm{KOC}\end{array}$

Sometimes AOC is stronger than ACC.

In diseases which cause degeneration of the nerres going to a muscle, stimulation causes results different from the above, and we get what is known as the reaction of degeneration. 


\section{Muscular and Nervous Metabolism.}

The question of the metabolism of muscle both in a resting and in an active condition has for many years occupied the attention of physiologists. It cannot be said even now to be thoroughly understood. Most of the facts with reference to the subject have been already mentioned. We may shortly recapitulate them here:-First, muscle during rest absorbs oxygen and gives out carbon dioxide. 'This has been shown by an analysis of the gases of the blood going to and leaving muscles. During activity, e.g., during tetanus, the same interchange of gases takes place: but the quantities of the oxygen absorbed and of the carbon dioxide given up are increased, and the proportion between them is altered thus:-

\begin{tabular}{|c|c|c|}
\hline Venous Blood. & $\begin{array}{l}\text { O, less than Arterial } \\
\text { Blood. }\end{array}$ & $\begin{array}{c}\mathrm{CO}_{2}, \text { more than Arterial } \\
\text { Blood. }\end{array}$ \\
\hline 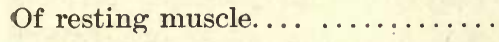 & 9 per cent. & 6.71 per cent. \\
\hline Of active muscle...$\ldots$. & 12.26 per cent. & 10.79 per cent. \\
\hline
\end{tabular}

There is then a greater proportion of carbon dioxide produced in muscle during activity than during rest.

During rigor mortis there is also an increased production of carbon dioxide.

Second, muscle during rest produces nitrogenous crystallizable substances, such as kreatin, from the metabolism which is constantly going on in it during life; in addition there is in all probability sarcolactic acid formed and other non-nitrogenous matters.

During activity the nitrogenous substances, such as kreatin, undergo very slight, if any, increase-about the amount produced during restbut the sarcolactic acid is distinctly increased; sugar (glucose) is also increased, whereas the glycogen is diminished.

During rigor mortis the sarcolactic acid is also increased, and in addition myosin is formed.

From these data it is assumed that the processes which take place in resting and active muscle are somewhat different, at any rate in degree. From actively contracting muscle, also, there are obtained an increased amount of heat and mechanical work, more potential is converted into kinetic energy.

Many theories have been proposed to explain the facts of muscular 
energy. It has been suggested by Herman that muscular activity depends upon the splitting up and subsequent re-formation of a complex nitrogenous body, called by him Inogen. When this body so splits up there result from its decomposition, carbon dioxide, sarcolactic acid, and a gelatino-albuminous body. Of these the carbon dioxide is carried away by the blood stream; the albuminous substance and possibly the acid, at any rate in part, go to re-form the inogen. The other materials of which the inogen is formed are supplied by the blood; of these materials we know that some carbohydrate substance and oxygen form a part. The decomposition, although taking place in resting muscle, reaches a climax in active muscle, but in that condition the destruction of inogen largely exceeds restoration, and so there must be a limit to muscular activity. But this is not the only change going on in muscle, there are others which affect the nitrogenous elements of the tissue, and from them result the nitrogenous bodies of which kreatin is the chief; these changes may be unusually large during severe exercise.

It has been further suggested that, as myosin is undoubtedly formed in rigor mortis, when the muscle becomes acid and gives off carbon dioxide, that myosin is also formed when muscle contracts, and that, in other words, contraction is a condition akin to partial death. The electrical reaction appears to justify this; both contracted and dead muscle are negative to living muscle, when at rest. What happens to the myosin which is formed when muscle contracts, if this view be the correct one, is unknown. Halliburton suggests that the myosin which can be made to clot and unclot easily enough outside the body, is able to do the same thing in the body. It is possible that the clotting of myosinogen which is supposed to occur during contraction, is not of the same intensity or extent as that which occurs post mortem. The relation of the hypothetical inogen to the rest of the muscle-fibre is undetermined. It may be that the inogen is formed by the activity of the muscle-protoplasm, and stored up within itself, and that during rest of muscle it is gradually used up, whereas in activity it is suddenly and explosively decomposed. In the rest of the fibre the nitrogenous metabolism continues much the same during rest as during activity.

Again, histologically, the question as to which is the contractile and which is the non-contractile part of muscle, has been, as we have seen (p. 86 et seq.), a matter of much controversy.

As regards nervous metabolism, we have little knowledge of anything except the electrical phenomena which have been already considered. For the maintenance of nervous irritability, oxygen is required; to form this, it has been suggested that the nervous impulse is the result of processes of an oxidative character, etc. The chief seat of the metabo- 
lism is no doubt the axis-cylinder. The question whether a nervous impulse is possibly an electrical change, as has been asserted by some, cannot be at present settled, but if it be so, at any rate it differs essentially from an ordinary current, if in no other respect, at any rate in the rate of transmission. 


\section{CHAPTER XV.}

\section{THE PRODUCTION OF THE VOICE}

BEFoRE commencing the consideration of the Nervous system and the Special Senses it will be convenient to consider first speech, the production of the human voice, and the physiology of the Larynx generally.

The Larynx. - In nearly all air-breathing vertebrate animals there are arrangements for the production of sound, or voice, in some parts of

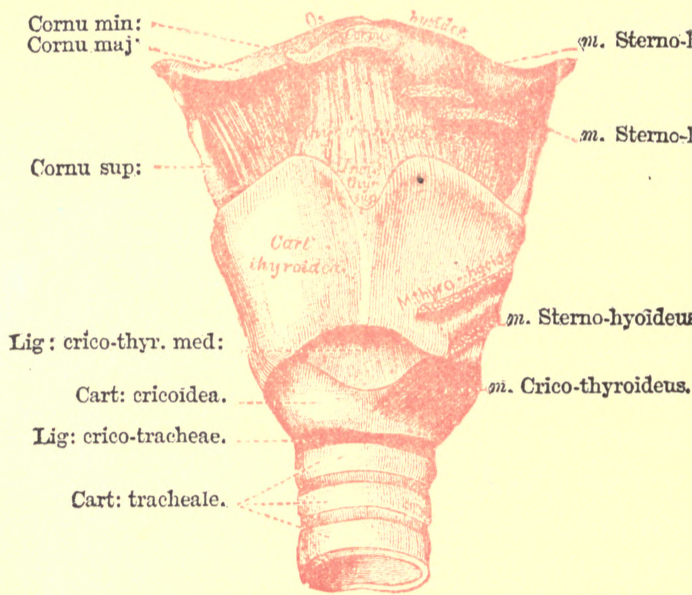

Fig. 341.-The Larynx, as seen from the front, showing the cartilages and ligaments. The muscles, with the exception of one crico-thyroid, are cut off short. (Stoerk.)

the respiratory apparatus. In many animals, the sound admits of being variously modified and altered during and after its production; and, in man, one such modification occurring in obedience to dictates of the cerebrum, is speech.

It has been proved by observations on living subjects, by means of the laryngoscope (p. 543), as well as by experiments on the larynx taken from the dead body, that the sound of the human voice is the result of the vibration of the inferior laryngeal ligaments, or the true vocal 
cords which bound the glottis, caused by currents of expired air impelled over their edges. If a free opening exists in the trachea, the sound of the voice ceases, but it returns if the opening is closed. An opening into the air-passages above the glottis, on the contrary, does not prevent the voice being produced. By forcing a current of air through the larynx in the dead subject, clear vocal sounds are elicited, though the epiglottis, the upper ligaments of the larynx or false vocal cords, the ventricles between them and the inferior ligaments or true vocal cords, and the upper part of the arytenoid cartilages, be all removed; provided the true vocal cords remain entire, with their points of attachment, and be kept tense and so approximated that the fissure of the glottis may be narrow.

The vocal ligaments or cords, therefore, are regarded as the proper organs for the production of rocal sounds: the modifications of these sounds being effected, as will be presently explained, by other parts, viz., by the tongue, teeth, lips, etc. The structure of the vocal cords is adapted to enable them to vibrate like tense membranes, for they are essentially composed of elastic tissue; and they are so attached to the cartilaginous parts of the larynx that their position and tension can be variously altered by the contraction of the muscles which act on these parts.

Thus it will be seen that the larynx is the organ of voice. It may be said to consist essentially of the two vocal cords and the various cartilaginous, muscular, and other apparatus by means of which not only can the aperture of the larynx (rima glottidis), of which they are the lateral boundaries, be closed against the entrance and exit of air to or from the lungs, but also by means of which the cords themselves can be stretched or relaxed, brought together and separated in accordance with the conditions that may be necessary for the air in passing over them, to set them vibrating to produce the various sounds. Their action in respiration has been already referred to.

Anatomy of the Larynx.-The principal parts entering into the formation of the larynx (figs. 342 and 343 ) are-the thyroid cartilage; the cricoid cartilage; the two arytenoid cartilages; and the two true vocal cords. The epiglottis (fig. 343), has but little to do with the voice, and is chiefly useful in protecting the upper part of the larynx from the entrance of food and drink in deglutition. It also probably guides mucus or other fluids in small amount from the mouth around the sides of the upper opening of the glottis into the pharynx and oesophagus: thus preventing them from entering the larynx. The false vocal cords and the ventricle of the larynx, which is a space between the false and the true cord of either side, need be here only referred to.

Cartilages. - (a) The thyroid cartilage (fig. 342,1 to 4 ) does not form a complete ring around the larynx, but only covers the front portion. (b) The cricoid cartilage (fig. $342,5,6$ ), on the other hand, is a complete ring; the 
back part of the ring being much broader than the front. On the top of this broad portion of the cricoid are (c) the arytenoid cartilages (fig. 342, 7 ), the connection between the cricoid below and arytenoid cartilages above being a joint with synovial membrane and ligaments, the latter permitting tolerably free
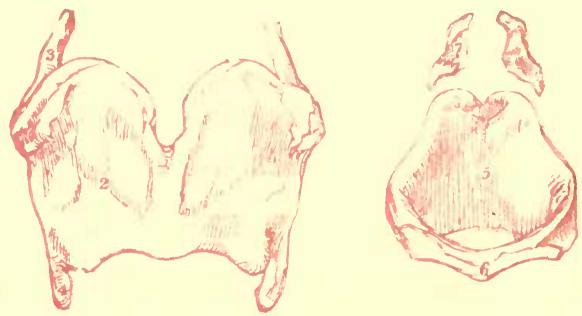

Fig. 342. - Cartilages of the larynx seen from the front. 1 to 4, thyroid cartilage; 1 , vertical ridge or pomum Adami; 2 , right ala ; 3 , superior, and 4, inferior cornu of the right side; 5,6 , cricoid cartilage; 5 , inside of the posterior part; 6 , anterior narrow part of the ring; 7 , arytenoid cartilages. $\times 3 / 4$.

motion between them. But although the arytenoid cartilages can move on the cricoid, they of course accompany the latter in all its movements, just as the head may nod or turn on the top of the spinal column, but must accompany it in all its morements as a whole.

Joints and Ligaments. - The thyroid cartilage is also connected with the cricoid, not only by ligaments, but also by joints with synovial membranes; the lower cornua of the thyroid clasping, or nipping, as it were, the cricoid between them, but not so tightly but that the thyroid can revolve, within a

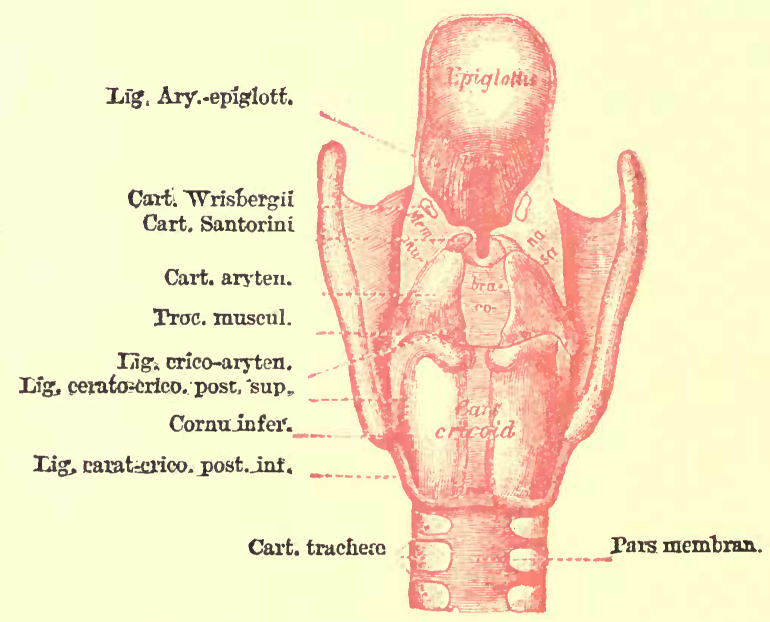

Fig. 343.-The larynx as seen from behind after removal of the muscles. The cartilages and ligaments only remain. (Stoerk.)

certain range, around an axis passing transversely through the two joints at which the cricoid is clasped. The vocal cords are attached (behind) to the front portion of the base of the arytenoid cartilages, and (in front) to the re-entering angle at the back part of the thyroid ; it is evident, therefore, that all 
movements of either of these cartilages must produce an effect on them of some kind or other. Inasmuch, too, as the arytenoid cartilages rest on the top of the back portion of the cricoid cartilage, and are connected with it by capsular and other ligaments, all movements of the cricoid cartilage must move the arytenoid cartilages, and also produce an effect on the vocal cords.

Intrinsic Muscles. - The intrinsic muscles of the larynx are so connected with the laryngeal cartilages that by their contraction alterations in the condition of the vocal cords and glottis are produced. They are usually divided into four classes according to their action, viz., into abductors, adductors, sphincters, and tensors. . The Abductors, the crico-arytenoidei, widen the glottis, by separating the cords; the Adductors, consisting of the thyro-ary-epiglottici, the arytenoideus posticus seu transversus, the thyro-arytenoidei externi, the cricoarytenoidei laterales, and the thyro-arytenoidei interni, approximate the vocal cords, dirninish the rima glottidis, and act generally as Sphincters and supporters of the glottis. Finally, the Tensors of the cords put the cords on the stretch, with or without elongating them; the tensors are the crico-thyroidei and the thyro-arytenoidei interni.

The attachments and the action of the muscles will be readily understood from the following table. All the muscles are in pairs except the arytenoideus posticus.

Table of the several Groups of the Intrinsic Muscles of the Larynx AND THEIR ATTACHMENTS.

\begin{tabular}{|c|c|c|}
\hline GROUP. & MUsclet. & AtTaCHMENTs. \\
\hline $\begin{array}{c}\text { I. } \\
\text { Abductors. }\end{array}$ & $\begin{array}{l}\text { Crico-aryte- } \\
\text { noidei pos- } \\
\text { tici. }\end{array}$ & $\begin{array}{l}\text { This pair of muscles arises, on } \\
\text { either side, from the posterior } \\
\text { surface of the corresponding half } \\
\text { of the cricoid cartilage. From } \\
\text { this depression their fibres con- } \\
\text { verge on either side upward and } \\
\text { outward to be inserted into the } \\
\text { outer angle of the base of the } \\
\text { arytenoid cartilages behind the } \\
\text { crico-arytenoid laterales. }\end{array}$ \\
\hline $\begin{array}{l}\text { II. and III. } \\
\text { Adductors } \\
\text { and } \\
\text { Sphincters. }\end{array}$ & $\begin{array}{l}\text { In three lay- } \\
\text { ers: } \\
\text { (a) Outer } \\
\text { layer, Thy- } \\
\text { ro-ar y - } \\
\text { ep i g lot- } \\
\text { tici. }\end{array}$ & $\begin{array}{l}\text { A pair of muscles. Flat and nar- } \\
\text { row, which arise on either side } \\
\text { from the processus muscularis of } \\
\text { the arytenoid cartilage, then pass- } \\
\text { ing upward and inward cross } \\
\text { one another in the middle line to } \\
\text { be inserted into the upper half of } \\
\text { the lateral border of the opposite } \\
\text { arytenoid cartilage and the poste- } \\
\text { rior border of the cartilage of } \\
\text { Santorini. The lower fibres run } \\
\text { forward and downward to be } \\
\text { inserted into the thyroid carti- } \\
\text { lage near the commissure. The } \\
\text { fibres attached to the cartilage of } \\
\text { Santorini are continued forward } \\
\text { and upward into the ary-epiglot- } \\
\text { tic fold. }\end{array}$ \\
\hline
\end{tabular}

ACtion.

Draw inward and backward the outer angle of arytenoid cartilages, and so rotate outward the processus vocalis and widen the glottis.

Help to narrow or close the rima glottidis. 


\begin{tabular}{|c|c|c|c|}
\hline Group. & Muscle. & AтTACHMENTS. & Action. \\
\hline
\end{tabular}

II. and III. Adductors and

Sphincters. -continued.

(b) Middle A single muscle. Half-q u a d r i-Draws together the layer. i. Aryteno id e us posticus. lateral, attached to the borders of the arytenoid cartilages, its fibres running horizontally between the two.

ii. Thyro-A pair of muscles. Each of which aryteno i - consists of three chief portions d e i ex- -lower, middle, and upper. terni.

iii. Crico-A aryteno i dei laterales.

(c) Inner-A most lay er, Thyroa r ytenoi. d e i in terni.
The lower and principal fibres may be further divided into two layers, internal and external. These fibres arise side by side from the lower half of the internal surface of the thyroid cartilage, close to the angle, and from the fibrous expansion of the cricothyroid ligament, and are inserted into the lateral border of the arytenoid cartilage. The inner fibres run horizontally, to be attached to the lower half of this border, and the outer fibres pass obliquely outward to be inserted into the upper half, while some pass to the cartilage of Wrisburg and the ary-epiglottic fold.

A pair of muscles.

They arise on Approximate the eith er side from the middle third of the upper border of the cricoid cartilage and are inserted into the whole anterior margin of the base of the arytenoid cartilage. Some of their fibres join the thyroidary-epiglottici.

A pair of muscles. They arise on $R$ either side, internally from the angle of the thyroid cartilage, internal to the last described muscle ( (b), iii.), and running parallel to and in the substance of the rocal cords are attached posteriorly to the processus rocalis along their whole length and to the adjacent part of the outer surface of the arytenoid cartilages. arytenoid cartilages and also depresses $\mathrm{them}$. When the nuscle is paralyzed, the inter-cartilaginous part of the cords cannot come together.

vocal cords by drawing the processus muscularis of the arytenoid cartilages forward and downward and so rotate the processus rocalis inward.

Render the rocal cords tense and rotate the arytenoid cartilages and approximate the processus vocalis. 


\begin{tabular}{|c|c|c|c|}
\hline Group. & Muscle. & Attachments. & Action. \\
\hline $\begin{array}{l}\text { IV. } \\
\text { Tensors. }\end{array}$ & $\begin{array}{l}\text { Crico - th y- } \\
\text { roidei. }\end{array}$ & $\begin{array}{l}\text { A pair of fan-shaped muscles at- } \\
\text { tached on either side to the cricoid } \\
\text { cartilage below ; from the mesial } \\
\text { line in front for nearly one-half of } \\
\text { its lateral circumference back- } \\
\text { ward the fibres pass upward and } \\
\text { outward to be attached to the low- } \\
\text { er border of the thyroid cartilage } \\
\text { and to the front border of its } \\
\text { lower cornea. }\end{array}$ & $\begin{array}{l}\text { The thyroid carti- } \\
\text { lage being fixed } \\
\text { by its extrinsic } \\
\text { m u c les, the } \\
\text { front of the cri- } \\
\text { coid cartilage is } \\
\text { drawn upward, } \\
\text { and its back, } \\
\text { with the aryte- } \\
\text { noids attached, } \\
\text { is drawn down. } \\
\text { Hence the vocal } \\
\text { cords are elon- } \\
\text { gated a } \mathrm{n} \text { e ro- } \\
\text { posteriorly and } \\
\text { put upon the } \\
\text { stretch. Paral- } \\
\text { ysis of these } \\
\text { muscles causes } \\
\text { an inability to } \\
\text { produce hi g h } \\
\text { notes. }\end{array}$ \\
\hline - & $\begin{array}{l}\text { Thyro - ary- } \\
\text { teno i d e i } \\
\text { interni. }\end{array}$ & $\begin{array}{l}\text { The most posterior part is almost } \\
\text { a distinct muscle and its fibres } \\
\text { are all but horizontal: some- } \\
\text { times this muscle is described as } \\
\text { consisting of two layers, super- } \\
\text { ficial with cortical fibres, deep } \\
\text { with oblique fibres, described } \\
\text { under Group III. }\end{array}$ & \\
\hline
\end{tabular}

Nerve Supply. - In the performance of the functions of the larynx the sensory filaments of the superior laryngeal branch of the vagus supply that acute sensibility by which the glottis is guarded against the ingress of foreign bodies, or of irrespirable gases. The contact of these stimulates the nerve filaments; and the impression conveyed to the medulla oblongata, whether it produce sensation or not, is reflected to the filaments of the recurrent or inferior laryngeal branch, and excites contraction of the muscles that close the glottis. Both these branches of the vagi co-operate also in the production and regulation of the voice; the inferior laryngeal determining the contraction of the muscles that vary the tension of the vocal cords, and the superior laryngeal conveying to the mind the sensation of the state of these muscles necessary for their continuous guidance. And both the branches co-operate in the actions of the larynx in the ordinary slight dilatation and contraction of the glottis in the acts of expiration and inspiration, and more evidently in those of coughing and other forcible respiratory movements.

The laryngoscope is an instrument employed in investigating during life the condition of the pharynx, larynx, and trachea. It consists of a large concave. mirror with perforated centre and of a smaller mirror fixed in a long handle. It is thus used : the patient is placed in a chair, a good light (argand burner, or lamp) is arranged on one side of, and a little above his head. The operator fixes the large mirror round his head in such a manner, that he looks through 
the central aperture with one eye. He then seats himself opposite the patient, and so alters the position of the mirror, which is for this purpose provided with a ball and socket joint, that a beam of light is reflected on the lips of the patient.

The patient is now directed to throw his head slightly backward, and to open his mouth; the reflection from the mirror lights up the cavity of the mouth, and by a little alteration of the distance between the operator and the patient the point at which the greatest amount of light is reflected by the mirror-in other words its focal length-is readily discorered. The small mirror fixed in the handle is then warmed, either by holding it over the lamp, or by putting it into a vessel of warm water; this is necessary to prevent the condensation of breath upon its surface. The degree of heat is regulated by

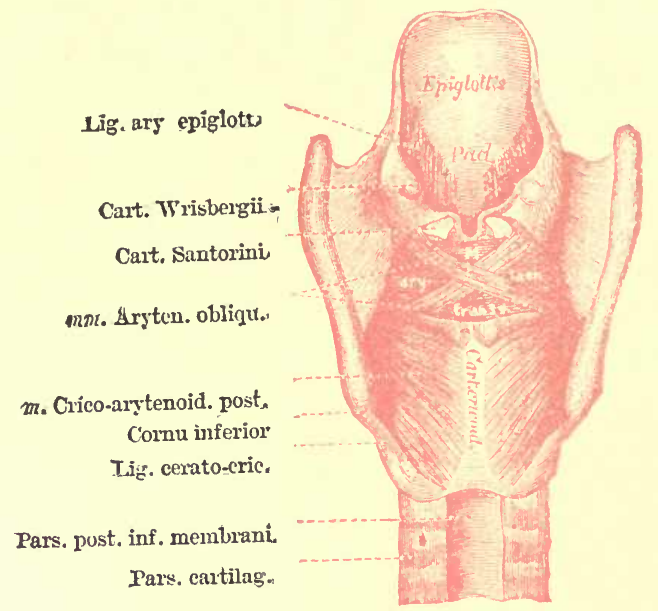

Fig. 344.-The larynx as seen from behind. To show the intrinsic muscles posteriorly. (Stoerk.)

applying the back of the mirror to the hand or cheek, when it should feel warm without being painful.

After these preliminaries the patient is directed to put out his tongue, which is held by the left hand gently but firmly against the lower teeth by means of a handkerchief. The warm mirror is passed to the back of the mouth, until it rests upon and slightly raises the base of the uvula, and at the same time the light is directed upon it: an inverted image of the larynx and trachea will be seen in the mirror. If the dorsum of the tongue be alone seen, the handle of the mirror must be slightly lowered until the larynx comes into view; care should be taken, howerer, not to more the mirror upon the urula, as it excites retching. The observation should not be prolonged, but should rather be repeated at short intervals.

The structures seen will vary somewhat according to the condition of the parts as to inspiration, expiration, phonation, etc.; they are (fig. 347) first, and apparently at the posterior part, the base of the tongue, immediately below which is the accurate outline of the epiglottis, with its cushion or tubercle. Then are seen in the central line the true vocal cords, white and shining in their normal condition. The cords approximate (in the inverted image) posteriorly ; 
between them is left a chink, narrow while a high note is being sung, wide during a deep inspiration. On each side of the true vocal cords, and on a higher level, are the pink false vocal cords. Still more externally than the

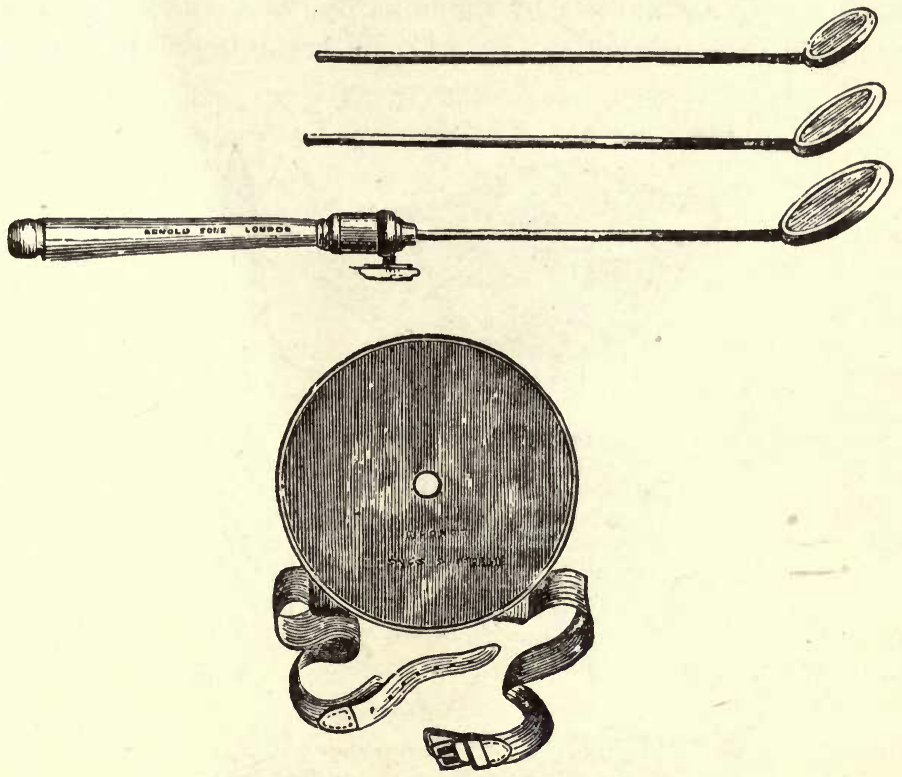

Fig. 345.-The parts of the Laryngoscope.

false vocal cords is the aryteno-epiglottidean fold, in which are situated upon each side three small elevations; of these the most external is the cartilage of Wrisberg, the intermediate is the cartilage of Santorini, while the summit of the arytenoid cartilage is in front, and somewhat below the preceding, being

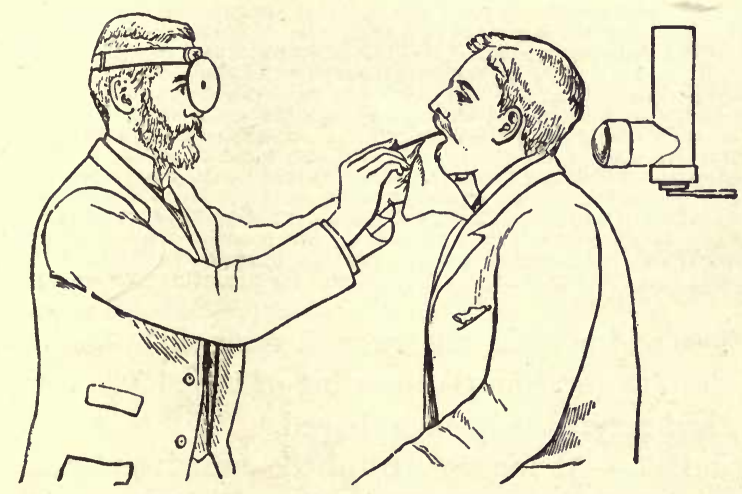

Fig. 346. -To show the position of the operator and patient when using the Laryngoscope.

only seen during deep inspiration. The rings of the trachea, and even the bifurcation of the trachea itself, if the patient be directed to draw a deep breath, may be seen in the interval between the true vocal cords. 


\section{Movements of the Vocal Cords.}

In Respiration.- The position of the vocal cords in ordinary tranquil breathing is so adapted by the muscles, that the opening of the glottis is wide and triangular (fig. $34 \%$, B) becoming a little wider at
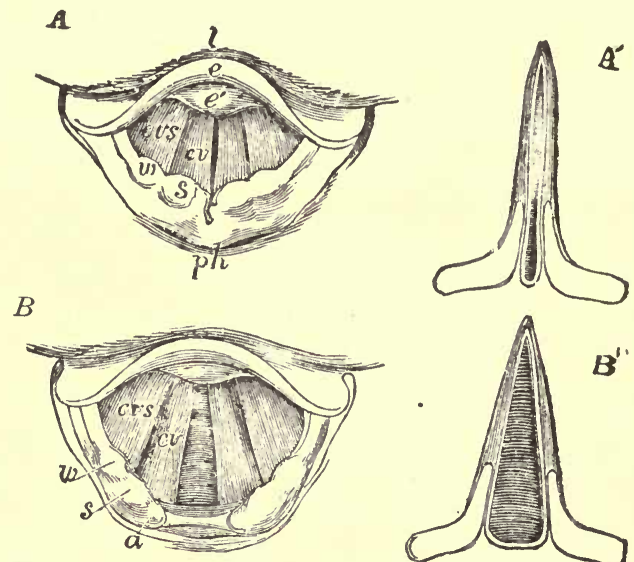

C
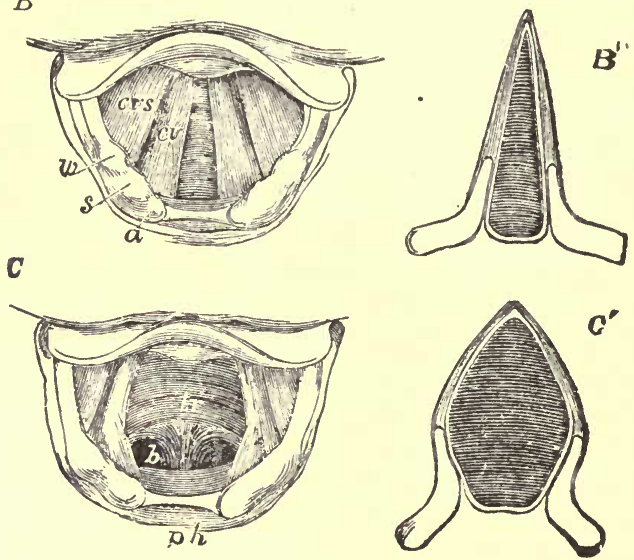

Fig. 347. - Three laryngoscopic views of the superior aperture of the larynx and surrounding parts. $A$, the glottis during the emission of a high note in singing; $\mathrm{B}$, in easy and quiet inhalation of air; $\mathrm{C}$, in the state of the widest possible dilatation, as in inhaling a very deep breath. The diagrams $\mathrm{A}^{\prime}, \mathrm{B}^{\prime}$, and $\mathrm{C}^{\prime}$, show in horizontal sections of the glottis the position of the vocal ligaments and arytenoid cartilages in the three several states represented in the other figures. In all the figures, so far as marked, the letters indicate the parts as follows, viz.: $l$, the base of the tongue; $e$, the upper free part of the epiglottis; $e^{\prime}$, the tubercle or cushion of the epiglottis; ph, part of the anterior wall of the pharynx behind the larynx; in the margin of the arytenoepiglottidean fold $w$, the swelling of the membrane caused by the cartilages of Wrisberg; $s$, that of the cartilages of Santorini; $a$, the tip or summit of the arytenoid cartilages; $c v$, the true vocal cords or lips of the rima glottidis; $c v s$, the superior or false vocal cords; between them the ventricle of the larynx; in $C, t r$ is placed on the anterior wall of the receding trachea, and $b$ indicates the commencement of the two bronchi beyond the bifurcation which may be brought into view in this state of extreme dilatation. (Quain after Czermak.)

each inspiration, and a little narrower at each expiration. On making a rapid and deep inspiration the opening of the glottis is widely dilated (fig. $347, \mathrm{c}$ ), and somewhat lozenge-shaped.

In Vocalization.-At the moment of the emission of a note, it is narrowed, the margins of the arytenoid cartilages being brought into contact and the edges of the vocal cords approximated and made parallel, at the same time that their tension is much increased. The higher the note produced, the tenser do the cords become (fig. $34 \%, \mathrm{~A}$ ); and the range of 
a voice depends, of course, in the main, on the extent to which the degree of tension of the vocal cords can be thus altered. In the production of a high note the vocal cords are brought well within sight, so as to be plainly visible with the help of the laryngoscope. In the utterance of grave tones, on the other hand, the epiglottis is depressed and brought over them, and the arytenoid cartilages look as if they were trying to hide themselves under it (fig. 348). The epiglottis, by being

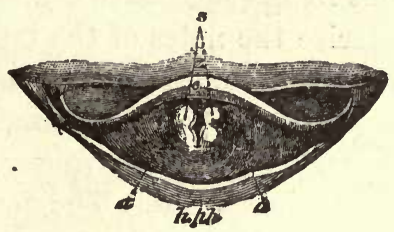

Fig. 348. - View of the upper part of the larynx as seen by means of the laryngoscope during the utterance of a grave note. $c$, Epiglottis; $s$, tubercles of the cartilages of Santorini; $a$, arytenoid cartilages; $z$, base of the tongue; $p h$, the posterior wall of the pharynx. (Czermak.)

somewhat pressed down so as to cover the superior cavity of the larynx, serves to render the notes deeper in tone and at the same time somewhat duller, just as covering the end of a short tube placed in front of caoutchouc tongues lowers the tone. In no other respect does the epiglottis appear to have any effect in modifying the rocal sounds.

The degree of approximation of the vocal cords also usually corresponds with the height of the note produced; but probably not always, for the width of the aperture has no essential influence on the height of the note, as long as the vocal cords have the same tension: only with a wide aperture the tone is more difficult to produce and is less perfect, the rushing of the air through the aperture being heard at the same time.

No true vocal sound is produced at the posterior part of the aperture of the glottis, that, viz., which is formed by the space between the arytenoid cartilages. For if the arytenoid cartilages be approximated in such a manner that their anterior processes touch each other, but yet leave an opening behind them as well as in front, no second vocal tone is produced by the passage of the air through the posterior opening, but merely a rustling or bubbling sound; and the height or pitch of the note produced is the same whether the posterior part of the glottis be open or not.

\section{'The Voice in Singing and Speaking.}

Varieties of Vocal Sounds. - The laryngeal notes may observe three different kinds of sequence. The first is the monotonous, in which the notes have nearly all the same pitch as in ordinary speaking; the variety of the sounds of speech being due to articulation in the mouth. 
In speaking, however, occasional syllables generally receive a higher intonation for the sake of accent. The second mode of sequence is the successive transition from high to low notes, and vice versâ, without intervals; such as is heard in the sounds, which, as expressions of passion, accompany crying in men, and in the howling and whining of dogs. The third mode of sequence of the vocal sounds is the musical, in which each sound has a determinate number of vibrations, and the numbers of the vibrations in the successive sounds have the same relative proportions that characterize the notes of the musical scale.

In different individuals this comprehends one, two, or three octaves. In singers-that is, in persons apt for singing-it extends to two or three octaves. But the male and female voices commence and end at different points of the musical scale. The lowest note of the female voice is about an octave higher than the lowest of the male voice; the highest note of the female voice about an octave higher than the highest of the male. The compass of the male and female voices taken together, or the entire scale of the human voice, includes about four octaves. The principal difference between the male and female voice is, therefore, in their pitch; but they are also distinguished by their tone,- - the male voice is not so soft. The voice presents other varieties besides that of male and female; there are two kinds of male voice, technically called the bass and tenor, and two kinds of female voice, the contralto and soprano, all differing from each other in tone. The bass voice usually reaches lower than the tenor, and its strength lies in the low notes; while the tenor voice extends ligher than the bass. The contralto voice has generally lower notes than the soprano, and is strongest in the lower notes of the female voice; while the soprano roice reaches higher in the scale. But the difference of compass, and of power in different parts of the scale, is not the essential distinction between the different voices; for bass singers can sometimes go very high, and the contralto frequently sings the high notes like soprano singers. The essential difference between the base and tenor voices, and between the contralto and soprano, consists in their tone or timbre, which distinguishes them even when they are singing the same note. The qualities of the barytone and mezzo-soprano voices are less marked; the barytone being intermediate between the bass and tenor, the mezzo-soprano between the contralto and soprano. They have also a middle position as to pitch in the scale of the male and female voices.

The differences in the pitch of the male and the female voices depends on the different length of the vocal cords in the two sexes; their relative length in men and women being as three to two. The difference of the two voices in tone or timbre, is owing to the different nature and form of the resounding walls, which in the male larynx are 
much more extensive, and form a more acute angle anteriorly. The different qualities of the tenor and bass, and of the alto and soprano voices, probably depend on some peeuliarities of the ligaments, and the membranous and cartilaginous parietes of the laryngeal cavity, which are not at present understood, but of which we may form some idea, by recollecting that musical instruments made of different materials, e.g., metallie and gut-strings, may be tuned to the same note, but that each will give it with a peculiar tone or timbre.

The boy's larynx resembles the female larynx; their rocal cords before puberty are not two-thirds the length of the adult cords; and the angle of their thyroid cartilage is as little prominent as in the female larynx. Boys' voices are alto and soprano, resembling in piteh those of women, but louder, and differing somewhat from them in tone. But, after the larynx has undergone the change produced during the period of development at puberty, the boy's voice becomes bass or tenor. While the change of form is taking place, the voice is said to crack; it becomes imperfect, frequently hoarse and crowing, and is unfitted for singing until the new tones are brought under command by practice. In eunuchs, who have been deprived of the testes before puberty, the voice does not undergo this change. The voice of most old people is deficient in tone, unsteady, and more restricted in extent: the first defect is owing to the ossification of the cartilages of the larynx and the altered condition of the vocal cords; the want of steadiness arises from the loss of nervous power and command over the muscles; the result of which is here, as in other parts, a tremulous movement. These two causes combined render the voices of old people roid of tone, unsteady, bleating, and weak.

In any class of persons arranged, as in an orchestra, according to the character of voices, each would possess, with the general characteristics of a bass, or tenor, or any other kind of voice, some peculiar character by which his voice would be recognized from all the rest. The conditions that determine these distinctions are, however, quite unknown. They are probably inherent in the tissues of the larynx, and are as indiscernible as the minute differences that characterize men's features; one often observes, in like manner, hereditary and family peculiarities of voice, as well marked as those of the limbs or face.

Most persons, particularly men, have the power, if at all capable of singing, of modulating their voices through a double series of notes of different character: namely, the notes of the natural voice, or chestnotes, and the falsetto notes. The natural voice, which alone has been hitherto considered, is fuller, and excites a distinct sensation of much stronger vibration and resonance than the falsetto voice, which has more a flute-like character. The deeper notes of the male voice can 
be produced only with the natural voice, the highest with the falsetto only; the notes of middle pitch can be produced either with the natural or falsetto voice; the two registers of the roice are therefore not limiterl in such a mamer as that one ends when the other begins, but they rum in part side by sicle.

'The natural or chest-notes are, as we have seen, produced by the ordinary vibrations of the rocal cords. The mode of production of the falsetto notes is still obscure.

By Mïller they were thought to be due to vibrations of only the inner borders of the roeal cords. In the opinion of Petrequin and Diday, they do not result from vibrations of the vocal cords at all, but from vibrations of the air passing through the aperture of the glottis, which they helieve assumes, at such times, the contour of the embouchure of a flute. Others, considering some degree of similarity which exists between the falsetto notes and the peculiar tones called harmonic, which are produced when, by touching or stopping a harp-string at a particular point, only a portion of its length is allowed to ribrate, have supposed that, in the falsetto notes, portions of the cords are thus isolated, and made to vibrate while the rest are held still. The question camnot yet be settled; but any one in the habit of singing may assure himself, both by the difficulty of passing smoothly from one set of notes to the other, and by the necessity of exercising himself in both registers, lest he should become very deficient in one, that there mist be some great difference in the modes in which their respective notes are produced.

The pitch of the note, which depends upon the rapidity of the vibrations, is altered by alterations of the rocal cords, and so the strength of the voice is in proportion $(a)$ to the degree to which the vocal cords can be made to vibrate; and partly $(b)$ to the fitness for resonance of the membranes and cartilages of the larynx, of the parietes of the thorax, lungs, and cavities of the mouth, nostrils, and communicating sinuses. It is diminished by anything which interferes with such eapability of vibration.

The intensity or loudness of a given note with maintenance of the same pitch, cannot be rendered greater by merely increasing the force of the current of air through the glottis; for increase of the force of the current of air, cceteris paribus, raises the pitch both of the natural and the falsetto notes. Yet, since a singer possesses the power of increasing the loudness of a note from the faintest piano to fortissimo without its pitch being altered, there must be some means of compensating the tendency of the vocal cords to emit a higher note when the force of the current of air is increased. This means evidently consists in modifying the tension of the vocal cords. When a note is rendered londer and more intense, the rocal cords must be relaxed by remission of the 
muscular action, in proportion as the force of the current of the breath through the glottis is increased. When a note is rendered fainter, the reverse of this must occur.

The arches of the palate and the uvula become contracted during the formation of the higher notes; but their contraction is the same for a note of given height, whether it be falsetto or not; and in either case the arches of the palate may be touched with the finger, without the note being altered. Their action, therefore, in the production of the higher notes seems to be merely the result of involuntary associate nervous action, excited by the voluntarily increased exertion of the muscles of the larynx. If the palatine arches contribute at all to the production of the higher notes of the natural voice and the falsetto, it can only be by their increased tension strengthening the resonance.

The office of the ventricles of the larynx is evidently to afford a free space for the vibrations of the lips of the glottis; they may be compared with the cavity at the commencement of the mouthpiece of trumpets, which allows the free vibration of the lips.

Speech.-Besides the musical tones formed in the larynx, a great number of other sounds can be produced in the vocal tubes, between the glottis and the external apertures of the air-passages, the combination of which sounds by the agency of the cerebrum into different groups to designate objects, properties, actions, etc., constitutes language. The languages do not employ all the sounds which can be produced in this manner, the combination of some with others being often difficult. Those sounds which are easy of combination enter, for the most part, into the formation of the greater number of languages. Each language contains a certain number of such sounds, but in no one are all brought together. On the contrary, different languages are characterized by the prevalence in them of certain classes of these sounds, while others are less frequent or altogether absent.

Articulate Sounds. - The sounds produced in speech, or the articulate sounds, are commonly divided into vowels and consonants: the distinction between which is, that the sounds for the former are generated by the larynx, while those for the latter are produced by interruption of the current of air in some part of the air-passages above the larynx. The term consonant has been given to these because several of them are not properly sounded, except consonantly with a vowel. Thus, if it be attempted to pronounce aloud the consonants $b$, d, and $g$, or their modifications, $p, t, k$, the intonation only follows them in their combination with a vowel. To recognize the essential properties of the articulate sounds, it is necessary first to examine them as they are produced in whispering, and then investigate which of them can also be uttered in a modified character conjoined with vocal tone. By this procedure we 
find two series of sounds: in one the sounds are mute, and cannot be uttered with a rocal tone; the sounds of the other series can be formed independently of voice, but are also capable of being uttered in conjunction with it.

All the rowels can be expressed in a whisper withont rocal tone, that is, mutely. These mute rowel-sounds differ, however, in some measure, as to their mode of production, from the consonants. All the mute consonants are formed in the vocal tube above the glottis, or in the eavity of the mouth or nose, by the mere rushing of the atir between the surfaces differently modified in disposition. But the sound of the rowels, even when mute, has its source in the glottis, though its roeal cords are not thrown into the ribrations necessary for the production of roice; and the somnd seems to be produced by the passage of the current of air between the relaxed rocal cords. 'The same rowel-sound can be produced in the larynx when the mouth is closed, the nostrils being open, and the utterance of all vocal tone avoided. The sound, when the mouth is open, is so modified by varied forms of the oral eavity, as to assume the characters of the rowels $\mathrm{a}, \mathrm{e}, \mathrm{i}, \mathrm{o}, \mathrm{u}$, in all their modifications.

The earity of the mouth assumes the same form for the articulation of each of the mute vowels as for the corresponding rowel when rocalized; the only difference in the two cases lies in the kind of sound (mitted by the larynx. It has been pointed out that the conditions necessary for changing one and the same sound into the different rowels, are differences in the size of two parts- the oral canal and the oral opening; and the same is the case with regard to the mute vowels. By oral canal, is meant here the space between the tongue and palate: for the pronunciation of certain vowels both the opening of the mouth and the space just mentioned are widened; for the pronunciation of other vowels both are contracted; and for others one is wide, the other contracted. Admitting five degrees of size, both of the opening of the month and of the space between the tongue and palate, Kempelen thus states the dimensions of these parts for the following rowel-sounds:-

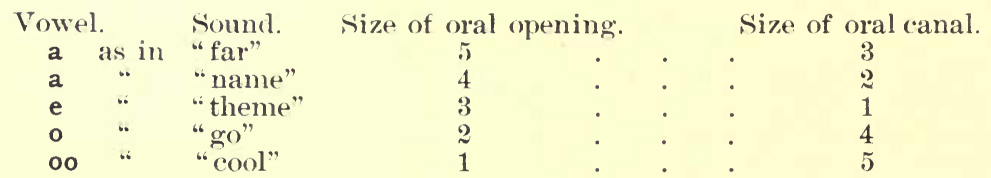

Another important distinction in articulate sounds is, that the utterance of some is only of momentary duration, taking place during a sudden change in the conformation of the mouth, and being incapable of prolongation by a continued expiration. 'T'o this class belong $\mathrm{b}, \mathrm{p}, \mathrm{d}$, and the hard $g$. In the utterance of other consonants the sounds may be continuous; they may be prolonged, ad libitum, as long as a particular disposition of the mouth and a constant expiration are maintained. 
Among these consonants are $\mathrm{h}, \mathrm{m}, \mathrm{n}, \mathrm{f}, \mathrm{s}, \mathrm{r}, \mathrm{l}$. Corresponding differences in respect to the time that may be occupied in their utterance exist in the vowel sounds, and principally constitute the differences of long and short syllables. Thus the $\mathbf{a}$ as in far and fate, the $\mathbf{o}$ as in go and fort, may be indefinitely prolonged; but the same vowels (or more properly different vowels expressed by the same letters), as in can and fact, in dog and rotten, cannot be prolonged.

All sounds of the first or explosive kind are insusceptible of combination with vocal tone (intonation), and are absolutely mute; nearly all the consonants of the second or continuous kind may be attended with intonation.

Ventriloquism.-The peculiarity of speaking, to which the term ventriloquism is applied, appears to consist merely in the varied modification of the sounds proauced in the larynx, in imitation of the modifications which voice ordinarily suffers from distance, etc. From the observations of Müller and Colombat, it seems that the essential mechanical parts of the process of ventriloquism consist in taking a full inspiration, then keeping the muscles of the chest and neck fixed, and speaking with the mouth almost closed, and the lips and lower jaw as motionless as possible, while air is very slowly expired through a very narrow glottis; care being taken also, that none of the expired air passes through the nose. But, as observed by Müller, much of the ventriloquist's skill in imitating the voices coming from particular directions, consists in deceiving other senses than hearing. We never distinguish very readily the direction in which sounds reach our ear; and, when our attention is directed to a particular point, our imagination is very apt to refer to that point whatever sounds we may hear.

Action of the Tongue in Speech. - The tongue, which is usually credited with the power of speech-language and speech being often employed as synonymous terms-plays only a subordinate, although very important part. This is well shown by cases in which nearly the whole organ has been removed on account of disease. Patients who recover from this operation talk imperfectly, and their voice is considerably modified; but the loss of speech is confined to those letters in the pronunciation of which the tongue is concerned.

Stammering depends on a want of harmony between the action of the muscles (chiefly abdominal) which expel air through the larynx, and that of the muscles which guard the orifice (rima glottidis) by which it escapes, and of those (of tongue, palate, etc.) which modulate the sound to the form of speech.

Over either of the groups of muscles, by itself, a stammerer may have as much power as other people. But he cannot harmoniously arrange their conjoint actions. 


\section{CHAPTER XVI.}

THE NERVOUS SYSTEM.

THE nervous system consists of the following parts: firstly, of large masses of nervous matter situated within the bony cranium and spinal column, and constituting the brain and spinal cord; secondly, of smaller masses of nervous matter, situated for the most part in the abdominal and thoracic cavities, but also in the neck and head, and constituting what are known as sympathetic ganglia; thirdly, of cords of nerve-fibres which connect the central nervous system with the periphery and with the so-called sympathetic ganglia, which are not in reality a system independent of the brain and cord as was formerly taught, but are really part and parcel of the same system; and fourthly, of peripheral organs in connection with the beginnings or endings of the nerves at the periphery of the body.

It will be necessary to consider these several parts of the nervous system seriatim; it will be most useful for the understanding of the subject, however, to proceed first of all with the consideration of the properties of nerve-fibres, as this forms the most elementary portion of the subject.

Nerve-fibres. - The structure of the different kinds of nerve-fibres has been already dealt with (p. 91, et seq.) ; their function remains to be considered here.

\section{Function of Nerve-fibres.}

The office of nerve-fibres is to conduct impressions. From the account of nervous action previously given (p. 52 r et seq.) it will be readily understood, that nerve-fibres may be stimulated to act by anything which, with sufficient suddenness, increases their irritability: they are incapable of originating of themselves the condition necessary for the manifestation of their own energy. The stimulus produces it: effect upon the termination of the nerve stimulated, being conducted to it by the nerve-fibre. The effect of the stimulus upon a nerve therefore depends upon the nature of its end-organ. A length of a nerve trunh when freshly removed from the body, if stimulated midway between its extremities, will, as shown by the deflection of the needle of a galvanomete: at either end, conduct the electrical impressions in either direction, and it may be considered therefore only an accidental circumstance as it were, whether when in situ it has conducted impressions to the central 
nervous system from the periphery, or from the central nervous system to the muscles or other tissues. The same fibre cannot be used for the one purpose at one time, and for the other at another, simply because of the nature of its terminal organs. Thus, when a cerebro-spinal nervefibre is irritated in the living body as by pinching, or by heat, or by electrifying it, there is, under ordinary circumstances, one of two effects, - either there is pain, or there is twitching of one or more muscles to which the nerve distributes its fibres. From various considerations it is certain that pain is always the result of a change in the nervecells of the brain. Therefore, in such an experiment as that referred to, the irritation of the nerve-fibre is conducted in one of two directions, i.e., either to the brain, which is the central termination of the fibre, when there is pain, or to a muscle, which is the peripheral termination, when there is movement.

The effect of this simple experiment is a type of what always occurs when nerve-fibres are engaged in the performance of their functions. The result of stimulating them, which roughly imitates what happens naturally in the body, is found to occur at one or other of their extremities, central or peripheral, never at both; and in accordance with this fact, and because, for any given nerve-fibre, the result is always the same, nerve-fibres have been commonly classed as sensory or motor.

This is not altogether accurate, and the terms centrifugal or efferent and centripetal or afferent are more properly used, since the result of stimulating a nerve of the former kind is not always the production of pain or other form of sensation, nor is motion the invariable result of stimulating the latter.

The term intercentral is applied to those nerve-fibres which connect more or less distinct nerve-centres, and may, therefore, be said to have no peripheral distribution, in the ordinary sense of the term.

Impressions made upon the terminations or upon the trunk of a centripetal nerve may cause $(a)$ pain, or some other kind of sensation; $(b)$ special sensation; or $(c)$ reflex action of some kind; or $(d)$ inhibition, restraint of action. Similarly impressions made upon a centrifugal nerve may cause $(a)$ contraction of muscle (motor nerve); (b) it may influence nutrition (trophic nerve); or (c) may influence secretion (secretory nerve); or $(d)$ inhibit, augment, or stop any other efferent action.

It is a law of action in all nerve-fibres, and corresponds with the continuity and simplicity of their course, that an impression made on any fibre, is simply and uninterruptedly transmitted along it, without itself being imparted or diffused to any of the fibres lying near it. It is possible that the mere passage of a nerve impulse along a nerve-fibre, however, may produce some effect upon the neighboring nerve-fibres. 
Their adaptation to the purpose of simple conduction is, perhaps, due to the contents of each fibre being completely isolated from those of adjacent fibres by the myelin sheath in which each is inclosed, and which acts, it may be supposed, just as silk, or other non-conductors of electricity do, which, when covering a wire, prevent the electric condition of the wire from being conducted into the surrounding medium.

Velocity of a Nervous Impulse. - The change which a stimulus sets up in a nerve, of the exact nature of which we are unacquainted, appears to travel along a nerve-fibre in both directions with considerable velocity in the form of a wave. Helmholtz and Baxt have estimated the average rate of conduction in human motor nerves at 111 feet (nearly 29 metres) per second; this result agreeing very closely with that previously obtained. It is probably rather under than over the average velocity. Rutherford's observations agree with those of Von Wittich, that the rate of transmission in sensory nerves is about 140 feet (42 metres) per second. The velocity of the nerve impulse in motor nerves has been calculated by noticing the duration of the interval between two contractions of the same muscle when stimulated by means of two pairs of electrodes, one placed behind the nerve close to the muscle, and the second placed at a known distance further away from the muscle. The contraction ensues when the stimulus is applied further from the muscle later than the other case, and the interval between the two contractions is occupied by the passage of the impulse down the nerve. With these data it is concluded that the velocity of the passage of the nerve impulse in a frog's motor nerve is 28 to 30 metres per second. In the human motor nerve, calculated by applying the stimulus through the skin instead of directly to the nerve, the velocity is greater, viz., about 33 to 50 metres per second. In sensory nerves the velocity is said to be about 30 to 33 metres per second. Various conditions modify the rate of transmission, of which temperature is one of the most important, a very low or a very high temperature diminishing it; fatigue of the nerve acting in the same direction, but increase of the stimulus up to a certain point increasing it, as does also the katelectrotonic condition of the nerve.

The Cerebro-Spinal Nervous System.-The parts of which this system is composed are the following: $(a)$ the spinal cord and its nerves; (b) the brain made up of cerebrum, crura cerebri and the ganglia in connection with them, pons varolii, cerebellum, and the medulla oblongata or bulb which connects the upper parts of the system with the spinal cord, or medulla spinalis.

All of these parts of the nervons system are nerve-centres, in contradistinction to nerve-trunks, and differ from the nerves in being made up of nerve-cells and their branchings as well as of nerve-fibres.

As now 
conceived, the nerve-centres are composed of neurons, while the nervetrunks are made up of the neuraxons with their various terminals. (See p. 91 et seq.) There are other ganglia besides these, distributed elsewhere and not within the cranium and spinal column, but these are, for the sake of convenience, considered apart, under the head of the sympathetic system, as they present some differences to the more central ganglia.

The cerebro-spinal centres then are distinguished from mere nervetrunks by the possession of nerve-cells; these are, as we have seen in a former chapter (p. 99 et seq.), of different kinds; they very possibly differ in function. It is, however, to the possession of ganglion-cells that the increase of the functions of nerve-centres over that of nervetrunks is credited. Before turning to the discussion of the functions of the spinal cord it will be as well to devote a little time therefore to the question of the functions of the nerve-centres in general. The ganglia of the sympathetic system also contain nerve-cells, but to these it is supposed a different use is to be assigned, and what is said as to the functions of nerve-ganglia in this place is only to be applied to those of the cerebro-spinal centres.

\section{Functions of Nerve-Centres.}

Reflex action.-One of the chief functions of nerve-cells appears to be the power of sending out impulses to the periphery along efferent nerves in response to impulses reaching them through afferent nerves. This power is sometimes called the conversion of an afferent into an efferent impulse. If may be supposed that an impulse passing to a nerve-cell may produce such a change in its metabolism that a discharge of energy ensues. This discharge is in some way passed down an efferent nerve as stimulus, and effects some change-motor, secretory, or nutritive, at the peripheral extremity of the latter-the difference in effect depending on the kind of peripheral-nerve termination. 'The reflex action may be limited in its effect, or it may be extensive. Reflex movements, occurring quite independently of sensation, are generally called excito-motor; those which are guided or acompanied by sensation, but not to the extent of a distinct perception, or intellectual process, are termed sensori-motor.

(a) For the manifestation of every reflex action, these things are necessary: (1), one or more perfect afferent fibres, to convey an impression; (2), a nervous centre for its reception, and by which it may be reflected; (3), one or more efferent nerve-fibres, along which the impression may be conducted to (4) the muscular or other tissue by which the effect is manifested. All this means, in simpler statement, that for the production of a reflex action there must be two perfect neurons, a sensory or afferent and a motor or efferent. This arrangement is shown in 
fig. 349. (b) All reflex actions are essentially involuntary, though most of them admit of being modified, controlled, or prevented by a voluntary effort.

(c) Reflex actions performed in health have, for the most part, a distinct purpose, and are adapted to secure

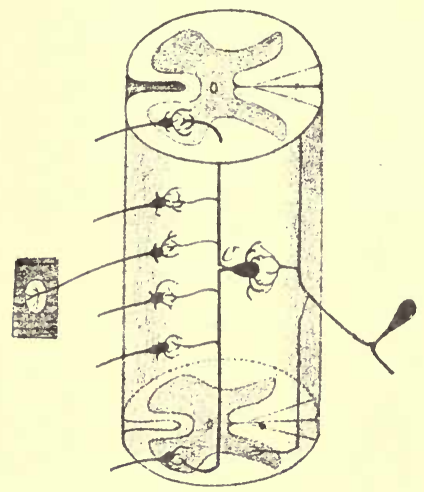

Fig. 349.-Showing the arrangement of the retlex mechanism, with a neurou intercalated between the sensory and motor neurons. some end desirable for the well-being of the body; but, in disease, many of them are irregular and purposeless.

(d) Muscular contractions produced by reflex action are often more sustained than those produced by the direct stimulus of motor nerves themselves. 'The irritation of a muscular organ, or its motor nerve, produces contraction lasting only so long as the irritation continues; but irritation applied to a nervous centre through one of its centripetal nerves may excite reflex anủ harmonious contractions, which last some time after the withdrawal of the stimulus.

Relations between the stimulus and the Effect frodured.-Certain rules showing the relation between the resulting reflex action and the stimulus have been drawn up by Pflüger as follows:-

1. Law of unilateral reflection.-A slight irritation of the surface supplied by certain sensory nerves is reflected along the motor nerves of the same region. 'Thus, if the skin of a frog's foot be tickled on the right side, the right leg is drawn up.

¿. Lan of symmetrical reflection.-A stronger irritation is reflected, not only on one sile, but also along the corresponding motor nerves of the opposite side.

3. Law of intensity.-In the above case, the contractions will be more violent on the side irritated, but it must not be assumed that the effect is always in proportion to the strength of the stimulus.

4. Lan of radiation. - If the irritation (afferent impulse) increases, it is reflected along other motor nerves till at length all the muscles of the body are thrown into action.

In the simplest form of reflex action a single sensory and single motor neuron may be supposed to be concerned, but in the majority of actual actions many neurons are probably engaged. 'The impulse is carried by collaterals up and down to different levels of the spinal cord, and thus a number of groups of cells are affected (fig. $349 \mathrm{~A}$ ).

The reflex effect produced by a stimulus applied to a sensory surface depends, however, not only upon the strength of the stimulus, but also upon other circumstances, the most important of which is the condition of the nerve-centre itself. Looking upon the effect produced as 
the result of the discharge as it were of energy from the centre, it may be supposed that sometimes the centre is in a more explosive condition than at another; this is shown for example in the case of a frog poisoned by strychnine, when the slightest stimulus applied to the skin will produce the most violent and general tetanic spasms, while under ordinary circumstances the contraction of a few muscles only would result. Wo must also suppose that the centres are particularly sensitive to particular kinds of stimuli, sometimes producing very extensive and violent

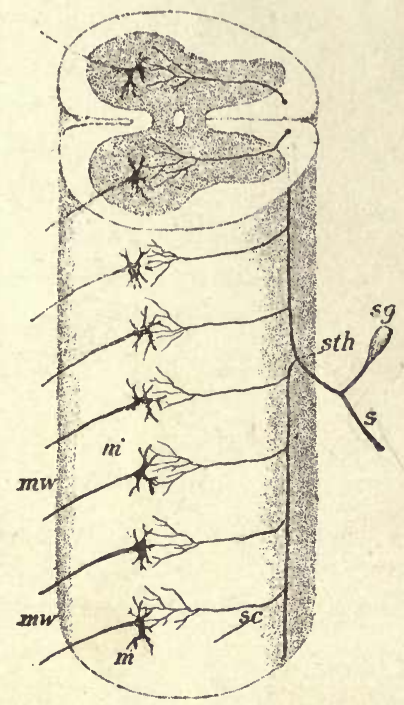

Fig. 349A.- Showing the arrangement of a simple reflex mechanism composed of a motor and sensory neuron. sg, Posterior spinal ganglion; $s$ and sth, sensory root; $m$, motor nerve cell; $m w$, motor root.

muscular actions in response to a slight stimulus of a special kind. Such a condition is illustrated in the violent and general muscular spasms occurring when a small particle of food passes into the larynx, violent expiratory spasms accompanied by contractions of other muscles taking place.

A nerve-centre must be considered as capable by its connections with efferent nerves of producing most extensive muscular movements, and when from any reason, either by the intensity of the afferent stimuli reaching it, or by the special nature, extent, or point of application of the afferent stimuli, or by special changes in its own metabolism brought about by poison or by some other means, a maximum discharge takes place, the resulting movements are most extensive. Under ordinary conditions, however, a slight stimulus produces, as above men- 
tioned only a moderate discharge from the centre, the movement being centre may be not only not to set it into activity, but to prevent or stop an action already going on. On the other hand, the action of afferent impulses upon a nerve-centre may be to augment, render more powerful or extensive, and increase in a certain direction an action already in course. Such may be well illustrated by the action of the to a certain extent co-extensive with the strength of the stimulus.

The time taken in a reflex action has been found to be .066 to .058 second, but this is only a rough and arbitrary estimation.

Automatism.-A second function which appears to be possessed by certain nerve-centres and not by others is that of automatic action or automatism. By this is meant that it is not dependent for its discharge upon any afferent stimuli, but that it is capable of sending ont of itself efferent impulses of various kinds. The centre may be supposed to do this by the nature of its own metabolism, anabolism or building up of the explosive substance being followed by katabolism or its discharge. So that the centre sends out its impulses to muscles rhythmically. Such a power of automatism we have seen is attributed to the respiratory centres in the bulb.

Inhibition and Augmentation.-Not only may movements of muscles, discharge of secretion from gland-cells and the like be produced by afferent impulses reaching nerve-centres, but also inhibition of action which is already taking place. 'This is well seen in the matter of the inhibitory action of the ragus upon the cardiac contractions. The vagi convey to the heart impulses from the cardio-inhibitory centres which have a restraining action upon the contractions of the heart, as is seen by the increase in the frequency of the heart-beats when the vagi are divided; but we have seen that appropriate afferent stimuli, as, for example, when applied to the abdominal sympathetic, may increase the action of the centre to such an extent that the heart may be altogether stopped in diastole. In such a case the result of the afferent stimuli upon the centre has been to produce complete inhibition and not muscular contraction. This is not the only example of inhibition which might be instanced; the action of almost any centre may be inhibited oy impulses reaching it; indeed the effect of afferent impulses upon a vagi upon the respiratory centres to which attention has been drawn in the chapter upon respiration.

Membranes of the Brain and Spinal Cord.-The Brain and Spinal Cord are enveloped in three membranes-(1) the Dura Mater, (2) the Arachnoid, (3) the Pia Mater.

(1) The Dura Mater, or external covering, is a tough membrane composed of bundles of connective-tissue which cross at various angles, and in whose interstices branched connective-tissue corpuscles lie: it is lined by a thin elastic membrane, and on the inner surface and where it is not adherent to the bone, on the 
outer surface also is a layer of endothelial cells very similar to those found in serous membranes. (2.) The Arachnoid is a much more delicate membrane, very similar in structure to the dura mater, and lined on its outer or free surface by an endothelial membrane.

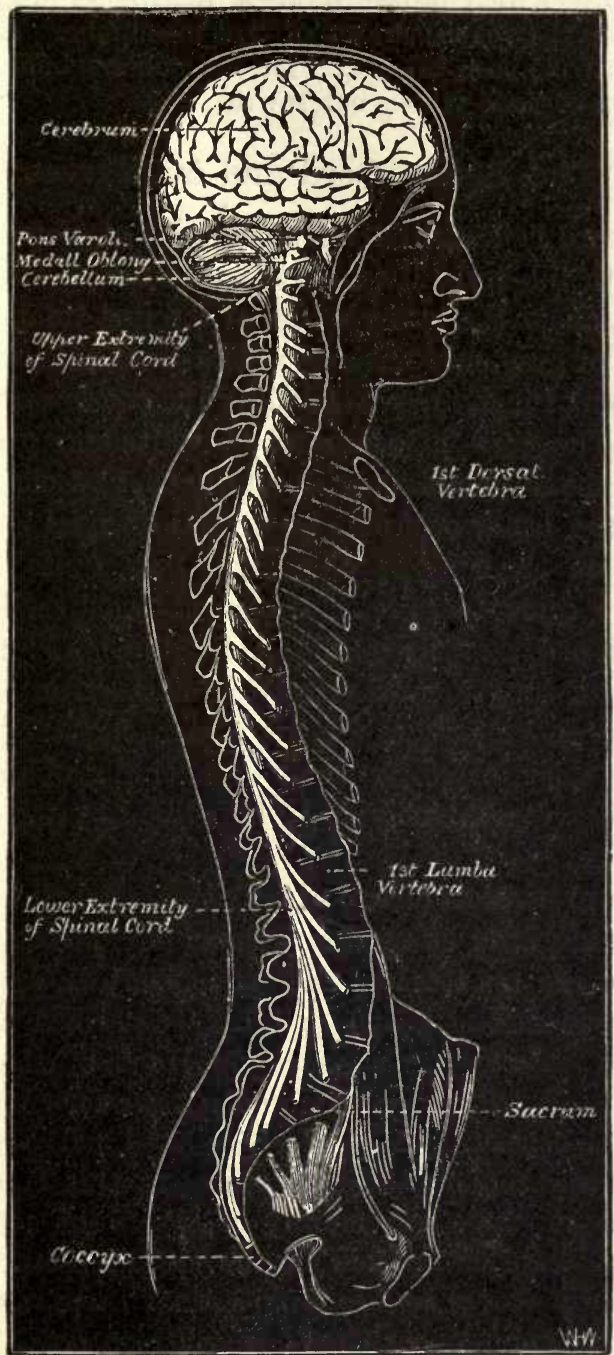

Fig. 350.- View of the cerebro-spinal axis of the nervous system. The right half of the cranium and.trunk of the body has been removed by a vertical section; the membranes of the brain and spinal cord have also been removed and the roots and first part of the fifth and ninth cranial, and of all spinal nerves of the right side, have been dissected out and laid separately on the wall of the skull and on the several vertebræ opposite to the place of their natural exit from the cranio-spinal cavity. (After Bourgery.)

(3.) The Pia Mater consists of two chief layers, between which numerous bloodvessels ramify. Between the arachnoid and pia mater is a network of fibroustissue trabeculæ sheathed with endothelial cells: these sub-arachnoid trabeculæ 
divide up the sub-arachnoid space into a number of irregular sinuses. There are some similar trabeculæ, but much fewer in number, traversing the sub-dural space, $i . e$. , the space between the dura mater and arachnoid.

Pacchionian bodies are growths from the sub-arachnoid network of connective-tissue trabeculæ which project through small holes in the inner layers of the dura mater into the renous sinuses of that membrane. The venous sinuses of the dura mater have been injected from the sub-arachnoidal space through the intermediation of these villous outgrowths.

\section{The Spinal Cord and its Nerves.}

The Spinal cord is a cylindriform column of nerve-substance connected above with the brain through the medium of the bulb, and terminating below, about the lower border of the first lumbar vertebra, in a slender filament of gray substance, the filum terminale, which lies in the midst of the loots of many nerves forming the cauda equina.

Structure.-The cord is composed of white and gray nervous substance, of which the former is situated externally, and constitutes its chief portion, while the latter occupies its central or axial portion, and is so arranged, that on the surface of a transverse section of the cord it appears like two somewhat crescentic masses comected together by a narrower portion or isthmus (fig. 350A). Passing through the centre of this isthmus in a longitudinal direction is a minute canal (central canal), which is continued through the whole length of the cord, and opens above into the space at the back of medulla oblongata and pons Varolii, called the fourth ventricle. It is lined by a layer of columnar ciliated epithelium.

The spinal cord consists of two exactly symmetrical halves, separated anteriorly and posteriorly by vertical fissures (the posterior fissure being deeper, but less wide and distinct than the anterior), and united in the middle by nervous matter which is usually described as forming two conmissures-an anterior commissure, in front of the central canai, consisting of morlullated nerve-fibres, and a posterior commissure behind the central canal consisting also of medullated nerve-fibres, but with more neuroglia, which gives the gray aspect to this commissure. 'The: fibres of the commissures are mainly composed of collaterals. Fach half of the spinal cord is marked on the sides (obscurely at the lower part, but distinctly above) by two longitudinal furrows, which divide it into three portions, columns, or tracts, an anterior, lateral, and posterior. From the groove between the anterior and lateral columns spring the anterior roots of the spinal nerves (4); and just in front of the groove between the lateral and posterior columns arise the posterior roots of the same; a pair of roots on each side corresponding to each vertebra.

White Matter.-The white matter of the cord is seen to be made up of nedullated nerve-fibres, of different sizes, arranged longi- 
tudinally, and of a supporting material of two kinds, viz.:-( $a$ ) ordinary fibrous connective tissue with elastic fibres, which is connected with septa from the pia mater which pass into the cord to carry the blood vessels. (b) Neuroglia; this material is made up of the branching cells (fig. 351A), the bodies of which, in consequence of the high development of the branchings, are small. The processes of the neuroglia-cells are arranged so as to support the nerve-fibres which are without the usual external nerve sheaths. Neuroglia was formerly considered to be a kind of connective tissue, but is now considered to be a distinct material.

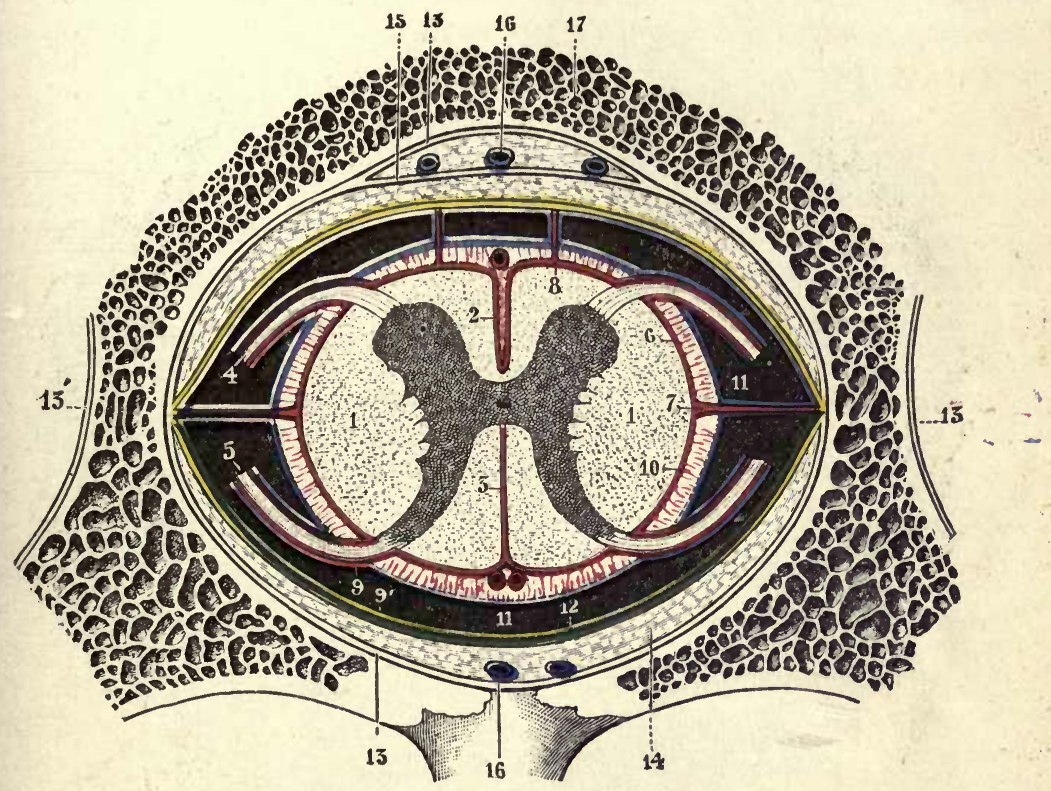

Fig. 350A.-Horizontal section of the cord and its envelnpes, at the middle of a vertebral body'. (Schematic). 1, Spinal cord with 2, its anterior median fissure; 3 , its posterior median fissure; 4 , anterior roots; 5 , posterior roots; 6 , pia mater (in red) $; 7$, ligamentum dentatum; 8 , connect? ing fibres passing from the pia to dura mater; 9 , visceral layer and 9', parietal layer of the arachnoid (in blue); 10, subarachnoid space; 11, arachnoid cavity; 12, dura mater (in yellow):-13, periosteum; 13', external periosteum; 14, cellular tissue situated between the dura mater and the wall of the vertebral canal; 15 , common posterior vertebral ligament; 16, intra-spinal veins; 17 . vertebra in section. (Testut.)

It is derived from the neural epiblast, and yields neuro-keratin. (See p:10\%.)

The general rule respecting the size of different parts of the cord appears to be, that each part is in direct proportion in this respect to the size and number of nerve-roots given off from it, and has but little relation to the size or number of those given off below it. Thus the cord is very large in the middle and lower part of its cervical portion, whence arise the large nerve-roots for the formation of the brachial plexuses and the supply of the upper extremities, and again enlarges at the lowest 
part of its dorsal portion and the upper part of its lumbar, at the origins of the large nerves which, after forming the lumbar and sacral plexuses, are distributed to the lower extremities. The chief cause of the greater size at these parts of the spinal cord is increase in the quantity of gray matter; for there seems reason to believe that the white part of the cord becomes gradually and progressively larger from below upward, doubtless from the addition of a certain number of upward passing fibres from each pair of nerves.

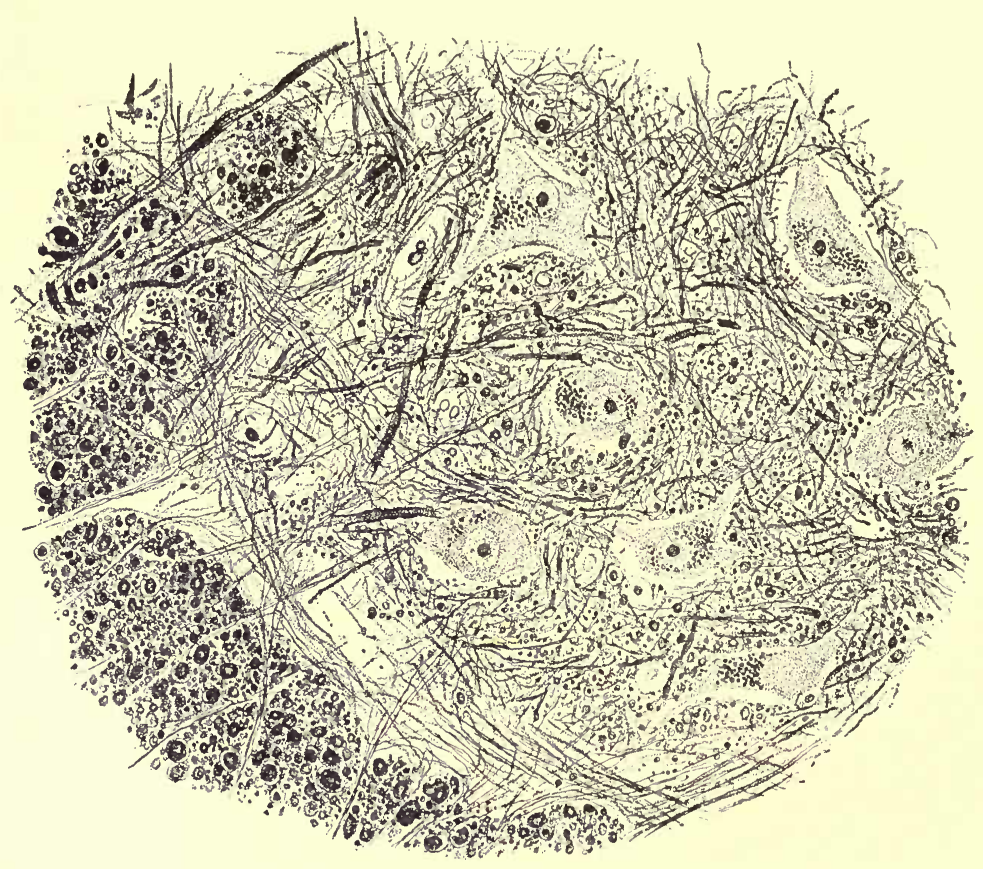

Fig. 351.-From the lower lumbar cord of man, after a preparation by Klönne and Müller, of Berlin ( $Y 0.11,153)$ stained by Weigert and Pal's method. A portion of the gray substance of the ventral cornu with the adjoining portions of the-lateral column is represented, showing anterior horn cells and the fine medullated fibres which enter the gray substance from the lateral column and surround the nerve-cells, which here are provided with fine pigmented granules. High power. (Koelliker.)

From careful estimates of the number of nerve-fibres in a transverse section of the cord toward its upper end, and the number entering or issuing from it by the anterior and posterior roots of each pair of nerves, it has been shown that in the human spinal cord not more than half of the total number of nerve-fibres of all the spinal nerves are contained in a transverse section near its upper end. It is obvious, therefore, that at least half of the nerve-fibres entering it must terminate somewhere in the cord itself. 
The gray matter of the spinal-cord consists of numerous groups of nerve-cells, of a close meshwork of medullated fibres, most of which are very fine and delicate, and of an extremely delicate network of axiscylinders. This latter fine plexus bas been called "Gerlach's network." Mingled with it and supporting it, is the meshwork of the neuroglia, which is finer even, in its structure, than that of the nerve-tissue, so that except under proper staining and illumination, it may appear granular. This is especially developed around the central canal, which is lined with columnar ciliated epithelium, the cells of which at their outer end terminate in fine processes, which join the neurogliar network surrounding the canal, and form the substantia gelatinosa centralis.
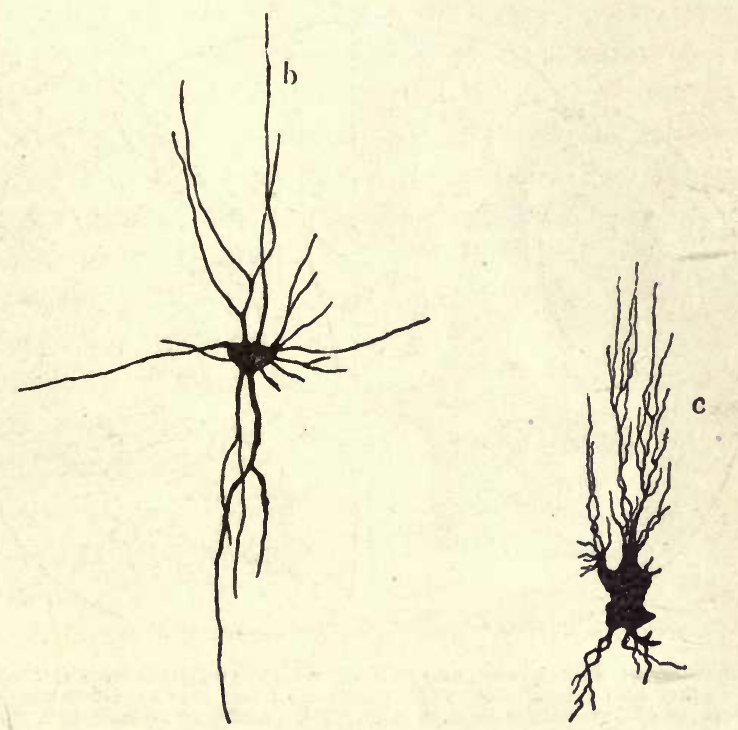

Fig. 351A.-Different types of neuroglia cells. (After v. Gehuchten.) b, Neuroglia cells of the white substance, and $c$, of the gray substance of the cord of an embryo calf.

Neuroglia was formerly thought to be mainly present in the tip of the posterior cornu of gray matter, forming what is known as the substantia gelatinosa lateralis of Rolando, through which the posterior nerve-roots pass. This is now known to be composed of very small nerve-cells and their processes.

Groups of cells in gray matter.-The multipolar cells are either scattered singly or arranged in groups, of which the following are to be distinguished on either side-certain of the groups being more or less marked in all of the regions of the cord, viz., those $(a)$ in the anterior cornu, $(b)$ those in the posterior cornu, and $(c)$ intrinsic cells distributed throughout the gray matter.

(a) The cells in the anterior cornu are large and branching, and 
each gives rise to an axis-cylinder process which passes out in the anterior nerve-root. These cells are everywhere conspicuous, but are particularly numerous in the cervical and lumbar enlargements. In these districts they may be divided into several groups-(i.) a group of large cells close to the tip of the inner part of the anterior cornu-all the cells of the anterior cornu in the dorsal or thoracic region are said to belong to this group; (ii.) several lateral groups (2, $a, b$, and $c$, fig. 353) on the outer side of the gray matter, and (iii.) a certain number of cells at the base of the inner part of the anterior cornu particularly well marked in the thoracic region. (b) Cells of the posterior cornu-these are not numerous; they are small and branched, and each has an axis-cylinder

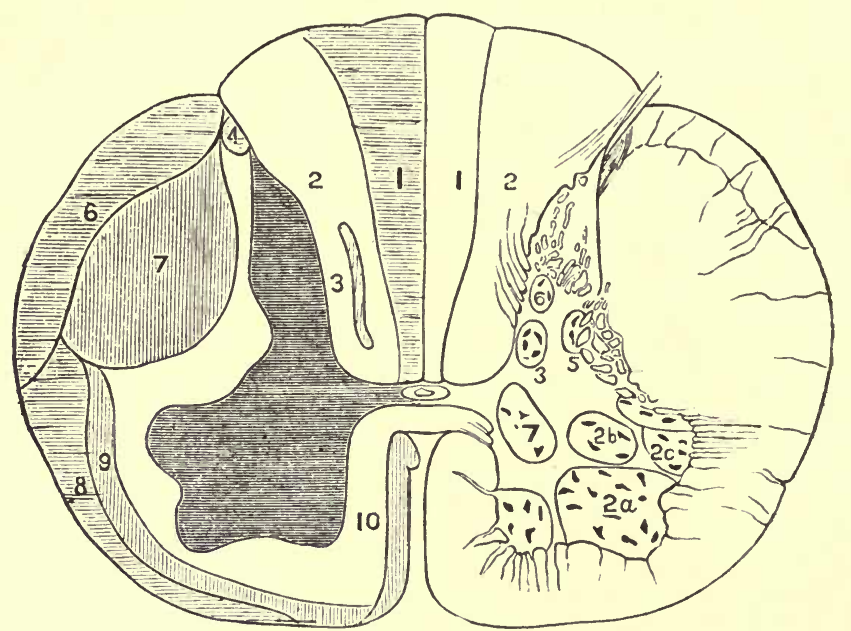

Fig. 352.- Section of spinal cord, one half of which (left) shows the tracts of the white matter, and the other half (right) shows the position of the nerve cells in the gray matter. 7 , 10,9 and 3 are tracts of descending degeneration, 1, 4,6 and 8, of ascending degeneration. Semidiagrammatic. (After Sherrington.)

process passing off; but these processes do not pass into the posterior nerve-roots. The groups are two at least in number, viz., (i.) in connection with the edge of the gray matter externally, where it is considerably broken up by the passage of bundles of fibres through it, and called the lateral reticular formation; and (ii.) in connection with a similar reticular formation, more at the tip of the gray matter of the posterior cornu; this is known as the posterior reticular formation.

A group of cells (No 3, fig. 352) is situated at the base and median side of the posterior cornu. It is formed of fairly large cells, fusiform in shape, and constitutes the posterior vesicular column, or Clarke's column. It extends from the upper lumbar to the lower cervical region. On the outer portion of the gray matter, midway between the anterior and posterior cornua, is a group of cells, known as the cells of the lateral gray column. These are small and spindle-shaped, and are nore or less 
marked in the lumbar region, as well as in the thoracic region (No. 5, fig. 532).

(c) Besides these groups, which have their names largely on account of their location, there are distributed throughout the gray matter a very large number of other cells, which are known as intrinsic cells. These send out neuraxons which pass into the white matter of the same or the opposite side, pass up and down the cord, enter the gray matter again, and connect there by their end-brushes with cells at a different level of the cord. The intrinsic cells are, therefore, in the main, commissural in their function, that is to say, they unite the two sides or different levels of the cord. They are also, themselves, in relation with the fibres and cells of the anterior and posterior cornua.

Columns and tracts in the white natter of the spinal cord.-In addition to the columns of the white matter which are marked out by the points from which the nerve-roots issue, and which are the anterior, the lateral and posterior, the posterior is further divided by a septum of the pia mater into two almost equal parts, constituting the postero-external column, or column of Burdach (fig. 353, 2), and the postero-median, or column of Goll (fig. 353, 1). In addition to these columns, however, it has been shown that the white matter can be still further subdivided. This subdivision has been accomplished by evidence of several kinds, that the parts or, as they are called, tracts in the white matter, perform different functions in the conduction of impulses.

The methods of observation are the following:-

(a) The embryological method. It has been found that if the development of the spinal cord be carefully observed at different stages that certain groups of the nerve-fibres put on their myelin sheath at earlier periods than others, and that the different groups of fibres can therefore be traced in various directions. This is known as the method of Flechsig.

(b) Wallerian or degeneration method.-This method depends upon the fact that if a nerve-fibre is separated from its nerve-cell, it wastes or degenerates. It consists in tracing the course of tracts of degenerated fibres, which result from an injury to any part of the central nervous system. When fibres degenerate below a lesion the tract is said to be of descending degeneration, and when the fibres degenerate in the opposite direction the tract is one of ascending degeneration. By modern methods of staining of the central nervous system it has proved comparatively easy to distinguish degenerated parts in sections of the cord and of other portions of the central nervous system. Degenerated fibres have a different staining reaction when the sections are stained by what are called Weigert's and Marchi's methods. Accidents to the central wervous system in man have given us much information upon this subject, but this has of late years been supplemented and largely extended by the experiments on animals, particularly upon monkeys; 
and considerable light has been by these means shed upon the conduction of impulses to and from the nervous system by the study of the results of section of different parts of the central nervous system, and of the spinal nerve-roots. Thus we have not only embryological evidence mapping out different tracts, but also confirmatory pathological and experimental observations.

The tracts which have been made out are the following:-

\section{(a) Of descending degeneration.}

(i.) The crossed pyramidal tract (fig. 352,7). - This tract is situated to the outer part of the posterior cornu of gray matter. It is found throughout the whole length of the spinal cord; at the lower part it extends to the margin of the cord, but higher up it becomes displaced from this position by the interpolation of another tract of fibres, to be presently described, viz., the direct cerebellar tract. 'The crossed pyramidal tract is large, and may touch the tip of gray matter of the posterior cornu, but is separated from it elsewhere. In shape on crosssection it is somewhat like a lens, but varies in different regions of the cord, and diminishes in size from the cervical region downward. The tract is particularly well marked out, both by the degeneration and the embryological methods. The fibres are supposed to pass off as they descend, and to join the various local nervous mechanisms of nerve cells and their branchings which are represented in the cord. The tract of degeneration may be traced upward beyond the cord, in a way to be presently described. The fibres of which this tract is composed are moderately large, but are mixed with some that are smaller.

(ii.) The direct or uncrossed pyramidal tract (fig. 352, 10).-This tract is situated in the anterior column by the sides of the anterior fissure. It is smaller than (i.), and is not present in all animals, though conspicuous in the human cord and in that of the monkey. It can be traced upward to the cerebral cortex, and downward as far as the mid or lower thoracic region, where it ends.

(iii.) Antero-lateral descending tract (fig. 33\%,9).- An extensive tract, elongated but narrow, and reaching from the crossed to the direct pyramidal tract. It is a mixed tract, since not all of its fibres degenerate below the lesions.

(iv.) Comma tract (fig. 352,3) is a small tract of fibres which degenerate below section or injury of the cord. Its presence has been demonstrated in the cervical and thoracic regions. It is supposed to consist of the descending collaterals of the posterior nerve-roots as they pass inte the postero-external columns.

\section{(b) Of ascending degeneration.}

(i.) Postero-median column (fig. 352,1). - This tract degenerates upward on injury or on section of the cord, as well as on section of the posterior nerve roots. It exists throughont the whole of the cord from below up, and can be traced into the bulb. It consists of fine fibres. 
(ii.) Direct cerebellar tract (fig. 352, 6).-This tract is situated on the outer part of the cord between the crossed pyramidal tract and the margin. It is found in the cervical, thoracic and upper lumbar regions of the cord, and increases in size from below upward. It degenerates on injury or section of the cord itself, but not on section of the posterior nerve-roots. As its name implies it is believed to pass up into the cerebellum. Its fibres are coarse.

(iii.) Antero-lateral ascending tract (Tract of Gowers and Tooth) (fig. $352,8)$. - This tract has been shown on injury to the spinal cord; it is situated at the margin of the cord outside of the corresponding descending tract. It is traceable throughout the whole length of the cord. Its fibres are composed of mixed, fine and coarse, elements.

(iv.) Tract of Lissauer, or posterior marginal zone (fig. 352, 4).-A small tract of fine white fibres, situated at the apex of the posterior horn, is made up of fibres from the posterior nerve-roots which enter the column and pass up and down for a short distance, finally entering the posterior horn where they terminate in fine end-brushes around the cells of the posterior horn.

It will thus be seen that the white matter of the spinal-cord has three general divisions, into the anterior, the lateral, and posterior columns. These columns are subdivided into columns in which the fibres degenerate upward, those in which the fibres degenerate downward, and other columns in which the fibres do not degenerate either way when the cord is cut across. These parts of the cord are composed of commissural fibres which connect different levels of the cord. These commissural columns are the antero-lateral columns, the lateral limiting layer, and the column of Burdach. The arrangement of these columns is shown well in the figure (fig. 352).

Spinal Nerves.-The spinal nerres consist of thirty-one pairs, isstiing from the sides of the whole length of the cord, their number corresponiing with the intervertebral foramina through which they pass. Each nerve arises by two roots, an anterior and posterior, the latter being the larger. The roots emerge through separate apertures of the sheath of dura mater surrounding the cord; and directly after their emergence, where the roots lie in the intervertebral foramen, a ganglion is found on the posterior root. The anterior root lies in contact with the anterior surface of the ganglion, but none of its fibres intermingle with those in the ganglion (fig. 350,4 ). Bnt immediately beyond the ganglion the two roots coalesce, and by the mingling of their fibres form a compound or mixed spinal nerve, which, after issuing from the intervertebral canal, gives off anterior and posterior or ventral and dorsal branches, each containing fibres from both the roots (fig. 350), as well as a third or visceral branch, ramus communicans, to the sympathetic.

The anterior roat of each spinal nerve arises by numerous separate 
and converging bundles from the anterior column of the cord; the posterior root by more numerous parallel bundles, from the posterior column, or, rather, from the posterior part of the lateral column (fig. 350), for if a fissure be directed inward from the groove between the middle and posterior columns, the posterior roots will remain attached to the former. The anterior roots of each spinal nerre consist chiefly of efferent fibres; the posterior exclusively of afferent fibres.

Course of the Fibres of the Spinal Nerve-Roots. - (a) The Anterior roots enter the cord in several bundles, which may be called:-(1) Internal; (2) Middle; (3) External; all being more or less connected with the groups of multipolar cells in the anterior cornua. 1. The internal fibres are partiy connected with internal group of verve-cells of the

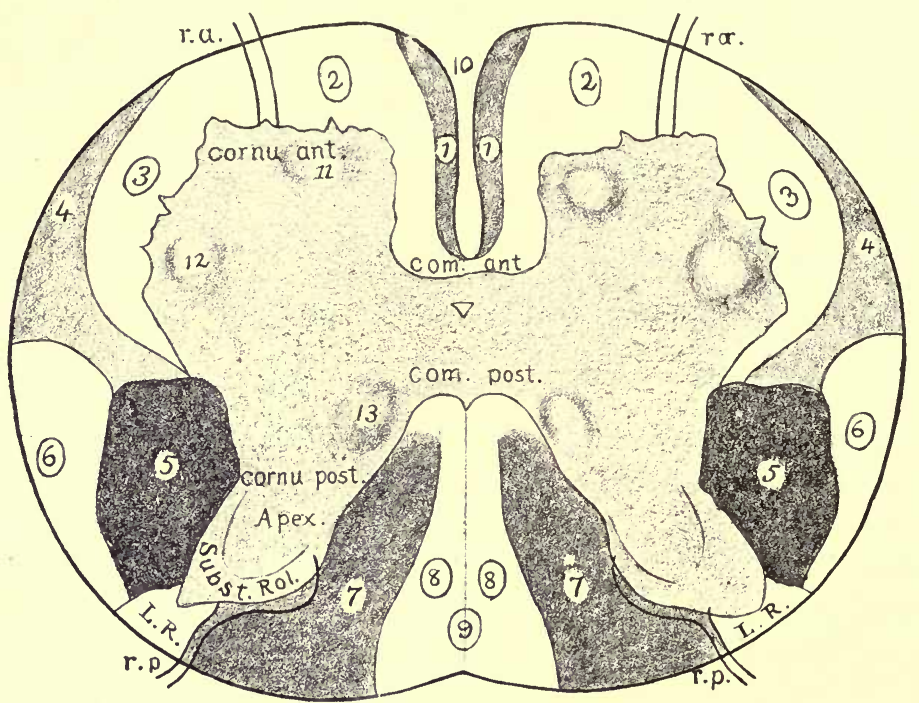

Fig. 352A. - Section of the spinal cord, showing the arrangement of the white and gray matter. 1, Direct pyramidal tract; 2,3 , antero-lateral column: 4, ascending lateral column; 5 , crossed pyramidal tract; 6 , direct cerebral tract; $\gamma$, column of Burdach; 8 . column of Goll; $\gamma$, posterior median fissure; 10, anterior median fissure; 11, 12 . anterior horn cells; 13 , Clarke's column; I. R., Lissauer's column; $r p$, posterior root; $r a$, anterior root.

anterior cornu of the same side; but some fibres send collaterals through the anterior commissure to end in the anterior cornu of opposite side, probably in the internal group of cells. 2. The middle fibres are partly in connection with the lateral group of cells in anterior cornu, and in part pass backward to the posterior cornu, having no immediate connection with cells. 3. The external fibres are partly in connection with the lateral group of cells in the anterior cornu, but some fibres proceed direct into the lateral column without connection with cells, and pass upward in it.

Besides these fibres, there are some which do not appear to have any connection with the anterior horn cells, but pass directly through to 
connect with groups of intrinsic cells in the median or posterior portion of the gray matter of the cord.

(b) The posterior roots enter the spinal cord to the inner or median side of the posterior cornu. The fibres, as soon as they reach the cord, divide in a fork-like fashion, ove branch passing down a short distance (only about three centimetres), the other branch passing up for a longer or shorter distance. This upper branch sometimes reaches nearly the whole extent of the cord, but generally it extends over only one or two segments of the cord. These divisions of the posterior root fibres give off in their course numerous collaterals. The nerve-fibres of the posterior roots are divided into two sets, an internal or median, an ex-

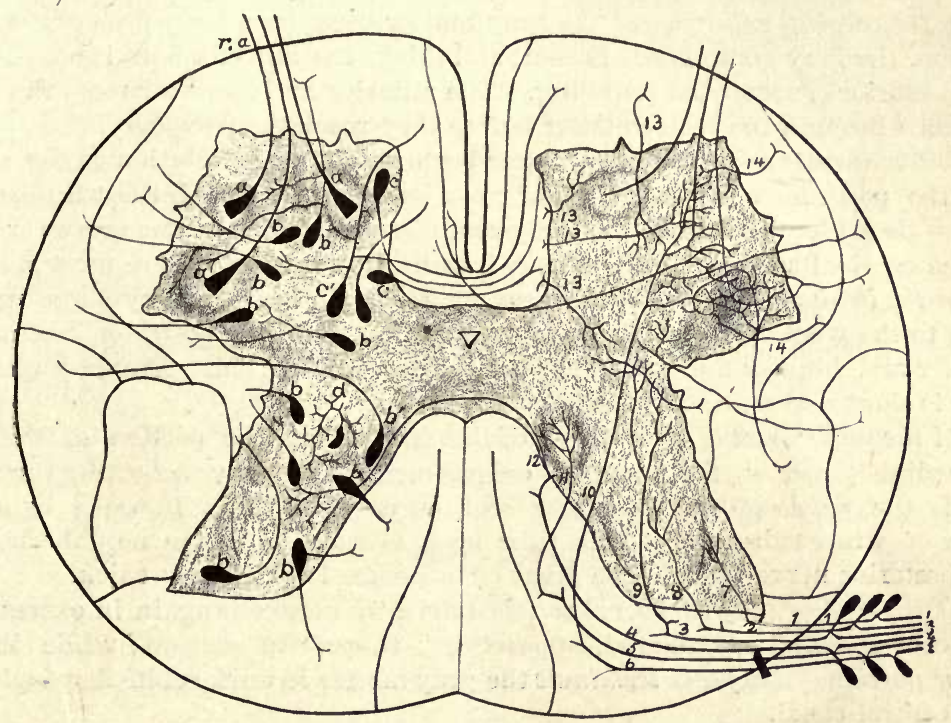

Fig. 353.-Section of the spinal cord showing the grouping of nerve-cells and the course of nervefibres entering in posterior and anterior roots.

ternal or lateral. The lateral set consists mostly of small fibres, and it enters the cord opposite the tip of the posterior horn. The fibres pass in part to the marginal column of Lissauer, where they ascend and descend; in part they penetrate the posterior horn, and come in relation with its cells. The median set sends some fibres which pass to Clarke's column of cells, others pass by way of the posterior commissure to the median cells of the other side. Some others pass through the median gray matter to the anterior horn cells of the same side. Thus the posterior root-fibres are connected with all the cell groups of the posterior horn, of the anterior horn of the same side, and the cells of the median gray of the opposite side. Besides this, they are connected through col- 
laterals with the intrinsic cells of the gray matter at different levels of the cord. One can realize that each nerve-root has, in this way, an effective grip upon a large extent of the cord. This is seen well by studying figs. $352 \mathrm{~A}$ and 353 .

The Peculiarities of different regions of the Spinal Cord.-The outline of the gray matter and the relatice proportion of the white matter varies in different regions of the spinal cord, and it is, therefore, possible to tell approximately from what region any given transverse section of the spinal cord has been taken. The white matter increases in amount from below upward. The amount of gray matter varies; it is greatest in the cerrical and lumbar enlargements, viz., at and about the 5th lumbar and 6th cervical nerre, and least in the thoracic region. The greatest development of gray matter corresponds with greatest number of nerre-fibres passing from the cord.

In the cervical enlargement the gray matter occupies a large proportion of the section, the gray commissure is short and thick, the anterior horn is blunt, while the posterior is somewhat tapering. The anterior and posterior roots run some distance through the white matter before they reach the periphery.

In the dorsal region the gray matter bears only a small relation to the white, and the posterior roots in particular run a long course through the white matter before they leave the cord; the gray commissure is thinner and narrower than in the cervical region. The tractus intermedio-lateralis is here most marked.

In the lumbar enlargement the gray matter again bears a very large proportion to the whole size of the transverse section, but its posterior cornua are shorter and blunter than they are in the cervical region. The gray commissure is short and extremely narrow.

At the upper part of the comus medullaris, which is the portion of the cord immediately below the lumbar enlargement, the gray substance occupies nearly the whole of the transverse section, as it is only invested by a thin layer of white substance. This thin layer is wanting in the neighborhood of the posterior nerve-roots. The great commissure is extremely thick.

At the level of the fifth sacral vertebra the gray matter is again in excess, and the central canal is enlarged, appearing T-shaped in section; while in the upper portion of the filum terminale the gray matter is uniform in shape without any central canal.

The shape of the cord changes from the sacral and lumbar region where it is circular to the thoracic where it is oval, and to the cervical where the lateral diameter considerably exceeds the antero-posterior; the change in shape is dne to a gradual increase of the lateral columns.

The Spixal Cord axd Nerve-Roots a Mass of Nerve-Units. We have, in the foregoing, described the spinal cord as being composed of white and gray matter, and these substances, in turn, being composed of nerve-fibres and nerve-cells, and a supporting substance called neuroglia. From the physiologist's point of view, the spinal cord is considered to be composed of a mass of nerve-units or neurons. These are divided into three great classes: the motor neurons, the sensory neurons, and the intermediate neurons. The motor neurons make up the larger part of the nerve-tissue in the anterior horns; their neuraxons pass out 
into the anterior roots. The sensory neurons have their cells or starting-points in the posterior spinal ganglia, these being large ganglionic masses which lie upon the posterior roots. These cells have a process which runs spineward through the posterior roots into the spinal cord, and another which runs peripherally, forming the sensory nerve. The intermediate neurons have their cells of origin in the posterior horus and median part of the gray matter, and, to a slight extent, in the anterior horns. Their cells form the intrinsic cells of the spinal cord, and also assist in the conduction of sensory and other afferent impulses. For example, the neurons, starting with the cells lying in Clarke's column, send their processes up into the cerebellum, and thus continue afferent impulses brought to the neurons through the posterior roots. On the other hand, other groups of cells lie in the lateral part of the gray matter and give rise to processes which pass out in to the lateral columns and then enter the gray matter again, to connect with cells at different levels. These are the intermediate neurons which are commissural in their functions.

\section{Functions of the Spinal Nerve-Roots.}

The anterior spinal nerve-roots are efferent in function: the posterior are afferent. The fact is proved in various ways. Division of the anterior roots of one or more nerves is followed by complete loss of motion in the parts supplied by the fibres of such roots; but the sensation of the same parts remains perfect. Division of the posterior roots destroys the sensibility of the parts supplied by their fibres, while the power of motion continues unimpaired. Moreover, irritation of the ends of the distal portions of the divided anterior roots of a nerve excites muscular movements; irritation of the ends of the proximal portions, which are still in connection with the cord, is followed by no appreciable effect. It must be remembered, however, that in the anterior or efferent nerves other besides motory are contained, e.g., vaso-motor, secretory, heat fibres, and it may be supposed that when the distal end of a divided nerve is stimulated, the effects would be exercised not only upon muscles, but upon glands, blood-vessels, etc. Irritation of the distal portions of the divided posterior roots, on the other hand, produces no muscular movements and no manifestations of pain; for, as already stated, sensory nerves convey impressions only toward the nervous centres: but irritation of the proximal portions of these roots elicits signs of intense suffering. Occasionally, under this last irritation, muscular movements also ensue; but these are either voluntary, or the result of the irritation being reflected from the sensory to the motor fibres. Occasionally, too, irritation of the distal ends of divided anterior roots elicits signs of pain, 
as well as producing muscular movements: the pain thus excited is probably the result either of cramp or of so-called recurrent sensibility.

Recurrent Sensibility. - If the anterior root of a spinal nerve be divided, and the peripheral end be irritated, not only movements of the muscles supplied by the nerve take place, but also of other muscles, indicative of pain. If the main trunk of the nerve (after the coalescence of the roots beyond the ganglion) be divided, and the anterior root be irritated as before, the general signs of pain still remain, although the contraction of the muscles does not occur. The signs of pain disappear when the posterior root is divided. From these experiments it is believed that the stimulus passes down the anterior root to the mixed nerve, and returns to the central nervous system through the posterior root by means of certain sensory fibres from the posterior root, which loop back into the anterior root before continuing their course into the mixed nerve-trunk. These fibres degenerate when the posterior nerveroot is divided beyond the ganglion.

Functions of the Ganglia on Posterior Roots. -The cells of the posterior ganglia act as centres for the nutrition of the nerve-fibres given off from them. When these are cut, the parts of the nerves so severed degenerate, while the parts which remain in connection with the cells do not. Thus on section of the posterior nerve-root beyond the ganglion the peripheral part wastes and the central does not, and on section of the root between the ganglion and the cord the central part to a great extent wastes and the peripheral remains unaffected.

\section{Functions of the Spinal Cord.}

The power of the spinal cord, as a nerve-centre, may be arranged under the heads of (1) Conduction; (2) Reflex action.

(1) Conduction.-The functions of the spinal cord in relation to conduction may be best remembered by considering its anatomical connections with other parts of the body. From these it is evident that there is no way by. which nerve-impulses can be conveyed from the trunk and extremities to the brain, or vice versa, other than that formed by the spinal cord. Through it, the impressions made upon the peripheral extremities or other parts of the spinal sensory nerves are conducted to the brain, where alone they can be perceived. Through it, also, the stimulus of the will, conducted from the brain, is capable of exciting the action of the muscles supplied from it with motor nerves. And for all these conductions of impressions to and fro between the brain and the spinal nerves, the perfect state of the cord is necessary; for when any part of it is destroyed, and its communication with the brain is interrupted, impressions on the sensory nerves given off from it below the 
seat of injury, cease to be propagated to the brain, and the brain loses the power of voluntarily exciting the motor nerves proceeding from the portion of cord isolated from it. Illustrations of this are furnished by various examples of paralysis, but by none better than by the common paraplegia, or loss of sensation and voluntary motion in the lower part of the body, in consequence of destructive disease or injury of a portion, including the whole thickness, of the spinal cord. Such lesions destroy the communication between the brain and all parts of the spinal cord below the seat of injury, and consequently cut off from their connection with the brain the various organs supplied with nerves issuing frnm those parts of the cord.

It is not probable that the conduction of motor or sensory impulses is effected under ordinary circumstances (to any great extent), as was fermerly supposed, through the gray substance, i.e., through the nervecorpuscles and filaments connecting them. All parts of the cord are not alike able to conduct all impressions; and as there are separate nervefibres for motor and for sensory impressions, so in the cord, separate and determinate tracts serve to conduct always the same kind of impression. The sensations of touch, temperature, and pain, however, do not appear to have such sharply limited tracts as the motor impulses.

Experimental and other observations point to the following conclusions regarding the conduction of sensory and motor impressions through the spinal cord. Many of these conclusions must, however, be received with considerable reserve.

a. Sensory Impressions.-By sensory impressions are here meant the sensations of touch and pain, of heat and cold, and of muscular sense. These impressions are conveyed to the spinal cord by the posterior nerveroots. Part of them are then carried directly into the postero-median column on the same side, and thence up to the nucleus of this column in the medulla. It is mainly the impulses of muscle sense that are thus carried. Other sensations are carried by the posterior root-fibres to the cells of the column of Clarke. From there the impulses are conveyed to the direct cerebellar tract on the same side, and thence up to the cerebellum. These are mainly sensations that subserve the sense of equilibrium, and are closely connected in function with those which pass np the column of Goll to its nucleus. The impressions of touch and pain, and of heat and cold, are conveyed to the nerve-cells in the posterior cornua of the same side in part, and in part to the nerve-cells in the posterior cornua and median gray of the opposite side. From this point, the impulse is taken up again by intermediary neurons and conveyed through the anterior and lateral columns of the cord, in the ascending tract of Gowers and Tooth, to the brain. By reason of the great number of collaterals and the interpolation in the course of the sensory impulse of many intermediary neurons, no very sharply defined tract has yet been satisfactorily made out in the spinal cord for the conduction of 
these sensations of temperature, pain, and touch. If one set of fibres is destroyed by disease, others seem able, through the collaterals, to take up its function. We can only say that most of these sensory impressions pass up in the lateral and anterior columns. It is probable, also, that pain and temperature sensations cross over at once, to a considerable extent, and pass up in the opposite side of the cord to which they enter. Touch and pressure sensations, as well as muscle-sense impressions, and sensations of equilibrium, pass up largely upon the same side until they reach the medulla or cerebellum.

The direct cerebellar tract is believed to commence in the cells of the posterior vesicular column of Clarke of the same side; it goes chiefly to the cerebellum, through the restiform body, but is said also to contain fibres which pass up as far as the corpora quadrigemina and then turn backward and lying near the brachium pass to the cerebellum. The fibres of the antero-lateral ascending tract are believed to arise from the gray matter of the posterior cornu. In the case of the ascending tracts, with the exception of the posterior median column, the connection with the posterior nerve-roots is not direct.

b. Motor Impressions.-Motor impressions are conveyed downward from the brain along the pyramidal tracts, viz., the direct or anterior, and the crossed or lateral, chiefly in the latter. Generally speaking, the impressions pass down on the side opposite to which they originate, having undergone decussation in the medulla; but some impressions do not cross in the medulla, but lower down, in the cord, being conveyed by the anterior or uncrossed pyramidal fibres, and decussate in the anterior commissure. The motor-fibres for the legs partially pass dowuward in the lateral columns of the same side. This is also probably the case with the bilateral muscles, i.e., muscles of the two sides acting together, such as the intercostal muscles and other muscles of the trunk, as well as the costo-humeral muscles.

It is quite certain, as was just now pointed out, that the fibres of the anterior nerve-roots are more numerous than the fibres proceeding downward from the brain in the pyramidal tracts, or the so-called pyramidal fibres. This is because each pyramidal fibre is really a very long nerve process or neuraxon, and is supplied in its course with a large number of collaterals, which go off at different points, and thus put it in relation with different groups of nerve-cells in the anterior cornua at various levels. Each nerve-fibre of the pyramidal tract, by means of its collaterals, can control a number of nerve-cells, and can thus co-ordinate the action of impulses sent out through the anterior roots to a number of groups of muscles. In other words, the gray matter of the anterior cornua contains an apparatus with various complicated co-ordinating powers, which apparatus is under the control of the neurons whose cells of origin are in the cortex of the brain. This apparatus is also reflexly influenced by sensory impressions passing to the cord. 
Division of the anterior pyramids of the medulla at the point of decussation is followed by paralysis of motion, never quite absolute, in all parts below. Disease or division of any part of the cerebro-spinal axis above the seat of decussation is followed by impaired or lost power of motion on the opposite side of the body; while a like injury inflicted below this part induces similar, never quite absolute no doubt, on the corresponding side.

When one half of the spinal cord is cut through in monkeys, the following results follow (Mott):-Motor paralysis of the muscles of the same side (never complete of muscles used in bilateral associated action), followed by gradual recovery of muscular movement, except of the finer movements of the hand and foot; wasting and flabbiness of the muscles; sensory paralysis of the same side (temperature, touch, pain and pressure); temporary vaso-motor paralysis on same side. The temperature of the affected side was depressed 1 to $3^{\circ}$ (F.).

Reflex Action. - In man the spinal cord is so much under the control of the higher nerve-centres, that its own individual functions in relation to reflex action are apt to be overlooked; so that the result of injury, by which the cord is cut off completely from the influence of the encephalon, is apt to lessen rather than increase our estimate of its importance and individual endowments. Thus, when the human spinal cord is divided, the lower extremities fall into any position that their weight and the resistance of surrounding objects combine to give them; and if the body is irritated, they do not move toward the irritation; and if they are touched, the consequent reflex movements are disorderly and purposeless; all power of voluntary movement is absolutely abolished. In other mammals, however, e.g., in the rabbit or dog, after recovery from the shock of the operation, which takes some time, reflex action will occur in the parts below after the spinal cord has been divided, a very feeble irritation being followed by extensive and co-ordinate movements. In the case of the frog, and many other cold-blooded animals, in which experimental and other injuries of the nerve-tissues are better borne, and in which the lower nerve-centres are less subordinate in their action to the higher, the reflex functions of the cord are still more clearly shown. When, for example, a frog's head is cut off, its limbs remain in, or assume a natural position; they resume it when disturbed; and when the abdomen or back is irritated, the feet are moved with the manifest purpose of pushing away the irritation. The main difference in the cold-blooded animals being that the reflex movements are more definite, complicated, and effective, although less energetic than in the case of mammals. It might indeed be thought, on superficial examination, that the mind of the animal was engaged in the acts; and yet all analogy would lead us to the belief that the spinal cord of the frog has no different endowment, in kind, from those which 
belong to the cord of the higher vertebrata: the difference is only in degree. And if this be granted, it may be assumed that, in man and the higher animals, many actions are performed as reflex movements occurring through and by means of the spinal cord, although the latter cannot by itself initiate or even direct them independently.

Cutaneous and Muscle Reflexes. - In the human subject two kinds of reflex actions dependent upon the spinal cord are usually distinguished, the alterations of which, either in the direction of increase or of diminution, are indications of some abnormality, and are used as a means of diagnosis in nervous and other disorders. They are termed respectively (a.) cutctneous reflexes, and (b.) muscle reflexes. (a.) Cutaneous reflexes are set up by a gentle'stimulus applied to the skin. The subjacent muscle or muscles contract in response. Although these cutaneous reflex actions may be demonstrated almost anywhere, yet certain of such actions as being most characteristic are distinguished, e.g., plantar reflex; glutear reflex, i.e., a contraction of the gluteus maximus when the skin over it is stimulated; cremaster reflex, retraction of the testicle when the skin of the inside of the thigh is stimulated, and the like. The ocular reflexes, too, are important. They are contraction of the iris on exposure to light, and its dilatation on stimulating the skin of the cervical region. All of these cutaneons reflexes are true reflex actions. They differ in different individuals, and are more easily elicited in the young. Muscle reflexes, or as they are often termed, tendon reflexes, consist of a contraction of a muscle under conditions of more or less tension, when its tendon is sharply tapped. The so-called patellartendon-reflex is the most well-known of this variety of reflexes. If one knee be slightly flexed, as by crossing it over the other, so that the quadriceps femoris is extended to a moderate degree, and the patella tendon be tapped with the fingers or the earpiece of a stethoscope, the muscle contracts and the foot is jerked forward.

Another variety of the same phenomenon is seen if the foot is flexed so as to stretch the calf muscles and the tendo Achillis is tapped; the foot is extended by the contraction of the stretched muscles. It appears, however, that the tendon reflexes are not exactly what their name implies. The interval between the tap and the contraction is said to be too short for the production of a true reflex action. It is suggested that the contraction is caused by local stimulation of the muscle, but that this would not occur unless the muscle had been reflexly stimulated previously by the tension applied, and placed in a condition of excessive irritability. It is further probable that the condition on which it depends is a reflex spinal irritability of the muscle or (exaggerated) muscular tone, which is admitted to be a reflex phenomenon-or an example of automatism-in the spinal cord. 
Inhibition of Reflex Actions. - Movements such as are produced by irritating the skin of the lower extremities in the human subject, after division or disorganization of a part of the spinal cord, do not follow the same irritation when the cerebrum is active and the connection between the cord and the brain is intact. This is, probably, due to the fact that the mind ordinarily perceives the irritation and instantly inhibits or controls the action; for, even when the cord is perfect, such involuntary movements may follow an irritation, applied when the cerebrum is inactive. When, for example, one is anxiously thinking, even slight stimuli may produce involuntary and reflex movements. So, also, during sleep, such reflex movements may be observed, when the skin is touched or tickled; for example, when one touches with the finger the palm of the hand of a sleeping child, the finger is grasped-the impression on the skin of the palm producing a reflex movement of the muscles which close the hand. But when the child is awake, no such effect is produced.

Further, many reflex actions are capable of being more or less controlled or even altogether prevented by the will: thus an inhibitory action may be exercised by the cerebrum over reflex functions of the cord and the other nerve-centres. The following may be quoted as familiar examples of this action:-

To prevent the reflex action of crying out when in pain, it is often sufficient firmly to clench the teeth or to grasp some object and hold it tight. When the feet are tickled we can, by an effort of will, prevent the reflex action of jerking them up. So, too, the involuntary closing of the eyes and starting, when a blow is aimed at the head, can be similarly restrained.

Darwin has mentioned an interesting example of the way in which, on the other hand, such an instinctive reflex act may override the strongest effort of the will. He placed his face close against the glass of the cobra's cage in the Reptile House at the Zoological Gardens, and though, of course, thoroughly convinced of his perfect security, could not by any effort of the will prevent himself from starting back when the snake struck with fury at the glass.

It has been found by experiment that in a frog the optic lobes and optic thalami have a distinct action in inhibiting or delaying reflex action, and also that more generally any afferent stimulus, if sufficiently strong, may inhibit or modify any reflex action even in the absence of these centres.

On the whole, therefore, it may, from these and like facts, be concluded that reflex acts, performed under the influence of the reflecting power of the spinal cord, are essentially independent of the brain and may be performed perfectly when the brain is separated from the cord: that 
these include a much larger number of the natural and purposive movements of the lower animals than of the warm-blooded animals including man: and that over nearly all of them the mind may exercise, through the higher nerve-centres, some control; determining, directing, hindering, or modifying them, either by direct action, or by its power over associated muscles.

To these instances of spinal reflex action, some add yet many more, including nearly all the acts which seem to be performed unconsciously, such as those of walking, running, writing, and the like: for these are really involuntary acts. It is true that at their first performances they are voluntary, that they require education for their perfection, and are at all times so constantly performed in obedience to a mandate of the will, that it is difficult to believe in their essentially involuntary nature. But the will really has only a controlling power over their performance; it can hasten or stay them, but it has little or nothing to do with the actual carrying out of the effect. And this is proved by the circumstance that these acts can be performed during complete mental abstraction: and, more than this, that the endeavor to carry them out entirely by the exercise of the will is not only not beneficial, but positively interferes with their harmonious and perfect performance. Any one may convince himself of this fact by trying to take each step as a voluntary act in walking downstairs, or to form each letter or word in writing by a distinct exercise of the will.

These actions, however, will be again referred to.

Morbid reflex actions. - The relation of the reflex action to the strength of the stimulus is the same as was shown generally to occur in nervecentres, a slight stinulus producing a slight morement, and a greater, a greater movement, and so on; but in instances in which we must assume that the cord is morbidly more irritable, i.e., apt to issue more nervous force than is proportionate to the stimulus applied to it, a slight impression on a sensory nerve produces extensive reflex movements. This appears to be the condition in the disease called tetanus, in which a slight touch on the skin may throw the whole body into convulsions.

Special Centres. - It may seem to have been implied that the spinal cord as a single nerve-centre, reflects alike from all parts all the impressions conducted to it. This, however, is not the case, and it should be regarded as we have indicated, as a collection of nervous centres united in a continuous column. This is well illustrated by the fact that segments of the cord may act as distinct nerve-centres, in which special co-ordinated muscular actions are represented, and excite muscular action in the parts supplied with nerves given off from them; as well as bv the analogy of certain cases in which the muscular movements of single 
organs are under the control of certain circumscribed portions of the cord. The special centres are the following (on each side) :-

(a.) The Defacation, or Ano-Spinal centre.-The mode of action of the ano-spinal centre appears to be this. The mucous membrane of the rectum is stimulated by the presence of fæces or of gas in the bowel. The stimulus passes up by the afferent nerves of the hæmorrhoidal and inferior mesenteric plexus to the centre in the cord, situated in the lumbar enlargement, and is reflected through the pudendal plexus to the anal sphincter on the one hand, and on the other to the muscular tissue in the wall of the lower bowel. In this way is produced a relaxation of the first and a contraction of the second, and expulsion of the contents of the bowel follows. 'The centre in the spinal cord is partially under the control of the will, so that its action may be either inhibited or augmented. The action may be helped by the abdominal muscles which are under the control of the will, although under a strong stimulus they may also be compelled to contract by reflex action.

(b.) The Micturition, or the Vesico-Spinal centre. - The vesico-spinal centre acts in a very similar way to that of the ano-spinal. The centre is also in the lumbar enlargement of the cord. It may be stimulated to action by impulses descending from the brain, or reflexly by the presence of urine in the bladder. The action of the brain may be voluntary, or it may be excited to action by the sensation of distention of the bladder by the urine. The sensory fibres concerned are the posterior roots of the lower sacral nerves. The action of the centre thus stimulated is double, or it may be supposed that the centre consists of two parts, one which is usually in action and maintains the tone of the sphincter, and the other which causes contraction of the bladder and other muscles. When evacuation of the bladder is to occur, impulses are sent to one part of the centre on the one hand, and from it to the bladder and to certain other muscles which cause their contraction, and on the other to the other part of the centre, inhibiting its action on the sphincter urethræ which procures its relaxation. The way having been opened by the relaxation of the sphincter, the urine is expelled by the combined action of the bladder and accessory muscles. The cerebrum may act not only in the way of stimulating the centre to action, but also in the way of inhibiting its action. The abdominal muscles may be called into action as in defæcation.

(c.) The Emission of Semen, or Genito-Spinal centre.-The centre situated in the lumbar enlargement of the spinal cord is stimulated to action by sensory impressions from the glans penis. Efferent impulses from the centre excite the successive and co-ordinate contractions of the muscular fibres of the vasa deferentia and vesiculæ seminales, and of the accelerator urinæ and other muscles of the urethra; and a forcible expul- 
sion of semen takes place, over which the mind has little or no control, and which, in cases of paraplegia, may be unfelt.

(d.) The Erection of the Penis centre.-This centre is also situated in the lumbar region. It is excited to action by the sensory nerves of the penis. Efferent impulses produce dilatation of the ressels of the penis, which also appears to be in part the result of a reflex contraction of the muscles by which the veins returning the blood from the penis are compressed.

(e.) Parturition centre.-The centre for the expulsion of the contents of the uterus in parturition is situated in the lumbar spinal cord rather higher up than the other centres already enumerated. The stimulation of the interior of the uterus by its contents may, under certain conditions, excite the centre to send out impulses which produce a contraction of the uterine walls and expulsion of the contents of the cavity. The centre is independent of the will since delivery can take place in paraplegic women, and also while a patient is under the influence of chloroform. Again, as in the cases of defæcation and micturition, the abdominal muscles assist; their action being for the most part reflex and involuntary.

(f.) The Centre for the Movements of Lymphatic Hearts of Frog.Volkmann has shown that the rhythmical movements of the anterior pair of lymphatic hearts in the frog depend upon nervous influence derived from the portion of spinal cord corresponding to the third vertebra, and those of the posterior pair on influence supplied by the portion of cord opposite the eighth vertebra. The movements of the heart continue, though the whole of the cord, except the above portions, be destroyed; but on the instant of destroying either of these portions, though all the rest of the cord be untouched, the movements of the corresponding hearts cease.

(y.) The Centre for the Tone of Muscles. - The influence of the spinal cord on the sphincter ani and sphincter urethræ has been already mentioned (see above). It maintains these muscles in permanent contraction. The condition of these sphincters, however, is not altogether exceptional. It is the same in kind, though it exceeds in degree that condition of muscles which has been called tone, or passive contraction; a state in which they always when not active appear to be during health, and in which, thongh called inactive, they are in slight contraction, and certainly are not relaxed, as they are soon after death, or when the spinal cord is destroyed. This tone of all the muscles of the trunk and limbs depends on the spinal cord, just as the contraction of the sphincters does. If an animal be killed by injury or removal of the brain, the muscles retain their tone; but if the spinal cord be destroyed, the sphincter ani relaxes, and all the muscles feel loose, flabby, and atonic, remaining so till rigor mortis commences. 
This kind of tone must be distinguished from that mere firmness and tension which it is customary to ascribe, under the name of tone, to all tissues that feel robust and not flabby, as well as to muscles. The tone peculiar to muscles has in it a degree of vital contraction: that of other tissues is only due to their being well nourished, and therefore compact and tense.

All the foregoing examples illustrate the fact that the spinal cord is a collection of reflex centres, upon which the higher centres act by sending down impulses to set in motion, modify or control them. The movements or other phenomena of reflex action are, as it were, the function of the ganglion cells to which an afferent impression is conveyed by the posterior nerve-trunks in connection with them. 'The extent of the movement depends upon the strength of the stimulus, the position in which it is applied as well as the condition of the nerve-cells; the connection between the cells being so intimate that a series of co-ordinated movements may result from a single stimulation. Whether the cells possess as well the power of originating impulses (automatism) is doubtful, but this is possible in the case of $(h)$ vaso-motor centres which are situated in the cord (p. 246), and of (i) sweating centres which must be closely related to them, and possibly in the case of $(j)$ the centres for maintaining the tone of muscles.

The Nutrition (a) of the muscles appears to be under the control of the spinal cord. When the nerve-cells of the anterior cornu are diseased the muscles atrophy. In the same way $(b)$ the bones and $(c)$ joints are seriously affected when the cord is diseased. The former when the anterior nerve-cells are implicated, do not grow, and the latter are disorganized in some cases when the posterior columns are affected. (dl) The skin, too, is evidently only maintained in a healthy condition as long as the cord and its nerves are intact. No doubt part of this influence which the cord exercises over nutrition is due to the relationship which it bears to the vaso-motor nerves.

Within the cord are contained, for some distance, fibres $(a)$ which regulate the dilatation of the pupil, (b) which have to do with the glycogenic function of the liver, $(c)$ which control the nerve-supply of the vessels of the face and head, $(d)$ which produce acceleration of the heart's action, and, $(e)$ have a termotaxic action on the muscles, etc.

\section{The Relations of the Different Parts of the Brain.}

Before considering the parts of the brain separately, it will be best for the comprehension of the plan of its construction to take a general survey of the whole. The brain on superficial examination presents four distinct parts, viz. (a.) The large and prominent masses of nervous 
matter divided by fissures into convolutions (fig. 354), and covering to a large extent the other parts, separated from one another by a deep fissure running from front to back. These constitute the cerebral hemispheres or cerebrum. (b) On the under or central surface of the brain can be seen a broad mass rounded on the surface more or less quadrilateral in shape; this is the pons Varolii (fig. 354, VI.). An-

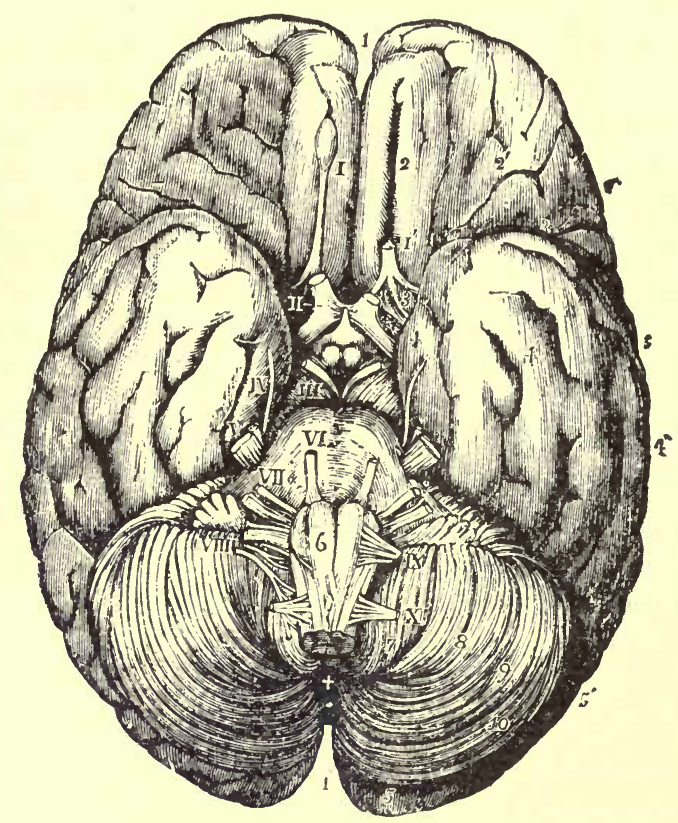

Fig. 354.-Base of the brain. 1, superior longitudinal fissure; 22, $2^{\prime}, 2^{n}$, anterior cerebral lobe; 3, fissure of Sylvius, between anterior and $4,4^{\prime}, 4^{\prime \prime}$. middle cerebral lobe; $5,5^{\prime}$, posterior lobe; 6 , medulla oblongata. The figure is in the right anterior pyramid; $7,8,9,10$, the cerebellum; + , the inferior verimform process. The figures from I. to IX. are placed against the corresponding cerebral nerves; III. is placed on the right crus cerebri. VI. and VII. on the pons Varolii; $\mathbf{X}$. the first cervical or suboccipital nerve. (Allen Thomson.) $1 / 2$.

teriorly it is seen to branch off into two strands, which are the crura cerebri; and posteriorly it joins with a narrower portion, which is the medulla oblongata or bulb. This latter is continuous with the spinal cord. In connection with the bulb and pons are seen many nerve-trunks passing off; these are the chief part of the cranial nerves. Two of the cranial nerves, however, are more interior, and one, the optic (fig. 354, 2), is seen to send off a broad band of fibres which apparently passes into the substance of the cerebrum. The most anterior nerve-root on either side, viz., the olfactory (fig. 354,1 ), extends for some distance upon the under surface of each cerebral hemisphere. (c.) The pons is seen to be connected laterally with a large mass of nervous matter, upon which in the position of the brain turned upward, the bulb also rests; 
this is the cerebellum, and $(d$.$) When the brain is viewed in the normal$ position at the bottom of the fissure, between the hemispheres is seen a broad band of white matter connecting one hemisphere with its fellow, the main commissure or corpus callosum (fig. 35\%). Such parts of the brain are evident even on superficial examination. On dissection, it is found that the central nervous system is not a solid mass of nerve material; it incloses certain cavities, the cerebral ventricles. Forming the walls and boundaries of these ventricles are very important masses of nervous matter. The cerebrum proper incloses a large central cavity, the lateral ventricle, but separated by a median partition into two. Into the cavity of each lateral ventricle (fig. 355) projects a rounded mass of gray matter anteriorly, which is the caudate nucleus of an important structure known as the corpus striatum, the more external part of which, the lenticular nucleus, is embedded in the mass of the cerebral hemisphere. Below, or more posterior to the caudate nucleus, and also projecting into the lateral ventricle, is a second mass of gray matter, called the optic thalamus; the upper part of this only, however, is seen in the lateral ventricle, the lower and more internal part approaching its fellow in the middle line leaves a space which on vertical section is more or less triangular, called the third ventricle. The lateral ventricles are separated from one another by means of a partition made of two layers of white matter, the septum lucidum. On section the septum is seen to be more or less triangular, and between the two layers there is the space of the fifth ventricle filled with fluid.

At the posterior part of the septum lucidum, and joining with it, is the fornix. This is a longitudinal commissure; it is arched and its edge is seen in the lateral ventricle on either side. Between its edge and the upper part of the optic thalamus projects a fringe of bloodvessels, which is the upper part of the septum of the vascular pia mater, which passes into the interior of the brain, and which is called the choroid plexus; the whole of the projection forming a roof for the third ventricle is called the velum interpositum.

The fornix (fig. $355, e$ ) is made up of two strands anteriorly, called the anterior pillars, and of two similar pillars posteriorly; the middle portion called the body consists of the parts of the two pillars which are joined together in the middle line. The body of the fornix is triangular in shape, broad and flat behind, where it is connected with the corpus callosum, and narrow in front where it is connected to the septum lucidum. The anterior pillars pass downward, separated from one another on either side of the third ventricle in front of the foramen, by which the lateral communicates with the third ventricle, called the foramen of Monro; each pillar then passes forward and down, and twisting upon itself forms the corpus albicans, and then passes in part to join the optic 
thalamus. The posterior pillars pass down and out and form part of the interior of that part of the lateral ventricle which descends into the posterior lobe of the cerebrum. 'Thus, when the fornix is reflected from the front, first of all the relum interpositum is seen, and when that is removed the third ventricle comes into sight.

'The third ventricle terminates at its posterior extremity in the pineal body. From this ventricle a short narrow passage, the iter a tertio al

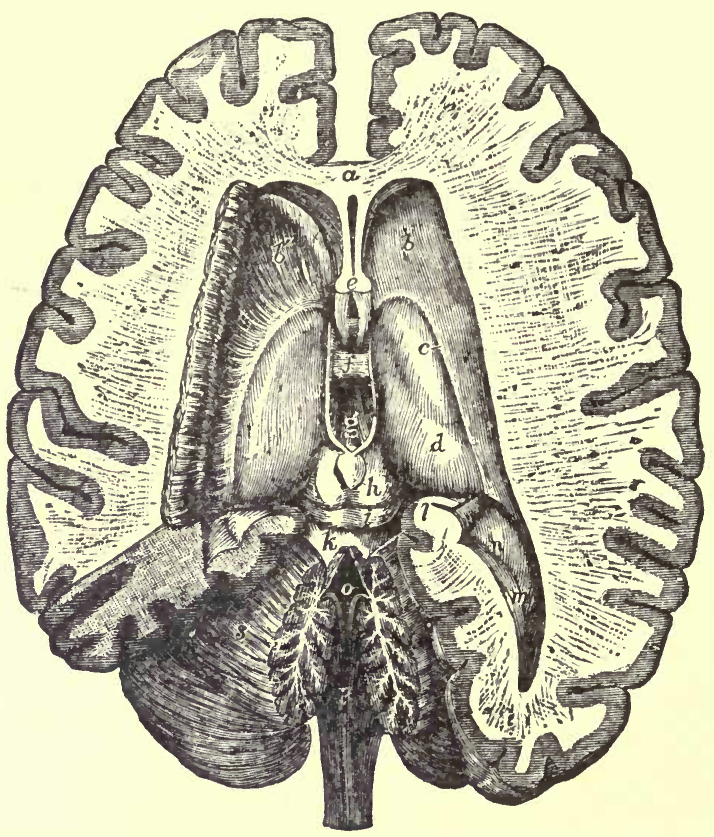

Fig. 355.-Dissection of brain, from above, exposing the lateral fourth and fifth ventricles with the surrounding parts. $1 / 2 . a$, Anterior part, or genu of corpus callosum; $b$, corpus striatum; $b^{\prime}$, the corpus striatum of left side, dissected so as to expose its gray substance; $c$, points by a line to the tænia semicircularis; $d$, optic thalamus; $e$, anterior pillars of fornix divided; below they are seen descending in front of the third ventricle, and between them is seen part of the anterior commissure; in front of the letter $e$ is seen the slit-like fifth ventricle, between the two laminæ of the septum lucidum; $f$, soft or middle commissure; $g$ is placed in the posterior part of the third ventricle; immediately behind the latter are the posterior commissure (just visible) and the pineal gland. the two crura of which extend forward along the inner and upper margins of the optic thalami; $h$ and $i$, the corpora quadrigemina; $k$, superior crus of cere. bellum; close to $k$ is the valve of Vieussens, which has, been divided so as to expose the fourth ventricle; $l$, hippocampus major and corpus fimbriatum, or tænia hippocampi ; $m$, hippocampus minor: $n$, eminentia collateralis; $o$, fourth ventricle; $p$, posterior surface of medulla oblongata; $r$ section of cerebellum; $s$, upper part of left hemisphere of cerebellum exposed by the removal of part of the posterior cerebral lobe. (Hirschfield and Leveille.)

quartum ventriculum, or aqueduct of Sylvius, passes through the next portion of the brain called the mid-brain. This part is covered in by two pairs of nerve-ganglia, the anterior and the posterior corpora quadrigemina, and the floor is formed by the crura cerebri. 'The aqueduct of Sylvius opens at the upper angle of a lozenge-shaped cavity, the fourth ventricle, which is situated on the dorsal aspect of the pons and bulb. The fourth ventricle has no roof of its own beyond a layer of 
epithelium, but it is covered in by the cerebellum, the superior peduncles of which, converging forward, form its anterior limits, and the inferior peduncles form its posterior boundaries on either side.

The lateral, third and fourth ventricles communicate, and through the last with the central canal of the spinal cord. They are all lined with columnar ciliated epithelium, beneath which is a development of neuroglia. This lining so formed is called the ependyma of the ventricles. Where the superior peduncles of the cerebellum are approaching each other at the upper part of the fourth ventricle, the interval between them is bridged over by a thin layer of gray matter called the valve of Vieussens.

The portions of the central nerrous system are thus classified:-

(i.) Cerebral hemispheres with the corpora striata, developed from the cerebral vesicles-and enclosing the lateral ventricles.

(ii.) Fore-brain, formed of the parts, including the optic thalami, which inclose the third ventricle.

(iii.) Mid-brain, consisting of the parts inclosing the aqueduct of

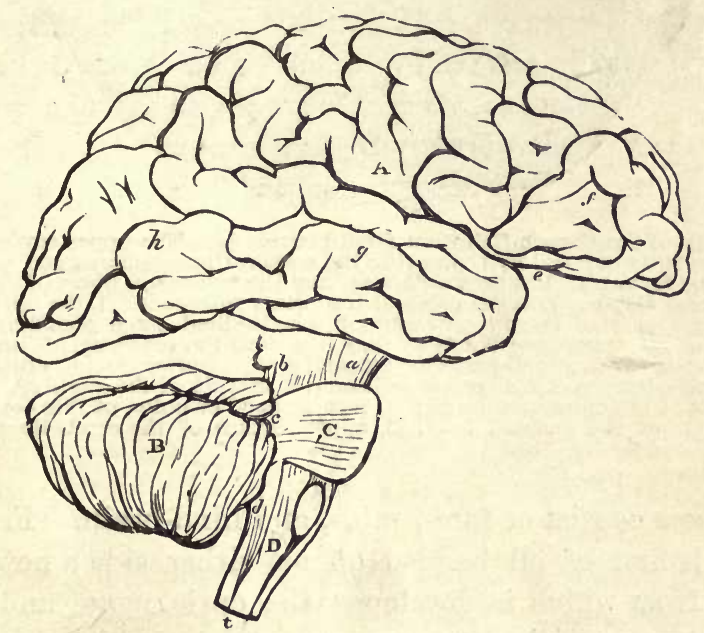

Fig. 356. - Plan in outline of the encephalon, as seen from the right side. 16. The parts are represented as separated from one another somewhat more than natural, so as to show their connections. A Cerebrum; $f, g, h$, its anterior, middle, and posterior lobes; $e$, fissure of Syl. vius: B, cerebellum; C, pons Varolii; D, medulla oblongata; $a$, peduncles of the cerebrum; $b, c, d$, superior, middle, and inferior pedincles of the cerebellum. (From Quain.)

Sylvius, viz., the corpora quadrigemina, which form the roof, and the crura cerebri which form the floor.

(iv.) Hind-brain, the pons Varolii and the cerebellum form respectively the floor and roof of the fore-part of the hind-brain, and the bulb the floor of the back part of the hind-brain, the roof being practically absent. 
This division of the brain into the four parts is justified by a consideration of its development. As will be seen later on, the brain consists originally of three cerebral vesicles, the dilated extremity of the neural

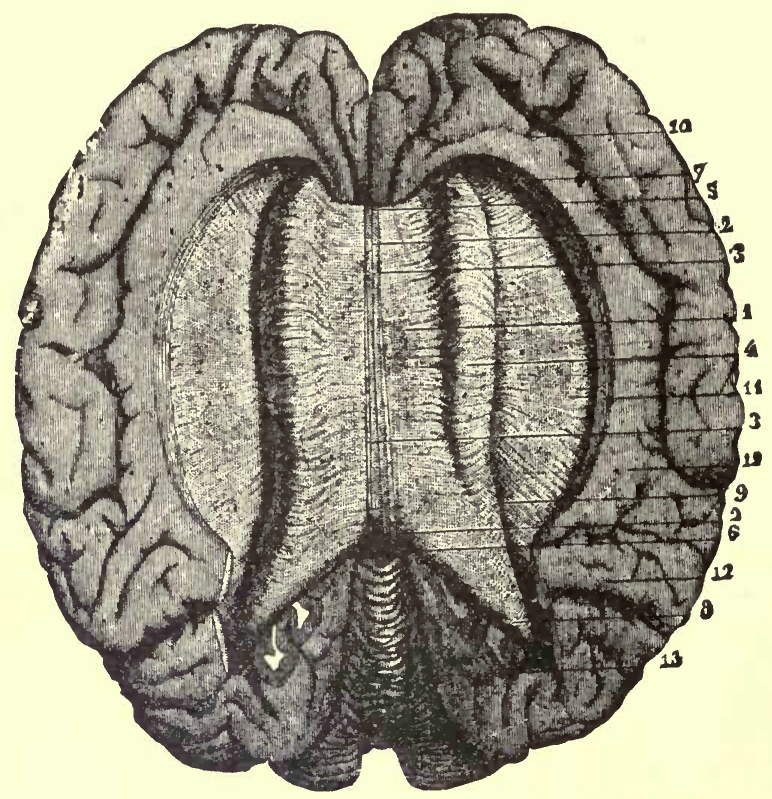

Fig. 35\%.-View of the Corpus Callosum from above. 1/2.-The upper surface of the corpus callosum has been fully exposed by separating the cerebral hemispheres and throwing them to the side; the gyrus fornicatus has been detached, and the transverse fibres of the corpus callosum traced for some distance into the cerebral medullary substance. 1, the upper surface of the corpus callosum; 2 , median furrow or raphe; 3 , longitudinal striæ bounding the furrow; 4 , swelling formed by the transverse bands as they pass into the cerebrum; 5 , anterior extremity or knee of the corpus callosum; 6 , posterior extremity; $\tau$, anterior, and 8, posterior part of the mass of fibres proceeding from the corpus callosum; 9, margin of the swelling; 10, anterior part of the convolution of the corpus callosum; 11 , hem or band of union of this convolution; 12 , internal convolutions of the parietal lobe; 13, upper surface of the cerebellum. (Sappey after Foville.)

canal, and these consist of fore-, mid-, and hind-brain. From the forebrain there is first of all budded off on either side a new vesicle, the optic vesicle from which is developed the optic nerve and retina, and afterward a large vesicle, the cerebral vesicle, which grows rapidly, becomes divided by a central partition into two, each of which incloses the lateral ventricle. The cerebral vesicles grow so quickly as to cover both the fore- and the mid-brain. The parts of which the fore-, mid-, and hind-brains are made up are developed from the corresponding cerebral vesicles.

It will be as well here to indicate briefly the structure of the brain. It consists of white and gray matter differently arranged in different districts. 


\section{Distribution of the Gray Matter.}

(i.) In the bulb, at the lower part the distribution of gray matter follows that which prevails in the cord. Higher up the chief part is found toward the posterior or dorsal aspect, surrounding the central canal. When the central canal opens out into the fourth ventricle the gray matter comes to that surface chiefly, and is found to consist more particularly, on either side, of the nuclei of origin of the cranial nerves, viz., the 12th, 11th, 10th, 9th, and 8th, and more externally of the nucleus gracilis and nucleus cuneatus (n.g., n.c., figs. 361, 362). In addition to these masses of gray matter, there are the olivary bodies ( 0 , figs. 361,362$)$ toward the ventral surface with the accessory otives $\left(o^{\prime}\right)$, and the external arcuate (n.ar. in figs.) nuclei, placed at the tip of the anterior fissure on either side on the ventral surface of the anterior pyramids.

(ii.) In the pons Varolii.-In addition to the origins of nerves in the floor of the fourth ventricle on the dorsal aspect of the pons, viz., of the $7 \mathrm{th}, 6 \mathrm{th}$, and 5 th nerves, there are several masses of gray matter, viz., in the back part, the superior alive (fig. 362), and in the front part the locus cceruleus, as well as small amounts of the same material mixed with fibres in the more ventral surface.

(iii.) In the mid-brain, the gray matter preponderates in the optic thalami, corpora quadrigemina, and corpora geniculata. It is also found surrounding the aqueduct of Sylvius, and in other parts of the crura, notably such masses as the red nucleus (fig. 363), locus niger (fig. 365).

(iv.) In the cerebral hemispheres, the cerebral cortex is made up of gray matter which incloses white matter, and the corpus striatum is made up more or less of the same material.

(v.) In the cerebellum, the gray matter forms the incasing material. In the interior too there are masses of gray matter forming the corpora dentata.

This then roughly indicates the localities in which gray matter is found; the arrangement of the fibres and their relationship to the gray matter will be dealt with later on.

\section{The Bulb or Medulla Oblongata.}

The medulla oblongata (figs. 358,359 ), is a column of gray and white matter formed by the prolongation upward of the spinal cord and connecting it with the brain.

Structure.-The gray substance which it contains is situated in the interior and variously divided into masses and laminæ by the white or fibrous substance which is arranged partly in external columns, and 
partly in fasciculi traversing the ceutral gray matter. The medulla oblongata is larger than any part of the spinal cord. Its columns are pyriform, enlarging as they proceed toward the brain, and are continuous with those of the spinal cord. Each half of the medulla, therefore, may be divided into three columns or tracts of fibres, continuous with the three tracts of which each half of the spinal cord is made up, - the columns more prominent than those of the spinal cord, and separated from each other by deeper grooves. The anterior, continuous with the anterior columns of the cord, are called the anterior pyramids, and the

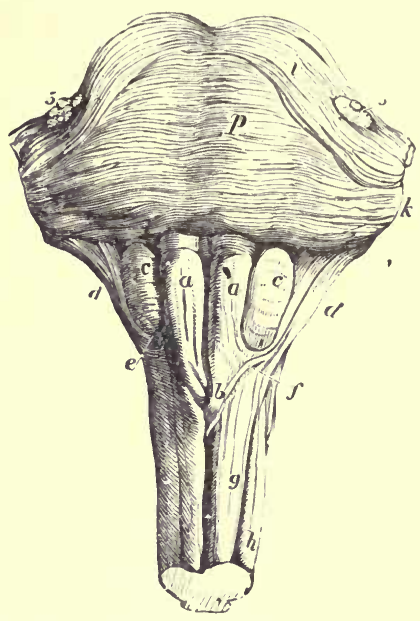

Fig. 358.

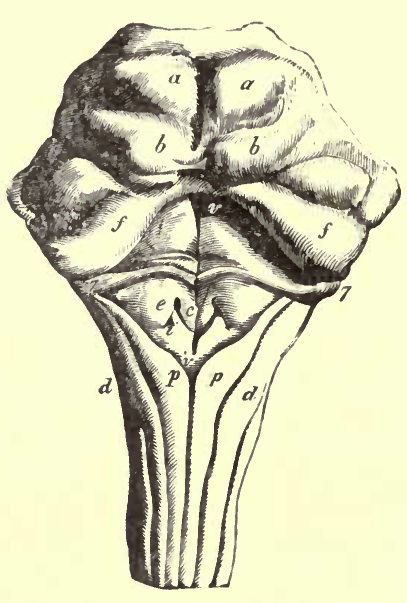

Fig. 359 .

Fig. 358. - Ventral or anterior surface of the pons Varolii, and medulla oblongata. $\boldsymbol{a}, \boldsymbol{a}$, anterior pyramids; $b$, their decussation; $c, c$, olivarv bodies; $d, d$, restiform bodies; $e$, arciform fibres; $f$, fibres passing from the anterior column of the cord to the cerebellum; $g$, anterior column of the spinal cord; $h$, lateral column; $p$, pons Varolii; $i$, its upper fibres; 5 , 5 , roots of the fifth pair of nerves.

Fig. 359.-Dorsal or posterior surface of the pons Varolii, corpora quadrigemina, and medulla oblongata. The peduncles of the cerebellum are cut short at the side. $a, a$, the upper pair of corpora quadrigemina; $b, b$, the lower: $f, f$, superior peduncles of the cerebellum; $c$, eminence connected with the nucleus of the hypoglossal nerve; $e$, that of the glosso-pharyngeal nerve; $i$, that of the vagus nerve; $d, d$, restiform bodies; $p, p$, posterior pyramids; $v, v$, groove in the middle of the fourth ventricle, ending below in the calamus scriptorius; $7, \gamma$, roots of the auditory nerves.

postero-median and postero-external columns are also represented at the posterior or dorsal aspect of the cord as the fasciculus gracilis and the fasciculus cuneatus. The posterior pyramids of the medulla which include these two columns of white matter soon become much increased in width by the addition of a new column of white matter outside the other two which is known as the fasciculus of Rolando. The lateral columns of the cord undergo considerable change and are scarcely represented as such in the bulb.

It may be said then that the bulb at its commencement differs only slightly in size from the cord with which it is continuous. It soon 
becomes larger both laterally and antero-posteriorly, and after a time opens out on the dorsal surface into a space which is known as the fourth ventricle, and from being a cylinder with a central canal, it is flattened out on one surface by the gradual approach of the central canal to that

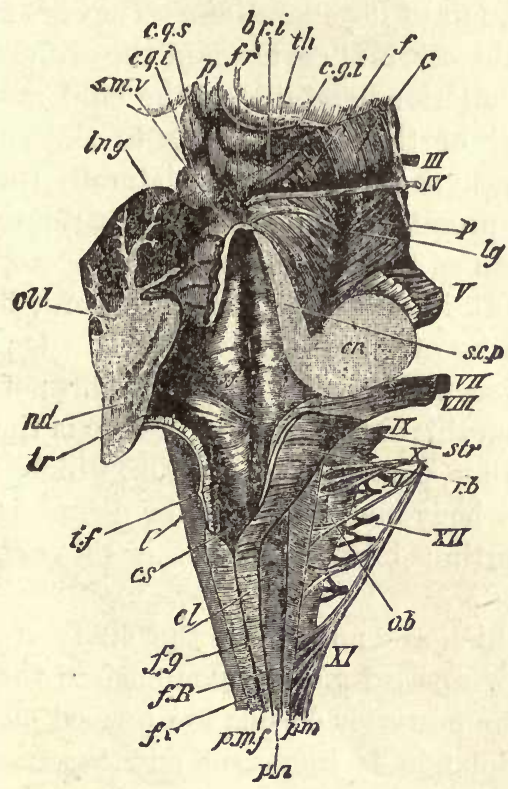

Fig. 360.-Dorgal or posterior view of the medulla, fourth ventricle, and mesencephalon (natural size). p.n., line of the posterior roots of the spinal nerves; $p . m . f .$, posterior median fissure; $f . g$. funiculus gracilis; $c l$, its clava; $f . c$. , funiculus cuneatus: $f . R$. , funiculus of Rolando; r.b., restiform body:c.s., calamus scriptorius: $l$, section of ligula or tænia; part of choroid plexus is seen beneath it; $l$.r., lateral recess of the ventricle; str., striæ acusticæ; $i . f$., inferior fossa; s.f., posterior fossa; between it and the median sulcus is the fasciculus teres; $c b l$., cut surface of the cerebellar hemisphere; $n d .$, central or gray matter; s.m. $v$., superior medullary velum; lng., ligula; s.c.p., superior cerebellar peduncle cut longitudinally; cr., combined section of the three cerebellar peduncles; c.q.s., c.q.i., corpora quadrigemina (superior and inferior); $f r$., frænulum; $f$., fibres of the fillet seen on the surface of the tegmentum; $c$. , crusti; l.g. l lateral groove; $c . g . i$. , corpus geniculum internus; th., posterior part of thalamus; $p$., pineal body. The Roman numbers indicate the corresponding cranial nerves. (E. A. Schäfer.)

surface. The central canal of the cord, therefore, is directly continuous with the fourth ventricle.

If the bulb be examined on its anterior or ventral surface it is found that the anterior fissure, which is a continuation of the same fissure in the cord, is occupied at the most posterior part by fibres which are crossing from one side to the other; the central canal being pushed now toward the posterior surface. This is what is known as the anterior decussation of the medulla oblongata. It is formed of the fibres which in the cord occupy the postero-lateral region and are called the crossed pyramidal fibres. The lateral pyramidal fibres of either side after crossing in the middle line in this way become part of the anterior pyramia 
of the opposite side; the rest of the pyramid being made up of the fiores from the anterior column of the cord known as the direct or uncrossed pyramidal fibres. These two pyramidal strands of fibres are those which degenerate on lesions of certain parts of the cerebrum which are known as the motor areas of the cortex. They can therefore be traced downward on such lesions as tracts of degeneration. They are the fibres of communication between the cerebral cortex and the different segments of the spinal cord. The anterior pyramids of the bulb are marked out by the exit from that part of the nervous axis to the outside of them, of a nerve, the 12th or hypoglossal. More laterally than this nerve, there soon becomes very prominent on either side a rounded elevation or column which is known as the olivary body. It is not seen at the beginning of the bulb at its junction with the cord, but begins at a lower level than the opening of the fourth ventricle. On the further side of the olivary body is seen the line of origin of fibres of the 11th, 10th, and 9 th nerves, and from this to the posterior fissure is the posterior pyramid.

The whole of that part of the medulla which is situated laterally between the olivary body and the posterior fissure is known as the restiform body; it is continued forward on either side as the inferior peduncle of the cerebellum.

The changes which are noticed by the study of series of sections of the bulb from below upward may be summarized thus: In the dorsal or posterior region, the posterior cornua are pushed more to each side, and the substance of Rolando is increased and becomes rounded, reaching almost to the surface of the bulb on each side, a small tract of longitudinal fibres of the ascending root of the 5th nerve only intervening. There is a great increase of the reticular formation around the central canal, and the lateral approaches the anterior cornu. Then at the ventral or anterior aspect the decussation of the lateral fibres begins. By this crossing over of the fibres, the tip of the gray anterior cornu is cut off from the rest of the gray matter. The central canal is pushed further toward the posterior surface, first of all by the decussation of the anterior pyramids just mentioned, and later on, i.e., above, by another decussation of fibres more dorsal. These fibres of the second decussation as they cross form a median raphe and also help to break up the remaining gray matter into what is called a reticular formation. There has been some little doubt as to the origin of these descussating fibres, but the best authorities now consider them to be, at any rate in part, the fibres from the nuclei of the fasciculus gracilis and fasciculus cuneatus of either side, and look upon them as a sensory decussation. At the posterior part soon there appear in the columns of white matter of the fasciculus gracilis and fasciculus cuneatus new masses of gray matter. The laterar norn approaches the anterior; but soon the latter is pushed 
further and further toward the centre, while the lateral horn remains near the lateral surface. The anterior gray matter becomes broken up and merged into the reticular formation. There is also a similar reticular formation both toward the centre and also laterally in the dorsal region. At the level where the central canal opens into the 4 th ventricle, the posterior pyramids diverging to form the lower and outside boundaries, and inclosing a space, the calamus scriptorius, between them, there are to be made out various masses of gray matter in addition to the reticular formation, viz., the nuclei of the fasciculus gracilis

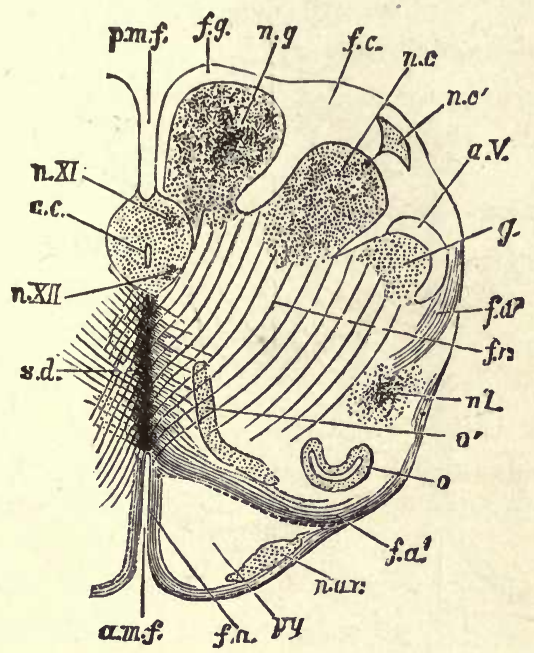

Fig. 361. - Anterior or dorsal section of the medulla oblongata in the region of the superior pyramidal decussation. a.m.f., anterior median fissure; $f . a .$, superficial arciform fibres emerging from the fissure; $p y$. , pyramid; $n$. ar., nuclei of arciform fibres; $f . a$. , deep arciform becoming superficial; o, lower end of olivary nucleus; n.l., nucleus lateralis; $f . r$. , formatio reticularis: $f . a .^{2}$, arciform fibres proceeding from the formatio reticularis; $g$., substantia gelatinosa of Rolando; $a . V$. , ascending root of fifth nerve; n.c., nucleus cuneatus; $n . c .^{\prime}$, external cuneate nucleus: $n . g$., nucleus gracilis; $f . g$., funiculus gracilis; $p . m . f$, posterior median fissure; c.c., central canal surrounded by gray matter, in which are $n$. $X I$., nucleus of the spinal accessory, and $n . X I I$., nucleus of the hypoglossal; s.d., superior pyramidal decussation. (Modified from Schwalbe.)

and fasciculus cuneatus $(361, n . g$. and n.c.), which are at this level, however, already diminishing and are lost at a level of the pons Varolii.

The olivary bodies extend forward almost to the level of the pons. They consist of gray and white matter. The gray matter consists of a plicated thinnish strand containing small nerve-cells, folded upon itself in the form of a loop, with the ends turned inward and slightly dorsal (Fig. 362,o). The gray loop is filled with and covered by white matter, part of the fibres passing through the gray.

Internal to the olivary body on either side are two small masses of gray matter, one more ventral to the other, called accessory olives, external and internal, and on the surface of the anterior pyramid on either 
side a small mass of gray matter, external arcuate nucleus; laterally another mass of the same material, the representative of the lateral nucleus of the cord, is seen, viz., the antero-lateral nucleus, which gives origin to the spinal accessory nerve.

It will be necessary to follow as shortly as possible the fibres of the spinal cord upward into the bulb and beyond:-

The crossed and direct pyramidal tracts have already been described. Nothing definite is known of the antero-lateral descending tracts. The cerebellar tracts pass laterally into the restiform bodies and go to the

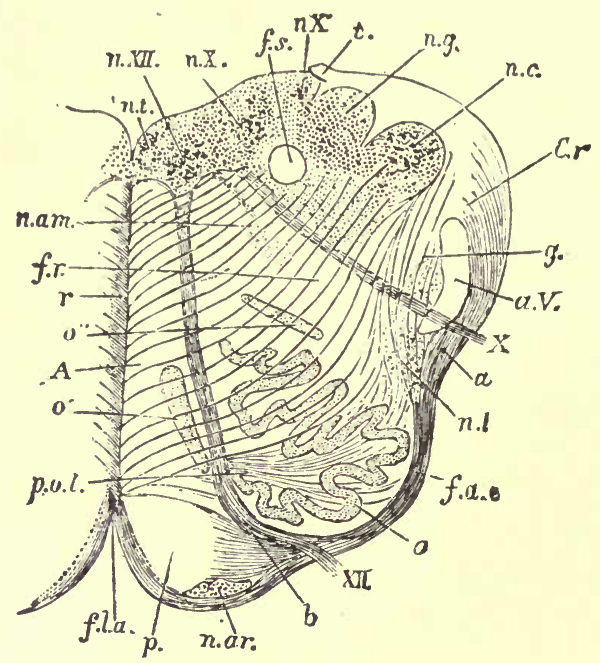

Fig. 362. - Section of the medulla oblongata at about the middle of the olivary body. f.l.a. anterior median fissure; $n$.ar., nucleus arciformis; $p$. , pyramid; XII., bundle of hypoglossal nerve emerging from the surface: at $b$, it is seen coursing between the pyramid and the olivary nucleus, o. ; f.a.e., external arciform fibres; $n . l$., nucleus lateralis; $a$., arciform fibres passing toward restiform body, partly through the substantia gelatinosa, $g$., partly superficial to the ascending root of the fifth nerve, $a, V$.: $X$, bundle of vagus root emerging; $f . r$. formatio reticularis; $c . r$, corpus restiform, beginning to be formed, chiefly by arciform fibres, superficial and deep; $n . c$. , nucleus cuneatus; $n . g$., nucleus gracilis; $t$, attachment of the ligula; $f$.s., funiculus solitarius; $n . X ., n . X .^{\prime}$, two parts of the vagus nucleus; n.XII., hypoglossal nucleus; n.t., nucleus of the funiculus teres; $n$. am., nucleus ambiguus; $r$. , raphe; $A$., continuation of the anterior column of cord; $o^{\prime}, o^{*}$, accessory olivary nucleus; $p . o$. , pedunculus olivæ. (Modiffed from Schwalbe.)

cerebellum. The antero-lateral ascending tracts appear to have the same destination and pass directly or indirectly into the cerebellum. The fibres of the postero-median and postero-external columns end in the nuclei of the fasciculus gracilis and cuneatus respectively, either in or about the cells contained in those nuclei; at any rate, ascending degeneration of these columns cannot be traced above these nuclei.

The rest of the fibres of the cord appear to end in the reticular formation of the bulb. The bundle of fibres constituting the ascending root of the 5th nerve appears to correspond with the tract of Lissauer.

Connections of the bulb with the cerebrum and cerebellum,-In addition 
to the pyramidal tracts connecting the bulb with the cerebrum and the direct cerebellar and the antero-lateral ascending tract connecting it with the cerebellum, there are other connections of the bulb with the cerebrum, and with the cerebellum, not actually direct.

(1.) Fibres from the nucleus gracilis and nucleus cuneatus, which, as we have said, are the bulbar endings of the fibres of the posteromedian and postero-external columns of the cord, pass in sets as it were in the following manner:-

(a.) Internal arcuate fibres. - Some pass down and inward to the other side in the reticular formation, forming in part the superior or sensory decussation, and in the inter-olivary region become longitudinal in a band of fibres called the fillet, which passes upward. These fibres are probably augmented by the addition of fibres from the anterior columns of the cord.

(b.) External arcuate fibres also decussate in the same way, pass down along the anterior fissure, and then running outward superficially over the anterior pyramid and olivary body, reach the restiform body and pass to the side of the cerebellum opposite to their nuclei of origin. These fibres appear to have some relation with the external arcuate nuclei. They connect one side of the spinal cord with the opposite side of the cerebellum through the gracile and cuneate nuclei.

(c.) Direct lateral fibres pass to the restiform body and so to the same side of the cerebellum.

(2.) Fibres from the olivary body pass to the opposite side of the cerebellum probably through the reticular formation.

(3.) Arciform fibres. - Fibres from the nucleus of the 8th or auditory nerve in the floor of the 4 th ventricle, pass to the same side of the cerebellum.

\section{Functions of the Bulb or Medulla Oblongata.}

The functions of the bulb are those of, (a.) conduction; (b.) reflex action; and $(c$.$) automatism.$

(a.) Conduction.-As a conductor of impressions, the medulla oblongata has a wider extent of function than any other part of the nervous system, since it is obvious that all impressions passing to and fro between the brain and the spinal cord must be transmitted through it.

(b.) Reflex Action.-As a nerre centre by which impressions are reflected, the medulla oblongata also resembles the spinal cord; the only difference between them consisting of the fact that many of the reflex actions performed by the former are much more complicated than any performed by the spinal cord.

It has been proved by repeated experiments on the lower animals that the entire brain may be gradually cut away in successive portions, 
and yet life may continue for a considerable time, and the respiratory movements be uninterrupted. Life may also continue when the spinal cord is cut away in successive portions from below upward as high as the point of origin of the phrenic nerve. In amphibia, the brain has been all removed from above, and the cord, as far as the medulla oblongata, from below; and so long as the medulla oblongata was intact, respiration and life were maintained. But if, in any animal, the medulla oblongata is wounded, particularly if it is wounded in its central part, opposite the origin of the vagi, the respiratory movements cease, and the animal dies asphyxiated. And this effect ensues even when all parts of the nervous system, except the medulla oblongata, are left intact.

Injury and disease in men prove the same as these experiments on animals. Numerous instances are recorded in which injury to the medulla oblongata has produced instantaneous death; and, indeed, it is through injury of it, or of the part of the cord connecting it with the origin of the phrenic nerve, that death is commonly produced in fractures attended by sudden displacement of the upper cervical vertebræ.

\section{Special Centres.}

In the medulla are contained a considerable number of centres which preside over many important and complicated co-ordinated movements of muscles. The majority of these centres are (a.) reflex centres simply, which are stimulated by afferent or by voluntary impressions. Some of them are $(b$.) automatic centres, being capable of sending out efferent impulses, generally rhythmical, without previous stimulation by afferent or by voluntary impressions. The automatic centres are, however, generally influenced by reflex or by voluntary impulses. Some again of the centres, whether reflex or automatic, are (c.) control centres, by which subsidiary spinal centres are governed. Finally the action of some of the centres is $(d$.$) tonic, i.e., they exercise their influence either directly or$ through another apparatus, continuously and uninterruptedly in maintaining a regular action.

\section{Simple Reflex centres.}

(1.) Bilateral centres for the co-ordinated movements of Mastication, the afferent and efferent nerves of which have been already enumerated (p. 326).

(2.) Bilateral centres for the movements of Deglutition. The medulla oblongata appears to contain the centre whence are derived the motor impulses enabling the muscles of the palate, pharynx, and œsophagus to produce the successive co-ordinate and adapted movements necessary to the act of deglutition (p. 353). This is proved by the persistence of swallowing in some of the lower animals after destruction of the cerebral 
hemispheres and cerebellum; its existence in anencephalous monsters; the power of swallowing possessed by the marsupial ambryo before the brain is developed; and by the complete arrest of the power of swallowing when the medulla oblongata is injured in experiments.

(3.) Bilateral centres for the combined muscular movements of Sucking, the motor nerves concerned being the facial for the lips and mouth, the hypoglossal for the tongue, and the inferior maxillary division of the 5th for the muscles of the jaw.

(4.) Bilateral centres for the Secretion of Saliva, which have been already mentioned (p. 333).

(5.) Bilateral centres for Vomiting (p. 369).

(6.) Bilateral centres for Coughing, which are said to be independent of the respiratory centre, being situated above the inspiratory part of that centre.

(\%) Bilateral centres for Sneezing, connected no doubt with the respiratory centre.

(8.) Bilateral centres for the Dilatation of the pupil, the fibres from which pass out partly in the third nerve and partly through the spinal cord (through the last two cervical and two upper dorsal nerves?) into the cervical sympathetic.

(b.) Automatic centres.

(1.) Respiratory centres. - The action of the respiratory centre has been already discussed. It is only necessary to repeat here that although it can be influenced by afferent impulses, it is also automatic in its action, being capable of direct stimulation, as by the condition of the blood circulating within it. It is also bilateral. It probably consists of an inspiratory part and of an expiratory part. The centre is capable of being influenced both reflexly and to a certain extent also by voluntary impulses. The vagus influence is probably constant in the direction of stimulating the inspiratory portion of the centre, whereas the influence of the superior laryngeal is not always in action, and is inhibitory.

(2.) Cardio-Inhibitory centres. The action of these centre in maintaining the proper rhythm of the heart through the vagus fibres, which terminate in a local intrinsic mechanism, has been already discussed. The centre can be directly stimulated, as by the condition of the blood circulating within it, and also indirectly by afferent stimuli, especially by stimulating the abdominal sympathetic nerves, but also by stimulating any sensory nerve, including the vagus itself.

(3.) Accelerator centres for the heart. The centres from which arise the accelerator fibres of the heart, in the medulla. They are antomatic but not tonic in action.

(4.) Vaso-motor centres, which control the unstriped muscle of the arteries, are also situated in the medulla. Like the respiratory centre, 
they are bilateral. As has already been pointed out, these centres may be directly or reflexly stimulated, as well as by impressions conveyed downward from the cerebrum to the medulla. The condition of the blood circulating in them is the direct stimulus. Its influence is no doubt a tonic or else a rhythmic one. It is also supposed that there is in the medulla a special vaso-dilator centre not acting tonically, stimulation of which produces vascular dilatation. The diabetic centre is probably a part of the raso-motor centre, at any rate stimulation of it causes dilatation of the ressels of the liver.

(5.) Bilateral chief centres for the secretion of Sweat exist in the medulla. 'The centres on either side control the subsidiary spinal sweat centres. They may be excited unequally so as to produce unilateral sweating. They are probably antomatic and reflex.

(6.) Bilateral Spasm centres are said to be present in the medulla, on the stimulation of which, as by suddenly produced excessive venosity of the blood, general spasms of the muscles of the body are produced.

(c.) Control centres. These are centres whose influence may be directed to controlling the action of subsidiary centres. They are-

(1.) The Respiratory centres, which probably control the action of other subordinate centres in the spinal cord.

(2.) The Cardio-Inhibitory centres, which act upon a local ganglionic mechanism in the heart.

(3.) The Accelerator centres, if they exist, probably act through a local mechanism in the heart.

(4.) The Vaso-motor centres control spinal as weil as local tonic centres.

(5.) The medullary Sweat centres control the spinal sweat centres.

(d.) Tonic centres. Of the centres whose action is tonic or continuous up to a certain degree, may be cited the vaso-motor and the cardio-inhibitory.

It should not be forgotten that in the medulla are the centres for the special senses, Hearing and Taste, and that other special centres are supposed to be localized there, of which may be mentioned one, the hypothetical Inhibitory heat centre, which controls the production of heat by the tissues, independently of the vaso-motor centre.

\section{The Cranial Nerves.}

The cranial nerves consist of twelve pairs; they appear to arise (superficial origin) from the base of the brain in a double series, which extends from the under surface of the anterior part of the cerebrum to the lower end of the medulla oblongata. Traced into the substance of the brain and medulla, the roots of the nerves are found to take origin from various masses of gray matter. 
The roots of the first or olfactory and of the second or optic nerves will be mentioned elsewhere. The third and fourth nerves arise from gray matter beneath the corpora quadrigemina; and the roots of origin of the remainder of the cranial nerves can be traced to gray matter in the floor of the fourth ventricle, and in the more central part of the medulla, around its central canal, as low down as the decussation of the pyramids.

According to their several functions the cranial nerves may be thus arranged :-

a. Nerves of special sense

b. Nerves of common sensation .

c. Nerves of motion

d. Mixed nerves
Olfactory, Optic, Auditory, part of the Glosso-pharyngeal, and part of the Fifth.

The greater portion of the Fifth.

Third, Fourth, lesser division of the Fifth, Sixth, Facial, and Hypoglossal.

Glosso-pharyngeal, Vagus, and Spinal accessory.

The physiology of the First, Second, and Eighth will be considered with the organs of Special sense.

\section{The IIIrd Nerve (Motor Oculi).}

Origin.-The third nerve arises in three distinct bands of fibres from the gray matter surrounding the aqueduct of Sylvius near the middle line ventral to the canal. The nucleus of origin consists of large multi-

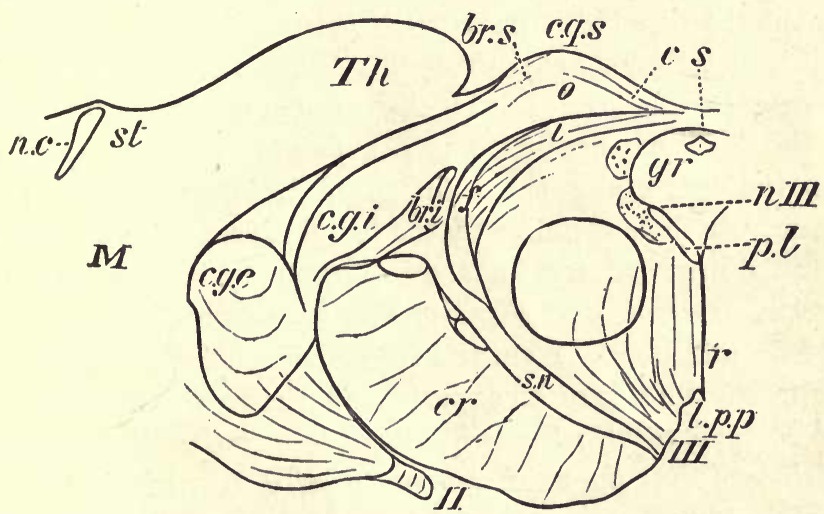

Fig. 363. - Section through anterior corpus quadrigeminum and part of optic thalamus. s., Aqueduct of Sylvius; gr., gray matter of the aqueduct; c.q.s., quadrigeminal eminence; $l$., stratum lemnisci; o., stratum opticum; $c$., stratum cinereum; Th., pulvinate of optic thalamus; c.g.e., c.g.i., lateral and median corpora geniculata; br.s., br.i., superior and inferior brachia: $f$., fillet; $p . \iota$., posterior longitudinal bundle: $r$., raphé; $I I I$., third nerve, and $n . I I I$., its nucleus; l.p.p., posterior perforated space; s.n., substantia nigra, above this is the tegmentum with the circular area of the red nucleus; $c r$. ., crusta; II., optic tract; $M$., medullary centre of hemisphere; n.c., nucleus caudatus; st., stria terminalis. (After Quain, from Meynert.)

polar ganglion-cells, and extends to the back part of the third ventricle as far as the level of the anterior corpus quadrigeminum. The fibres pass from their origin partly through the red nucleus to their superficial 
origin in front of the pons, at the median side of each crus. 'They decussate with their fellows in the middle raphe. The nerve is connected with the optic nerve.

Function.-It supplies the levator palpebræ superioris muscle, and all of the muscles of the eyeball, except the superior oblique to which

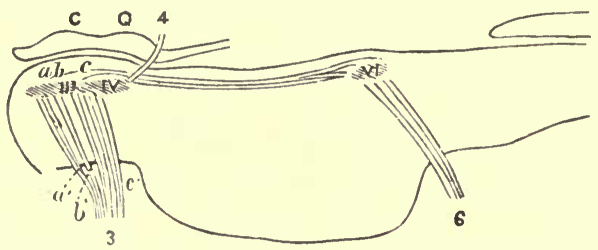

Fig. 364-Diagram of a longitudinal section through the pons, showing the relation of the nuclei for the ocular muscles. CQ, corpora quadrigemina; 3 , third nerve; III, its nucleus; 4 , fourth nerve; IV, its nucleus, the posterior part of the third; 6 , sixth nerve. The probable position of the centre and nerve fibres for accommodation is shown at $a$ and $\alpha^{\prime}$, for the reflex action of iris, at $b$, and $b^{\prime}$; for the external rectus muscles, at $c, c^{\prime}$. The lines beneath the Hoor of the fourth ventricle indicate fibres, which connect the nuclei. (Gowers.)

the fourth nerve is appropriated, and the rectus externus which receives the sixth nerve. Through the medium of the ophthalmic or lenticular ganglion, of which it forms what is called the short root, it also supplies motor filaments to the iris and ciliary muscle. The fibres which subserve the three functions, accommodation, contraction of the pupil, and nerve-supply to the external ocular muscles, arise from three distinct groups of cells.

When the third nerve is irritated within the skull, all those muscles to which it is distributed are convulsed. When it is paralyzed or divided the following effects ensue:-(1) the upper eyelid can be no longer raised by the levator palpebræ, but droops (ptosis) and remains gently closed over the eye, under the unbalanced influence of the orbicularis palpebrarum, which is supplied by the facial nerve: (2) the eye is turned outward and downward (external strabismus) by the unbalanced action of the rectus externus and superior oblique to which the sixth nerve is appropriated; and hence, from the irregularity of the axes of the eyes, double sight, diplopia, is often experienced when a single object is within view of both the eyes: (3) the eye cannot be moved either upuard, downward, or inward: (4) the mupil becomes dilated (mydriasis): (5) the eye cannot accommodate for short distances.

\section{The IVth Nerve (Trochlearis).}

Origin.-The IVth nerve arises from a nucleus consisting of large multipolar ganglion cells situated below, i.e., ventral to the aqueductus of Sylvius, which extends from the back part of the nucleus of the third nerve to the hind level of the posterior corpus quadrigeminum. The fibres from either side sweep round the central gray matter, and 
reach the valve of Vieussens, where they decussate in the middle line and appear at the front of the pons at the lateral edge of the crus. The

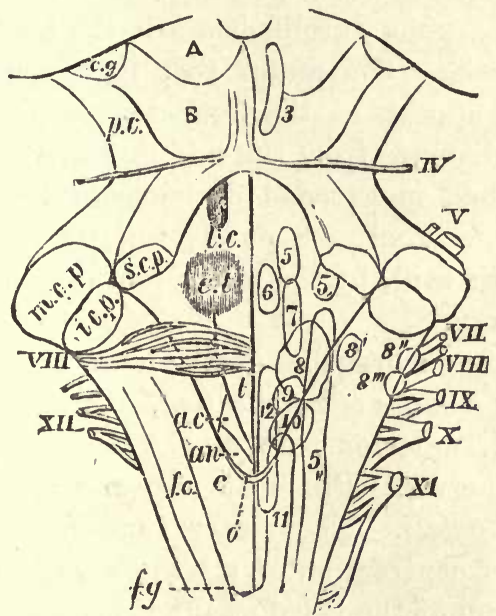

Fig. 365. - Fourth ventricle with the medulla oblongata and the corpora quadrigemina. The roman numbers indicate superficial origins of the cranial nerves, while the other numbers indicate their deep origins, or the position of their central nuclei. $8,8^{\prime}, 8^{\prime \prime}, 8^{\prime \prime \prime}$, auditory nuclei nerves; $t$, funiculus teres; $\mathrm{A}, \mathrm{B}$, corpora quadrigemina; $c . g$, corpus geniculatum; $p, c$, pedunculus cerebri : $m, c, p$, middle cerebellar peduncle; $s, c, p$, superior cerebellar peduncle; $i, c, p$, inferior cerebellar peduncle; $l, c$, locus cæruleus; $e, t$, eminentia teres; $a, c$, ala cinerea; $a, n$, accessory nucleus; $o$, obex; $c$, clava; $f, c$, funiculus cuneatus; $f, g$, funiculus gracilis.

nucleus of the fourth nerve on either side is connected with those of the third and sixth nerves.

Functions. - The IVth nerve is exclusively motor, and supplies only the trochlearis or obliquus superior muscle of the eyeball.

\section{The Vth Nerve (Trigeminus).}

Origin. - The Vth or Trigeminal nerve resembles, as already stated, the spinal nerves, in that its branches are derived through two roots; namely, the larger or sensory, in connection with which is the Gasserian ganglion, and the smaller or motor root which has no ganglion, and which passes under the ganglion of the sensory root to join the third branch or division which ensues from it. The fibres of origin of the fifth nerve come from the floor of the fourth ventricle. The motor root to the inside of the sensory, about the middle of each lateral half. The sensory fibres, however, can be traced down in the medulla oblongata as far as the upper part of the cord. From the motor nucleus there stretches forward as far as the anterior corpus quadrigeminum a bundle of long fibres termed the descending root, which has attached to it sparse spheroidal nerve-cells. It is also connected with the locus cæruleus. The sensory nucleus outside the motor has connected with it a tract of 
fibres from the cord as low as the second cervical nerve, and this forms a tract at the tip of the posterior cornu, between it and the restiform body. No nerve cells are connected with it. The roots can be traced obliquely through the pons Varolii, beneath the floor of the front part of the fourth ventricle. The motor root is in a position median to sensory. The nerve appears at the ventral surface of the pons near its front edge, at some distance from the middle line.

Function.-The first and second divisions of the nerve, which arise wholly from the larger root, are purely sensory. The third division being joined, as before said, by the motor root of the nerve, is of course both motor and sensory.

(a.) Motor.-Through branches of the lesser or non-ganglionic portion of the fifth, the muscles of mastication, namely, the temporal, masseter, two pterygoid, anterior part of the digastric, and mylohyoid, derive their motor nerves. Filaments are also supplied to the tensor tympani and tensor palati. The motor function of these branches is proved by the violent contraction of all the muscles of mastication in experimental irritation of the third or inferior maxillary division of the nerve; by paralysis of the same muscle, when it is divided or disorgan-

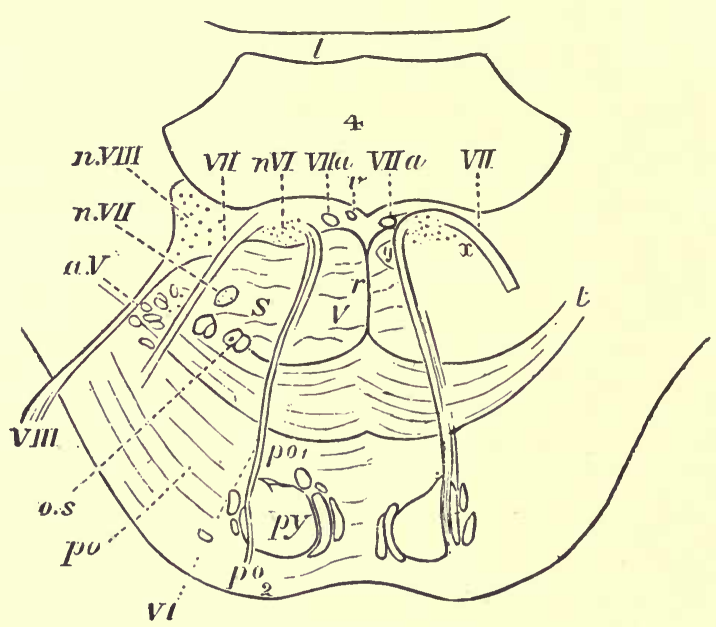

Fig. 366. - Section across the pons, about the middle of the fourth ventricle. py., pyramidal bundles; $p o$. , transverse fibres passing $p o_{1}$, behind, and $p \sigma_{2}$, in front of $p y . ; r$., raphe; o.s., superior olive; $a . V_{\text {. }}$, bundles of ascending root of $V$. nerve inclosed in a prolongation of the substance of Rolando; VI., the sixth nerve, $n V I$., its nucleus; VII., facial nerve; VII. $a$., intermediate portion, $n . V I I$., its nucleus; VIII., auditory nerve, $n V I I I$. , lateral nucleus of the auditory. (After Quain.)

ized, or from any reason deprived of power; and by the retention of the power of these muscles, when all those supplied by the facial nerve lose their power through paralysis of that nerve. The last instance proves best, that though the buccinator muscle gives passage to, and receives 
some filaments from, a buccal branch of the inferior division of the fifth nerve, yet it derives its motor power from the facial, for it is paralyzed together with the other muscles that are supplied by the facial, but retains its power when the other muscles of mastication are paralyzed. Whether, however, the branch of the fifth nerve which is supplied to the buccinator muscle is entirely sensory, or in part motor also, must remain for the present doubtful. From the fact that this muscle, besides its other functions, acts in concert or harmony with the muscles of

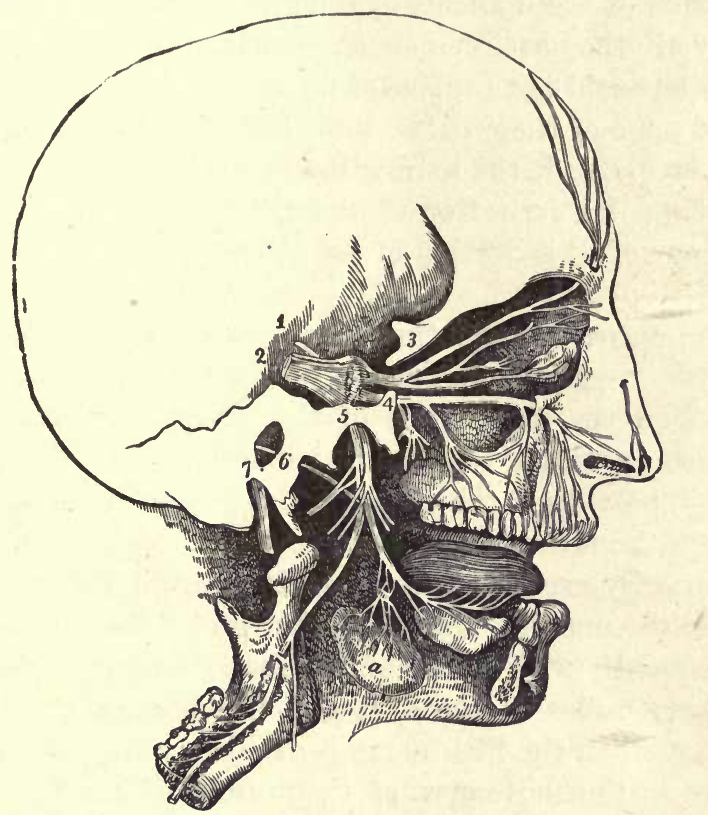

Fig. 367.-General plan of the branches of the fifth pair. 1/3.-1, lesser root of the fifth pair; 2 , greater root passing forward into the Gasserian ganglion; 3, placed on the bone above the ophthalmic nerve, which is seen dividing into the supra-orbital, lachrymal, and nasal branches, the latter connected with the ophthalmic ganglion; 4 , placed on the bone close to the foranien rotundum, marks the superior maxillary division, which is connected below with the spheno-palatine ganglion, and passes forward to the infra-orbital foramen; 5 , placed on the bone over the foramen ovale, marks the inferior maxillary nerve, giving off the anterior auricular and muscular branches, and continued by the inferior dental to the lower jaw, and by the gustatory to the tongue; $a$, the submaxillary gland, the submaxillary ganglion placed above it in connection with the gustatory nerve; 6 , the chorda tympani; 7 , the facial nerve issuing from the stylomastoid foramen. (Charles Beli.)

mastication, in keeping the food between the teeth, it might be supposed from analogy, that it would have a motor branch from the same nerve that supplies them. There can be no doubt, however, that the so-called buccal branch of the fifth is, in the main, sensory; although it is not quite certain that it does not give a few motor filaments to the buccinator muscle.

(b.) Sensory.-Through the branches of the greater or ganglionic portion of the fifth nerve, all the anterior and antero-lateral parts of the 
face and head, with the exception of the skin of the parotid region (which derives branches from the cervical spinal nerves), acquire common sensibility; and among these parts may be included the organs of special sense, from which common sensations are conveyed through the fifth nerve, and their special sensations through their several nerves of special sense. 'The muscles, also, of the face and lower jaw acquire muscular sensibility, through the filaments of the ganglionic portion of the fifth nerve distributed to them with their proper motor nerves. The sensory function of the branches of the greater division of the fifth nerve is proved, by all the usual evidences, such as their distribution in parts that are sensitive and not capable of muscular contraction, the exceeding sensibility of some of these parts, their loss of sensation when the nerve is paralyzed or divided, the pain withont convulsions produced by morbid or experimental irritation of the trunk or branches of the nerve, and the analogy of this portion of the fifth to the posterior root of the spinal nerve.

Other Functions.-In relation to muscular movements, the branches of the greater or ganglionic portion of the fifth nerve exercise a manifold influence on the movements of the muscles of the head and face and other parts in which they are distributed. 'They do so, in the first place $(a)$, by providing the muscles themselves with that sensibility without which the mind, being unconscious of their position and state, cannot voluntarily exercise them. It is, probably, for conferring this sensibility on the muscles, that the branches of the fifth nerve communicate so frequently with those of the facial and hypoglossal, and the nerves of the muscles of the eye; and it is because of the loss of this sensibility that when the fifth nerve is divided, animals are always slow and awkward in the movement of the muscles of the face and head, or hold them still, or guide their movements by the sight of the objects toward which they wish to move.

(b.) Again, the fifth nerve has an indirect influence on the muscular movements, by conveying sensations of the state and position of the skin and other parts: which the mind perceiving, is enabled to determine appropriate acts. Thus, when the fifth nerve or the infra-orbital branch is divided, the movement of the lips in feeding may cease, or be imperfect.

(c.) An intimate connection with muscular movements through the many reflex acts of muscles of which it is the necessary excitant. Hence, when it is divided and can no longer convey impressions to the nervous centres to be thence reflected, the irritation of the conjunctiva produces no closure of the eye, the mechanical irritation of the nose excites no sneezing.

(d.) Through its ciliary branches and the branch which forms the 
long root of the ciliary or ophthalmic ganglion, it exercises also some influence on the movements of the iris. When the trunk of the ophthalmic portion is divided, the pupil becomes, according to Valentin, contracted in men and rabbits, and dilated in cats and dogs; but in all cases, becomes immovable even under all the varieties of the stimulus of light. How the fifth nerve thus affects the iris is unexplained; it has been ingeniously suggested the influence of the fifth nerve on the movements of the iris may be ascribed to the affection of vision in consequence of the disturbed circulation or nutrition in the retina, when the normal influence of the fifth nerve is disturbed. In such disturbance, increased circulation making the retina more irritable might induce extreme contraction of the iris.

Trophic influence. - The morbid effects which division of the fifth nerve produces in the organs of special sense, make it probable that, in the normal state, the fifth nerve exercises some special or trophic influence on the nutrition of all these organs; although, in part, the effect of the section of the nerve is only indirectly destructive by abolishing sensation, and therefore the natural safeguard which leads to the protection of parts from external injury. Thus, after such division, within a period varying from twenty-four hours to a week, the cornea begins to be opaque; then it grows completely white; a low destructive inflammatory process ensues in the conjunctiva, sclerotica, and interior parts of the eye; and within one or a few weeks, the whole eye may be quite disorganized, and the cornea may slough or be penetrated by a large ulcer. The sense of smell (and not merely that of mechanical irritation of the nose), may be at the same time lost or gravely impaired; so may the hearing, and commonly, whenever the fifth nerve is paralyzed, the tongue loses the sense of taste in its anterior and lateral parts, and according to Gowers in the posterior part as well.

In relation to Taste.-The loss of tactile sensibility as well as the sense of taste, is no doubt due $(a)$ to the lingual branch of the fifth nerve being a nerve of tactile sense, and also because with it runs the chorda tympani, which is one of the nerves of taste; partly, also, it is due (b), to the fact that this branch supplies, in the anterior and lateral parts of the tongue, a necessary condition for the proper nutrition of that part; while $(c)$, it forms also one chief link in the nervous circle for reflex action, in the secretion of saliva. But, deferring this question until the glosso-pharyngeal nerve is to be considered, it may be observed that in some brief time after complete paralysis or division of the fifth nerve, the power of all the organs of the special senses may be lost; they may lose not merely their sensibility to common impressions, for which they all depend directly on the fifth nerve, but also their sensibility to their several peculiar impressions for the reception and conduction of which 
they are purposely constructed and supplied with special nerves besides the fifth. The facts observed in these cases can, perhaps, be only explained by the influence which the fifth nerve exercises on the nutritive processes in the organs of the special senses. It is not unreasonable to believe, that, in paralysis of the fifth nerve, their tissues may be the seats of such changes as are seen in the laxity, the vascular congestion, wdema, and other affections of the skin of the face and other tegumentary parts which also accompany the paralysis; and that these changes, which may appear unimportant when they affect external parts, are sufficient to destroy that refinement of structure by which the organs of the special senses are adapted to their functions.

\section{The VIth Nerve (Ablucens).}

Origin.-The VIth nerve arises from a compact oval nucleus, situated somewhat deeply at the back part of the pons near the middle of the floor of the fourth ventricle. The eminentia teres marks its position. It contains moderately large nerve-cells with distinct axis cylin. der processes. It is connected (fig. 364) with the nuclei of the third, fourth, and seventh nerves. It is nearer the middle line than the nuclei of the fifth and seventh. The root is thin, and passes ventrally and laterally through the reticular formation, to the surface, which it reaches at the hind end of the pons opposite the front end of anterior pyramid.

Functions. - The sixth nerve is exclusively motor, and supplies only the rectus externus muscle of the eye.

The rectus externus is convulsed, and the eye is turned outward, when the sixth nerve is irritated; and the muscle is paralyzed when the nerve is divided. In all such cases of paralysis, the eye squints inward, and cannot be moved outward.

In its course through the cavernous sinus, the sixth nerve forms larger communications with the sympathetic nerve than any other nerve within the cavity of the skull does. But the import of these communications with the sympathetic, and the subsequent distribution of its filiments after joining the sixth nerve, are quite unknown.

\section{The VIIth Nerve (Facial).}

Origin.-The facial, or portio dura of the seventh pair of nerves, arises from the floor of the central part of the fourth ventricle behind and in line with the motor nucleus of the fifth, to the outside of and deeper down than the nucleus of the sixth. 'The nucleus is narrower in front than behind, and consists of large cells with well marked axis cylinder-processes, which are gathered up at the dorsal surface of the nucleus to form a root. The root describes a loop round the nucleus of 
the sixth nerve, running forward for some little distance dorsal to the nucleus, then descending vertically, passing to outside of its own nucleus between it and the ascending root of fifth nerve. It emerges at the hinder margin of the pons lateral to the sixth nerve, opposite the front edge of the groove between the olivary and restiform bodies. It may be connected with the hypoglossal nucleus. There are two roots; the lower and smaller is called the portio intermedia.

Functions. - The seventh nerve is the motor nerve of all the muscles of the face, including the platysma, but not including any of the muscles of mastication already enumerated; it supplies, also, the parotid gland, and through the connection of its trunk with the Vidian nerve, by the petrosal nerves, some of the muscles of the soft palate, probably the levator palati and azygos uvulæ. By its tympanic branches it supplies the stapedius and laxator tympani ; and through the optic ganglion, the tensor tympani; through the chorda tympani it sends branches to the submaxillary gland and to the lingualis and some other muscular fibres of the tongue, and to the mucous membrane of its anterior twothirds; and by branches given off before it comes upon the face, it supplies the muscles of the external ear, the posterior part of the digastricus, and the stylo-hyoideus.

Beside its motor influence, the facial is also, by means of the fibres which are supplied to the submaxillary and parotid glands, a secretory nerve. For, through the last-named branches, impressions may be conveyed which excite increased secretion of saliva.

Paralysis of Facial Nerve. - When the facial nerve is divided, or in any other way paralyzed, the loss of power in the muscles which it supplies, while proving the nature and extent of its functions, displays also the necessity of its perfection for the perfect exercise of all the organs of the special senses. Thus, in paralysis of the facial nerve, the orbicularis palpebrarum being powerless, the eye remains open through the unbalanced action of the levator palpebræ; and the conjunctiva, thus continually exposed to the air and the contact of dust, is liable to repeated inflammation, which may end in thickening and opacity of the cornea. These changes, however, ensue much more slowly than those which follow paralysis of the fifth nerve, and never bear the same destructive character.

The sense of hearing, also, is impaired in many cases of paralysis of the facial nerve; not only in such as are instances of simultaneous disease in the auditory nerves, but in such as may be explained by the loss of power in the muscles of the internal ear. The sense of smell is commonly at the same time impaired through the inability to draw air briskly toward the upper part of the nasal cavities in which part alone the olfactory nerve is distributed; because, to draw the air perfectly in 
this direction, the action of the dilators and compressors of the nostrils should be perfect.

Lastly, the sense of taste is impaired, or may be wholly lost in paralrsis of the facial nerve, provided the source of the paralysis be in some part of the nerve between its origin and the giving off of the chorda tympani. This result, which has been observed in many instances of disease of the facial nerve in man, appears explicable on the supposition that the chorda tympani is the nerve of taste to the anterior two-thirds of the tongue, its fibres being distributed with the so-called gustatory or lingual branch of the fifth. Some look upon the chorda as partly or entirely made up of fibres from the fifth nerve, and not strictly speaking as a branch of the facial; other's consider that it receives its taste fibres from communications with the glosso-pharyngeal.

Together with these effects of paralysis of the facial nerve, the muscles of the face being all powerless, the countenance acquires on the paralyzed side a characteristic, vacant look, from the absence of all expression: the angle of the mouth is lower, and the paralyzed half of the mouth looks longer than that on the other side; the eye has an unmeaning stare. All these peculiarities increase, the longer the paralysis lasts; and their appearance is exaggerated when at any time the muscles of the opposite side of the face are made active in any expression, or in any of their ordinary functions. In an attempt to blow or whistle, one side of the mouth and cheeks acts properly, but the other side is motionless, or flaps loosely at the impulse of the expired air; so in trying to suck, one side only of the mouth acts; in feeding, the lips and cheeks are powerless, and on account of paralysis of the buccinator muscle food lodges between the cheek and gums.

\section{The VIIIth Nerve (Auditory).}

Origin.-The VIIIth nerve arises from two nuclei, median and lateral, in the floor of the fourth ventricle, in the anterior part of the bulb in front and to the side of the twelfth nerve; it extends from the middle line to the outside margin of the ventricle. There is also an accessory nucleus situated on the ventral surface of the restiform body. The nerve leaves the surface of the brain from the ventral surface of the fore-part of the restiform body at the hind margin of the pons in two roots. One winds round the restiform body dorsal to it and the other passes median to it. The former is called the dorsal root. The latter is called the ventral root. Most of the fibres of the dorsal root (cochlear) end in cells of the accessory nuclens, but fibres emerging from this nucleus pass inward to the bulb, superficially, forming the strice acustice in the floor of the fourth ventricle and end in the median nucleus. Most 
of the fibres of the ventral root (vestibular) end in cells of the lateral nucleus. The cells of the median nucleus are small, those of the lateral nucleus large.

Functions. - The cochlear branch is the auditory nerve proper, and the vestibular is distributed to the semicircular canals, the utricule and saccule, parts of the internal ear not directly concerned with hearing.

\section{The IXth Nerve (Glosso-Pharyngeal).}

Origin.-The glosso-pharyngeal nerves (ix., fig. 364), in the enumeration of the cerebral nerves by numbers according to the position in which they leave the cranium, are considered as divisions of the eighth pair of nerves, the vagus and spinal accessory nerves being included with them. The union of the nuclei is indeed so intimate that it will be as well to take the origins of the ninth, tenth, and eleventh nerves together.

These three nerves emerge from the bulb and spinal cord in their numerical order from above downward, the bulbar portion from the lateral aspect of the bulb in a line between the olivary and restiform bodies; and the spinal portion from a line intermediate between the anterior and posterior nerve roots as far down as the sixth or seventh cervical.

The combined glosso-pharyngeal-accessory-vagus nucleus appears to consist of two parts, viz., one median or common origin, having conspicuous nerve-cells of moderate size, and three lateral origins, having but few cells of small size. These are-i. the nucleus ambiguus, which lies on the lateral side of the reticular formation and is the origin of the vagus; ii. the fasciculus solitarius, situated in the bulb, ventral and a little lateral to the combined nucleus, is also called the ascending root of the glosso-pharyngeal nerve or the respiratory bundle; and iii. the spinal portion which takes origin from a group of cells lying in the extreme lateral margin of the anterior cornu. This is the origin of the spinal accessory; it corresponds to the antero-lateral nucleus of the bulb, and the lateral part of the gray matter of the spinal cord.

The fibres of the spinal origin of the nerve pass from these cells through the lateral column to the surface of the cord.

The fibres from the combined nucleus, chiefly from the median part, pass in a ventral and lateral direction through the reticular formation, then ventral to or through the gelatinous substance and strand of fibres connected with the fifth nerve, to the surface of bulb.

The fibres from the nucleus ambiguus join the combined nerve, but especially the vagus.

The bundles of fibres of the fasciculus solitarius start in the lateral gray matter of the cervical cord and higher in the reticular formation of the bulb, run longitudinally forward to pass into the roots of the ninth nerve. 
IXth Nerve.-Distribution. - The glosso-pharyngeal nerve gives filaments through its tympanic branch (Jacobson's nerve), to the fenestra ovalis and fenestra rotunda, and the Eustachian tube, parts of the middle ear; also, to the carotid plexus, and through the petrosal nerve, to the spheno-palatine ganglion. After communicating, either within or without the cranium, with the vagus, and soon after it leaves the cranium, with the sympathetic, digastric branch of the facial, and the accessory nerve, the glosso-pharyngeal nerve parts into the two principal divisions indicated by its name, and supplies the mucous membrane of the posterior and lateral walls of the upper part of the pharynx, the Eustachian tube, the arches of the palate, the tonsils and their mucous membrane, and the tongue as far forward as the foramen cæcum in the middle line, and to near the tip at the sides and inferior part.

Functions. - The glosso-pharyngeal nerve contains some motor fibres, together with those of common sensation and the sense of taste.

1. Motor fibres are distributed to the palato-pharyngeus, the stylopharyngeus, palato-glossus, and constrictors of the pharynx.

2. Sensory fibres in the parts which it supplies, and a centripetal nerve through which impressions are conveyed to be reflected to the adjacent muscles.

3. Fibres for the SPECIAL NERVE of taste (from its fibres derived from the fifth, Gowers), in all the parts of the tongue and palate to which it is distributed. After many discussions, the question, Which is the nerve of taste?-the chorda tympani, the gustatory, or the glosso-pharyngeal? - may be most probably answered by stating that they are not themselves, strictly speaking, nerres of this special function, but through their connection with the fifth nerve. For very numerous experiments and cases have shown that when the trunk of the fifth nerve is paralyzed or divided, the sense of taste is completely lost in the superior surface of the anterior and lateral parts of the tongue, at the back of the tongue, and on the soft palate and palatine arches. The loss is instantaneous after division of the nerve, and, therefore, cannot be ascribed wholly to the defective nutrition of the part, though to this, perhaps, may be ascribed the more complete and general loss of the sense of taste when the whole of the fifth nerve has been paralyzed.

\section{The Xth Nerve (Vagus or Pneumogastric).}

The origin of the Vagus nerve is, as we have just seen, situated in the lower half of the calamus scriptorius in the ala cinerea (fig. 365). Its mucleus is said to represent the cells of Clarke's (posterior vesicular) column of the spinal cord. In origin it is closely connected with the ninth, eleventh, and the twelfth. The combined glosso-pharyngeal- 
vago-accessory nuclei lie outside of, close to, and parallel with the nucleus of the twelfth.

Distribution. - It supplies sensory branches, which accompany the sympathetic on the middle meningeal artery, and others which supply the back part of the meatus and the adjoining part of the external ear. It is connected with the petrous ganglion of the glosso-pharyngeal, by means of fibres to its jugular ganglion; with the spinal accessory which supplies it with its motor fibres for the larger and upper portion of the asophagus, and with its inhibitory fibres for the heart; also with the twelfth; with the superior cervical ganglion of the sympathetic; and with the cervical plexus. It has, of all the nerves, the most varied distribution and functions, either through its own filaments, or through those which, derived from other nerves, are mingled in its branches. The parts supplied by the branches of the vagus are as follows:-

(1.) By its pharyngeal branches, which enter the pharyngeal plexus, a large portion of the mucous membrane, and, probably, all the muscles of the pharynx.

(2.) By the superior laryngeal nerve, the mucous membrane of the under service of the epiglottis, the glottis, and the greater part of the larynx, and the crico-thyroid muscle.

(3.) By the inferior laryngeal nerve, the mucous membrane and muscular fibres of the trachea, the lower part of the pharynx and larynx, and all the muscles of the larynx except the crico-thyroid.

(4.) By its cesophageal branches, the mucous membrane and muscular coats of the œsophagus.

(5.) Through the cardiac nerves, moreover, the branches of the vagus form a large portion of the supply of nerves to the heart and the great arteries.

(6.) Through the anterior and the posterior pulmonary plexuses to the lungs.

(7.) Through its gastric branches to the stomach; and to the intestines, and kidneys, by its terminal branches.

(8.) Through its hepatic and splenic branches, the liver and the spleen are partly supplied with nerves.

Functions.-Throughout its whole course, the vagus contains both sensory and motor fibres. To summarize the many functions of this nerve, which have been for the most part considered in the preceding chapters, it may be said that it supplies (1) motor influence to the pharynx and œsophagus, stomach and intestines, to the larynx, trachea, bronchi, and lung; (2) sensory and, in part, (3) vaso-motor influence, to the same regions; (4) inhibitory influence to the heart; (5) inhibi- 
tory afferent impulses to the vaso-motor centre; (6) excito-secretory to the salivary glands; $(\%)$ excito-motor in coughing, vomiting, etc.

Effects of Section.-Division of both vagi, or of both their recurrent

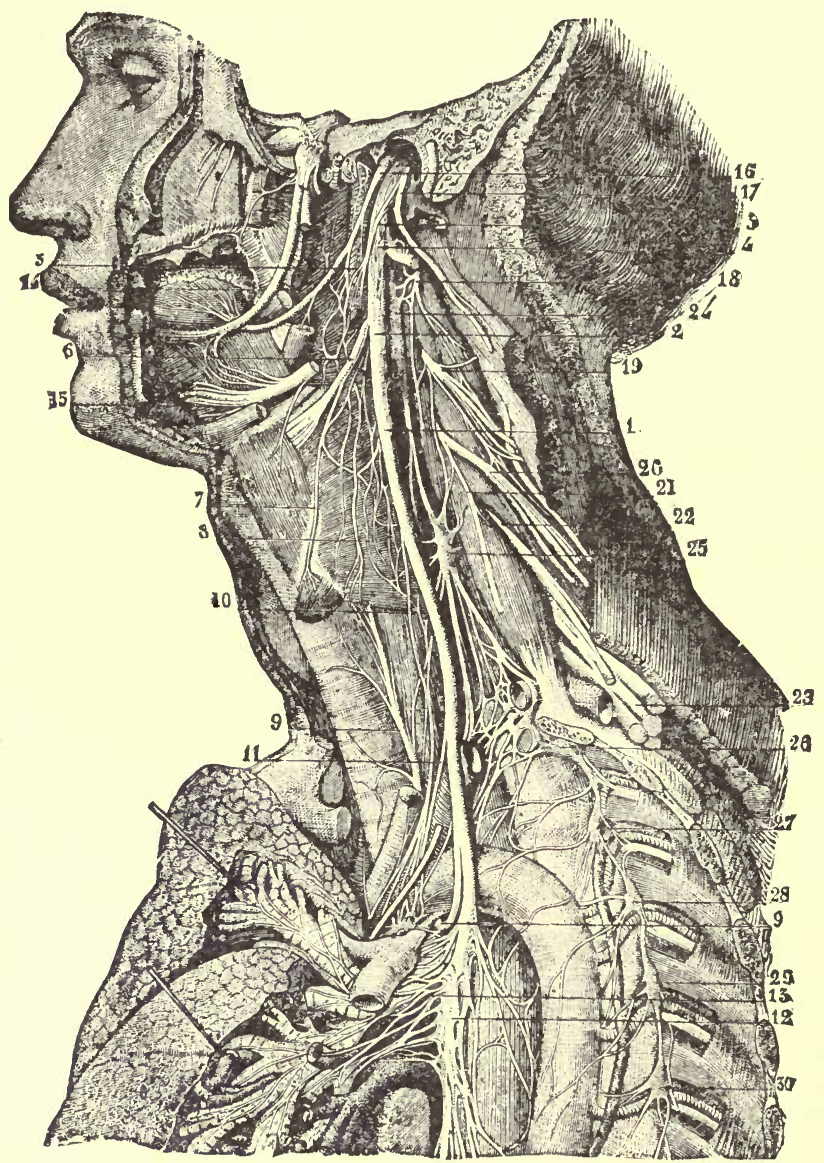

Fig. 368.-View of the nerves IX, $\mathrm{X}$, and XI, their distribution and connections on the left side. 2-5.-1, Pneumogastric nerve in the neck; 2, ganglion of its trunk; 3 , its union with the spinal accessory; 4, its union with the hypoglossal: 5, pharyngeal branch; 6, superior laryngeal nerve; 7, external laryngeal; 8, laryngeal plexus; 9. inferior or recurrent laryngeal; 10 , superior cardiac branch; 11 , middle cardiac; 12 , plexiform part of the nerve in the thorax; 13, posterior pulmonary plexus; 14 , lingual or gustatory nerve of the inferior maxillary; 15 , bypoglossal, passing into the muscles of the tongue, giving its thyro-hyoid branch, and uniting with twigs of the lingual; 16 , glosso-pharyngeal nerve; $1 \%$, spinal accessory nerve, uniting by its inner branch with the pneumogastric, and by its outer, passing into the sterno-mastoid muscle; 18 , second cervical nerve; 19 , third; 20 , fourth; 21 , origin of the phrenic nerve, 22,23 , fifth, sixth, seventh, and eighth cervical nerves, forming with the first dorsal the brachial plexus; 24 , superior cervical ganglion of the sympathetic; 25 , middle cervical ganglion; 26 , inferior cervical ganglion united with the first dorsal ganglion; $27,28,29,30$, second, third, fourth, and fifth dorsal ganglia. (From Sappey after Hirschfeld and Leveillé.)

branches, is often very quickly fatal in young animals; but in old animals the division of the recurrent nerve is not generally, and that of both the vagi is not always, fatal, and, when it is so, death ensues slowly. 
This difference is, that the yielding of the cartilages of the larynx in young animals permits the glottis to be closed by the atmospheric pressure in inspiration, and so they are quickly suffocated unless tracheotomy be performed. In old animals, the rigidity and prominence of the arytenoid cartilages prevent the glottis from being completely closed by the atmospheric pressure; even when all the muscles are paralyzed, a portion at its posterior part remains open, and through this the animal continues to breathe.

In the case of slower death, after division of both the vagi, the lungs are commonly found gorged with blood, œdematous, or nearly solid, from a kind of low pneumonia, and the bronchial tubes full of frothy bloody fluid and mucus, to which, in general, the death may be ascribed. These changes are due, in part, to the passage of food and of the various secretions of the mouth and fauces through the glottis, which, being deprived of its sensibility, is no longer stimulated or closed in consequence of their contact.

\section{Tlie XIth Nerve (Spinal Accessory).}

Origin and Connections. - The nerve arises by two distinct originsone from a centre in the floor of the fourth ventricle, partly but chiefly in the medulla, and connected with the glosso-pharyngeal-vagus-nucleus; the other, from the outer side of the anterior cornu of the spinal cord as low down as the fifth or sixth cervical nerve. The fibres from the two origins come together at the jugular foramen, but separate again into two branches, the inner of which, arising from the medulla, joins the vagus, to which it supplies its motor fibres, consisting of small medullated or visceral nerve-fibres, while the outer consisting of large medullated fibres, supplies the trapezius and sterno-mastoid muscles. The small-fibred branch is said to arise from a nucleus corresponding to the posterior vesicular column of Clarke.

The principal branch of the accessory nerve, its external branch, then supplies the sterno-mastoid and trapezius muscles; and, though pain is produced by irritating it, is composed almost exclusively of motor fibres. The internal branch of the accessory nerve supplies chiefly viscero-motor filaments to the vagus. The muscles of the larynx, all of which, as already stated, are supplied, apparently, by branches of the vagus, are said to derive their motor nerves from the accessory; and (which is a very significant fact) Vrolik states that in the chimpanzee the internal branch of the accessory does not join the vagus at all, but goes direct to the larynx.

Among the roots of the accessory nerve, the lower or external, arising from the spinal cord, appears to be composed exclusively of motor 
fibres, and to be destined entirely to the trapezius and extending from the back of the fourth ventricle to the level of the olivary bodies close to the middle line, inside the combined nucleus of the ninth, tenth, and eleventh nerves.

\section{The XIIth Nerve (Hypoglossal).}

Origin and Connections. - The nerve arises from a large-celled and very long nucleus in the bulb, extending from the back of the fourth ventricle to the level of the olivary bodies close to the middle line, inside the combined nucleus of the ninth, tenth, and eleventh nerves. Fibres from this nucleus run from the ventral surface through the reticular formation in a series of bundles passing between the olivary nucleus laterally and the anterior pyramid and accessory olive medially, to gain the surface. The nerve emerges from a groove between the anterior pyramid and olivary body. The fibres of origin are continuous with the anterior roots of the spinal nerves. It is connected with the vagus, the superior cervical ganglion of the sympathetic and with the upper cervical nerves.

Distribution.-This nerve is the motor nerve to the muscles connected with the hyoid bone, including those of the tongue. It supplies through its descending branch (descendens noni), the sterno-hyoid, sterno-thyroid, and omo-hyoid; through a special branch, the thyrohyoid, and through its lingual branches, the genio-hyoid, stylo-glossus, hyo-glossus, and genio-hyo-glossus and linguales.

Functions. - The function of the hypoglossal is exclusively motor. As a motor nerve, its influence on all the muscles enumerated above is shown by their convulsions when it is irritated, and by their loss of power when it is paralyzed. The effects of the paralysis of one hypoglossal nerve are, however, not very striking. Often, in cases of hemiplegia involving the functions of the hypoglossal nerve, it is not possible to observe any deviation in the direction of the protruded tongue; probably because the tongue is so compact and firm that the muscles on either side, their insertion being nearly parallel to the median line, can push it straight forward or turn it for some distance toward either side.

\section{The Pons Varolii.}

The pons Varolii is generally spoken of as a great commissure of fibres; of fibres which connect the two halves of the cerebellum and of fibres which connect the bulb and spinal cord with the upper part of the brain. Although this is true it must not be forgotten that the pons contains several masses of gray matter, and also in addition smaller collections of nerve-cells. It is found that on section the following parts 
may be made out in its structure, beginning from the anterior or ventral surface.

(a.) Transverse or commissural fibres connecting the one side of the cerebellum with the other, forming the middle peduncle. These fibres emerge from the lateral parts of the white substance of the hemispheres, having come from the superficial gray matter of the whole surface, from the median vermis, and from the lateral hemispheres. Some of these fibres are truly commissural and probably connect the same points on the surfaces of the two halves; some end in the gray matter of the same side of the pons on the ventral surface, and others cross to the opposite side of the pons and then become longitudinal, passing on to the tegmentum, a system of fibres and gray matter to be immediately described.

(b.) Fibres longitudinal in direction which are arranged in larger or smaller bundles separated by gray matter; some of these fibres are what are called the pyramidal fibres, which pass down to the anterior pyramids of the bulb.

(c.) The dorsal portion of the pons is made up to a considerable extent of the reticular formation of the tegmental region together with one or two distinct bundles of longitudinal fibres: i., the chief, situated toward the junction of the ventral two thirds with the dorsal third, is the fillet, which consists of two portions, outer and median; and ii., the second, a bundle of similar fibres, posterior longitudinal bundles, is situated between the two divisions of the fillet below the lateral and to the outer side of the median.

(d.) In the fore part of the pons, a mass of gray matter containing pigment, the locus coruleus, possibly forming the origin of the fifth nerve, and in the back part a second mass of gray matter, the superior olive.

\section{The Crura Cerebri.}

The crura cerebri (III, fig. 354) diverge from the anterior edge of the pons Varolii and pass upward on either side toward the cerebral hemispheres. At their anterior termination each of them appears to have upon its dorsal surface, to the inner and outer sides respectively, two large masses of gray matter which have been already spoken of, viz., the optic thalamus and the corpus striatum. These bodies are not only as it were placed upon the surface of each crus, but are also deeply embedded in its substance.

The crus is found to be made up of two principal parts:-

(a.) The one, the tegmentum, situated for the most part on the dorsal aspect, is composed chiefly of gray matter and some longitudinal fibres.

And (b.) the other, the crusta, situated toward the other surface, is composed almost entirelv of longitudinal fibres. It is known also as the 
pes. Separating these two parts, is a mass of gray matter of the shape of a lens, called the locus or nucleus niger or substantia nigra.

The tegmentum situated dorsally ends for the most part in the neighborhood of the optic thalamus and the parts beneath. In consequence of this the fibres of the pes are allowed to come dorsally and to proceed between the optic thalamus and the more posterior part (the lenticular nucleus) of the corpus striatum, on their course to the cerebral cortex. When in this situation they form a compact mass of fibres. As they pass more dorsally the fibres spread out in the form of a fan, and this arrangement is called the corona radiata. The fibres of the pes

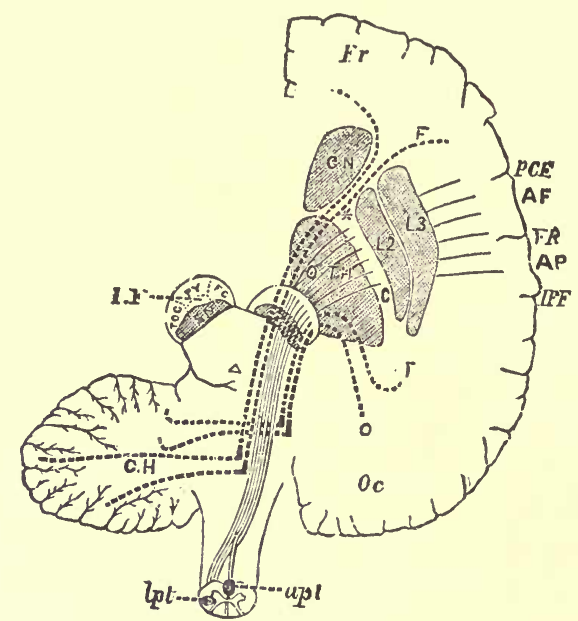

Fig. 369.-Diagram of the motor tract as shown in a diagrammatic horizontal section through the cerebral hemispheres, Crura, Pons, and Medulla. Fr., Frontal lobe; Oc., occipital lobe; AF., ascending frontal, AP., ascending parietal convolutions; PCF., pre-central fissure, in front of the ascending frontal convolution; FR. , fissure of Rolando; IPF., inter-parietal fissure, a section of crus is lettered on the left side. 'SN., Substantia nigra; Py., pyramidal motor fibre, which on the right is shown as continuous lines converging to pass through the posterior limb of IC. internal capsule (the knee or elbow of which is shown thus*) upward into the hemisphere and downward through the pons to cross the medulla in the anterior pyramids. (Gowers.)

are found to stretch not only between the optic thalamus and the lenticular nucleus, but also more anteriorly between the former and the caudate nucleus of the corpus striatum which, as we have seen, is to be seen in the floor of the lateral ventricle. The fibres of the pes thus spread out, have the form of a fan bent upon itself as they rise to pass into the cerebral hemisphere. This constitutes the internal capsule, and that portion of it which forms the angle at which the fibres are bent is called the genu of the capsule, that in front of it being the front, and that behind, the hind limb. The fibres constituting the internal capsule are distributed to different districts of the cerebral cortex. They are made up of fibres not only constituting the pyramidal system, but 
also of others which end in the masses of gray matter in the pons or erus itself; but the function of all of the fibres is believed to be to carry impulses downward from the cerebrum either to the spinal cord and so to the cranial nerves, or to the cerebellum.

The tegmentum of either side, on the other hand, is supposed to be concerned, for the most part at any rate, with afferent impulses. It is made up to a very considerable extent of collections of gray matter, the most important of which are $(a)$ the locus or nucleus niger, separating the pes and tegmentum; (b) the nucleus ruber, which is a rounded mass situated more toward the aqueduct of Sylvius; this' extends from the third ventricle to the anterior corpus quadrigeminum. The locus niger extends back as far as the posterior corpus quadrigeminum. (c) A third mass of gray matter is situated beneath the optic thalamus, and is the corpus subthalamicum. Posteriorly the tegmentum is made up chiefly of the reticular material so often spoken of, and in the pons consists almost entirely of that kind of structure, but with the two additional masess of gray matter already indicated, viz., the locus cœruleus and superior olive.

It will be as well here to indicate briefly the other collections of gray matter in the neighborhood of the crura, viz., the corpus striata, optic thalami, corpora quadrigemina, corpora geniculata, and the corpora dentata of the cerebellum.

Corpora Striata. - The corpora striata are situated in front and to the outside of the optic thalami, partly within and partly without the lateral ventricle.

Each corpus striatum consists of two parts:-

(a.) An intraventricular portion (caudate nucleus) which is conical in shape, with the base of the cone forward; it consists of gray matter, with white substance in its centre. (b.) An extraventricular portion (lenticular nucleus), which is separated from the other portion by a layer of white material, which forms a portion of the internal capsule,- - the anterior limb. The lenticular nucleus is seen, on a horizontal section of the hemisphere, to consist of three parts.(the two internal called globus pallidus, major and minor, and the outer called the putamen), separated from one another by white matter, of which the smallest of the three is inside. Each part somewhat resembles a wedge in shape. The upper and internal surface is in relation with the caudate nucleus, being separated from it by the anterior limb of the internal capsule. The remainder of the internal surface is in relation to the optic thalamus, being separated from it by the posterior limb of the internal capsule. The horizontal section is wider in the centre than at the ends. On the outside is the gray lamina (claustrum) separated by a thin white layerexternal capsule-from the lenticular nucleus. 
The cells of the corpora striata are evenly distributed, and not grouped in nuclei. Their neuraxuns pass, for the most part, into the internal capsule. The corpora striata are connected with the cerebellum through these fibres. It is doubtful if these ganglia have any anatomical relations with the cortex of the brain.

Optic Thalami.-The optic thalami are oval in shape, and rest upon the inner and dorsal surfaces of the crura cerebri. The upper surface of each thalamus is free, and of white substance; it projects into the lateral ventricle. The posterior surface is also white. 'The inner sides of the two optic thalami form the outer borders of the third ventricle, are in partial contact, and are composed of gray material uncovered by white and are, as a rule, connected together by a transverse portion.

The optic thalamus is composed of several collections of gray matter, forming somewhat indistinctly defined masses separated by white fibres. These masses of gray matter are known as the nuclei of the thatamus, and they are six in number. They are called the anterior tubercle, the median nucleus, the lateral nucleus, the ventral nucleus, the pulvinar, and the posterior nucleus. The anterior tubercle is composed of large nerve-cells whose neuraxons pass down to the corpora mammillaria at the base of the brain. There they meet the fibres of the fornix which connect this tubercle of the thalamus with the hippocampal convolution. The median nucleus is connected by its neuraxons with the cortex of the Island of Reil and the second and third convolutions. The lateral nucleus is quite large and lies against the internal capsule, into which it sends fibres. It is connected with the central convolutions. The ventral nucleus lies beneath the preceding; it is small in size. It is connected with the cortex of the frontal lobe and with the operculum, the central convolutions, and the supramarginal gyrus. The fifth nucleus, known as the pulvinar, forms the posterior tip of the thalamus, and is connected with the optic tract. The posterior nucleus, lying just below the pulvinar, is a small mass and is connected with the cortex of the inferior parietal convolution. The cells of the optic thalamus are thus seen to be connected with a large area of the cerebral cortex. They are also connected with the sensory, and probably, to some extent, with the motor tracts coming from below.

Corpora Quadrigemina.-There are two on each side, anterior and posterior; they form prominences on the dorsal surface of the pons and crura above the aqueduct of Sylvius. They are composed of alternate layers of white and gray matter. The posterior bodies receive fibres from the eighth nerve and the sensory tract, known as the fillet. They send fibres out to the temporal region of the brain. They are closely associated with the lateral corpora geniculata. The anterior corpora quadrigemina are connected by fibres with the optic nerve and 
also the fillet, and send fibres to the occipital cortex of the brain. They are closely associated with the median corpora geniculata.

Corpora Geniculata. - These are two on either side, lateral or outer and median or inner; the former is developed from the fore-brain, the latter from the mid-brain. The lateral corpus geniculatum is at the side of the crus and appears to be a swelling on the lateral division of the optic tract. Similarly the median appears to be the termination of the median division of the optic tract. They both contain gray matter (fig. 363).

Corpora Dentata are plicated areas of gray matter in the interior of the cerebellum, not unlike the olivary body of the bulb. The fibres from each pass chiefly to the superior peduncle of its own side.

The Cerebrum.-For convenience of description, the surface of the brain has been divided into five lobes (Gratiolet).

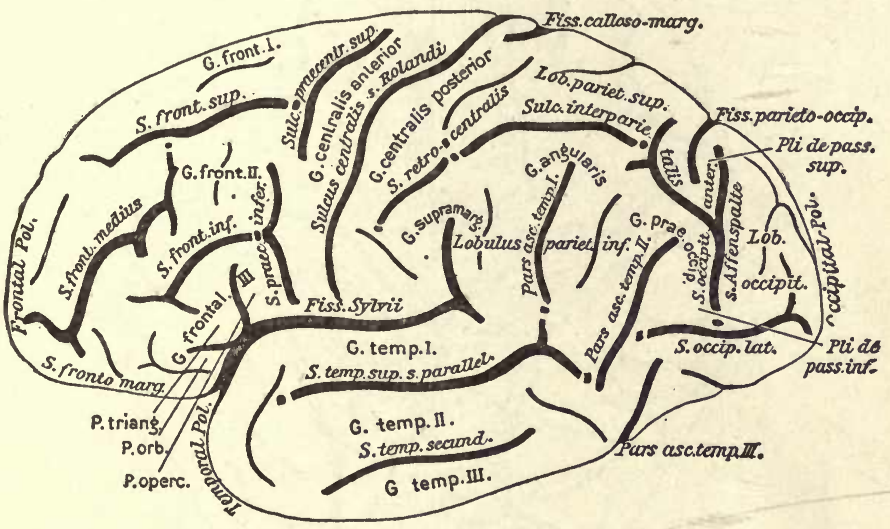

Fig. 3r0.-Left hemisphere, from without. (After Eberstaller.)

1. Frontal (fig. $3 \% 0$ ), limited behind by the fissure of Rolando (central fissure), and beneath by the fissure of Sylvius. Its surface consists of three main convolutions, which are approximately horizontal in direction, and are broken up into numerous secondary gyri. They are termed the superior, middle, and inferior frontal convolutions. In addition, the frontal lobe contains, at its posterior part, a convolution which runs upward almost vertically (ascending frontal), and is bounded in front by a fissure termed the præcentral, behind by that of Rolando.

2 Parietal. This lobe is bounded in front by the fissure of Rolando, behind by the external perpendicular fissure (parieto-occipital), and below by the fissure of Sylvius. Behind the fissure of Rolando is the ascending parietal convolution, which swells out at its upper end into what is termed the superior parietal lobule. The superior parietal lobule is separated from the inferior parietal lobule by the intra-parietal 
sulcus. The inferior parietal lobule (pli courbe) is situated at the pos. terior and upper end of the fissure of Sylvius; it consists of $(a)$ an anterior part (supra-marginal convolution) which hooks round the end of the fissure of Sylvius, and joins the superior temporal convolution, and a posterior part (b) (angular gyrus) which hooks round into the middle temporal convolution.

3. Temporal contains three well-marked convolutions, parallel to each other, termed the superior, middle, and inferior temporal. The superior and middle are separated by the parallel fissure.

4. Occipital. This lobe lies behind the external perpendicular

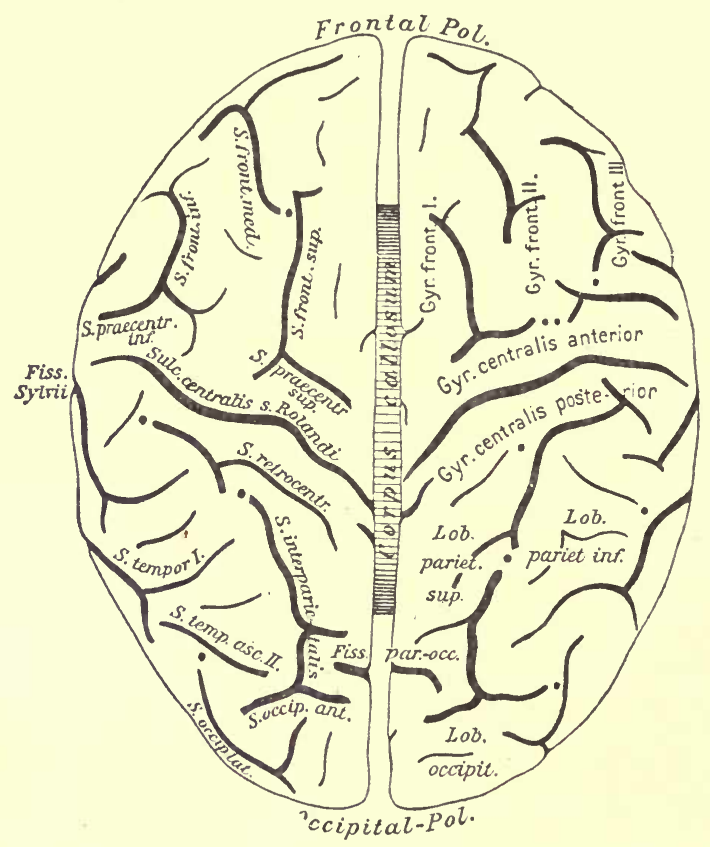

Fig. 3ĩ.-The cerebrum, from above. (After Eberstaller.)

or parieto-occipital fissure, and contains three convolutions, termed the superior, middle, and inferior occipital. They are often not well marked. In man, the external parieto-occipital fissure is only to be distinguished as a notch in the inner edge of the hemisphere; below this it is quite obliterated by the four annectant gyri (plis de passage) which run nearly horizontally. The upper two connect the parietal, and the lower two the temporal with the occipital lobe.

5. Central lobe, or island of Reil, which contains a number of radiating convolutions (gyri operti).

The fig. 372 shows the following gyri and sulci:-

Gyrus fornicatus, a long curved convolution, parallel to and curving 
round the corpus callosum, and swelling out at its hinder and upper end into the quadrate lobule (præcuneus), which is continuous with the superior parietal lobule on the external surface. Marginal convolution runs parallel to the preceding, and occupies the space between it and the edge of the longitudinal fissure. The two convolutions are separated by the calloso-marginal fissure. The internal perpendicular fissure is well marked, and runs downward to its junction with the calcarine fissure: the wedge-shaped mass intervening between these two is termed the cuneus. The calcarine fissure corresponds to the projection into the posterior cornu of the lateral ventricle, termed the Hippocampus minor. The temporal lobe on its internal aspect is seen to end in a hook (uncinate gyrus). The notch round which it curves is continued up and back as the dentate or hippocampal sulcus: this fissure underlies the

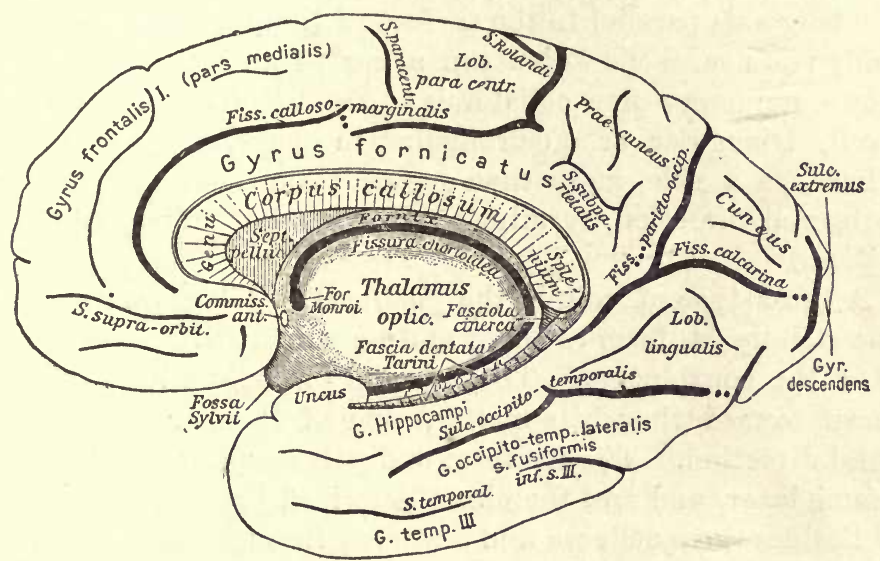

Fig, 872.-Right hemisphere, from within. (After Eberstaller.)

projection of the hippocampus major within the brain. There are three internal temporo-occipital convolutions, of which the superior and inferior ones are usually well marked, the middle one generally less so.

The collateral fissure (corresponding to the eminentia collateralis) forms the lower boundary of the superior temporo-occipital convolution.

All the above details will be found indicated in the diagrams (figs. $371,372)$.

Structure.-The cerebrum is constructed like the other chief divisions of the cerebro-spinal system, of gray and white matter; and, as in the case of the Cerebellum (and unlike the spinal cord and medulla oblongata) the gray matter (cortex) is external, and forms a capsule or covering for the white substance. For the evident purpose of increasing its amount without undue occupation of space, the gray matter is variously infolded so as to form the cerebral convolutions. 
The cortical gray matter of the cerebral cortex has an average thickness of about $\frac{1}{8}$ inch $\left(3 \mathrm{~mm}\right.$.), being thin in the occipital lobe, $\frac{1}{12}$ inch $(2 \mathrm{~mm}$.$) , and thick in the pre-central, \frac{1}{6}$ inch $(4 \mathrm{~mm}$.). The cells of which the substance is composed are of different kinds: $(a)$ The apical process is very long and reaches up often nearly to the surface. It gives off lateral branches, and is studded along its course with little projections called gemmules. This process is a protoplasmic process or dendrite; the cell has other dendrites given off from the angles of the body of the cell. It always has an axis-cylinder process or neuraxon which passes off usually from about the middle of the base. There are, besides these large pyramidal cells, other's practically of the same shape and structure but smaller. They are the small pyramidal cells.

(b) In the superficial layer of the cortex there is a peculiar type of cell, first described by Cajal. Most of these bodies are fusiform in shape, with the long axis parallel to the surface of the convolution. They give off usually two neuraxons which run along parallel to the surface and send down numerous fine collaterals at right angles. Another form of Cajal cell, triangular or quadrangular in shape, is also seen. Both forms have, as a rule, more than one neuraxon. Their collaterals pass in a horizontal direction, forming a fine band of fibres, known as tangential fibres.

(c) A third type of cell is the fusiform or polymorphous. Some of these are strictly fusiform in shape and lie with their axis parallel to the surface of the convolution. They give off protoplasmic processes which pass down toward the white matter, some of them turning to run in a horizontal direction. The fusiform and polymorphous cells are grouped in the same layer, and are, therefore, described together.

(d) Besides these cells we find scattered through the cortex a considerable number of the neuroglia-cells. The character and position of these are shown in fig. 373 .

The general arrangement of the layers of the cortex is described very differently by different authors, and it differs in different parts of the brain. The simplest and most representative type, however of the arrangement is that in which the cortex is divided into four layers. The outermost, or superficial, known as the molecular layer, contains relatively few cells. It is composed of neuroglia tissue, embedded in which are a number of cells of the Cajal type, which have just been described. There are also in this layer many neuroglia-cells. In the superficial part of the layer of some areas of the cortex are many tangential fibres. The second layer is composed of small pyramidal cells. In parts of the brain there are here interposed what are known as the vertical fusiform cells. The third layer is composed of large pyramidal cells, in which, however, one sees many small pyramids also. The fourth layer is composed of the fusiform and polymorphous cells, and beneath this is the white sub- 
stance. This arrangement is shown in the accompanying figures ( 373 and $373 \mathrm{~A})$. The gray matter of the brain contains, however, not only these layers and cells, but an infinitely rich mass of fibres, which can be shown by various stains to have a certain definite arrangement. Some of the fibres are vertical in direction, passing directly up to the most superficial layers of cells; others have a horizontal direction, dividing

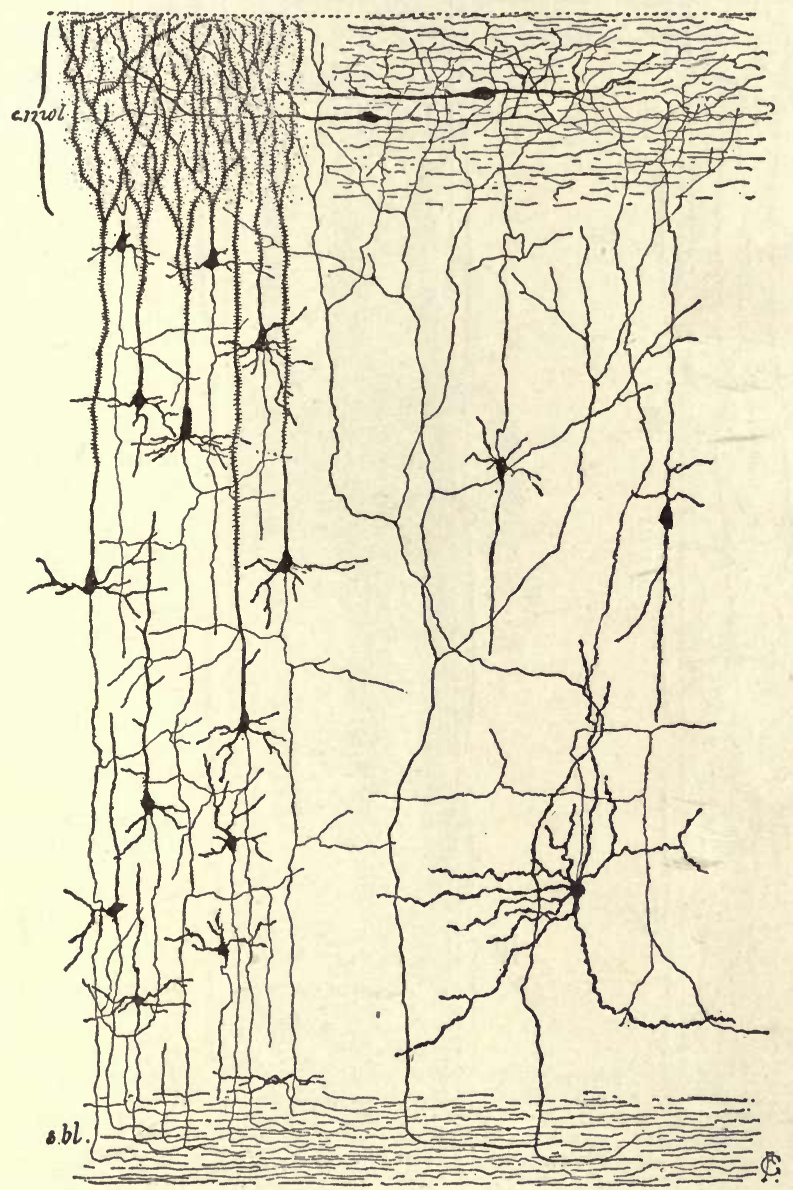

Fig. 3\%3.-The principal constituent elements of the gray cortical layer of the anterior cerebrum. (After Ramon y Cajal.)

the gray matter into different layers. These layers of fibres have received different names. They vary somewhat in accordance with the area of the cortex examined. A typical arrangement is shown in fig. $3 \%$. The most conspicuous are certain large triangular or pyramidal 


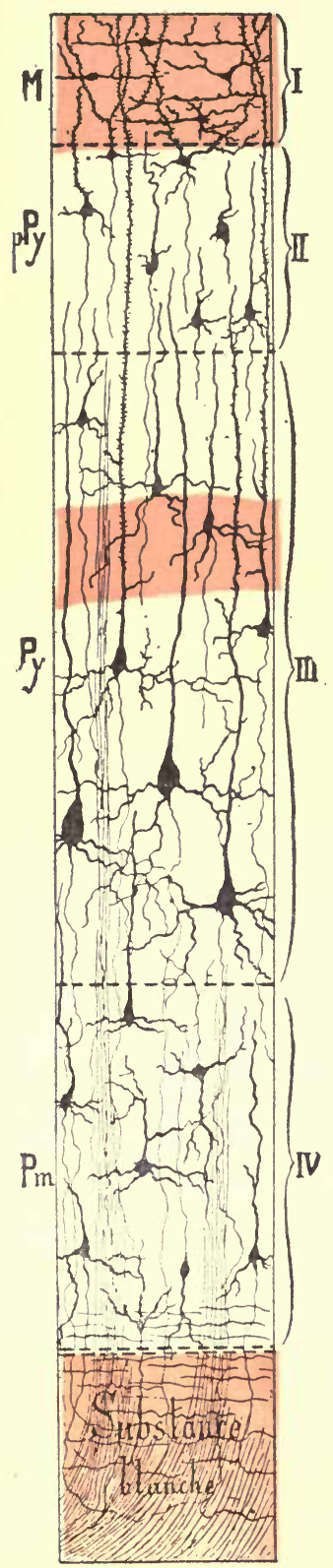

Fig. $3 \pi 3 \mathrm{~A}$.

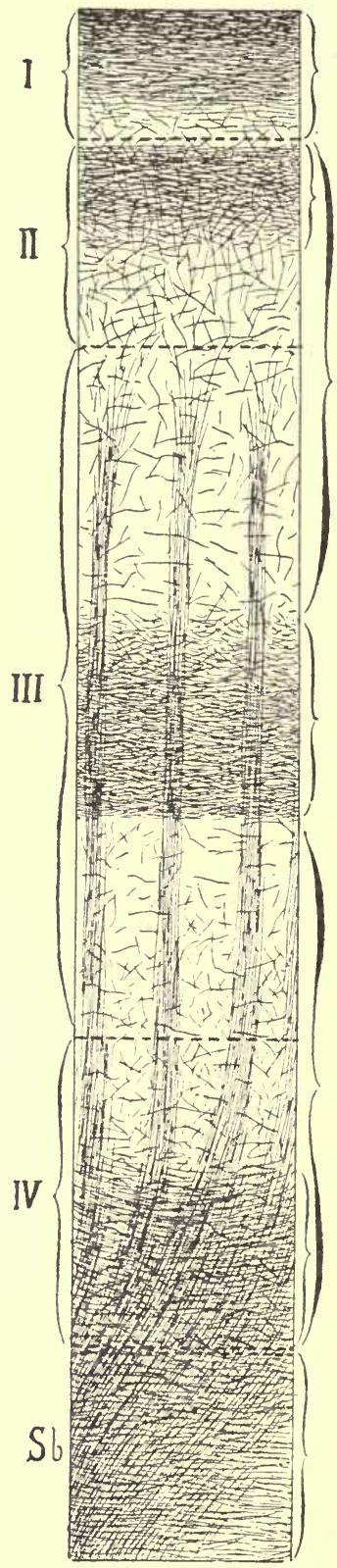

Fig. $3 \pi 4$.
Tangential fibres.

Striæ of Bechterew and de Kaes.

Superradiary network (of the second and third layers).

Striæ of Baillarger.

Interradiary network (of the third and fourth layers).

Meynert's intracortical association fibres.

Subcortical association fibres.

Fig. 3r3A. - Schematic diagram of the different layers of the cerebral cortex. (After Ramon y Tajal, 1890.) The tangential fibles, Vicq d'Azyr's ribbon, Baillarger's internal and external striæ, and the white substance are stained red; $M$, molecular layer; $p P y$. layer of small pyramidal cells; $g P y$, layer of large pyramidal cells; $P m$, layer of polymorphous cells.

Fig. 3r4.-Schematic diagram showing the arrangement of the nerve fibres in the cerebral cortex. The dotted lines separate the four cellular layers of Cajal. $S b$, white substance. 
cells, granular or fibrillated, with large and distinct nuclei, arranged with their apices toward the surface.

Chemical Composition. - The chemistry of nerves and nerve-cells has been chiefly studied in the brain and spinal cord. Nerve matter contains several albuminous and fatty bodies (cerebrin, lecithin, and some others), also fat matter which can be extracted by ether (including cholesterin) and various salts, especially Potassium and Magnesium phosphates, which exist in larger quantity than those of Sodium and Calcium.

Arrangement of the parts of the cerebrum.-The great relative and absolute size of the Cerebral hemispheres in the adult man, masks to a

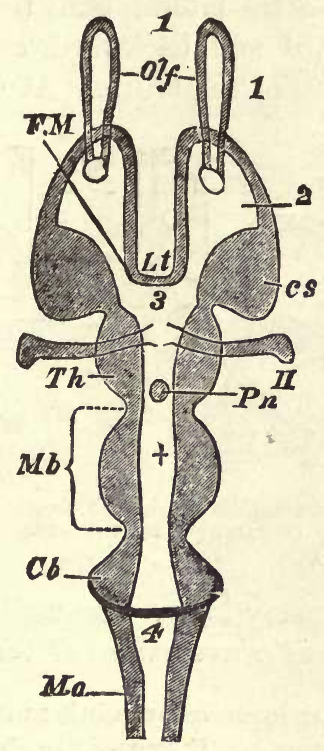

Fig. 375.-Diagrammatic horizontal section of a vertebrate brain. The figures serve both for this and the next diagram. $M b$, mid-brain: what lies in front of this is the fore-, and what lies behind, the hind-brain; $L t$, lamina terminalis; Olf, olfactory lobes; Hmp, hemispheres; $T h$. $E$, thalamencephalon; $P n$, pineal gland; $P y$, pituitary body; $F . M$, foramen of Munro; $c s$, corpus striatum; Th, optic thalamus; $C C$, crura cerebri: the mass lying above the canal represents the corpora quadrigemina; $C b$, cerebellum; $I-I X$, the nine pairs of cranial nerves; 1 , olfactory ventricle; 2 , lateral ventricle; 3 , third ventricle; 4 , fourth ventricle; + , iter a tertio ad quartum ventriculum. (Huxley.)

great extent the real arrangement of the several parts of the brain, which is illustrated in the two accompanying diagrams (figs. 375,376 ).

From these it is apparent that the parts of the brain are disposed in a linear series, as follows (from before backward): olfactory lobes, cere- 
bral hemispheres, optic thalami, and third rentricle, corpora quadrigemina, or optic lobes, cerebellum, medulla oblongata.

This linear arrangement of parts actually occurs in the human fœtus; and it is permanent in some of the lower Vertebrata, e.g., Fishes, in which the cerebral hemispheres are represented by a pair of ganglia intervening between the olfactory and the optic lobes, and considerably smaller than the latter. In Amphibia the cerebral lobes are further developed, and are larger than any of the other ganglia.

In reptiles and birds the cerebral ganglia attain a still further development, and in mammalia the cerebral hemispheres exceed in weight all the rest of the brain. As we ascend the scale, the relative size of the cerebrum increases, till in the higher apes and man the hemispheres, which commenced as two little lateral buds from the anterior cerebral vesicle, have grown upward and backward, completely covering in and hiding from view all the rest of the brain. At the same time the smooth

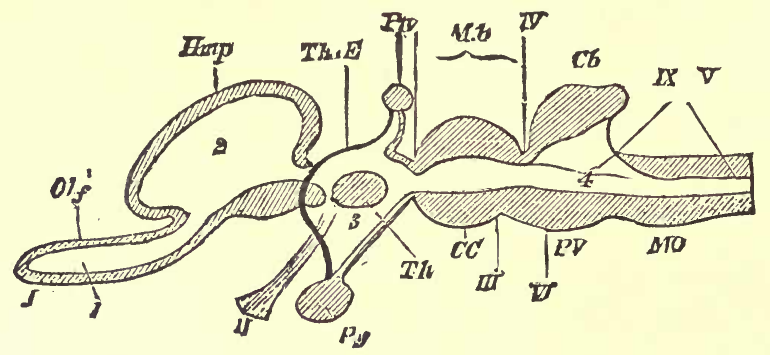

Fig. 376. - Longitudinal and vertical diagrammatic section of a vertebrate brain. Letters as before. Lamina terminalis is represented by the strong black line joining $P n$ and $P y$. (Huxley.)

surface of the brain, in many lower mammalia, such as the rabbit, is replaced by the labyrinth of convolutions of the human brain.

Weight of the Brain.-The brain of an adult man weighs from 48 to $50 \mathrm{oz}$.or about 3 lbs. (about 1550 grms.). It exceeds in absolute weight that of all the lower animals except the elephant and whale. Its weight, relatively to that of the body, is only exceeded by that of a few small birds, and some of the smaller monkeys. In the adult man it ranges from $\frac{1}{30}-\frac{1}{50}$ of the body weight.

Variations. Age.-In a new-born child the brain (weighing 10 to $14 \mathrm{oz}$.) is $\frac{1}{10}$ of the body weight. At the age of 7 years the weight of the brain already averages $40 \mathrm{oz}$., and about 14 years the brain not infrequently reaches the weight of $48 \mathrm{oz}$. Beyond the age of forty years the weight slowly but steadily declines at the rate of about $1 \mathrm{oz}$. in 10 years.

Sex. - The average weight of the female brain is less than the male : and this difference persists from birth throughout life. In the adult it amounts to about $5 \mathrm{oz}$. Thus the average weight of an adult woman's brain is about $44 \mathrm{oz}$.

Intelligence. - The brains of idiots are generally much below the average, some weighing less than $16 \mathrm{oz}$. Still the facts at present collected do not warrant more than a very general statement, to which there are numerous exceptions, that the brain weight corresponds to some extent with the degree of intelligence. There can be little doubt that the complexity and depth of the 
convolutions, which indicate the area of the gray matter of the cortex, correspond with the degree of intelligence.

Weight of the Spinal Cord. - The spinal cord of man weighs from 1-1 $\frac{1}{2} \mathrm{oz}$; its weight relatively to the brain is about $1: 36$. As we descend the scale, this ratio constantly increases till in the mouse it is $1: 4$. In cold-blooded animals the relation is reversed, the spinal cord is the heavier and the more important organ. In the newt, 2:1; and in the lamprey, $75: 1$.

Distinctive Characters of the Human Brain.-The following characters distinguish the brain of man and apes from those of all other animals. (a.) The rudimentary condition of the olfactory lobes. (b.) A perfectly defined fissure of Sylvius. (c.) A posterior lobe completely covering the cerebellum. The presence of posterior cornua in the lateral ventricles.

The most distinctive points in the luman brain, as contrasted with that of apes, are:-(1.) The much greater size and weight of the whole brain. The brain of a full-grown gorilla weighs only about $15 \mathrm{oz}$. (450 grms.), which is less than $\frac{1}{3}$ the weight of the human adult male brain, and barely exceeds that of the human infant at birth. (2.) The much greater complexity of the convolutions, especially the existence in the human brain of tertiary convolutions

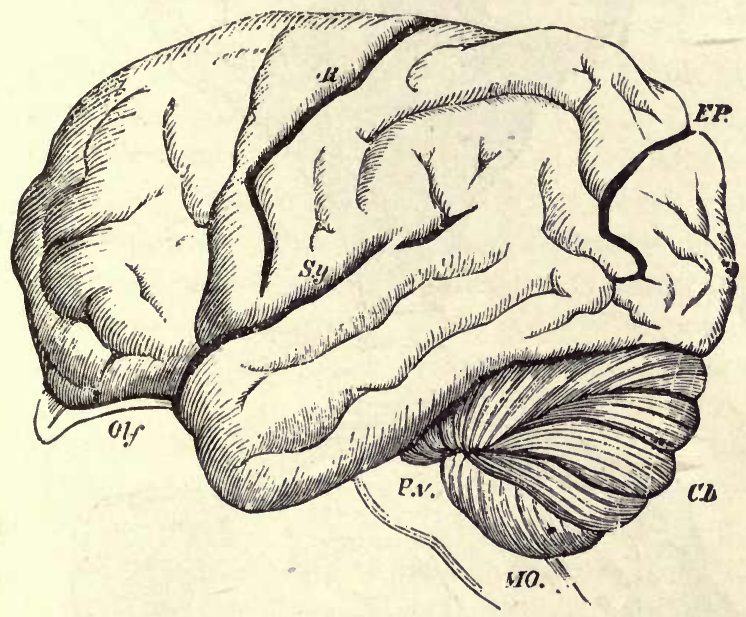

Fig. 377.-Brain of the Orang, 2/3 natural size, showing the arrangement of the convolutions. $S y$, fissure of Sylvius; $R$, fissure of Rolando; $E \dot{P}$, external perpendicular fissure; Olf, olfactory lobe; $C b$, cerebellum; $P V$, pons Varolii; $M O$, medulla oblongata. As contrasted with the human brain, the frontal lobe is short and small relatively, the fissure of Sylvius is oblique, the temporo-sphenoidal lobe very prominent, and the external perpendicular fissure very well
marked. (Gratiolet.)

in the sides of the fissures. (3.) The greater relative size and complexity, and the blunted quadrangular contour of the frontal lobes in man, which are relatively both broader, longer, and higher, than in apes. In apes the frontal lobes project keel-like (rostrum) between the olfactory bulbs. (4.) The much greater prominence of the temporo-sphenoidal lobes in apes. (5.) The fissure of Sylvius is nearly horizontal in man, while in apes it slants considerably up. ward. (6.) The distinctness of the external perpendicular fissure, which in apes is a well-defined almost vertical "slash," while in man it is almost obscured by the annectent gyri.

Most of the above points are shown in the accompanying figure of the brain of the Qrang. 


\section{The Motor areas of the Cerebral Cortex.}

The experiments upon the brains of various animals by means of electrical stimulation have demonstrated that there are definite re-

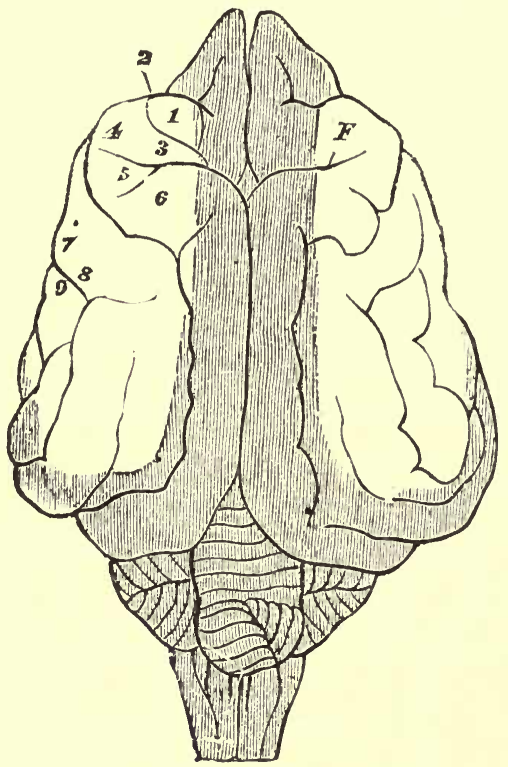

Fig. 3\%8.

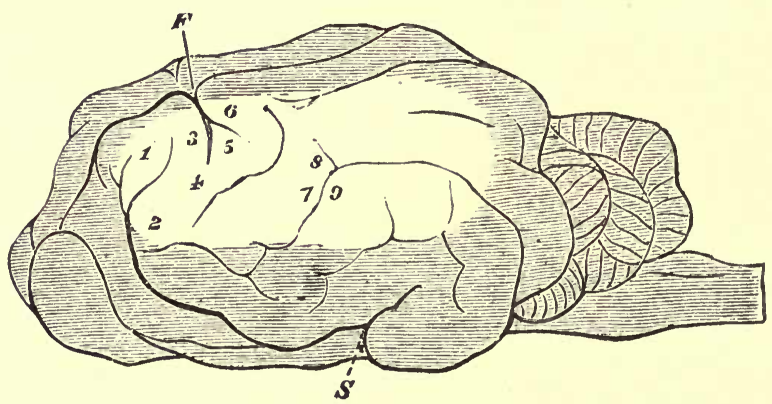

Fig. 3\%9.

Figs. 378 and 3\%9.-Brain of dog, viewed from above and in profile. $F$, frontal fissure some. times termed crucial sulcus, corresponding to the fissure of Rolando in man. $S$, fissure of Sylvius, around which the four longitudinal convolutions are concentrically arranged; 1 , flexion of head on the neck, in the median line; 2 , flexion of head on the neck with rotation toward the side of the stimulus; 3,4 , flexion and extension of anterior limb; 5,6 , flexion and extension of posterior limb; $7,8,9$, contraction of orbicularis oculi. and the facial muscles in general. The unshaded part is that exposed by opening the skull. (Dalton.)

gions of the cerebral cortex the stimulation of which produces definite movements of co-ordinated groups of muscle of the opposite side of the body. Fritsch and Hitzig were the first to show that the cere- 
bral cortex responded to electric irritation. They employed a weak constant current in their experiments, applying a pair of fine electrodes not more than $\frac{1}{12}$ in. apart to different parts of the cerebral cortex. The results thus obtained have been confirmed and extended by Ferrier and many others, chiefly with induction currents.

The fundamental phenomena observed in all these cases may be thus epitomized:-

(1). Excitation of the same spot is always followed by the same movement in the same animal. (2). The area of excitability for any given movement is extremely small, and admits of very accurate definition. (3). In different animals excitations of anatomically corresponding spots produce similar or corresponding results.

The various definite movements resulting from the electric stimulation of circumscribed areas of the cerebral cortex, are enumerated in the description of the accompanying figures of the dog and monkey's brain.

In the case of the dog, the results obtained are summed up as follows, by Hitzig:-

(a.) One portion (anterior) of the convexity of the cerebrum is motor; another portion (posterior) is non-motor. (b.) Electric stimulation of the motor portion produces co-ordinated muscular contraction on the opposite side of the body. (c.) With very weak currents, the contractions produced are distinctly limited to particular groups of muscles; with stronger currents the stimulus is communicated to other muscles of the same or neighboring parts. (d.) The portions of the brain intervening between these motor centres are inexcitable by similar means.

Motorial area of the Monkey's Brain.-According to the observations of Ferrier, confirmed and extended by later experimenters, stimulation of various parts of the monkey's brain, as indicated by the numbers in. figs. 380,381 , produces movements of definite muscles, thus:-

Stimulation of the district marked 1 , causes movement of hind foot: of 2, chiefly adduction of the foot; of 3 , movements of hind foot and tail; of 4, of latissimus dorsi; of 5 , extension forward of arm; $a$, $b, c, d$, movements of hand and wrist; of 6 , supination and flexion of forearm; of $\%$, elevation of the upper lip; of 8 , conjoint action of elevation of upper lip and depression of lower; of 9, opening of mouth and protrusion of tongue; of 10, retraction of tongue; of 11, action of platysma; of 12, elevation of eyebrows and eyelids, dilatation of pupils, and turning head to opposite side; of 13 , eyes directed to opposite side and upward, with usually contraction of the pupils; of $13^{\prime}$, similar action, but eyes usually directed downward; of 14, retraction of opposite ear, head turns to the opposite side, the eyes widely opened, and pupils dilated; of 15 , stimulation of this region, which corresponds to 
the tip of the uncinate convolution, canses torsion of the lip and nostril of the same side.

It is thus seen that the motor areas chiefly correspond with the ascending frontal and ascending parietal convolutions, and.that the movements of the leg are represented at the upper part of these convolutions, then follow from above downward the centres for the arms, the face, the lips, and the tongue.

According to the further researches of Schäfer and Horsley, electrical stimulation of the marginal convolution internally at the parts corresponding with the ascending frontal and parietal convolutions, from

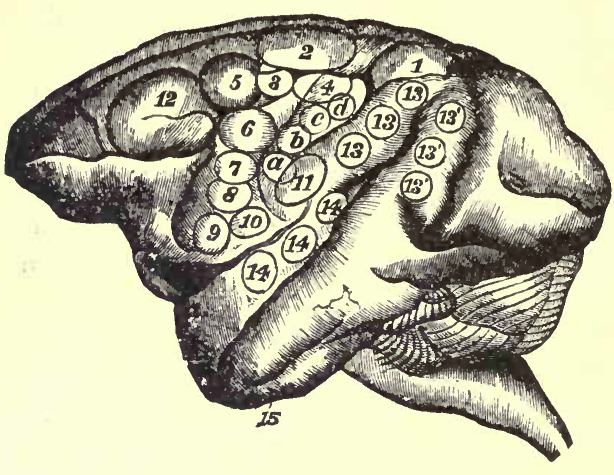

Fig. 380.

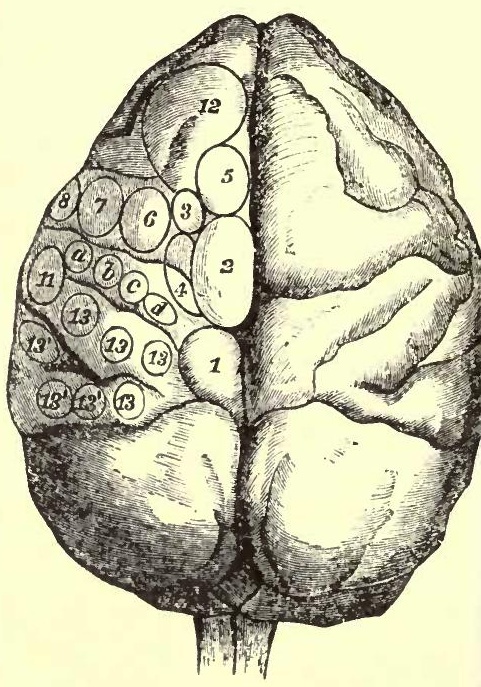

Fig. 381.

Figs. 380 and 381.-Diagrams of monkey's brain to show the effects of electric stimulation of certain spots. (According to Ferrier.)

before backward, produces movements of the arm, of the trunk, and of the leg.

A good deal of doubt was thrown upon the experiments of Ferrier by Goltz and other observers, from the results of excising the so-called motor areas of the dog's brain. It was found that the part might be sliced away or washed away with a stream of water, but that no perma. nent paralysis ensued.

More extensive observations however, have confirmed Ferrier's original statement, at any rate with regard to the monkey's brain. Destruction of the motor areas for the arm produces at any rate some permanent paralysis of the arm of the opposite side, and similarly of that for the leg, paralysis of the opposite leg. If both areas are destroyed permanent hemiplegia ensues. Paralysis of so extensive and permanent character 
does not, however, appear the rule when the brain of a dog is used instead of that of the monkey. It is suggested that in the animal lower in the scale, the functions which in the monkey are discharged by the cortical centres may be subserved by the basal ganglia.

Motorial Areas of the Human Brain.-It is naturally of great importance to discover how far the result of experiments upon the dog and monkey hold good with regard to the human brain. Evidence furnished by diseased conditions is not wanting to support the general idea of the existence of cortical motorial centres in the human brain (fig. 382).

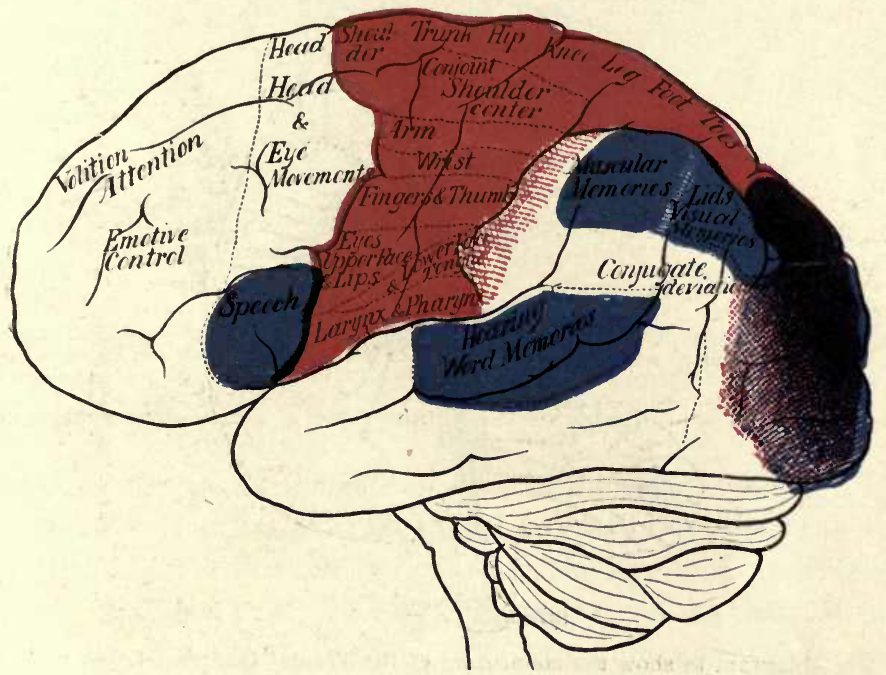

Fig. 382. - The Cortical Centres. (Dana.)

So far, however, it has been possible to localize motor functions in the frontal and ascending parietal convolutions only, to the convolutions which bound the fissure of Rolando, and to those on the inner side of the hemispheres which correspond thereto, and possibly to the frontal lobe in front of the ascending convolution.

The position of the centres is probably much the same as in the monkey's brain-those for the leg above, those for the arm, face, lips, and tongue from above downward. Destruction of these parts causes paralysis, corresponding to the district affected, and irritation causes convulsions of the muscles of the same part. - Again, a number of cases are on record in which aphasia, or the loss of power of expressing ideas in words, has been associated with disease of the posterior part of the lower or third frontal convolution on the left side. This condition is usually associated with paralysis of the right side (right hemiplegia).

This district of the brain is now generally known as the motor area; and there seems no doubt whatever that from this area pass the nerve- 
fibres which proceed to the spinal cord, and are there represented as the pyramidal tracts.

This is the reason, no doubt, that movements are produced on stimulation of the white matter after the superficial gray matter of the animal's brain has been sliced off.

Motor tracts in the brain.-These motor fibres are connected with the pyramidal cells of the cortex, and are indeed their continuations.

It will be necessary, therefore, to trace them from the cortex downward. From the motor area of the cortex they converge to the inter-

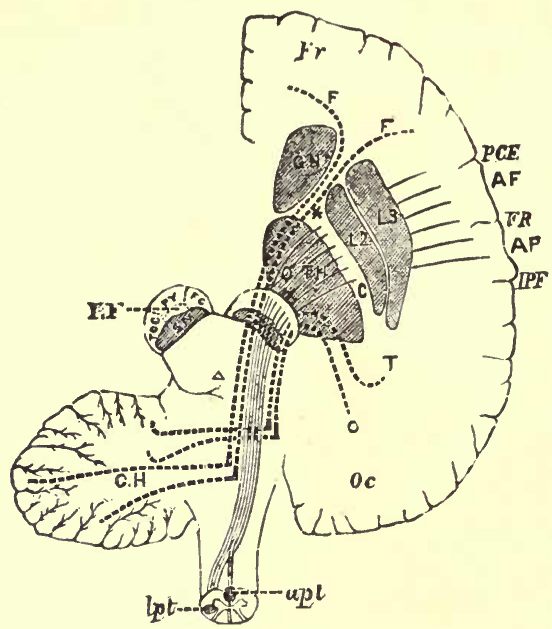

Fig. 383. -Diagram to show the connecting of the Frontal Occipital Lobes with the Cerebellum, etc. The dotted lines passing in the crusta (TOC), outside the motor fibres, indicate the connection between the temporo-occipital lobe and the cerebellum. F.c., the fronto-cerebellar fibres, which pass internally to the motor tract in the crusta; 1 . F., fibres from the caudate nucleus to the pons. FR., frontal lobe; Oc., occipital lobe; $\mathbf{A F}$., ascending frontal; $\mathbf{A P}$., ascend. ing parietal convolutions; PCF., precentral fissure in front of the ascending frontal convolution; FR., fissure of Rolando; IPF., interparietal fissure, a section of crus is lettered on the left side. SN., substantia nigra; PY., pyramidal motor fibre, which on the right is shown as continuous lines converging to pass through the posterior limb of Ic. internal capsule (the knee or elbow of which is shown thus*) upward into the hemisphere and downward through the pons to cross at the medulla in the anterior pyramids. (Gowers.)

nal capsules, and pass down to the crusta of the crus in the way already indicated.

In the internal capsule the fibres which pass onward and downward to the pyramidal tracts of the spinal cord do not occupy more than a small section, namely, that part known as the knee, and the anterior two-thirds of the posterior segment (fig. 384). In this district the fibres for the face, arm, and leg, are in this relation: those for the face and tongue are just at the knee, and below or behind them come first the fibres for the arm and then those for the leg.

The more accurate arrangement of these fibres in the monkey's brain from above down are those for the eye, head, tongue, moutin, shoulder, 
elbow, digits, abdomen, lip, knee, digits. These fibres come for the most part from the part of the cortex on either side of the fissure of Rolando, hence called the Rolandic area on either side. But the areas

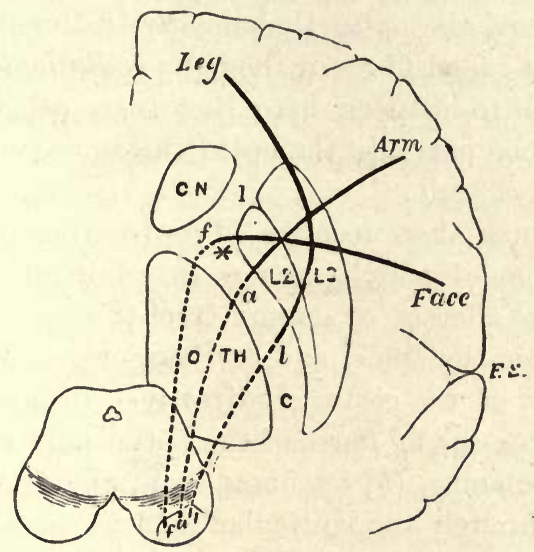

Fig. 384.-Diagram to show the relative positions of the several motor tracts in their course from the cortex to the crus. The section through the convolution is vertical; that through the internal capsule, I, C, horizontal; that through the crus again vertical. C, N, caudate nucleus; $O$, TH, optic thalamus; $L 2$ and L3, middle and outer part of lenticular nucleus; $f, a, l$, face, arm, and leg fibres. The words in italic indicate corresponding cortical centres. (Gowers.)

for the head and eyes lie more anterior in the frontal lobe, to the front of the precentral sulcus, that for the head above that for the eyes, and an area for the trunk (not indicated in the fig. 383), is situated more toward the middle line of the hemisphere, internal to that for the leg.

But there are other fibres which are arranged in front of the pyramidal fibres in the front limb of the capsule, as well as others behind them in the hind limb of the capsule. Those in front are from the anterior part of the frontal lobe, and these in passing into the crus are found on the median side of the pyramidal fibres (fig. 383). They appear to end in the gray matter of the pons, and there to be connected with fibres from the middle peduncle of the opposite side of the cerebellum. Those behind the pyramidal fibres in the hind limb of the capsule are from the temporal-occipital lobe. These fibres pass into the crus to the outer side of the pyramidal fibres (fig. 383), they probably also end in the gray matter in the same way. There are other fibres from the corpus striatum, from both nuclei, but particularly from the caudate nucleus, which pass to the crus, and are situated between the pyramidal tract and the locus niger (fig. 383), some of which terminate in that nucleus, while others terminate in the pons. Besides the above fibres, all of which are believed to be efferent fibres, and are at any rate fibres of descending degeneration, there are fibres which pass from the cortex to the optic thalamus and tegmentum, fibres of ascending degeneration 
found in the internal capsule, viz., those from the frontal lobes are situated at the extreme tip of the front limb, in front of the motor fibres from the same district, and others from the temporal-occipital district converge to the posterior part of the hind limb. Those passing between the occipital lobe and the optic thalamus are believed to be concerned with vision, and are called fibres of the optic radiation.

It may be as well to mention here that some other fibres from the temporo-occipital lobe pass into the optic thalamus, withont forming a part of the internal capsule.

The optic thalamus then receives fibres from nearly all parts of the cerebral cortex, some of which are not found in the internal capsule. The tegmentum, the afferent or sensory tract of the crus to a great extent ends in the optic thalamus, and is, therefore, connected through it with nearly all parts of the cortex, indirectly. It is also more directly connected with cortex $(a)$ by fibres of the optic radiation which do not go to the optic thalamus, $(b)$ by fibres from the frontal and parietal lobes, which pass through the lenticular nucleus, and $(c)$ by fibres from both the lenticular and caudate nuclei of the corpus striatum.

In the tegmentum the longitudinal fibres may be thus enumerated:(a.) The fillet, which consists of fibres from the sensory decussation of

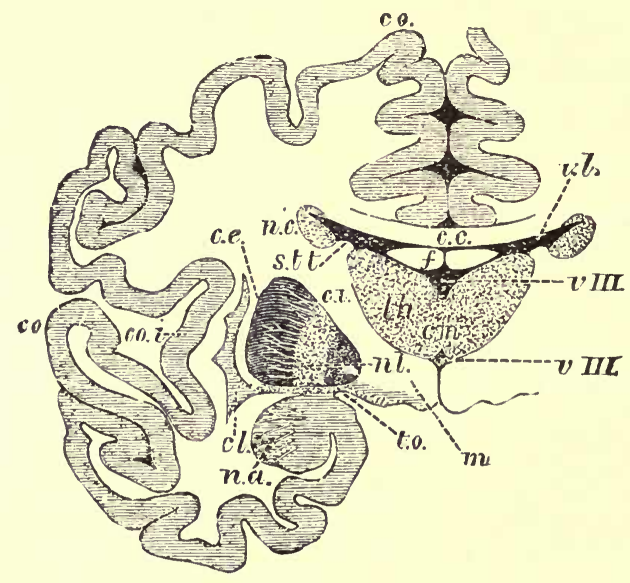

Fig. 385. - Vertical section through the cerebrum and basic ganglia to show the relations of the latter. co, cerebral convolutions; $c, c$, corpus callosum; v.l., lateral ventricle; $f$, fornix; vIII., third ventricle; n.c., caudate nucleus; th, optic thalamus; n.l., lenticular nucleus; c.i., internal capsule; c.l., claustrum; c.e., external capsule; $m$, corpus mammillare; $t . o$. , optic tract; s.t.t., stria terminalis; n.a., nucleus amygdalæ; cm, soft commissure. (Schwalbe.)

the bulb, which becomes longitudinal in the inter-olivary region, and in its course upward, from masses of gray matter, such as the superior olive; it divides into two bundles. (i.) Lateral, ends in gray matter of posterior corpus quadrigeminum and in white matter beneath the anterior, and (ii.) median, ends in anterior corpus quadrigeminum and 
in the corpus subthalamicum, thence to the optic thalamus and the cerebral cortex.

(b.) Posterior longitudinal bundles.-A bundle of fibres which appear to begin the bulb as certain fibres of the anterior column of the cord, which are the short longitudinal commissures between segments of the cord. It is traceable upward as far as the nucleus of the third nerve. It is supposed to connect the nuclei of the fourth and sixth nerves with the third, and with the anterior corpus quadrigeminum.

(c.) Superior peduncle of the cerebellum. - This arises on either side from the superficial gray matter, but chiefly from the corpus dentatum, and passes forward outward beneath the posterior corpus quadrigeminum, and beneath it and the anterior corpus quadrigeminum decussates with its fellow; the fibres then pass forward in the anterior district of the tegmentum and end in the red nucleus.

(d.) Fibres from the corpora quadrigemina.-From each corpus quadrigeminum passes forward and downward a tract called the brachium. The anterior brachium goes to the lateral corpus geniculatum, and then to the optic tract, other fibres pass into the tegmentum, and thence directly to the occipital cortex. The posterior brachium goes to the median corpus geniculatum, thence to the tegmentum, and through it possibly to the temporal region of the cerebral cortex.

Commissural fibres. - In addition to the fibres of the corpus callosum, which connect all parts of the hemispheres, and fornix, there are three other commissures, the anterior white commissure, and the posterior white commissure in the third ventricle connect by white fibres the two sides of the brain. The fibres in the anterior come from the temporosphenoidal convolution chiefly, but a few are part of the olfactory tract. The posterior connects the optic thalami and tegmenta. The middle is chiefly composed of gray matter, but also contains some transverse fibres.

\section{Functions of the Cerebrum.}

Speaking in the most general way, and for the present omitting the accumulating evidence in favor of the direct representation of the various co-ordinated movements of the muscles of the body in ganglia situated in different parts of the cerebral cortex, it may be said that:(1.) The cerebral hemispheres are the organs by which are perceived those clear and more impressive sensations which can be retained, and regarding which we can judge. (2.) The cerebrum is the organ of the will, in so far at least as each act of the will requires a deliberate, however quick determination. (3.) It is the means of retaining impressions of sensible things, and reproducing them in subjective sensations and ideas. (4.) It is the medium of all the higher emotions and feelings, and 
of the faculties of judgment, understanding, memory, reflection, induction, imagination and the like.

Evidence regarding the physiology of the cerebral hemispheres, has been obtained, as in the case of other parts of the nervous system, from the study of Comparative Anatomy, from Pathology, and from Experiments on the lower animals. The chief evidences regarding the functions of the cerebral hemispheres derived from these varions sources, are briefly these:-1. Any severe injury of them, such as a general concussion, or sudden pressure by apoplexy, may instantly deprive a man of all power of manifesting externaliy any mental faculty. 2. In the same general proportion as the higher mental faculties are developed in the Vertebrate animals, and in man at different ages and in different individuals, the more is the size of the cerebral hemispheres developed in comparison with the rest of the cerebro-spinal system. 3. No other part of the nervous system bears a corresponding proportion to the development of the mental faculties. 4. Congenital and other morbid defects of the cerebral hemisphere are, in general, accompanied by corresponding deficiency in the range or power of the intellectual faculties and the higher instincts. 5. Removal of the cerebral hemispheres in one of the lower animals produces effects corresponding with what might be anticipated from the foregoing facts.

Effects of the Removal of the Cerebrum. - The removal of the cerebrum in the lower animals appears to reduce them to the condition of a mechanism without spontaneity.

In the case of the frog, when the cerebral lobes have been removed, the animal appears similarly deprived of all power of spontaneous movement. But it sits up in a natural attitude, breathing quietly; when pricked it jumps away; when thrown into the water it swims; when placed upon the palm of the hand it remains motionless, although, if the hand be gradually tilted over till the frog is on the point of losing his balance, he will crawl up till he regains his equilibrium, and comes to be perched quite on the edge of the hand. This condition contrasts with that resulting from the removal of the entire brain, leaving only the spinal cord; in this case only the simpler reflex actions can take place. The frog does not breathe, he lies flat on the table instead of sitting up; when thrown into a vessel of water he sinks to the bottom; when his legs are pinched he kicks out, but does not leap away.

A pigeon from which the cerebrum has been removed will remain motionless and apparently unconscious unless disturbed. When disturbed in any way it soon recovers its former position; when thrown into the air it flies.

In mammals it is difficult to remove the cerebral hemispheres, but in those animals in which the operation has been carried out, as for example 
in the rabbit and rat, a result very similar to those observed in the case of the frog and pigeon has been obtained. The animal is able to maintain its equilibrium, to run or jump, and in fact carry out all the most complicated co-ordinated movements, but it is unable to originate them without stimulation. In the case of the dog, however, it has been found impossible to remove the whole brain, but when it has been removed piecemeal the animal may be kept alive for some time, and can carry out coordinated movements well, and even manifest intelligence.

It is quite evident, therefore, that the apparatus for carrying out coordiuated movements is in these animals not localized either in the cerebrum or in the spinal cord, and must therefore be connected in some way with the parts of the brain below the cerebrum and above the cord. There is no reason why such an arrangement may not be supposed to exist in the human brain.

We must look upon the cerebrum, however, for the originator of voluntary movements.

As regards the theory of the localization of different movements in different parts of the cerebral cortex which as we have seen has received so much support from observation on animals such as the dog and the monkey, at any rate, we may say that certain parts of the cerebral cortex appear to be highly sensitive to electrical stimuli, particularly the Rolandic area and the frontal lobe in front of it. Stimulation of certain other regions, viz., of the occipital region, of the parietal and temporal region, and of the gyrus fornicatus and the frontal region in front of the motor area, does not give rise to such movements. Such observations as it has been possible to make on man show that the localization of movement on the human cerebral cortex is, if anything, superior to that observed in monkeys. We have, of course, but few data upon which to base our conclusion, except such as have been obtained from the observation of the symptoms of disease, but with the help of these we may assume that in the cerebral cortex the co-ordinated movements of the body in some way are represented. The cases which have given us most of our knowledge upon the subject are those in which hæmorrhages have occurred in different parts of the brain, followed by paralysis of the opposite side of the body. These hæmorrhages chiefly occur in the neighborhood of the corpus striatum. The paralysis of the extremities is practically permanent, although, as a rule, the muscles connected witl the trunk are not paralyzed. This means that some interruption has taken place between the cerebral cortex and the paralyzed muscles, and if the lesion is a destroying one, the connection is never re-established. In the case of the animals, such as the $\mathrm{dog}$, this is not the case, as the paralysis is temporary. It is supposed that in man not only the more highly skilled movements but all voluntary movements of the muscles are 
actually represented in the cortical areas, and that the pyramidal tracts are actually essential for voluntary movements. If the pyramidal tracts be partially or wholly destroyed, anywhere in their course, a paralysis corresponding with the amount destroyed invariably follows. In the dog experiments have shown that this is not the ease, and the conduction of voluntary impulse to muscles may take place, for example, in other parts of the cord besides the pyramidal tract, after hemisection.

The pyramidal tracts in man, however, must be considered also as the only path connecting the cortical centres with the co-ordinated centres lower down in the brain, as, for example, in the bulb. The impulses which pass down from the cortex, whatever they may be, are not however of necessity connected with consciousness, and many voluntary movements of a complicated nature may take place really better without consciousness than with it. This is shown in such co-ordinated movements as writing, walking, marching, and the like, all of which are acquired with time and much labor, but when once perfect in the individual, can best be performed without voluntary effort. Such movements must be represented by impulses passing in the pyramidal tracts, for if they are interrupted, the movements are no longer performed.

What actually originates a voluntary action, or one performed by an effort of the will, we are unable to say. No doubt impulses from the periphery conducted to the cerebral cortex along all kinds of afferent channels must have something to do with it; directly or indirectly, sooner or later. In the human cortex it would seem that the apparatus for performing all manner of possible co-ordinated movements which may result in speech or action, are stored. This apparatus is capable of being set in action either in the absence of consciousness by afferent stimuli of some kind directly, or by what may be, indirectly or remotely, in some way the result of afferent stimuli, viz., the will. It is also probable that the will of another may take the place of the man's own will, and may call for the movements, actions, and speech, all of which are, as it were, ready to be called forth by a stimulus of some kind. It may be supposed that the condition of development of the brain inherited by the individual has something to do both with the potentialities of the apparatus for co-ordinated acts, which he receives at birth, and with the way in which the apparatus is set in motion.

Unilateral Action.-Respecting the mode in which the brain discharges its functions, there is no evidence whatever. But it appears that, for all but its highest intellectual acts, one of the cerebral hemispheres is sufficient. For numerous cases are recorded in which no mental defect was observed, although one cerebral hemisphere was so disorganized or atrophied that it could not be supposed capable of dis- 
charging its functions. The remaining hemisphere was, in these cases, adequate to the functions generally discharged by both; but the mind does not seem in any of these cases to have been tested in very high intellectual exercises; so that it is not certain that one hemisphere will suffice for these. In general, the brain combines, as one sensation, the impressions which it derives from one object through both hemispheres, and the ideas to which the two such impressions give rise are single. In relation to common sensation and the efforts of the will, it must always be remembered that the impressions to and from the hemispheres of the brain are carried across the middle line; so that in destruction or compression of either hemisphere, whatever effects are produced in loss of sensation or voluntary motion, are observed on the side of the body opposite to that on which the brain is injured.

Sleep.-All parts of the body which are the seat of active change require periods of rest. The alternation of work and rest is a necessary condition of their maintenance, and of the healthy performance of their functions. These alternating periods, however, differ much in duration in different cases; but, for any individual instance, they preserve a general and rather close uniformity. Thus, as before mentioned, the periods of rest and work, in the case of the heart, occupy, each of them, about half a second ; in the case of the ordinary respiratory muscles the periods are about four or five times as long. In many cases, again (as of the voluntary muscles during violent exercise), while the periods during active exertion alternate very frequently, yet the expenditure goes far ahead of the repair, and, to compensate for this, an after repose of some hours becomes necessary; the rhythm being less perfect as to time, than in the case of the muscles concerned in circulation and respiration.

Obviously, it would be impossible that, in the case of the brain, there should be short periods of activity and repose, or in other words, of consciousness and unconsciousness. The repose must occur at long intervals; and it must therefore be proportionately long. Hence the necessity for that condition which we call Sleep; a condition which seeming at first sight exceptional, is only an unusually perfect example of what occurs, at varying intervals, in every actively working portion of our bodies.

A temporary abrogation of the functions of the cerebrum imitating sleep, may occur, in the case of injury or disease, as the consequence of two apparently widely different conditions. Insensibility is equally produced by a deficient and an excessive quantity of blood within the cranium (coma); but it was once supposed that the latter offered the truest analogy to the normal condition of the brain in sleep, and in the absence of any proof to the contrary, the brain was said to be during sleep congested. Direct experimental inquiry has led, however, to the opposite conclusion.

By exposing, at a circumscribed spot, the surface of the brain of living animals, and protecting the exposed part by a watch-glass, Durham was able to prove that the brain becomes visibly paler (anæmic) during sleep; and the anæmia of the optic disc during sleep, observed by Hughlings Jackson, may be taken as a strong confirmation, by analogy, of the same fact.

A very little consideration will show that these experimental results correspond exactly with what might have been foretold from the analogy of other 
physiological conditions. Blood is supplied to the brain for two partly distinct purposes. (1.) It is supplied for mere nutrition's sake. (2.) It is necessary for bringing supplies of potential or active energy (i.e., combustible matter or heat) which may be transformed by the cerebral corpuscles into the various manifestations of nerve-force. During sleep blood is requisite for only the first of these purposes; and its supply in greater quantity would be not only useless, but by supplying an excitement to work, when rest is needed, would be positively harmful. In this respect the varying circulation of blood in the brain exactly resembles that which occurs in all other energy-transforming parts of the body; e.g., glands or muscles.

At the same time, it is necessary to remember that the normal anæmia of the brain which accompanies sleep is probably a result, and not a cause of the quiescence of the cerebral functions. What the immediate cause of this periodical partial abrogation of functions is, however, we do not know.

Somnambulism and Dreams. - What we term sleep occurs often in very different degrees in different parts of the nerrous system; and in some parts the expression cannot be used in the ordinary sense.

The phenomena of dreams and somnambulism are examples of differing degrees of sleep in different parts of the cerebro-spinal nervous system. In the former case the cerebrum is still partially active; but the mind-products of its action are no longer corrected by the reception, on the part of the sleeping sensorium, of impressions of objects belonging to the outer world; neither can the cerebrum, in this half-awake condition, act on the centres of reflex action of the voluntary muscles, so as to cause the latter to contract-a fact within the painful experience of all who have suffered from nightmare.

In somnambulism the cerebrum is capable of exciting that train of reflex nervous action which is necessary for progression, while the nerve-centre of muscular sense (in the cerebellum?) is, presumably, fully awake; but the sensorium is still asleep, and impressions made on it are not sufficiently felt to rouse the cerebrum to a comparison of the difference between mere ideas or memories and sensations derived from external objects.

The centres for muscular co-ordinations. - In asserting that the coordination of complicated muscular movements is connected with the middle parts of the brain below the cerebrum and above the bulb, we were stating a fact deduced from experiments upon animals. It is difficult to understand the exact way in which these parts of the brain are concerned. It appears, however, that co-ordinated movements such as standing, walking, and the maintenance of the equilibrium generally, require to be guided and governed by afferent impulses, which tell of the condition of the body and of its relations to its environment ("its position in space"). The afferent impulses are firstly visual and tactile sensations, secondly sensations by which we appreciate the condition of our muscles (muscular sense), and thirdly, as appears from experiments on pigeons and other animals, sensations produced by the pressure, in different directions, of the fluid in the semicircular canals of the internal ear.

Experiments show that when the horizontal semicircular canal is 
divided in a pigeon, inco-ordination occurs, with a constant movement of the head from side to side, and similarly, when one of the vertical canals is operated upon, up and down movements of the head are observed. The bird is unable to fly in an orderly manner, flutters and falls when thrown into the air, and, moreover, is able to feed with difficulty. Hearing remains unimpaired. So that inco-ordination depends upon deficiency or disorder of normal ampullar influences. It will be recollected that the semicircular canals are supplied with a nerve, the vestibular branch of the anditory, which is connected with the bulb.

It is probable that the various afferent impulses upon which co-ordination and the maintenance of the equilibrium depend are gathered up, as it were, in the tegmental system from the bulb upward, since this region is so intimately connected with the bulb and cord posteriorly, and with the optic thalamus and corpora quadrigemina anteriorly. In addition to the tegmentum, however, the cerebellum and pons are in some way concerned, because of their intimate connection with the spinal cord and bulb, the cerebellum being further connected with the auditory nerve on the one hand, and with the gray matter in-connection with the tegmentum on the other hand.

\section{Sensory Centres.}

There is evidence that fibres from the nerves of special sense are specially connected with definite and distinct parts of the cerebrum.

Visual or Optic Centre.-The termination of the optic nerve in each eye, the retina, to the structure of which we shall return when treating of the eye, is so arranged that when we look at an object with both eyes symmetrical parts of each retina are used. For example, if we look at an object to the left, an image of that object is focussed upon the right half of both retinæ, viz., upon the temporal side of the right retina, and upon the nasal side of the left retina. The optic nervefibres of these symmetrical parts of the retina are gathered together behind where the optic nerves decussate, viz., in the optic chiasma. The fibres which come from the right side of both eyes are contained in the optic tract of the same side, viz., the right, those from the right eye being outside of the others. In the same way the left optic tract contains internally fibres from the left side of the right eye and externally those from the left side of the left eye. On the inner border of the optic chiasma and tract there are also commissural fibres which pass from one side of the brain to the other; these are fibres which connect one median corpus geniculatum with the other. They are called the inferior or arcuate commissure. The optic tract thus formed then passes back- 
ward and terminates in three distinct nuclei, viz., the pulvinar of the optic thalamus, the anterior corpus quadrigeminum and the latera corpus geniculatum. These nuclei waste if the eyes are removed fron an adult animal; and if from a newly born animsl they do not develop. The optic chiasma in its course gives off fibres which are connected with the nucleus of the third nerve.

It appears that some of the fibres of the optic tract pass directly inte the cerebral cortex without joining with the optic thalamus, corpus quadrigeminum or corpus geniculatum.

It was shown above that the fibres of the cerebral cortex, known as the optic radiation, pass from the occipital region to the three nuclei about which we are speaking, riz., into the pulvinar of the optic thalamus, the anterior corpus quadrigeminum and lateral corpus geniculatum,

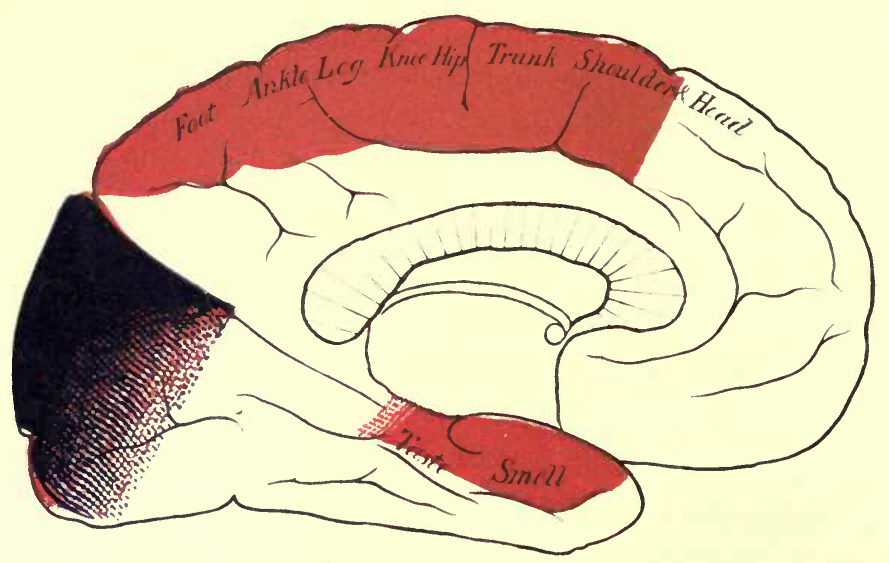

Fig. $386 .-$ The Cortical Centres.

and it is known that when the oceipital cortex is removed, these three waste. It has been further shown that in a newly born animal the removal of such a region is followed by imperfect development of the parts in question.

If one optic nerve be divided blindness of the corresponding eye results, but if one optic tract be divided there is a half blindness, which is called hemianopsia, hemianopia, or hemiopia, right or left, according as the right or left field of vision is cut off. It is highly probable that the occipital lobe (figs. 382, 386), and particularly the cuneus, is concerned as a so-called visual centre, since not only is it connected with the optic nerves, as we have seen, but also because the removal of the right occipital lobe in an animal (monkey), is followed by left hemiopia, removal of the left by right hemiopia, and removal of both occipital lobes by total blindness. Some have connected the angular 
gyrus also with vision as the centre, while others look upon it merely as an accessory centre.

Olfactory centre.-The olfactory nerve differs from the other cranial nerves. In reality it is a representative of the olfactory lobes of other . animals, which are part of the cerebrum. It originates as an off-shoot from the cerebral vesicle, the front part of which is developed into the bulb of the olfactory nerve, while the back forms its peduncle. The nerve, the cavity of which is filled up in the fully developed condition with neurogliar substance, lies upon the cribriform plate of the ethmoid bone, and is contained in a groove of the frontal lobe on its under surface. On examination of the bulb it is found to be thus made up. Beneath the neurogliar layer is a layer of longitudinal fibres and a few nerve-cells, next to this is a layer of small cells (nuclear layer), fibres from the layer of nerve-fibres passing through it.

The nuclear layer is also separated into groups of cells by an interlacing of the fibres. The next layer is thick and is composed of neuroglia and some fibres, some of which are medullated, as well as of cells more or less pyramidal in shape. Below this layer is the layer of olfactory glomeruti. These glomeruli are small coils of olfactory fibres inclosing small cells and granular matter. A full description of the anatomy of these parts is given later (see Olfactory nerve).

Fibres of the olfactory nerve proper are found below this layer and pass to be distributed to the olfactory mucous membrane. They are thought to have origin in the glomeruli. The peduncle of the nerve or the olfactory tract as it is sometimes called, is made up of longitudinal fibres originating in the bulb, with neuroglia and some nerve-cells.

The fibres of the olfactory tract have been traced into the nucleus amygdalæ and its junction with the hippocampal gyrus in the temporal lobe (fig. 386). The hippocampus must be in some way connected with smell, since a lesion of it, leaving the olfactory tract uninjured, seriously interferes with that sense.

Taste centre. - It is very uncertain where the taste centre is situated, if such exist. It has been placed in the temporal lobe, not far from that of smell (fig. 386).

Auditory Centre.-This centre has been localized in the superior temporal convolution (fig. 382). Experiments have been made which connect auditory impulses on either side with the posterior corpus quadrigeminum and the median corpus geniculatum, for when the internal ear is destroyed there results atrophy of these bodies as well as of the lateral fillet of the opposite side; and on the other hand, destruction of the part of the temporal lobe above indicated is similarly followed by atrophy of the nuclei of the same side. If these results be confirmed by additional experiments, it would make it plain that these nuclei bear much the same relation to the sense of hearing as do the anterior corpus 
quadrigeminum and the lateral corpus geniculatum to the sense of sight.

Centre for Cutaneous Sensations.--Physiological experiments, as well as clinical and pathological observations, now show pretty certainly that the cortical centre for sensations of touch, and probably of pain and temperature, are essentially identical with the motor areas, that is to say, in the central convolutions. Owing, howerer, to the wide distribution of afferent impulses, through the multiplication of their means of getting to the brain, the area of these sensory centres is not as strictly limited as that of other special centres.

The Centre for Muscular Sensations. - A great deal of evidence is accumulated to show that the most important area in which these sensations are bronght to conscionsness is in the inferior parietal lobule.

\section{Furctions of Corpora Striata axd Optic 'Thalayi.}

The Corpora Striata.--The idea formerly held that the corpora striata are concerned in the transmission of motor impulses, or that they are the great motor ganglia at the base of the brain, rests upon insufficient evidence. Lesions of the corpora striata produce hemiplegia only because of the pressure-effects they exercise upon the internal capsule close by.

The candate nucleus is comnected with the opposite side of the cerebellum by fibres which conduct downward, and the lenticular nucleus is connected with the cerebellum by fibres from the tegmentum and superior cerebellar peduncles which conduct upward. It is suggested that the corpora striata are central organs analogous to the cerebral cortex itself. "The analogy to those parts of the cortex that are connected with the cerebellum is rendered still greater by the fact that a lesion, even an extensive lesion, may exist in either the caudate or lenticular nucleus, and so long as it does not interfere with the functions of the motor or sensory parts of the internal capsules it causes no persistent symptoms." (Gowers.)

On the whole, however, it must be said that the functions of the corpora striata are unknown, and it is possible that in man they are very subsidiary, if not even rudimentary, bodies.

The Optic Thatami. - That the optic thalami are the great sensory centres at the base of the brain-which was a view held by many until recently-does not seem to be based upon sufficiently accurate observations. The important relation to the tegmentum of its own side would make it appear as being specially concerned with the sensory fibres passing to the cerebrum, for which it probably forms a relay.

Its connection with the optic nerves bas been commented upon above. Fibres connect the optic thalamus too with the superior peduncle of the cerebellum of the opposite side. 
Lesions of the optic thalamus do not of themselves produce entire loss of sensation. If such a symptom follows, it is due to pressure upon, or injury to, the posterior limb of the internal capsule. The optic thalamus is connected with visual sensations and may be a reflex-centre for some of the higher reflex actions.

The optic thalamus is so closely connected with a large area of the cortex that it undoubtedly must have some function in connection with the mechanical or muscular movements and of expression. It is probable that it is the organ to which automatic activities are relegated in states of partial consciousness. The automatic walking, writing, speak-

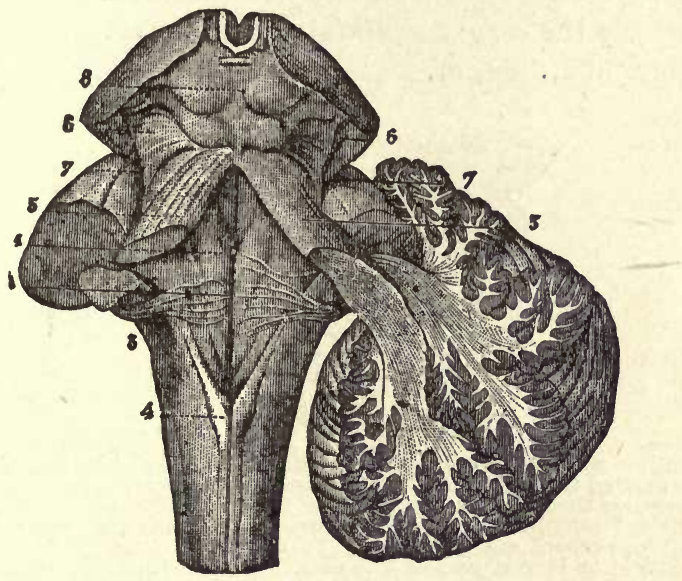

Fig. 387.-Cerebellum in section and fourth ventricle, with the neighboring parts. 1 , Median groove of fourth ventricle, ending below in the calamus scriptorius, with the longitudinal eminences formed by the fasciculi teretes, one on each side; 2 , the same groove, at the place where the white streaks of the auditory nerve emerge from it to cross the floor of the ventricle; 3 , inferior crus or peduncle of the cerebellum, formed by the restiform body; 4 , posterior pyramid; above this is the calamus scriptorius; 5 , superior crus of cerebellum, or processus cerebello ad cerebrum (or ad testes); 6,6 , fillet to the side of the crura cerebri; 7,7 , latera grooves of the crura cerebri; 8, corpora quadrigemina. (From Sappey after Hirschfeld and Leveille.)

ing, and emotional expressions, for example, that are done by men in hypnotic states or in sleep, are very probably largely under the control of the optic thalamus in connection with the cerebellum and associated .ganglia.

Of the functions of the external capsule and of the claustrum nothing definite is known.

\section{The Cerebellum.}

The cerebellum $(7,8,9,10$, fig. 354$)$ is composed of an elongated central portion or lobe, called the vermiform processes, and two hemispheres. Each hemisphere is connected with its fellow, not only by means of the vermiform processes, but also by a bundle of fibres called the middle crus or peduncle (the latter forming the greater part of the 
pons Varolii), while the superior crura with the valve of Vieussens con. nect it with the cerebrum (5, fig. 38\%), and the inferior crura (formed by the prolonged restiform bodies) connect it with the medulla oblongata (3, fig. $38 \%)$.

Structure.-The cerebellum is composed of white and gray matter, the latter being external, like that of the cerebrum, and like it infolded, so that a larger area may be contained in a given space. The convolutions of the gray matter, however, are arranged after a different pattern, as shown in fig. 38\%. Besides the gray substance on the surface, there is, near the centre of the white substance of each hemisphere, a small capsule of gray matter called the corpus dentatum (fig. $388, c l$ ), resembling very closely the corpus dentatum of the olivary body of the medulla oblongata (figs. $362,388, o$ ).

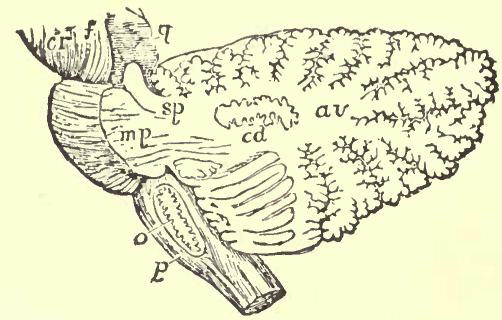

Fig. 388.-Outline sketch of a section of the cerebellum, showing the corpus dentatum. The section has been carried through the left lateral part of the pons, so as to divide the superior peduncle and pass nearly through the middle of the left cerebellar hemisphere. The olivary body has also been divided longitudinally so as to expose in section its corpus dentatum. cr, crus cerebri : $f$, fillet; $q$, corpora quadrigemina; $s p$, superior peduncle of the cerebellum divided; $m p$, middle peduncle or lateral part of the pons Varolii, with fibres passing from it into the white stem; $a v$, continuation of the white stem radiating toward the arbor vitæ of the folia; $c d$, corpus dentatum; $o$, olivary body with its corpus dentatum; $p$, anterior pyramid. (Allen Thomson.) $2 / 3$.

If a section be taken through the gray matter of the cerebellum, it will be found to be composed of two layers, an onter, or molecular, and an inner, or granular, layer. Each of these layers contains a large number of peculiar shaped nerve-cells, and very rich plexuses of nerve-fibres. Recent studies of the cortex of the cerebellum by modern methods have revealed a most complex and beautiful arrangement of the parts, which we shall describe briefly here.

The molecular layer contains two kinds of cells, one large and known as Purkinjé's cell, the other smaller and known as stellate cells. 'The cells of Purkinjé lie along the internal margin of the layer, being, in fact, practically at the boundary of the molecular and granular layers. They measure 40×30 $\mu$, and have large, round nuclei. Each cell gives off an enormous number of branching dendrites, which run up toward the surface of the cerebellum in the shape of a bush. Each little branch sends off from the side small buds, which are called the gemmules or thorns. These branching dendrites do not pass up altogether like the branches of a round bush, but are flattened like a bush that has been 
pressed, so that if one cuts the cell in one direction, only the profile is shown. The Purkinjé cells are arranged so that the axis of these flattened branches is transverse to the longitudinal surface of the convolu-

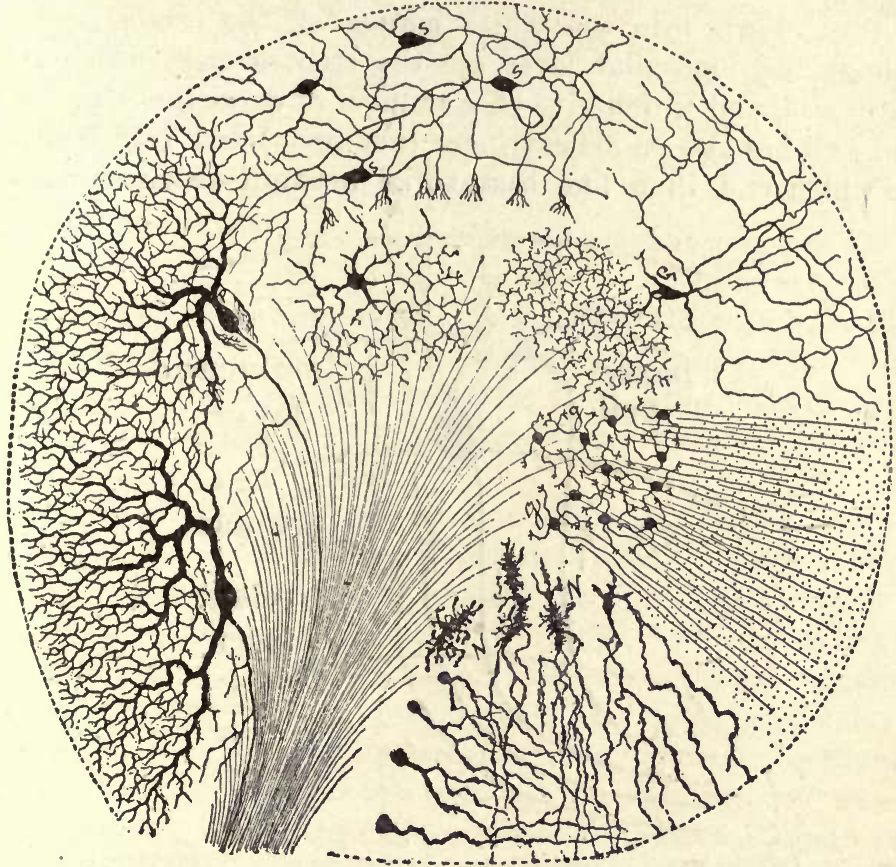

Fig. 388A.-The different constituent elements of the gray cortical layer of the cerebellum.

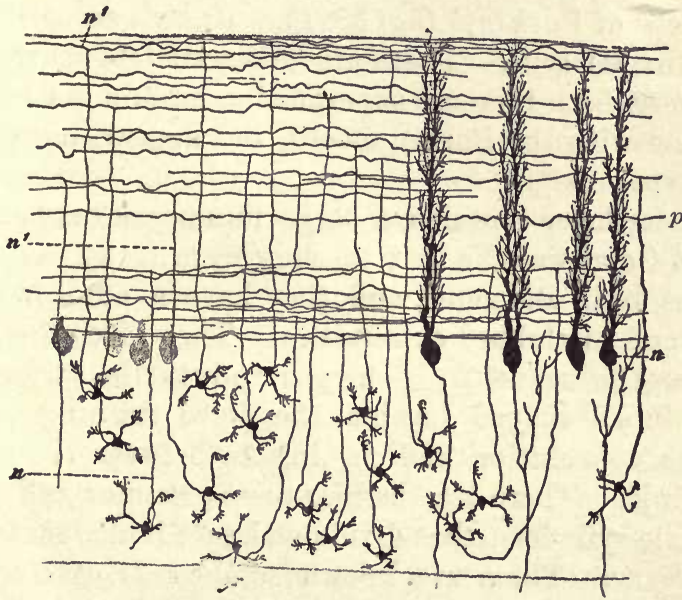

Fig. 389.-Longitudinal section of the gray substance of a cerebellar convolution. Schematic. $g r$, Granula; $n$, its nervous processes: $n^{\prime}$, divisions of the latter in the molecular layer and each separating into two longitudinal fine fibres; $p$, cells of Purkinjé. 
tion, and if one makes a section down through the centre of the couvolution, in its longitudinal conrse, a side view of the cell only is shown (fig. 389).

The cells of Purkinjé give off at their under surface a neuraxon which runs down into the white matter of the cerebellum. Lying throughout the molecular layer are the stellate cells, which are much smaller in size, and which give off a number of dendrites (fig. 388 A).

Each cell has also an axis-cylinder (neuraxon) and this sends off collaterals which end in a fine basket-like network which surrounds the

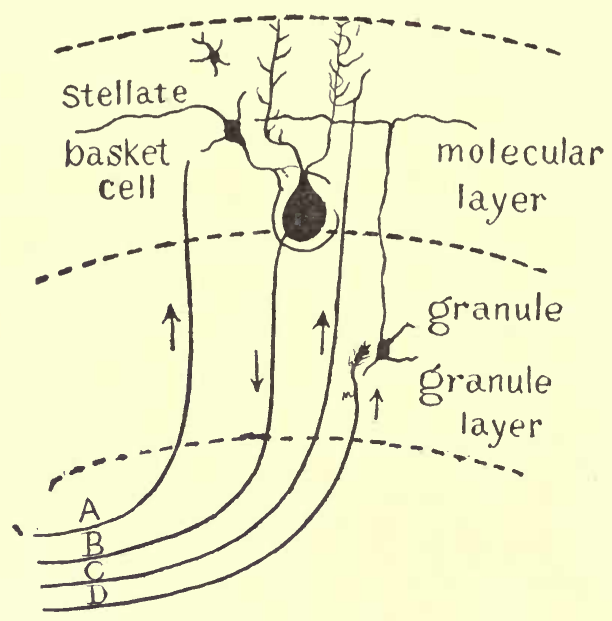

Fig. 389A.-A, Afferent fibre to basket (stellate) cell; B, neuraxon of Purkinje cell; C, afferent fibre to Purkinjé cell; D, afferent (mossy) fibre to granule cell.

body of the cells of Purkiujé (fig. $389 \Lambda)$ ). On this account they are some times called basket-cells. There are other stellate-shaped cells in the molecular layer which lie more superficially, and do not have this particular connection with the Purkinjé cells, but appear, however, to belong to the same type.

The granular layer contains a large number of very small granularlike cells that Golgi was the first to show were really nerve cells. They are only about $5 \mu$ in diameter, and they have a number of short dendrites which end in clubbed extremities. They give off a very fine axiscylinder process (neuraxon) which ruus up into the molecular layer and there divides in a T-shaped fashion, the fibres running parallel to the surface of the convolution and passing in between the branches of the cells of Purkinjé. There are, besides these granular cells, a few larger cells, with axis-cylinders, that divide and subdivide, ending in a finely ramifying plexus. These are known as the cells of Golgi. They are found in other parts of the brain.

The white substance of the cerebellum consists of nerve-fibres, which 
are of three kinds: 1st, Descending fibres, that are made up of the axiscylinders of the cells of Purkinjé carrying impulses down from the cerebellar cortex. 2d, Ascending fibres, which pass into the granular layer, and there end in a number of very short, finely split fibres, presenting a mossy appearance, so that these are known as the mossy fibres. These connect with the granular cells of this layer. $3 \mathrm{~d}$, Ascending fibres, which pass up through the granular into the molecular layer and there break up into a fine network, which interlaces with and coils among the protoplasmic branches of the cells of Purkinjé.

It will be seen that the arrangements for the transmission and diffusion of nerve-impulses and for the cooperation of different cells with each other are extremely complicated and delicate, as would be needed for so important an organ. It is not possible to indicate absolutely by any scheme the course of fibres and the course of impulses through the cerebellum, but, approximately, it is somewhat like that in the accompanying figure (fig. 389A).

Impulses pass up along those ascending fibres called "mossy" to the granular cells. These cells, being stimulated, send the impulses by their axis-cylinders to the molecular layer, and through their T-shaped divisions to the dendrites of the cells of Purkinjé. Thence an impulse is send out by the axis-cylinder process of this cell. Other ascending impulses are brought up by those fibres which pass to the molecular layer and send their terminals winding around among the dendrites of the cells of Purkinjé. Probably impulses pass up also through the ascending fibres, and affect the stellate cells, and through them and their basketlike terminals the cells of Purkinjé.

\section{Functions of the Cerebellum.}

(1.) With the exception of its middle lobe, the cerebellum is itself insensible to irritation and may be all cut away without eliciting signs of pain (Longet). Its removal or disorganization by disease is also generally unaccompanied by loss or 'disorder of sensibility; animals from which it is removed can smell, see, hear, and feel pain, to all appearances, as perfectly as before (Flourens; Magendie). It cannot, therefore, be regarded as a principal organ of sensation. Yet, if any of its crura be touched, pain is indicated; and, if the restiform tracts of the medulla oblongata bo irritated, the most acute suffering appears to be produced.

(2.) Co-ordination of Movements. - In reference to motion, the experiments of Longet and many others agree that no irritation of the cerebellum produces movement of any kind. Remarkable results, however, are produced by removing parts of its substance. Flourens (whose experiments have been confirmed by those of Bouillaud, Longet, and others) extirpated the cerebellum in birds by successive layers. Feebleness and 
want of harmony of muscular movements were the consequence of removing the superficial layers. When he reacher the middle layers, the animals became restless without being convulsed; their movements were violent and irregular, but their sight and hearing were perfect. By the time that the last portion of the organ was cut away, the animals had entirely lost the powers of springing, flying, walking, standing, and preserving their equilibrium. When an animal in this state was laid npon its back, it could not recover its former posture, but it fluttered its wings, and did not lie in a state of stupor; it saw the blow that threatened it, and endeavored to aroid it. Volition and sensation, therefore, were not lost, but merely the faculty of combining the actions of the muscles; and the endeavors of the animal to maintain its balance were like those of a drunken man.

The experiments afforded the same results when repeated on all classes of animals; and from them and the others before referred to, Flourens inferred that the cerebellum belongs neither to the sensory nor the intellectual apparatus; and that it is not the source of voluntary movements, although it belongs to the motor apparatus; but is the organ for the coordination of the voluntary movements, or for the excitement of the combined action of muscles.

Such evidence as can be obtained from cases of disease of this organ confirms the view taken by Flourens: and, on the whole, it gains support from comparative anatomy; animals whose natural movements require most frequent and exact combinations of muscular actions being those whose cerebella are most developed in proportion to the spinal cord.

We must remember, too, that the cerebellum is connected with the posterior columns of the cord as well as with the direct cerebellar tract, both of which probably convey to the middle lobe muscular sensations. It is also connected with the auditory nerves and bulb by the internal and external acute fibres; and with the tegmentum through the red nuclei. Its connection with the efferent tracts from the different cerebral lobes through the pons is also highly important. Movements of the eyes also occur on direct stimulation of the middle lobe. It seems, therefore, to be connected in some way with all of the chief sensory impulses which have to do with the maintenance of the equilibrium, and is generally included in the nervous apparatus which is supposed to govern this function of our bodies.

Foville supposed that the cerebellum is the organ of muscular sense, i.e., the organ by which the mind acquires that knowledge of the actual state and position of the muscles which is essential to the exercise of the will upon them; and it must be admitted that all the facts just referred to are as well explained on this hypothesis as on that of the cerebellum being the organ for combining 
morements. A harmonious combination of muscular actions must depend as much on the capability of appreciating the condition of the muscles with regard to their tension, and to the force with which they are contracting, as on the power which any special nerve-centre may possess of exciting them to contraction. And it is because the power of such harmonious movement would be equally lost, whether the injury to the cerebellum involved injury to the seat of muscular sense, or to the centre for combining muscular actions, that experiments on the subject afford no proof in one direction more than the other.

Forced Movements. - The influence of each half of the cerebellum is directed to muscles on the opposite side of the body; and it would appear that for the right ordering of movements, the actions of its two halves must be always mutually balanced and adjusted. For if one of its crura, or if the pons on either side of the middle line, be divided, so as to cut off from the medulla oblongata and spinal cord the influence of one of the hemispheres of the cerebellum, strangely disordered movements ensue (forced movements). The animals fall down on the side opposite to that on which the crus cerebelli has been divided, and then roll over continuously and repeatedly; the rotation being always round the long axis of their bodies, and generally from the side on which the injury has been inflicted. The rotations sometimes take place with much rapidity; as often, according to Magendie, as sixty times in a minute, and may last for several days. Similar movements have been observed in men; as by Serres in a man in whom there was apoplectic effusion in the right crus cerebelli; and by Belhomme in a woman, in whom an exostosis pressed on the left crus. They may, perhaps, be explained by assuming that the division or injury of the crus cerebelli produces paralysis or imperfect and disorderly movements of the opposite side of the body; the animal falls, and then, struggling with the disordered side on the ground, and striving to rise with the other, pushes itself over; and so again and again, with the same act, rotates itself. Such movements cease when the other crus cerebelli is divided; but probably only because the paralysis of the body is thus made almost complete. Other varieties of forced movements have been observed, especially those named "circus movements," when the animal operated upon moves round and round in a circle; and again those in which the animal turns over and over in a series of somersaults. Nearly all these movements may result on section of one or other of the following parts; viz., crura cerebri, medulla, pons, cerebellum, corpora quadrigemina, corpora striata, optic thalami, and even, it is said, of the cerebral hemispheres.

\section{Functions of the Corpora Quadrigemina and Geniculata.}

The corpora quadrigemina are the homologues of the optic lobes in birds, amphibia, and fishes. The anterior pair may be regarded as the. 
principal nerve-centres for visual sensations, the posterior possibly with auditory sensation.

Functions.-(1) The experiments show that removal of the anterior corpora quadrigemina wholly destroys the power of seeing; and diseases in which they are disorganized are usually accompanied by blindness. Atrophy of them is also often a consequence of removal of the eyes. Destruction of one of the anterior corpora quadrigemina (or of one optic lobe in birds) produces hemiopia of opposite field of vision. 'This loss of sight is the only apparent injury of sensibility sustained by the removal of the corpora quadrigemina.

The (2) removal of one of them affects the movements of the body, so that animals rotate, as after division of the crus cerebri, only more slowly: but this may be due to giddiness and partial loss of sight.

(3) The more evident and direct influence is that produced on the iris. It contracts when the anterior corpora quadrigemina are irritated: it is always dilated when they are removed: so that they may be regarded, in some measure at least, as the nervous centres governing its movements, and adapting them to the impressions derived from the retina through the optic nerves and tracts.

(4) The centre for the co-ordination of the movements of the eyes is also contained in them. This centre is closely associated with that for contraction of the pupil, and so it follows that contraction or dilatation follows upon certain definite ocular movements.

As we have seen, the lateral corpus geniculatum is associated on either side with the anterior corpus quadrigeminum, and the median corpus geniculatum with the posterior corpus quadrigeminum.

T'he Sympathetic System.-Having in the preceding chapters completed the description of the Cerebro-spinal nervous system proper, there remains to be considered the structure and functions of the so-called Sympathetic nervous system, and to this it is now necessary to direct attention.

It should, however, be distinctly borne in mind that the cerebrospinal and sympathetic systems are not distinct from one another. The separation of the one from the other may be considered to be purely for the sake of convenience.

Distribution.-It consists of: (1) A double chain of ganglia and fibres, which extends from the cranium to the pelvis, along each side of the vertebral column, and from which branches are distributed both to the cerebro-spinal system, and to other parts of the sympathetic system. With these may be included the small ganglia in connection with those branches of the fifth cerebral nerve which are distributed in the neighborhood of the organs of special sense: namely, the Ophthalmic, Otic, 
Fig. 390.-Diagrammatic view of the Sympathetic cord of the right side, showing its connections with the principa cerebro-spinal nerves and the main præaortic plexuses. 1/4. (From Quain's Anatomy.)

Cerebro-spinal nerves.-VI., a portion of the sixth cranial as it passes through the cavernous sinus, receiving two twigs from the carotid plexus of the sympathetic nerve; $\mathrm{O}$, ophthalmic ganglion connected by a twig with the carotid plexus; M, connection of the spheno-palatine ganglion by the Vidian nerve with the carotid plexus; C, cervical plexus; $\mathrm{Br}$, brachial plexus; D 6 , sixth intercostal nerve; D 12, twelfth; L 3, third lumbar nerve; S 1, first sacral nerve; S 3, third; S 5, fifth; Cr, anterior crural nerve; Cr' great sciatic; $p n$, vagus in the lower part of the neck; $r$, recurrent nerve winding round the subclavian artery.

Sympathetic Cord.-c, superior cervical ganglion; $c^{\prime}$, second, or middle; $c^{\prime \prime}$ inferior: from each of these ganglia cardiac nerves (all deep on this side) are seen descending to the cardiac plexus; $d 1$ placed immediately below the first dorsal sympathetic ganglion; $d 6$, is opposite the sixth; $l 1$, first lumbar ganglion; $c g$, the terminal or coccygeal ganglion.

Proeartic and $V$ isceral Plexuses.-pp, pharyngeal, and, lower down, laryngeal plexus; $p l$, post-pulmonary plexus spreading from the vagus on the back of the right bronchus; $c a$, on the aorta, the cardiac plexus, towards which, in addition to the cardiac nerves from the three cervical sympathetic ganglia, other branches are seen descending from the vagus and recurrent nerves; co, right or posterior and $c o^{\prime}$, left or ant. coronary plexus; $o$, œsophageal plexus in long meshes on the gullet; $s p$, great splanchnic nerve formed by branches from the fifth, sixth, seventh, eighth, and ninth dorsal ganglia: + , small splanchnic from the ninth and tenth; +- , smallest or third splanchnic from the eleventh; the first and second of these are shown joining the solar plexus, $s o$; the third descending to the renal plexus, re; connecting branches between the solar plexus and the vagi are also rep. resented; $p n^{\prime}$, above the place where the right vagus passes to the lower or posterior surface of the stomach; $p n^{\prime \prime}$ the left distributed on the anterior or upper surface of the cardiac portion of the organ: from the solar plexus large branches are seen surrounding the arteries of the coliac axis, and descending to $m s$, the sup. mesenteric plexus; opposite to this is an indication of the suprarenal plexus; below $r e$ (the renal plexus), the spermatic plexus is also indicated; $a O$, on the front of the aorta, marks the aortic plexus, formed by nerves descending from the solar and sup. mesenteric plexuses and from the lumbar ganglia; $m i$, the inf. mesenteric plexus surrounding thecorresponding artery; $h y$, hypogastric plexus placed between the common iliac vessels, connected above with the aortic plexus, receiving nerves from the lower lumbar ganglia, and dividing below into the right and left pelvic or inf. hypogas tric plexuses; $p l$, the right pelvic plexus; from this the nerves descending are join. ed by those from the plexus on the sup. hemorrhoidal vessels, $m i^{\prime}$, by nerves from the sacral ganglia, and by visceral nerves from the third and fourth sacra spinal nerves, and there are thus formed

the rectal, vesical, and other plexuses, which ramify upon the viscera, as towards $i r$, and $v$, the rectum and bladder.

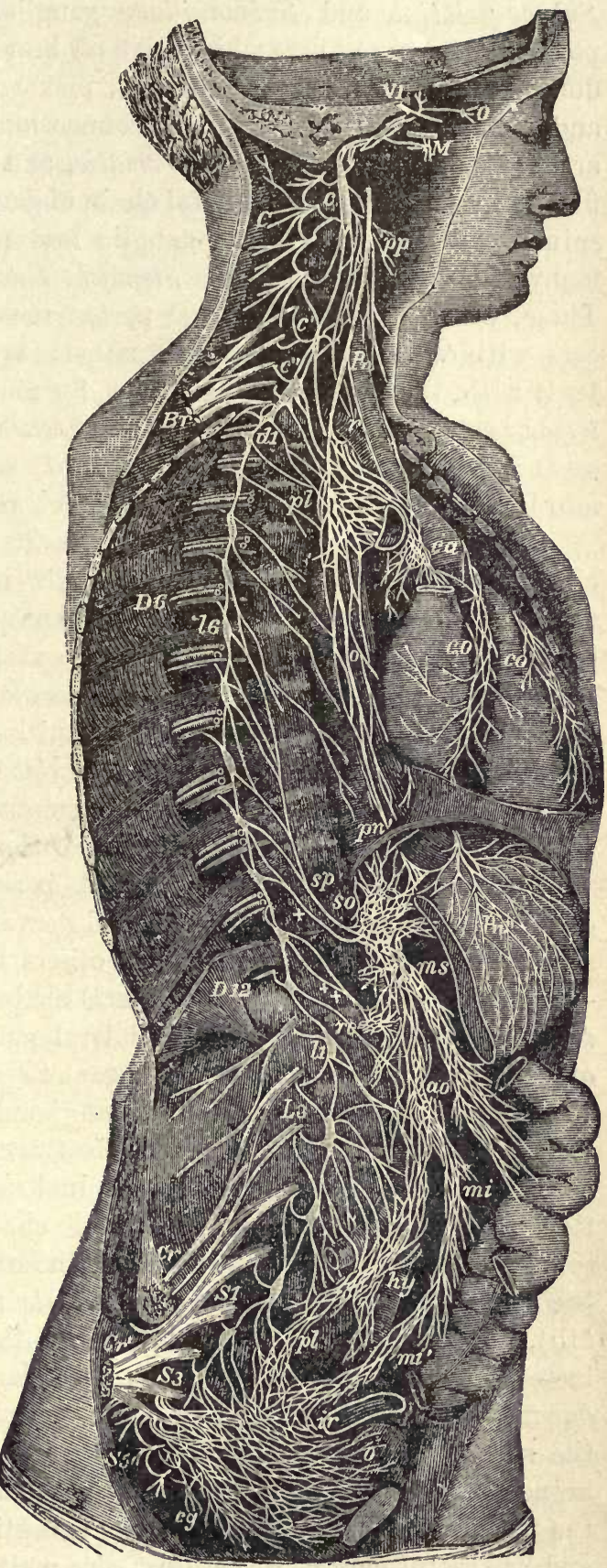


Spheno-palatine and Submaxillary ganglia.

(2) Various ganglia and plexuses of nerve-fibres which give off branches to the thoracic and abdominal viscera, the chief of such plexuses being the Cardiac, Solar, and Hypogastric; but inintimate connection with these are many secondary plexuses, as the Aortic, Spermatic, and Renal. To these plexuses, fibres pass from the prævertebral chain of ganglia, as well as from cerebrospinal nerves. (3) Various ganglia and plexuses in the substance of many of the viscera, as in the Stomach, Intestines, and Urinary bladder. These, which are, for the most part, microscopic, also freely communicate with other parts of the sympathetic system, as well as, to some extent, with the cerebro-spinal. (4) By many, the ganglia on the Posterior roots of the spinal nerves, on the Glossopharyngeal and Vagus, and on the Sensory root of the Fifth cerebral nerve (Gasserian ganglion), are also included as sympathetic-nerve structures.

Classification.-Gaskell's researches have suggested a convenient classification for the sympathetic ganglia into: (1.) The main sympathetic chain, extending from above downward, in the form of connected ganglia lying upon the bodies of the vertebræ, which may be called lateral or vertebral ganglia. (2). A more or less distinct chain, prævertebral in position, consisting of the semi-lunar, inferior mesenteric and similar plexuses, which may be called collateral ganglia. (3.) Ganglia situated in the organs and tissues themselves, called terminal ganglia. (4.) The ganglia of the posterior roots of the spinal nerves.

The connection between these parts is as follows: the visceral branch or ramus communicans of each spinal nerve, which is one of the divisions of a typical spinal nerve-the others being the dorsal and ventral - passes first of all into the lateral chain; from this chain branches, rami efferentes, pass into the collateral ganglia, and from these again other branches pass off into the organs to end in the terminal ganglia. In the thoracic region the rami communicantes are composed of two parts, white and gray. The former can be traced backward into both spinal nerve-roots of their corresponding spinal nerve; and in the other direction partly into the lateral sympathetic chain, and partly into the great splanchnic nerves and so into the collateral ganglia without entering the lateral chain at all. The upper white rami (from the 2nd to the 5th), however, proceed upward and join the superior cervical ganglion instead of passing downward into the splanchnics. Other branches go downward into the lumbar and sacral plexuses. The gray rami of all the spinal nerves are the only apparent representatives of the visceral branches in the regions above the 2 nd thoracic nerve-root, and below the 2 nd lumbar nerve-root, with the exception of the roots of the 2 nd and 3rd sacral nerves, which have also white rami, and consist of nonmedullated fibres, and pass from the ganglia to be distributed chiefly to 
the spinal column, to the spinal membranes and to the spinal nerve-roots themselves. We must look upon the white rami then as the visceral branches proper.

A peculiarity in the structure of these white medullated visceral nerves is the fineness of their fibres. They are a third or a fourth of the diameter of ordinary medullated fibres, measuring $1.8 \mu$ to $2.7 \mu$ instead of $14.4 \mu$ to $19 \mu$. Such fibres are a peculiarity of the spinal nerve-roots chiefly in the thoracic region, but they are also found in the second and third sacral nerves, and constitute there the nervi erigentes which pass directly to the hypogastric plexus, and not first of all into the lateral chain. From this plexus branches pass upward into the inferior mesenteric ganglia and downward to the bladder, rectum and generative organs. These nerves, called by Gaskell pelvic splanchnic nerves, differ from the rami viscerales of the thoracic region only in not communicating with the lateral ganglia; the branches which pass upward from the thoracic region to the neck, he calls cervical splanchnics, and the splanchnics proper abdominal splanchnics. The white rami viscerales of the upper cervical and cervico-cranial regions do not run with their corresponding gray rami, butform, Gaskell thinks, the internal branch of the spinal accessory nerve, which contains small medullated fibres similar to those of the visceral branches in the thoracic region. This branch passes into the ganglion of the trunk of the vagus. Small visceral fibres exist too in the roots of the vagus, and in those of the glosso-pharyngeal in connection with the ganglion of the trunk and ganglion petrosum, as well as in the chorda tympani, in the small petrosal and in other cranial visceral nerves.

Functions. - The researches of Gaskell have, however, done much to clear up the former confusion as to the functions of the sympathetic; and in the following account the description of the functions, as given by that observer, is followed.

The efferent nerve fibres of the sympathetic system supply $(a)$ the muscles of the vascular system, to which they send vaso-motor fibres, i.e., vaso-constrictor and cardiac augmentor or accelerator, and vaso-inhibitory fibres, i.e., vaso-dilator and cardiac inhibitory; (b) the visceral muscles, to which they send both viscero-motor and viscero-inhibitory fibres. (c) The secretory gland-cells.

(a) i. Vaso-motor or Vaso-constrictor and Cardio-augmentor Fibres. The vaso-motor nerves for all parts of the body come from the central nervous system, and pass out from the spinal cord in the white rami viscerales of the thoracic region from the second thoracic to the second lumbar nerveroots inclusive, as fine medullated fibres; they then pass to the lateral or main sympathetic chain, become non-medullated, and are distributed to their muscles either directly or through terminal ganglia. Thus the aug- 
mentor nerves of the heart arise in the thoracic rami, pass upward through the ganglion stellatum (first thoracic ganglion), the annulus of Vieussens and the inferior cervical ganglion, and are distributed to the heart; the raso-motor roots of the brachial plexus, in the anterior roots of the second and lower thoracic nerves, and reach that plexus by the same ganglion; the vaso-motor nerves of the foot leave the spinal cord high up, and reach the sympathetic lateral ganglia above the origin of the sciatic nerve, into which they pass through the abdominal sympathetic. In all cases the nerves lose their medulla in the ganglia. Similarly the vaso-motor nerve supply for the blood-ressels of the head and neck and of the abdomen is derived from the cervical and abdominal splanchnics respectively, or from the corresponding rami efferentes of the upper lumbar ganglia.

The lateral sympathetic chain Gaskell proposes to call the chain of vaso-motor ganglia.

ii. Vaso-inhibitory or Vaso-dilator, and Cardio-inhibitory Fibres.Of these, which are doubtless as widely distributed as the vaso-motor fibres, we have distinct proof in the existence of fibres separate from vaso-motor, e.g., in the inhibitory nerve of the heart, the cardio-vagus; in the chorda tympani; in the small petrosal, and in the nervi erigentes.

These nerve-fibres, as far as we know at present, leave the central nervous system among the fine medullated nerves of the cervico-cranial and sacral rami communicantes, do not enter the lateral ganglia, but pass without losing their medulla into the collateral or terminal ganglia.

(b.) i. Viscero-motor Fibres. - These fibres, upon which depend the peristaltic movements of the thoracic portion of the œsophagus, and of the stomach and intestines, arise from the central nervous system, as the fine medullated fibres of the upper portion of the cervical region, not in the spinal nerve-roots of that region, but as the bundles of fibres which may be called the rami viscerales of the vagus and accessory nerves. They pass to the ganglion of the trunk of the ragus, where they lose their medulla.

ii. Viscero-Inhibitory Fibres. - It appear's that the nerve supply to the circular muscles of the alimentary canal and its appendages, is contained in the abdomiual splanchnics, and consists of those fibres which have not passed through the lateral chain, and which therefore retain their medulla until they reach the proximal or collateral chain.

(c.) Glandular Nerve-Fibres. - A double nerve supply, in all probability coinciding with the supply to the visceral muscles, has been demonstrated in the cases of the submaxillary, parotid, and lachrymal glands, and in these cases the course of the fibres is very similar to that of the corresponding fibres for the vaso-muscular supply. Thus 
the sympathetic supply for these glands passes along with the vasomotor fibres from the cervical splanchnic (or sympathetic trunk), and superior cervical ganglion; while the cerebro-spinal supply comes from the rami viscerales of the cranial nerves in conjunction with the vasodilator fibres.

Central Origin of the Rami Viscerales. - There appears to be the strongest presumption that the white rami of the thoracic region arise in the spinal cord in, or are connected with, the cells of the posterior vesicular column of Clarke. This conclusion is based upon the fact that these special cells are found in the three regions already mentioned, and in those only where the white rami of fine medullated fibres exist, viz., in the cervico-cranial regions, in the spinal accessory, in the thoracic region, and in the sacral region. But it is probable that the fibres are also connected with the cells of the lateral horn of the gray matter of the spinal cord, and its representative in the medulla, the antero-lateral nucleus of Clarke.

In a paper supplementary to his first account of the sympathetic system, Gaskell traced the nerve fibres of the anterior nerve roots to the various groups of nerve cells in the spinal cord thus: (i.) Efferent nerves to somatic muscles arise from group of cells of anterior cornua; (ii.) efferent nerves to striated splanchnic muscles from cells of the tractus intermedio-lateralis.

muscles of viscera, and vessels of splanchnic system from cells of Clarke's column; (iv.) motor nerves to visceral muscles from solitary cells at the base of the posterior cornu; and (v.) motor or catabolic nerves to glands and vascular muscles from small cells of the lateral cornu.

Structure and Functions of the Ganglia.-The sympathetic ganglia all contain-(1.) nerve-fibres tiaversing them; (2.) nerve-fibres originating in them, (3.) nerve or ganglion-corpuscles, giving origin to these fibres; and (4.) other corpuscles that appear free. In the sympathetic ganglia of the frog, ganglion-cells of a very complicated structure have been described by Beale, and subsequently by Arnold. The cells are inclosed each in a nucleated capsule: they are pyriform in shape, and from the pointed end two fibres are given off, which gradually acquire the characters of nerve-fibres: one of them is straight, and the other (which sometimes arises from the cell by two roots) is spirally coiled around it.

According to Gaskell the functions of the main sympathetic gauglia are the following:-(1.) They effect the conversion of medullated into non-medullated fibres; (2.) They possess a nutritive influence over the nerves which pass from them to the periphery; (3.) They increase the number of fibres at the same time as they cause the removal of the medulla. As regards their possession of the usual properties of nerve- 
centres little or nothing is certainly known. It appears unlikely that they possess the reflex functions of the spinal centres.

As a contribution toward the explanation of the nervous mechanism of nutrition comes in Gaskell's theory of katabolic and anabolic nerves. He supposes that every tissue is supplied with two sets of nerves, the former of which corresponds with the motor nerve, the viscero-motor and the cardio-augmentor, by the stimulation of which an increase of the metabolism takes place, and which is followed by exhaustion. It may be accompanied either by contraction of a muscle or by an increase of contraction. Such a nerve is excellently illustrated by the sympathetic augmentor or accelerator nerve of the heart, on stimulation of which an increase in the force and frequency of the heart takes place, followed after a time by exhaustion. A katabolic nerve stimulates the destructive metabolism which is always going on in a tissue. The anabolic nerve is the exact opposite of the katabolic nerve in function. It subserves constructive metabolism. Stimulation of the nerve produces diminished activity, repair of tissue and building up. An example of this kind of nerve is seen in the cardiac vagus, stimulation of which produces inhibition. Inhibition must generally be looked upon as an anabolic process.

It will be seen that the results of stimulation of the nerves to the salivary glands, discussed in a former chapter, appear to support the theory, that the processes of constructive and destructive metabolism are under the control of separate nerve-fibres. In the case of the submaxillary gland for example, if the sympathetic fibres be stimulated, a katabolic effect is produced, and the materials of secretion are formed at the expense of the protoplasm (this action in the case of the gland Heidenhain calls trophic); if on the other hand the chorda tympani or the secretory nerve be stimulated, two things happen, one being the discharge of water and the materials of secretion from the gland cells, and the other the building up or reconstruction of the protoplasm of the cells. A part of this action at any rate is anabolic, and similar to the action of inhibitory nerves. 


\section{CHAPTER XVII.}

\section{THE SENSES.}

General Considerations. - Through the medium of the nervous system the mind obtains a knowledge of the existence both of the various parts of the body, and of the external world. This knowledge is based upon sensations resulting from the stimulation of certain centres in the brain, by irritations conveyed to them by afferent nerves. Under normal circumstances, the following structures are necessary for sensation: $(a)$ A peripheral organ for the reception of the impression; $(b)$ a nerve for conducting it; $(c)$ a nerve-centre for feeling or perceiving it.

Classification of Sensations.-Sensations may be conveniently classed as (1) common and (2) special.

(1.) Common Sensations.-Under this head fall all those general sensations which cannot be distinctly localized in any particular part of the body, such as fatigue, discomfort, faintness, satiety, together with hunger and thirst, in which, in addition to a general discomfort, there is in many persons a distinct sensation referred to the stomach or fauces. In this class must also be placed the various irritations of the mucous membrane of the bronchi, which give rise to coughing, and also the sensations derived from various viscera indicating the necessity of expelling their contents; e.g., the desire to defæcate, to urinate, and, in the female, the sensations which precede the expulsion of the foetus. We must also include such sensations as itching, creeping, tickling, tingling, burning, aching, etc., some of which come under the head of pain: they will be again referred to in describing the tactile sense. It is impossible to draw a very clear line of demarcation between many of the common sensations above mentioned, and the sense of touch, which forms the connecting link between the general and special sensations. Touch is, indeed, usually classed with the special senses, and will be considered in the same group with them; yet it differs from them in being common to many nerves. Among common sensations some would rank the muscular sense, which has been already alluded to. It is by means of this sense that we become aware of the condition of the muscles, and thus obtain the information necessary for their adjustment to various purposes-standing, walking, grasping, etc. This muscular sensibility (to which we shall again refer) is shown in our power to esti- 
mate the differences between weights by the different muscular efforts necessary to raise them. It must be carefully distinguished from the sense of contact and of pressure, of which the skin is the organ. When standing erect, we can feel the ground (contact), and further there is a sense of pressure, due to our feet being pressed against the ground by the weight of the body. Both these are derived from the skin of the sole of the foot. If now we raise the body on the toes, we are conscious (muscular sense) of a muscular effort made by the muscles of the calf, which overcomes a certain resistance.

(2.) Special Sensations. - Including the sense of touch, the special senses are five in number-Touch, Taste, Smell, Hearing, Sight.

The most important distinction between common and special sensations is that by the former we are made aware of certain conditions of various parts of our bodies, while from the latter we gain our knowledge of the external world also. This difference will be clear if we compare the sensations of pain and touch, the former of which is a common, the latter a special sensation. "If we place the edge of a sharp knife on the skin, we feel the elge by means of our sense of touch; we perceive a sensation, and refer it to the object which has caused it. But as soon as we cut the skin with the knife, we feel pain, a feeling which we no longer refer to the entting knife, but which we feel within ourselves, and which communicates to us the fact of a change of condition in our own body. By the sensation of pain we are neither able to recognize the object which caused it, nor its nature."

In studying the phenomena of sensation, it is important clearly to understand that the sensorim, or seat of sensation, is in the brain, and not in the particular organ through which the sensory impression is received. In common parlance we are said to see with the eye, hear with the ear, etc., but in reality these organs are only adapted to receive impressions which, being conducted to the sensorium, through their respective nerves give rise to sensation.

Hence, if the optic nerve is severed, vision is no longer possible: since, although the image falls on the retina as before, the sensory impression can no longer be conveyed to the sensorium. When any given sensation is felt, all that we can with certainty affirm is that some part of the brain is excited. The exeiting cause may be some object of the external world, producing an objective sensation; or the condition of the sensorium may be due to some excitement within the brain itself, in which case the sensation is termed subjective. The mind habitually refers sensations to external causes; and hence, whenever they are subjective we can hardly divest ourselves of the idea of an external cause, and an illusion is the result.

Numberless examples of such illusions might be quoted. As familiar 
cases may be mentioned, humming and buzzing in the ears caused by some irritation of the auditory nerve or centre, and even musical sounds and voices (sometimes termed auditory spectra); also so-called optical illusions: objects are deseribed as seen, although not present. Such illusions are most strikingly exemplified in cases of delirium tremens or other forms of delirium, and may take the form of cats, rats, creeping loathsome forms, etc.

Causes of Illusions.-One uniform internal cause, which may act on all the nerves of the senses in the same manner, is capillary congestion. 'This one cause excites in the retina, while the eyes are closed, the sensaions of light and luminous flashes; in the auditory nerve, the sensation of humming and ringing sounds; in the olfactory nerve, the sense of odors; and in the nerves of feeling, the sensation of pain. In the same way, also, a narcotic substance introduced into the blood, excites in the nerves of each sense peculiar symptoms: in the optic nerves, the appearance of luminous sparks before the eyes; in the auditory nerves, tinnitus aurium; and in the common sensory nerves, the sensations of creeping over the surface. So, also, among external causes, the stimulus of electricity, or the mechanical influence of a blow, concussion, or pressure, excites in the eye the sensation of light and colors; in the ear, a sense of a loud sound or of ringing; in the tongue, a saline or acid taste; and in the other parts of the body, a perception of peculiar jarring or of the mechanical impression, or a shock like it.

Experiments seem to have proved, however, that none of the nerves of special sense possess the faculty of common sensibility.

Perceptions. - The habit of constantly referring our sensations to external causes, leads us to interpret the various modifications which external objects produce in our sensations, as properties of the external bodies themselves. Thus we speak of certain substances as possessing a disagreeable taste and smell; whereas, the fact is, their taste and smell are only disagreeable to $u s$. It is evident, however, that on this habit of referring our sensations to causes outside ourselves (perception), depends the reality of the external world to us; and more especially is this the case with the senses of touch and sight. By the co-operation of these two senses, aided by the others, we are enabled gradually to attain a knowledge of external objects which daily experience confirms, until we come to place unbounded confidence in what is termed the evidence of the senses.

Judgments. - We must draw a distinction between mere sensations, and the judgments based, often unconsciously, upon them. Thus, in looking at a near object, we unconsciously estimate its distance and say it seems to be ten or twelve feet off: but the estimate of its distance is in reality a judgment based on many things besides the appearance of 
the object itself; among which may be mentioned the number of interrening objects, the number of steps which from past experience we know we must take before we could touch it, and many others.

\section{The Special Sexses.}

\section{Touch.}

Sect.-The sense of touch is not confined to particular parts of the body of small extent, like the other senses; on the contrary, all parts capable of perceiving the presence of a stimulus by ordinary sensation are, in a certain degrees, the seat of this sense; but touch should not be considered as a mere modification or exaltation of eommon sensation or sensibility. For although the nerves on which the sense of touch depends, are the same as those which confer ordinary sensation on the different parts of the body, viz., those derived from the posterior roots of the nerves of the spinal cord, and the sensory cerebral nerves, yet it seems probable that the nerve-fibres which subserve the special sense of touch are provided with special end organs.

All parts of the body supplied with sensory nerves are thus, in some degree, organs of touch, yet the sense is exercised in perfection only in those parts the sensibility of which is extremely delicate, e.g., the skin, the tongue, and the lips, which are provided with abundant papillæ. A peculiar and, of its own kind in each ease, a very acute sense of touch is exercised through the medium of the nails and teeth. To a less extent the hair may be reckoned an organ of tonch; as in the ease of the eyelashes. The sense of touch renders us conscious of the presence of a stimulus, from the slightest to the most intense degree of its action, by that indescribable something which we eall feeling, or eommon sensation. The modifications of this sense often depend on the extent of the parts affecter. The sensation of pricking, for example, informs us that the sensitive fibres are intensely affected in a small extent; the sensation of pressure indicates a slighter affection of the parts in the greater extent, and to a greater depth. It is by the depth to which the parts are affected that the feeling of pressure is distinguished from that of mere contact.

Varieties. - $(a)$ 'The sense of touch proper, taetile sensibility or pressure, (b) temperature. These when earried beyond a certain degree are merged in the sensation of $(c)$ pain.

Touch proper.-In almost all parts of the body which have delicate tactile sensibility the epidermis, immediately over the papillæ, is moderately thin. When its thickness is much increased, as over the heel, the sense of touch is very much dulled. On the other hand, when it is altogether removed, and the cutis laid bare, the sensation of con- 
tact is replaced by one of pain. Further, in all highly sensitive parts, the papillæ are numerous and highly vascular, and the sensory nerves are connected with special end-organs which have been described p. 99 et seq.

The special endings of the nerves which have to do with touch may, however, be here again mentioned. They are of two kinds, viz., (a) touch corpuscles, which are found chiefly in the hands and feet, particularly on the palmar surface of the hands and fingers, but also on the under surface of the forearm, nipple, eyelids, lips, and genital organs. Touch corpuscles are situated in the cutis vera. (b) end bulbs, which are found in conjunctivæ and other mucous membranes, the lips, genital organs, tongue, rectum, and elsewhere, but not in the skin proper. As regards the Pacinian corpuscles and similar end-organs, which are so widely distributed, and which may be in some way connected with the sensation, when they are found in the skin they are situated very deeply in the cutis vera or in the subcutaneous tissue. They are extremely numerous on the nerves of the palmar surface of the fingers. In all of these endings, and in similar ones found in other animals, the nerve ends, as in axis cylinder, in a special development of the connective tissue sheath. In addition to these special nerve-endings, nerve-fibres appear to terminate everywhere in the skin between the cells of the Malpighian stratum of the epidermis in the ends, and in certain animals some of them appear to end in special and rather large cells.

It is practically impossible to distinguish between what is called mere contact and touch in which the element of pressure comes in. The acuteness of the sense of touch depends very largely on the cutaneous circulation, which is of course largely influenced by external temperature. Hence the numbness, familiar to every one, produced by the application of cold to the skin.

Acuteness of the Sense.-The perfection of the sense of touch on different parts of the surface is proportioned to the power which such parts possess of distinguishing and isolating the sensations produced by two points placed close together. This power depends, at least in part, on the number of primitive nerve-fibres distributed to the part; for the fewer the primitive fibres which an organ receives, the more likely is it that several impressions on different contiguous points will act on only one nervous fibre, and hence be confounded, and perhaps produce but one sensation. Experiments have been made to determine the tactile properties of different parts of the skin, as measured by this power of distinguishing distances. These consist in touching the skin, while the eyes are closed, with the points of a pair of compasses sheathed with cork, and in ascertaining how close the points of compasses might be brought to each other, and still be felt as two bodies. 
Table of variations in the tactile sensibility of the different parts. - The measurement indicates the least distance at which the two blunted points of a pair of compasses could be separately distinguished. (E. H. Weber.)

Tip of tongue

Palmar surface of third phalanx of foretinger

Palmar surface of second phalanges of fingers

Red surface of under-lip

Tip of nose

Middle of dorsum of tongue

Palm of hand

Centre of hard palate

Dorsal surface of first phalanges of fingers

Back of hand

Dorsum of foot near toes

Gluteal region

Sacral region

Upper and lower parts of forearm

Back of neck near occiput

Upper dorsal and mid-lumbar regions .

Middle part of forearm

Middle of thigh

Mid-cervical region.

Mid-dorsal region

\begin{tabular}{|c|c|c|c|}
\hline & nch & & Imn \\
\hline & $"$ & 2 & \\
\hline & " & 4 & " \\
\hline & " & 4 & " \\
\hline & " & 6 & "• \\
\hline & $"$ & 8 & \\
\hline 12 & " & 10 & $"$ \\
\hline 2 & $"$ & 12 & " \\
\hline$\overline{1}^{7} 2$ & ". & 14 & $"$ \\
\hline $1 \frac{1}{6}$ & $"$ & $2 \pi$ & \\
\hline $1 \frac{1}{2}$ & “" & 37 & ." \\
\hline $1 \frac{1}{2}$ & .. & 3 & .. \\
\hline $1 \frac{1}{2}$ & .. & 37 & " \\
\hline $2^{-}$ & " & 50 & $\because$ \\
\hline 2 & " & 50 & ". \\
\hline 2. & " & 62 & \\
\hline & " & 62 & \\
\hline & $"$ & 62 & \\
\hline & " & 62 & \\
\hline
\end{tabular}

Moreover, in the case of the limbs, it was found that before they were recognized as two, the points of the compasses had to be further separated when the line joining them was in the long axis of the limb, than when in the transverse direction.

According to Weber the mind estimates the distance between two points by the number of nnexcited nerve-endings which intervene between the two points tonched. It would appear that a certain number of intervening unexcited nerve-endings are necessary before two points tonched can be recognized as separate, and the greater this number the more clearly are the points of contact distinguished as separate. By practice the delicacy of a sense of touch may be very much increased. $\Lambda$ familiar illustration occurs in the case of the blind, who, by constant practice, can acquire the power of reading raised letters the forms of which are almost if not quite undistinguishable by the sense of touch to an ordinary person.

Loculization.- The power of correctly localizing sensations of touch is gradually derived from experience. Thus infants when in pain simply cry, but make no effort to remove the cause of irritation, as an older child or adult would, doubtless on account of their imperfect knowledge of its exact situation.

Illusions. - The different degrees of sensitiveness possessed by different parts may give rise to errors of judgment in estimating the distance between two points where the skin is touched. Thus, if blunted points of a pair of compasses (maintained at a constant distance apart) be slowly drawn over the skin of the cheek toward the lips, it is almost impossible to resist the conclusion that the distance between the points is 
gradually increasing. When they reach the lips they seem to be considerably further apart than on the cheek. Thus, too, our estimate of the size of a cavity in a tooth is usually exaggerated when based upon sensation derived from the tongue alone. Another curious illusion may here be mentioned. If we close the eyes, and place a small marble or pea between the crossed fore and middle fingers, we seem to be touching two marbles. This illusion is due to an error of judgment. The marble is touched by two surfaces which, under ordinary circumstances, could only be touched by two separate marbles, hence the mind, taking no cognizance of the fact that the fingers are crossed, forms the conclusion that two sensations are due to two marbles.

Temperature. - The whole surface of the body is more or less sensitive to differences of temperature. The sensation of heat is distinct from that of touch: and it would seem reasonable to suppose that there are special nerves and nerve-endings for temperature. At any rate the power of discriminating temperature may remain unimpaired when the sense of touch is temporarily in abeyance. Thus if the ulnar nerve be compressed at the elbow till the sense of touch is very much dulled in the fingers which it supplies, the sense of temperature remains quite unaffected.

The sensations of heat and cold are often exceedingly fallacious, and in many cases are no guide at all to the absolute temperature as indicated by a thermometer. All that we can with safety infer from our sensations of temperature, is that a given object is warmer or cooler than the skin. Thus the temperature of our skin is the standard; and as this varies from hour to hour according to the activity of the cutaneous circulation, our estimate of the absolute temperature of any body must necessarily vary too. If we put the left hand into water at $5^{\circ} \mathrm{C}$. $\left(40^{\circ} \mathrm{F}\right.$.) and the right into water at $45^{\circ} \mathrm{C}$. $\left(110^{\circ} \mathrm{F}\right.$.), and then immerse both in water at $27^{\circ} \mathrm{C}$. $\left(80^{\circ} \mathrm{F}\right.$.), it will feel warm to the left hand but cool to the right. Again, a piece of metal which has really the same temperature as a given piece of wood will feel much colder, since it conducts away the heat much more rapidly. For the same reason air in motion feels very much cooler than air of the same temperature at rest.

In some cases we are able to form a fairly accurate estimate of absolute temperature. Thus, by plunging the elbow into a bath, a practised bath-attendant can tell the temperature sometimes within half a degree centigrade.

The temperatures which can be readily discriminated are between $10^{\circ}-45^{\circ} \mathrm{C} .\left(50^{\circ}-115^{\circ} \mathrm{F}\right.$. $)$; very low and very high temperatures alike produce a burning sensation. A temperature appears higher according to the extent of cutaneous surface exposed to it. Thus, water of a temperature which can be readily borne by the hand, is quite intolerable if 
the whole body be immersed. So, too, water appears much hotter to the hand than to a single finger.

The delicacy of the sense of temperature coincides in the main with that of touch, and appears to depend largely on the thickness of the skin; hence, in the elbow, where the skin is thin, the sense of temperature is delicate, though that of touch is not remarkably so. Weber has further ascertained the following facts: two compass points so near together on the skin that they produce but a single impression, at once give rise to two sensations, when one is hotter than the other. Moreover, of two bodies of equal weight, that which is the colder feels heavier than the other.

As every sensation is attended with an idea, and leaves behind it an idea in the mind which can be reproduced at will, we are enabled to compare the idea of a past sensation with another sensation really present. Thus we can compare the weight of one body with another which we had previously felt, of which the idea is retained in our mind. Weber was indeed able to distinguish in this manner between temperatures, experienced one after the other, better than between temperatures to which the two hands were simultaneously subjected. This power of comparing present with past sensations diminishes, howerer, in proportion to the time which has elapsed between them. After-sensations left by impressions on nerves of common sensibility or touch are very vivid and durable. As long as the condition into which the stimulus has thrown the organ endures, the sensation also remains, though the exciting cause should have long ceased to act. Both painful and pleasurable sensations afford many examples of this fact.

Subjective sensations, or sensations dependent on internal causes, are in no sense more frequent than in the sense of touch. All the sensations of pleasure and pain, of heat and cold, of lightness and weight, of fatigue, etc., may be produced by internal causes. Neuralgic pains, the sensation of rigor, formication or the creeping of ants, and the states of the sexual organs occurring during sleep, afford striking examples of subjective sensations. The mind has a remarkable power of exciting sensations in the nerves of common sensibility: just as the thought of the nauseous excites sometimes the sensation of nausea, so the idea of pain gives rise to the actual sensation of pain in a part predisposed to it; numerous examples of this influence might be quoted.

Pain.-As regards painful sensations, three riews can be taken: 1 , that it is a special sensation provided with a special conducting apparatus in each part of the body; $⿱$, that it is produced by an over-stimulation of the special nerves concerned with touch or temperature, or of the other nerves of special sense; or 3, that it is an over-stimulation of the nerves of common sensation, which tell us of the condition of our own bodies, both of the surface aud also of the internal organs. There 
seems to be much in favor of all of these views. The weight of evidence is, however, rather against there being any special pain sense with a special end-organ and fibres. It is, however, certain that even if any variety of pain be a special sensation, some kind of pain may be produced by stimulation of the bare sensory nerves apart from any special form of nerve termination. It is said that the main difference between the common sensation which tells us of the condition of all parts of the body and of which thirst and hunger are but examples, the one informing us of the condition of the palate and the other of the state of our stomach, and the special sense of touch and temperature, is that the latter are provided with special apparatus. By means of this apparatus we are able to localize the sensation from which it is possible to form judgments. Such a special apparatus is evidently not absolutely essential for the sensation of pain, but this does not exclude the idea that pain may result from over-stimulation of a nerve of special sense or of its termination.

The Muscular Sense.-The estimate of a weight is usually based on two sensations: 1, of pressure on the skin, and 2, the muscular sense.

The estimate of weight derived from a combination of these two sensations (as in lifting a weight) is more accurate than that derived from the former alone (as when a weight is laid on the hand); thus Weber found that by the former method he could generally distinguish $19 \frac{1}{2}$ oz. from 20 oz., but not $19 \frac{3}{4}$ oz. from 20 , while by the latter he could at most only distinguish $14 \frac{1}{2} \mathrm{oz}$. from $15 \mathrm{oz}$.

It is not the absolute, but the relative, amount of the difference of weight which we have thus the faculty of perceiving.

It is not, however, certain, that our idea of the amount of muscular force used is derived solely from the muscular sense. We have the power of estimating very accurately beforehand, and of regulating, the amount of nervous influence necessary for the production of a certain degree of movement. When we raise a ressel, with the contents of which we are not acquainted, the force we employ is determined by the idea we have conceived of its weight. If it should happen to contain some very heavy substance, as quicksilver, we shall probably let it fall; the amount of muscular action, or of nervous energy, which we had exerted being insufficient. The same thing occurs sometimes to a person descending stairs in the dark; he makes the movement for the descent of a step which does not exist. It is possible that in the same way the idea of weight and pressure in raising bodies, or in resisting forces, may in part arise from a consciousness of the amount of nervous energy transmitted from the brain rather than from a sensation in the muscles themselves. The mental conviction of the inability longer to support a weight must also be distinguished from the actual sensation of fatigue in the muscles. 
So, with regard to the ideas derived from sensations of touch combined with movements, it is doubtful how far the consciousness of the extent of muscular movement is obtained from sensations in the muscles themselves. The sensation of movement attending the motions of the hand is very slight; and jersons who do not know that the action of particular muscles is necessary for the production of given morements, do not suspect that the morement of the fingers, for example, depends on an action in the forearm. The mind has, nevertheless, a very definite knowledge of the changes of position produced by movements; and it is on this that the ideas which it eonceives of the extension and form of a body are in great measure founded.

There is no marked development of common sensibility to be made out in muscles: they may be cut without the production of pain. On the other hand, there is no doubt that afferent impulses must pass upward from muscles and tendons acquainting the brain with their condition. This, then, must be a special sense. It has been suggested that the minute end-bulbs of Golgi found in tendons, and that the Pacinian eorpuscles in the neighborhood of joints, are the terminal organs of this special sense.

Judgment of the Form and size of Bodies.-By the sense of touch the mind is made acquainted with the size, form, and other external characters of bodies. And in order that these characters may be easily ascertained, the sense of touch is especially developed in those parts which can be readily mored over the surface of bodies. Touch, in its more limited sense, or the act of examining a body by the touch, consists merely in a voluntary employment of this sense combined with movement, and stands in the same relation to the sense of touch, or common sensibility, generally, as the act of seeking, following, or examining odors, does to the sense of smell. The hand is the best adapted for it, by reason of its peculiarities of structure, - namely, its capability of pronation and supination, which enables it, by the movement of rotation, to examine the whole circumference of the body; the power it possesses of opposing the thumb to the rest of the hand, and the relative mobility of the fingers; and lastly from the abundance of the sensory terminal organs which it possesses. In forming a conception of the figure and extent of a surface, the mind multiplies the size of the hand or fingers used in the inquiry by the number of times which it is contained in the surface traversed; and by repeating this process with regard to the different dimensions of a solid body, acquires a notion of its cubical extent, but, of course, only an imperfect notion, as other senses, e.g., the sight, are required to make it complete.

It is impossible in this consideration to say how much of our knowledge of the thing touched depends upon pressure and how much upon the muscular sense. 


\section{Taste.}

Conditions necessary. - The conditions for the perceptions of taste are:-1, the presence of a nerve and nerve-centre with special endowments; 2, the excitation of the nerve by the sapid matters, which for this purpose must be in a state of solution; 3 , a temperature of about $37^{\circ}$ to $40^{\circ} \mathrm{C}$. $\left(98^{\circ}\right.$ to $100^{\circ} \mathrm{F}$.). The nerves concerned in the production of the sense of taste have been already considered (p. 349 et seq.) The mode of action of the substances which excite taste consists in the production of a change in the condition of the gustatory nerves, and the conduction of the stimulus thus produced to the nerve-centre; and, according to the difference of the susbtances, an infinite variety of changes of condition of the nerves, and consequently of stimulations of the gustatory centre, may be induced. The matters to be tasted must either be in solution or be soluble in the moisture covering the tongue; hence insoluble substances are usually tasteless, and produce merely sensations of touch. Moreover, for the perfect action of a sapid, as of an odorous substance, it is necessary that the sentient surface should be moist. Hence, when the tongue and fauces are dry, sapid substances, even in solution, are with difficulty tasted.

The nerves of taste, like the nerves of other special senses, may have their peculiar properties excited by various other kinds of irritation, such as electricity and mechanical impressions. Thus, a small current of air directed upon the tongue gives rise to a cool saline taste, like that of saltpetre; and a distinct sensation of taste similar to that caused by electricity, may be produced by a smart tap applied to the papillæe of the tongue. Moreover, the mechanical irritation of the fauces and palate produces the sensation of nausea, which is probably only a modification of taste.

Seat.- The prineipal seat (apparent seat, that is, to our senses) of the sense of taste is the tongue. But the result of experiments as well as ordinary experience show that the soft palate and its arches, the uvula, tonsils, and probably the upper part of the pharynx, are also endowed with taste. 'These parts, together with the base and posterior parts of the tongue, are supplied with branches of the glosso-pharyngeal nerve, and evidence has been already adduced that the sense of taste is conferred upon them by this nerve. In most, though not in all persons, the anterior parts of the tongue, especially the edges and tip, are endowed with the sense of taste. The middle of the dorsum is only feebly endowed with this sense, probably because of the density and thickness of the epithelium covering the filiform papillæ of this part of the tongue, which will prevent the sapid substances from penetrating to their sensitive parts.

Other Functions. - Beside the sense of taste, the tongue, by means 
also of its papillæ, is endued (2) especially at its side and tip, with a very delicate and accurate sense of touch, which renders it sensible of the impressions of heat and cold, pain and mechanical pressure, and consequently of the form of surfaces. The tongue may lose its common sensibility, and still retain the sense of taste, and vice versit. This fact renders it probable that, although the senses of taste and of touch may be exercised by the same papillæ supplied by the same nerves, yet the nervous conductors for these two different sensations are distinct, just as the nerves for smell and common sensibility in the nostrils are distinct; and it is quite conceivable that the same nervous trunk may contain fibres differing essentially in their specific properties. Facts already detailed seem to prove that the lingual branch of the fifth nerve is the conductor of sensations of taste in the anterior part of the tongue; and it is also certain, from the marked manifestations of pain to which its division in animals gires rise, that it is likewise a nerve of common sensibility. The glosso-pharyngeal also seems to contain fibres both of common sensation and of the special sense of taste.

The functions of the tongue in connection with (3) speech, (4) mastication, (5) deglutition, (6) suction, have been referred to in other chapters.

Taste and Smell: Perceptions. - The concurrence of common and two kinds of special sensibility, i.e., touch and taste in the same part, makes it sometimes difficult to determine whether the impression produced by a substance is perceived through the ordinary sensitive fibres, or through those of the sense of taste. In many cases, indeed, it is probable that both sets of nerve-fibres are concerned, as when irritating acrid substances are introduced into the mouth.

Much of the perfection of the sense of taste is often due to the sapid substances being also odorous, and exciting the simultaneous action of the sense of smell. This is shown by the imperfection of the taste of such substances when their action on the olfactory nerves is prevented by closing the nostrils. Many fine wines lose much of their apparent excellence if the nostrils are held close while they are drunk.

Varieties of Tastes.-Among the most clearly defined tastes are the sweet and bitter (which are more or less opposed to each other), the acid, alkaline, salt, and metallic tastes. Acid and alkaline taste may be excited by electricity. If a piece of zinc be placed beneath and a piece of copper above the tongue, and their ends brought into contact, an acid taste (due to the feeble galvanic current) is produced. The delicacy of the sense of taste is sufficient to discern 1 part of sulphuric acid in 1000 of water; but it is far surpassed in acuteness by the sense of smell. Experiments have shown that it is possible to entirely do away with the power of tasting bitters and sweets while the taste for acids and salts 
remains. This is done by chewing the leaves of an Indian plant (Gymnema sylvestre). It has also been shown that the power of tasting sweet substances disappears before that of tasting bitter. Other experiments have shown that the apparatus for salt and for acid tastes are distinct. It is also demonstrable that bitters are most appreciated at the back and sweets at the tip of the tongue, that salts are also most potent at the tip, and acids at the sides of the tongue. All these tastes then, are almost certainly provided with a distinct apparatus. It is clear therefore that the taste buds cannot be the only terminal organs for the sense of taste, if from no other reason, at any rate from their exceedingly limited distribution in the human tongue.

Although the taste apparatus is bilateral the sensation or perception is single, and in this respect taste resembles vision.

After-taste. - Very distinct sensations of taste are frequently left after the substances which excited them have ceased to act on the nerve; and such sensations often endure for a long time, and modify the taste of other substances applied to the tongue afterward. Thus, the taste of sweet substances spoils the flavor of wine, the taste of cheese improves it. There appears, therefore, to exist the same relation between tastes as between colors, of which those that are opposed or complementary render each other more vivid, though no general principles governing this relation have been discovered in the case of tastes. In the art of cooking, however, attention has at all times been paid to the consonance or harmony of flavors in their combination or order of succession, just as in painting and music the fundamental principles of harmony have been employed empirically while the theoretical laws were unknown.

Frequent and continued repetitions of the same taste render the perception of it less and less distinct, in the same way that a color becomes more and more dull and indistinct the longer the eye is fixed upon it. Thus, after frequently tasting first one and then the other of two kinds of wine, it becomes impossible to discriminate between them.

The simple contact of a sapid substance with the surface of the gustatory organ seldom gives rise to a distinct sensation of taste; it needs to be diffused over the surface, and brought into intimate contact with the sensitive parts by compression, friction, and motion between the tongue and palate.

Subjective Sensations of Taste.-The sense of taste seems capable of being excited only by external causes, such as changes in the conditions of the nerves or nerve-centres, produced by congestion or other causes, which excite subjective sensations in the other organs of sense. But little is known of the subjective sensations of taste; for it is difficult to distinguish the phenomena from the effects of external causes, such as changes in the nature of the secretions of the mouth. 


\section{Smell.}

Conditions necessary.-(1.) 'The first conditions essential to the sense of smell are a special nerve and nerve-terminations in the form of special cells, the changes in whose condition stimulate a special nerve-centre, and are perceived in sensations of odor, for no other nervous structure is capable of these sensations, eren though acted on by the same causes. The same substance which excites the sensation of smell in the olfactory centre may cause another peculiar sensation through the nerves of taste, and may produce an irritating and burning sensation on the nerres of touch; but the sensation of odor is yet separate and distinct from these, though it may be simultaneously perceived. (2.) The material canses of odors are, usually, in the case of animals living in the air,

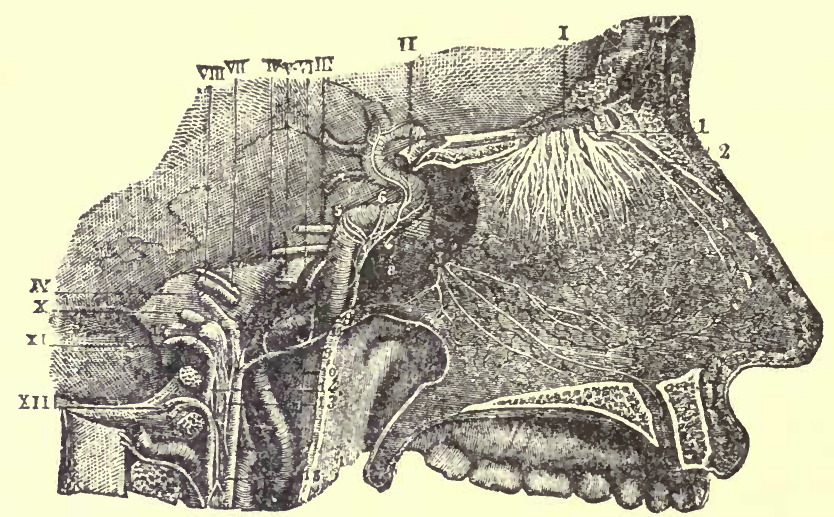

Fig. 391.-Nerves of the septum nasi. seen from the right sirle. 2/3.- I. the olfactory bulb: 1 , the olfactory nerves passing through the foramina of the cribriform plate, and descending to be distributed on the septum: 2, the internal or septal twig of the nasal branch of the ophthalmic nerve; 3, naso-palatine nerves. (From Sappey, after Hirschfeld and Leveillé.)

either solids suspended in a state of extremely fine division in the atmosphere; or gaseous exhalations often of so subtle a nature that they can be detected by no other reagent than the sense of smell itself. The matters of odor must, in all cases, be dissolved in the mucus of the mucous membrane before they can be immediately applied to, or affect the olfactory nerves; therefore a further condition necessary for the perception of odors is, that the mucous membrane of the nasal cavity be moist. When the Schneiderian membrane is dry, the sense of smell is impaired or lost; in the first stage of catarrh, when the secretion of mucus within the nostrils is lessened, the faculty of perceiving odor is either lost, or rendered very imperfect. (3.) In animals living in the air, it is also requisite that the odorous matter should be transmitted in a current through the mostrils. This is effected by an inspiratory move- 
ment, the mouth being closed; hence we have voluntary influence over the sense of smell; for by interrupting respiration we prevent the perception of odors, and by repeated quick inspiration, assisted, as in the act of sniffing, by the action of the nostrils, we render the impression more intense. An odorous substance in a liquid form injected into the nostrils appears incapable of giving rise to the sensation of smell; thus Weber could not smell the slightest odor when his nostrils were completely filled with water containing a large quantity of eau-de-Cologne.

The nose is not entirely an organ for the seat of smell. In fact the nasal cavities are divided into three districts called respectively - $(a)$ Regio vestibularis, which is the entrance to the cavity. It is lined with a mucous membrane very closely resembling the skin, and contains hair (vibris$s(c)$ with sebaceous glands. (b) Regio respiratoria, which includes the lower meatus of the nose, and all the rest of the nasal passages except $(c)$; it is covered with mucous membrane covered by stratified columnar ciliated epithelium. The mucosa is thick and consists of fibrous connective tissue; it contains a certain number of tubular mucous and serous glands. (c) Regio olfactoria. This includes the anterior twothirds of the superior meatus, the middle meatus, and the upper half of the septum nasi. It is of a yellowish color. It consists of a thicker mucous membrane than in $(b)$, made up of loose areolar connective tissue covered by epithelium of a special variety, resting upon a basement membrane. The cells of the epithelium are of two principal kinds: (a) columnar epithelial cells whose function is to support $(b)$ the bipolar olfactory cells. (a) The epithelial cells are prismatic in shape and have upon their surfaces facets into which the olfactory cells fit themselves. They are thus analogous to the cells of Müller of the retina (fig. 392e). (b) The olfactory cells have an oblong or fusiform shape, which is mainly determined by the large nucleus. The

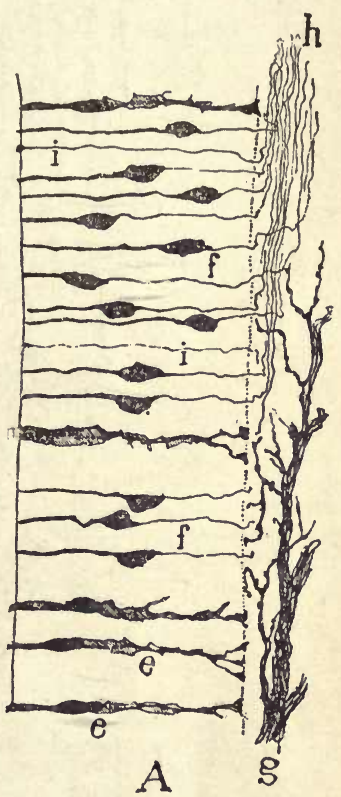

Fig. 392. - Bipolar olfactory cells from the vasal fossæ of the rat (full-term foetus). $A$, Epithelium of the olfactory mucosa; $e$, epithelial cells; $f, f$, nerve-cells ; $i$, nerve-fibres terminating freely on the epithelial surface; $h$, olfactory nerve-fibres : $g$, sensory nerve derived from the trigeminus. (Cajal.) thin protoplasmic body has two processes, an external and an internal. The external is large and passes up to the free surface to end in a small bunch of fibrils that are not vibratile. The internal process is very fine, often varicose, and passes through the mucous membrane to become continuous with the fibres of the olfactory bulb.

The olfactory bulb must be studied in relation with the nerve- 
fibres and olfactory cells with which it is connected. These parts together form a sensory end-organ which resembles in many respects the retina. The discovery of its true structure has thrown a flood of light on the architecture of the nerve-centres as a whole.

The olfactory bulb is not a nerve, but a modification of the brain cortex. A transection shows it to be made up of four layers:

1st. Peripheral fibres.

2d. Olfactory glomerules.

3d. Layer of mitral celle.

Zone of Glomerules.

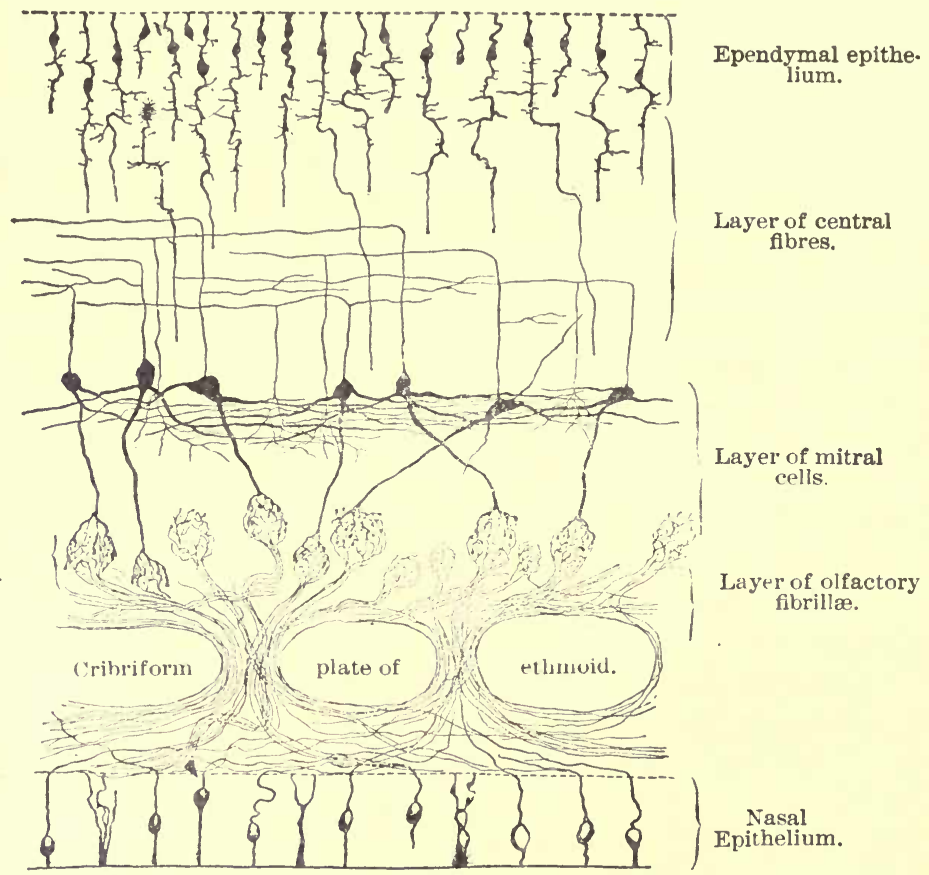

Fig. 393. - Principal constituent elements of the olfactory bulb of a mammal. (Van Gehuchten.)

4th. Layer of granular cells and deep nerre-fibres.

1st. The first and external layer is composed of the fine nerve-fibrils of the olfactory nerves. They pass through the cribriform plate of the ethmoid and continue on, ending in the olfactory cells.

2d. The glomerular layer contains numbers of small round bodies whose structure is now known to be nervous. They are made up of the expansions of the olfactory fibres on the one hand and of the "mitral" cells on the other. These are mingled in a close network, but do not anastonose. It was by the study of these bodies in part that the fact of 
the non-continuity of the neurons .was demonstrated (fig. 393). This layer also contains small fusiform cells with branching dendrites that extend outward to the glomeruli. Each has an axis-cylinder process which passes inward to join the fibres of the internal olfactory nerves.

3d. 'The layer of mitral cells contains large cells, some of them triangular and some in the shape of a mitre. They have numerous dendrites, one of which passes into a glomerule and then breaks up in a fine arborization. An axis-cylinder process (neuraxon) passes off from the inner surface and is continued as an internal olfactory nerve-fibre.

4 th. The layer of granules and central fibres. This contains a large number of very small nerve-cells, which are peculiar in that they

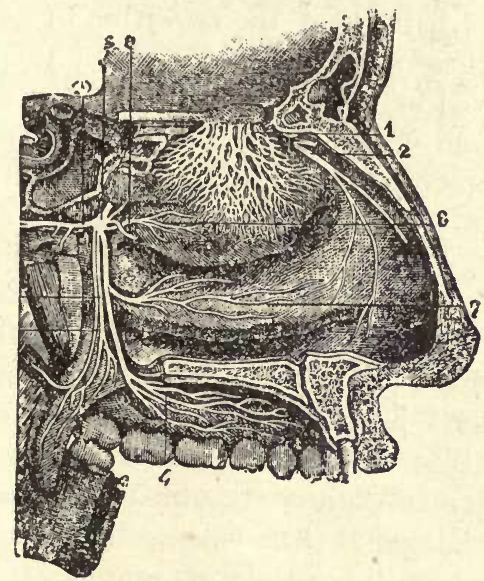

Fig. 394.--Nerves of the outer walls of the nasal fossæ. 3-5.-1, network of the branches of the olfactory nerve, descending upon the region of the superior and middle turbinated bones; 2 , external twig of the ethmoidal branch of the nasal nerves; 3 , spheno-palatine ganglion; 4, ramification of the anterior palatine ierves; 5 , posterior, and 6 , middle divisions of the palatine nerves; 7, branch to the region of the inferior turbinated bone; 8 , branch to the region of the Superior and middle turbinated bones: 9, naso-palatine branch to the septum cut short. (Frow

have no axis-cylinder. Their dendrites extend chiefly into the layer of mitral cells. They resemble the spongioblasts of the retina and probably have commissural functions. This layer has also some small starshaped cells whose dendrites end in the mitral cell-layer. Among these cells run numerous fibres, chiefly from the mitral cells and the fusiform cells of the glomerular layer.

The general arrangement is shown in fig. 393 .

The sense of smell is derived exclusively through those parts of the nasal cavities in which the olfactory nerves are distributed; the accessory cavities or sinuses communicating with the nostrils seen to have no relation to it. Air impregnated with the vapor of camplor was injected 
into the frontal sinus through a fistulous opening and odorous substances have been injected into the autrum of Highmore; but in neither case was any odor pereeived by the patient. 'The purposes of these sinuses appear to be that the bones, necessarily large for the action of the muscles and other parts connected with them, may be as light as possible, and that there may be more room for the resonance of the air in rocalizing. 'The former purpose, which is in other bones obtained by filling their cavities with fat, is here attained, as it is in many bones of bircls, by their being tilled with air.

Other Functions of the Vusel heyjun.-All parts of the nasal calvities, whether or not they can be the seats of the sense of smell, ane endowed with common sensibility by the nasil hranches of the first and second divisions of the fifth nerve. Ilence the sensitions of cold, heat, jtehing, tickling, and piain; and the sensation of tension or pressure in the nostrils. That these nerves annot perform the funetion of the olfactory nerves is proved by cases in which the sense of smell is lost, while the nucous nembrane of the nose remains susceptible of the varions modifications of common sensation and of tonch. But it is oftendifficult to distinguish the sensation of smell from that of mere feeling, and to ascertain what belongs to each separately. This is the case particularly with the sensations excited in the nose by acrid vapors, as of ammonia, horse-radish, mustarl, ete, which resemble much the sensations of the nerves of tonch; and the difficulty is the greater when it is rememberect that these acrid valors hare nearly the same action upon the mucons membrane of the eyelicls. It was beanse the common sensibility of the nose to these irritating substances remained after the destruction of the olfactory nerves that Inagendie wis led to the erroneous belief that the fifth nerve might excreise this special sense.

Turieties of Odorous Sensutions. - Animals do not all equally perceive the same odors; the odors most plainly perceived by an herbivorous animal and by a carnivorons animal are different. The Camivora have the power of detecting most aceurately by the smell the special peculiarities of animal matters and of tracking other animals by the scent; but have apparently very little sensibility to the odors of plants and flowers. Herbivorous animals are peculiarly sensitive to the latter, and have a narrower sensibility to animal oilors, especially to such as proceen from other individuals than their own species. Man is far inferior to many animals of both classes (which appear to have a special epithelial arrangement called Jacobson's organ, for the purpose of "scent"), in respect of the acuteness of smell; but his sphere of susceptibility to various odors is more uniform and extended. The cause of this difference lies probably in the endowments of the cerebral parts of the olfactory apparatus. The delicacy of the sense of smell is most remarkable; it can dis- 
cern the presence of bodies in quantities so minute as to be undiscoverable even by spectrum analysis; $\frac{100,000,000}{100}$ of grain of musk can be distinctly smelt (Valentin). Opposed to the sensation of an agreeable odor is that of a disagreeable or disgusting odor, which corresponds to the sencations of pain, dazzling and disharmony of colors, and dissonance in the other senses. The cause of this difference in the effect of different odors is unknown; but this much is certain, that odors are pleasant or offensive in a relative sense only, for many animals pass their existence in the midst of odors which to us are highly disagreeable. A great difference in this respect is, indeed, observed amongst men : many odors, generally thought agreeable, are to some persons intolerable; and different persons describe differently the sensations that they severally derive from the same odorous substances. There seems also to be in some persons an insensibility to certain odors, comparable with that of the eye to certain colors; and among different persons, as great a difference in the acuteness of the sense of smell as among others in the acuteness of sight. We have no exact proof that a relation of harmony and disharmony exists between odors as between colors and sounds; though it is probable that such is the case, since it certainly is so with regard to the sense of taste; and since such a relation would account in some measure for the different degrees of perceptive power in different persons; for as some have no ear for music (as it is said), so others have no clear appreciation of the relation of odors, and therefore little pleasure in them.

Subjective sensations. - The sensations of the olfactory nerves, inde. pendent of the external application of odorous substances, have hitherto been little studied. The friction of the electric machine produces a smell like that of phosphorus. Ritter, too, has observed, that when a galvanic current is applied to the organ of smell, besides the impulse to sneeze, and the tickling sensation excited in the filaments of the fifth. nerve, a smell like that of ammonia was excited by the negative pole, and. an acid odor by the positive pole; whichever of these sensations were pro-duced, it remained constant as long as the circle was closed, and changed to the other at the moment of the circle being opened. Subjective sen. sations occur frequently in connection with the sense of smell. Frequently a person smells something which is not present, and which other persons cannot smell; this is very frequent with nervous people, but it oc. casionally happens to every one. In a man who was constantly conscious of a bad odor, the arachnoid was found after death to be beset with deposits of bone, and a lesion in the middle of the cerebral hemispheres was also discovered. Dubois was acquainted with a man who, ever after a fall from his horse, which occurred several years before his death, believed that he smelt a bad odor. 


\section{Hearing.}

Anatomy of the Ear.-For descriptive purposes, the Ear, or Organ of Hearing, is divided into three parts, (1) the external, (2) the middle, and (3) the internal ear. The two first are only accessory to the third

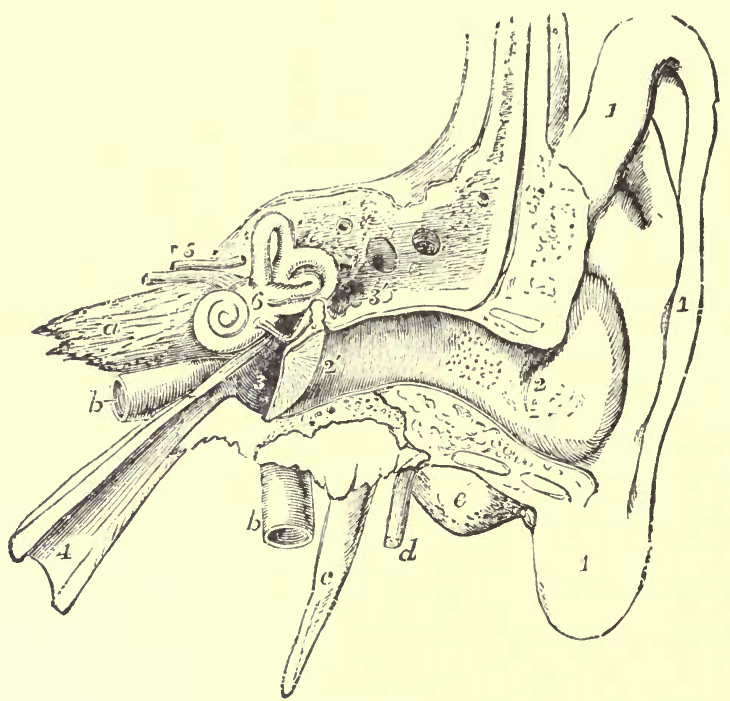

Fig. 395. - Diagrammatic view from before of the parts composing the organ of hearing of the left side. The temporal bone of the left side, with the accompanying soft parts, has been detached from the head, and a section has been carried through it transversely, so as to remove the front of the meatus externus, half the tympanic membrane, the upper and anterior wall of the tympanum and Eustachian tube. The meatus internus has also been opened, and the bony labylinth exposed by the removal of the surrounding parts of the petrous bone. 1, the pinna and lobe: 2,2 ', meatus externus; $2^{\prime}$, membrana tympani; 3 , eavity of the tympanum; 3 , its opening backward into the mastoid cells; between 3 and $3^{\prime}$, the chain of small hones: 4 , Eustachian tube; 5 , meatus internus, containing the facial (uppermost) and the auditory nerves; 6 . placed on the vestibule of the labvinth above the fenestra ovalis; $a$, apex of the petrous bone: 5 , internal carotid artery; $c$, styloid process; $d$, facial nerve issuing from the stylo-mastoid :oramen; $e$, mastoid process; $f$, squamous part of the bone covered by integument, etc. (Arnold.)

or internal ear, which contains the essential parts of an organ of hearing. The accompanying figure shows very well the relation of these divisions, one to the other (fig. 395).

External Ear.-The external ear consists of the pinna or auricle and the external auditory canal or meatus.

'The principal parts of the pinna (fig. 395) are two prominent rims inclosed one within the other (helix and antihelix), and inclosing a central hollow named the concha; in front of the concha, a prominence directed backward, the tragus, and opposite to this one directed forvard, the antitragus. From the conchia, the auditory canal, with a 
slight arch directed upward, passes inward and a little forward to the membrana tympani, to which it thus serves to convey the vibrating air. Its outer part consists of fibro-cartilage continued from the concha; its inner part of bone. Both are lined by skin continuous with that of the pinna, and extending over the outer part of the membrana tympani.

Toward the outer part of the canal are fine hairs and sebaceous glands, while deeper in the canal are small glands, resembling the sweatglands in structure, which secrete the cerumen.

Middle Ear or Tympanum.- The middle ear, or tympanum (3, fig. 395), is separated by the membrana tympani from the external auditory canal. It is a cavity in the temporal bone, opening through its anterior and inner wall into the Eustachian tube, a cylindriform

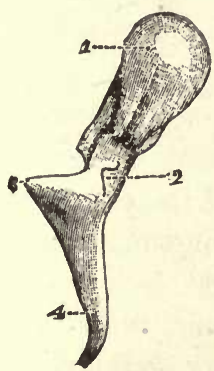

Fig. 396.

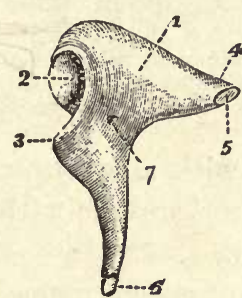

Fig. 397 .

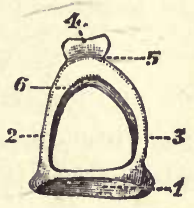

Fig. 398.

Fig. 396.-The hammer-bone or malleus, seen from the front. 1, the head; 2 , neck; 3 , short process; 4 , long process. (Schwalbe.)

Fig. 397 . - The incus, or anvil-bone. 1, body ; 2 , ridged articulation for the malleus; 4, processus brevis, with 5 , rough articular surface for ligament of incus; 6 , processus magnus, with articulating surface for stapes; 7 , nutrient foramen. (Schwalbe.)

Fig. 398. - The stapes, or stirrup-bone. 1, base; 2 and 3 , arch; 4 , head of bone, which articulates with orbicular process of the incus; 5 , constricted part of neck; 6 , one of the crura.
(Schwalbe.)

flattened canal, dilated at both ends, composed partly of bone and partly of elastic cartilage, and lined with mucous membrane, which forms a communication between the tympanum and the pharynx. It opens into the cavity of the pharynx just behind the posterior aperture of the nostrils. The carity of the tympanum communicates posteriorly with air cavities, the mastoid cells in the mastoid process of the temporal bone; but its only opening to the external air is through the Eustachian tube (4, fig. 395). The walls of the tympanum are osseous, except where apertures in them are closed with membrane, as at the fenestra rotunda and fenestra ovalis, and at the outer part where the bone is replaced by the membrana tympani. The cavity of the tympanum is lined with mucous membrane, the epithelium of which is ciliated and continuous with that of the pharynx. It contains a chain of small bones (ossicula auditus) which extends from the membrana tympani to the fenestra ovalis. 
The membrame tympani is placed in a slanting direction at the bottom of the external auditory canal, its plane being at an angle of about $45^{\circ}$ with the lower wall of the canal. It is formed chiefly of a tough and tense fihrous membrane, the edges of which are set in a bony groove;

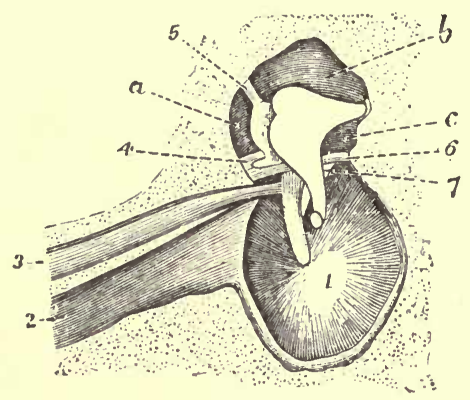

Fig. 399.-Interior view of the tympanum, with membrana tympani and bones in natural position. 1, Membrana tympani; 2, Eustachian tube: 3 , tensor tympani muscle; 4, lig. mallei super.; 6 , corda-tympani nerve; $a, b$, and $c$, sinuses about ossicula. (Schwalbe.)

its outer surface is covered with a continuation of the cutaneous lining of the auditory canal, its inner surface with part of the ciliated mucous membrane of the tympanum.

The ossicles are three in number; named malleus, incus, and stapes. 'The malleus, or hammer-bone, is attached by a long slightly-curved process, called its handle, to the membrana tympani; the line of attachment being vertical, including the whole length of the handle, and extending from the upper border to the centre of the membrane. The head of the malleus is irregularly rounded; its neck, or the line of boundary between it and the handle, supports two processes; a short conical one, which receires the insertion of the tensor tympani, and a stender one, processus gracitis, which extends forward, and to which the laxator tympani muscle is attached. The incus, or anvil-bone, shaped like a bicuspid molar tooth, is articulated by its broader part, corresponding with the surface of the crown of a tooth, to the malleus. Of its two fang-like processes, one, directed backward, has a free end lodged in a depression in the mastoid bone; the other, curved downward and more pointed, articulates by means of a roundish tubercle, formerly called os orbiculare, with the stapes, a little bone shaped exactly like a stirrup, of which the base or bar fits into the fenestra ovalis. To the neck of the stapes, a short process, corresponding with the loop of the stirrup, is attached the stapedius muscle.

The bones of the ear are covered with mucous membrane reflected over them from the wall of the tympanum; and are movable both altogether and one upon the other. The malleus moves and vibrates with every movement and vibration of the membrana tympani, and its movements are communicated through the incus to the stapes, and through 
it to the membrane closing the fenestra ovalis. The malleus, also, is movable in its articulation with the incus; and the membrana tympani moving with it is altered in its degree of tension by the laxator and tensor tympani muscles. The stapes is movable on the process of the incus, when the stapedius muscle acting, draws it backward. The axis round which the malleus and incus rotate is the line joining the processus gracilis of the malleus and the posterior (short) process of the incus.

The Internal Ear.-The proper organ of hearing is formed by the distribution of the auditory nerve within the internal ear, or labyrinth, a set of cavities within the petrous portion of the temporal bone. The bone which forms the walls of these cavities is denser than that around it, and forms the osseous labyrinth; the membrane within the cavities forms the membranous labyrinth. The membranous labyrinth contains a fluid called endolymph; while outside it, between it and the osseous labyrinth, is a fluid called perilymph. This fluid is not pure lymph; as it contains mucin.

The osseous labyrinth consists of three principal parts, namely the vestibule, the cochlea, and the semicircular canals.

The vestibule is the middle cavity of the labyrinth, and the central organ of the whole auditory apparatus. It presents, in its inner wall,

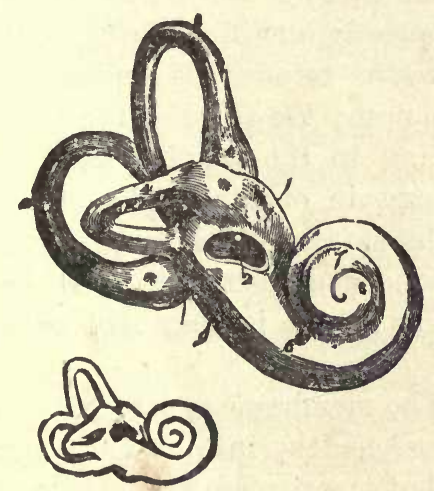

Fig. 400 .

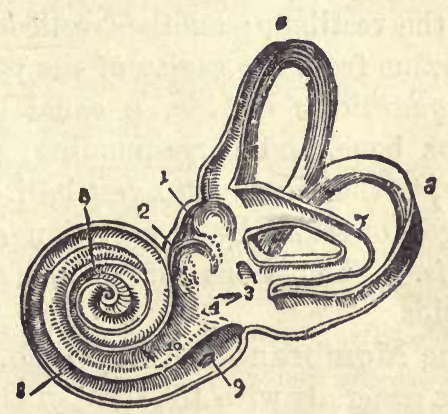

Fig. 401.

Fig. 400.-Right bony labyrinth, viewed from the outer side. The specimen here represented is prepared by separating piecemeal the looser substance of the petrous bone from the dense walls which immediately inclose the labyrinth. 1 , the vestibule; 2 , fenestra ovalis; 3 , superior semicircular canal; 4 , horizontal or external canal; 5 , posterior canal; *, ampullæ of the semicircular canals; 6 , first turn of the cochlea; 7 , second turn; 8 , apex; 9 , fenestra rotunda. The smaller figure in outline below shows the natural size. $\frac{21 / 2}{1}$ (Sömmering.)

Fig. 401.-Viex of the interior of the left labyrinth. The bony wall of the labyrinth is removed st periorly and externally. 1, Fovea hemielliptica: 2, fovea hemispherica; 3, common opening of the superior and posterior semicircular canals; 4, opening of the aqueduct of the vestibule: 5 , the superior, 6 , the posterior, and $\tau$, the external semicircular canals; 8 , spiral tube of the cochlea (scala tympani);9, opening of the aqueduct of the cochlea; 10 , placed on the lamina spiralis in the scala vestibuli. $\frac{21 / 2}{1}$ (Sômmering.)

several openings for the entrance of the divisions of the anditory nerve; in its outer wall, the fenestra ovalis (2, fig. 400), an open- 
ing filled by the base of the stapes; in its posterior and superior walls, five openings by which the semicircular canals communicate with it: in its anterior wall, an opening leading into the cochlea. 'The hinder part of the inner wall of the vestibule also presents an opening, the orifice of the aquceductus vestibuli, a canal leading to the posterior margin of the petrous bone, with uncertain contents and unknown purpose.

The semicircular canals (figs. 400, 401) are three arched cylindriform bony canals, set in the substance of the petrous bone. They all open at both ends into the restibule (two of them first coalescing). 'The ends of each are dilated just before opening into the restibule; and one enil being more dilated than the other is called an ampulla. Two of the canals form nearly vertical arches; of these the superior is also anterior; the posterior is inferior; the third canal is horizontal, and lower and shorter than the others.

The cochlea (6, 7, 8, figs. 400 and 401), a small organ, shaped like a common snail-shell, is situated in front of the vestibule, its base resting on the bottom of the internal meatus, where some apertures transmit to it the cochlear filaments of the auditory nerve. In its axis, the cochlea is traversed by a conical column, the modiolus, round which a spiral canal winds with about two turns and a half from the base to the apex. At the apex of the cochlea the canal is closed; at the base it presents three openings, of which one, already mentioned, communicates with the vestibule; another called fenestra rotunda, is separated by a membrane from the cavity of the tympanum; the third is the orifice of the aquacductus cochlece, a canal leading to the jugular fossa of the petrous bone, and corresponding, at least in obscurity of purpose and origin, to the aquæductus vestibuli. The spiral canal is divided into two passages, or scalæ, by a partition of bone and membrane, the lamina spiralis. The osseous part or zone of this lamina is connected with the modiolus.

The Membranous Labyrinth.-The membranous labyrinth corresponds generally with the form of the osseous labyrinth, so far as regards the vestibule and semicircular canals, but is separated from the walls of these parts by perilymph, except where the nerves enter into connection within it. The labyrinth is a closed membrane containing endolymph, which is of much the same composition as perilymph, but contains less solid matter. It is somewhat viscid, as is the perilymph, and it is secreted by the epithelium lining its cavity; all the sonorous vibrations impressing the auditory nerves in these parts of the internal ear, are conducted through fluid to a membrane suspended in and containing fluid. In the cochlea, the membranous labyrinth completes the septum between the two scalce, and incloses a spiral canal, previously mentioned, called canalis membranaceus or canalis cochlece (fig. 403). The fluid in 
the scalce of the cochlea is continuous with the perilymph in the vestibule and semicircular canals, and there is no fluid external to its lining membrane. The vestibular portion of the membranous labyrinth comprises two, probably communicating cavities, of which the larger and upper is named the utriculus; the lower, the sacculus. They are lodged in depressions in the bony labyrinth, termed respectively forca hemielliptica and fovea hemispherica. In to the former open the orifices of the membranous semicircular canals; into the latter the canalis cochlece. The membranous labyrinth of all these parts is laminated, transparent, very vascular, and covered on the inner surface with nucleated cells, of which those that line the ampullø are prolonged into stiff hair-like procosses; the same appearance, but to a much less degree, being visible in the utricule and saccule. In the cavities of the utriculus and sacculus are small masses of calcareous particles, otoconia or otoliths; and the

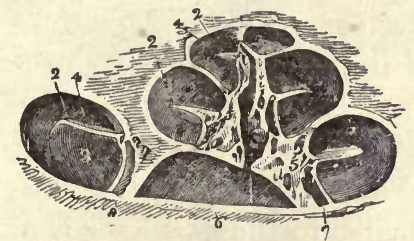

Fig. 402. - View of the osseous cochlea divided through the middle. 1, central canal of the trodiolus; 2 , lamina spiralis ossea; 3 , scala tympani ; 4 , scala vestibuli; 5 , porous substance of

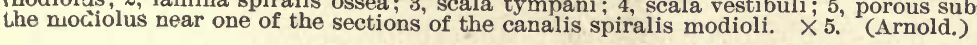

same, although in more minute quantities, are to be found in the interior of some other parts of the membranous labyrinth.

Auditory Nerve.-All the organs now described are provided for the appropriate exposure of the filaments of the auditory nerve to sonorous vibrations. It is characterized as a nerve of special sense by its softness (whence it derived its name of portio mollis of the seventh pair), and by the fineness of its component fibres. It enters the bony canal (the meatus auditorius internus), with the facial nerve and the nervus intermedius, and, traversing the bone, enters the labyrinth at the angle between the base of the cochlea and the vestibule, in two divisions; one for the vestibule and semicircular canals, and the other for the cochlea.

There are two branches for the vestibule, one, superior, distributed to the utricule and to the superior and horizontal semicircular canals, and the other, inferior, ending in the saccule and posterior semicircular canal. Where the nerve comes in connection with the utricule and saccule, the structure of the membrane is modified somewhat and the places are called maculoe acustica. The epithelium in this region is, as ve shall see directly, considerably specialized, and where the nerve is in connection with the ampullæ of the semicircular canals, too, the structare is altered, becoming elevated into a horse-shoe ridge, which projects 
into the interior of the cavity, forming the crista acustica. Here, too, the epithelium is of a special kind. The nerve fibres spread out and radiate on the inner surface of the membranous labyrinth: their exact termination is uncertain. The distribution of the other division of the auditory nerve, the cochlear, will be more clearly understood after the description of the cochlea itself.

Structure.-The structure of the membranous labyrinth consists of three coats, externally a layer of areolar tissue, next a hyaloid membrane, elevated into minute papillæ, and internally a layer of flattened epithelium. At the position where the branches of the vestibular branch of the auditory nerve join it, viz., at the saccule, utricule, and ampullæ of the semicircular canals, there is a marked difference in the structure, the external and middle layers are thicker and the epithelium becomes columnar. 'The epithelium in which the fibres of the vestibular' nerve are said to terminate are of two kinds, called cylinder or hair cells, and rorl cells. The hair cells occupy only one-half' of the thickness of the membrane; from their inner end hair-like processes project into the cavity of the labyrinth. Their outer end is rounded and contains 2 large round nucleus. 'To these cells the primitive fibrille of the axis eylinders pass up, some of them being distinctly raricose. The exact relation of the nerve fibrillæ to the hair-cells is unknown; by some they are believed actuaily to enter the cells, by others they are stated to form a kind of nest of fibrillie into which the cells fit. The rod-cells are of somewhat rarying form. They are elongated cells extending from the surface to the basement membrane, broad at the upper or surface end, and containing oral nuclei toward their attached end, but not exactly at the same level in ali cases. These nuclei, therefore, form a distinct broad nuclear layer on a rertical section of the membrane, as the cells are numerous, much more so, indeed, than the other variety of cell. The lower or attached part of the cell may be branched.

The membranous part of the cochlea, with a muscular zone, forming its outer margin, is attached to the outer wall of the canal. Commens:ing at the base of the cochlea, between its restibular and tympanic openings, it forms a partition between these apertures; the two scalæ are, therefore, in correspondence with this arrangement, named scala vestibuli and scala tympani (fig. 403). At the apex of the cochlea, the lamina spiralis ends in a small hamulus, the inner and concave part of which, being detached from the summit of the modiolus, leaves a small aperture named helicotrema, by which the two scalæ, separated in all the rest of their length, communicate.

Besides the scala vestibuli and scala tympani, there is a third space between them, calied scala media or canal membranaceus (CC, fig. 403). In section it is triangular, its external wall being formed by the wall of 
the cochlea, its upper wall (separating it from the scala vestibuli) by the membrane of Reissner, and its lower wall (separating it from the scala tympani) by the basilar membrane, these two meeting at the outer edge of the bony lamina spiralis. Following the turns of the cochlea to its apex, the scala media there terminates blindly; while toward the base of the cochlea it is also closed with the exception of a very narrow passage (canalis reuniens) uniting it with the sacculus. The scala media (like the rest of the membranous labyrinth) contains endolymph.

Organ of Corti.-Upon the basilar membrane are arranged cells of various shapes. About midway between the outer edge of the lamina

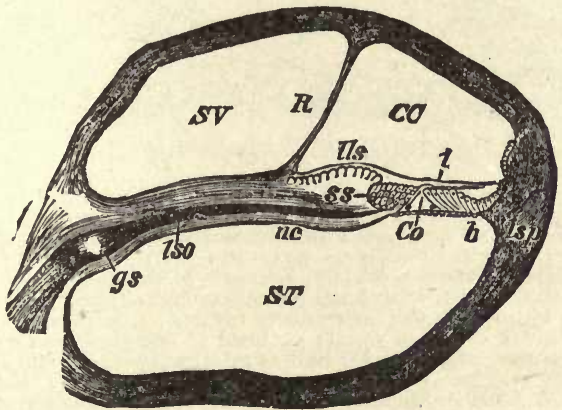

Fig. 403. - Section through one of the coils of the cochlea (diagrammatic). ST, scala tympani: SV scala vestibuli: $C C$, canalis cochlem or canalis membranaceus: $R$, membrane of Reissner; lso, lamina spiralis ossea; $l l s$, limbus laminæ spiralis; ss, sulcus spiralis ; $n c$, cochlear nerve; $g s$, ganglion spirale; $t$. membrana tectoria (below the membrana tectoria is the lamina recticularis); $b$, membrana basilaris; $C o$, rods of Corti ; lsp, ligamentum spirale. (Quain.)

spiralis and the outer wall of the cochlea are situated the rods of Corti. Viewed sideways, they are seen to consist of an external and internal pillar, each rising from an expanded foot or base on the basilar membrane ( $0, n$, fig. 404). They slant inward toward each other, and each ends in a swelling termed the head; the head of the inner pillar overlying that of the outer (fig. 404). Each pair of pillars forms, as it were, a pointed roof arching over a space, and by a succession of them a little tunnel is formed.

It has been estimated that there are about 3000 of these pairs of pillars, in proceeding from the base of the cochlea toward its apex. They are found progressively to increase in length, and become more oblique; in other words the tunnel becomes wider, but diminishes in height as we approach the apex of the cochlea. Leaning, as it were, against these external and internal piliars are certain other cells, of which the external ones, hair cells, terminate in small hair-like processes. Most of the above details are shown in the accompanying figure (fig. 404). This complicated structure rests, as we have seen, upon the basilar membrane; it is roofed in by a remarkable fenestrated membrane or lamina reticu- 
laris into the fenestræ of which the tops of the various rods and cells are received. When viewed from above, the organ of Corti shows a remarkable resemblance to the key-board of a piano. In close relation

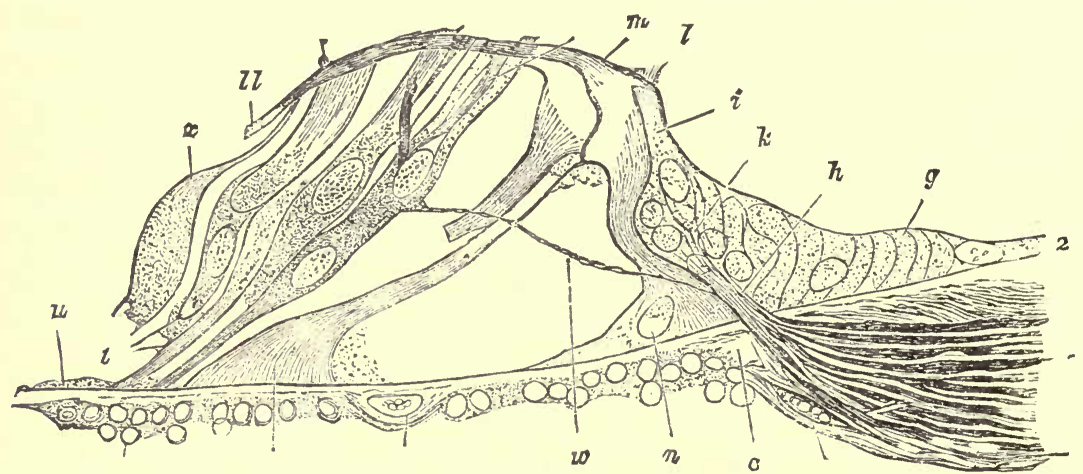

Fig. 404.- Vertical section of the organ of Corti from the dog. 1 to 2. Homogeneous layer of the so-called membrana basilaris: $u$, vestibular layer; $v$, tympanal layer, with nuclei and protoplasm; $a$, prolongation of tympanal periosteum of lamina spiralis ossea: $c$, thickened commencement of the membrana basilaris near the point of perforation of the nerves $h: d$, blood-vessel (vas spirale): $e$ blood-ressel: $f$, nerves: $g$. the epithelium of the sulcus spiralis internus: $i$, internal or tufted cell. with basil process $k$, surrounded with nuclei and protoplasm (of the granular layer). into which the nerre-fibres radiate: $l$, hairs of the internal hair-cell; $n$, base or foot of inner pillar of organ of Corti ; $m$, head of the same uniting with the correspond. ing part of an external pillar. whose under half is missing, while the next pillar beyond, o, presents both middle portion and base; $r s d$, three external hair-cells; $t$, bases of two neighboring hair or tufted cells: $x$. so-called supporting cell of Hensen: $w$, nerve-fibre terminating in the first of the external hair-cells: $l l$ to $l$, lamina reticularis. $\times 800$. (Waldeyer.)

with the rods of Corti and the cells inside and outside them, and proba bly projecting by free ends into the little tunnel containing fluid (roofed in by them), are filaments of the anditory nerve. These are derived from the cochlear division already mentioned. This passes up the axis of the cochlea, and in its course gives off fibres to the lamina spiralis. These fibres are thick at their origin, but thin out peripherally, and containing bipolar ganglion cells form the ganglion spirale. Beyond the ganglion at the edge of the lamina the fibres pass up and become connected with the organ of Corti.

\section{'The Physiology of Hearixg.}

All the acoustic contrivances of the organ of hearing are means for conducting sound. Since all matter is capable of propagating sonorous vibrations, the simplest conditions must be sufficient for mere hearing; for all substances surrounding the auditory nerve would stimulate it. The whole development of the organ of hearing, therefore, can have for its object merely the rendering more perfect the propagation of the sonorous vibrations, and their multiplication by resonance; and, in fact, the whole of the acoustic apparatus may be shown to have reference to these principles.

The external auditory passages influence the propagation of sound 
to the tympanum in three ways:-1, by causing the sonorous undulations, entering directly from the atmosphere, to be transmitted by the air in the passage immediately to the membrana tympani, and thus preventing them from being dispersed; 2 , by the walls of the passage conducting the sonorous undulations imparted to the external ear itself, by the shortest path to the attachment of the membrana tympani, and so to this membrane; 3 , by the resonance of the column of air contained within the passage; 4 , the external ear, especially when the tragus is provided with hairs, is also, doubtless, of service in protecting the meatus and membrana tympani against dust, insects, and the like.

Regarding the cartilage of the external ear, therefore, as a conductor of sonorous vibrations, all its inequalities, elevations, and depressions, become of evident importance; for those elevations and depressions upon which the undulations fall perpendicularly, will be affected by them in the most intense degree; and, in consequence of-the varions form and position of these inequalities, sonorous undulations, in whatever direction they may come, must fall perpendicularly upon the tangent of some one of them. This affords an explanation of the extraordinary form given to this part.

In animals living in the atmosphere, the sonorous vibrations are conveyed to the anditory nerve by three different media in succession; namely, the air, the solid parts of the body of the animal and of the auditory apparatus, and the fluid of the labyrinth. Sonorous vibrations are imparted too imperfectly from air to solid bodies, for the propagation of sound to the internal ear to be adequately effected by that means alone; yet already an instance of its being thus propagated has been mentioned. In passing from air directly into water, sonorous vibrations suffer also a considerable diminution of their strength; but if a tense membrane exists between the air and the water, the sonorous ribrations are communicated from the former to the latter medium with very great intensity. This fact, of which Müller gives experimental proof, furnishes at once an explanation of the use of the fenestra rotunda, and of the membrane closing it. They are the means of communicating, in full intensity, the vibrations of the air in the tympanum to the fluid of the labyrinth. This peculiar property of membranes is the result, not of their tenuity alone, but of the elasticity and capability of displacement of their particles; and it is not impaired when, like the membrane of the fenestra rotunda, they are not impregnated with moisture.

Sonorous vibrations are also communicated without any perceptible loss of intensity from the air to the water, when to the membrane forming the medium of communication, there is attached a short, solid body, which occupies the greater part of its surface, and is alone in contact 
with the water. This fact elucidates the action of the fenestra ovalis, and of the plate of the stapes which occupies it, and, with the preceding fact, shows that both fenestræ- that closed by membrane only, and that with which the movable stapes is connected-transmit very freely the sonorous vibrations from the air to the fluid of the labyrinth.

A small, solid body, fixed in an opening by means of a border of membrane, so as to be movable, communieates sonorous vibrations from air on the one side, to water, or the fluid of the labyrinth, on the other side, much better than solid media not so constructed. But the propigation of sound to the fluid is rendered much more perfect if the solid conductor thus occupying the opening, or fenestra oralis, is by its other end fixed to the middle of a tense membrane, which has atmospherie air on both sides. A tense membrane is a much better conductor of the vibrations of air than any other solid body bounded by definite surfaces: and the vibrations are also communicated very readily by tense membranes to solid bodies in contact with them. Thus, then, the membrana tympani serves for the transmission of sound from the air to the chain of ossicles. Stretched tightly in its osseous ring, it vibrates with the air in the auditory passage, as any thin tense membrane will, when the air near it is thrown into vibrations by the sounding of a tuning-fork or a musical string. And, from such a tense vibrating membrane, the vibrations are communicated with great intensity to solid bodies which touch it at any point. If, for example, one end of a flat piece of wood be applied to the membrane of a drum, while the other end is held in the hand, vibrations are felt distinctly when the vibrating tuning-fork is held over the membrane without touching it; but the wood alone, isolated from the membrane, will only very feebly propagate the vibrations of the air to the hand.

In comparing the membrana tympani to the membrane of a drum, however, it is necessary to point out certain important differences.

When a drum is struck, a certain definite tone is elicited (fundamental tone); similarly a drum is thrown into vibration when certain tones are sounded in its neighborhood, while it is quite unaffected by others. In other words it can only take up and vibrate in response to those tones whose vibrations nearly correspond in number with those of its own fundamental tone. The tympanic membrane can take up ans immense range of tones produced by vibrations ranging from 30 to 4000 or 5000 per second. This would be clearly impossible if it were an evenly stretched membrane.

The fact is, that the membrana tympani is by no means evenly stretched, and this is due partly to its slightly funnel-like form, and partly to its being connected with the chain of auditory ossieles. Further, if the membrane were quite free in its centre, it would go on 
vibrating as a drum does some time after it is struck, and each sound would be prolonged, leading to considerable confusion. This evil is obviated by the ear-bones, which check the continuance of the vibrations like the "dampers" in a pianoforte.

The ossicles of the ear are the better conductors of the sonorous vibrations communicated to them, on account of being isolated by an atmosphere of air, and not continuous with the bones of the cranium; for every solid body thus isolated by a different medium, propagates vibrations with more intensity through its own substance than it communicates them to the surrounding medium, which thus prevents a depression of the sound; just as the vibrations of the air in the tubes used for conducting the voice from one apartment to another are prevented from being dispersed by the solid walls of the tube. The vibrations of the membrana tympani are transmitted, therefore, by the chain of ossicula to the fenestra ovalis and fluid of the labyrinth, their dispersion in the tympanum being prevented by the difficulty of the transition of vibrations from solid to gaseous bodies.

The necessity of the presence of air on the inner side of the membrana tympani, in order to enable it and the ossicula auditus to fulfil the objects just described, is obvious. Without this provision, neither would the vibrations of the membrane be free, nor the chain of bones isolated, so as to propagate the sonorous undulations with concentration of their intensity. But while the oscillations of the membrana tympani are readily communicated to the air in the cavity of the tympanum, those of the solid ossicula will not be conducted away by the air, but will be propagated to the labyrinth without being dispersed in the tympanum.

The propagation of sound through the ossicula tympani to the labyrinth, must be affected either by oscillations of the bones, or by a kind of molecular vibration of their particles, or, most probably, by both these kinds of motion.

It has been shown that the existence of the membrane over the fenestra rotunda will permit approximation and removal of the stapes to and from the labyrinth. When by the stapes the membrane of the fenestra ovalis is pressed toward the labyrinth, the

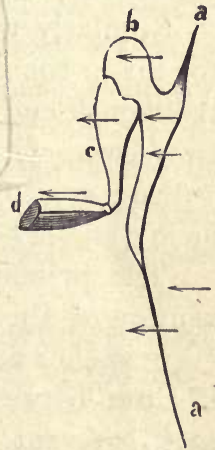

Fig. 405. - Diagram to illustrate the action of the ossicles of the middle ear in the conduction of sound to the internal ear. membrane of the fenestra rotunda may, by the pressure communicated through the fluid of the labyrinth, be pressed toward the cavity of the tympanum.

The long process of the malleus receives the undulations of the membrana tympani (fig. 405, $a, a$ ) and of the air in a direction indicated by 
the arrows, nearly perpendicular to itself. From the long process of the malleus they are propagated to its head $(b)$ : thence into the incus $(c)$, the long process of which is parallel with the long process of the malleus. From the long process of the incus the undulations are communicated to the stapes $(d)$, which is united to the incus at right angles. The several changes in the direction of the chain of bones have, however, no influence in changing the character of the undulations, which remain the same as in the meatus externus. From the long process of the malleus, the undulations are communicated by the stapes to the fenestra ovalis in a perpendicular direction.

Increasing tension of the membrana tympani diminishes the facility of transmission of sonorous undulations from the air to it.

The dry membrana tympani, on the approach of a body emits a loud sound, rejects particles of sand strewn upon it more strongly when lax than when very tense; and it has been inferred, therefore, that hearing is rendered less acute by increasing the tension of the membrana tympani.

The pharyngeal orifice of the Eustachian tube is usually shut; during swallowing, however, it is opened; this may be shown as follows:If the nose and mouth be closed and the cheeks blown out, a sense of pressure is produced in both ears the moment we swallow; this is due, doubtless, to the bulging out of the tympanic membrane by the compressed air, which at that moment enters the Eustachian tube.

Similarly the tympanic membrane may be pressed in by rarefying the air in the tympanum. This can be readily accomplished by closing the mouth and nose, and making an inspiratory effort and at the same time swallowing. In both cases the sense of hearing is temporarily dulled; proving that equality of pressure on both sides of the tympanic membrane is necessary for its full efficiency.

The principal office of the Eustachian tube has relation to the prevention of these effects of increased tension of the membrana tympani. Its existence and opemness will provide for the maintenance of the equilibrium between the air within the tympanum and the external air, so as to prevent the inordinate tension of the membrana tympani which would be produced by too great or too little pressure on either side. While discharging this office, however, it will serve to render sounds clearer, as the apertures in violins do; to supply the trmpanum with air; and to be an outlet for mucus. If the tube were permanently open, the sound of one's own voice would probably be greatly intensified, a condition which would of course interfere with the perception of other sounds. At any rate, it is certain that sonorous vibrations can be propagated up the tube to the tympanum by means of a catheter inserted into the pharyngeal orifice of the Eustachian tube. 
The influence of the tensor tympani muscle in modifying hearing may also be probably explained in connection with the regulation of the tension of the membrana tympani. If, through reflex nervous action, it can be excited to contraction by a very loud sound, then it is manifest that a very intense sound would, through the action of this muscle, induce a deafening or muffling of the ears. In favor of this supposition we have the fact that a loud sound excites, by reflection, nervous action, winking of the eyelids, and, in persons of irritable nervous system, a sudden contraction of many muscles.

The exact influence of the stapedius muscle in hearing is unknown. It acts upon the stapes in such a manner as to make it rest obliquely in the fenestra ovalis, depressing that side of it on which it acts, and elevating the other side to the same extent. It prevents too great a movement of the bone.

The fluid of the labyrinth is the most general and constant of the acoustic provisions of the labyrinth. In all forms of organs of hearing, the sonorous vibrations affect the auditory nerve through the medium of liquid-the most convenient medium, on many accounts, for such a purpose.

The otoliths in the labyrinth would reinforce the sonorous vibrations by their resonance, even if they did not actually touch the membranes upon which the nerves are expanded; but, inasmuch as these bodies lie in contact with the membranous parts of the labyrinth, and the vestibular nerve-fibres are imbedded in them, they communicate to these membranes and the nerves, vibratory impulses of greater intensity than the fluid of the labyrinth can impart. This appears to be their office. Sonorous undulations in water are not perceived by the hand itself immersed in the water, but are felt distinctly through the medium of a rod held in the hand. The fine hair-like prolongations from the epithelial cells of the ampullæ have, probably, the same function.

The function of the semicircular canals in the co-ordination of movements necessary to the maintenance of the equilibrium of the body has already been indicated.

The cochlea seems to be constructed for the spreading out of the nerve-fibres over a wide extent of surface, upon a solid lamina which communicates with the solid.walls of the labyrinth and cranium, at the ame time that it is in contact with the fluid of the labyrinth, and which, besides exposing the nerve-fibres to the influence of sonorous indulations, by two media, is itself insulated by fluid on either side.

The connection of the lamina spiralis with the solid walls of the abyrinth, adapts the cochlea for the perception of the sonorous undulainns propagated by the solid parts of the head and the walls of the labyinth. The membranous labyrinth of the vestibule and semicircular 
canals is suspended free in the perilymph, and is destined more particularly for the perception of sounds through the medium of that fluid, whether the sonorous undulations be imparted to the fluid through the fenestræ, or by the intervention of the cranial bones, as when sounding bodies are brought into communication with the head or teeth. The spiral lamina on which the nervous fibres are expanded in the cochlea, is, on the contrary, continuous with the solid walls of the labyrinth, and receives directly from them the impulses which they transmit. This is an important advantage; for the impulses imparted by solid bodies, have, coeteris paribus, a greater absolute intensity than those communicated by water. And, even when a sound is excited in the water, the sonorous undulations are more intense in the water near the surface of the vessel containing it, than in other parts of the water equally distant from the point of origin of the sound; thus we may conclude that, cceteris paribus, the sonorous undulations of solid bodies act with greater intensity than those of water. Hence, we perceive at once an important use of the cochlea.

This is not, however, the sole office of the cochlea; the spiral lamina, as well as the membranous labyrinth, receives sonorous impulses through the medium of the fluid of the labyrinth from the cavity of the vestibule, and from the fenestra rotunda. The lamina spiralis is, indeed, much better calculated to render the action of these undulations upon the auditory nerve efficient, than the membranous labyrinth is; for as a solid body insulated by a different medium, it is capable of resonance.

The rods of Corti are probably arranged so that each is set to vibrate in unison with a particular tone, and thus strike a particular note, the sensation of which is carried to the brain by those filaments of the auditory nerve with which the little vibrating rod is connected. The distinctive function, therefore, of these minute bodies is, probably, to render sensible to the brain the various musical notes and tones, one of them answering to one tone, and one to another; while perhaps the other parts of the organ of hearing discriminate between the intensities of different sounds, rathe" than their qualities.

"In the cochlea we have to do with a series of apparatus adapted for performing sympathetic vibrations with wonderful exactness. We have here before us a musical instrument which is designed, not to create musical sounds, but to render them perceptible, and which is similar in construction to artificial musical instruments, but which far surpasses them in the delicacy as well as the simplicity of its execution. For, while in a piano every string must have a separate hammer by means of which it is sounded the ear possesses a single hammer of an ingenious form in its ear bones, which can make every string of the organ of Corti sound separately." (Bernstein.) 
Since about 3000 rods of Corti are present in the human ear, this would give about 400 to each of the seven octaves which are within the compass of the ear. Thus about 32 would go to each semi-tone. Weber asserts that accomplished musicians can appreciate differences in pitch as small as $\frac{1}{64}$ th of a tone. Thus on the theory above advanced, the delicacy of discrimination would, in this case, appear to have reached its limits.

\section{Sounds.}

Any elastic body, e.g., air, a membrane, or a string performing a certain number of regular vibrations in the second, gives rise to what is termed a musical sound or tone. We must, however, distinguish between a musical sound and a mere noise; the latter being due to irregular vibrations.

Musical sounds are distinguished from each other by three qualities. 1. Strength or intensity, which is due to the amplitude or length of the vibrations. 2. Pitch, which depends upon the number of vibrations in a second. 3. Quality, Color, or Timbre. It is by this property that we distinguish the same note sounded on two instruments, e.g., a piano and a flute. It has been proved by Helmholtz to depend on the number of secondary tones, termed harmonics, which are present with the predominating or fundamental tone.

It would appear that two impulses, which are equivalent to four single or half vibrations, are sufficient to produce a definite note, audible as such through the auditory nerve.

The maximum and minimum of the intervals of successive impulses still appreeiable through the auditory nerve as determinate sounds, have been determined by Savart. If their intensity is sufficiently great, sounds are still audible which result from the succession of 48,000 half vibrations, or 24,000 impulses in a second; and this, probably, is not the extreme limit in acuteness of sounds perceptible by the ear. For the opposite extreme, he has succeeded in rendering sounds audible which were produced by only fourteen or eighteen half vibrations, or seven or eight impulses in a seeond; and sounds still deeper might probably be lieard, if the individual impulses could be sufficiently prolonged.

Direction.-The power of perceiving the direction of sounds is not a faculty of the sense of hearing itself, but is an act of the mind judging on experience previously acquired. From the modifications which the sensation of sound undergoes aceording to the direction in which the sound reaches us, the mind infers the position of the sounding body. The only true guide for this inference is the more intense action of the sound upon one than upon the other ear. But even here there is room for much deception, by the influence of reflexion or resonance, and by 
the propagation of sound from a distance, without loss of intensity, through curved conducting tubes filled with air. By means of such tubes, or of solid conductors, which convey the sonorous vibrations from their source to a distant resonant body, sounds may be made to appear to originate in a new situation. The direction of sound may also be judged of by means of one ear only; the position of the ear and head being varied, so that the sonorous undulations at one moment fall upon the ear in a perpendicular direction, at another moment obliquely. But when neither of these circumstances can guide us in distinguishing the direction of sound, as when it falls equally upon both ears, its source being, for example, either directly in front or behind us, it becomes impossible to determine whence the sound comes.

Distance. - The distance of the source of sounds is not recognized by the sense itself, but is inferred from their intensity. The sense itself is always seated but in one place, namely, in our ear; but it is interpreted as coming from an exterior soniferous body. When the intensity of the voice is modified in imitation of the effect of distance, it excites the idea of its originating at a distance. Ventriloquists take advantage of the difficulty with which the direction of sound is recognized, and also the influence of the imagination over our judgment, when they direct their voice in a certain direction, and at the same time pretend, themselves, to hear the sounds as coming from thence.

Intensity.-By removing one or several teeth from the toothed wheel the fact has been demonstrated that in the case of the auditory nerve, as in that of the optic nerve, the sensation continues longer than the impression which causes it; for a removal of a tooth from the wheel produced no interruption of the sound. The gradual cessation of the sensation of sound renders it difficult, however, to determine its exact duration beyond that of the impression of the sonorous impulses.

So we see that the effect of the action of sonorous undulations upon the nerve of hearing, endures somewhat longer than the period during which the undulations are passing through the ear. If, however, the impressions of the same sound be very long continued, or constantly repeated for a long time, then the sensation produced may continue for a very long time, more than twelve or twenty-four hours even, after the original cause of the sound has ceased.

Binaural Sensations. - Corresponding to the double vision of the same object with the two eyes, is the double hearing with the two ears; and anaiogous to the double vision with one eye, dependent on unequal refraction, is the double hearing of a single sound with one ear, owing to the sound coming to the ear through media of unequal conducting power. The first kind of double hearing is very rare; instances of it, however, have been recorded. The second kind which depends on the 
unequal conducting power of two media through which the same sound is transmitted to the ear, may easily be experienced. If a small bell be sounded in water, while the ears are closed by plugs, and a solid conductor be interposed between the water and the ear, two sounds will be heard differing in intensity and tone; one being conveyed to the ear through the medium of the atmosphere, the other through the conducting-rod.

Subjective Sensations. - Subjective sounds are the result of a state of irritation or excitement of the auditory nerve produced by other causes than sonorous impulses. A state of excitement of this nerve, however induced, gives rise to the sensation of sound. Hence the ringing and buzzing in the ears heard by persons of irritable and exhausted nervous system, and by patients with cerebral disease, or disease of the auditory nerve itself; hence also the noise in the ears heard for some time after a long journey in a rattling, noisy vehicle. Ritter found that electric currents also excite sounds in the ears. From the above truly subjective sound we must distinguish those dependent, not on a state of the auditory nerve itself merely, but on sonorous vibrations excited in the auditory apparatus. Such are the buzzing sounds attendant on vascular congestion of the head and ear, or on aneurismal dilatation of the vessels. Frequently even the simple pulsatory circulation of the blood in the ear is heard. To the sounds of this class belong also the buzz or hum, heard during the contraction of the palatine muscles in the act of yawning, during the forcing of air into the tympanum so as to make tense the membrana tympani, and in the act of blowing the nose, as well as during the forcible depression of the lower jaw.

Irritation or excitement of the auditory nerve is capable of giving rise to movements in the body, and to sensations in other organs of sense. In both cases it is probable that the laws of reflex action, through the medium of the brain, come into play. An intense and sudden noise excites, in every person, closure of the eyelids, and, in nervous individuals, a start of the whole body or an unpleasant sensation, like that produced by an electric shock, throughout the body, and sometimes a particular feeling in the external ear. Various sounds cause in many people a disagreeable feeling in the teeth, or a sensation of cold tickling through the body, and, in some people, intense sounds are said to make the saliva collect.

\section{Sight.}

Anatomy of the Optical Apparatus. - The eyelids consist of two movable folds of skin, each of which is kept in shape by a thin plate of yellow elastic tissue. Along their free edges are inserted a number of curved hairs (eyelashes), which, when the lids are half closed, serve 
to protect the eye from dust and other foreign bodies: their tactile sensibility is also very delicate.

On the inner surface of the elastic tissue are disposed a number of small racemose glands (Meibomian), whose ducts open near the free edge of the lid.

The orbital surface of each lid is lined by a delicate, highly sensitive mucous membrane (conjunctiva), which is continuous with the skin at the free edge of each lid, and after lining the inner surface of the eyelid is reflected on to the eyeball, being somewhat loosely adherent to the sclerotic coat. The epithelial layer is continued over the cornea at its anterior epithelium. At the inner edge of the eve the conjunctira becomes continuous with the mucous lining of the lachrymal sac and duct, which again is continuous with the mucous membrane of the inferior meatus of the nose.

The lachrymal gland, composed of several lobules made up of acini resembling the serous salivary glands, is lodged in the upper and outer angle of the orbit. Its secretion, which issues from several ducts on the inner surface of the upper lid, under ordinary circumstances just suffices to keep the conjunctiva moist. It passes out through two small openings (puncta lachrymalia) near the inner angle of the eye, one in each lid, into the lachrymal sac, and thence along the nasal duct into the inferior meatus of the nose. The excessive secretions poured out under the influence of any irritating vapor or painful emotion overflows the lower lid in the form of tears.

The eyelids are closed by the contraction of a sphincter muscle (orticularis), supplied by the facial nerve; the upper lid is raised by the levator palpebrce superioris, which is supplied by the third nerve.

\section{The Eyeball.}

The eyeball or the organ of vision (fig. 406) consists of a variety of structures which may be thus enumerated:-

The sclerotic, or outermost coat, envelops about fire-sixths of the eyeball: continuous with it, in front, and occupying the remaining sixth, is the cornea. Immediately within the sclerotic is the choroid coat, and within the choroid is the retina. The interior of the eyeball is well-nigh filled by the aqueous and vitreous humors and the crystalline lens; but, also, there is suspended in the interior a contractile and perforated curtain,-the iris, for regulating the admission of light, and behind at the junction of the sclerotic and cornea is the ciliary muscle, the function of which is to adapt the eye for seeing objects at various distances.

Structure of the Sclerotic Cout.-The sclerotic coat is composed of 
white fibrous tissue, with some elastic fibres near the inner surface, arranged in variously disposed and interlacing layers. Many of the bundles of fibres cross the others almost at right angles. It is strong,

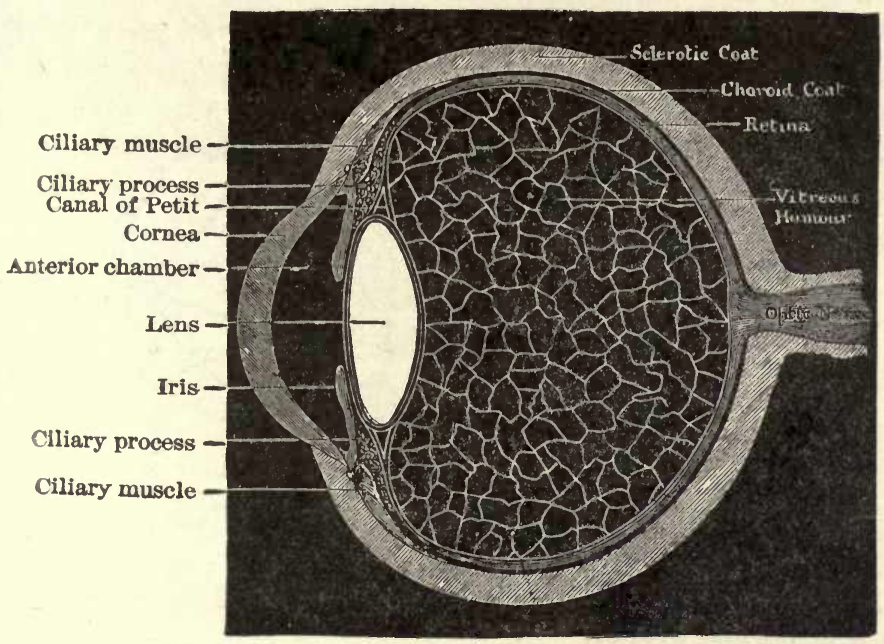

Fig. 406. -Seciton of the anterior four-fifths of the eyeball.

tough, and opaque, and not very elastic. It is separated from the choroid by a considerable lymphatic space (perichoroidal), and this is in connection with smaller spaces lined with endothelium in the sclerotic coat itself. There is a lymphatic space also outside the sclerotic separating it from a loose investment of connective tissue called the capsule of Tenon. The innermost layer is made up of loose connective tissue and pigment-cells, and is called the lamina fusca.

Structure of the Cornea. - The cornea is a transparent membrane which forms a segment of a smaller sphere than the rest of the eyeball,
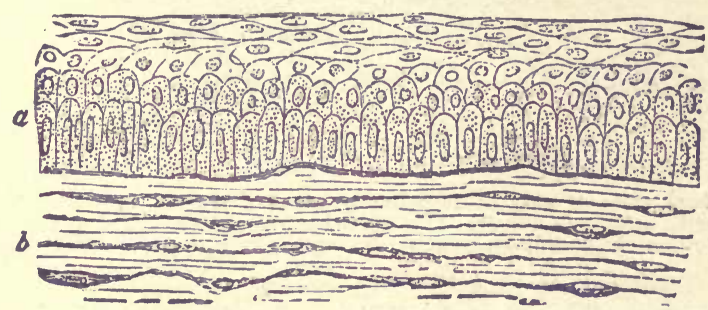

Fig. 407.-Vertical section of rabbit's cornea. a, Anterior epithelium, showing the different shapes of the cells at various depths from the free surface; $b$, portion of the substance of cornea. (Klein.)

and is let in, as it were, into the sclerotic with which it is continuous all round. It is covered by laminated epithelium ( $a$, fig. $40 \%)$, consist- 
ing of seven or eight layers of cells, of which the superficial ones are flattened and scaly, and the deeper ones more or less columnar. Immediately beneath this is the anterior elastic lamina of Bowman, which

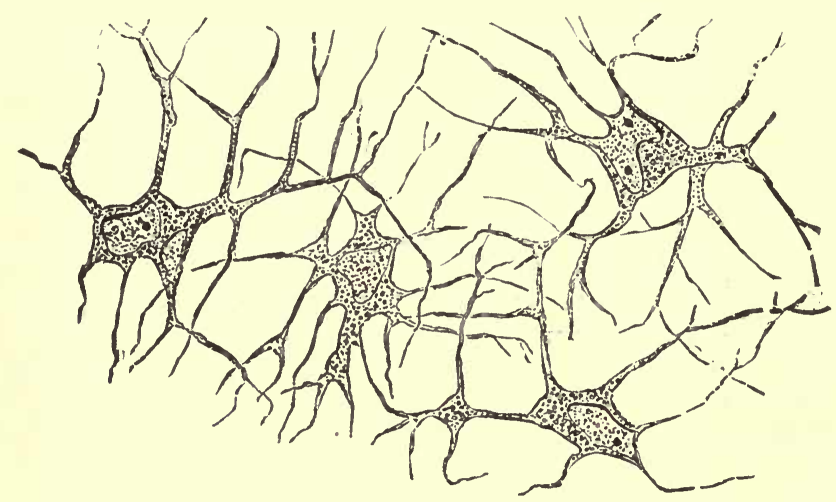

Fig. 408. - Horizontal preparation of cornea of frog; showing the network of branched mrneacorpuscles. The ground substance is completely colorless. $\times 400$. (Klein.)

differs, only in being more condensed tissue, from the general structure of the cornea or cornea proper.

This latter tissue, as well as its epithelium is, in the adult, completely destitute of blood-vessels; it consists of an intercellular groundsubstance of rather obscurely fibrillated flattened bundles of connective tissue, arranged parallel to the free surface, and forming the boundaries of branched anastomosing spaces in which the cornea-corpuscles lie. These branched cornea-corpuscles have been seen to creep by amøboid

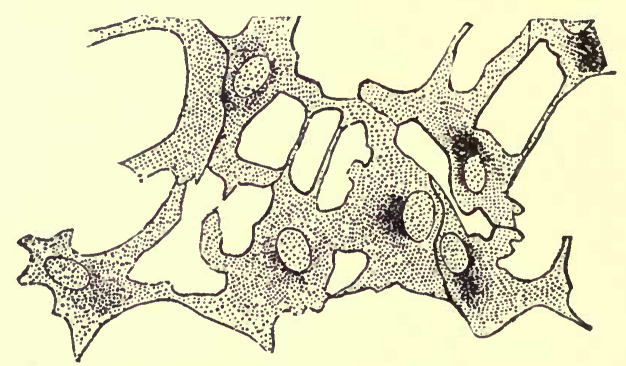

Fig, 409. - Surface view of part of lamella of kitten's cornea, prepared first with caustic potash and then with nitrate of silver. (By this method the branched cornea-corpuscles with their granular protoplasm and large oval nuclei are brought out.) $\times 450$. (Klein and Noble Smith.)

movement from one branched space into another. At its posterior surface the cornea is limited by the posterior elastic lamina, or membrane of Descemet, similar in structure to the anterior elastic lamina, the inner layer of which consists of a single stratum of epithelial cells (fig. $410, d$ ). Nerves. - The nerves of the cornea are both large and numerous: they 
are derived from the ciliary nerves. They traverse the substance of the cornea, in which some of them near the anterior surface break up into axis cylinders, and their primitive fibrillæ. The latter form a plexus immediately beneath the epithelium, from which delicate fibrils pass up between the cells anastomosing with horizontal branches, and forming a deep intra-epithelial plexus, from which still finer fibres ascend, till near the surface they form a superficial intra-epithelial net-work. Most of the primitive fibrillæ have a beaded or varicose appearance. The cornea

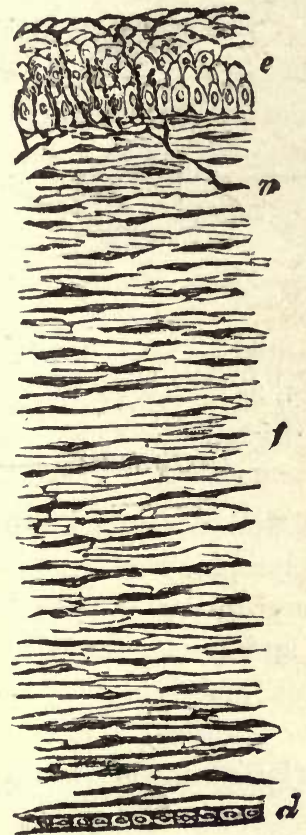

Fig. 410 .

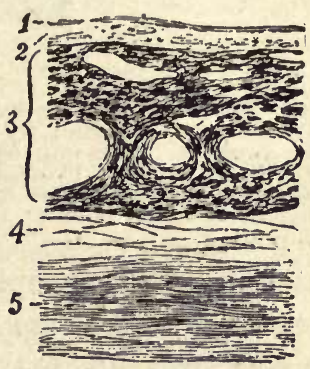

Fig. 411.

Fig. 410.-Vertical section of rabbit's cornea, stained with gold chloride. $e$, Laminated anterior epithellum. Immediately beneath this is the anterior elastic lamina of Bowman. $n$, Nerves forming a delicate sub-epithelial plexus, and sending up fine twigs between the epithelial cells to end in a second plexus on the free surface; $d$, Descemet's membrane, consisting of a fine elastic layer, and a single layer of epithelial cells; the substance of the cornea, $f$, is seen to be fibrillated, and contains many layers of branched corpuscles, arranged parallel to the free surface, and here seen edgewise. (Schofield.)

Fig. 411. - Section through the choroid coat of the human eye. 1, elastic membrane, structureless or finely fibrillated; 2, chorio-capillaris or tunica Ruyschiana; 3, Proper substance of the choroid with large vessels cut through; 4, suprachoroidea; 5, sclerotic. (Schwalbe.)

has no blood-vessels penetrating its structure, nor yet lymphatic vessels proper. It is nourished by the circulation of lymph in the spaces in which the cornea corpuscles lie. These communicate-freely and form a lymph-canalicular system.

Structure of the Choroid Coat (tunica vasculosa). -This coat is atteched to the inner layer of the sclerotic in front at the corneo-scleral junction and behind at the entrance of the optic nerve, elsewhere it is 
connected to it only by loose connective tissue. Its external coat is formed chiefly of elastic fibres and large pigment corpuscles loosely arranged and containing lymphatic spaces lined with endothelium. This is the suprachoroidea. More internally is a layer of arteries and veins arranged in a system of venous whorls, together with elastic fibres and

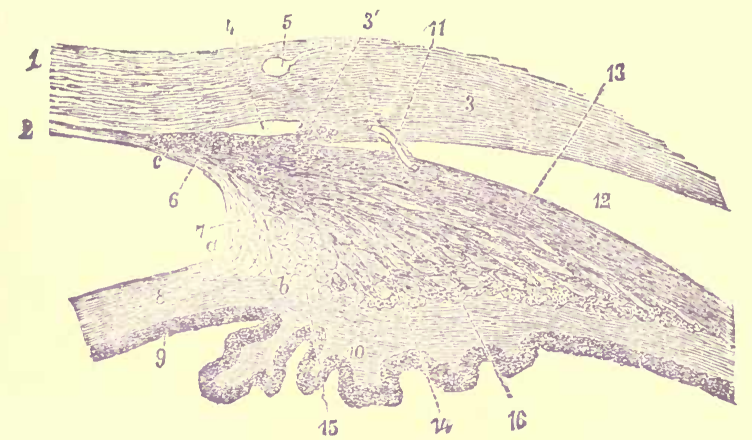

Fig. 412. - Section through the eye carried through the c1liary processes. 1 , Cornea; 2 , membrane of Descemet; 3 , sclerotic; 3 , corneo-scleral junction; 4 , canal of Schlemm; 5 , vein; 6 , nucleated network on inner wall of canal of Schlemm; 7 , lig. pectinatum iridis, $a b c ; 8$, iris stroma; 9, pigment of iris: 10, ciliary processes; 11, ciliary muscle; 12 , choroid tissue; 13 . meridional and 14, radiating fibres of ciliary muscle; 15, ring muscle of Müller; 16, circular or angular bundles of ciliary muscle. (Schwalbe.)

pigment cells. The lymphatics, too, are well dereloped around the blood-ressels, and there are besides distinct lymph spaces lined with endothelium. Internally to this is a layer of fine capillaries, very dense and derived from the arteries of the onter coat and ending in veins in that coat. It contains corpuscles without pigment, and lymph spaces which surround the blood-ressels. (membrana chorio-capillaris). It is separated from the retina by a fine elastic membrane (membrane of Bruch), which is either structureless or finely fibrillated.

The choroid coat ends in front in what are called the ciliary processes (fig. 412). These consist of from 70 to 80 meridionally arranged radiating plaits, which consist of blood-vessels, fibrous connective tissue, and pigment corpuscles. They are lined by a continuation of the membrane of Bruch. The ciliary processes terminate abruptly at the margin of the lens. The ciliary muscle (13, 14 and 15, fig. 412), which may be considered to form part of the processes, is situated between the sclerotic (at the corneo-scleral junction) and the folds of the ciliary processes. It is a ring of muscle, $3 \mathrm{~mm}$. broad and $8 \mathrm{~mm}$. thick, made up of fibres running in two or three directions. (a) Meridional fibres near the sclerotic and passing to the choroid; $(b)$ radial fibres, passing toward the centre; and $(c)$ circular fibres, more internal, and constituting the so-called ciliary sphincter.

The Iris. - The iris is a continuation of the choroid inward beyond 
the ciliary processes. It is a fibro-muscular membrane perforated by a central aperture, the pupil. It is made up chiefly of blood-vessels and connective tissue with pigment and unstriated muscle.

Posteriorly are two layers of pigment cells (uvea), in which are represented the two layers of cells of which the optic vesicle is originally formed, and behind which are the retina proper and its pigment layer. In the iris representatives of both layers are deeply pigmented. The structure of the iris proper is made of connective tissue in front with corpuscles which may or may not be pigmented, and behind of similar tissue supporting blood-vessels inclosed in connective tissue. The pigment cells are usually well developed here, as are also many nerve-fibres radiating toward the pupil. Surrounding the pupil is a layer of circular unstriped muscle, the sphincter pupilloe. In some animals there are also muscle-fibres which radiate from the sphincter in the substance of the iris forming the dilator pupilla. The iris is covered anteriorly by a layer of endothelium continued upon it from the posterior surface of the cornea; posteriorly there is a very fine layer which is a continuation of the membrana limitans interna of the retina.

The Lens. - The lens is situated behind the iris, being inclosed in a distinct capsule, the posterior surface of which is less thick than the anterior. It is supported in place by the suspensory ligament, fused to the anterior surface of the capsule. The suspensory ligament is derived from the hyaloid membrane, which incloses the vitreous humor.

Structure. - The lens is made up of a series of concentric laminæ (fig. 414), which when it has been hardened, can be peeled off like the

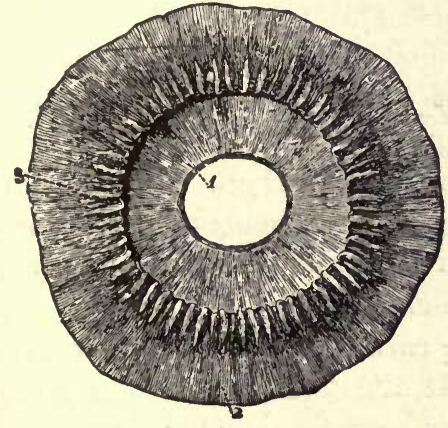

Fig. 413.

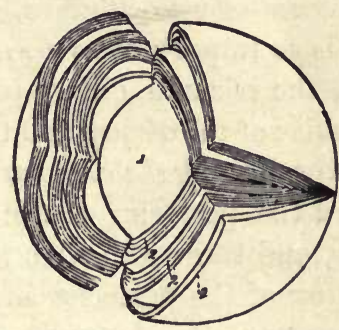

Fig. 414.

Fig. 413.-Ciliary processes, as seen from behind. 1, posterior surface of the iris, with the sphincter muscle of the pupil; 2 , anterior part of the choroid coat; 3 , one of the ciliary processes, of which about seventy are represented. $1 / 2$.

Fig. 414.- Laminated structure of the crystalline lens. The laminæ are split up after hardening in alcohol. 1, the denser central part or nucleus; 2 , the successive external layers. $\times 4$. (Arnold.)

leaves of an onion. The laminæ consist of long ribbon-shaped fibres, which in the course of development have originated from cells. 
The lens itself is made up of transparent longitudinal fibres, hexagonal and prismatic, thickened posteriorly. Those fibres at the cortex have nuclei and are smooth, those near the centre are without nuclei and have serrated edges. The fibres are united together by a scanty amount of cement substance.

The arrangement is such that no fibres run the whole half of the lens, from front to back, since, if a fibre starts near the anterior pole, its other end is far from the posterior pole (fig. 415.)

The epithelium of the lens consists of a layer of cubical cells anteriorly, which merges at the equator into the lens fibres. The development of the lens explains this transition. The lens at first consists of a closed sac composed of a single layer of epithelium. The cells of the posterior part soon elongate forward and obliterate the cavity, the anterior cells do

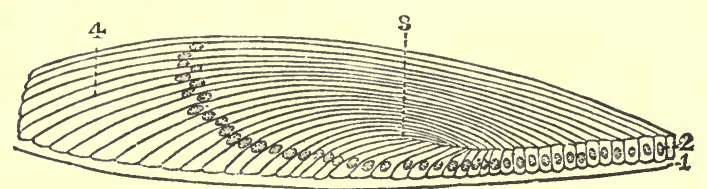

Fig. 415.-Meridional section through the lens of a rabbit. 1, Lens capsule; 2 , epithelium of lens; 3 , transition of the epithelium into the fibres: 4 , lens fibres. (Bubuchin.)

not grow, but at the edge they become contiuuous with the posterior cells, which are gradually developed into fibres. The lens contains globulin or crystallin, but no native-albumin; it also contains cholesterin. The capsule is a homogeneous transparent elastic membrane. The hardest portion of the lens is that which is most internal. It forms the so-called nucleus of the lens (fig. 414, 1).

Corneo-scleral junction. - At this junction the relation of parts (fig. 412 ) is so important as to need a short description. In the neighborhood, the iris and ciliary processes join with the cornea. The proper substance of the cornea and the posterior elastic lamina become continuous with the iris, at the angle of the iris, and the iris sends forward processes toward the posterior elastic lamina, forming the ligamentum pectinatum iridis, and these join with fibres of the elastic lamina. The endothelial covering of the posterior surface of the cornea is, as we have seen, continuous over the front of the iris. At the iridic angle, the compact inner substance of the cornea is looser, and between the bundles are lymph spaces filled with fluid, called the spaces of Fontana. They are little developed in the human cornea. Where the cornea and scleratic join, there is an intermediate part which resembles both, but which is still not transparent, as the internal part remains scleral in structure.

The spaces which are present in the broken up bundles of corneal tissue at the angle of the iris, are continuous with the larger lymphatic 
space of the anterior chamber. Above the angle at the corneo-scleral junction is a canal, which is called the canal of Schlemm. It is a lymphatic channel, but appears to be in communication with blood-vessels, as it may be under certain circumstances filled with blood.

Structure of the Retina. - The retina (fig. 416) is a delicate membrane, concave with the concavity directed forward and apparently ending in front, near the outer part of the ciliary processes, in a finely notched edge, - the ora serrata, but really represented to the very margin of the pupil. Semitransparent when fresh, it soon becomes clouded and opaque, with a pinkish tint from the blood in its minute vessels. It results from the sudden spreading out or expansion of the optic nerve, of whose ter-

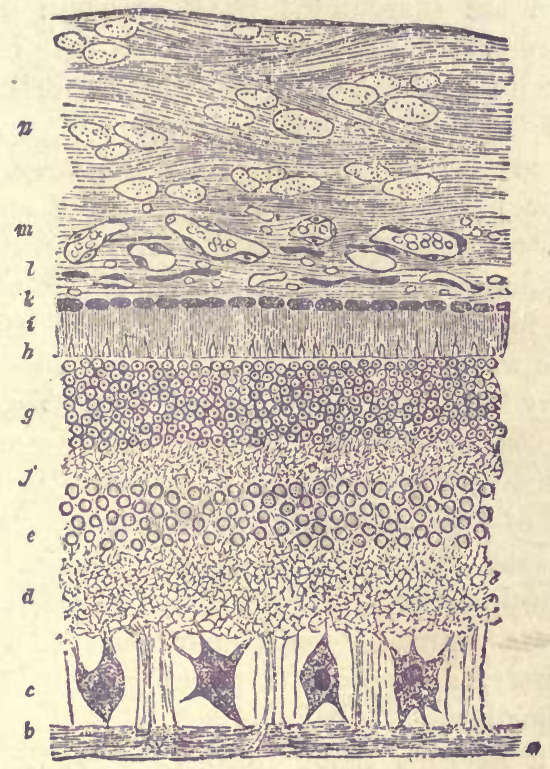

Fig. 416. A section of the retina, choroid, and part of the sclerotic, moderately magnified. $a$, Membrana limitaus interna; $b$, nerve-fibre layer traversed by Muller's sustentacular fibres; $c$, ganglion-cell layer; $d$, molecular layer; $e$, internal nuclear layer; $f$, internuclear layer; $g$, external nuclear layer; $h$, membrana limitans externa, running along the lower part of $i$, the layer of rods and cones; $k$. pigment-cell layer; $l m$, internal and external vascular portions of the choroid, the first containing capillaries, the second larger blood-vessels, cut in transverse section; $n$, sclerotic. (W. Pye.)

minal fibres, apparently deprived of their external white substance, together with nerve cells, it is essentially composed.

Exactly in the centre of the retina is a round yellowish elevated spot, about $\frac{1}{24}$ of an inch $(1 \mathrm{~mm}$.) in diameter, having a minute depression in the centre, called after its discoverer the macula lutea, or yellow spot of Scemmering. The minute depression in its centre is called the fovea centralis. About $\frac{1}{10}$ of an inch $(2.5 \mathrm{~mm}$.) to the inner side of the yel- 
low spot, is the point at which the optic nerve enters the eyeball, and begins to spread out its fibres into the retina.

'The optic nerve passes forward from the ventral surface of the cerebrum toward the orbit inclosed in prolongations of the membranes, the dura mater, arachnoid and pia mater, which cover the brain. The external sheath at the entrance of the nerve into the eyeball becomes continuous with the sclerotic, which at this part is perforated by holes to allow of passage of the optic nerve-fibres and the pia mater with the choroid, the perforated part being the lamina cribrost. The pia mater here becomes incomplete, and the subarachnoid and the superarachnoid spaces become continuous. 'The pia mater sends in processes into the nerve to support the fibres. The fibres of the nerve themselves are exceedingly fine, and are surrounded by the myelin sheath, but do not possess the ordinary external nerve-sheath. As they pass into the retina they lose their myeliu sheaths and proceed as axis-cylinders. Neuroglia supports the nerve-fibres in the optic nerve-trunk. In the centre of the nerve is a small artery, the arteria centralis retince. The number of fibres in the optic nerve is said to be upward of 500,000. The axiscylinders pass on to the retina, turning over the edges of the porus opticus, to be distributed on the inner surface of the retina, as far as the ora serrata, as a layer of optic nerve-fibres, and separated from the hyaloid membrane which contains the vitreous humor to be presently described, by a very thin layer, the membrana limitans interna.

T'he retina consists of eertain nervous elements arranged in several layers and supported by a very delicate connective tissne.

The researches of Cajal upon the structure of the retina of rertebrates has shown that this membrane is a much simpler structure than has heretofore been described. Cajal's observations being confirmed by other observers and accepted by neuro-anatomists, it will be safe to give the descriptions here, as representing our present knowledge of the structure of this membrane.

The retiua is a nervous tissue formed essentially of three layers of nerve-cells. From without inward they are: the layer of visual cells, the layer of bipolar cells, and the layer of ganglionic cells. This subdivision is shown in the diagram (fig. 41\%). These different layers may be subdivided so as to give the following layers from without inward:

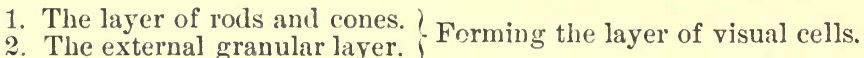

3. The external molecular layer.
4. Internal granular layer.

5. Internal molecular layer.

6. Ganglionic layer, with the fibres of the optic nerve. $\left\{\begin{array}{c}\text { ganglion cells. } \\ \text { ganing }\end{array}\right.$

The layer of visual cells is subdivided, as seen in the figure, into that of the rods and cones externally and that of the external granular inter. 
nally. This is, however, practically a layer made up simply of bipolar nerve-cells with prolongations more or less long which run to the external surface of the retina and there form a series of bodies known as the rods and cones.

1. The rods and cones are really a kind of secretion from the protoplasm of the bipolar cell beneath, and are not distinct nerve-cells. They consist of bodies more or less alike, which extend up through the exterual limiting membrane from the cells beneath.

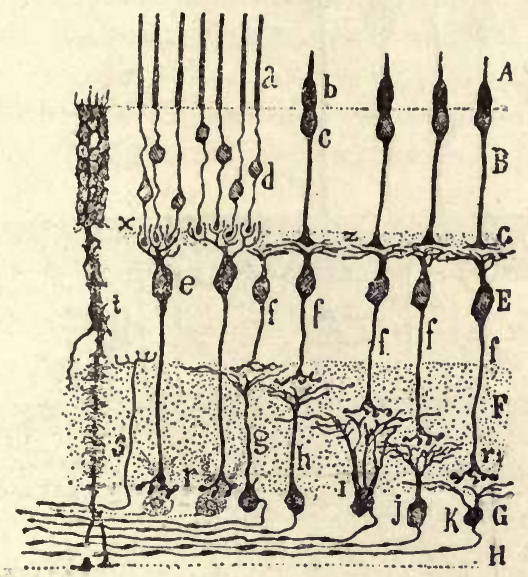

Fig. 417.-Transverse section of a mammalian retina. $A$, Layer of rods and cones; $B$, bodies of visual cells (external granular); $C$, external molecular layer; $E$, layer of blpolar cells (internal granular); $F$, internal molecular layer; $G$, layer of ganglionic cells; $H$, layer of optic-nerve fibres; $a$, rod; $b$, cone; $c$, body of the cone celi; $d$, body of the rod cell; $e$, bipolar rod cells; $f$, bipolar cone cells; $g, h, i, j, k$, ganglionic cells ramifylng in the various strata of the internal molecular zone; $r$, inferior arborization of the bipolar rod cells, connecting with the ganglionic cells; $r_{1}$ inferior arborization of the bipolar cone cells; $t$, epithelial or Muller cells; $x$, point of contact between the rods and their bipolar cells; $z$, point of contact between the cones and their bipolar cells; $s$, centrifugal nerve-fibre. (Cajal.)

The Rods. - Each rod (fig. $41 \%, a$ ) is made up of two parts, very different in structure, called the outer and inner limbs. The outer limb of the rods is about $30 \mu \frac{1}{000}$ inch long and $2 \mu$ broad, is transparent, and doubly refractive. It is said to be made up of fine superimposed discs. It resembles in some ways the myelin sheath of a medullated nerve. It swells up on exposure to light, and is part of the layer in which the pigment called visual purple is found. The inner limb is about as long but slightly broader than the outer, is longitudinally striated at its outer and granular at its inner part. Each rod is connected by a fine hair-like process to a nerve-cell in the external granular layer below (figs. $41 \%, d ; 41 \% \mathrm{~A}, 2)$.

The Cones.-Each cone (fig. $41 \%, c$ ), like the rods, is made up of two limbs, outer and inner. The outer limb is tapering and not cylindrical like the corresponding part of the rod, and about one-third only of its 
length, but it resembles this in structmre. 'There is, however, no visua' purple found in the cone. The inner limb of the cone is broader in the centre; each cone is in connection by its internal end with a cone fibre, which has much the same structure as the rod fibre, but is much stouter. This connects with a nerve-cell of the layer below (fig. $41 \% \mathrm{~A}, 4$ ).

In the rod and cone layer of birds, the cones usually predominate

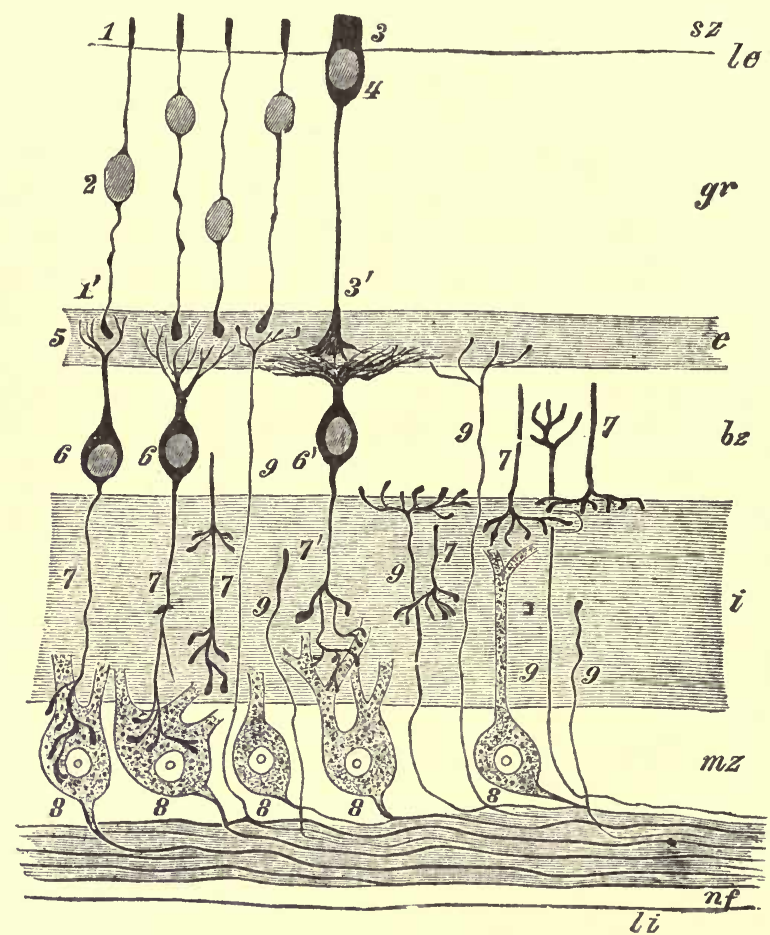

Fig. $417 \mathrm{~A}$. - Schematic diagram of the elementary structure of the retina. $s z$, Rods and cones; $\iota$, membrana limitans externa; $g r$, external granules; $e$, external molecular layer); $b z$, internal granular layer; $i$, internal molecular layer; $m z$, multipolar cell layer (ganglion optici); $n f$, nervefibre layer; $l i$, membrana limitans interna.

1. Rod; 2 , rod granule; 3 , cone; 4, cone granule; $1-1$ ', rod visual cell; $3-3$ ', cone visual cell; 5 , central termination of the visual cells and peripheral terminal arborization of the bipolar cells; 6,6 , two bipolar cells for rods; 6 , oue bipolar cell for cone; $7,7,7,7, \tau, 7$, the central processes of bipolar cells with the terminal arborizations situated in the various layers of the internal molecular layer; $\tau^{\prime}$, central process of a bipolar cell for cone; 8 , multipolar cells with their peripheral dendrites and central neuraxons; 9, 9, 9, nerve-fibres and terminal arborizations of remote cells.

largely in number, whereas in man the rods are by far the more numerous, except in the fovea centralis, where cones only are present, as is the case at the anterior part of the retina near the ora serrata. The number of cones has been estimated at 3,000,000. In nocturnal birds, however, such as the owl, only rods are present, and the same appears to be the case in many nocturnal and burrowing mammalia, e.g., bat, hedgehog, mouse, and mole. The rods are absent in reptiles.

External Limiting Membrane.-A delicate membrane lies beneath 
the rods and cones and separates them from the layer beneath. This is called the external limiting membrane (fig. $41 \% \mathrm{~A}, l e$ ).

2. External Cranular iayer.-The cells of the external granular layer are the bipolar or visual cells which contain the protoplasm not yet transformed into rods and cones in the layer above. The cells whose bodies are continued upward as cones are different in shape from those which are connected with the rods. The cells of the cones are situated close to the external limiting membrane. They have a large ovoid nucleus. From the inner side of the cell-body a process descends toward the external molecular layer where it ends in a slight dilatation (see fig. 417). On its outer side a process of the body ascends through into the external limiting membrane and swells into a cone (fig. $41 \%, c$ ).

The bipolar cells giving birth to the rods lie at deeper levels in the

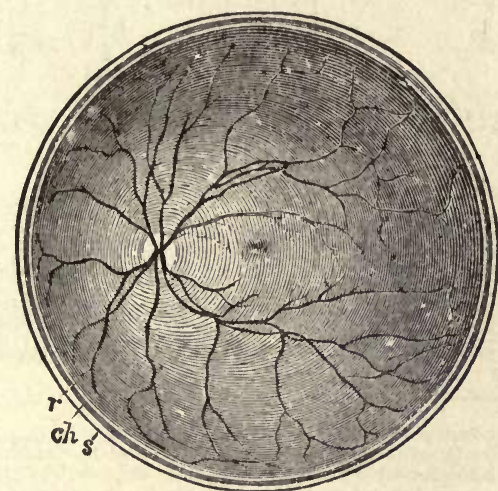

Fig. 418.-The posterior half of the retina of the left eye, vlewed from before; $s$, the cut edge of the sclerotic coat: $c h$, the choroid; $r$, the retina; in the interior at the middle the macula lutea with the depression of the fovea centralis is represented by a slight oval shade: toward the left side the light spot indicates the colliculus or eminence at the entrance of the optic nerve, from the centre of which the arteria centralis is seen spreading its branches into the retina, leaving the part occupied by the macula comparatively free. (After Henle.)

grantular layer. They contain an ovoid nucleus of a smaller volume than those of the cone cells. The protoplasm of the cell-body gives off two fibres, one ascending, and the other descending. The ascending fibre runs up through the limiting membrane and is continued as a rod. The descending fibre goes into the molecular layer and ends here in a small nodule. According to Cajal, these cells of the visual layer have no direct anatomical continuity with the cells of the bipolar layer below, though Dogiel and others have denied this.

3. The external molecular layer or external plexiform layer (fig. $41 \%, C$ ) is composed of numerous protoplasmic processes (dendrites) which come from the cells of the internal granular layer below and from the visual cells above. Some subdivisions of this layer are made, there being an outer part in which the rod cells meet the branching fibres of the bi- 
polar layer, and a slightly deeper layer in which the cone cells come in contact with the dendrites of the bipolar cells.

4. The internal granular layer (fig. $41 \%, E$ ) is an inner subdivision of a layer of bipolar cells, and is the most complicated of any of the layers of the retina. It is made up, however, mainly of bipolar cells, which are fusiform in shape, vertical in arrangement, and have two processes, one ascending and the other descending. 'The descending fibre is always single and ends at different levels in the internal molecular or plexiform layer, where it forms flattened and brush-like expansions. The ascending process is often multiple, and it ends in a large number of different branches, which arrange themselves in something like a horizontal layer in the lower part of the external molecular layer.

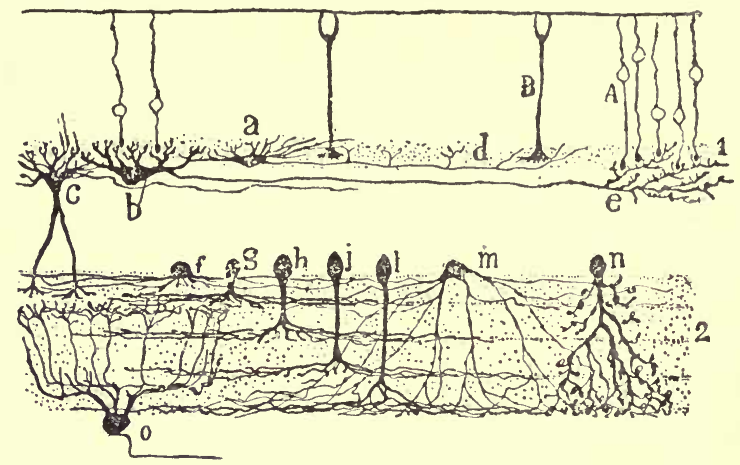

Fig. 418 A.-Perpendicular section of the retina of a mammal. A, External grains or bodies of rods; $B$, bodies of cones; $a$, horizontal external or small cell: $b$, horizontal int+1nal or large cell; $c$, horizontal internal cell with descending protoplasmic appendages; $e$, flattented arborization of one of the large cells; $f, a, h, j, l$, spongioblasts ramifying in the various st rata of the internal molecular zone; $m, n$, diffuse spougioblasts; $o$, ganglionic cell; 1 , external molecular zone; 2 , internal molecular zone. (Cajal.)

Besides these vertical bipolar cells there are flattened star-shaped cells lying just beneath the external molecular layer, sending out branches parallel to the periphery and ending in numerous ramifying expansions which come in contact with the different descending branches of the cone cells. Their general arrangement is horizontal. These little cells appear to have as their function the connecting of the visual cells with each other (fig. $418 \AA, c, b$ ). There are other horizontal cells, larger than these, but having practically the same shape and arrangement, and lying somewhat more deeply in the layer; these connect the processes of the rod cells with each other and have thus an associative function. There is, in addition, in this layer, a series of larger cells, called by Cajal spongioblasts, which lie deep in the internal granular layer, and whose branches take a horizontal direction and appear to have the function of associating the cells of the ganglionic layer below (see fig. 418 A).

5. The internal molecular layer is composed of a plexus of fibres 
formed by the processes of the bipolar cells from above and of the ganglionic cells below, and of fibres from the spongioblasts.

6. The most internal of the nervous layers is a layer of ganglionic cells, consisting of large multipolar verve-cells, with large round nuclei. In some parts of the retina, especially near the macula lutea, this layer is very thick and consists of several distinct strata of nerve-cells. These cells lie in the spaces of the connective-tissue framework. They are arranged with their single neuraxon or axis-cylinder processes directed inward. These pass into and are continuous with the layer of optic fibres. Externally the cells send up numerous branching processes or dendrites which interlace with the fibres of the bipolar cells and the horizontal processes of the spongioblasts.

All the elements of the retina are sustained and isolated by large cells lying vertically which are known as the fibres of Müller, or epithelial retinal cells. Like the corresponding cells of the olfactory mucous membrane, these fibres have upon their sides an infinite number of facets which serve as receptacles to the nerve-corpuscles and fibres of the retina. The nucleus of the fibre of Müller is found at the level of the internal granular layer, and the two extremities of the protoplasm or cell-body are condensed in two homogeneous layers, known as the external and internal limiting layer. The external limiting layer is placed, as already described, just between the layer of rods and cones and that of the visual cells. The other is situated upon the internal surface of the retina. The fibres of Müller are completely independent of each other, having between themselves and the nerve elements only the relation of contact. It is believed that their function is that of supporting the nerve-tissues and also isolating them.

It will be seen now that the retina is composed essentially of three layers of vertical cells, whose processes have a vertical direction, and which are connected with each other by contact of these processes; that there are also two other sets of cells which form horizontal layers of nerve-processes, these being in the inner and outer parts of the internal granular layer. There are, therefore, strictly speaking, five layers of nerve-cells, three vertical and two horizontal. Two other layers are made up by the modification of the protoplasm of the fibres of Müller and are purely mechanical in function. They are the external and internal limiting layers.

Pigment-cell layer, which was formerly considered part of the choroid, consists of cells which cover and entirely surround the outer limbs of the rods and cones.

The further subdivisions of the retina are more for purposes of fine anatomy than of functional importance.

Differences in Structure of Different Parts. - Toward the centre of the macula lutea all the layers of the retina become greatly thiuned out and 
almost disappear, except the rod and cone layer, which considerably increases in thickness but at the fovea centralis comes to consist almost entirely of long slender cones and cone-fibres, which curve toward the periphery. They are supported by neuroglia, which is also found internally as a thin layer, the rods being absent. There are capillaries here, but none of the larger branches of the retinal arteries.

Toward the edge of the macula lutea, not only are all the layers present, but the ganglionic layer consists of many strata of cells ( 7 or 8 ), and with this increase there is also an increase in the thickness of the inner granular layer. The cells are generally bipolar. Toward the centre the layers diminish in this order: optic nerve-fibres, ganglionic layer, inner molecular layer, and inner granular layer. The rods grow scanty and then are absent.

At the ora serrata the layers are not perfect and disappear in this order: nerve-fibres and ganglion cells, then the rods, leaving only the inner limbs of the cones, these cease, then the inner molecular layer. The Müllerian fibres persist.

At the pars-ciliaris retinæ, the retina is represented by a layer of columnar cells, derived from the fusion of the nuclear layers. The cells are covered by the membrana limitans interna, and externally are in contact with the pigment layers of the retina, which is continued over the ciliary processes.

The chambers of the eye.-The anterior chamber is the space behind the cornea and in front of the lens. It is filled with aqueous humor, which is essentially a diluted lymph with a small amount of proteid in it, viz., of fibrinogen, serum-globulin, and septum-albumin. It is seldom spontaneously coagulable. It contains salts, chiefly sodium chloride, sometimes a substance which reduces copper sulphate, but is not sugar, and a trace of urea and sarcolactic acid. There are no formed elements in the fluid. It is stated that the aqueous humor is secreted by glands in the ciliary region, but the cavity is itself obviously a lymph sac.

The posterior chamber, or that behind the lens, contains the vitreous humor, which is a semifluid substance contained in the meshes of an indistinct connective tissue. It is inclosed in a distinct membrane called membrana hyaloidea, from the anterior surface of this membrane at the ora serrata fibres pass off to the back of the lens capsule, forming an incomplete canal, called the Canal of Petit, the membrane itself being the Zonule of Zinn. The hyaloid membrane separates the vitreous from the retina.

Blood-vessels of the Eyeball.-The eye is very richly supplied with blood-vessels. In addition to the conjunctival vessels which are derived from the palpebral and lachrymal arteries, there are at least two 
other distinct sets of vessels supplying the tunics of the eyeball. (1) The vessels of the sclerotic, choroid, and iris, and (2) the vessels of the retina.

(1.) These are the short and long posterior ciliary arteries which pierce the sclerotic in the posterior half of the eyeball, and the anterior ciliary which enter near the insertions of the recti. These vessels anastomose and form a very rich choroidal plexus; they also supply the iris and

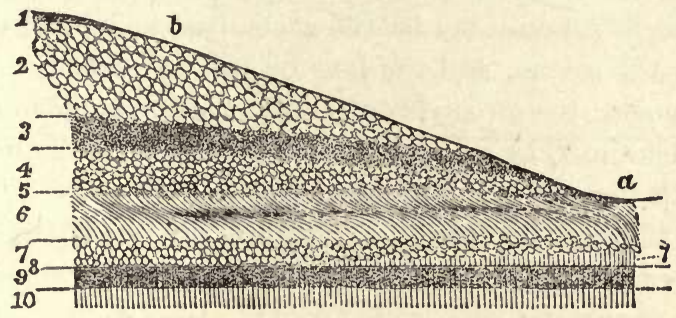

Fig. 419.--Section through the macula lutea and fovea centralis of human retina. $a$, fovea; $b$, descent of the macula toward fovea. The numbers indicate the layers of the retina.' (Kuhnt.)

ciliary processes, forming a very highly vascular circle round the outer margin of the iris and adjoining portion of the sclerotic.

The distinctness of these vessels from those of the conjunctiva is well seen in the difference between the bright red of blood-shot eyes (conjunctival congestion), and the pink zone surrounding the cornea which indicates deep seated ciliary congestion.

(2.) The retinal vessels (fig. 418) are derived from the arteria centralis retince, which enters the eyeball along the centre of the optic nerve. They ramify all over the retina, chicfly in its inner layers. They can be seen by direct ophthalmoscopic examination.

\section{The Optical Apparatus.}

The optical apparatus may be supposed, for the sake of description, to consist of several parts. Firstly, of a system of transparent refracting surfaces and media by means of which images of external objects are brought to a focus upon the back of the eye; and secondly, of a sensitive screen, the retina, which is a specialized termination of the optic nerve, capable of being stimulated by luminous objects, and of sending through the optic nerve, such an impression as to produce in the brain visual sensations. To these main parts may be added, thirdly, an apparatus for focussing objects at different distances from the eye, called accommodation. Even this does not complete the description of the whole organ of vision, since both eyes are usually employed in vision, and fourthly, an arrangement exists by means of which the eyes may be turned in the same direction by a system of muscles, so that binocular vision is possible. 
The arrangement of the optic nerve-fibres, and of the continuation of these backward in the optic chiasma, and thence to special districts of the brain, have already been discussed.

The eye may be compared to a photographic camera, and the transparent media corresponds to the photographic lens. In such a camera images of external objects are thrown upon a ground-glass screen at the back of a box, the interior of which is painted black. In the eye, the camera proper is represented by the eyeball with its choroidal pigment, the screen by the retina, and the lens by the refracting media. In the case of the camera, the screen is enabled to receive clear images of objects at different distances, by an apparatus for focussing. The corresponding contrivance in the eye is the accommodation.

The iris, which is capable of allowing more or less light to pass into the eye, corresponds with the different sized diaphragms used in the protographic apparatus.

Refractive media and surfaces. - At first sight it would seem as if the refracting apparatus of the eye were very complicated, seeing that it consists of so many parts. These parts are: the anterior surface of the cornea itself, the posterior surface of the cornea, the aqueous humor, the anterior surface of the lens, the substance of the lens itself (which is also unequally refractive), the posterior surface of the lens, and the vitreous humor. Thus there are four surfuces, and at least including the air, five media. For all practical purposes, however, these may be resolved into a somewhat simpler form, and the cornea may be considered as one surface, the anterior, and one medium; the aqueous and vitreous humors as one medium; the lens, as two surfaces and one medium. It will be as well to consider the laws which govern the refraction of light under such circumstances.

In its simplest form, we may consider the refraction through a simple transparent spherical surface, separating two media of different density.

The rays of light which fall upon the surface exactly perpendicularly do not suffer refraction, but pass through, cutting the optic axis (O A, fig. 420), a line which passes exactly through the centre of the surface, at a certain point, the nodal point (fig. $420, \mathrm{~N}$ ), or centre of curvature. Any rays which do not so strike the curved surface are refracted toward the optical axis. Rays which impinge upon the spherical surface parallel to the optical axis, will meet at a point behind, upon the said axis which is called the chief posterior focus (fig. $420, \mathrm{~F}_{1}$ ); and again there is a point in the optical axis in front of the surface, rays of light from which so strike the surface that they are refracted in a line parallel with the axis $d f^{\prime \prime}$; such a point (fig. $420, \mathrm{~F}_{2}$ ) is called the chief anterior focus. The optic axis cuts the surface at what is called the principal point. 
It is quite obvious that the eye, even in the simplified form above indicated, is a much more complicated optical apparatus than the one described in the figure. It is, however, possible to reduce the refractive

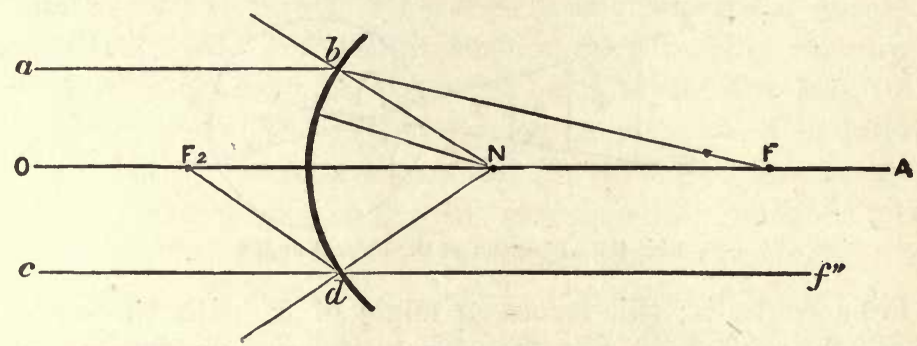

Fig. 420.-Diagram of a simple optical system (after M. Foster). The curved surface, $b, d$, is supposed to separate a less refractive medium toward the left from a more refractive medium toward the right.

surfaces and media to a simpler form when the refractive indices of the different media and the curvature of each surface are known. All of these data have been very carefully collected. They are as follows:-

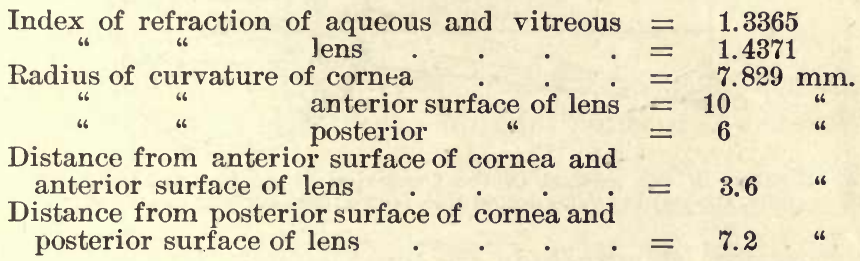

With these data, it has been found comparatively easy to reduce by calculation the different surfaces of different curvatures, into one mean curved surface of known curvature, and the differently refracting media into one mean medium the refractive power of which is known.

The simplest so-called schematic eye formed upon this principle, suggested by Listing as the reduced eye, has the following dimensions:-

From anterior surface of cornea to the principal point

From the nodal point to the posterior surface of lens

Anterior chief focus in front of cornea - : = 12.8237 "

Radius of curvature of ideal surface $: \vdots=5.1248$ "

In this reduced or simplified eye the principal posterior focus, about $23 \mathrm{~mm}$. behind the spherical surface, would correspond to the position of the retina behind anterior surface of cornea. The refracting surface would be situated about midway between the posterior surface of the cornea and the anterior surface of the lens.

The optical axis of the eye is a line drawn through the centres of 
curvature of the cornea and lens, prolonged backward to touch the retina between the porus opticus and fovea centralis, and this differs from the visual axis which passes through the nodal point of the reduced eye to

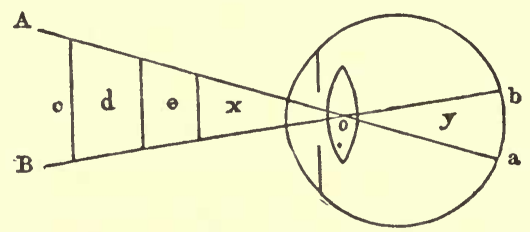

Fig. 421.-Diagram of the optical angle.

the fovea centralis; this forms an angle of $5^{\circ}$ with the optical axis. By some the optical axis and the visual axis are considered to be identical. The visual or optical angle is included between the lines drawn from the borders of any object to the nodial point; if the lines be pro-

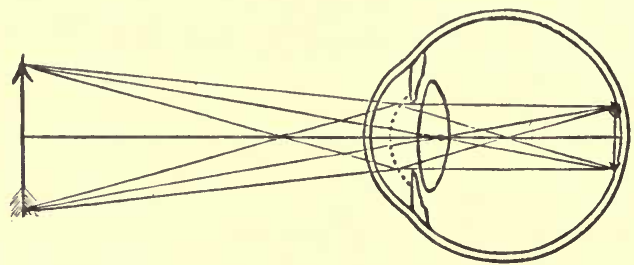

Fig. 421 4.-Diagram of the method of the formation of an inverted image exactly focussed upon the retina. The dotted line is the ideal surface of curvature.

longed backward they include an equal angle. It has been shown by Helmholtz that the smallest angular distance between two points which can be appreciated $=50$ seconds, the size of the retinal image being $3.65_{2}$; this practically corresponds to the diameter of the cones at the

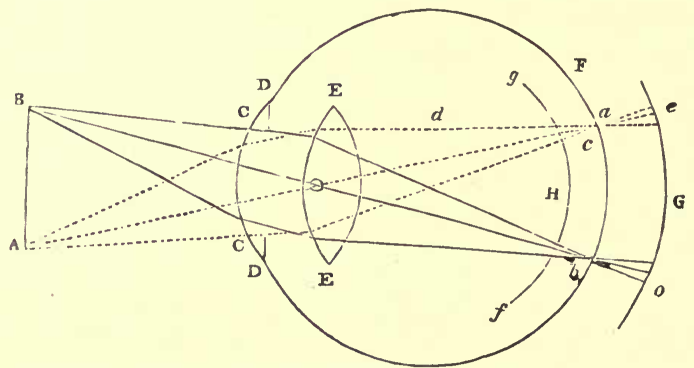

Fig. 422.-Diagram of the course of a ray of light, to show how a blurred or indistinct image is formed if the object be not exactly focussed upon retina. The surface $C \mathrm{C}$ should be supposed to represent the ideal curvature. The nodal point should be nearer the posterior surface of lens as in fig. $421 \mathrm{~A}$.

fovea centralis which $=3 \mu$, the distance between the centres of two adjacent cones being $=4 \%$.

The image of an object, then, is thus formed upon the retina. An 
object may be considered as a series of points, from each of which a pencil of light diverges to the eye, and this pencil has for its centre or axis, a ray which impinging upon the refractive surface perpendicularly to the surface is not refracted, but passes through the nodal point, and is prolonged backward to the retina, whereas the diverging rays are also made to converge to a principal posterior focus behind the lens, or the chief axis of the pencil of light proceeding from the point in question, and this focus, if the image is to be clear, should fall on the retina.

Thus from each point of an object a corresponding image is formed on the retina, so that an image of the distal object is produced. It is an inverted image. Whether the image is blurred or not depends upon the refractive power of the media, and upon the distance of the anterior surface of the cornea from the retina. If the refractive media are too powerful, or the eye too long, the image is formed in front of the retina (fig. 422); if the reverse, the image is formed behind the retina, and in both cases an indistinct and blurred image is the result.

\section{Accommodation.}

The distinctness of the image formed upon the retina, is mainly dependent on the rays emitted by each luminous point of the object being brought to a perfect focus upon the retina. If this focus occur at a point either in front of, or behind the retina, indistinctness of vision ensues, in the way we have already described, with the production of a halo. The focal distance, i.e., the distance from a lens of the point at which the luminous rays are collected, besides being regulated by the degree of convexity and density of the lens, varies with the distance of the object from the lens, being greater as this is shorter, and vice versa. Hence, since objects placed at various distances from the eye can within a certain range, different in different persons, be seen with almost equal distinctness, there must be some provision by which the eye is enabled to adapt itself, so that whatever length the focal distance may be, the focal point may always fall exactly upon the retina.

This power of accommodation, or the adaptation of the eye to vision at different distances, has received the most varied explanations. It is obvious that the effect might be produced in either of two ways, viz., (a) by altering the convexity, and thus the refracting power, either of the cornea or of the lens; or (b) by changing the position either of the retina or of the lens, so that whether the object be near or distant, the focal points to which the rays are converged by the lens may always fall exactly on the retina. The amount of either of these changes, which would be required in even the widest range of vision, would be extremely small. For, from the refractive powers of the media of the eye, the dif- 
ference between the focal distances of the images of an object at a distance, and of one at the distance of four inches, is only about 0.143 of an inch $(3.5 \mathrm{~mm}$.). On this calculation the change in the distance of the retina from the lens required for vision at all distances, supposing the cornea and lens to remain the same, would not be more than about one line.

The adaptation of the eye for objects at different distances is primarily due to a varying shape of the lens, its front surface becoming more or less convex, according as the distance of the object looked at is near or far. 'The nearer the object, the more convex, up to a certain limit, the front surface of the lens, and vice versa ; the back surface taking little or no share in the production of the effect required. And this surface, which during rest is more convex than the anterior, becomes the less convex of the two during accommodation. The following simple experiment illustrates this point: If a lighted candle be held a little to one side of a person's eye, an observer looking at the eye from the other side sees three distinct images of the flame (fig. 423). The first and brightest is (1) a small erect image formed by the anterior convex surface of the cornea; the second ( 2$)$ is also crect, but larger and less distinct than the preceding, and is formed at the anterior convex surface of the lens; the third (3) is smaller, inverted, and indistinct; it is formed at the posterior surface of the lens, which is concave forward, and therefore, like all concave mirrors, gives an inverted image. If now the eye under observation be made to look at a near object, the second image becomes smaller, clearer, and approaches the first. If the eye be now adjusted for a far point, the second image enlarges again, becomes less distinct, and recedes from the first. In both cases alike the first and third images

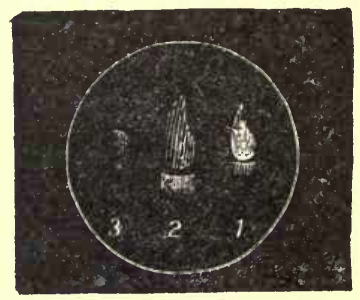

Fig. 423.-Diagram showing three reflections of a candle. 1, From the anterior surface of cornea; 2 , from the anterior surface of lens; 3 , from the posterior surface of lens. For further explanation, see text. The experiment is best performed by employing an instrument invented by Helmholtz, termed a Phakoscope.

remain unaltered in size, distinctness, and relative position. This proves that during accommodation for near objects the curvature of the cornea, and of the posterior of the lens, remains unaltered, while the anterior surface of the lens becomes more convex and approaches the cornea. 
The experiment (fig. $423 \mathrm{~A}$ ) is more striking when two candles are used, and the images of the two candles from the front surface of the lens during accommodation not only approach those from the cornea,

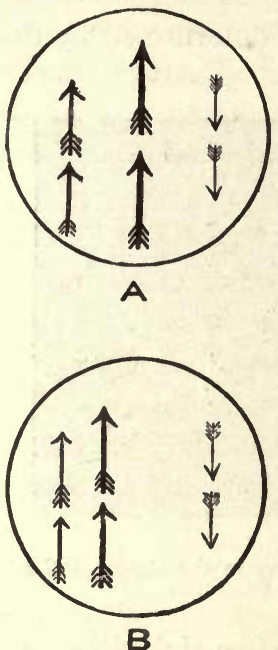

Fig. $423 \mathrm{~A}$.

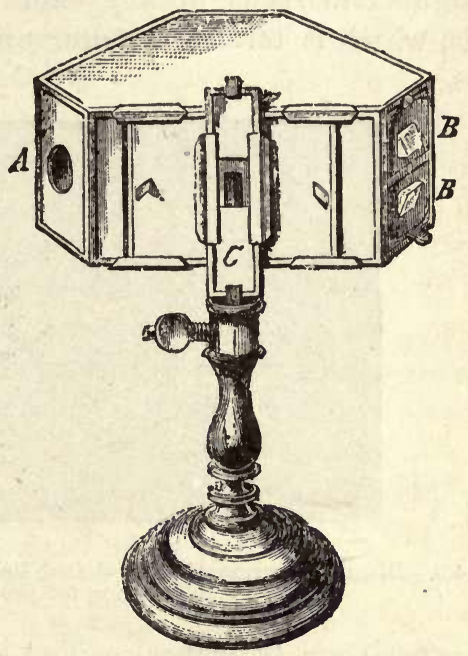

Fig. 424.

Fig. 423 A.-Diagram of Sanson's images. A, when the eyes are not, and B, when they are focussed for near objects. The fig. to the right in $A$ and $B$ is the inverted image from the posterior surface of the lens.

Fig. 424,- Phakoscope of Helmholtz. At $B B^{\prime}$ are two prisms, by which the light of a candle is concentrated on the eye of the person experimented with at $C$. $A$ is the aperture for the eye of the observer. The observer notices three double images, as in fig. 423, reflected from the eye under examination when the eye is fixed upon a distant object; the position of the images having been noticed, the eye is then made to focus a near object, such as a reed pushed up by $C$; the images from the anterior surface of the lens will be observed to move toward each other, in consequence of the lens becoming more convex.

but also approach one another, and become somewhat smaller. (Sanson's images.)

Mechanism of accommodation.-The lens having no inherent power of contraction, its changes of outlines must be produced by some power from without; this power is supplied by the ciliary muscle. It is sometimes termed the tensor choroidece. Its action is to draw forward the choroid, and by so doing to slacken the tension of the suspensory ligament of the lens which arises from it. The anterior surface of the lens is kept flattened by the action of this ligament. The ciliary muscle during accommodation by diminishing its tension, diminishes to a proportional degree the flattening of which it is the cause. On diminution or cessation of the action of the ciliary muscle, the lens returns to its former shape, by virtue of the elasticity of the suspensory ligament (fig. 425). From this it will appear that the eye is usually focussed for distant objects... In viewing near objects the pupil contracts; the opposite effect taking place on withdrawal of the attention from near objects, and fixing it on those distant. 
Range of Distinct Vision. Near-point. - In every eye there is a limit to the power of accommodation. If a book be brought nearer and nearer to the eye, the type at last becomes indistinct, and cannot be brought into focus by any effort of accommodation, however strong. This, which is termed the near-point, can be determined by the follow-

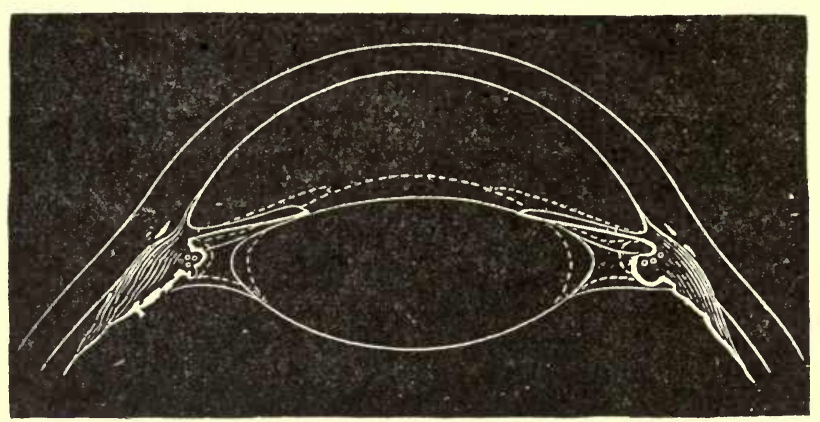

Fig. 425.-Diagram representing by dotted lines the alteration in the shape of the lens on accommodation for near objects. (E. Landolt.)

ing experiment (Scheiner). Two small holes are pricked in a card with a pin not more than a twelfth of an inch $(2 \mathrm{~mm}$.$) apart, at any rate$ their distance from each other must not exceed the diameter of the pupil. The card is held close in front of the eye, and a small needle viewed through the pin-holes. At a moderate distance it can be clearly focussed, but when brought nearer, beyond a certain point, the image appears double or at any rate blurred. This point where the needle ceases to appear single is the near-point. Its distance from the eye can of course be readily measured. It is usually about 5 or 6 inches (13 cm.). In the accompanying figure (fig. 426) the lens $b$ represents the

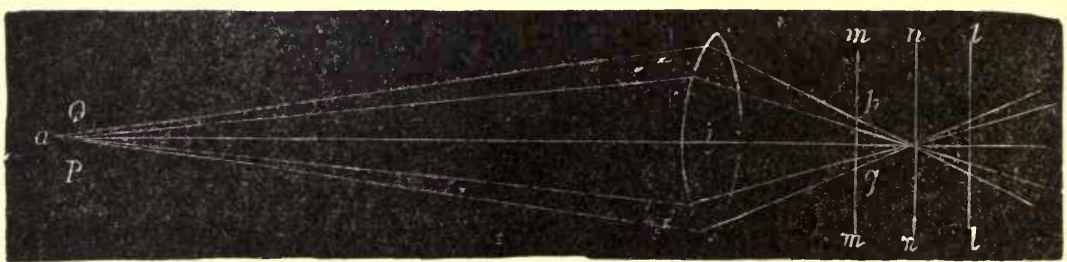

Fig. 426.-Diagram of experiment to ascertain the minimum distance of distinct vision.

eye; ef the two pin-holes in the card, $n n$ the retina; $a$ represents the position of the needle. When the needle is at a moderate distance, the two pencils of light coming from $e$ and $f$, are focussed at a single point on the retina $n n$. If the needle be brought nearer than the near-point, the strongest effort of accommodation is not sufficient to focus the two pen- 
cils, they meet at a point behind the retina. The effect is the same as if the retina were shifted forward to $\mathrm{mm}$. Two inages h.g. are formed, one from each hole. It is interesting to note that when two images are produced, the lower one $g$ really appears in the position $Q$, while the upper one appears in the position $P$. This may be readily verified by covering the holes in succession.

During accommodation two other changes take place in the eyes, (1) The eyes converge by the action of the extra-ocular muscles chiefly by the internal and inferior recti, or internal and superior recti. The superior oblique and the inferior oblique may also be used to turn the eye upward or downward.

Movements of the Eye.-The eyeball possesses movement around three axes indicated in fig. 427, viz., an antero-posterior, a vertical, and a transverse, passing through a centre of rotation a little behind the centre of the optic axis. The movements are accomplished by pairs of muscles.

\section{Inward}

Direction of Movement.

Outward .

Upward

Downward

Inward and upward .

Inward and downward .

Outward and upward

Outward and downward

\section{By what muscles accomplished.}

Internal rectus.

External rectus.

$\{$ Superior rectus.

Inferior oblique.

$\{$ Inferior rectus.

$\{$ Superior oblique.

Internal and superior rectus.

$\{$ Inferior oblique.

Internal and inferior rectus.

$\{$ Superior oblique.

$\{$ External and superior rectus.

Inferior oblique.

$\{$ External and inferior rectus.

\{ Superior oblique.

(2) The second change which takes place in the eyes is, that the pupils contract: The contraction of all of the muscles which have to do with accommodation, viz., of the ciliary muscle, of the recti muscles, and of the sphincter pupillæ is under the control of the third nerve. But the superior oblique may also be employed, in which case the fourth nerve is also concerned.

Contraction of the pupil may also occur under the following circumstances: (1) On exposure of the eye to a bright light; (2) on the local application of eserine (active principle of Calabar bean); (3) on the administration internally of opium, aconite, and in the early stages of ehloroform and alcohol poisoning; (4) on division of the cervical sympathetic or stimulation of the third nerve, and dilatation of the pupil occurs (1) in a dim light; (2) when the eye is focussed for distant objects; (3) on the local application of atropine and its allied alkaloids; (4) on the internal administration of atropine and its allies; (5) in the later stages of poisoning by chloroform, opium, and other drugs: (6) on paralysis of the third nerve; (7) on stimulation of the cervical 
sympathetic, or of its centre in the floor of the front of the aqueduct of Sylvius. The contraction of the pupil appears to be under the control of a centre in the bulb or in the corpora quadrigemina, and this is reflexly stimulated by a bright light, and the dilatation when the reflex centre is not in action is due to the more powerful sympathetic action; but in addition, it appears that both contraction and dilatation may be

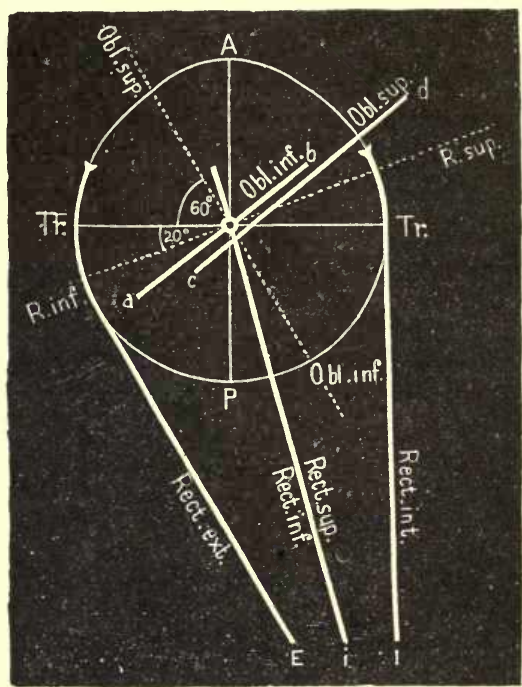

Fig. 42\%.-Diagram of the axes of rotation to the eye. The thin lines indicate axes of rotation, the thick the position of muscular attachment.

produced by a local mechanism, upon which certain drugs can act, which is independent of and probably often antagonistic to the action of the central apparatus of the third and sympathetic nerve. The action of the fifth nerve upon the pupil is not well understood, but its apparent effect in producing dilatation is due to the mixture of sympathetic fibres with its nasal branch. The sympathetic influence upon the radiating fibres is believed to be conveyed not by the long ciliary branches of that nerve, but by the short ciliary branches from the ophthalmic ganglion.

The close sympathy subsisting between the two eyes is nowhere better shown than by the condition of the pupil. If one eye be shaded by the hand its pupil will of course dilate; but the pupil of the other eye will also dilate, though it is unshaded.

\section{Defects in the Optical Apparatus.}

Defects in the Refracting Media.-Under this head we may consider the defects known as (1) Myopia, (2) Hypermetropia, (3) Astigmatism, (4) Spherical Aberration, (5) Chromatic Aberration. 
The normal (emmetropic) eye is so adjusted that parallel rays are brought exactly to a focus on the retina without any effort of accommodation (1, fig. 428). Hence all objects except near ones (practically all objects more than twenty feet off) are seen without any effort of accommodation; in other words, the far-point of the normal eye is at an infinite distance. In viewing near objects we are conscious of the effort (the contraction of the ciliary muscle) by which the anterior surface of the lens is rendered more convex, and rays which would otherwise be focussed behind the retina are converged upon the retina (see dotted lines 2 , fig. 428).

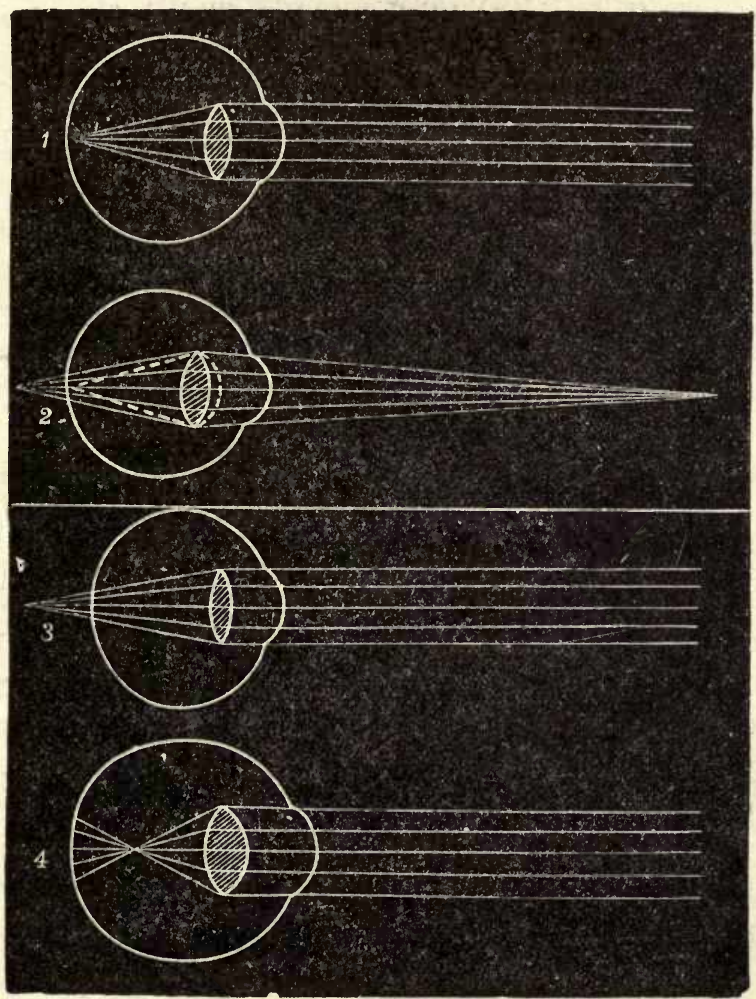

Fig. 428.-Diagram showing-1, normal (emmetropic) eye bringing parallel rays exactly to a focus on the retina; 2 , normal eye adapted to a near point; without accommodation the rays would be focussed behind the retina, but by increasing the curvature of the anterior surface of the lens (shown by a dotted line) the rays are focussed on the retina (as indicated by the meeting of the two dotted lines); 3, hypermetropic eye, in this case the axis of the eye is shorter, and the lens flatter, than normal; parallel rays are focussed behind the retina; 4, myopic eye; in this case the axis of the eye is abnormally long, and the lens too convex; parallel rays are focussed in front of the retina.

1. Myopia (short-sight) (4, fig. 428). - This defect is due to an abnormal elongation of the eyeball. The eye is usually larger than normal and is always longer than normal; the lens is also probably too convex. The retina is too far from the lens and consequently parallel rays are 
focussed in front of the retina, and, crossing, form little circles on the retina; thus the images of distant objects are blurred and indistinct. The eye is, as it were, permanently adjusted for a near-point. Rays from a point near the eye are exactly focussed in the retina. But those which issue from any object beyond a certain distance (far-point) cannot be distinctly focussed. This defect is corrected by concave glasses which cause the rays entering the eye to diverge; hence they do not come to a focus so soon. Such glasses of course are only needed to give a clear vision of distant objects. For near objects, except in extreme cases, they are not required.

Hypermetropia (long-sight) (3, fig. 428).-This is the reverse defect. The eye is too short and the lens too flat. Parallel rays are focussed behind the retina: an effort of accommodation is required to focus even parallel rays on the retina; and when they are divergent, as in viewing a near object, the accommodation is insufficient to focus them. Thus in well-marked cases distant objects require an efiort of accommodation and near ones a very powerful effort. Thus the ciliary muscle is constantly acting. This defect is obviated by the use of convex glasses, which renders the pencils of light more convergent. Such glasses are of course especially needed for near objects, as in reading, etc. 'They rest the eye by relieving the eiliary muscle from excessive work.

3. Astigmatism.-This defect, which was first discovered by Airy, is due to a greater curvature of the eye in one meridian than in others. The eye may be even myopic in one plane and hypermetropic in others. Thus vertical and horizontal lines crossing each other cannot both be focussed at once; one set stands out clearly and the others are blurred and indistinct. This defect, which is present in a slight degree in all eyes, is generally seated in the cornea, but occasionally in the lens as well; it may be corrected by the nse of cylindrical glasses (i.e., curved only in one direction).

4. Spherical Aberration. - The rays of a cone of light from an object situated at the side of the field of vision do not meet all in the same point, owing to their unequal refraction; for the refraction of the rays which pass through the circumference of a lens is greater than that of those traversing its central portion. This defect is known as spherical aberration, and in the camera, telescope, microscope, and other optical instruments, it is remedied by the interposition of a screen with a circular aperture in the path of the rays of light, cutting off all the marginal rays and only allowing the passage of those near the centre. Such correction is effected in the eye by the iris, which forms an annular diaphragm to cover the circumference of the lens, and to prevent the rays from passing through any part of the lens but its centre which corresponds to the pupil. The posterior surface of the iris is coated with 
pigment, to prevent the passage of rays of light through its substance. The image of an object will be most defined and distinct when the pupil is narrow, the object at the proper distance for vision, and the light abundant; so that, while a sufficient number of rays are admitted, the narrowness of the pupil may prevent the production of indistinctness of the image by spherical aberration. But even the image formed by the rays passing through the circumference of the lens, when the pupil is much dilated, as in the dark, or in a feeble light, may, under certain circumstances, be well defined.

Distinctness of vision is further secured by the pigment of the outer surface of the retina, the posterior surface of the iris and the ciliary processes, which absorbs any rays of light that may be reflected within the eye, and prevents their being thrown again upon the retina so as to interfere with the images there formed. The pigment of the retina is especially important in this respect; for with the exception of its outer layer the retina is very transparent, and if the surface behind it were not of a dark color, but capable of reflecting the light, the luminous rays which had already acted on the retina would be reflected again through it, and would fall upon other parts of the same membrane, producing both dazzling from excessive light, and indistinctness of the images.

5. Chromatic Aberration. - In the passage of light through an ordinary convex lens, decomposition of each ray into its elementary colored part, commonly ensues, and a colored margin appears around the image, owing to the unequal refraction which the elementary colors undergo. In optical instruments this, which is termed chromatic aberration, is connected by the use of two or more lenses, differing in shape and density, the second of which continues or increases the refraction of the rays produced by the first, but by recombining the individual parts of each ray into its original white light, corrects any chromatic aberration which may have resulted from the first. It is probable that the unequal refractive power of the transparent media in front of the retina may be the means by which the eye is enabled to guard against the effect of chromatic aberration. The human eye is achromatic, however, only so long as the image is received at its focal distance upon the retina, or so long as the eye adapts itself to the different distances of sight. If either of these conditions be interfered with, a more or less distinct appearance of colors is produced.

An ordinary ray of white light in passing through a prism, is refracted, i.e., bent out of its course, but the different colored rays which go to make up white light are refracted in different degrees, and therefore appear as colored bands fading off into each other: thus a colored band known as the "spectrum" is produced, the colors of which are arranged, as follows-red, orange, yellow, green, blue, indigo, violet; of these 
the red ray is the least, and the violet the most refracted. Hence, as Helmholtz has shown, a small white object cannot be accurately focussed on the retina, for if we focus for the red rays, the violet are out of focus, and vice versâ. such objects, if not exactly focussed, are often seen surrounded by a pale yellowish or bluish fringe.

For similar reasons a red surface looks nearer than a blue one at an equal distance, because, the red rays being less refrangible, a stronger effort of accommodation is necessary to focus them, and the eye is adjusted as if for a nearer object, and therefore the red surface appears nearer.

From the insufficient adjustment of the image of a small white object, it appears surrounded by a sort of halo or fringe. This phenomenon is termed irradiation. It is from this reason that a white square on a black ground appears larger than a black square of the same size on a white ground.

As an optical instrument, the eye is superior to the camera in the following, among many other particulars, which may be enumerated in detail. 1. The correctness of images even in a large field of view. 2. The simplicity and efficiency of the means by which chromatic aberration is avoided. 3. The perfect efficiency of its adaptation to different distances. In the photographic camera, it is well known that only a comparatively small object can be accurately focussed. In the photograph of a large object near at hand, the upper and lower limits are always more or less hazy, and vertical lines appear curved. This is due to the fact that the image produced by a convex lens is really slightly curved and can only be received without distortion on a slightly curved concave screen, hence the distortion on a flat surface of ground glass. It is different with the eye, since it possesses a concave background, upon which the field of vision is depicted, and with which the curved form of the image coincides exactly. Thus, the defect of the camera obscura is entirely avoided; for the eye is able to embrace a large field of vision, the margins of which are depicted distinctly and withont distortion. If the retina had a plane surface like the ground glass plate in a camera, it must necessarily be much larger than is really the case if we were to see as much; moreover, the central portion of the field of vision alone would give a good clear picture (Bernstein).

Defective Accommodation-Presbyopia.-This condition is due to the gradual loss of the power of accommodation which is part of the general decay of old age. In consequence the patient would be obliged in reading to hold his book further and further away in order to focus the letters, till at last the letters are held too far for distinct vision. The defect is remedied by weak convex glasses, which are very commonly worn by old people. It is due chiefly to the gradual increase in density of the lens, which is unable to swell out and become convex when near 
objects are looked at, and also to a weakening of the ciliary muscle, and a general loss of elasticity in the parts concerned in the mechanism.

\section{Visual Sensations.}

Excitation of the Retina.-Light is the normal agent in the excitation of the retina. The only layer of the retina capable of reacting to the stimulus is the rods and cones. The proofs of this statement may be summed up thus:-

(1.) The point of entrance of the optic nerve into the retina, where the rods and cones are absent, is insensitive to light and is called the blind spot. The phenomenon itself is very readily demonstrated. If we direct one eye, the other being closed, upon a point at such a distance to the side of any object, that the image of the latter must fall upon the retina at the point of entrance of the optic nerve, this image is lost either instantaneously, or very soon, If, for example, we close the left eye, and direct the axis of the right eye steadily toward the circular

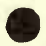

spot here represented, while the page is held at a distance of about six inches from the eye, both dot and cross are visible. On gradually increasing the distance between the eye and the object, by removing 'the book farther and farther from the face, and still keeping the right eye steadily on the dot, it will be found that suddenly the cross disappears from view, while on removing the book still farther, it suddenly comes in sight again. The cause of this phenomenon is simply that the porcion of retina which is occupied by the entrance of the optic nerve, is quite blind; and therefore that when it alone occupies the field of vision, objects cease to be visible. (2.) In the fovea centralis and macula lutea which contain rods and cones but no optic nerve-fibres, light produces the greatest effect. In the latter, cones occur in large numbers, and in the former cones without rods are found, whereas in the rest of the retina which is not so sensitive to light, there are fewer cones than rorks. We may conclude, therefore, that cones are even more important to vision than rods. (3.) If a small lighted candle be moved to and fro at the side of and close to one eye in a dark room while the eyes look steadily, forward into the darkness, a remarkable branching figure (Purkinje's figures) is seen floating before the eye, consisting of durk lines on a reddish ground. As the candle moves, the figure moves in the opposite direction, and from its whole appearance there can be no doubt that it is a reversed picture of the retinal vessels projected before the eye. The two large branching arteries passing up and down from the optic disc are clearly visible together with their minutest branches. 
A little to one side of the disc, in a part free from vessels, is seen the yellow spot in the form of a slight depression. 'This remarkable appearance is due to shadows of the retinal vessels cast by the candle. The branches of these vessels are chiefly distributed in the nerve-fibre and ganglionic layers; and since the light of the candle falls on the retinal vessels from in front, the shadow is cast behind them, and hence those elements of the retina which perceive the shadows must also lie behind the vessels. Here, then, we have a clear proof that the light-perceiving elements of the retina are not the fibres of the optic nerve forming the innermost layer of the retina, but the external layers of the retina, rods and cones, which indeed appear to be the special terminations of the optic nerve-fibres.

Duration of Visual Sensations. - The duration of the sensation produced by a luminous impression on the retina is always greater than that of the impression which produces it. However brief the luminous impression, the effect on the retina always lasts for about one-eighth of a second. 'Thus, supposing an object in motion, say a horse, to be revealed on a dark night by a flash of lightning. The object would be seen apparently for an eighth of a second, but it would not appear in motion; because, although the image remained on the retina for this time, it was really revealed for such an extremely short period (a flash of lightning being almost instantaneous) that no appreciable movement on the part of the object could have taken place in the period during which it was revealed to the retina of the observer. And the same fact is proved in a reverse way. The spokes of a rapidly revolving wheel are not seen as distinct objects, because at every point of the field of vision over which the revolving spokes pass, a given impression has not faded before another comes to replace it. Thus every part of the interior of the wheel appears occupied.

The duration of the after-sensation, produced by an object, is greater in a direct ratio with the duration of the impression which caused it. Hence the image of a bright object, as of the panes of a wiudow through which the light is shining, may be perceived in the retina for a considerable period, if we have previonsly kept our eyes fixed for some time on it. But the image in this case is negative. If, however, after shutting the eyes for some time, we open them and look at an object for an instant, and again close them, the after-image is positive.

Intensity of Visual Sensations. - It is quite evident that the more luminous a body the more intense is the sensation it produces. But the intensity of the sensation is not directly proportional to the intensity of the luminosity of the object. It is necessary for light to have a certain intensity before it can excite the retina, but it is impossible to fix an arbitrary limit to the power of excitability. As in other sensations, so 
also in visual sensations, a stimulus may be too feeble to produce a sensation. If it be increased in amount sufficiently it begins to produce an effect which is increased on the increase of the stimulation; this increase in the effect is not directly proportional to the increase in the excitation, but, according to Fechner's law, "as the logarithm of the stimulus," i.e., in each sensation, there is a constant ratio between the increase in the stimulus and the increase in the sensation, this constant ratio for each sensation expresses the least perceptible increase in the sensation or minimal increment of excitation.

This law, which is true only within certain limits, may be best understood by an example. When the retina has been stimulated by the light of one candle, the light of two candles will produce a difference in sensation which can be distinctly felt. If, however, the first stimulus had been that of an electric light, the addition of the light of a candle would make no difference in the sensation. So, generally, for an additional stimulus to be felt, it may be proportionately small if the original stimulus have been small, and must be greater if the original stimulus have been great. 'The stimulus increases as the ordinary numbers, while the sensation increases as the logarithm.

Part of the light which enters the eye is absorbed and produces some change in the retina, of which we shall treat further on; the rest is reflected.

Every one is perfectly familiar with the fact, that it is quite impossible to see the fundus or back of another person's eye by simply looking into it. The interior of the eye forms a perfectly black background to the pupil. The same remark applies to an ordinary photographic camera, and may be illustrated by the difficulty we experience in seeing into a room from the street through the window, unless the room be lighted within. In the case of the eye this fact is partly due to the feebleness of the light reflected from the retina, most of it being absorbed by the retinal pigment, as mentioned above; but far more to the fact that every such ray is reflected straight to the source of light (e.g., candle), and cannot, therefore, be seen by the unaided eye without intercepting the incident light from the candle, as well as the reflected rays from the retina. This difficulty is surmounted by the use of the ophthalmoscope.

The ophthalmoscope, brought into use by Helmholtz, consists in its simplest form of $a$, a slightly concave mirror of metal or silvered glass perforated in the centre, and fixed into a handle; and $b$, a biconvex lens of about $2 \frac{1}{2}-5$ inches focal length. Two methods of examining the eye with this instrument are in common use-the direct and the indirect: both methods of investigation should be employed. A normal eye should be examined ; a drop of a solution of atropia (two grains to the ounce) or of homatropia hydrobromate, should be instilled about twenty minutes before the examination is commenced; the ciliary muscle is thereby paralyzed, the power of accommodation is abolished, and the 
pupil is dilated. This will materially facilitate the examination; but it is quite possible to observe all the details to be presently described without the use of this drug. The room being now darkened, the observer seats himself in front of the person whose eye he is about to examine, placing himself upon a somewhat higher level. A brilliant and steady light is placed close to the left ear of the patient. The atropia having been put into the right eye only of the patient, this eye is examined. Taking the mirror in his right hand, and looking through the central hole, the operator directs a beam of light into the eye of

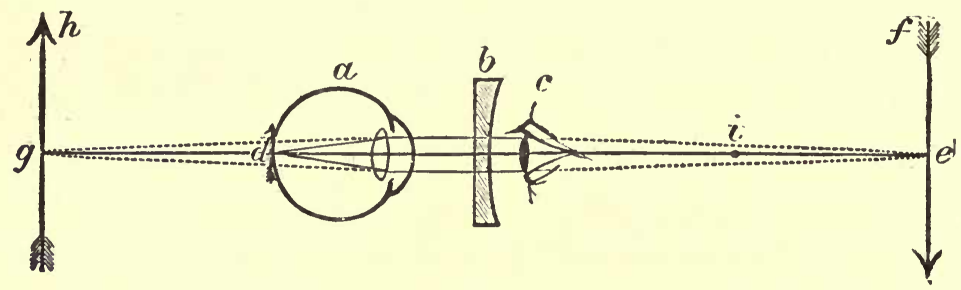

Fig. 429.-Diagram to illustrate the action of the Ophthalmoscope, when a plane concave glass is used. $c$. observer's eye. The light reflected from any point, $d$, on retina of $a$, would naturally be focussed at $e$; if the lens $b$ is used it would be focussed at $i$, in other words, at back of $c$. The image would be enlarged, as though of $g$, and would be inverted. (After McGregor Robertson.)

the patient. A red glare, known as the reflex, is seen; it is due to the illumination of the retina. The patient is then told to look at the little finger of the observer's right hand as he holds the mirror; to effect this the eye is rotated somewhat inward, and at the same time the reflex changes from red to a lighter color, owing to the reflection from the optic dise. The observer now approximates the mirror, and with it his eye to the eye of the patient, taking care to keep the light fixed upon the pupil, so as not to lose the reflex. At a certain point, which raries with different eyes, but is usually when there is an interval of about two or three inches between the observed and the observing eye, the vessels of the retina will become visible as lines running in different directions. Distinguish the smaller and brighter red arteries from the larger and darker colored reins. Examine carefully the fundus of the eye, i.e., the red surface-until the optic dise is seen; trace its circular outline, and observe

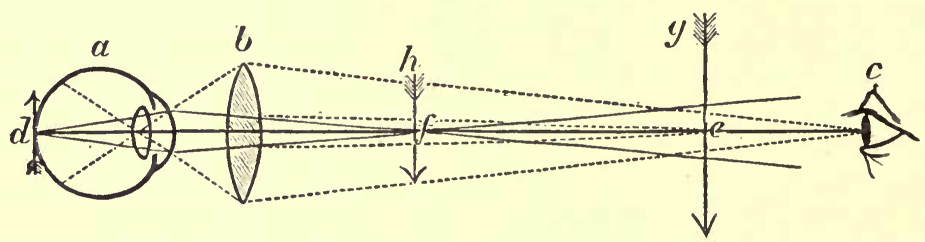

Fig. 430.-Diagram to illustrate action of ophthalmoscope when a bi-convex glass is used. The flg. $d$ on retina of $a$ is under ordinary conditions focussed at $f$ and inverted. If the lens $b$ be placed between eyes, the image $h$ is seen by the eye $c$ as an enlarged image. (After McGregor Robertson.)

the small central white spot, the porus opticus, physiological pit: near the centre is the central artery of the retina breaking up upon the disc into branches ; veins also are present, and correspond roughly to the course of the arteries. Trace the vessels orer the disc on to the retina. The optic disc is bounded by two delicate rings, the more external being the choroidal. while the more internal is the sclerotic opening. Somewhat to the outer side, and only visible 
after some practice, is the yellow spot, with the smaller lighter-colored fovea centralis in its centre. This constitutes the direct method of examination (fig. $429)$; by it the various details of the fundus are seen as they really exist, and it is this method which should be adopted for ordinary use.

If the observer is ametropic, i.e., is myopic or hypermetropic, he will be unable to employ the direct method of examination until he has remedied his defective vision by the use of proper glasses.

In the indirect method (fig. 430) the patient is placed as before, and the operator holds the mirror in his right hand at a distance of twelve to eighteen inches from the patient's right eye. At the same time he rests his left little finger lightly upon the right temple, and holding the lens between his thumb and forefinger, two or three inches in front of the patient's eye, directs the light through the lens into the eye. The red reflex, and subsequently the white one, having been gained, the operator slowly moves his mirror, and with it his eye, toward or away from the face of the patient, until the outline of one of the retinal vessels becomes visible, when very slight movements on the part of the operator will suffice to bring into view the details of the fundus above described, but the image will be much smaller and inverted. The lens should be kept fixed at a distance of two or three inches, the mirror being alone moved until the disc becomes visible: should the image of the mirror, however, obscure the disc, the lens may be slightly tilted.

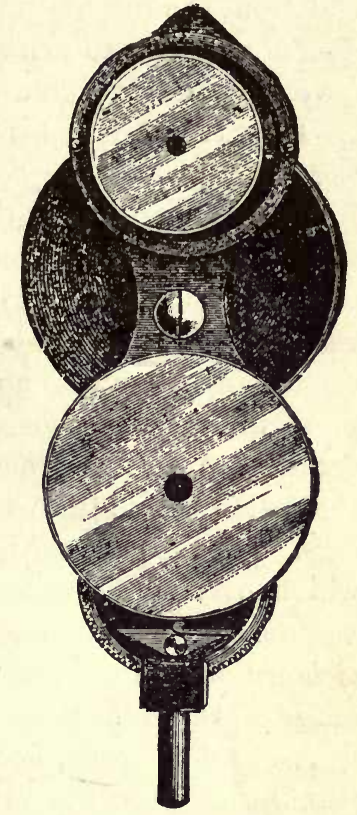

Fig. 431. - The ophthalmoscope. The small upper mirror is for direct, the larger for indirect illumination.

Visual Purple.-The method by which a ray of light is able to stimulate the endings of the optic nerve in the retina in such a manner that a visual sensation is perceived by the cerebrum is not yet understood. It is supposed that the change effected by the agency of the light which falls upon the retina is in fact a chemical alteration in the protoplasm, and that this change stimulates the optic nerve-endings. The discovery of a certain temporary reddish-purple pigmentation of the outer limbs of the retinal rods in certain animals (e.g., frogs) which had been killed in the dark, forming the so-called rhodopsin or visual purple, appeared likely to offer some explanation of the matter, especially as it was also found that the pigmentation disappeared when the retina was exposed to light, and reappeared when the light was removed, and also that it underwent distinct changes of color when other than white light was used. It was also found that if the operation were performed quickly enough, the image of an object (optogram) might be fixed in the pigment on the retina by soaking the retina of an animal, which has been killed in the dark, in alum solution. 
The visual purple cannot however be absolutely essential to the due production of visual sensations, as it is absent from the retinal cones, and from the macula lutea and fovea centralis of the human retina, and does not appear to exist at all in the retinæ of some animals, e.g., bat, dove, and hen, which are, nevertheless, possessed of good vision.

However the fact remains that light falling upon the retina $(a)$ bleaches the visual purple, and this must be considered as one of its effects. It has been found that certain pigments, also sensitive to light, are contained in the inner segments of the cones. These colored bodies are said to be oil globules of various colors, red, green, and yellow, called chromophanes, and are found only in the retinas of animals not mammals. The rhodopsin at any rate appears to be derived in some way from the retinal pigment, since the color is not renewed after bleaching if the retina be detached from its pigment layer. (b) The second change produced by the action of the light upon the retina is the moiement of the pigment cells. On the stimulation of light the granules of pigment in the cells which overlie the outer part of the rod and cone layer of the retina become diffused in the parts of the cells between the rods and cones, the melanin or fuscin granules, as they are called, passing down into the processes of the cells. (c) A movement of the cones and possibly of the rods is also said to occur, as has been already incidentally mentioned; on the stimulus of light the outer parts of the cones, which in an eye protected from light extend to the pigment layer, are retracted. It is even thought that the contraction is under the control of the nervous system; and finally, according to the careful researches of Dewar and McKendrick, and of Holmgren, it appears that the stimulus of light is able to produce $(d)$ a variation of the natural electrical currents of the retina. The current is at first increased and then diminished. McKendrick believes that this is the electrical expression of those chemical changes in the retina of which we have already spoken.

\section{Visual Perceptions and Judgments.}

Reversion of the Image.-It will be as well to repeat here that the direction given to the rays by their refraction is regulated by that of the central ray, or axis of the cone, toward which the rays are bent. The image of any point of an object is, therefore, as a rule (the exceptions to which need not here be stated), always formed in a line identical with the axis of the cone of light, as in the line of в $b$, or $\mathrm{A} a$ (fig. 432), so that the spot where the image of any point will be formed upon the retina may be determined by prolonging the central ray of the cone of light, or that ray which traverses the centre of the pupil. Thus $\mathrm{A} a$ is the axis or central ray of the cone of light issuing from $\mathrm{A} ;$ в $b$ the central 
ray of the cone of light issuing from $\mathrm{B}$; the image of $\mathbf{A}$ is formed at $a$, the image of $\mathrm{B}$ at $b$, in the inverted position: therefore what in the object was above is in the image below, and vice versâ, - the right-hand part of the object is in the image to the left, the left-hand to the right. If an opening be made in an eye at its superior surface, so that the retina can be seen through the vitreous humor, this image of any bright object, such as the windows of the room, may be perceived inverted upon the retina. Or still better, if the eye of any albino animal, such as a white rabbit, in which the coats, from the absence of pigment, are transparent, is dissected clean, and held with the cornea toward the window, a very distinct image of the window completely inverted is seen depicted on the posterior translucent wall of the eye. Volkmann has also shown

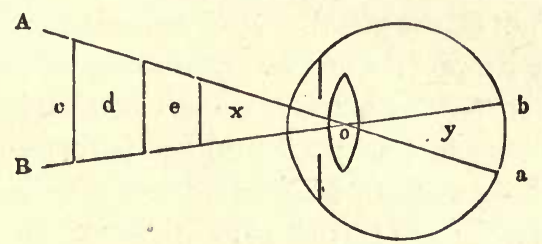

Fig 432.-Diagram of the formation of the image on the retina.

that a similar experiment may be successfully performed in a living person possessed of large prominent eyes, and an unusually transparent sclerotic.

An image formed at any point on the retina is referred to a point outside the eye, lying on a straight line drawn from the point on the retina outward through the centre of the pupil. Thus an image on the left side of the retina is referred by the mind to an object on the right side of the eye, and vice versâ. Thus all images on the retina are mentally, as it were, projected in front of the eye, and the objects are seen erect though the image on the retina is inverted. Much needless confusion and difficulty have been raised on this subject for want of remembering that when we are said to see an object, the min l is merely conscious of the picture on the retina, and when it refers it to the external object, or "projects" it outside the eye, it necessarily reverses it and sees the object as erect, though the retinal image is inverted. This is further corroborated by the sense of touch. Thus an object whose picture falls on the left half of the retina is reached by the right hand, and hence is said to lie to the right. Or, again, an object whose image is formed on the upper part of the retina is readily touched by the feet, and is therefore said to be in the lower part of the field, and so on.

Hence it is also, that no discordance arises between the sensations of inverted vision and those of touch, which perceives everything in its 
erect position; for the images of all objects, even of our own limbs, on the retina, are equally inverted, and therefore maintain the same relative position.

Even the image of our hand, while used in touch, is seen inverted. The position in which we see objects, we call, therefore, the erect position. A mere lateral inversion of our body in a mirror, where the right hand occupies the left of the image, is indeed scarcely remarked: and there is but little discordance between the sensations acquired by touch in regulating our movements by the image in the mirror, and those of sight, as, for example, in tying a knot in the cravat. There is some want of harmony here, on account of the inversion being only lateral, and not complete in all directions.

The perception of the erect position of objects appears, therefore, to be the result of an act of the mind. And this leads us to a consideration of the several other properties of the retina, and of the co-operation of the mind in the several other parts of the act of vision. To these belong not merely the act of sensation itself and the perception of the changes produced in the retina, as light and colors, but also the conversion of the mere images depicted in the retina into ideas of an extended field of vision, of proximity and distance, of the form and size of objects, of the reciprocal influence of different parts of the retina upon each other, the simultaneous action of the two eyes, and some other phenomena.

Field of Vision.-The actual size of the field of vision depends on the extent of the retina, for only so many images can be seen at any one time as can occupy the retina to the same time; and thus considered, the retina, the conditions of which are perceived by the brain, is itself the field of vision. But to the mind of the individual the size of the field of vision has no determinate limits; sometimes it appears very small, at another time very large; for the mind has the power of projecting images on the retina toward the exterior. Hence the mental field of vision is very small when the sphere of the action of the mind is limited to impediments near the eye: on the contrary, it is very extensive when the projection of the images on the retina toward the exterior, by the influence of the mind, is not impeded. It is very small when we look into a hollow body of small capacity held before the eyes; large when we look out upon the landscape through a small opening; more extensive when we look at the landscape through a window; and most so when our view is not confined by any near object. In all these cases the idea which we receive of the size of the field of vision is very different, although its absolute size is in all the same, being dependent on the extent of the retina. Hence it follows, that the mind is constantly cooperating in the acts of vision, so that at last it becomes difficult to say what belongs to mere sensation, and what to the influence of the mind. 
By a mental operation of this kind, we obtain a correct idea of the size of individual objects, as well as of the extent of the field of vision. To illustrate this, it will be well to refer to fig. 433.

The angle $x$, included between the decussating central rays of two cones of light issuing from different points of an object, is called the

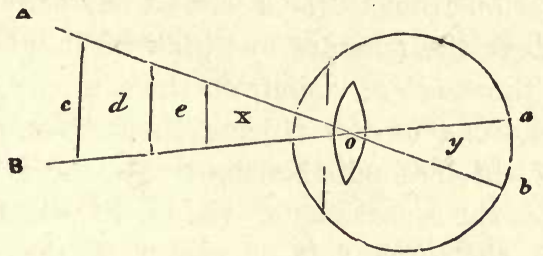

Fig. 433.

optical angle-angulus opticus seu visorius. This angle becomes larger, the greater the distance between the points A and $\mathrm{B}$; and since the angles $x$ and $y$ are equal, the distance between the points $a$ and $b$ in the image on the retina increases as the angle becomes larger. Objects at different distances from the eye, but having the same optical angle $x$-for example, the objects, $c, d$, and $e,-$ must also throw images of equal size upon the retina; and, if they occupy the same angle of the field of vision, their image must occupy the same spot in the retina.

Nevertheless, these images appear to the mind to be of very unequal size when the ideas of distance and proximity come into play; for, from the image $a b$, the mind forms the conception of a visual space extending to $e, d$, or $c$, and of an object of the size which that represented by the image on the retina appears to have when viewed close to the eye, or under the most usual circumstances.

Estimation of Size.-Our estimate of the size of various objects is based partly on the visual angle under which they are seen, but much more on the estimate we form of their distance. Thus a lofty mountain many miles off may be seen under the same visual angle as a small hill near at hand, but we infer that the former is much the larger object because we know it is much further off than the hill. Our estimate of distance is often erroneous, and consequently the estimate of size also. Thus persons seen walking on the top of a small hill againts a clear twilight sky appear unusually large, because we over-estimate their distance, and for similar reasons most objects in a fog appear immensely magnified. The same mental process gives rise to the idea of depth in the field of vision; this idea being fixed in our mind principally by the circumstance that, as we ourselves move forward, different images in succession become depicted on our retina, so that we seem to pass between these images, which to the mind is the same thing as passing between the objects themselves. 
The action of the sense of vision in relation to external objects is, therefore, quite different from that of the sense of touch. The objects of the latter sense are immediately present to it; and our own body, with which they come in contact, is the measure of their size. The part of a table touched by the hand appears as large as the part of the hand receiving an impression from it, for a part of our body in which a sensation is excited, is here the measure by which we judge of the magnitude of the object. In the sense of vision, on the contrary, the images of objects are mere fractions of the objects themselves realized upon the retina, the extent of which remains constantly the same. But the imagination, which analyzes the sensations of vision, invests the images of objects, together with the whole field of vision in the retina, with very varying dimensions; the relative size of the image in proportion to the whole field of vision, or of the affected parts of the retina to the whole retina, alone remaining unaltered.

Estimation of Direction.-The direction in which an object is seen, depends on the part of the retina which receives the image, and on the distance of this part from, and its relation to, the central point of the retina. Thus, objects of which the images fall upon the same parts of the retina lie in the same visual direction; and when, by the action of the mind, the images or affections of the retina are projected into the exterior world, the relation of the images to each other remains the same.

Estimation of Form.-The estimation of the form of bodies by sight is the result partly of the mere sensation, and partly of the association of ideas. Since the form of the images perceived by the retina depends wholly on the outline of the part of the retina affected, the sensation alone is adequate to the distinction of only superficial forms of each other, as of a square from a circle. But the idea of a solid body as a sphere, or a body of three or more dimensions, e.g., a cube, can only be attained by the action of the mind constructing it from the different superficial images seen in different positions of the eye with regard to the object, and, as shown by Wheatstone and illustrated in the stereoscope, from two different perspective projections of the body being present simultaneously to the mind by the two eyes. Hence, when, in adult age, sight is suddenly restored to persons blind from infancy, all objects in the field of vision appear at first as if painted flat on one surface; and no idea of solidity is formed until after long exercise of the sense of vision combined with that of touch.

The clearness with which an object is perceived irrespective of accommodation, would appear to depend largely on the number of rods and cones which its retinal image covers. Hence the nearer an object is to the eye (within moderate limits) the more clearly are all its details 
seen. Moreover, if we want carefully to examine any object, we always direct the eyes straight to it, so that its image shall fall on the yellow spot where an image of a given area will cover a larger number of cones than anywhere else in the retina. It has been found that the images of two points must be at least $3 \mu$ apart on the yellow spot in order to be distinguished separately; if the images are nearer together, the points appear as one. The diameter of each cone in this part of the retina is about $3 \mu$.

Estimation of Movement.-We judge of the motion of an object, partly from the motion of its image over the surface of the retina, and partly from the motion of our eyes following it. If the image upon the retina moves while our eyes and our body are at rest, we conclude that the object is changing its relative position with regard to ourselves. In such a case the movement of the object may be apparent only, as when we are standing upon a body which is in motion, such as a ship. If, on the other hand, the image does not move with regard to the retina, but remains fixed upon the same spot of that membrane, while our eyes follow the moving body, we judge of the motion of the object by the sensation of the muscles in action to move the eye. If the image moves over the surface of the retina while the muscles of the eye are acting at the same time in a manner corresponding to this motion, as in reading, we infer that the object is stationary, and we know that we are merely altering the relations of our eyes to the object. Sometimes the object appears to move when both object and eye are fixed, as in vertigo.

The mind can, by the faculty of attention, concentrate its activity more or less exclusively upon the sense of sight, hearing, and touch alternately. When exclusively occupied with the action of one sense, it is scarcely conscious of the sensations of the others. The mind, when deeply immersed in contemplations of another nature, is indifferent to the actions of the sense of sight, as of every other sense. We often, when deep in thought, have our eyes open and fixed, but see nothing, because of the stimulus of ordinary light being unable to excite the brain to perception, when otherwise engaged. The attention which is thus necessary for vision, is necessary also to analyze what the field of vision presents. The mind does not perceive all the objects presented by the field of vision at the same time with equal acuteness, but directs itself first to one and then to another. The sensation becomes more intense, according as the particular object is at the time the principal object of mental contemplation. Any compound mathematical figure produces a different impression according as the attention is directed exclusively to one or the other part of it. Thus in fig. $433 \mathrm{~A}$, we may in succession have a vivid perception of the whole, or of distinct parts only; of the six triangles near the outer circle, of the hexagon in the middle, of the 
three large triangles. The more numerous and varied the parts of which a figure is composed the more scope does it afford for the play of the attention. Hence it is that architectural ornaments have an enlivening

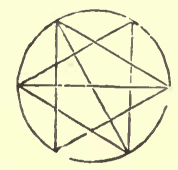

Fig. 433 ^.

effect on the sense of rision, since they afford constantly fresh subject for the action of the mind.

Color Sensations. - If a ray of sunlight be allowed to pass through a prism, it is decomposed by its passage into rays of different colors, which are called the colors of the spectrum; they are red, orange, yellow, green, blue, indigo, and violet. The red rays are the least turned out of their course by the prism, and the violet the most, while the other colors occupy in order places between these two extremes. The differences in the color of the rays depend upon the number of vibrations producing each, the red rays being the least rapid and the violet the most. In addition to the colored rays of the spectrum, there are others which are invisible, but which have definite properties, those to the left of the red, and less refrangible, being the calorific rays which act upon the thermometer, and those to the right of the violet, which are called the actinic or chemical rays, which have a powerful chemical action. The rays which can be perceived by the brain, i.e., the colored rays, must stimulate the retina in some special manner in order that colored vision may result, and two chief explanations of the method of stimulation have been suggested.

(1.) 'The one, originated by Young and elaborated by Helmholtz, holds that there are three primary colors, viz., red, green, and violet, and that in the retina are contained rods or cones which answer to each of these primary colors, whereas the innumerable intermediate shades of color are produced by stimulation of the three primary eolor terminals in different degrees, the sensation of white being produced at the same time when the three elements are equally excited. Thus if the retina be stimulated by rays of certain wave length, at the red end of the spectrum, the terminals of the other colors, green and violet, are hardly stimulated at all, but the red terminals are strongly stimulated, the resulting sensation being red. The orange rays excite the red terminals considerably, the green rather more, and the violet slightly, the resulting sensation being that of orange, and so on (fig. 434).

(2.) The second theory of color (Hering's) supposes that there are six 
primary color sensations, of three pair of antagonistic or complemental colors, black and white, red and green, and yellow and blue, and that these are produced by the changes either of disintegration or of assimulation taking place in certain substances, somewhat it may be supposed of the nature of the visual purple, which (the theory supposes to) exist in the retina. Each of the substances corresponding to a pair of colors, being capable of undergoing two changes, one of construction and the other of disintegration, with the result of producing one or other color. For instance, in the white-black substance, when disintegration is in excess of construction or assimilation, the sensation is white, and when assimilation is in excess of disintegration the reverse is the case; and similarly with the red-green substance, and with the yellow-blue substance. When the repair and disintegration are equal with the first

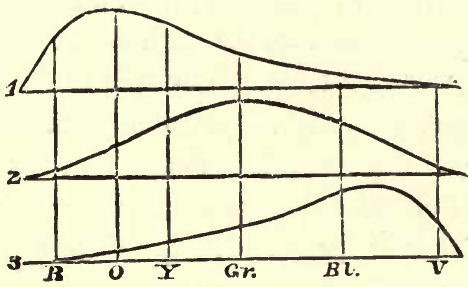

Fig. 434 .

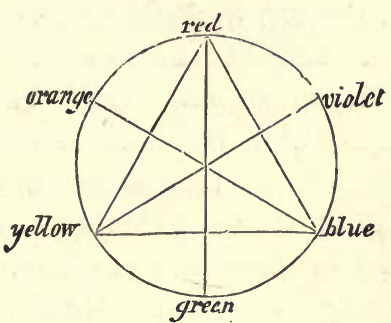

Fi. 455 .

Fig. 434. - Diagram of the three primary color sensations. (Young-Helmholtz theory ) 1, is the red; 2 , green, and 3, violet, primary color sensations. The lettering indicates the colors of the spectrum. The diagram indicates by the height of the curve to what extent the several primary sensations of color are excited by vibrations of different wave lengths.

Fig. 435.-Diagram of the various simple and compound colors of light, and those which are complemental of each other, i.e., which, when mixed, produce a neutral gray tint. The three simple colors, red, yellow, and blue, are placed at the angles of an equilateral triangle, which are connected together by means of a circle; the mixed colors, green, orange, and violet, are placed intermediate between the corresponding simple or homogeneous colors; and the conplemental colors, of which the pigments, wher mixed, would constitute a gray, and of which the prismatic spectra would together produce a white light, will be found to be placed in each case opposite to each other, but connected by a line passing through the centre of the circle. The figure is also useful in showing the further shades of color which are complementary of each other. If the circle be supposed to contain every transition of color between the six marked down, those which, when united, yield a white or gray color, will always be found directly opposite to each other; thus, for example, the intermediate tint between orange and red is com. plemental of the middle tint between green and blue.

substance, the visual sensation is gray; but in the other pairs when this is the case, no sensation occurs. The rays of the spectrum to the left produce changes in the red-green substance only, with a resulting sensation of red, while the (orange) rays further to the right affect both the red-green and the yellow-blue substances; blue rays cause constructive changes in the yellow-blue substances but none in the red-green and so on. These changes produced in the visual substances in the retina are perceived by the brain as sensations of color.

The spectra left by the images of white or luminous objects are ordinarily white or luminous; those left by dark objects are dark. Sometimes, however, the relation of the light and dark parts in the image 
may, under certain circumstances, be reversed in the spectrum; what was bright may be dark, and what was dark may appear light. This occurs whenever the eye, which is the seat of the spectrum of a luminous object, is not closed, but fixed upon another bright or white surface, as a white wall, or a sheet of white paper. Hence the spectrum of the sun, which, while light is excluded from the eye, is luminous, appears black or gray when the eye is directed upon a white surface. The explanation of this is, that the part of the retina which has received the luminous image remains for a certain period afterward in an exhausted or less sensitive state, while that which has received a dark image is in an unexhausted, and therefore much more excitable condition.

The ocular spectra which remain after the impression of colored objects upon the retina are always colored; and their color is not that of the object, or of the image produced directly by the object, but the opposite, or complemental color. The spectrum of a red object is, therefore, green; that of a green object, red; that of violet, yellow; that of yellow, violet, and so on. The reason of this is obrious. The part of the retina which receives, say, a red image, is wearied by that particular color, but remains sensitive to the other rays which with red make up white light; and, therefore, these by themselves reflected from a white object produce a green hue. If, on the other hand, the first object looked at be green, the retina being tired of green rays, receires a red image when the eye is turned to a white object. And so with the other colors; the retina while fatigned by yellow rays will suppose an object to be violet, and vice versî; the size and shape of the spectrum corresponding with the size and shape of the original object looked at. The colors which thus reciprocally excite each other in the retina are those placed at opposite points of the circle in fig. 435. The peripheral parts of the retina do not react to rays of red. The area of the retiua which is capable of receiving impressions of color, and therefore the field of vision, is slightly different for each color.

Color Blindness or Daltonism.-Daltonism or color-blindness is a by no means uncommon visual defect. One of the commonest forms is the inability to distinguish between red and green. The simplest explanation of such a condition is, that the elements of the retina which receive the impression of red, etc., are absent, or very imperfectly dereloped, or, according to the other theory, that the red-green substance is absent from the retina. Other varieties of color blindness in which the other color-perceiving elements are absent have been shown to exist occasionally.

\section{The Reciprocal Action of Different Parts of the Retina.}

Although each elementary part of the retina represents a distinct portion of the field of vision, yet the different elementary parts, or sensi- 
tive points of that membrane, have a certain influence on each other; the particular condition of one influencing the other, so that the image perceived by one part is modified by the image depicted in the other. The phenomena which result from this relation between the different parts of the retina, may be arranged in two classes: the one including those where the condition existing in the greater extent of the retina is imfarted to the remainder of that membrane; the other, consisting of those in which the condition of the larger portion of the retina excites, in the less extensive portion, the opposite condition.

1. When two opposite impressions occur in contiguous parts of an image on the retina, the one impression is, under certain circumstances, modified by the other. If the impressions occupy each one-half of the image, this does not take place; for in that case, their actions are equally balanced. But if one of the impressions occupies only a small part of the retina, and the other the greater part of its surface, the latter may, if long continued, extend its influence over the whole retina, so that the opposite less extensive impression is no longer perceived, and its place becomes occupied by the same sensation as the rest of the field of rision. Thus, if we fix the eye for some time upon a strip of colored paper lying upon a white surface, the image of the colored object, especially when it falls on the lateral parts of the retina will gradually disappear, and the white surface be seen in its place.

2. In the second class of phenomena, the affection of one part of the retina influences that of another part, not in such a manner as to obliterate it, but so as to cause it to become the contrast or opposite of itself. Thus a gray spot upon a white ground appears darker than the same tint of gray would do if it alone occupied the whole field of vision, and a shadow is always rendered deeper when the light which gives rise to it becomes more intense, owing to the greater contrast.

The former phenomena ensue gradually, and only after the images have been long fixed on the retina; the latter are instantaneous in their production, and are permanent.

In the same way, also, colors may be produced by contrast. Thus, a very small dull gray strip of paper, lying upon an extensire surface of any bright color, does not appear gray, but has a faint tint of the color which is the complement of that of the surrounding surface. A strip of gray paper upon a green field, for example, often appears to hare a tint of red, and when lying npon a red surface, a greenish tint; it has an orange-colored tint upon a bright blue surface, and a bluish tint upon an orange-colored surface; a yellowish color upon a bright violet, and a violet tint upon a bright yellow surface. The color excited thus, as a contrast to the exciting color, being wholly independent of any rays of the corresponding color acting from without upon the retina, must arise as 
an opposite or antagonistic condition of that membrane; and the opposite conditions of which the retina thus becomes the subject would seem to balance each other by their reciprocal reaction. A necessary condition for the production of the contrasted colors is, that the part of the retina in which the new color is to be excited, shall be in a state of comparative repose; hence the small object itself must be gray. A second condition is, that the color of the surrounding surface shall be very bright, that is, shall contain much white light.

\section{Bixoctlar Vision.}

Although the sense of sight is exercised by the two eyes, yet the impression of an object conveyed to the mind is single. Various theories have been advanced to account for this phenomenon.

By Gall it was supposed that we do not really employ both eyes simultaneously in vision, but always see with only one at a time. This especial employment of one eye in vision certainly occurs in persons whose eyes are of very unequal focal distance, but in the majority of individuals both eyes are simultaneously in action, in the perception of the same object; this is shown by the double images seen under certain conditions. If two fingers be held up before the eyes, one in front of the other, and rision be directed to the more distant, so that it is seen singly, the nearer will appear double; while, if the nearer one be regarded, the most distant will be seen double; and one of the double images in each case will be found to belong to one eye, the other to the other eye.

Diplopia.-Single vision results only when certain parts of the two retinæ are affected simultaneously; if different parts of the retinæ receive the image of the object, it is seen double. This may be readily illustrated as follows:- the eyes are fixed upon some near object, and one of them is pressed by the thumb so as to be turned slightly in or out; two images of the object (Diplopia) are at once perceived, just as is frequently the case in persons who squint. This diplopia is due to the fact that the images of the object do not fall on corresponding points in the two retinæ.

The parts of the retinæ in the two eyes which thus correspond to each other in the property of referring the images which affect them simultaneously to the same spoi in the field of vision, are, in man, just those parts which would correspond to each other, if one retina were placed exactly in front of, and over the other (as in fig. 436). Thus, as we have noticed in speaking of the distribution of the optic nervefibres, the temporal portion of one eye corresponds to, or, to use a better term, is identical with the nasal portion of the other eye; or $a$ of the 
eye A (fig. 436), with $\alpha^{\prime}$ of the eye B. The upper part of one retina is also identical with the upper part of the other; and the lower parts of the two eyes are identical with each other. The distribution of the optic nerve-fibres correspond with their distribution. The identical points on the upper and lower parts of the retinæ may also be shown by the following simple experiment.

Pressure upon any part of the ball of the eye, so as to affect the retina, produces a luminous circle, seen at the opposite side of the field of vision to that on which the pressure is made. If, now, in a dark room, we press with the finger at the upper part of one eye, and at the lower part of the other, two luminous circles are seen, one above the other; so, also, two figures are seen when pressure is made simultaneously on the two outer or the two inner sides of both eyes. It is certain, therefore, that neither the upper part of one retina and the lower part of the other are identical, nor the outer lateral parts of the two retinæ, nor their inner lateral portions. But if pressure be made with the fingers upon

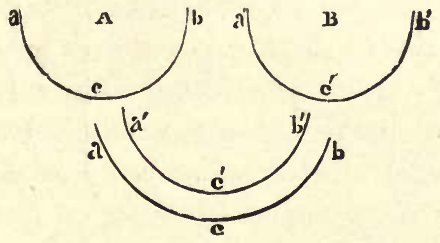

Fig. 436.-Diagram to show the corresponding parts of both retina.

both eyes simultaneously at their lower part, one luminous ring is seen at the middle of the upper part of the field of vision; if the pressure be applied to the upper part of both eyes a single luminous circle is seen in the middle of the field of vision below. So, also, if we press upon the outer side $a$ of the eye $A$, and upon the inner side $a^{\prime}$ of the eye B, a single spectrum is produced, and is apparent at the extreme right of the field of vision; if upon the point $b$ of one eye, and the point $b^{\prime}$ of the other, a single spectrum is seen to the extreme left.

The spheres of the two retinæ may, therefore, be regarded as lying one over the other, as in c, fig. 436 ; so that the left portion of one eye lies over the identical left portion of the other eye, the right portion of one eye over the identical right portion of the other eye; and with the upper and lower portions of the two eyes, $a$ lies over $a^{\prime}, b$ over $b^{\prime}$, and $c$ over $c^{\prime}$. The points of the one retina intermediate between $a$ and $c$ are again identical with the corresponding points of the other retina between $a^{\prime}$ and $c^{\prime}$; those between $b$ and $c$ of the one retina, with those between $b^{\prime}$ and $c^{\prime}$ of the other. If the axes of the eyes, A and B (fig. 43\%), be so directed that they meet at $a$, an object at $a$ will be seen singly, for the 
point $a$ of the one retina, and $\alpha^{\prime}$ of the other are identical. So, also, if the object $\beta$ be so situated that its image falls in both eyes at the same distance from the central point of the retina,-namely, at $b$ in the one eye, and at $b^{\prime}$ in the other, $-\beta$ will be seen single, for it affects identical parts of the two retinæ. The same will apply to the object $\gamma$.

In quadrupeds, the relation between the identical and non-identical parts of the retina cannot be the same as in man; for the axes of their eyes generally diverge, and can never be made to meet in one point of an object. When such an animal regards an object situated directly in front of it, the image of the object must fall, in both eyes, on the outer portion of the retinæ. Thus the image of the object $a$ (fig. 438) will fall at $a^{\prime}$ in one, and at $a^{\prime \prime}$ in the other: and these points $a^{\prime}$ and $a^{\prime \prime}$ must be identical. So, also, for distinct and single vision of objects, $b$ or $c$, the

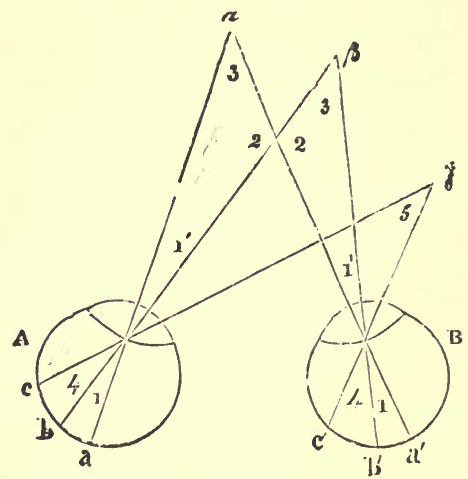

Fig. 437 .

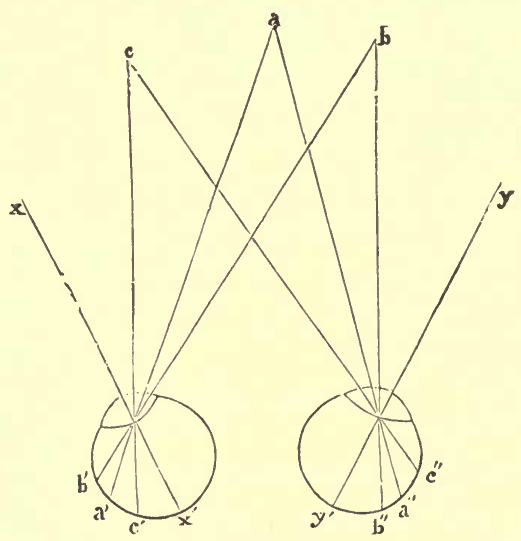

Fig. 438.

Fig. 437.-Diagram to show the simultaneous action of the eyes in viewing objects in different directions.

Fig. 438.-Diagram to show the corresponding parts of the retina in the horse.

points $b^{\prime}$ and $b^{\prime \prime}$ or $c^{\prime} c^{\prime \prime}$, in the two retinæ, on which the images of these objects fall, must be identical. All points of the retina in each eye which receive rays of light from lateral objects only, can have no corresponding identical points in the retina of the other eye; for otherwise two objects, one situated to the right and the other to the left, would appear to lie in the same spot of the field of vision. It is probable, therefore, that there are in the eyes of animals, parts of the retinæ which are identical, and parts which are not identical, i.e., parts in one which have no corresponding parts in the other eye. And the relation of the two retinæ to each other in the field of vision may be represented as in fig. 439.

The cause of the impressions on the identical points of the two retinæ giving rise to but one sensation, and the perception of a single image, 
must either lie in the structural organization of the deeper or cerebral portion of the visual apparatus, or be the result of a mental operation; for in no other case is it the property of the corresponding nerves of the two sides of the body to refer their sensations as one to one spot.

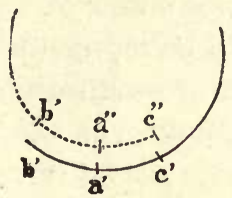

Fig. 439.

Many attempts have been made to explain this remarkable relation between the eyes, by referring it to anatomical relation between the optic nerres. The circumstance of the inner portion of the fibres of the two optic nerves decussating at the commissure, and passing to the eye of the opposite side, while the outer portion of the fibres continue their course to the eye of the same side, so that the left side of both retinæ is formed from one root of the nerves, and the right side of both retinæ from the outer root, naturally led to an attempt to explain the phenomenon by this distribution of the fibres of the nerves. And this explanation is favored by cases in which the entire of one side of the retina, as far as the central point in both eyes, sometimes becomes insensible. But Müller has endeavored to show the inadequateness of this theory to explain the phenomenon, unless it be supposed that each fibre in each cerebral portion of the optic nerves divides in the optic commissure in to two

A

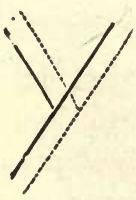

B

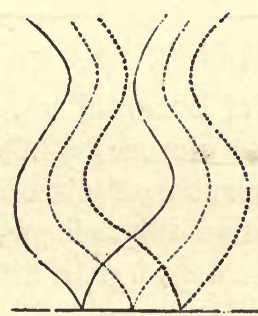

$\mathrm{C}$

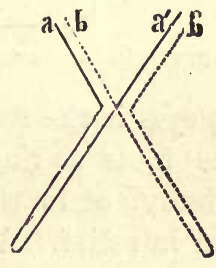

Fig. 440:-Diagrams to illustrate three theories to explain the action of symmetrical parte of branches for the identical points of the two retinæ, as is shown in A, fig. 440. But there is no foundation for such supposition.

By another theory it is assumed that each optic nerve contains exactly the same number of fibres as the other, and that the corresponding fibres of the two nerves are united in the sensorium (as in fig. 440, B). But in this theory no account is taken of the partial decussation of the fibres of the nerves in the optic commissure. 
According to a third theory, the fibres $a$ and $a^{\prime}$, fig. 440, c, coming from identical points of the two retinæ, are in the optic commissure brought into one optic nerve, and in the brain either are united by a loop, or spring from the same point. The same disposition prevails in the case of the identical fibres $b$ and $b^{\prime}$. According to this theory, the left half of each retina would be represented in the left hemisphere of the brain, and the right half of each retina in the right hemisphere.

Another explanation is founded on the fact, that at the anterior part of the commissure of the optic nerve, certain fibres pass across from the distal portion of one nerve to the corresponding portion of the other nerves, as if they were commissural fibres forming a connection between the retinæ of the two eyes. It is supposed, indeed, that these fibres may connect the corresponding parts of the two retinæ, and may thus explain their unity of action; in the same way that corresponding parts of the cerebral hemispheres are believed to be connected together by the commissural fibres of the corpus callosum, and so enabled to exercise unity of function.

Judgment of Solidity.-On the whole, it is probable, that the power of forming a single idea of an object from a double impression conveyed

A

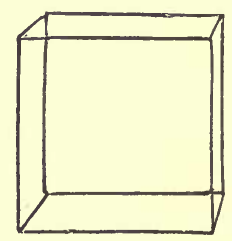

B

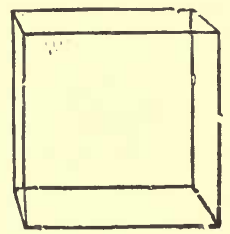

Fig. 441. - Diagrams to illustrate how a judgment of a figure of three dimensions is obtained.

by it to the eyes is the result of a mental act. 'This view is supported' by the same facts as those employed by Wheatstone to show that this. power is subservient to the purpose of obtaining a right perception of bodies raised in relief. When an object is placed so near the eyes that to view it the optic axes must converge, a different perspective projection of it is seen by each eye, these perspectives being more dissimilar as the convergence of the optic axes becomes greater. Thus, if any figure of three dimensions, an outline cube, for example, be held at a moderate distance before the eyes, and viewed with each eye successively while the head is kept perfectly steady, A (fig. 441) will be the picture presented to the right eye, and в that seen by the left eye. Wheatstone has shown that on this circumstance depends in a great measure our conviction. of the solidity of an object, or of its projection in relief. If different perspective drawings of a solid body, one representing the image seen by the right eye, the other that seen by the left (for example, the drawing: 
of a cube, A, B, fig. 441) be presented to corresponding parts of the two retinæ, as may be readily done by means of the stereoscope, the mind will perceive not merely a single representation of the object, but a body projecting in relief, the exact counterpart of that from which the drawings were made.

By transposing two stereoscopic pictures a reverse effect is produced; the elevated parts appear to be depressed, and vice versa. An instrument contrived with this purpose is termed a pseudoscope. Viewed with this instrument a bust appears as a hollow mask, and as may readily be imagined the effect is most bewildering.

There can be no doubt in order that the image of an object should fall upon corresponding points in the two retinæ, it is essential that the movements of the eyes should be accurately co-ordinated, and the method of this co-ordination is not so easily understood when examined carefully. Thus, suppose the eyes be directed downward and to the left. On the left side, the inferior rectus, the external rectus, and the superior oblique would contract, and, on the right side the inferior rectus, internal rectus, and superior oblique. In other words, a different set of muscles on either side, and supplied to a certain extent by different nerves. There must be some co-ordinating centre for these binocular movements. It is thought that this centre is localized in the anterior corpus quadrigeminum, since stimulation of it causes conjugal lateral movement of the visual axes to the opposite side, and stimulation at another spot produces movements downward and inward. The posterior longitudinal bundle of fibres described as found in the pons and crus, appears to be concerned in some way with the simultaneous movement of the eyes; it appears to unite the nuclei of the three nerves to the ocular muscles, the sixth, fourth, and third. In it are said to be contained fibres from the sixth nerve of the opposite side which go to the nucleus of the third nerve of the same side; and this would serve to connect the nerve supply of the internal rectus of one side, and the external rectus of the other side. It appears, however, that there is no evidence to assume that the fibres of the sixth nerve decussate, but those of the fourth nerve do entirely, and those of the third, partially. 


\section{CHAPTER XVIII.}

\section{THE REPRODUCTIVE ORGANS.}

BEFore describing the method of Reproduction, or the way which the species is propagated, it will be adrisable to describe

\section{The Genital Orgaxs of the Female.}

The female organs of generation (fig. 442) consist of two ovaries, the function of which is the formation of ova; of a Fallopian tube, or oviduct, connected with each ovary, for the purpose of conducting the ovum from the ovary to the uterus in the cavity of which, if impreg-

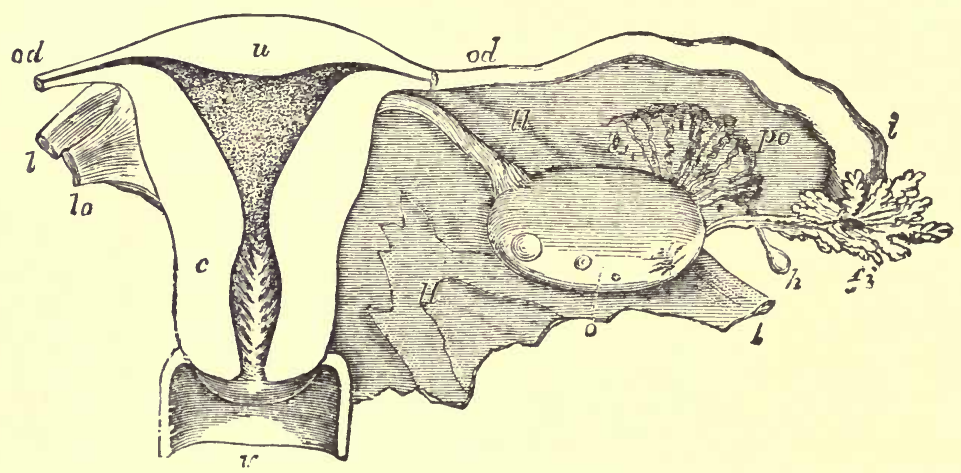

Fig. 442. - Diagrammatic view of the uterus and its appendages, as seen from behind. The uterus and upper part of the vagina have been laid open by removing the posterior wall; the Fallopian tube, round ligament, and ovarian ligament have been cut short, and the broad ligament removed on the left side; $u$, the upper part of the uterus; $c$, the cervix opposite the os in. ternum; the triangular shape of the uterine cavity is shown, and the dilatation of the cervical cavity with the rugæ termed arbor vitæ; $v$, upper part of the vagina; od, Fallopian tube or oviduct; the narrow communication of its cavity with that of the cornu of the uterus on each sicle is seen; $l$, round ligament; lo, ligament of the ovary; o, ovary; $i$, wide outer part of the right Fallopian tube; $f$, its fimbriated extremity; po, parovarium; $h$, one of the hydatids frequently found connected with the broad ligament. 1/2. (Allen Thomson.)

nated, it is retained until the embryo is fully developed, and fitted to maintain its existence independently of internal connection with the parent; and, lastly, of a canal, or vagina, with its appendages, for the reception of a male organ in the act of copulation, and for the subsequent discharge of the fotus.

The Ovaries. - The ovaries are two oval compressed bodies, situated in the cavity of the pelvis, one on each side, and are adherent to the posterior surface of the broad ligament by their anterior border. This 
border of the ovary is called the hilum, and it is at this point that the blood-vessels and nerves enter it. Each ovary measures about an inch and a half in length $(3.75 \mathrm{~cm}$.$) , three quarters of an inch in width$ $(1.86 \mathrm{~cm}$.$) , and nearly half an inch (1.25 \mathrm{~cm}$.$) in thickness, and is$ attached to the uterus by a narrow fibrous cord (the ligament of the ovary), and, more slightly, to the Fallopian tubes, by one of the fimbriæ into which the walls of the extremity of the tube expand.

Structure.-A layer of condensed connective tissue, called the tunica albuginea, surrounds the ovary, and this is covered on the outside by epithelium (germ-epithelium), the cells of which although continuous with,

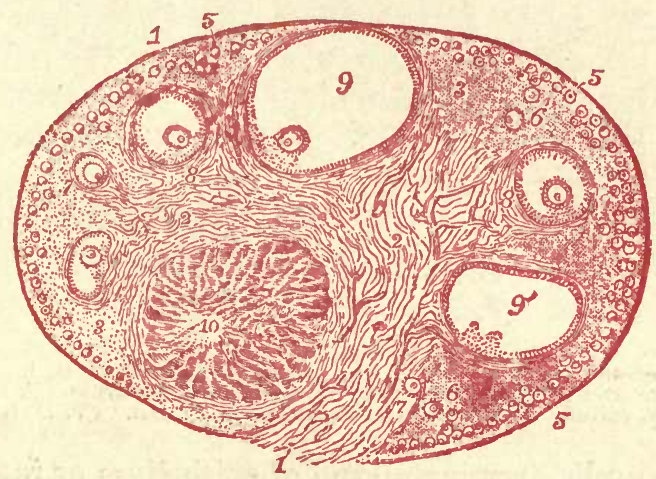

Fig. 443. - View of a section of the ovary of the cat. 1, outer covering and free border of the ovary; $1^{\prime}$, attached border; 2, the ovarian stroma, presenting a fibrous and vascular structure; 3 , granular substance lying external to the fibrous stroma; 4 , blood-vessels; 5 , ovigerms in their earliest stages occupying a part of the granular layer near the surface; 6 , ovigerms which have begun to enlarge and to pass more deeply into the ovary; 7 , ovigerms round which the Graafian follicle and tunica granulosa are now formed, and which have passed somewhat deeper into the ovary and are surrounded by the fibrous stroma; 8 , more advanced Graafian follicle with the ovum imbedded in the layer of cells constituting the proligerous disc; 9 , the most ad. vanced follicle containing the ovum, etc. ; $9^{\prime}$, a follicle from which the ovum has accidentally escaped; 10, corpus luteum. $\times 6$. (Schrön.)

and originally derived from, the squamous epithelium of the peritoneum, are short columnar (A, fig. 444).

The internal structure of the organ consists of a peculiar soft fibrous tissue-a kind of undeveloped connective tissue, with long nuclei closely resembling unstriped muscle (C, fig.444) - or stroma, abundantly supplied with blood-vessels, and having embedded in it,in various stages of development, numerous minute follicles or vesicles, the Graafian follicles, or sacculi, containing the ova (fig. 444).

If the ovary be examined at any period between early infancy and advanced age, but especially during that period of life in which the power of conception exists, it will be found to contain a number of these vesicles. Immediately after the tunica albuginea (fig. 444) they are small and numerous, either arranged as a continuous layer, as in the cat or rabbit, or in groups, as in the human ovary. These small follicles 
embedded in the soft stroma of fine connective tissue and unstriped muscle form here the cortical layer; they are sometimes called ovisacs.

Each of the small follicles of this layer has an external membranous envelope, or membrana propria. This envelope or tunic is lined with a

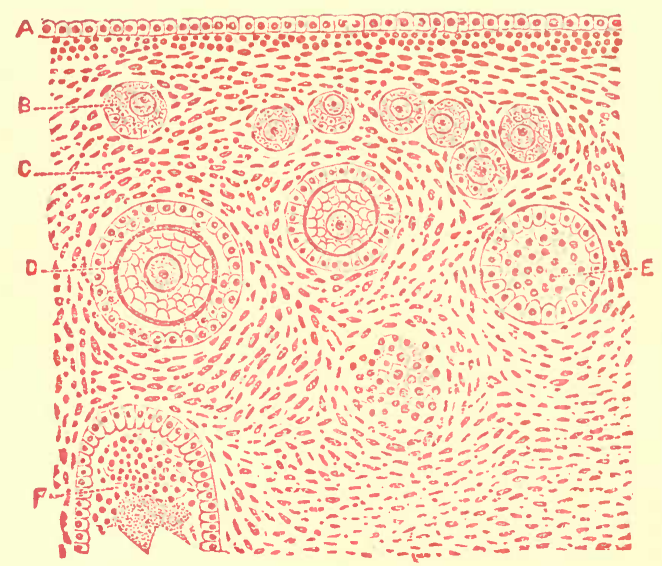

Fig. 444.- Section of the ovary of a cat. A, germinal epithelium; B, immature Graafian follicle; C, stroma of ovary; D, vitelline membrane containing the ovum; E, Graafian follicle showing lining cells; F, follicle from which the ovum has fallen out. (V.'D. Harris.)

layer of nucleated cells, forming a kind of epithelium or internal tunic, and named the membrana granulosa. The cavity of the follicle is filled up by a nucleated mass of protoplasm inclosed in a very delicate membrane, which is the Ovum. The large spherical nucleus contains one or more nucleoli. The nucleus is known as the germinal vesicle, and the nucleolus as the germinal spot.

The central portion of the stroma of the ovary extends from the cortical layer to the hilum of the organ, at which enter the numerous arteries, fibrous tissue, and unstriped muscle, forming a highly vascular zona vasculosa. Within this central zone are contained the fully-developed Graafian follicles, varying in size however, but considerably larger than those of the cortical layer. In these follicles the cavity is not nearly filled by the ovum, which is attached at one side to the zona granulosa by a collection of small cells, the discus proligerus, the remainder of the cavity being filled with fluid, the liquor folliculi. The envelope of the ovum, or zona pellucida, is much thicker. The zona granulosa is formed of several layers of cells, instead of one only. Its membrana propria is much thicker, so as to form a distinct fibrous investment; the membrana fibrosa and the blood-vessels surrounding it are numerous, and may be said to form a membrana vasculosa about it.

The human ovum measures about $\frac{1}{120}$ of an inch (about $.2 \mathrm{~mm}$.) in diameter. Its external investment, or the zona pellucida, or vitelline 
membrane, is a transparent membrane, about $\frac{1}{2500}$ of an inch $(10 \mu)$ in thickness, which under the microscopic appears as a bright ring (fig. 445), bounded externally and internally by a dark outline. Within this transparent investment or zona pellucida, and usually in close contact with it, lies the yolk or vitellus, which is composed of granules and globules of various sizes, imbedded in a more or less fluid substance. The smaller granules, which are the more numerous, resemble in their appearance, as well as their constant motion, pigment-granules. The larger granules or globules, which have the aspect of fat-globules, are in greatest number at the periphery of the yolk. The number of the granules is greatest in the ova of carnivorous animals. In the human ovum their quantity is comparatively small.

In the substance of the yolk is imbedded the germinal vesicle, or vesicula germinativa, $\frac{1}{500}$ of an inch $(.05 \mathrm{~mm}$.) (fig. 445$)$. The vesicle is of greatest relative size in the smallest ova, and is in them surrounded closely by the yolk, nearly in the centre of which it lies. During the development of the ovum, the germinal vesicle increases in size much less rapidly than the yolk, and comes to be placed near to its surface. It consists of a fine, transparent, structureless membrane, containing a clear, watery fluid, in which are sometimes a few granules; and at that part of the periphery of the germinal vesicle which is nearest to the periphery of the yolk is situated the germinal spot, or macula germinativa, of a finely

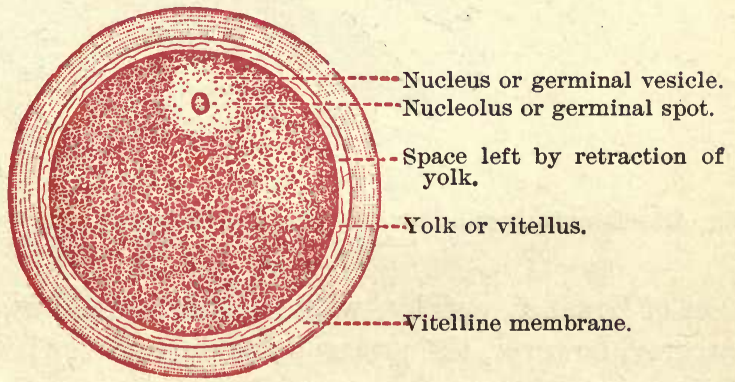

Fig. 445. - Semidiagrammatic represention of a human ovum, showing the parts of an animal cell. (Cadiat.)

granulated appearance and of a yellowish color, strongly refracting the rays of light.

Such are the parts of which the Graafian follicle and its contents, including the ovum, are composed. With regard to the mode and order of development of these parts there is considerable uncertainty.

The Graafian follicles are formed in the following manner:-The embryonic ovary is covered with short columnar cells, or the so-called germinal epithelium. The cells of this layer undergo proliferation, so as to form several strata, and grow into the ovarian stroma as longer or shorter 
columns or tubes. By degrees these tubes become cut off from the surface epithelium, and form cell nests, small, if near the surface, larger if in the depth of the stroma. The nests increase in size from multiplication of their cells, and may even give off new nests laterally by constriction of them in various directions. Certain of the cells of the germinal epithelium enlarge, and form ova; and the formation of ova also takes place in the nests within the stroma. The ova of a nest may multiply by division. The small cells of a nest surround the ova, and form their membrana granulosa, and the stroma growing up separates the surrounded ova into so many Graafian follicles. The other layers, namely, the membrana fibrosa and the membrana vasculosa, are derived from the stroma.

The smallest follicles are formed at the surface, and make up the cortical layer. It is said by some that the superficial follicles as they ripen become more deeply placed in the ovarian stroma; and, again, that as they increase in size, they make their way toward the surface (fig. 443).

When mature, they form little prominences on the exterior of the ovary, covered only by a thin layer of condensed fibrous tissue and epithelium. Only a few follicles ever reach maturity.

From the earliest infancy, and through the whole fruitful period of life, there appears to be a constant formation, development, and matura-

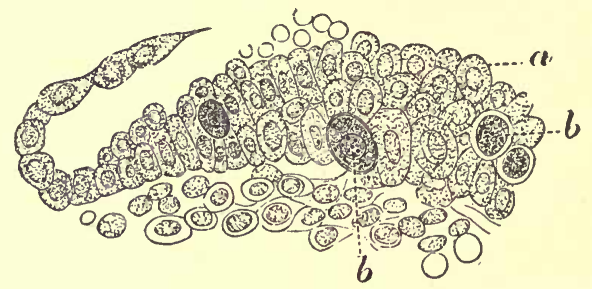

Fig. 446. - Germinal epithelium of the surface of the ovary of five days' chick. a, small oroblasts; $b$, larger ovoblasts. (Cadiat.)

tion of Graafian vesicles, with their contained ova. Until the period of puberty, however, the process is comparatively inactive; for, previous to this period, the ovaries are small and pale, the Graafian vesicles in them are very minute, and probably never attain full development, but soon shrivel and disappear, instead of bursting, as matured follicles do; the contained ova are also incapable of being impregnated. But, coincident with the other changes which occur in the body at the time of puberty, the ovaries enlarge, and become very vascular, the formation of Graafian vesicles is more abundant, the size and degree of development attained by them are greater, and the ova are capable of being fecundated.

The Fallopian Tubes (Oviducts). - The Fallopian tubes are about four inches in length $(10 \mathrm{~cm}$.), and extend between the ovaries and the 
upper angles of the uterus. At the point of attachment to the uterus, each tube is very nariow; but in its course to the ovary it increases to about an eighth of an inch ( $3 \mathrm{~mm}$.) in thickness; at its distal extremity, which is free and floating, it bears a number of fimbrice, one of which, longer than the rest, is attached to the ovary. The canal by which each tube is traversed is narrow, especially at its point of entrance into the uterus, at which it will scarcely admit a bristle; its other extremity is wider, and opens into the cavity of the abdomen, surrounded by the zone of fimbriæ. Externally, the Fallopian tube is invested with peritoneum; internally, its canal is lined with mucous membrane, which is apt to be thrown into numerous longitudinal folds, covered with ciliated epithelium: between the peritoneal and mucous coats the walls are composed, like those of the uterus, of fibrous tissue and unstriped muscular fibros, chiefly circular in arrangement.

The Uterus.- The uterus ( $u . c$, fig. 442) is a somewhat pyriform shaped organ, and in the unimpregnated state is about three inches (\%.5 $\mathrm{cm}$.) in length, two $(5 \mathrm{~cm}$. $)$ in breadth at its upper part or fundus, but at its lower pointed part, neck or cervix, only about half an inch $(1.25 \mathrm{~cm}$.). The part between the fundus and neck is termed the body of the uterus: it is about an inch $(2.5 \mathrm{~cm}$.) in thickness.

Structure.-The uterus is constructed of three principal layers, or coats-serous, fibrous and muscular, and mucous. (a) The serous coat, which has the same general structure as the peritoneum, covers the organ before and behind, but is absent from the front surface of the neck. (b) The middle coat is composed of unstriped muscle, arranged in the human uterus in three layers from without inward, longitudinal, circular, oblique and circular. They become enormously developed during pregnancy. The arteries and veins are found in large numbers in the outer part of their coat, so as to form almost a special vascular coat. (c) The mucous membrane of the uterus is lined by columnar ciliated epithelium, which extends also to the interior of the tubular glands, of which the mucous membrane is largely made up.

In the cervix uteri the mucous membrane is arranged in permanent longitudinal folds, palmoe plicatce. The glands of this part are of the tubulo-racemose type, branching repeatedly and extending deeply into the substance of the cervix. They are lined by columnar epithelium, and open on the ridges and furrows of the mucous membrane. They secrete a thick glairy mucus, resembling unboiled white of egg.

The mucous membrane of the cavity of the body of the uterus forms a thin membrane about $\frac{1}{25}$ inch $(1 \mathrm{~mm}$.) thick, and is covered on its surface by columnar ciliated epithelium. Imbedded in its substance are numerous simple tubular glands set somewhat obliquely and lined with columnar ciliated epithelium. These glands often bifurcate at their 
lower ends. The glands are imbedded in a delicate connective tissue, consisting of round and spindle-shaped cells.

The cavity of the uterus corresponds in form to that of the organ itself: it is very small in the unimpregnated state, the sides of its mucous surface being almost in contact. Into its upper part, at each side, opens the canal of the corresponding Fallopian tube: below, it communicates with the vagina by a fissure-like opening in its neck, the os uteri, the margins of which are distinguished into two lips, an anterior and posterior.

The Vagina is a membranous canal, five or six inches (12.5 to 15 $\mathrm{cm}$.) long, extending obliquely downward and forward from the neck of the uterus, which it embraces, to the external organs of generation. It is lined with mucous membrane, covered with stratified squamous epithelium, which in the ordinary contracted state of the canal is thrown into transverse folds. External to the mucous membrane the walls of the vagina are constructed of unstriped muscle and fibrous tissue, within which in the submucosa, especially around the lower part of the tube, is a layer of erectile tissue. This exists also in the mucosa. The lower extremity of the vagina is embraced by an orbicular muscle, the sphincter vagino; its external orifice, in the virgin, is partially closed by a fold or ring of mucous membrane, termed the hymen. The external organs of generation consist of the clitoris, a small elongated body, situated above and in the middle line, and constructed of two erectile masses or corpora cavernosa. They are not perforated by the urethra; of two folds of mucous membrane, termed labia interna, or nymphac; and, in front of these, of two other folds, the labia externa, or pudenda, formed of the external integument, and lined internally by mucous membrane. Between the nymphæ and beneath the clitoris is an angular space, termed the vestibule, at the centre of whose base is the orifice of the meatus urinarius. Numerous mucous follicles are scattered beneath the mucous membrane composing these parts of the external organs of generation; and at the side of the lower part of the ragina are two larger lobulated glands, vulvo-vaginal or Duverney's glands, which are analogous to Cowper's glands in the male. The ducts of these glands are about $\frac{1}{2}$ inch (12.5 mm.) long and open immediately external to the hymen at the mid-point of the lateral wall of the vaginal orifice. The vulvo-vaginal glands secrete a thick brownish mucus.

\section{The Genital Organs of the Male.}

The male organs of generation comprise the two Testes, in which the semen is formed; each with a duct, the Vas Deferens, and accessory Vesicula Seminalis; the Penis, an erectile organ, through which the 
semen as well as the trine is discharged. The Prostate gland, the exact function of which is not understood, is generally included in the same class.

The Testes.-The secreting structure of the testicle and its duct are disposed of in two contiguous, parts (1) the body of the testicle proper, inclosed within a thick and tough white fibrous membrane, the tunica albuginea, on the outer surface of which is the serous covering formed by the tunica vaginalis, and (2) the epididymis and vas deferens.

The Vas deferens, or duct of the testicle, which is about two feet $(60 \mathrm{~cm}$.) in length, is constructed externally of connective tissue, and internally is lined by a mucous membrane, covered with columnar epithelium; while between these two coats is a middle coat, very firm and tough, made up of unstriped muscle, chiefly arranged longitudinally, but also containing some circular fibres. When followed back to its origin, the vas deferens is found to pass to the lower part of the epididymis, with

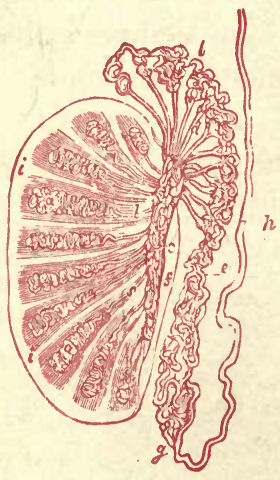

Fig. 447.

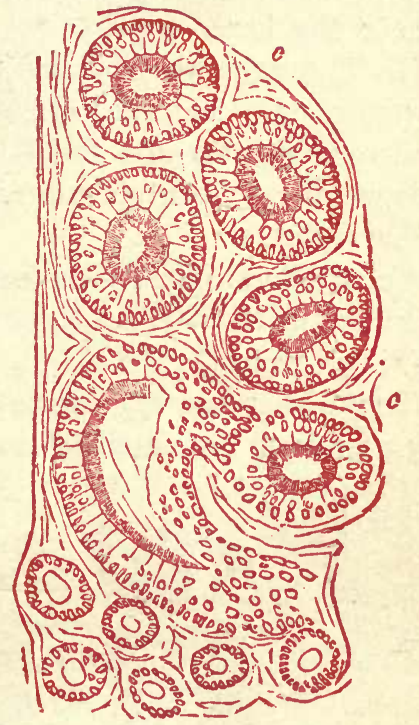

Fig. 448.

Fig. 447.-Plan of a vertical section of the testicle, showing the arrangement of the ducts. The true length and diameter of the ducts have been disregarded. $a \alpha$, tubuli seminiferi coiled up in the separate lobes; $b$, tubuli recti or vasa recta; $c$, rete testis; $d$, vasa efferentia ending in the coni vasculosi; $l, e, g$, convoluted canal of the epididymis; $h$, vas deferens; $f$, section of the back part of the tunica albuginea; $i, i$, fibrous processes running between the lobes; $s$, mediastinum.

Fig, 448. - Section of the epididymis of a dog. - The tube is cut in several places, both transversely and obliquely; it is seen to be lined by a ciliated epithelium, the nuclei of which are well shown. $c$, connective tissue. (Schofield.)

which it is directly continuous (fig. $44^{\%}$ ), and assumes there a much smaller diameter with an exceedingly tortuous course.

The Epididymis, which is lined, except at its lowest part, by co- 
lumnar ciliated epithelium (fig. 447), is commonly described as consisting (fig. 447) of aglobus minor $(g)$, the body $(e)$, and the globus major (l.) When unravelled it is found to be constructed of a single tube, measuring about twenty feet in length.

At the globus major this duct divides into ten or twelve small branches, the convolutions of which form coniform masses, named Coni vasculosi; and the ducts continued from these, the Vasa efferentia, after anastomosing, one with another in what is called the Rete testis, lead finally as the Tubuli rectior Vasa recta to the seminal tubules (tubuli seminiferi), which form the proper substance of the testicle. The epithelium lining the coni vasculosi and rasa efferentia is columnar and ciliated; that of the rete testis is squamous.

The seminal tubules are arranged in lobules, separated from one another by incomplete fibrous septa or cords, which pass from the front of the tunica albuginea internally to a firm incomplete vertical septum of thick extending fibrous tissue at the posterior border, from the upper to near the lower part, called the corpus Highmori, or mediastinum testis. Through this very firm fibrous tissue pass the seminal tubes from the vasa recta. The tunica albuginea is covered by a very fine plexus of blood-vessels internally, derived from the spermatic vessels. The fibrous cords which may contain unstriped muscle are also covered with a similar capillary plexus.

Tubuli Seminiferi.-The seminal tubes, which compose the parenchyma of the testicle, are loosely arranged in lobules between the connective tissue septa.

They are relatively large, very wavy, and much convoluted; and they possess a few lateral branches, by which they become connected

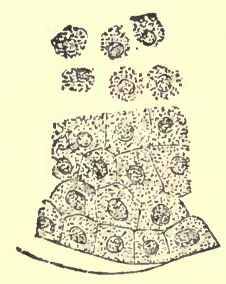

A

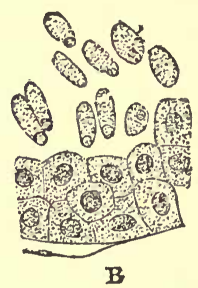

B

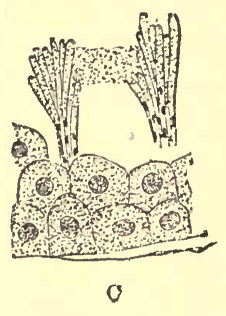

Fig. 449.-From a section of the testis of dog, showing portions of seminal tubes. A, seminal epithelial cells, and numerous small cells loosely arranged; B, the small cells or spermatoblasts converted into spermatozoa; groups of these in a further stage of development. (Klein.)

into a network. They form terminal loops, and in the peripheral portion of the testis the tubules are possessed of minute lateral cæcal branchlets.

Each seminal tubule in the adult testis is limited by a membrana 
propria, which appears as a hyaline elastic membrane, but which is really made up of several incomplete layers of flattened cells, containing oval flattened nuclei at regular intervals. Inside this membrana propria are several layers of epithelial cells, the seminal cells (fig. 449). These consist of two or more layers, the outermost being situated next the membrana propria. These cells are of two kinds, those that are in

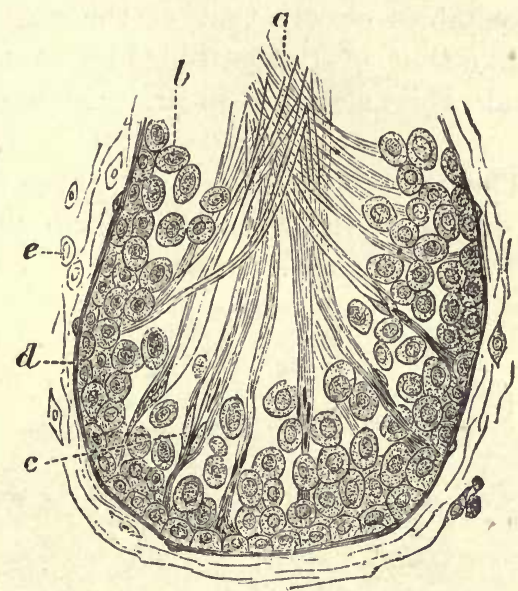

Fig 450.-Section of a tubule of the testicle of a rat, to show the formation of the spermatozoa; $a$ spermatozoa; $b$, seminal cells; $c$, spermatoblasts, to which the spermatozoa are still adherent; $d$, membrana propria; $e$, fibro-plastic elements of the connective tissue. (Cadiat.)

a resting state, which generally form a complete layer, and those that are in a state of division, of which there may be two layers. The latter are called mother cells, and the smaller cells resulting from their division are called daughter cells or spermatoblasts. From these the spermatozoa are formed, their head corresponding with the nuclei of the daughter cells; and during their development they lie in groups (figs. 449,450 ), and are supported by irregular masses of so-called nutritive cells; but when fully formed, they become detached, and fill the lumen of the seminiferous tubule (fig. 450). This detachment is effected by the liquefaction of the nutritive cells in which the groups of spermatozoa are imbedded.

In the fine connective tissue which supports the tubules of the testis, are to be found flattened and nucleated epithelial cells, probably the remains of the Wolffian body. The lymphatics of the testes are numerous, and may be injected by inserting the needle of an injecting syringe into the tunica albuginea, and pressing in the injection with slight effort.

The Vesiculæ Seminales. - The vesiculæ seminales have the appearance of outgrowths from the vasa deferentia. Each vas deferens, just 
before it enters the prostate gland, through part of which it passes to terminate in the urethra, gives off a side branch, which bends back from it at an acute angle: and this branch dilating, variously branching, and pursuing in both itself and its branches a tortuous course, forms the vesicula seminalis.

Structure. - Each vesicula may be unravelled into a single branching tube sacculated, convoluted, and folded up.

The structure resembles closely that of the vasa deferentia. The mucous membrane, like that of the gall-bladder, is minutely wrinkled and set with folds and ridges arranged so as to give it a finely reticulated appearance.

The Penis.-The penis is composed of three long more or less cylindrical masses, inclosed in remarkably firm fibrous sheaths, of

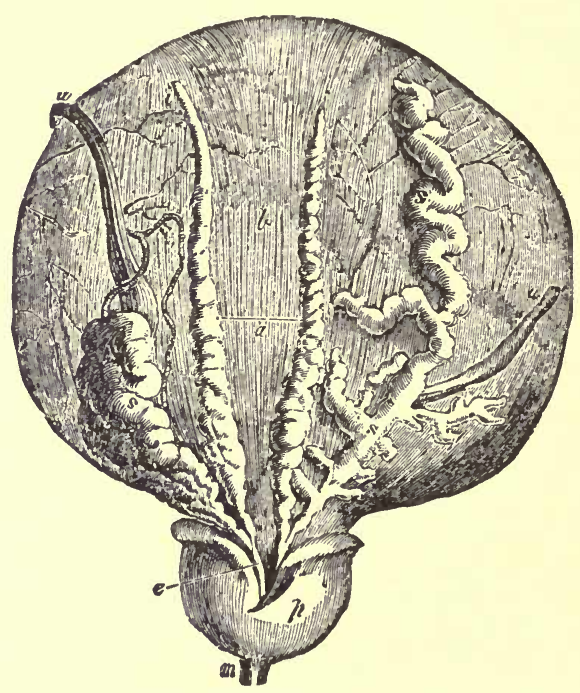

Fyg. 451. -Dissection of the base of the bladder and prostate gland, showing the vesiculæ seminales and vasa deferentia. $a$, lower surface of the bladder at the place of reflection of the peritoneum; $b$, the part above covered by the peritoneum; $i$, left vas deferens, ending in $e$, the ejaculatory duct; the vas deferens has been divided near $i$, and all except the vesical portion has been taken away; $s$, left vesicula seminalis joining the same duct; $s, s$, the right vas deferens and right vesicula seminalis, which has been unravelled; $p$, under side of the prostate gland; $m$, part of the urethra; $u, u$, the ureters (cut short), the right one turned aside. (Haller.)

which two, the corpora cavernosa, are alike, and are firmly joined together, and receive below and between them the third part, or corpus spongiosum. The urethra passes through the corpus spongiosum. The penis is attached to the symphysis pubis by its root. The enlarged extremity or glans penis is continuous with the corpus spongiosum. The integument covering the penis forms a loose fold from the junction of the glans with the body, called the prepuce or foreskin. 
Structure.-(a.) The urethra is lined by stratified pavement epithelium in the prostatic portion; in front of the bulb the epithelium becomes columnar, while at the fossa navicularis it is again lined with stratified pavement epithelium. The mucous membrane consists chiefly of fibrous connective-tissue, intermixed with which are many elastic fibres. It is surrounded by unstriped muscular tissue. In the inter-

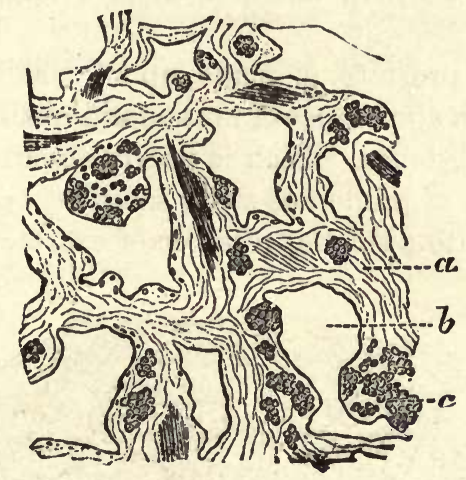

Fig. 452.-Erectile tissue of the human penis. $a$, fibrous trabeculæ with their crdinary capillaries; $b$, section of the venous sinuses; $c$, muscular tissue. (Cadiat.)

mediate portion many large veins run amongst the bundles of museular tissue. Many mucous glands, glands of Littré, are present.

(b.) The corpora cavernosa, a true erectile structure, are surrounded by a dense fibrous and elastic sheath, and from the inner surface of this, and from the septum which separates the two corpora cavernosa, pass numerous bundles of fibrous, elastic, and plain muscular fibres, called trabeculoe, and these by their anastomosis form a series of irregular spaces. These spaces are lined with endothelium, and are filled with venous blood. The inter-trabecular spaces or sinuses of one corpus cavernosum anastomose with those of the other, especially in front where the dividing septum is incomplete.

(c.) The corpus spongiosum urethræ consists of an inner portion or plexus of longitudinal veins, and of an outer or really cavernous portion identical in structure with that which has just been described. The lymphatics of the penis are very numerous, both superficially and also around the urethra. They join the inguinal glands.

The nerves, derired from the pudic nerves and hypogastric plexus, are distributed to the skin and mucous membrane and to the corpora cavernosa and spongiosum respectively. The nerves are provided with end bulbs and Pacinian corpuscles in the glans penis, and form also a dense subepithelial plexus.

Cowper's glands are two small glands, the ducts of which open into 
the second part of the urethra. They are small round bodies, of the size of a pea, yellow in color, resembling the sublingual gland; in structure they are compound tubular mucous glands.

The Prostate Gland.-The prostate is situated (fig. 451) at the neck of the urinary bladder, and incloses the commencement of the urethra. It is somewhat chestnut-shaped. It measures an inch and a half in breadth, and an inch and a quarter long, and half an inch in thickness.

Structure. -The prostate is made up of small compound tubular glands imbedded in an abundance of muscular fibres and connective tissue.

The glandular substance, which is nearly absent from the front part of the organ, consists of numerous small saccules, opening into elongated ducts, which unite into a smaller number of excretory ducts. The acini

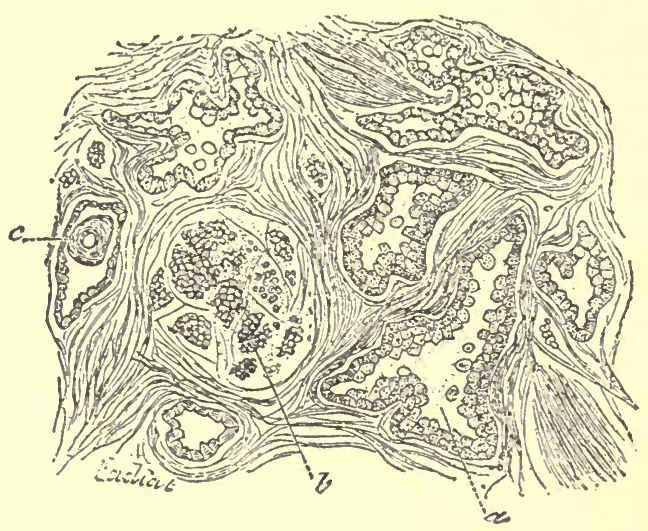

Fig. 453.-Section of a small portion of the prostate. $a$, gland duct cut across obliquely; $b$, gland structure; $c$, prostatic calculus. (Cadiat.)

of the upper part of the prostate are small and hemispherical; while in the middle and lower parts the tubes are longer and more convoluted. The acini are of two kinds, namely, those $(\alpha)$ lined with a single layer of thin and long columnar cells, each with an oval nucleus in outer part of wall; and those (b) acini resembling the foregoing, but with a second layer of small cortical, polyhedral, or fusiform cells between the membrana propria and the columnar cells. The ducts, twelve to twenty in number, open into the urethra. They are lined by a layer of columnar cells, beneath which is a layer of small polyhedral cells.

The tunica adventitia consists of dense fibrous tissue of two layers, between which is situated a plexus of veins. Large vessels pass into the interior of the organ, to form a broad, meshed, capillary system. Nerves with numerous large ganglion-cells surround the cortex. Pacinian bodies are sometimes found in the substance of the organ. 
The muscular tissue of the prostate not only forms the chief part of the stroma of the gland, but also forms a continuous layer inside the fibrous sheath, as well as a layer surrounding the urethra, which is continous with the sphincter resicæ.

\section{Physiology of the Sexual Organs.}

Of the Female. - In the process of derelopment in the orary of individual Graafian resicles, it has been already observed, that as each increases in size, it gradually approaches the surface of the ovary, and when fully ripe or mature, forms a little projection on the exterior. Coincident with the increase in size, caused by the augmentation of its liquid contents, the external envelope of the distended resicle becomes very thin and eventually bursts. By these means, the ovum and fluid contents of the resicle are liberated, and escape on the exterior of the ovary, whence they pass into the Fallopian tube or oriduct, the fimbriated processes of the extremity of which are supposed coincidentally to grasp the orary, while the aperture of the tube is applied to the part corresponding to the matured and bursting resicle.

In animals whose special capability of being impregnated occurs at regular periods, as in the human subject, and most mammalia, the Graafian vesicles and their contained ova appear to arrive at maturity, and the latter to be discharged at such periods only. But in other animals, e.g., the common fowl, the formation, maturation, and discharge of ova appear to take place almost constantly.

It has long been known, that in the so-called oviparous animals, the separation of ora from the ovary may take place independently of impregnation by the male, or eren of sexual union. And it is now established that a like maturation and discharge of ora, independently of coition, occurs in mammalia, the periods at which the matured ora are separated from the oraries and receired into the Fallopian tubes being indicated in the lower mammalia by the phenomena of heat or rut: in the human female, although not always with exact coincidence, by the phenomena of menstruation. If the union of the sexes take place, the orum may be fecundated, and if no union occur it perishes.

That this maturation and discharge occur periodically, and only during the phenomena of heat in the lower mammalia, is made probable by the facts that, in all instances in which Graafian resicles have been found presenting the appearance of recent rupture, the animals were at the time, or had recently been, in heat; that on the other hand, there is no authentic and detailed account of Graafian resicles being found ruptured in the intervals of the period of heat; and that female animals do not admit the males, and never become impregnated, except at those periods. 
Relation of Menstruation to the Discharge of Ova.-The human female is subject to the same law as the females of other mammiferous animals; her ova are matured and discharged from the ovary independent of sexual union. This maturation and discharge occur, moreover, periodically at or about the epochs of menstruation.

The evidence of the periodical discharge of ova at the menstrual periods is that in most cases in which signs of menstruation have been found in the uterus, follicles in a state of maturity or of rupture have been seen in the ovary; and although conception is not confined to the periods of menstruation, yet it is more likely to occur about a menstrual epoch than at other times.

The exact relation between the discharge of ova and menstruation is not very clear. It was formerly believed that the monthly flux was the result of a congestion of the uterus arising from the enlargement and rupture of a Graafian follicle; but though a Graafian follicle is, as a rule, ruptured at each menstrual epoch, yet several instances are recorded in which menstruation has occurred where no Graafian follicle can have been ruptured, and on the other hand cases are known where ova have been discharged in amenorrhæic women. It must therefore be admitted that menstruation is not dependent on the maturation and discharge of ova.

It was, moreover, formerly understood that ova were discharged toward the close or soon after the cessation of a menstrual flow. Observations made after death, and facts obtained by clinical investigation, however, do not support this view. Rupture of a Graafian follicle does not happen on the same day of the monthly period in all women. It may occur toward the close or soon after the cessation of a flow; but only in a small minority of the subjects examined after death was this the case. On the other hand, in almost all such subjects of which there is record, rupture of the follicle appears to have taken place before the commencement of the catamenial flow. Moreover, the custom of the Jews-a prolific race, to whom by the Levitical law sexual intercourse during the week following menstruation was forbidden-militates strongly in favor of the view that conception usually occurs before and not soon after a menstrual epoch, and necessarily, therefore, for the view that ova are usually discharged before the catamenial flow. This, together with the anatomical condition of the uterus just before the catamenia, seems to indicate that the orum fertilized is that which is discharged in connection with the first absent, and not that with the last present menstruation.

Though menstruation does not appear to depend upon the discharge of ova, yet the presence of the ovaries seems necessary for the performance of the function; for women do not menstruate when both ovaries 
have been removed by operation. Some instances have been recently recorded, indeed, of a sanguineous discharge occurring periodically from the vagina after both ovaries have been previously removed for disease; and it has been inferred from this that menstruation is a function independent of the ovary: but this evidence is not conclusive, inasmuch as it is possible that portions of ovarian tissue were left after the operation.

Source and Characters of Menstrual Discharge.-The menstrual discharge is a thin sanguineous fluid, having a peculiar odor. It is of a dark color, and consists of blood, epithelium, and mucus from the

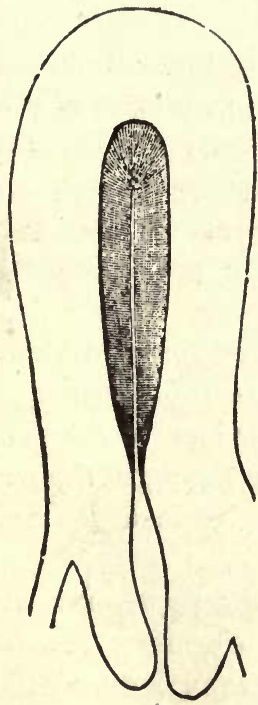

Fig. 454.

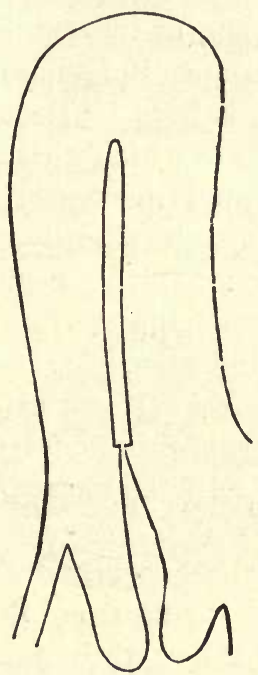

Fig. 455 .

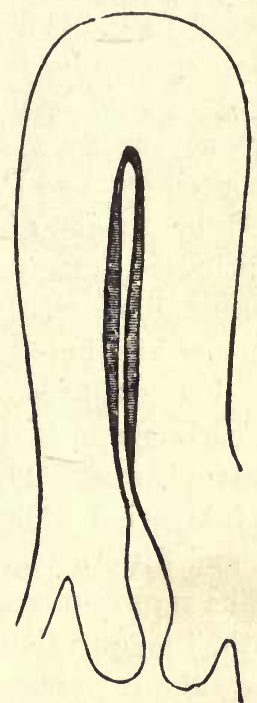

Fig. 456.

Fig. 454.-Diagram of uterus just before menstruation; the shaded portion represents the thickened mucous membrane.

Fig. 455.-Diagram of uterus when menstruation has just ceased, showing the cavity of the uterus deprived of mucous membrane.

Fig. 456. - Diagram of uterus a week after the menstrual flux has ceased: the shaded portion represents renewed mucous membrane. (J. Williams.)

uterus and vagina. The menstrual flow is preceded by a general engorgment of all the pelvic organs with blood. The cervix and vagina become darker in color and softer in texture, and the quantity of mucus secreted by the glands of the cervix and body is increased. The uterine mucous membrane is swollen and the glands are elongated and tortuous. The discharge of blood, the source of which is the mucous membrane of the body of the nterus, is probably associated with uterine contractions. There is great difference of opinion as to whether or not any of the uterine mucous membrane is normally shed during the process of menstruation. John Williams believes that the whole of the mucous membrane of the body of the uterus is thrown off at each monthly period, 
forming a true decidua menstruatis (fig. 454), while Möricke and others believe that the mucous membrane remains intact. Leopold believes that red blood corpuscles escape from the congested capillaries and undermine the superficial epithelium, and that in this way the superficial layer of the mucous membrane is eroded and subsequently regenerated. It is probable that menstruation is not a sign of the capability of being impregnated, as much as of disappointed impregnation.

Menstrual Life.-The occurrence of a menstrual discharge is one of the most prominent indications of the commencement of puberty in the female sex; though its absence even for several year's is not necessarily attended with arrest of the other characters of this period of life, or with inaptness for sexual union, or incapability of impregnation. The average time of its first appearance in females of this country and others of about the same latitude, is from fourteen to fifteen; but it is much influenced by the kind of life to which girls are subjected, being accelerated by habits of luxury and indolence, and retarded by contrary conditions. Its appearance may be slightly earlier in persons dwelling in warm climes than in those inhabiting colder latitudes. Much of the influence attributed to climate appears due to the custom prevalent in many hot countries, as in Hindostan, of giving girls in marriage at a very early age, and inducing sexual excitement previous to the proper menstrual time. The menstrual functions continue through the whole fruitful period of a woman's life and usually cease between the fortyfifth and fiftieth years.

The several menstrual periods usually occur at intervals of a lunar month, the duration of each being from three to six days. In some women the intervals are so short as three weeks or even less; while in others they are longer than a montl.. The periodical return is usually attended by pain in the loins, a sense of fatigue in the lower limbs, and other symptoms, which are different in different individuals. Menstruation does not usually occur in pregnant women, or in those who are suckling; but instances of its occurrence in both these conditions are by no means rare.

Corpus Luteum.-Immediately before, as well as subsequent to, the rupture of a Graafian follicle, and the escape of its ovum, certain changes ensue in the interior of the vesicle, which result in the production of a yellowish mass, termed a Corpus luteum.

When fully formed the corpus luteum of mammiferous animals is a roundish solid body, of a yellowish or orange color, and composed of a number of lobules, which surround, sometimes a small cavity, but more frequently a small stelliform mass of white substance, from which delicate processes pass as septa between the several lobules. Very often, in the cow and sheep, there is no white substance in the centre; and the 
lobules projecting from the opposite walls of the Graafian follicle appear in a section to be separated by the thinnest possible lamina of semitransparent tissue.

When a follicle is about to burst and expel the ovum, it becomes highly vascular and opaque; and, immediately before the rupture takes place, its walls appear thickened on the interior by a reddish glutinous or fleshy-looking substance. Immediately after the rupture, the inner layer of the wall of the vesicle appears pulpy and flocculent. It is thrown into wrinkles by the contraction of the outer layer, and, soon, red fleshy mammillary processes grow from it, and gradually enlarge till they nearly fill the vesicle, and even protrude from the orifice in the external covering of the ovary. Subsequently this orifice closes, but the fleshy growth within still increases during the earlier period of pregnancy, the color of the substance gradually changing from red to yellow, and its consistence becoming firmer.

The human corpus luteum (fig. $45 \%$ ) differs from that of the domestic quadruped in being of a firmer texture, and having more frequently a
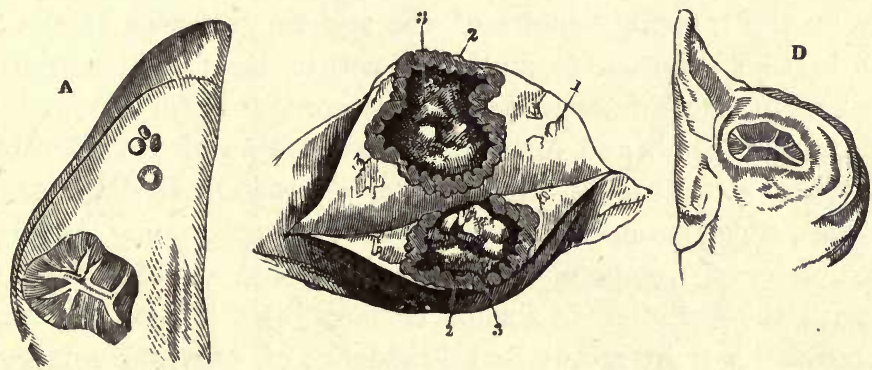

Fig. 457.-Corpora lutea of different periods. B, corpus luteum of about the sixth week after impregnation, showing its plicated form at that period. 1, substance of the ovary; 2, substance of the corpus luteum; 3, a grayish coagulum in its cavity. (Paterson.) A, corpus luteum two days after delivery; D, in the twelfth week after delivery. (Montgomery.)

persistent cavity at its centre, and in the stelliform cicatrix, which remains in the cases where the cavity is obliterated, being proportionately of much larger buik. The quantity of yellow substance formed is also much less: and although the deposit increases after the vesicle has burst, yet it does not usually form mammillary growths projecting into the cavity of the vesicle, and never protrudes from the orifice, as is the case in other Mammalia. It maintains the character of a uniform, or nearly uniform, layer, which is thrown into wrinkles, in consequence of the contraction of the external tunic of the vesicle. After the orifice of the resicle has closed, the growth of the yellow substance continues during the first half of pregnancy, till the cavity is reduced to a comparatively small size, or is obliterated; in the latter case, merely a white stelliform cicatrix remains in the centre of the corpus luteum.

An effusion of blood generally takes place into the cavity of the fol- 
licle at the time of its rupture, esperially in the human subject, but it has no share in forming the yellow body; it gradually loses its coloring matter. 'The serum of the blood sometimes remains included within a cavity in the centre of the coagulum, and then the decolorized fibrin forms a membraniform sac, lining the corpus luteum. At other times the serum is removed, and the fibrin constitutes a solid stelliform mass.

The yellow substance of which the corpus luteum consists, both in the human subject and in the domestic animals, is a growth from the inner surface of the ruptured follicle, the result of an increased development of the membrana granulosa.

The first changes of the internal coat of the Graafian vesicle in the process of formation of a corpus luteum seem to occur in every case in which an ovum escapes; as well in the human subject as in the domestic quadrupeds. If the ovum is impregnated, the growth of the yellow substance continues during nearly the whoie period of gestation and forms the large corpus luteum commonly described as a characteristic mark of impregnation. If the ovum is not impregnated, the growth of yellow substance on the internal surface of the vesicle proceeds, in the human ovary, no further than the formation of a thin layer, which shortly disappears; but in the domestic animals it continues for some time after the ovum has perished, and forms a corpus Juteum of considerable size. The fact that a structure, in its essential characters similar to, though smaller than, a corpus luteum observed during pregnancy, is formed in the human subject, independent of impregnation or of sexual union, coupled with the varieties in size of corpora lutea formed during pregnancy, necessarily renders unsafe all evidence of previous impregnation founded on the existence of a corpus luteum in the ovary.

The following table by Dalton, expresses well the differences between the corpus luteum of the pregnant and unimpregnated condition respectively :-

\section{Corpus Luteum of Menstru- ation. \\ Corpus Luteum of Pregnancy.}

At the end of | Three-quarters of an inch in diameter ; central clot reddish ; con

three weeks roluted wall pale.

One month . Smaller; convoluted wall Larger; convoluted wall bright yelbright yellow; clot still low ; clot still reddish. reddish.

Two months Reduced to the condition of an insignificant cicatrix.

Six months . Absent.

Seven-eighths of an inch in diameter; convoluted wall bright yellow; clot perfectly decolorized.

Still as large as at end of second month ; clot fibrinous ; convoluted wall paler.

Nine months Absent.

One-half an inch in diameter; central clot converted into a radiating cicatrix; the external wall tolerably thick and convoluted, but without any bright yellow color. 
Of the Male.-In order that the ovum should be fecundated, it is necessary that it should meet with the seminal fluid of the male. This is accomplished by the junction of the sexes in the act of coition, whereby the seminal fluid is discharged into the neighborhood of, if not within, the cervix uteri. Before considering the changes which are produced in the ovum by impregnation, it will be as well to describe the nature of the seminal fluid. This consists essentially of the semen secreted by the testes, and to this are added a material secreted by the vesiculæ seminales, as well as the secretion of the prostate gland, and of Cowper's glands. Portions of these several fluids are discharged, together with the proper secretion of the testicles.

The semen is a viscid, whitish, albuminous fluid of a peculiar odor. It contains epithelium, granules or colorless particles, and large numbers of spermatozoa, which are the characteristic and essential elements.

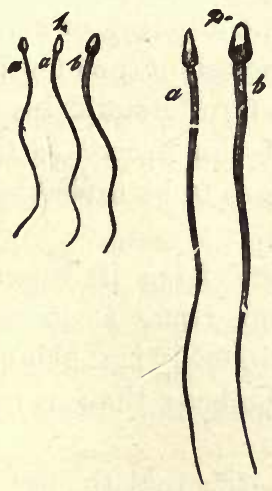

Fig. 458.

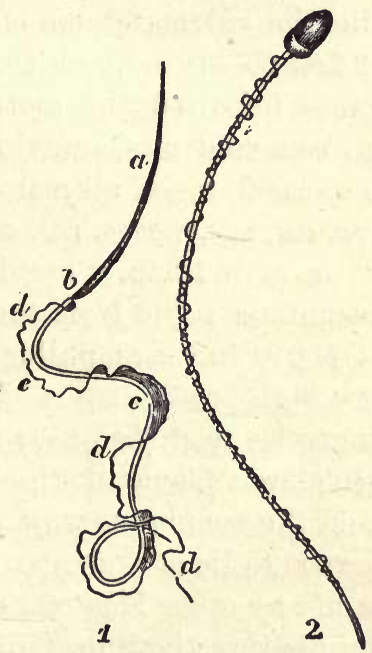

Fig. 459.

Fig. 458. - Spermatic filaments from the human vas deferens. 1, magnified 300 diameters; 2, magnified 800 diameters; $a$, from the side; $b$, from above. (From Kölliker.)

Fig. 459.-Spermatozoa. 1, Of salamander; 2, human. (H. Gibbes.)

The spermatozoa are minute bodies each consisting of a flattened oval head and attached to it a long slender tapering mobile flagellum or tail.

In some forms of spermatozoa there is a small middle piece interposed between the head and the tail. The head is about $\frac{1}{6000}$ th inch (about $4 \mu$ ) long and $\frac{1}{10000}$ th inch (about $2.5 \mu$ ) broad. The tail is about $\frac{1}{5000}$ th to $\frac{1}{4000}$ th inch $(5 \mu-6 \mu)$ long. The spermatozoa possess the power of active movement, and it is by this sinuous, cilia-like movement that they are propelled in the female and so helped in their progress to meet the ovum. The lashing cilium-like movement of a spermatozoon may 
go on for hours or days in the alkaline fluids of the body. It is stopped by any of the agencies which stop ciliary movement, e.g., acids, or strong alkalies, alcohol, chloroform, cold to $0^{\circ} \mathrm{C}$., and heat above $50^{\circ} \mathrm{C}$.

On examining the spermatozoon of Triton cristatus, one of the amphibia which possess the largest spermatozoa of all vertebrate animals, $\mathrm{H}$. Gibbes found that the organism consisted of $(a)$ a long pointed head, at the base of which is $(b)$, an elliptical structure joining the head to (c), a long filiform body; $(d)$, a fine filament, much longer than the body, is connected with this latter by $(e)$, a homogeneous membrane.

The head, as it appears in the fresh specimen, has a different refractive power from that of the rest of the organism, and with a high power appears to be a light green color; there is also a central line running up it, from which it appears to be hollow.

The elliptical structure at the base of the head connects it with the Iong threadlike body, and the filament springs from it.

While the spermatozoon is living, this filament is in constant motion; at first this is so quick that it is difficult to see it, but as its vitality becomes impaired the motion gets slower, and it is then easily perceived to be a continuous waving from side to side.

The spermatozoa of all mammalia examined, consisting of man, bull, dog, horse, cat, pig, mouse, rat, guinea-pig, had instead of the long-pointed head of the amphibian, a blunt thick process of different shapes in the different animals; and from the root or neck of this proceeded the long filament just as in the amphibia, only so delicate as to be invisible except with very high powers.

In man the head (fig. 459) is club-shaped, and from its base springs the very delicate filament, which is three or four times as long as the body; and the membrane which attaches it to the body is much broader, and allows it to lie at a greater distance from the body than in the spermatozoa of any other Mammal examined.

From his investigation, Gibbes concluded:-1st, that the head of the spermatozoon is inclosed in a sheath, which is a continuation of the membrane which surrounds the filament, and connects it to the body, acting in fact the part of a mesentery. 2ndly. That the substance of the head is quite distinct in its composition from the elliptical structure, the filament and the long body, and that it is readily acted on by alkalies; these reagents have no effect, however, on the other part, exempting the membraneous sheath. 3rdly. That this elliptical structure has its analogue in the mammalian spermatozoon; in the one case the head is drawn out as a long pointed process, in the other it is of a globular form, and surrounds the elliptical structure. 4thly. That the motive power lies, in a great measure, in the filament and the membrane attaching it to the body. 
'The spermatozoa are derived from the breaking up of the seminal cells or daughter cells. They must be looked upon as modified cells.

The occurrence of spermatozoa in the impregnating fluid of nearly all classes of animals, proves that they are essential to the process of impregnation, and their actual contact with the ovum is necessary for its development.

The seminal fluid is, probably, after the period of puberty secreted constantly, though, except under excitement, very slowly, in the tubules of the testicles. From these it passes along the vasa deferentia into the vesiculæ seminales, whence, if not expelled in emission; it may be discharged, as slowly as it enters them, either with the urine, which may remove minute quantities, mingled with the mucus of the bladder and the secretion of the prostate, or from the urethra in the act of defæcation.

To the vesiculæ seminales a double function may be assigned; for they both secrete some fluid to be added to that of the testicles, and serve as reservoirs for the seminal fluid. The former is their most constant and probably most important office; for in the horse, bear, guineapig, and several other animals, in whom the vesiculæ seminales are large and of apparently active functions, they do not communicate with the vasa deferentia, but pour their secretions, separately, though it may be simultaneously, into the urethra.

There is a complete want of information respecting the nature and purposes of the secretions of the prostate and Cowper's glands. That they contribute to the right composition of the impregnating fluid, is shown both by the position of the glands and by their enlarging with the testicles at the approach of an animal's breeding time. But that they contribute only a subordinate part is shown by the fact, that, when the testicles are lost, though these other organs be perfect, all procreative power ceases.

The fluid part of the semen or liquor seminis has not been satisfactorily analyzed: but Henle says it contains fibrin, because shortly after being discharged, flocculi form in it by spontaneous coagulation, and leave the rest of it thinner and more liquid, so that the filaments move in it more actively. The chief constituents of the semen are said to be a variety of nuclein, which does not contain sulphur; certain proteids, one of which contains four per cent. of sulphur; lecithin; cholesterin; fat, and extractives. 


\section{CHAPTER XIX.}

DEVELOPMENT.

\section{Changes which occur in the Ovum.}

OF the changes which take place in the ovum, some occur before and are as it were preparatory to impregnation, and others ensue after impregnation. It will be as well to consider the respective changes separately.

Changes prior to Impregnation.-These changes especially concern the germinal vesicle, and have been observed chiefly in the ova of low types. The ovum when ripe and detached from the ovary consists, it will be remembered, of a granular yolk inclosed within the protoplasmic zona pellucida, and containing the germinal vesicle and germinal spot situated eccentrically. The yolk granules are of differen $t$ sizes, from the minutest molecules up to a diameter of $\frac{1}{1000}$ th to $\frac{1}{1500}$ th of an inch (about $25 \%$ ). The germinal vesicle consists of reticulated protoplasm inclosed in a distinct membrane, and containing one or more nucleoli or germinal spots. The primary change observed in the ovum consists in the travelling of the germinal vesicle to the surface, and the disappearance of its inclosing membrane, with a consequent indentation and indistinctness of its outline. Its protoplasm becomes to a considerable extent confounded with the yolk substance, and its germinal spot disappears. The next step in the process is the appearance in the yolk of two stars in a clear space near the poles of the resicle elongated to a certain extent, and from this results a nuclear spindle, with the stars at either end lying near the surface of the yolk. This spindle next becomes vertical, the nucleus divides into two parts, and that nearer the surface protrudes from the ovum enveloped in a protoplasmic mass, which by constriction forms the first polar cell. A second polar cell arises in the same way. The remaining daughter nucleus again divides-one-half of it is extruded from the ovum, forming a second polar cell; the other half remains behind and is called the female pro-nucleus. This is clearly derived from the original germinal vesicle. It must be remembered that these changes have been so far observed only in a certain number of instances. It is very possible, not to say probable, that such changes are universal in the animal kingdom (Balfour).

Balfour's view as to the formation of the polar bodies may be given 
in his own words:- "My view amounts to the following, viz., that after the formation of the polar-cells, the remainder of the germinal vesicle within the ovum (the female pro-nucleus) is incapable of further development without the addition of the nuclear part of the male element (spermatozoon), and that if polar-cells were not formed, parthenogenesis might normally occur.'

Changes following Impregnation.-The process of impregnation of the ovum has been observed most accurately in the lower types. In mammalia, although spermatozoa pass in numbers through the yolk envelope, yet their further progress is only inferred from observations on the lower animals. The process in asterias glacialis, according to Balfour, is as follows:-The head of a single spermatozoon joins with an elevation of the yolk substance, the tail remaining motionless, and then disappearing. The head enveloped in the protoplasm then sinks into the yolk and becomes a nucleus, from which the yolk substance is arranged in radiating lines. This is the male pro-nucleus. At first, at some distance from the female pro-nucleus, it after a while approaches nearer, and the female pro-nucleus, which was before inactive, becomes active. The nuclei at last meet and unite. The result of their union is the first segmentation sphere, or blasto-sphere. It is a nucleated protoplasmic cell. The changes which have resulted in the formation of the blasto-sphere or primitive segmentation germ are followed by the process known as segmentation of the yolk.

This process and the earlier stages in development are so fundamentally similar in all vertebrate animals, from fishes up to man, that the gaps existing in our knowledge of the process in the higher mammalia, such as man, may be, in part, at any rate, filled up by the more accurate knowledge which we possess of the development of the ovum in such animals as the trout, frog, and fowl.

One important distinction between the ora of various vertebrata should be remembered. In the hen's egg, besides the shell and the white or albumen, two other structures are to be distinguished-the germ, often called the cicatricula or "tread," and the yolk, inclosed in its vitelline membrane.

The germ is (as was mentioned in the description already given) essentially a cell, consisting of protoplasm inclosing a nucleus and nucleolus. It alone participates in the process of segmentation, the great mass of the yolk (foodyolk) remaining quite unaffected by it. Since only the germ, which forms but a small portion of the yolk, undergoes segmentation, the ovum is called meroblastic.

In the mammalia, on the other hand, there is no large unsegmented mass corresponding to the food-yolk of birds ; the entire ovum undergoes segmentation, and is hence termed holoblastic.

The eggs of fishes, reptiles, and birds, are meroblastic, while those of amphibia and mammalia are holoblastic.

Of the changes which the mammalian ovum undergoes previous to 
the formation of the embryo, those which occur while it is still in the ovary are independent of impregnation: others take place after it has reached the Fallopian tube. The knowledge we possess of these changes is derived almost exclusively from obser-

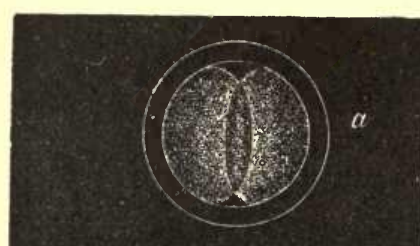
vations on the ova of the bitch and rabbit: but it may be inferred that analogous changes ensue in the human ovum.

As the ovum approaches the middle of the Fallopian tube, it begins to receive a new investment, consisting of a layer of transparent albuminous or glutinous substance, which forms upon the exterior of the zona pellucida. It is at first exceedingly fine, and owing to this, and to its transparency, is not easily recognized, but at the lower part of the Fallopian tube it acquires considerable thickness.

Segmentation. - The first visible result of fertilization is a slight amoboid movement in the protoplasm of the ovum: this has been observed in some fish, in the frog, and in some mammals. Immediately succeeding to this the process of segmentation commences, and is completed during the passage of the ovum through the Fallopian tube. In mammals, in which the process is an example of complete segmentation, the yolk becomes constricted in the middle, and is surrounded by a furrow which, gradually deepening, at length cuts it in half, while the same process begins almost immediately in each half of the yolk, and cuts it also in two. The same process is repeated in each of the quarters, and so on, until at last by continual cleavings, the whole yolk is changed into a mulberry-like mass of small and more or less rounded bodies, sometimes called vitelline spheres, the ous stages of cleavage of the yolk. (Dalton.)

whole still inclosed by the zona pellucida (fig. 460). Each of these little spherules contains a transparent vesicle, like an oil-globule, which is seen with difficulty, on account of its being enveloped by the yolk-granules which adhere closely to its surface. 
The cause of this singular subdivision of the yolk is quite obscure: though the immediate agent in its production seems to be the central vesicle contained in each division of the yolk. Originally there was probably but one vesicle, situated in the centre of the entire granular mass of the yolk, and probably derived in the manner already described from the germinal vesicle. This divides and subdivides: each successive division and subdivision of the vesicle being accompanied by a corresponding division of the yolk.

About the time at which the mammalian ovum reaches the uterus, the process of division and subdivision of the yolk appears to have ceased, its substance having been resolved into its ultimate and smallest divisions, while its surface presents a uniform finely-granular aspect, instead of its late mulberry-like appearance. The ovum, indeed, appears at first sight to have lost all trace of the cleavage process, and, with the exception of being paler and more translucent, almost exactly resembles the ovarian ovum, its yolk consisting apparently of a confused mass of finely granular substance. But on a more careful examination, it is found that these granules are aggregated into numerous minute spheroidal masses, each of which contains a clear vesicle or nucleus in its centre, and is, in fact, an embryonal cell. The zona pellucida, and the layers of albuminious matter.surrounding it, have at this time the same character as when at the lower part of the Fallopian tube.

The passage of the ovum, from the ovary to the uterus, oceupies probably eight or ten days in the human female.

When the peripheral cells, which are formed first, are fully developed, they arrange themselves at the surface of the yolk into a kind of membrane, and at the same time assume a polyhedral shape from mutual pressure, so as to resemble pavement epithelium. The deeper cells of the interior pass gradually to the surface and accumulate there, thus increasing the thickness of the membrane already formed by the more superficial layer of cells, while the central part of the yolk remains filled only with a clear fluid. By this means the yolk is shortly converted into a kind of secondary vesicle, the walls of which are composed externally of the original vitelline membrane, and within by the newly formed cellular layer, the blastoderm or germinal membrane, as it is called.

Segmentation in the Chick. - The embryo chick affords an illustration of what is known as incomplete or partial segmentation, or meroblastic segmentation. In the youngest ova the germinal vesicle is situated subcentrally, but as development proceeds it passes to the periphery, and the protoplasm surrounding it remaining free from yolk granules, the germinal disc is formed. This germinal disc is not marked out by any sharp line from the remaining protoplasm, but passes insensibly into it. The first change consists in the appearance of a furrow run- 
ning across the disc dividing it into two; it does not extend across the whole breadth. A second furrow, at right angles, cutting the first a little eccentrically, next appears, and the disc is thus cut into four quadrants. The furrows do not extend through the whole thickness of the disc, and the segments are not separated out on the lower aspect. The quadrants are next bisected by radiating furrows, and the disc is thus divided into eight parts. The central portion of each segment is now cut off from the peripheral furrow, so that a number of smaller central and larger peripheral portions result. As the primary division was eccentric and the succeeding followed the same plan, there results a bilateral symmetry; but the relation of the axis of symmetry and the long axis of the embryo is not known. Rapid division of the segments by furrows in various directions now ensues, and the small central portions are more rapidly broken up than the larger, and therefore become more numerous. During this superficial segmentation a similar process goes on throughout the whole mass, and division goes on not only by vertical but also by horizontal furrows. The result of this process of segmentation is that the original germinal disc is cut into a large number of small rounded protoplasmic cells, small in the centre, larger to the periphery, and that the superficial cells are smaller than those below: the two original layers of the blastoderm are thus early represented.

The process of segmentation proceeds at the periphery of the germinal disc, and at the same time further division of the cells at the

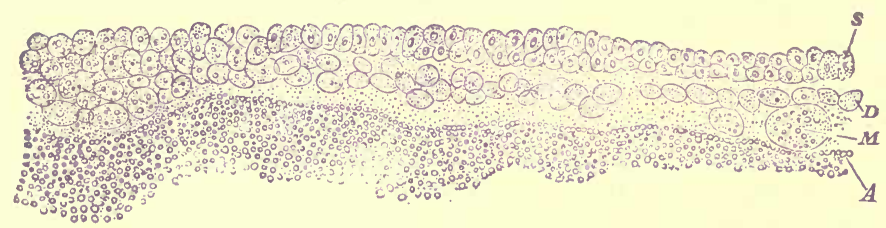

Fig. 461.-Vertical section of area pellucida and area opaca (left extremity of figure) of blastoderm of a fresh-laid egg (unincubated). $S$, superficial layer corresponding to epiblast; $D$, deeper layer, corresponding to hypoblast, and probably in part to mesoblast; $M$, large "formative cells," filled with yolk granules, and lying on the floor of the segmentation cavity; $A$, the white yolk immediately underlying the segmentation cavity. (Stricker.)

centre proceeds. The nucleus of the original cell divides coincidently with the protoplasm, and so it comes that the protoplasmic masses are nucleated; and besides this, nuclei derived from the original nucleus are found in the ovum below the area of segmentation, and from these by the protoplasm which surrounds them being constricted off with them, supplementary segmentation masses come to be formed. The blastoderm is thus formed as the result of segmentation, and between it and the subjacent white yolk is a cavity containing fluid. The segmentation having been completed toward the centre, although it still proceeds at the periphery, the superficial layer of the blastoderm becomes 
a layer of columnar nucleated cells, and the lower layer consists of larger masses indistinctly nucleated, still granular and rounded, irregularly disposed. In the segmentation cavity are the supplementary segmentation masses or formative cells.

When the egg is incubated, rapid changes take place in the blastoderm, resulting in the formation first of all of two, then of the three layers, which have been already mentioned in the first chapter. The superficial, or epiblast, does not at first enter into these changes, but

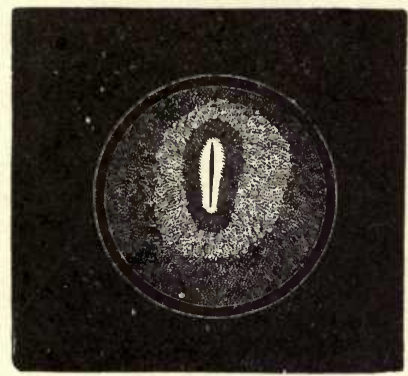

Fig. 462.-Impregnated egg, with commencement of formation of embryo; showing the area germinativa or embryonic spot, the area pellucida, and the primitive.groove or trace. (Dalton.)

continues to be a layer of nucleated columnar cells. But in the lower layer of larger rounded cells certain of the cells become flattened horizontally, their granules disappear, and the nuclei become distinct. A membrane of flattened nucleated cells is then formed, first of all toward the centre of the area, afterward peripherally also: this is the hypoblast. Between the two layers some cells, not belonging to either layer, remain. These cells are almost entirely at the back part of the area. The formation of the intermediate layer of mesoblast is more complicated, and will now be described.

At this period it is necessary to return to the surface view of the blastoderm. Before incubation it is seen to consist of a more or less circular transparent area, the area pellucida, surrounded by an opaque rim, which is called the area opaca. The area opaca rests upon the white yolk: beneath the area pellucida is a cavity containing fluid. In the centre of the area pellucida is a white shining spot, or nucleus of Pander, shining through. This is the upper dilated extremity of the flask-shaped accumulation of white yolk upon which the blastoderm rests.

The yellow yolk consists of spheres $25 \mu$ to $100 \mu$ in diameter, filled with highly refractive granules of an albuminous nature, and the white yelk being distinguished from the yellow not only by its lighter color, but also because its vesicles are smaller than those of the yellow. Each con- 
tains a highly refractive body. Some large spheres contain a number of spherules. Some of these are vacuolated. The white yolk not only envelopes the yellow yolk in a thin layer, and merges with the central flask-shaped mass, already mentioned, but also is found in the yellow yolk, forming with it alternate layers.

Except that the central shining opacity of the pellucid area has disappeared, that the size of the area has increased, and that the opaque

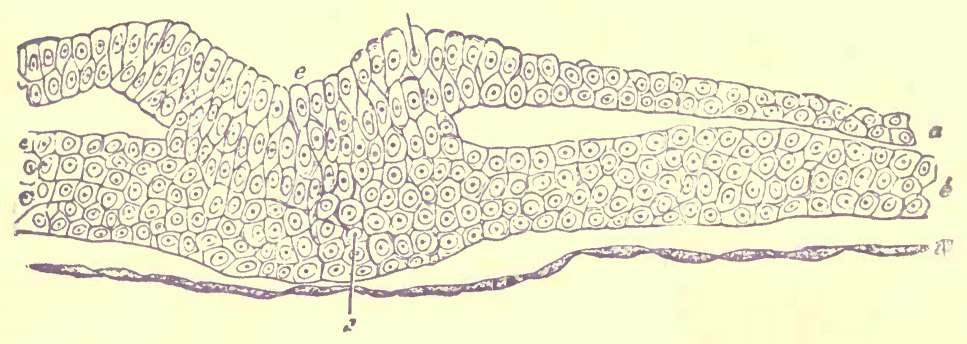

Fig. 463,-Transverse section through embryo chick (26 hours). $a$, epiblast; $b$, mesoblast; $c$, hypoblast; $d$, central portion of mesoblast, which is here fused with epiblast; $e$, primitive groove; $f$, dorsal ridge. (Klein.)

area has also increased, no other change can be remarked up to the formation of the two complete layers. There is, however, a slight illdefined opacity at the posterior part of the area pellucida, known as the embryonic shield. This opacity is probably due to the intermediate cells already mentioned as existing between the epiblast and hypoblast.

In the posterior part of the area pellucida now appears an opaque streak which extends about a third of the diameter of the area toward the middle line. This is the Primitive streak. It is found on transverse section of the blastoderm in this neighborhood to be due to a proliferation downward of cells two or more deep from the epiblast. The area pellucida now becomes oval. As the primitive streak becomes more

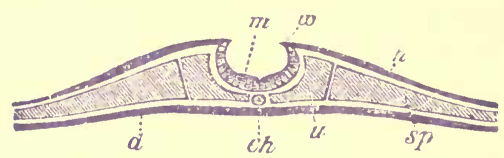

Fig, 464.-Diagram of transverse section through an embryo before the closing-in of the medullary groove. $m$, cells of epiblast lining the medullary groove which will form the spinal cord; $h$. epiblast; $d$, hypoblast; $c h$, notochord; $u$, protovertebra; $s p$, mesoblast; $w$, edge of lamina dorsalis, folding over medullary groove. (Kölliker.)

defined the area pellucida changes its oval for a pear shape, but the streak increases in size faster than the area, and so after a time is about two-thirds of its length. In the primitive streak a groove, the primitive groove, runs along its axis. From the primitive streak the cells from the under surface of the epiblast now extend as lateral wings to the edge of the pellucid area; they are not joined with the hypoblast.

The 
intermediate layer of cells in this position producing the primitive streak is a portion of the intermediate layer or mesoblast. It is formed chiefly from the epiblast, but laterally, especially in the front part of the primitive streak, it appears to be derived at any rate in part from the cells of the primitive lower layer. At the most anterior part of the primitive streak, at the point which corresponds to the future posterior end of the embryo, the three layers are all joined together. The next important change which occurs is found in the hypoblast in front of the primitive streak. The irregular layer of primitive cells of which it is composed, split into two layers, the lower consisting of flat-

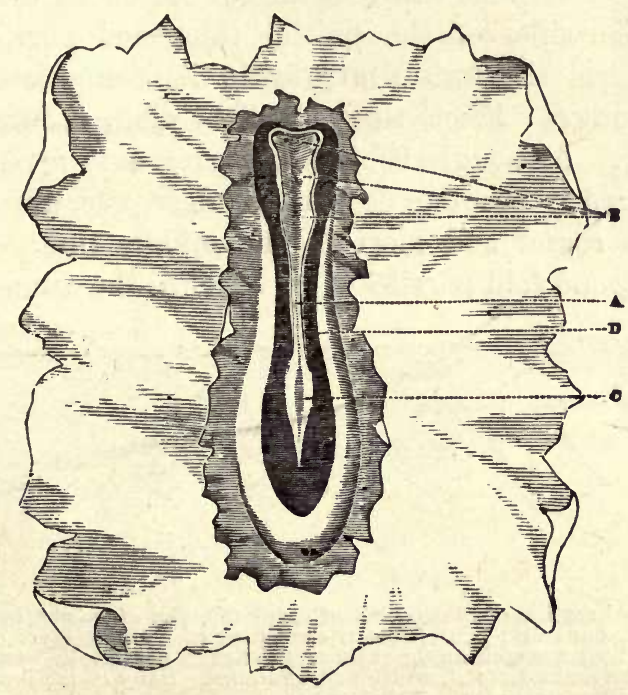

Fig. 465.-Portion of the germinal membrane, with rudiments of the embryo; from the ovum of a bitch. The primitive groove, $A$, is not yet closed, and at its upper or cephalic end presents three dilatations, B, which correspond to the three divisions or veslcles of the brain. At its lower extremity the groove presents a lancet-shaped dilatation (sinus rhomboidalis) $\mathrm{a}$ The margins of the groove consist of clear pellucid nerve-substance. Along the bottom of the groove is observed a faint streak, which is probably the chorda dorsalis. D. Vertebral plates. (Bischoff.)

tened cells which forms the hypoblast proper and an upper consisting of several layers of stellate cells, the mesoblast.

In the preceding account of the formation of the blastodermic layers, Balfour's description has been chiefly followed. It differs somewhat from that which was formerly given. The mesoblast was described as arising from the hypoblast, together with some of the large formative cells, which migrate by amœboid movement round the edge of the hypoblast (fig. $466, M$ ), and no difference was made in the formation of the mesoblast in the primitive streak and elsewhere.

There now appears in the middle line extending forward from the primitive streak an opaque line, which proceeds almost to the anterior 
edge of the area pellucida, stopping short at a transverse crescent-shaped line, the future headfold. This line is the commencing notochord. It is a collection of mesoblastic cells from the hypoblast in the middle line, and remains connected with the latter after the lateral portions of the mesoblast have become quite detached from it. The notochord and the hypoblast from which it arises are continued posteriorly into the primitive streak. Thus the mesoblast of the area on either side of the middle line in which the embryo is formed arises from the hypoblast, as does also the notochord. In the formation of the medullary plate which now appears, the epiblast is concerned. In the middle line above the collection of cells that will become the notochord that layer becomes thickened. The sides of the central thickened portion are elevated somewhat to form the medullary folds inclosing between them the medullary groove. From this medullary plate is formed the central nervous system. Although behind the groove is a shallow one, if it be traced forward it becomes deeper and narrower, and at the headfold the folds curve round and meet in the middle line. Anterior to the headfold is a second fold parallel to it, which is the commencing amnion.

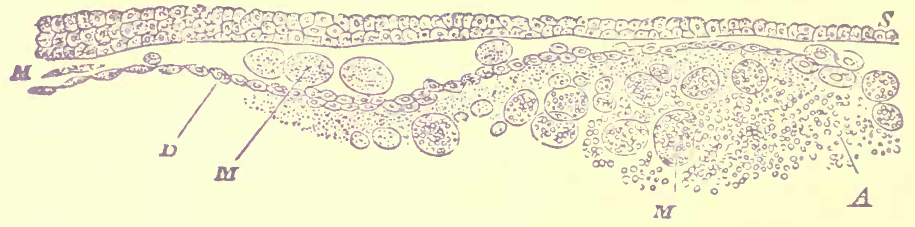

Fig. 466.-Vertical section of blastoderm of chick (1st day of incubation). $S$, epiblast consisting of short columnar cells; $D$, hypoblast, consisting of a single layer of flattened cells; $\boldsymbol{M}$. "formative cells." They are seen on the right of the figure, passing in between the epiblast and hypoblast to form the mesoblast; $A$, white yolk granules. Many of the large "formative cells" are seen containing these granules. (Stricker.)

The medullary canal is bounded by its two folds or longitudinal elevations, laminæ dorsales, which are folds consisting entirely of cells of the epiblast: these grow up and arch over the medullary groove (fig. 464) till after some time they coalesce in the middle line, converting it from an open furrow into a closed tube-the neural canal or the primitive cerebro-spinal axis. Over this closed tube, the walls of which consist of more or less cylindrical cells, the superficial layer of the epiblast is now continued as a distinct membrane.

The union of the medullary folds or laminæ dorsalis takes place first about the neck of the future embryo; they soon after unite over the region of the head, while the closing in of the groove progresses much more slowly toward the hinder extremity of the embryo. The medullary groove is by no means of uniform diameter throughout, but even before the dorsal laminæ have united over it, is seen to be dilated at the ante- 
rior extremity and obscurely divided by constrictions into the three primary cerebral vesicles.

The part from which the spinal cord is formed is of nearly uniform calibre, while toward the posterior extremity is a lozenge-shaped dilatation, sinus rhomboidalis, which is the last part to close in (fig. 465).

While the changes which have been described are taking place in the area pellucida, which has enlarged to a certain extent, the area opaca has also considerably extended. The hypoblast and mesoblast have also been prolonged laterally, not by mere extension, but also from the germinal wall, which is made up of the thickened edge of the blastoderm, together with formative cells of the yolk; on each side of the notochord and medullary canal, the mesoblast remains as a longitudinal thickening.

It now however splits horizontally into two layers or laminæ (parietal and visceral): of these the former, when traced out from the central axis, is seen to be in close apposition with the epiblast, and gives origin to the parietes of the trunk, while the latter adheres more or less closely to the hypoblast, and gives rise to the serous and muscular walls of the alimentary canal and several other parts.

The united parietal layer of the mesoblast with the epiblast is termed somatopleure, the united visceral layer and hypoblast, splanchnopleure. The space between them is the pleuro-peritoneal cavity, which becomes subdivided by subsequent partitions into pericardium, pleura, and peritoneum.

The splitting of the mesoblast extends almost to the medullary canal, but a portion on either side ( $P . v$. fig. 468) remains undi-

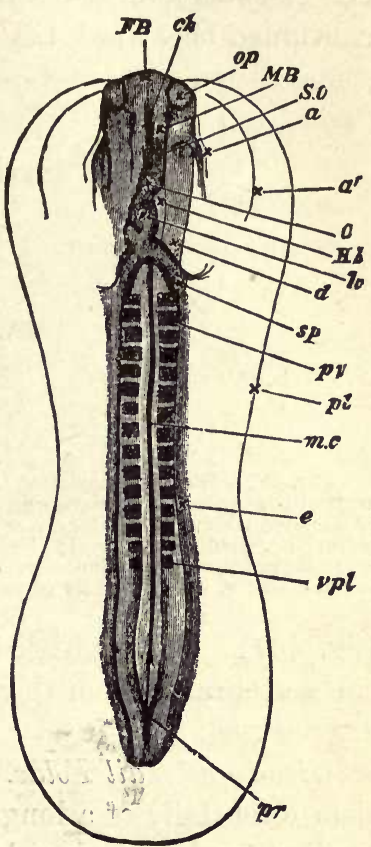

Fig. 467.-Embryo chick (36 hours), viewed from beneath as a transparent object (magnified). $p l$, outline of pellucid area, $F B$, fore-brain, or first cerebral vesicle: from its sides project op, the optic vesicle; $S O$, backward limit of somatopleure fold, "tucked in" under head; $a$, head-fold of true amnion; $a^{\prime}$, reflected layer of amnion, sometimes termed "false amnion;" sp, backward limit of splanchnopleure folds, a l ong which run the omphalomesaraic veins uniting to form $h$, the heart, which is continued forward into $b a$, the bulbus arteriosus; $d$, the fore-gut, lying behind the heart, and having a wide crescentic opening between the splanchnopleure folds; $H B$, hind-brain; $M B$, mid-brain; $p v$, protovertebræ lying behind the fore-gut; $m c$, line of junction of medullary folds and of notochord; vpl, vertebral plates; $p r$, the primitive groove at its caudal end. (Foster and Balfour.) vided, the vertebral plate. The divided portion is known as the lateral plate. The longitudinal thickening of the vertebral plate is seen after a while to be divided at right angles to the medullary canal by bright transverse lines into a number of square segments. These seg- 
ments, which are the surface appearance of cubes of mesoblast, are the mesoblastic somites or protovertebræ. The first three or four of the protovertebræ make their appearance in the cervical region, while one or two more are formed in front of this point: and the series is continued backward till the whole medullary canal is flanked by them

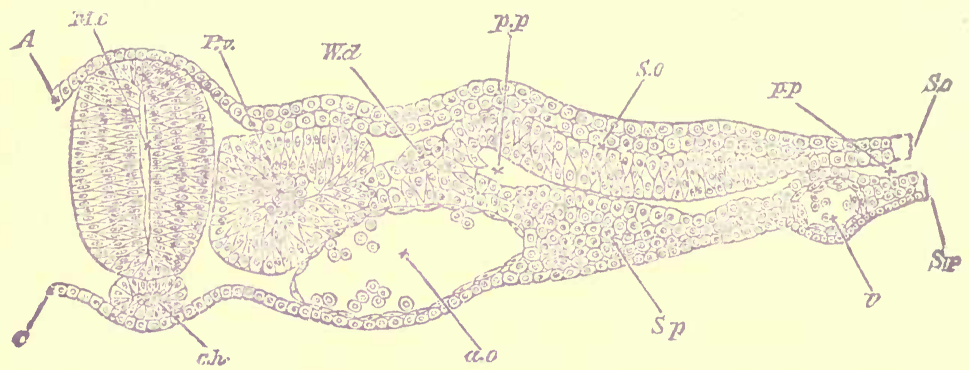

Fig. 468. - Transverse section through dorsal region of embryo chick ( $45 \mathrm{hrs}$.). One half of the section is represented; if completed it would extend as far to the left as to the right of the line of the medullary canal (MC). $A$, epiblast; $C$, hypoblast, consisting of a single layer of flattened cells; $M c$, medullary canal; $P v$, protovertebra; $W d$, Wolffian duct; $S o$, somatopleure: $S p$, splanchnopleure; $p$, pleuro-peritoneal cavity; ch, notochord; ao, dorsal aorta, containing blood cells; $v$, blood-vessels of the yolk-sac. (Foster and Balfour.)

(fig. 467). That which is first formed corresponds to the second cervical vertebra. From these somites the vertebræ and the trunk muscles are deriveld.

Head and Tail Folds. Body Cavity.-Every vertebrate animal consists essentially of a longitudinal axis (vertebral column) with a neural canal above it, and a body-cavity (containing the alimentary canal) beneath.

We have seen how the earliest rudiments of the central axis and the neural canal are formed; we must now consider how the general body-

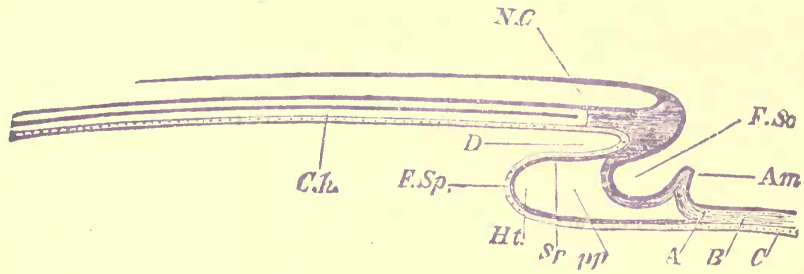

Fig. 469. - Diagrammatic longitudinal section through the axis of an embryo. The head-fold has commenced, but the tail-fold has not yet appeared. FSo, fold of the somatopleure: $F s p$, fold of the splanchnopleure; the line of reference, Fso, lies outside the embryo in the "moat, which marks off the overhanging head from the amnion; $D$, inside the embryo, is that part which is to become the fore-gut: $F s o$ and $F s p$, are both parts of the head-fold, and travel to the left of the figure as development proceeds; $p p$, space between somatopleure and splanchnopleure, pleuro-peritoneal cavity; $A m$, commencing head-fold of amnion; $N C$. neural canal; $C h$, notochord; $\boldsymbol{H t}$, heart: $\boldsymbol{A}, \boldsymbol{B}, \boldsymbol{C}$, epiblast, mesoblast, hypoblast. (Foster and Balfour.)

cavity is developed. In the earliest stages the embryo lies flat on the surface of the yolk, and is not clearly marked off from the rest of the blastoderm: but gradually the head-fold or crescentic depression (with 
its concavity backward) is formed in the blastoderm, limiting the head of the embryo; the blastoderm is, as it were, tucked in under the head, which thus comes to project above the general surface of the membrane: a similar tucking in of blastoderm takes place at the caudal extremity, and thus the head and tail folds are formed.

Similar depressions mark off the embryo laterally, until it is completely surrounded by a sort of moat which it overhangs on all sides, and which clearly defines it from the yolk.

This moat runs in further and further all round beneath the overhanging embryo, till the latter comes to resemble a canoe turned upside-

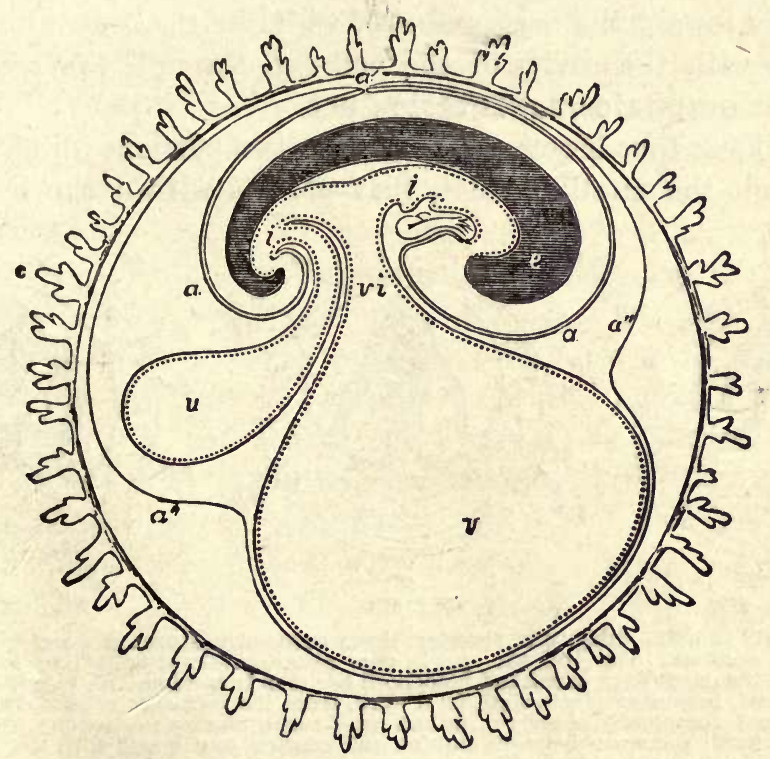

Fig. 470. - Diagrammatic section showing the relation in a mammal between the primitive alimentary canal and the membranes of the ovum. The stage represented in this diagram corresponds to that of the fifteenth or seventeenth day in the human embryo, previous to the expansion of the allantois; $c$, the villous chorion; $a$, the amnion; $a^{\prime}$, the place of convergence of the amnion and reflection of the false amnion $a^{n} a^{n}$, or outer or corneous layer; $e$, the head and trunk of the embryo, comprising the primative vertebræ and cerebro-spinal axis; $i, i$, the simple alimentary canal in its upper and lower portions. Immediately beneath the right hand is seen the foetal heart, lying in the anterior part of the pleuro-peritoneal cavity; $v$, the yolk-sac or umbilical vesicle; $v i$, the vitello-intestinal opening; $u$, the allantois connected by a pedicle vith the anal portion of the alimentary canal. (Quain.)

down, the ends and middle being, as it were, decked in by the folding or tucking in of the blastoderm, while on the ventral surface there is still a large communication with the yolk, corresponding to the well or undecked portion of the canoe.

This communication between the embryo and the yolk is gradually contracted by the further tucking in of the blastoderm from all sides, till it becomes narrowed down, as by an invisible constricting band, to 
a mere pedicle which passes out of the body of the embryo at the point of the future umbilicus.

The downwardly folded portions of blastoderm are termed the vis-

\section{ceral plates.}

Thus we see that the body-cavity is formed by the downward folding of the visceral plates, just as the neural carity is produced by the upward growth of the dorsal laminæ, the difference being that, in the visceral or ventral laminæ, all three layers of the blastoderm are concerned.

The folding in of the splanchnopleure, lined by hypoblast, pinches off, as it were, a portion of the yelk-sac, inclosing it in the body-cavity. This forms the rudiment of the alimentary canal, which at this period ends blindly toward the head and tail, while in the centre it communicates freely with the cavity of the yolk-sac through the canal termed vitelline or omphalo-mesenteric duct.

The yolk-sac thus becomes divided into two portions which communicate through the vitelline duct, that portion within the body giving

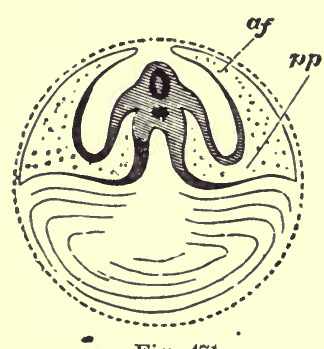

Fig. 471.

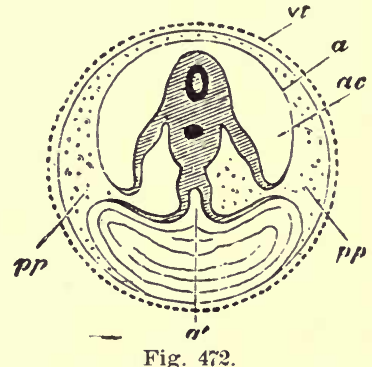

Fig. 4\%2.

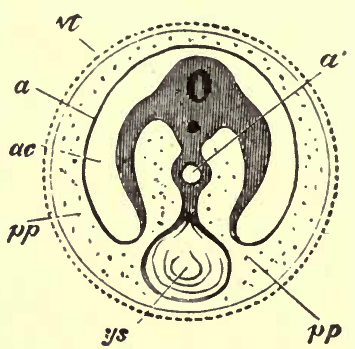

Fig. 473.

Figs. 471, 472 and 473. - Diagrams showing three successive stages of development. Transverse vertical sections. The yolk-sac, $y s$, is seen progressively diminishing in size. In the embryo itself the medullary canal and notochord are seen in section. $a^{\prime}$, in middle figure, the alimentary canal, becoming pinched off, as it were, from the yolk-sac; $a^{\prime}$ in right-hand figure, alimentary canal completely closed; $a$, in last two figures, amnion; $a c$, cavity of amnion filled with amniotic fluid; $p p$, space between amnion and chorion continuous with the pleuro-peritoneal cavity inside the body; $v t$. vitelline membrane; $y s$, yolk-sac, or umbilical vesicle. (Foster and Balfour.)

rise, as above stated, to the digestive canal, and that outside the body remaining for some time as the umbilical vesicle (fig. $473, y s$.). The hypoblast forming the epithelium of the intestine is of course continuous with the lining membrane of the umbilical vesicle, while the visceral plate of the mesoblast is continuous with the outer layer of the umbilical vesicle.

All the above details will be clear on reference to the accompanying diagrams.

At the posterior end of the embryo chick, when the amniotic fold is commencing to be formed, and the hind fold of the splanchnopleure has commenced, there remains for a time a communication between the neural canal and the hind gut, which is called the neurenteric canal. 
It passes in at the point where the notochord falls into the primitive streak. The anterior part of the primitive streak becomes the tail swelling, the posterior part atrophies, and the corresponding lateral part of the blastoderm forms part of the body-wall of the embryo.

The anterior part of the medullary canal having been completely roofed in, the foremost portion undergoes dilatation, and a bulb, the first or anterior cerebral vesicle, results. From either side of this dilatation a process, the cavity of which is in communication with it, is separated off, which is called the optic vesicle.

Behind the first cerebral vesicle two other vesicles now arise, the second or middle, and the third or posterior cerebral vesicle, and at the posterior part of the head.two small pits, the auditory vesicles or pits, are to be seen. The folding of the head, it should be recollected, is the cause of the inclosure below the neural canal (fig. 469) of a canal ending blindly, which has in front the splanchnopleure, and which is just as long as the involution of that membrane. This canal is the fore-gut. In the interior of the splanchnopleure fold below it (as seen in fig. 469) in the pleuro-peritoneal cavity the heart is formed, at the point where the splanchnopleure makes its turn forward. It arises as a thickening of the mesoblast on either side as the two splanchnopleure folds diverge, and of a thickening of the mesoblast at the point of divergence. So that at first the rudiment of the heart is like an inverted $\mathrm{V}$, which by the gradual coming together of the diverging cords is converted into an inverted $\mathrm{Y}$.

The cylinders become hollowed out, and are thus converted into tubes, which then coalesce. Layers are separated off toward the interior, which become the epithelial lining, and the mass of the mesoblast surrounding this, afterward form the muscle and serous covering, while at first the rudimentary organ is attached to the gut by a mesoblastic mesentery, the mesocardium.

Fotal Membranes.

Umbilical Vesicle (Yolk-sac). - The splanchnopleure, lined by hypoblast, forms the yolk-sac in reptiles, birds, and mammals; but in amphibia and fishes, since there is neither amnion nor allantois, the wall of the yolk-sac consists of all three layers of the blastoderm, inclosed, of course, by the original vitelline membrane.

The body of the embryo becomes in great measure detached from the yolk-sac or umbilical vesicle, which contains, however, the greater part of the substance of the yolk, and furnishes a source whence nutriment is derived for the embryo. This nutriment is absorbed by the numerous vessels (omphalo-mesenteric) which ramify in the walls of the yolk-sac, forming what in birds is termed the area vasculosa. In 
birds, the contents of the yolk-sac afford nourishment until the end of incubation, and the omphalo-mesenteric vessels are developed to a corresponding degree; but in mammalia the office of the umbilical vesicle ceases at a very early period, as the quantity of the yolk is small, and the embryo soon becomes independent of it by the connections it forms with the parent. Moreover, in birds as the sac is emptied, it is gradually drawn into the abdomen through the umbilical opening, which then

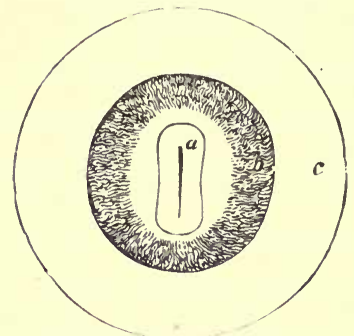

Fig. 474.

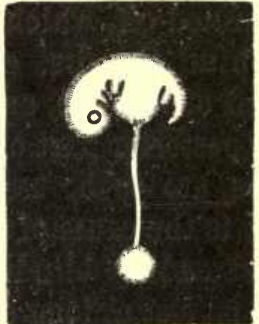

Fig. 475 .

Fig, 474.-Diagram showing vascular area in the chick. $a$, area pellucida; $b$, area rasculosa; c, area vitellina.

Fig. 475. - Human embryo of fifth week with umbilical vesicle; abont natural size. (Dalton.) The human umbilical vesicle never exceeds the size of a small pea.

closes over it: but in mammalia it always remains on the outside; and as it is emptied it contracts (fig. 473), shrivels up, and together with the part of its duct external to the abdomen, is detached and disappears, either before or at the termination of intra-uterine life, the period of its disappearance varying in different orders of mammalia.

When blood-vessels begin to be developed, they ramify largely over the walls of the umbilical vesicle, and are actively concerned in absorbing its contents and conveying them away for the nutrition of the embryo.

At an early stage of development of the fotus, and some time before the completion of the changes which have been just described, two important structures, called respectively the amnion and the allantois, begin to be formed.

Amnion. - The amnion is produced as follows:-Beyond the headand tail-folds before described (p. 776 ), the somatopleure coated by epiblast, is raised into folds, which grow up, arching over the embryo, not only anteriorly and posteriorly but also laterally, and all converging toward one point over its dorsal surface (fig. 476). The growing up of these folds from all sides and their convergence toward one point very closely resembles the folding inward of the visceral plates already described, and hence, by some, the point at which the amniotic folds meet over the back has been termed the amniotic umbilicus.

The folds not only come into contact but coalesce. The inner of 
the two layers forms the true amnion, while the outer or reflected layer, sometimes termed the false amnion, coalesces with the inner surface of the original vitelline membrane to form the subzonal membrane or false chorion. This growth of the amniotic folds must of course be clearly distinguished from the very similar process, already described, by which at a much earlier stage the walls of the neural canal are formed.

"The cavity between the true amuion and the external surface of the embryo becomes a closed space, termed the amniotic cavity (ac, fig. 473).

At first, the amnion closely invests the embryo, but it becomes gradually distended with fluid (liquor amnii), which, as pregnancy advances, reaches a considerable quantity.

This fluid consists of water containing small quantities of albumen and urea. Its chief function during gestation appears to be the mechanical one of affording equal support to the embryo on all sides, and of protecting it as far as possible from the effects of blows and other injuries to the abdomen of the mother.

The embryo up to the end of pregnancy is thus immersed in fluid, which during parturition serves the important purpose of gradually and evenly dilating the neck of the uterus to allow of the passage of the fœtus: when this is accomplished the amniotic sac bursts, and the waters escape.

On referring to figs. 471,472 and 473 , it will be obvious that the cavity outside the amnion, between it and the false amnion, is continuous with the pleuro-peritoneal cavity at the umbilicus. This cavity is not entirely obliterated even at birth, and contains a small quantity of fluid, which is discharged during parturition either before, or at the same time as the amniotic fluid.

Allantois.-Into the pleuro-peritoneal space the allantois sprouts out, its formation commencing during the development of the amnion.

Growing out from or near the hinder portion of the intestinal canal ( $c$, fig. 476), with which it communicates, the allantois is at first a solid pear-shaped mass of splanchnopleure; but becoming vesicular by the projection into it of a hollow outgrowth of hypoblast, and very soon simply membraneous and vascular, it insinuates itself between the amniotic folds, just described, and comes into close contact and union with the outer of the two folds, which has itself, as before said, become one with the external investing membrane of the egg. As it grows, the allantois develops muscular tissue in its external wall and becomes exceedingly vascular; in birds (fig. $47 \%$ ) it envelops the whole embryotaking up vessels, so to speak, to the outer investing membrane of the egg, and lining the inner surface of the shell with a vascular membrane, by these means affording an extensive surface in which the blood may be aërated. In the human subject and in other mammalia, the vessels carried out by the allantois are distributed only to a special part of the 
outer membrane or false chorion, where, by interlacement with the vascular system of the mother, a structure called the placenta is developed.

In mammalia, as the visceral laminæ close in the abdominal cavity, the allantois is thereby divided at the umbilicus into two portions; the outer part, extending from the umbilicus to the chorion, soon shrivelling; while the inner part remaining in the abdomen, is in part converted into the urinary bladder; the portion of the inner part not so converted, extending from the bladder to the umbilicus, under the name of the urachus. After birth the umbilical cord, and with it the external and shrivelled portion of the allantois, are cast off at the umbilicus, while the urachus remains as an impervious cord stretched from the top of the urinary bladder to the umbilicus, in the middle line of the body,

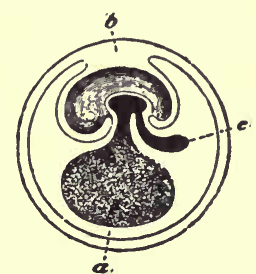

Fig. 476 .

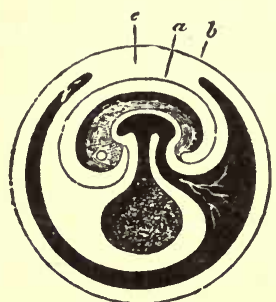

Fig. 477 .

Fig. 476.-Diagram of fecundated egg. $a$, unbilical vesicle; $b$, amniotic cavity ; $c$, allantois. 'Dalton.)

Fig. 477.-Fecundated egg with allantois nearly complete. $\alpha$, inner layer of amniotic fold; $b$, outer layer of ditto; $c$, point where the amniotic folds come in contact. The allantois is seen penetrating between the outer and inner layers of the amniotic folds. This figure, which represents only the amniotic folds and the parts within them, should be compared with figs. 478,479 , in which will be found the structures external to these folds. (Dalton.).

immediately beneath the parietal layer of the peritoneum. It is sometimes enumerated among the ligaments of the bladder.

It must not be supposed that the phenomena which have been successively described, occur in any regular order one after another. On the contrary, the development of one part is going on side by side with that of another.

The Chorion.-It has been already remarked that the allantois is a structure which extends from the body of the fotus to the outer investing membrane of the ovum, that it insinuates itself between the two layers of the amniotic fold, and becomes fused with the outer layer, which has itself become previously joined with the vitelline membrane. By these means the external investing membrane of the ovum, or the true chorion, as it is now called, represents three layers, namely, the original vitelline membrane, the outer layer of the amniotic fold, and the allantois.

Very soon after the entrance of the ovum into the uterus, in the human subject, the outer surface of the chorion is found beset with fine 
processes, the so-called chorion villi (a, figs. 478, 479), which give it a rough and shaggy appearance. At first only cellular in structure, these little outgrowths subsequently become vascular by the development in

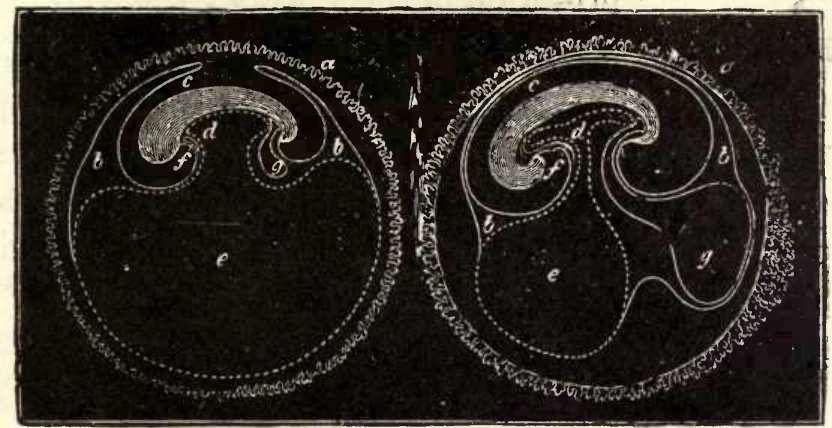

Fig. 478.

Fig. 479.

Figs. 478 and 479. $-a$, chorion with villi. The villi are shown to be best developed in the part of the chorion to which the allantois is extending; this portion ultimately becomes the placenta; $b$, space between the two layers of the amnion; $c$, amniotic cavity; $d$, situation of the intestine, showing its connection with the umbilical vesicle; $e$, umbilical vesicle; $f$, situation of heart and vessels; $g$, allantois.

them of loops of capillaries (fig. 480); and the latter at length form the minute extremities of the blood-vessels which are, so to speak, conducted from the foetus to the chorion by the allantois. The function of the villi of the chorion is evidently the absorption of nutrient matter for the fotus; and this is probably supplied to them at first from the fluid matter, secreted by the follicular glands of the uterus, in which they are soaked. Soon, however, the fœtal vessels of the villi come into. more intimate relation with the vessels of the uterus. The part at which this relation between the vessels of the fœtus and those of the parent ensues, is not, however, over the whole surface of the chorion; for, although all the villi become vascular, yet they become indistinct or disappear except at one part where they are greatly developed, and by their branching give rise, with the vessels of the uterus, to the formation of the placenta.

To understand the manner in which the foetal and maternal blood-vessels come into relation with each other in the placenta, it is necessary

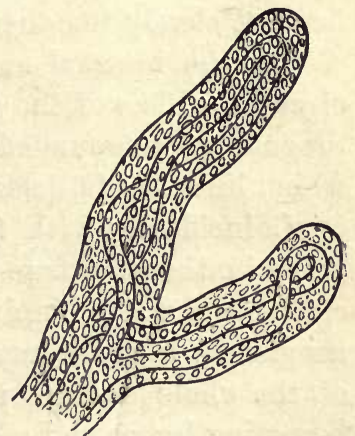

Fig. 480. briefly to notice the changes which the uterus undergoes after impregnation. These changes consist especially of alterations in structure of the superficial part of the mucous membrane which lines the interior of the uterus, and which forms, after a kind of development to be imme- 
diately described, the membrana decidua, so called on account of its being discharged from the uterus at birth.

\section{Formation of the Placenta.}

The mucous membrane of the human uterus, which consists of a matrix of connective tissue containing numerous corpuscles, and is lined internally by columnar ciliated epithelium, is abundantly beset with tubular glands, arranged perpendicularly to the surface (fig. 481). These

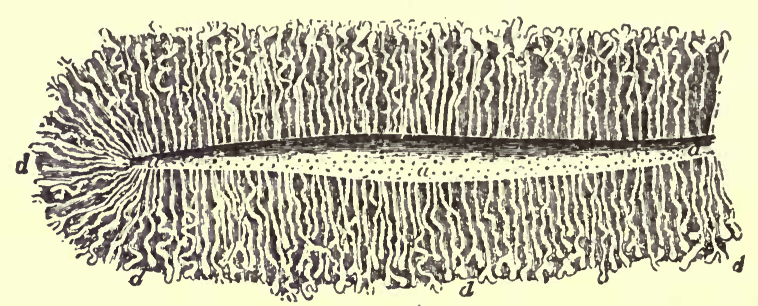

Fig. 481. - Section of the lining membrane of a human uterus at the period of commencing pregnancy showing the arrangement and other peculiarities of the glands, $d, d, d$, with their orifices, $a, a, a$, on the internal surface of the organ. Twice the natural size.

follicles are very small in the unimpregnated uterus; but when examined shortly after impregnation, they are found elongated, enlarged, and much waved and contorted toward their deep and closed extremity, which is planted at some depth in the tissue of the uterus, and may dilate into two or three closed sacculi.

The glands are lined by columnar ( (?) ciliated) epithelium and they open on the inner surface of the mucous membrane by small round orifices set closely together ( $a, a$, fig. 481).

On the internal surface of the mucous membrane may be seen the circular orifices of the glands, many of wlich are, in the early period of pregnancy, surrounded by a whitish ring, formed of the epithelium which lines the follicles.

Coincidently with the occurrence of pregnancy, important changes occur in the structure of the mucous membrane of the uterus. The epithelium and sub-epithelial connective tissue, together with the tubular glands, increase rapidly, and there is a greatly increased vascularity of the whole mucous membrane, the vessels of the mucous membrane becoming larger and more numerous; while a substance composed chiefly of nucleated cells fills up the interfollicular spaces in which the bloodvessels are contained. The effect of these changes is an increased thicknes, softness, and vascularity of the mucous membrane, the superficial part of which itself forms the membrana decidua.

The object of this increased development seems to be the production 
of nutritive materials for the ovum; for the cavity of the uterus shortly becomes filled with secreted fluid, consisting almost entirely of nucleated cells in which the chorion villi are imbedded.

When the ovum first enters the uterus it becomes imbedded in the structure of the deciủua, which is yet quite soft, and in which soon afterward three portions are distinguishable. These have been named the decidua vera, the decidua reflexa, and the decidua serotina.

The first of these, the decidua vera, lines the cavity of the uterus; the second, or decidua reflexa, is a part of the decidua vera which grows up around the ovum, and wrapping it closely, forms its immediate investment.

The third, or decidua serotina, is the part of the decidua vera which becomes especially developed in connection with those villi of the chorion, which, instead of disappearing, remain to form the fœtal part of the placenta.

In connection with these villous processes of the chorion, there are developed depressions or crypts in the decidual mucous membrane, which correspond in shape with the villi they are to lodge; and thus the chorionic villi become more or less imbedded in the maternal structures.

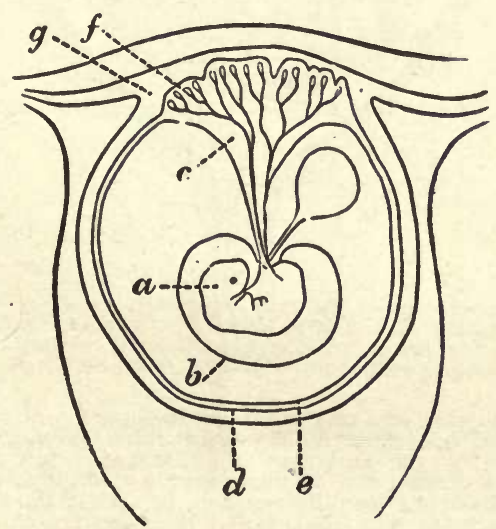

Fig. 482. - Diagram of an early stage of the formation of the human placenta. $a$, embryo; $b$, amnion; $c$, placental vessels; $d$, decidua reflexa; $e$, allantois; $f$, placental villi; $g$, mucous membrane. (Cadiat.)

These uterine crypts, it is important to note, are not, as was once supposed, merely the open mouths of the uterine follicles.

As the ovum increases in size, the decidua vera and the decidua reflexa gradually come into contact, and in the third month of pregnancy the cavity between them has almost disappeared. Though the two layers come into contact at the third month, they are not closely amalgamated until the end of the sixth month.

The Placenta.-During these changes the deeper part of the mu- 
cous membrane of the uterus, at and near the region where the placenta is placed, becomes hollowed out by sinuses, or cavernous spaces, which communicate on the one hand with arteries and on the other with veins of the uterus. Into these sinuses the villi of the chorion protrude, pushing the thin wall of the sinus before them, and so come into intimate relation with the blood contained in them. There is no direct communication between the blood-vessels of the mother and those of the fcetus; but the layer or layers of membrane intervening between the

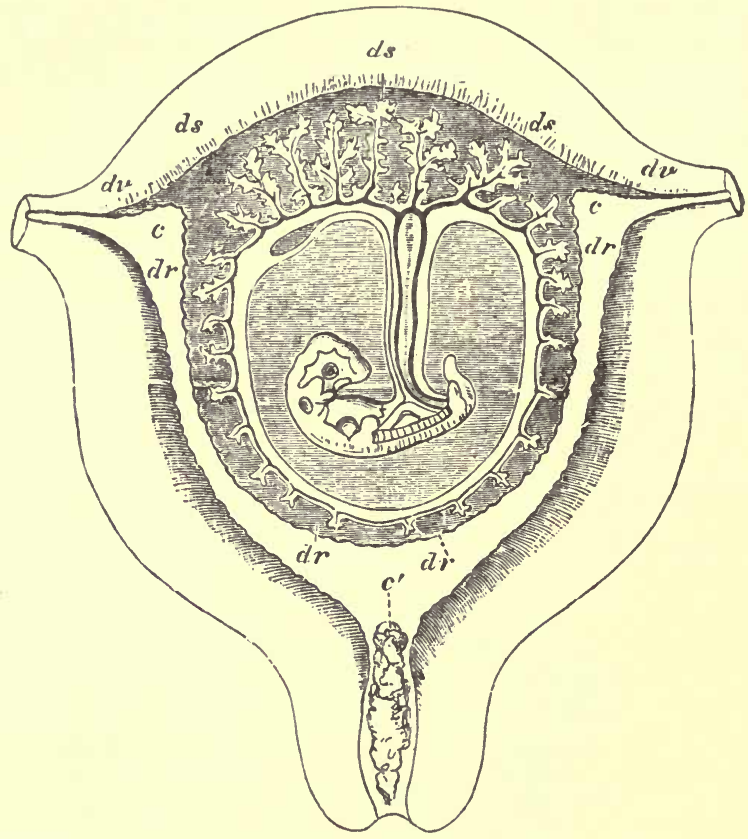

Fig. 483. - Diagrammatic view of a vertical transverse section of the uterus at the serenth or eighth week of pregnancy. $c, c, c^{\prime}$, cavity of uterus, which becomes the cavity of the decidua, opening at $c, c$, the cornua, into the Fallopian tubes, and at $c^{\prime}$ into the cavity of the cervix, which is closed by a plug of mucus; $d v$, decidua vera; $d r$, decidua reflexa, with the sparser villi imbedded in its substance; $d s$, decidua serotina, involving the more developed chorionic villi of the commencing placenta. The fotus is seen lying in the amniotic sac; passing up from the umbilicus is seen the umbilical cord and its vessels, passing to their distribution in the villi of the chorion; also the pedicle of the yolk-sac, which lies in the cavity between the amnion and chorion. (Allen Thomson.)

blood of the one and of the other offer no obstacle to a free interchange of matters between them by diffusion and osmosis. Thus the villi of the chorion containing fœtal blood, are bathed or soaked in maternal blood contained in the uterine sinuses. The arrangement may be roughly compared to filling a glove with fotal blood, and dipping its fingers into a vessel containing maternal blood. But in the fœtal villi there is a constant stream of blood into and out of the loop of capillary blood-vessels contained in it, as there is also into and out of the maternal sinuses. 
It would seem that, at the villi of the placental tufts, where the fotal and maternal portions of the placenta are brought into close relation with each other, the blood in the ressels of the mother is separated from that in the ressels of the fœtus by the intervention of two distinct sets of nucleated cells (fig. 484). One of these (b) belongs to the maternal portion of the placenta, is placed between the membrane of the villus and that of the vascular system of the mother, and is probably designed to separate from the blood of the parent the materials destined for the blood of the foetus; the other $(f)$ belongs to the fœtal portion of the placenta, is situated between the membrane of the villus and the loop of ressels contained within, and probably serves for the absorption of the material secreted by the other sets of cells, and for its conveyance into the blood-vessels of the fœtus. Between the two sets of cells with their investing membrane there exists a space $(d)$, into which it is possible that the materials secreted by the one set of cells of the villus are poured in order that they may be absorbed by the other set, and thus conveyed into a fotal vessel.

Not only, however, is there a passage of materials from the blood of the mother into that of the fœtus, but there is a mutual interchange of

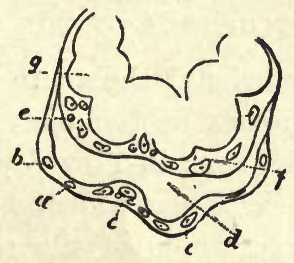

Fig. 484. - Extremity of a placental villus. $a$, lining membrane of the vascular system of the mother; $b$, cells immediately lining $a ; d$, space between the maternal and foetal portions of the villus; $e$, internal membrane of the villus, or external membrane of the chorion; $f$, internal cells of the villus, or cells of the chorion; $g$, loop of umbilical vessels. (Goodsir.)

materials between the blood both of fœtus and of parent; the latter supplying the former with nutriment, and in turn abstracting from it materials which require to be removed.

The placenta, therefore, of the human subject is composed of a foetal part and a maternal part,- - the term placenta properly including all that entanglement of fotal villi and maternal sinuses, by means of which the blood of the fœtus is enriched and purified after the fashion necessary for the proper growth and development of those parts which it is designed to nourish.

The whole of this structure is not, as might be imagined, thrown off immediately after birth. The greater part, indeed, comes away at that time, as the after-birth; and the separation of this portion takes place by a rending or crushing through of that part at which its cohesion is least strong, namely, where it is most burrowed and undermined 
by the cavernous spaces before referred to. In this way it is cast off with the fotal membrane and the decidua vera and reflexa, together with a part of the decidua serotina. The remaining portion withers, and disappears by being gradually either absorbed, or thrown off in the uterine discharges or the loclia, which occur at this period.

A new mucous membrane is of course gradually developed, as the old one, by its transformation into the decidua, ceases to perform its original functions.

The umbilical cord, which in the latter part of fœtal life is almost solely composed of the two arteries and the single vein which respectively convey fotal blood to and from the placenta, contains the remnants of other structures which in the early stages of the development of the embryo were, as already related, of great comparative importance. Thus, in early fœtal life, it is composed of the following parts:-(1.) Externally, a layer of the amnion, reflected over it from the umbilicus. (2) The umbilical vesicle with its duct and appertaining omphalo-mesenteric blood vessels. (3.) The remains of the allantois, and continuous with it the urachus. (4.) The umbilical vessels, which, as just remarked, ultimately form the greater part of the cord.

\section{The Development of the Organs.}

Before considering very briefly* the main points in the development of the chief organs and tissues of the body, it will be useful to have before us the following table, compiled by Schäfer, $\uparrow$ showing the different parts derived from the three blastodermic layers:-

From the Epiblast.-The whole of the nervous system, including not only the central organs (brain and spinal cord), but also the peripheral nerves and sympathetic.

The epithelial structures of the organs of special sense.

The epidermis and its appendages, including the hair and nails.

The epithelium of all the glands opening upon the surface of the skin, including the mammary glands, the sweat glands and the sebaceous glands. The muscular fibres of the sweat glands.

The epithelium of the mouth (except that covering the tongue, and the adjacent posterior part of the floor of the mouth, which is derived from the hypoblast), and that of the glands opening into it.

The enamel of the teeth.

The epithelium of the nasal passages, of the adjacent upper part of the pharynx and of all the cavities and glands opening into the nasal passages.

* For a more detailed account the reader is referred to special text-books of embryology.

† Quain's Anatomy, Xth Ed., Vol. I., Part I., p. 25. 
From the Mesoblast. - The urinary and generative organs (except the epithelium of the urinary bladder and urethra).

All the voluntary and involuntary muscles of the body (except the muscular fibres of the sweat glands).

The whole of the vascular and lymphatic system, including the serous membranes and spleen.

The skeleton and all the connective tissues and structures of the body.

From the Hypoblast. - The epithelium of the alimentary canal from the back of the mouth to the anus, and that of all the glands which open into this part of the alimentary tube.

The epithelium of the Eustachian tube and tympanum.

The epithelium of the bronchial tubes and air sacs of the lungs.

The epithelium lining the vesicles of the thyroid body.

The epithelial nests of the thymus.

The epithelium of the urinary bladder and urethra.

It remains now to consider in succession the development of the several organs and systems of organs in the further progress of the

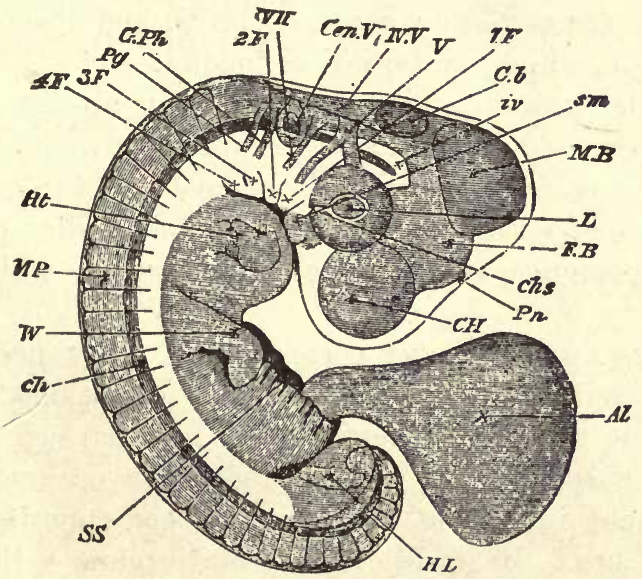

Fig. 485. - Embryo chick (4th day), vlewed as a transparent object, lying on its left side (magnified). $C H$, cerebral hemispheres; $F B$, fore-brain or vesicle of third ventricle, with $P n$, pineal gland projecting from its summit; $M B$, mid-brain; $C b$, cerebellum; $I V$. $V$, fourth ventricle; $L$, lens; $c h s$, choroidal slit; $C e n ' V$, auditory vesicle; $s m$, superior maxiliary process; $1 F, 2 F$, etc., first, second, third, and fourth visceral folds: $\vec{V}$, fifth nerve, sending one branch (ophthalmic) to the eye, and another to the first visceral arch; $\dot{V} I I$, seventh nerve, passing to the second visceral arch; GPh, glosso-pharyngeal nerve, passing to the third visceral arch; $P g$, pneumogastric nerve, passing toward the fourth visceral arch; $i v$, investing mass; $c h$, notochord; its front end cannot be seen in the living embryo, and it does not end as shown in the figure, but takes a sudden bend downward, and then terminates in a point; $H t$, heart seen through the walls of the chest: $M P$, muscle plates; $W$, wing, showing commencing differentiation of segments, corresponding to arm, forearm, and hand; $H L$, hind-limb, as yet a shapeless bud, showing no differentiation. Beneath it is seen the curved tail. (Foster and Balfour.)

embryo. The accompanying figure (fig. 485) shows the chief organs of the body in a moderately early stage of development.

The Vertebral Column and Cranium.-The primitive part of the vertebral column in all the vertebrata is the chorda dorsalis or noto- 
chord, which consists entirely of soft cellular cartilage. 'This cord tapers to a point at the cranial and caudal extremities of the animal. In the progress of its development, it is found to become inclosed in a membranous sheath, which at length acquires a fibrous structure, composed of transverse annular fibres. The chorda dorsalis is to be regarded as the azygos axis of the spinal column, and, in particular, of the future bodies of the vertebræ, although it never itself passes into the state of hyaline cartilage or bone, but remains inclosed as in a case within the persistent parts of the vertebral column which are developed around it. It is permanent, however, only in a few animals: in the majority only traces of it persist in the adult animal.

In many fish no true vertebræ are developed, and there is every graduation from the amphioxus, in which the notochord persists through life and there are no vertebræ, through the lampreys in which there are a few scattered cartilaginous vertebræ, and the sharks, in which many of the vertebræ are partly ossified, to the bony fishes, such as the cod and herring, in which the vertebral column consists of a number of distinct ossified vertebræ, with remnants of the notochord between them. In amphibia, reptiles, birds, and mammals, there are distinct vertebræ, which are formed as follows:-

The mesoblastic somites, which have been already mentioned ( $p$. 776), send processes downward and inward to surround the notochord, and also upward between the medullary canal and the epiblast covering it. In the former situation, the cartilaginous bodies of the vertebræ make their appearance, in the latter their arches, which inclose the neural canal.

The vertebræ do not exactly correspond in their position with the protovertebræ: but each permanent vertebra is developed from the contiguous halves of two protovertebræ. The original segmentation of the protovertebræ disappears and a fresh subdivision occurs in such a way that a permanent invertebral disc is developed opposite the centre of each protovertebra. Meanwhile the protovertebræ split into a dorsal and ventral portion. The former is termed the musculo-cutaneous plate, and from it are developed all the muscles of the back together with the cutis of the dorsal region (the epidermis being derived from the epiblast). 'The ventral portions of the protovertebræ, as we have already seen, give rise to the vertebræ and heads of the ribs.

The chorda is now inclosed in a case, formed by the bodies of the vertebræ, but it gradually wastes and disappears. Before the disappearance of the chorda, the ossification of the bodies and arches of the vertebræ begins at distinct points.

The ossification of the body of a vertebra is first observed at the point where the two primitive elements of the vertebræ have united 
inferiorly. Those vertebræ which do not bear ribs, such as the cervical vertebræ, have generally an additional centre of ossification in the transverse process, which is to be regarded as an abortive rudiment of a rib. In the fœtal bird, these additional ossified portions exist in all the cervical vertebræ, and gradually become so much developed in the lower part of the cervical region as to form the upper false ribs of this class of animals. The same parts exist in mammalia and man; those of the last cervical vertebræ are the most developed, and in children may, for a considerable period, be distinguished as a separate part on each side like the root or head of a rib.

The true cranium is a prolongation of the vertebral column, and is developed at a much earlier period than the facial bones. Originally, it is formed of but one mass, a cerebral capsule, the chorda dorsalis being continued into its base, and ending there with a tapering point. At an early period the head is bent downward and forward round the end of the chorda dorsalis in such a way that the middle cerebral vesicle, and not the anterior, comes to occupy the highest position in the head.

Pituitary Body.-In connection with this must be mentioned the development of the pituitary body. It is formed by the meeting of two outgrowths, one from the fœtal brain, which grows downward, and the other from the epiblast of the buccal cavity, which grows up toward it. The surrounding mesoblast also takes part in its formation. The connection of the first process with the brain becomes narrowed, and persists as the infundibulum, while that of the other process with the buccal cavity disappears completely at a spot corresponding with the future position of the body of the sphenoid.

Cranium.-The first appearance of a solid support at the base of the cranium observed by Muller in fish, consists of two elongated bands of cartilage (trabeculæ cranii), one on the right and the other on the left side, which are connected with the cartilaginous capsule of the auditory apparatus, and which diverge to inclose the pituitary body uniting in front to form the septum nasi beneath the anterior end of the cerebral capsule. Hence, in the cranium, as in the spinal column, there are at first developed at the sides of the chorda dorsalis two symmetrical elements, which subsequently coalesce, and may wholly inclose the chorda.

The brain-case consists of three segments: occipital, parietal, and frontal, corresponding in their relative position to the three primitive cerebral vesicles; it may also be noted that in front of each segment is developed a sense-organ (auditory, ocular, and olfactory, from behind forward). The basis cranii consists at an early period of an unsegmented cartilaginous rod, developed round the notochora, and continued forward beyond its termination into the trabeculce cranii, which bound the pituitary fossa on either side. 
In this cartilaginous rod three centres of ossification appear: basioccipital, basi-sphenoid, and pre-sphenoid, one corresponding to each segment.

The bones forming the vault of the skull, viz., the frontal, parietal, squamous portion of temporal and the squamo-occipital, are ossified in membrane.

\section{The Visceral Clefts and Arches.}

As the embryo enlarges, the heart, which at first occupied a position close to the cranial flexure, is carried further and further back ward until a considerable part, in which the mesoblast is undivided, intervenes between
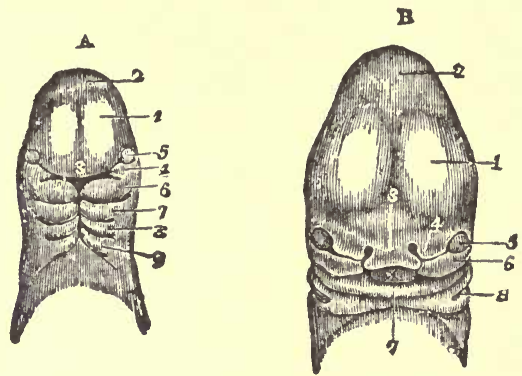

Fig. 486. - A. Magnified view from before of the head and neck of a human embryo of about three weeks (from Ecker.)-1, anterior cerebral vesicle or cerebrum; 2 , middle ditto; 3 , middlo or fronto-nasal process; 4, superior maxillary process; 5, eye; 6 , inferior maxillary process, or first visceral arch, and below it the first cleft; $\gamma, 8,9$, second, third, and fourth arches and elefts. B. Anterior view of the head of a human foetus of about the fifth week (from Ecker, as before, fig. IV.). 1, 2, 3, 5, the same parts as in $\mathrm{A} ; 4$, the external nasal or lateral frontal process: 6, the superior maxillary process; $\tau$, the lower jaw; $\times$, the tongue; 8 , first branchial cleft becoming the meatus auditorius externus.

it and the head. This becomes the neck. On section it is seen that in it the whole three layers are represented in order, and that there is no interval between them. In the neck thus formed soon appear the visceral or branchial clefts on either side, in series, across the axis of the gut not quite at right angles. 'They are four in number, the most anterior being first found. At their edges the hypoblast and their epiblast are continuous. The anterior border of each cleft forms a fold or lip, the branchial or visceral fold. The posterior border of the last cleft is also formed into a fold, so that there are four clefts and five folds, but the three most anterior are far more prominent than the others, and of these the second is the most conspicuous. The first fold nearly meets its fellow in the middle line, the second less nearly, and the others in order still less so. Thus in the neck there is a triangular interval, into which by the splitting of the mesoblast at that part the pleuroperitoneal cavity extends. The branchial clefts and arches are not all permanent. The first arch gives off a branch from its front edge, which passes forward to meet its fellow, but these offshoots do not quite meet, being separated 
by a process which grows downward from the head. Between the branches and the main first fold is the cavity of the mouth. The branches represent the superior maxilla, and the main folds the mandible or lower jaw. The central process, which grows down, is the fronto-nasal process.

In this way the so-called visceral arches and clefts are formed, four on each side (fig. $486, \mathrm{~A}$ ).

From or in connection with these arches the following parts are developed:-

The first arch (mandibular) contains a cartilaginous rod (Meckel's cartilage), around the distal end of which the lower jaw is developed, while the malleus is ossified from the proximal end.

When the maxillary processes on the two sides fail partially or completely to unite in the middle line, the well-known condition termed cleft palate results. When the integument of the face presents a similar deficiency, we have the deformity known as hare-lip. Though these two

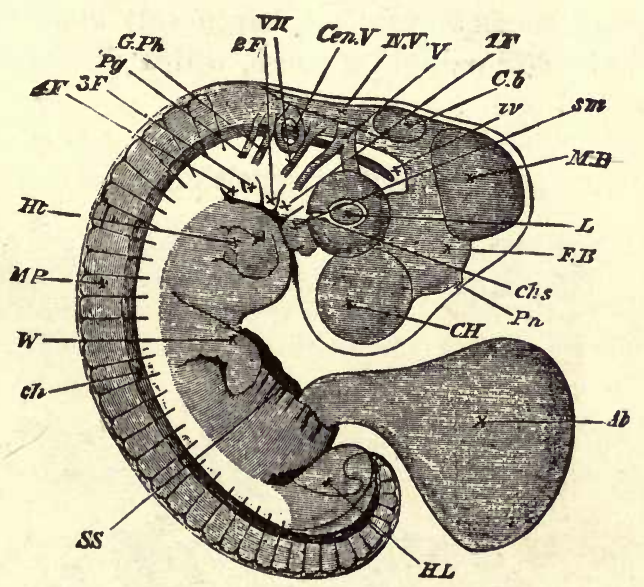

Fig. 487.- Embryo chick (4th day), viewed as a transparent object, lying on its left side (magnifled). $C H$, cerebral hemispheres; $F B$, fore-brain or vesicle of third ventricle, with $P n$, tricle; $L$, lens; $c h s$, choroidal slit: $C e n$. $V$, auditory vesicle; $s \mathrm{~m}$, superior maxillary process: $1 F, 2 F$, etc., first, second, third, and fourth visceral folds; $\dot{V}$, fifth nerve, sending one branch (ophthalmic) to the eye, and another to the first visceral arch; VII, seventh nerve, passing to the second visceral arch; $G$. $P h$, glosso-pharyngeal nerve, passing to the third visceral arch; $P g$, pneumogastric nerve, passing toward the fourth visceral arch; $i v$, investing mass; $c h$, notochord; its front end cannot be seen in the living embryo, and it does not end as shown in the figure, but takes a sudden bend downward, and then terminates in a point; $H t$, heart seen through the walls of the chest; $M P$, muscle-plates; $W$, wing, showing commencing differentiation of segments, corresponding to arm, forearm, and hand; $S S$, somatic stalk; $A l$, allantois; $H L$, hind-limb, as yet a shapeless bud, showing no differentiation. Beneath it is seen the curved tail. (Foster and Balfour.)

deformities frequently co-exist, they are by no means always necessarily associated.

The upper part of the face in the middle line is developed from the so-called frontal-nasal process (A, 3, fig. 486.) From the second arch 
are developed the incus, stapes, and stapedius muscle, the styloid process of the temporal bone, the stylo-hyoid ligament, and the smaller cornu of the hyoid bone. From the third visceral arch, the greater cornu and body of the hyoid bone. In man and other mammalia the fourth visceral arch is indistinct. It occupies the position where the neck is afterward dereloped.

A distinct connection is traceable between these visceral arches and certain cranial nerves: the trigeminal, the facial, the glcsso-pharyngeal, and the vagus. 'The ophthalmic division of the trigeminal supplies the fronto-nasal process; the superior and inferior maxillary divisions supply the maxillary and mandibular arches respectively.

The facial nerve distributes one branch (chorda tympani) to the first visceral arch, and others to the second visceral arch. Thus it divides, inclosing the first risceral cleft.

Similarly, the glosso-pharyngeal divides to inclose the second risceral cleft, its lingual branch being distributed to the second, and its pharyngeal branch to the third arch.

The ragus, too, sends a branch (pharyngeal) along the third arch, and in fishes it gives off paired branches, which divide to inclose several successive branchial clefts.

\section{The Extremities.}

The extremities are developed in a uniform manner in all vertebrate animals. 'They appear in the form of leaf-like elevations from the

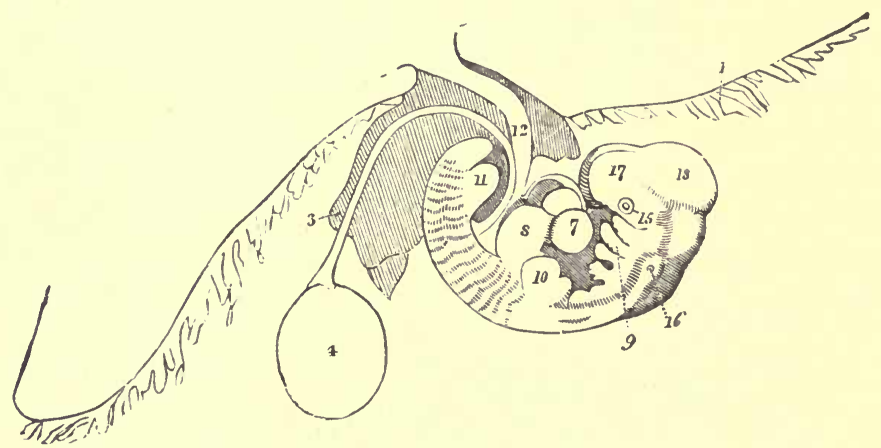

Fig. 488.-A human embryo of the fourth week, $31 / 2$ lines in length. -1 , the chorion; 3 , part of the amnion; 4, umbilical vesicle with its long pedicle passing into the abdomen; 7 , thr heart; 8 , the liver; 9 , the visceral arch destined to form the lower jaw, beneath which are two other visceral arches separated by the branchial clefts; 10 , rudiment of the upper extremity; 11, that of the lower extremity; 12 , the umbilical cord; 15 , the eye; 16 , the ear; 17 , cerebral hemispheres; 18, optic lobes, corpora quadrigemina. (Müller.)

parieties of the trunk (see fig. 488), at points where more or less of an arch will be produced for them within. The primitive form of the extremity is nearly the same in all vertebrata, whether it be destined for 
swimming, crawling, walking, or flying. In the human foetus the fingers are at first united, as if webbed for swimming; but this is to be regarded not so much as an approximation to the form of aquatic animals, as the primitive form of the hand, the individual parts of which subsequently become more completely isolated.

The fore-limb always appears before the hind-limb, and for some time continues in a more advanced state of development. In both limbs alike, the distal segment (hand or foot) is separated by a slight notch from the proximal part of the limb, and this part is subsequently divided again by a second notch (knee or elbow-joint).

The Vascular System.-At an early stage in the development of the embryo-chick, the so-called area vasculosa begins to make its appearance. A number of branched cells in the mesoblast send out processes which unite so as to form a network of protoplasm with nuclei at the nodal points. A large number of nuclei acquire red color; these form the red blood-corpuscles. The protoplasmic processes become hollowed out in the centre so as to form a closed system of branching canals, in the walls of which the rest of the nuclei remain imbedded. In the blood-ressels thus formed, the circulation of the embryonic blood commences.

According to Klein, the first blood-vessels in the chick are developed from embryonic cells of the mesoblast, which swell up and become vacuolated, while their nuclei undergo segmentation. These cells send out protoplasmic processes, which unite with corresponding ones from other cells, and become hollowed, give rise to the capillary wall composed of endothelial cells; the blood corpuscles being budded off from the endothelial wall by a process of gemmation.

Heart.-About the same early period the heart makes its appearance as a solid mass of cells of the splanchnopleure in the manner before indicated.

At this period the anterior part of the alimentary tube ends blindly beneath the notochord. It is beneath the posterior end of this fore-gut that the heart begins to be developed. The heart when first formed is made up of two not quite complete tubes which coalesce to form one, and so when the cavity is hollowed out in the mass of cells, the central cells float freely in the fluid, which soon begins to circulate by means of the rhythmic pulsations of the embryonic heart.

These pulsations take place even before the appearance of a cavity, and immediately after the first laying down of the cells from which the heart is formed, and long before muscular fibres or ganglia have been formed in the cardiac walls. At first they seldom exceed from fifteen to eighteen in the minute. The fluid within the cavity of the heart shortly assumes the characters of blood. At the same time, the cavity 
itself forms a communication with the great vessels in contact with it, and the cells of which its walls are comprised are transformed into fibrous and muscular tissues, and into epithelium. In the developing chick

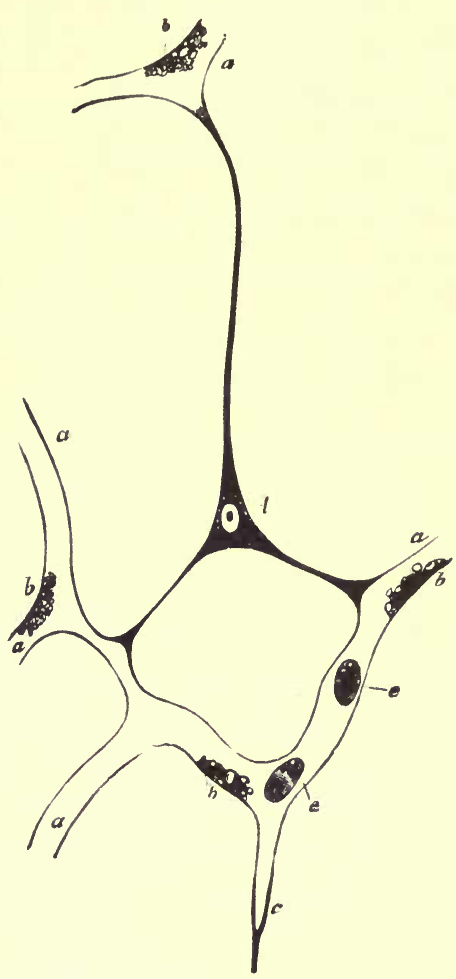

Fig. 489.

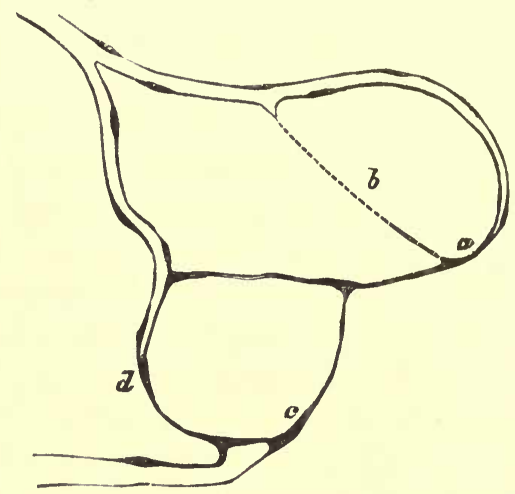

Fig. 490 .

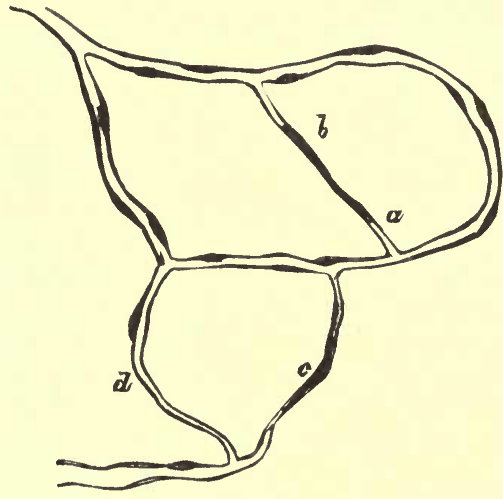

Fig. 491 .

Fig. 489. - Capillary blood-vessels of the tail of a young larval frog. $a$, capillaries permeable to blood; $b$, fat granules attached to the walls of the vessels, and concealing the nuclei; $c$, hollow prolongation of a capillary, ending in a point; $d$, a branching cell with nucleus and fatgranules; it communicates by three branches with prolongation of capillaries already formed; $e, e$, blood corpuscles still containing granules of fat. $\times 350$ times. (Kölliker.)

Fig. 490. - Development of capillaries in the regenerating tail of a tadpole. $a b c d$. sprouts and cords of protoplasm. (Arnold.)

Fig. 491.-The same region after the lapse of 24 hours. The "sprouts and cords of protoplasm" have become channelled out into capillaries. (Arnold.)

it can be observed with the naked eye as a minute red pulsating little mass before the end of the second day of incubation.

Blood-vessels. - Blood-ressels appear to be developed in two ways, according to their size. In the formation of large blood-vessels, masses of embryonic cells similar to those from which the heart and other structures of the embryo are developed, arrange themselves in the position, form, and thickness of the developing vessel. Shortly afterward the cells in the interior of a column of this kind seem to be developed into blood- 
corpuscles, while the external layer of cells is converted into the walls of the vessel.

In the development of capillaries another plan is pursued. This has been well illustrated by Kölliker, as observed in the tails of tadpoles. The first lateral vessels of the tail have the form of simple arches, passing between the main artery and vein, and are produced by the junction of prolongations, sent from both the artery and vein, with certain elongated or star-shaped cells, in the substance of the tail. When these arches are formed and are permeable to blood, new prolongations pass from them, join other radiated cells, and thus form secondary arches. In this manner, the capillary network extends in proportion as the tail increases in length and breadth, and it, at the same time, becomes more dense by the formation, according to the same plan, of fresh vessels within its meshes. The prolongations by which the vessels communicate with the star-shaped cells, consist at first of narrow pointed projections from the side of the vessels, which gradually elongate until they come in contact with the radiated processes of the cells. The thickness of such a prolongation often does not exceed that of a fibril of fibrous tissue, and at first it is perfectly solid; but, by degrees, especially after its junction with a cell, or with another prolongation, or with a vessel already permeable to blood, it enlarges, and a cavity then forms in its interior (see figs. 491, 492). This tissue is well calculated to illustrate the various steps in the development of blood-vessels from elongating and branching cells.

In many cases a whole network of capillaries is developed from a network of branched, embryonic connective-tissue corpuscles by the join-

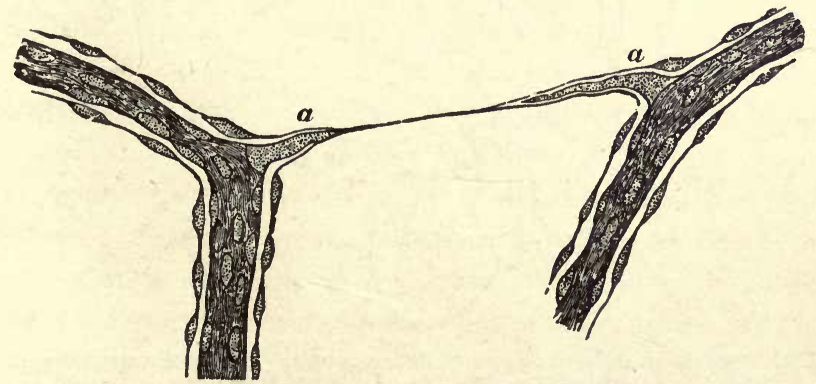

Fig. 492.-Capillaries from the vitreous humor of a fœtal calf. Two vessels are seen connected by a "cord" of protoplasm, and clothed with an adventitia, containing numerous nuclei. $a$, insertion of this "cord "into the primary walls of the vessels. (Frey.)

ing of their processes, the multiplication of their nuclei, and the vacuolation of the cell-substance. The vacuoles gradually coalesce till all the partitions are broken down, and the originally solid protoplasmic cellsubstance is, so to speak, tunnelled out into a number of tubes.

Capillaries may also be developed from cells which are originally spheroidal, vacuoles form in the interior of the cells gradually becoming 
united by fine protoplasmic processes: by the extension of the vacuoles into them, capillary tubes are gradually formed.

Morphology. Heart. - When it first appears, the heart is approximately tubular in form, being at first a double tube then a single one. It receives at its two posterior angles the two omphalo-mesenteric or vitelline veins, and gives off anteriorly the primitive aorta (fig. 493). The junction of the two veins which pass into the auricle becomes removed farther and farther away from the heart, and the ressel thus formed is called sinus venosus near to the auricle, and ductus venosus farther away, or if it be called by one name that of meatus venosus may be used.

It soon, however, becomes curved somewhat in the shape of a horseshoe, with the convexity toward the right, the venous end being at the same time drawn up toward the head, so that it finally lies behind and somewhat to the right, of the arterial. It also becomes partly divided by constrictions into three cavities.

Of these three cavities which are developed in all vertebrata, that at the venous end is the simple auricle, with the sinus venosus, that at the arterial end the bulbus arteriosus, and the middle one is the simple rentricle.

These three parts of the heart contract in succession. The auricle and the bulbus arteriosus at this period lie at the extremities of the

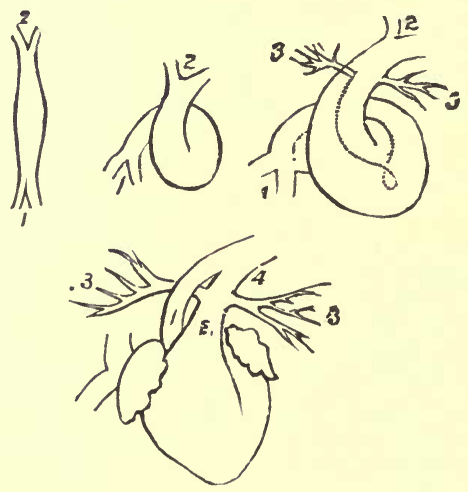

Fig. 493. - Fœtal heart in successive stages of development. 1, venous extremity; 2, arterial extremity; 3, 3, pulmonary branches; 4, ductus arteriosus. (Dalton.)

horse-shoe. The bulging out of the middle portion inferiorly gives the first indication of the future form of the ventricle (fig. 493). The great curvature of the horse-shoe by the same means becomes much more developed than the smaller curvature between the auricle and bulbus; and the two extremities, the auricle and bulb, approach each other superiorly, so as to produce a greater resemblance to the later form of the heart, while the ventricle becomes more and more developed in- 
feriorly. The heart of fishes retains these cavities, no further division by internal septa into right and left chambers taking place. In amphibia, also, the heart throughout life consists of the three muscular divisions which are so early formed in the embryo and the sinus venosus; but the auricle is divided internally by a septum into a pulmonary and systemic auricle. In reptiles, not merely the auricle is thus divided into two cavities, but a similar septum but incomplete is more or less developed

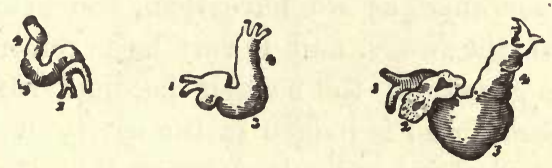

Fig. 494.-Heart of the chick at the 45th, 65th, and 85th hours of incubation. 1, the venous trunks; 2 , the auricle; 3 , the ventricle; 4 , the bulbus arteriosus. (Allen Thomson.)

in the ventricle. In birds and mammals, both auricle and ventricle undergo complete division by septa; while in these animals as well as in reptiles, the bulbus aortæ is not permanent, but becomes lost in the ventricles. The septum dividing the ventricle commences at the apex and extends upward. The subdivision of the auricles is very early foreshadowed by the outgrowth of the two auricular appendages, which occurs before any septum is formed externally. The septum of the auricles is developed from a semilunar fold, which extends from above downward. In man, the septum between the ventricles, according to Meckel, begins to be formed about the fourth week, and at the end of eight weeks is complete. The septum of the auricles, in man and all animals which possess it, remains imperfect throughout fotal life. When the partition of the auricles is first commencing, the two venæ cavæ have different relations to the two cavities. The superior cava enters, as in the adult, into the right auricle; but the inferior cava is so placed that it appears to enter the left auricle, and the posterior part of the septum of the auricles is formed by the Eustachian valve, which extends from the point of entrance of the inferior cava. Subsequently, however, the septum, growing from the anterior wall close to the upper end of the ventricular septum, becomes directed more and more to the left of the vena cava inferior. During the entire period of fotal life, there remains an opening in the septum, which the valve of the foramen ovale, developed in the third month, imperfectly closes.

The bulbus arteriosus, which is originally a single tube, becomes gradually divided into two by the growth of an internal septum, which springs from the posterior wall, and extends forward toward the front wall and downward toward the ventricles. This partition takes a somewhat spinal direction, so that the two tubes (aorta and pulmonary artery) 
which result from its completion, do not run side by side, but are twisted round each other.

As the septum grows down toward the ventricles, it meets and coalesces with the upwardly growing ventricular septum, and thus from the right and left ventricles, which are now completely separate, arise respectively the pulmonary artery and aorta, which are also quite distinct. The auriculo-ventricular and semi-lunar valves are formed by the folds of the endocardium.

At its first appearance, as we have seen, the heart is placed just beneath the head of the fotus, and is very large relatively to the whole body; but with the growth of the neck it becomes further and further removed from the head, and is lodged in the cavity of the thorax.

Up to a certain period the auricular is larger than the ventricular division of the heart; but this relation is gradually reversed as development proceeds. Moreover, all through fotal life, the walls of the right ventricle are of very much the same thickness as those of the left, which may probably be explained by the fact that in the fœtus the right ventricle has to propel the blood from the pulmonary artery into the aorta, and thence into the placenta, while in the adult it only drives the blood through the lungs.

Arteries. - The primitive aorta arises from the bulbus arteriosus and divides into two branches which arch backward, one on each side of the foregut and unite again behind it, and in front of the notochord into a single vessel.

This gives off the two omphalo-mesenteric arteries, which distribute branches all orer the yolk-sac; this area vasculosa in the chick attaining a large development, and being limited all round by a vessel known as the sinus terminalis.

The blood is collected by the venous channels, and returned through the omphalo-mesenteric veins to the heart.

Behind this pair of primitive aortic arches, four more pairs make their appearance sucessively, so that there are five pairs in all, each one running along one of the visceral arches.

These five are never all to be seen at once in the embryo of higher animals, for the two anterior pairs gradually disappear, while the posterior ones are making their appearance, so that at length only three remain.

In fishes, however, they all persist throughout life as the branchial arteries supplying the gills, while in amphibia three pairs persist throughout life.

In reptiles, birds, and mammals, further transformations occur.

In reptiles the fourth pair remains throughout life as the permanent right and left aorta; in birds the right one remains as the permanent 
aorta, curving over the right bronchus instead of the left as in mammals.

In mammals the left fourth aortic arch develops into the permanent aorta, the right one remaining as the subclavian artery of that side. Thus the subclavian artery on the right side corresponds to the aortic arch on the left, and this homology is further confirmed by the fact that

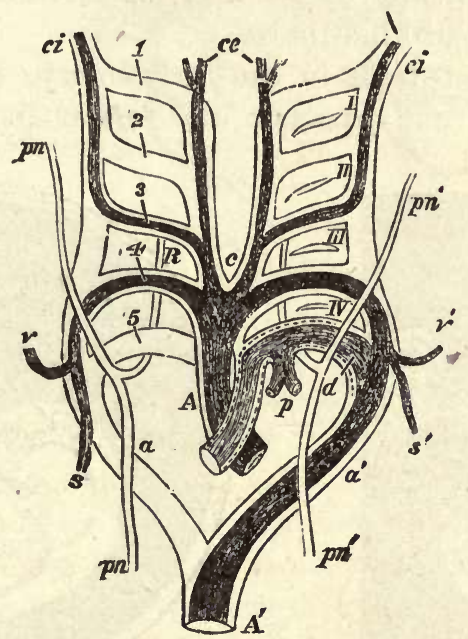

Fig. 495.-Diagram of the aortic arches in a mammal, showing transformations which give rise to the permanent arterial vessels. $A$, primitive arterial stem or aortic bulb, now divided into $A$, the ascending part of the aortic arch, and $p$, the pulmonary; $a a^{\prime}$, right and left aortic roots; $A^{\prime}$, descending aorta; $1,2,3,4,5$, the flve primitive aortic or branchial arches; $I, I I, I I I, I V$, the four branchial clefts which, for the sake of clearness, have been omitted on the right side. The permanent systemic vessels are deeply, the pulmonary arteries lightly, shaded; the parts of the primitive arches which are transitory are simply outlined; $c$, placed between the permanent common carotid arteries; $c e$, external carotid arteries ; $c i$, internal carotid arteries; $s$, right subclavian, rising from the right aortic root beyond the fifth arch; $v$, right vertebral from the same, opposite the fourth arch; $v^{\prime} s^{\prime}$, left vertebral and subclavian arteries rising together from the left or permanent aortic root, opposite the fourth arch; $p$, pulmonary arteries rising together from the left fifth arch; $d$, outer or back part of the left fifth arch, forming ductus arteriosis; $p n, p n^{\prime}$, right and left pneumogastric nerves descending in front of aortic arch with their recurrent branches represented diagrammetically as passing behind, to illustrate the relations of these nerves respectively to the right subclavian artery (4) and the arch of the aorta and ductus arteriosus $(d)$. (Allen Thomson, after Rathke.)

the recurrent laryngeal nerve hooks under the subclavian on the right side, and the aortic arch on the left.

The third aortic arch remains as the internal carotid artery, while the fifth disappears on the right side, but on the left forms the pulmonary artery. The distal end of this arch originally opens into the descending aorta, and this communication (which is permanent throughout life in many reptiles on both sides of the body) remains throughout fœtal life under the name of cluctus arteriosus: the branches of the pulmonary artery, to the right and left lung, are very small, and most of the blood which is forced into the pulmonary artery passes through the wide ductus arteriosus into the descending aorta. All these points will become clear on reference to the accompanying diagram (fig. 495). 
As the umbilical vesicle $d$ windles in size, the portion of the omphalomesenteric arteries outside the body gradually disappears, the part inside the body remaining as the mesenteric arteries.

Meanwhile with the growth of the allantois two new arteries (umbilical) appear, and rapidly increase in size till they are the largest branches of the aorta: they are given off from the internal iliac arteries, and for a long time are considerably larger than the external iliacs which supply the comparatively small hind-limbs.

Veins. - The chief veins in the early embryo may be divided into two groups, visceral and parietal: the former includes the omphalo-

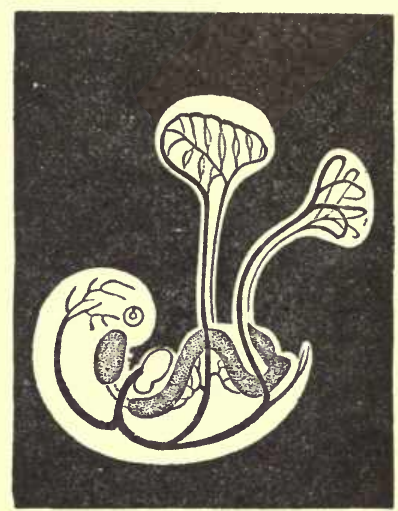

Fig. 496.

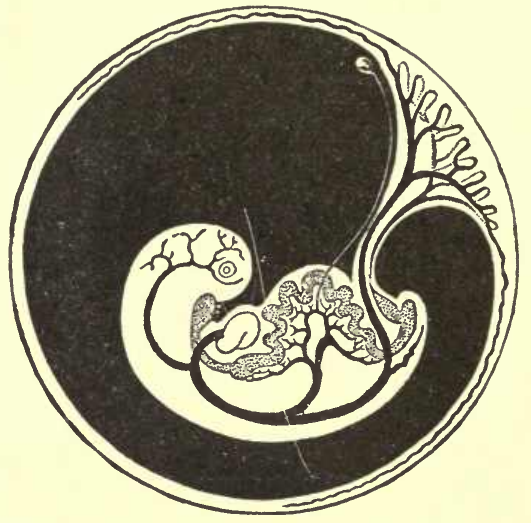

Fig. 497 .

Fig. 496.-Diagram of young emtryo and its vessels, showing course of circulation in the umbilical resicle; and also that of the allantois (near the caudal extremity), which is just commencing. (Dalton.)

Fig, 497. - Diagram of embryo and its vessels at a later stage, showing the second circulation. The pharynx, œsophagus, and intestinal canal have become further developed, and the mesenteric arteries have enlarged, while the umbilical vesicle and its vascular branches are very much reduced in size. The large umbilical arteries are seen passing out in the placenta. (Dalton.)

mesenteric and umbilical, the latter the jugular and cardinal veins. The former may be first considered.

The earliest veins to appear in the fœtus are the omphalo-mesenteric or vitelline, which return the blood from the yolk-sac to the developing auricle. As soon as the placenta with its umbilical veins is developed, these unite with the omphalo-mesenteric, and thus the blood which reaches the auricle comes partly from the yolk-sac and partly from the placenta. The right omphalo-mesenteric and the right umbilical veins soon disappear, and the united left omphalo-mesenteric and umbilical veins pass through the developing liver on the way to the auricle. Two sets of vessels make their appearance in connection with the liver (venæ hepaticæ advehentes, and revehentes), both opening into the united omphalo-mesenteric and umbilical veins, in such a way that a portion of the venous blood traversing the latter is diverted into the developing 
liver, and, having passed through its capillaries, returns to the umbilical vein through the venæ hepaticæ revehentes at a point nearer the heart (see fig. 498). The portion of vein between the afferent and efferent veins of the liver becomes the ductus venosus. The venæ hepaticæ
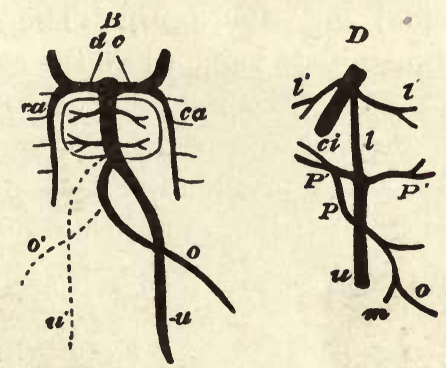

Fig. 498-Diagrams illustrating the development of veins about the liver. $B, d c$, ducts of Cuvier, right and left; $c a$, right and left cardinal veins; $o$. left omphalo-mesenteric vein; $o^{\prime}$, right omphalo-mesenteric vein, almost shrivelled up; $u u^{\prime}$, umbilical veins, of which $u^{\prime}$, the right one, has almost disappeared. Between the venæ cardinales is seen the outline of the rudimentary liver with its venæ hepaticæ advehentes, and revehentes. $D$, ductus venosus; $l^{\prime}$, hepatic veins; $c$, vena cava inferior; $P$, portal vein; $P^{\prime} P^{\prime}$, venæ advehentes; $m$, mesenteric veins. (Kölliker.)

advehentes become the right and Jeft branches of the portal vein, the venæ hepaticæ revehentes become the hepatic veins, which open just at the junction of the ductus venosus with another large vein (vena cava inferior), which is now being developed. The mesenteric portion of the omphalo-mesenteric vein returning blood from the developing intestines remains as the mesenteric vein, which, by its union with the splenic vein, forms the portal.

Thus the fotal liver is supplied with venous blood from two sources, through the umbilical and portal vein respectively. At birth the circulation through the umbilical vein of course completely ceases and the vessel begins at once to dwindle, so that now the only venous supply of the liver is through the portal vein. The earliest appearance of the parietal system of veins is the formation of two short transverse veins (ducts of Cuvier) opening into the auricle on either side, which result from the union of an anterior cardinal, afterward forming a jugular, vein, collecting blood from the head and neck, and a posterior cardinal vein which returns the blood from the Wolffian bodies, the vertebral column, and the parieties of the trunk. This arrangement persists throughout life in fishes, but in mammals the following transformations occur.

As the kidneys are developing a new vein appears (vena cava inferior), formed by the junction of their efferent veins. It receives branches from the legs (iliac) and increases rapidly in size as they grow; further up it receives the hepatic veins, which by now have lost their original opening into the ductus venosus. The heart gradually descends into 
the thorax, causing the ducts of Cuvier to become oblique instead of transverse. As the fore-limbs develop, the subclavian veins are formed.

A transverse communicating trunk now unites the two ducts of Cuvier, and gradually increases, while the left duct of Cuvier becomes almost entirely obliterated (all its blood passing by the communicating trunk to the right side) (fig. 499, c.I.). The right duct of Cuvier remains as the right innominate vein, while the communicating branch forms the left innominate. The remnant of the left duct of Cuvier generally remains as a fibrous band, running obliquely down to the coronary vein, which is really the proximal part of the left duct of Cuvier. In front of the root of the left lung, another relic may be found in the
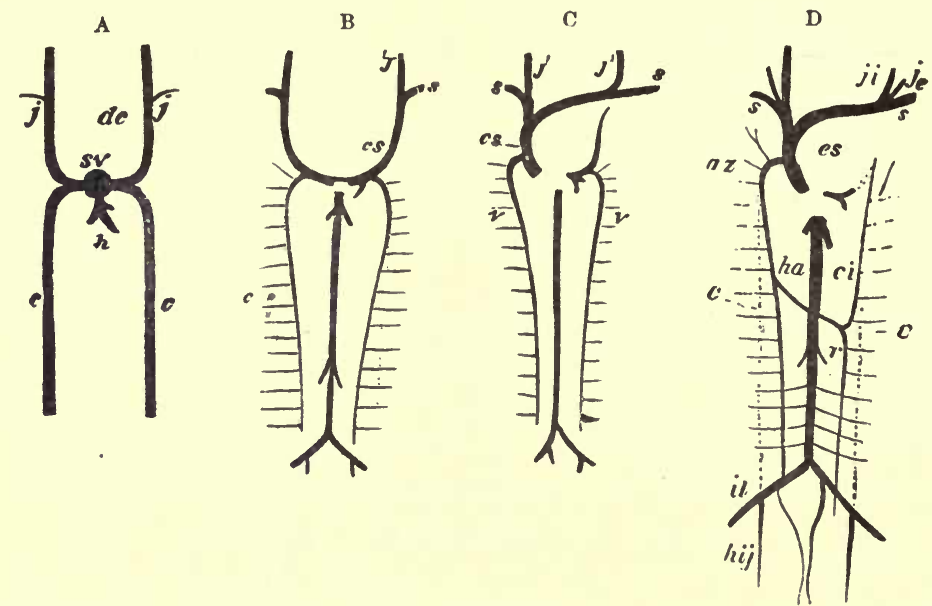

Fig. 499.-Diagrams illustrating the development of the great veins, $d c$ ducts of Cuvier: $j$, jugular veins; $h$, hepatic veins; $c$, cardinal veins; $s$, subclavian vein; $j i$, internal jugular vein; $j e$, external jugular vein; $a z$, azygos vein; $c$, inferior vena cava; $r$, renal veins; $i l$, iliac veins; $h i j$, hypogastric veins. (Gegenbaur.)

form of the so-called vestigial fold of Marshall, which is a fold of pericardium running in the same direction.

In many of the lower mammals, such as the rat, the left ductus Cuvieri remains as a left superior cava.

Meanwhile, a transverse branch carries across most of the blood of the left posterior cardinal vein into the right; and by this union the great azygos vein is formed.

The upper portions of the left posterior cardinal vein remains as the left superior intercostal and vena azygos minor.

\section{Circulation of Blood in the Fetus.}

The circulation of blood in the fœetus differs considerably from that of the adult. It will be well, perhaps, to begin its description by trac- 
ing the course of the blood, which, after being carried out to the placenta by the two umbilical arteries, has returned, cleansed and replenished, to the foetus by the umbilical vein.

It is at first conveyed to the under surface of the liver, and there the stream is divided, - a part of the blood passing straight on to the in-

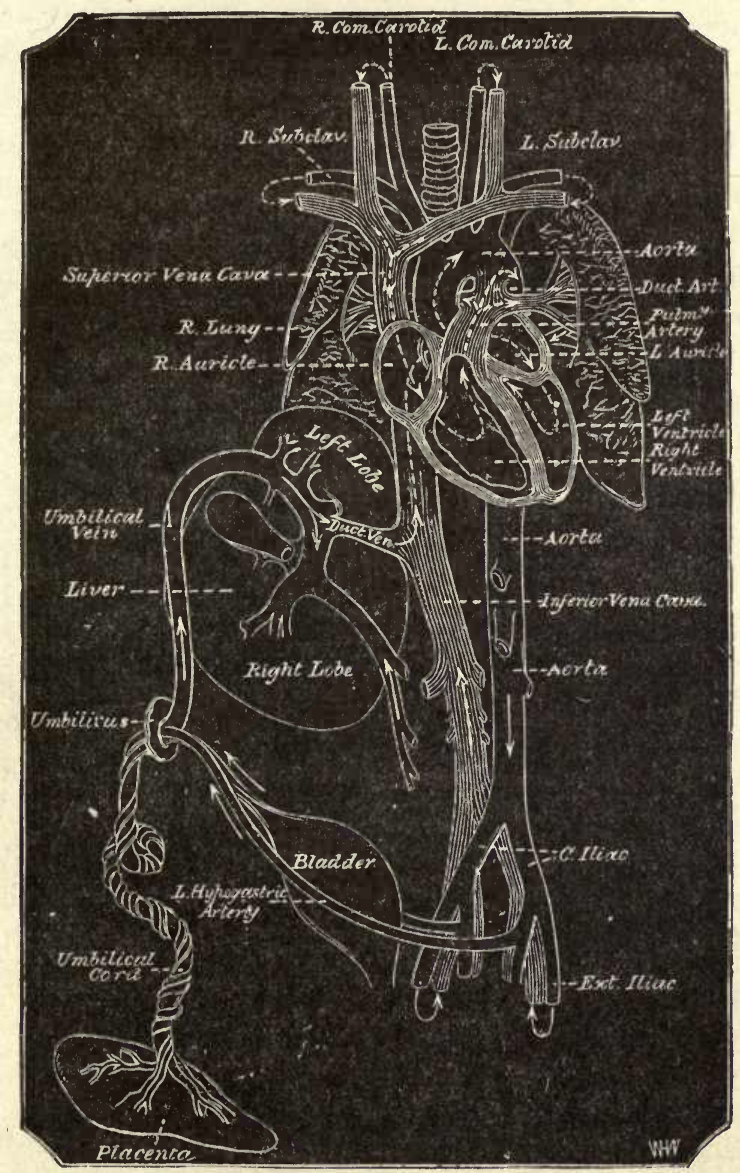

Fig. 500.-Diagram of the Fotal Circulation.

ferior vena cava, through a venous canal called the ductus venosus, while the remainder passes into the portal vein, and reaches the inferior vena cava only after circulating throngh the liver. Whether, however, by the direct route through the ductus venosus or by the roundabout way through the liver,-all the blood which is returned from the placenta by the umbilical vein reaches the inferior vena cava at last, and is carried by it to the right auricle of the heart, into which cavity is also pouring 
the blood that has circulated in the head and neck and arms, and has been brought to the auricle by the superior rena cava. It might be naturally expected that the two streams of blood would be mingled in the right auricle, but such is not the case, or only to a slight extent. The blood from the superior vena cara-the less pure fluid of the twopasses almost exclusively into the right reutricle, through the auriculoventricular opening, just as it does in the adult; while the blood of the inferior vena cava is directed by a fold of the lining membrane of the heart, called the Eustactian valve, through the foramen ovale into the left auricle, whence it passes into the left rentricle, and out of this into the aorta, and thence to all the body, but chiefly to the head and neck. The blood of the superior vena eara, which, as before said, passes into the right ventricle, is sent out thence in small amount though the pulmonary artery to the lungs, and thence to the left auricle, as in the adult. The greater part, however, by far, does not go to the lungs, but instead, passes through a canal, the ductus arteriosus, leading from the pulmonary artery into the aorta just below the origin of the three great ressels which supply the upper parts of the body; and there meeting that part of the blood of the inferior rena cara which has not gone into these large ressels, it is distributed with it to the trunk and lower parts, - a portion passing out by way of the two umbilical arteries to the placenta. From the placenta it is returned by the umbilical vein to the under surface of the liver, from which the description started.

Changes after Birth.-After birth the foramen orale closes, and so do the ductus arteriosus and ductus renosus, as well as the umbilical vessels; so that the two streams of blood which arrive at the right auricle by the superior and inferior vena cava respectively, thenceforth mingle in this cavity of the heart, and passing into the right ventricle, go by way of the pulmonary artery to the lungs, and through these after purification, to the left auricle and ventricle, to be distributed orer the body.

\section{The Nervous System.}

The Cranial and Spinal Nerves.-The cranial nerves are derived from a continuous band, called the neural band. They are formed before the neural canal is complete. The neural band is made up of two laminæ going from the dorsal edges of the neural groove to the external epiblast. It becomes separated from the epiblast, and then forms a crest attached to the upper surface of the brain. The posterior roots of the spinal nerves arise as outgrowths of median processes of cells from the dorsal dde of the spinal cord, which become attached laterally to the spinal cord as their original point of attachment disappears. The anterior roots probably arise from the ventral part of the cord as a number of 
strands for each nerve. They appear later than the posterior roots. The rudiment of the posterior root is differentiated into a proximal round nerve connected to the cord, a ganglionic portion and a distal portion. To the last the anterior nerve-root becomes attached.

The Spinal Cord. - The spinal cord consists at first of the undifferentiated epiblast of the walls of the neural canal, the cavity of which is large, with almost parallel sides. The walls are at first composed of elongated irregular nucleated columnar cells, arranged in a radiate manner. The cavity then becomes narrow in the middle and of an hour-glass shape (fig. 501). When the spinal nerves make their first

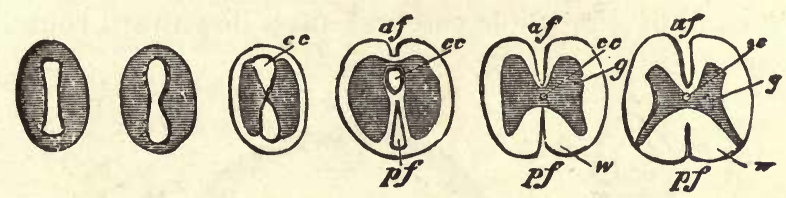

Fig. 501.-Diagram of development of spinal cord, $c c$, central canal; $a f$, anterior fissure; $p f$, posterior fissure; $g$, gray matter; $w$, white matter. For further explanation, see text.

appearance, about the fourth day in the chick, the epiblastic walls become differentiated into three parts: $(a)$ the epithelium lining the central canal; $(b)$ the gray matter; $(c)$ the external white matter. The last is derived from the outermost part of the epiblastic walls by the conversion of the cells into longitudinal nerve-fibres. The fibres being without any myelin sheath, are for a time gray in appearance. The white matter corresponds in position to the anterior and posterior nerve-roots, and are the anterior and posterior white columns. It is at first a very thin layer, but increases in thickness until it covers the whole cord. The gray matter too arises from the cells by their being prolonged into fibres. The change in the central cells is sufficiently obvious. The anterior and posterior cornua of gray matter and the anterior gray commissure then appear. The anterior fissure is formed on the fifth day by the growth downward of the anterior cornua of gray matter toward the middle line. The posterior fissure is formed later. The whole cord now becomes circular. The posterior gray commissure is then formed.

When it first appears, the spinal cord occupies the whole length of the medullary canal, but as development proceeds, the spinal column grows more rapidly than the contained cord, so that the latter appears as if drawn up till, at birth, it is opposite the third lumbar vertebra, and in the adult opposite the first lumbar. In the same way the increasing obliquity of the spinal nerves in the neural canal, as we approach the lumbar region, and the cauda equina at the lower end of the cord, are accounted for.

Brain. - We have seen that the front portion of the medullary canal 
is almost from the first widened out and divided into three vesicles. From the anterior vesicle (thalamencephalon) the two primary optic vesicles are budded off laterally: their further history will be traced in the next section. Somewhat later, from the same vesicle the rudiments of the hemispheres appear in the form of two outgrowths at a higher level, which grow upward and backward. These form the prosencrphaton.

In the walls of the posterior (third) cerebral vesicle, a thickening appears (rudimentary cerebellum) which becomes separated from the rest of the vesicle by a deep inflection.

At this time there are two chief curvatures of the brain (fig. 502). (1.) A sharp bend of the whole cerebral mass downward round the end
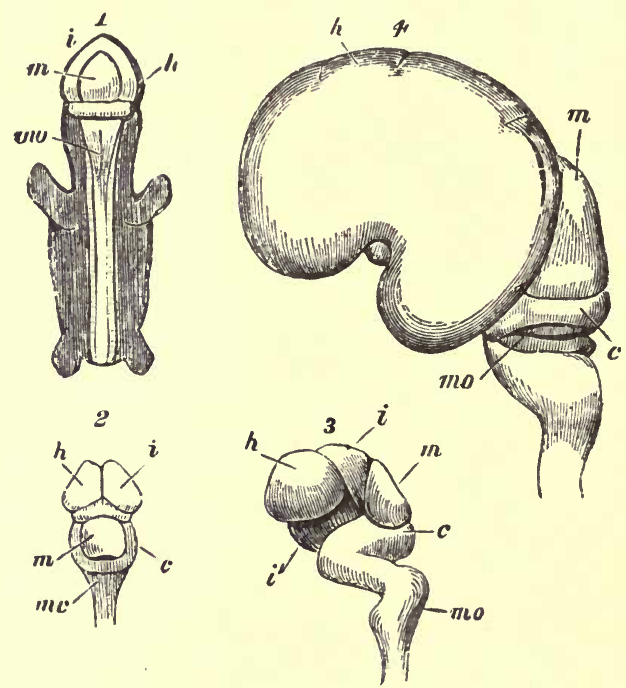

Fig. 502.- Early stages in development of human brain (magnified). 1, 2, 3, are from an embryo about seven weeks old; 4 , about three months old. $m$, middle cerebral vesicle (niesencephalon); $c$, cerebellum ; $m o$, medulla oblongata: $i$, thalamencephalon; $h$, hemispheres; $i^{\prime}$, infundibulum; Fig. 3 shows the several curves which occur in the course of development; Fig. 4 is a lateral view, showing the great enlargement of the cerebral hemispheres which have covered in the thalami, leaving the optic lobes, $m$, uncovered. (Kölliker.)

N. B.-In Fig. 2 the line $i$ terminates in the right hemisphere; it ought to be continued into the thalamencephalon.

of the notochord, by which the anterior vesicle, which was the highest of the three, is bent downward, and the middle one comes to occupy the highest position. (2.) A sharp bend, with the convexity forward, which runs in from behind beneath the rudimentary cerebellum separating it from the medulla.

Thus, five fundamental parts of the foetal brain may be distinguished, which, together with the parts developed from them, may be presented in the following tabular view:- 
Table of Parts developed from fundamental Parts of Brain.

I. Anterior

Primary

Vesicle, or Forebrain.

II. Middle Primary Vesicle, or Midbrain.

III. Posterior Primary Vesicle, or Hindbrain.
First Secondary Vesicle of Prosencephalon.

Second Secondary Vesicle or Thalamencephalon (Diencephalon).

Third Secondary Vesicle or Mesencephalon.

Fourth Secondary Vesicle or Epencephalon.

Fifth Secondary Vesicle or Metencephalon.

Anterior end of third ventricle, foramen of Monro, lateral ven. tricles, cerebral hemispheres, corpora striata, corpus callosum, fornix, lateral ventricles, olfactory bulb.

Thalami optici, pineal gland, part of pituitary body, third ventricle, optic nerve and retina, infundibulum.

Corpora quadrigemina, crura cerebri, aqueduct of Sylvius.

Fourth ven- $\{$ Cerebellum, pons, tricle. $\left\{\begin{array}{l}\text { medulla oblon- } \\ \text { gata. }\end{array}\right.$

(Quain.)

The cerebral hemispheres grow rapidly upward and backward, while from their inferior surface the olfactory bulbs are budded off, and the prosencephalon, from which they spring, remains to form the third ventricle and optic thalami. The middile cerebral vesicle (mesencephalon) for some time is the most prominent part of the fœtal brain, and in fishes, amphibia, and reptiles, it remains uncovered through life as the optic lobes. But in birds the growth of the cerebral hemispheres thrusts the optic lobes down laterally, and in mammalia completely overlaps them.

In the lower mammalia the backward growth of the hemispheres ceases as it were, but in the higher groups, such as the monkeys and man, they grow still further back, until they completely cover in the

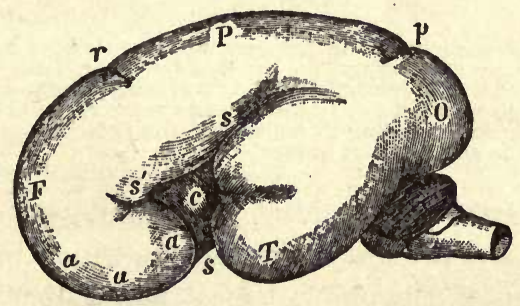

Fig. 503.-Side view of foetal brain at six months, showing commencement of formation of the principal fissures and convolutions. $F$, frontal lobe; $P$, parietal; $O$, occipital ; $T$, temporal; a $a$, commencing frontal convolutions; $s$, Sylvian fissure; $s^{\prime}$, its anterior division; $c$, within it the central lobe or island of Reil; $r$, fissure of Rolando; $p$, perpendicular fissure. (R. Wagner.)

cerebellum, so that on looking down on the brain from above, the cerebellum is quite concealed from view. The surface of the hemispheres is at first quite smooth, but as early as the third month the great Sylvian fissure begins to be formed (fig. 503). 
The next to appear is the parieto-occipital or perpendicular fissure; these two great fissures, unlike the rest of the sulci, are formed by a curving round of the whole cerebral mass.

In the sixth month the fissure of Rolando appears: from this time till the end of fotal life the brain grows rapidly in size, and the convolutions appear in quick succession; first the great primary ones are sketched out, then the secondary, and lastly the tertiary ones in the sides of the fissures. The commissures of the brain (anterior, middle, and posterior), and the corpus callosum, are developed by the growth of fibres across the middle line.

The Hippocampus major is formed by the folding in of the gray matter from the exterior into the lateral ventricles. The essential points in the structure and arrangement of the various parts of the brain, are diagrammatically shown in the two accompanying figures (figs. 502, 503).

\section{The Special Sense Organs.}

The Eye.-Soon after the first three cerebral vesicles have become distinct from each other, the anterior one sends out a lateral vesicle from each side (primary optic resicle), which grows out toward the free surface, its cavity of course communicating with that of the cerebral vesicle through the canal in its pedicle. It is soon met and invaginated by an
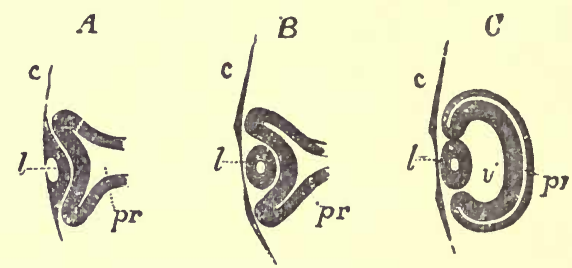

Fig. 504.-Longitudinal section of the primary optic vesicle in the chick magnified (from Remak). - A, from an embryo of sixty-five hours; B, a few hours later; C, of the fourth day; $c$, the corneous layer or epidermis, presenting in A the open depression for the lens, which is closed in B and C; $l$, the lens follicle and lens; pr, the primary optic vesicle; in A and B, the pedicle is shown; in C, the section being to the side of the pedicle, the latter is not shown; $v$, the secondary ocular vesicle and vitreous humor.

ingrowing process from the epiblast (fig. 504), very much as the growing tooth is met by the process of epithelium which produces the enamel organ. This process of the epiblast is at first a depression, which ultimately becomes closed in at the edges so as to produce a hollow ball, which is thus completely severed from the epithelium with which it was originally continuous. From this hollow ball the crystalline lens is developed. The way in which this occurs has been indicated in a previous chapter under the head of structure of the lens. By the ingrowth of the lens the anterior wall of the primary optic vesicle is forced back nearly into contact with the posterior, and thus the primary optic vesi- 
ele is almost obliterated. The cells in the anterior wall are much longer than those of the posterior wall; from the former the retina proper is developed, from the latter the retinal pigment.

The cup-shaped hollow in which the lens is now lodged is termed the secondary optic vesicle: its walls grow up all round, leaving, however, a slit at the lower part.

Choroidal Fissure. - Through this slit (fig. 506), often termed the choroidal fissure, a process of mesoblast containing numerous blood-

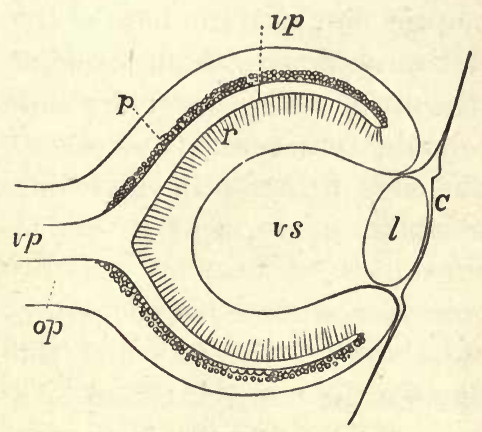

Fig. 505 .

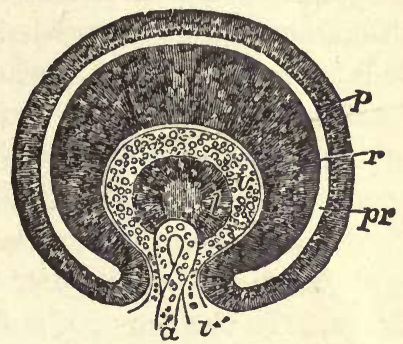

Fig. 506 .

Fig. 505.-Diagrammatic sketch of a vertical longitudinal section through the eyeball of a human foetus of four weeks. The section is a little to the side, so as to avoid passing through the ocular cleft; $c$, the cuticle where it becomes later the corneal epithelium; $l$, the lens; op, optic nerve formed by the pedicle of the primary optic vesicle; vp, primary medullary cavity or optic vesicle; $p$, the pigment layer of the retina; $r$, the inner wall forming the retina proper; $v s$, secondary optic vesicle containing the rudiment of the vitreous humor. $\times 100$. (Kölliker.)

Fig. 506. - Transverse vertical section of the eyeball of a human embryo of four weeks. The anterior half of the section is represented: $p r$, the remains of the cavity of the primary optic vesicle; $p$, the inner part of the outer layer forming the retinal pigment; $r$, the thickened inner part giving rise to the columnar and other structures of the retina; $v$, the commencing vitreous humor within the secondary optic vesicle; $v^{\prime}$, the ocular cleft through which the loop of the central blood-vessel, $a$, projects from below; $l$, the lens with a central cavity. $\times 100$. (Kölliker.)

vessels projects, and occupies the cavity of the secondary optic vesicle behind the lens, filling it with vitreous humor and furnishing the lens capsule and the capsulo-pupillary membrane. This process in mammals projects, not only into the secondary optic vesicle, but also into the pedicle of the primary optic vesicle invaginating it for some distance from beneath, and thus carrying up the arteria centralis retinoe into its permanent position in the centre of the optic nerve.

This invagination of the optic nerve does not occur in birds, and consequently no arteria centralis retinæ exists in them. But they possess an important permanent relic of the original protrusion of the mesoblast through the choroidal fissure, in the pecten, while a remnant of the same fissure sometimes occurs in man under the name coloboma iridis. The cavity of the primary optic vesicle becomes completely obliterated, and the rods and cones growing up from the external limiting membrane, get into apposition with the pigment layer of the retina. 
The inner segments of the rods become the first formed, then the outer. The cavity of its pedicle disappears and the solid optic nerve is formed. Meanwhile the cavity which existed in the centre of the primitive lens becomes filled up by the growth of fibres from its posterior wall. The epithelium of the cornea is developed from the epiblast, while the corneal tissue proper is derived from the mesoblast which intervenes between the epiblast and the primitive lens which was originally continuous with it. The sclerotic coat is developed round the eyeball from the general mesoblast in which it is embedded. The choroid is developed from the mesoblast on the outside of the optic cup and the iris by the growing forward of the anterior edge of the optic cup, both layers of which becoming pigmented remain as the uvea. Externally the choroidal mesoblast grows inward to form the main structure. The ciliary processes arise from the hypertrophy of the edge of the optic cup which forms folds into which the choroidal mesoblast grows, and in which blood-vessels and pigment-cells develop.

The iris is formed rather late, as a circular septum projecting inward, from the fore part of the choroid, between the lens and the cornea. In the eye of the fotus of mammalia, the pupil is closed by a delicate membrane, the membrana pupillaris, which forms the front portion of a highly vascular membrane that, in the fœtus, surrounds the

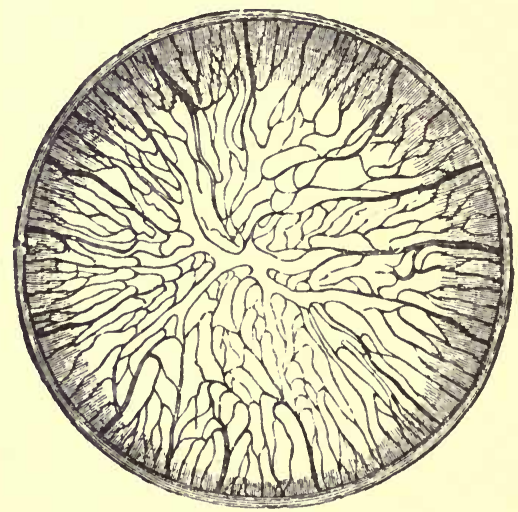

Fig. 50\%.-Blood-vessels of the capsulo-pupillary membrane of a new-born kitten, magnified. The drawing is taken from a preparation injected by Tiersch, and shows in the central part the convergence of the net-work of vessels in the pupillary membrane. (Kölliker.)

lens, and is named the membrana capsulo-pupillaris (fig. 507). It is supplied with blood by a branch of the arteria centralis retina, which, passing forward to the back of the lens, there subdivides. The membrana capsulo-pupillaris withers and disappears in the human subject a short time before birth.

The eyelids of the human subject and mammiferous animals, like 
those of birds, are first developed in the form of a ring. They then extend over the globe of the eye until they meet and become firmly agglutinated to each other. But before birth, or in the carnivora after birth, they again separate.

The Ear.-Very early in the development of the embryo a depression or ingrowth of the epiblast occurs on each side of the head which deepens and soon becomes a closed follicle. This primary optic vesicle, which closely corresponds in its formation to the lens follicle in the eye, sinks down to some distance from the free surface; from it are developed the epithelial lining of the membranous labyrinth of the internal ear, consisting of the vestibule and its semicircular canals and the scala media of the cochlea. The surrounding mesoblast gives rise to the various fibrous bony and cartilaginous parts which complete and inclose this membranous labyrinth, the bony semicircular canals, the walls of the cochlea with its scala vestibuli and scala tympani. In the mesoblast between the primary optic vesicle and the hrain, the auditory nerve is gradually differentiated and forms its central and peripheral attachments to the brain and internal ear respectively. According to some authorities, however, it is said to take its origin from and grow out of the hind brain.

The Eustachian tube, the cavity of the tympanum, and the external auditory passage, are remains of the first branchial cleft. The membrana tympani divides the cavity of this cleft into an internal space, the tympanum, and the external meatus. The mucous membrane of the mouth, which is prolonged in the form of a diverticulum through the Eustachian tube into the tympanum, and the external cutaneous system come into relation with each other at this point; the two membranes being separated only by the proper membrane of the tympanum.

The pinna or external ear is developed from a process of integument in the neighborhood of the first and second visceral arches, and probably corresponds to the gill-cover (operculum) in fishes.

The Nose. - The nose originates like the eye and ear in a depression of the superficial epiblast at each side of the fronto-nasal process (primary olfactory groove), which is at first completely separated from the cavity of the mouth, and gradually extends backward and downward till it opens into the mouth.

The outer angles of the fronto-nasal process, uniting with the maxillary process on each side, convert what was at first a groove into a closed canal.

\section{The Alimentary Canal.}

The alimentary canal in the earliest stages of its development consists of three distinct parts-the fore and hind gut ending blindly at 
each end of the body, and a middle segment which communicates freely on its ventral surface with the cavity of the yolk-sac through the vitelline or omphalo-mesenteric duct.

From the fore-gut are formed the pharynx, osophagus, and stomach; from the hind-gut, the lower end of the colon and the rectum. The mouth is developed by an involution of the epiblast between the maxillary and mandibular processes, which becomes deeper and deeper till it reaches the blind end of the fore-gut, and at length communicates freely with the pharynx by the absorption of the partition between the two.

At the other end of the alimentary canal the anus is formed in a precisely similar way by an involution from the free surface, which at length

A

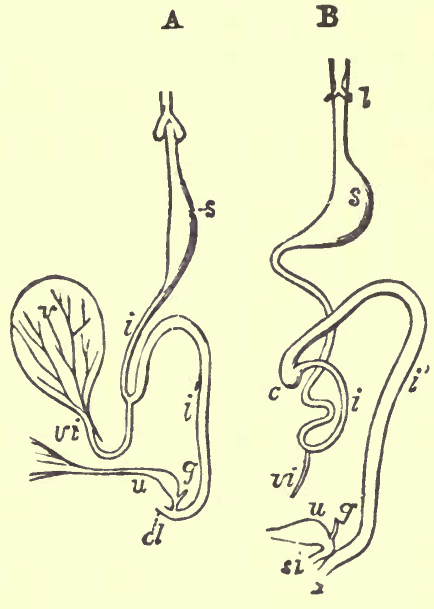

$\mathrm{c}$

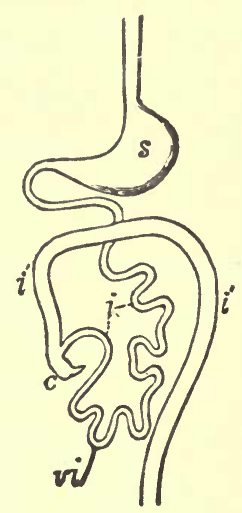

D

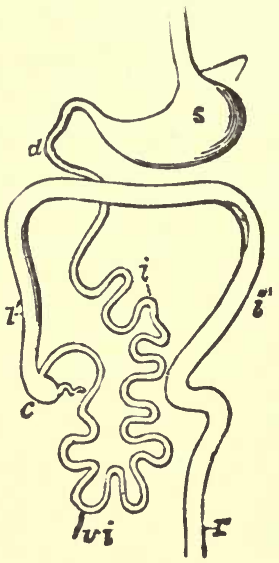

Fig. 508.- Outlines of the form and position of the alimentary canal in successive stages of its development. A, alimentary canal, etc., in an embryo of four weeks; B, at six weeks; C, at eight weeks; D, at ten weeks; $l$, the primitive lungs connected with the pharynx; $s$, the stomach; $d$, duodenum; $i$, the small intestine: $i^{\prime}$, the large; $c$, the cæecum and vermiform appendage; $r$, the rectum; $c l$, in $\mathrm{A}$, the cloaca; $a$, in $\mathrm{B}$, the anus distinct from $s i$, the sinus uro-genitalis; $v$, the yolk-sac; $v i$, the vitello-intestinal duct; $u$, the urinary bladder and urachus leading to the allantois; $g$, genital ducts. (Allen Thomson.)

opens into the hind-gut. When the depression from the free surface does not reach the intestine, the condition known as imperforate anus results. A similar condition may exist at the other end of the alimentary canal from the failure of the involution which forms the mouth, to meet the fore-gut. The middle portion of the digestive canal becomes more more and closed in till its originally wide communication with the yolk-sac becomes narrowed down to a small duct (vitelline). This duct usually completely disappears in the adult, but occasionally the proximal portion remains as a diverticulum from the intestine. Sometimes a fibrous cord attaching some part of the intestine to the umbilicus, remains to represent the vitelline duct. Such a cord has been known to cause in after-life strangulation of the bowel and death. 
The alimentary canal lies in the form of a straight tube close beneath the vertebral column, but it gradually becomes divided into its special parts, stomach, small intestine, and large intestine (fig. 508), and at the same time comes to be suspended in the abdominal cavity by means of a lengthening mesentery formed from the splanchnopleure which attaches it to the vertebral column. The stomach originally has the same direction as the rest of the canal; its cardiac extremity being superior, its pylorus inferior. The changes of position which the alimentary canal undergoes may be readily gathered from the accompanying figures (fig. 508).

Pancreas and Salivary Glands.-The principal glands in connection with the intestinal canal are the salivary, pancreas, and the liver. In mammalia, each salivary gland first appears as a simple canal with bud-

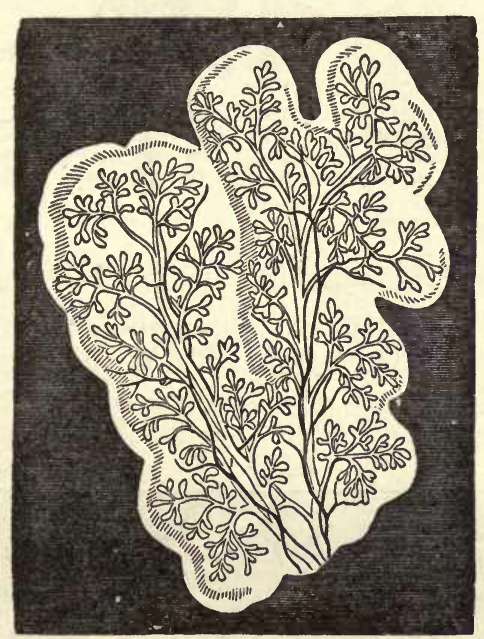

Fig. 509. - Lobules of the parotid, with the salivary ducts, in the embryo of the sheep, at a more advanced stage.

like processes (fig. 509), lying in a gelatinous nidus or blastema, and communicating with the cavity of the mouth. As the development of the gland advances, the canal becomes more and more ramified, increasing at the expense of the blastema in which it is still inclosed. The branches or salivary ducts constitute an independent system of closed tubes (fig. 509). The pancreas is developed exactly as the salivary glands, but is developed from the hypoblast lining the intestine, while the salivary glands are formed from the epiblast lining the mouth.

The Liver.-The liver is developed by the protrusion, as it were, of a part of the walls of the fore-gut, in the form of two conical hollow branches, which embrace the common venous stem (figs. 510, 511). The 
outer part of these cones involves the omphalo-mesenteric vein, which breaks up in its interior into a plexus of capillaries, ending in venous trunks for the conveyance of the blood to the heart. The inner portion of the cones consists of a number of solid cylindrical masses of cells,

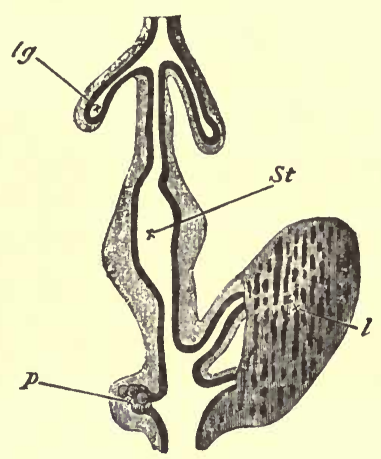

Fig. 510.-Diagram of part of digestive tract of a chick (4th day). The black line represents hypoblast, the outer shading mesoblast; $l g$, lung diverticulum with expanded end forming primary lung-vesicle; $S t$, stomach; $l$, two hepatic diverticula, with their terminations united by solid rows of hypoblast cells; $p$, diverticulum of the pancreas with the vesicular diverticula coming from it. (Gotte.)

derived probably from the hypoblast, which become gradually hollowed by the formation of the hepatic ducts, and among which blood-vessels are rapidly developed. The gland cells of the organ are derived from the hypoblast, the connective tissue and vessels without doubt from the

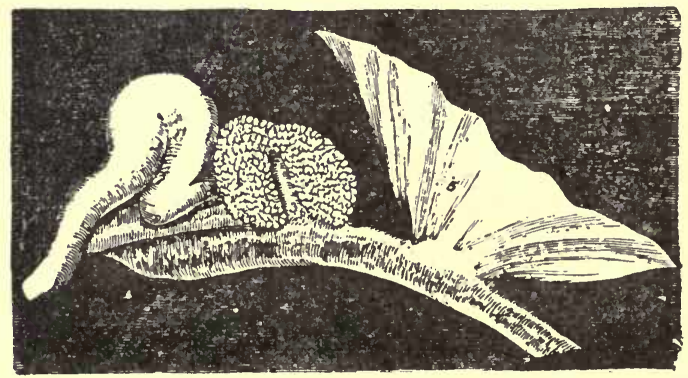

Fig. 511. - Kudiments of the liver on the intestine of a chick at the fifth day of incubation 1 , heart; 2 , intestine; 3 , diverticulum of the intestine in which the liver (4) is developed: 5 , part of the mucous layer of the germinal membrane. (Miuller.)

mesoblast. The gall-bladder is developed as a diverticulum from the hepatic duct. The spleen, lymphatic, and thymus glands are developed from the mesoblast: the thyroid partly also from the hypoblast, which grows into it as a diverticulum from the fore-gut. 


\section{The Respiratory Appákatus.}

The Lungs, at their first development, appear as small tubercles or diverticula from the abdominal surface of the œsophagus.

'The two diverticula at first open directly into the œsophagus, but as they grow, a separate tube (the future trachea) is formed at their point of fusion, opening into the œsophagus on its anterior surface. These primary diverticula of the hypoblast of the alimentary canal send off secondary branches into the surrounding mesoblast, and these again give off tertiary branches, forming the air-cells. Thus we have the lungs formed: the epithelium lining their air-cells, bronchi, and trachea being derived from the hypoblast, and all the rest of the lung-tissue,

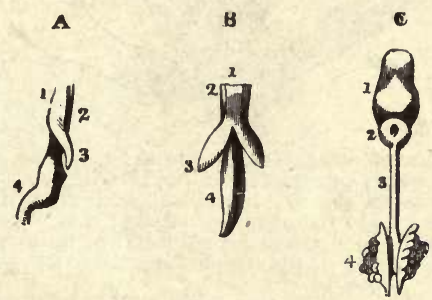

Fig. 512 illustrates the development of the respiratory organs. A, is the oesophagus of a chick on the fourth day of incubation, with the rudiments of the trachea on the lung of the left side, viewed laterally; 1 , the inferior wall of the osophagus; 2 , the upper portion of the same tube; 3 , the rudimentary lung; 4, the stomach; $B$, is the same object seen from below, so that both lungs are visible. c, shows the tongue and respiratory organs of the embryo of a horse; 1 , the tongue; 2 , the larynx; 3 , the trachea; 4, the lungs viewed from the upper side. (After Rathke.)

nerves, lymphatics, and blood-vessels, cartilaginous rings, and muscular fibres of the bronchi from the mesoblast. The diaphragm is early developed.

\section{The Genito-Urinary Apparatus.}

'The Wolffian bodies are organs peculiar to the embryonic state, and may be regarded as temporary, rather than rudimental, kidneys; for although they seem to discharge the functions of these latter organs, they are not developed into them.

The Wolffian duct makes its appearance at an early stage in the history of the embryo, as a cord running longitudinally on each side in the mass of mesoblast, which lies just externally to the intermediate cellmass (ung, fig. 513). This cord, at first solid, becomes gradually hollowed out to form a tube (Wolffian) which sinks down till it projects beneath the lining membrane into the pleuro-peritoneal cavity.

The primitive tube thus formed sends off secondary diverticula at frequent intervals which grow into the surrounding mesoblast: tufts of vessels grow into the blind ends of these tubes, invaginating them and producing Malpighian bodies very similar in appearance to those of the 
permanent kidney, which constitute the substauce of the Wolffian body. Meanwhile another portion of mesoblast between the Wolffian body and the mesentery projects in the form of a ridge, covered on its free surface

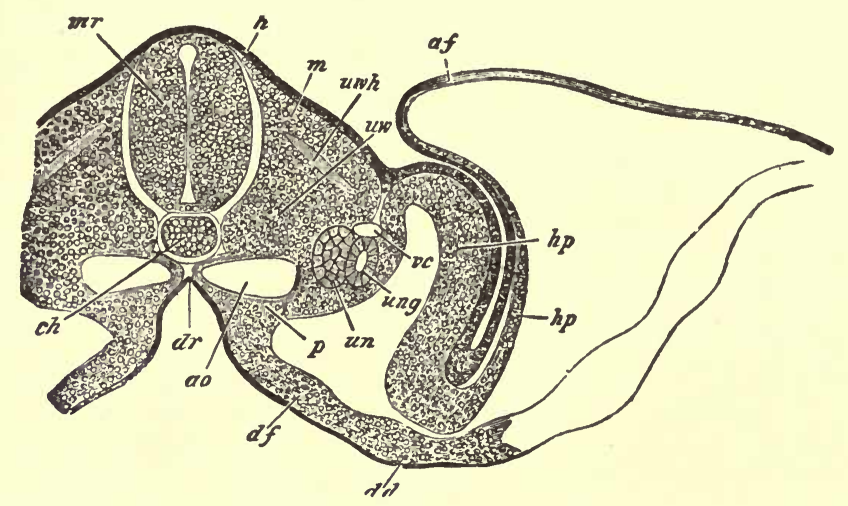

Fig. 513.-Transverse section of embryo chick (third day). $m r$, rudimentary spinal cord; the primitive central canal has become constricted in the middle; $c h$, notochord; $u w h$, primordial vertebral mass; $m$, muscle-plate; $d r, d f$, hypoblast and visceral layer of mescblast lining groove, which is not yet closed in to form the intestines; $a o$, one of the primitive aortæ; $u n$, Wolffian body; $u n g$, Wolffian duct; $v c$, vena cardinalis; $h$, epiblast; $h p$, somatopleure and its reflection to form $a f$, amniotic fold; $p$, pleuro-peritoneal cavity. (Kölliker.)

with epithelium termed germ epithelium. From this projection is developed the reproductive gland (ovary or testis as the case may be).

Simultaneously, on the outer wall of the Wolffian body, between it and the body-wall on each side, an involution is formed from the pleuroperitoneal cavity in the form of a longitudinal furrow, whose edges soon close over to form a duct (Müller's duct).

All the above points are shown in the accompanying figures, 513, 514.

The Wolffian bodies, or temporary kidneys, as they may be termed, give place at an early period in the human fotus to their successors, the permanent kidneys, which are developed behind them. They diminish rapidly in size, and by the end of the third month have almost entirely disappeared. In connection, however, with their upper part, in the male, there are developed from a new mass of blastema, the vasa efferentia, coni vasculosi, and globus major of the epididymis; and thus is brought about a direct connection between the secreting part of the testicle and its duct. The Wolffian ducts persist in the male, and are developed to form the body and globus minor of the epididymis, the vas deferens, and ejaculatory duct on each side, the vesiculæ seminales forming diverticula from their lower part. In the female a small relic of the Wolffian body persists as the parovarium; in the male a similar relic is termed the organ of Giraldès. The lower end of the Wolffian duct remains in the female as the duct of Gaertner which descends towara, and is lost upon, the anterior wall of the vagina. 
From the lower end of the Wolffian duct a diverticulum grows back along the body of the embryo toward its anterior extremity, and ultimately forms the ureter. Secondary diverticula are given off from it and grow into the surrounding blastema of blood-vessels and cells.

Malpighian bodies are formed just as in the Wolffian body, by the invagination of the blind knobbed end of these diverticula by a tuft of vessels. This process is precisely similar to the invagination of the primary optic vesicle by the rudimentary lens. Thus the kidney is developed, consisting at first of a number of separate lobules; this condition remaining throughout life in many of the lower animals, e.g., seals and whales, and traces of this lobulation being visible in the human fœtus at birth. In the adult all the lobules are fused into a compact solid organ.

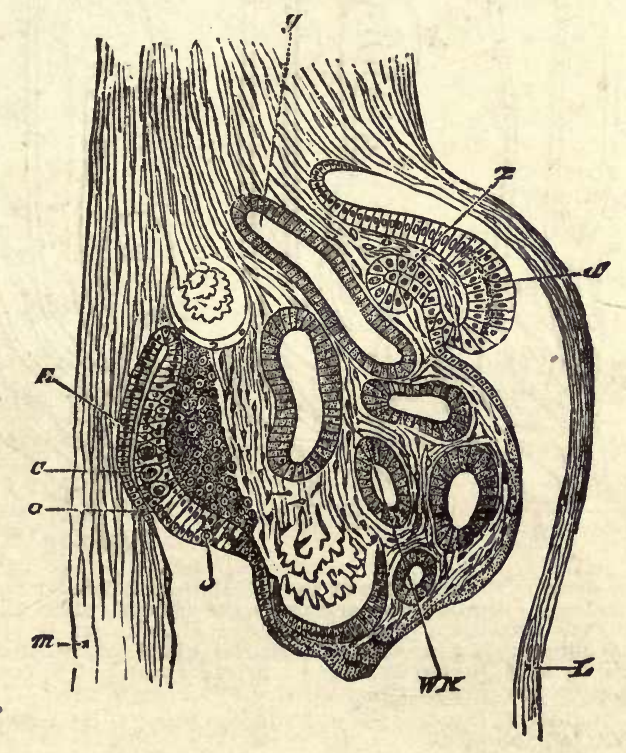

Fig. 514. - Section of intermediate cell-mass on the fourth day. $m$, mesentery; $L$, somatopleure; $a$, germinal epithelium, from which $z$, the duct of Müller, becomes involuted; $a$, thickened part of germinal epithelium in which the primitive ova $O$ and $o$, are lying: $E$, modified mesoblast, which will form the stroma of the ovary; $W K$, Wolffian body; $y$, Wolffian duct; $X$ 160. (Waldeyer.)

The supra-renal capsules originate in a mass of mesoblast just above the kidneys; soon after their first appearance they are very much larger than the kidneys (see fig. 516), but by the more rapid growth of the latter this relation is soon reversed.

The first appearance of the generative gland has been already described: for some time it is impossible to determine whether an ovary or testis will be developed from it; gradually however the special characters belonging to one of them appear, and in either case the organ 
soon begins to assume a relatively lower position in the body; the ovaries being ultimately placed in the pelvis; while toward the end of fœtal existence the testicles descend into the scrotum, the testicle entering the internal inguinal ring in the seventh month of fœtal life, and completing its descent through the inguinal canal and external ring into the scrotum by the end of the eighth month. A pouch of peritoneum, the processus vaginalis, precedes it in its descent, and ultimately forms

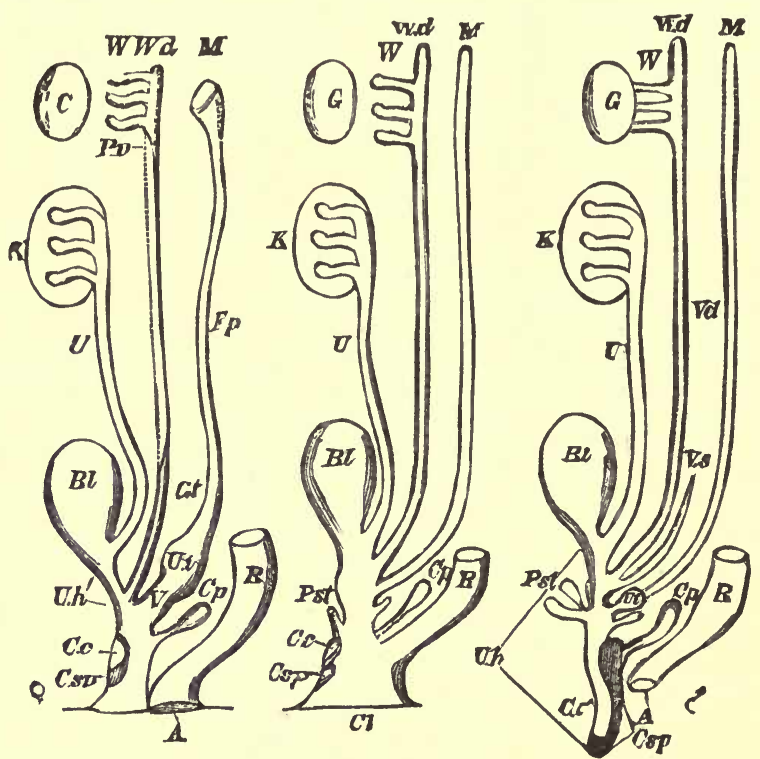

Fig. 515.-Diagram showing the relations of the female (the left-haud figure \&) and of the male (the right-hand figure $\delta$ ) reproductive organs to the general plan (the middle figure of these organs in the higher vertebrata (including man). $C l$, cloaca: $R$, rectum: $B l$, urinary bladder: $U$, ureter; $K$, kidney; $U h$, urethra; $G$, genital gland, ovary, or testis; $W$, Wolffian body; $W d$, Wolffian duct; $M$, Müllerian duct; $P s t$, prostate gland; $C p$, Cowper's gland; $C s p$, corpus spongiosum; $C c$, corpus cavernosum.

In the female. $-V$, vagina; $U t$, uterus; $F$, Fallopian tube; $G t$, Gaertnèr's duct; $P v$, parovarium; $A$, anus; $C c, C s p$, clitoris.

In the male.-Cs $p, C c$, penis: $U t$, uterus masculinis; $V s$, vesicula seminalis; $V d$, vas deferens. (Huxley.)

the tunica vaginalis or serous covering of the organ; the communication between the tunica vaginalis and the cavity of the peritoneum being closed only a short time before birth. In its descent, the testicle or ovary of course retains the blood-vessels, nerves, and lymphatics, which were supplied to it while in the lumbar region, and which are compelled to accompany it, so to speak, as it assumes a lower position in the body. Hence the explanation of the otherwise strange fact of the origin of these parts at so considerable a distance from the organ to which they are distributed.

Descent of the Testicles into the Scrotum. - The means by which the 
descent of the testicles into the scrotum is effected are not fully and exactly known. It was formerly believed that a membraneous and partly muscular cord, called the gubernaculum testis, which extends while the testicle is yet high in the abdomen, from its lower part, through the abdominal wall (in the situation of the inguinal canal) to the front of the pubes and lower part of the scrotum, was the agent by the contraction of which the descent was effected. It is now generally thought, however, that such is not the case, and that the descent of the testicle and ovary is rather the result of a general process of development in these and neighboring parts, the tendency of which is to produce this change in the relative position of these organs. In other words, the descent is not the result of a mere mechanical action, by which the organ is dragged down to a lower position, but rather one change out of many which attend the gradual development and re-arrangement of these organs. It may be repeated, however, that the details of the process by which the descent of the testicle into the scrotum is affected are not accurately known.

The homologue, in the female, of the gubernaculum testis is a structure called the round ligament of the uterus, which extends through the inguinal canal, from the outer and upper part of the uterus to the subcutaneous tissue in front of the symphysis pubis.

At a very early stage of fotal life, the Wolffian ducts, ureters, and Müllerian ducts, open into a receptacle formed by the lower end of the allantois, or rudimentary bladder; and as this communicates with the lower extremity of the intestine, there is for the time, a common receptacle or cloaca for all these parts, which opens to the exterior of the body through a part corresponding with the future anus, an arrangement which is permanent in reptiles, birds, and some of the lower mammalia. In the human fœtus, however, the intestinal portion of the cloaca is cut off from that which belongs to the urinary and generative organs; a separate passage or canal to the exterior of the body, belonging to these parts, being called the sinus uro-genitalis. Subsequently, this canal is divided, by a process of division extending from before backward or from above downward, into a 'pars urinaria' and a 'pars genitalis.' The former, continuous with the urachus, is converted into the urinary bladder.

The Fallopian tubes, the uterus, and the vagina are developed from the Müllerian ducts (fig. 516, m), whose first appearance has been already described. The two Müllerian ducts are united below into a single cord, called the genital cord, and from this are developed the vagina, as well as the cervix and the lower portion of the body of the nterus; while the ununited portion of the duct on each side forms the upper part of the uterus, and the Fallopian tube. In certain cases of arrested 
or abnormal development, these portions of the Müllerian ducts may not become fused together at their lower extremities, and there is left a cleft or horned condition of the upper part of the uterus resembling a condition which is permanent in certain of the lower animals.

In the male, the Müllerian ducts have no special function, and are but slightly dereloped. The hydatid of Morgagni is the remnant of the upper part of the Müllerian duct. The small prostatic pouch, uterus masculinus, or sinus pocularis, forms the atrophied remnant of the dis-

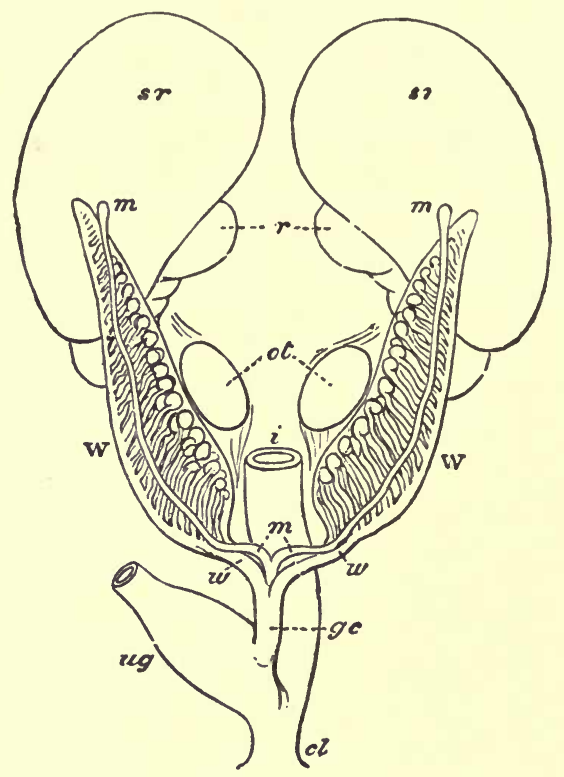

Fig. 516.-Diagram of the Wolffian bodies, Müllerian ducts and adjacent parts previous to sexual distinction, as seen from before. $s r$, the supra-renal bodies; $r$, the kidneys; ot, common blastema of ovaries or testicles; W, Wolffian bodies; $w$, Wolffian ducts; $m m$, Müllerian ducts; $g$, genital cord; $u g$, sinus urogenitalis; $i$, intestine; $c l$, cloaca. (Allen Thomson.)

tal end of the genital cord, and is, of course, therefore, the homologue, in the male, of the vagina and uterus in the female.

The external parts of generation are at first the same in both sexes.

The opening of the genito-urinary apparatus is, in both sexes, bounded by two folds of skin, while in front of it there is formed a penis-like body surmounted by a glans, and cleft or furrowed along its under surface. The borders of the furrows diverge posteriorly, running at the sides of the genito-urinary orifice internally to the cutaneous folds just mentioned. In the female, this body becoming retracted, forms the clitoris, and the margins of the furrow on its under surface are converted into the nymphæ or labia minora, the labia majora pudendæ being constituted by the great cutaneous folds. In the male fœtus, the margins 
of the furrow at the under surface of the penis unite at about the fourteenth week, and form that part of the urethra which is included in the penis. The large cutaneous folds form the scrotum, and later (in the eighth month of derelopment), receive the testicles, which descend into them from the abdominal cavity. Sometimes the urethra is not closed, and the deformity called hypospadias then results. The appearance of hermaphroditism may, in these cases, be increased by the retention of the testes within the abdomen. 



\section{APPENDIX.}

\section{CLASSIFICATION OF THE ANIMAL KINGDOM}

\section{Mammalia}

Monodelphia

Primates

Cheiroptera

Insectivora

Carnivora

Proboscidea

Hyracoidea

Ungulata

Sirenia

Cetacea

Rodentia

Edentata

Didelphia

Ornithodelphia Aves

Carinatæ

Ratitæ .

Reptilia

Crocodilia

Ophidia

Chelonia

Lacertilia

AMPHIBIA

Anura

Pisces
A.-VERTEBRATA.

Typical examples.

Man, ape.

Bat.

Hedgehog.

Cat, dog, bear.

Elephant.

Hyrax.

Horse, sheep, pig

Dugong.

Whale.

Rabbit, rat.

Armadillo.

Kangaroo.

Duck-billed platypus

Fowl, duck.

Ostrich.

Crocodile.

Snake.

Tortoise.

Lizard.

Frog.

Newt.

Lamprey, shark, cod

\section{B. -INVERTEBRATA.}

\section{Mollusca}

Odontophora

Lamellibranchiata

Brachiopoda

Polyzoa

ARTHROPODA

Crustacea

Arachnida

Insecta

Myriapoda

ECHINODERMATA .

Vermes

Annelida

Platyhelminthes

Nemathelminthes
Actinozoa

Hydrozoa

Protozoa
Whelk, snail.

Mussel, oyster.

Terebratula.

Sea mat.

Lobster.

Scorpion, spider.

Bee, fly.

Centipede.

Sea stars.

Earthworm.

Tapeworm, fluke.

Round-worm, thread-worm

Sea anemone.

Hydra.

Amœba, Vorticella. 


\section{Organic Chemical Substances.}

Nearly all of the most important substances found in the animal body have been mentioned and described in the preceding pages. It will be only necessary here to add some brief notes.

Certain terms have been used without explanation.

Hydrocarbons.-Compounds of carbon and hydrogen. Carbon being a tetrad element, the simplest hydrocarbon is $\mathrm{C}^{\mathrm{i} v} \mathrm{H}_{4}^{\mathrm{i}}$, methane or marsh gas. It is found in the gases of the alimentary canal (intestiues) (p. 406). It is the first of the series known as paraffins. The different members of the series increase by $\mathrm{CH}_{2}$, so that the next paraffin is $\mathrm{C}_{2} \mathrm{H}_{6}$, ethane; $\mathrm{C}_{3} \mathrm{H}_{r}$, propane, and so on. The general formula being $\mathrm{C}_{\mathrm{n}} \mathrm{H}_{2 \mathrm{n}+2}$.

Alcohols.-From a hydrocarbon, by substituting $\mathrm{OH}$ (hydroxyl) for $\mathrm{H}$, we obtain the corresponding alcohol; thus from $\mathrm{CH}_{3} \mathrm{H}$ we obtain $\mathrm{CH}_{3} \mathrm{OH}$, methyl alcohol; from $\mathrm{C}_{2} \mathrm{H}_{5} \mathrm{H}_{2} \mathrm{C}_{2} \mathrm{H}_{5} \mathrm{OH}$, ethyl alcohol; from $\mathrm{C}_{3} \mathrm{H}_{2} \mathrm{H}, \mathrm{C}_{3} \mathrm{H}_{7} \mathrm{OH}$, propyl alcohol and the like. They are hydrates of the hydrocarbons.

Ethers. - The ethers are obtained from their corresponding alcohols by dehydration; e.g., $2\left(\mathrm{C}_{2} \mathrm{H}_{5}\right) \mathrm{OH}-\mathrm{H}_{2} \mathrm{O}=\left(\mathrm{C}_{2} \mathrm{H}_{5}\right)_{2} \mathrm{O}$, ethylic ether.

Aldehydes. - The aldehydes are obtained by oxidation of alcohols thus : $-\mathrm{C}_{2} \mathrm{H}_{5} \mathrm{OH}+\mathrm{O}=\mathrm{CH}_{3} \mathrm{COH}+\mathrm{H}_{2}$, ethyl aldehyde.

Acids. - The acids are obtained by further oxidation, one atom of $\mathrm{O}$ being substituted for $\mathrm{H}_{2}$, e.g., $\mathrm{CH}_{3} \mathrm{CO} \mathrm{OH}$, acetic acid.

The series of acids obtained from the first series of paraffins is known as fatty acids; those which are most familiar as fatty acids being $\mathrm{C}_{4} \mathrm{H}_{8} \mathrm{O}_{2}$, lutyrie acid; $\mathrm{C}_{6} \mathrm{H}_{12} \mathrm{O}_{2}$, caproic acid; $\mathrm{C}_{16} \mathrm{H}_{32} \mathrm{O}_{2}$, palmitic, and $\mathrm{C}_{1 \times} \mathrm{H}_{36} \mathrm{O}_{2}$, steriric, derived from $\mathrm{C}_{4} \mathrm{H}_{10}$ (butane), $\mathrm{C}_{6} \mathrm{H}_{14}$ (hexane), $\mathrm{C}_{16} \mathrm{H}_{34}$ (hecdecane), and $\mathrm{C}_{1 \times} \mathrm{H}_{3 \mathrm{~B}}$, respectively.

Soaps and Fats. - The fatty acids in combination with soda or potash, or similar bases, form sorps, and when combined with glycerine form futs.

Other series of hydrocarbons. - The first series of paraffins consists of saturated hydrocarbons; many other series exist, however, in which the $C$ is unsaturated. Their general formulæ are as follows: $\mathrm{C}_{n} \mathrm{H}_{2 n}$; $\mathrm{C}_{n} \mathrm{H}_{2 n-2} ; \mathrm{C}_{n} \mathrm{H}_{2 n-4} ; \mathrm{C}_{n} \mathrm{H}_{2 n-6}$, and so on.

From each series of hydrocarbons, the corresponding alcohols, acids, aldehydes, and ethers are obtainable. The alcohols derived from series of ethene, $\mathrm{C}_{2} \mathrm{H}_{4}$, are called glycols. But in glycols there are two $\mathrm{OH}$ united to the radicle instead of one-these are therefore called diatomic alcohols; and similarly acirls, of two kinds, may be obtained, by the sub- 
stitution of one or of two atoms of $\mathrm{O}$ for the corresponding $\mathrm{H}_{2} \mathrm{Or} \mathrm{H}_{4}$. An example or two may be cited:-

$\mathrm{C}_{2} \mathrm{H}_{4}$, ethene; $\mathrm{C}_{2} \mathrm{H}_{4} \mathrm{OH}$, ethene glyeol; $\mathrm{C}_{2} \mathrm{H}_{4} \mathrm{O}_{3}$, glycolic acid; $\mathrm{C}_{2} \mathrm{H}_{2} \mathrm{O}_{4}$, oxalic acid; and $\mathrm{C}_{3} \mathrm{H}_{6}$, propene; $\mathrm{C}_{3} \mathrm{H}_{6} \mathrm{OH}_{2}$, propene glycol; $\mathrm{C}_{5} \mathrm{H}_{8} \mathrm{O}_{3}$, lactic acid; $\mathrm{C}_{8} \mathrm{H}_{4} \mathrm{O}_{4}$, malonic acid.

The next series of hydrocarbons, $\mathrm{C}_{\mathrm{n}} \mathrm{H}_{2 \mathrm{n}-2}$, is represented by $\mathrm{C}_{2} \mathrm{H}_{2}$, acetylene; the next $\mathrm{C}_{\mathrm{n}} \mathrm{H}_{2 \mathrm{n}-4}$, by terebinthene, $\mathrm{C}_{10} \mathrm{H}_{16}$; the next $\mathrm{C}_{\mathrm{n}} \mathrm{H}_{2 \mathrm{n}-6}$, by benzene, $\mathrm{C}_{6} \mathrm{H}_{6}$.

From these we obtain triatomic alcohols, e.g., glycerine, $\mathrm{C}_{8} \mathrm{H}_{8} \mathrm{OH}_{3}$, tetratomic alcohols, e.g., erythrite, $\mathrm{C}_{4} \mathrm{H}_{6} \mathrm{OH}_{4}$, and hexatomic alcohols, e.g., mannite, $\mathrm{C}_{6} \mathrm{H}_{8} \mathrm{OH}_{6}$; from the last, the carbohydrates are derived.

Of the hydrocarbons, only one is, as we have said, found in the body, viz., methane; of the alcohols, cholesterine, $\mathrm{C}_{26} \mathrm{H}_{43} \mathrm{OH}$, a monatomic, and glycerine, $\mathrm{C}_{3} \mathrm{H}_{3} \mathrm{OH}_{3}$, a triatomic alcohol.

Of the aldehydes and ketones (analogous products to aldehyde, obtained from isomeric alcohols), acetone, or propyl ketone, is found in blood and in urine, particularly in diabetes. The glucoses are aldehydes of mannite, and the other carbohydrates are derived from that class.

Fatty Acids.-Formic, acetic, propionic, butyric, caproic and caprylic, are all more or less represented in the secretions and tissues of the body. Palmitic and stearic in fats.

Glycol Acids. - Lactic acid, of which there are three isomeric bodies, and leucic acid and two other acids, oxalic and succinic.

Aromatic Series.-The foundation is the benzol ring, $\mathrm{C}_{6} \mathrm{H}_{6}$, and all bodies containing this radicle are closely related. They differ in regard to the position in the ring of the $\mathrm{H}$ atoms which are replaced, as well as in regard to the substances which replace them; the derivatives often occur in the decomposition of proteids. Phenol or oxybenzol $\left(\mathrm{C}_{6} \mathrm{H}_{6} \mathrm{O}\right)$ is found in combination in the urine and fæces. Oxybenzoic acid $\left(\mathrm{C}_{6} \mathrm{H}_{4}, \mathrm{OH}, \mathrm{COOH}\right)$ is a common decomposition product of proteids; one atom of $\mathrm{H}$ is replaced by hydroxyl and another by carboxyl. The action of Millon's reagent is due to the benzol ring.

\section{Nitrogenous Products of Proteid Decomposition.}

Amines.-These are bodies of the ammonia type $\left(\mathrm{NH}_{3}\right)$ in which one or more of the $\mathrm{H}$ atoms of the ammonia are replaced by hydrocarbon radicles; e.g., $\mathrm{NH}_{2}, \mathrm{CH}_{3}=$ methylamine or mono-methylamine. Trimethylamine, $\mathrm{N}\left(\mathrm{CH}_{3}\right)_{3}$, often occurs in putrefaction.

Protamines. - These are basic proteid bodies which give the Biuret reaction; on decomposition they yield the nitrogenous bases but no leucin or tyrosin. They occur in the decomposition of all proteids and also as 
mimary constituents of cells, especially in spermatozod. In this group are urgenim, lysin, and hystidin.

Amides. - These are bodies of the ammonia type $\left(\mathrm{NH}_{3}\right)$ in which one or more of the $\mathrm{H}$ atoms are replaced by organic acirl residues (an acid residue $=$ the acid minus hydroxyl ; e.y., CH(C) is the residue of $\left.\mathrm{CH}_{3} \mathrm{COOH}\right)$. Monacetamide $=\mathrm{NH}_{2}, \mathrm{CH}_{3} \mathrm{CO}$ There are also more complicated amides which are built up from two molecules of ammonia; e./., urea or carbamide, which is formed from carbonic acirl and is usually written $\mathrm{CO}, \mathrm{NH}_{2}, \mathrm{NH}_{2}$.

Amido-acids. - These are bodies of the ammonia type $\left(\mathrm{NH}_{3}\right)$ in which one or more of the $\mathrm{H}$ atoms of the ammonia are replaced by organic acid radicles; they may also be regarded as acids in which one or more of the $\mathrm{H}$ atoms of the acid radicle are replaced by amidogen, $\mathrm{NH}_{2}$. As the term implies that they are acids, it is necessary that they contain the carboxyl group $(\mathrm{COOH})$ intact. For example, glycin (amido-acetic acid) in either $\mathrm{NH}_{2}, \mathrm{CH}, \mathrm{COOH}$ or $\mathrm{CH}_{2}\left(\mathrm{NH}_{2}\right)$, COOH.

Nitrogenous or Nuclein Bases. - The members of this group are very closely related and consist of hypoxanthin $\left(\mathrm{C}_{5} \mathrm{H}_{4} \mathrm{~N}_{4} \mathrm{O}\right)$, xanthin $\left(\mathrm{C}_{5} \mathrm{H}_{4} \mathrm{~N}_{4} \mathrm{O}_{2}\right)$, adenin $\left(\mathrm{C}_{5} \mathrm{H}_{5} \mathrm{~N}_{5}\right)$, and guanin $\left(\mathrm{C}_{5} \mathrm{H}_{5} \mathrm{~N}_{5} \mathrm{O}\right)$. Besides occurring in ordinary proteid decomposition, they are also always present in all downward chemical changes in the cells. Uric acid $\left(\mathrm{C}_{5} \mathrm{H}_{4} \mathrm{~N}_{4} \mathrm{O}_{3}\right)$, though not a member of the group, is shown to be closely related by studying their chemical composition. By oxidation uric acid yields urea and alloxan $\left(\mathrm{C}_{4} \mathrm{H}_{2} \mathrm{~N}_{2} \mathrm{O}_{4}\right)$; it has been found that in alloxan there is present a radicle $\mathrm{C}_{5} \mathrm{~N}_{4}$ known as the purin or alloxan-uric nucleus; purin is formed from this radicle by the substitution of $\mathrm{H}$ atoms. Both uric acid and the nuclein bases can be derived from this base: hypoxanthin is oxypurin, uric acid is tri-oxypurin, adenin is amino-oxypurin, etc.

Protargons.-These are very complex phosphorus-containing bodies which are chiefly obtained from nervous tissues. Protargon was at one time considered an entity, but, according to the most recent views, it is merely a mixture of cerebrin and lecithin.

Of the bodies which constitute the above-mentioned groups, ouly the following need be described:

$$
\begin{aligned}
& \text { Glycin, Glycocol, Glycocin, or }\} \\
& \text { Amido-acetic acid. }
\end{aligned}
$$

This substance occurs in the body in combination as in the biliary acids, but is never free. Glycocholic acid, when treated with weak acids, with alkalies, or with baryta water, splits up into cholic acid and glycin, or amido-acetic acid. Thus: $\mathrm{C}_{26} \mathrm{H}_{13} \mathrm{NO}_{6}+\mathrm{H}_{2} \mathrm{O}=\mathrm{C}_{26} \mathrm{H}_{40} \mathrm{O}_{3}+\mathrm{C}_{2} \mathrm{H}_{5} \mathrm{NO}_{2}$. Glycocholic acid + water $=$ cholic acid + glycin, and under similar circumstances Taurocholic acid splits up into cholic acid and taurin: 
$\mathrm{C}_{26} \mathrm{H}_{45} \mathrm{O}_{3} \mathrm{NSO}_{2}+\mathrm{H}_{2} \mathrm{O}=\mathrm{C}_{26} \mathrm{H}_{40} \mathrm{O}_{5}+\mathrm{C}_{2} \mathrm{H}_{7} \mathrm{NSO}_{3}$, or amido-isethionic acid. Taurocholic acid + water $=$ cholic acid and taurin. Glycin occurs also in hippuric acid. It can be prepared from gelatin by the action of acids or alkalies, and can also be obtained from hippuric acid.

Sarcosin or Methyl , amido-acetic acid.

$$
\mathrm{C}_{3} \mathrm{H}_{7} \mathrm{NO}_{2}\left(=\mathrm{CH}_{2}<\underset{\mathrm{CO} \mathrm{OH}}{\mathrm{NH} \mathrm{CH}_{3}}\right)
$$

It is a constituent of kreatin, and also of eaffeine, but has never been found free in the human body. It may be obtained from these bodies by boiling with baryta water.

$\left.\begin{array}{l}\text { Leucin or Amido- } \\ \text { caproic acid, }\end{array}\right\} \mathrm{C}_{6} \mathrm{H}_{13} \mathrm{NO}_{2}\left(=\mathrm{CH}_{3} \cdot \mathrm{CH}_{2} \mathrm{CH}_{2} \mathrm{CH}_{2} \cdot \mathrm{CH}\left(\mathrm{NH}_{2}\right) \mathrm{CO} \mathrm{OH}\right.$ occurs normally in many of the organs of the body, and is a product of the pancreatic digestion of proteids. It is present in the urine in certain diseases of the liver in which there is loss of substance, especially in acute yellow atrophy. It occurs in circular oily dises or erystallizes in plates, and can be prepared either by boiling horn shavings, or any of the gelatins with sulphuric acid, or out of the products of pancreatic digestion.

Guanidin, $\mathrm{CN}_{3} \mathrm{H}_{5}\left(=\mathrm{CNH}<\frac{\mathrm{NH}_{2}}{\mathrm{NH}_{2}}\right)$ is a derivative of urea, the atom of $\mathrm{O}$ being replaced by $\mathrm{NH}$.

Kreatin, or Methyl $\quad$ guanidin acetic arid, $\int_{4}^{1} \mathrm{C}_{4} \mathrm{H}_{2} \mathrm{~N}_{3} \mathrm{O}_{2}\left(=\mathrm{CNH}<\mathrm{NCH}_{3}, \mathrm{CH}_{2}, \mathrm{COOH}\right)$ is one of the primary products of muscular disintegration. It is always found in the juice of muscle. It is formed by the action of guanidin on methyl amido-acetic acid. It is generally decomposed in the blood into urea and sarcosin, and only appears in the urine as kreatinin. Treated with either sulphuric or hydrochloric acid, it is converted into kreatinin; thus-

$$
\mathrm{C}_{4} \mathrm{H}_{9} \mathrm{~N}_{3} \mathrm{O}_{2}=\mathrm{C}_{4} \mathrm{H}_{7} \mathrm{~N}_{3} \mathrm{O}+\mathrm{H}_{2} \mathrm{O} \text {. }
$$

It has been made synthetically by bringing together cyanimide and sarcosine.

Kreatinin, $\mathrm{C}_{4} \mathrm{H}_{7} \mathrm{~N}_{3} \mathrm{O}\left(=\mathrm{CNH}<\begin{array}{l}\mathrm{NH}-\mathrm{CO} \\ \mathrm{N}\left(\mathrm{CH}_{3}\right)_{\mathrm{CH}} \mathrm{CH}_{2}\end{array}\right)$ is present in human urine, derived from dehydration of kreatin. It does not appear to be present in muscle. It is basic, having lost the $\mathrm{COOH}$ group, and reacts as an alkaline body, combining with salts to form double salts, etc. On decomposition it yields urea, sareosin, and methyl guanidin.

Taurin or Amido-
isethionic acid, $\mathrm{C}_{2} \mathrm{H}_{7} \mathrm{NSO}_{3}\left(=\mathrm{C}_{2} \mathrm{H}_{4}<\mathrm{NH}_{2} \mathrm{SO}^{\mathrm{H}}\right)$ is a constituent of the bile acid, taurocholic acid, and is found also in traces in the muscles and lungs. It has been prepared synthetically from isethionic acid. It is a crystalline substance, very stable. 


\section{$\begin{aligned} & \text { Hippuric Acid or } \\ & \text { zol amido-acetic acid }\end{aligned} \int_{9} \mathrm{C}_{9} \mathrm{H}_{9} \mathrm{NO}_{3}=\left(\mathrm{C}_{6} \mathrm{H}_{5} \mathrm{CONH} \mathrm{CH} \mathrm{CH}_{2} \mathrm{COH}\right)$,}

a normal constituent of luman urine, the quantity excreted being increased by a vegetable diet, and therefore it is present in greater amount in the urine of herbivora. It may be decomposed by acids into glycin and benzoic acid. It crystallizes in semi-transparent rhombic prisms, almost insoluble in cold water, soluble in boiling water.

\section{Tyrosin or Para-oxyphen- 1 yl-amido-proprionic acid,

$$
\int_{9}^{1} \mathrm{C}_{9} \mathrm{H}_{11} \mathrm{NO}_{3}\left(=\mathrm{C}_{6} \mathrm{H}_{4}<\underset{\mathrm{C}_{2} \mathrm{H}_{3}}{\mathrm{OH}}, \mathrm{NH}_{2}, \mathrm{COOH}\right) \text {. }
$$

This is found generally together with leucin, in certain glands, e.\%., pancreas and spleen; and chiefly in the products of pancreatic digestion or of the putrefaction of proteids. It is found in the urine in some diseases of the liver, especially acute yellow atrophy. It crystallizes in fine needles, which collect into feathery masses. It gives the proteid test with Millon's reagent, and heated with strong sulphuric acid, on the addition of ferric chloride gives a violet color.

$$
\text { Lerithin, } \mathrm{C}_{42} \mathrm{H}_{84} \mathrm{PNO}_{9}\left(\begin{array}{l}
=\mathrm{CH}_{2}, \mathrm{O} . \mathrm{C}_{17} \mathrm{H}_{33} \mathrm{CO} \\
\mathrm{C} \mathrm{H}, \mathrm{O} . \mathrm{C}_{15} \mathrm{H}_{31} \mathrm{CO} \\
\vdots \\
\mathrm{CH}_{22}, \mathrm{O} . \mathrm{PO}<{ }_{\mathrm{O}}, \mathrm{CH}_{2}, \mathrm{CH}_{2} \mathrm{~N}\left(\mathrm{CH}_{3}\right)_{3} \mathrm{OH} .
\end{array}\right)
$$

It is a combination of cholin with glycero-phosphoric acid in which the two $\mathrm{H}$ atoms of the glycerine are replaced by fatty acid radicles. The chemical formula varies in accordance with the kind of fatty acid; in the above formula one radicle is that of oleic acid and the other that of palmitic. In character it is a complex nitrogenous fatty body, containing phosphorus, which has been found mixed with cerebrin and oleo-phosphoric acid in the brain. It is also found in blood, bile, and serous fluids, and in larger quantities in nerves, pus, yolk of egg, semen, and white blood-corpuscles. On boiling with acids it yields cholin, glycero-phosphoric acid, and fatty acids.

Cerebrin, $\mathrm{C}_{17} \mathrm{H}_{33} \mathrm{NO}_{3}$, is a light amorphous powder, tasteless and odorless, which is found in nerves, pus corpuscles, and in the brain. It is a nitrogenous body whose chemical constitution is not known, though the large amount of $\mathrm{C}$ which it contains indicates the presence of a fatty acid. It swells up like starch when boiled with water. When decomposed it yields, besides other substances, a sugar (gelactose).

Uric Acid or Tri-oxypurin, $\mathrm{C}_{5} \mathrm{H}_{4} \mathrm{~N}_{4} \mathrm{O}_{3}$, occurs in the urine, sparingly in human urine, abundantly in that of birds and reptiles, where it represents the chief nitrogenous decomposition product. It occurs also in the blood, spleen, liver, and sometimes is the only constituent of urinary calculi. It is probably converted in the blood into urea and carbonic acid. It generally occurs in urine in combination with bases, forming 
urates, and never free unless under abnormal conditions. A deposit of urates may occur when the urine is concentrated or extremely acid, and in febrile disorders.

Xanthin or Di-oxypurin, $\mathrm{C}_{5} \mathrm{H}_{4} \mathrm{~N}_{4} \mathrm{O}_{2}$, has been obtained from the liver, spleen, thymus, muscle, and the blood. It is found in normal urine, and is a constituent of certain rare urinary calculi.

Hypoxanthin or Oxypurin, $\mathrm{C}_{5} \mathrm{H}_{4} \mathrm{~N}_{4} \mathrm{O}$, is found in juice of flesh, in the spleen, thymus, and thyroid.

Guanin or Amino-oxypurin, $\mathrm{C}_{5} \mathrm{H}_{5} \mathrm{~N}_{5} \mathrm{O}$, has been found in the human liver, spleen, and fæces, but does not occur as a constant product.

Adenin or Amino-purin, $\mathrm{C}_{5} \mathrm{H}_{5} \mathrm{~N}_{5}$, is the simplest member of the purin group. It exists abundantly in the liver and urine of leucocythemic patients.

Allantoin, $\mathrm{C}_{4} \mathrm{H}_{6} \mathrm{~N}_{4} \mathrm{O}_{3}$, found in the allantoic fluid of the fœetus, and in the urine of animals for a short period after their birth. It is one of the oxidation products of uric açid, which on oxidation gives urea.

In addition to the above compounds and probably related to them, are certain coloring and excrementitious matters, which are also most likely distinct decomposition compounds.

\section{Pigments, Etc.}

Bilirubin, $\mathrm{C}_{16} \mathrm{H}_{18} \mathrm{~N}_{2} \mathrm{O}_{3}$, is the best known of the bile pigments. It is best made by extracting inspissated bile or gall stones with water (which dissolves the salts, etc.), then with alcohol, which takes out cholesterin, fatty and biliary acids. Hydrochloric acid is then added, which decomposes the lime salt of bilirubin and removes the lime After extracting with alcohol and ether, the residue is dried and finally extracted with chloroform. It crystallizes of a bluish-red color. It is allied in composition to hæmatin, as has been described.

Biliverdin, $\mathrm{C}_{16} \mathrm{H}_{18} \mathrm{~N}_{2} \mathrm{O}_{4}$, is made by passing a current of air through an alkaline solution of bilirubin, and by precipitation with hydrochloric acid. It is a green pigment which is an oxidation product of bilirubin.

Bilifuscin, $\mathrm{C}_{9} \mathrm{H}_{11} \mathrm{NO}_{3}$, is made by treating gall stones with ether, then with dilute acid, and extracting with absolute alcohol. It is a non-crystallizable brown pigment.

Biliprasin is a pigment of a green color, which can be obtained from gall stones, and from bile which has been allowed to decompose.

Bilihumin (Staedeler) is a dark brown earthy-looking substance, of which the formula is unknown.

Urochrome and Urobilin occur in bile and in urine; the latter is prob- 
ably identical with stercobilin, which is found in the fæces. Uroprythrin is one of the coloring matters of the urine. It is orange red and contains iron, as is also Choletelin

Melanin is a lark brown or black pigment which occurs especially in epidermal tissues, where it is associated with keratin. It is found in the lungs, bronchial glands, hair, choroid, skin of negroes, etc.; also in the urine and in melanotic diseases, e.!., sarcoma. It is a transformation product of proteids, to which it is closely related, and can be made artificially by boiling proteid with sulphuric acid. It contains $\mathrm{C}, \mathrm{H}, \mathrm{O}, \mathrm{N}, \mathrm{S}$, and (rarely) Fe

Lipochromes are pigments, usually yellow or yellowish-red, which are associated with fat, being almost always present in adipose tissue. Little is known about them, but they are thought to consist only of $\mathrm{C}, \mathrm{H}$, and 0 .

Hrematin has been fully treated of, p. 1620 st sey.

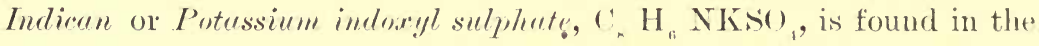
urine and is derived from proteid putrefaction in the intestines. It is colorless.

Indigo or Indiyg-blue, $\mathrm{C}_{16} \mathrm{H}_{10} \mathrm{~N}_{20}{ }_{0}$, is formed from indiean. It is usually found free in sinall amounts in ilecomposing urine, where it may give a bluish color to the sedinent; in very rare instances it makes the whole urine blue.

Indol, $\mathrm{C}_{n} \mathrm{H}_{3} \mathrm{~N}$, belongs to the aromatio, series and is a product of proteid putrefaction in the intestine. It is fomol in the fieces and helps cause their odor. When absorbed, it is excreted in the urine as potassium indoxyl sulphate (indican).

Skrotol, $\mathrm{C}_{9} \mathrm{H}_{9} \mathrm{~N}$, is also one of the aromatic series, a product of proteid putrefaction in the intestines. It is fomd in the faecs and contril)ntes to their oxlor. When absorberl, it is excreted in the urine as sodium or potassium skatoxyl sulphate. Both indol anel skatol are crystalline and volatile.

\section{Nitrogenous Bodies of Uneertrin Nuture.}

Ferments are boties which possess the property of exciting ehemical rhanges in matter with which they (onme in contact. They are at present rliviled inte two slasses, ealled (1) organized, and (2) unorganized or soluble.

(1.) Of the organized, yeast may be taken as an example. Its activity depends upon the vitality of the yeast cell, and disappears as soon as the cell dies, neither can any substance be obtained from the yeast by means of precipitation with alcohol or in any other way which has the 
power of exciting the ordinary change produced by the plant itself. The action of micro-organisms in the alimentary canal and elsewhere is also an example of the same nature.

(2.) Unorganized or soluble ferments are those which are found in secretions of glands, or are produced by chemical changes in animal or vegetable cells in general; when isolated they are colorless, tasteless, amorphous solids soluble in water and glycerin, and precipitated from the aqueous solutions by alcohol and acetate of lead. Chemically many of these are said to contain nitrogen.

Mode of action.-Without going into the theories of how these unorganized ferments act, it will suffice to mention that:

(1.) Their activity beyond a certain point does not depend upon the actual amount of the ferment present. (2.) That the activity is destroyed by high temperature, and various concentrated chemical reagents, but increased by moderate heat, about $40^{\circ} \mathrm{C}$., and by weak solutions of either an acid or alkaline fluid. (3.) The ferments themselves appear to undergo no change in their own composition, and waste very slightly during the process.

The chief classes of unorganized ferments are:-

(1.) Amylolytic, which possess the property of converting starch into glucose. They add a molecule of water, and may be called hydrolytic. The principal amylolytic ferments are Ptyalin, found in the saliva, and a ferment, probably distinct, in the pancreatic juice, called Amylopsin. These both act in an alkaline medium. Amylolytic ferments have been found in the blood and elsewhere.

(2.) Proteolytic convert proteids into peptones. The nature of their action is probably hydrolytic. The proteolytic ferments of the body are called Pepsin, from the gastric juice acting in an acid medium. Trypsin, from the pancreatic juice acting in alkaline, neutral, or acid media. The Succus entericus is said to contain a third such ferment.

(3.) Inversive, which convert cane sugar or saccharose into grape sugar or glucose. Such a ferment was found by Claude Bernard in the Succus entericus; and probably exists also in the stomach mucus.

(4.) Ferments which act upon fats. - Such a body, called Steapsin, has been found in pancreatic juice.

(5.) Milk-curdling ferments. - It has been long known that rennet, a decoction of the fourth stomach of a calf, in brine, possessed the power of curdling milk. This power does not depend upon the acidity of the gastric juice, since the curdling will take place in a neutral or alkaline medium; neither does it depend upon the pepsin, as pure pepsin scarcely curdles milk at all, and the rennet which rapidly curdles milk has no proteolytic action. From this and other evidence it is believed that a 
distinct milk-curdling ferment exists in the stomach. W. Roberts has shown that a similar but distinct ferment exists in pancreatic extract, which acts best in an alkaline medium, next best in an acid medium, and worst in a nentral medium. The ferment of rennet acts best in an acid medium, and worst in an alkaline, the reaction ceasing if the alkalinity be more than slight. Also in the Succus entericus.

In addition to the above ferments, many others most likely exist in the body, of which the following are the most important:

(6.) Fibrin-forming ferment (Schmidt), (see p. 136 et seq.), found in the blood, lymph and chyle.

(7.) A ferment which converts glycogen into glucose in the liver; being therefore an amylolytic ferment.

(8.) Myosin ferment.

\section{Carbo-hydrates or AMyloids.}

The divisions of carbo-hydrates, and the chief substances forming each class with their properties, have been already given (p. 122 et seq). The following additional information may be useful.

The glucoses may be considered as the aldehydes of mannite, thus:

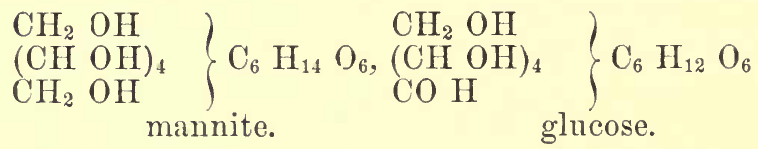

The Saccharoses or sucroses are made up of two volumes of glucose minus one molecule of water.

$$
\mathrm{C}_{6} \mathrm{H}_{12} \mathrm{O}_{6}+\mathrm{C}_{6} \mathrm{H}_{12} \mathrm{O}_{6}-\mathrm{H}_{2} \mathrm{O}=\mathrm{C}_{12} \mathrm{H}_{22} \mathrm{O}_{11} \text {. }
$$

The amyloids are anhydrides of the glucoses, $\mathrm{C}_{6} \quad \mathrm{H}_{12} \mathrm{O}_{6}-\mathrm{H}_{2} \mathrm{O}=$ $\mathrm{C}_{6} \mathrm{H}_{10} \mathrm{O}_{5}$.

Tests for Glucose.-(i.) Trommer's.-This test depends upon the power sugar possesses of reducing copper salts to their sub-oxide. It is done in the following way:--An excess of caustic potash and then a solution of copper sulphate, drop by drop, are added to the solution containing the sugar in a test-tube, as long as the blue precipitate which forms redissolves on shaking the tube. The upper portion of the fluid is then heated, and a yellowish-brown precipitate of copper suboxide appears. The test may also be done by taking only a drop or two of the copper sulphate solution.

(ii.) Moore's.-If a solution of sugar in a test-tube is boiled with caustic potash, a brown coloration appears.

(iii.). Fermentation.-If a solution of sugar be kept in the warm plate for a time after the addition of yeast, the sugar is converted into alcohol and carbon dioxide. $\left(\mathrm{C}_{6} \mathrm{H}_{12} \mathrm{O}_{6}=2 \mathrm{C}_{2} \mathrm{H}_{5} \mathrm{OH}+2 \mathrm{CO}_{2}\right.$. $)$

(iv.) Böttcher's test.-A little bismuth oxide or subnitrate and an excess of caustic potash are added to the solution in a test-tube, and the mixture is heated; the solution becomes at first gray and then black. 
(v.) Picric acid test. - To the solution about a fourth of its bulk of picric acid (saturated solution) and an equal quantity of caustic potash are added, and the solution is boiled; the liquid becomes of a very deep coffee-brown.

(vi.) Indigo-carmine test.-Add a solution of indigo carmine to color sugar solution distinctly blue, and add solution of sodium carbonate, and heat. The blue color changes to purple and then to brown and yellow, but is restored on shaking the solution.

(vii.) Phenyl hydrazine test.-A solution of phenyl hydrazine hydrochloride and sodium acetate is added. Keep in water-bath at boiling for some minutes, then cool. Yellow crystals result.

\section{Quantitative Estimation of Grape Sugar.}

1. Fehling's Method.-Solution required = copper sulphate and caustic soda, with some sodic potassic tartrate of such a strength that 10 c.c. of solution contain the amount of cupric oxide which 0.5 grm. of sugar can reduce to cuprots oxide. (This solution should be freshly prepared.) It is made as follows: Take of sulphate of copper, $40 \mathrm{grms}$; neutral tartrate of potash, 160 grms.; caustic soda (sp. gr. 1.12), 750 grms.; add distilled water to 1154.5 c.c. Each 10 c.c. contains .05 grm. of sugar.

Method.-Take 10 c.c. of the saccharine solution free from albumen, and add 90 c.c. of distilled water. Place this in a burette. Put into a flask or dish 10 c.c. of the standard solution, and dilute with four times its bulk of water and boil. Run into it, from burette, some of the diluted urine, say 20 c.c., and boil. Allow precipitate to settle, and if supernatant fluid is still blue, add, say, 5 c.c. from burette, and boil again, and so on, till the fluid ceases to have a blue tinge, taking care, toward the end of the process, to add only a few drops each time. If, after adding 20 c.c. of diluted urine and boiling, the fluid has been decolorized, too much of the solution has been added, and another estimation with a second 10 c.c. of standard solution must be made, but less than 20 c.c. of the saccharine solution should be added (say 10 c.c.) in first instance.

When the number of c.c. of diluted urine required to decolorize the solution has been determined, that volume contains the amount of sugar necessary to reduce 10 c.c. of standard solution, i.e., $.05 \mathrm{grm}$. But onetenth only of this is the saccharine solution, $\therefore$ one-tenth of number of c.c. used contains .05 grm. of sugar. From this, the percentage can be easily calculated.

2. Pavy's Modification of Fehling's Method.-By Fehling's method it is difficult and tedious to judge of the point of complete reduction of the cupric oxide. Dr. Pavy, accordingly, uses a strongly ammoniacal solution of the above. A certain amount is introduced into a small 
flask, which is then heated till the vapor of ammonia escapes by a narrow tube. The sugar solution is then allowed to flow from a burette into the flask until the blueness has disappeared, the solution being kept boiling all the time. The blueness is apt to disappear suddenly, and care should therefore be taken toward the end of the process.

Calculate as in Fehling's method.

3. Estimation of sugar by fermentation.-In the case of saccharine urine, it is allowable as a single test to use the following method:- Take specific gravity of urine before and after fermentation. Each degree of specific gravity lost by the urine represents one grain of sugar per ounce of urine.

4. Sugar may also be estimated by adding yeast to urine, and collecting the carbon dioxide evolved. The carbon dioxide is a measure of the amount of sugar present.

5. The estimation may also be done by the saccharimeter, an instrument for the estimation of the degree of polarization which a ray of light undergoes in passing through a solution of sugar, either to the left or to the right.

Urea, $\mathrm{CO}\left(\mathrm{NH}_{2}\right)_{2}$. The properties and relations of urea have been treated of at some length in the chapter upon excretion. There remains to be described the method of its quantitative estimation in the urine. There are two chief methods, viz.:-

(i.) Hypobromite Method.--One of the forms of apparatus employed in this method (Russell and West's) consists of $(a)$ a water-bath supported by three iron bands, arranged as a tripod. The bath is provided with a cylindrical depression, and with a hole, into which fits a perforated india-rubber cork; $(b)$ a bulb tube with a constricted neck; $(c)$ a glass rod provided with an india-rubber band at one extremity; $(d)$ a pipette of five cubic centimetres capacity; $(e)$ a graduated glass collecting tube; $(f)$ a spirit lamp; $(g)$ a wash-bottle with distilled water; $(h)$ hypobromous solution. The hypobromous solution is made in the following way: three and a half ounces (100 grm.) of solid caustic soda is dissolved in nine ounces ( $250 \mathrm{grm}$.) of distilled water. When the solution is cold, seven drachms (25 c.c.) of pure bromine are to be added carefully and gradually. The mixture is not to be filtered; it keeps badly, and for this reason it should be made shortly before it is required; or the solution of caustic soda in water may be made in large quantities as it does not undergo any change, the bromine in the proper proportion being added at the time it is required for use.

Method.-Fill the pipette to the mark on the stem with the urine to be examined; pour the 5 c.c. of urine thus measured out into the bulb; fill up the bulb tube as far as the constricted neck with distilled water from the wash-bottle; insert the glass rod $(c)$ in such a way that the india-rubber band at the extremity fills up the constricted neck; the 
diluted urine should exactly occupy the bulb and neck of the tube, no bubble of air being below the elastic band on the one hand, while on the other the fluid should not rise above the band; in the former case a little more water should be added, in the latter a fresh portion of urine must be used, and the experiment repeated. After adjusting the glass rod, fill up the rest of the bulb tube with hypobromous solution; it will not mix with the urine so long as the rod is in place. The water-bath having been previously erected, and the india-rubber cork fixed firmly into the aperture, the bulb tube is to be thrust from below through the perforation in the cork. The greater part of the tube is then beneath the water-bath, the upper extremity alone being grasped by the cork. Fill the water-bath half full of water, fill also the graduated glass tube $(e)$ with water, and invert it in the bath; in doing this no air must enter the tube, which when inverted should be completely filled with water. Now slide the graduated tube toward the orifice of the bulb tube, at the same time withdrawing the glass rod which projects into the bath through the cork. At the instant that the rod is withdrawn the hypobromous solution mixes with the diluted urine, and a decomposition takes place represented thus: $\mathrm{CON}_{2} \mathrm{H}_{4}+$ $3 \mathrm{NaBrO}+2 \mathrm{NaHO}=3 \mathrm{NaBr}+3 \mathrm{H}_{2} \mathrm{O}+\mathrm{Na}_{2} \mathrm{CO}_{3}+\mathrm{N}_{2}$. Urea + sodium hypobromite + -caustic soda $=$ sodium bromide + water + sodium carbonate + nitrogen. The nitrogen produced is given off as gas, and displaces the water in the graduated tube, which is held over it. The gas is at first evolved briskly, but afterward more slowly; to facilitate its evolution, the bulb of the tube may be slightly warmed with a spirit lamp; as a rule, however, this is unnecessary. After ten minutes, the amount of water displaced by the gas should be read off on the tube, which is divided into tenths. Each number on the tube represents one gram of urea in 100 c.c. of urine. Normal urine should yield roughly 1.5-2.5 parts of nitrogen by this test. If 5 c.c. of urine gives off more nitrogen than fills the tube to iii., dilute the urine with an equal volume of water, and take 5 c.c.; read off and multiply by two.*

Several apparatus may be employed instead of the one described, viz., those of Dupré, Gerard, and Squibb. The chemical reactions in each case are the same.

(ii.) Liebig's Method.-This method is of greater accuracy. The solutions required are $(a)$ baryta mixture $=2$ vols. of saturated solution of barium nitrate and 1 vol. of saturated solution of barium hydrate; (b) standard solution of mercuric nitrate, such that 1 c.c. will precipitate $.01 \mathrm{grm}$. of urea, and (c) a solution of carbonate of soda.

Method.-Take 40 c.c. of urine, add 20 c.c. of $(a)$, filter off the precipitate of sulphates and phosphates; keep the filtrate. Fill a burette

* Several corrections have to be made before the result can be considered as accurate; for these the detailed accounts in practical handbooks of Physiology should be consulted. 
with $(b)$, and take 15 c.c. of the filtrate in a dish. Let $(b)$ fall drop by drop into the 15 c.c. in the dish, stirring constantly. Have ready a glass plate with several separate drops of $(c)$, and from time to time add a drop of the urine mixture by means of a glass rod to one of the drops. When a yellow color first appears in a drop of the $\mathrm{NaCO}_{3}$, the mercuric nitrate is just in excess. Read the burette. Calculate as follows:

1 c.c. of mercuric solution precipitates $.01 \mathrm{grm}$ of urea, $\therefore$ the No. of c.c. used $\times .01=$ amount of urea in 15 c.c. of filtrate, i.e., in 10 c.c. of urine. But 10 c.c. of urine usually contains enough $\mathrm{NaCl}$ to act on 2 c.c. of mercury solution.* Hence, when reckoning the number of c.c. of standing mercury solution used, a deduction of 2 c.c. must always be made.

\section{Quantitative Estimation of Chlorides.}

Liebig's Method.-The solutions required are a baryta mixture as above; and $(b)$ standard solution of mercuric nitrate, such that 1 c.c. would be capable of decomposing .01 grm. of sodium chloride.

Method.-Take 40 c.c. of urine free from albumen, and add 20 c.c. of (a). Filter. Take 15 c.c. of filtrate and place in a flask or dish, adding a drop or two of nitric acid. Fill a burette with (b), and slowly rum some of this solution into the filtrate in the dish, stirring constantly. As soon as a distinct cloud appears in the diluted urine, and does not disappear on stirring, then all the sodium chloride in urine has been decomposed. Read burette. Calculate as follows:

1 c.c. of mercury solution decomposed $01 \mathrm{grm}$. of $\mathrm{NaCl}, \therefore$ the number of c.c. used $\times .01 \mathrm{grm}$. = number of grms. of $\mathrm{NaCl}$ in 15 c.c. of filtrate, i.e., 10 c.c. of urine.

\section{Quantitative Estimation of Phosphates.}

The solutions required are ( $($ ) solution of sodium acetate, containing $100 \mathrm{grm}$. of sodium acetate, 100 c.c. of acetic acid, and 900 c.c. of distilled water; $(b)$ a solution of uranium acetate or nitrate, such that 1 c.c. will precipitate .005 grm. of phosphoric acid; and $(c)$ a solution of ferrocyanide of potassium.

Method.-CTake 50 c.c. of urine. Add some (a) solution, and heat on water-bath to nearly $100^{\circ} \mathrm{C}$. Fill burette with $(b)$, and allow this to fall into the urine slowly. Have ready a gliss plate with several distinct drops of potassium ferro-cyanide solution. From time to time add a drop of urine mixture to one of the drops; and when there FIRST appears a reddish-brown color in a drop of potassium ferro-cyanide, all the phosphates are precipitated. Read burette. Calculate thus:

1 c.c. precipitates $.005 \mathrm{grm}$. of phosphoric acid, $\therefore$ the number of c.c. nsed $\times .005$ grm. $=$ number of grms. of phosphoric acid in 50 c.c. of urine.

* This is only a rough estimate. 


\section{NDEX.}

Abducens nerve, 604

Absorption, 408

channels of, 422

conditions for, 411

in the alimentary canal, 424

in the large intestine, 425

in the small intestine, 425

in the stomach, 424

rapidity of, 410

through the lungs, 426

the skin, 425

where it may occur, 424

Accommodation of vision, 713

Achromatic spindle, 15

Achromatin, 12

Achroödextrin, 339

Acid albumin, 115

Acids, 826

Addison's disease, 317

Adenin, 831

Adenoid tissue, 46

Adipose tissue, 48

developnent of, 49

uses of, 50

After-birth, the, 787

$\Lambda$ ir cells, 259

Akinesis, 13

Albuminates, 115

Albumin molecule, action of gastric juice on, 361

Albuminoids, 120

Albumose, 362

Alcohols, 826

Aldehyres, 826

Alimentary canal, development of, 813

Alkali albumin, 116

Allantoin, 831

Allantois, 781

Amides, 828

Amido-acids, 828

Amidulin, 339

Amines, 828
Amitosis, 13

Amitotic division, 13

Ammonium carbamate, 432

carbonate, 431

Amnion, 780

Amoboid movement, 4, 146

Amylopsin, 383

Amyloses, 122

Anabolism, 7

Anacrotic wave, 219

Anaphases, 17

Anclectrotonus, 530

Animal heat, 449

Animal kingdom, classification of, 825

Anode, 531

Ano-spinal centre, 579

Antipeptone, 382

A pnoea, 286

A ppendices epiploicr, 377

Appendix, 825

Areolar tissue, 45

Aromatic series, 827

Arteries, 180

development of, 800

structure of, 180

tone of, 245

Articulate sounds, 549

Asphyxia, 292

cause of death in, 294

Astigmatism, 720

Atmosphere, composition of, 271

Attraction sphere, 12

Auditory centre, 641

nerve, 606

vesicles, 779

Auerbach's plexus, 371

Augmentor nerve, 239

Auricles, action of, 189

Axis-cylinder, 92

Basophil, 146

Beef, composition of, 327 
Bezold's ganglion, 234

Bidder's ganglion, 2:34

Bile as an antiseptic, 393

as an excretion, 394

as a purgative, 394

discharge of, 394

disposal of, 396

pigments, 391

test for, 392

properties of, 390

Bile salts, 390

test for, 391

secretion of, 394

Bilifulvin, 391

Bilifuscin, 831

Bilihumin, 831

Bilin, 390

Biliprasin, 391, 831

Bilirubin, 391, 831

Biliverdin, 391, 831

Bioplasm, 2

Biuret reaction, 113

Bladder, urinary, 469

Blastema, 2

Blastoderm, 22, 769

Blastosphere, 767

Blind spot, 723

Blood, 129

arterial flow, 213

capillary flow of, 222

carbon dioxile of, 164

chemical composition of, 150

circulation of, 171

in foetus, 804

coagulation of, 131

colorless corpuscles, 145

corpuscles, hyaline, 146

corpuscles, varieties, 146

corpuscles, 140

chemical composition of, 154

development of, 167

enumeration of, 148

red, 140

red. varieties, 142

spieen in formation of, 322

differences between arterial and venous, 165

flow, regulation of, 231

gases of, 155

oxygen of, 156

physical characters, 129
Blood plasma, 150

chemical composition of, 151

plates, 147

pressure, 20.5

proofs of circulation of, 249

quantity of, 130

respiratory changes in, 2\%6

serum, chemical composition of, 1.52

uses of, 170

variations in composition, 164

velocity of flow, 225

venous flow, 224

Blood-vessels, development of, $\% 96$

Blushing, 247

Body, chemical composition of, 110

Bone, 5j

canaliculi of, 57

development of, 60

functions of, 69

growth of, 68

Haversian canals of, 58

lacunæ of, 57,59

marrow, 56

ossification in cartilage, 62

in memibrane, 61

periosteum of, 57

Brain, 581

development of, 807

distinctive characters of human, 625

fore-, 809

gray matter in, $58 \%, 620$

gyri of, 618

hind-, 809

lobes of, 617

mid-, 809

motor areas of, 626

areas of human, 629

areas of monkey's, $62 \tau$

tracts in, 630

relation of different parts, 581

sulci of, 618

weight of, 624

Branchial clefts, $\% 92$

folds, 792

Bronchi, 254

Brownian movement, 3

Brunner's glands, 374

Buffy coat, 133

Bulb, the, 587

centres in, 594 
Bulb, connections with cerebrum and cerebellum, 592

functions of, 593

Bulbus arteriosus, 799

CAecun, 376

Calorimeter, 452

Cane-sugar, 123

Capillaries, development of, 797 structure of, 183

Capillary flow, 222

Carbohydrates, 122, 834

Carbon dioxide in expired air, 271

Cardiac cycle, 193

Cardio-accelerator centres, 595

Cardiogram, 198

Cardiograph, 198

Cardio-inhibitory centre, 240, 595

Carotid gland, 325

Cartilage, 51

development of, 55

functions of, 55

liyaline, 51

white fibro-, 54

yellow elastic, 53

Casein, 120, 311

insoluble calcium-, 120

soluble, 120

Caseinogen, 119, 311

Caudate nucleus, 583, 615

Cell, differences between plant and animal, 17

division of, 13

functions of, 18

nucleus of, 11

reticulum of, 9

structure of, 9

Cells, decay and death of, 28

fixed, 40

functions of, 22

migratory, 41

modes of connection, 27

plasma, 41

shapes of, 26

Cellulose, 338

Centres, sensory, 639

Centrosome, 13

Cerebellum, 583, 643

connection with bulb, 592

functions of, 647

Cerebral ventricles, 583
Cerebrin, 830

Cerebrum, 582, 617

arrangement of parts of, 623

connection with bulb, 592

effects of removal of, 634

functions of, 633

motor areas of cortex, 626

unilateral action of, 636

Cheyne-Stokes breathing, 285, 292

Chlorides, quantitative estimation of, 838

Choletelin, 478

Chondrin, 121

Chordæ tendineæ, 179

Chorda tympani, 342,605

Chorion, 782

villi, 783

Choroidal fissure, 811

Choroid plexus, 583

Chromatin, 12

Chromoplasm, 12

Chromo-proteids, 118

Chromosome, 16

Chyle, 414, 421

corpuscles, 421

Chyme, 361

Cilia, 35

Ciliary motion, 36

Circulation, coronary, 244 effect of respiration on, 287

in brain, 228

in erectile structures, 230

local peculiarities of, 228

of blood, 171

velocity of, 219,225

Claustrum, 615

Coagulation, calcium salts in, 136 conditions affecting, 138 schema of, 132, 137

theories of, 136

Coccygeal gland, 325

Cochlea, 679

Cohnheim's fields, 85

Cold, influence of extreme, 459

Collagen, 55, 120

Collaterals, 98

Colloids, 410

Colon, 376

Color blindness, 736

Color, Hering's theory of, 734 sensations, 734 
Coior, Young's and IIelmholtz's theory of, 734

Colorless corpuscles, 14i and thymus gland, 324

Colostrum, 310 corpuscles, 308

Columna carnex, 179

Column of Burlach, 565 of Goll, 565

(common sensations, 657

('omplemental air, 268

Compound proteids, 118

Concentric corpuscles of Iassall, 324

Conjunctiva, 694

Connective tissues, 40

classification of, 43

cells of, 40

development of, 47

white fibrous, 43

fibrous, development of, $4 \tau$

yellow elastic, 44

Consonants, 549

Contractility of muscle, 505

Cornea, structure of, 695

Corona raliata, 614

Coronary circulation, 244

Corpora cavernosa, 755

dentata, 58\%, 617

geniculata, 617

functions of, 649

quatrigemina, 616

functions of, 649

striata, 61j

functions of, 642

Corpus albicans, 583

callosum, 583

luteum, $\approx 60$

striatum, 583

Corpuscles of Krause, 106

Coughing, 279

Cowper's glands, 755

Cranial nerves, 596

development of, 806

Cranium, development of, 791

Crassamentum, 132

Crula (erebri, 582, 613

Crusta, 613

$$
\text { petrosa, } \% 6
$$

phlogistica, 133

Crypts of Lieberktihn, 373

Crystalloids, 410 ('utaneous sensations, centre for, 642

Cutis vera, 491

Cystin in urine, 481

DAltonisu, 736

Daniel's battery, 508

Decidua reflexa, 785

serotina, 78.5

vera, 785

Defecation, 407

centre, 579

Deglutition, 352

nervous mechanism of, 353

time occupied in, 353

Dendrite, 91

Dental papilla, 78

Depressor nerve, 247

Descemet. membrane of, 696

Deutero-proteose, 362, 382

Development, 22,766

Dextrin, 123

Dextrose, 124

1) iabetes mellitus, 438

Diabetic centre, 596

Diapedesis, 224

Diastole of heart, 189

Dicrotic notch, 219

pulse, 220

wave, 219

1)icrotism, causes of, 221

1)iencephalon, 809

1)iet, effect of albuminoid, 430

carbohyclrate, 434

fatty, 434

proteid, 429

requisites of a normal, 441

tables, 442

variations in, 444

Digestion, 331

in intestines, 379

Diplopia, 738

Direct cell division, 13

Direction, visual estimation of, 732

Dobie's line, 85

Iogiel's cells, 234

Dreams, 638

Du Bois Reymond's induction coil, 509 key, 509

I) uctless glands, 312

Ducts of Cuvier, 803

Ductus arteriosus, 801 
Ductus renosus, 805

Dura mater, 558

Dyspnoa, 285, 292

FAlr, cochlea of, 679

development of, 813

external, 667

internal, 679

membranous labyrinth of, 680

middle, 678

osseous labyrinth of, $6 \% 9$

ossicles of, 678

vestibule of, 679

Edestrine, 117

Egg-albumin, 114

Figgs, chemical composition of, $3: 8$

Eighth nerve, 606

Elastin, 43, 121

Electrodes, 508

non polarizable, 503

Electrotonus, 529

Eleventh nerve, 611

Emission centre, 579

Enamel cap, 79

organ, 76

Enchylema, 9

Endocardiac pressure, 198

Eudocardium, 178

Endomysium, 83

Endoneurium, 97

Endosmometer, 499

Findothelium, 29

Enzymes, 331

Eosinophil, 146

Epencephalon, 809

Epiblast, 22, 7\%1

organs developed from, 788

Epicardium, 172

Epidermis, 28

structure of, 489

Epididymis, 751

Epiglottis, 253

Epimysium, 82

Epinephrin, 317

Epineurium, 97

Epithelial tissues, 28

Epithelium, 29

functions of, 39

simple, 29

stratified, 37

transitional, 37
Erection centre, 580

Ery throblasts, 169

Erythrodextrin, 339

Ethers, 826

Excretion, 460

definition of, 297

Expiration, 264

Expired air, 271

how changes effected in, 275

volume of, 273

External capsule, 615

Extremities, development of, 794

Eye, anatomy of, 793 and the camera, 722

chambers of, 708

chromatic aberration of, 721

development of, 810

movements of, 717

muscles eoncerned in movement, 717

optical axis of, 711

refractive surfaces and media of, 710

spherical aberration of, 720

Eyeball, 694

blood vessels of, 708

iris of, 698

lens of, 699

retina of, 701

structure of ehoroid coat, 697

of cornea, 695

of selerotic coat, 694

Facial nerve, 604

paralysis of, 605

Fuces, composition of, 405

Fallopian tubes, 748

Fasciculus cuneatus, 588

gracilis, 588

of Rolando, 588

solitarius, 607

Fats, 122

digestion of, 381,393

emulsitication of, 383,393

saponification of, 383,393

Fatty acids, 826

Fermentation in intestines, 402

Ferments, 832

Fibres of Remak, 95

Fibrin, 118, 132, 154

ferment, 136

formation of, 133

sources of, 138 
Fibrinogen, 135

Fifth nerve, 599

Fillet, 593, 616, 632

Filtration, 410

Fission, 9

Fixed cells, 40

Fœtal membranes, 779

Food and digestion, 326

effects of deprivation of, 439

of too much, 438

mastication of, 332

salts of, 329

Foods, 326

effects of cooking, 330

inorganic, 326, 329

liquid, 330

nitrogenous, 326

compcsition of, 327

organic, 326

non-nitrogenous, 329

Foramen of Munro, 583

Forced movements, 649

Fore-brain, 585, 809

Fore-gut, 779

Form, estimation of, 732

Fornix, 583

Fourth nerve, 598

Fovea centralis, 701

Galactophorous ducts, 307

Galactose, 125

Gall-bladder, 389

Galvanic currents, 508

Gastric digestion, conditions affecting, 363

products of, 362

time of, 364

juice, 358

chemical compositior of, 351

pepsin of, 359,360

Gelatin, 120

Gelatinous tissue, 46

Gemmation, 9

Genital organs of female, 744

of male, 750

Genito-spinal centre, $5 \% 9$

Germinal matter, 2

Glands, secreting, 302

varieties of, 302

Globulins, 116

Globus pallidus, $61 j$
Glosso-pharyngeal nerve, 607

Glucase, 338, 359, 364, 381, 383

Gluco-nucleo-proteids, 118

Gluco-proteids, 118.

Glucose, 124

tests for, 834

Glucoses, 122, 834

Glycin, 828

Glycogen, 123

destination of, 436

effect of different diets on, 436

formation of, $43 \pi$

source of, 435

Glycogenesis, 435

Glycol acids, 827

Glycosuria, 437

Gmelin's test, 382, 392

Graafian follicles, 74.5

Gramme-calorie, 452

Granulose, 338

Grape sugar, quantitative estimation 835

Guanidin, 829

Guanin, 831

Gullet, 351

Gustatory buds, 349

cells, 349

HAMACHRONOGEN, 162

Hæmacy tometer; 148

Hæmatin, 162

Hæmatochometer, 221

Hæmatoidin, 163

IIæmatoporphyrin, 162

IIæmin, 163

Hæmoglobin, 15\%

action of gases on, 159

derivatives of, 162

estimation of, 160

reduced, 159

Hiemoglobinometer, 161

Hair, structure of, 494

Haversian canals, 58

Heart, 1\%2

action of, 189

automaticity of, 233

capacity of, 178

chambers of, 174

development of, 795

effect of poisons on, 241

of temperature on, 241 
Heart, force of action, 204

frequency of action, 203

impulse of, 196

influence of central nervous system on, 237

of sympathetic on, 239

of vagus on, $23 \%$

metabolism of, 243

morphology of, 798

muscle, 88 properties of, 232

nerves of, 234

origin of nerve-fibres, 239

regulation of force and frequency of contraction, 232

size of, 177

sounds of, 194

structure of, 177

valves of, 178

action of, 190

weight of, 177

work of, 205

IIeart-beat, electrical phenomena of, 243 method of investigating, 241

Heat, animal, 449

influence of extreme, 459

of nervous system on, 457

loss from surface of body, 454

through lungs, 455

-producing tissues, 452

production of body-, 451

regulation of body-, 453

variations in loss of, 453

in production of, 456

Hearing, 676

physiology of, 684

Hemianopsia, 640

Henle's membrane, 45

Henson's disc, 85

Hetero-proteose, 362,382

Hiccough, 279

Hind-brain, 585, 809

Hippocampus minor, 619

Hippuric acid, 477, 828

formation of, 434

Homoiothermal animals, 450

Hyaloplasm, 9

Hydrobilirubin, 392, 478

Hydrocarbons, 826

Hydrochloric acid, combined, 341, 360

test for free, 360
Hypermetropia, 720

Hyperpnoa, 292

Hypoblast, 22, 771

organs developed from, 789

Hypoglossal nerve, 612

Hypoxanthin, 831

ILEO-C.ECAL valve, 376, 378

Income of energy, 444

Indican, 478,832

Indigo, 832

in urine, 478

Indirect cell division, 14

Indol, 403, 832

Indoxyl, 403

Induced electric currents, $\mathbf{5 0 8}$

Induction coil, 508

Inorganic foods, 329

Inosite, 125

Insalivation, 333

Inspiration, 261

forced, 263

muscles of, 262

quiet, 263

Intercellular substance, 27,41

Interlobular veins, 387

Internal capsule, 614, 630

secretions, 312

Intestinal digestion, influence of nervous system on, 404 duration of, 404

secretion, 398

Intestine, large, 376

$$
\text { glands of, } 377
$$

structure of, 377

small, villi of, 375

Intestines, 370

action of bacteria in, 401

digestion in, 379

fermentation in, 402

gases in, 405

movements of, 403

putrefaction in, 403

Invertebratæ, 825

Invertin, 398

Iodothyrin, 314

Iris, 698

Island of Reil, 618

JUDGMENTS, 659

KARYOKINESIS, 14 
Karyoplasm, 12

Katabolism, 7

Katacratic waves, 219

Katelectrotonus, 530

Kathode, 531

Keratin, 122

Kidneys, blood-vessels of, 46.5

loop of Henle of, 463

Malpighian bodies of, 461

nerves of, 468

structure and functions of, 460

tubuli uriniferi of, 461

vasa efferentia of, 467

vasa recta of, 467

Kilogramme-calorie, 462

Krause's membrane, 86

Kreatin, 829

Kreatinin, 829

formation of, 434

in urine, 479

Kymograph, 208

LACirYMAL gland, 694

Lact-albumin, 311

Lacteal, 375,422

Lactiferous ducts, 307

Lacto-globulin, 311

Lactose, 124, 311

Lævulose, 124

Laminæ dorsalis, $;$;

Large intestine, $3 r 6$

summary of digestion in, 400

Laryngoscope, $\check{5} 41$

Larynx, 253, 536

anatomy of, 537

Laughing, 281

Lecithin, 830

Leguminous fruits, 329

Lenticular nucleus, 615

Leucin, 829

Leucocytes, 145

Lieberkühn's jelly, 116

Life, phenomena of, 1

Linin, 12

Lipochromes, 832

Liquid foods, 330

Liquor sanguinis, 129

Liver, 385

development of, 815

functions of, 389

internal secretion of, 318
Liver, structure of, 385

Locus cæruleus, 587

niger, 614

Lungs and pleuræ, 256

blood supply of, 260

development of, 817

lymphatics of, 261

nerves of, 261

structure of, 256

Luxus consumption, 430

Lymph, 421

capillaries, 412

origin of, 414

chemical composition of, 422

flow, 416

heart, 417

quantity of, 422

Lymphatic glands, 417

system. 412

tissue, 46

Lymphatics, 423

communications of, 415

Lymphocyte, 146

Lymphoid tissue, 46

Malpighax bodies, 461

Maltose, 124

Mammary glands, 306 areola of, 308

Manometer, 207

Mastication, 332 muscles of, 333 nervous mechanism of, 333

Mastoid cells, 677

Meconium, 394

Medulla oblongata, 582, 587 functions of, 593

Medullary folds, 774 groove, 774 plate, $7 \% 4$

sheath, 92

Meissner's plexus, 371

Melanin, 832

Membrana decidua, 784

propria, 27 tympani, $67 \%$

Menstrual discharge, 759

Menstruation, 757

Mesencephalon, 809

Mesoblast, 22, 771, 773

organs developed from, 789 
Mesoblastic somites, 776

Metabolism, 7 nutrition and diet, 427

Metaphases, 16

Metencephalon, 809

Methæmoglobin, 160

Micturition, 488 centre, 579

Mid-brain, 585, 809

Milk, 309

as a food, 328

chemical composition of, 310,328

coagulation of, 310

digestion of, 363

globules, 309

salts of, 311

Millon's reaction, 113

Mineral foods, 329

Mitosis, 14

Motor oculi nerve, 597

Mouth, description of, 332

Movement, visual estimation of, 733

Mucin, 118

Mucous membranes, 300

Mucus, 301

in urine, 479

Murexide test, 477

Muscle at rest, 502

blood supply of, 89

-caskets theory, 85

chemical composition of, 500

-clot, 500

conditions affecting irritability, 505

contractility of, 505

currents, 502, 504

negative variation of, 516

of action, 516

of rest, 505

curve, 513

effect of blood supply on, 505

of disuse on, 506

of separation from the nervous system on, 506

of single induction shock on, 512

of temperature on, 507

of use on, 506

fatigue of, 506,519

heart, 88

in activity, 505

natural currents, 505
Muscle-nerve physiology, 500

plain, 81

plasma, 500

reticulum theory, 86

serum, 500

sound, 514

stimuli of, 507

striated, 82

Muscles, action of involuntary, 52\% of voluntary, 522

as heat producers, 452

centre for tone of, 570

Muscular contraction, accompaniments of, 514

conditions affecting character of, 516

differences between voluntary and involuntary, $\tilde{5} 19$

latent period of, 513

phenomena of, 507

record of, 510

single, 513

stage of contraction, 513

stage of elastic after-vibration, 514

stage of elongation, 513

coördination centres for, 638

metabolism, 533

sensations, centre for, 642

sense, 665

tissue, 81

work, 518

Musculi papillares, 179

Mutton, composition of, 327

Myelin sheath, 92

Myelocyte, 146

Myeloplaxe, 56

Myograph, pendulum, 512

Myohæmatin, 501

Myopia, 719

Myosin, 116

ferment, 501

Myхœdema, 314

NaILs, structure of, 495

Native albumins, 114

Neural canal, 774

Neuraxon, 91

Neurenteric canal, 778

Neurilemma, 93

Neuroglia, 107 
Neuron, 91

Neutrophil, 146

Nerve-cells, 99

Nerve-centres, automatism of, 558

functions of, 555

inhibition and augmentation of, 558

Nerve collaterals, 98

-corpuscles, 93

effect of constant current on, 528

electrotonus in, 529

-fibres, 91

functions of, 552

medullated, 91

impulse, velocity of, $5 \tilde{5} 4$

plexuses, 99

stimuli, 528

terminations, 102

trunks, 97

Nerves, effect of battery currents on human, 531

electrical currents in, $52 \%$

vaso-motor, 246

Nervous metabolism, 534

system, 5 52

cerebro-spinal, 554

development of, 806

Neuro-keratin, 122

Ninth nerve, 607

Nitrogenous bases, 828

Nitrogenous equilibrium, 429

Nodes of Ranvier, 95

Nœud vital, 282

Normal saline solution, 35, 134

Nose, development of, 813

Notochord, 774

Nuclear matrix, 13

Nucleic acid, 119

Nuclein bases, 119, 828

Nucleins, 119

Nucleoli, 12

Nucleo-proteids, 119

Nucleus, 11

ambiguus, 607

of Pander, 771

structure of, 12

ODONTOBLASTS, 72

Esophagus, 351

Oils, 122

Olfactory bulb, 671

centre, 641
Olfactory tract, 641

Olivary bodies, 587

Omphalo-mesenteric duct, 778

Oncograph, 483

Oncometer, 483

Ophthalmoscope, 725

Optic centre, 639

lobes, 649

thalami, 583, 616

functions of, 642

Optical apparatus, 709

anatomy of, 693

defects of, 718

Organ of Corti, 683

Organic chemical substances, 826 substances, 111

Organized ferments, 401

Organs, development of, 788

Osmosis, 408

Osseous labyrinth, 679

Osteoclasts, 65

Osteogenetic fibres, 61

Output of energy, 444

Ovaries, 744

Oviducts, 748

Orum, 746

clianges in, 766

following impregnation, $76 \%$

prior to impregnation, 766

Oxygen in expired air, 273

Oxyhæmoglobin, 158

Oxyntic cells, $3 \tilde{5} 6$

Oxyphil, 146

Pacinian corpuscles, 104

Pain. 661

Pancreas, 379

development of, 815

internal secretion of, 318

structure of, 379

Pancreatic diabetes, 318

juice, 381

conditions favoring action of, 384

functions, 382

nervous mechanism of secretion, 384

Paraglobulin, 117, 135

Parathyroids, 313

Parotid gland, secretion of, 342

Parturition centre, 580 
Penis, 754

Pepsin, 3

action of, 361

functions of, 361

how obtained, 361

- Pepsinogen, 357

Peptone, 117, 361

characteristics of, 362

Perceptions, 659

Perforating fibres of Sharpey, 60

Perfusion canula, 241

Pericardium, 172

Perimysium, 82

Perineurium, 97

Periosteum, 57

Peripheral resistance, 206, 245

Perspiration, 496

Pettenkofer's test, 391

Peyer's patches, 374

Pflüger's law of contractions, 530

Phagocyte, 146

Pharynx, 349

Phenol formed in intestine, 403

Phosphates, quantitative estimation of, 838

Phosphoric acid in urine, 480

Phrenograph, 266

Pia mater, 5̃59

Pineal gland, 325

Pigments, 831

Pituitary body, 317 development of, 791

Placenta, 785 formation of, 784

Plasma, 129, 150

cells, 41

salted, 134

Plasmine, 134

Pleura, 257

Pneumogastric nerve, 608

Pneumograph, 265

Poikilothermal animals, 450

Polar cell, 766

Pons Varolii, 582, 612

Pork, composition of, 327

Post-dicrotic wave, 219

Potassium indoxyl sulphate, 832

Poultry, composition of, 327

Predicrotic wave, 219

Presbyopia, 722

Pressor nerves, 246
Primary areolx, 64

Primitive groove, 772

streak, 772

Pro-nucleus, female, 754

Prophases, 15

Prosencephalon, 808

Prostate gland, 756

Protamines, 827

Protargons, 828

Proteids, 111

chemical reactions of, 112, 11 .

circulating, 430

coagulated, 117

digestion of, 362

floating, 430

morphotic, 430

tissue, 430

varieties, 114

Proteoids, 120

Proteoses, 117, 362, 382

primary, 362,382

reactions of, 363

secondary, 362, 382

Protoplasm, 2

chemistry of, 3

definition of, 3

growth of, 7

irritability of, 6

movement of, 4

properties of, 3

reproduction of, 8

stimuli of, 6

- vital characteristics of, 4

Proto-proteose, 362, 382

Protovertebræ, $\mathbf{7 7 6}$

Pseudoscope, 743

Pseudo-stomata, 32

Ptyalin, 338

action of, 339

Pulse, 206, 215

Pulvinar, 616

Pupil, 699

movements of, 717

Purin base, 119 nucleus, 119, 828

Purkinje's cell, 644 figures, 723

Putamen, 615

Putrefaction in intestines, 403

REACTION of degeneration, 532 
Recurrent sensibility, $5 \approx 2$

Red corpuscles, action of reagents on, 142

destruction of, in spleen, $: 322$

formation in spleen, 322

varieties, 142

Red nucleus, 587

Reflex action, 5ij

inhibition of, $5 \% 7$

morbid, $5 \% 8$

relation of stimulus to, 556

Reflexes, cutaneous, 576

Remak's fibres, 95

ganglion, $2: 34$

Rennin, 363

Reproductive organs, 744

Reserve air, 268

Resiclual air, 268

Respiration, 251

effect of altitude on, 296

effect on circulation, 287

effect of various gases on, 295

effect of vitiated air on, 287

influence of general sensory nerves on, 284

of glosso-pharyngeal nerves on, 284

of superior laryngeal nerve on, 283

of vagi on, 283

inspiration, 261

mechanism of, 261

movements of vocal cords in, .541

nervous apparatus of, 281

rhythm of, 267

special acts, 278

Respirations, number of, 269

Respiratory apparatus, 252

capacity, 268

conditions affecting, 269

centre, 282

automatic action of, 284

method of stimulating, 28.5

centres, 595

changes in air breathed, $2 \approx 1$

in the blood, 276

in the tissues, 277

movements, methods of recording, 265

of nostrils and glottis, 267

murmur, 267
Respiratory muscles, force of, $: 269$

quotient, 224

Restiform body, 590

Retiform tissue, 46

Retina, $\approx 01$

cones of, $\pi 03$

excitation of, $i 2: 3$

layers of, 702

reciprocal artion of parts of, $\tau 36$

rods of, $\pi 03$

Rheoscopic frog, $52 \tau$

Rhythmical contratetility, 2:32

Ribs, movements of, in respiration, 262

Rigor mortis of muscle, 521

Rima glottidis, 25:)

Ritter's tetanus, 5.81

Roy's tonometer, 242

Rumning, 52\%

SACCHAROSE, 122, 834

Saliva, 33 i

action of, on starch. 33s

chemical composition of, 337

conditions atfecting action of, 340

nervous mechanism of secretion.

341

properties of, $3: 3 \pi, 3: 38$

ptyalin of, 338

quantity of, 338

rate of sereretion, $: 338$

uses of, 338

Salivary digestion in stomach, 341,360 glands, 333

changes in colls during secretion, 344

development of, 815

nerves of, 336

structure of, 333

varieties of, 335

Sanson's images, 715

Sarcode, 2

Sarcolemma, 83

Sarcomeres, 87

Sarcoplasm, 8.5

Sarcosin, 829

Schiff's test for uric acid, 477

Sebaceous glands, 493

Secreting glands, 302

Secretion, 297

circumstances influencing, 30.j

discharge of, 304 
Secretion, organs and tissues of, 298 process of, 304

Secretions, internal, 312 relations between, 306

Segmentation in chick, 769

Semicircular canals, 679

Semilunes of Heidenhain, 335

Sensations, common, $65 \%$ of color, 734 special, 658

Sense, muscular, 665 of pain, 664 of sight, 693 of smell, $6 \% 0$ of taste, 667 of temperature, 663 of touch, 660 organs, development of, 810

Senses, 657

Sensory centres, 639

septum lucidum, 563

Serous membranes, functions of, 299 in secretion, 298

Serum, 132, 152

-albumin, 115, 152

-globulin, 135, 153

Seventh nerve, 604

Sexual organs, female, physiology of, $75 \%$

male, physiology of, 763

Sighing, 279

Sight, 693

Singing, 280

Sixth nerve, 604

Size (of objects), estimation of, 731

Skatol, 403, 832

Skatoxyl, 403

Skein, 15

Skeletal muscle, 82

Skin, functions of, 496 glands of, 492 papillæ of, 491 structure and functions of, 489

Sleep, 637

Small intestine, 371

glands of, 373

structure of, 371

summary of digestion, 398

Smell, 670

Sneezing, 280

Sniffing, 280
Soaps, 826

Sobbing, 281

Solidity, judgment of, 742

Somatopleure, 775

Somnambulism, 638

Sounds, 691

speaking, 280

Special respiratory acts, 278 sensations, 658

Speech, 549

action of tongue in, 551

Spermatoblasts, 753

Spermatozoa, 763

Sphygmogram, 219

Sphygmograph, 216, 218

Sphygmometer, 218

Spinal accessory nerve, 611

bulb, 582

centres, 578

cord, 560

antero-lateral ascending tract, 567

antero-lateral descending tract, 566

ascending degeneration of, 565

columns of, 565

comma tract, 566

conduction in, 572

course of motor impressions in, 574

course of sensory impressions in, 573

descending degeneration of, 565

development of, 807

direct cerebellar tract, 567

direct pyramidal tract, 566

functions of, 572

gray matter of, 563

peculiarities of different regions, 570

postero-median columu, 566 posterior marginal zone, 567

reflex action in, 575

white matter of, 560

nerve-roots, functions of, 571

nerves, 567

anterior roots, 568

course of fibres of, 568

development of, 806

posterior roots, 569

Spirem, 15 
Spirometer, 268

Splanchnopleura, $7 \% 5$

Spleen, 318

functions of, 321

influence of drugs on, 323

of nervous system on, 323

lobules of, 320

Malpighian corpuscles of, 321

pulp, 320

structure of, 319

Spongioplasm, 9

Stammering, 5.j1

Stannius' experiment, 236

Starch, 123

action of saliva on, 339

formation of, 19

granules, structure of, 338

Starvation, 439

Steapsin, 384

Stercobilin, 392

Stercorin, 397

Stereoscope, 732

Stethograph, 265

Stethometer, 265

Stokes' fluid, 159

Stomach, 3.54

blood-vessels of, 357

changes in gland cells during secretion, 357

digestion of, after death, 368

gases in, 406

glands of, 356

lymphatics of, 357

movements of, 364

nerves of, 358

nervous control of secretion, 367

influence on movements, 366

structure of, 354

Stratum intermedium of Hannover, 79

Striated muscle, 82

Stromuhr, Ludwig's, 226

Sublobular veins, 387

Submaxillary gland, action of atropine on, 343

paralytic secretion, 343

secretion of, 342

Succus entericus, 398

Sucking, 281

Sudoriferous glands, 492

Sugar, test for, 340

Suprarenal capsules, 314
Suprarenal capsules and Addison's disease, 317

functions of, 316

nerves of, 315

structure of, 314

Swallowing, 352

Sweat, 496

chemical composition of, 497

glands, 49:

influence of nervous system on secretion, 498

Sympathetic ganglia, functions and structure, 6.5.

nervous system, 650

functions of, 653

Synovial fluid, 300

membranes, 298

Systole of heart, 189

Tactile corpuscles, 105

menisques, 107

Taste, 667

after-, 669

centre, 641

-goblets, 349

varieties of, 668

Taurin, 829

Tecth, 69

dentine of, $; 3$

development of, $\approx 6$

enamel of, i.j

permanenț, $\tau_{0}$

structure of, 72

temporary, $\sigma_{0}$

wislom. 71

Tegmentum, 613

Telophases, 17

Temperature, regulation of body, 45:3

sense of, 663

variations in body, 449

Tenth nerve, 608

Testes, $\%$. 1

Testicles, descent into scrotum, 820

Tetanus, 518

Ritter's, 531

Thalameneephalon, 809

Third eye, 325

nerve, 597

Thoracic duct, 413

Thorax, respiratory changes in diamet'r', 262 
Thrombin, 136

Thymus gland, 3\%4

Thyroid gland, 312

function of, 314

Thyroids, accessory, 313

Tidal air, 268

Tissue elements, derived, 27

Tissues, connective, 40

elementary, 26

respiratory changes in, $27 \%$

Tongue, epithelium of, 348

papillæ of, 347

structure of, 346

Tonometer, 242

Tonsils, 350

Touch corpuscles, 105

sense of, 660

Trachea, 254

Tract of Gowers and Tooth, 567 of Lissauer, $\tilde{567}$

Traube-Hering curves, 292

Trigeminus, 599

Trochlearis, 598

Trypsin, 382, 833 action of, 382,383

Tubuli seminiferi, 752 uriniferi, 461

Twelfth nerve, 612

Tympanum, 677

Tyrosin, 830

Umbilical vesicle, 779

Unstriped muscle, 81

Urea, biuret reaction, 474

chemical nature, 474

formation of, 433

in the urine, 472

properties of, 473

quantitative estimation of, 475

variations in quantity excreted, 474

Uretèrs, 468

Ureters, structure of, 468

Urethra, 755

Uric acid, 475, 830

condition of, in urine, 476

formation of, 433

properties of, 475

tests for, 476

variations in quantity of, 476

Urina cibi, 472
Urina potus, 472

sanguinis, 472

Urinary bladder, 469

Urine, 469

abnormal constituents of, 472

average daily quantity of constituents, 471

chemical composition, 469

chlorine in, 481

colored by medicines, 478

cystine in, 481

extractives of, 479

filtration theory of secretion, 482

gases in, 482

hippuric acid in, 477

indican in, 478, 832

indigo in, 478, 832

kreatinin in, 479

method of secretion, 482

mucus in, 479

passage of, into bladder, 488

pigments of, $47 \%$

phosphoric acid in, 480

physical properties of, 469

quantity of, 472

reaction of, 470

relation of secretion to arterial pressure, 485

saline matter in, 479

secretion theory, 486

solids of, 472

sulphuric acid in, 479

urea in, 472

uric acid of, 475

variations in specific gravity, 471

xanthin in, 479

Urobilin, 392, 47\%, 831

Urochrome, 477, 831

Uroëry thrin, 478, 832

Uromelanin, 478

Uterus, 749

Vagina, 750

Vagus nerve, 608 effects of section, 610

functions of, 609

Valvulæ conniventes, 372

Vascular system, development of, 795

Vas deferens, 751

Vaso-constrictor nerves, 246

Vaso-dilator nerves, 246 
Vaso-motor centres, 246,595

nerves, 246

course of, 248

reflexes, 246

Veins, 186

development of, 802

valves of, 187

Velum interpositum, 583

Venous flow, 224

Ventilation, 287

Ventricles of heart, action of, 189

Ventriloquism, 551

Vernix caseosa, 497

Vertebral columns, development of, 789 plate, 775

Vertebratæ, 825

Vesico-spinal centre, 579

Vesiculæ seminales, 753

Vesicular breathing, 267

Villi, 372

Visceral arches, 792

clefts, 792

folds, 792

Vision, accommodation of, 713

binocular, 738

field of, 730

mechanism of accommodation, 715

range of distinct, 716

reversion of image, 728

Visual axis, 712
Visual centre, 639 judgments, 728 perceptions, 728 purple, 727

sensations, 723 duration of, 724 intensity of, 724

Vital capacity, 268

Vitellin, 120

Vitelline duct, $\approx$ \%

Vocal cords, movements of, 544

Voice, 536

difference between male and female, 536

in singing and speaking, 545

Vomiting, 369

Vowels, j49

WALKING, 524

Wallerian degeneration, 565

Wharton's jelly, 46

Wolffian bodies, 817

Xanthis, 831

base, 119

in urine, $4 \pi 9$

Xantho-proteic reaction, 113

YAWNING, 281

Yolk-sac, 789

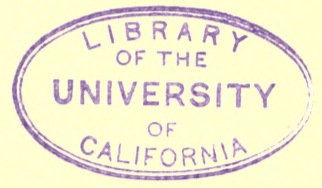






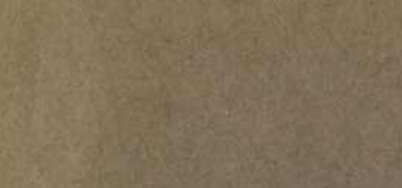

H.

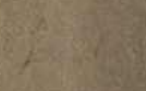

(⿻)

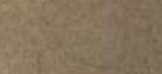

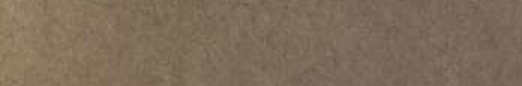

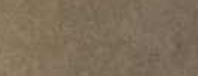

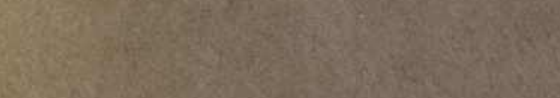

(3)

sis

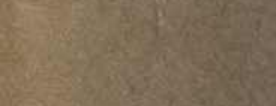

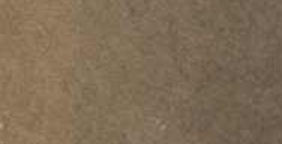

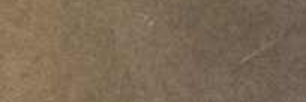

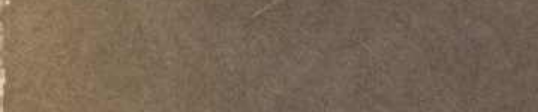

sing

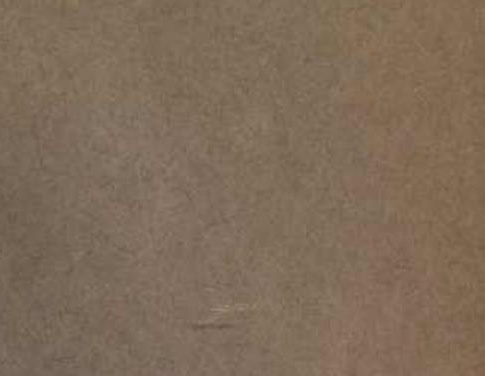

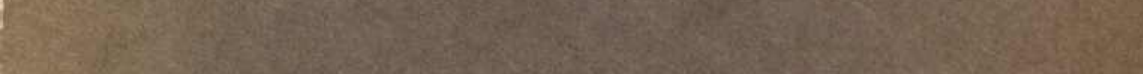

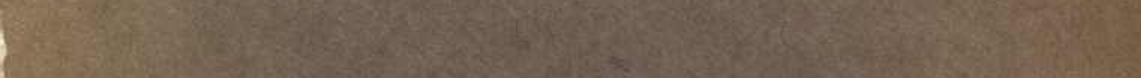

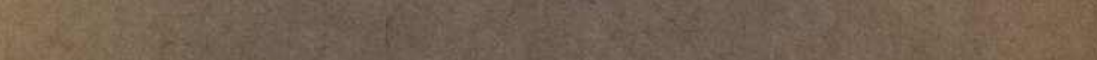

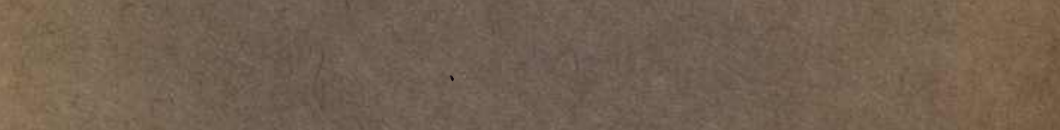

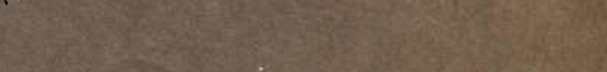

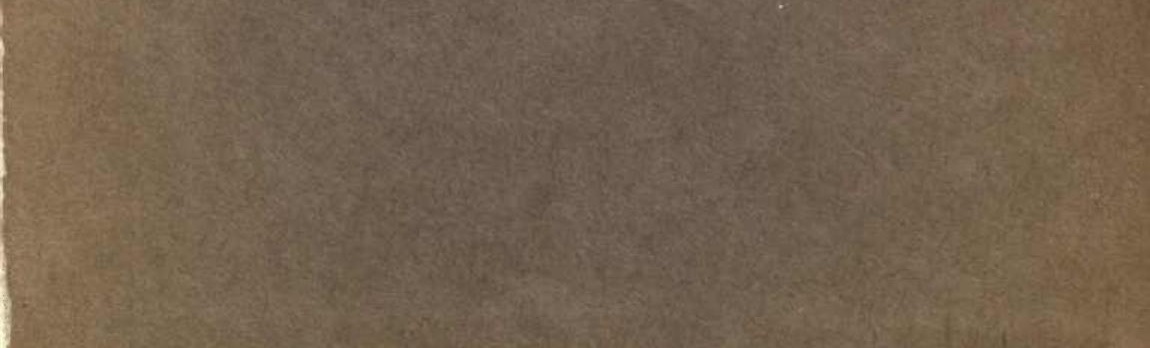


THIS BOOK IS DUE ON THE IAST DATE STAMPED BELOW

AN INITIAL FINE OF 25 CENTS WILL BE ASSESSED FOR FAILURE TO RETURN THIS BOOK ON THE DATE DUE. THE PENALTY WILL INCREASE TO 50 CENTS ON THE FOURTH DAY AND TO $\$ 1.00$ ON THE SEVENTH DAY OVERDUE.

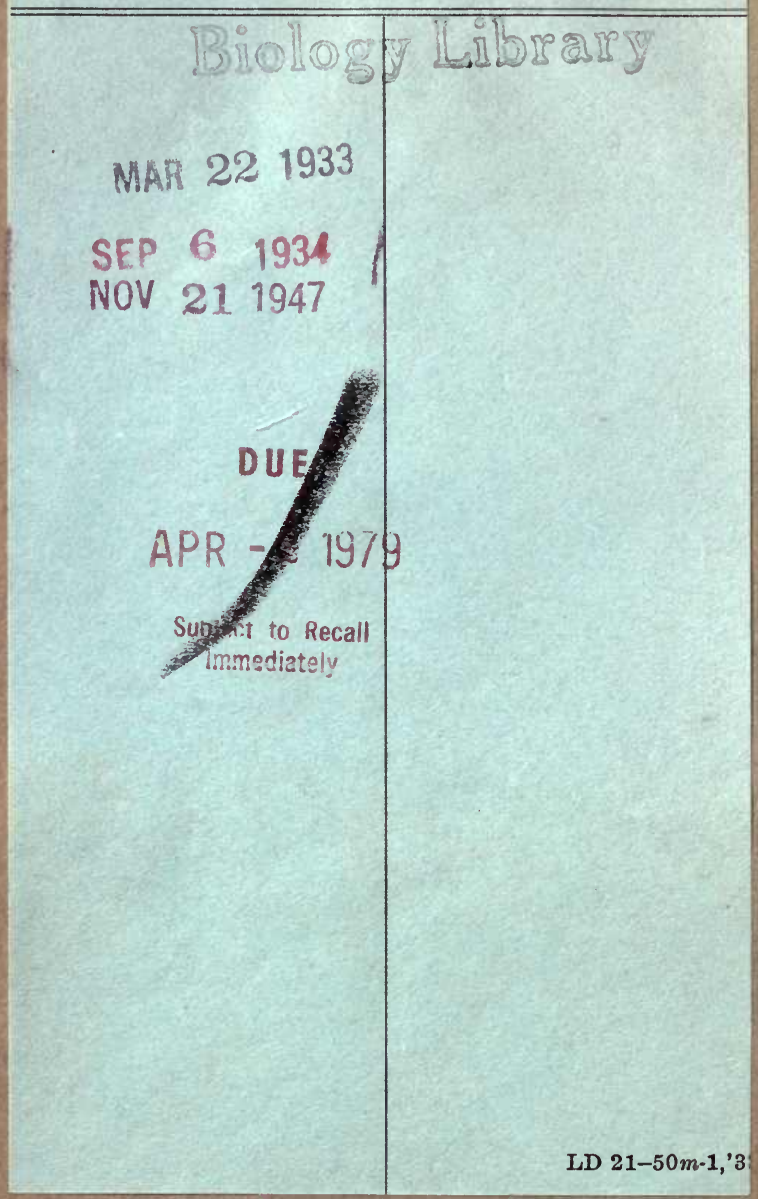



